\title{
SUPPORTING DOCUMENT FOR THE HISTORICAL TANK CONTENT ESTIMATE FOR
}

\author{
A TANK FARM
}

\section{WORK ORDER ER4945}

\author{
Prepared for \\ Westinghouse Hanford Company
}

Jume 1994

\section{DISCLAIMER}

\begin{abstract}
This report was prepared as an account of work sponsored by an aponcy of the Uhited Stales Govemment. Neither the Unfed Stales Government nor any aptency thased, por any of thetr employat, makes any wartanty, express or implied, or asomes any kgal liability or responsibility for the accuracy, compleleness, or usefulness of any information, apparatus, prodoct, or proces dieclosed, or represtals that is use would not infinge privately awned righis Reference berein to any specific conmercilal peoduct, peocest, or service by trado name, 1rodemark. manplectorer, or otheturise dow pot pocessirily comstilute or fmply its endocsement, tecommendation, or favwing by the Uniled Strites Governmeal of any agency thereot. The rem and optaions of authors expressed bereip do not necessarily state at reflect those of the United States Goworomerh or ang genticy thereof.
\end{abstract}

\section{Prepared by}

\section{ICF Kaiser Hanfori Company \\ Richland, Washington}




\section{DISCLAIMER}

Portions of this document may be illegible in electronic image products. Images are produced from the best available original document. 


\section{DISTRIBUTION SHEET}

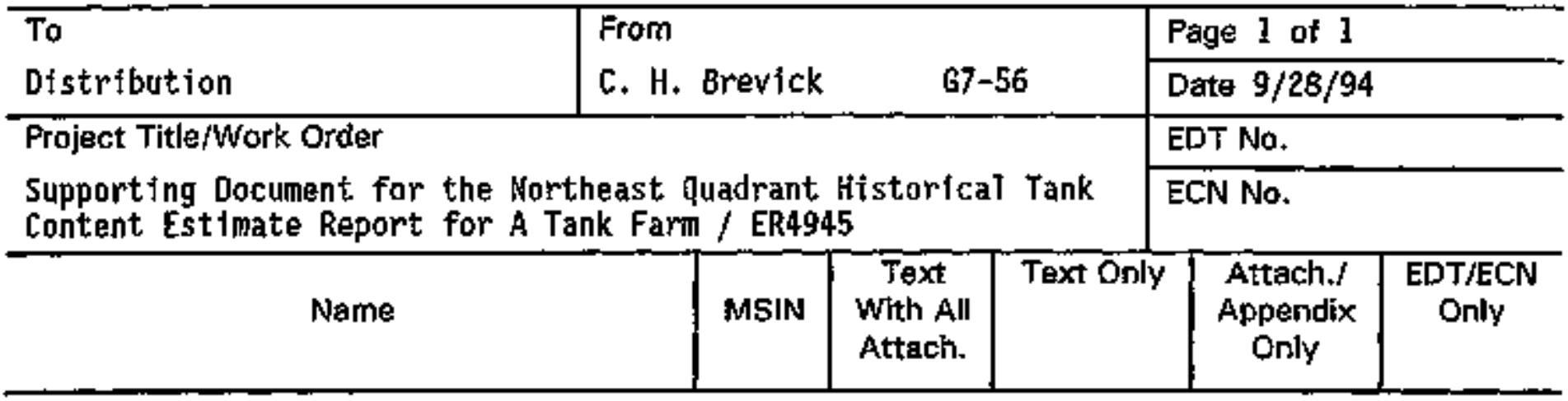

Westinghouse Hanford Company

T. H. Brown

$G$. Jansen

P. Sathyanarayana

B. C. Stmpson -

TFIC (Tank Farm Information Center)

Central files

OSTI

$\begin{array}{ll}R 2-12 & X \\ H 6-33 & X \\ R 2-12 & X(2) \\ R 2-12 & X \\ R 1-20 & X \\ L 8-04 & X(2) \\ L 8-07 & X(2)\end{array}$

ICF-Kaiser Hanford Company

c. H. Brevick

L. A. Gaddis

M. W. Hoffmann

E. D. Johnson

R. L. Newel1

H. W. Pickett

A. C. Valsh

ICF-KH Publications

ICF-KH DOC Control

$\begin{array}{ll}67-56 & X \\ 67-57 & X \\ 52-54 & X \\ 67-57 & X \\ 66-46 & X \\ 67-57 & X \\ 67-57 & X \\ E 6-63 & 0 \\ 63-11 & X\end{array}$

$X$

$x$

$x$

$x$

$x$

$\stackrel{0}{x}$

\section{$x$}

Department of Energy - Richland_Operations

J. M. Clark

Los A] amos Nationa? Laboratory

5. F. Agnew

Los Alamos Mationat Laboratory

MS-J586 - CST-14

P.0. Box 1663

Los Alamos, NM 87545.

Los Al amos Technical Associates

D. S. Delorenzo

Los Alamos Technical Associates

750 Swift, Suite 4

Richland, WA 99352
$X(2)$

$X$ 
2. Ta; (Recelving Organization)

THARS

5, Proj./Prog./Dept,/0tv, : ER4945

8. Originator Remerks:

Supporting Document produced under W.0. ER4945, provides supporting data and in-depth characterization information for ME Quadrant Historical Tank Content Estimate Report for A-Tank Farm. For original release.

11. Receiver Remerks:
3. Froar toriginating organization) ICF Kalser Hanforf

6. cog. Engr.:

L.A. Gaddis

4. Related EDT *o,:

7. Purchase Order Mo.:

9. Equip./Component Hi.: $N / A$

10. Systen/gtde./Factlty:

Tank Farms

12. Hejor Assm. Ung. Ho, N/A

13. Pemit/Permit Application ho.; N/A

14. Requited Response oate:

$N / A$

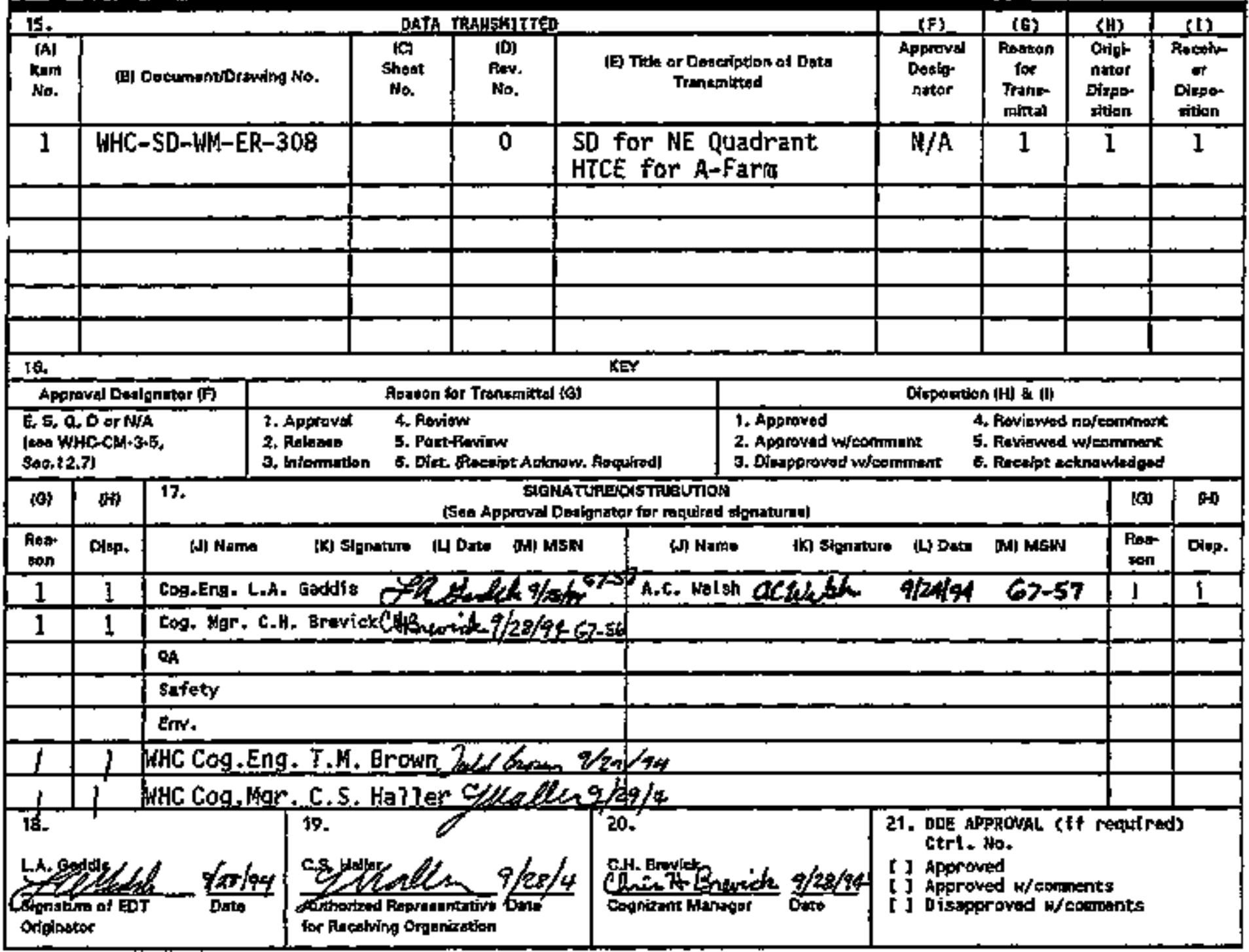

(00-7400-172-2 (04/94) GEF 097 
2. Title

Supporting Document for Northeast Quadrant Historical Tank Content Estimate Report for A-Tank Farm.

5. Key words

A-Tank Farm, Tank Waste Characterization, Historical Tank Content Estimate, Histortcal Waste Transfer, tank waste Tevel data, tank physica? information, drywe11, Ijquid observation wel1, waste zemperature, waste sampling data, analyticai data.

\section{APPROVED FOR}

3. Numbet

WHC-SD-WM-ER-308

4. Rat Ha.

0

\section{tros $\% / 28 / 94$ PUBLIC RELEASE}

\section{Huthor}

Xant: Brevick, C.H.

L.A. Gaddis, A.C. Walsh

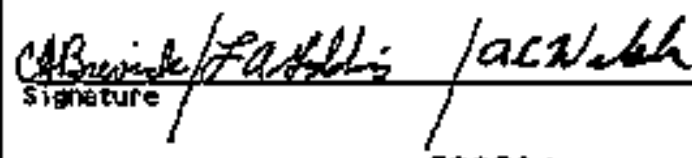

Organizatiah/Charge Codn $58250 /$

ER4945/Tank Farm Technical

Support

\section{Abstract -}

This Supporting Document provides historical in-depth characterization information gathered on A-Tank Farm, such as historical waste transfer and level data, tank physica] information, temperature data, sampling data, and dry well and liquid observation well data for Historical Tank Content. Estimate Report for the HE Quadrant and the Hanford 200 East Areas.

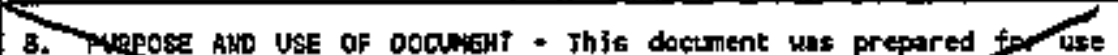

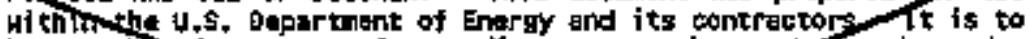
be used paly to parform, direct, of integryte work under U.S. Departmentes Energy contracts. Th5s dogynitit Is not approved for putic retease uncil rewiowed.

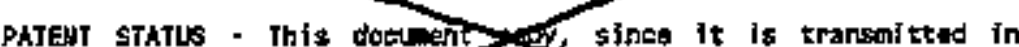

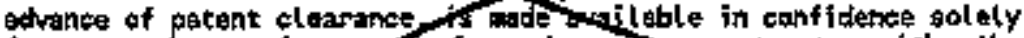
for use in perfornapot of work urbem contracts with the U.S. Departant of Er.ifgy. This doctment is notwa pe published nor its contente ontsfikiso disseningted or used for purposengother then epecified coove before outent approvat far stuch relesse ormug has

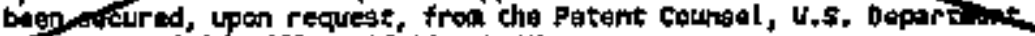
of Energy field off Jee, Richland, Wh.

DISCLALAER * This report was propaced as an account of work sponsored by en agency of the tiril ted states fovermint. Hoittur the inlited states Governoent nor any egenty thereaf, nar any of thair encloyees, nor any of their conterectors, stexeontrectors of their enployes, makes ary warranty, express or Impl iet, or sosume any logel liablitity or respons foflity for the acturacy, completeness or any thind party's wee or the resul ts of such use of any inforfation. apparatus, product, or proftiss disclosed, or repcesents that its use woutd not infeinge privateir ownd rights. Referescte herein to any

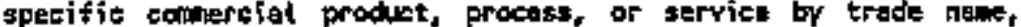
tradenark, manufacturer, or otherifise, does not necessarily canetitute or feply its endersenant, racomentation, or fovoring by the thifed States Goverment or any agtency therest of its contractors or stubcontractors. The views and oplatons of authors expressed horoin do not nectssarily state or reflect those of the

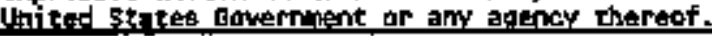

9. Inpart Leval N/A

to. RELEMSE STAMP

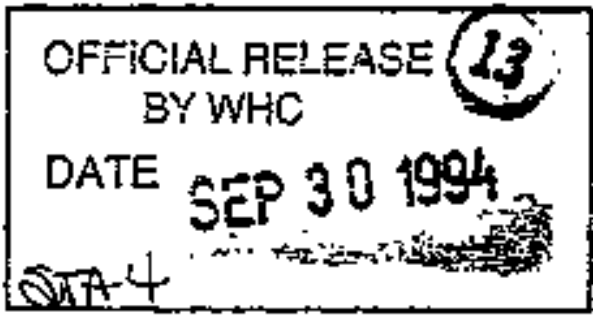




\section{RELEASE AUTHORIZATION}

\begin{tabular}{|ll}
\hline Document Number: & WHC-SD-WM-ER-308, REV.O \\
\hline Document Tite: & $\begin{array}{l}\text { Supporting Document for the Northeast Quadrant } \\
\text { Historical Tank Content Est imate Report for A-Tank } \\
\text { Farm }\end{array}$
\end{tabular}

Release Date:

September 28, 1994

This document was reviewed following the procedures described in WHC-CM-3-4 and is:

APPROVED FOR PUBLIC RELEASE

WHC Information Release Administration Specialist:

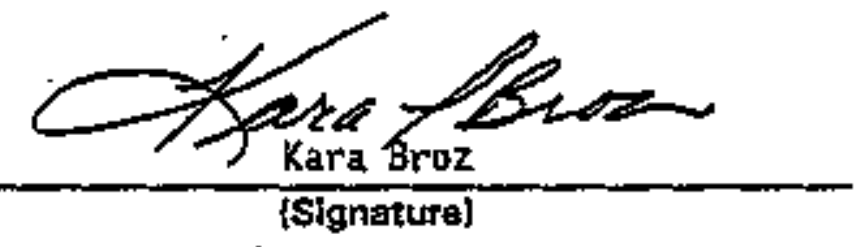




\title{
SUPPORTING DOCUMENT FOR THE HISTORICAL TANK CONTENT ESTIMATE FOR
}

\author{
A TANK FARM
}

WORK ORDER ER4945

\section{Prepared by}
ICF Kaiser Hanford Company
Richland, Washington

for

\author{
Westinghouse Hanford Company
}

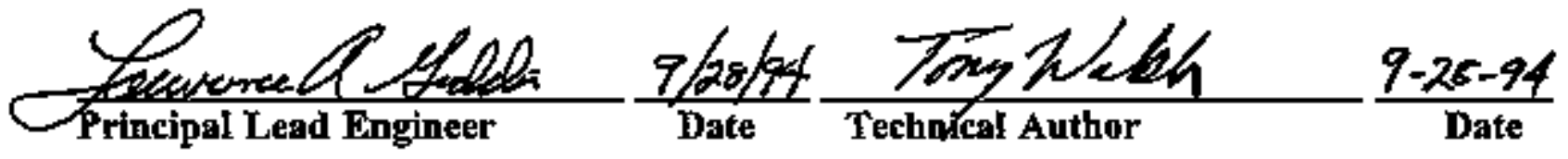

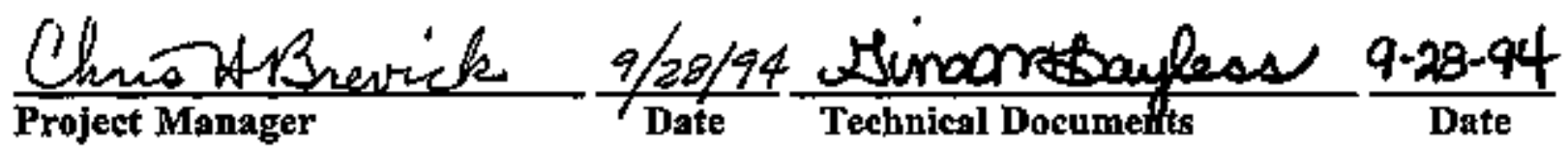

Westinghouse Hanford Company

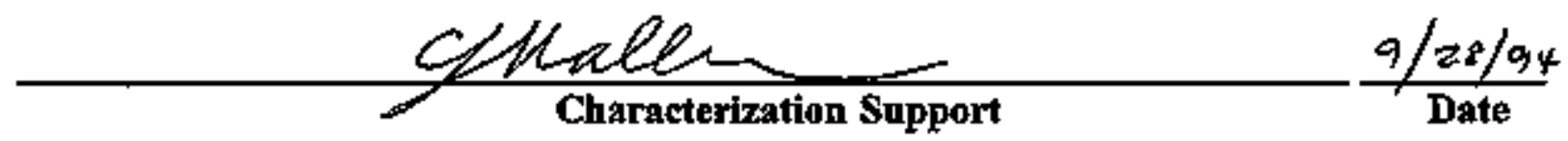


TABLE OF CONTENTS

1.0 Introduction ................... 1

1.1 Purpose . . . . . . . . . . . . . . . . . . 1

1.2 Scope . . . . . . . . . . . . . . . . . . . . . - 1

1.3 Approach . . . . . . . . . . . . . . . 1

1.3.1 Surveillance Techniques for the Hanford

Radioactive Mixed Waste Storage Tanks . . 3

1.3..2 Approach and Development of supporting Information Contained in the Historical

1.3.3 Approach and Development of the Process-

Approach and Development of the Process-

1.3.4 Assumptions... . . . . . . . . . . 10

1.4 Historical Information Quality . . . . . . . . . 13

1.5 Background $\mathrm{NE}$ Quadrant . . . . . . . . . . . 16

1.5.1 Waste Generating Processes . . . . . . 17

1.5.2 Waste Management Operations ........ 21

1.5.3 Miscellaneous Waste Sources and

Equipment ................. 23

1.6 Safety Issues $\begin{aligned} & 1.5 .4 \\ & \text { Timeline }\end{aligned}$

1.6.1 Watch List Safety Issues ........ 27

1.6.2 Non-Watch List Safety Issues . . . . . 28

2.0 A Tank Farm . . . . . . . . . . . . . . . . 31

2.0.1 A Tank Farm History . . . . . . . . 31

2.0.1.1 A Tank Farm Waste History . . . . 31

2.0.1.2 A Tank Parm Temperature History : . 32

2.0.1.3 A Tank Farm Integrity . . . . . 32

2.0.1.4 A Tank Farm Historical Sample., 33

2.0.2 Current status of A Tank Farm . . . . . 34

2.0.2.1 A Tank Farm In-Tank Photographs . . 34

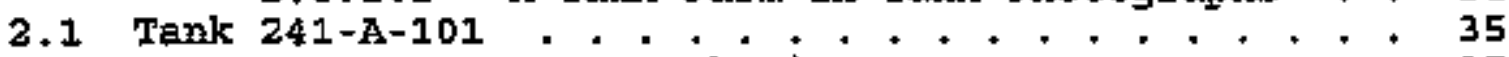

$2.1 .1241-\mathrm{A}-101$ Tank History . . . . . 35

2.1.1.1 Waste History Tank 241-A-101... 35

2.1.1.2 Temperature History 241-A-101 . . . 36

2.1.1.3 Integrity of Tank 241-A-101 . . . 37

2.1.1.4 Historical Sample Analysio Data for Tank 241-A-101....... 37

2.1 .2 Current Status of Tank 241-A-101 . . . 38

2.1.2.1 Waste Layer Volume Approximation

for Tank 241-A-101 . . 38

2.1.2.2 In-Tank Photograph 241-A-101... 38

2.1.3 Synopgis Tank 241-A-101 . . . . . . . . . 39

2.2 Tank 241-A-102 . . . . . . . . . . . . 40

$2.2 .1241-\mathrm{A}-102$ Tank History . . . . . . . 40

2.2 .1 .1 Waste History Tank $24 \hat{1}-\hat{A}-102 . \cdots 40$

2.2.1.2 Temperature History 241-A-102 *. . 41

2.2.1.3 Integrity of Tank 241-A-102 ... . 41

2.2.1.4 Histor 1 cal Sample Analysis Data for

Tank 241-A-102......... 42 
2.2.2 Current Status of Tank 241-A-102

2.2.2.1 Waste Layer Volume Approximation

for Tank 241-A-102 . + + + + . . . . . 42

2.2.2.2 In-Tank Photograph 241+A-102.*. 42

2.3 Tank 241-2ynopsis Tank 241-A-102,***.*.**.. 42

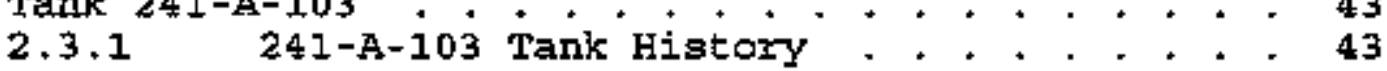

2.3.1.1 Waste History Tank 241-A-103... . . 43

2.3 .1 .2 Temperature History 241-A-103 . . . 44

2.3 .1 .3 Integrity of Tank 241-A-103 . . . . 44

2.3.1.4 Historical Sample Analysis Data for

Tank 241-A-103............ 45

2.3.2 Current Status of Tank 241-A-103 . +. . 46

2.3.2.1 Waste Layer Volume Approximation

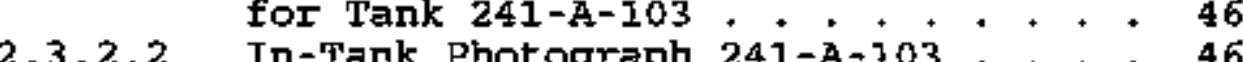

2.3.3 Synopsis Tank 241-A-103.+.+.+.+.+. 46

2.4 Tank 24l-A-104..................... . 47

2.4.1 241-A-104 Tank History . . . . . . . + . 47

2.4.1.I Waste History Tank 241-A-104. . + . 47

2.4 .1 .2 Temperature History $241-\mathrm{A}-104 .+.48$

2.4 .1 .3 Integrity of Tank $241-A-104 .+.+48$

2.4.1.4 Historical Sample Aralysis Data for

Tank 241-A-104..........+. 49

2.4 .2 Current Statas of Tank $243-\mathrm{A}-104++_{+}++^{+} 49$

2.4.2.1 Haste Layer Volume Approximation

for Tank 241-A-104.. . + . . . . . 49

2.4.2.2 In-Tank Photograph 241-A-104. + + . 49

2.4.3 Synopsis Tank 241-Ä-104.......... . 50

2.5 Tank 24l-A-105 . . . . . . . . . . . . . . . . . 51

2.5.1 241-A-105 Tank History . . . . . . . 51

2.5.1.1 Waste History Tank 241-A-105... . 51

2.5.1.2 Temperatire History 241-A-105... 51

2.5.1.3 Integrity of Tank 241-A-105 . . . . 52

2.5.1.4 Historical Sample Analysis Data for

Tank 241-A-105............ 53

2.5.2 Current Status of Tank 241-A-105. . . . 53

2.5.2.1 Waste Layer Volume Approximation

for Tank 241-A-105.......... . 54

2.5 .2 .2 In-Tank Photograph $24 I-A-105 . .2 .54$

2.5.3 Bynopsis Tank 241-A-105. . . . . . . . . 54

2.6 Tank 241-A-106 . . . . . . . + . . . . . . . . . . 55

2.6.1 241-A-106 Tank History . . . . . . . . . 55

2.6 .1 .1 Waste History Tank 241-A-106. . . . 55

2.6 .1 .2 Tenperature History 241-A-106 . . . 56

2.6 .1 .3 Integrity of Tank 241-A-106... . 57

2.6.1.4 Historical Sample Analysis Data for Tark 241-A-106........... 57

2.6 .2 current status of Tank 241-A-106 . . . . 57

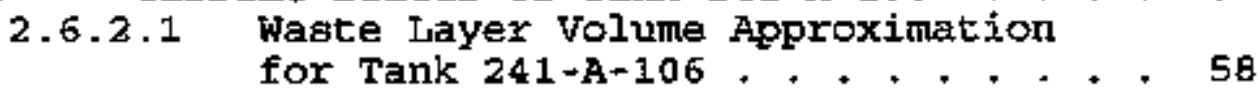

2.6.2.2 In-Tank Photagraph 241-A-106... . 5B

2.6.3 Synopsis Tank 241-A-106......... . 58 
WHC-SD-WM-ER-308, Rev, 0

\section{APPENDICES}

Appendix A. Glossary

Appendi, $\mathrm{B}$. References

Appendix C. Tank Ievel Histories and Data

Appendix D. Temperature Graphs and Data

Appendix E. Surface Level Graphs and Data

Appendix F. Drywell Graphs and Data

Appendix G. Riger Configuration and Tank Cross Section

Appendix H. , Sampling Data

Appendix I. Tank Photographs

Appendix J. Unknown Tank Transfers

Appendix K. Tark Layering Comparison and Data 
HHC-SD-WM-ER-30B, ReV.O

\section{INFORMATION FEEDBACK CARD}

\section{SUPPORTING DOCUMENT FOR THE HISTORICAL TANK CONTENT ESTIMATE FOR A TANK FARM ER4945}

\section{COMMRNTS AND CONTRIBUTIONS}

The reader is requested to utilize this card to comment on this woxking document, report any discrepancies, or contribute new information to improve the accuracy and content of the report. please use the space provided below, add additional pages if necessary, and return to the addressee on the reverse side. 

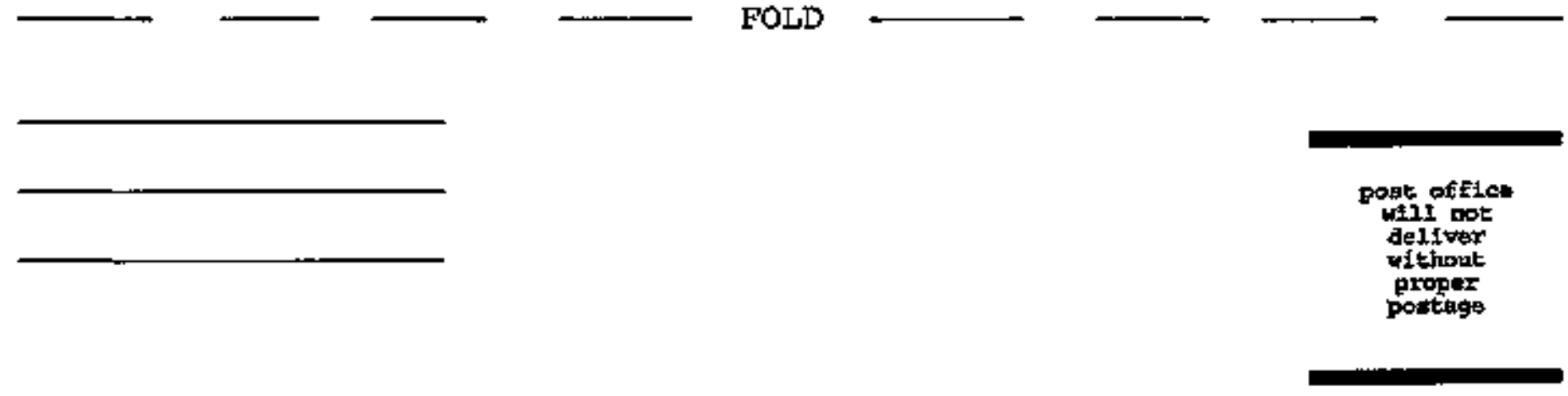

Mr. Carl S. Haller

Manager, Characterization Support

Westinghouge Hanford Company

P.O. Box 1970

Richland, WA 99352 
1.0 Introduction

1.1 Purpose

Thts document provides historical evaluations of the radioactive mixed wastes stored in the Hanford Site 200-East Area underground stingle-shell tanks (SSTs). A Historical Tank Content Estimate has been developed by reviewing the process histories, waste transfer data, and available physical and chemical characterization data from various Department of Enexgy (DOE) and Department of Defense (DOD) contractors.

The historlcal data will supplement information gathered from in-tank core sampling activities that are currently underway. $A$ tank history review that is accompanied by current characterization data creates a complete and reliable inventory estimate. Additionally, historical review of the tanks may reveal anomalies or unusual contents that are critical to characterization and post characterization activities. Complete and accurate tank waste characterizations are critical first steps for DOE and Hestinghouse Hanford Company safety programs, waste pretreatment, and waste retrieval activities.

\subsection{Scope}

The scope of this document is limited to the SSTs in the A Tank Farm of the northeast quadrant of the 200 East Area. For oummarized characterization data on all SST's in the NE Quadrant, refer to the Historical Tank Content Estimate Report Brevick 1994. The northeaet and southeast quadrants represent an approximate geographical division of the 200-East Area tank farms. The diviaion was made as follows:

- NE Quadrant: $\mathrm{A}, \mathrm{AX}, \mathrm{B}, \mathrm{BX}, \mathrm{BY}, \mathrm{C}$

- SE Quadrant: $\mathrm{AN}, \mathrm{AZ}, \mathrm{AP}, \mathrm{AH}, \mathrm{AY}, \mathrm{SY}$

The following map depicts the 200-East Area.

\subsection{Approach}

This report is a compilation of work performed by ICF Kaiser Hanford Company, Los Alamos National Laboratories, and Westinghouse Hanford Company.

Hestinghouse Hanford Company requested that ICF Kaiser Hanford Company review the history of the tanke in each of the four quadrants and incorporate the tank layering models and inventory estimates being developed by Los Alamos National Laboratories into the report. Historical waste transfer and level data, tank physical information, temperature data, sampling data, and drywell and 11quid observation well data have been compiled for this report. 
HHC-SD-WM-ER-308, ReV * 0

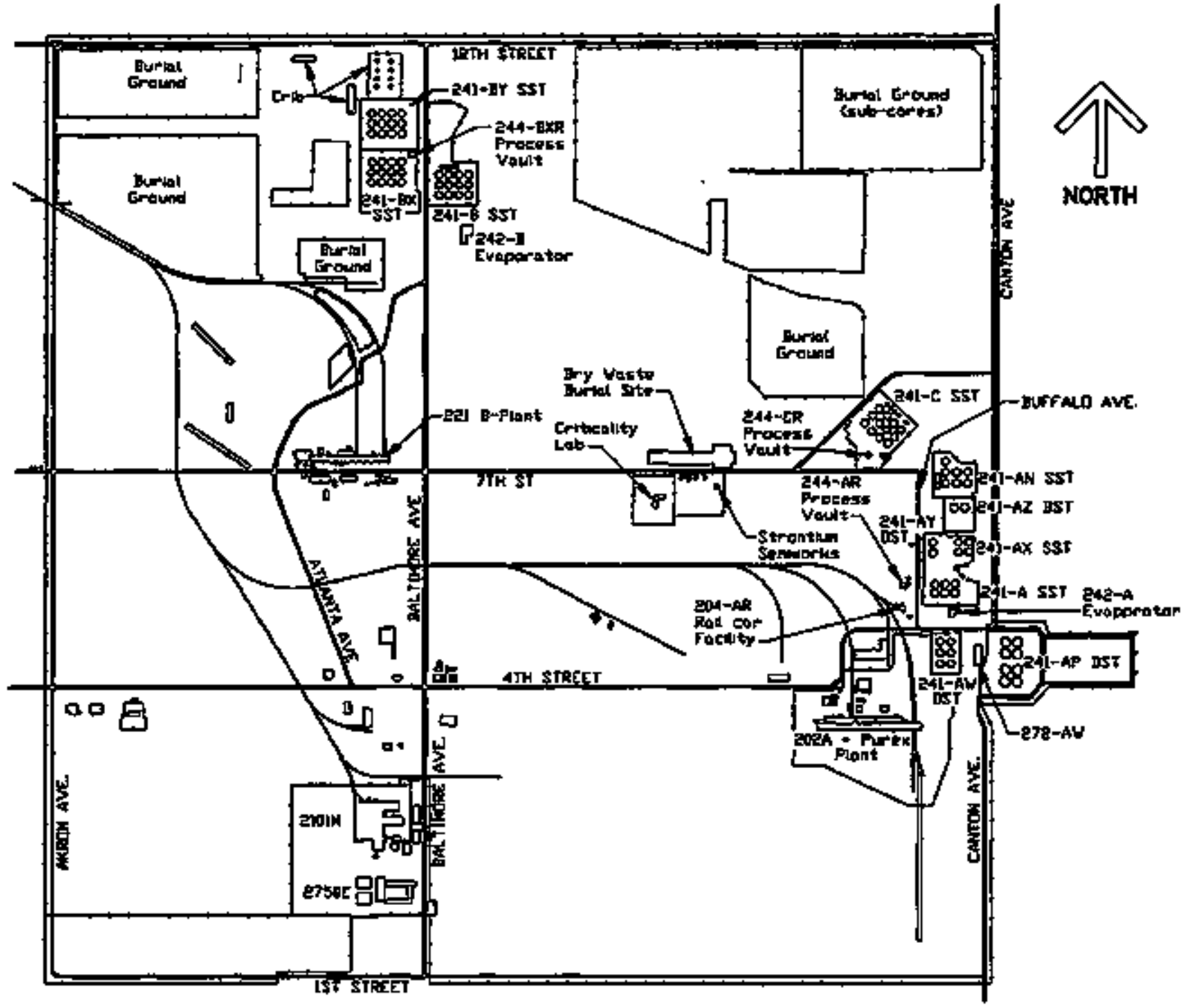

200 East Area

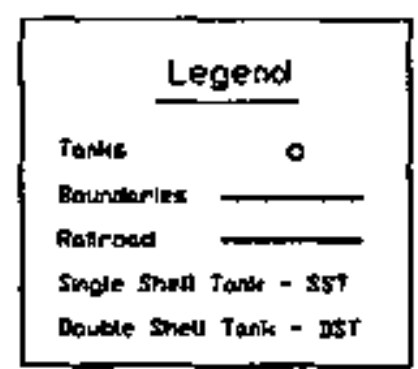




$$
\text { WHC-SD-WM-ER-308, Rev. O }
$$

1.3.1 Surveillance Techniques for the Hanford Radioactive Mixed Waste storage Tanks

Surveillance information is used for tank characterization. Tank integrity (i.e., sourid, assumed leaker, assumed re-leaker) is investigated by comparing the changing liquid levels to liquid observation well data and drywell activity. Haste temperatures can be used to correlate the inventories of heat-generating radionuclides ( ${ }^{137} \mathrm{Cs}$ and ${ }^{91} \mathrm{Sr}$ ) in the tanks. The instrumentation and methods used. for. tank surveillance are described in the following sectiong.

\section{- In-Tank Surface Ievela}

Surface levels within the SSTs are monitored by instruments consisting of calibrated steel tapes with an electrode piummet on the end that will complete a circuit when it contacts a conducting surface (i.e., liquid). The surface level meagurements are usually taken with Food Industry Corporation gauges and manual tapes. The tank monitoring device, manufactured by the Food Industry Corporation, automatically moves up and down and contacts the waste. The gauge is often set to the intrusion mode by placing the plummet a short distance above the waste. If an intrusion occurs in the tank, the Food Industry Corporation gauge will record the event when the surface level rises to meet the plummet. The manual tapes are similar to the Food Industry corporation gauge, except that manual operation of the tape and manual recording of the readings are required. Some tanks contain both types of level monitoring devices. The measurements are recorded on a computer automated surveillance system (CASs).

Intoratitial Iiquid Level (Itquid Observation Wells)

Food Instrument corporation gauges and manual tapes are limited to ourface level measurement; therefore, liquid observation wells in many tanks have been installed specifically to monitor the interstitial liquid level within the SSTs. The interstitial liquid is the liquid trapped within and underneath the solid waste. Fiberglase or TEFZEL-reinforced epoxy-polyester resin liquid observation well tubes (by E.I. du Pont de Numours and Company) are gealed on the botton and vertically installed epproximately 1 in. from the tank bottom. Gamma, neatron, and acoustic probes may be lowered into the liquid observation wells to determine the interstitlal licuid levels within the tank. A gamuna probe detects the difference between saltcake and galtcake containing liquid, based on the principle that the liguid ts the primary source of gamma emissions. A neutron probe distinguishes between the existing dry saltcake, wet saltcake, and liquid phases. Distinct differences exist in the seutron count rates of each material type. An acougtic probe emits a pulse that is reflected of the interface between the outer wall of-the liquid observation well and the waste. A fiberglass/liquíd interface produces a minimal reflected pulse and a fíberglass/air interface produces a maximum pulse reflection. Wet saltcake will fall between the limits appropriately; therefore, interpretation of the records will determine the liquid level. Each probe acan is fully automatic and 
WHC-SD-WM-ER-308, Rev, 0

succegsful scans are input to the computer automated surveillance syotem.

Internal Tank Temperatureg (Thermocouples)

Temperatures usually are recorded automaticaliy in the Computer Automated Surveillance syster (CASS). Temperatures are monitored with thermocouple trees that are comprised of stainless steel pipe with 4 to 18 thermocouples. Usually, the lowest sensor is located approximately 4 in. from the tank bottom and the other sensors progress up the tree at-2-ft intervals. More frequent temperature readings are available for a few select tanks due to an upgrade in the surveillance data acquisition system to the tank monitor and control system (TMACS) in 1991.

Generally, a single thermocouple sensor is sent down the liquid observation well to take semiannual readings in tanks that do not contain a thermocouple tree. The temperatures are recorded manuaily in degrees Fahrenheit and are entered into the surveiliance analysis computer system (SACS) that is currently using the ORACLE database.

\section{External Ireak Detection (Drywella)}

Drywells are located around the circumference of each SST and throughout the tank farms to determ 1 ne if tank wastes have leaked. The drywells are generally 6-in. diameter carbon steel plpe, between 75- and 250-ft deep. The wells are termed dry because they are not deep enough to reach ground water. Scan profiles of radiation or moisture in the soil as a function of well depth are obtained with gamma radiation or neutron sensorg. Scintillation monitors, Gelger-Mueller Instruments, and neutron sensors are used to monitor the drywells. Scintillation monitors are radiation detection instruments based on the principle that light pulses are produced in some materials when they are exposed to radiation. Geiger-Mueller instruments detect low-level beta and gamma radiation. Neutron probes are equipped with a neutron source and detector that determine if moisture is present in the soll. The frequency of and probe used for drywell monitoring are specifled in a schedule from the "Waste Storage Tank Status and Leak Detection Criteria" (Welty 1999).

Drywell readings are recorded at 1 -ft intervala. The peak readings and the depth of the readings are recorded on a summary sheet. If a drywell indicates a new or unusual increase in ectivity, a rerun is requested and the drywell is retested. The wells are sealed when they are not being tested.

The drywells described in this report have a 6-digit computer identification number: the first two digits denote the tank farm (i.e., $\mathrm{A}=10, \mathrm{AX}=11, \mathrm{~B}=20, \mathrm{BX}=21, \mathrm{BY}=22$, and $\mathrm{C}=30$ ), the third and fourth are the last two digits of the tank number (i.e., 241-A-105 world be 10-05 and 00 indicates a non-specific tank), and the fifth and sixth describe the clockwise location of the well from due north. For example, the identification number of the first drywell for Tank 241-BY-102 would be 22-02-01. 
Laterals (i.e., horizontal drywelis) are positioned under some ssTs to detect radionuclides in the soil which could be indicative of tank leakage. The drywells are monitored by radiation detection probes and occasionally by a temperature probe. Laterals have 4 in. inside diameter steel pipes located 8 to 30 ft below the concrete base of the tank, and each tank has three laterals. Laterals are located only in the A Tank Fartn and a portion of the sX Tank Farm.

In-Tank. Photography

In-tank photography is another waste volume determination method used to resolve in-tank measurement anomalies and determine tank integrity. Photographs can help determine sludge and liquid levels, provide a degree of corroboration with other waste management processes, and are a source of information on foreign objects contained in the tanks. The photos can be compared to see if tank wagteg with similar histories have similax characteristicg.

1.3.2 Approach and Development of Supporting Information contained in the Historical Tank content Estimate and Supporting Documents

Extensive information was gathered for each tank. This document contains a comprehensive data set on each tank, that are summarized in the Historical Tank Characterization Estimates.

Tank Level Higtories

The tank level historiss are graphical representations of waste levels over the lifetime of a tank. They include information such as $\mathrm{pH}$, in-tank photo dates, waste types, transfer data, and physical data. Information from the mid $1940 s$ to 1980 was compiled irom two Weatinghouse Hanford Company documents (Anderson 1990 and Welty 1988). Data from 1977 to the present were obtained from the monthly waste atatug stumary reports. The following Hanford site operating contractors prepared sumaries: Atlantic Richfield Hanford Company from 1973 to 1977, Rockwell Hanford Operations from 1977 to 1987, and Westinghouse Hanford Company Eron 1987 to present. Script $£$ iles were imported from a spreadiheet to AutocAD 12 to graph the surface level data. Liquid observation well data wexe obtained from the Westinghouse Hanford Company Tank Farm Survelliance group in a spreadsheet format. Additional information was gathered from various documents and comments were added directly to each drawing.

The liquid, solid, and interstitial liguid levels (liquid obeervation wells data) are shown on the tark level hiatory graphs as volumes and tank elevations. The following formulas were used to relate the volumes to the respective elevation for each tank farm design: 
FORMULAS

$\frac{\text { Total Gallons }}{2750 \frac{\text { Gallons }}{\text { Inch }}}=$ Total Inches

$\frac{\text { Total Gallons }-12,500 \text { Gallons }}{2750 \frac{\text { Gallons }}{\text { Inch }}}+12$ Inches $=$ Total Inches

Total Gallons - 12,500 Gallons = Total Inches

$2750 \frac{\text { Gallons }}{\text { Inch }}$

Total Gallons $-18,500$ Gallons +12 Inches $=$ Total Inches $2750 \frac{\text { Gallons }}{\text { Inch }}$.

Total Gallons - 590 Gallons $* 6$ Inches = Total Inches $196 \frac{\text { Gallons }}{\text { Inch }}$

\begin{tabular}{||l|l|c||}
\hline Equation No. & Applicable Tank Farms: & Tank Diameter \\
\hline 1 & A, AN, AP, AW, AX, AY, AZ, SY & $75 \mathrm{ft}$ \\
\hline 2 & BY, S, TX, TY & $75 \mathrm{ft}$ \\
\hline 3 & B, BX, C, T, U & $75 \mathrm{ft}$ \\
\hline 4 & SX & $75 \mathrm{ft}$ \\
\hline 5 & B, C, T, U (200 Series) & $20 \mathrm{ft}$ \\
\hline
\end{tabular}


WHC-SO-WM-ER-308, Rev, 0

Temperature Rlots

There are two categories of temperature data, one or few temperature readings per day and thermocouple temperature data. The documentation used for the first category includes waste status Summary Reports (Roberts, R.E. 2/58 - 10/58, Thress, H.A. 11/58 2/59, Lentz, J.E. 3/59-12/60, and Roberts, R.E. 6/61 - 6/62), RHOCD-1172, and HH-83906. The thermocouple temperature data were obtained from the Westinghouse Hanford Company ORACIE database commonly known as the Surveillance Analysis Computer system (sAcs) and various . retrieved hand-entered tencoerature data sheets. Temperature versus time plots were developed for both categories of temperature data. Historical temperature plots cover the time span between January 1957 and January 1973. The comon temperature span used for these plots is 350 degrees. There are no tanks that have temperature data outside the sliding 350 degree temperature range.

No thermocouple information has been located prior to 1973. The thermocouple temperature infortnation was parsed in an ExCEL spreadsheet and graphed to show each individual thermocouple probe. January of 1973 is then the starting date for all thermocouple plots. The temperature range for all tank thermocouple plots in all tank farms was kept to a 120 degree $F$ span, except where the data exceeded this range. In the few tanks where the temperature apan exceeds this sliding range the data are shown using the smallest temperature range possible. A consistent time frame and temperature range is essential in comparing temperature information from one tank to another. If documentation was available, thermocouple elevations were included in the plots for tanks currently containing thermocouple trees. Thermocouples are ordered from the bottom to the top of the tank. Therefore, a plot of thermocouple 1 over time would indicate the temperature near the bottom of the tank. In-tank temperature information is available in Appendix D.

\section{Surface Level Plots}

The surface level data from 1991 to 1993 were obtained from the Westinghouse Hanford Company ORACLE database. The information was parsed in an EXCEL spreadsheet and graphed to show the waste level versus time. Current in-tank surface level information is available in Appendix E.

\section{Drywell Plots}

The arywell information was obtained fron the manual records of the Weetinghouse Hanford Company Tank Farm Surveillance Group. Data from 1990 to the present were input into an EXCEL spreadsheet and graphed to show the peaks in counts/eecond $(\mathrm{c} / \mathrm{s})$ and the peak depths in feet (ft) versus the date on the same chart. Tank drywell information is available in Appendix F.

Tank Protiles and Other Drawinge

The drawings included in this report have been compiled from several sources including as-built drawings, the vaste storage Tank statug and Leak Detection Criterla (Welty 1988), and the Riser Configuration Document for single-shell waste Tanks (Alstad 1991). 
The risers and penetrations throughout the tank dome are used for many purposes. Some risers were installed during the original tank construction and others were added later.

Through the years, sleeves or smaller risers were installed in many of the larger risers. For the riser configurations in this report, a tank may have a riser number followed by a letter which means that a larger riser was fitted with smaller sleeves. Depending on the intended use, the riser sleeves can be as large as 12 in. or as small as 4 in. For example, a saltwell pump and screen require a 12-in. riser but a thermocouple tree requires only a 4-in. riser. The tank profile drawings can be found in Appendix K.

\section{Photographs}

The tank farm photos were labeled to indicate the relative position of each tank in the farm and arrows were added to show the cascade series of each tank farm.

The latest in-tank and tank farm photos were obtained from Pacific Northwest Iaboratory. In-tank photos have been arranged to show the contents of each tank. The auxiliary equipment within each photo collage was identified if possible and labeled appropriately. The latest in-tank and tank farm photos axe available in Appendix I.

\section{H1atorteal Sample Analysis Data}

The sampling data were obtained from the WHC Tank Waste and Retrieval Group. Tables were made for the samples that were taken prior to September 1989. The information was compiled in EXCEL to present the data in as similar a manner as possible. The tables include physical data, chemical analysis data by component, and radiological analysis data by component. Any other data included with the sample references are summerized as text. Tank historical. sample analysis data are available in Appendix $\mathrm{H}$.

1.3.3 Approach and Development of the Process-Based Historical Tank Content Estimate

The process-based historical tank content estimates presented in this report were taken from work being pexformed by Los Alamos Fational iaboratories. The tank content estimates are the primary source of tank characterization data until the tank characterization reports are completed. The tank characterization reports will incorporate current core sampling and analysis results with historical-based tank content estimates to present the most current evaluation of the tank waste constituents.

Generating historical tank content estimates involves management and evaluation of large quantities of data. Several smalier components of the data management effort make up the system of generating historical tank estimates. The components are described in the following sections. 
- Waste Statur and Transaction Record Summary Database

The waste status and transaction record sutmary database (i.e., transaction summary) is a database of all known waste transfer recorda. H1storically, when waste was pumped from a process plant to tank or tank to tank, a record of the pumped waste volume and type was kept. The transaction suntuary is the recorded waste level of the solids and supernatant.

The waste status and transaction record sutmary database is being part1tioned into four separate sections. (i.e., quadrants). The waste status and transaction record summary database for the northeast quadrant is available in WHC-SD-WM-TI-615 Rev. 0 (Agnew $1994)$.

- Tank Layering Model

The tank layering model is derived from the waste status and transaction record gummary database. The purpose of the tank layering model is to predict the waste types and volumes in each tank. The transaction summary database records the types of waste that enter each tank and the corresponding increase in the solids volume from each waste entry. Each waste type that increases the solida volume in the tank is recorded in the tank layering model. Therefore, the completeness and accuracy of the information in the tank layering model is directly related to the completeness and accuracy of the transaction summary database.

The tank layering model was created to develop historjcal tank content estimates. The results of the tank layering model are reported in this document. The tank layering model is presented graphically with the inventory estimate described below in Appendix $\mathrm{K}$.

Defined wagte Types

Early in the development of the histortcal tank content entimates, a need to compile the chemical compositions af all known wagte typeg 1nto one source document was recognized. This compilation was performed by Los Alamos National Laboratories. The Hanford defined waste document was developed independently of the waste atatus and transaction record summaries database and the tank layerting model. However, the Hanford defined waste list will be uged with the tank layering model to obtain the actual tank waste inventory estimates as described bolow.

The Hanford defined waste document gives the compositions for all known Hanford waste types that went into the double-shell and single-shell tanks. Eor more details of the methods used to develop these waste types, refer to the Hanford Defined Wastes Document (Agnew 1994).

Inventory gatimato

The end result of combining the tank layer model and the Hanford defined waste types is the tank inventory estimate. When the volumes and compositions of each waste type in a particular tank are known from the tank layering model and the Hanford defined 
wastes document, a calculation of the total chemical inventory in the tank is performed. The individual tank results are presented in tabular form in Appendix $\mathrm{K}$.

The inventory estimates developed by Los Alamos National Iaboratory are quality checked against existing data from current sample analyses, thermal and physical models, and historical records. The checking constitutes a large portion of the time recuired to develop the estimater. When estimates are checked against existing data, the models used may be reevaluated. Thus, the process of generating estimates will iterate and the associated errors will be reduced over time as the historical knowledge of the tank waste operations increases.

\section{I.3.4 Assumptions}

The quality of the historical information avallable for the tanks often requires that assimptions and judgement calls be made on the applicability and validity of the data. Some assumptions were used to interpret the data.

\section{Temperature Data}

The lines joining the data points on the temperature piots were detached to show gaps in data of more than three years.

The single thermocouple tree in some tanks was removed and replaced with two trees. The original single thermocouple tree and tree 1 data were plotted on the same graph, and the tree 2 data wexe graphed separately.

No conclusion has been made on the status of thermocouples with less than 5 data points. In general, when there is thermocouple data with less than 5 data points, the data are retained on the temperature data sheets, but have not been plotted. Some possible explanations for the limited data are that the thermocouple failed, it was inoperable when readings were taken, or the operator misrepresented the number of thermocouples and the temperature data in the tank.

The thermocouples used in the trees were installed as purchased. There were no field calibrations before 1991. In 1991, a survey of Ferrocyanide Watch List thermocouple trees was conducted. The thermocouple tree probe temperatures were tested against probes inserted in liquid observation wells or by other methods as specified in Engineering Evaluation of Thermocouples in FeCN Watchlist Tanks, (Bussell 1992). Thermocouples, with equipment that could be repaired outside the tank, were made operable at this time.

It is assumed that the sinusoidal variances in the data can be attributed to oeasonal and diurnal trends which are clear aftex 1991 due to an upgrade in thermocouple surveillance to a computerized data acquisition system, i.e., Tank Monitor and 
WHC-SD-WM-ER-308, Rev. O

Control Systerm (TMACS). Also, in general, the temperatures decrease from the bottom to the top of the tanks.

All the temperature data received by ICF Kaiser Hanford Company was reviewed for suspect data points. Data that obviously deviate from the temperature trend for a any tank are marked as suspect and designated in the comment column as either "guspect data (low)" or "gtuspect data (high)". Typically, suspect data sharply deviates from the data trend 15 degrees $F$ or more (See historical and thermocouple temperature data in Appendix D Temperature Graphs and Data).

After review of the data, it was determined that some data was recorded in degrees Celsius rather than in degrees Fahrenheit as it was electronically received. The data are converted to Fahrenheit and evaluated against the rest of the data set. Where the converted data are found to be consistent with the temperature trend, they axe designated in the comment column as "raw data assumed degree C". Other delineations used to flag suspect data or substantiate the degree Celsius conversions are found from the Tank Waste Information Network System (TwINS) database, property of Battelle - Pacific Northwest Laboratory. Data recorded in degree $c$ or input incorrectly and confirmed with TwINs are delineated in the comutents column as: "degree c/TwINs confirm" and "wrong input/TWINS confirm", respectively (See historical and thermocouple temperature data in Appendix D - Temperature Graphs and Data).

In addition, some of the lines of data were flagged in the comment column by Westinghouse Hanford Company (WHC) before transmiasion of the data. Some of the HHC comment flags include "cags" or "SETf2 FROH CASs". It is assumed that "cass", an acronym for Computer Autonated Surveillance System, applies to all the data taken on that particular day, and that "SET\#2 FROM CASS" applies to the contiguous data designated under the TREE SET \# Column as 2 (See historical and thermocouple temperature data in Appendix D Temperature Graphs and Data).

Sources of temperature data are located in the footnotes of both the temperature plots and data. Some Historical Temperature plotg uge data from Waste. Status summary Reports (Roberts, R.E. $2 / 58$ - 10/58, Thress, M.A. 11/58 - 2/59, Lentz, J.B. 3/59-12/60, and Roberts, R.E. $5 / 61-6 / 62$ ). If these reports are used, footnotes on the tempertutue plots indicate by including the source of data concieely as from "Waste Status sumary Reports" (See htgtorical temperature plots in Appendix D - Temperature Graphs and Data).

Drywall Data

The drywell data did not appear to have had a quality review; therefore, the following assumptions were made about the data:

-A data point is considered suppect if an apparent rerun has been performed. 
-A data point is considered to be a rerun if the preceding data are erratic and the rerun is performed on the sarke day if monitored daily, within one or two days if monitored weekly, or within several days if monitored monthly or yearly.

-If suspect data were indeed relevant, the appropriate surveillance organizations would have followed the appropriate procedures to identify any problems.

Sampling - Data

The data obtained from the historical analytical information are understood to be widely varlable in type, quality, and scope. The data range from process control data obtained from a grab sample on a few selected analytes to a total characterization suite performed on a core sample. There are no specific quantitative data quality criteria for the information incorporated into the historical tank content estimate. A qualitative assessment of the information sources is done during construction of the historical tank content estimate. The available data are assessed for suitability and consistency in the study and each data source is weighted appropriately for jts contribution to the overall estimates.

When the sampling data were compiled, a sample was considered a solida sample $1 f$ it was greater than $30^{\circ}$ solids. sludge/slurry aamples were included.

\section{Inventory Ettimater}

Solid waste fortuations vary by type and are determined individually by examination of the historical fill records. Further refinement and bounding of the solids formation are provided by conformation to physically attainable systems (e.g., waste volumes contained in the tarks do not exceed the tank capacity and negative vold fractiong are not possible).

Haste stream compositions were based on process flow sheet information and previous analytical data.

Solids were evenly distributed throughout tank. The solids were assumed to be evenly distributed throughout the slurry during their transfer and their settling rate was assumed to be uniform throughout the tank.

Complete settling of the sludge occurred and the solids settled in a pseudo-pancake formation (1.e., flat layers conforming to the botundaries of the tank) which was a simplifying assumption. Localized mounding and pocketing in various areas of the tank may have occurred due to inlet/outlet phenomena $(e . g .$, cascades and transfers from the floating suction pumps that remove or deposit sludge). This phenomenon would have affected only a small fraction of the tank area and does not impact the overall results of this study. However, spatial variability withir physical configuration and process history is acknowiedged. No method is currently available that would adequately model this discrepancy. An effort 
is underway to develop this concept and incorporate it into the tank characterization process, but it is incomplete at this time.

Solids from a specifically designated waste type are homogeneous, which is a simplifying assumption. Variation and layering of the sludge due to component concentration differencea between batcheg, prectpitate particle size, and particle density were quite likely. The effect of these Eactors on the distribution of analytes within the solids is unknown; however, the effect was probably omall and not detectable with the present gample collection and analysis process.

Solids compaction did not occur and was not accounted for in the atudy which is a simplifying assumption. Although compaction probably occurred in the history of the tanks because of the formation of additional solids layer on top of each other, the degree of compression was not known and, therefore, was not incoxporated.

Density and other physical characteristics of the solids were determined by individual waste type using available information or were based on approximate physical analogues.

The capacity of the tanks was never exceeded during transfers. Tank capacity is dependent on fill status, fill order, tank location in a cascade, and tank type.

Leaking of the tanks was not incorporated during the time of the study but was accounted for at the end.

Cascades, tank transfers, and crib line effluents were normally free of particulate; therefore, the majority of solids were confined to their initial receiver tanks and were not cribbed or traneferred extensively fron tank to tank. Thiss assumption based on cases of plugged cascade lines when appreciable anounts of solids tried to cascade, and the pumping equipment typically was not designed to transfer of substantial guantities of solids (except when sluicing or specifically transferring solids). Monitors on the crib discharge lines should have indicated significant solids contamination, and the tank farm operating procechures would have prevented solids discharges to the ground.

\subsection{Historical Information Quality}

The quality of historical data compiled for this report varied greatly. Sone data came from published documentation. Other data were retrieved electronically from various Hestinghouse Hanford Company detabases. The quality of data used in the estimates is described in the following section.

\section{Level Histories}

The level history narrative is designed to give a general description of the waste types contained in a specific tank from startup to the present. The history presents physical 
characteristics of the waste such as waste pH values and the latest. maximum temperature of the waste. It also presents information on when the tank was removed from service, if $\neq t$ was stabilized, the method of stabilization, if it ever leaked, and the current stabilization status. It is not designed to be a detailed summary of every waste type and transaction for that tank. The waste types are general and usually are given only for large transactions (i.e., if a waste is not of sufficient volume to significantly impact the total volume of the tark then it was not reported in the narrative). For a more complete record of transactions and types of wastes involved, see the waste status and transaction record summary (Agnew 1994).

The accuracy of the tank level histories relies on the completeness of the Hestinghouse Hanford company document "A History of the 200 Area Tank Farms" (Anderson 1990); the monthly Hestinghouse Hanford Company, Atlantic Richfield Hanford Company, and Rockwell Harford Operations waste summary reports; and the waste status and transaction record sumnary (Agnew 1994).

Surface level readings may be affected by several factora: plummet error, flushing watex accumulation, and waste surface irregularities. Crystalifine wastes (i.e., saltcake) can build up on the end of the plummet gradually and contact the waste which indicates a false surface level increase. Significant level diecrepancies occur when the buildup breaks off or when the measuring instrument plummet is flushed to remove the saltcake buildups. Flushing the Food Industry Corporation Gauge, manual tapes, or any other equipment may cause accumulated wash water to collect under the plummet which indicates a false increase in the overall volume of waste within the tank. Swrface level readinga often are difficult to obtain from tanks that have a relatively dry saltcake surface. Some tanks have crystalline buildup on in-tank equipment (i.e., pumps, thermocouples, liciud observation welis, and other protruding equipment). As the supernatant is pumped from the tanks, the crystalline structure may remain attached to the equipment and be suppended above the liquid. Therefore, an accurate surface level measurement would be diffjcult it the breakup of the crystalline structure was inconsistent and a nonuniform waste surface was created. Steel tapes that are bent or warped from operation or those dincarded on the waste surface are other sources of altered surface level readings.

Routine measurements of solids and sludges in most SSTs were not xeported in the Anderson document until 1965. The solids measurements are taken with a steel donut on a string and operators interpret the sludge level. The interpretation could introduce a wide range of variability in the recorded solids level. Sometimes estimates taken from photographic data and saltwell pumping activitieg are the only indications of the solids levels.

Licuia observation Fell Data

The liquid observation well data were obtained electronically from the Westinghouse Hanford Company Surveillance Group. Some 
discrepancies exist in the liquid observation well readings for tanks with liquid waste ourfaces. The discrepancies were supposed to be addressed in a December 1993 rebaselining of the liquid observation well data. Agreement between the liquid observation well plots and the data obtained from the surveillance group has been verified; however, errors in the data prior to the exchange of information could still exist. The liquid observation well data were understood to be unverified prior to the exchange. Therefore, the data should be reviewed before being used for any design purposes or safety evaluations.

\section{Temperature Readings}

The single-shell tank temperature profiles are provided by electronic data from Westinghouse Hanford Company. Agreement between the thermocouple plots and the data obtalned from the surveiliance group has been verified; however, errors in the data prior to the exchange of information could still exist. Thermocouples used in the thexmocouple trees were purchased and installed according to the American society of Testing and Materials (ASTM) standard. There were no field calibrations befoxe 1991 when a survey of Ferrocyanide Watch List thermocouple trees was conducted and $a$ few of the thermocouple trees were compared against a thermocouple probe inserted in a liquid observation well.

During a review of the plots, some thermocouples wexe found to have outlying data points and many of them have only one or two readings. The unusual readings may be the result of the thermocouples functioning on only one occasion, the operator misrepresenting the number of thermocouples in the tank. unnecessary thermocouples readings, or possible thermocouple failure.

Occasionally the operator would read the temperature $\pm n$ degrees celsius instead of degrees fabrenheit and would not indicate this on the data sheets. The thermocouple data were understood to be unverified prior to the exchange. An attempt has been made by ICF Kaiser Hanford to validate and verify the data, however, the data should be checked before being used for any design purposes or safety evaluations.

Surface Ievel Data

The gurveillance automated computer systen data were obtained electronically from the Westinghouse Hanford Company surveillance Group and were plotted. The data are actual surface levels recorded from the surveillance equipment. If the surveillance equipment in a particular tank riger was removed from service, the readings may show a level change when a new instrument and/or riser is used, especially if the waste surface shows severe heterogeneity.

Agreement between the plots and the data obtained from the Eurveililance group has been verified; however, errors in the data prior to the exchange of information could still exist. The surveillance automated computer system data were understood to be 
unverified prior to the exchange. Therefore, the data should be reviewed before being used for any design purpoess or safety evaluations.

\section{Drywell Date}

The drywell information is received on data sheets for each drywell. If the readings are questionable, Westinghouse Hanford Company often will request a rerun of the drywell scan. Because the reruns are not indicated on the data sheets, it can be difficult to determine if the data sheet has been superseded. In some wells, several scans occurred on the same day with no explanation; therefore, the scan with the accepted results is not identifiable.

\section{Risex Configuration}

The riser configuration was determined from the WHC Riser Configuration Document for Single-Shell Waste Tanks (Alstad 1991). The information was current as of June 1991 ; therefore, any changes made after that date would not be included.

\section{Photographs}

The photo collages were made from the latest available in-tank photographs. In some tanks, photos have not been taken since the early 1970s. Some tanks had fogging problems in the vapor space which prevented use of the latest photos. Sometimes a review of the older photos was useful to determine the waste changes. Collages made from older photos may not be indicative of current tank contents, especially if the tank has been pumped since the last photo date.

\section{Historical Sample Analyaio Data}

The historical sample results only refer to sample and analysis information obtained before August 1989. The historical sample and analysis resulta have not been validated by the characterization program. When validation occurs, they will be relssued in a later revision of the Historical Tank content Eatimate report and supporting documentation. The gample data often were retrieved from memos that indicated the results of the sample analysis. The analytical methods, holding times, and quality control information are unavailable. The location at which the sample was taken was not provided which leads to Integration of some uncertainty into the sampling results. The analytical scope performed in many cases was quite narrow which limited the available data set. However, the data set is the only available historical chemical characterization information.

\subsection{Background NE Quadrant}

The SSTs located on the Hanford site near Richland, Washington were built between 1943 and 1964 to provide interim storage for high-level nuclear wastes. Processes such as plutoniun separation from spent nuclear fuel and uranium metal recovery generated the millions of gallons of mixed radioactive hazardous waste solutions stored within these tanks. 
Several waste tanks are on watch lists. The definition of a watch liat tank is explained in a later section of this report. Wastes stored in the NE quadrant were generated mostly from Plutonium Uranium Extraction (PUREX) and a Bismuth phosphate plant (B Plant); however, some tanks in the quadrant received wastes from the other onsite separation processes. The separation processes went through many changes as new technologies became available. Liguid waste volumes were reduced by evaporators, in-tank golidification units, or chemical precipitation of radionuclides. The resulting -supernatant was disposed of in the ground. Vaults were used for temporary storage or treatment of waste and they were the intermediate storage point for tank waste or waste awaiting further processing. A special rail car facility in the NE quadrant was used to unload onsite waste that was distributed to an evaporator, tanke, or processing facilitiea. The facilities were sources of the wagte that exists in the SSTs of the NE quadrant.

\subsubsection{Waste Generating Processes}

Although not all of the processes listed below contributed waste directly to tanks in the NE quadrant, the waste they generated could have been indirectly transferred to the quadrant through tank to tank transferg. The plants and processes that generated waste now contained in the SSTs and the DSTs are pxesented in chronological oxder.

T Plant

T Plant was the first-full scale separations plant at Hanford. It was constructed in 1944 and was used as a separation facility for irradiated production reactor fuel until 1956. The bismuth phosphate separation process used at $T$ plant was identical to the one used at B Plant. Since 1957, T Plant has been used as a decontamination and repair facility. The facility was modified in 1978 for storage of pressurized water reactor (PWR) core II fuel assemblies. T Plant provided facilities for decontamination, decomposition, repair, testing, ahipping, and burial of contaminated tools and equipment from onsite and offsite locations.

The following wasteg were sent to sSTs: waste solution from equipment decontamination effortg at $T$ Plant, decontamination waste (DW), metal waste (MW) from the bismuth phosphate process, firstcycle (1c) decontamination waste from the bismuth phosphate process, and second-cycle (2C) decontamination waste from the bismuth phosphate process.

- B Plant

B Plant was constructed between August: 1943 and February 1945. The bismuth phosphate process ran until 1952. One of the first plants built along with T Plant, $B$ Plant was designed to separate plutonium from uranium and the bulk of fission products in irradiated fuel via the bismuth phosphate separation process. In 1968, B plant became a waste fractionation plant and was retrofitted to remove cesium 137 and strontium 90 Exom PUREX teid 
WHC-SD-WM-ER-308, Rev. 0

waste, high-level supernatant liouids, and sludge from self-boiling liquid wastes.

B Plant contained 40 processing cells that served several functions \{e.g. equipment storage, radionuclide purification, liquid waste concentration, and solvent extraction). Cells 5, 23, 24 and 25 were used as liquid waste concentrators for the $B$ plant processing. Cell 23 of $\mathrm{B}$ Plant was used as a waste concentrator from July 28, 1967 to February 2, 1968. Because B Plant was being upgraded to a-waste fractionation plant, the Cell 23 concentrator was running specifically to concentrate intermediate level waste in the single-shell tanks.

In 1974, the Waste Encapsulation and Storage Facility (WESF) was added to $\mathrm{B}$ plant to encapsulate strontium and cesiun that was recovered from the $B$ plant procens. The cesium capsules were leased commercially for irradiation of food, medical supplies, and lumber. The strontium and cesium capsules are stored in water basins for future retrieval. Currently most of $B$ plant is going through closure except for the plant sections that are needed to support the WESF mission. The closures result from a 1991. evaluation of the future $B$ Plant mission.

The following B Plant waste streams were sent to SSTB: B Plant high-level waste (B), B Plant flush (BFSH), low-level waste from the waste fractionation plant (BL), complex concentrate (CC) or (CCPL), complexed waste (CPLX), fission products waste (FP), metal waste (MW), REDOX ion exchange waste processed at $B$ Plant (RIX), ion exchange (IX), strontium sludge (SRS), cell 5 tank 6 waste (56), Eirst-cycle waste (1C), and second-cycle waste (2c).

\section{Biamuth Phosphate Process}

The $B$ and $T$ Plants used $a$ bismith phosphate extraction to remove plutonium from uranium fuel elements. The extraction waste that resulted was a metal waste that still contained $90 \%$ of the fission products and 99 \& of the original uraniun. The metal waste was sent to specific tank cascades in the 200-East and 200-West Areas. First-cycle decontamination waste (1c), which contained approximately 10 \% of the fission products, was also stored in ather underground waste tanks in the 200 Areas.

\section{Plutonium Finiehing plant}

The Plutonitu Finiohing Plant (PFP or z Plant), previously called Plutonium Recovery and Finishing Operations, began operation in late 1949 to process plutonium and prepare plutonium products. PFP operations included plutonium handling, plutonium reclamation, plutonium conversion, and decontamination and decommissioning.

Waste from this plant contained minor amounts of fission products and low concentrations of plutonium and other transuranic elements, but was high in metallic nitrates. Initially, the waste was discharged via cribs to soil columns, which absorbed the txansuranic elements and retained them close to the point of 
WHC-SD-WM-ER-308, Rev, 0

diecharge. Later waste from $Z$ plant was stored along with other wagte in underground tanka.

The following waste was sent to tanks: waste from the Plutonium Reclamation Facility and the remote mechanical c line (z) .

Jranium Oxide Plant

The 224-0 Building was completed in 1944 as part of the U plant complex. The building was converted to a vo plant in 1951. The 224-t/A building was constructed in 1957 with six installed calciners. The $\mathrm{UO}_{3}$ plant was able to handle the uranyl nitrate hexahydrate (UNH) stream from RBDOX, $u$ Plant, and PUREX. The UO, plant shut down in 1972 and restarted in 1984 with the feed lines Erom REDOX and the U Plant canyon disconnected. Since 1984, there have been 17 campaigns at the plant that averaged 8 days each. The plant can calclne UnH much faster than the PUREX plant can produce it. Final deactivation was ordered for the plants in 1992. In April 1993, the $00_{3}$ plant regumed operations to convert 200,000 gal of remaining UNH to uranium oxide powder. A final deactivation plan for the facility was prepared in the summex of 1993 and will be carried out in the next few years.

In the basic $\mathrm{HO}_{3}$ process, calcining consisted of concentrating and heating liquid uinh until it was converted to a stable orangeyellow powder. The nitric acid in the UNH solution was also recovered in the same process. The $\mathrm{DO}_{3}$ powder was the base material needed to manutacture urantum hexaflouride (UF ${ }_{6}$ ) which is the primary feed material for gaseous diftusion plants in the United States.

\section{RFDOX}

The Reduction and Oxidation extraction (REDOX) plant was built between May 1950 and August 1951 and operated until July 1967. Uranium and plutonium were extracted from solid uranium metal slugs that were irradiated in the Hanford reactors or piles into a methyl isobutyl ketone (hexone) solvent by a continuous solvent extraction process. The REDOX process was the first at Hanford to recovex both plutonitum and uranium.

The waste stream which was slightly acidic contałned fission products and large quantitieg of aluminum nitrate that were used to promote the extraction of plutonium and uranium. The waste was then neutralized and stored in SSTs.

The following wastes were sent to SSTs: REDOX process waste resulting from the dissolution of aluminum and zircaloy fuel element cladding, REDOX coating waste (CWR), REDOX high-level waste (R), and the supernatant liquid portion of waste generated by the REDOX pxocess, REDOX supernatant (RSN). RSN is found above the sludge in the underground storage tanks. 
O Plant

U Plant (221-U) was built as one of three original bismuth phosphate process facilities although it was not used for that purpose. U Plant was modifled extensively and used for the uranium recovery process. U Plant operated from 1952 to 1958 . The main carlyon at $U$ Plant currently stores failed equipment.

Uranium in waste from the BiPO, process inftially was stored in the SSTs. Iater it was mined by sluicing, dissolved in nitric acid, and processed through a solvent extraction process. For every gallon of metal wagte originally stored, the uranium extraction process yielded approximately $1.4 \mathrm{gal}$ of UR waste. The waste volume increase made development of a technology that would reduce the volume of the stored waste a priority. This need resulted in the development/implenentation of the ferrocyanide scavenging process.

The waste sent to the SSTs from U Plant was called tributyl phosphate (TBP). In reality it contained very little TBP; therefore, to ellminate confusion, it will be referred to as uranium recovery (UR) waste in this report.

\section{Strontium Semiworke}

The strontium or hot aemiworkg processing facility (i.e., C Plant) was built in 1949 as a hot pilot plant for the REDOX procesa, but was later converted to a pilot plant for the PUREX process. In 1960, the plant was reactivated and used later as a process demonstration for the conversion of $B$ plant to a waste fractionation facllity and was used to recover strontium 90, promethium 147, and cesium 144 from PuREX waste. The butlding and building site have been decontaminated and decomissioned.

The strontium semiworks waste sent to SSTs was known as hot semiworks (HS), strontium semiworks (SSH), and fission products waste (FP).

\section{PUREX}

The Plutonium Urantum Extraction (PUREX) plant (i.e., A Plant) was an advanced solvent extraction process that used tributy phosphate in a paraffin hydrocarbon solvent to recover uranium and plutonium from nitric acid solutions of irradiated uranium. PUREX was built between April 1953 and April 1955, it operated until 1972 when it was closed for 11 years. Two thorium campaigns were conducted in the PUREx plant between 1966 and 1971 . The irradiated waste was similar to the PUREX waste but it contained thorium and uranium 233 rather than uranium and plutonium which was extracted from typical PUREX waste. It began operating again in November 1983 and was shut down in 1988 after a safety violation. The PUREX Plant was shut down several times between 1988 and 1990 for various reasons. In October 1990, the PUREX Plant was pist in standby mode and was eventually identified for closure in December 1992 by Secretary of Energy James Hatkins. 
The following PURBX waste streams were sent to SSTs: cladding waste (CW) or (CWP), organic solvent wash waste (OWW), neutralized PUREX plant acid waste (P), low-level waste From PUREX (PL), PUREX eludge eupernatant liquid (PSS), organic wash waste using sodium carbonate (CARB), cesium feed (CF), and concentrated neutralized high-level waste (1Ww) .

\subsubsection{Haste Management Operations}

This section describes the different waste concentrating methods used in the 200 Areas. Evaporating, concentrating, and scavenging are all methods uged to reduce liquid volumes or precipitate solids out of the supernatant solutions. The operations are presented in chronological order.

\section{2-T Evaporator}

The 242-T Evaporator was built in the early 1950 s to reclaim nonbolling waste storage capacity in existing tanks. The evaporator was shut down in the sumer of 1955 and modified for tributyl phosphate scavenging, although scavenging was never performed in this evaporator. The 242-T Evaporator restarted in 1965 and operated until 1976. Tank 241-TX-118 was the feed tank for the evaporator.

The following wastes were sent to tanks from the $242 \mathrm{~T}$ Evaporator: Slurry product from the evaporator bottoms (EB), evaporator feed (EF), and terminal liquor (TL).

\section{2-B Evaporator}

The 242-B Evaporator was built south of the B Tank Farm and started operating in December 1951. It received feed waste until November 1954 and was shut down in September 1955. The evaporator was never reactivated. Tanks 241-B-105 and -106 were used as the bottoms and feed tank respectively. The evaporator operated at atmospheric pressure.

\section{Ferroeyanide Sonvenging}

Ferrocyanide sctuenging was developed to reduce waste volumes. The ferrocyanide flow sheet was firet tested in $U$ Plant in October 1953. In-farm scavenging was completed in March 1956 and December 1957 for the 200-West and 200-East Areas respectively. The ferrocyanide scavenging program was designed to remove fission products such as ${ }^{137 \mathrm{Cs}}$ (half 1 ife: $28.6 \mathrm{Yr}$ ) and ${ }^{90} \mathrm{Sr}$ (half life: $30.2 \mathrm{yr}$ ) from the UR and $1 \mathrm{C}$ wastes as a precipitate. Becauge the ${ }^{9} \mathrm{gr}$ was essentially ingoluble in the neutralized UR waste, the atrontium compounds would precipitate as phosphates, sulfates, or hydrated oxides when the acidic waste was neutralized. However, common cesium compounds are quite soluble even at alkaline pHs. Development of the nickel ferrocyanide scavenging process provided a relatively simple process to precipitate ${ }^{10} \mathrm{Cs}$ and enhance the precipitation of ${ }^{90} \mathrm{~S} x$. The precipitating chemicals were added in stainless steel process vessels with agitation (i.e. U Plant, T plant, or the $C R$ vault) and the waste slurry was routed 
to opecific sSTs for settling. After the ${ }^{157} \mathrm{Cs}$ and ${ }^{90} \mathrm{Sr}$ precipitates settled, the clarified supernatant was sampled and disposed of in cribs. After the ${ }^{103} \mathrm{Cg}$ and ${ }^{90} \mathrm{Sr}$ precipitates settled, the clarified supernatant was sampled and disposed of in cribs.

\section{In-Tank Solidification}

The primary function of the in-tank solidification systems was to concentrate the nonboiling waste directly inside of specially designed tanks that would produced a saltcake slurry. Each in-tank solidification system included a heat exchanger for water evaporation and a serjes of bottoms tanks. The first in-tank golidification unit (ITS \#1) operated with an airlift circulator through Tank 241-BY-102 and the second in-tank solidification unit (ITS \#2) operated with a similar circulator through Tank 241-BY112. Tank 24l-BY-112 contained a 4,000 kW electric immersion heater. Tank 24l-BY-101 contained an in-tank solidification prototype that was used only for demonstration purposes. In-tank solidification units 1 and 2 began operating in 1965 and 1968, respectively. In 1971 in-tank solidification unit 1 became the cooler for in-tank solidification unit 2, Both units were shut down in 1974 .

\section{REDOX Concentrator}

The REDOX concentrator was used for volume reduction of dilute 200-Vest Area tank Earm wastes by removing water. The concentrator recelved tank farm wastes from July 28, 1967 until June 30, 1972. Cell $D$ in the REDOX process was the concentration and neutralization section of the plant.

\section{2-8 Evaporator/Cryatallizer}

The 242-S Evaporator/Crystallizer began operating in November 1973 and shut down in 1981. It was depigned as a reduced pressure (i.e., partial vacuum) evaporator/cryotallizer. Aqueous salt wastes were converted in the evaporator to balt crystals for storage in undergxound tanks in the $\mathrm{S}$ and sX Tank Farms.

The following waste typez were sent to sSTs from the 242-s Evaporator: noncomplexed waste concentrated until the solution was nearly saturated with sodium aluminate known as double-shell slurry feed (DSSF), slurry product from the evaportor bottoms (EB). evaporator feed (EF), Hanford defense residual liquor (HDRL), partial neutralized feed watte (PNF), and terminal liquor (TL) .

\section{2-A Evaporator/Cryatallizer}

Construction of the 242-A Evaporator/Crystallizer was completed in 1976. The evaporator began operation in March 1977 with a design life of 10 years. In 1987, design changes were developed to extend the facilities operating life by 10 years. The evaporator was shut down in April 1989 because of regulatory issues and restarted in May 1994 after extensive modifications.

The following evaporated wasteg were sent to SSTs: evaporator bottoms from B Plant low-level waste feed (BLEB), noncomplexed waste concentrated in evaporators known as double-shell slurry feed 
WHC-SD-WM-ER-3Da, Rev, 0

(DSSF), evaporator feed (EF) or (BVAP), evaporator bottoms (BB), and terminal liquor (TL) .

1.5.3 Miscellaneous Waste Sources and Equipment

Thexe are various other sources of waste on the Hanford site, as well as other material added to the tanks. Some wastes are from the 300 Area, 100 Area production reactors, various laborataries, and catch tanks. Unique contenta added to SSTs included laboratory wastes, diatomaceous. earth, Portland-cement, shroud tubes, ceramic balls, experimental tuel elements, and relatively small amounts of enriched uranium, plutoniuk, cobalt, and natural uraniun.

The following wastes contribute to SST waste: diatonaceous earth (DE), Hanford defense reaidual liquor (HDRL), Hanford laboratory operations (HLO), filtered Hanford water $\left(\mathrm{H}_{2} \mathrm{O}\right)$, phosphate decontamination waste from $\mathrm{N}$ reactor (N), and noncouplexed waste (NCPL).

\section{Critical Mans Lab}

The critical mass lab was used to develop data on the factors that are necessary to make a criticality or self-sugtained nuclear reaction occur. Because plutonium is the key radioactive element on the Hanford site, the research was based on plutonium criticality safety in various fuel cycles. This facility was located next to the strontium semiworks and ran from the early $1950 \mathrm{~g}$ to the early 19B0s. The plutonium used in the lab was reprocessed in PUREX.

244-AR, -BXR, and-CR Procese Vaults

There are three process vaults in the NE quadrant: the AR Vault, the BXR Vault, and the CR Vault. The vaults were composed of several process vessels or tanks used to prepare the waste for treatment or storage. Specific wastes from tanks can be pumped temporarily to the vaults and the wastes can be sent directly to desired tanks or processing facilities later.

The AR Vault was designed and constructed between 1964 and 1968 and is located next to the $A$ and AX Tank Farms. The AR Vault has been in standby mode since 1978 .

The 244-BXR Vault is located south of the 241-BX Tank Farm and was constructed between 1950 and 1951. The BXR Vault began operating in 1952 and became inactive in 1956. The waste in the vault was difficult to handle, so the vault was high-pressure steam jetted in 1976. The vault was interim isolated after 1976 and interim stabilized in March 1985. Tank BXR-004 was removed in 1963.

The CR Vault was constructed in 1952 and is located next to the C Tank Farm. saltwell waste from the C Tank Farm is interim stored in the CR vault. The 244-BXR and $-C R$ Vaults were constructed to aid in the recovery of uranium from bismuth 
WHC-SD-WM-ER-308， Rev. 0

phosphate metal waste. The CR Vault received waste from the Stxontiun Semiworks Plant en route to the 241-C Tank Farm.

\section{4-AR and 204-S Railroad Car Faciliticn}

The 204-AR rail car urloading facility was built in 1981 and replaced the 204-S rail car unloading Eacility. The facilities were built for unloading radioactive liquid waste tank cars.

Itiquids or slurrieg from the 1314-N Building in the I00 Area and the 340 Building in the 300 Area are unloaded at the 204-AR unloading facility.

\subsubsection{Timeline}

The following timeline represents the times and spans of different events that occurred during the lifetime of major Hanford plants in the 200-East and 200-West Areas. The plants were the main contributors of waste currently contained in SSTs. The following list of abbreviations and corresponding meanings are used on the timeline:

PUREX: Plutonium uranium extraction process

$\mathrm{DO}_{3}$ : Uranium trioxide

PFP: $\quad$ Plutonium finishing plant

RG-RB : Rubber glove - remote button line

RAA: Remote mechanical "A" I1ne

RSE : Recoplex solvent extraction

RMC: $\quad$ Remote mechanical "C" Iine

PRF : $\quad$ Plutonium reclamation facility

PCB : Polychiorinated biphynels

WESF : Waste encapsulation and storage Eacility

ITS: In-tank solidification (units 1 and 2)

PWR: Pressurized water reactor

R\&DOX: Reduction oxidation process 



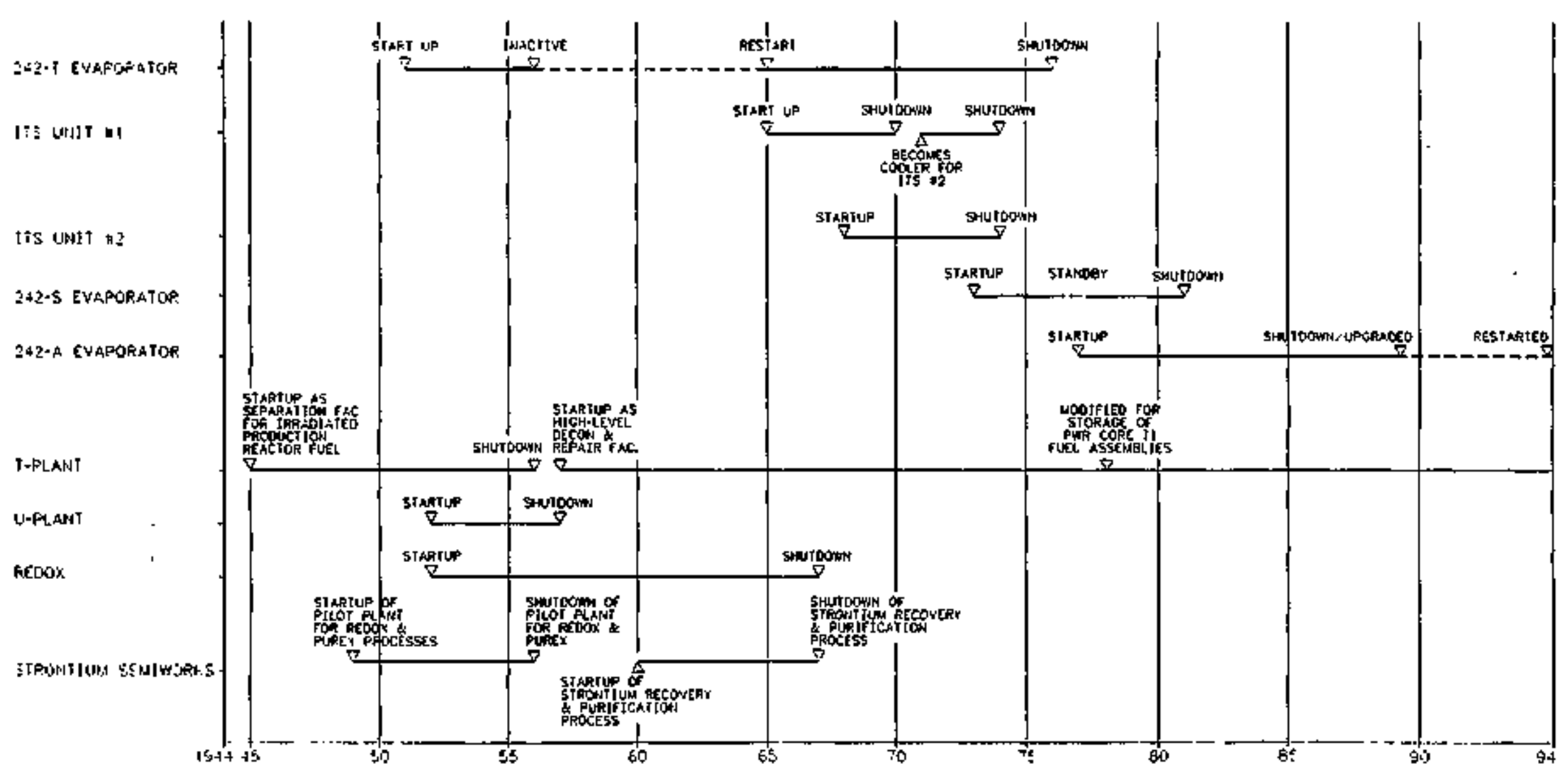




$$
\text { WHC-SD-TM-ER-308，Rev. } 0
$$

\subsection{Safety Issues}

The safety 1ssues that effect the tanks can be divided into two groups: watch list and non-watch list. The watch Iista are listings of the tanks believed to pose potential safety hazards to the environment and the public. Non-watch list issues are of concern because they may impact the environment.

\subsubsection{Watch List Safety Issues}

Issues in these tanks were identified as nissues/situations that contain most necessary conditions that could lead to worker (onsite) or offsite radiation exposure through an uncontrolled release of fission proctucts" under Eublic Law 101-510, Section 31.37, of the National Defense Authorization Act of Fiacal Year 1991 (i.e., the Wyden Amendment). As of October 1993, 45 SSTs and 6 double-shell tanks are on a watch list. In the ws quadrant, 21 of the 66 SSTs are on a watch list. The four watch list designations described in this report are: ferrocyanide, hydrogen/flammable gas, organic salts, and high-heat load. The following sections provide a general description and criteria of the different wateh listz. For a more complete explanation, refer to the Hanford Site Tank Farm Facilities Interim Safety Basis (WHC 1993).

\section{Ferrocyaniale}

A tank containing or believed to contain greater than 1,000 gram moles of ferrocyanide (ary basia) is on the Ferrocyanide watch List.

\section{Hydrogen and Flammable Gas}

Most tanks were placed on the Hydrogen/Flammable Gas Watch Liat because of the potential to contain flamuble gases rather than the verified presence of hazardous concentrations. Hydrogen/Flammable Gas Watch List tanks have been identified as unreviewed safety questions due to the concern of a flaminable gas burn resulting in a radiological release.

Oxganio salte

Tanks containing or believed to contain more than the equivalent of $10 \%$ by weight of sodium acetate or 3 wto total organic caxion (TOC) on a dry basis recelved a watch liat designation as in the Tank Farm surveillance and Waste status Suminaxy Report (Hanlon 1993).

\section{High-Ëbt Loads}

Tanks with a heat load greater thar 40,000 Btu/hr are designated as high-heat load tanks. Tank 241-c-106 is the only high-heat load tank on the High-Heat Ioad Watch List.

A tank that does not appear on a watch list but has a heat load less than $40,000 \mathrm{Btu} / \mathrm{hr}$ is considered a low-heat load tank by default. 
The following table lists the tanks in the NE quadrant that appeared on a watch list as of october 1993. A current list is published monthly in the Tank Farm Surveillance and waste status Summary Report (Hanlon 1993).

NE Quadrant Watch List Tanks

\begin{tabular}{|c|c|c|c|}
\hline Tank & Category & Tank & Category \\
\hline$A_{-}-101$ & Hydrogen & BY-108 & Ferrocyanide \\
\hline $\mathrm{AX}-101$ & Hydrogen & BY -110 & Ferrocyanide \\
\hline$A X-103$ & Hycrogen & $B Y-111$ & Ferrocyanide \\
\hline $\mathrm{B}-103$ & Organic Salts & BY -112 & Ferrocyanide \\
\hline $\mathrm{Bx}-102$ & Ferrocyanide & $\mathrm{C}-103$ & Organic salto \\
\hline $\mathrm{BX}- \pm 06$ & Ferrocyanide & $c-106$ & High-Heat Load \\
\hline $\mathrm{BY}-103$ & Ferrocyanide & $c-108$ & Ferrocyanide \\
\hline BY -104 & Ferrocyanide & $c-109$ & Ferrocyanide \\
\hline BY -105 & Ferrocyanide & $c-111$ & Ferrocyanide \\
\hline BY -106 & Ferrocyanide & $\mathrm{C}-112$ & Ferrocyanide \\
\hline$B Y-107$ & Ferrocyanide & & \\
\hline
\end{tabular}

1.6.2 Non-Watch List Safety Issues

Tank leaks are a safety hazard because of the potential chemical and radioactive liquid releases to the ground. Corrosion is the main cause of tank leaks and stabilization, integrity, and intrusion prevention are discussed in the corrosion section of this report. Criticality, tank bumps, and toxic vapor releases are other safety issues that do not require a watch list designation and/or continual monitcoring under the wyden Amendment.

Corrogion

Corrosion is believed to be caused by localized or general reduction in SST mild caxbon liner thickness. Localized liner thickness reduction is caused by three types of corrosion: pitting corrosion, stress corrosion cracking, and crevice cracking. General liner thickness reduction may be caused by uniform corrosion.

The SSTz were removed from service on or before November 21 , 1980 and no longer accept wastes. When the ssis are deactivated, an effort is made to minimize potential leaks into the earth by reducing the liquid waste contained in the ssTs. Primary atabilization is the first deactivation process of removing the supernatant or free liquid above the solid wastes within the SSTs. supernatant is removed by turbine or jet pumps in a saltwell system, low-volume submersible pumps, or in situ drying. In 
saltwell systems, the liquid is puruped from the waste through a pipe screen in a manner similar to ground water pumping. Tanks with less than $50,000 \mathrm{gal}$ of drainable interstitial liquid and less than 5,000 gal of supernatant liquid are known as interim stabilized tanks. Liquid waste 1 s sent to the evaporator/crystallizer concentrating facilities and the concentrated waste is deposited into double-shell tanks (DSTa).

Eventually, ssTs will be interim stabilized and prepared for intrusion prevention. (formexly known as interim isolation). stabilization efforts will be completed prior to final closure of the SSTs. Partial isolation of the tanks includes sealing the piping and risers that are not required for pumping or other stabilization methods. Intrusion prevention is the total physical and administrative effort involved in sealing inactive storage tank accesses against liguld additions while still allowing for longterm surveillance.

There are 67 assumed/confirmed leaking SSTs in the 200 East and Weat Areas with 32 in the NE quadrant. The remaining 34 tanks in the NE quadrant are sound. There are 51 interim stabilized tanks in the NE quadrant and 15 non-interim stabilized tanks. Intrugion prevention is in place for 46 SSTs in the NE quadrant, and 20 tanks are at the partial interim isolation stage.

\section{- Critionlity}

Criticality is an inadvertent self-sustained nuclear chain reaction. Studies have concluded that a nuclear criticality accident in the tank farms is probably not an imuinent risk. However, definitlve knowledge of the fissile material inventory and distribution within the tanks is lacking. Therefore, criticality remains a safety isaue. The key criticality radionuclide on the Hanford site is plutonium. A fissile material criticality pxevention apecification of $125 \mathrm{~kg}$ has been placed on the tanks. An unusual. occurrence report involving nuclear criticality was filed in June 1991 for Tank 241-C-104. An inventory of $56 \mathrm{~kg}$ was calculated later which indicated a low probability for criticality. Criticality is considered an unreviewed safety question but is not affected by the Wyden Amendment according to the Hanford Site Tank Farm Facilities Interin Safety Basia (WHC 1993).

\section{Tank Bumpa}

Tank bumps have been an issue at Banford since 1953 with the last bump occurring in 1968. The A Tank Farm is known to have had tank bumps. Tank bumps occur when heat from the hotter solid waste in the lower portion of the tank is suddenly transferred to a near boiling supernatant, Mixing can be achieved by "roll aver" or sudden reatart of airlift clrculators which results in rapid Elujd vaporization and a sudden. Internal tank.pressurization that causeg a bump. The three documented bumps that have occurred were due to airlift circulator failure followed by rapid startup of the aixlift circulators which causes rapid mixing. Two key factors have been identified to control bumping: liquid and solid temperatures must be maintalned as far away from the local boiling point as possible 
and airlift cixculators and ventilation systems must be operated as designed. A tank bump is extremely unlikely if airlift circulators and vent flow rates are maintained at 500 to $1,000 \mathrm{ft}^{3} / \mathrm{min}$ per tank and $50 \mathrm{ft}^{3} / \mathrm{min}$ to the airlift circulators, according to the Hanford Site Tank Farm Facilities Interim Safety Basis (hHC 1993).

\section{Toxic Vapor Releases}

The safety concern of toxic vapor releases have been analyzed recently at Henford. Odors have been released from the C Tank Farm in the NE quadrant and from Tank 241-SY-101. Tank 241-C-105 has experienced releases that have resulted in worker exposure. If toxic vapors are determined to exist in a tank, three safety recommendations should be followed: determine a release rate. determine offsite and onsite exposure concentrations, and determine if the releage is a spray effect. The entixe issue of toxic gas releases at the tank farms has just begun to be understood, Hanford site Tank Farm Facilities Intexim Safety Basis (WHC 1993). 


\subsubsection{A Tank Farm History}

The A Tank Farm is located east of Buffalo Avenue and west of Canton Avenue in the 200-East Area. The A Tank Farm contains six 100 series; 1,000,000-gal; 75-ft diameter single-shell tanks. The A Tank Farm was considered a fourth generation design for holding boiling or self-concentrating waste. Each tank was allowed to boil or aelf-concentrate over a 5 to 10 year period with a $250^{\circ} \mathrm{F}$ fluid temperature. Cascade overflows are connected fron Tank 241-A-101 to -102 to -103 to -106 , and from Tank 241-A-104 to -105 to -106 . The cascade overflow helght is approximately 371 in. from the tank bottom. Use of the cascade lines has been reported but cannot be verified.

Three tanks contain double-ghell slurry feed, two tanks contain noncomplexed waste, and one tank contains concentrated phosphate waste in the A Tank Farm. One tank is on the Fydrogen Watch List and two are high-heat load tanks. A 1993 color aerial photograph of the $\mathbb{A}$ Tank Farm shows the 100 series tank orientation, leak detection caisoons, a thermocouple probe caisson, a transfer box, valve pits, and a north axrow (see Appendix I). The arrows between the tanks represent the cascade overflow lines and the flow directions.

The A Tank Farm was constructed under project ca-513A using apecification HW-5614. The tanks are 75-ft diameter cylindrical tanks with flat bottons, no knuckle, and a 31-ft operating depth. Similar to all other single-shell tanks, the A Tank Farm tanks were designed with primary steel iiners and concrete domes with varioug risers. The tanks are covered with approximately 6 ft of overburden. Because the tanks are used specifically for boiling waste, aixlift circulators were installed to control waste tempertures.

Various coatings and materials were used to seal the tanks. One coat of red lead paint was sprayed on the carbon steel inside each tank. A lead flashing was used to seal the tank steel liner to the concrete dome. Some exterior paints and coatings were used. The construction joints of the concrete lifts contain copper water stops. The riser exteriors were covered with thres layers of asphalt paint. The pump pit and sample pit steel were covered with zinc chromate primer and two coats of metal paint. Some chenical resistant coatings were applted to pump pits, diversion boxes, cover alabs, process buildings, flow stopper boxes, de-entrainer tanks, and miscellaneous carbon steel pipes and joints.

\subsubsection{A Tank Fartn Waste History}

Aging PUREX high-level waste was stored in Tanks 241-A-101, -102, -103, -104, and -106. Tank 241-A-105 stored PUREX acid concentrator waste for approximately three years. In January 1965, the steel liner bulged on the tank bottom due to a bulldup of steam 
between the tank bottom and concrete foundation. Organic wash waste from the PUREX Plant was stored 保 Tank 24I-A-104 and was sluiced in 1975 when the tank began to leak. Tanks 241-A-104 and 105 were no longer used atter they were determined to be leakers.

Tank sizucing was used to rechuce the amount of strontium in the sludge portion and the amount of cesium in the liquid portion of the tank. These two radioactive isotopes were found to be the main heat generating sources in the A and AX Tank Farms. Early sluicing operations in the $A$ Tank Farm began in the mid 1960 s and continued into the 1970s. Early sluiding from Tanks 241-A-102 and -103 began in 1964. The waste was sent to the $C R$ vault until the $A R$ Vault was completed in 1968. Often, the sluiced sludge waste was returned to the tank after the strontium was removed.

The early goal was to sluice the tanks to a 4- to 6-in. heel before startup of the 242-A Evaporator in 1976. The 241-A tanks were expected to be used for saltcake storage. The sluicing goal was reduced and the A Tank Farm was sluiced to a smaller 1- to 2in. heel in the late 1970 s because the tank temperatures would be maintained better wtth the smaller heel. Tanks 241-A-101, -102, and -103 were sluiced and released for saltcake storage in 1978 because of the small heel remaining in them. Tank 24l-A-102 was used as the 242-A Evaporator feed tank before it received saltcake.

\subsubsection{A Tank Farm Temperature ffistory}

Tanks 241-A-101, -102, -103 , and -106 contain a single thermocouple tree. Tanks with one thermocouple tree have a tree designed to contain 18 thermocouple probes. Tanks 241-A-104 and 105 have two and eight thermocouple trees respectively. of the two trees in Tanks 241-A-104, one has two thermocouple probes to record temperature data and the other has only one thermocouple probe.

All eight of the trees in Tank 241-A-105 are single-sensor temperature probes that have been staggered at varying depths in the tank. Tank 241-A-101, a Hydrogen Watch List tank, and Tanks 241-A-104 and -105, which are high-heat load tanks, have weekly temperature reading requirements. The remaining tanks in the A Tank Farm have semiannual reading requirements.

\subsubsection{A Tank Farm Integrity}

The six 1-Mgal tanks in the A Tank Farm are out of service. As of July 1993, Tanks 241-A-101, -102, and -106 are categorized as sound and Tanks 241-A-103, -104, and -105 are categorized as assumed leakers. Tank integrity aurveillance methods include liquid observation wells, surface level measurements, leak detection wells (i.e., drywells), and laterals.

Tanks 241-A-101 and -103 have liquid observation wells to monitor the interstitial liquid levels. The maximum allowable deviation from the established baseline is a $0.3-\mathrm{ft}$ increase or a 0.4-ft decrease. The interstitial liquid level is monitored weekly with a neutron probe and on request with a gamma probe. 
Surface level monitoring with manual measurement devices in the A Tank Farm is conducted quarterly. The maximum allowable increase from the established baseline is 1 to 3 in. depending on the tank. There is no criterion for an allowable decrease in surface level. The no criterion designation was established for waste tanks containing sludge with little or no solution. For these tanks, a surface level decrease is not a valid means of leak detection because the level may decrease due to sludge slumping (espectally during salt well pumping), tape flushes, and perforation of the exposed sludge at the point of probe contact. Tanks with a saltcake surface and an automatic Food Industry Coxporation gauge may have a 10-in. decrease criteria which was established to alert the operating personnel if the gauge unwinds in search of a conductive 1iquid surface.

The A Tank Farm has 51 leak detection wells that were drilled frotm 1955 to 1981. Five wells are not tank-specific and none of them have a background radiation of more than 50 counts/second (c/s). The drywells are monitored yearly or on request using scintillation probes with the following action criteria for increasing activity: if the normal readings are less than $200 \mathrm{c} / \mathrm{s}$, the readings must double and exceed $200 \mathrm{c} / \mathrm{s}$; if the normal readings are greater than $200 \mathrm{c} / \mathrm{s}$, the readinge must double. Drywells associated with assumed leaking tanks are monitored for contamination migration. The criteria Iimits do not apply except where new radtation peaks occur. If increasing dryweil activity has not exceeded the action criteria stated above, appropriate personnel will determine if increased monitoring is required.

Each tank in the A Tank Farm has three laterals positioned approxfmately 8 ft below the bottom of the tank. The laterals extend from two leak detection calssons and are monitored with Geiger-Mueller Probes (see Appendix F). For the past three years, the laterals beneath Tank 241-A-10S have had readings greater than the $50 \mathrm{c} / \mathrm{s}$ background radiation. Lateral data are not included in this report.

The A Tank Farm layout includes the peak arywell information, lateral layotit, tank integrity, tank status, and isolation -information (see Appendix F). The drywell information is based on summarized data prior to 1987 and data collected from January 1990 to the prestent.

\subsubsection{A Tank Farm Elstorical Sample Analysis Data}

Histarically, SST waste samples have been analyzed to characterize the supernatant, sludge, and saltcake in each tank. The data for this study have been compiled from samples obtained between the late 1950 a and the present. The data from supernatant samples or aolids analyges performed before 1990 were reviewed and tabulated in Appendix $\mathrm{H}$. 
2.0.2 Current status of A Tank Farm

Tank 241-A-101 was added to the Hydrogen watch List officially in January 1991. It has been declared an unreviewed safety question because of the potential consequences of a radiological release resulting from a flammable gas burn. Previous safety studies did not 1nvestigate the burn potential. The Tank 241-A-101 temperature is monitored on a weekly basia.

Tanks 241-A-104 and -105 are high-heat load tanks $(>40,000$ Btu/hr). The tanks are actively ventilated and the temperatures are monitored weekly.

Three types of waste are stored in the A Tank Farm: double-shell slurry feed in Tanks 241-A-101, -102 , and -103 ; noncomplexed waste in Tanks 241-A-104 and -105; and concentrated phosphate waste in Tark 241-A-106. The total waste volume is 1,536,000 gal: $8,000 \mathrm{gal}$ of supernatant; $972,000 \mathrm{gal}$ of saltcake; and $556,000 \mathrm{gal}$ of sludge.

\subsubsection{A Tank Farm In-Tank Photographs}

The A Tank Farm is the second most recently constructed tank fartm and it consists of six tanks built in 1954-55. Because the fartm was one of the later ones installed, it benefited from experience gained in operating the earlier farms which resulted in improved tank construction and instrumentation. Four airlift circulators were bolted to the floor of each tank to maintain waste homogeneity. A large 42-in. riger extends down from the top center of the dome, sometimes protruding into the waste. The small pipes hanging from the dome are air lines for the airlift circulatars. The latest in-tank photographs for the A Tank Farm date from August 1985 to July 1989. The photographs are an accurate representation of the current status of the tanks except where noted. 
2.1.1 241-A-101 Tank History

2.1.1.1 Waste H1story Tank 241-A-101

The waste history narrative in the following paragraph is from "A Hiatory of The 200 Area Tank Farms" (Anderson 1990). Tank 241A-101 was filled with PUREX high-level waste and organic wash waste during the Eirst-quarter of 1956. The tank held the high-level waste unt1l the first quarter of 1975. During 1963 and 1964, carbonate was added to the tank. Organlc wash waste was added Erom the second quarter of 1965 through the first quarter of 1968 . In the firgt quarter of 1969, the tank was sluiced for strontium and cesium recovery and then a leak test was performed. Hater from the leak tegt was held in the tank to maintain temperature and the tank was intended to be used as a spare for energencies. The tank recelved B Plant waste from the second quarter of 1973 until the third quarter of 1975. The tank was sluiced to reduce the sludge hesl for saltcake storage from the fourth quarter of 1975 until the first quarter of 1976 . The tank began receiving evaporator feed in the third quaxter of 1976. The tank was a salt and slurry receiver for the 242-A Evaporator until the second quarter of 1977. From the third quarter of 1977 until the first quarter of 1978, the tank received residual liquor from the 242-A Evaporator. Between the second and fourth quarters of 1978, the tank contained double-shell slurry feed. A level adjuatment was made during the second and fourth quarterg of 1979. The tank received noncomplexed waste throughout 1979. In the first, third, and fourth guarters of 1980 , the tank contained double-shell slurrxy feed. During the second ctuarter of 1980 , the tank received complexed waste from B PIant. Eresently, the tank waste is classified as double-shell slurry feed.

The waste history narrative of the primary additions that follow in this paragraph are from the waste status and Transaction Record Sumuary" (Agnew 1994). Tank 241-A-101 was filled with PUREX organic wash waste from the first quarter of 1956 until the first quarter of 1968. From the first quarter of 1956 until the last quarter of 1970, the tank received PUREX high-level waste. From the second quarter of 1958 until the fourth quarter of 1973 , the tank received PUREx low-level waste. From the first quarter of 1960 until the third quarter of 1975, the tank received various amounts of water waste. From the first quarter of 1973 until the third quarter of 1975, the tank received strontium recovery waste. The Waste Status and Transaction Record summary kept track of all the unexplained tank additions and transfers. A record of unexplained transfers is shown in Appendix $J$.

The tark was deactivated in November 1980 and partial
isolation was completed in 1982 . The tank is awaiting
stabilization. Liquid observation well data used to detertuine
interstitial levels appear to be off baseline. Although the tank
is considered sound, it is not stabilized. In-tank photographs


were taken on the following dates: Notober 1986; two in 1969, 1975, 1976, and 1977; May 1978; July 1979; Novenber 1980; March 1981, and August 1985. Three pH readings are listed on the level filstory: 9.8 in May 1973, $>14.0$ in October 1990, and $>14.0$ in October 1984. The tank was placed on the Hydrogen Watch Ligt during January 1991. The maximum current temperature reading from October 1993 is $154^{\circ}$ F. Sketch ES-TKS-E1, which shows a gxaphical representation of the Tank 241-A-103 level history, and the historical waste level data used to make it are in Appendix $C$.

\subsubsection{Temperature History 241-A-101}

The single theruocouple tree in Tank 241-A-101 has 18 thermocouple probes to monitor the waste temperature through riser 12. Risers 2 and 10 have been used to record temperature data. Elevations are available for all the thermocouple probes. Recorded temperature readings from the tank in-gervice date unti,1 1976 are sporadic. Thermocouples 1 through 14 have fairly congigtent and similar temperature readings that span from october 1976 to the present. Thermocouples 15 and 16 indicate significantiy lower temperatures during the same time period. Limited but similar data for thermocouples 17 and 18 are available from 1994 to the present.

Review of the Tank 241-A-101 level history over the time span of the readings indicates that thermocouples 1 through 14 were in or near the liquid level and thermocouples 25 through 19 appear to have been in the vapor space from October 1976 to october 1977. From October 1977 to April 1978, thermocouples 1 through 6 were in or near the solids level, thermocouplee 7 through 15 were in or near the liquid level, and thermocouples 16 through 18 appear to have been in the vapor space. From April 1978 to January 1979, solid and liquid level spikes indicated that thertoocouples 1 through 8 were in the solids level, thermocouples 9 through 15 were in the liquid level, and the rest were in the vapor space. Another liquid spike occurs from January to October 1979 indicating that thermocouples 1 through 7 were in or near the solids level, thermocouplee a through 15 were in or near the liquid level, and the rest were in the vapor space. A small liquid spike from october 1979 to April 1980 indicates that thermocouples 1 through 6 were in or near the solids level, thermocouple 7 was in or near the liquid ievel, and the reet were in the vapor space. Thereafter, a solid/liquid level change occurs indicating that thermocouples 1 through 15 were in or near the solids level and the rest were in the vapor space.

The Eiret available vapor space temperature reading in January 1957 was approximately $180^{\circ} \mathrm{F}$. A sum of the squares regression line fit for the first 16 thermocouples starting in 1976 shows an overall zero slope $\pm 9.8^{\circ} \mathrm{F}$ with an average $r$ square value of 0.27 . Thermocouples 17 and 18 also have an overall zero slope \pm 4.4 with an average $r$ square value of 0.24 . Less deviation in slope is apparent in thermocouples 15 to 18 . The temperature readings for the first 1.6 temperature probes increase approximately $50^{\circ} \mathrm{F}$ from 
January to June 1977. A temperature decrease of $20^{\circ} \mathrm{F}$ to $45^{\circ} \mathrm{F}$ is observed from late 1978 to 1980 . The moxe dranatic temperature decrease in thermocouples 5 to 14 shows an increase of the same magnitude from late 19B0 to 1981. An annual temperature undulation is more evident in thermocouples 17 and 18 after January 1991. From 1976 to the present, the median temperature is $147^{\circ} \mathrm{F}$ with a minimum of $75^{\circ} \mathrm{F}$ and a maximum of $180^{\circ} \mathrm{F}$. Current analysis of the tank ehows that the waste has a nominal bulk temperature in the range of $155^{\circ} \mathrm{F}$. Tank 241-A-101 is on the Hydrogen watch List and is monitored weekly.

The data and plots of the thermocouple xeadings in Tank 241-A101 are in Appendix D.

2.1.1.3 Integrity of Tank 241-A-101

Tank 241-A-101 is categorized as sound and is partially isolated. The Tank 241-A-101 gurface level is mont tored guarterly with a manual tape. Liquid waste volume is determined by a photographic evaluation end the solid waste volume is determined with a Food Inatrument Corporation gauge. The maximum allowable increase from the 348-in. baseline value is 3 in. There is no criterion for a decrease. The surface level for the past three years has been steady with the readings ranging between 341 and 345.5 in. Data and graphical representations of the surface level measurements from January 1991 to the present are in Appendix E.

The Tank 241-A-101 liquid observation well is located in riser 19. The tank is monitored weekly with a neutron probe and on request with a gamma probe. The maximum allowable deviations from the established bageline are a 0.3-ft decrease or a 0.4-ft increase. Sketch ES-TKS-B1 in Appendix $C$ has a graphjcal representation of the liquid observation tell date.

Eleven drywells are identified for Tank 241-A-101. Drywells 10-01-01, (not active prior to 1986 , one reading > 50 c/s), 10-0104, 10-01-16, and 10-01-28 (all active prior to 1990, current readings $>200 \mathrm{c} / \mathrm{s})$ have readings greater than the $50 \mathrm{c} / \mathrm{s}$ background radiation.

An occurrence report was iseued in July 1980 due to increased activity in drywell 10-01-04. The source of activity for drywells 10-01-04 and 10-01-16 was attributed to contamination leaching in the soil around the $241-\mathrm{A}-01 \mathrm{~B}$ pit area.

Data and graphical representations of the active drywell from January 1990 to the present are in Appendix $F$. Each graph includea the peak $\mathrm{c} / \mathrm{s}$ (sotne wells have multiple peaks), the depth at which the peak occurred, and the date of the reading.

2.1.1.4 Historical Sample Analysis Data for Tank 241-A-101

In April 1976, a sludge sample with coarse, granular, sandlike cryetals intermixed with small particles and hard chunks about 
the size of quarters was analyzed. The coarse granules were gray and the smaller particles were reddish-brown. Neither type of granule would soften in water. The chemical analysis showed that approximately three times as much aluminum as silicon was in the sludge. In September 1980, three samples were obtained from different areas of the tank: at the surface, near the sludge, and in the sludge. The samples showed that the waste consisted mostly of carbonates with lower percentages of nitrates and aluminates. In October 1980, samples from 1 ft and 6 ft below the waste surface were found to contain approximately 20 cryotalline solids. The solids were dissolved, analyzed, and found to contain approximately 50 sodium nitrate with sodium caxbonate and podium phosphate. Analysis of another sample from october showed solids consisting primarily of sodium nitrate and sodium carbonate. The viscosity of three samples analyzed in November 1980 indicated that the waste would need to be diluted if a transfer was attempted. Two boildown studies were run in 1979 which showed that more than 70 \% of the waste could be boiled away by raibing the temperature of the waste to between 50 and $60^{\circ} \mathrm{C}$. Two samples were received in the analytical laboratory in October 1983 that contained significant amounts of sodium azuminate and organic carbon. TOC was determined to be $13.5 \mathrm{~g} / \mathrm{l}$. The second sample bad a more concentrated supernatant liquor. Appendix $\mathrm{H}$ contains the tables that represent the complete analyses results.

\section{1 .2 Current status of Tank 241-A-101}

Tank 241-A-101 entered service in January 1956 and currently stores 953,000 gal of waste categorized as double-shell sluxry feed. The waste is comprised of $950,000 \mathrm{gal}$ of saltcake and 3,000 gal of sludge with 390,000 gal of pumpable interstitial liquid remaining. The tank is identified on the Hydrogen Watch List, is passively ventilated, and is categorized as sound with partial interim isolation completed. Tank 241-A-101 is equipped to cascade to Tank 241-A-I02 and is first in a four-tank cascade series. The plan view and tank cross-section that depict the approximate waste level and riser configuration are in Appendix G. Tank 241-A-101 has 22 tank dome risers and three are available for use: one 6-in. riser (no. 10), one 42-in. riser (no. 13), and one 12-in. riser (no. 16).

\subsubsection{Waste Layer Volume Approximation for Tank 241-A-101}

A comparative analysis of all layer approximations for the entire 241-A Tank Farm and the data used to derive the tank layer approximations are located in Appendix $\mathrm{K}$.

\section{1 .2 .2 In-Tank Photograph 241-A-101}

The 1988 photographic montage of the Tank 241-A-101 interior shows a white to grayish-yellow galtcake gurface with no viaible liquid (see Appendix I). Picture quality is very good and all equipment can be easily identified. The waste is about 29 ft deep. The heating coil in the top right corner kept the waste from 
cooling too quickly which prevented thermal gradient limitations of the tanks steel and concrete shell from being exceeded. The debris near the center of the photograph is an old flange gasket. No waste has been added to or removed from this tank since these photographs were taken; therefore, the picture should represent the current tank contents.

\subsubsection{Symopels Tank 241-A-101}

(To be completed.) 
WHC-SD-WM-ER-308, Rev. 0

2.2 Tank 241-A-102

2.2.1 241-A-102 Tank History

2.2.1.1 Waste History Tank 241-A-102

The waste history narrative in the following paragraph is from "A History of The 200 Area Tank Farms" (Anderson 1990). Tank 241A-102 was filled with PURRX waste from the firet quarter of 1956 unt1l the third quarter of 1970. During the third guarter of 1957, the tank received some carbonate wash. The tank was sluiced in 1964 for strontium and cesium removal. The tank received and contained PUREX sludge supernatant waste from the fourth quarter of 1970 until the fourth quarter of 1972. The tank was olufced to reduce the sludge heel for saltcake storage from the fourth quarter of 1972 until February 1974. From the third quarter of 1974 until the first guarter of 1976, the tank received B Plant high-level waste. The tank was sluiced to prepare for saltcake waste in the first two quarters of 1976 and it received evaporator feed until the first quarter of 1978. Noncomplexed waste was sent to the tank during the second and third quarters of 1978 , the fourth quarter of 1979, and the second and thind quarters of 1980. The tark received complexed waste during $B$ Plant from the first through third quarters of 1979. The tank contained double-shell slurry feed in the fourth quarter of 1978 and the firet quarter of 1980. Presently, the tank waste is classified as double-shell slurry feed.

The waste history narrative of the primary additions that follow in this paragraph are from the "waste statur and Transaction Record Summary" (Agnew 1994). Tank 241-A-102 received puREX highlevel waste and PUREX organic wash waste from the first quarter of 1956 until the third quarter of 1961 and the third quarter of 1963 reapectively. During the second guaxter of 1960, the tank received PUREX low-level waste. The tank received wastewater frot the third quarter of 1960 until the first quarter of 1976 . From the third guarter of 1974 until the second quarter of 1975 , the tank contained strontium recovery waste. From the fourth guarter of 1974 until the second quarter of 1975, the tank contained washed PURFX sludge. The Waste status and Transaction Record summary kept track of ali the unexplained tank additions and transfers. A record of the unexplained transfers are in Appendix $J$.

The tank was deciared deactivated in November 1980 and intrusion prevention was conpleted during 1982. The tank was Interim stabilized in August 1989 after most of the supernatant was pumped. A level adjustment was made in July 1969. The tank integrity is considered sound. In-tank photographs were taken on the following dates: several in 1973, two in 1977, May 1979, August 1986, and July 1989. Five pe readings are 1isted on sketch ES-TKSE2: 10.3 in August 1975, 13.9 in October 1979, 13.8 in July 1980, $>14.0$ in August 1980, and 12.0 in March 1986. The current maximum temperature reading from July 1993 is $92^{\circ}$ F. Sketch ES-TKS-B2, which shows a graphical representation of the Tank 24l-A-103 level 
WHC-SD-WM-ER-308, ReV. 0

history, and the historical waste level data used to make it are in Appendix $\mathrm{C}$.

\subsubsection{Temperature History 241-A-102}

The single thermocouple tree In Tank 241-A-102 has 18 probes to monitor the waste temperatuxe through riser 7 . Specific thermocouple elevations are unavaileble at this time. In-tank temperature readings from the tank in-service date to Narch 1977 are sporadic. Thermocouples 1 through.5 and 7 through 16 have very aimilar temperature readings that span from 1977 to 1993 . Limited data are available for thermocouples 6, 17, and 18. Data are available only from 1977 to 1988 and from 1984 to 1989 for thermocouple 6 . For thermocouples 17 and 18 , data are available fxom 1984 to 1993 .

The first available sludge temperature reading in March 1958 was 210'F. A sum of the squares regression line fit for all the thermocouples starting in 1977 shows an overall zero slope $\pm 8.5^{\circ} \mathrm{F}$ with an average $x$ square value of 0.02 . A low point of approximately $29^{\circ} \mathrm{F}$ was common for all the probes in February 1981. From March 1977 to the present, the median temperature is $89^{\circ} \mathrm{F}$ with a minimum of $63^{\circ} \mathrm{F}$ and a maximum of $137^{\circ} \mathrm{F}$. Because Tank 241-A-102 is a low-heat load tank, it is scheduled to have temperature readings taken semianmually in January and July.

Data and plots of the Tank 241-A-102 thermocouple readings are in Appendix D.

\subsubsection{Integrity of Tank 241-A-102}

Tank 241-A-102 is categorized as sound, interim stabilized, and partially isolated. The Tank 241-A-102 surface level ig monitored with a Food Instrument corporation gauge through riser 6 . Liquid waste volume is determined by a photographic evaluation and the solid waste volume is determined with a Food Instrument Corportition gauge and a photographic evaluation. The gauge is set in the intrusion mode for a 1-in. increase. If the Food Instrument corpoxation gauge fails, manual field measurements will be conducted quarterly. The maximum allowable increase from the 16.5in. baseline is 3 in. There in no criterion for a decrease and no data are available for a ouxface level plot due to the Food Instrument Corporation gauge setting.

In February 1989, the Tank 241-A-102 decrease criteria limit of 1 in. was exceeded and an unusual occurrence report was issued. In-tank photographs showed no evidence of a liquid level increase or decrease. Tank 241-A-102 does not have a liquid observation well.

Seven drywella are identified for Tank 241-A-102. Drywells 10-02-03 (activity prior to 1990, current readings $<200 \mathrm{c} / \mathrm{s}$ ) and 10-02-08 (not active prior to 1986, current readings $<200 \mathrm{c} / \mathrm{s}$ ) have readings greater than the $50 \mathrm{c} / \mathrm{s}$ background radiation. 
Data for and graphical representations of the active drywell from January 1990 to the present are in Appendix $F$. Each graph includes the peak $c / s$, the depth at which the peak occurred, and the date of the reading.

\subsubsection{Historical Sample Analysis Data for Tank 241-A-102}

The results from the waste analyses for Tank 241-A-102 conducted between May 1963 and May 1989 are included in this report. In.May. 1963, a chemical and radiochemical analysis was conducted on a siludge sample. In December 1980, two samples were analyzed and the results indicated that the solids were primarily sodiun nitrate, Both samples had approximately 30 \% by volume of large crystalline solids. The deep sample had 15 wts solids and the shallow sample had $9 \%$. A series of hot boildow samples were run in 1980. Four samples were analyzed in fiscal year 1986 to characterize the waste. The samples varied in color and texture. The most recent data are from a sample of the supermatant liquid analyzed in May 1989. The tank has been found to contain organic carbon; it has a foc of $12.4 \mathrm{~g} / 1$. Tables presenting the results of these and the other sample analyses can be found in Appendix $H$.

\subsubsection{Current Status of Tanik 241-A-102}

Tank 241-A-102 currently stores 41,000 gal of waste categorized as double-shell slurry feed. The waste is comprised of $4,000 \mathrm{gal}$ of supernatant; $22,000 \mathrm{gal}$ of saltcake; and 15,000 gal of sludge with no pumpable liquid remaining. The tank is aategorized as sound with interim stabilization and partial interim isolation completed. A plan view and tank cross-section that depict the approximate waste level and riser configuration can be found in Appendix G. Tank 241-A-102 has 20 tank dome risers and two are available for use: one $8-i n$. riser (no. 2) and one 12-in. riser (no. 19).

\subsubsection{Waste Layer Volume Approximation for Tank 241-A-102}

A comparative analysis of all layer approximations for the entire 241-A Tank Farm and the data used to derive the tank layer approximations are located in Appendix $\mathrm{K}$.

\subsubsection{In-Tank Photograph 241-A-102}

The picture of the Tank 241-A-102 interior is made up of photographs taken in 1989 shows a thin grey saltcake surface layer that is broken up with supernatant showing through (see Appendix I). Equipment features in the tank are hazy and indistinct because the quality of the picture is poor; however, all the important items have been identified and label. The waste is about 2 ft deep. Because the photographs were taken before supernatant was pumped, the current status of the tank is not represented.

\subsubsection{Synopsis Tank 241-A-102}

(To be completed.) 
WHC-SD-WH-ER-308, REV. 0

2.3 Tank 241-A-103

2.3.1 241-A-103 Tank History

2.3.1.1 Waste History Tank 241-A-103

The waste history narrative in the following paragraph is from "A History of The 200 Area Tank Farns" (Anderson 1990). Tank 241A-103 was filled with gelf-concentrating PURRX waste from the second guarter of. 1956 until the fourth guarter of 1969. During 1964, the tank sludge was sluiced for atrontium and cesium recovery. The tank was declared to be a backup in the event of tank failure. The tank was sluiced for cesium and strontium recovery again in 1966. From the first quarter of 1970 through the last quarter of 1973, the tank received ion exchange waste and PUREX waste. From the first quarter of 1974 to the second quarter of 1975, the tank was sluiced to prepare for saltcake waste. The tank received PUREX sludge supernatant and $B$ Plant waste from the third quarter of 1975 until the second quarter of 1976. The tank was sluiced to prepare for ealtcake waste and it became an evaporator feed and residual liquor tank from the third quarter of 1976 until the first quarter of 1978. Between the second quarter of $197 \mathrm{~B}$ and the fourth guarter of 1979, the tank contained doubleshell slurry feed, noncomplexed waste, and complexed waste. The tank received a cross-site transfer in the fourth quarter of 1978 . Presently, the tank waste is classified as double-shell slurry feed.

The waste history narrative of the primary additions that follow in this paragraph are from the "Waste status and Transaction Record Summary" (Agnew 1994). The tank received PURBX high-level waste from the second guarter of 1956 until the third quarter of 1960. From the first quarter of 1957 until the fourth guarter of 1975, the tank received wastewater. From the third quarter of 1957 until the eecond quarter of 1967, the tank received PUREX organic wash waste. During the first quarter of 1965, the tank received decontamination waste. During the fourth quarter of 1973 , the tank received cesium recovery waste. From the fourth quarter of 1974 until the fourth quarter of 1975, the tank received washed EUREX sludge. During the fourth quarter of 1975, the tank received atrontium recovery waste. The Haste Status and Transaction Record Summary kept track of a.1 the unexplained tank additiong and transfers. A record of the unexplained transfers is in Appendix $J$.

Tank 241-A-103 was declared inactive in August 1980 . Intrusion prevention was completed in 1982. Liquid observation well data used to determine interstitial levels correspond with the liquid surface level data (see sketch ES-TKS-E3). The tank was declared an assumed leaker in 1987 with a leak volume of 5,550 gal. Interim stabilization was administratively completed in August 1988. In-tank photographs were taken on the following dates: January 1975, October 1976, January 1977, January 1978, September 1978, December 1979, three in 1980, two in 1981, Jume 1986, two in 1987, December 1988, and July 1989. Two pH readings 
HHC-SD-HM-ER-308, ReV. 0

of $>14.0$ in October 1980 and 12.9 in Apxil 1986 are on the Tank 241-A-103 level history (sketch ES-TKS-E3). The current maximum temperature reading in July 1993 if $117^{\circ} \mathrm{F}$. Sketch ES-TKS-E3, which shows a graphical representation of the Tank 241-A-103 level history, and the historical waste level data used to make it axe in Appendix C.

\subsubsection{Temperature History 241-A-103}

The single thermocouple tree, in Tank 241-A-103 has 18 thermocouple probes to monjtor the waste temperature through riser 15. Risers 2 and 19 were also used to record temperature data. Specific thermocouple elevations are unavailable at this time. Recorded temperature readings from the tank in-service date to october 1976 are sporadic. Thermocouples I through 16 have fairly consistent and similar temperature readings that span from october 1976 to 1993. Data are available from August 1977, 1984 to 1988, and 1991 to 1993 for thermocotuples 17 and $z 8$. There is $a$ slgnificant historical temperature decrease and the highest temperatures were recorded from thermocouples 1 through 4 . In relation to the lower elevation probes, the temperatures recorded For thermocouples 6 through 18 are approximately $25^{\circ} \mathrm{F}$ cooler.

The first available vapor space temperature reading in May 1956 was $80^{\circ} \mathrm{F}$. A sum of the squares regression line fit for all the thermocouples starting in 1976 shows a slightly negative near zero slope $\pm 16.7^{\circ} \mathrm{F}$ with an average $r$ square value of 0.07 . Initial temperature fluctuations are followed by a temperature increase of $45^{\circ} \mathrm{F}$ between May and August 1977. There are a few temperature increase and decrease spikes from 1988 to 1990 . From october 1976 to the present, the median temperature is $90^{\circ} \mathrm{F}$ with a minimum of $50^{\circ} \mathrm{F}$ and a maximum of $143^{\circ} \mathrm{F}$. Tank $241-\mathrm{A}-102$ is scheduled to have semiannual temperature readings taken in January and July.

Plots and data for the thermocouple readings from Tank 241-A103 are in Appendix $D$.

\section{3 .1 .3 Integrity of Tank $241-A-103$}

Tank 24l-A-103 is categorized as an assumed leaker and is interim stabilized with intrusion prevention completed. Becauge there is no supernatant, the liquid waste volume is determined by a liquid observation weli and the solid waste volume (i.e., surface level) is determined by a food Instrument Corporation gauge through riser 6 and a photographic evaluation. The gauge is set in the intrusion mode for a $1-i n$, increase. If the food Instrument Corporation gauge fails, manual field measurements will be conducted quarterly and the maximum allowable increase from the 137.4-in. baseline is 3 in. There is no criterion for a decrease. Data are not available for a surface level plot due to the Food Instrument Corporation gauge setting.

The Tank 241-A-103 liquid observation well is located in riser 19. The interstitial liquid level is monitored weekly with a 
WHC-SD-WM-ER-308, Ret, 0

neutron probe and on request with a gamma probe. The maximum allowable deviations from the established baseline are $0.3-\mathrm{ft}$ decrease and a 0.4-ft increase. Sketch ES-TKS-E3 in Appendix C has a graphical representation of the liquid observation well data.

An occurrence report was issued in September 1980 due to a liquid level decrease. The decrease was attributed to mixing disaimilar solids within the tank.

An occurrence report was issued in December 1979 due to a liquid level decrease. The decrease was attributed to crust slumping.

Seven drywellg are identified for Tank 241-A-103. Drywellg 10-03-01 (no longer active) and 10-03-07 (active prior to 1990, current readings $<200 \mathrm{c} / \mathrm{s}$ ) have or had readings greater than the $50 \mathrm{c} / \mathrm{s}$ background radiation.

An occurrence report was isgted in May 1978 due to a small peak in drywell 10-03-01. The activity was associated with contamination leaching in the soil etused by a 60,000-gal raw water leak.

Graphical representations of the active drywell from January 1990 to the present are in Appendix F. Each graph includes the peak c/s (some welia have multiple peaks), the depth at which the peak occurred, and the date of the reading.

\subsubsection{Historical Bample Analysis Data for Tank 241-A-103}

In December 1972, a core sample of Tank 241-A-103 sludge was analyzed to identify the characteristics of the waste for processing through solvent extraction. The botton 5-in. portion of a core aample was observed to be very hard and the upper 6-in. portion was a fine, soft, chocolate brown material. After determining the solubility of the hard material, it was determined that the sludge could be sluiced by maintaining continuous agitation within the tank.

In August 1979, a hot boildown sample was analyzed to determine slurry behavior. A sample from 1 ft below the waste surface and another from $6 \mathrm{ft}$ below the surface were analyzed in September 1980 to detexuine solids content. This analysis indicated only a trace amount of solids in the waste. The low percentage of solids was considered unlikely because of the pumping behavior and other characteristics of Tank 241-A-103. Another sample fxom 3 ft below the surface was analyzed in october 1980. The oanple yielded 20\% well-settled solids. The solids were primarily carbonates with. significant amounte of nitrate. Two composite gamples were analyzed in fiscal year 1986 that were made up from 10 core samples each. The individual samples were all grey with the appearance and consistency of wet cement, but the composites turned greenish-brown during handling. Another set of samples was analyzed in 1987 and as a repult of this sampling, 
WHC-SD-WM-ER-308, Rev. 0

65,000 gal of pumpable supermatant were determined to be in the tank. Previous samples indicated that no pumpable liquid was in the tank. The tank has been found to contain organic carbon; it has a TOC of $10.8 \mathrm{~g} / 1$. Tables that present the reaults of these and other analyses are in Appendix $\mathrm{H}$.

2.3.2 Current Status of Tank 241-A-103

Tank 241-A-103 entered service in May 1956 and currentiy stores 370,000 gal of waste categorized as double-shell slurry feed. The waste material is comprised of 4,000 gal of supernatant and $366,000 \mathrm{gal}$ of sludge with no pumpable liquid remaining. The tank is categorized as an assumed leaker with interim stabilization and intrusion prevention completed. Tank 241-A-103 is equipoed to cascade to Tank 241-A-106 and is third in a four-tank cascade series. The plan view and tank cross-section that depict the approximate waste level and riser configuration are in Appendix $G$. Tank 241-A-103 has 19 tank dome risers and three are available for isse : one 4-in. riser (no. 5), one 8-in. riser (no. 11), and one 12 in. riser (no. 12).

\subsubsection{Waste Layer Volume Approximation for Tank 241-A-103}

A comparative analyais of all layer approximations for the entire A Tank Farm and the data used to derive the tank layer approximations are located in Appendix $\mathrm{K}$.

\subsubsection{In-Tank Photograph 241-A-103}

The 1988 photographic montage of the Tank 24l-A-103 interior shows a grey sludge surface with pockets of supermatant to the right and bottom (see Appendix I). The waste is about 11.5 ft deep. Only two of the airlift circulators show through the waste because one pair is shorter than other two. The quality of the photographs are good so all visible equipment is clearly presented. The picture does not represent the current status of the tank because the level was adjusted by about 6 in. after the photographs were taken. The change in level was due to a liquid/solids method of evaluation update in April 1990.

2.3.3 Symopeis Tank 241-A-103

(To be completed.) 

0

\subsection{Tank 241-A-104}

\subsubsection{1-ㅈ-104 Tank History}

\subsubsection{Waste History Tank 241-A-104}

The waste history narrative in the following paragraph is from "I History of The 200 Area Tank Farms" (Anderson 1990). Tank 241A-104 contained test water from the second quarter of 1957 until the second quarter of 1958. From the third quarter of 1958 until the third quarter of 1969, the tank received waste similar to carbonate weste and organic wash waste from PUREX. The tank waste was a self-concentrating or boiling type. The tank was sluiced for strontium and cesium recovery between the first and third quarters of 1.969. The tank was checked for leaks and declared a spare in the fourth quarter of 1969. The tank contained sluicing water from the fourth quarter of 1969 until the third quarter of 1972. The tank received $B$ Plant waste in the fourth quarter of 1972. From the first quarter of 1973 until the first quarter of 1975, the tank received PUREX sludge supernatant. The tark was sluiced to reduce the sludge heel for saltcake storage in the first quarter of 1975 . In the second quarter of 1975 , the tank appeared to develop a leak and transfers into the tank were stopped. Because the leak occurred during sluicing, the tank was not completely slufced. Presently, the tank contains noncomplexed waste.

The waste history narrative of the primary additions that follow in this paragxaph are from the waste status and Transaction Record Summary" (Agnew 1994). Tank 24I-A-104 received wastewater from the second quarter of 1957 until the fourth quarter of 1969. From the third quarter of 1959 until the fourth quarter of 1962 , the tank received puREx high-level waste. From the third quarter of 1960 until the first quarter of 1968, the tank received PUREX organic wash waste. From the third quarter of 1972 until the first: guarter of 1975, the tank received washed PUREX sludge. During the fourth quarter of 1972 , the tark received cesium recovery waste. The Waste status and Transaction Record summary kept track of all the unexplained tank additions and transfers. A record of the unexplained transfers is in Appendix $T$.

A level adjustment was made in october 1977 and in January 1978. Intrusion prevention was completed in 1983. The tank was declared an assumed leaker in 1975 and was interim stabilized administratively in September 1.978. In-tank photographs were taken on the following dates: October 1969, three in 1975, January 1976, October 1977, and Jure 1996. Three pH readings are shown on sketch ES-TKS-E4: 10.2 in January 1964, 10.3 in February 1969, and 10.5 in March 1974. The current maximum temperature reading in October 1993 is $196^{\circ} \mathrm{F}$. sketch. ES-TKS-E4, which. showg a graphical representation of the Tank 241-A-104 level history, and the historical waste level data used to make it are in Appendix $c$. 
WHC-SD-WM-ER-308, ReV , 0

\subsubsection{Temperature Eistory 241-A-104}

Tank 241-A-104 has two thermocouple trees. Trees 12 and 18 are single-sensor probes in rlsers 12 and 18 . Specific thermocouple elevations are not avallable for thege probes. Recorded temperature readings from the tank in-service date to Aligust 1974 are sporadic. Both probes have fairly consistent and similar readings that span from 1974 to the present. However, the probe in riaer 12 has more exratic low temperature spikes. Suspect data for a second probe in tree 1B. show two data points in october 1987 and May 1993.

The firat availabie sludge and liquid temperature readings in March 1959 were $170.6^{\circ} \mathrm{F}$ and $172.4^{\circ} \mathrm{F}$, respectively. A sum of the squares regression line fit for both thermocouple trees starting in 1974 shows an overall zero slope $\pm 12.7^{\circ} \mathrm{F}$ with an average $r$ square value of 0.08 . From August 1974 to the present, the median temperature is $188^{\circ} \mathrm{F}$ with a minimum of $100^{\circ} \mathrm{F}$ and maximum of $236^{\circ} \mathrm{F}$. Current isotopic analysis results indicate that the heat generation rate of the sludge is $14.3 \mathrm{Btu} / \mathrm{hr}$; therefore, cooling is required for the tank. Tank 241-A-104 is a high-heat load tank and is scheduled to have temperature data monitored weekly.

Plots of the thermocouple readings in Tark 241-A-104 are in Appendix D.

\subsubsection{Integrity of Tank 241-A-104}

Tank 241-A-104 is categorized as ar assumed leaker and is interim stabilized with intrusion prevention completed. The Tank 241-A-104 surface level is monitored quarterly with a manual tape through riser 6 . The liquid waste volume is determined by a manual tape and the solid waste volume is determined by a photographic evaluation and a sludge measurement device. The maximum allowable increase from the 12.5-in. baseline value is 1 in. There is no criterion for a decrease. Graphical representations of the surface level measurements from January 1991 to the present are in Appendix E. The plot shows an inaccurate data point in the second quarter of 1993 which is attributed to input error of the field data sheets into the computer automated surveillance system. The surface level has not exceeded the increase criteria and the readings range from 11.5 to 3 in.

Tank 241-A-104 does not have a liquid observation well. Seven drywells are identified for Tank 241-A-104. Drywell 10-04-07 (not active prior to 3990 , one reading $>50 \mathrm{c} / \mathrm{s}$ ) has readings greater than the $50 \mathrm{c} / \mathrm{s}$ background radiation.

An occurrence report was 1ssued in July 1976 due to increasing radiation levels in the laterals. Evaluation indicated a minor leak migration.

Graphical representations of data for the active drywell from January 1990 to the present are in Appendix F. The graph includes 
WHC-SD-WM-ER-308, Rev, 0

the peak $\mathrm{c} / \mathrm{s}$, the depth at which the peak occurred, and the date of the reading.

\subsubsection{Historical Sample Analysis Data for Tank 241-A-104}

Tank 241-A-104 was used to store PUREX sludge supernatant (PSS) generated during the sluicing of Tanks 241-A-106, -101, and -103. In 1974, the waste had a very low solids content and the eolid material was fluid with a consistency similar to heavy cream. In one analysis, the fluid was described as yellow and containing less than $1 \%$ solids. From a second sample, particle size distribution was characterized as 4 wt: less than $10 \mu \mathrm{m}$ and $90 \%$ less than $50 \mathrm{\mu m}$. In 1975, a leak was discovered in the tank and the liquid remaining in the tank aftex the leak was discovered was punped out. At that tine, the tank was estimated to contain approximately 2.5 megacuries of ${ }^{90} \mathrm{Sr}$ and $10 \mathrm{kilograms}$ of plutonium. $\mathrm{A}{ }^{9} \mathrm{Sr}$ content of $43.9 \mathrm{Ci} / 1$ and a ${ }^{137} \mathrm{Cs}$ content were found in 1976 . Heat load in the tank was estimated at $50,000 \mathrm{BT} / \mathrm{hr}$ in 1989. Tables presenting early analyses results are in Appendix $H$. More recent efforts to sample the tank were unsuccessitu, appaxently because the material in the tank was too dry.

\subsubsection{Curcent Status of Tank 241-A-104}

Tank 241-A-104 entered gervice in June 1959 and stoxes 20,000 gal of noncomplexed waste. The waste is comprised entixely of sludge with no pumpable liquid remaining. It is identified as a non-watch list high-heat load tank with active ventilation. Tank 241-A-104 cascades to Tank 241-A-105 and is first in a three-tank cascade series. A plan view and tank cross-section that depict the approximate waste level and riser configuration are in Appendix $G$. Tank 241-A-104 has 2I tank done risers and 12-in, risexs (nos, 4, 7, and 17) are avallable for use.

2.4.2.1 Waste Layer Volume Approximation for Tank 241-A-104

A comparative analysig of all layer approximations for the entire 241-A Tank Farm and the data used to derive the tank layer approximations are located in Appendix $\mathrm{K}$.

\subsubsection{In-Tank Photograph 241-A-104}

The 1986 photographic montage of the Tank 241-A-104 interiox indicates a grey to dark-brown dry cracked sludge surface (see Appendix I). The waste is about 1 ft deep. The light blue to white discoloration is damage on the negat ive that may be caused by xadiation overexposure. The lines shown hanging in the top right of the photo are air lines to the airlift circulator that have loosened from their brackets in the dome. - The rest of the equipment in the tank is shown clearly in the picture and it bas been identified and labelled. It appears that there have been no changes in the tank since 1986; therefore, this picture should be representative of conditions in the tark at present. 
WHC-SD-WM-ER-308, Rev, 0

2.4.3 Synopgin Tank 241-A-104

(To be completed.) 
2.5.1 241-A-105 Tank History

2.5.1.1 Waste History Tank 241-A-105

The waste history narrative in the following paragraph is from "A History of The 200 Area Tank Farms" (Anderson 1990). Tank 241A-105 was filled with test water in the second quarter of 1957. Test water was left in the tank until the tank received a transfer of PUREX high-level waste in the first quarter of 1962. This tyge of waste was contained in the tank until the second quarter of 1968. The tank was suspected of leaking in 1963 and was declared an assumed leaking tank. Ieaking occurred in the fourth quarter of 1967 and the first quarter of 1968, and a atean explosion occurred between the carbon steel liner and the concrete base in January 1965. The tank contained PUREX and ion exchange waste from the second quarter of 1970 until the third quarter of 1971. The tank was sluiced for strontium and cesium recovery from the third quarter of 1968 until the first quarter of 1971 . Presently, the tank waste if classified as noncomplexed.

The waste history narrative of the primary additions that follow in this paragraph are from the "Waste status and Transaction Record Sunmary" (Agnew 1994). Tank 241-A-105 received wastewater from the second quarter of 1957 until the second guarter of 1965. From the fourth quarter of 1962 until the fourth quarter of 1964 , the tank received PUREX high-level waste. During the second guarter of 1968 , the tank received ceglum recovery waste. The Wagte status and Transaction Record summary kept track of all the unexplained tank additions and transfers. A record of those unexplained transfers can be found in Appendix $J$.

The tank was interim stabilized in 1979 and intrusion prevention was completed in 1985. A level adjustment was made in 1979. After the water additions to cool the tank were stopped in January 1979, a portable exhauster was used to control aludge temperatures. The portable exhauster falled in 1988 and was restarted. The exhauster ran for approximately 2 years and is slated to be removed from the A Tank Farm. The removal has been approved due to low heat generation of the tank waste. In-tank photographs were taken on the following dates: veveral in 1968, three in 1969, September 1970, Decenber 1971, two in 1972, two in 1976, two in 1977, June 1979, two in February 1981, and August 1986. The current maximum temperature reading in October $19931 \mathrm{~s}$ 155 F. Sketch ES-TKS-E5, which shows a graphical representation of the Tank 241-A-105 level history, and the historical waste level data used to make it are in Appendix $C$.

\subsubsection{Temperature History 241-A-105}

Tank 241-A-105 has 6 elngle-sensor probes (i.e., 9, 15, 16, 17, 19, and 22) that contain one thermocouple each in xisers 9, 15, 16, 17, 19, and 22. Specific thermocouple elevations are 
unavailable at the present time. Recorded temperature readings from the tank in-service date to December 1985 are sporadic. All the single-sensor probes have fairly consistent and very bimilar temperature readings that span from 1985 to the present. Trees 16 , 17, and 19 are the most similar with virtually no abrupt low or high temperature spikes. Hore pronounced high and low spiken can be found for tree 9 in 1986 and between 1989 and 1991 . There is an abrupt low data point of $41.4^{\circ} \mathrm{F}$ for tree 9 in August 1993 . Two abrupt low data points of $43.2^{\circ} \mathrm{F}$ and $35.7^{\circ} \mathrm{F}$ exist for tree 15 in August and December 1993. Tree 22 has a discontinuity in the temperatures taken before 1993 with those taken in 1993 and after. Temperatures after 1993 for this tree are $65^{\circ} \mathrm{F}$ lower than similar trees. Records show one data point of $181.9^{\circ} \mathrm{F}$ in May 1991 for tree 12 which is higher than most other data points taken on the same date. Tree 18 has three data points between 1988 and 1991 , and for and two of them are higher than other data points recorded on the same dater.

The fixat avallable vapor space temperature reading in Nay 1958 was approximately $60^{\circ} \mathrm{F}$. A sum of the actuares regression line fit starting in 1985 for trees $9,15,16,17,19$, and 22 shows a 0.03 overall alope $\pm 10.7^{\circ} \mathrm{F}$ with an average $I$ square value of 0.2 . The tank has an annual temperature undulation that is nore evident after October 1989. From December 1985 to the present, the median temperature is $128^{\circ} \mathrm{F}$ with a minimum of $47.9^{\circ} \mathrm{F}$ and a maximum of $189^{\circ} \mathrm{F}$.

Historical information indicates that a steam release in January 1965 caused an 8.5-in. bulge in the bottom liner. Compositional anaiysis and determination of specific heat generated by both isotropic and calorimetric analyses show a heat generation of 38 to 42 Btu/hr. A liquid level drop in october 1973 caused a temperature incxease in the tank that was followed by water additiong to cool the tank until october 1979. Tank 241-A-105 is a high-heat load tank and is scheduled to have temperature data monitored weekly.

An unusual occurrence report was issued in December 1990 due to an increasing temperature trend in the thermocouple lateral under Tank 241-A-105. The cause of the trend was deterzined to be faulty wiring.

Plots of the thermocouple readings in Tank 241-A-105 can be found in Appendix D.

\subsubsection{Integxity of Tank 241-A-105}

Tank 241-A-105 is categorized as an assumed leaker and is interim stabilized with intruston prevention completed. The Tank 241-A-105 surface level is monitored quarterly with a manual tape through riser 5. Liquid waste volume is determined by a photographic evaluation and solid waste volume is determined by a manual tape and a photographic evaluation. The maximum allowable increase frow the 16-in. baseline value is 1 in. There is no 
criterion for a decrease. A graphical representation of the surface level measurements from January 1991 to the present are in Appendix E. The surface level plot has an increasing trend for the past 3 yeare: the surface level during the third quarter of 1991 was 13 in. and steadily increased to 15 in. during the fourth quarter of 1993. An inaccurate data polnt with an apparent "rerun" is included on the plot.

Tank 241-A-105 does not have a liquid observation well. Seven drywells are identified for Tank 241-A-105. Drywells 10-05-02 (no longer active) and 10-05-10 (active prior to 1990, current readings $<200 \mathrm{c} / \mathrm{s}$ ) have or had readings greater than the $50 \mathrm{c} / \mathrm{s}$ background radiation.

Eraphical representations of data for the active arywell from January 1990 to the present are in Appendix F. The graph includes the peak c/s (aome wells have multiple peaks), the depth at which the peak occurred, and the date of the reading.

An unusual occurrence report (upgraded off-normal report via downgraded occurrence report) was issued in December 1990 due to an increasing temperature trend in the thermocouple lateral under Tank 241-A-105. The cause of the trend was determined to be faulty wiring.

\subsubsection{Historical Sample Analysis Data for Tank 241-A-105}

Two samples were analyzed in 1972 after a bulge was discovered in the bottom of Tank 241-A-105. The samples were taken to gain information on the composition, volume, and heat generation properties of the waste material contained in the tank. One of the samples was from dry solid waste and the other was from wet solid waste. The dry solids were hard, dry, brown lumps. In 1976, it was found that Tank 241-A-105 contained $44.8 \mathrm{Ci} / 1$ of ${ }^{90} \mathrm{Sr}$ and $2.24 \mathrm{Ci} / 1$ of ${ }^{137} \mathrm{Cs}$.

Because of the gross tank failure which occurred in 1967, most of the solid waste was removed from the tank by sluicing. A topographic study was pexformed in 1978 in which the waste surface was carefully studied by stereo photography. This study concluded that the tank waste depogits probably ranged from clay-sized through medium sand-sized materials. Chemical and property data are given in Appendix $\mathrm{H}$ for samples taken from the solid material in 1972 .

\section{5 .2 Current Status of Tank 241-A-105}

Tank 241-A-105 entered service in 1962 and currently stores 19,000 gal of noncomplexed waste. The waste is comprised entirely of sludge with no pumpable liquid remaining. It is identified as a high heat-load tank with active ventilation. The tank is categorized as an assumed leaker with interim gtabilization and intruaion prevention completed. Tank 241-A-10S is equipped to cascade to Tank $241-\mathrm{A}-106$ and is second in the three-tank cascade 
flow series. A plan view and tank cross-section that depict the approximate waste level and riser configuration are in Appendix $G$. Tank 24l-A-105 has 25 tank dome risers and six risers are available For use: one 12-in. riser (no, 4) and five 4-in. risers (nos. 7, $8,14,18$, and 20).

\subsubsection{Waste Layer Volume Approximation for Tank 241-A-105}

A comparative analysis of all layer approximations for the entire 241-A Tank Farm and the data used to derive the tank layer approximations are located in Appendix $\mathrm{K}$.

\subsubsection{In+Tank Photograph 241-A-105}

The 1986 photographic montage of the Tank 241-A-105 interior shows a grey sludge surface that is just under 1 ft deep (see Appendix I). The acid milling machine to the right of the phot: was used to drill through the bulge in the steel bottom. Operators were able to take a sample from the air space under the tank to detexmine if a combustible gas was present. A large amount of debrit is visible on the bottom of the tank. Mary of the inotrument lines and other equipment are loose.

2.5.3 Synopsis Tank 241-A-105

(To be completed.) 
2.6.1 241-A-106 Tank History

2.6.1.1 Waste History Tenk 241-A-106

The waste history narrative in the following paragraph is from "A History of The 200 Area Tank Farms" (Anderson 3990). Tank 241A-106 recelved de-entrained and condensate waste from the boiling waste tanks in the A Tank Farm from the first quarter of 1957 until the third quarter of 1960. Fxom the fourth quarter of 1960 until the third quarter of 1973, the tank recelved PUREX waste such as organic wash waste, carbonate waste, and high-level waste. The tank received sludge waste from Tank 24l-A-104 in 1969. From the first quarter of 1970 until the second quarter of 1972, the tank was sluiced for strontium and cesium recovery. From the first quarter of 1974 until the second quarter of 1976, the tank received B Plant waste. The tank contained evaporator waste between the third quarter of 1976 and the firet quarter of 1978. During this time, the tank was part of an in-farm sluicing operation and the sludge waste from Tanks 241-A-101, -102, and -103 was sent to Tank 241-A-106. The in-farm scavenging opexation was created to remove enough sludge for the storage of saltcake in Tanks 241-A-101, -102, and -103. The sluiced waste held in Tank 241-A-106 was then going to be sent to the $A R$ process vallt, but the sluicing operation was stopped before the sluiced waste was completely removed from Tank 241-A-106. From the second quarter of 1978 until the third quarter of 1980, the tark contained noncomplexed waste or concentrated complexed waste. Presently, the tank waste is classified as concentrated phosphate waste.

The waste history narrative of the primary additions that follow in this paragraph are from the "Wagte Status and Transaction Record summary" (Agnew 1994). Tank 241-A-106 received wastewater from the first quarter of 1957 until the third quarter of 1975. During the fourth quarter of 1969, the tank received PUREX lowlevel waste. From the fourth quarter of 1961 until the first quarter of 1963 , the tank received PUREX high-level waste. The tank received PUREX organic wash waste from the third quarter of 1962 until the second quarter of 1968. From the first quarter of 1974 until the third quarter of 1975, the tank received strontium recovery waste. From the second quarter of 1974 until the third quarter of 1975, the taak received washed PUREX gludge. The waste Status and Transaction Record Sumnary kept track of all the unexplained tank additions and transfers. A record of the unexplained transfers is in Appendix $J$.

Tank 241-A-106 was declared inactive in August 1980. Interim stabilization was administratively completed in August 1982 and intrusion prevention was completed in December 1982. The tank is categorized as sound. In-tark photographs were taken on the following dates: January 1973, January 1974, February 1978, May 1979, August 1980, April 1982, and August 1986. Four pH readings are ifsted on Sketch ES-TKS-E6: 10.6 in January $1964,12.6$ in June 
1974, >14.0 in May 1980, and $>12.0$ in March 1986. The current maximum temperature reading in July 1993 is $137^{\circ} \mathrm{F}$. Sketch ES-TKSB6. which shows a graphical representation of the Tank 241-A-106 level history, and the historical waste level data used to make it are in Appendix $c$.

\subsubsection{Temperature Hiatory 241-A-106}

The single thermocouple tree in Tank 24l-A-106 has 18 thermocouples to monitor the waste temperature through riser 14. Specific thermocouple elevations axe available for all probes. Recorded temperature readings from the tank in-service date to October 1976 are sporadic. Thermocouples 1 through 16 have fairly consistent and very similar readings that spar from october 1976 to the preaent. Thermocouples $I$ and 2, usually submerged within the solid waste, record the highest temperatures for the tree. The thermocouples whow an initial increase of $50^{\circ} \mathrm{F}$ until February 1977 and a large decrease of $100^{\circ} \mathrm{F}$ between September 1977 and April $197 \mathrm{~B}$. Data are available for thermocouples 17 and 18 in 1977 and from 1985 to the present.

Review of the Tank 241-A-106 level history indicates that from october to January 1977, thermocouples $I$ and 2 were in or neax the solids level, thermocouples 3 through 13 were in or near the liguid level, and thermocouples 14 through 18 appear to have been in the vapor space. From January to October 1977, a liquid level spike indicates that thermocouples 1 and 2 wexe still in or near the solids level, thermocouples 3 through 15 were in or near the liguid level, and the rest appear to have been in the vapor apace. From October 1977 to April 1978, a solids level drop and small liquid spike showed only thermocouple 1 in or near the solids level, thertocouples 2 and 3 in or near the liquid level, and the rest appear to have been in the vapor epace. Between April 1978 and April 1980, a soljds level increase and liquid level spike indicate that thermocouples $I$ and 2 were in or near the solids level, thermocouples 3 through 11 were in or near the licuid level, and the rest appear to have been in the vapor space. Thereafter, thermocouples 1 and 2 were in or neax the solids level and the rest appear to have been in the vapor space.

The first available sludge and liquid temperature readings in December 1962 were $327^{\circ} \mathrm{F}$ and $273^{\circ} \mathrm{F}$, respectively. A sum of the squares regression line fit for all thermocouples starting in 1976 showg a slightly negative, near zero slope $\pm 25.86^{\circ} \mathrm{F}$ with an average $r$ square value of 0.14 . Abrupt high and low temperatures are observed between 1989 and 1991 for all thernocouples. From October 1976 to present, the median temperature is $104^{\circ} \mathrm{F}$ with a minimum of $50.2^{\circ} \mathrm{F}$ and a maximum of $246^{\circ} \mathrm{F}$. The tank contains high strontium heat generating sludge which is not a heat problem if it is ventilated. Tank 24l-A-106 is a low-heat load tank and is scheduled to have semtannual temperature data taken in January and July. 
Plots and data of the thermocouples readings for Tank 241-A106 are in Appendix D.

\subsubsection{Integrity of Tank 24I-A-106}

Tank 241-A-106 is categorized as sound and is interim stabilized with intrusion prevention completed. The Tank 241-A-106 surface level is monitored with a Food Instrument Corporation gauge through riser 6 . Liquid waste volume is determined by a photographic evaluation and the solid waste volume is determined by a manual tape. The gauge is get in the intrusion mode for a 1 in. increase. If the Food Instrument comporation gauge fails, manual field measurements will be conducted on a quarterly basis and the maximum allowable increage from the 46.5-in. baseline value in 3 in. There is no criterion for a decrease. Data are not available for the surface level plot due to the Food Induatry corporation gauge setting.

Tank 241-A-106 does not have a liquid observation well. Seven drywells are identified for Tank 241-A-106. Drywell 10-06-10 had readings greater than the $50 \mathrm{c} / \mathrm{s}$ background radiation.

\subsubsection{Historical Sample Analysis Data for Tank 241-A-106}

Waste damples from Tank 241-A-106 were analyzed four times Erom June 1974 to January 1988 . The data reported in 1988 was actually ampled in fiscal year 1986. The analytical regults are from two composites made up from six individual core samples. The core sampleg apparently varied considerably in appearance. Soms were grey, some were white and crystalline, and some were dark brown or black. Both composites were brown although one was smooth and the other was deseribed as having a sandy texture. The 1980 manple was drawn for a "hot botldown" analysis. The sample was approximately 90\% solids and viscous. A sample analyzed in 1977 was described as dark brown, gooey, and mixed with some liquids. Analytical results are not available for this sample. The tank has been found to contain organic carbon; it has a Toc of $10.7 \mathrm{~g} / 1$. Data obtained from the samples analyzed can be found in Appendix $H$.

\subsubsection{Current Status of Tank 241-A-106}

Tank 241-A-106 entered service in 1957 and currently atores 125,000 gal of concentrated phosphate waste. The waste is comprised entirely of sludge with no pumpable liquid remaining. It is identified as a low-heat load tank with active ventilation. Tank 241-A-106 is equipped to receive cascaded waste from Tanks 241-A-103 and -105 and is last in the cascade aeries. A plan view and tank cross-section that depict the approximate waste level and riger configuration are in Appendix G. Tank 241-A-106 has 21 tank dome risers and four are avtilable for use: two 12-in. risers (nos. 4 and 17), one 4-in. riser (no. 7), and one 6-in. riser (no. 20). 
WHC-SD-WM-ER-309, Rev. 0

2.6.2.1 Waste Layer Volume Approximation for Tank 241-A-106

A comparative analysis of all layer approximations for the entire 241-A Tank Farm and the data used to derive the tank layer approximations are located in Appendix $\mathrm{K}$.

2.6.2.2 In-Tank Photograph 241-A-106

The 1986 photographic montage indicates a grey to yellow sludge surface with no visible supernatant (see Appendix I). The quality of the photographs is good and the equipment can be seen clearly. All of the equipment has been labelled and identified. The waste in the tank is about 4 ft deep. There have been no changes in the tank that would affect the waste since these photographs were taken; therefore, this picture should represent the current tank contents.

2.6.3 Synopsis Tank 241-A-106

(To be completed.) 
WHC-SD-WM-ER-308, Rev. 0

\section{GLOSSARY}

0.17

0.33

1

1AYIN

IAZIN

$1 \mathrm{C}$

1CEB

ICS

1st Generation Tank

222-B

$222-C$

222-T

222-U

224

224-F

2312

242-A

242-5
Transaction flag key-monthly volumes derived from semianmual reports.

Transaction ilag key-monthly volumes derived from quarterly reports.

Monthly report.

See Waste Abbreviations.

See Waste Abbreviations.

See Waste Abbreviations.

See Wate Abreviations.

See Waste Abbreviations.

The original tank design encompassing

Tank Farms B, C, T, U (excluding the 200 series tanks), and BX. These tanks have an operating capacity of 530,000 gal, a 75-ft diatneter, a 32-in. dish bottom, and a 4-ft knuckle. Also see Type II tanks.

See Facilities.

See Facilities.

See Facilities.

See Facilities.

See Waste Abbreviations.

See Waste Abbreviations.

See Waste Abbreviations.

See Facilities.

see Facilities. 
$242-3$

$242-\mathrm{T}$

2AYIN

2AZIN

2c

2nd Generation Tank

2 SYIN

3

3AWIN

$3 r d$ Generation Tank

4th Generation Tank

5

$5-6 \#$

5AWIN

5th Generation Tank
See Facilities.

See Facilities.

See Naste Abbreviations.

See Waste Abbreviations.

See Waste Abbreviations.

Same as original tank design (1st generation, or type II) except the operating capacity was increased to 758,000 gal. Also see Type III tanks.

See waste Abbreviations.

Quarteriy report.

See Haste Abbreviations.

The first generation of the type IV tanks, contains the SX Tark Farm only. These tanks have a $1,000,000$ gal operating capacity, a 75-ft diameter, a 14.875-in. dish bottom, and no knuckie. Also see Type IV tanks.

The second generation of the type IV tanks, contains the A Tank Farm only. These tanks are the same as the 3 rd generation except they have a Elat bottom. Also see Type IV tanks.

See Waste Abbreviations.

Cells 5 and 6 from B-plant.

See Waste Abbreviations.

The third generation of the tyos IV tanks, contains the $\mathrm{AX}$ Tank Rarm only. These tanks are the same as the $4 \mathrm{th}$ generation with the addition of grid drain slots beneath the steel liner battom.

Semianrual report. 
WHC-SD-FM-ER-308, Rev. 0

6Atin

A Plant (PUREX)

A1 SITCK

A2 SLTSERY

Active Tank

Active Drywell

$A D D$

$\mathrm{ADJ}$

AGE, AGING

Aging Waste

Airlift Circulator

Annulus

AR Vault

$\mathbf{A R}$
See Naste Abbreviations.

See Facilities.

See IANL Defined Waste List.

See LAN Defined Waste Iist.

A tank that contains more than 33,000 gal of waste and/or is still involved in waste managerrent operations.

Drywell in which radiation readings of greater than 50 counts/second are detected. To be considered active, the readings must be consistent as to depth and radiation level for repeated readings.

Add primary waste from process.

Adjustment to waste amount-see CORR.

Waste Abbreviation.

High-level, first-cycle solvent extraction waste from the PUREX Plant \{NCAW\}.

The airlift circulators are installed in tanks used to age wastes. The circulators promote mixing of the superate and prevent agglomeration of radionucildes. By maintalning motion within the body of liquid, the circulators minimize superheat buildup and, consequently, minimize bumping.

A vessel space in the form of a ring; the space between concentric walls.

A structure containing tanks and chemical processing equipment that is used for waste processing ox storage operations.

See LANL Defined waste List . 
Assumed Leaker

Assumed Re-leaker

Assumed Leaking Tank

B SITCK

B

B Plant $(222-8)$

B860N

Background Radietion

Baseline

BFSH

BL

BLEB

BLIX

BLIXB

BNW

BNW
A waste storage tank for which surveillance data, in the past, has indicated a loss of liquid attributed to a breach of integrity.

A designation that exists after a tank has been declared an "assumed leaker" and then the surveillance data indicate a new loss of liquid attributed to a breach of integrity.

In 1984 , the criteria designations of "suspect leaker," "questionable integrity," "confirmed leaker,"

"declared leaker," "borderline," and "dormant" were merged into one category now reported as "assuned lekker."

See I+ANL Defined Waste List.

See Waste Abbreviations.

See Facilities.

See Waste Abbreviations.

Radioactivity from raturally occurring sources; prinarily radiation from cosmogentc and naturally occurring radionucilices.

A refexence; for example, a specified liquid level or radiation level against which new information is compared.

See Waste Abbreviations.

See Waste Abbreviations.

Se* Waste Abbreviations.

See Waste Abbreviations.

See Waste Abbreviations.

Battelle Northwest Iaboratory.

See Faste Abbreviations. 
BPDCC

BpDcs

BEDCV

BPFPS

BELCS

BELDC

BEIDN

Bumping, Tank Bump

BVCLN

BY SLTCK

C Plant (222-c)

C PLANT

Caisson

Calcine

CARB

cas
See Haste Abbreviations.

See Waste Aboreviations.

See haste Abbreviations.

See Waste Abbreviations.

See Waste Abbreviations.

See Taste Abbreviations.

See Waste Abbreviations.

A tank bump occurs when solids overheat in the lower portion of the tank. The hot solids rapidly transfer heat to the liguid, some of which guickly vaporizes. The sudden pressurization caused by vapor generation is called a "bump".

See Waste Abbreviations.

See LANL Defined Waste List.

See Facilities.

See SSW.

An underground structure used to store high-level waste; typical designs include corrogated metal or concrete cylinders, 55-gal drums weldea end-toend, and vertical steel pipes below grade.

To heat a substance to a high temperature, but below its melting point, causing loss of volatile constituents such as moisture; refers also to the material produced by this process.

See Waste Abioreviations.

Cascade-see SET and BND. This process filled three tanks with one puxp using overtlow siphoning. Normal use was with 
Cascade

CASS

Catch Tanks

CAH

$\mathrm{cc}$

CCGI

$\operatorname{CCGR}$

CCPI

$\operatorname{CCPLX}$

CCH

CDF

$\mathrm{CB}$

CELL 23

CEM a sequence of tanks such as 101, 102, 103, or 110, 111, 112 .

Eleven of the single-sheli tank fartus (all except the AX Tank Farn) were equipped with overflow lines between tanks. The tanks were connected in series and were placed at different elevations creating a downhilil gradient. for liquids to flow from one tank to another.

Acronya for Computer Automated Surveillance System.

Small capacity single-sheli tanks associated with diversion boxes and diverter stations. The tanks are designed to receive any transfer line cleanout, spills or leakage from the boxes, or leakage from the adjacent pipe encasement.

Current acid waste; this is PUREX acid HIN.

See Waste Abbreviations.

See Waste Abbreviations.

See Haste Abbreviations.

See Haste Abbreviations.

See Waste Abbreviations.

Concentrated custorner waste.

Composition data file or transaction Elag key-unit volume assumed to make strean active.

See Waste Abbreviations.

Waste from cell 23 at B-Plant. Cell 23 contained an evaporator.

See Waste Abbreviations. 
CF

$\mathrm{CON}$

COND

Condeneate

Conductivity Prabe

Confirmed or Storage Declared Leaker
See Waste Abbreviations.

See Waste Abbreviations.

Condensate-see EVAP, EB.

Liquid formed from cooling vapors.

A device that completes an electrical circuit when contacted by a conductive material.

The designation of any underground waste tank where the data are considered sufficient to support a conclusion with 95 percent confidence that the tank has leaked.

COOL

CORR

CP

CPLX

CR vault

Crib

Crust

CSFD
Change in waste volume due to cooling. See CORR.

Correction to waste amount-see ADJ, LBAK, COOL.

See Waste Anbreviations.

See Waste Abbreviations.

Facility located adjacent to C Farm, used for scavenging campaign following uraniurn recovery. Ferrocyanide was added to tank supernatants in $C R$ vault and the slurry was returned to C-Farm.

A linear excavation approximately $15 \mathrm{ft}$ in depth, with a perforated pipe in the bottom. The ditch is backfilled with broken rock or other Ioose material and then covered by soil and by a liquid impermeable membrane; the pipe is then used to distribute intermediate level liquid wastes along the crib.

A haxd surface layer that has formed on top of the liquid in many waste tanks that contain concentrated solutions.

see Waste Abbreviations. 
WHC-5D-WM-ER-308, Rev. O

CSR

CsT

CSWLB

CSWLW

CTW

CN

CNP

CWP/ZR

CWP2

CWR

$\operatorname{Cx} 70$

D

DBP

DC

DCS

DE

DE

Deliquescent

Desiccant

Diatomaceous Earth
See Waste Abbreviations.

See Waste Abbreviations.

See Waste Abbreviations.

See Waste Abbreviations.

Caustic waste for makeup.

See Waste Abbreviations

See Waste Abbreviations.

See Haste Abbreviations.

See LANL Defined Waste List.

See Waste Abbreviations.

See Hast Abbreviations.

Transaction Flag Key-Amount by difference.

Dibutyl phosphate.

See Waste Abbreviations.

Acroynm for dilute caustic solutions

Acronym for diatomaceous earth that was adched to Tanks 241-BX-102, -SX-113, $-\mathrm{TX}-106$, and $-\mathrm{U} \times 104$ from 1970 to 1972 .

See LaNL Defined waste List.

A solid that is capable of absorbing moisture from the air and becoming a 1 iquid.

A drying agent such as diatomaceous earth.

Diatomite, a light friable siliceous material, derived chiefly from diatom (algae) remains that is added to selected underground waste storage tanks 
DBP

DIL

DILFD

Ditch

Diveraion Box

DN/PT

$\mathrm{DN} / \mathrm{PD}$

DN

Double-Shell Tank

Double-shel1 slurry

Double-shell slurry Feed (DSSF)

Drywe11 to absorb and, thereby, immobilize residual liquids (i.e help to stabilize the tank) .

Dibutyl phosphate.

Dilution

\section{Dizute feed}

A linearly oriented excavation often used for the temporary diversion or disposal of process waste streans.

A below grade concrete enclosure containing the remotely maintained jumpers and spare nozzles for the routing of waste solution to storage tank Eaxms.

See Waste Abbreviations.

See Waste Abbreviations.

See Waste Abbreviations.

The newer underground waste storage tanks consisting of a concrete sheld and two concentric carbon steel liners with an annular space between the liners.

Faste that exceeds the sodium aluminate saturation boundary in the evaporator without exceeding receiver tank composition limits. For reporting purposes, DSs is considered a solid.

Waste concentrated jut before reaching the sodium altminate saturation boundary in the evaporator without exceeding receiver tank composition 1imits. This form is not as concentrated as DSS.

A steel casing, generally 6 inches in diameter drilied into the ground to various depths, and used to insert monitoring instruments for measuring the presence of radioactivity or moisture 
content.

Dryoll (in-tank)

DSS

DSSF

DUMM

DUMMY

DF

DWBIX

$\mathbf{B}$

$\mathrm{EB}$

BOTA

EF

BFD

END

Environs

EV

BVA?

EVAPF

Evaporator Feed
A sealed casing within a tank that is attached to a riser. It is used to insert experimental equipment, such as neutron or acoustical probes, to determine the level of arainable interstitial liquor.

See Waste Abbreviations.

See Waste Abbreviations.

See Waste Abbreviations.

See Haste Abbreviations.

See Waste Abbreviations.

See Waste Abbreviations.

Transaction flag key-waste transferred through evaporator.

See Waste Abbreviations.

Ethylenedianinetetraacetic acia.

See Waste Abbreviations.

See Waste Abbreviations.

Disconnect Cascaded Tanks, see CAS, Set.

surrounding area, vicinity.

Evaporation.

See Waste Abbreviations.

See Waste Abbreviations.

Any waste liquid that can be concentrated to form saltcake; e.g., low heat waste, dilute interstitial liquor, aged waste, and other Iadioactive waste solutions. 
WHC-SD-WM-ER-308, Rev. 0

Evaporatox-Crystallizer $242-A$ and $242-S$ waste concentration facilities that operate at a reduced pressure (vacuum) and are capable of producing a slurry containing about 30 volume percent solids at a specific gravity of greater than 1.6 .

EVS

Partial neutralization in the 242-s Evaporator.

EVT

HEDTA destruction in the 242-B or 242-T Evaporators .

FD

See Waste Abbreviations.

FECN

Ferrocyanide

Ferrocyanide wastes created during a scavenging campaign in 1953-57, see SCAV, POO, TOO.

An ion composed of iron and cyanide with FIC the chemical formula of $\mathrm{Fe}(\mathrm{CN})^{-4}$.

A Food Instrument Corporation autonatic liquid level gauge based on a conductivity probe. At tanford, they are electrically connected to a computer for data transmiesion, analysis, and reporting. Local readings may also be obtained from a dial.

First- And Second-Cycle Decontamination Wastes

Waste contained 10 percent of the original tission product activity and 2 percent of the products. By"product cake solution was mixed with product waste and neutralized with 50 percent caustic. The waste contained a mixture of suspended solids, hydroxides, carbonate and phosphate, scavenger metals, chromium, iron, sodium, and silicofluoride.

FLSH

See Waste Abbreviations.

FP

Fission Product Waste. Waste produced at $B$ Plant and Hot Semiworks in campaigns for $\mathrm{Cs}$ and $\mathrm{Sr}$ recovery during the $1960 \mathrm{~s}$. Cs was removed from PUREX supernatant and $\mathrm{Sr}$ was removed from 
WHC-SD-WM-ER-308, Rev. 0

PP

GA

Garima Ray

GAS

GM Instrument

GROTP

Gunite

$\mathrm{H}_{2} \mathrm{O}$

HDRI.

HEAT

HEDTA

Heel

Hexone

High-Level Waste

Historical Information
PUREX sludge; both were removed fIon acidic waste.

See Waste Abbreviations.

Gain to tank.

Electronagnetic radiation sometimes emitted by the nuclei of radioactive substances during decay, similar in natiare to $\mathrm{x}$-rays.

slurry growth as a result of gas generation.

Instrument for detecting low-level beta and gattina radiation using t GeigerMuelier tube.

A group of tanks where ITs averaged the supernatant phases-see ITS.

A building material consisting of a mixture of cement, sand, and water that is sprayed onto a mold.

Hater.

See Waste Abbreviations.

A tank correction, see CORR, COOL.

N hydroxy-ethylenediaminetriacetic acid.

The amount remaining in a vessel or container after most of the contents have been removed.

Methyl isobutyl ketone, an organic solvent used in the RRDOX solvent extraction process. Also known as Isobutyl methyl ketone, Methyl isobutyl ketone, 4-Methyl 2-pentanone, MIBK.

Waste from the fuel reprocessing operations in separations plants.

All information obtained from material

$$
A-12
$$


referenced in published documentation and unpublished sources. Unpublished sources include electronically retrieved data (i.e. temperatures, Iiguid observation well, bampling, and surtace level) and photographs.

HLO

HLO

EIH

BOT-SBMI

Fis

HS

HWVP

I\&S

Inactive Tank

INST

Interim Isolatea

Interin Stabilized
See Waste Abbreviations.

Hanford Laboratory Operations.

See Faste Abbreviations.

See HS, SSW.

See Waste Abbreviations.

Hot Semi-Works. A pilot facility that had a variety of operations. c plant.

Dilute, non-complexed waste from the vitrification plant.

Tank isolated and stabilized.

A tank that has been removed erom liquid-processing service, has been pumped to less than 33,000 gal of waste, and is waiting to be, or is in the process of being, stabilized and interim isolated. Includes all tanks not in active or active-restricted categories. Otherwise inactive contingency spares that would be used if an active tank railed are also included.

Change in tank level due to change in instrumentation.

An administrative designation reflecting the conpletion of the physical effort required to minimize the adaition of liquids into an inactive storage tank, process vault, sump, catch tank, or diversion box.

A tank which contains less than 50,000 gallons of drainable interstitial iiquid 
Interstitial Liguor

Interstitial

Intrusion Mode

FIC Setting

Intrusion Prevention

Iso

ITS

IWW

IX

Jet Pump

Knuckle and has less than 5,000 gallons of supernatant liquid. If the tank was jet pumped to achieve interim stabilization, then the jet pump flow must also have been at or below 0.05 gallons per minute before interim stabilization is completed.

The liquio that fills the interstices or the void volume in the solid material in a waste tank.

The volume within a solid matrix made up of the crevices, cracks, and void spaces.

The FIC probe is positioned a short distance above the waste surface. If the surface level of the waste in the tank increases, thereby touching the probe tip, a positive indication is received.

This is an administrative designation reflecting the completion of the physical effort required to minimize the addition of liquid into an inactive storage tank, process vault, catch tank, surm, or diversion box.

Tank is Interim-isolated.

The In-Tank Solidification Program was a program used to reduce waste volume directly by evaporating the wastes from inside certain tanks in the $\mathrm{BX}$ and $\mathrm{BY}$ Farms. The process used both hot air (ITS-1) and an electric heater (ITS-2).

See Waste Abbreviations.

See Waste Abbreviations.

A modified commercially avalable low capacity jet punp used as a salt well pump.

point where the side wall and the bottom 
WFC-SD-WM-ER-308, Rev. 0

curved surface of a tank meet.

L2225

$\operatorname{L3A4A}$

IaF

Lateral

Leak Detection Pit

LEAR

LETT

Level Adjustment

Level History

Liquid Observation Hell (LOW)

Liguid Level Best Engineering Judgement Line
See Maste Abbreviations.

See Waste Abbreviations.

See Waste Abbreviations.

Forizontal drywell under $A$ Farm and certain SX Farn waste storage tanks.

Collection point for any leakage from AX Farm tanks. The pits are equipped with radiation and liguid detection instruments.

Tank leak volume, see CORR.

Liquid efeluent treatment facility from N-Reactor.

Any update in the waste inventory (or tark level) in a tank. The adjustments usually result from surveillance observations or historical investigations.

A diagram that shows the history of the waste level and waste level changes in a tank. The diagram also includes other related data.

Liquid observation wells are used to monitor the interstitial liquid level (ILL) in single-shell waste storage tanks. Three probes are used to monitor changes in the ILL: acoustic, gamma, and neutron. Each method can indicate intrusions or leakage by increases or decreases in the ILI.

During the initial fill of certain single-shell tanks, only the liquid level was reported. To adjust for the big increase in level height, which occurred when solids were added to the record, a sloped line was used to reflect solids volume between the 
IO

IUNC

LW

Metal waste (MW)

Molarity

Mole

MN

MNF

$\mathbf{N}$

NCAW

NCEL

NCPLEX

NCPLX

NCRH

Neutralized BUREX Açia Waste initial fill and the time the solids data was recorded.

Loss from tank.

See Waste Abbreviations.

See Waste Abbreviations.

Waste from the extraction containing all the uranium, approximately 90 percent of the original fission product activity, and approximetely $i$ percent of the product. This waste vas brought just to the neutral point with 50 percent caustic and then treated with an excess of sodium carbonate. This procedure yielded almost completely soluble waste at. a minimum total volume. The exact composition of the carbonate compounds was not known but was assumed to be an uranium phosphate caxbonate atixture.

Number of gram molecular weights (moles) per liter of solution.

The amount of a sibstance with weight equal to the molecular weight in grams.

See Waste Rbbreviations.

See Waste Abbreviations.

See Waste Abbreviations.

See Waste Abbreviations.

See Waste Abbreviations.

See Waste Abbreviations.

See Waste Abbreviations.

See Waste Abbreviations.

The original plant in 1956 neutralized all the high-level waste and sent it to the 241-A Tank Fastn. As fission product 
WEC-SD-WM-BR-308, Rev, 0

Neutron Probe

NFAH

NHAW

NIT

Noise At The Bottom of Wel1-Drywell Probe

Non-Complexed (NCPLX)

NPH

NRAH

NRP82

SRPO4

NRSO4

NTA

Nuclear reactor

Open Hole Salt Well recovery started, a portion of the waste was treated for strontium recovery and then neutralized. As of 1967, all of the high-level waste left PUREX as an acia solution for treatment at $B$ plant.

Probe equipped with a neutron source and detector. They are used in dry well monitoring to detemine the moisture content of the soit as one means for detecting leaks in uderground waste storage tanks of pipelines.

See Waste Abbreviations.

See Waste Abbreviations.

$\mathrm{HNO}_{3} / \mathrm{KHNO}_{4}$ solution added during evaporator operation.

Erroneous reading caused by probe coming contact with foreign objects at the bottom of a well.

General waste term applied to all Hanford Site liquors not identified as complexed (containing organics).

Normal parafein hydrocarbon.

See Waste Abbreviations.

See Waste Abbreviations.

See Wuste Abbreviations.

See Waste Abbreviations.

Nitrilotriacetic acid.

A device for creating a controlled nuclear chain reaction using atomic fued, as for the production of energy.

A well in which a pump is inserted in solid waste. Frequently used to remove the liquid from tanks containing less than 2 feet of sludge. 
Organic Wash Waste(OWW) The solvent used in PUREX was treated before reuse by washing with potassium permanganate and sodium carbonate, followed by dilute nitric acid and then a sodiun carbonate wash.

Out-of-Service-Tank

OWW

P

P1 :

P2:

PADFG

RADWG

Partially Interim Isolated

PAS

PASF

DAF

PD

EDENG

PDBSU

PDETG

PDCSS
A tank that does not meet the definition of an in-service tank. Before September 1988 , these tanks were defined as inactive. (Note: all single-shell tanks (SSTs) are out of service.)

See Waste Abbreviations.

See Waste Abbreviations.

See LAM Defined Waste List.

See band Defined waste List.

See Faste Abbreviations.

See Waste Abbreviations.

The administrative designation reflecting the completion of the phyoical effort required for Interim Isolation except for the isolation of risers and piping that will be required EOr jet pumping or for other methods of stabilization.

See Waste Abbreviations.

See Naste Abbreviations.

See Wate Abbreviations.

See Waste Abbreviations.

See Nate Abbreviations.

See Waste Abbreviations.

See Waste Abbreviations .

See Waste Abbreviations. 


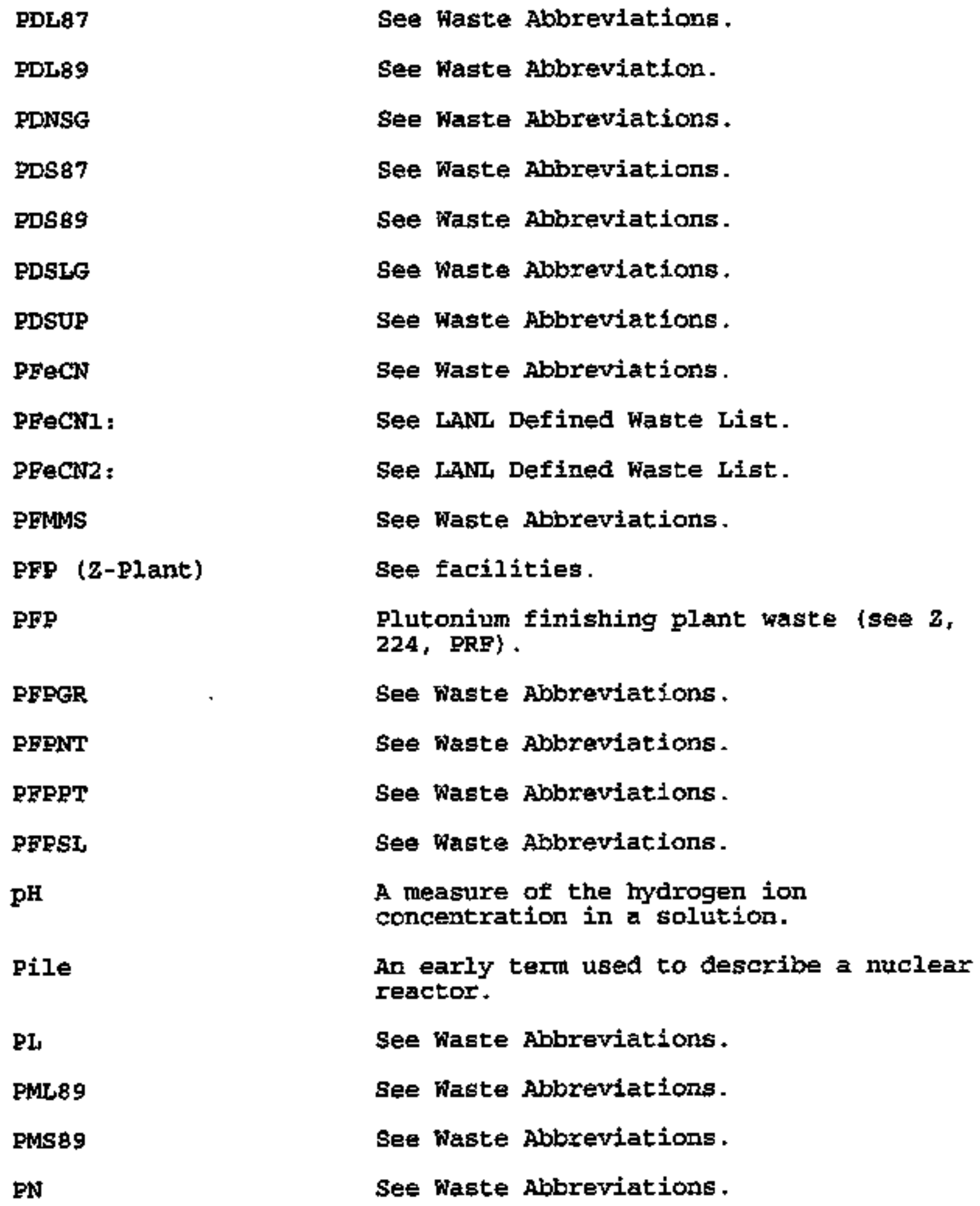


WHC-SD-WM-ER-308, Rev, 0

PNF

POO-P\#\#

Portland Cement

PRE

Primary Addition

Primary Stabilization

Probe

PSL

PSs

PSSE

Psychrometry
See Waste Abbreviations.

In-Plant scavenging with FeCl-see SCAV, TOO-T\#\#.

A hydraulic cement made by finely pulverizing the clinker produced by calcining to incipient fusion a mixture of clay and limestone or sibilar materiels.

Plutorium Reclaimation Facility-Type of waste generated in z-Plant for "finishing wastes". Solvent based extraction process using $\mathrm{CCl} / \mathrm{TBP}$.

An addition of waste from a specific plant or process vault. These additions cone from the "waste status and Transaction Record Sumuary", WHC-SD-WMTI-614\&-615 Rev. O DRAFT.

The condition of an inactive waste storage tank after all Iiquid above the solide, other than isolated surface pockets, has been removed. Isolated surface pockets of liquid are those not pumpable by conventional techniques.

A device used to get information about the environment. In this report, an instrument package designed to be inserted in drywells, risers, or ports to measure waste characteristics.

See Vaste Abbreviations.

See Waste Abbreviations.

See Waste Abbreviations.

Determination of bumidity or dew point from wet and dry bulb temperatures with the difference in the two used as a measure of aryness in the atmosphere.

See Waste Abbreviations. 
WHC-SD-WM-BR-30B, Rev. 0

RUREX

PX865

PXBAF

PXBSG

PXFTR

EXLOW

PXMET

EXMSC

PXNAW

Questionable Integrity

R

R SLTCK:

R1 :

R2:

Radiation zone

Radiation

Readily availabie

have

Risers
See Pacilities.

See Waste Abibreviations.

See Haste Abbreviations.

See Waste Abbreviations.

See Waste Abbreviations.

See Waste Abbreviations.

See Waste Abbreviations.

See Waste Abbreviations.

See Waste Abbreviations.

Any tank that has a small decrease in liquid level or a radiation increase in an associated dry well, for which the data are insufficient to support a conclusion with 95 percent confidence that the tank is sound.

See Waste Abbreviations.

See LANL Defined Waste List.

See LANL Defined Waste List.

See LaNd Defined Waste List.

An area containing radioactive materials in quantities significant enough to require control of personnel entry to the area.

Particles and electromagnetic energy emitted by nuclear transformations that are capable of producing ions when interacting with matter.

R1sers located above grade which do not apparatus attached to or contained in them. 
FEC

REDOX

Removed From Service

is not (Tanks)

Rerun Drywell Data

RESD

RIX

RMC

RSN

RSS

RTX

S

S-Plant (REDOX)

S1 SITCK :

S2 SLTSERY:

salt Cake

Salt Hell

scav

Scavenged
Receive waste Erom another tank-see XFER.

See Facilities.

Any tank that is a conflimed leaker or Intended for reuse.

Surveillance data which supersedes "suspect data".

See Haste Abbreviations.

See Waste Abbreviations.

Remote Mechanical C-Line -Process used in z-plant.

See Waste Abbreviations.

See Waste Abbrevintions.

See Naste Abbreviations.

Transaction Flag Key-partial neutralization (PNF).

See Facklities.

See IANE Defined Faste Iist.

See LaNL Defined Waste Eist.

Cryetallized nitrate and other salts deposited in waste tanks, uevally after active measures are taken to remove moisture.

A hole drilled or sluiced into a salt cake and lined with a cylindrical screen to permit arainage and jet pumping of interstitial liquors.

Scavenging campaign with FECN on TBP, 1952-57. See TOO-T\#\#, POO-P\#\#.

Waste which has been treated with ferrocyanide to remove cesium from the 
supernatant by precipitating it into the sludge.

Scintillation Monitor

Self Concentrating

Self-Evaporation

SET

$\operatorname{six}$

SL

SL3SY

sludge

Slugs

sluicing, or sluiced

slurry

Sound Tank
A radiation detection instrument based on the principle that light pulses are produced in some materials when they are exposed to radiation.

High-level Iiquid radioactive waste whose constituent radionuclides contribute sufficient decay heat to cause the solution to boil and/or selfconcentrate.

A waste material in which moisture is being lost as the moisture changes to a vapor and diffuses into the atmosphere.

Connect cascaded tanks together-see CAS and END.

See Haste Abbreviations.

See Waste Abbreviations.

See Waste Abbreviations.

Primarily insoluble metal hydroxides and oxides precipitated from neutralized waste.

An early term for uranium fuel elements which had been machined or extruded into bhort cylinders which were then clad or encased in corrosion-resistant metals.

To wash with water. At Hanford, this has meant to dissolve or suspend in solution by action of a high pressure water stream.

Insoluble material suspended in water or aqueous solution.

The classification of a waste storage tank for which surveillance data indicates there has been no loss of liquid attributed to a breach of 
integrity.

SERG

$S R R$

SRS

SRS

SSH

STAB

Stabilization

Static Tank

SU

Supernatant

(Supernatant Liquid)

surveillance

Suspect Data
Sparge-transfer of water or volume

See Faste Abbreviations.

See Waste Abbreviations.

See Waste Abbreviations.

See Haste Abbreviations.

Tank stabilized by removal of liquid. Both floating suction and salt-well jet pumps used to remove 11 quid.

The removal, or imrnobilization, as completely as possible, of the liquid contained in a radioactive waste storage tank by salt well pumping, open hole salt well pumping, diatomaceous earth addition, etc.

A tank with no significant change in liquid level or involvement in transfer operations during a stated period of time.

Strontium Semi-Works

Called C-Plant or Hot Semi-Horks, was a pilot plant for both Redox and Purex. then reconfigured for strontium recovery.

See Waste Abbreviat1ons.

In waste storage tanks, the 1iquid quantity defined by the difference between the measured liquid level and the measured average solids level in a tank.

Regular or systematic watch kept over someone or something.

Surveillance data which does not fall within the expected range and is superseded by "reruns." 
SV

SW RCR

SWLIQ

SWLON

T-Plant $(222-T)$

T1 SIFCK:

T2 SLTCK:

Tank Earm

TBP

TBP

TCO

Terminal Liquor

TFeCN

THE

Thermocouple Tree

Thermocouple,
Transaction Flag Key-Amount by difference in solids.

Salt-weli Receiver.

See Waste Abbreviations.

See Haste Abbreviation.

See Facilities.

See LaNL Defined Waste Iist.

See IAWL Derined Haste List.

An area containing a number of storage tanks; i.e., a chemical tank farth for storage of chemtcals used in a plant, or underground waste tank storage of radioactive waste.

Tributyl phosphate, a solvent used in the FURBX solvent extraction process.

See Waste Abbreviations.

See Waste Abbreviations.

The liquid product fron the evaporationcrystallization process which, upon further concentration, forms an unacceptable solid for storage in single-shell tanks. Terminal liguor is characterized by a caustic concentration of approximately $5.5 \mathrm{M}$ (the caustic molerity will be lower if the aluminum salt saturation is reached first).

see Waste Abbreviations.

See Waste Abbreviations.

A group of thermocouples assembled in a pipe and inserted into a waste tank for measuring temperatures at regular (normally 2 feet) vertical intervals.

A probe for measuring tertperature, 
Thermocouple probe

Thermoweld

TEI

Thorium

TR

TL

TOO-TH

TPLAI

TPLAN

TPLAS

TR

Trench

trFlag

Tributy 1 Phosphate (TBP) consisting of two dissimilar metal wires joined at one end (hot junction) with the free ends joined to a measuring instrument. Electrical potential changes due to temperature changes at the hot end axe measured and calibrated to read out as temperature.

A well in a waste tank which contains thermocouples.

See Waste Abbreviations.

A chemical element which is also a tertile material. By fertile is meant that when subjected to radiation in a nuclear reactor it will be converted, in this case, to ${ }^{233}$ uranium, a potential fuel.

Tank. TK-17-2, however, was an early designation for B-plant.

See Waste Abbreviations.

In-Tank scavenging with FECN, see SCAV, P\#\#.

See Waste Abbreviations.

See Waste Abbzeviations.

See Waste Abbreviations.

Transfer from taak.

A deep turyow in the ground. At Hanford they are used for the disposal of solid waste.

Transaction Blag Keys-used by W-Trac-see CDF, D, E, S, SV, I, 3, 6, 0.17, 0.33.

A chemical compound, also an organic solvent used in the PUREX solvent extraction process. 
Type I Tank

Type II Tank

Type III Tark

Type IV Tank

Type V Tank

$\mathrm{U}$-Plant (222-U)

U1U2

UNKN

UNKANOWN :

UR

UR:

Fatch ILst Tank
These are the 200 series $B, C, T$, and $U$ Farm tanks. They have an operating capacity of 55,000 gal, a $20-\mathrm{ft}$ diameter, a 6-in. dish bottom, and a 3-ft knuckle. There is no generation associated with type $I$ tanks.

These are the original, lst generation, tank designs, encompassing $B, C, T, U$ (excluaing the 200 geries tanks), and $\mathrm{BX}$ Tank Farms, Also see Ist Generation Tank.

These are the 2nd Generation tank destgns, encompasing BY, $S, T X$, and TY Tank Earins. Also see 2nd seneration Tank.

These include 3rd, 4th, and 5th generation tank designs, encompasing $s x$, $A$, and AX Tank Farms respectively. Also see $3 r d$ Generation Tank, 4th Generation Tank, and 5th Gneration Tank.

These are the first double shell tank designs, encompasing $\mathrm{AY}, \mathrm{AZ}$, and SY Tank Farms .

see Facilities.

See Waste Abbreviations.

trnknown waste origin sink.

See IAML Defined Waste List.

Uranium recovery operation in 222-U, 1952-57. Created TBP (primary waste) and FECN (scavenging wastes). See TEeCN, EFeCN, ROO, TOO, FeCN.

See IANI Defined Waste List.

An underground storage tank containing waste that recpuires special safety precautions because it may have a serious potential for release of highlevel radioactive waste because of 


$$
\text { WHC-SD-WM-ER-308, Rev . } 0
$$

uncontrolled increases in temperature or pressure. Special restrictions have been placed on these tanks by "Safety Measures for Waste Tanks at Hanford Niclear Reservation, "Section 3137 of the National Defense Authorization Act for Fiscal Year 1991, Novenber 5, 1990, public Law 101-501 (also known as the Wyden Bmendment) .

WATER

Flush water from various sources.

WTR

water.

WVP

Waste volume projections

XFER

Transfer of waste out of tank.

z

See Waste Abbreviations

z-Plant

See Facilities

Z-PLANT

PFP. Plutonium Finishing Plant.

ZAW

See Waste Abbreviations.

ZEIGH

See Waste Abbreviations.

ZLAB

See Waste Abbreviations.

ZLOW

See Waste Abbreviations.

ZPRFL

See Waste Abbreviations.

ZPRFS

See Waste Rbbreviations.

ZRM

See waste Abbreviations.

ZRMCL

See Waste Abbreviations.

ZRMCS

See Maste Abbreviations. 
WFC-SD-WM-ER-308, Rev. O

\section{FASTE ABERBVIATIONS}

IAYIN

IAZIN

IC

$1 \mathrm{CBB}$

ICS

224

224-F

2312

2AYIN

2AZIN

$2 c$

2SYIN

3AMIN

$5-6$

5AHIN

GAVIN

AGE, AGING

B

BE60N

BFSH

BL
Concentrated complex waste from 101 AY inventory

Pre 2-91 201Az inventory

First cycle waste

1st cycle evaporator bottons

1st cycle scavenging waste

224- w waste

224-U waste. LaF Pu rinishing plant

Dilute, phosphate waste from 2318 laboratories

Pre 2-81 102AY inventory

Pre 2-81 concentrated complex waste from 102Az inventory

second cycle waste

Pre 2-8I 102Sx inventory

Pre 2-8I 103AW inventory

B-Plant tank 5,6 waste

Pre 2-B1 105Aw inventory

Concentrated phosphate waste in 106Aw inventory

Aging waste

B-plant high- level waste

Dilute, non-complexed waste from 3 plant celi drainage

B-plant flush waste water

B-plant low-1evel waste 
WHC-SD-WM-ER-308，Rev , 0

BLEB

BLIX

BLIXB

BNT

BPDCC

BPDCS

BPDCV

BPFPS

BPLCS

BPLDC

BPLDN

BVCLN

CARB

CC

CCGL

CCGR

CCPL

CCPIX
B-Plant low-level evaporator bottoms

B-Plant low leve? ion exchange?

B-Plant low levei ion exchange bottoms?

Battelle Northwest Laboratory waste

Dilute, complexed waste from B Plant. cesium processing

Dilute, complexed waste from B Plant strontium processing

Dilute, complexed waste from B plant vessel clean-out

B Plant high Tro solids from retrieved PPP solids

Dilute, non-complexed waste from B Plant strontium processing

Dilute, complexed waste from B Plant cesium processing

Dilute, non-complexed waste from B Plant cesium processing

Dilute, non-complexed waste from B Plant vessel clean-out

PUREX organic wash waste

Complexant concentrate waste

B-Plant high Tru solids from retrieved complexed concentrate

Dilute, non-complexed waste from Ietrieved complexed

concentrate

Complexant concentrate

Complexant concentrate-see CPLX 


\begin{tabular}{|c|c|}
\hline $\mathrm{CF}$ & Bvaporator concentrate \\
\hline CEM & Concrete-see CON \\
\hline CF & Cesium feed \\
\hline CON & Concrete-see CEM \\
\hline$C P$ & $\begin{array}{l}\text { Concentrated phosphate waste from } \mathrm{N} \\
\text { Reactor decontatuination }\end{array}$ \\
\hline CPLXX & Conclex waste \\
\hline CSFD & Cesium feed \\
\hline $\operatorname{CsR}$ & $\begin{array}{l}\text { Waste sent to B-Plant for cesium } \\
\text { recovery }\end{array}$ \\
\hline CST & Caustic solution \\
\hline CSWLE & Conglexed salt well liquid east area \\
\hline $\operatorname{cswL} w$ & Complexed salt well liquid west area \\
\hline $\mathrm{Cw}$ & Coating waste \\
\hline CWE/ZR & $\begin{array}{l}\text { Coating waste (PUREX), zirconium } \\
\text { cladding }\end{array}$ \\
\hline CWP & Coating waste (PUREX) \\
\hline CWR & Coating waste (REDOX) \\
\hline $\operatorname{cx} 70$ & $\begin{array}{l}\text { Dilute, complexed mixture hot-semiworks } \\
\text { Tru solids }\end{array}$ \\
\hline DC & Dilute complexed waste \\
\hline $\mathrm{DN} / \mathrm{PD}$ & DN with P Tru solids \\
\hline DN & Dilute non-complexed waste \\
\hline $\mathrm{DN} / \mathrm{PT}$ & DN with PFP Tru solids \\
\hline DSS & Double-shell slurry \\
\hline DSSE & Double-shell Blurry feed \\
\hline
\end{tabular}


DOMIM

DUMMY

DW

DWBIX

BE

EF

EFD

EVAP

EVAPF

FD

FLSH

FP

HDRL

HEO

HLW

HS

IWW

IX

L222S

L3A4A

LaF
Dunmy waste

Dummy waste

Decontamination waste

Decontamination waste and B-Plant ion exchange

Evaporator bottorus

Bvaporator feed

Evaporator Eeed dilute

Evaporator feed (post 1976)

Dilute, non-complexed waste from evaporator pad flush

Feed ailute

Flush water

Fission product waste

Hanford defense residual liquor

Hanford laboratory operations waste

High level waste-generic for all Hanford tank wastes

Hat semiworke waste

PUREX \#1 acid concentrator waste

Ion exchange waste

2225 laboratory, dilute non-coinplexed waste

Dilute non-complexed laboratory wastes from 300 \&s 400 areas

Lanthanum fluoride waste generated in PFP 
LONC

LN

NW

MNF

N

NCAW

NCPLEX

NCPLX

NCEL

NCRW

NFAY

NEAF

NRAW

NRPB2

NRPO4

NRSO4

OWพ

$\boldsymbol{P}$

PADFG

PADWG
Dilute, non-complexed waste from tnc fuels fabrication

Laboratory waste

Metaz waste

Metal waste feed

w-Reactor waste

Neutralized current acid waste, primary HLW stream from PUREX process

Non-complexed waste-see NCPLX

Non-complexed waste-see NCPLEX

fon-Complexed waste

Neutralized cladding removal waste-same as CWP.

Aglng waste from PUREx/EFM high level waste

Aging waste Erom PUREX/PFM processing of NPR fuel

Aging waste troin PUREX/PFM reslaue acid waste

Dilute, non-complexed waste from FYs2 100-is area waste transfer

Dilute, phosphate waste from looN area

Dilute, non-complexed wate from $100 \mathrm{~N}$ area

Organic wash waste

PUREX wagte

Purex amonia destruction waste, from Euel: grade fuel

Purex ammonia destruction waste, from 
WHC-SD-WM-ER-308, Rev. 0

weapons grade fuel

PAS

PASE

PAW

PD

PDBNE

PDBSU

PDETG

pDCsS

PDL87

PDL89

PDNSG

PDS87

FDS89

PDSLG

PDSUP

PFeCN

PFMMS

PFPGR

purex acidtfied sludge

Purex anuonia scrubber feed

Purex acidified waste

Purex decladding taste

Decladding sludge (non-Tru) from B plant processing

Dilute, non-complexed waste from B Plant decladding waste

B Plant aging waste solids from Purex decladding waste

Dilute non-complexed Purex decladding waste, FY1986 only

purex decladding supernatant, 1987

Purex decladding supernatant, non-Tru, spent metathesis removed

Non-Tru decladding sludge from Purex

Purex decladding sludge

Furex decladding sludge after FY89

Purex decladding sludge sol Furex

Dilute, non-complexed waste, Purex decladding waste

Ferrocyanide sludge produced by in-plant scavenging of waste from uranium recovery

Dilute, non-complexed waste from shear/leach processing of NPR fuel

Dilute, non-complexed waste from retrieved PFP solids 
WHC-SD-FM-ER-308, Rev. 0

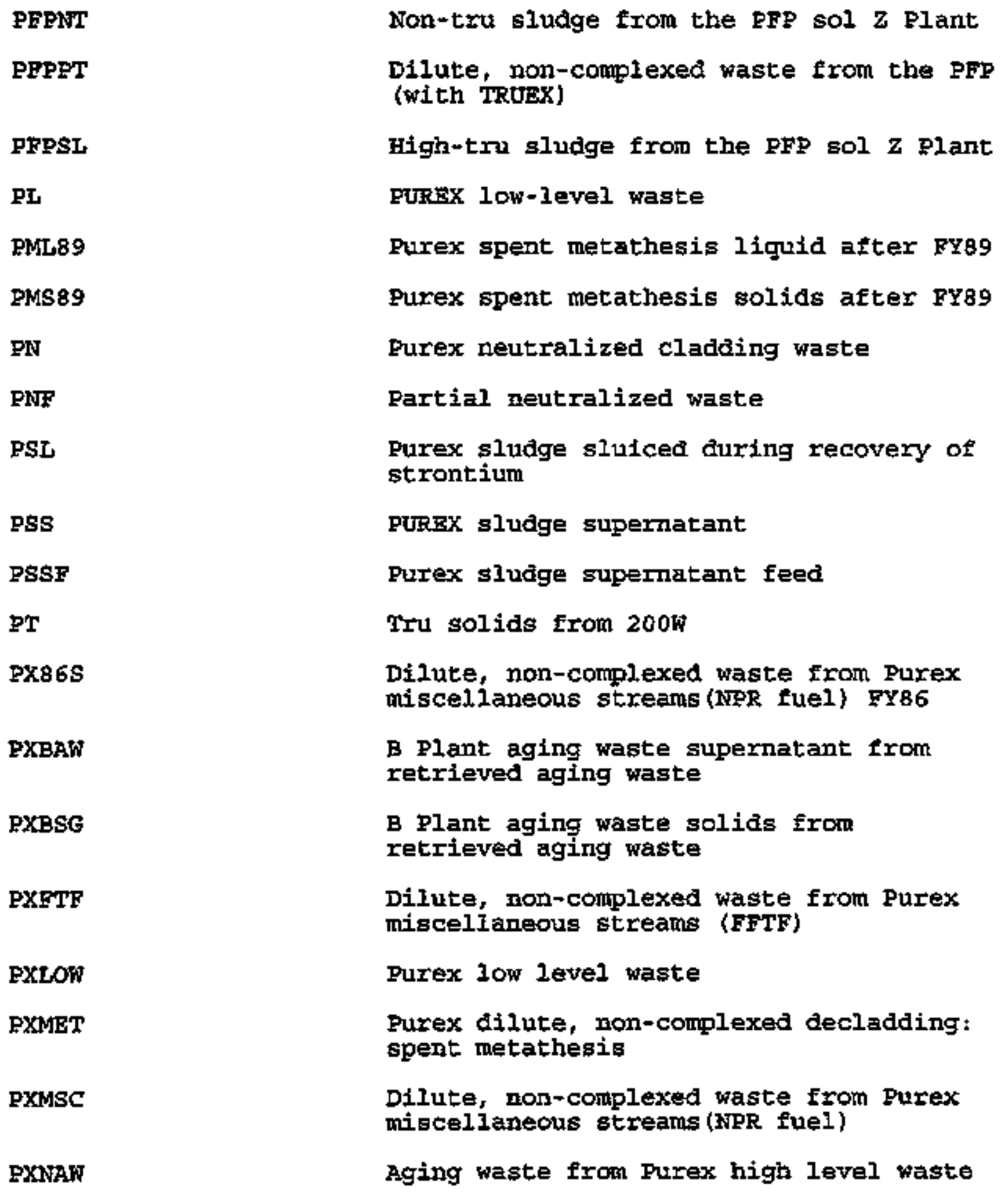


WEC-SD-WM-ER-308， Rev. 0

$\mathbf{R}$

RESD

RIX

RSN

RSS

RTX

SIX

SL

SL3SY

SRR

SRS

SRS

SSW

SU

SWIIQ

SWLOW

TBP

TCO

TFeCN

TH

THL
REDOX waste

Residual evaporator liquor

REDOX ion exchange waste

REDOX supernatant

Redox sludge supernatant

Redox ion exchange

PUREX ion exchange waste

sludge

Double shell slurry from end of FY80, 103SY inventory

Waste sent to B-Plant for strontium recovery

strontium sludge

Strontium recovery supernatant

Strontium semiworks waste

Supernatant

Dilute, non-complexed waste from east area single shell tanks

Dilute, non-complexed waste Irom west area single shell tanks

Tri-Butyl Phosphate

Dilute non-complexed waste from terminal cleanout

Ferrocyanide sluage produced by in-tank or in-Farm scavenging

Thoria HLW or cladding waste

Thoria low level waste 
WEC-SD-WM-ER-30B，REV. 0

TH

TPLAT

TELAN

TPLAS

J1U2

z

ZAพ

ZHIGH

ZIAAB

ZIOW

ZPRFL

ZPRFS

ZRMCL

ERMCS
Terminal 1iquor

DiIute, non-complexed waste from $T$ Plant

Dizute, non-complexed waste from $T$ Plant

Sludge from T Rlant operations

Dilute, non-complexed waste from U1/U2 groundwater pumping

z-Plant waste

Burex waste stream from zirconium cladded Euel

Dilute, non-complexed waste from the PFP (without Truex)

Dilute, non-complexed waste from the PFP laboratories

Dilute, non-complexed waste from PreFYB5 z plant operations

Dilute, non-complexed waste from PRF processing

PFP Tru solids from PRF processing

Dilute, non-complexed waste from PFP RMC processing

pFP Tru solids from PFP RMC processing 
WHC-SD-WM-ER-308，Rev. O

A. LANL Defined Wastes

IANT DEFINGD MASTE IIST

A1 SLTCK

A2 SLTSLRY

$A R$

B SLTCK

BY SLTCK

CWP2

DE

P1

P2

PFeCN1

PFeCR2

R SLTCK

R1

R2
Saltcake waste generated from the 242-A evaporator-crystellizer from 1977 until 1980 .

Salt slurry waste generated from the 242-A evaporator-crystallizer from 1981 until 1994.

"Washed" PURRX sludge fromthe AR vault.

Saltcake waste generated from the 242-B evaporator from 1951 until 1955.

Saltcake waste generated from in-tank solidification unts 1 and 2 between 1965 and 1974 .

Cladding waste-PUREX 2???

Diatonaceous earth.

FUREX high-level waste gererated between 1955 and 1962.

EUREX high-level waste genersted between 1963 and 1967.

Ferrocyanide sludge generated from inplant scavenging of waste from uranitm recovery.

Same as PFeCN1, except used $0.0025 \mathrm{M}$ ferrocyanide.

Salt cake waste from the RBDOX concentrator.

REDOX waste generated between 1952 and 3957 .

REDOX waste generated between 1958 and 1966 . 
WHC-SD-WM - ER-308, Rev. 0

S1 SLTCK

S2 SLTSLRY

T1 SLTCK

T2 SLTCK

UNKNOWN

UR
Saltcake waste generated from the 242-S evaporator/crystallizer from 1973 until 1976.

Salt slurry waste generated from the 242-s evaporatox-crystallizer from 1977 until 1980 .

Saltcake waste generated from the 242-T evaporator from 1951 until 1955.

Saltcake waste generated from the 242-T evaporator trom 1965 until 1955.

Unkown waste type.

Draniun Iecovery waste (also known as tributyl phosphate (TBP) waste). 
WHC-SD-WM-BR-308，Rev. 0

\section{FACIIITIES}

A Plane (PUREX)

B Plant (222-B)

C Plant (222-C)

S Plant (REDOX)

T plant (222-T)

t Plant (222-j)

PFP (z Plant)

$242-A$

$242-B$

242-S

$242-T$
The facility at Hanford which contains the latest solvent extraction process for recovery of both piutonium and uranium.

On of the three original bismuthphosphate processing facilities. Later converted to a waste Eractionation plant.

Initially a pilot plant for Redox, later a pilot plant for Purex and B-Plant waste partitioning.

The facility at Hanford which contains the original extraction process for recovery of both plutonium and uraniurn.

One of the three original bismuthphosphate processing facilities. Later converted to a decontamination facility.

One of the three original bismuthphosphate processing facilities. Later converted to a uranium recovery plant.

Plutonium Finishing Plant. The final operations for production of plutonium products are carried out in this facility.

A forced circulation vacuum evaporation systern.

An evaporator that operates at atmospheric pressure. Also referred to as an open air type evaporator.

A forced circulation vacuum evaporation systern.

An evaporator that operates at atmospheric pressure. Also referred to as an open air type evaporator. 
WHC-SD-MM-ER-308, Rev.

0

\section{REFERENCES}

Agnew, S.F., February 1994, Waste Status and Transaction Record Stmmary for the Northeast Quadrant, WHC-SD-WM-TI-615, REV. O, Westinghouse Hanford Company, Richland, Hashington.

Agnew, S.F., February 1994, Waste status and Transaction Record Sumary for the southwest Quadrant, WHC-SD-WM-TI-614, REV. 0 , Westinghouse Hanford Company, Richland, Washington.

Agnew, S.F. April 1994, Hanford Defined Wastes: Chemical and Radionuclide Compositions, LA-UR-94-2657, REV. 0, Los Alatios National Laboratory, Los Alamos, New Mexico.

Alien, G.K., March 1976, Estimated Inventory of Chemicals Adied to Underground Waste Tanka, 1944 through I975, ARH-CD-610B, Atlantic Richfield Hanford Company, Richland, Washington.

Alatad, A.T., September 19, 1991, Riser Configuration Docunent for single-shell Waste Tanks, WHC-SD-Rg-TT-053, Rev. 8, Westinghouse Hanford Company, Richland, Washington.

Anderson, J.D., January, 1972- December 1972, Waste Status Summary - Chemical Processing Division, ARH-2456, Atlantic Richfield Hanford Company, Richland, Washington.

Anderson, J.D., January, 1973-December 1973, Waste Status Summary - Chemical Processing Division, ARH-2794, Atlantic Richfield Hanford Company, Richland, Washington.

Anderson, J.D., January, 1974-December 1974, Waste Status Stmmaxy - Operations Division, ARH-CD-133, Atlantic Richfield Hanford Company, Richland, Washington.

Anderson, J.D., January, 1974-December 1974, Waste Status Stumary - Chemical Processing Division, ARH-CD-133, Atlantic Richfield Hanford Company, Richland, Washington.

Anderson, J.D., January, 1975- December 1975, Waste Status Summary - Production and Waste Management Division, ARH-CD-336, Atlantic Richfield Hanford Company, Richland, Washington.

Anderson, J.D., Jaruary, 1976- September 1976, Waste status Summary - Production and Waste Masagement Divisfon, ARH-CD-702, Atlantic Richfield Hânford Company, Richland, Washington.

Anderson, J.D., Octobex, 1976- May 1977, Waste Status summary Production and Waste Management Division, ARH-CD-822, Atlantic Richfield Hanford Company, Richland, Washington.

Anderson, J.D., June 1990, A History of the 200 Areas Tank Farms, WHC-MR-0132, Westinghouse Hanford Company, Richland, Washington. 
ARCHO, October, 1967, wonthly Report, 200 Areas Operation, ARH-60 DEL, Atlantic Richfield Hanford Company, Richland, Washington.

ARHCO, June, 1969, SX Tank Farm Air Cooling Safety Analyois, ARH-1291, Atlantic Richfield Hanford Company, Richland, Washington.

ARHCO, July, 1972 through December, Chemical Processing Division Waste Status Sumpary, Quarterly Reports, ARH-2456 C and D, Atlantic Richfield Hanford Company, Richland, Washington.

ARHCO, January, 1973 through December 1973, Chemical Processing Division Waste Status Sumary, QuarterIy Reports, ARH-2694 A, B, C, and D, Atlantic Richfield Hanford Company, Richland, Washington.

ARHCO, January, 1973 through September 1975, Operations Division Waste Status Summary, QuarterIy Reports, ARH-CD-133 A, B, C, and D, At lantic Richfield Hanford Company, Richland, Washington.

ARCHO, September, 1976, Production and Waste Management Division Waste Statug Sumbary, Monthly Reports ARH-CD-702, Atlantic Richfield Hanford Company, Richland, Washington.

ARCHO, October, 1976 through June 1977. Production and waste Management Division Waste Status Summary, Monthly Report, ARH-CD-822, Atlantic Richfield Hanford Company, Richland, Washington.

Babad, H., D. M. Camaioni, M. A. Lilga, W. D. SamreIs, and D. M. Strachan, Eebruary 1993, Tank Waste Chemistry-A New Understanding of Waste Aging, WHC-SA-1694-FP, Westinghouse Hanford Company, Richland, Washington.

Boomer, R.D., June 1990, Functional Reguirement Baseline for the Closure of Single-ShelI Tanks, WHC-EP-0338, Westinghouse Hanford Company, Richland, washington.

Borsheim, G.L., and B. C. Simpgon, October 1991. An Assessment of the Inventories of the Ferrocyanide Watchlist Tanks, WHC-SD-WMER-133 Rev. 0, Westinghouse Hanford Company, Richland, Washington.

Borsheim, G.I., August 2, 1989, Single-Shell Tank Isolation Safety Analysis Repört, SD-WM-SAR-006-Rev 2, Wegtinghouse Hanford Company, Richland, Washington.

Borshiem, G. I. and N. W. Kirch, March 1991, Summary of Single-Shell Tank Waste Stability, WHC-EP-0347, Westinghouse Hanford Company, Richland, Washington. 
Boyles, V.C., June 1981, Safety Analysis Report; Strbilization of Single-Shell Waste storage Tanks by Saltwell Jet Pumping, RHO-SD-WM-SAR-034, Rev.0, Rockwell Hanford Operations, Richland, Washington.

Brevick, C.H., L. A. Gaddis, and W. W. Pickett, Juse, 1994, Historical Tank Content Estimate Report for the Northeast Quadrant of the Hanford 200 East Area, WHC-SD-WM-ER-349, Rev,0, ICF Kaiser Hanford Company, Richland, Washington.

Brevick, C.H., L. A. Gaddis, and W. W. Pickett, June, 1994, Hiatorical Tank Content Estimate Report for the Southwest Quadrant of the Hanford 200 West Area, WHC-SD-WM-ER-352, Rev.0, ICF Kaiser Hanford Company, Richland, Washington.

Brown, W.G., April 26.1993, Hazard rcientification and Evaluation for Non-Stabilized Single Shell Tanks, SD-WM-SAR-022, Rev. 0, Westingholsse Hanford Company, Richland, Washington.

Bussel1, J.F, February 3, 1992, Engineering Evaluation of Thenmocouples in FeCN Watchlist Tanks, WHC-SD-WM-ER-134, Rev O-A, Westinghouse Hanford Company, Richiand, Washington.

Cain, R.J., I977, Isolation and stabilization of Special Tanks, (Internal Letter to J.C. Womack) Rockwell Hanford Company, Richland, washington.

Carpenter, G.K., April, 1953 - June 1953, Waste Status Summary Separations Section, HW-28043 etc., General Electric Company, Richland, Washington.

Carpenter, G.K., September, 1953 - May 1954, Waste Status Summary - Separations Section, H-29624 etc., General Blectric Company, Richland, Washington.

Carter, George J., April, 1986 - September 1986 , Waste Status Sumary, RHO-RE-SR-14, Rockwel1 Hanford Operatjons, Richland, Washington.

Cooley, C.R., and G.L. Richardson, July 17, 1963, Hot Semiworks strontium-90 Recovery program, HW-72666, General Electric Company, Richland, Vashington.

BRDA, Decembex, 1,975, Waste Management Operations, BRDA-1538, Energy Research andobelopment Administration, Richland, Washington.

Escobar, Glenn A., October, 1986-January 1987, Waste status Sumpary, RHO-RE-SR-14, Rockwell Hanford Operations, Richland, Washington. 
WHC-SD-WM-ER-308, Rev, O

Faxley, W.G., May 20, 1992, Safety Assessment for Thermocouple Tree System Installation and Operation in Nonleaking Ferrocyanide Tanks, WHC-SD-WM-SAD-014, Rev, 1, Weetinghouse Hanford Company, Richland, Washington.

GE Co., July 10, 1951, REDOX TechnicaI Manual, HW-18700-Del, General Electric Company, Richland, Washington.

Gerber, M.S., September, 1992, Legend and Legacy: Fifty Years of Defense Production at the Hanford site, WHC-MR-0293, Rev 2, Westinghouse Hanford Company, Richland, Washington

Gerber, M.S., Novenber, 1993, A Brief History of the Purex and $\mathrm{VO}_{3}$ Facilities, WHC-MR-0437, Westinghouse Hanford Company,

Richland, Washington.

Gerber, M.s., September,1993, Multiple Missions: The 300 Area in Hanford Site History, WHC-MR+0440, Westinghouse Hanford Company, Richland, Washington.

Hanlon, B.M., November, 1989- December, 1990, Tank Farm Surveillance and Waste Status Sumary Report, WHC-EP-0182, Westinghouse Hanford Company, Richland, Washington.

Hanlon, B.M., January, 1991-December, 1991, Tank Farth Surveillance and Waste Status Summary Report, WHC-EP-0182, Hestinghouse Hanford Company, Richland, Washington.

Hanlon, B.M., January, 1992-December, 1992, Tank Farm Survejliance and Waste Status Summary Report, WHC-EP-0182, Hestinghouse Hanford Company, Richland, Washington.

Hanlon, B.M., July, 1993, Tank Farm Surveillance and Waste Status Summary Report for JuIy I993, WHC-EP-0182-64, Hestinghouse Hanford Company, Richland, Washington.

Hanlon, B.M., January, 1993-October, 1993, Tank Farm Surveillance and Waste Status Sumary Report, WHC-BP-0182, Westinghouse Hanford Company, Richland, Washington.

Hatch, P., December 18, 1969, Limitations for use of Underground Waste Tanks, ARH-951, Atlantic Richfield Hanford Company, Richland, Washington.

HEW, January 13, 1944, CVI, "Specifications for Construction of Composite storage Tanks Blag. No. 241," Project 9536, CVI 73550, Hanford Bngineer Works, Richl,and, Washington.

Jenkins, C., M. Danielson, B. Rarig, R. Campbell, T. Ambalam, C. Kenoyer, and L. Hall, February, 1993, Engineering Evaluation of Alternatives Managing the Assumed Leak from SST 241-T-IOI, EEA93-1, Kaiser Engineers Hanford, Richland, Washington. 
Jungfleisch, F. M., March 1984, Preliminary Estimation of the Wate Inventories in Hanford Tanks through 1980, SD-WM-TI-057, Rev.0, Rockwell Hanford Operations, Richland, Washington.

KEH, February 1993, EBP Managing the Assumed Leak From SST, KEEA93-1, Kaiser Engineers Fanford, Richland, Washington.

Kirkman, M.J., G. L. Ritter, and P. H. Smith, March 6, 1969, Bplant Produation Schedule and In-Tank Solidification

Alternatives, ARH-900, Atlantic Richfield Hanford Company, Richland, Hashington.

Kreig, S.A., W.W. Jenkins, K.J. Leist, K. G. Squires, and J. F. Thompson, June 1990, Single-Shell Tark Waste Retrieval study, WHC-EP-0352, UC-721, Westinghouse Hanford Company, Richland, Washington.

Lentz, J.E., Apri.I 1959- December 1959, Waste Statis Sumnary Chemical Procesaing Department, HW-58579 etc., General Electric Company, Richland, Washington.

Ientz, J.E., January 1960- December 1960, Waste Status Summary Chemical Procesaing Depaxtment, HW-63896 etc., General Electric Company, Richland, Washington.

Ij.verman, J.L., December 1975, Final Envirommental statement, Waste Management Opexationg Volume $I$ and 2, ERDA-1538, Energy Research and Development Administration, Richland, Washington.

Lucas, G.E., June 1989, Waste Types in Hanford Single-shell Tanks, WHC-SD-ER-TI-001, Rev. 0, Hestinghouse Hanford Company, Richland, Washington.

McBride, J.P., July, 1953, Waste Status Stmmery - Separations Section, HW-29054, General Electric Company, Richland, Washington.

McCann, D.C., July, 1981-October 1981, Waste status Sumary, RHO-CD-14, Rockwell Hanfoxd Operations, Richland, Washington.

McCann, D.C., October, 2981- December 1982, Waste Status Summary, RHO-RE-SR-14, Rockwell Hanford operations, Richland, Washiagton.

McCann, D.C., January, 1983- Decenber 1983, Waste Status Summary, RHO-RE-SR-14, Rockwëll Hanford Operations, Richland, Washington.

MeCamn, D.C., January, 1984- February 1985, Waste Status Sumary, RFO-RE-SR-14, Rockwell Hanford Operatione, Richland, Washington.

Morganthaler, A.C., February, 1952 - March 1953, Waste Status Summary - Separations Section, Hw-27897 etc., General Electric Company, Richland, Washington. 
Mudd, o.C., January, 1979- December 1979, Waste Status Stmmary, RHO-CD-14, Rockwell Hanford Operations, Richland, Washington.

Mudd, 0.C., Januaxy, 1980-December 1980, Waste status Surmary, RHO-CD-14, Rockweli. Hanford Operations, Richland, Washington.

Mudd, O.C., January, 1981- June 1981, Waste Status Summary, RHoCD-14, Rockwell Banford Operations, Richland, washington.

Mulvey, C.A., June, 1977- December 1977, Waste Status Sumary, RHO-CD-14, Rockwell Hanford Operations, Richland, Washington.

Mulvey, C.A., January, 1978- December 1978, Waste Status Summary, RHO-CD-14, Rockwell Hanford Operations, Richland, Washington.

Peterson, D.E., June, 1954-May 1955, Waste Status Sumary Separations section, HW-32389 etc., General Electric Company, Richland, Washington.

Peterson, D.E., June, 1955- May 1956, Waste status Sumary Separations section, HW-38000 etc., General Electric Company, Richland, Washington.

Peterson, D. $\mathbf{.}$, Septembex, 1956-December 1956, Waste Status Summary - Chemical processing Department, HW-45738 etc., General Electric Company, Richland, Washington.

Peterson, D.B., June, 1956-August 1956, Waste Status Summary Separations Section, HW-43895 etc., Ceneral Electric Company. Richland, Washington.

Pineg, A.G., January 4, 1991, 244-AR Vault Safety Analysig Repoxt, SD-WM-SAR 018 Rev, 0 , Westinghouse Hanford Company, Richliand, Washington.

Prosk, W., and D.A. Smith, January 1986, Tank Isolation Safety Anelysis Report, SD-WM-SAR-006 Rev. I, Rockwell Hanford Operations, Richland, Washington.

RHO, June 1977 through October 1981, Waste status Summary, Monthly Reports RHO-CD-14, Rockwell Hanford Operations, Richland, Washington.

RHO, November 1981 through June 1987, Waste Status Sumpary, Nonthly Reports RHO'RE-SR-014, Rockwell Hanford Operations, Richland, Washington.

Roberts, R.E., January, 1957-December 1957, waste Status Sumary - Chemical Processing Department, HW-48144 etc., General Electric Company, Richland, Washington.

Roberts, R.E., January, 1958- October 1958, Waste status Summary - Chemical Procesaing Department, HW-54916 etc., General Electric company, Richland, Washington. 
Roberts, R.E., January, 1961- December 1961, Waste status Summary - Chemical Processing Department, Hw-71610 etc., General Electric Company, Richland, Hashington.

Roberts, R.E., January, 1962- December 1962, Waste Status Summary - Chemical Processing Department, HW-74647 etc. General Electric Company, Richland, Washington.

Roberts, R.E., January, 1963- December 3963, Waste Status Summary - Chemical Processing Department, HW-78279 etc., General Electric Company, Richland, Washington.

Roberts, R.E., January, 1964-December 1964, Wagte Status Sumnary - Chemical Processing Department, HH-83308 etc., General Electric Company, Richland, washington.

Roberts, R.E., January, 1965- December 1965, Weste status Sumary - Chemical Processing Department, HW-83906 etc., General Electric Company, Richland, Washington.

Robexts, R.E., October, 1966- December 1966, Waste Status Summary - Chemical Procegsing Division, Is0-674, Isochem Inc., Richland, Washington.

Roberts, R.E., January, 1966-September 1966, Waste Status Summary - Chemical Proceseing Department, HW-83906 etc., General Blectric Company, Richland, Washington.

Rodenhizer, D.G., September 30, 1987, Hanford Waste Tank SIuicing History, SD-WM-TI-302, Westinghouse Hanford Company, Richland, Washington.

Rutherford, M.J., August 31, 1948, Additional Waste Storage Facilitieg 200-East Area, Specification No. HW 3783, General Electrise Company, Richland, Hashington.

Scaief, C.C. III, October 19, 3993, TMACS I/O Termination Point Listing, WHC-BD-WM-TI-594, Rev, 0, Westinghouse Hanford Company, Richland, Fashington.

Smith, D.A., August 1986, SAR, Hazard Identification and Evaluation fox Nonstabilized single-Shell Tanks, SD-WM-SAR 022 Rev. 0, Rockwell Hanford Operations, Richland, Washington.

Stahl, S.M., Novembéri 22, 3993, Hanford Site Tank Faxa Pacilities Interim safety Basis, Volume $I$ and 2, WHC-SD-WM-ISB-001, Hestinghouse Hanford Company, Richland, Washington.

Stong, F.S., September 29, 19860, Drywell Van In-Tank Liquid Observation Wel I Survelllance Data Interpretation, RHO-SD-WM-TI-237, Rockwell. Hanford Operations, Richland, Washington. 
ThanMai, T. T., Apxil 27, 1993, Thermocouple status Single-shell and Double-Shell waste Tanks, WHC-SD-WM-TI-553, Rev. 0 , Westinghouse Hanford Company, Richland, Washington.

Thress, M A., Novenber 1958-February 1959, Waste Status Sumary - Chemical Processing Department, Hw-58579 etc., General Electric Company, Richland, Washington.

Thurman, Jack M., June, 1987 through December 1987, Tank Farm Surveiliance and Waste Status Sumary Report, Monthly Reporta WHC-SP 0038, Westinghouse Hanford Company, Richland, Washington.

Thurman, Jack M., December, 1987 through June 1993, Tank Farm Surveillance and Waste Status Summary Report, Manthly Reports WHC-EP-0182-55, Westinghouse Hanford Company, Richland, Washington.

Thurman, Jack M., February, 1987- June 1987, Waste Status Sunnary, RHO-RE-SR-14, Rockwell Hanford Operations, Richland, Washington.

Thurman, Jack M., July, 1987-March, 1988, Waste Status Sumnary, wHC-\$p-0038, Westinghouse Hanford Company, Richland, Washington.

Thurman, Jack M., April, 1988- December, 1988, Tank Farm Surveiliance and Waste Status Sumary Report, HHC-EP-0182, Westinghouse Hanford Company, Richland, Washington.

Thurman, Jack M., January, 1989- October, 1989, Tank Farm Surveillance and Waste Statug Stumaxy Report, WHC-EP-0182, Wentinghouse Hanford Company, Richland, Washington.

U.S.DOE, December 1987, EIS, Disposal of Hanford Defense High Level, Transuranic, and Tank Wastes, DOE/EIS-011.3 Volume 1-5, Department of Energy, Richland, Washington.

Uebelacker, D.I., October, 1968-Decembex 1968, Waste Statre Sumnary - Chenical Processing Division, ARH-1061, Atlantic Richfield Hanford Company, Richland, Nashington.

Uebelacker, D.L., January, 1969-December 1969, Waste Status Summary - Chemical Processing Division, ARH-1200, Atlantic Richfield Hanford Company, Ríchland, Washington.

Uebelacker, D.I., Jänuary, 1970- December 1970, Waste Status Summary - Chemical Processing Dtvision, ARH-1666, Atlantic Richeield Hanford Company, Richland, Washington.

Va11, Texry S., January, 1985- March 1986, Wagte Status Sumary, RHO-RE-SR-14, Rockwell Hanford Operations, Richland, Washington.

Wagoner, J.D., March 18, 1993, Wyden Request, Document No. $9301156 \mathrm{~B} \mathrm{R3}$, U.S. Department of Energy, Richland, Washington. 
Waite, J.I., April 1991, Tank Wastes Discharged Directly to the Soil at the Hanford $g_{i}$ te, WHC-MR-227, Westinghouse Hanford Company, Richland, Washington.

Welty, R. K., September 1988, Waste Storage Tank Status and Leak Detection Criteria, WHC-SD-WM-TI-356, Testinghouse Hanford Company, Vol. 1 and 2, Richland, Washington.

Welty, R.K. and Norma J. Vermeulen, September 12, 1989 with ECNs through November 08, 1993, Waste storage Tank Status and Leak Detection Criteria, WHC-SD-WM-TI-357, Rev. 1K, Westinghouse Hanford Company, Richland, Washington.

HHC, September, 1991, Fallure to Continuously Man Control Room During Transfer Per Procedire, Occurrence Report RI--WHC--Tank Farm-1991-1019, Vestinghouse Hanford Company, Richland, Washington.

WHC, July, 1991, High Radiation Readings in 241-BY Drywells, occurrence Report RI--WHC-Tank Farm-1991-1022, Westinghouse Hanford Company, Richland, Washington.

WHC, Auguet 1993, Hanford Site Tank Farm Factlities Interim Safety Basis, HHC-SD-WM-ISB-001 Rev, 1 , volume 1 and volume 2. Westinghouse Hanford Compary.

WHC, March, 1993, Hanford Watchlist Tank Survelliance Trends, Letter to the Honorable Ron Wyden, Westinghouse Hanford Conpany, Richland, Washington.

WHC, July, 1993, Unauthorized Water Usage at 24I-BY Tank Farm Results in Exceeding Raw Water Operational Limit, Occurrence Report RI--mHC-Tank Farm-1993-0050, Westinghouse Hanford Company, Rjchland, Hashington.

Winters, W.I., I. Jensen, L.M. Sasaki, R.L. Weiss, J.F. Keller, A.J. Schmidt, and M.G. Woodruff, May 1989, Waste Characterization Plan for the Hanford site single-shell Tanka, HHC-BP-0210, Westlnghouse Hanford Company, Richland, Waghington. 


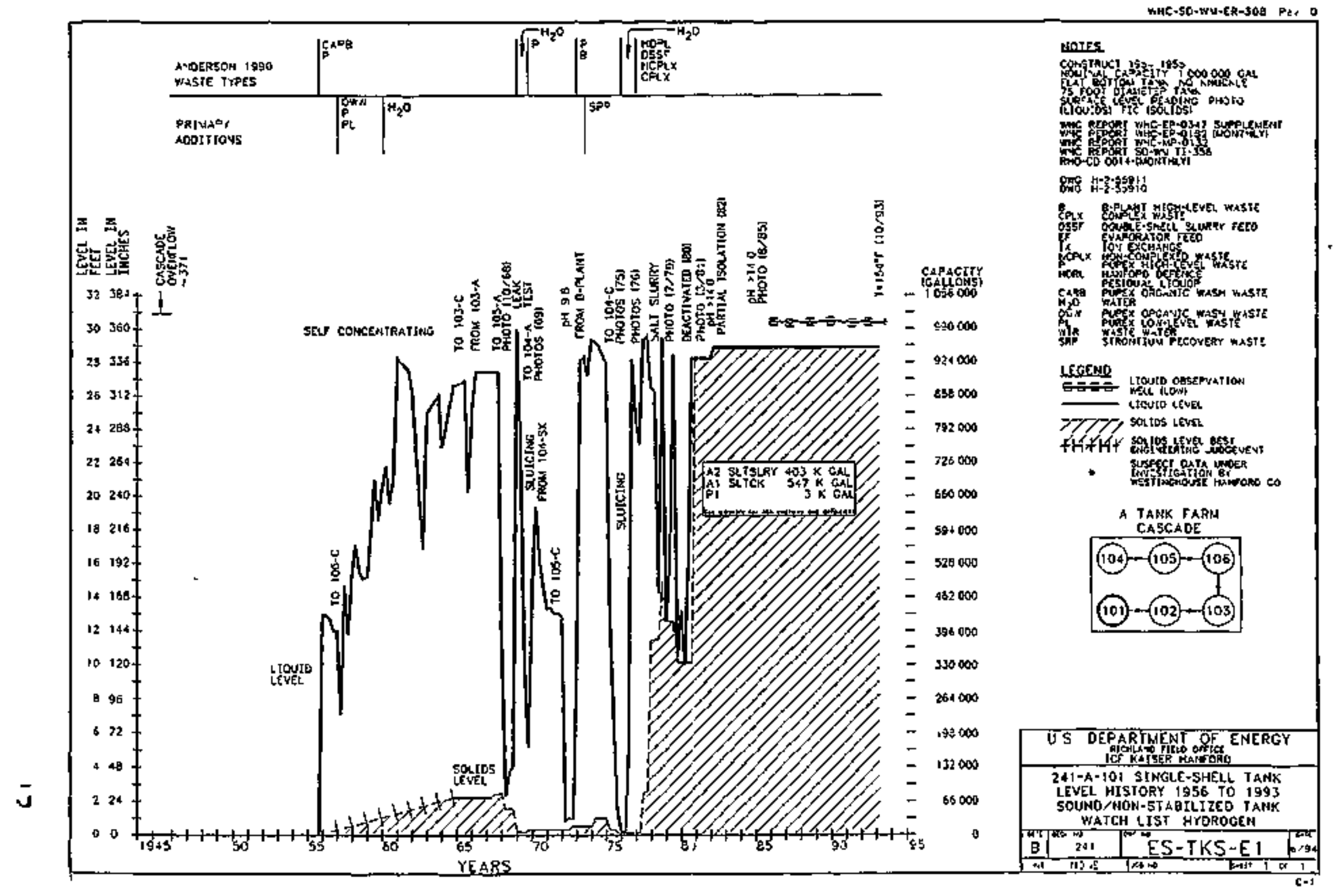


- - -

6

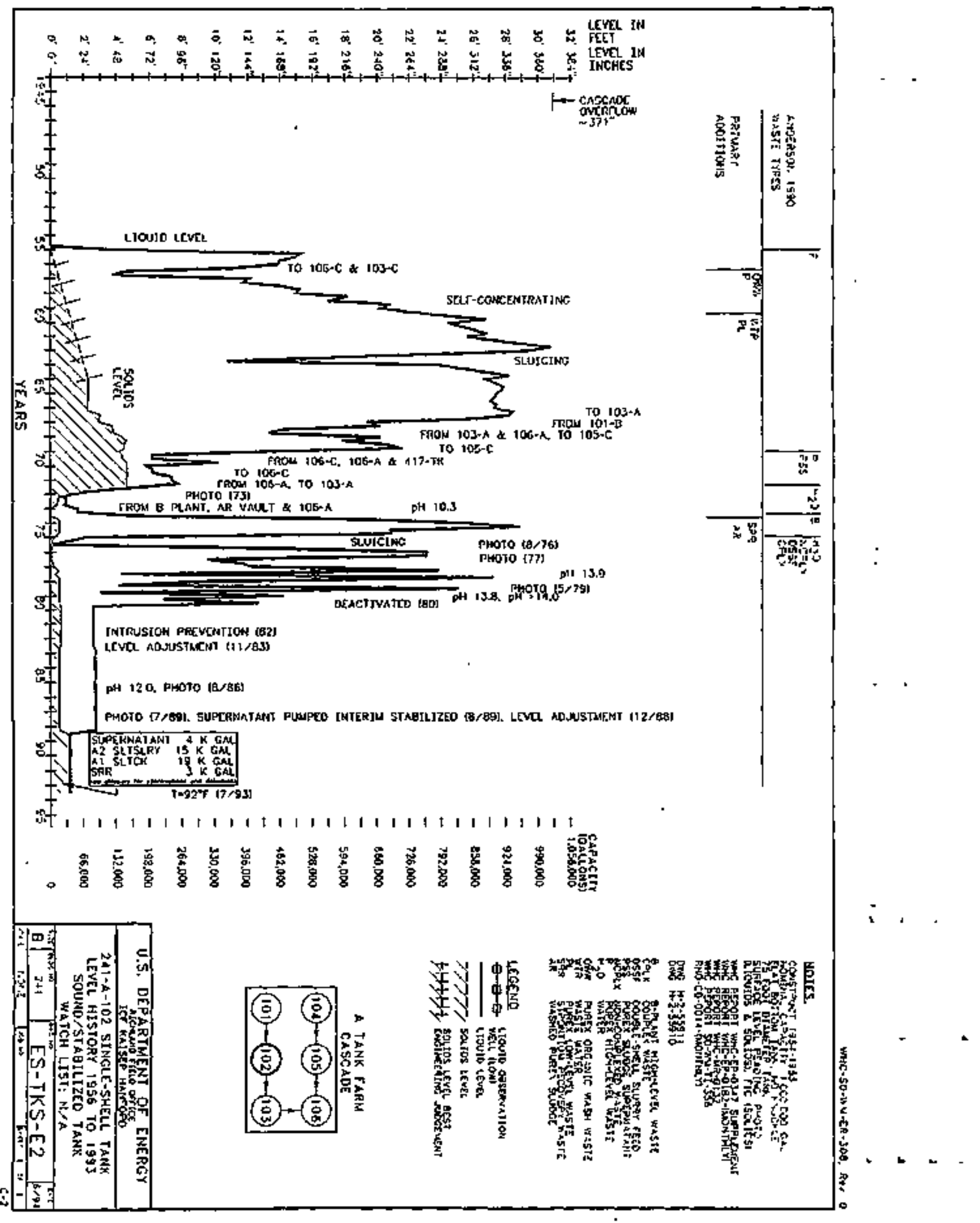




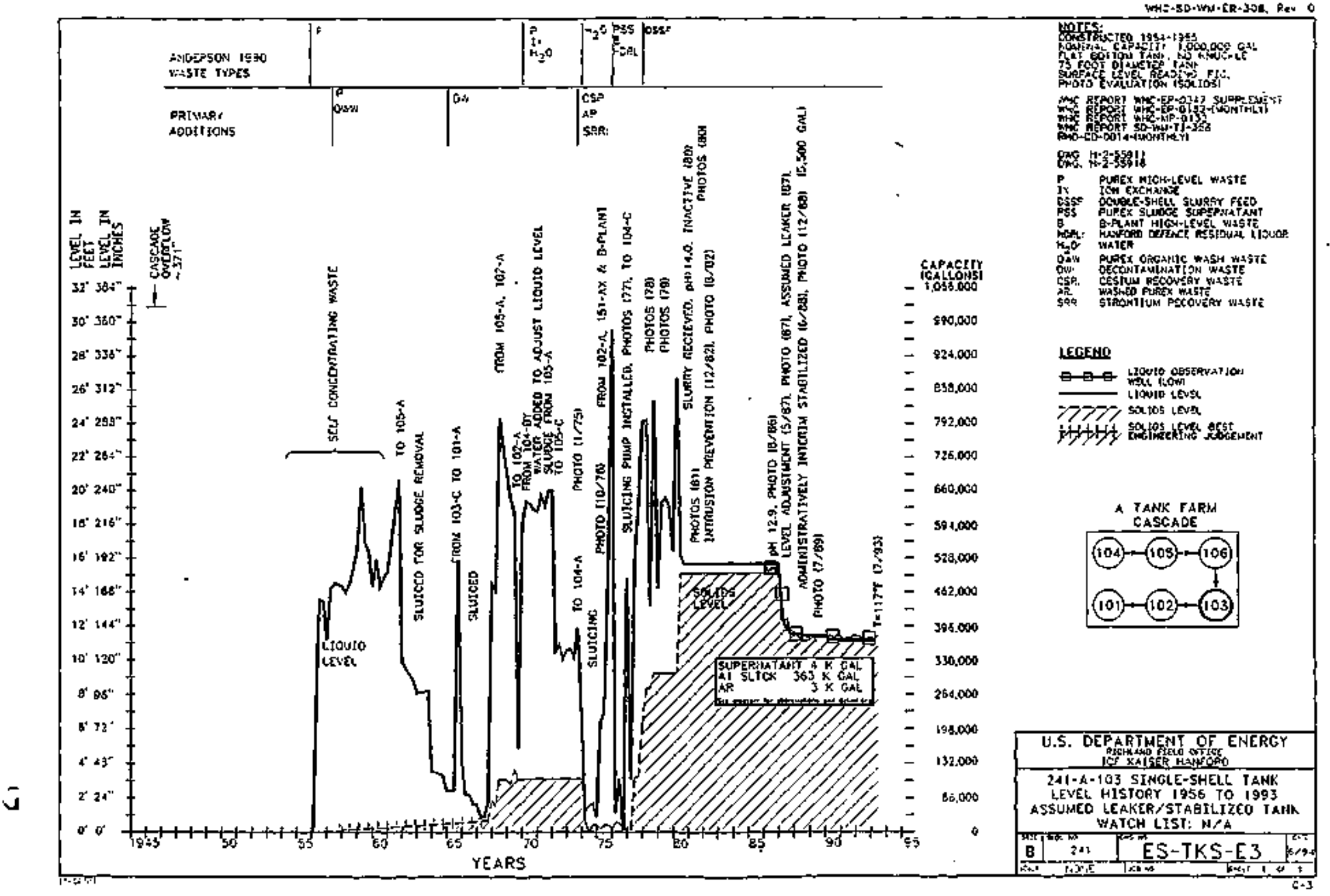




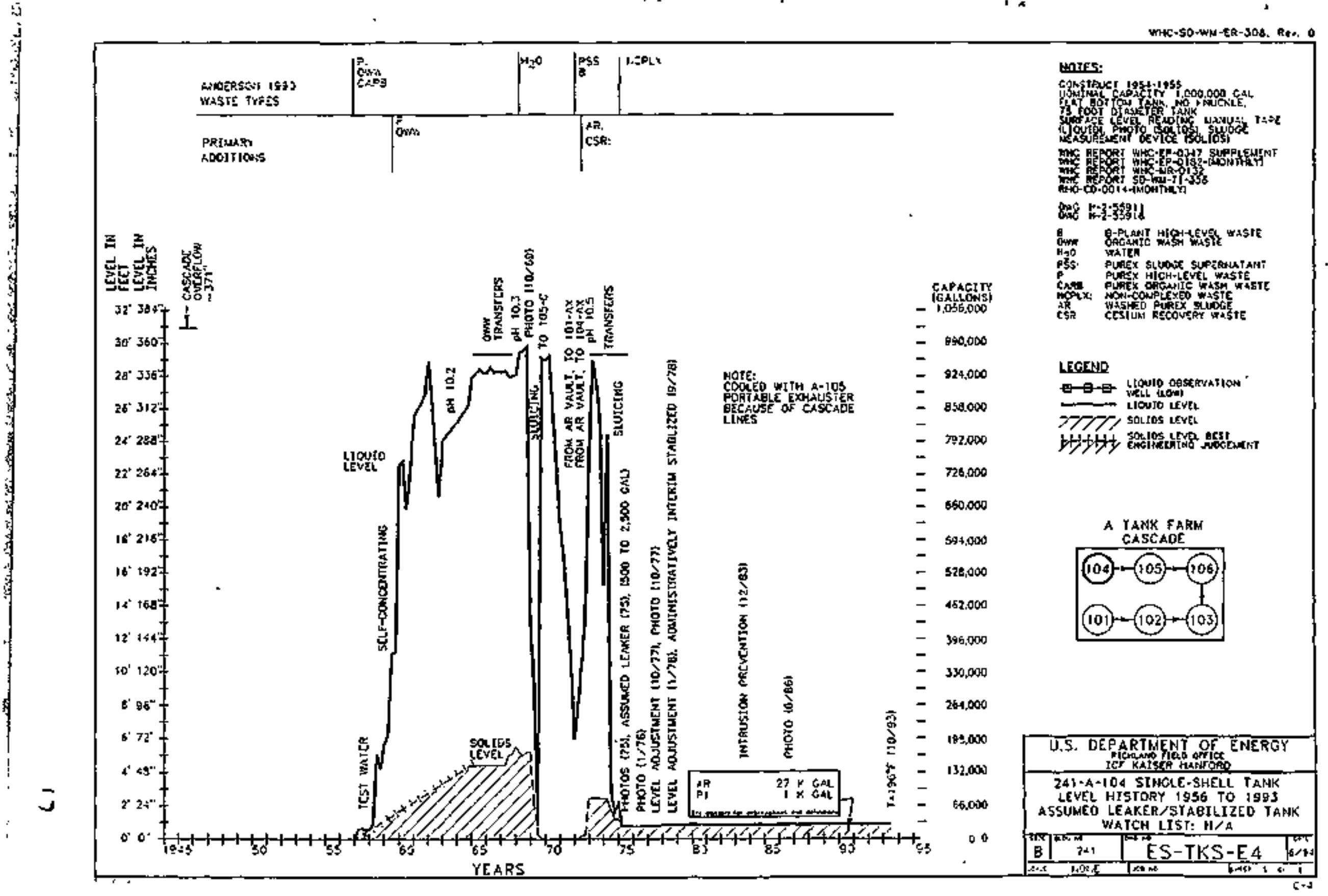




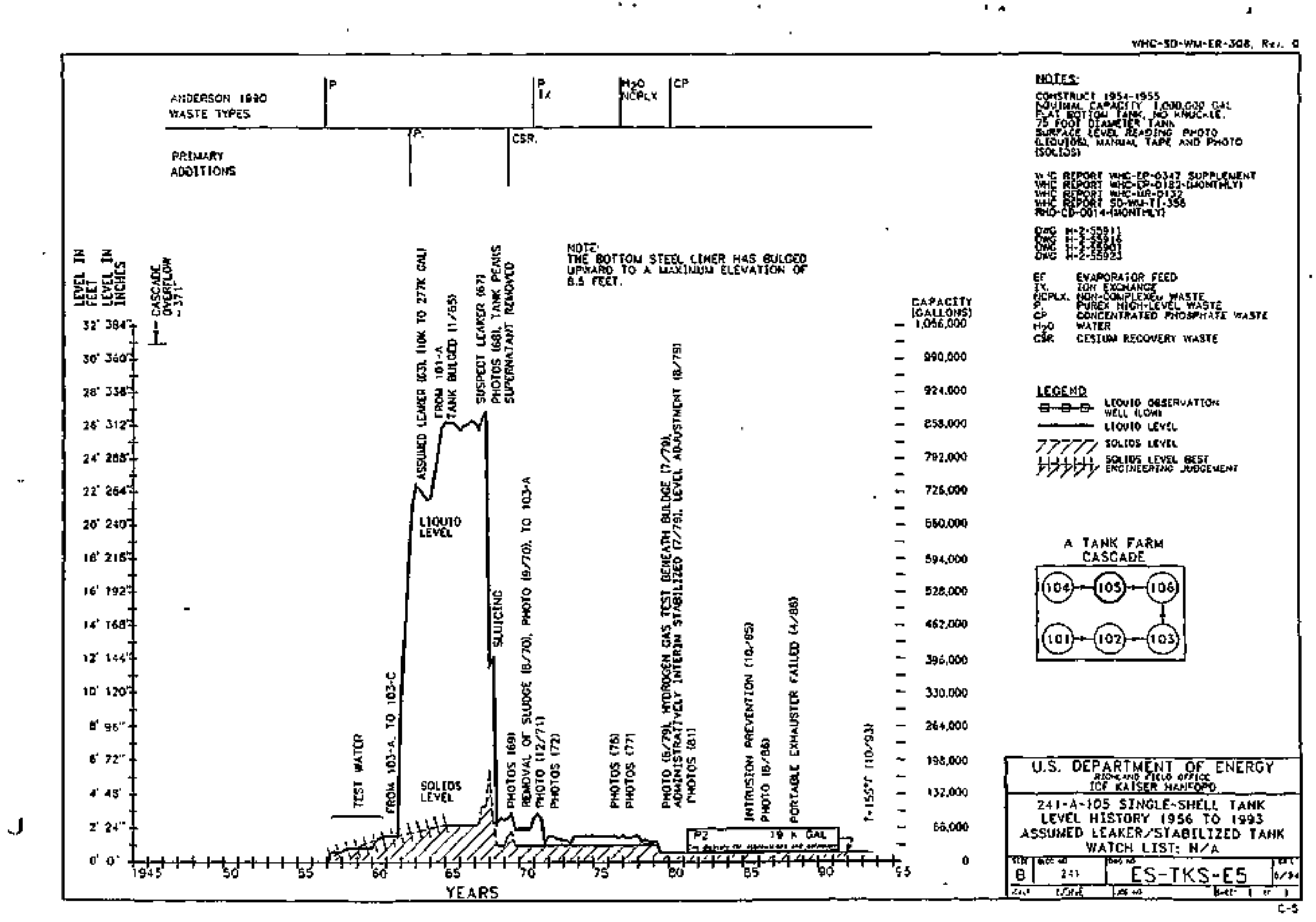




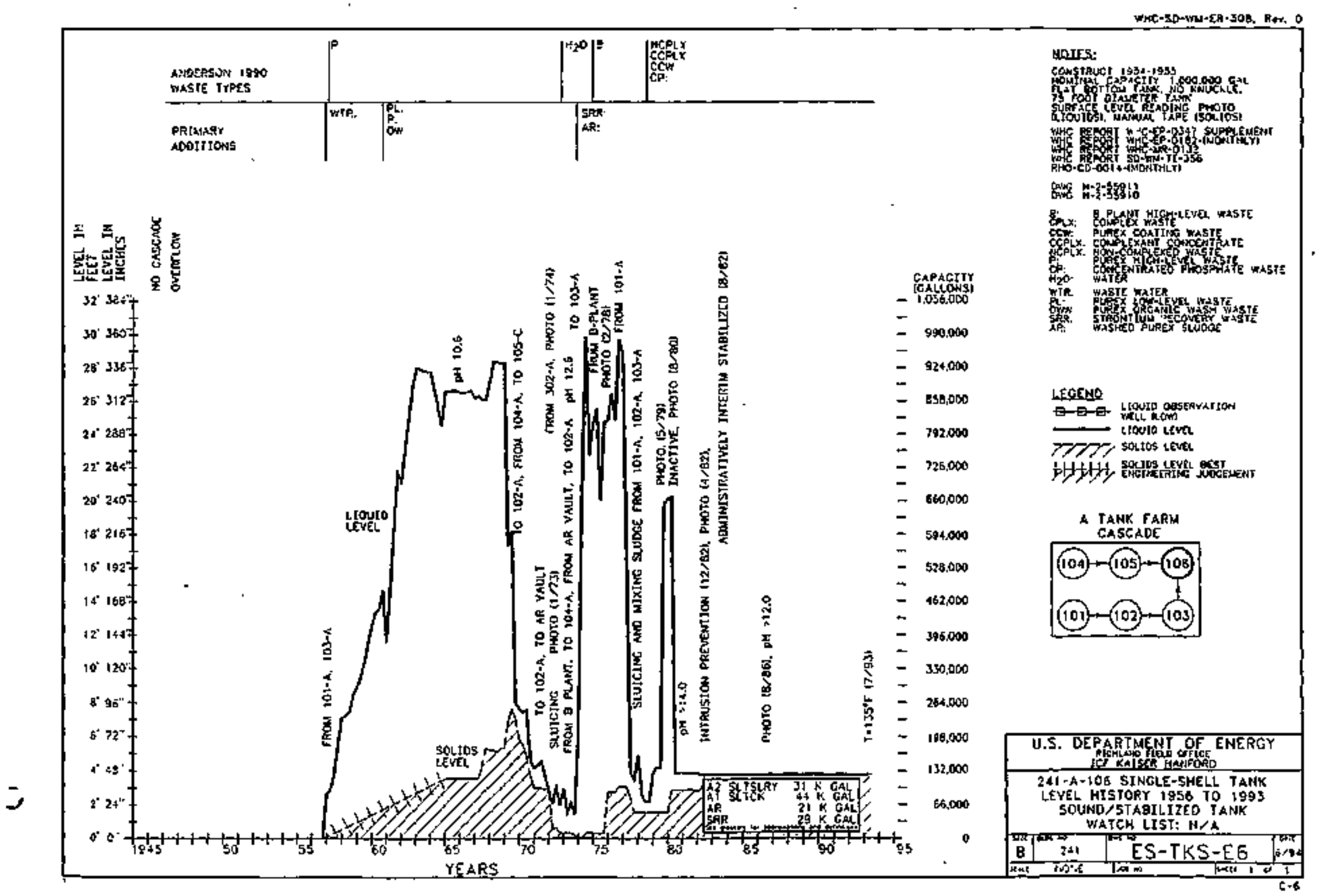


241-A-101 SINGLE-SHELL WHC-SD-WM-ER-308, REV. 0 TANK LEVEL HISTORY

\begin{tabular}{|c|c|c|c|c|c|}
\hline Yeer & Totel & Toto여 & Solids & Solids & LOW \\
\hline & [K gal] & $(\ln )$ & \{K gal\} & (tn) & $(\mathrm{In})$ \\
\hline $1-7956$ & 424 & 154 & & & \\
\hline 2 & 424 & 154 & & & \\
\hline 3 & 419 & 152 & & & \\
\hline 4 & 397 & 144 & & & \\
\hline$t-1957$ & 397 & 144 & & & \\
\hline 2 & 234 & 85 & & & \\
\hline 3 & 484 & 176 & & & \\
\hline 4 & 388 & 141 & & & \\
\hline $1-1958$ & 509 & 185 & & & \\
\hline 2 & 564 & 205 & & & \\
\hline 3 & 509 & 185 & & & \\
\hline$\overline{4}$ & 498 & $\overline{1 B T}$ & & & \\
\hline 1-1959 & 600 & 182 & & & \\
\hline 2 & 578 & 210 & & & \\
\hline 3 & 694 & 252 & & & \\
\hline 4 & 614 & 223 & & & \\
\hline $1-1960$ & 674 & 245 & & & \\
\hline 2 & 719 & 261 & & & \\
\hline 3 & 644 & 234 & & & \\
\hline 4 & 697 & 253 & & & \\
\hline $1-1961$ & 930 & 338 & & & \\
\hline \multicolumn{6}{|l|}{2} \\
\hline \multicolumn{6}{|l|}{3} \\
\hline 4 & 910 & 331 & & & \\
\hline 1.1962 & 861 & 313 & & & \\
\hline \multicolumn{6}{|l|}{2} \\
\hline \multicolumn{6}{|l|}{3} \\
\hline 4 & 558 & 203 & & & \\
\hline$t-1963$ & 825 & 300 & & & \\
\hline \multicolumn{6}{|l|}{2} \\
\hline \multicolumn{6}{|l|}{3} \\
\hline 4 & 857 & 312 & & & \\
\hline 1-1964 & 754 & 274 & & & \\
\hline \multicolumn{6}{|l|}{2} \\
\hline \multicolumn{6}{|l|}{3} \\
\hline 4 & 880 & 320 & 72 & 26 & \\
\hline $1-1965$ & 880 & 320 & 72 & 26 & \\
\hline \multicolumn{6}{|l|}{2} \\
\hline 3 & 886 & 322 & 72 & 26 & \\
\hline 4 & 668 & 243 & 72 & 26 & \\
\hline $1-1966$ & 833 & 303 & 72 & 26 & \\
\hline 2 & 905 & 329 & 72 & 26 & \\
\hline 3 & 905 & 329 & 72 & 26 & \\
\hline 4 & 905 & 329 & 72 & 26 & \\
\hline $1-1967$ & 905 & 329 & 72 & 26 & \\
\hline 2 & 905 & 329 & 72 & 26 & \\
\hline
\end{tabular}


241.A-101 \$INGLE-SHEL.L WHC-SD-WM-BR-308, Rev. 0 TANK LEVEL HISTORY

\begin{tabular}{|c|c|c|c|c|c|}
\hline Year & Total & Total & Solids & Solids & LOW \\
\hline & (र gal) & (in) & (K g日i) & (in) & [in] \\
\hline & & & & & \\
\hline 3 & $\overline{904}$ & $3 \overline{29}$ & 79 & $\overline{29}$ & \\
\hline 4 & 905 & 329 & 80 & 29 & \\
\hline $1+1968$ & 358 & 130 & 83 & 30 & \\
\hline 2 & 77 & 28 & $50^{\circ}$ & 18 & \\
\hline 3 & 124 & $\overline{45}$ & 50 & $T B$ & \\
\hline 4 & 135 & 49 & 50 & 18 & \\
\hline $1-1969$ & 990 & 360 & 8 & 3 & \\
\hline 2 & 749 & $2 \overline{272}$ & 8 & $\overline{3}$ & \\
\hline 3 & 333 & $1 \overline{21}$ & 3 & 1 & \\
\hline$\overline{4}$ & 173 & 63 & 17 & 4 & \\
\hline $1-1970$ & 462 & 168 & $1 i$ & 4 & \\
\hline 2 & 639 & 232 & 11 & 4 & \\
\hline 3 & 531 & $1 \overline{93}$ & 11 & 4 & \\
\hline 4 & 483 & 176 & 11 & 4 & \\
\hline $1-1971$ & 441 & 160 & 11 & 4 & \\
\hline 2 & 440 & 160 & 11 & 4 & \\
\hline 3 & $430^{\circ}$ & $1 \overline{156}$ & 11 & 4 & \\
\hline$\overline{4}$ & 430 & 158 & 11 & 4 & \\
\hline $1-1972$ & 422 & 153 & 11 & 4 & \\
\hline 2 & 28 & 10 & 11 & 4 & \\
\hline$\overline{3}$ & 32 & 12 & 11 & $\overline{4}$ & \\
\hline 4 & 33 & 12 & 16 & 6 & \\
\hline 1.1973 & 302 & 110 & 16 & 6 & \\
\hline 2 & 928 & 337 & 16 & 6 & \\
\hline 3 & 934 & 340 & 16 & 8 & \\
\hline 4 & 900 & 327 & 16 & $\overline{6}$ & \\
\hline $1-1974$ & 968 & 352 & 16 & 6 & \\
\hline 2 & 962 & 350 & 33 & 12 & \\
\hline 3 & 957 & 348 & 33 & 12 & \\
\hline 4 & 938 & 341 & 33 & $\overline{12}$ & \\
\hline $1-1975$ & 924 & $\overline{336}$ & 33 & 12 & \\
\hline 2 & 432 & 757 & 11 & 4 & \\
\hline 3 & 217 & 79 & 11 & 4 & \\
\hline 4 & 52 & 19 & 8 & 3 & \\
\hline $1-19 \overline{76}$ & 6 & 2 & 1 & $\overline{0.4}$ & \\
\hline 2 & 6 & 2 & 3 & 1 & \\
\hline 3 & 157 & 57 & 3 & 1 & \\
\hline 4 & 930 & $\overline{338}$ & 3 & 1 & \\
\hline 1.1977 & 809 & 294 & 3 & 1 & \\
\hline 2 & 762 & 277 & 3 & 1 & \\
\hline 3 & 968 & 352 & 85 & 31 & \\
\hline 4 & $97 \overline{4}$ & $\overline{354}$ & 85 & 31 & \\
\hline $1-1978$ & 877 & 319 & 377 & 137 & \\
\hline 2 & 866 & 315 & 377 & 137 & \\
\hline$\overline{3}$ & $\overline{468}$ & 170 & 377 & 137 & \\
\hline 4 & 971 & 353 & 490 & 178 & \\
\hline
\end{tabular}


241-A-101 SINGLE-SHELL WHC-SD-WM-ER-308, REV. 0 TANK LEVEL HISTORY

\begin{tabular}{|c|c|c|c|c|c|}
\hline Year & Total & Total & Soldds & Sollds & LoW \\
\hline & (K qgal) & (in) & IK gal & (in) & (in) \\
\hline $1-1979$ & $4 \overline{415}$ & 151 & 415 & 161 & \\
\hline 2 & 545 & 198 & 415 & 151 & \\
\hline 3 & $93 \overline{8}$ & 341 & 415 & 151 & \\
\hline 4 & 344 & 125 & 333 & 121 & \\
\hline 1.1980 & 435 & 158 & 333 & $1 \overline{21}$ & \\
\hline 2 & 329 & 120 & 333 & 121 & \\
\hline 3 & 530 & 193 & 333 & 121 & \\
\hline 4 & $93 t$ & 339 & 333 & 121 & \\
\hline $1-1981$ & 931 & 339 & 931 & 339 & \\
\hline 2 & $9 \overline{31}$ & 339 & 931 & $\overline{339}$ & \\
\hline 3 & 931 & 339 & 931 & 339 & \\
\hline 4 & 931 & 339 & 931 & 339 & \\
\hline $1-1982$ & 931 & 339 & 931 & 339 & \\
\hline$\overline{2}$ & 953 & 347 & 953 & 347 & \\
\hline 3 & 953 & $34 \overline{7}$ & $\overline{953}$ & 347 & \\
\hline 4 & 953 & 347 & 953 & 347 & \\
\hline $7-1983$ & $95 \overline{3}$ & 347 & $\overline{953}$ & $3 \overline{47}$ & \\
\hline 2 & 953 & 347 & 953 & 347 & \\
\hline$\overline{3}$ & 953 & 347 & 553 & 347 & \\
\hline 4 & 953 & 347 & 953 & $\overline{347}$ & \\
\hline $1-1984$ & 953 & 347 & 953 & 347 & \\
\hline$\overline{2}$ & 953 & 347 & 953 & 347 & \\
\hline 3 & 953 & 347 & 953 & 347 & \\
\hline 4 & 953 & 347 & 953 & $\overline{347}$ & \\
\hline $1-1985$ & 953 & 347 & 963 & 347 & \\
\hline$\overline{2}$ & 953 & 347 & 953 & 347 & \\
\hline 3 & 953 & 347 & 953 & $\overline{347}$ & \\
\hline 4 & 953 & 347 & 953 & 347 & \\
\hline $1-1986$ & 953 & 347 & 953 & 347 & 364 \\
\hline 2 & 953 & 347 & 953 & $\overline{347}$ & $3 \overline{65}$ \\
\hline 3 & 953 & 347 & 953 & 347 & 364 \\
\hline 4 & 953 & 347 & 953 & 347 & 365 \\
\hline $1-1987$ & 953 & 347 & 953 & 347 & 364 \\
\hline 2 & $9 \overline{53}$ & 347 & 953 & $\overline{347}$ & $3 \overline{65}$ \\
\hline 3 & 953 & $34 \overline{7}$ & 953 & 347 & 363 \\
\hline 4 & 953 & 347 & 953 & 347 & 364 \\
\hline $1-1988$ & 953 & 347 & 953 & 347 & 365 \\
\hline 2 & $9 \overline{53}$ & 347 & 953 & 347 & 366 \\
\hline 3 & 953 & 347 & 953 & 347 & 364 \\
\hline 4 & 953 & 347 & 953 & 347 & 365 \\
\hline $1-1989$ & 953 & 347 & 953 & 347 & 364 \\
\hline$\overline{2}$ & 963 & 347 & 953 & 347 & 365 \\
\hline 3 & 953 & 347 & 953 & 347 & 365 \\
\hline 4 & 953 & 347 & 953 & 347 & 365 \\
\hline 1.1990 & 953 & 347 & 953 & 347 & \\
\hline 2 & 953 & $34 \overline{7}$ & 953 & 347 & \\
\hline
\end{tabular}


241-A-101 SINGLE-SHELL WHC-SD-FM-ER-308, ReV, 0 TANK LEVEL HISTORY

\begin{tabular}{|l|c|c|c|c|c|}
\hline Year & Total & Total & Solids & Soljds & Low \\
\hline & (K ggl] & (in) & (K ggi) & (in) & (in) \\
\hline & & & & & \\
\hline 3 & 953 & 347 & 953 & 347 & \\
\hline 4 & 953 & 347 & 953 & 347 & 365 \\
\hline $1-1991$ & 953 & 347 & 953 & 347 & 365 \\
\hline 2 & 953 & 347 & 953 & 347 & 365 \\
\hline 3 & 953 & 347 & 953 & 347 & 364 \\
\hline 4 & 963 & 347 & 953 & 347 & 364 \\
\hline $1-1992$ & 953 & 347 & 953 & 347 & \\
\hline 2 & 953 & 347 & 953 & 347 & \\
\hline 3 & 953 & 347 & 953 & 347 & 365 \\
\hline 4 & 953 & 347 & 953 & 347 & 364 \\
\hline $1-1993$ & 953 & 347 & 953 & 347 & \\
\hline 2 & 953 & 347 & 953 & 347 & 364 \\
\hline 3 & & & & & 365 \\
\hline 4 & & & & & \\
\hline
\end{tabular}


241-A-702 \$INGLE-SHEA. WHC-SD-WM-ER-308, Rev . 0 TANK LEVEL. HISTORY

\begin{tabular}{|c|c|c|c|c|}
\hline Year & Total & Total & Solids & Solids \\
\hline & (K galf & (in) & (K gal) & (in) \\
\hline 11956 & 130 & 47 & & \\
\hline 2 & 505 & 184 & & \\
\hline 3 & 495 & $18 \overline{0}$ & & \\
\hline 4 & 463 & 168 & & \\
\hline $1-1957$ & 457 & 166 & & \\
\hline 2 & 374 & 136 & & \\
\hline$\overline{3}$ & 149 & 54 & & \\
\hline 4 & 124 & 46 & & \\
\hline 1.1958 & 399 & $14 \overline{5}$ & & \\
\hline 2 & 388 & 141 & & \\
\hline 3 & 465 & 169 & & \\
\hline 4 & 504 & $18 \overline{3}$ & & \\
\hline 1-1959 & 495 & 180 & & \\
\hline 2 & 602 & 219 & & \\
\hline 3 & 562 & 204 & & \\
\hline 4 & 689 & 251 & & \\
\hline $1-1960$ & 671 & 244 & - & \\
\hline 2 & 727 & 264 & & \\
\hline 3 & 800 & 291 & & \\
\hline 4 & 882 & 321 & & \\
\hline $1-1961$ & 806 & 293 & & \\
\hline \multicolumn{5}{|l|}{2} \\
\hline \multicolumn{5}{|l|}{$\overline{3}$} \\
\hline 4 & 888 & $32 \overline{3}$ & & \\
\hline $1-1962$ & 847 & 308 & & \\
\hline \multicolumn{5}{|l|}{2} \\
\hline \multicolumn{5}{|l|}{$\overline{3}$} \\
\hline 4 & 1015 & 369 & & \\
\hline 1.1963 & 965 & 351 & & \\
\hline \multicolumn{5}{|l|}{2} \\
\hline \multicolumn{5}{|l|}{$\overline{3}$} \\
\hline 4 & 356 & 129 & & \\
\hline 1.1964 & 787 & $28 \overline{6}$ & & \\
\hline \multicolumn{5}{|l|}{2} \\
\hline \multicolumn{5}{|l|}{$\overline{3}$} \\
\hline 4 & 930 & 338 & & \\
\hline $1-1965$ & 888 & 323 & 77 & 28 \\
\hline \multicolumn{5}{|l|}{2} \\
\hline 3 & 921 & 335 & 77 & 28 \\
\hline 4 & 956 & 333 & 77 & 28 \\
\hline $1-1966$ & 905 & 329 & 77 & 28 \\
\hline 2 & 902 & 328 & 77 & 28 \\
\hline 3 & 894 & 325 & 77 & 28 \\
\hline 4 & 905 & 329 & 77 & 28 \\
\hline 1.1967 & 902 & $32 \overline{8}$ & 77 & 28 \\
\hline 2 & 939 & 341 & 77 & 28 \\
\hline
\end{tabular}


241-A-102 SINGLE-SHELI. WHC-SD-WM-ER-308, Rev. 0 TANK LEVEE HISTOAY

\begin{tabular}{|c|c|c|c|c|}
\hline Year & Total & Total & Solids & Solids \\
\hline & (K gat) & (in) & (K g䪨) & (in) \\
\hline & & & & \\
\hline 3 & 932 & $33 \overline{9}$ & 102 & 37 \\
\hline 4 & 862 & 333 & 99 & 36 \\
\hline 7.1968 & 641 & 233 & 99 & 36 \\
\hline 2 & 668 & 243 & 124 & 45 \\
\hline 3 & 465 & 169 & 127 & 46 \\
\hline 4 & 440 & 160 & 127 & 46 \\
\hline $\bar{t}+1969$ & 666 & 242 & 146 & 53 \\
\hline 2 & 591 & 215 & 154 & 56 \\
\hline 3 & 679 & 247 & 149 & 54 \\
\hline 4 & 710 & 258 & 149 & 54 \\
\hline 1.7970 & 415 & $15 \overline{1}$ & $1 \overline{38}$ & 50 \\
\hline 2 & 206 & 75 & 146 & 53 \\
\hline 3 & 205 & 75 & 146 & 53 \\
\hline 4 & 335 & 122 & 154 & 56 \\
\hline 1.1971 & 193 & 70 & 154 & 56 \\
\hline 2 & 206 & 75 & 154 & 56 \\
\hline 3 & 206 & 75 & 154. & 56 \\
\hline 4 & 242 & 88 & $1 \overline{54}$ & $\overline{56}$ \\
\hline 1.1972 & 246 & 89 & 154 & 68 \\
\hline 2 & 256 & 93 & 154 & 56 \\
\hline 3 & 175 & 64 & 154 & 56 \\
\hline 4 & 96 & 35 & 69 & 25 \\
\hline 1.1973 & 39 & 14 & 39 & 14 \\
\hline 2 & 30 & 11 & 15 & 5 \\
\hline 3 & 32 & 12 & 15 & 5 \\
\hline 4 & 30 & $1 \mathrm{~T}$ & 15 & 5 \\
\hline$i+1974$ & 56 & 20 & 0 & 0 \\
\hline 2 & 56 & 20 & 0 & 0 \\
\hline 3 & 173 & $6 \overline{3}$ & $\overline{0}$ & 0 \\
\hline 4 & 652 & 237 & 17 & 6 \\
\hline $1-1975$ & 839 & 305 & 17 & 6 \\
\hline 2 & 952 & $3 \overline{46}$ & $\overline{17}$ & 6 \\
\hline 3 & 690 & 251 & 17 & 6 \\
\hline 4 & 690 & 251 & 17 & 6 \\
\hline $\bar{t}-1 \overline{976}$ & 72 & $2 \overline{6}$ & $\overline{1}$ & 0.4 \\
\hline 2 & 39 & 14 & 2 & 1 \\
\hline 3 & 6 & 2 & 6 & 2 \\
\hline 4 & 429 & 156 & 3 & 1 \\
\hline $7-1977$ & 762 & 277 & 3 & 1 \\
\hline 2 & 762 & 277 & 3 & 1 \\
\hline 3 & 316 & 115 & 3 & 1 \\
\hline 4 & 385 & 140 & 6 & 2 \\
\hline $1-1 \overline{978}$ & 407 & $1 \overline{48}$ & $\overline{8}$ & $\overline{3}$ \\
\hline 2 & 789 & 287 & 17 & 6 \\
\hline 3 & 143 & 52 & 11 & 4 \\
\hline 4 & 897 & 326 & 17 & 6 \\
\hline
\end{tabular}


241-A-102 SINGLE-SHELL WHC-SD-WM-ER-308, Rev. 0 TANK LEVEL HISTORY

\begin{tabular}{|c|c|c|c|c|}
\hline Year & Total & Totdid & Solids & Solids \\
\hline & $\overline{\text { [K gali) }}$ & (in) & (K gal) & \{in\} \\
\hline 1.1979 & $31 \overline{9}$ & 176 & 17 & 6 \\
\hline 2 & 138 & 50 & 17 & 6 \\
\hline 3 & 828 & 301 & 17 & 6 \\
\hline 4 & 102 & 37 & 17 & 6 \\
\hline $1=1980$ & $47 \overline{0}$ & $\overline{171}$ & 17 & 6 \\
\hline 2 & 228 & 83 & 17 & 6 \\
\hline$\overline{3}$ & $4 \uparrow 7$ & 152 & 17 & 6 \\
\hline 4 & 89 & 32 & 22 & 8 \\
\hline $1-1981$ & 89 & 32 & 22 & 8 \\
\hline 2 & 89 & 32 & $2 \overline{2}$ & $\overline{\mathrm{B}}$ \\
\hline$\overline{3}$ & 89 & 32 & 22 & 8 \\
\hline 4 & 89 & 32 & 22 & 8 \\
\hline 1-1982 & 89 & 32 & 22 & 8 \\
\hline 2 & 89 & 32 & $2 \overline{2}$ & 8 \\
\hline 3 & 89 & 32 & 22 & 8 \\
\hline 4 & 89 & 32 & 22 & $\mathbf{8}$ \\
\hline 1.1983 & $8 \overline{9}$ & 32 & $2 \overline{2}$ & 8 \\
\hline 2 & 89 & 32 & 22 & 8 \\
\hline 3 & 89 & 32 & 22 & 8 \\
\hline 4 & 91 & 33 & 19 & 7 \\
\hline 1.1984 & 91 & 33 & 19 & 7 \\
\hline 2 & $9 \overline{7}$ & 33 & 19 & $\overline{7}$ \\
\hline 3 & 91 & 33 & 19 & 7 \\
\hline 4 & 91 & 33 & 19 & $\overline{7}$ \\
\hline 1.1985 & 91 & $\overline{33}$ & 19 & $\overline{7}$ \\
\hline 2 & 91 & 33 & 19 & 7 \\
\hline$\dot{3}$ & 91 & 33 & 19 & 7 \\
\hline 4 & $9 \overline{1}$ & 33 & $1 \overline{9}$ & 7 \\
\hline $1-1986$ & 91 & 33 & 19 & 7 \\
\hline 2 & 91 & 33 & 19 & 7 \\
\hline 3 & 91 & $\overrightarrow{33}$ & 19 & $\overline{7}$ \\
\hline 4 & 91 & 33 & 19 & 7 \\
\hline $1-1987$ & 91 & 33 & 19 & 7 \\
\hline 2 & 91 & 33 & 19 & 7 \\
\hline 3 & 91 & 33 & 19 & 7 \\
\hline 4 & 91 & 33 & 19 & 7 \\
\hline 3.1988 & 91 & 33 & 19 & 7 \\
\hline 2 & 91 & 33 & 19 & 7 \\
\hline$\overline{3}$ & 91 & 33 & 19 & 7 \\
\hline 4 & 91 & 33 & 19 & 7 \\
\hline $7-1 \overline{989}$ & 91 & 33 & 19 & 7 \\
\hline 2 & $9 \overline{1}$ & 33 & 19 & $\overline{7}$ \\
\hline 3 & 41 & 95 & 37 & 13 \\
\hline 4 & 41 & 15 & 37 & 13 \\
\hline 1.1990 & 41 & 15 & 37 & 13 \\
\hline 2 & 41 & 15 & 37 & 13 \\
\hline
\end{tabular}


241-A+102 SINGLE-SHELL WHC-SD-WM-ER-308, ReV . 0 TANK LEVEL HISTOAY

\begin{tabular}{|l|c|c|c|c|}
\hline \multicolumn{1}{|c|}{$Y_{\text {eas }}$} & Total & Total & Solids & Solids \\
\hline & (K gal) & (in) & (K gali & (in) \\
\hline 3 & & & & \\
\hline 4 & 41 & 15 & 37 & 13 \\
\hline $1-5991$ & 41 & 15 & 37 & 13 \\
\hline 2 & 41 & 15 & 37 & 13 \\
\hline 3 & 41 & 15 & 37 & $\frac{13}{13}$ \\
\hline 4 & 41 & 15 & 37 & 13 \\
\hline $1-1992$ & 41 & 15 & 37 & 13 \\
\hline 2 & 41 & 15 & 37 & 13 \\
\hline 3 & 41 & 15 & 37 & 13 \\
\hline 4 & 41 & 15 & 37 & 13 \\
\hline $1-1993$ & 41 & 15 & 37 & 13 \\
\hline 2 & 41 & 15 & 37 & 13 \\
\hline 3 & & & & \\
\hline 4 & & & & \\
\hline
\end{tabular}


241-A-103 SINGLE-SHELL WHC-SD-WM-ER-308, ReV.0 TANX LEVEL HISTOAY

\begin{tabular}{|c|c|c|c|c|c|}
\hline Year & Totel & Total & Solids & Solids & LOW \\
\hline & \{K gal\} & (lin) & [K gald] & (n) & (in) \\
\hline & & & & & \\
\hline \multicolumn{6}{|l|}{1.1956} \\
\hline 2 & 171 & 62 & & & \\
\hline 3 & 449 & 163 & & & \\
\hline 4 & $\overline{444}$ & 161 & & & \\
\hline $1-1957$ & 369 & 134 & & & \\
\hline$\overline{2}$ & $4 \overline{70}$ & 171 & & & \\
\hline 3 & 481 & 175 & & & \\
\hline \multicolumn{6}{|l|}{4} \\
\hline$t-\overline{958}$ & 473 & 172 & & & \\
\hline 2 & 462 & 168 & & & \\
\hline 3 & $4 \overline{81}$ & 175 & & & \\
\hline 4 & 509 & 185 & & & \\
\hline $1-1959$ & $5 \overline{48}$ & $\overline{199}$ & & & \\
\hline 2 & 666 & 242 & & & \\
\hline 3 & 562 & 204 & & & \\
\hline 4 & 539 & 196 & & & \\
\hline $1-1960$ & 473 & 172 & & & \\
\hline 2 & $5 \overline{26}$ & 191 & & & \\
\hline 3 & 473 & 172 & & & \\
\hline 4 & 490 & 178 & & & \\
\hline 1.1961 & $5 \overline{01}$ & 182 & & & \\
\hline \multicolumn{6}{|l|}{2} \\
\hline \multicolumn{6}{|l|}{3} \\
\hline 4 & 682 & 248 & & & \\
\hline $1-1962$ & 325 & 118 & & & \\
\hline \multicolumn{6}{|l|}{2} \\
\hline \multicolumn{6}{|l|}{3} \\
\hline 4 & 294 & 107 & & & \\
\hline 1.1963 & 267 & 97 & & & \\
\hline \multicolumn{6}{|l|}{2} \\
\hline \multicolumn{6}{|l|}{3} \\
\hline 4 & 270 & 98 & & & \\
\hline $1-1964$ & $1 \overline{16}$ & $\overline{42}$ & & & \\
\hline \multicolumn{6}{|l|}{2} \\
\hline \multicolumn{6}{|l|}{3} \\
\hline 4 & 110 & 40 & & & \\
\hline $1-1965$ & 80 & 29 & & & \\
\hline \multicolumn{6}{|l|}{2} \\
\hline 3 & 80 & 29 & & & \\
\hline 4 & 525 & 191 & & & \\
\hline $1-1966$ & 140 & 51 & & & \\
\hline 2 & 74 & 27 & & & \\
\hline 3 & 72 & 26 & & & \\
\hline 4 & 55 & 20 & & & \\
\hline $1-1967$ & 55 & 20 & & & \\
\hline 2 & 33 & 12 & & & \\
\hline
\end{tabular}


241-A-103 SINGLE-SHELL WHC-SD-WM-ER-308, Rev. 0 TANK LEVEL HISTORY

\begin{tabular}{|c|c|c|c|c|c|}
\hline Year & Total & Total & Sollids & Solids & Low \\
\hline & (K ged) & (in) & (K gal) & (in) & (in) \\
\hline & & & & & \\
\hline 3 & 30 & 11 & & & \\
\hline 4 & 54 & $2 \overline{0}$ & 22 & 8 & \\
\hline $1-1968$ & 476 & 173 & 61 & 22 & \\
\hline 2 & 465 & 169 & 52 & 19 & \\
\hline 3 & 803 & 292 & $\overline{102}$ & $\overline{37}$ & \\
\hline 4 & $74 \overline{1}$ & 269 & 102 & 37 & \\
\hline $1-1969$ & 690 & 251 & 102 & 37 & \\
\hline 2 & 641 & 233 & 91 & 33 & \\
\hline 3 & 617 & 224 & 121 & 44 & \\
\hline 4 & 160 & 58 & 102 & 37 & \\
\hline $1-1970$ & 594 & 216 & 102 & 37 & \\
\hline 2 & 637 & 232 & 102 & 37 & \\
\hline 3 & 635 & 231 & 102 & 37 & \\
\hline 4 & 622 & $2 \overline{26}$ & $10 \overline{02}$ & $\overline{37}$ & \\
\hline i-1971 & 620 & 226 & 102 & 37 & \\
\hline 2 & 649 & 236 & 102 & 37 & \\
\hline 3 & 630 & 229 & 102 & 37 & \\
\hline 4 & 660 & 240 & 102 & 37 & \\
\hline $1-1972$ & 661 & 240 & 102 & 37 & \\
\hline 2 & 346 & 126 & 102 & 37 & \\
\hline 3 & 358 & 130 & 102 & 37 & \\
\hline 4 & 333 & 121 & 102 & 37 & \\
\hline $1-1973$ & $34 \overline{9}$ & 127 & $1 \overline{02}$ & $\overline{37}$ & \\
\hline 2 & 352 & 128 & 102 & 37 & \\
\hline 3 & 336 & 122 & 102 & 37 & \\
\hline 4 & 392 & $1 \overline{43}$ & $1 \overline{02}$ & $\overline{37}$ & \\
\hline $1-1974$ & 227 & 83 & 102 & 37 & \\
\hline 2 & 41 & 15 & 22 & $g$ & \\
\hline 3 & 50 & 18 & 0 & 0 & \\
\hline 4 & $5 \overline{5}$ & 20 & 14 & $\overline{5}$ & \\
\hline $1-1975$ & 28 & 10 & 14 & 5 & \\
\hline 2 & 212 & 77 & 6 & 2 & \\
\hline 3 & $2 \overline{3 t}$ & 94 & $\overline{14}$ & $\overline{5}$ & \\
\hline 4 & 481 & 176 & 14 & 5 & \\
\hline 1-1976 & 974 & 354 & 6 & 2 & \\
\hline 2 & 35 & 13 & 16 & 6 & \\
\hline 3 & 102 & 37 & 17 & 6 & \\
\hline 4 & 3 & 1 & 3 & 1 & \\
\hline 1.1977 & 487 & 177 & 3 & 1 & \\
\hline$\overline{2}$ & $1 \overline{02}$ & $3 \overline{7}$ & $\overline{3}$ & 1 & \\
\hline 3 & $5 \overline{20}$ & $1 \overline{89}$ & 105 & $\overline{38}$ & \\
\hline 4 & 641 & 233 & 105 & 38 & \\
\hline $1-1978$ & 795 & 289 & 208 & 75 & \\
\hline 2 & 800 & 291 & 275 & 100 & \\
\hline 3 & $4 \overline{37}$ & $1 \overline{59}$ & 275 & 100 & \\
\hline 4 & 836 & 304 & 303 & 110 & \\
\hline
\end{tabular}


24i-A-103 SINGLF-SHELL WHC-SD-WM-ER-308, Rev. 0 TANK LEVEL HISTORY

\begin{tabular}{|c|c|c|c|c|c|}
\hline Year & Total & Total & Solids & Solids & LOW \\
\hline & [K gal] & (tn) & \{K gal\}) & (in) & (in) \\
\hline $1-1979$ & 470 & 171 & $30 \overline{2}$ & 510 & \\
\hline 2 & 638 & 232 & $30 \overline{3}$ & 110 & \\
\hline 3 & 644 & 234 & 303 & 110 & \\
\hline 4 & 627 & 228 & $30 \overline{3}$ & 110 & \\
\hline 1.1980 & 642 & 197 & 303 & 110 & \\
\hline 2 & 879 & 320 & $30 \overline{3}$ & 110 & \\
\hline 3 & 532 & 193 & 499 & 181 & \\
\hline 4 & 516 & 188 & 499 & 181 & \\
\hline 1.1981 & $\overline{516}$ & 188 & $49 \overline{9}$ & 181 & \\
\hline 2 & 516 & 188 & 499 & 181 & \\
\hline 3 & $\overline{516}$ & 188 & $49 \overline{9}$ & 181 & \\
\hline 4 & 516 & 188 & 499 & 181 & \\
\hline 1-1982 & 516 & 188 & 499 & 181 & \\
\hline 2 & $\overline{516}$ & 188 & $49 \overline{9}$ & 181 & \\
\hline 3 & 516 & 188 & 499 & 181 & \\
\hline 4 & $\overline{516}$ & $1 \overline{8} 8$ & $49 \overline{9}$ & 181 & \\
\hline t-i983 & 516 & 188 & 499 & 181 & \\
\hline$\overline{2}$ & 516 & 188 & 499 & 181 & \\
\hline 3 & 516 & 188 & 499 & 181 & \\
\hline 4 & 516 & 188 & 499 & 181 & \\
\hline 1-1984 & 516 & $18 \mathrm{~B}$ & 499 & 181 & \\
\hline$\overline{2}$ & 516 & 188 & 499 & 181 & \\
\hline 3 & 516 & 188 & $49 \overline{9}$ & 181 & \\
\hline 4 & 516 & 188 & 499 & 181 & \\
\hline $1-1985$ & 516 & 188 & 499 & 181 & \\
\hline 2 & 516 & 188 & 499 & 181 & \\
\hline 3 & 516 & $1 \overline{8} 8$ & $49 \overline{9}$ & 181 & \\
\hline 4 & 516 & 188 & 499 & 181 & \\
\hline 1.1986 & 516 & 188 & 499 & 181 & 186 \\
\hline 2 & 516 & 188 & 499 & 181 & 186 \\
\hline 3 & 516 & 188 & 499 & 181 & 184 \\
\hline 4 & 516 & 188 & 499 & 181 & 186 \\
\hline 1.1987 & 516 & 188 & 499 & 181 & 184 \\
\hline 2 & 409 & 149 & 406 & 148 & 185 \\
\hline 3 & 394 & 143 & 389 & 141 & 147 \\
\hline 4 & 380 & 138 & 388 & $\overline{141}$ & 138 \\
\hline $1-1988$ & 380 & 138 & 386 & 140 & 138 \\
\hline 2 & 379 & 138 & 373 & 136 & 137 \\
\hline 3 & 377 & 137 & 368 & 133 & 135 \\
\hline 4 & 377 & 137 & 366 & 133 & 137 \\
\hline $1-1989$ & 377 & 137 & 371 & 135 & 136 \\
\hline 2 & 377 & $1 \overline{37}$ & $\overline{373}$ & 136 & 136 \\
\hline 3 & 377 & 137 & 373 & 136 & 137 \\
\hline 4 & 377 & 137 & 373 & 136 & 137 \\
\hline $1-1990$ & 377 & 137 & 373 & 136 & 137 \\
\hline 2 & 371 & 135 & $3 \overline{66}$ & $13 \overline{3}$ & 136 \\
\hline
\end{tabular}


241-A-103 SINGLE-SHEL WHC-SD-WM-ER-30B, Rev. 0 TANK LEVEL HISTORY

\begin{tabular}{|l|c|c|c|c|c|}
\hline Year & Total & Total & Solids & Solids & LOW \\
\hline & (K gal) & (in) & (K gal) & [in) & [if] \\
\hline & & & & & \\
\hline 3 & 370 & 135 & 366 & 133 & 136 \\
\hline 4 & 370 & 135 & 366 & 133 & 137 \\
\hline $1-1991$ & 370 & 135 & 366 & 133 & 137 \\
\hline 2 & 370 & 135 & 366 & 133 & 135 \\
\hline 3 & 370 & 135 & 366 & 133 & 136 \\
\hline 4 & 370 & 135 & 366 & 133 & 136 \\
\hline 1.1992 & 370 & 135 & 366 & 133 & 137 \\
\hline 2 & 370 & 135 & 366 & 133 & \\
\hline 3 & 370 & 135 & 366 & 133 & 136 \\
\hline 4 & 370 & 135 & 366 & 133 & 134 \\
\hline $1-1993$ & 370 & 135 & 366 & 133 & 136 \\
\hline 2 & 370 & 135 & 366 & 133 & 135 \\
\hline 3 & & & & & 137 \\
\hline 4 & & & & & 135 \\
\hline
\end{tabular}


241-A-104 SINGLE-SHEL WHC-SD-WM-ER-308, Rev + 0 TANK LEVEL HISTORY

\begin{tabular}{|c|c|c|c|c|}
\hline Year & Total & Total & Sollds & Soltds \\
\hline & (K gal & (in) & (K g익) & [in] \\
\hline & & & & \\
\hline \multicolumn{5}{|l|}{$1 . \overline{1956}$} \\
\hline \multicolumn{5}{|l|}{2} \\
\hline \multicolumn{5}{|l|}{3} \\
\hline \multicolumn{5}{|l|}{4} \\
\hline \multicolumn{5}{|l|}{$1-1957$} \\
\hline 2 & 17 & 6 & & \\
\hline 3 & 22 & 8 & & \\
\hline 4 & 17 & 6 & & \\
\hline $1-1958$ & 22 & 8 & $\overline{0}$ & 0 \\
\hline 2 & 28 & $1 \overline{0}$ & 0 & 0 \\
\hline 3 & $16 \overline{8}$ & 61 & 0 & 0 \\
\hline 4 & 138 & 50 & 0 & 0 \\
\hline $1-1959$ & 187 & 68 & 0 & 0 \\
\hline 2 & $20 \overline{4}$ & 74 & 0 & 0 \\
\hline 3 & 366 & 133 & 0 & $\overline{0}$ \\
\hline 4 & 369 & 134 & 0 & 0 \\
\hline $1-1960$ & 743 & $2 \overline{70}$ & 0. & $\overline{0}$ \\
\hline 2 & 750 & 273 & 0 & 0 \\
\hline 3 & 655 & 238 & 0 & 0 \\
\hline 4 & 725 & 264 & $\overline{0}$ & $\overline{0}$ \\
\hline $1-1961$ & 842 & 306 & 0 & 0 \\
\hline \multicolumn{5}{|l|}{2} \\
\hline 3 & & & 0 & 0 \\
\hline 4 & 886 & 322 & & \\
\hline $1-1962$ & 952 & $3 \overline{46}$ & 0 & 0 \\
\hline \multicolumn{5}{|l|}{2} \\
\hline & & & & \\
\hline 4 & 682 & 248 & 0 & 0 \\
\hline $1-1963$ & 795 & 289 & 0 & 0 \\
\hline \multicolumn{5}{|l|}{2} \\
\hline \multicolumn{5}{|l|}{3} \\
\hline 4 & 824 & 300 & $\overline{0}$ & 0 \\
\hline $1-1964$ & 831 & 302 & 0 & 0 \\
\hline \multicolumn{5}{|l|}{2} \\
\hline \multicolumn{5}{|l|}{3} \\
\hline 4 & 864 & $3 \overline{14}$ & 0 & 0 \\
\hline $1-1965$ & 919 & 334 & $\overline{146}$ & 53 \\
\hline \multicolumn{5}{|l|}{2} \\
\hline 3 & 938 & 341 & 146 & 53 \\
\hline 4 & 932 & 339 & 146 & 53 \\
\hline $1-1966$ & 932 & 339 & 146 & 53 \\
\hline 2 & 941 & 342 & 146 & 53 \\
\hline 3 & 932 & 339 & 146 & 53 \\
\hline 4 & 935 & 340 & $\overline{146}$ & 53 \\
\hline $1-1967$ & 932 & 339 & 146 & 53 \\
\hline 2 & 935 & 340 & 146 & 53 \\
\hline
\end{tabular}


241-A-104 SINGLE-SHELL WHC-SD-MM-BR-308, REV. 0 TANK LEVEL HISTORY

\begin{tabular}{|c|c|c|c|c|}
\hline Year & Totel & Total & Solids & Solids \\
\hline & (K gal) & (in) & (K gal) & [in] \\
\hline & & & & \\
\hline 3 & 924 & 336 & 165 & 60 \\
\hline 4 & 924 & 336 & 168 & 61 \\
\hline$\overline{1}-1968$ & $\overline{927}$ & 337 & 182 & 66 \\
\hline 2 & 971 & 353 & 171 & 62 \\
\hline 3 & 976 & $35 \overline{\bar{b}}$ & 165 & $60^{-}$ \\
\hline 4 & $\overline{983}$ & 357 & $17 t$ & 62 \\
\hline $1-1969$ & 428 & 156 & 171 & 62 \\
\hline 2 & 308 & 112 & 74 & 27 \\
\hline 3 & 28 & 10 & 11 & 4 \\
\hline 4 & 960 & 349 & 3 & 1 \\
\hline 1.1970 & 957 & 348 & 1 & $0 . \overline{4}$ \\
\hline 2 & $\overline{964}$ & 357 & 1 & 0.4 \\
\hline 3 & 861 & 313 & $t$ & 0.4 \\
\hline 4 & 743 & 270 & 1 & 0.4 \\
\hline $1-1971$ & 627 & 228 & 1 & 0.4 \\
\hline 2 & 561 & 204 & 1 & 0.4 \\
\hline 3 & 454 & 165 & 1 & 0.4 \\
\hline 4 & 349 & 127 & 1 & 0.4 \\
\hline $1-1972$ & $19 \mathrm{~B}$ & 72 & 1 & 0.4 \\
\hline 2 & 275 & 100 & 1 & 0.4 \\
\hline 3 & 346 & 126 & 8 & 3 \\
\hline 4 & 440 & 160 & 8 & 3 \\
\hline $1-197 \sqrt{3}$ & 773 & 281 & 80 & 29 \\
\hline 2 & 956 & 348 & 80 & 29 \\
\hline 3 & 912 & 332 & 80 & 29 \\
\hline 4 & 837 & 304 & 80 & 29 \\
\hline$t-1974$ & 503 & 183 & 80 & 29 \\
\hline 2 & 806 & 293 & 80 & 29 \\
\hline 3 & 96 & 35 & 57 & 21 \\
\hline 4 & 55 & 20 & 39 & $1 \overline{4}$ \\
\hline I-1975 & 69 & 25 & 39 & 14 \\
\hline 2 & 25 & 9 & 25 & 9 \\
\hline 3 & 25 & 9 & 25 & 9 \\
\hline 4 & 25 & 9 & 25 & 9 \\
\hline $1-1976$ & 25 & 9 & 25 & 9 \\
\hline 2 & 25 & 9 & 25 & 9 \\
\hline 3 & 25 & 9 & $\overline{25}$ & 9 \\
\hline 4 & 25 & 9 & 25 & $\overline{9}$ \\
\hline $7-1977$ & 25 & 9 & 25 & 9 \\
\hline 2 & 25 & 9 & 25 & 9 \\
\hline$\overline{3}$ & 25 & 9 & 25 & 9 \\
\hline 4 & 25 & 9 & 25 & 9 \\
\hline १-१978 & 28 & 20 & 28 & 10 \\
\hline 2 & 28 & 70 & 28 & 10 \\
\hline 3 & 28 & 10 & 28 & $1 \overline{0}$ \\
\hline 4 & 28 & 10 & $2 \dot{8}$ & 10 \\
\hline
\end{tabular}


241-A-104 SINGLE-SHELL WHC-SD-WM-ER-308， REV. 0 TANK LEVEL, HISTORY

\begin{tabular}{|c|c|c|c|c|}
\hline Year & Total & Total & Solids & Solids \\
\hline & [K gal) & (in) & [K gal] & (in) \\
\hline & & $\cdot$ & & \\
\hline 1-1979 & 28 & 10 & 28 & $1 \overline{0}$ \\
\hline$\overline{2}$ & 28 & 10 & 28 & $1 \overline{0}$ \\
\hline 3 & 28 & 10 & 28 & 10 \\
\hline 4 & $\overline{28}$ & 10 & 28 & 10 \\
\hline $1-1980$ & 28 & 10 & 28 & 10 \\
\hline 2 & 28 & 10 & 28 & 10 \\
\hline 3 & 28 & 10 & 28 & 10 \\
\hline 4 & 28 & 10 & 28 & 10 \\
\hline $1-1981$ & 28 & 10 & 28 & 10 \\
\hline 2 & 28 & 10 & 28 & 10 \\
\hline$\overline{3}$ & 28 & 10 & 28 & 10 \\
\hline 4 & 28 & 10 & 28 & 10 \\
\hline $1-1982$ & 28 & 10 & 28 & to \\
\hline$\overline{2}$ & $2 B$ & 10 & 28 & 10 \\
\hline 3 & 28 & 10 & 28 & 10 \\
\hline 4 & 28 & 10 & 28 & 10 \\
\hline $1-1983$ & 28 & 10 & 28 & 10 \\
\hline 2 & 28 & 10 & 28 & 10 \\
\hline$\overline{3}$ & 28 & 10 & 28 & 10 \\
\hline 4 & 28 & 10 & 28 & 10 \\
\hline $1-1984$ & 28 & 10 & 28 & 10 \\
\hline 2 & 28 & 10 & 28 & 10 \\
\hline 3 & 28 & 10 & 28 & 10 \\
\hline 4 & $\overline{28}$ & 10 & 28 & $1 \overline{0}$ \\
\hline $1-1985$ & 28 & 10 & 28 & 10 \\
\hline 2 & 28 & 10 & 28 & 10 \\
\hline 3 & 28 & 10 & 28 & 10 \\
\hline 4 & 28 & 10 & 28 & 10 \\
\hline $1-1986$ & 28 & 10 & 28 & 10 \\
\hline 2 & 28 & 10 & 28 & 10 \\
\hline 3 & 28 & 10 & 28 & $1 \overline{0}$ \\
\hline 4 & 28 & 10 & 28 & 10 \\
\hline $1-1987$ & 28 & 10 & 28 & 10 \\
\hline$\overline{2}$ & 28 & 10 & 28 & 10 \\
\hline 3 & 28 & 10 & 28 & 10 \\
\hline 4 & 28 & 10 & 28 & 10 \\
\hline 1-1988 & 28 & 10 & 28 & 10 \\
\hline$\overline{2}$ & 28 & 10 & 28 & 10 \\
\hline 3 & 28 & 10 & 28 & 10 \\
\hline 4 & 28 & 10 & 28 & 10 \\
\hline $1-1989$ & 28 & 10 & 28 & 10 \\
\hline 2 & 28 & 10 & 28 & 10 \\
\hline 3 & 28 & 10 & 28 & $\overline{10}$ \\
\hline 4 & 28 & 10 & 28 & 10 \\
\hline $1-1990$ & 28 & 10 & 28 & 10 \\
\hline 2 & 28 & 10 & 28 & 10 \\
\hline
\end{tabular}


241-A-104 \$INGLE-SHELL WHC-SD-WM-ER-308, REV.0 TANK LEVEL, HISTORY

\begin{tabular}{|l|c|c|c|c|}
\hline Year & Total & Total & Sollds & Solids \\
\hline & (K gil) & (in) & (K gal) & (in) \\
\hline 3 & & & & \\
\hline 4 & 28 & 10 & 28 & 10 \\
\hline $1+1991$ & 28 & 10 & 28 & 10 \\
\hline 2 & 28 & 10 & 28 & 10 \\
\hline 3 & 28 & 10 & 28 & 10 \\
\hline 4 & 28 & 10 & 28 & 10 \\
\hline $1-1992$ & 28 & 10 & 28 & 10 \\
\hline 2 & 28 & 10 & 28 & 10 \\
\hline 3 & 28 & 10 & 28 & 10 \\
\hline 4 & 28 & 10 & 28 & 10 \\
\hline $1-1993$ & 28 & 10 & 28 & 10 \\
\hline 2 & 28 & 10 & 28 & 10 \\
\hline 3 & & & & \\
\hline 4 & & & & \\
\hline
\end{tabular}


241-A-TOS SINGLE-SHEL WHC-SD-WM-ER-308, Rev. 0 TANK LEVEL HISTORY

\begin{tabular}{|c|c|c|c|c|}
\hline Year & Total & Total & Solids & Solids \\
\hline & \{k gal\} & (in) & (K ged] & (in) \\
\hline & & & & \\
\hline \multicolumn{5}{|l|}{$1-1956$} \\
\hline \multicolumn{5}{|l|}{2} \\
\hline \multicolumn{5}{|l|}{3} \\
\hline \multicolumn{5}{|l|}{4} \\
\hline \multicolumn{5}{|l|}{ 1-1957 } \\
\hline 2 & 17 & 6 & & \\
\hline 3 & 22 & 8 & & \\
\hline 4 & 17 & 6 & 17 & 6 \\
\hline 1.1958 & 22 & 8 & & \\
\hline 2 & 25 & 9 & & \\
\hline 3 & 30 & 11 & & \\
\hline 4 & 30 & 11 & & \\
\hline 1-1959 & 30 & 11 & & \\
\hline 2 & 30 & 11 & & \\
\hline 3 & 30 & 11 & & \\
\hline 4 & 30 & 11 & & \\
\hline $1-1960$ & 30 & 11 & & \\
\hline 2 & 41 & 15 & & \\
\hline 3 & 47 & 17 & & \\
\hline 4 & 50 & 18 & & \\
\hline $1-1961$ & 50 & 18 & 50 & 18 \\
\hline \multicolumn{5}{|l|}{$\underline{\mathbf{2}}$} \\
\hline \multicolumn{5}{|l|}{3} \\
\hline 4 & 50 & 18 & 50 & 18 \\
\hline 1.1962 & 333 & 121 & & \\
\hline \multicolumn{5}{|l|}{2} \\
\hline \multicolumn{5}{|l|}{3} \\
\hline 4 & 699 & 254 & & \\
\hline 7.1963 & 740 & 269 & & \\
\hline \multicolumn{5}{|l|}{2} \\
\hline \multicolumn{5}{|l|}{3} \\
\hline 4 & 721 & 259 & & \\
\hline $1-1964$ & 715 & 260 & & \\
\hline \multicolumn{5}{|l|}{2} \\
\hline \multicolumn{5}{|l|}{3} \\
\hline 4 & 853 & 310 & & \\
\hline $1-7965$ & 866 & 315 & 72 & $2 B$ \\
\hline \multicolumn{5}{|l|}{2} \\
\hline 3 & 864 & 314 & 72 & 26 \\
\hline 4 & 858 & 312 & 72 & 26 \\
\hline 1.1966 & 850 & 309 & 72 & 26 \\
\hline 2 & 858 & 312 & $\overline{72}$ & 26 \\
\hline 3 & 861 & 313 & 72 & 26 \\
\hline 4 & 869 & 316 & 72 & 26 \\
\hline 7.7967 & 864 & 314 & 72 & 26 \\
\hline 2 & 855 & 311 & 72 & 26 \\
\hline
\end{tabular}


241-A-105 SINGLE-SHELL WHC-SD-WM-ER-308, REV.0 TANK LEVEL HISTORY

\begin{tabular}{|c|c|c|c|c|}
\hline Year. & Total & Total & Solids & Solids \\
\hline & (K gal) & [in] & [K gal) & (in) \\
\hline & & & & \\
\hline 3 & 872 & 317 & 110 & 40 \\
\hline 4 & 687 & 323 & 110 & 40 \\
\hline$\overline{1.1968}$ & $\overline{385}$ & $14 \overline{0}$ & 180 & 65 \\
\hline 2 & 396 & 144 & 82 & 30 \\
\hline 3 & 77 & 28 & 33 & 12 \\
\hline 4 & 88 & 32 & 33 & 12 \\
\hline $1+1969$ & 85 & 31 & 33 & 12 \\
\hline 2 & 89 & 32 & 52 & 19 \\
\hline 3 & 94 & 34 & 63 & 23 \\
\hline 4 & 66 & 24 & 33 & 12 \\
\hline 1.1970 & $\overrightarrow{66}$ & 24 & 33 & 12 \\
\hline 2 & 68 & 24 & 33 & 12 \\
\hline 3 & 66 & 24 & 33 & 12 \\
\hline 4 & 68 & 24 & 33 & 12 \\
\hline 1-1971 & 89 & 32 & 33 & 12 \\
\hline 2 & $\overline{94}$ & $3 \overline{4}$ & 33 & 12 \\
\hline 3 & 87 & 32 & 33 & 12 \\
\hline 4 & 37 & 13 & 33 & 12 \\
\hline$\overline{t .1972}$ & 50 & 18 & 33 & 12 \\
\hline 2 & 50 & 18 & 33 & 12 \\
\hline 3 & 47 & 17 & 33 & 12 \\
\hline 4 & 44 & 16 & 33 & 12 \\
\hline$\overline{1.1973}$ & 44 & $1 \overline{6}$ & 33 & 12 \\
\hline 2 & 43 & 16 & 33 & 12 \\
\hline 3 & 36 & 13 & 33 & 12 \\
\hline 4 & 50 & $1 \overline{8}$ & $\overline{33}$ & 12 \\
\hline $1-1974$ & 50 & 18 & 33 & 12 \\
\hline 2 & 50 & 18 & 33 & 12 \\
\hline$\overline{3}$ & 50 & 18 & 33 & 12 \\
\hline 4 & 52 & 19 & 33 & 12 \\
\hline $1-1975$ & 52 & 19 & 33 & 12 \\
\hline$\overline{2}$ & 52 & 19 & 33 & 12 \\
\hline 3 & 52 & $1 \overline{9}$ & 33 & $1 \overline{2}$ \\
\hline 4 & 50 & $1 \overline{8}$ & 33 & $1 \overline{2}$ \\
\hline 1.1976 & 50 & $1 \overline{8}$ & 33 & $1 \overline{2}$ \\
\hline 2 & 50 & 18 & 33 & 12 \\
\hline 3 & 50 & 18 & 33 & 12 \\
\hline 4 & 47 & 17 & 33 & 12 \\
\hline 1-1977 & 50 & 18 & 33 & 12 \\
\hline 2 & 47 & 17 & 33 & 12 \\
\hline 3 & 47 & 17 & 33 & 12 \\
\hline 4 & 50 & $1 \overline{8}$ & 33 & 12 \\
\hline $1-1978$ & 50 & 18 & 33 & 12 \\
\hline 2 & 41 & 15 & 33 & 12 \\
\hline 3 & 44 & $1 \overline{6}$ & 33 & 12 \\
\hline 4 & 39 & 14 & 33 & 12 \\
\hline
\end{tabular}


241-A-T05 SWGLE-SHELL WHC-SD-WM-ER-308, ReV.0 TANK LEVEL HISTORY

\begin{tabular}{|c|c|c|c|c|}
\hline Year & Total & Totel & Solids & Solids \\
\hline & (K gal) & (in) & (K gal) & (in) \\
\hline 1.1979 & 39 & 14 & 33 & 12 \\
\hline 2 & 39 & 14 & 33 & 12 \\
\hline 3 & 19 & 7 & 19 & 7 \\
\hline 4 & 19 & 7 & 19 & 7 \\
\hline $1-1980$ & 19 & 7 & 19 & 7 \\
\hline 2 & 19 & 7 & 19 & 7 \\
\hline 3 & 19 & 7 & 19 & 7 \\
\hline 4 & 19 & 7 & 19 & 7 \\
\hline $1-1981$ & 19 & 7 & 19 & 7 \\
\hline 2 & 19 & 7 & 19 & 7 \\
\hline 3 & 19 & 7 & 19 & 7 \\
\hline 4 & 19 & 7 & 19 & 7 \\
\hline 1.1982 & 19 & 7 & 19 & 7 \\
\hline 2 & 19 & 7 & 19 & 7 \\
\hline 3 & 19 & 7 & 19 & 7 \\
\hline 4 & 19 & 7 & 19 & 7 \\
\hline $1-1983$ & 19 & 7 & 19 & 7 \\
\hline 2 & $1 \overline{9}$ & 7 & 19 & 7 \\
\hline 3 & 19 & 7 & 19 & 7 \\
\hline 4 & 19 & 7 & 19 & 7 \\
\hline$i=1984$ & 19 & 7 & 19 & 7 \\
\hline 2 & 19 & 7 & 19 & 7 \\
\hline$\overline{3}$ & 19 & 7 & 19 & 7 \\
\hline 4 & 19 & 7 & 19 & 7 \\
\hline $1-1985$ & 19 & 7 & 19 & 7 \\
\hline 2 & 19 & 7 & 19 & 7 \\
\hline 3 & 19 & 7 & 19 & 7 \\
\hline 4 & 19 & 7 & 19 & 7 \\
\hline 1.1986 & 19 & 7 & 19 & 7 \\
\hline 2 & 19 & 7 & 19 & 7 \\
\hline 3 & 19 & 7 & 19 & 7 \\
\hline 4 & 19 & 7 & 19 & 7 \\
\hline $1-1987$ & 19 & 7 & 19 & 7 \\
\hline 2 & 19 & 7 & 19 & 7 \\
\hline 3 & 19 & 7 & 19 & 7 \\
\hline 4 & 19 & 7 & 19 & 7 \\
\hline 1-1988 & 19 & 7 & 19 & 7 \\
\hline 2 & 19 & 7 & 19 & 7 \\
\hline 3 & 19 & 7 & 19 & 7 \\
\hline 4 & 19 & 7 & 19 & 7 \\
\hline 1-1989 & 19 & 7 & 19 & 7 \\
\hline 2 & 19 & 7 & 19 & 7 \\
\hline 3 & 19 & 7 & 19 & 7 \\
\hline 4 & 19 & 7 & 19 & 7 \\
\hline $1-1990$ & 19 & 7 & 19 & 7 \\
\hline 2 & 19 & 7 & 19 & 7 \\
\hline
\end{tabular}


241.A-105 SINGLE-SHELL WHC-SD-WM-ER-308, REv, 0 TANK LEVEL HISTORY

\begin{tabular}{|l|c|c|c|c|}
\hline \multicolumn{1}{|c|}{ Year } & Total & Total & Solids & Solids \\
\hline & (K gal) & (in) & (K gal) & (in) \\
\hline 3 & & & & \\
\hline 4 & 19 & 7 & 19 & 7 \\
\hline $1-1991$ & 19 & 7 & 19 & 7 \\
\hline 2 & 19 & 7 & 19 & 7 \\
\hline 3 & 19 & 7 & 19 & 7 \\
\hline 4 & 19 & 7 & 19 & 7 \\
\hline $1-1992$ & 19 & 7 & 19 & 7 \\
\hline 2 & 19 & 7 & 19 & 7 \\
\hline 3 & 19 & 7 & 19 & 7 \\
\hline 4 & 19 & 7 & 19 & 7 \\
\hline $1-1993$ & 19 & 7 & 19 & 7 \\
\hline 2 & 19 & 7 & 19 & 7 \\
\hline 3 & & & & \\
\hline 4 & & & & \\
\hline
\end{tabular}


241-A-106 SINGLE-SHELL WHC-SD-WM-ER-308, Rev + D TANK LEVEL HISTORY

\begin{tabular}{|c|c|c|c|c|}
\hline Yeat & Total & Totel & Solids & Salids \\
\hline & (K gal) & (in) & (K gal) & (in) \\
\hline \multirow{2}{*}{\multicolumn{5}{|c|}{$1-1956$}} \\
\hline & & & & \\
\hline \multirow{2}{*}{\multicolumn{4}{|c|}{3}} & \\
\hline \multirow{2}{*}{\multicolumn{4}{|c|}{4}} & \\
\hline $1-1967$ & $\overline{8 \mathrm{~B}}$ & 32 & & \\
\hline 2 & 94 & 34 & & \\
\hline 3 & 124 & 45 & & \\
\hline 4 & $\sqrt{84}$ & & & \\
\hline $1-1958$ & 234 & 67 & & \\
\hline 2 & & 85 & & \\
\hline 3 & & 87 & & \\
\hline 4 & $\leq 40$ & 89 & & \\
\hline $1-1959$ & 278 & 101 & & \\
\hline 2 & 295 & 107 & & \\
\hline & 380 & 138 & & \\
\hline$\frac{1+7960}{2}$ & 416 & 151 & & \\
\hline & 440 & 160 & & \\
\hline & 449 & 163 & & \\
\hline 4 & 485 & 176 & & \\
\hline $1-1961$ & 380 & 138 & & \\
\hline \multicolumn{5}{|l|}{2} \\
\hline \multicolumn{5}{|l|}{3} \\
\hline 4 & 715 & 260 & & \\
\hline $1-1962$ & 699 & 254 & & \\
\hline \multicolumn{5}{|l|}{12} \\
\hline \multicolumn{5}{|l|}{3} \\
\hline 4 & 883 & 327 & & \\
\hline $1-1963$ & 924 & 336 & & \\
\hline \multicolumn{5}{|l|}{2} \\
\hline \multicolumn{5}{|l|}{3} \\
\hline 4 & 915 & 333 & & \\
\hline $7+7964$ & 916 & 333 & & \\
\hline \multicolumn{5}{|l|}{$\underline{z}$} \\
\hline \multicolumn{5}{|l|}{3} \\
\hline 4 & 811 & 295 & & \\
\hline $1-1965$ & 876 & 318 & 118 & 43 \\
\hline \multicolumn{5}{|l|}{2} \\
\hline 3 & 880 & 320 & 118 & 43 \\
\hline 4 & 880 & 320 & 118 & 43 \\
\hline 1.1966 & 877 & 319 & 118 & 43 \\
\hline 2 & 877 & 319 & 118 & 43 \\
\hline 3 & 877 & 319 & 118 & 43 \\
\hline 4 & 880 & 320 & 118 & 43 \\
\hline 1.1967 & $\overline{864}$ & $3 \overline{14}$ & 118 & $\overline{43}$ \\
\hline 2 & 870 & 316 & 118 & 43 \\
\hline
\end{tabular}


24t-A-106 SINGLE-SHELL WHC-SD-FM-ER-308, REV. 0 TANK LEVEL HISTORY

\begin{tabular}{|c|c|c|c|c|}
\hline Year & Total & Totel & Solids & Solids \\
\hline & (K gal) & (in) & (KK gal) & (in) \\
\hline 3 & 861 & 313 & 940 & 5.1 \\
\hline 4 & 851 & 313 & 176 & 64 \\
\hline$t .19 \overline{68}$ & 894 & 325 & $17 \overline{3}$ & $6 \overline{3}$ \\
\hline 2 & 936 & 340 & $17 \overline{1}$ & 62 \\
\hline 3 & 935 & 340 & 168 & 61 \\
\hline 4 & 936 & 340 & 176 & 64 \\
\hline$t-1969$ & 935 & 340 & 175 & 64 \\
\hline 2 & 572 & 208 & $2 \overline{7}$ & $8 \dot{3}$ \\
\hline 3 & $6 \overline{00}$ & $27 \overline{8}$ & 254 & 92 \\
\hline 4 & 263 & 96 & 234 & 85 \\
\hline 1.1970 & 252 & 92 & 195 & 71 \\
\hline 2 & 245 & 89 & 185 & 67 \\
\hline 3 & 249 & 91 & 163 & 69 \\
\hline 4 & 154 & 56 & 124 & 45 \\
\hline$\overline{1-1971}$ & 139 & 51 & $9 \overline{8}$ & $3 \overline{6}$ \\
\hline 2 & $1 \overline{42}$ & 52 & 98 & 36 \\
\hline 3 & 151 & 65 & 98 & 36 \\
\hline 4 & 122 & 44 & $9 \overline{8}$ & 36 \\
\hline 1.1972 & 96 & $3 \overline{5}$ & 96 & 35 \\
\hline 2 & 70 & 25 & 19 & 7 \\
\hline 3 & 107 & 39 & 22 & 8 \\
\hline 4 & 66 & 24 & 11 & 4 \\
\hline $1-1973$ & 99 & 36 & $1 \overline{1}$ & 4 \\
\hline 2 & $\overline{51}$ & 19 & 11 & 4 \\
\hline 3 & 69 & 25 & 11 & 4 \\
\hline 4 & 48 & 17 & $\overline{6}$ & 2 \\
\hline $1-1974$ & 206 & 75 & $\overline{0}$ & $\overline{0}$ \\
\hline$\underline{z}$ & 686 & 249 & 0 & 0 \\
\hline 3 & 987 & 359 & 12 & 4 \\
\hline 4 & 751 & 273 & 11 & 4 \\
\hline 1.1975 & 814 & 296 & 11 & 4 \\
\hline 2 & $\overline{842}$ & 306 & 11 & 4 \\
\hline 3 & 663 & 241 & 11 & 4 \\
\hline 4 & 817 & 297 & 11 & 4 \\
\hline 1.1976 & 820 & 298 & 91 & 33 \\
\hline 2 & $8 \overline{72}$ & 317 & 91 & 33 \\
\hline 3 & 822 & 299 & 91 & 33 \\
\hline 4 & 979 & 366 & 102 & 37 \\
\hline $7-1977$ & 949 & 345 & 102 & 37 \\
\hline 2 & 561 & 204 & 102 & 37 \\
\hline 3 & 129 & 47 & $\overline{80}$ & 29 \\
\hline 4 & 116 & 42 & $\overline{52}$ & 19 \\
\hline$\overline{1.1978}$ & 162 & $\overline{\mathbf{5 9}}$ & 52 & $\overline{19}$ \\
\hline 2 & $\overline{83}$ & 30 & 50 & 18 \\
\hline 3 & 72 & 26 & 50 & 18 \\
\hline 4 & 72 & 26 & 50 & 18 \\
\hline
\end{tabular}


241-A.106 SENGLE-SHEL WHC-SD-WM-ER-308, ReV.0 TANK LEVEL. HISTORY

\begin{tabular}{|c|c|c|c|c|}
\hline Year & Total & Total & Solidis & Solids \\
\hline & (K gal) & (in) & (K gal) & (in) \\
\hline & & & & \\
\hline $1-1979$ & 127 & 46 & 50 & 18 \\
\hline 2 & 140 & 51 & 50 & 18 \\
\hline 3 & 140 & 51 & 50 & 18 \\
\hline 4 & 657 & 239 & 50 & 18 \\
\hline 1.1980 & 663 & 241 & 50 & 18 \\
\hline 2 & 666 & 242 & 96 & 35 \\
\hline 3 & 128 & 47 & 94 & 34 \\
\hline 4 & 128 & 47 & 94 & 34 \\
\hline $1-1981$ & 128 & 47 & 94 & 34 \\
\hline 2 & $12 \mathrm{~B}$ & 47 & 94 & 34 \\
\hline 3 & 128 & 47 & 94 & 34 \\
\hline 4 & 128 & 47 & 94 & 34 \\
\hline $1-1982$ & 128 & 47 & 94 & 34 \\
\hline$\overline{2}$ & 126 & 45 & 95 & 35 \\
\hline 3 & 125 & 45 & 125 & 45 \\
\hline 4 & 125 & 45 & 125 & 45 \\
\hline $7-1983$ & 125 & 45 & 125 & 45 \\
\hline 2 & 125 & 45 & 125 & 45 \\
\hline 3 & 125 & 45 & 125 & 45 \\
\hline 4 & 125 & 45 & 725 & 45 \\
\hline $1-1984$ & 125 & 45 & 125 & 45 \\
\hline 2 & 125 & 45 & 725 & 45 \\
\hline 3 & 125 & 45 & 125 & 45 \\
\hline 4 & 125 & 45 & 125 & 45 \\
\hline $1-1985$ & 125 & 45 & 125 & 45 \\
\hline 2 & 125 & 45 & 125 & 45 \\
\hline 3 & 125 & 45 & 125 & 45 \\
\hline 4 & 125 & 45 & 125 & 45 \\
\hline $1-1986$ & 125 & 45 & 125 & 45 \\
\hline 2 & 125 & 45 & 125 & 45 \\
\hline 3 & 125 & 45 & 125 & 45 \\
\hline 4 & 125 & 45 & 125 & 45 \\
\hline $1-1987$ & 125 & 45 & 125 & 45 \\
\hline 2 & 125 & 45 & 125 & 45 \\
\hline 3 & 125 & 45 & 125 & 45 \\
\hline 4 & 125 & 45 & 125 & 45 \\
\hline 1.1988 & 125 & 45 & 125 & 45 \\
\hline 2 & 125 & 45 & 125 & 45 \\
\hline 3 & 125 & 45 & 125 & 45 \\
\hline 4 & 125 & 45 & 125 & 45 \\
\hline $1-1989$ & $\overline{125}$ & $\overline{45}$ & 125 & 45 \\
\hline 2 & 125 & 45 & 125 & 45 \\
\hline 3 & 125 & 46 & 125 & 45 \\
\hline 4 & 125 & 45 & 125 & 45 \\
\hline $1-1990$ & 125 & 45 & 125 & 45 \\
\hline 2 & 125 & 45 & 125 & 45 \\
\hline
\end{tabular}


241-A-106 SINGLE-SHELL WHC-SD-WM-ER-308, REV. 0 TANK LEVEL HISTOAY

\begin{tabular}{|l|c|c|c|c|}
\hline Year & Total & Total & Solids & Solids \\
\hline & (K gali & (tn) & (K gal) & (in) \\
\hline 3 & & & & \\
\hline 4 & 125 & 45 & 125 & 45 \\
\hline $1-1991$ & 125 & 45 & 125 & 45 \\
\hline 2 & 125 & 45 & 125 & 45 \\
\hline 3 & 125 & 45 & 125 & 45 \\
\hline 4 & 125 & 45 & 125 & 45 \\
\hline $1-1992$ & 125 & 45 & 125 & 45 \\
\hline 2 & 125 & 45 & 125 & 45 \\
\hline 3 & 125 & 45 & 125 & 45 \\
\hline 4 & $\overline{125}$ & 45 & 125 & 45 \\
\hline $1-1993$ & 125 & 45 & 125 & 45 \\
\hline 2 & 125 & 45 & 125 & 45 \\
\hline 3 & & & & \\
\hline 4 & & & & \\
\hline
\end{tabular}


241-A-101

Historical Vapor Space Temperature

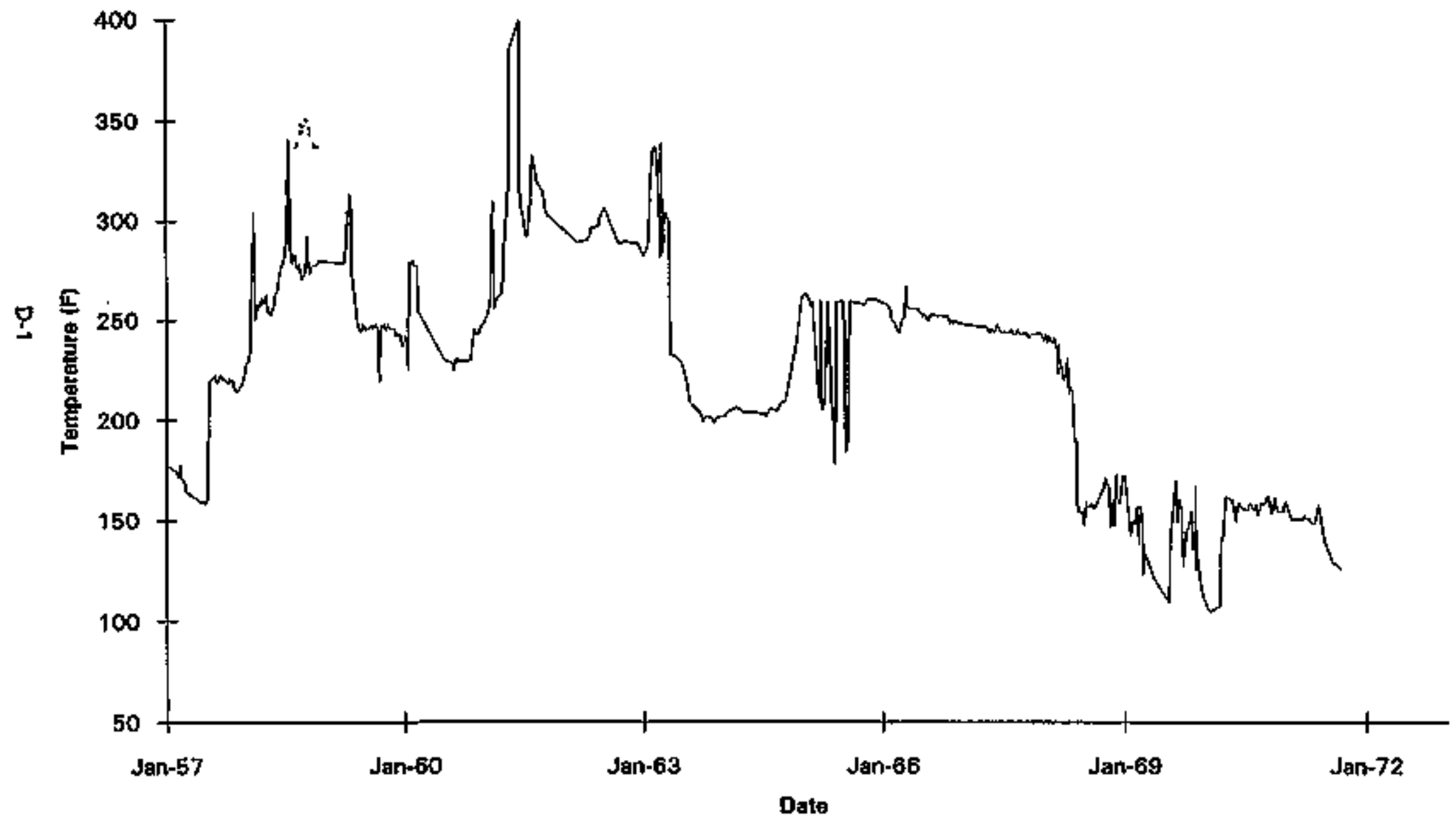


241-A-101

Historical Average Waste Temperature

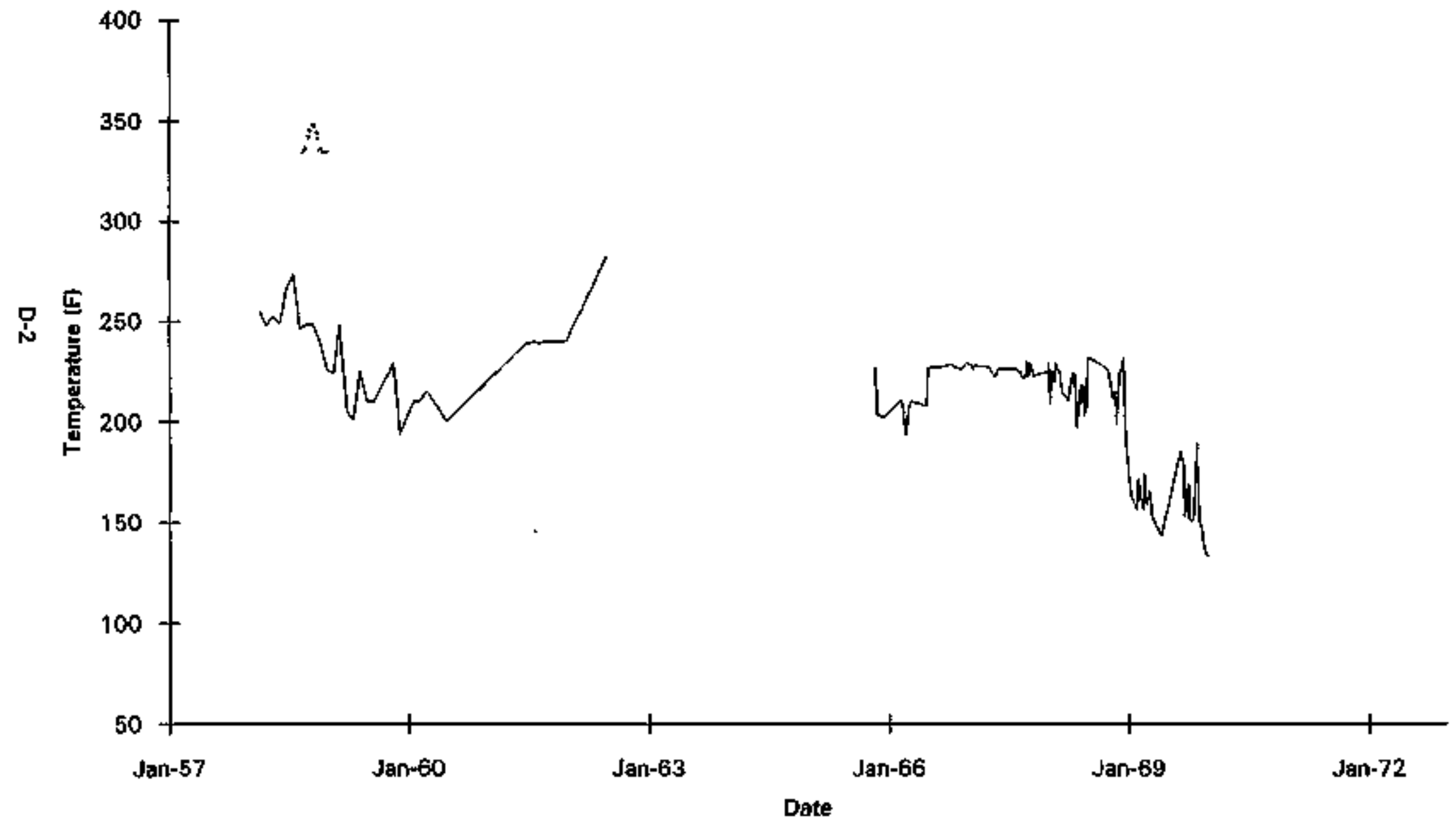

Data obtained from RHO-CO-1172 and Waste Status Summary Reports. 
Tank 241-A-101

Historical High Or Only Waste Temperature

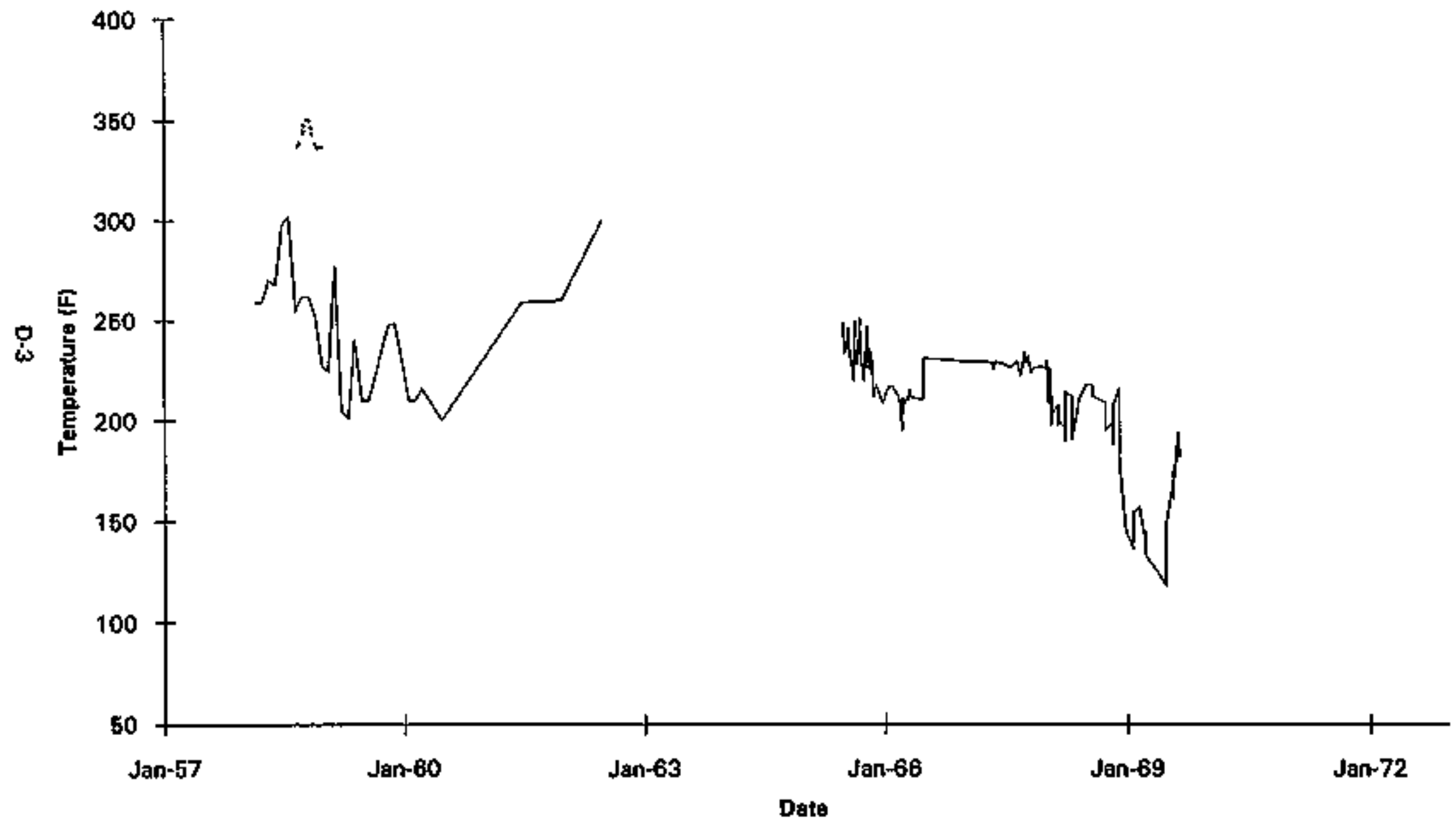


Tank 241-A-102

Historical Vapor Space Temperature

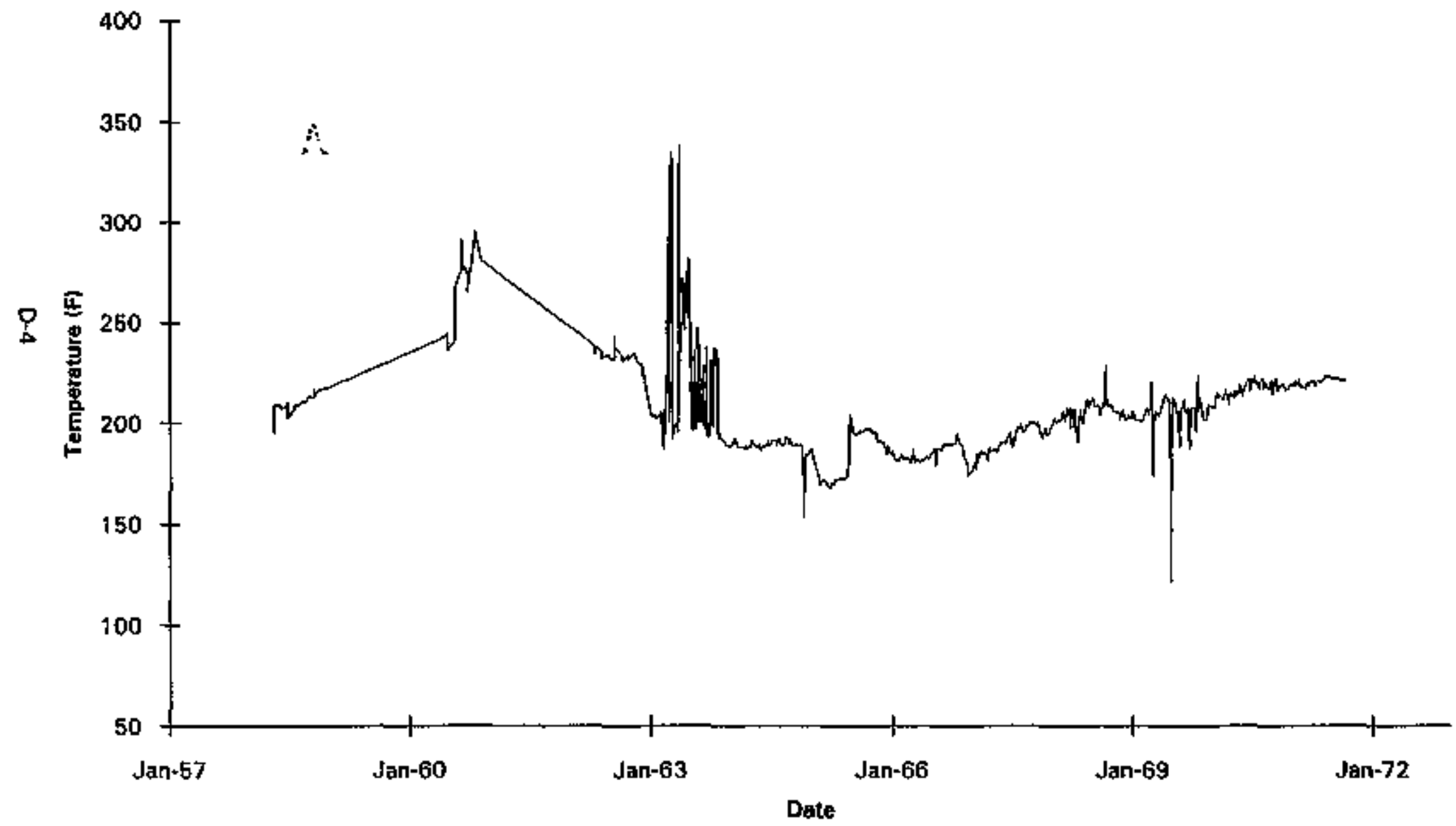


Tank 241-A-102

Historical Average Waste Temperature

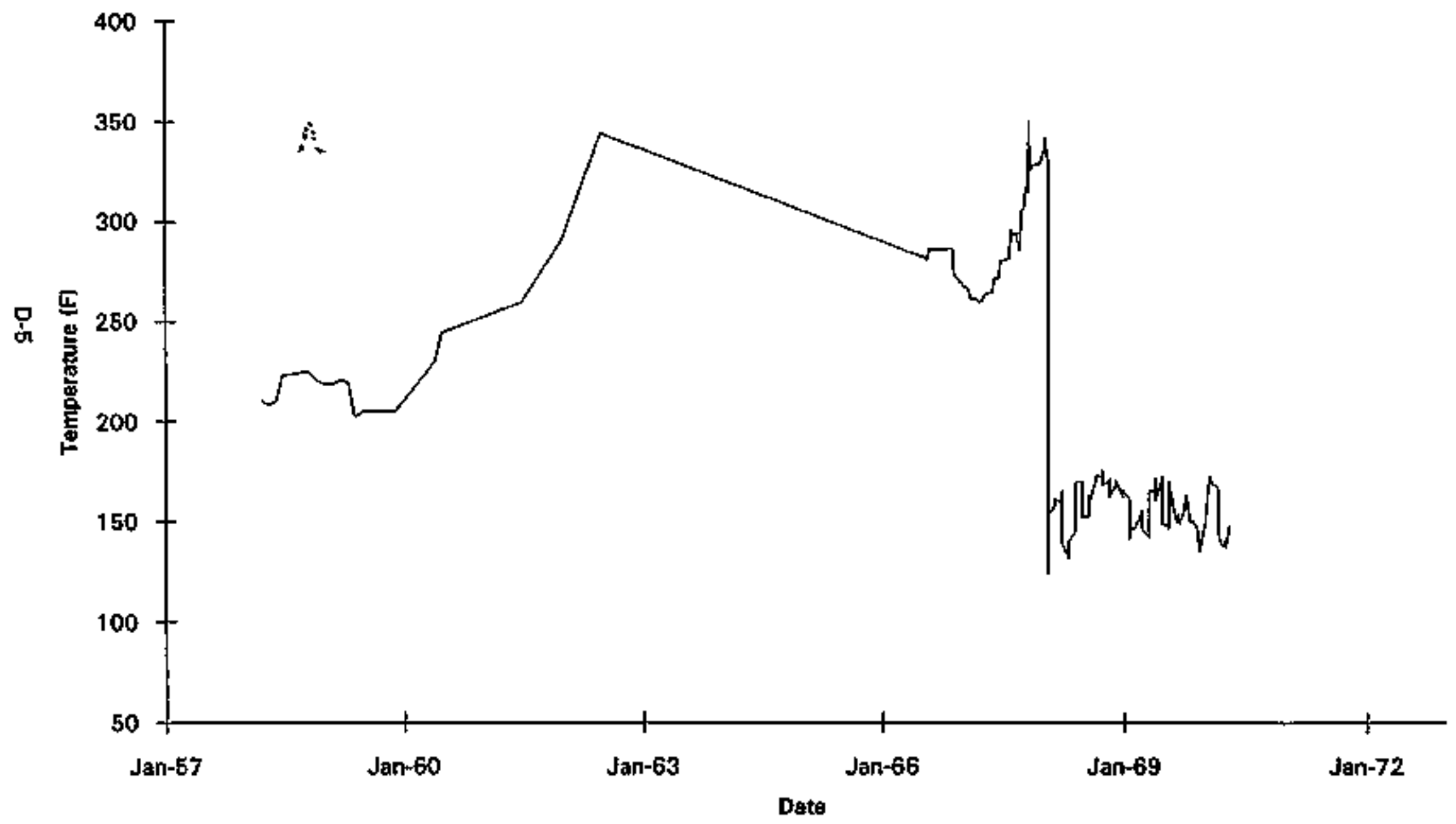


Tank 241-A-102

Historical High Or Only Waste Temperature

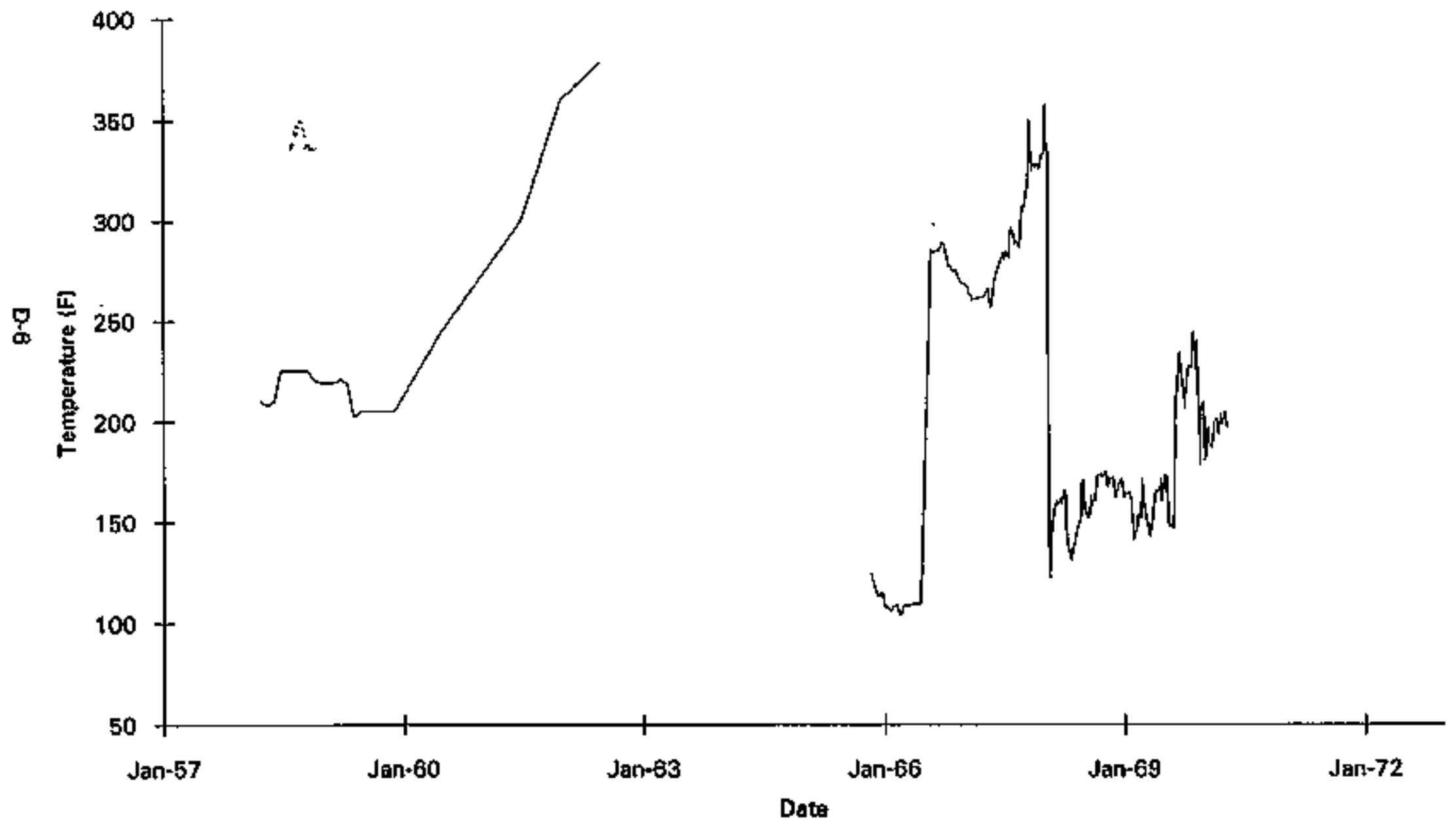


Tank 241-A-103

Historical Vapor Space Temperature

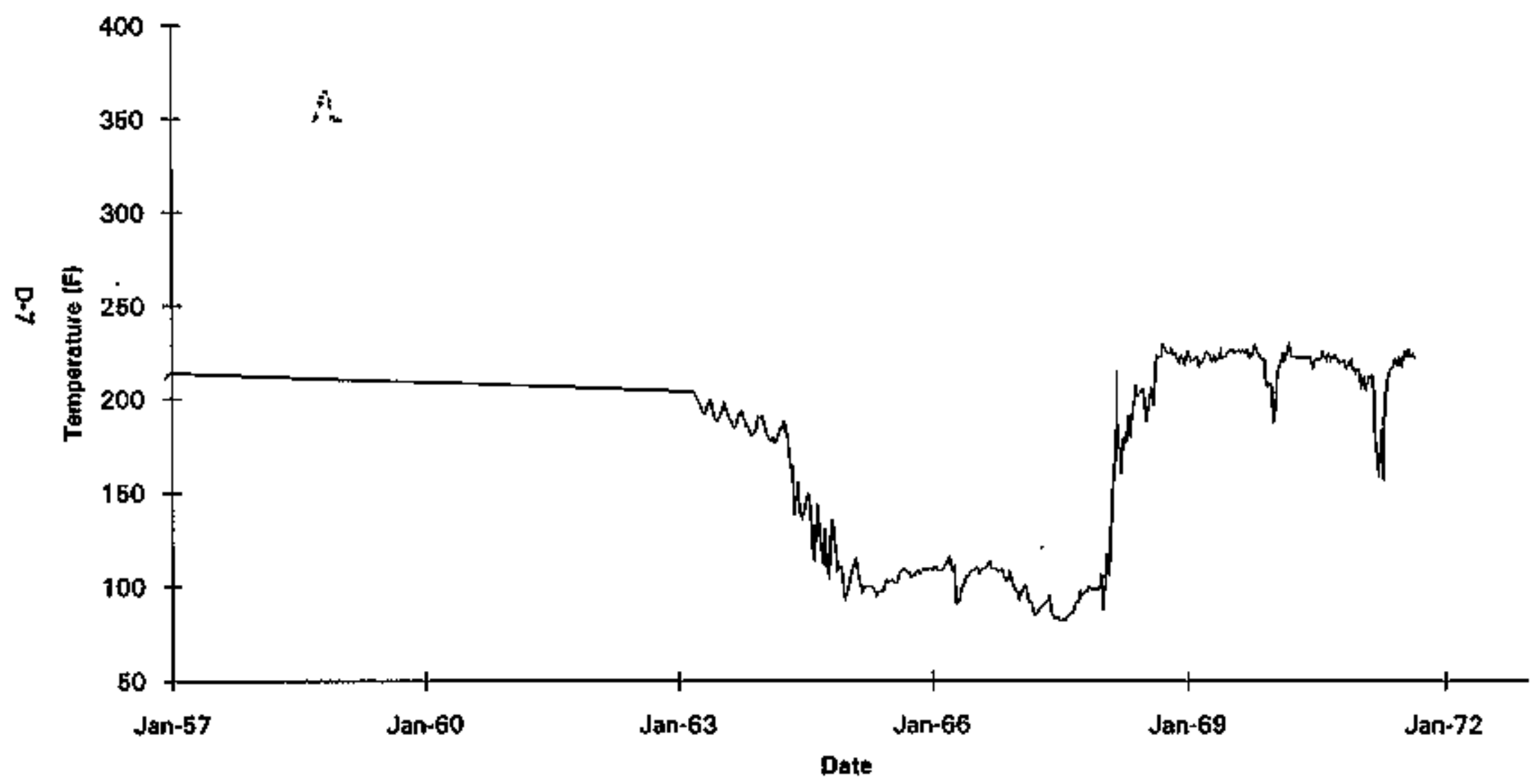


Tank 241-A-103

Historical Average Waste Temperature

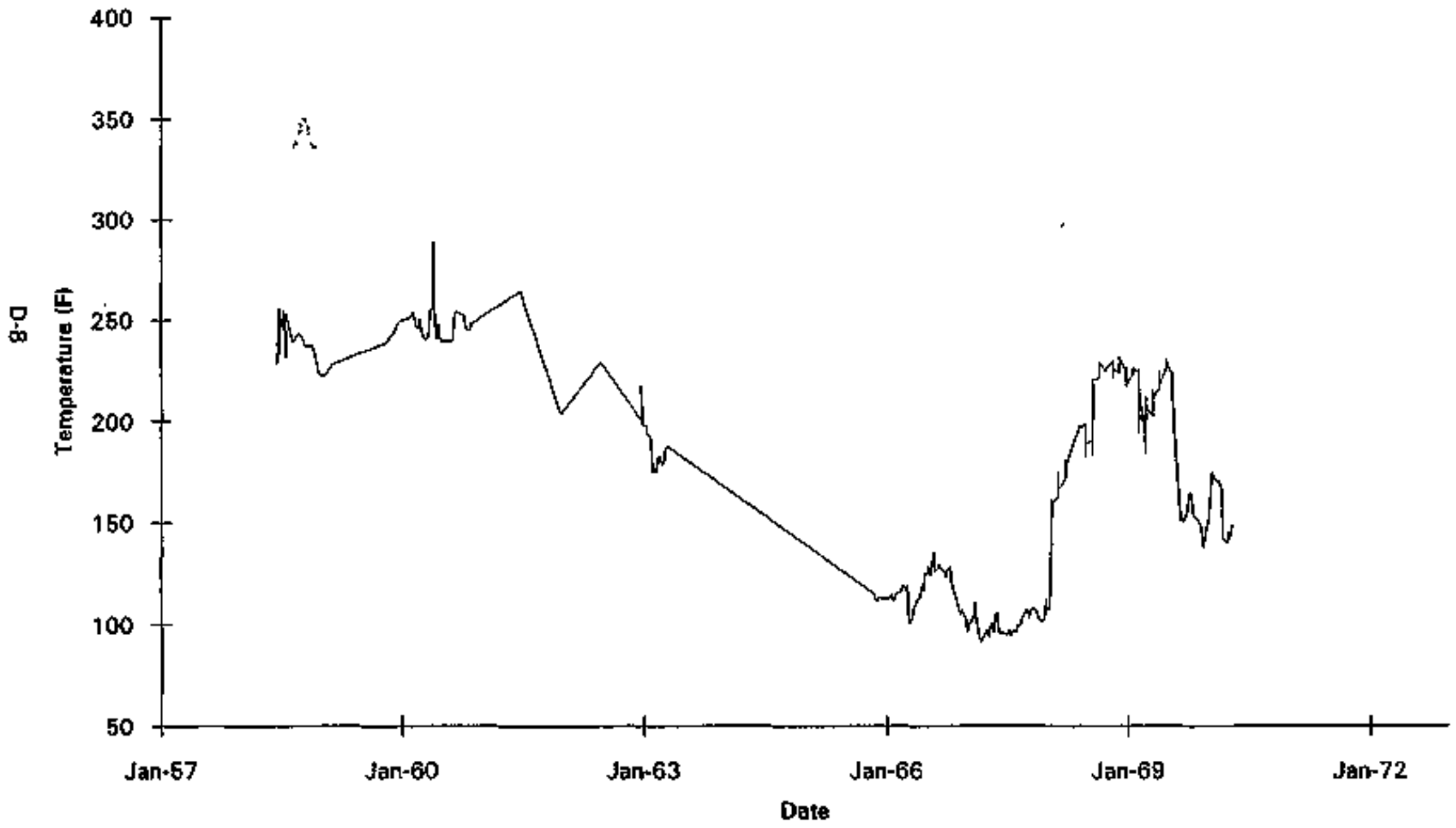

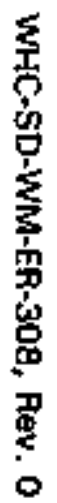

Data obtained from RHO-CO-1172 and Waste Status Summary Reports. 
Tank 241-A-103

Historical High Or Only Waste Temperature

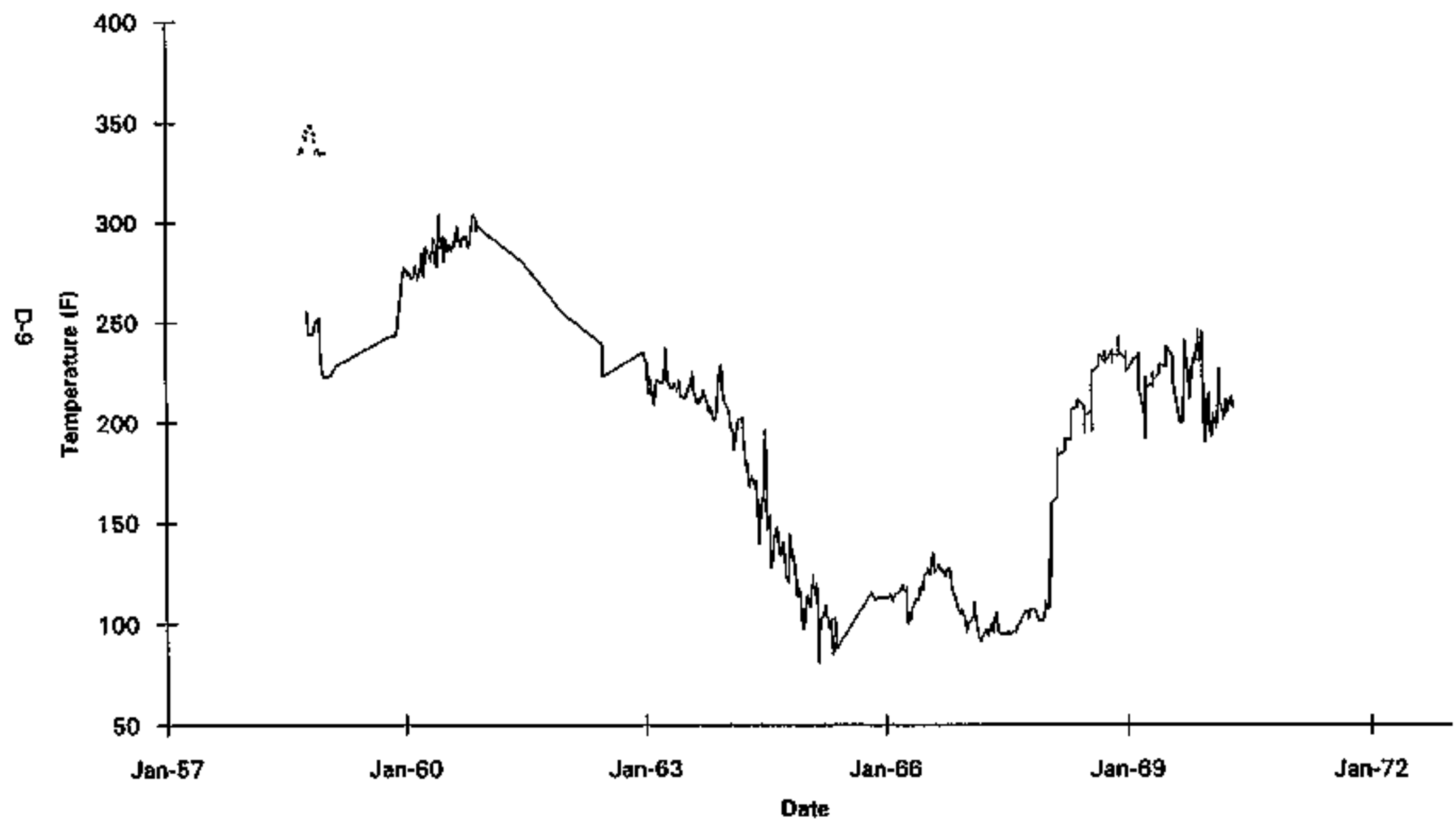

Data abtained from RHO-CD-1172 and Waste Status Summsry Reports. 
Tank 241-A-104

Historical Vapor Space Temperature

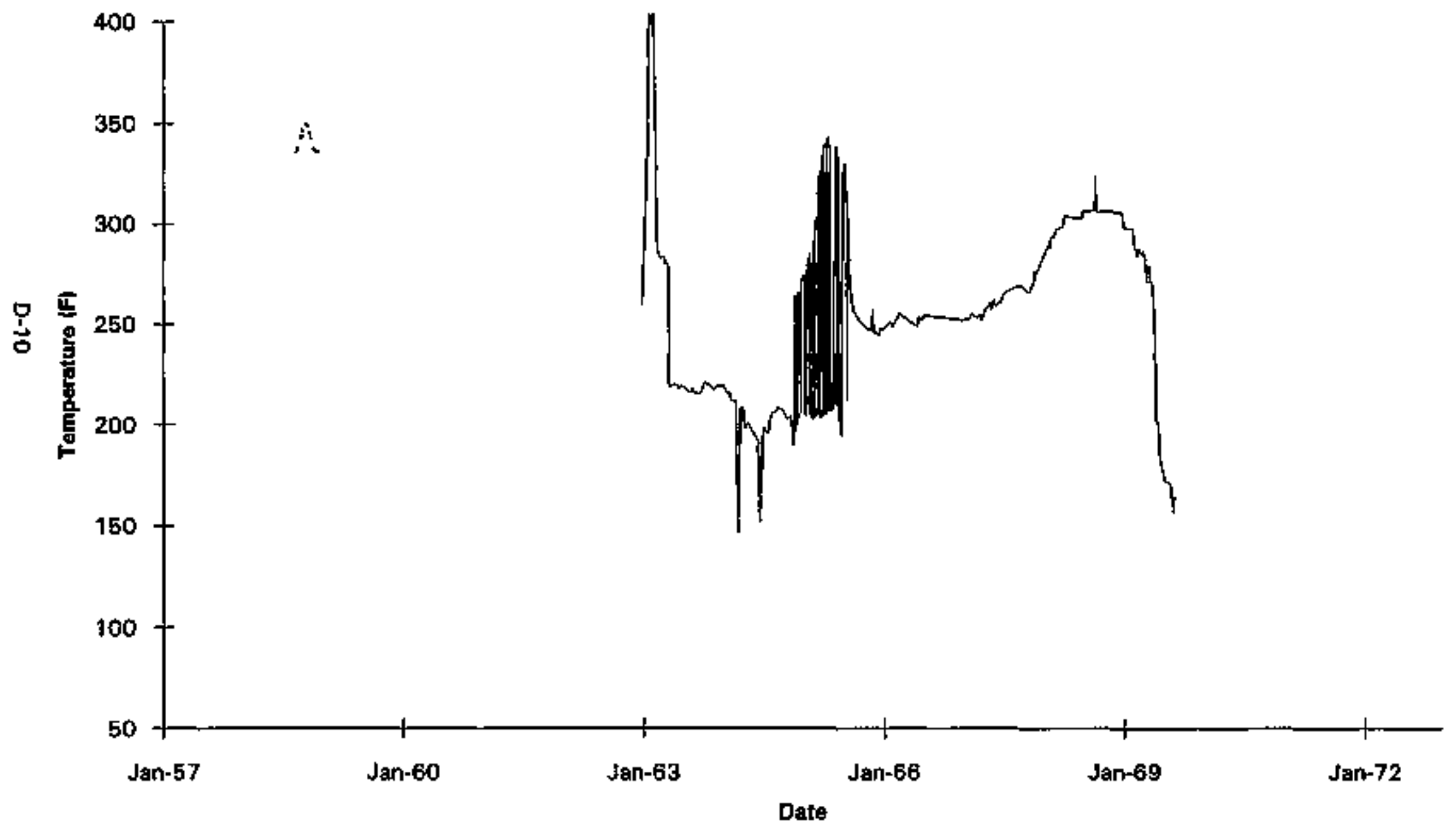

Data obtained from RHC-CD-1172 and Waste Status Summary Reports. 
Tank 241-A-104

Historical Average Waste Temperature

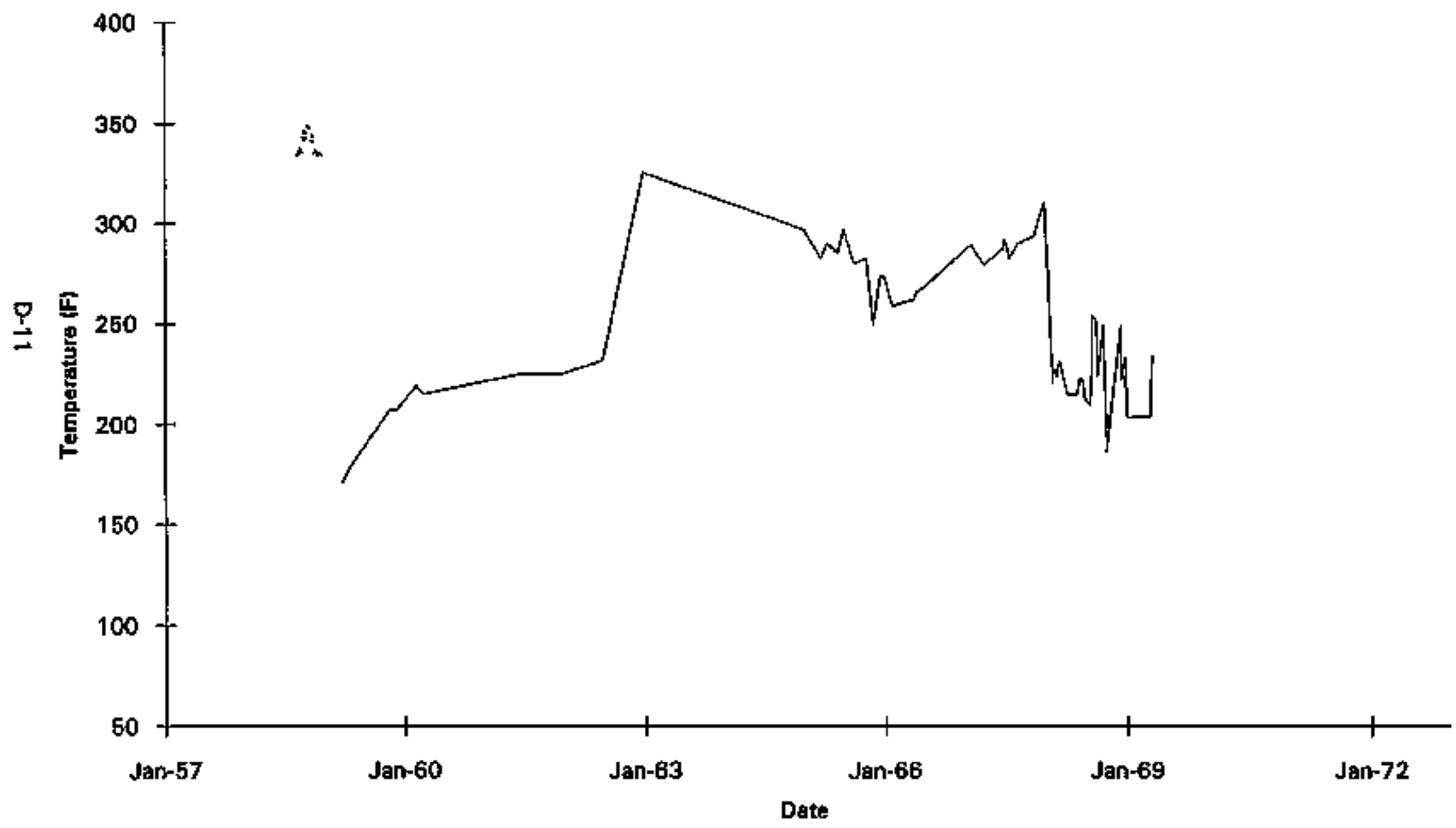


Tank 241-A-104

Historical High Or Only Waste Temperature

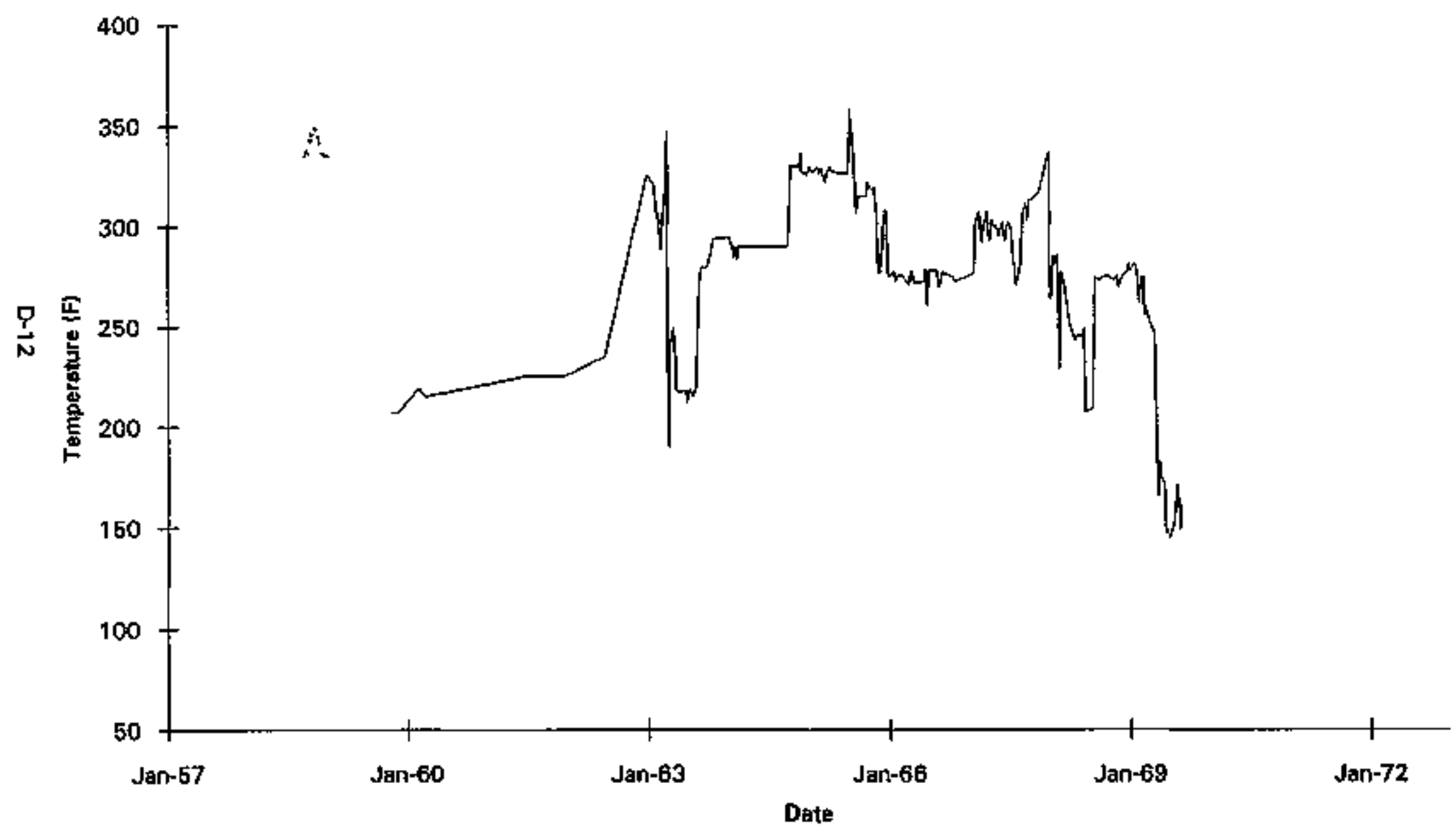

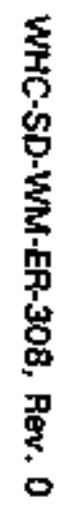

Data obtained from RHO-CD-1172 and Waste Stațus Summary Reports. 
Tank 241-A-105

Historical Vapor Space Temperature

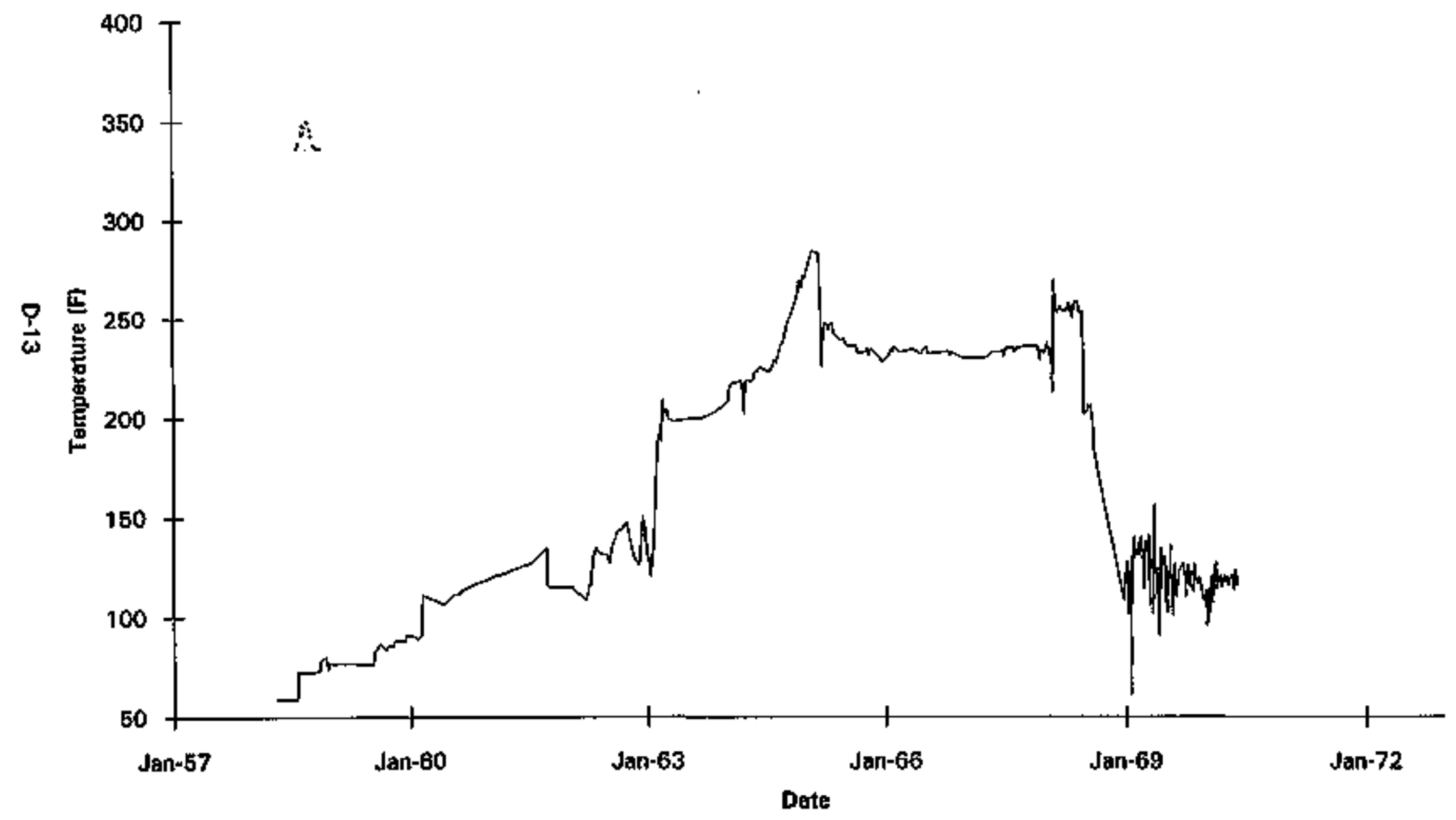

Data obtained from Document FHO-CD-1172 and Waste Status Summary Reports. 
Tank 241-A-105

Historical Average Waste Temperature

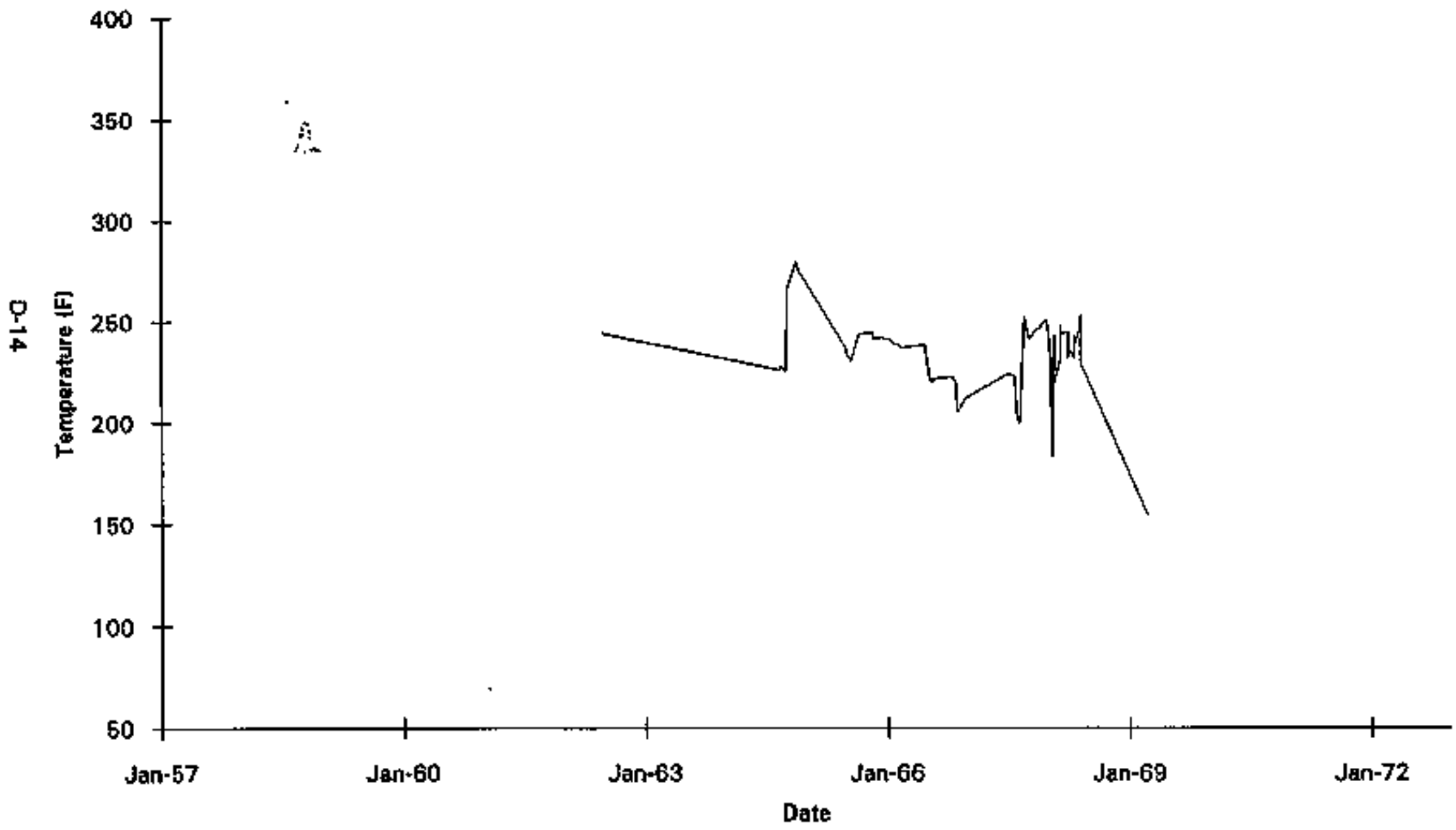

Data obtained from RHO-CD*1172 and Waste Status Summary Reports. 
Tank 241-A-105

Historical High Or Only Waste Temperature

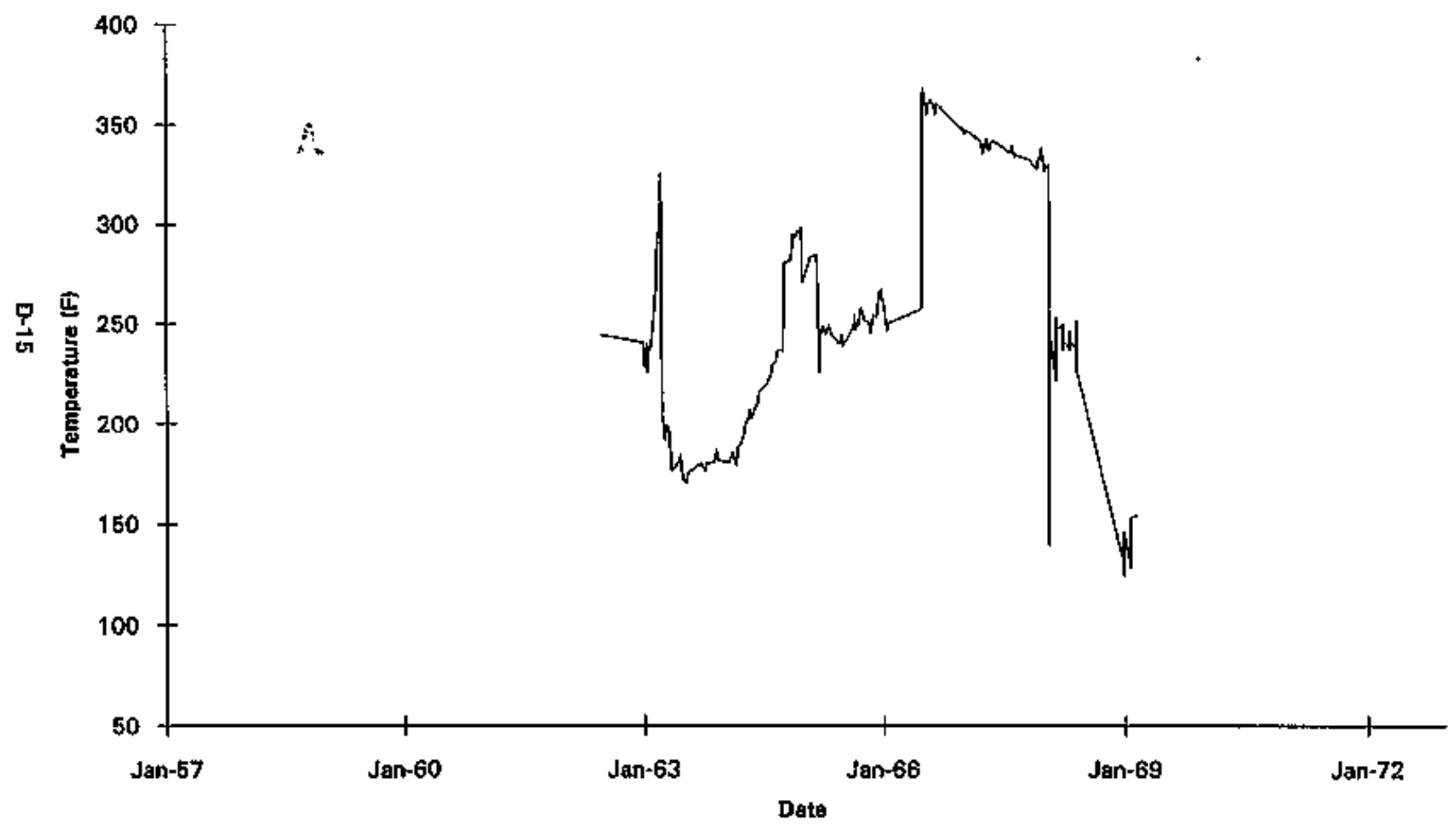


Tank 241-A-106

Historical Vapor Space Temperature

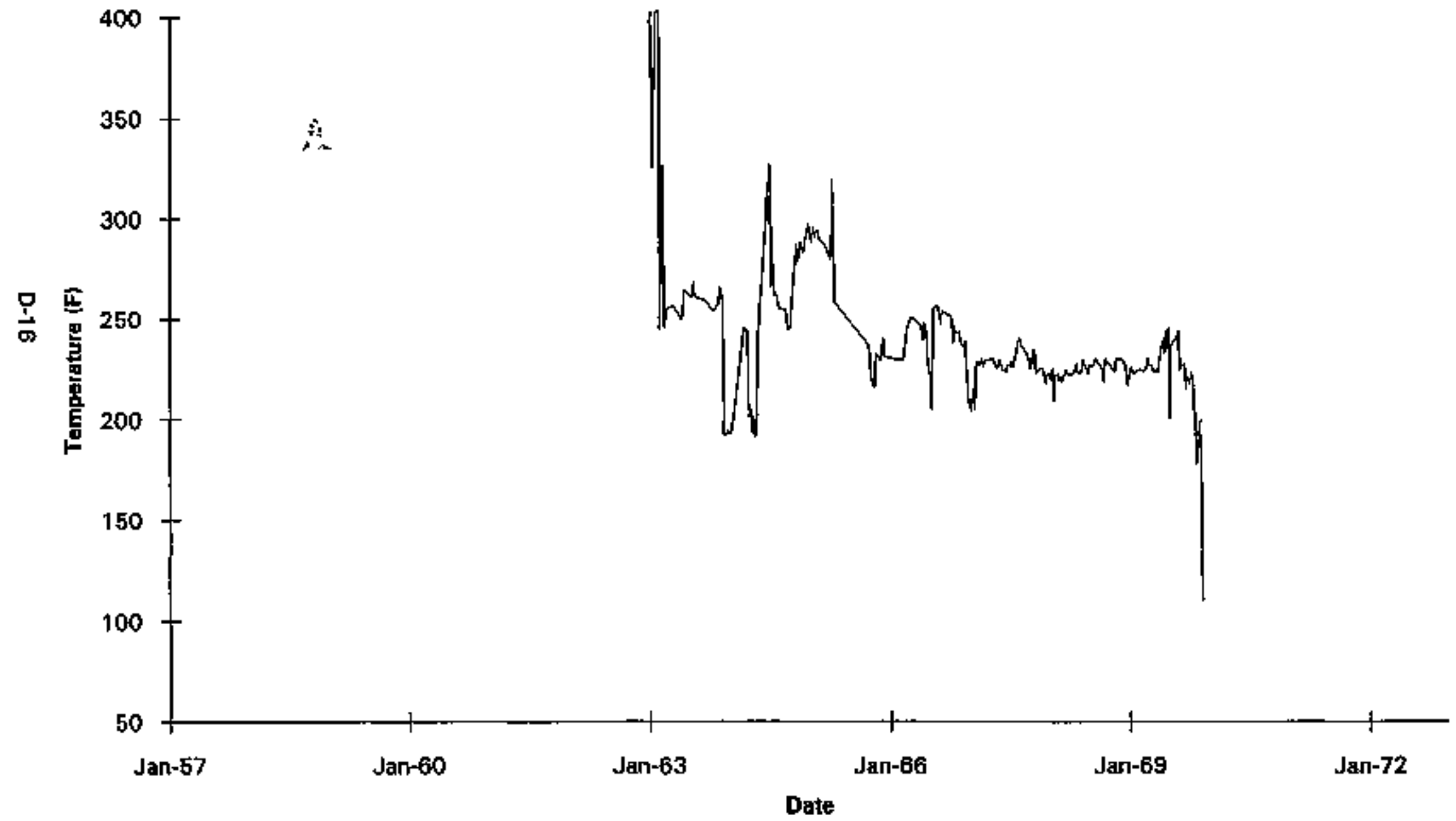


Tank 241-A-106

Historical Average Waste Temperature

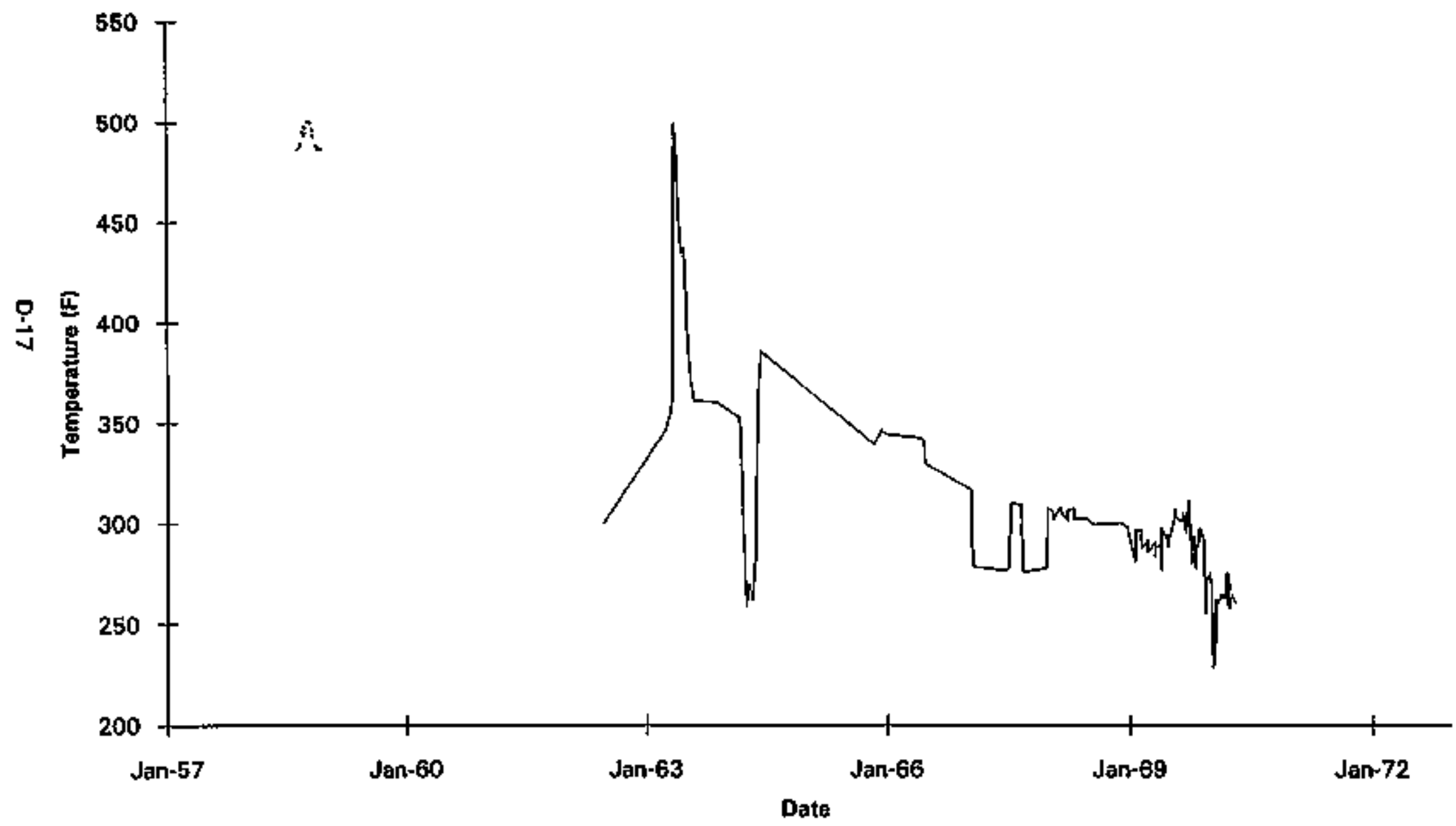

Data abtained from RHO-CO-\$172 and Waste Status Summary Reports. 
Tank 241-A-106

Historical High Or Only Waste Temperature

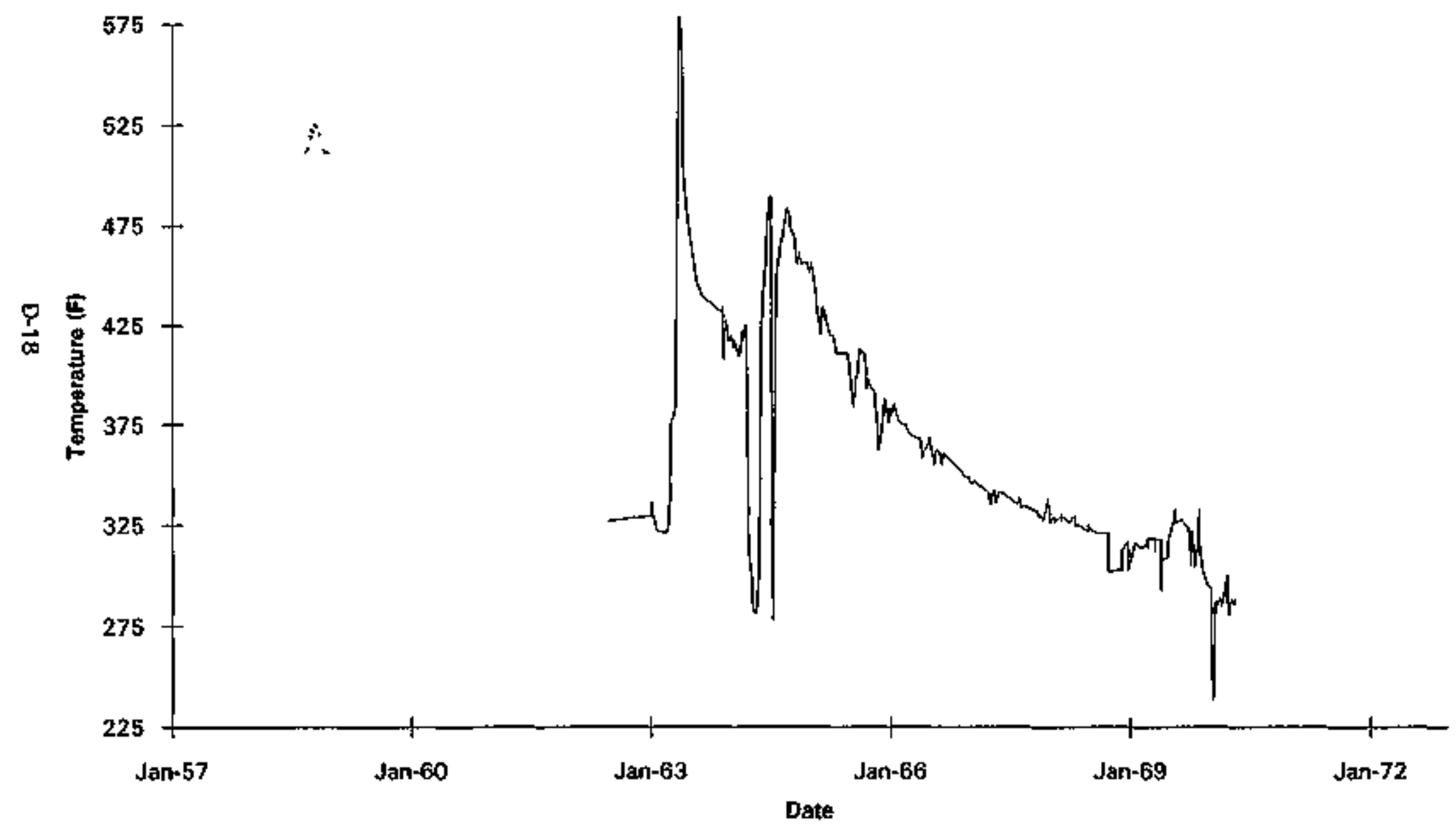




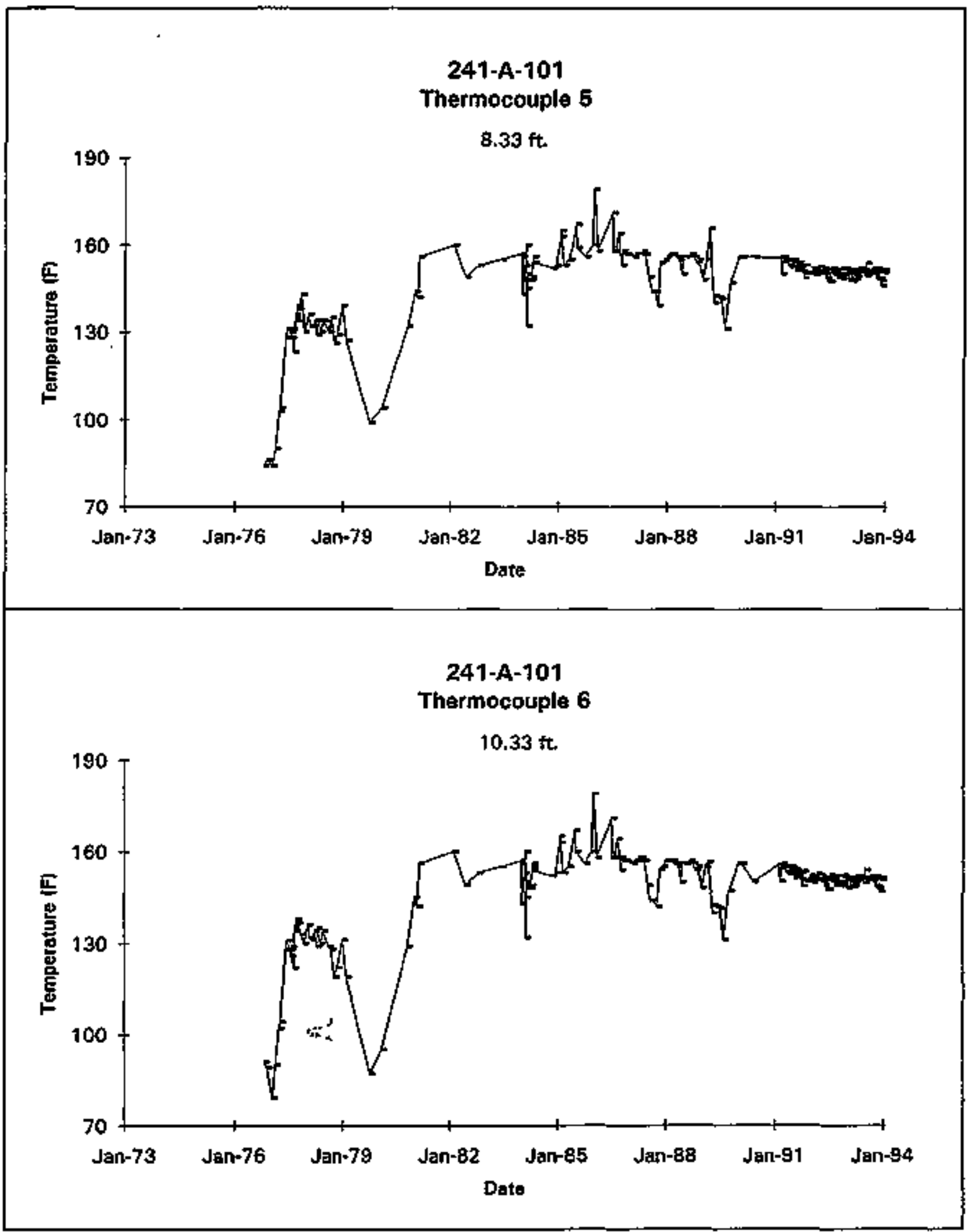

Data obtained from WHC Surveillence Analysis Computer System (SACS), November 12, 1993.

D-2t 


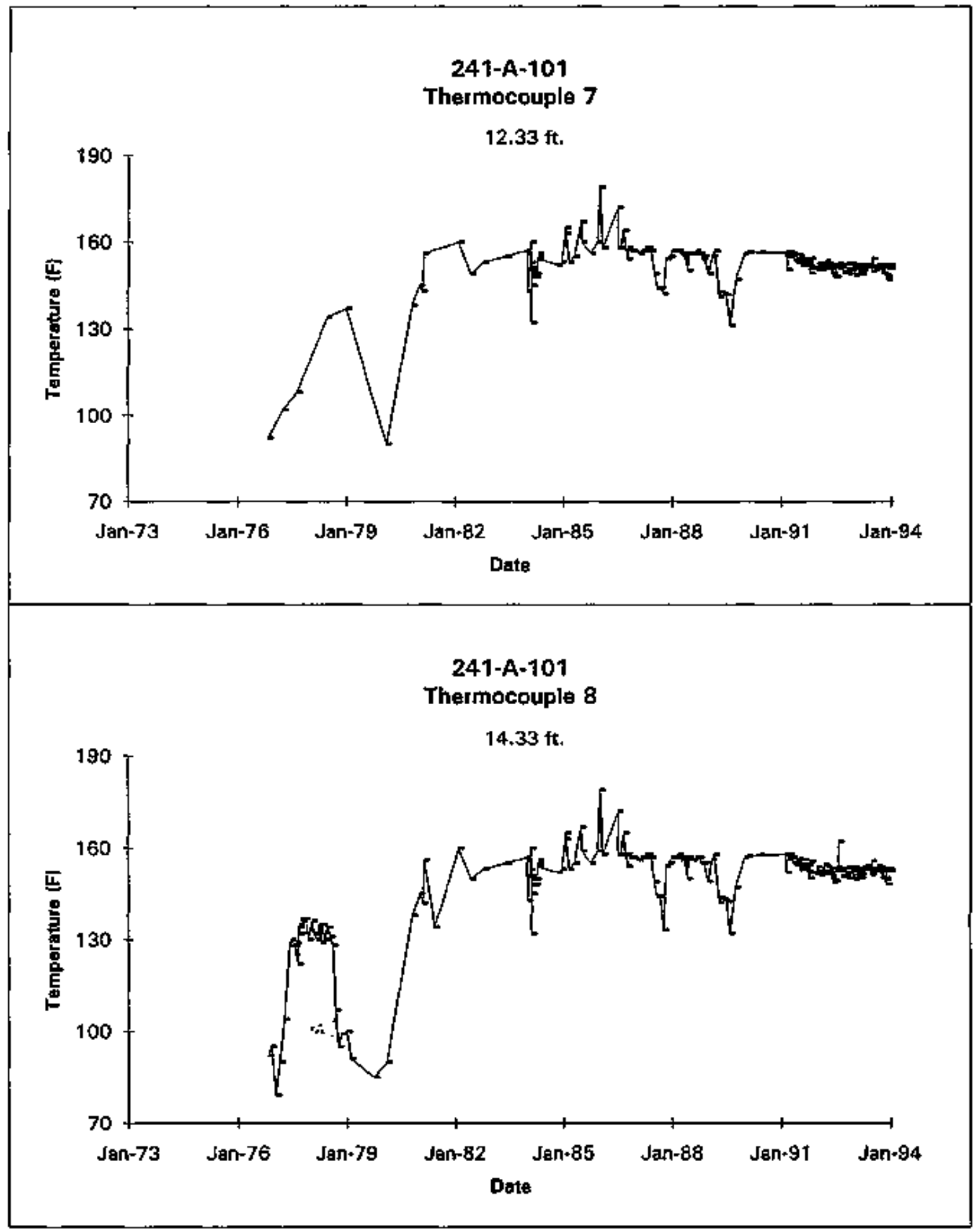

Data obtained from WHC Surveillance Analysis Computer System (SACS), Novernber 12, 1993.

D-22 


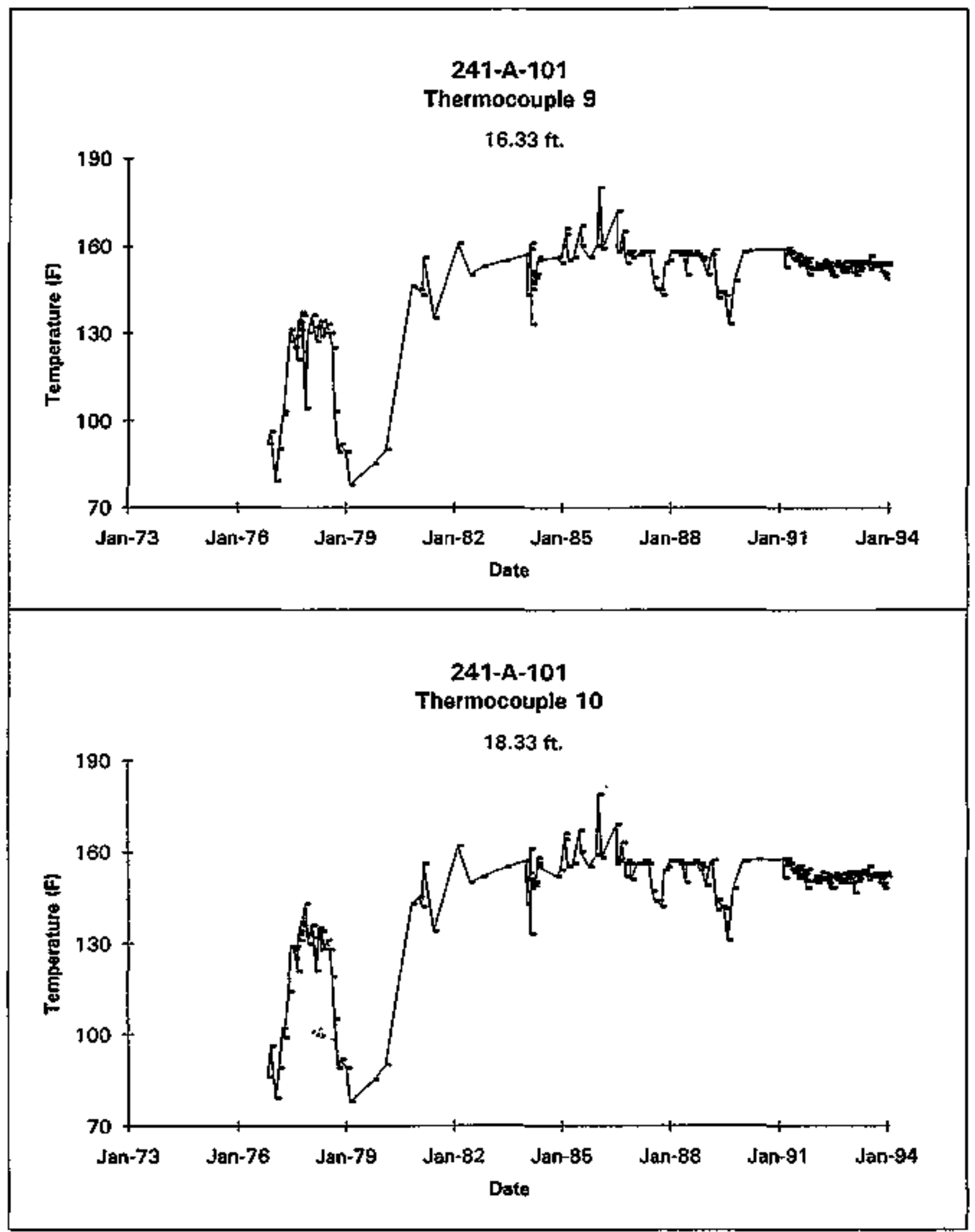

Data obtained from WHC Surveillance Arialysis Computer System (SACS), Noverrber 12, 1993. 


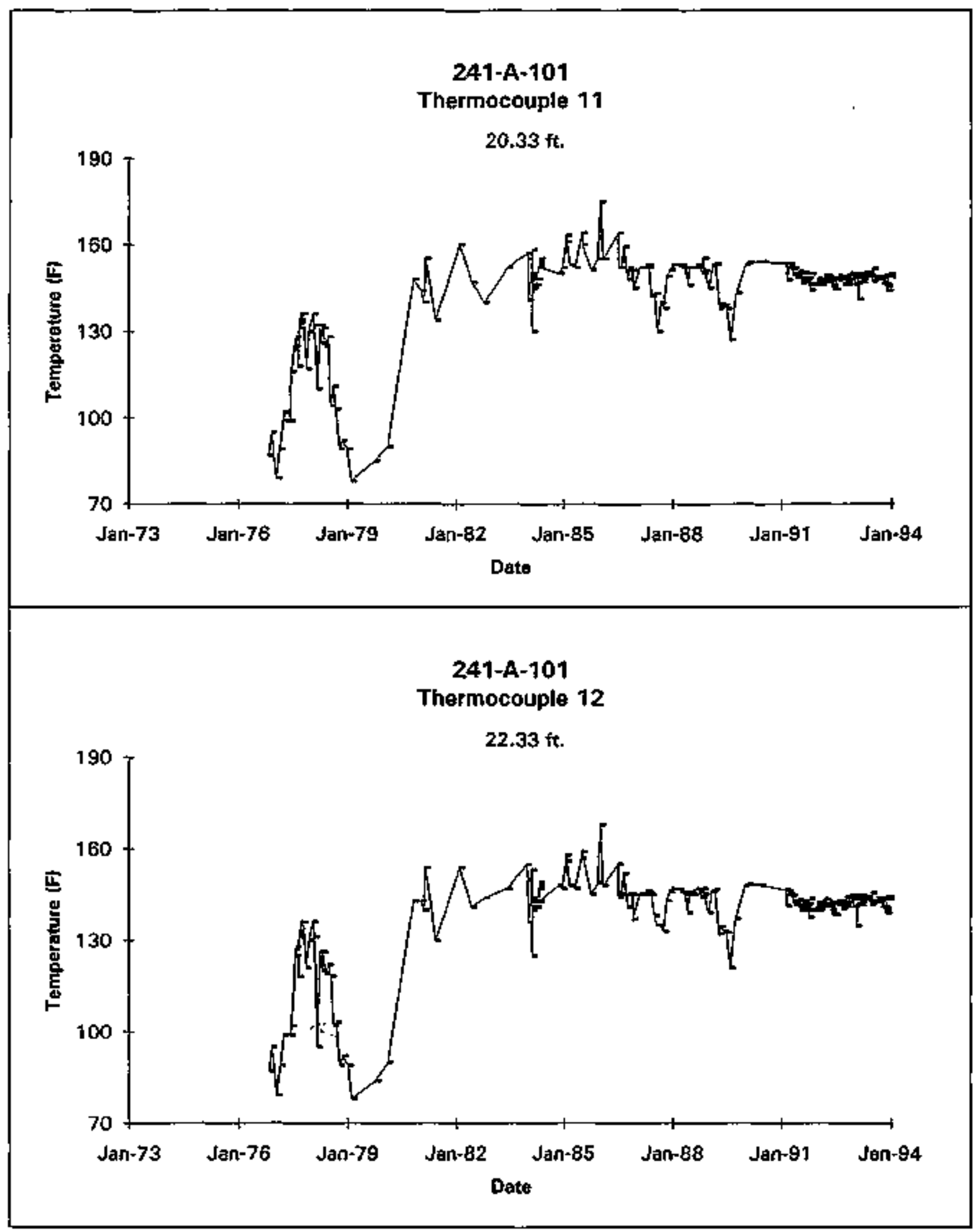

Data obtained from WHC Surveillance Analysis Computer System (SACS), November 12, 1993. 


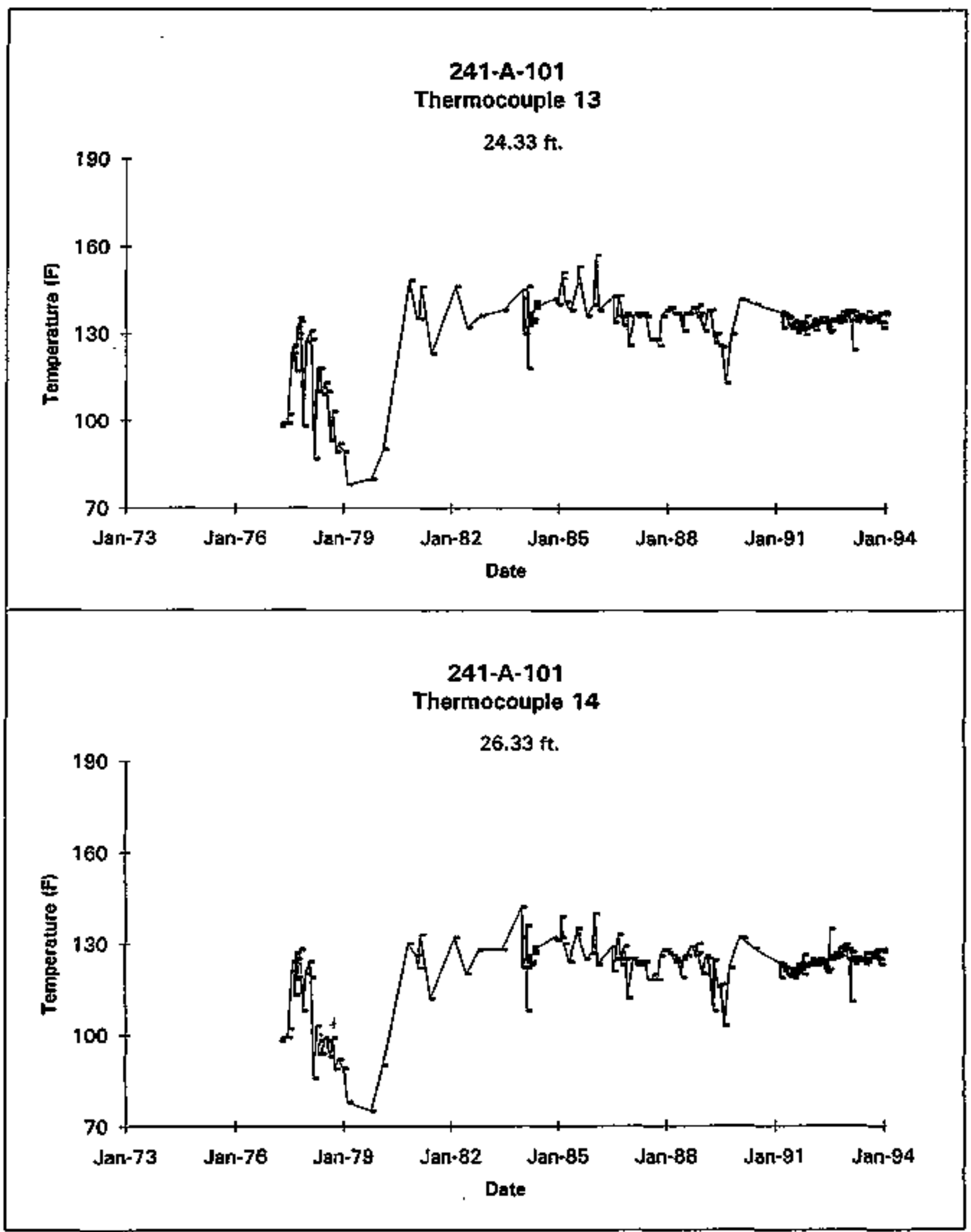

Data obtained from WHC Surveillance Analysis Computer System (SACS), November 12, 1993. 


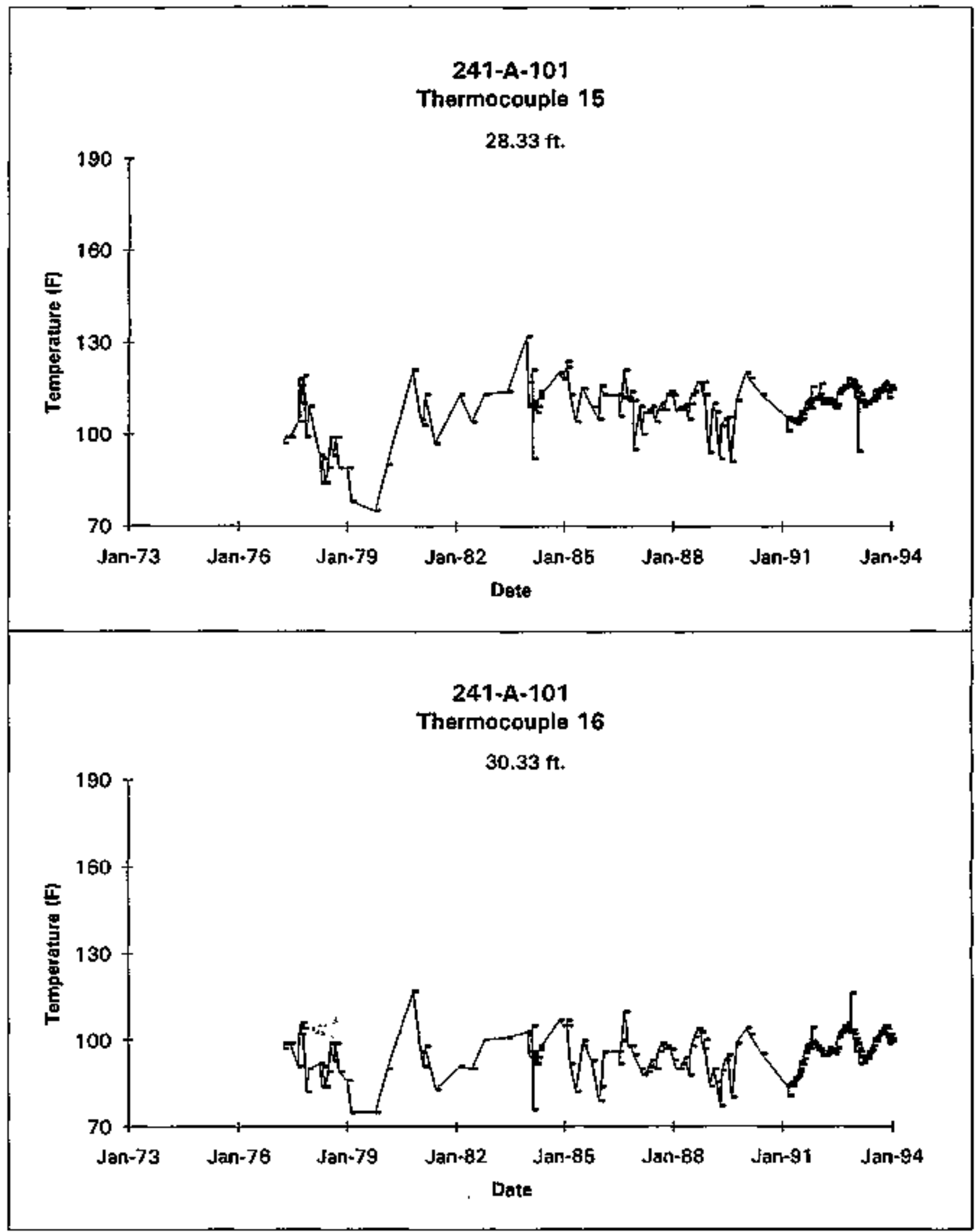

Data obtained from WHC Surveillance Analysis Computer System (SACS), November 12, 1993. 


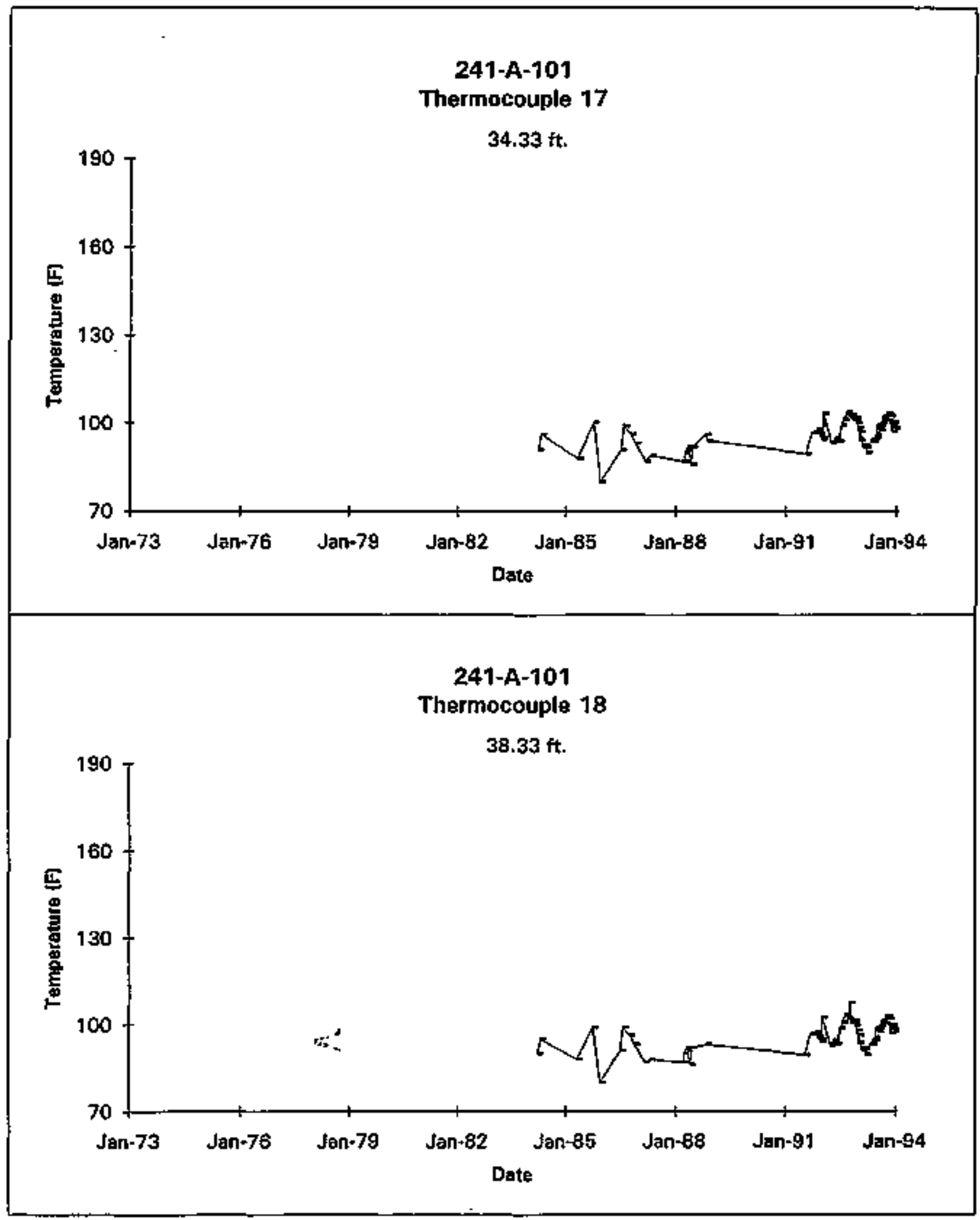

Data obtained from WilC Surveillance Analysis Computer System (SACS), November 12, 1993. 


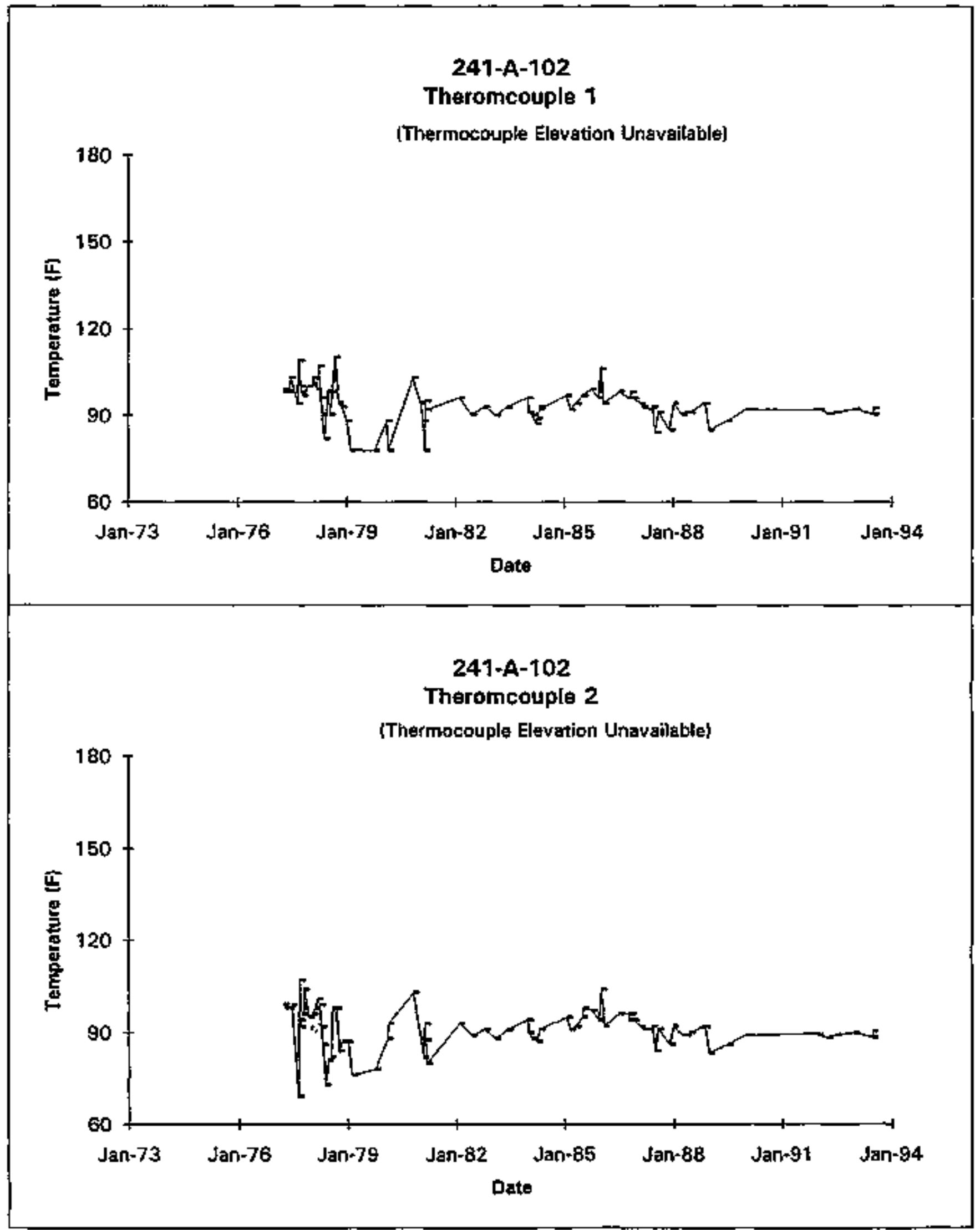

Dała obtained from WHC Surveillance Analysis Computer System (SACSI, November 12, 1993. 


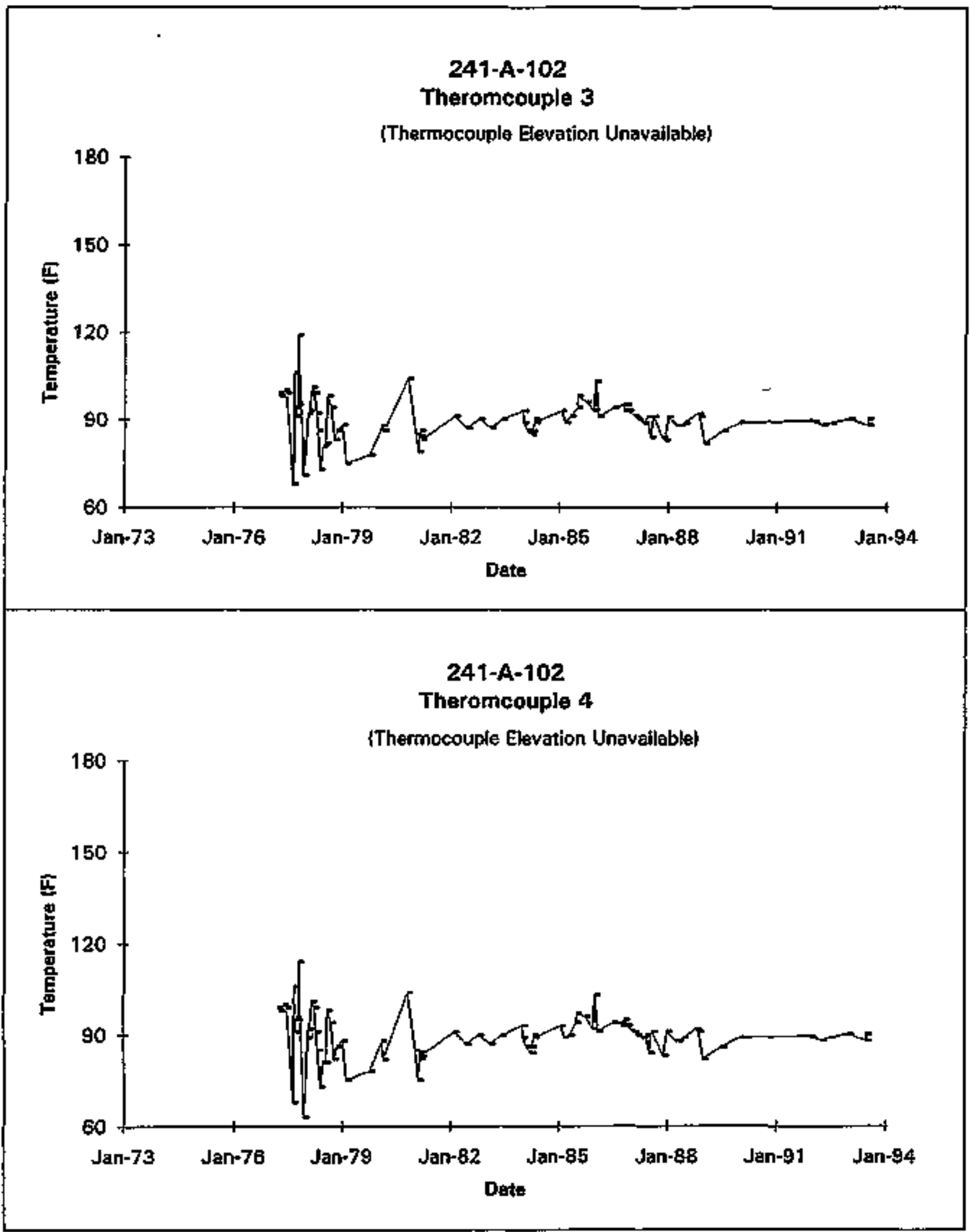

Data obtained from WHC Surveillance Analysis Computer Systen (SACS), November 12, 1993. 


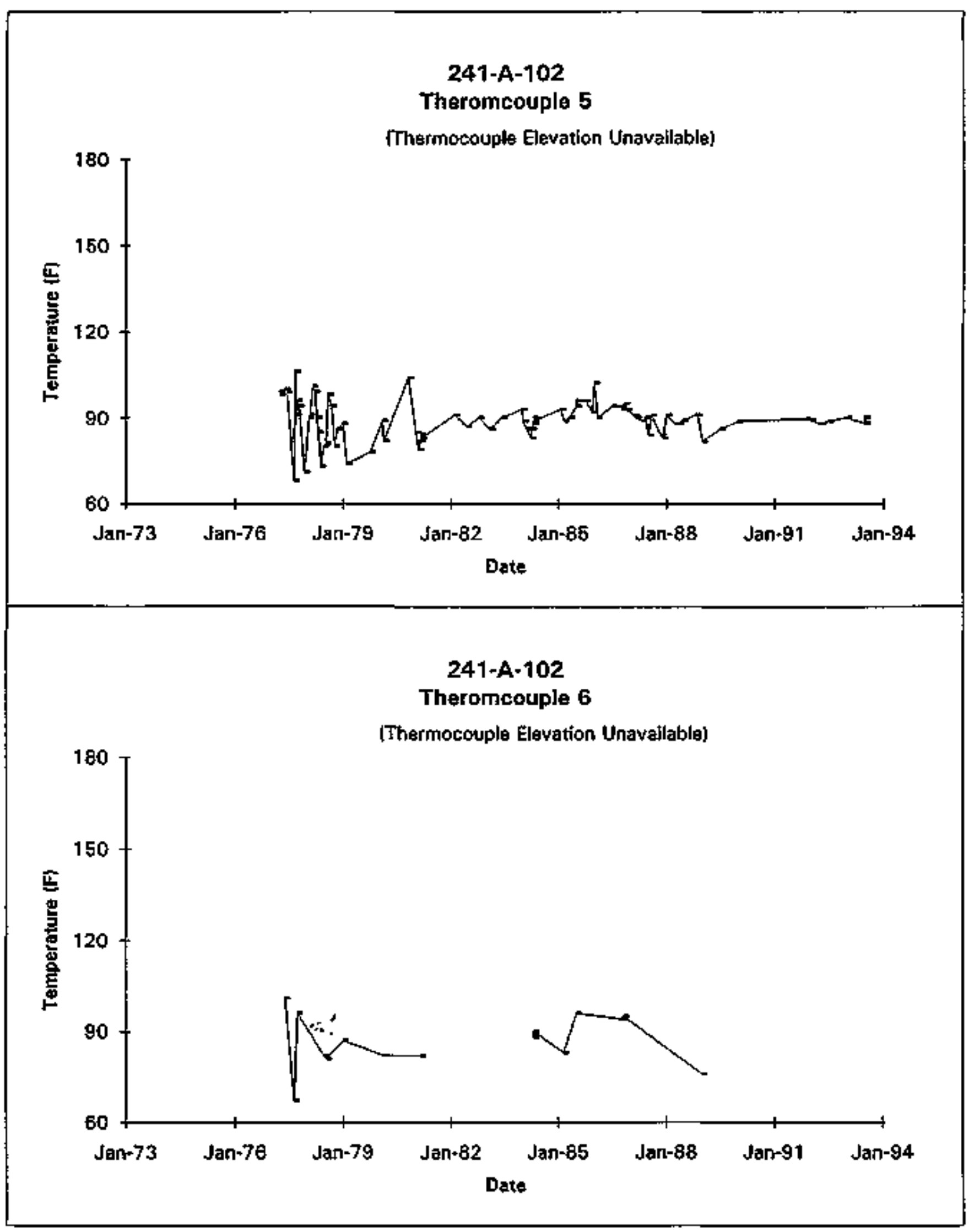

Data obtained from WHC Surveillance Analysis Computer System (SACS), November 12, 1993. 


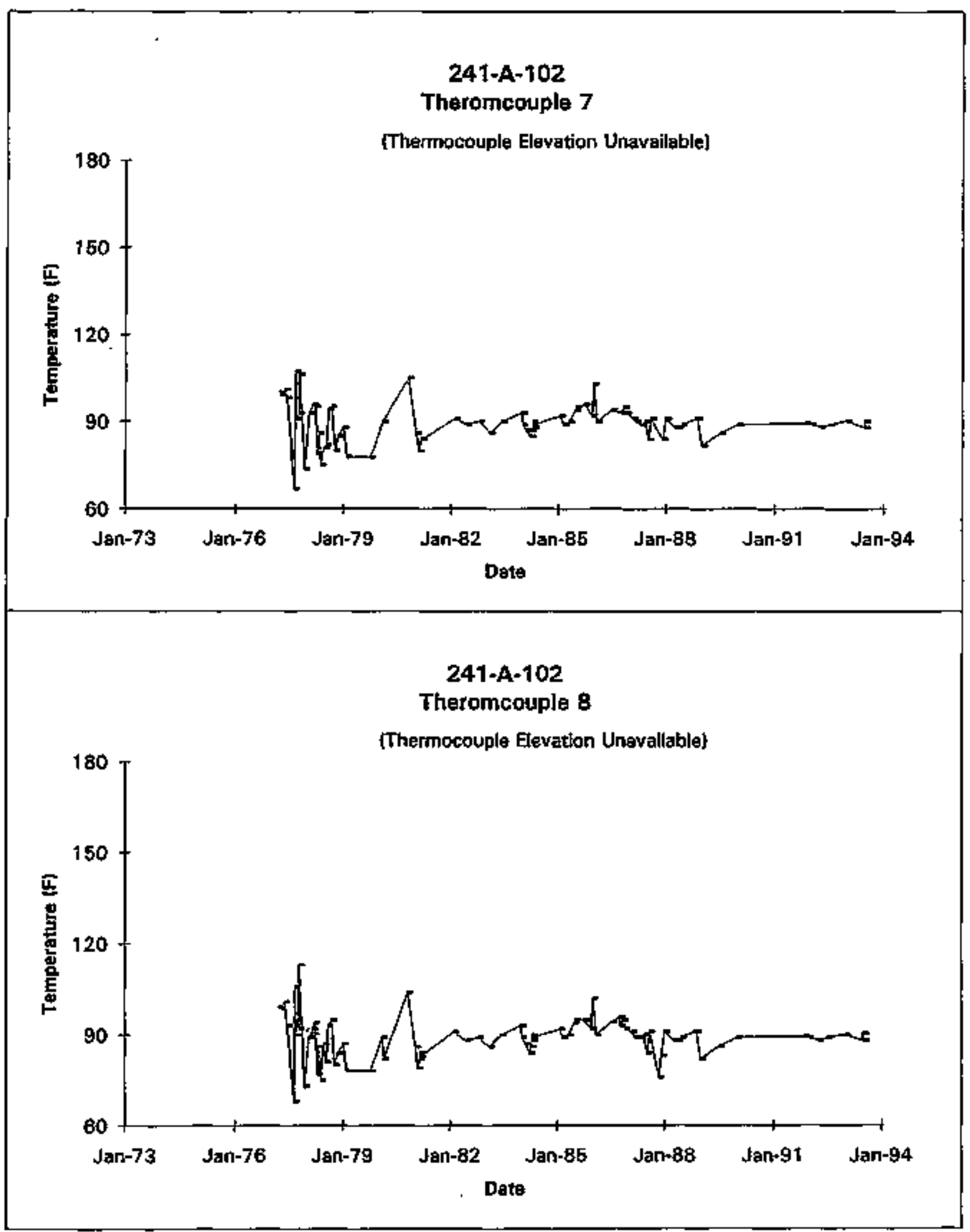

Data obtained from WHC Surveillence Analysis Computer Systen (SACS\}, November 12, 1993. 


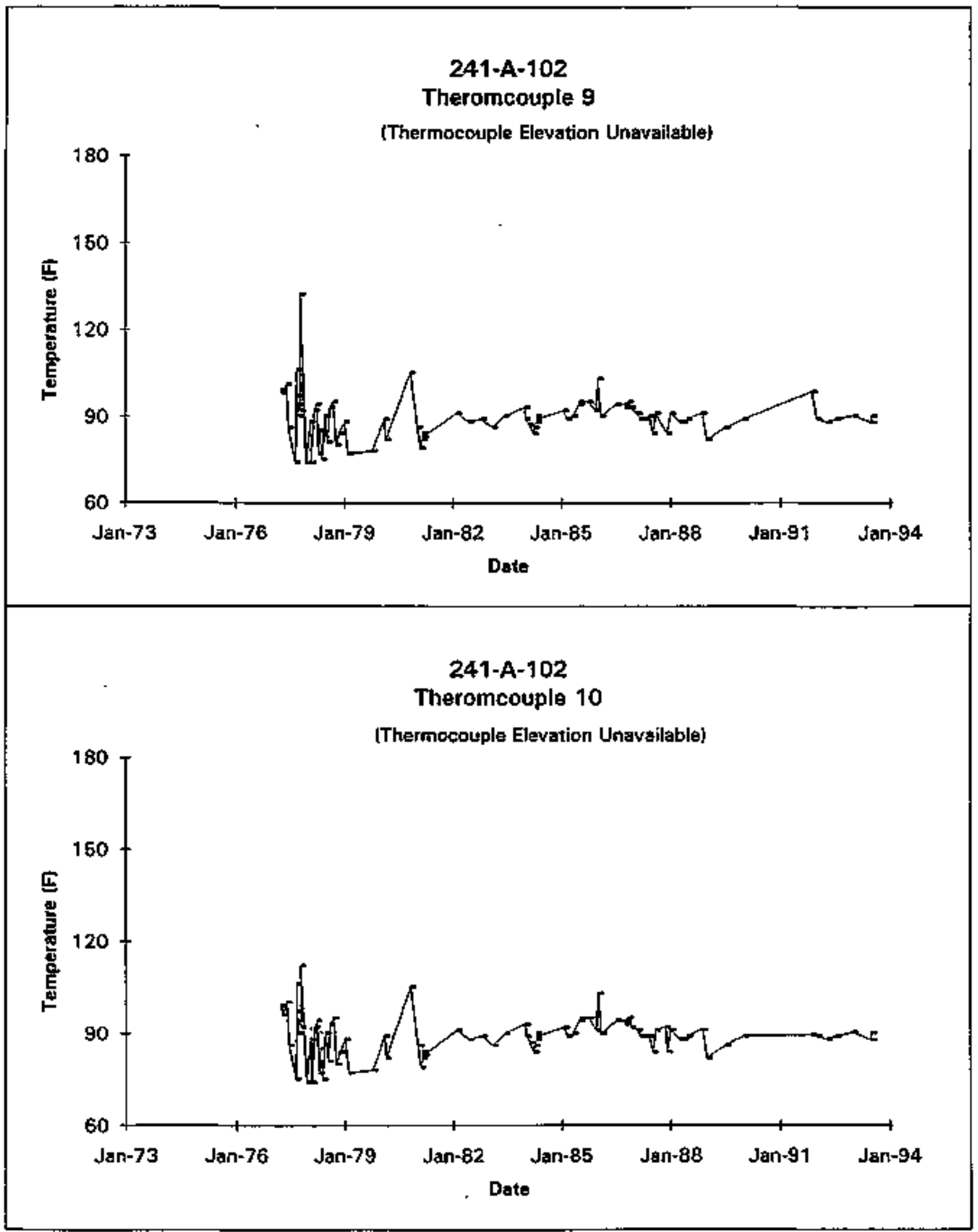

Data obtained frorn WHC Surveiltence Analysts Computer System (SACS), November 12, 1993. 


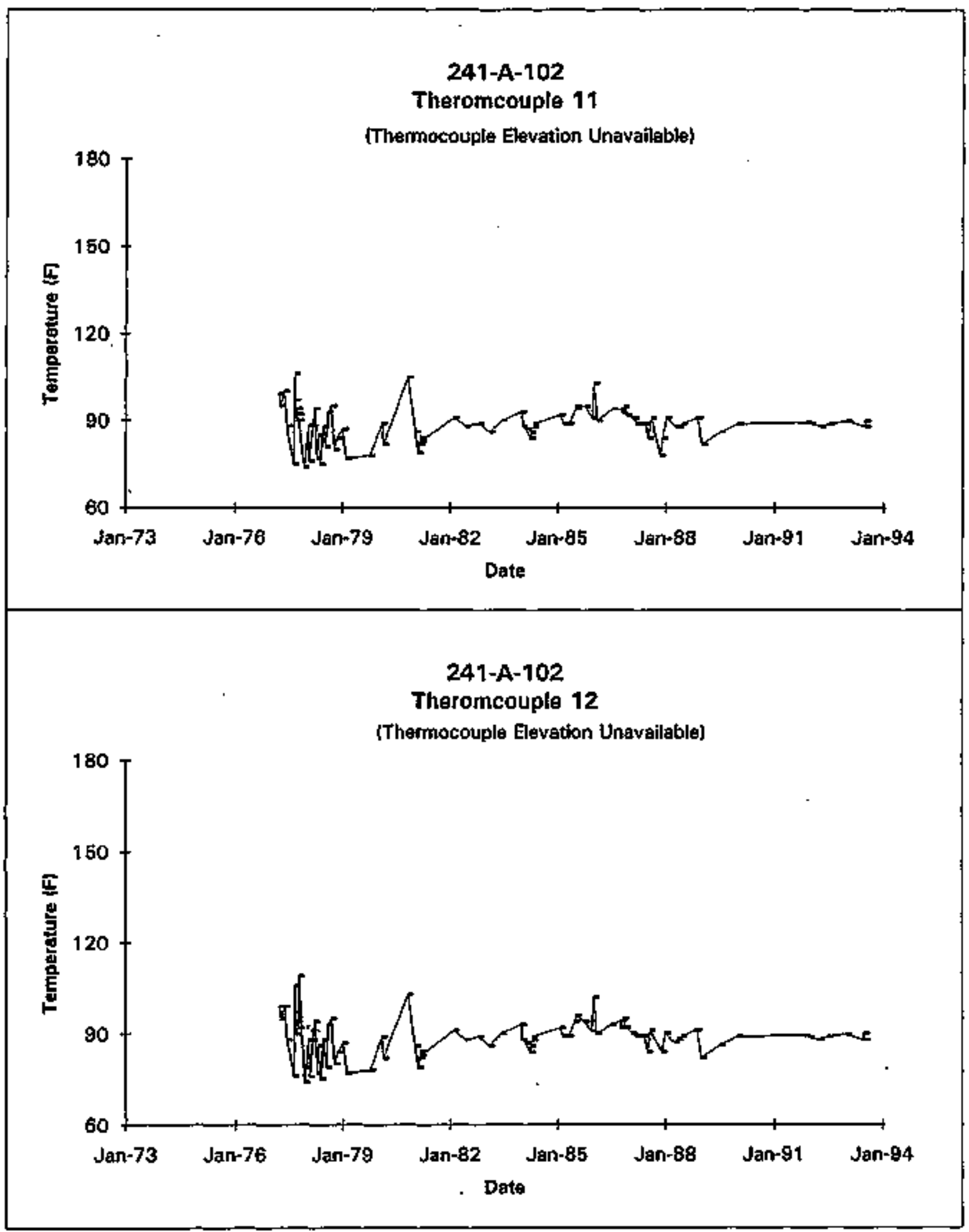

Data obtained from WHC Survellence Analysts Computer System (SACSl, November 12, 1993. 


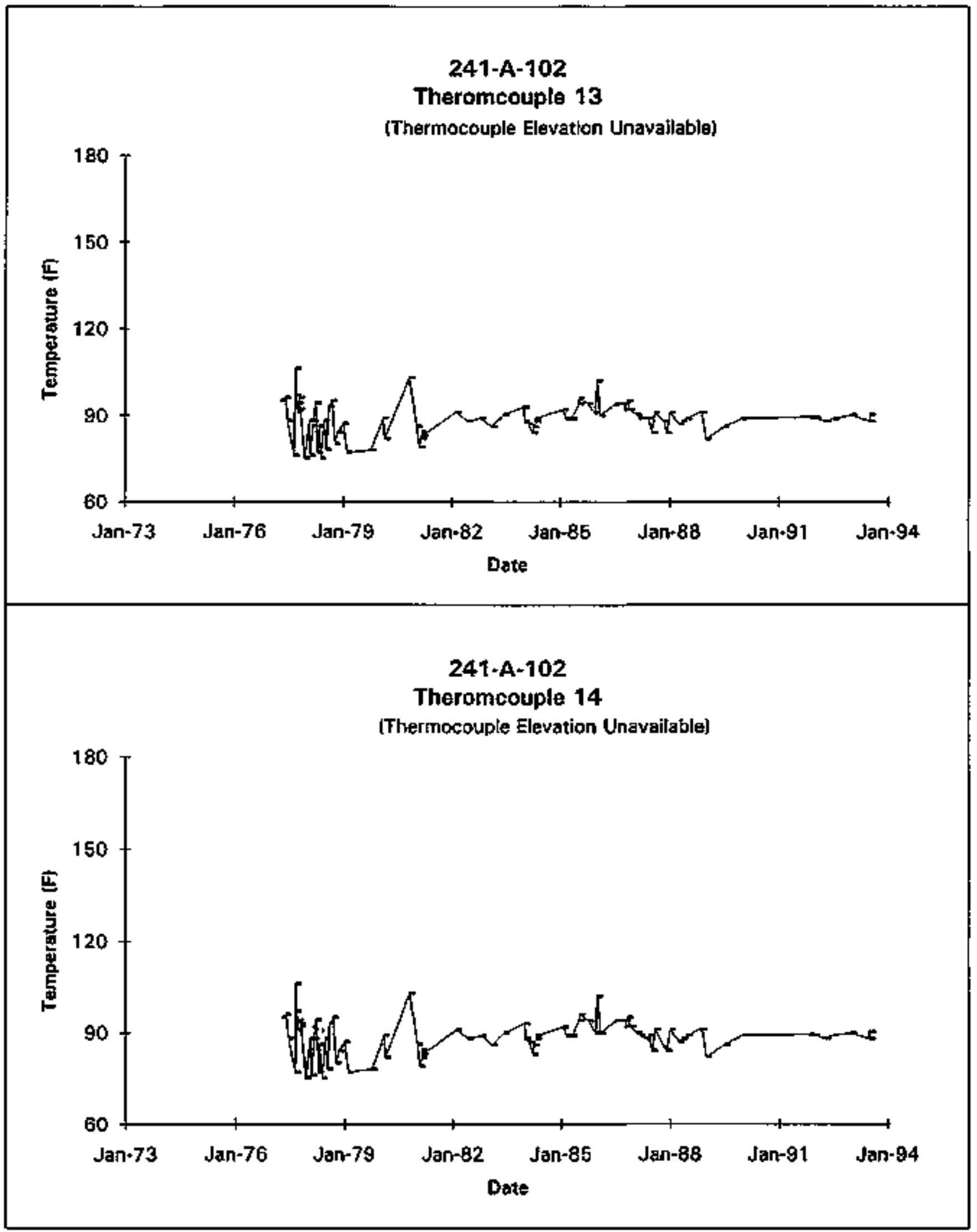

Data obtained from WHC Surveillance Analysis Computer System (SACS), November 12, 1993. 


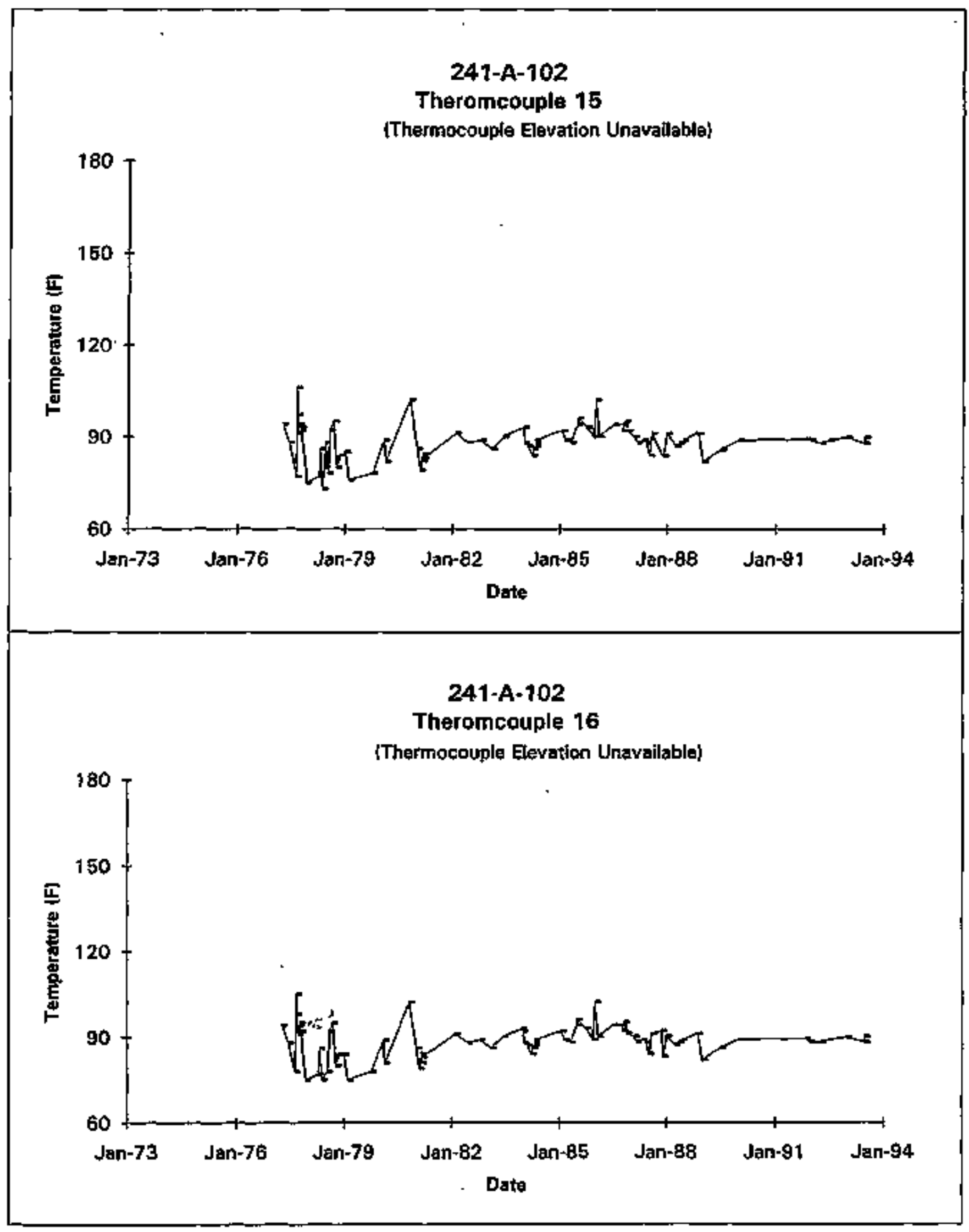

Data obtained from WHC Surveiltance Analysass Computer System (SACS), November 12, 1993. 


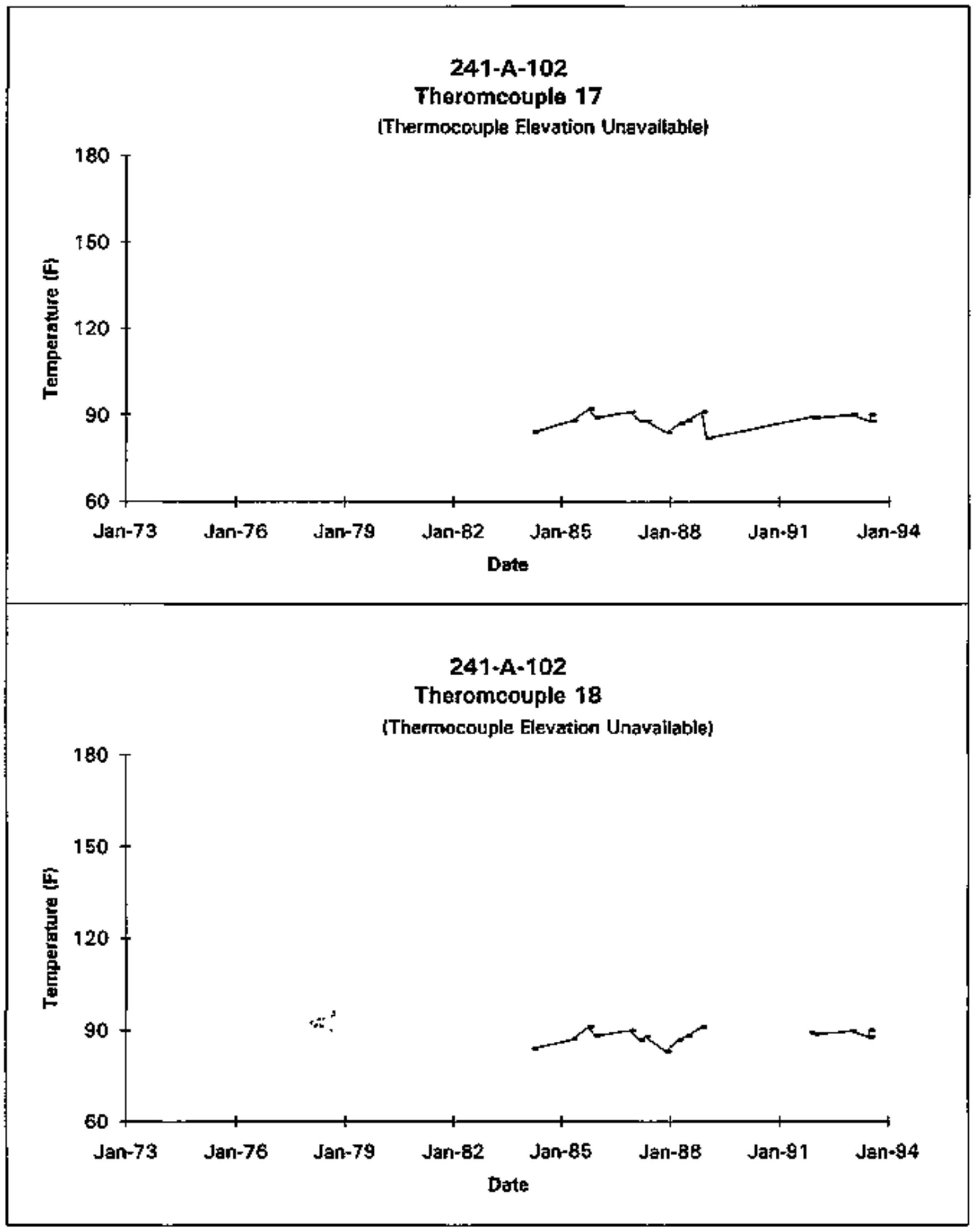

Data obtained from WHC Surveillance Analysis Computer System (SACS), November 12, 1993. 


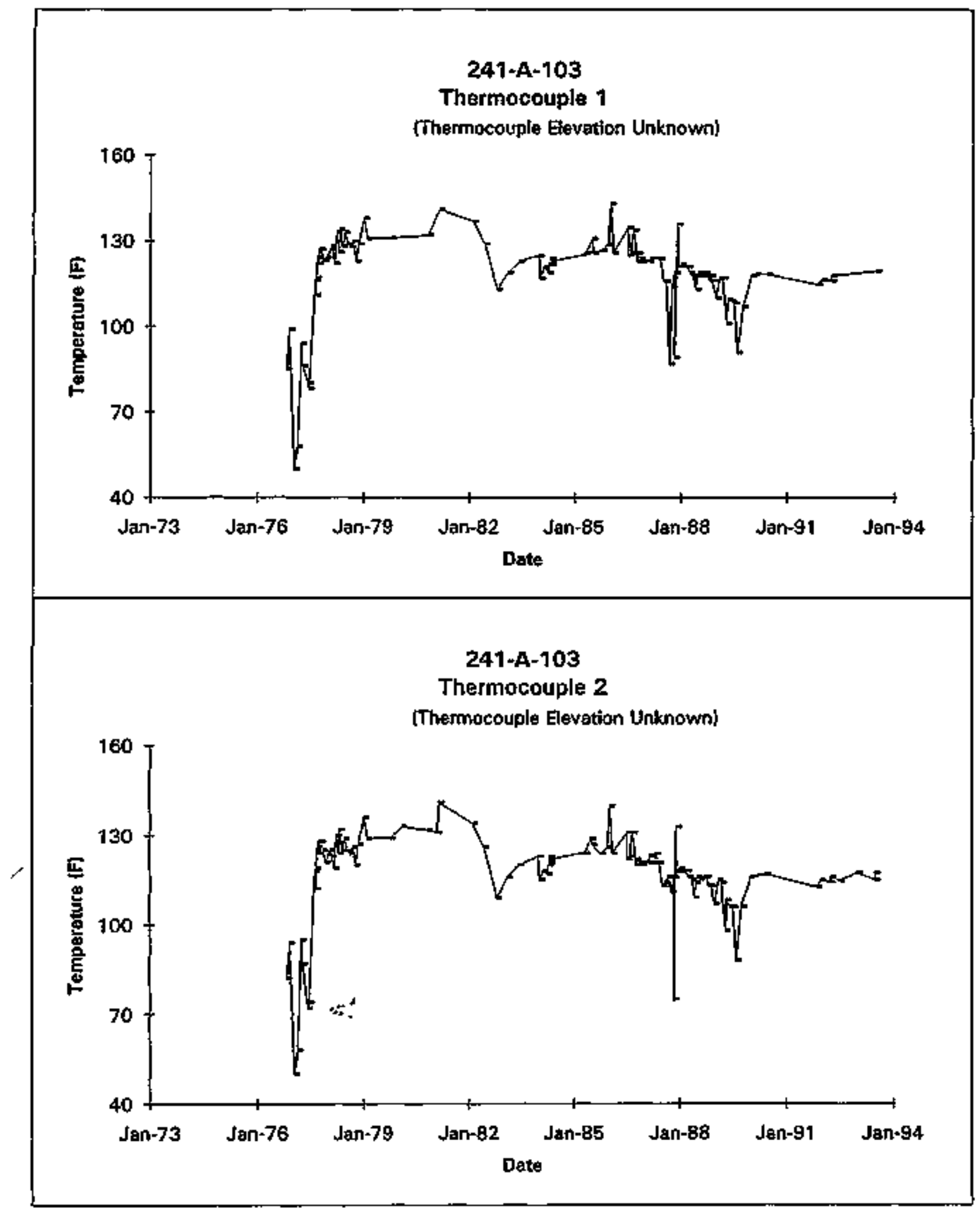

Dats obtained from WHC Surveillance Analysis Computer System (SACS\}, November 12, 1993. 


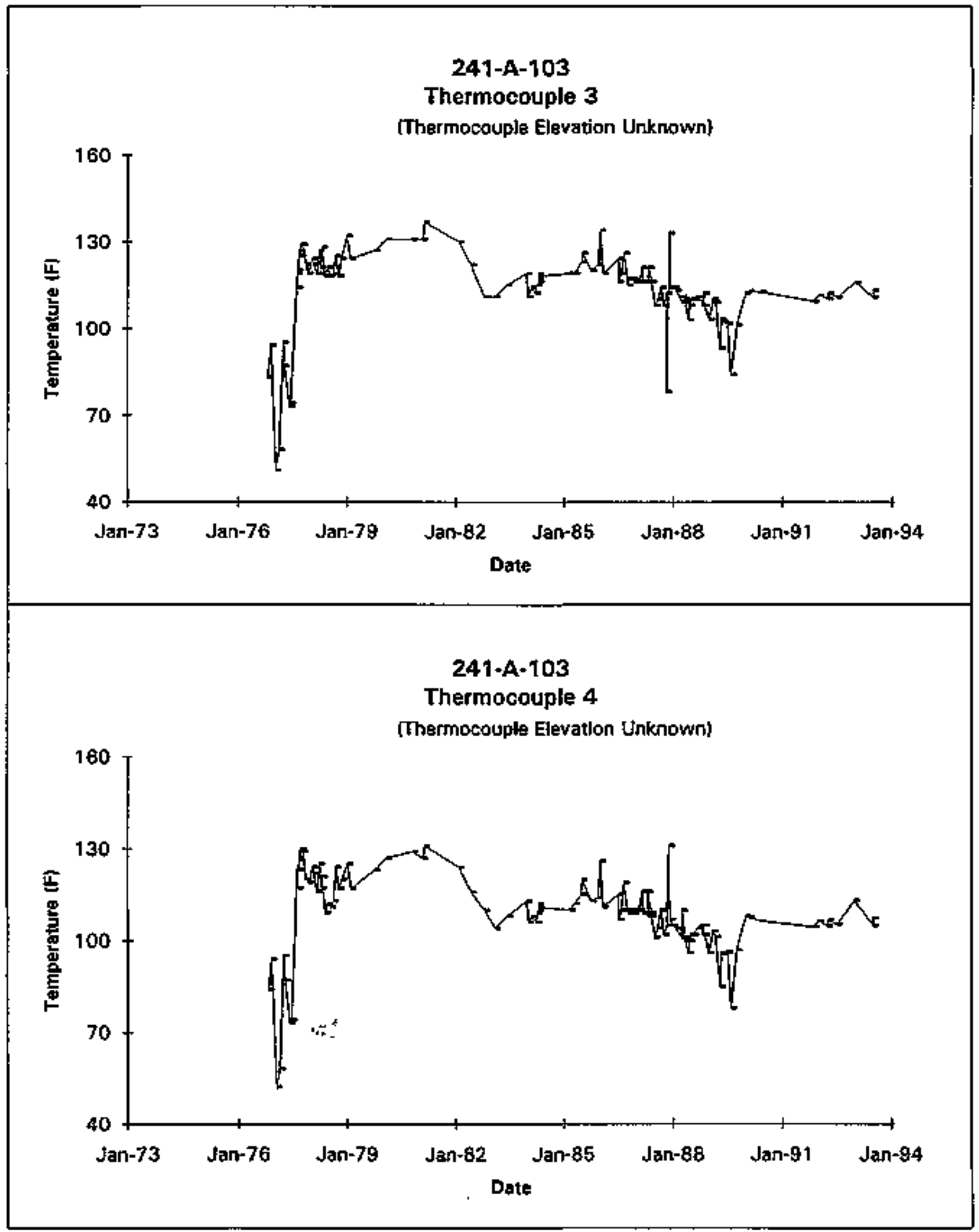

Data obtained from WHC Surveillance Analysis Computer System (SACS), November 12, 1993. 


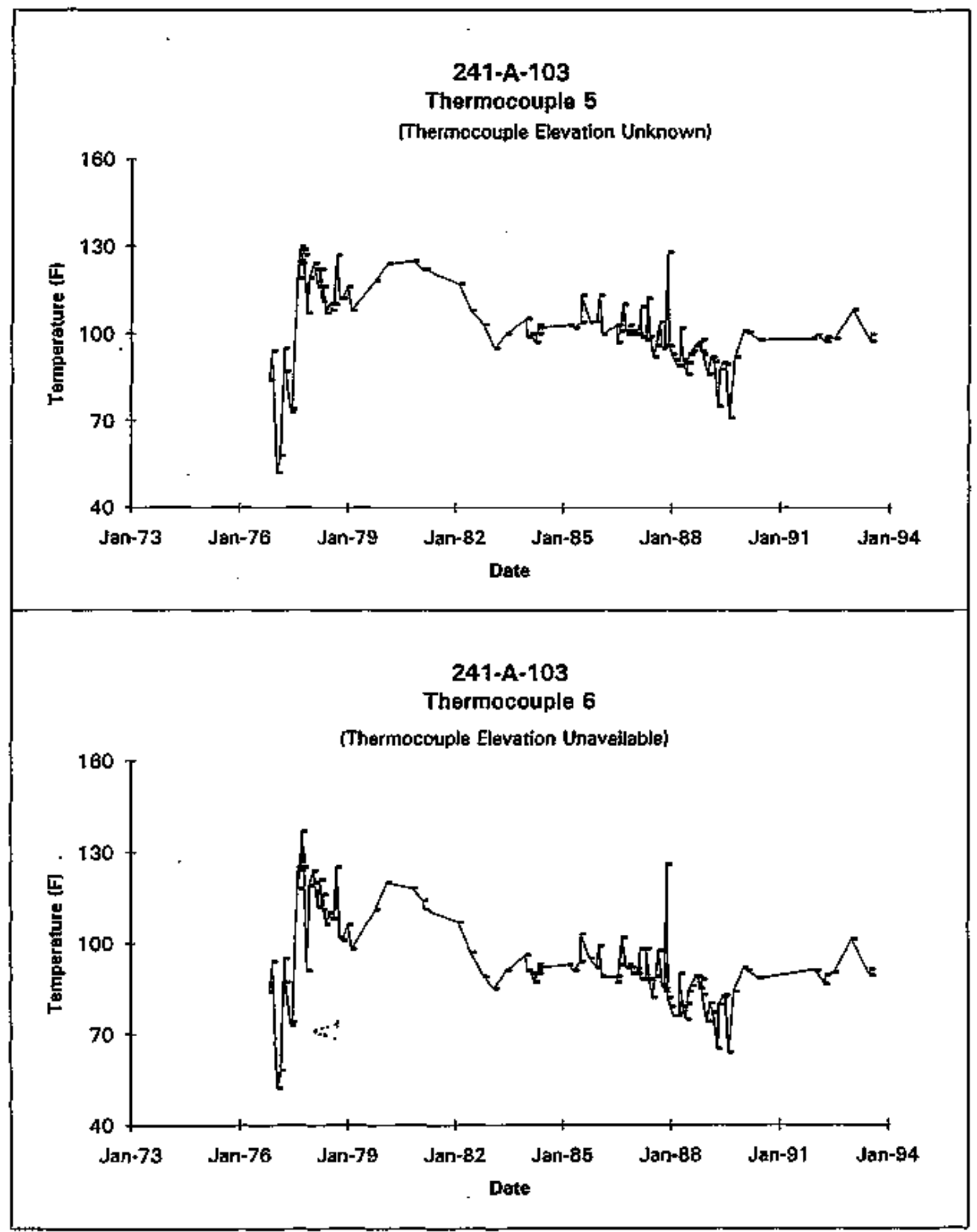

Data obtained from WHC Surveillance Analysis Computer System (SACS). November 12, 1993. 


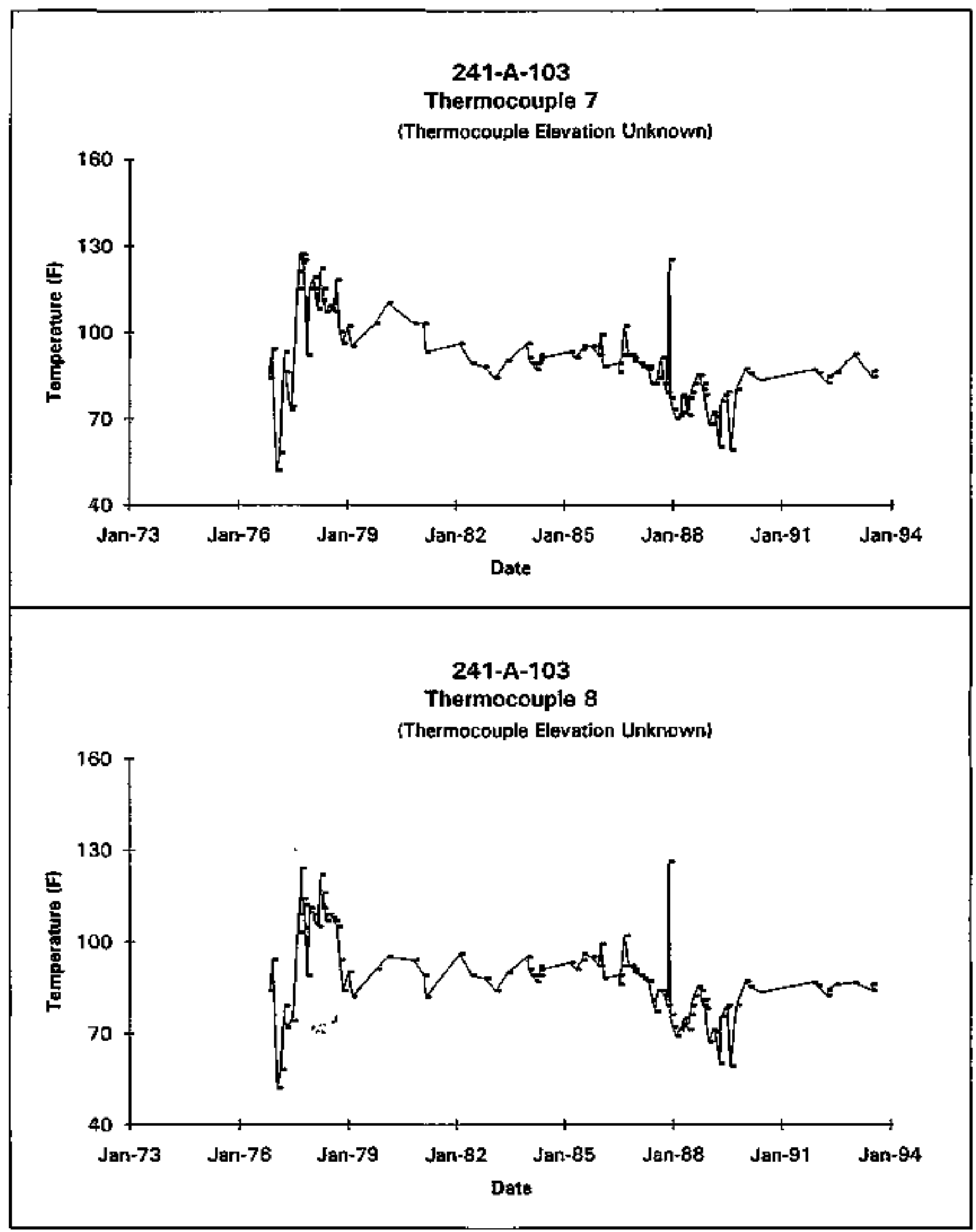

Data obtained from WHC Surveillance Analysis Computer System (SACS), November 12, 1993. 


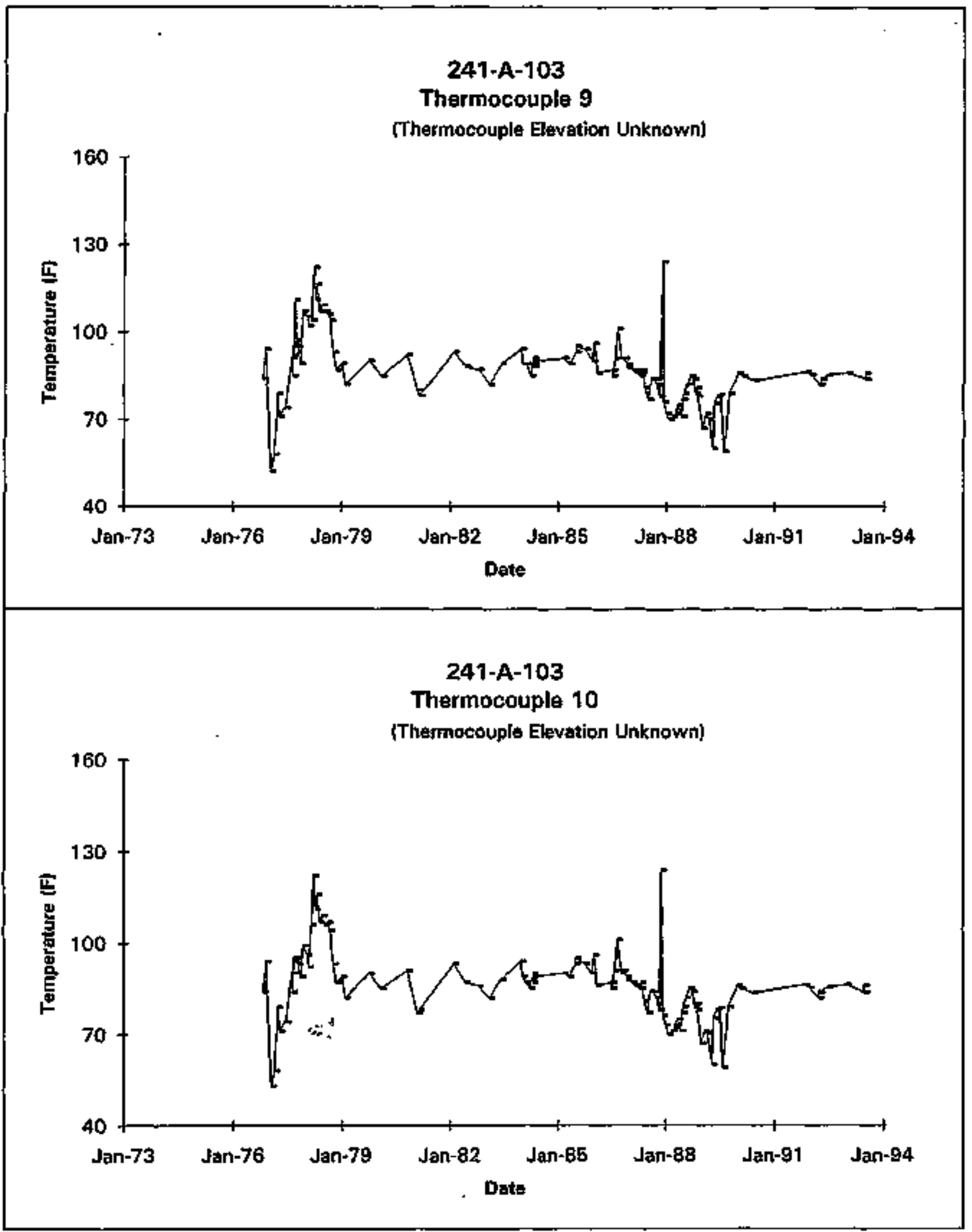

Data obtained from WHC Surveillance Analysis Computer System ISACS\}, November 12, 1993. 


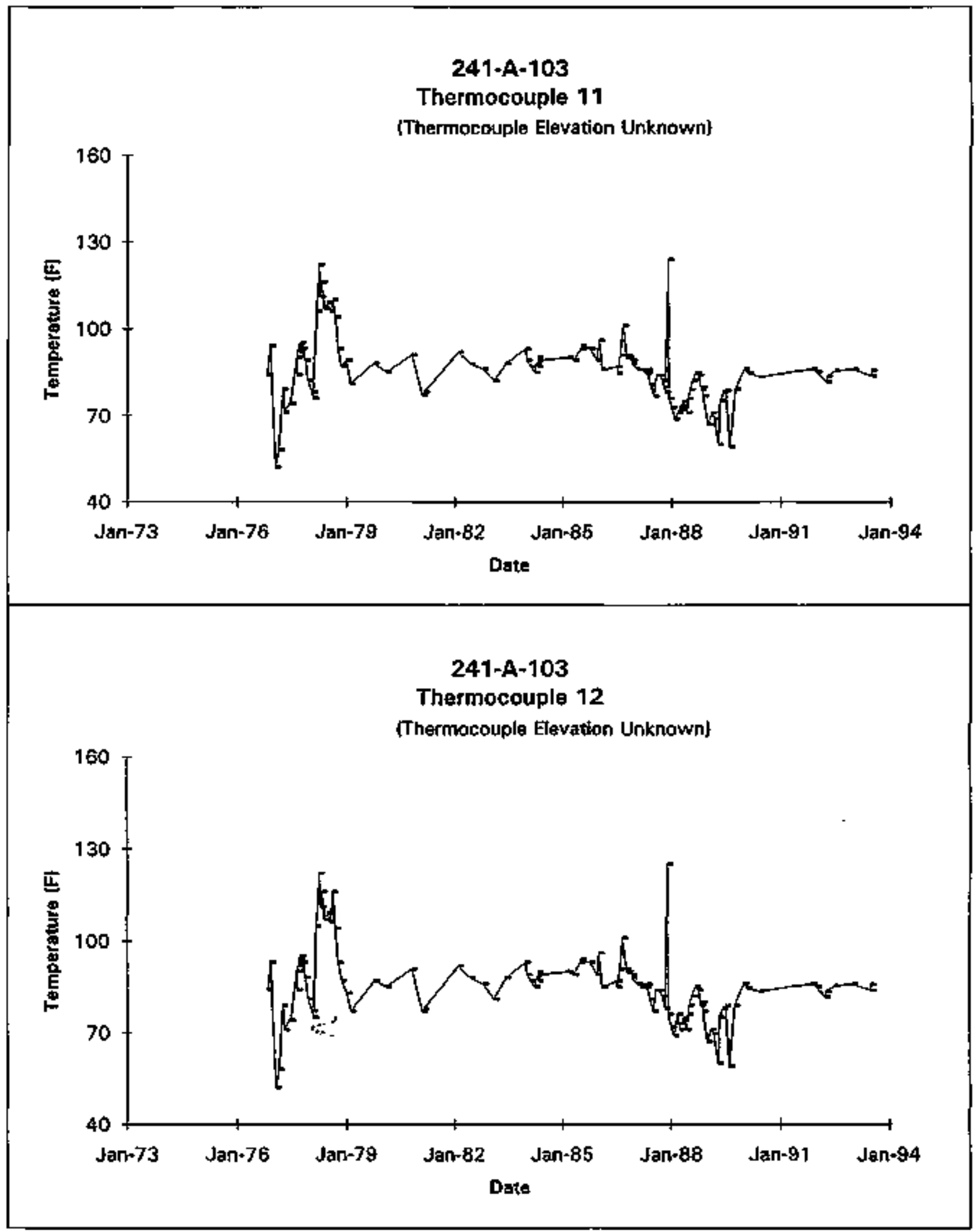

Data obtained from WHC Surveillance Analysis Computer System (SACS\}, November 12, 1993. 


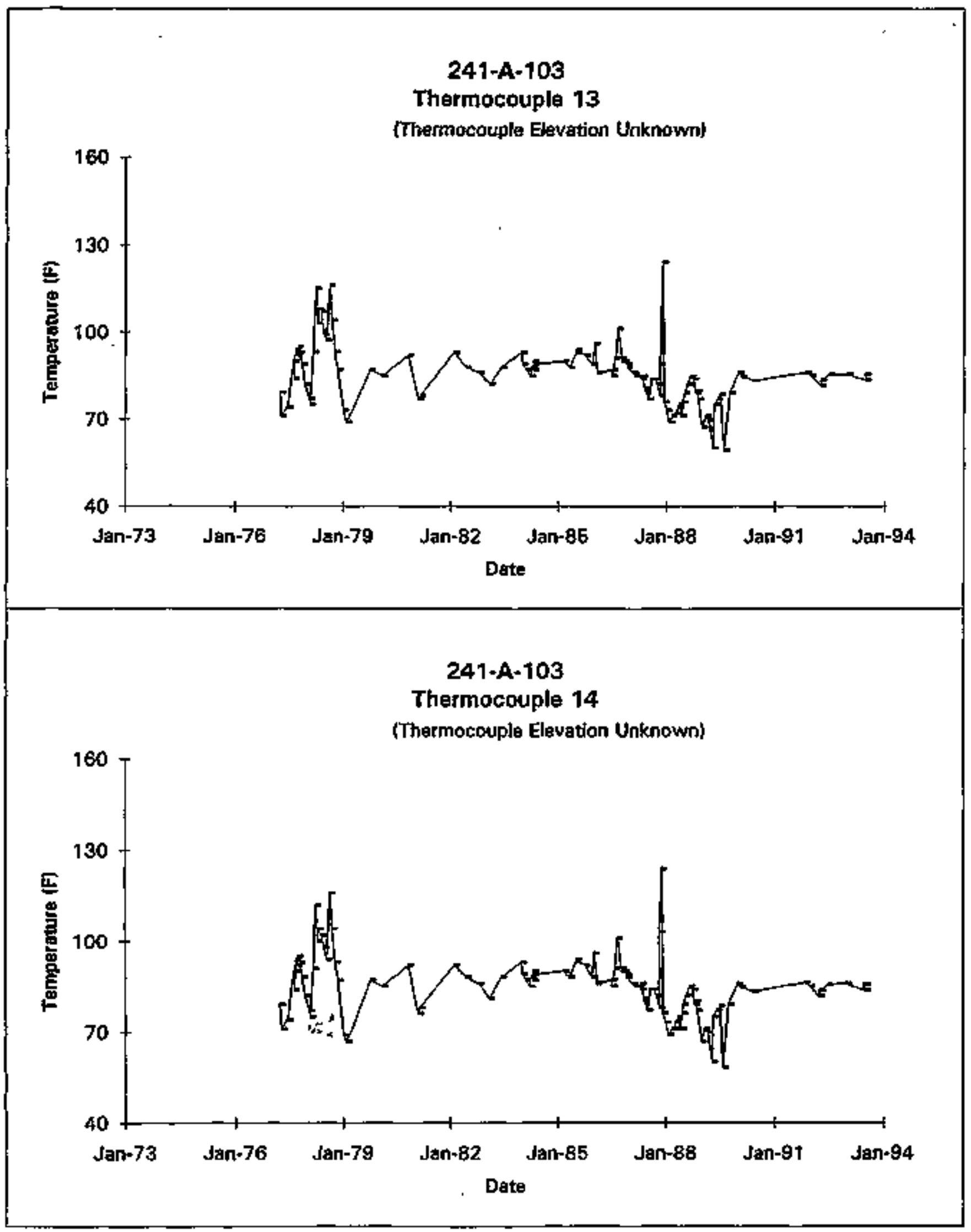

Data obtained from WHC Survefllance Analysis Computer System (SACS), November 12, 1993. 


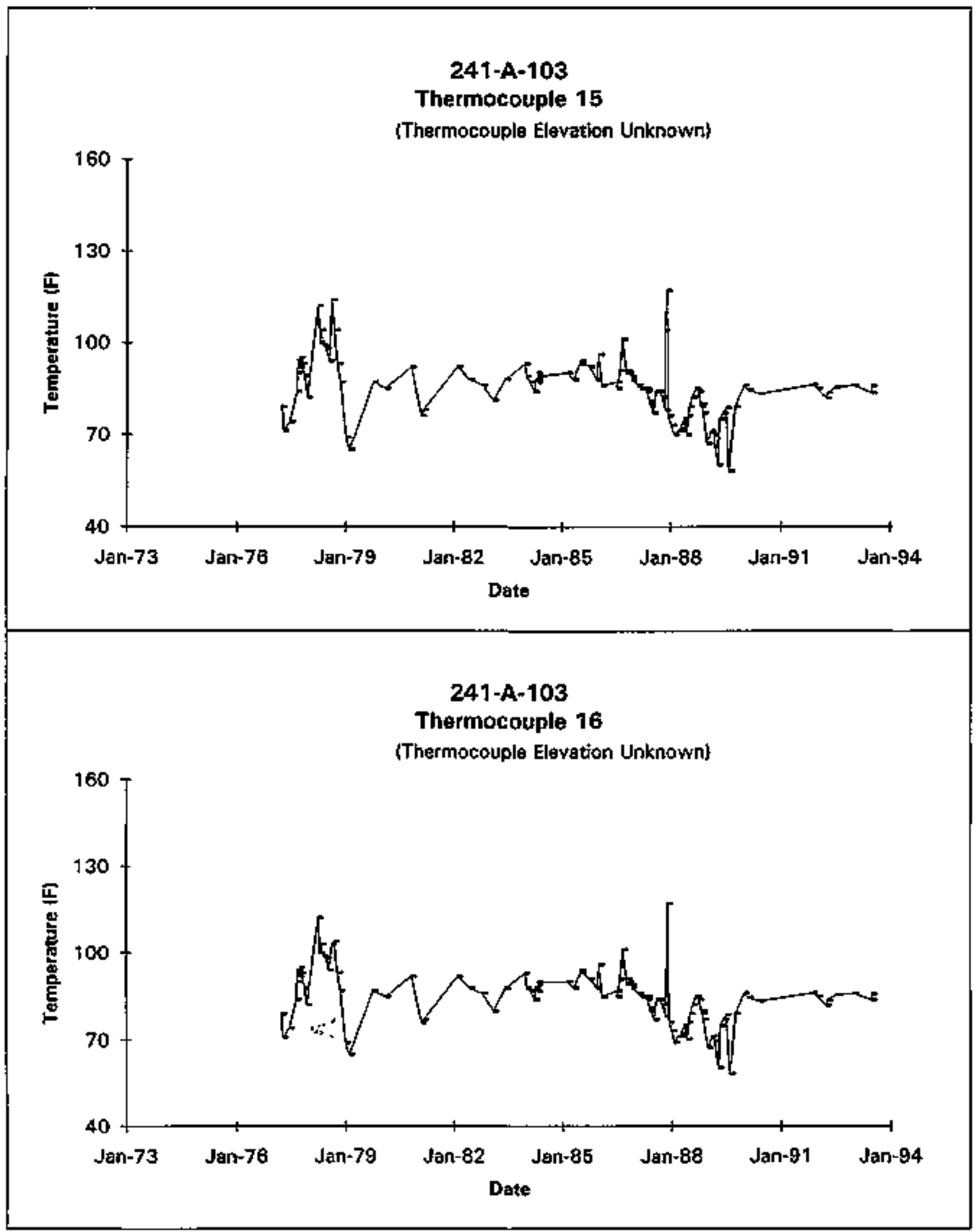

Data obtained from WHC Surveillance Analysis Computer System (SACS), November 12, 1993. 


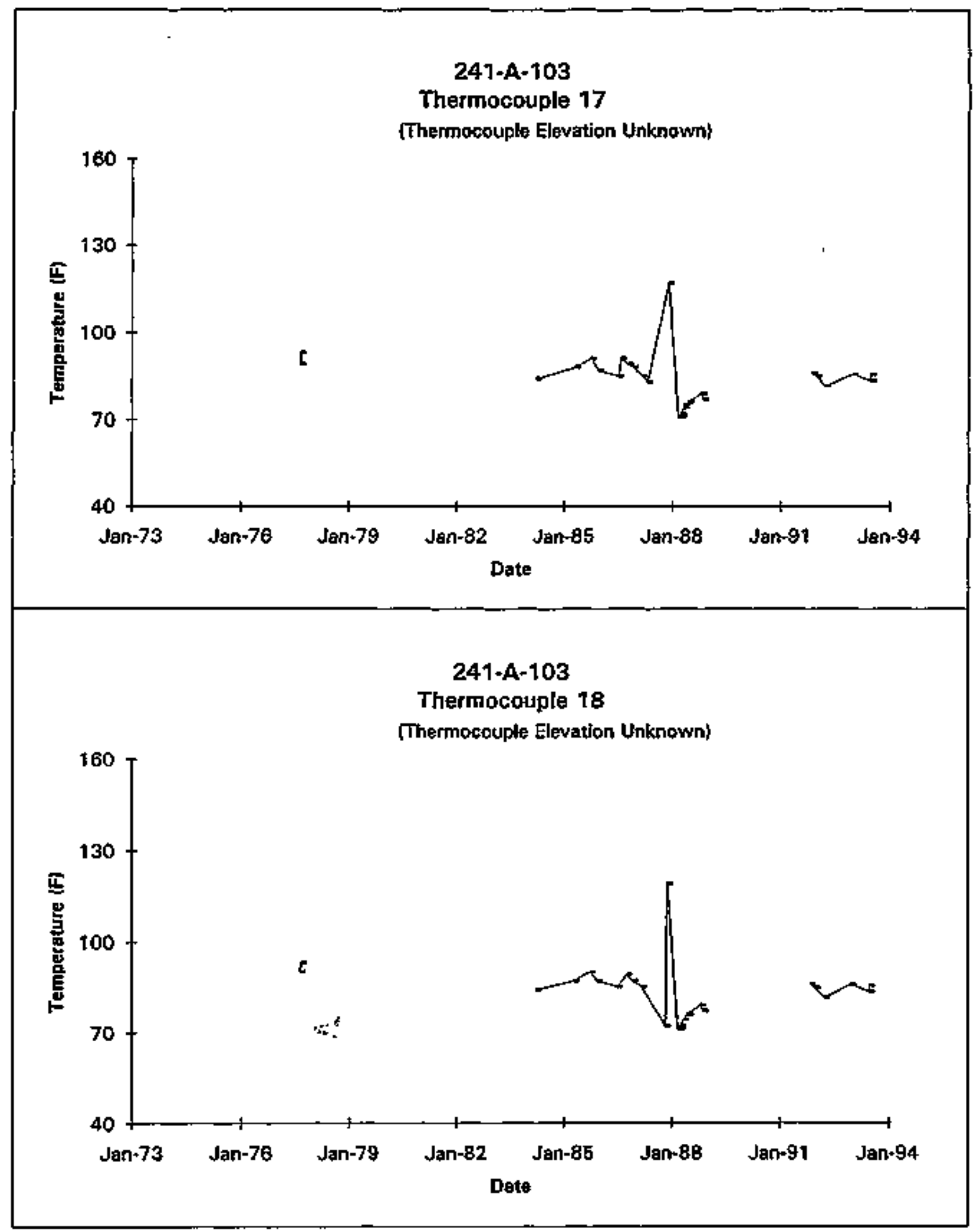

Data obtained from WHC Surveillance Analysis Computer System (SACS), November 12, 1993. 


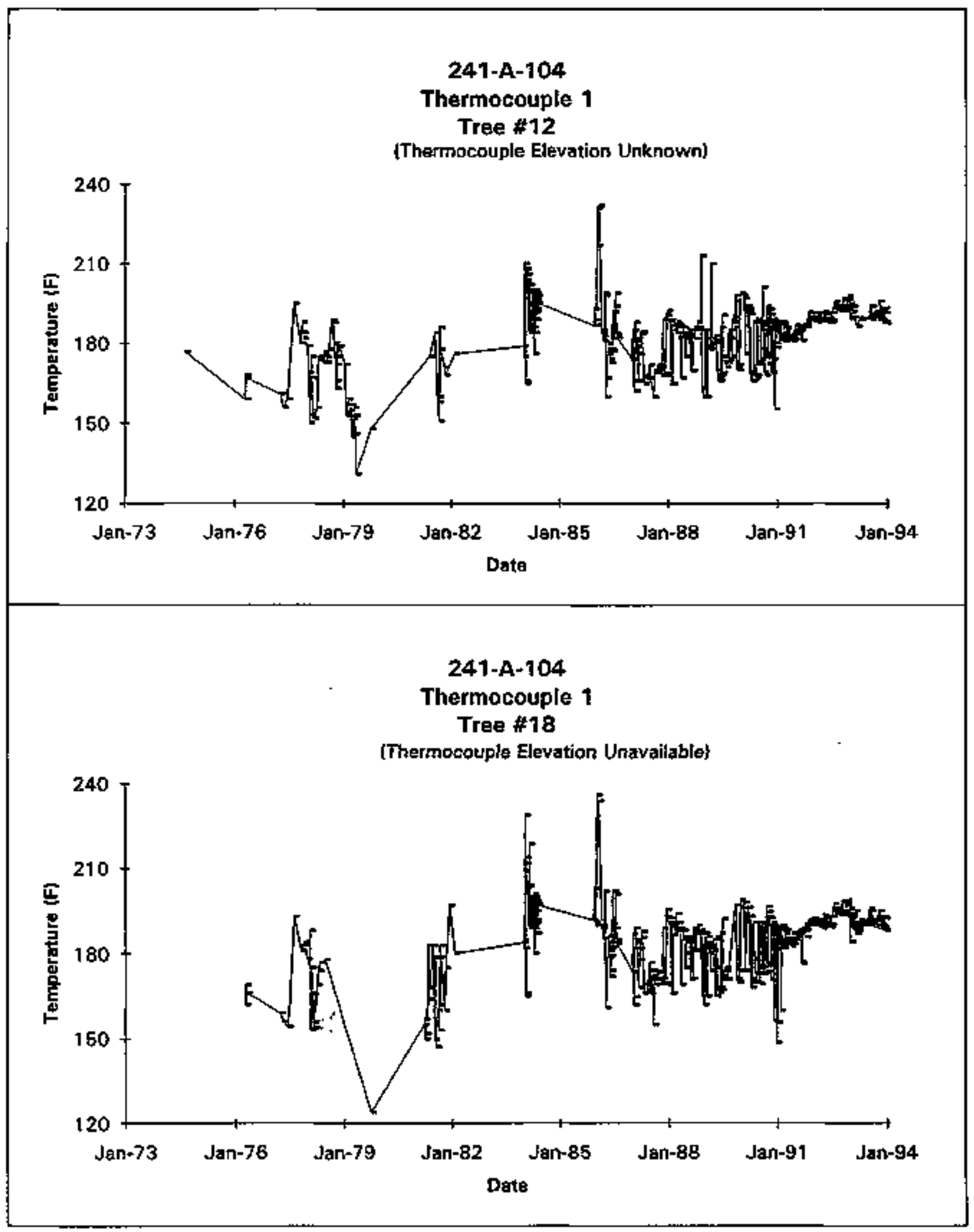

Data obtained from WHC Surveillance Analysis Computer System [SACS], November 12, 1993. 


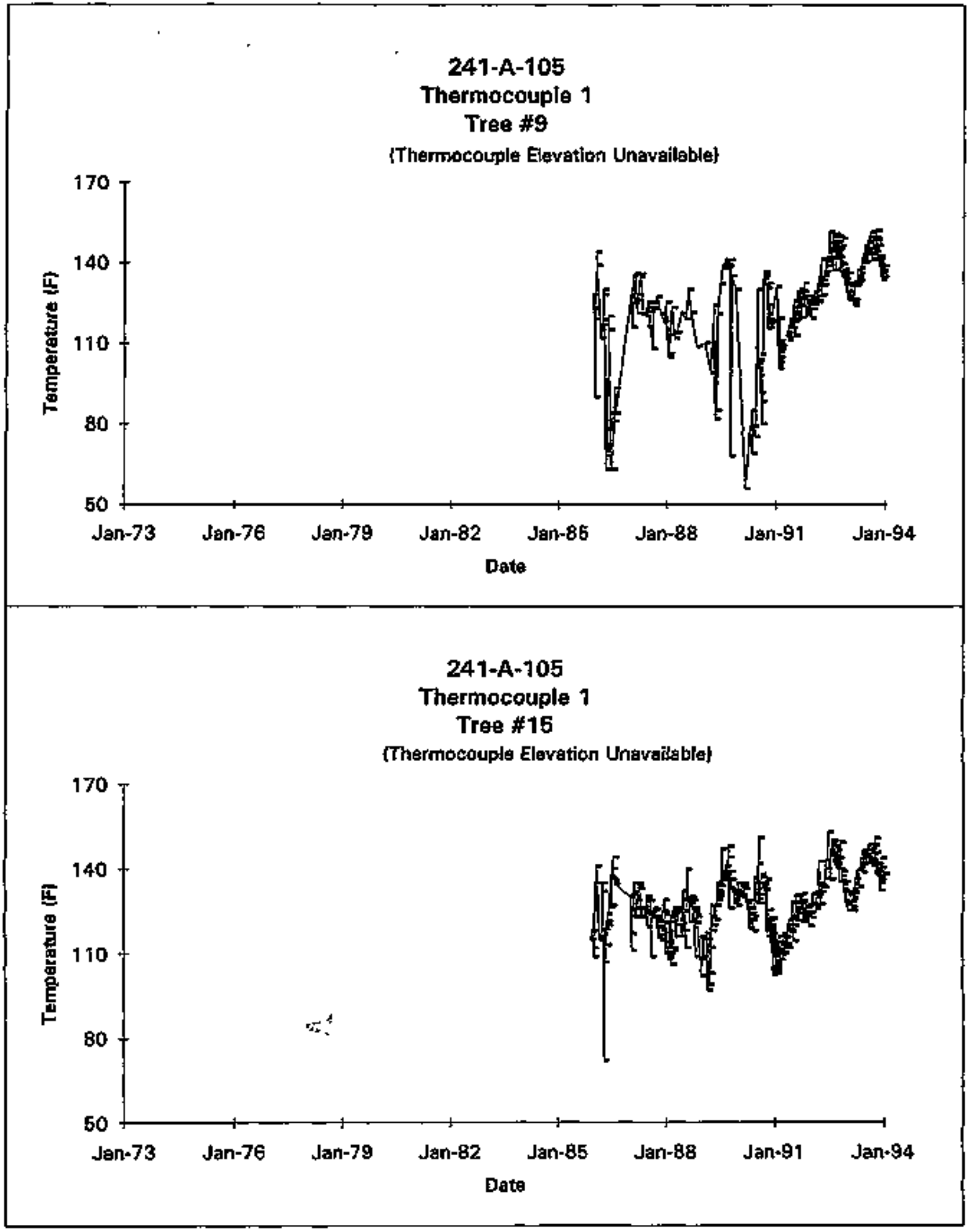

Data obtained from WHC Surveillence Analysis Computer System(SACS), November 12, 1993.

$$
\text { D. } 47
$$




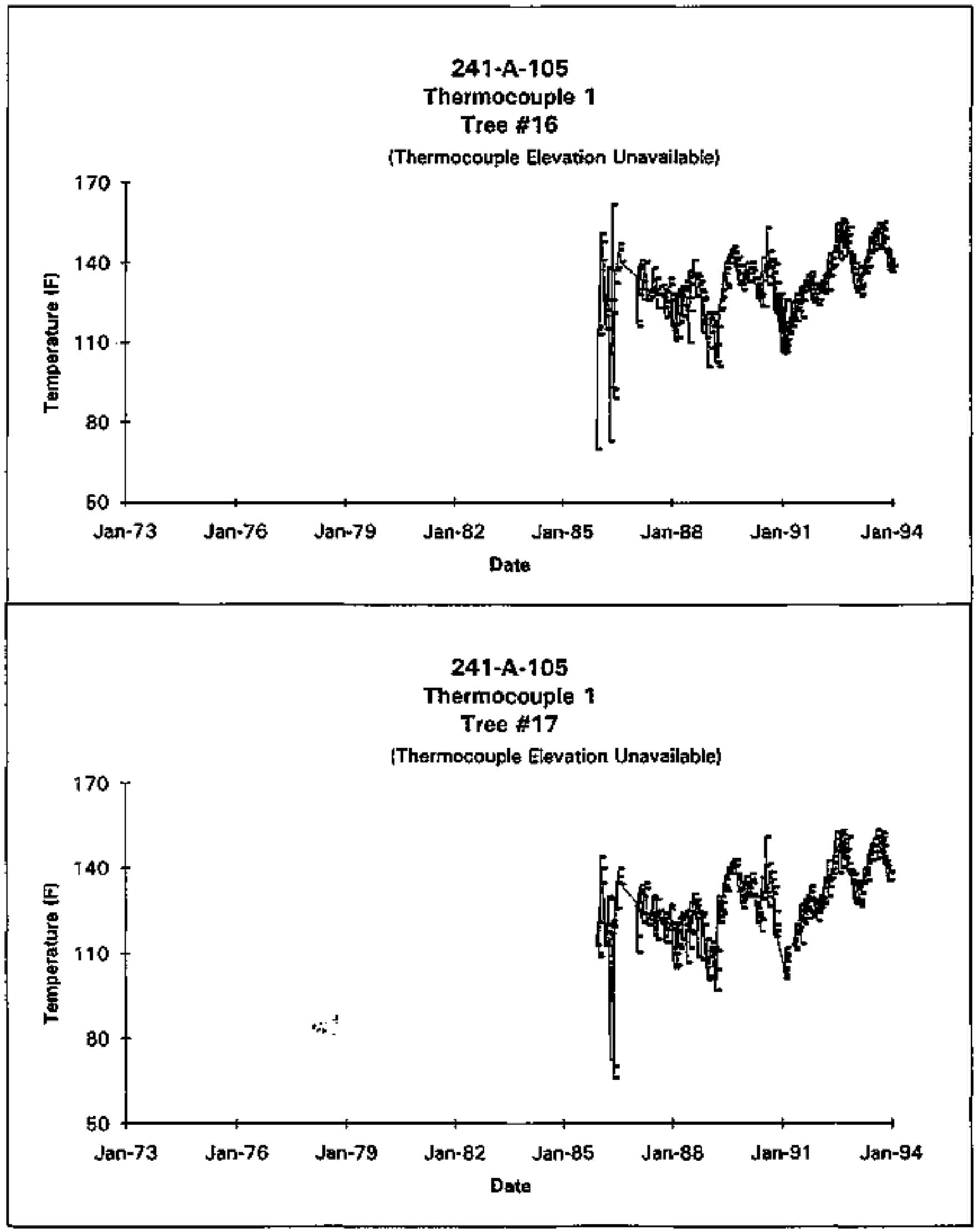

Data obtained from WHC Surveillance Anelysis Computer System(SACS), November 12، 1993. 


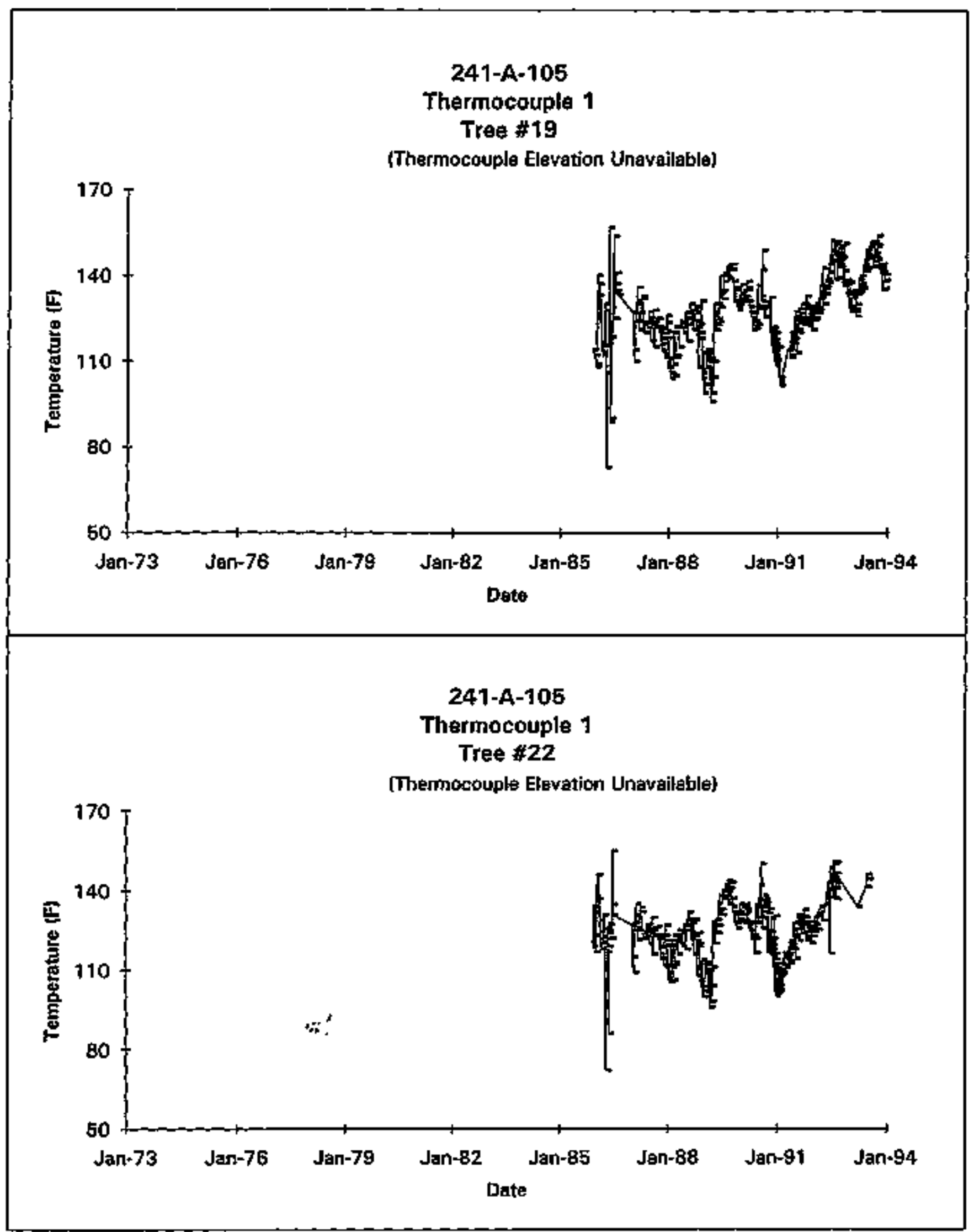

Data obtained from WHAC Surveillance Analysis Computer System(SACS), November 12, 1993. 


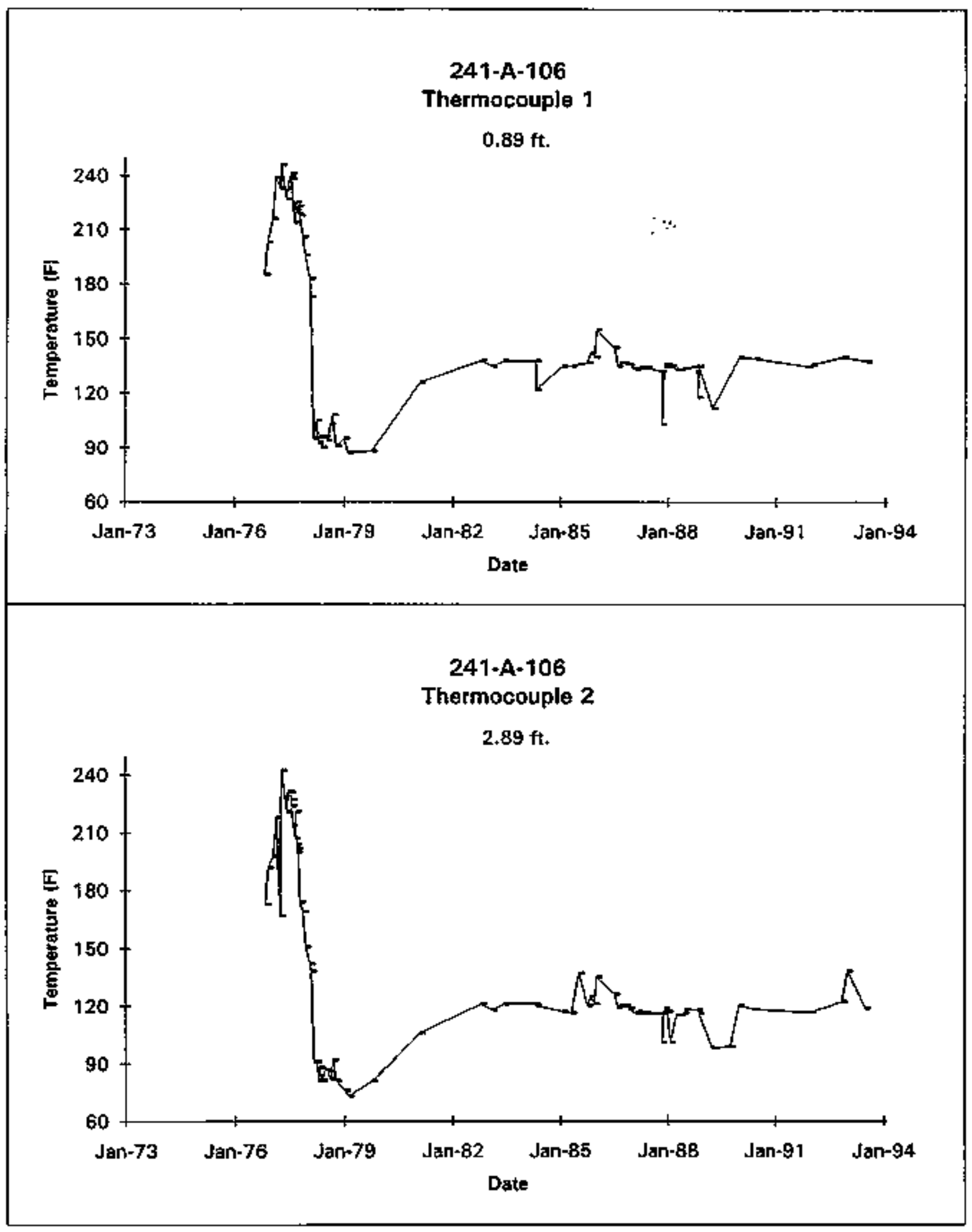

Data obtained from WHC Surveillance Analysis Computer System (SACS), November 12, 1993. 


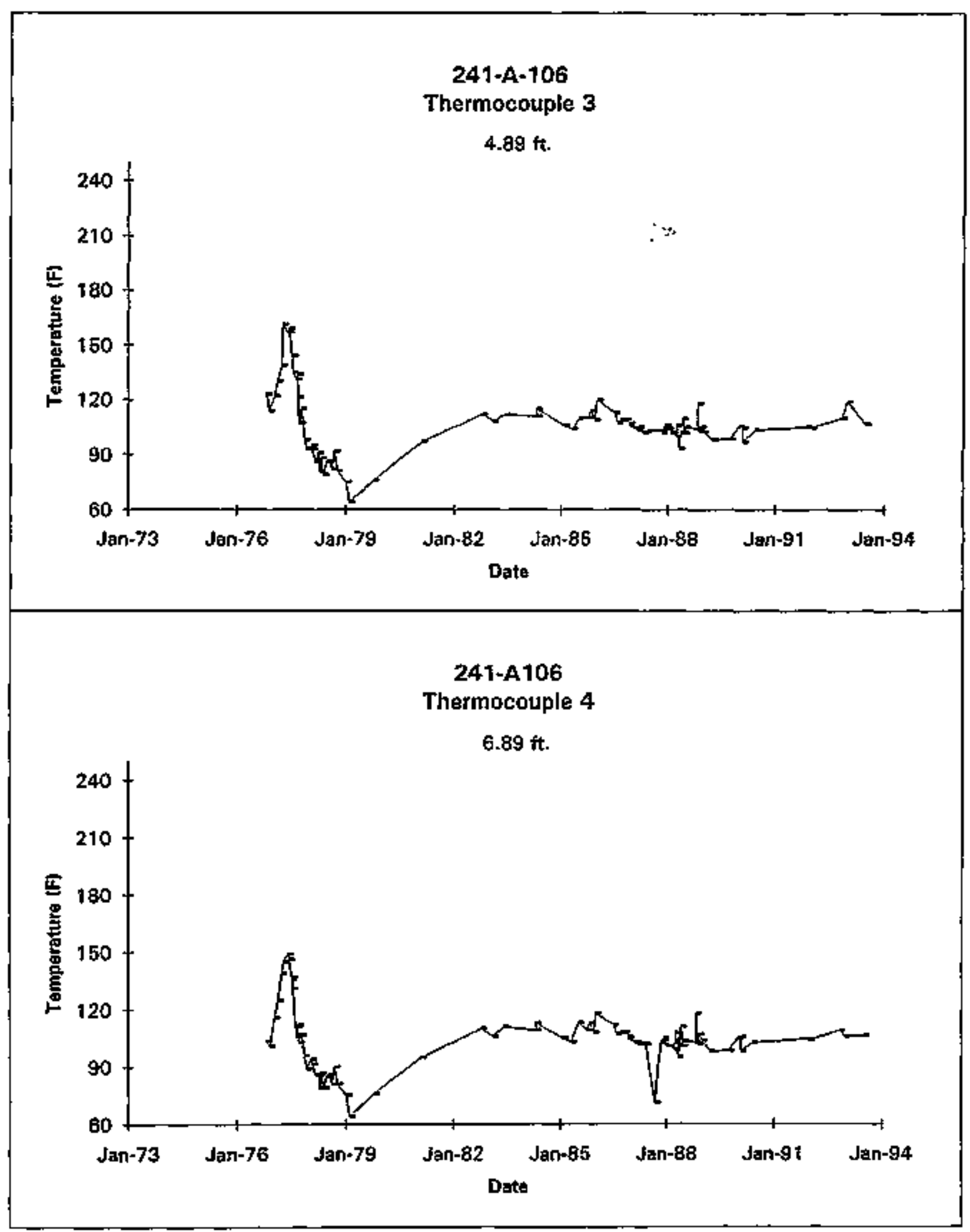

Data obtained from WHC Surveillance Analysis Computer System [SACS), November 12, 1993.

D-51 


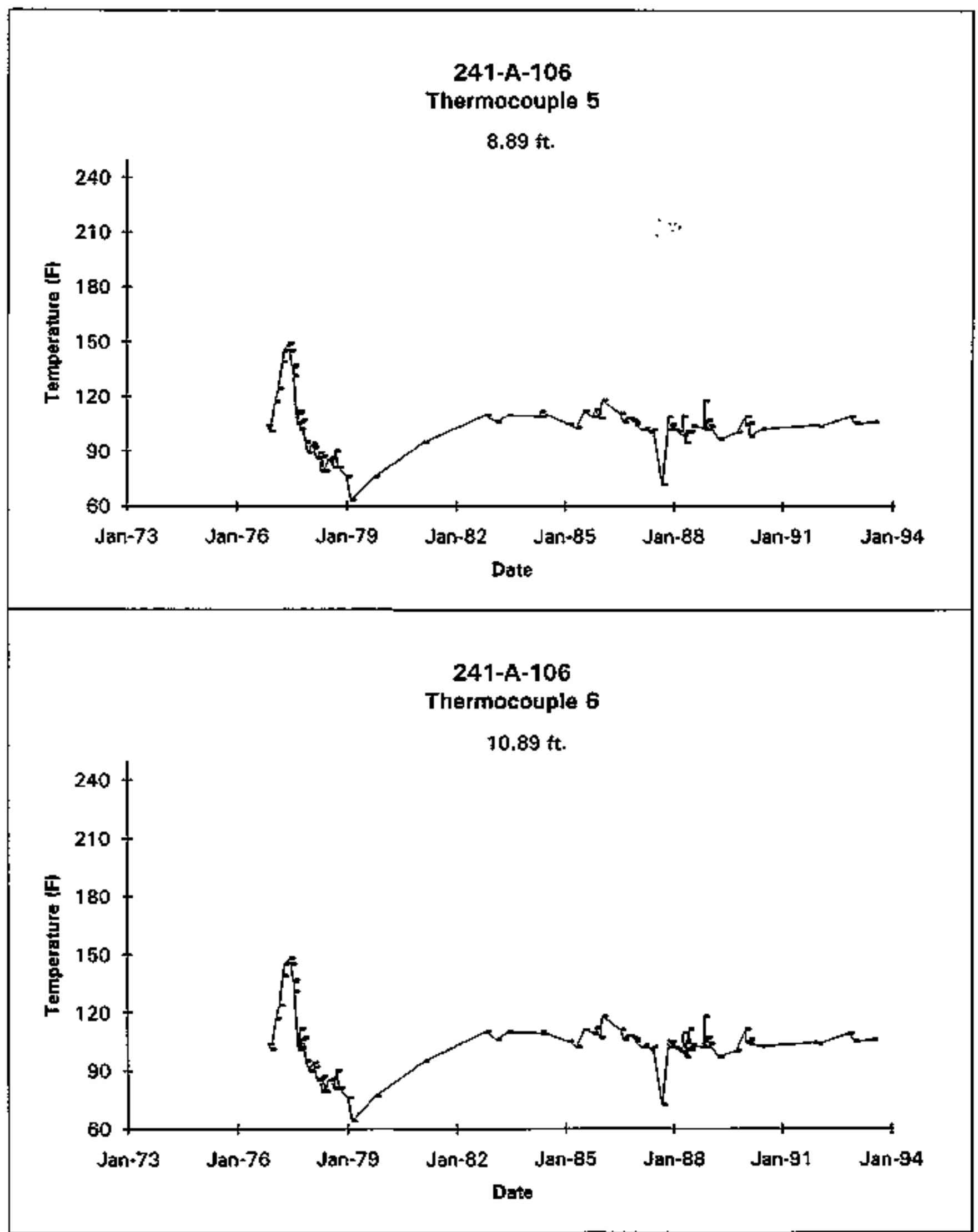

Data pbtained from WHC Surveillance Analysis Computer System (SACS), November 12, 1993. 


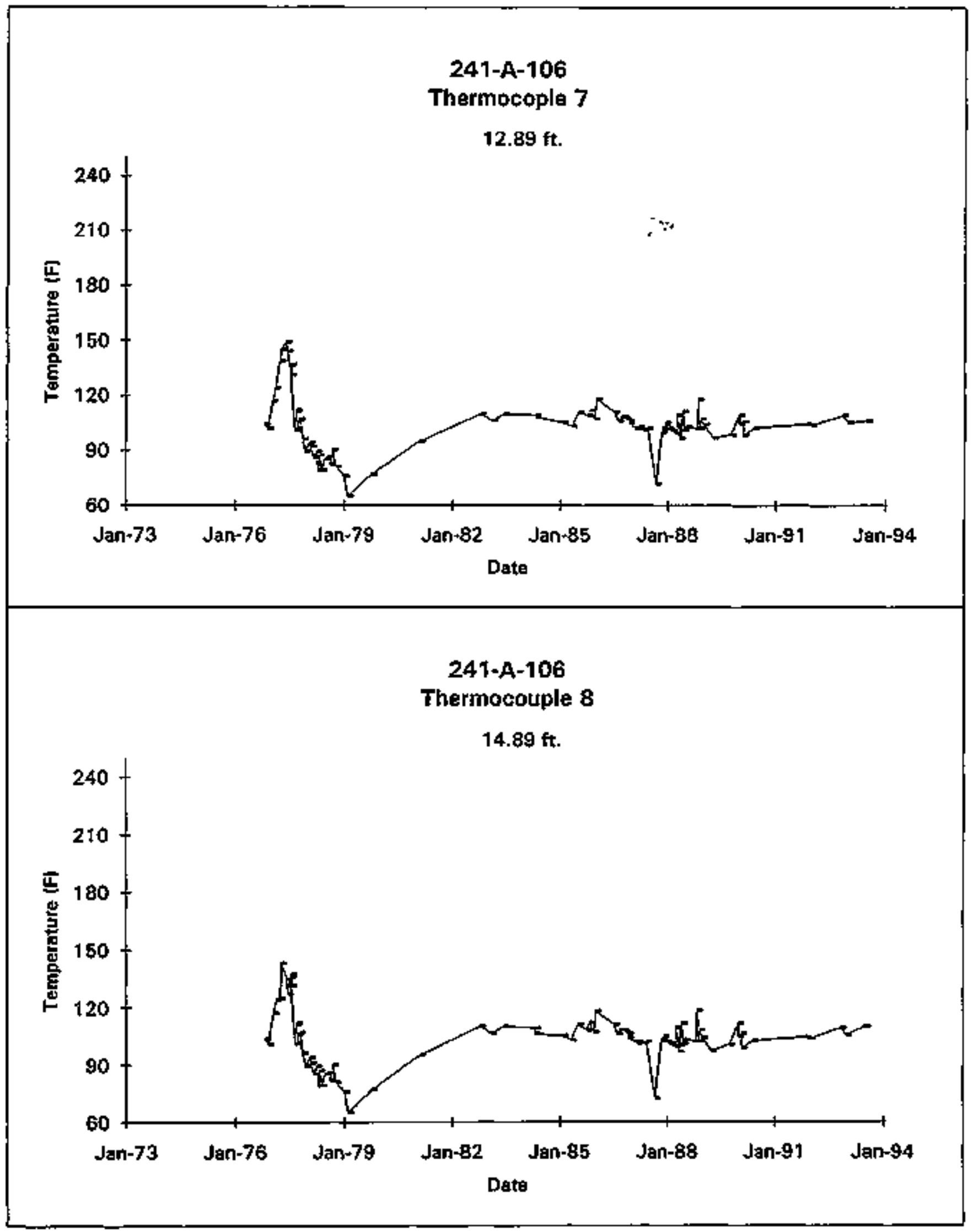

Data obtained from WHC Surveillance Analysis Computer System (SACS), November 12, 1993.

D-63 


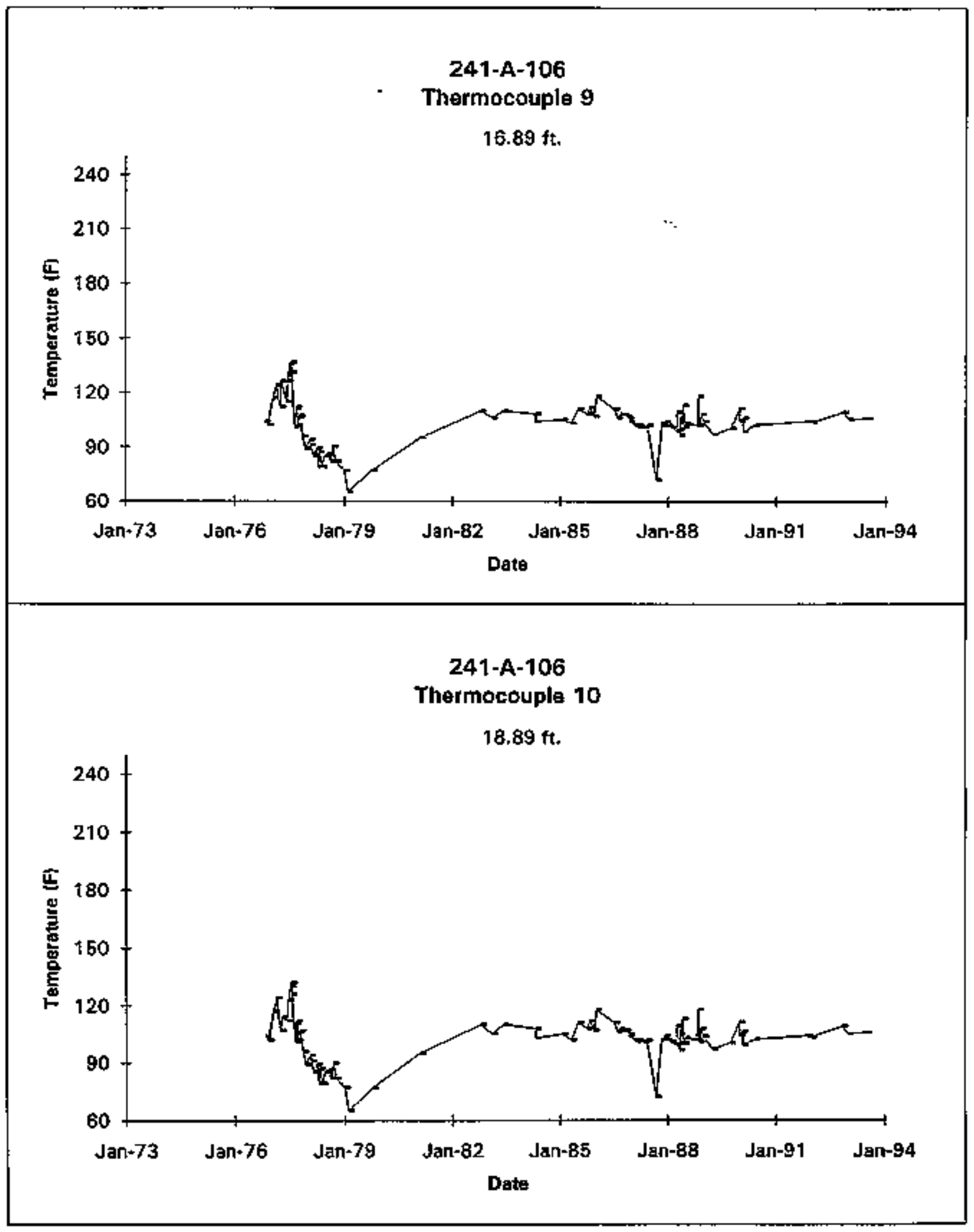

Data obtained from WHC Surveillance Analysis Computer Systern ISACS), November 12, 1993. 


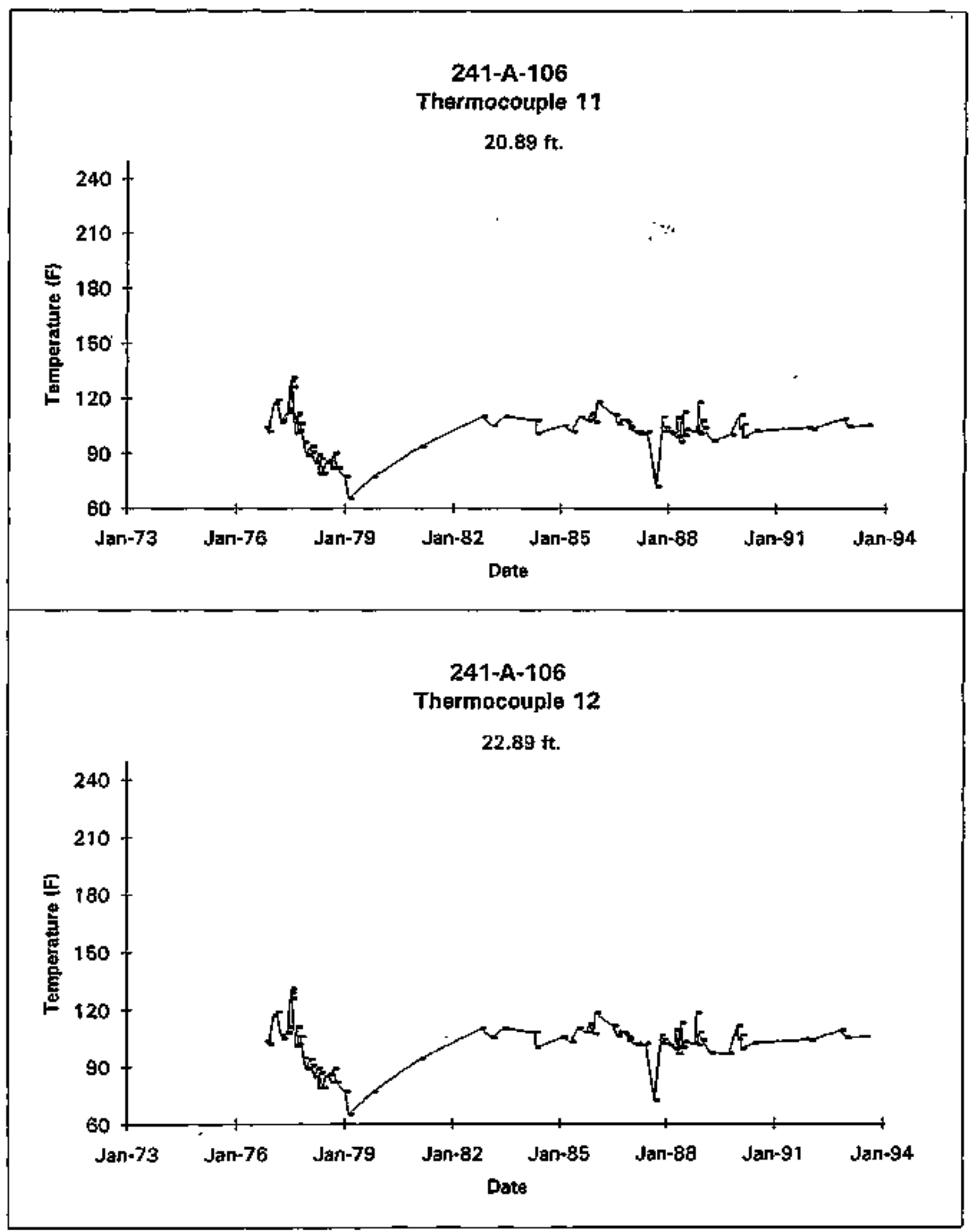

Data obtained from WHC Surveillance Analysis Computer System (SACS). November 12, 1993.

$$
\text { D-56 }
$$




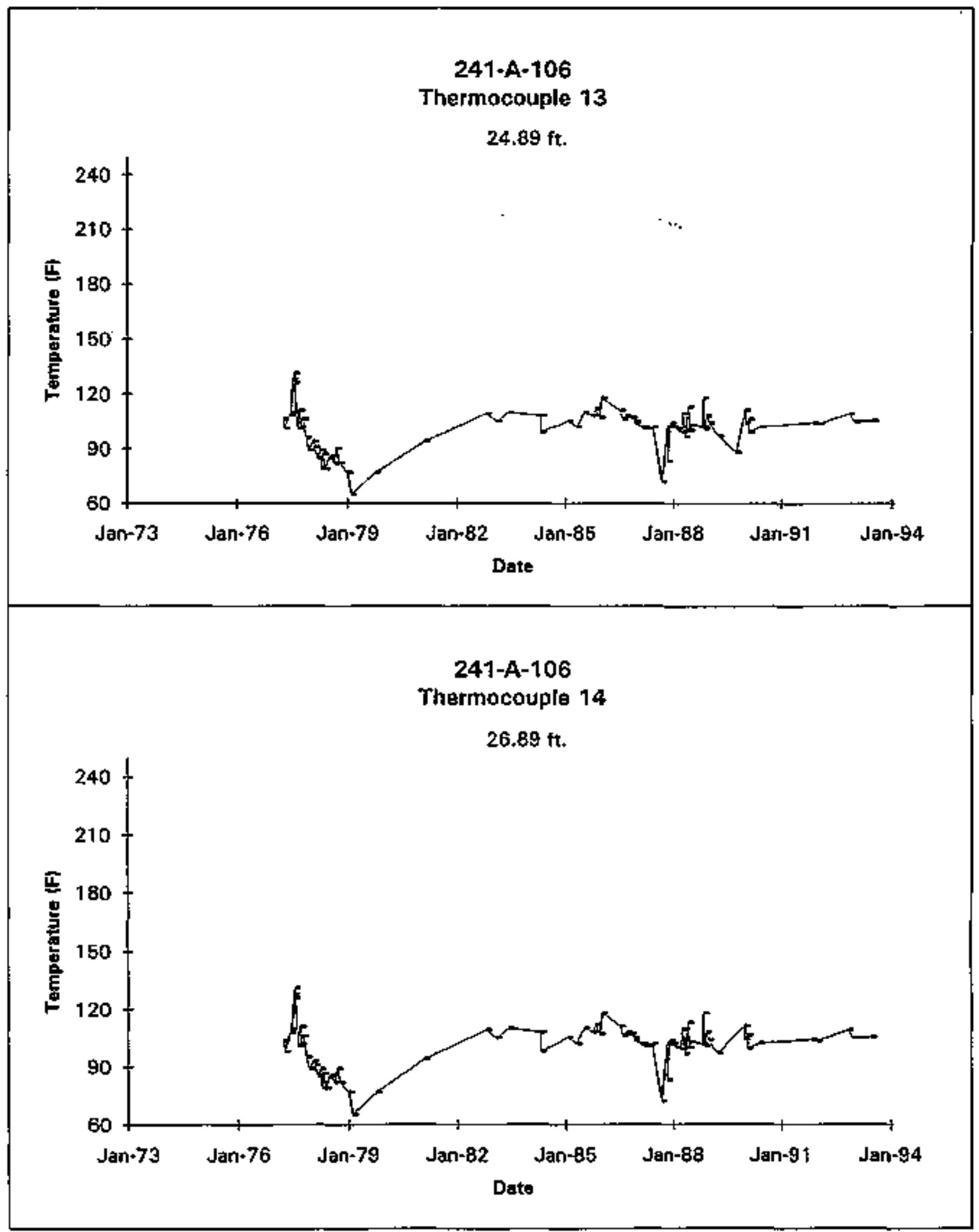

Data obtained from WHC Surveillance Analysis Computer System (SACSt, November 12, 1993. 
241-A-106

Thermocouple 15

$28.89 \mathrm{ft}$.

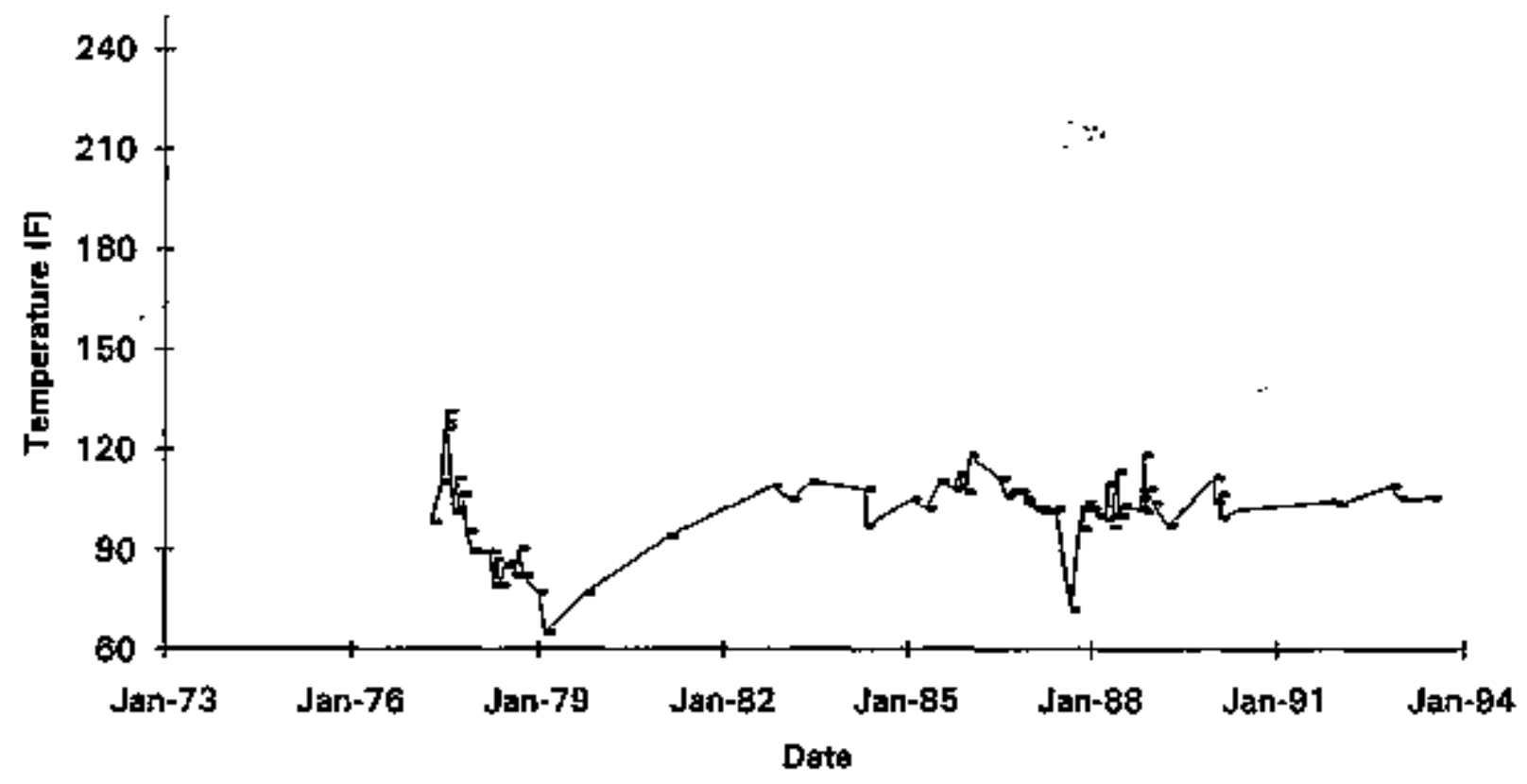

241-A-106

Thermocouple 16

$30.89 \mathrm{ft}$.

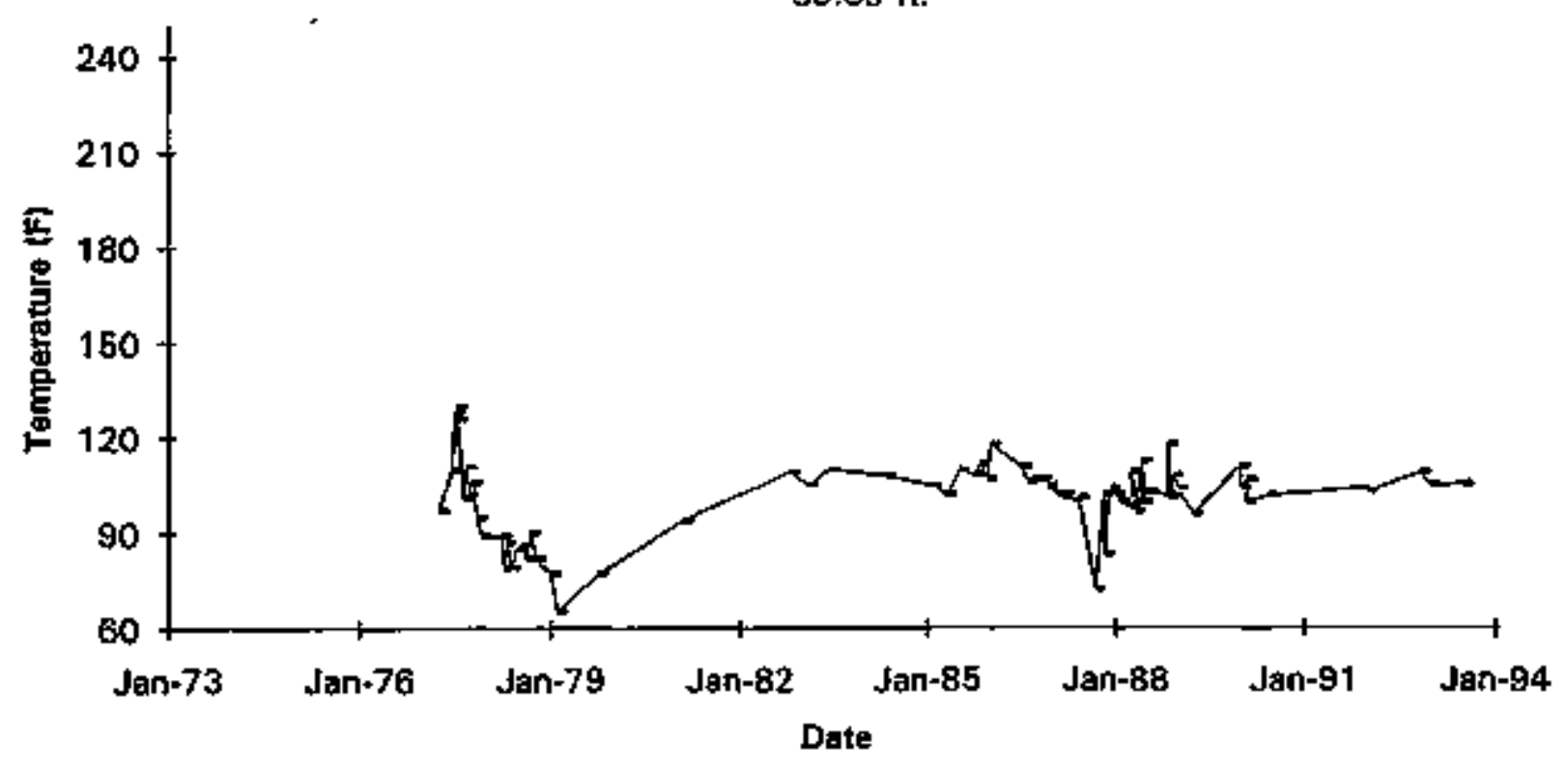

Data obtained from WHC Surveiltance Analysis Computer System [SACS], November 12, 1993. 
241-A-106

Thermocouple 17

$34.89 \mathrm{ft}$.

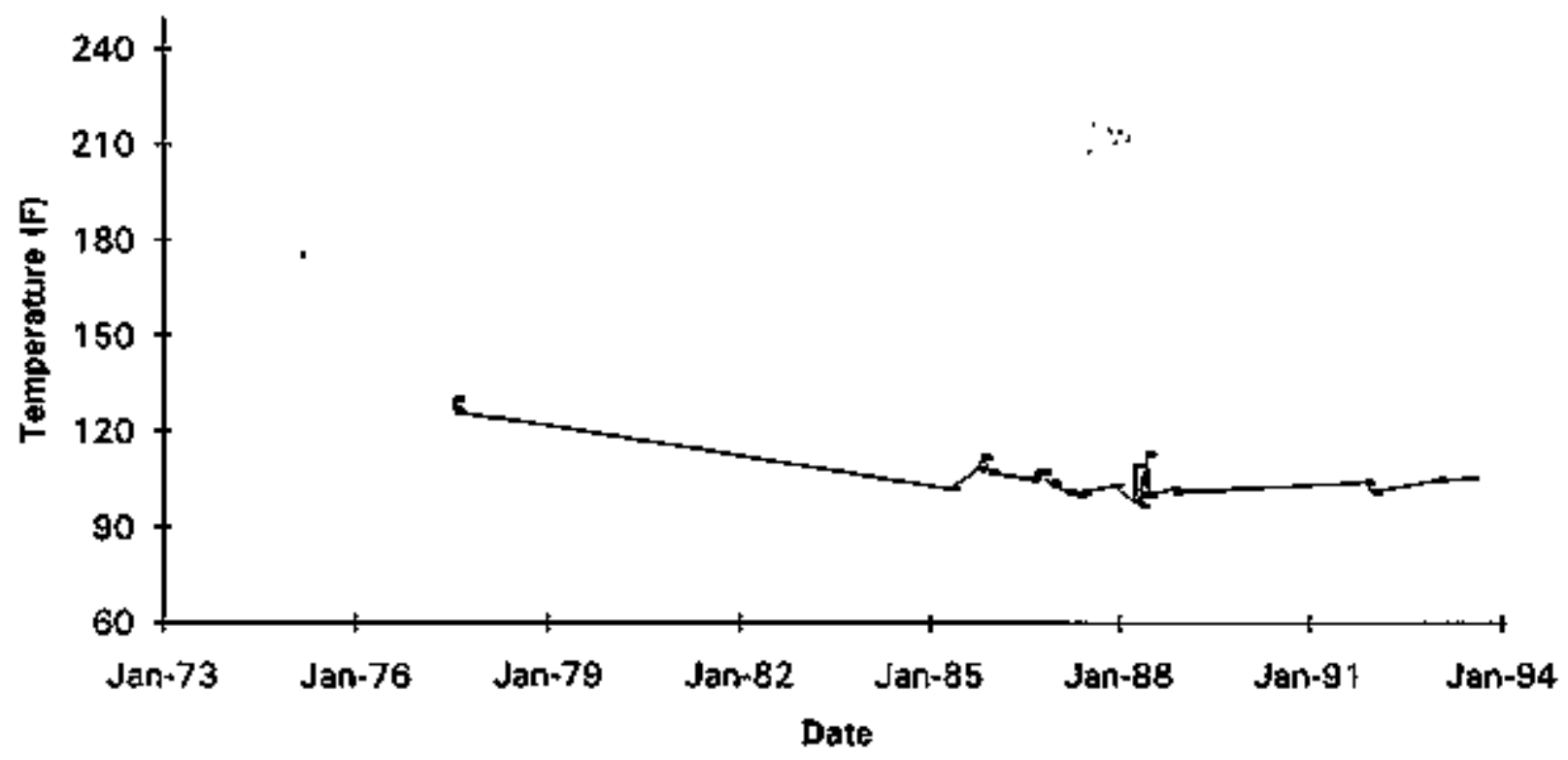

241-A-106

Thermocouple 18

$38.89 \mathrm{ft}$.

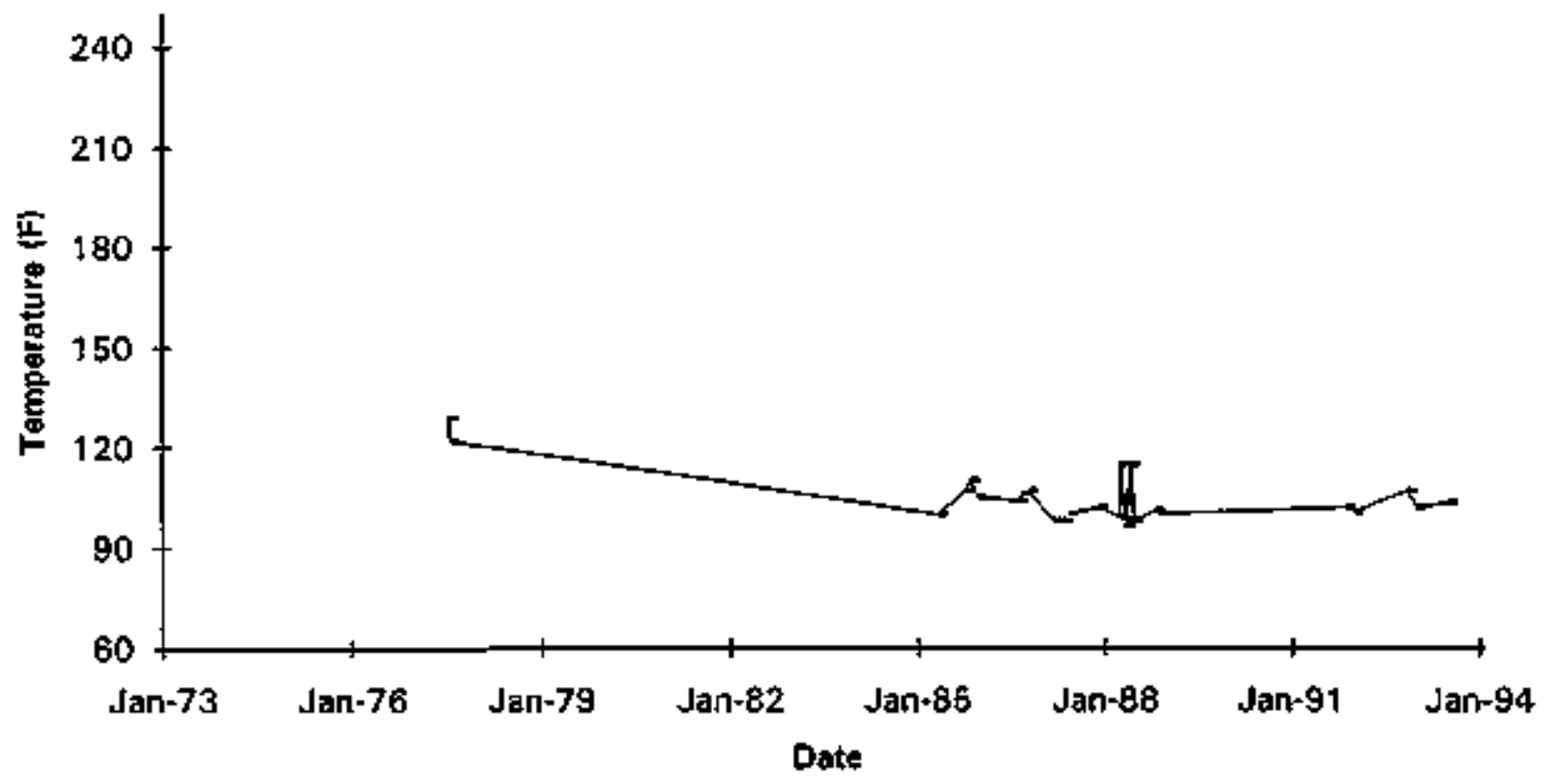

Data obtained from WHC Surveillance Analysis Computer System (SACS), November 12, 1993. 


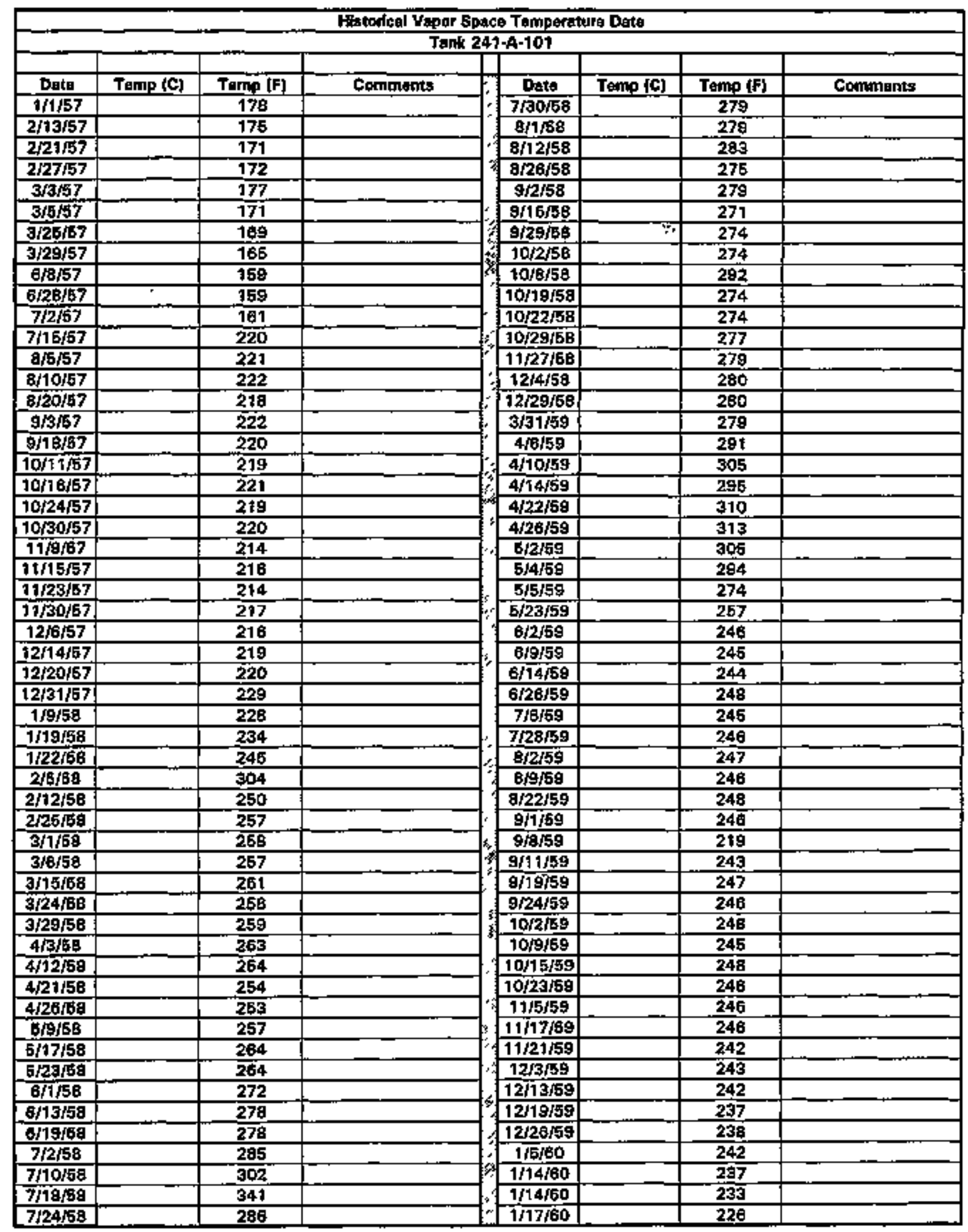

Oata obtained from Document RHO-CD-1172 and Waste Status Summary Reports. 


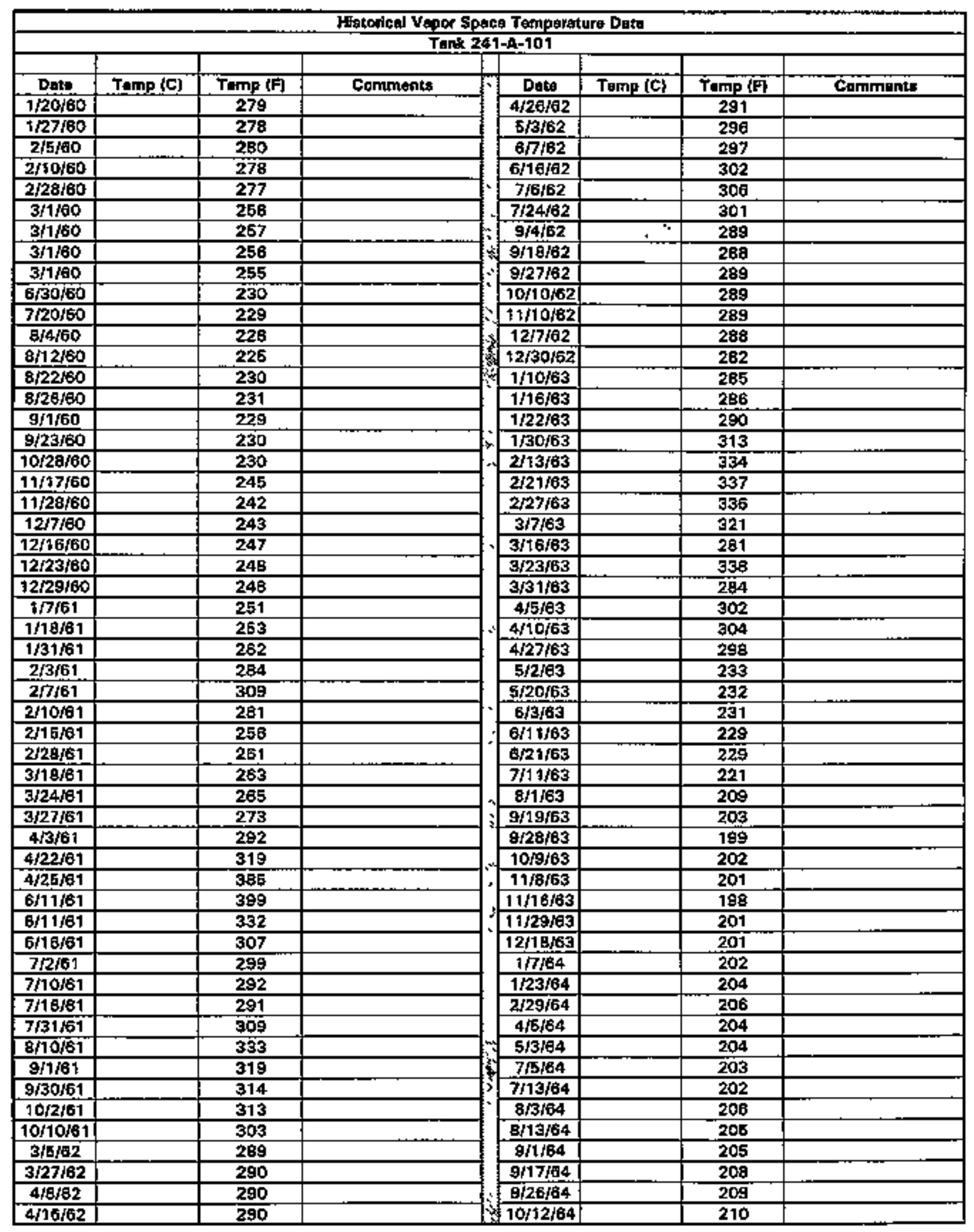

Date abtained fram Decumant RHO-CD-1172 and Waste Status Summary Reports. 


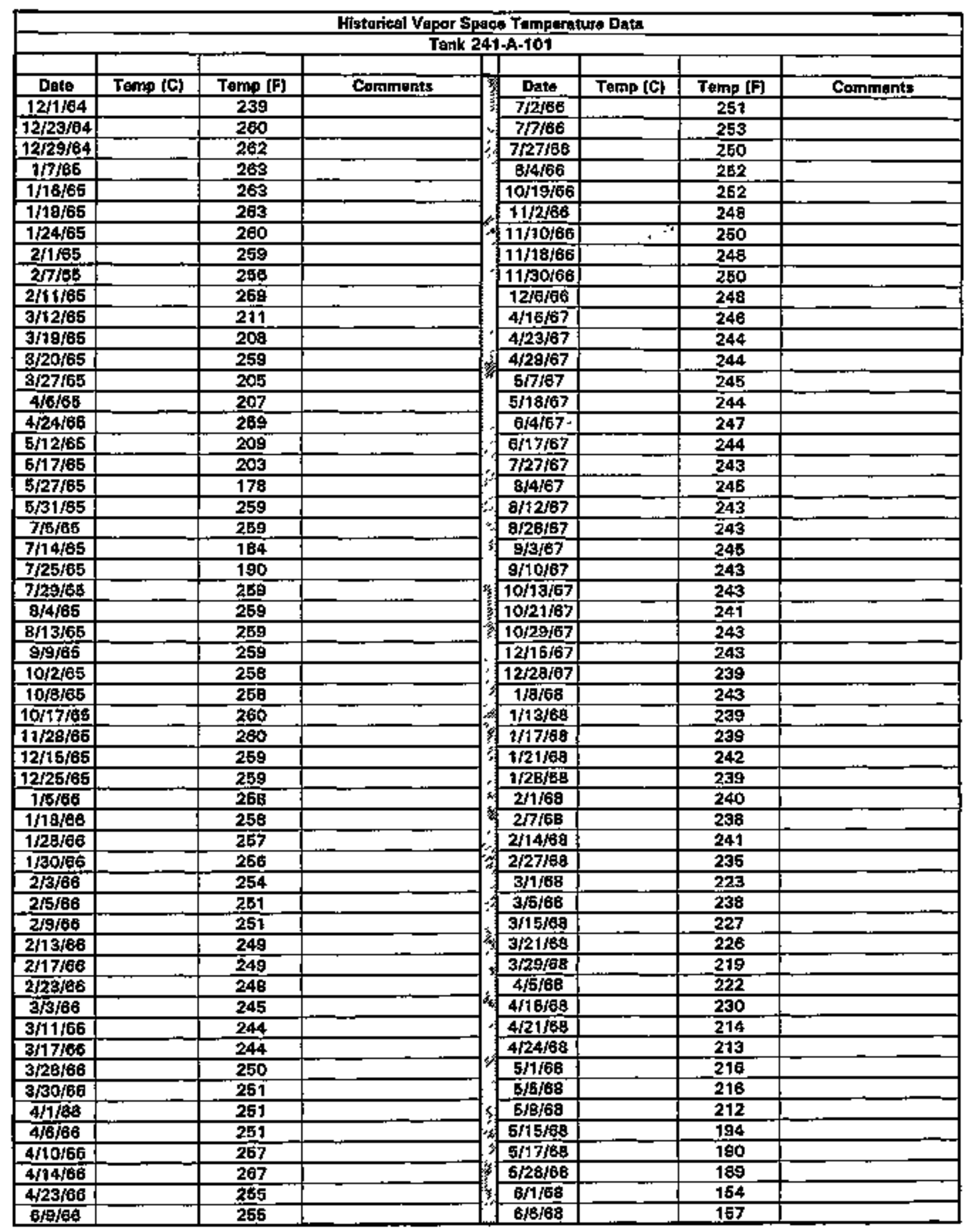

Data obtained from Decument futo-co-1172 and Waste Status Surnmary Reports. 


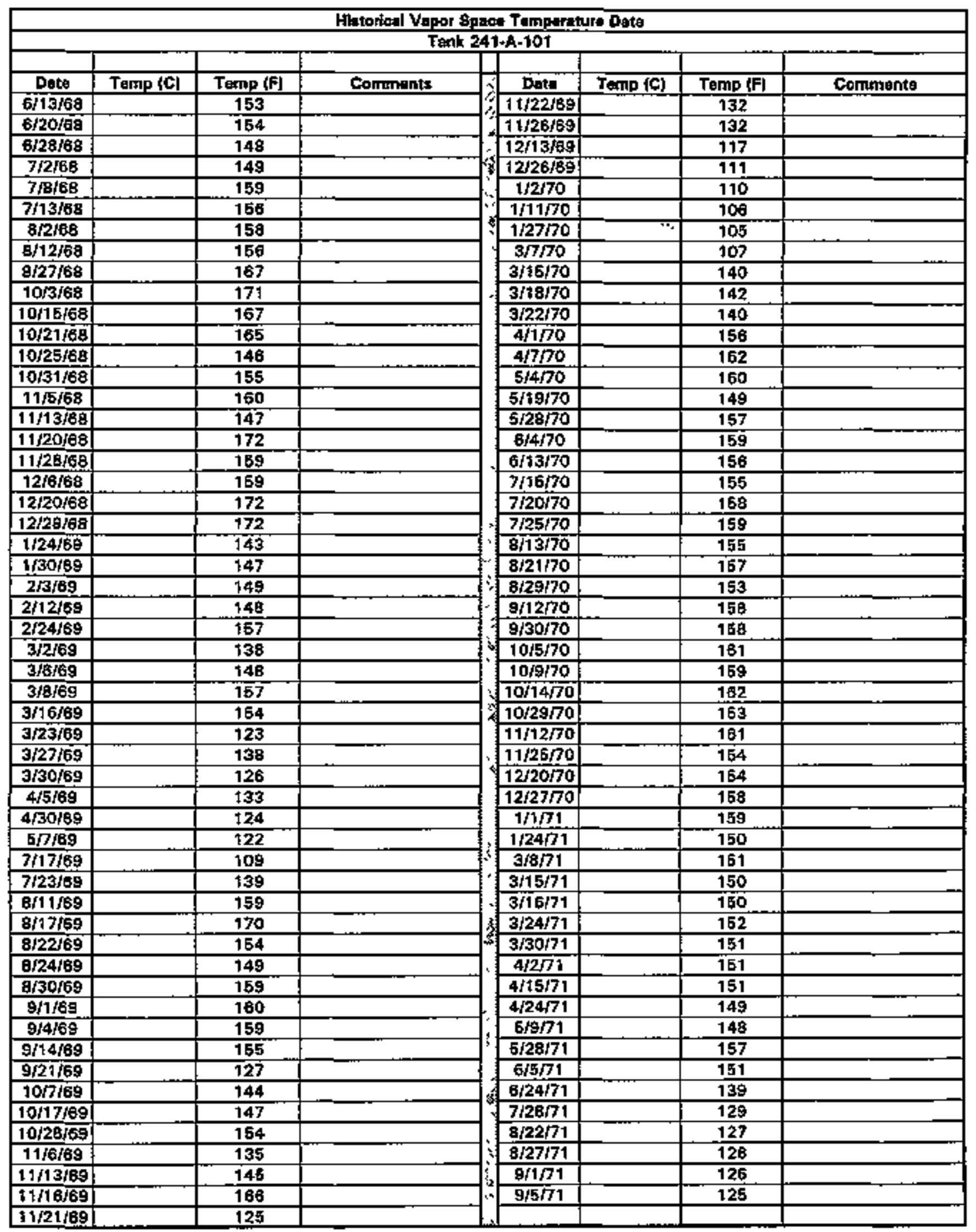

Date obtained from Doctument RHO-CD.1172 and Weste Status Summery Reports. 


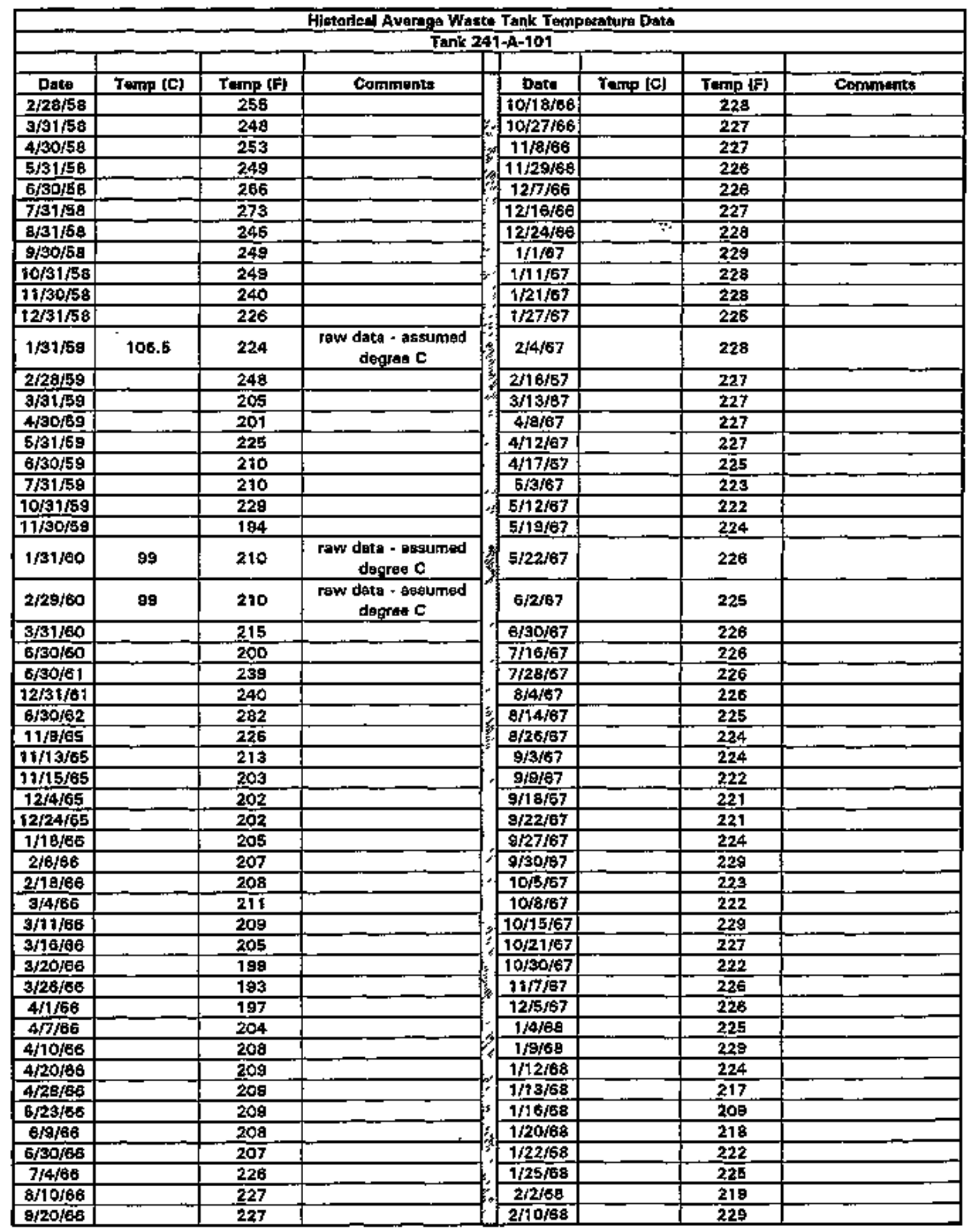

Data obtained from Doctiment RHO-CO-1172 and Weste Stetue Summery Reports. 


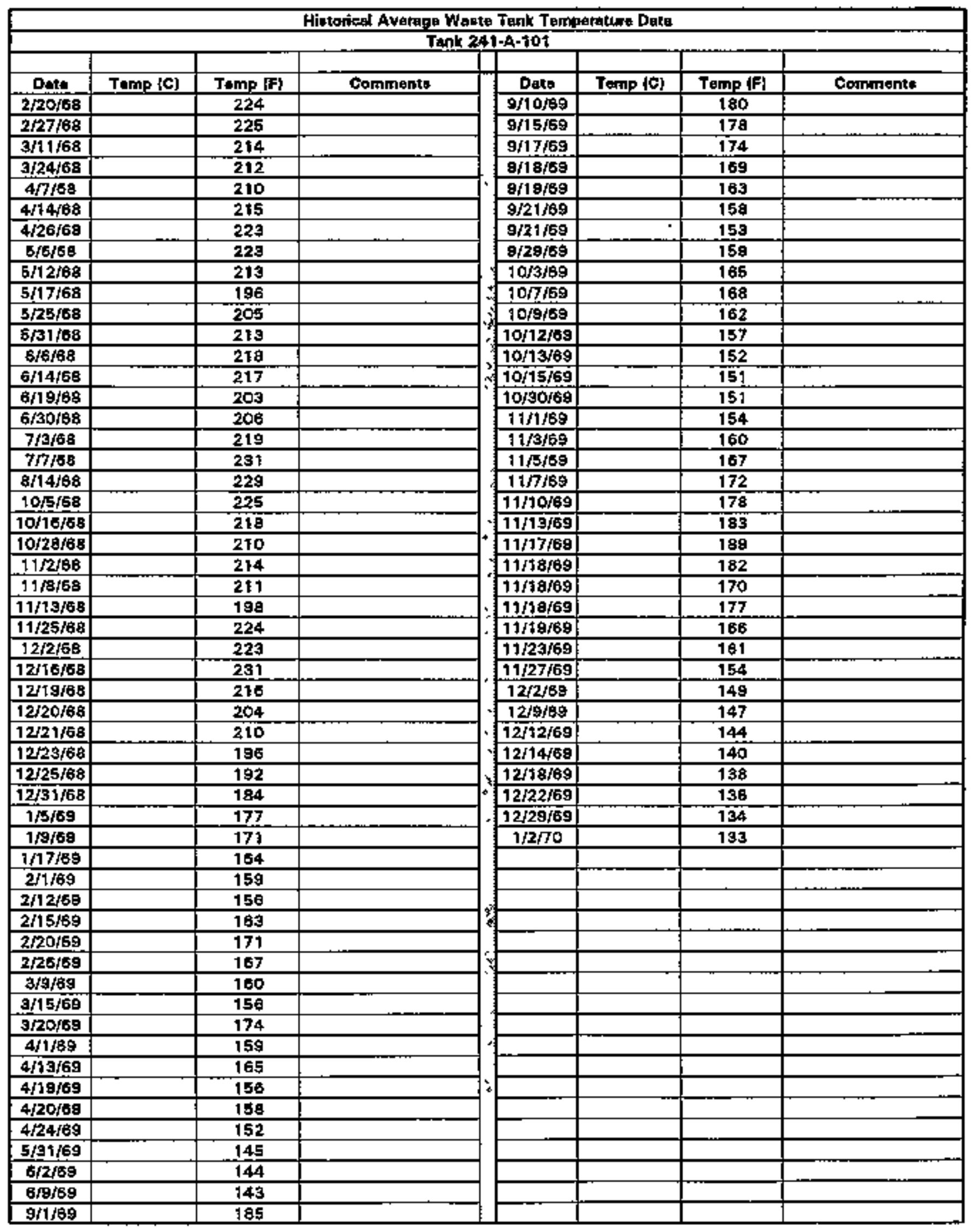

Date obtained from Dooument RHO-CO- $t 172$ ant We 


\begin{tabular}{|c|c|c|c|c|c|c|c|c|}
\hline \multicolumn{9}{|c|}{ Histowical High or Only Temperature Dente } \\
\hline \multicolumn{9}{|c|}{ Tank 241-A-10t } \\
\hline Data & Temp (c) & 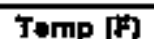 & Comment: & & Dato & Tamp (C) & Temp (F) & Camments: \\
\hline $2 / 28 / 5 B$ & & 259 & & & $1 / 31 / 86$ & & 216 & \\
\hline $3 / 31 / 56$ & & 259 & & & $2 / 10 / 88$ & & 217 & \\
\hline $4 / 30 / 58$ & & 270 & & & $3 / 17 / 68$ & & 211 & \\
\hline $5 / 31 / 58$ & & 268 & & & $3 / 29 / 88$ & & 188 & \\
\hline $6 / 30 / 58$ & & 298 & & & $4 / 2 / 6 B$ & & 211 & \\
\hline $7 / 31 / \sqrt{S B}$ & & 302 & & & $4 / 10 / 66$ & & 208 & \\
\hline $8 / 31 / 83$ & & 255 & & & $4 / 23 / 69$ & & 212 & \\
\hline $5 / 30 / 58$ & & 262 & & & $4 / 23 / 60$ & & 210 & \\
\hline $10 / 31 / 58$ & & 262 & & & $4 / 29 / 66$ & & 215 & \\
\hline $11 / 30 / 58$ & & 253 & & & $6 / B / B B$ & & 212 & \\
\hline $12 / 31 / 58$ & & 227 & & & $6 / 29 / 66$ & & 210 & \\
\hline $1 / 31 / 59$ & 107 & 224.6 & $\begin{array}{c}\text { raw data - assumed } \\
\text { degres C }\end{array}$ & 8 & $7 / 5 / 66$ & & 231 & \\
\hline $2 / 29 / 50$ & & 277 & & & $10 \sqrt{7 / 66}$ & & 230 & \\
\hline $3 / 31 / 59$ & & 205 & & & $12 / 18 / 66$ & & 229 & \\
\hline 4/30/59 & & 201 & & & $3 / 22 / 67$ & & 229 & \\
\hline $5 / 37 / 58$ & & 240 & & & $8 / / 677$ & & 228 & \\
\hline $6 / 30 / 59$ & & 210 & & & $5 / 16 / 67$ & & 228 & \\
\hline $7 / 31 / 59$ & & 210 & & & $5 / 23 / 67$ & & 229 & \\
\hline $10 / 31 / 59$ & & 248 & & & $6 / 2 / 67$ & & 229 & \\
\hline $11 / 30 / 5 \bar{\theta}$ & & 248 & & & $6 / 17 / 87$ & & 228 & \\
\hline $1 / 31 / 50$ & 99 & 210.2 & $\begin{array}{c}\text { raw deta + agsumed } \\
\text { degrap C }\end{array}$ & & $7 / 30 / 67$ & & 220 & \\
\hline $2 / 29 / 80$ & 98 & 210.2 & $\begin{array}{c}\text { Paw data - asaumed } \\
\text { degree } \mathrm{C}\end{array}$ & & $8 / 9 / 67$ & & 227 & \\
\hline $3 / 31 / 50$ & & 213 & & & $8 / 26 / 67$ & & 229 & \\
\hline $8 / 30 / 80$ & & 200 & & & $9 / / 187$ & & 223 & \\
\hline $6 / 30 / 61$ & & 259 & & & $9 / 11 / 97$ & & 222 & \\
\hline 12/31/21 & & 260 & & & $8 / 17 / 07$ & & $\overline{222}$ & \\
\hline $6 / 30 / 22$ & & 300 & & & $9 / 25 / 67$ & & 231 & \\
\hline $7 / 2 / 5 B$ & & 248.772 & & & $10 / 1 / 67$ & & 234 & \\
\hline $7 / 8 / 65$ & & 233.167 & & & $10 / 9 / 67$ & & 229 & \\
\hline $7 / 18 / 65$ & & 236,729 & & & $10 / 16 / 87$ & & $23 \overline{2}$ & \\
\hline $7 / 24 / 86$ & & 245.888 & & & $10 / 30 / 87$ & & 223 & \\
\hline $7 / 29 / 85$ & & 230.792 & & & $11 / 7 / 67$ & & 226 & \\
\hline $8 / 4 / 65$ & & 235.202 & & & $12 / 31 / 57$ & & 226 & \\
\hline $8 / 20 / 65$ & & 218.258 & & & $1 / 3 / 68$ & & 225 & \\
\hline $\mathrm{g} / 2 \mathrm{/} / \mathrm{65}$ & & 248.856 & & & $1 / 9 / 68$ & & 230 & \\
\hline $8 / 30 / 05$ & & 248,802 & & & 1/1/4/68 & & 225 & \\
\hline $9 / 9 / 55$ & & 227.569 & & & $1 / 16 / 68$ & & 206 & \\
\hline $9 / 17 / 86$ & & 250.977 & & & $1 / 21 / 68$ & & 226 & \\
\hline $9 / 22 / 85$ & & 226.721 & & & $1 / 26 / 68$ & & 225 & \\
\hline $0 / 20 / 65$ & & 228.332 & & & $2 / 1 / 68$ & & 198 & \\
\hline $10 / 8 / 85$ & & 219.943 & & & $2 / 1 / 68$ & & 207 & \\
\hline $10 / 22 / 65$ & & 246.651 & & & $2 / 1 / 89$ & & 208 & \\
\hline $10 / 29 / 65$ & & 225.364 & & & $3 / 1 / 68$ & & 208 & \\
\hline $11 / 4 / 65$ & & 232,081 & & & $3 / 1 / 68$ & & 197 & \\
\hline $11 / 6 / 85$ & & 233.804 & & & $3 / 1 / 28$ & & 200 & \\
\hline $11 / 10 / 65$ & & 233.011 & & & $4 / 1 / 68$ & & $19 B$ & \\
\hline $11 / 17 / 65$ & & 212.098 & & & $4 / 1 / 68$ & & 195 & \\
\hline $11 / 24 / 65$ & & 212.098 & & & $4 / 1 / 68$ & & 189 & \\
\hline $12 / 1 / 85$ & & 217.485 & & & $4 / 1 / / 68$ & & 214 & \\
\hline $12 / 30 / 05$ & & 208.454 & & & $5 / 1 / 68$ & & 212 & \\
\hline $1 / 26 / 66$ & & 216.634 & & & $5 / 1 / 68$ & & 206 & \\
\hline
\end{tabular}

Date obtalnad from Daoument RHO-CO-1172 and Waste Stetus Summary Roports. 


\begin{tabular}{|c|c|c|c|c|c|c|c|}
\hline \multicolumn{8}{|c|}{ Hintoried High of Only Temporature Dato } \\
\hline \multicolumn{8}{|c|}{ Tank 241-A-701 } \\
\hline & & & & & & & \\
\hline Date & $|\operatorname{simp}| \mathrm{L} \mid$ & Emp (F) & Comingents & Dats & inmp $\{C\}$ & Tomp \{F\} & Comments \\
\hline $5 / 1 / 68$ & & 199 & & & & & \\
\hline $5 / \overline{1 / 8 B}$ & & 180 & & & & & \\
\hline $6 / 1 / 68$ & & 209 & & & & & \\
\hline $6 / 1 / 68$ & & 205 & & & & & \\
\hline $6 / 1 / 88$ & & 209 & & & & & \\
\hline $6 / \overline{108}$ & & 203 & & & & & \\
\hline $6 / 1 / 68$ & & 210 & & & & & \\
\hline $7 / 1 / 88$ & & 218 & & & & & \\
\hline $7 / \overline{1 / 88}$ & & 217 & & & & & \\
\hline $8 / 1 / 68$ & & 218 & & & & & \\
\hline $8 / 1 / 88$ & & 217 & & & & & \\
\hline $8 / 1 / 68$ & & 212 & & & & & \\
\hline $10 / 1 / 68$ & & 209 & & & & & \\
\hline $10 / 1 / 68$ & & $\mathbf{t 9 5}$ & & & & & \\
\hline $11 / 1 / 68$ & & 199 & & & & & \\
\hline$1 1 \longdiv { 1 / 6 8 }$ & & $\$ 88$ & & & & & \\
\hline $11 / \overline{1 / 68}$ & & 200 & & & & & \\
\hline $11 / 1 / 68$ & & 207 & & & & & \\
\hline $12 / 1 / 68$ & & 218 & & & & & \\
\hline $12 / 1 / 68$ & & 212 & & & & & \\
\hline $12 / 1 / 68$ & & 180 & & & & & \\
\hline $1 / \overline{1 / 69}$ & & 145 & & & & & \\
\hline $2 / 1 / 69$ & & 135 & & & & & \\
\hline $2 / 1 / 69$ & & 154 & & & & & \\
\hline $3 / 1 / 69$ & & 157 & & & & & \\
\hline $4 / 1 / 8 B$ & & $13 \mathrm{~B}$ & & & & & \\
\hline $4 \sqrt{1 / 69}$ & & 145 & & & & & \\
\hline $4 / 1 / 69$ & & 133 & & & & & \\
\hline $6 / 1 / 69$ & & 123 & & & & & \\
\hline $7 / 1 / 69$ & & 118 & & & & & \\
\hline $7 / 1 / 69$ & & $1 \overrightarrow{48}$ & & & & & \\
\hline $7 / 1 / 69$ & & 150 & & & & & \\
\hline $8 / 1 / 69$ & & 169 & & & & & \\
\hline $9 / \overline{1 / 69}$ & & $\overline{174}$ & & & & & \\
\hline $8 / 1 \sqrt{69}$ & & 101 & & & & & \\
\hline g/1/A9 & & 162 & & & & & \\
\hline $7 / 16 / 69$ & & 137 & & & & & \\
\hline $7 / 19 / 69$ & & 147 & & & & & \\
\hline $7 / 22 / 69$ & & 160 & & & & & \\
\hline $7 / 28 / 69$ & & 169 & & & & & \\
\hline $7 / 30 / 69$ & & 169 & & & & & \\
\hline $8 / 27 / 69$ & & 1 여 & & & & & \\
\hline $9 / 28 / 69$ & & 181 & & & & & \\
\hline
\end{tabular}




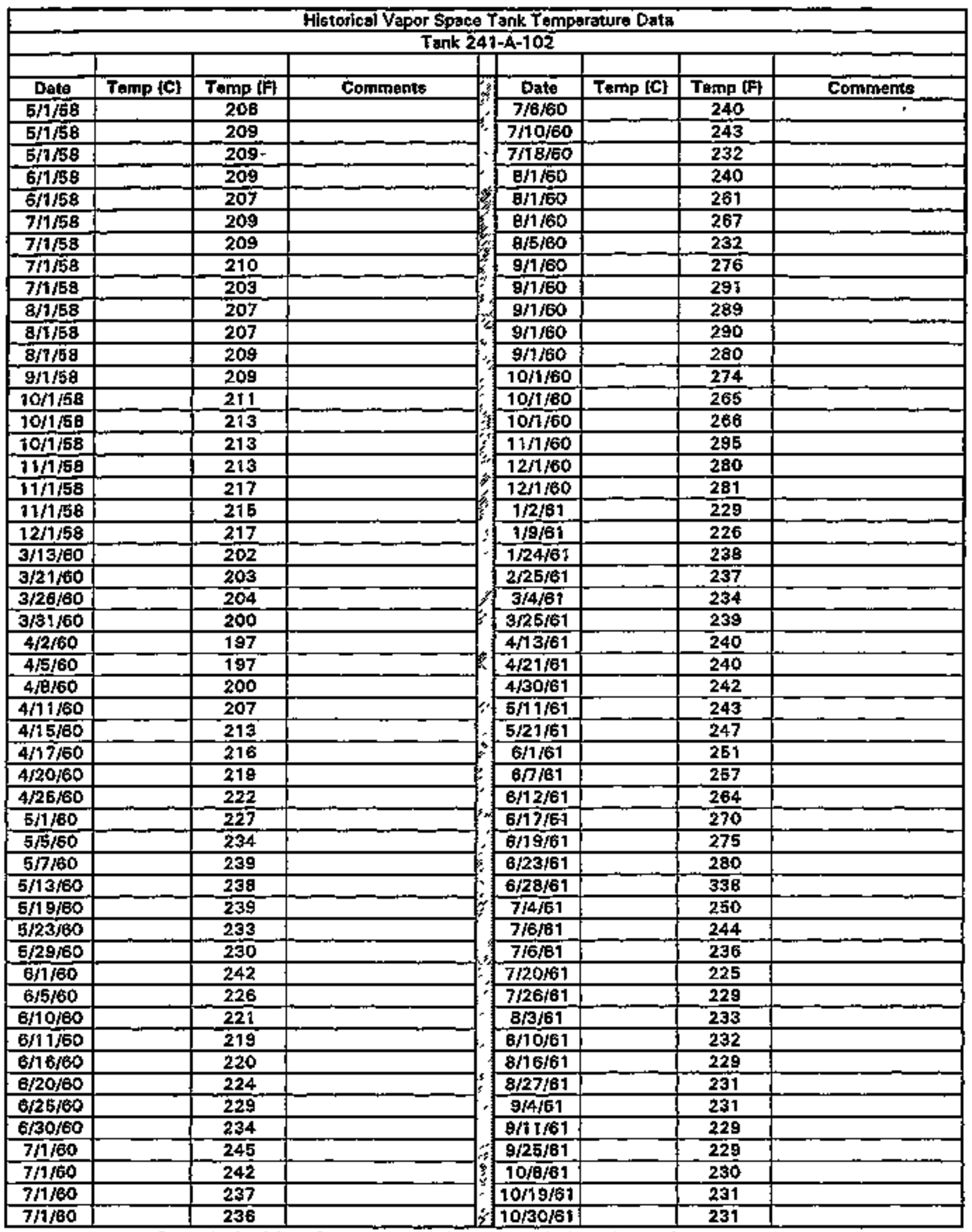

Data obtained feom Document FHO-CD-1172 and Waste Status Summsry Reports. 


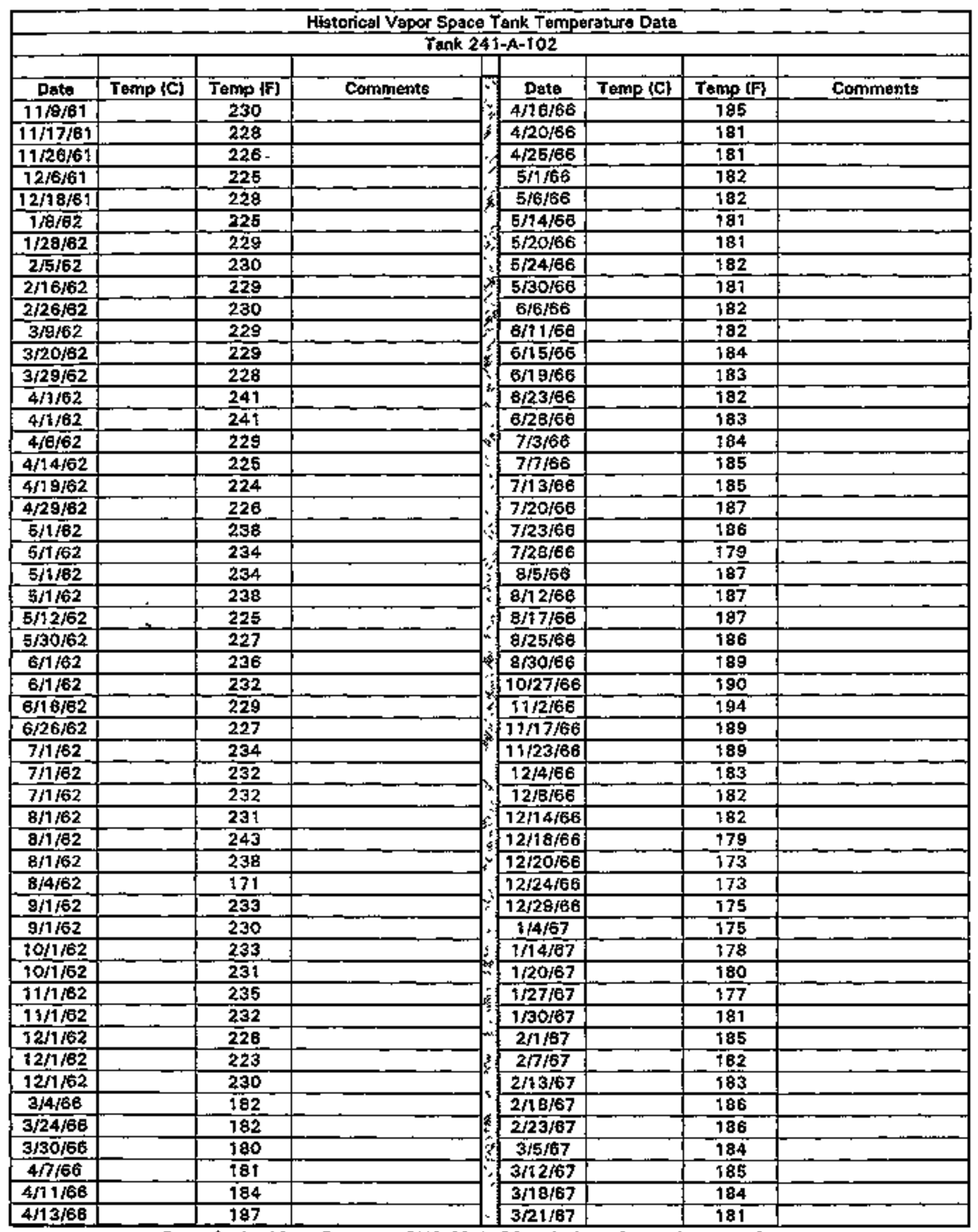




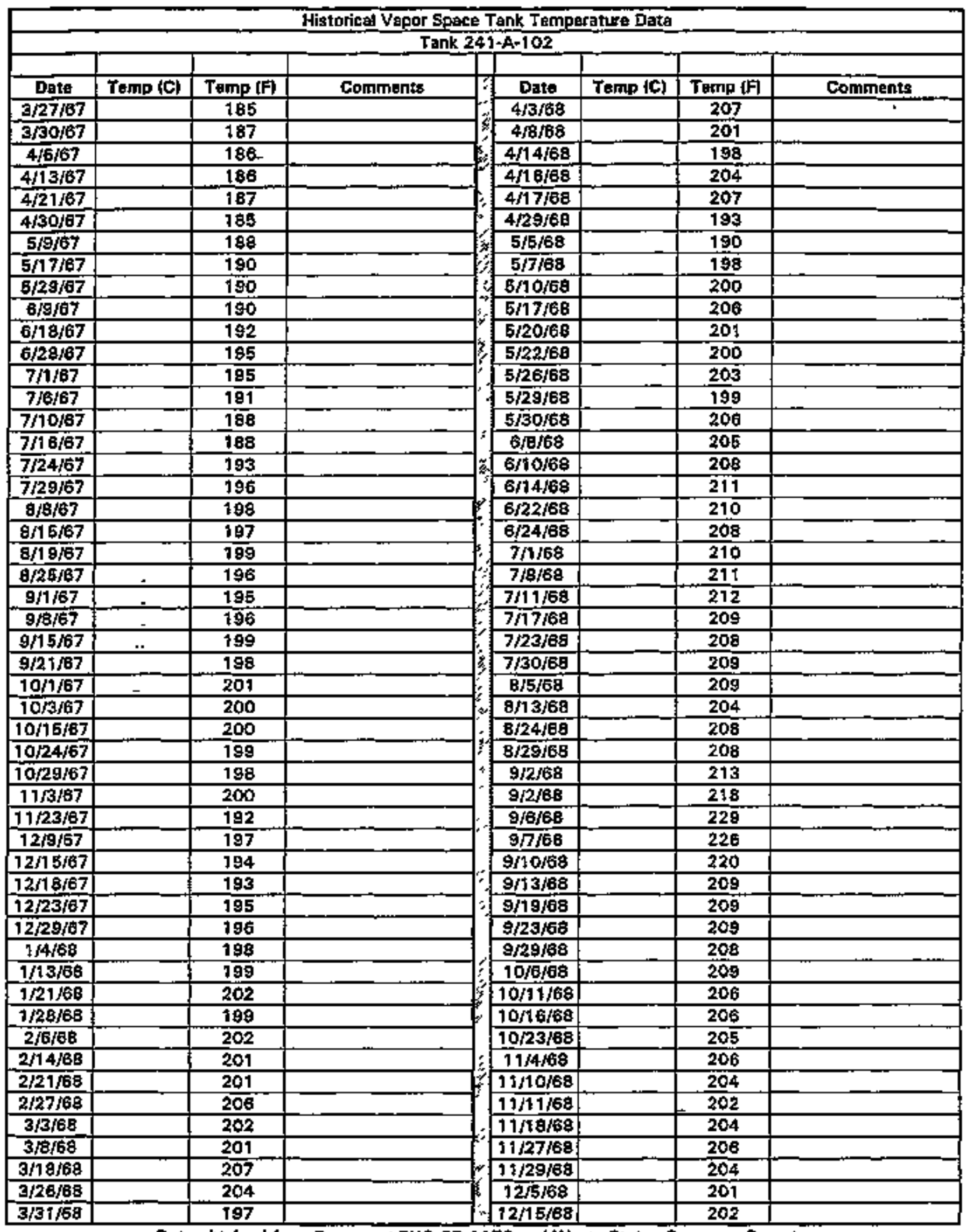

Data obtained from Document RHO-CD-1172 and Waste Status Summary Reports. 


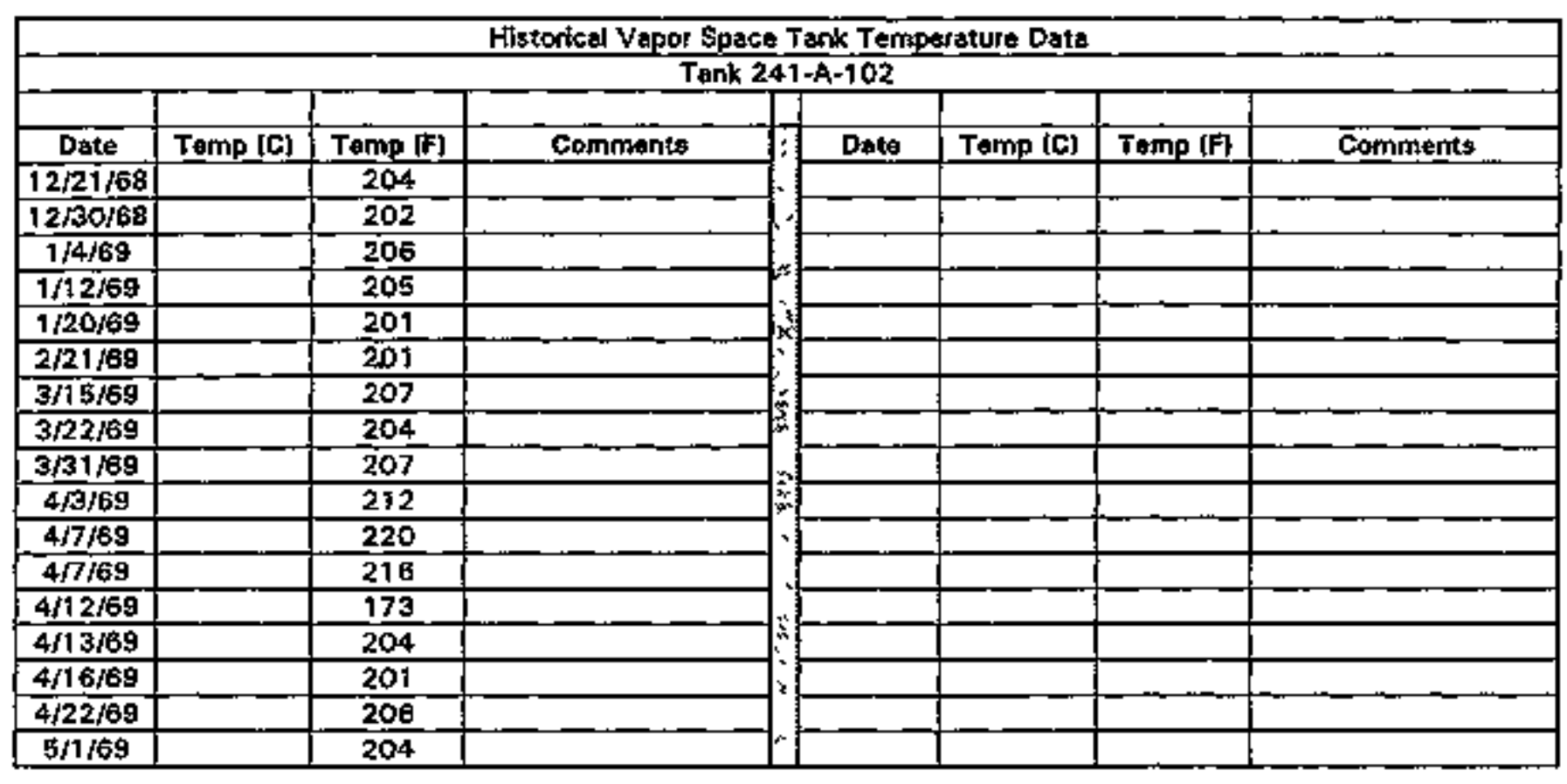

Data obteined from Documemt RHO-CD-1 172 and Waste Status Sumrnary Reports. 


\begin{tabular}{|c|c|c|c|c|c|c|c|}
\hline \multirow{2}{*}{\multicolumn{8}{|c|}{ Hestoflenil Averags Watte Temponature Date }} \\
\hline & & & & $41-A-102$ & & & \\
\hline Dute & $T$ then (C) & Temp (F) & Coxmmente & Date & Temp (C) & Tenge (F) & Comments \\
\hline $3 / 31 / 58$ & & 210 & & $10 / 23 / 87$ & & 316 & \\
\hline $4 / 30 / 58$ & & 208 & & $10 / 30 / 67$ & & 314 & \\
\hline $5 / 31 / 58$ & & 210 & & $11 / 5 / 67$ & & 360 & \\
\hline $8 / 30 / 58$ & & 223 & & $11 / 12 / 67$ & & 326 & \\
\hline $10 / 31 / 58$ & & 225 & & $11 / 22 / 67$ & & 328 & \\
\hline $11 / 30 / 5 \mathrm{~B}$ & & 221 & & $12 / 2467$ & & 329 & \\
\hline $12 / 31 / 68$ & & 219 & & $1 / 1 / 68$ & $\because$ & 332 & \\
\hline $1 / 31 / 69$ & 104 & 219 & $\begin{array}{c}\text { raw dota * esstmed } \\
\text { degrege C } \\
\end{array}$ & $1 / 14 / 68$ & & 396 & \\
\hline $2 / 20 / 59$ & & 215 & & $1 / 1 \mathrm{~b}^{2} \mathrm{ca}$ & & $\mathbf{3 4 2}$ & \\
\hline $3 / 31 / 59$ & & 221 & & $1 / 24 / 69$ & & 332 & \\
\hline $4 \sqrt{30 / 50}$ & & 219 & & $1 / 31 / 68$ & & 334 & \\
\hline$\overline{5} / 31 / 59$ & & 202 & & $2 / 1 / 8 B$ & & 124 & \\
\hline $6 / 30 / 59$ & & 205 & & $2 / 1 / 58$ & & 143 & \\
\hline $10 / 3+/ 59$ & & 205 & & $2 / 1 / 68$ & & 153 & \\
\hline $91 / 30 / 59$ & & 205 & & $3 / 1 / 8 g$ & & 158 & \\
\hline $2 / 29 / 80$ & & & $\begin{array}{c}107 \text { - suspect data, } \\
\text { low }\end{array}$ & $3 / 1 / 88$ & & 159 & \\
\hline $5 / 31 / 60$ & & 230 & & $3 / 1 / 68$ & & 150 & \\
\hline 6/30/60 & 118 & 244 & $\begin{array}{l}\text { raw data + aseumed } \\
\text { degres } C\end{array}$ & $3 / 1 / 68$ & & 181 & \\
\hline s/30/61 & & 260 & & $4 / 1 / 68$ & & 159 & \\
\hline $12 / 31 / 81$ & & 290 & & $4 / 1 / 68$ & & 183 & \\
\hline B/30/62 & & 344 & & $4 / 1 / 68$ & & 781 & \\
\hline $7 / 3 / 83$ & & 282 & & $4 / 1 / 68$ & & 165 & \\
\hline $7 / 19 / 66$ & & 282 & & $4 / 1 / 68$ & & $7 \overline{83}$ & \\
\hline $7 / 31 / 66$ & & 280 & & $4 / 1 / 68$ & & 139 & \\
\hline $8 / 10 / 66$ & & 286 & & $5 / 1 / 68$ & & 939 & \\
\hline $10 / 3 / 60$ & & 285 & & $5 / 1 / 68$ & & 137 & \\
\hline $11 / 23 / 8 B$ & & 285 & & $5 / 1 / 68$ & & 140 & \\
\hline $12 / 2 / 66$ & & 272 & & $6 / 1 / 68$ & & $\overline{145}$ & \\
\hline $12 / 15 / 80$ & & $\overline{272}$ & & $6 / 1 / 68$ & & $\overline{153}$ & \\
\hline $12 / 23 / 88$ & & 270 & & 6/1/B8 & & 169 & \\
\hline 1/e/67 & & 289 & & $7 / 1 / 68$ & & 170 & \\
\hline $1 / 20 / 67$ & & 267 & & $7 / 1 / 68$ & & 155 & \\
\hline $1 / 29 / 67$ & & 287 & & $7 / 1 / 68$ & & 152 & \\
\hline $2 / 12 / 67$ & & 261 & & g/1/80 & & 152 & \\
\hline $3 / 7 / 67$ & & 261 & & $\overline{8} / 1 / 6 \mathrm{~B}$ & & 161 & \\
\hline $3 / 22 / 67$ & & 250 & & $8 / 1 / 89$ & & 157 & \\
\hline $4 / 9 / 67$ & & 280 & & $8 / 1 / 58$ & & $\overline{153}$ & \\
\hline $4 / 18 / 67$ & & 264 & & $8 / 1 / 68$ & & 158 & \\
\hline $5 / 22 / 67$ & & 264 & & $9 / 1 / 6 a$ & & $\overline{171}$ & \\
\hline $6 / 2 / 67$ & & 272 & & $9 / 1 / \theta 8$ & & 173 & \\
\hline $6 / 20 / 67$ & & 271 & & $10 / 4 / 68$ & & 172 & \\
\hline 6/28/67 & & 280 & & $50 / 1 / 68$ & & $\sqrt{73}$ & \\
\hline $7 / 20 / 67$ & & 280 & & $10 / 1 / 88$ & & 175 & \\
\hline $8 / 5 / 67$ & & 281 & & $10 / 7 / 68$ & & $\overline{187}$ & \\
\hline $8 / 13 / 67$ & & 296 & & $\overline{I 1 / 1 / 68}$ & & 170 & \\
\hline $8 / 30 / 67$ & & 299 & & $11 / 1 / 68$ & & 171 & \\
\hline $9 / 6 / 87$ & & 294 & & $11 / 1 / 68$ & & $\overline{182}$ & \\
\hline $9 / 12 / 67$ & & 290 & & $-12 / 1 / 68$ & & 168 & \\
\hline $9 / 22 / 67$ & & 285 & & $12 / 1 / 68$ & & 170 & \\
\hline $10 / 1 / 67$ & & 308 & & $1 / 1 / 89$ & & 182 & \\
\hline $10 / 10 / 67$ & & 306 & & $1 / 1 / 89$ & & 164 & \\
\hline
\end{tabular}

Data ebtelned feam Dooument RHO-CO-1172 and Weste Status Summmary Raports. 


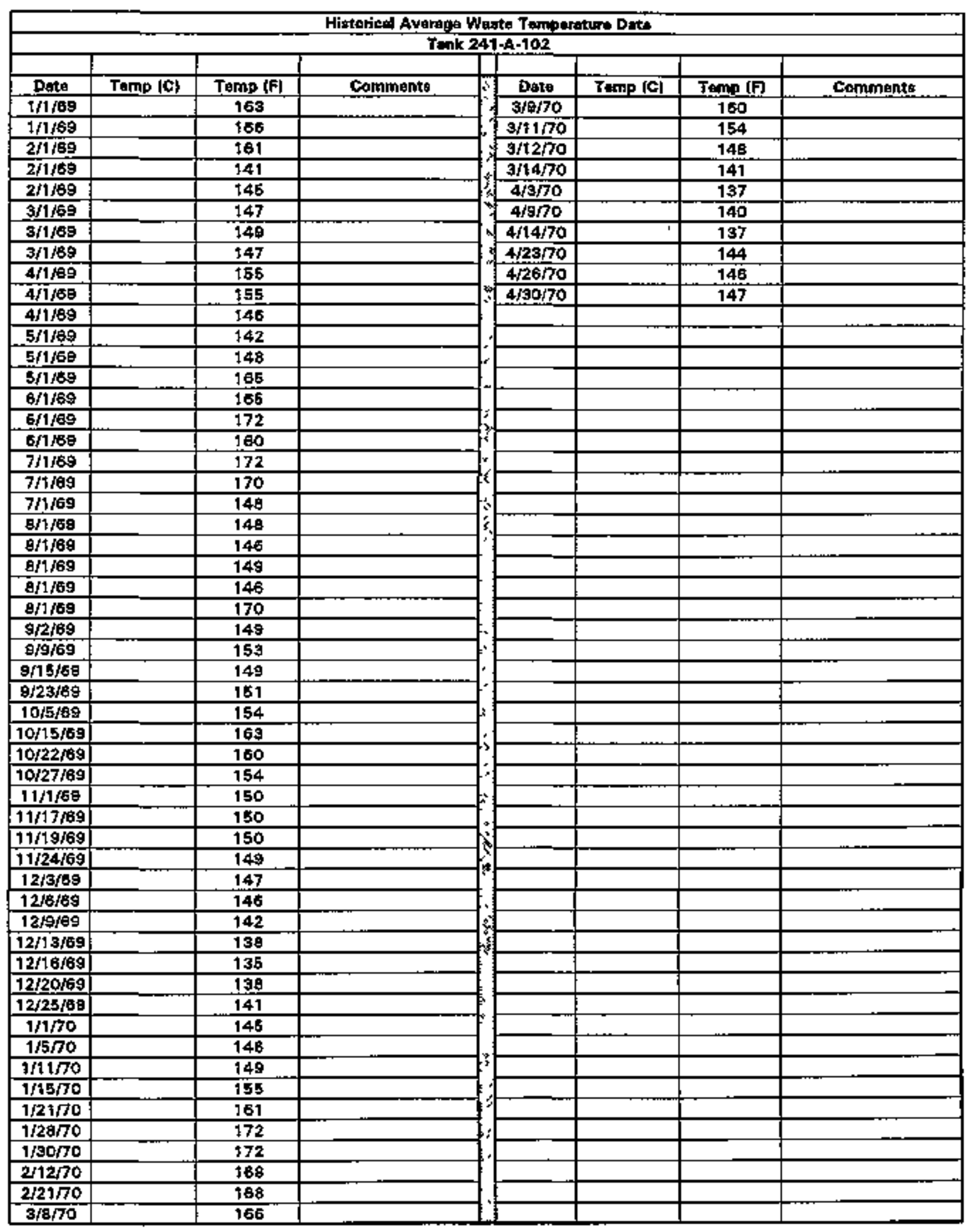

Beta obtained from Dopument Fto-CD-1172 and Waste Status Summmery Reports. 


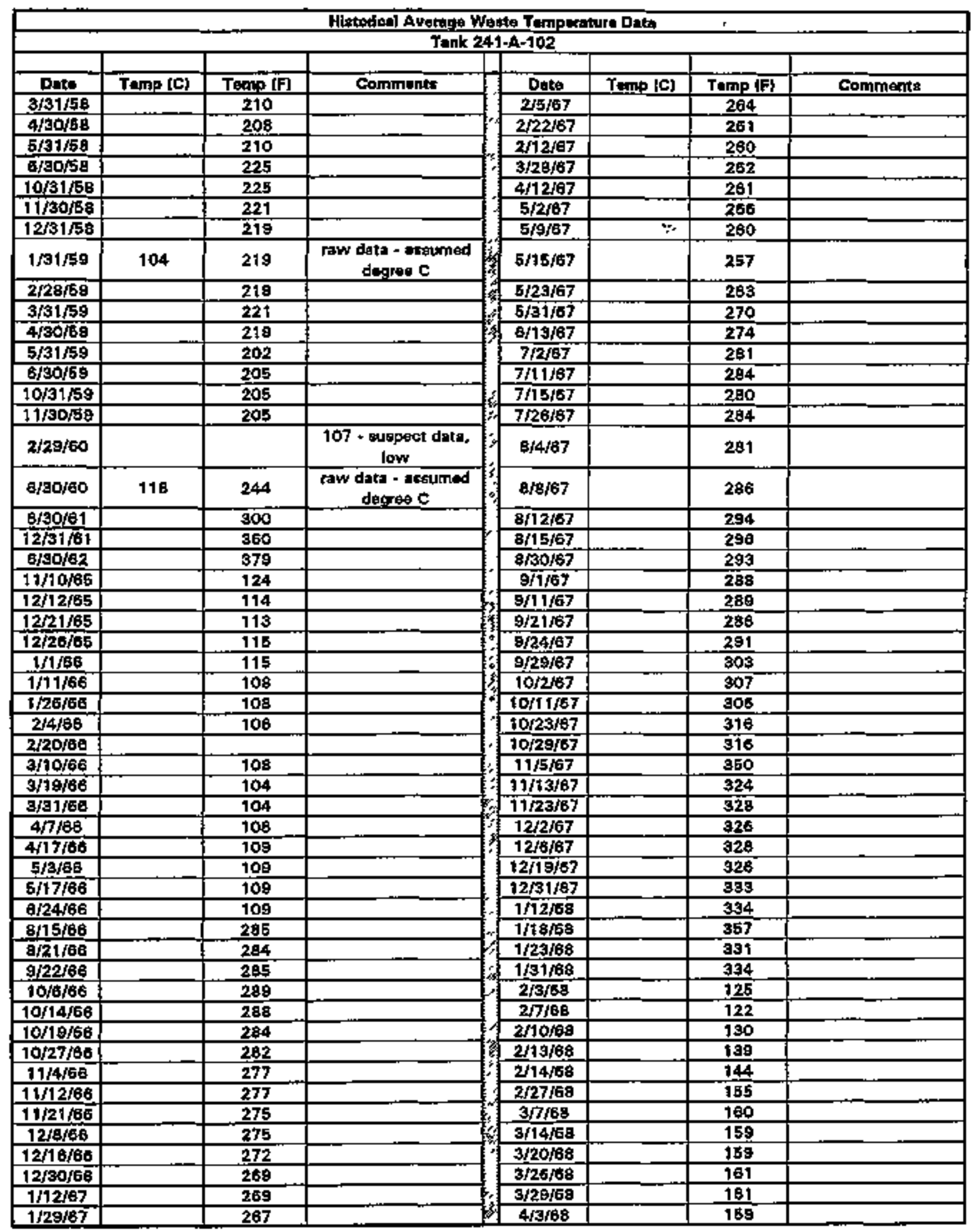

Data obtained from Documant RHO-CD-1172 and Waste Statư Summar Reparts. 


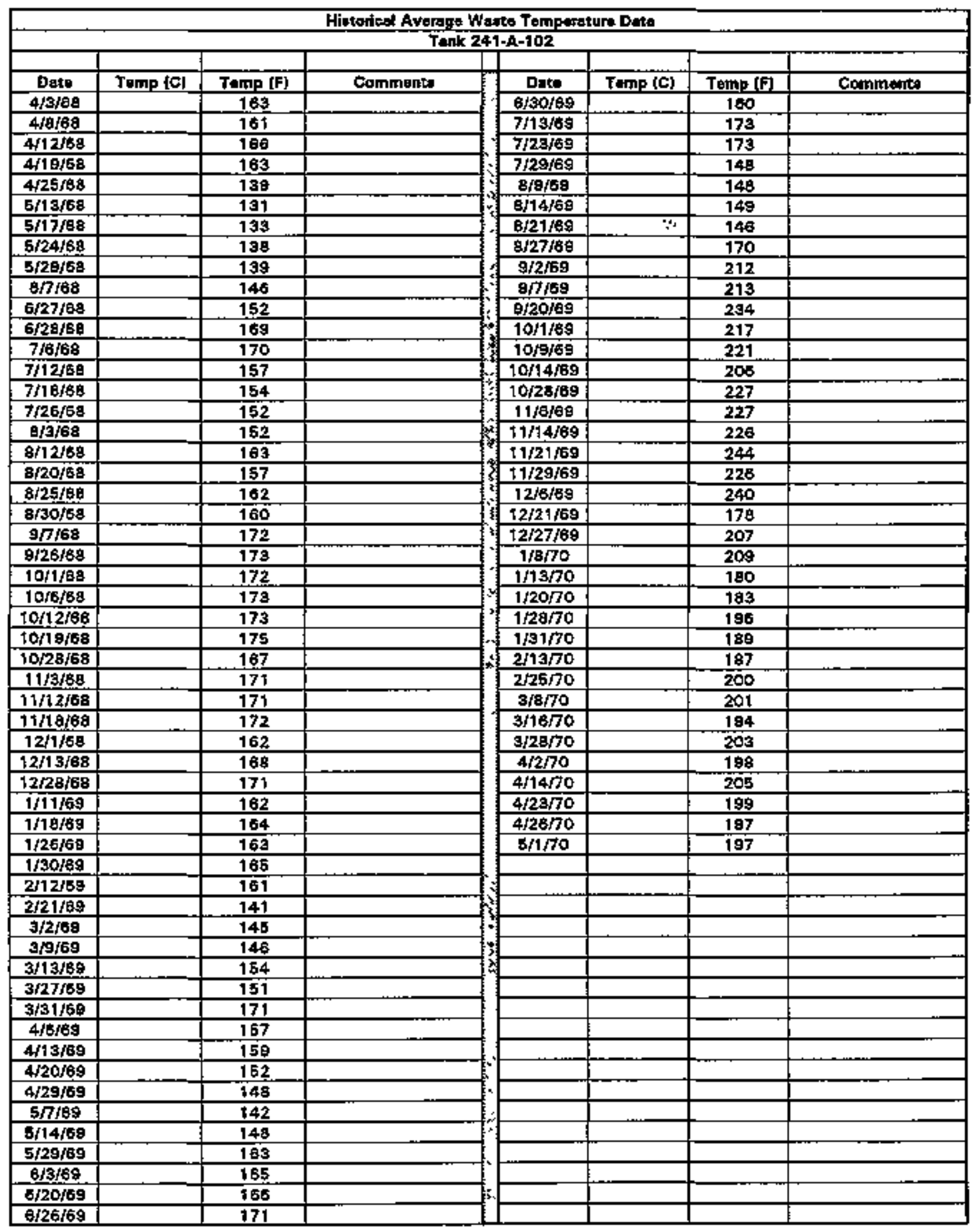

Data obtained trom Dasument RHO-CD-1172 and Waste \$tatus Summary Reports. 


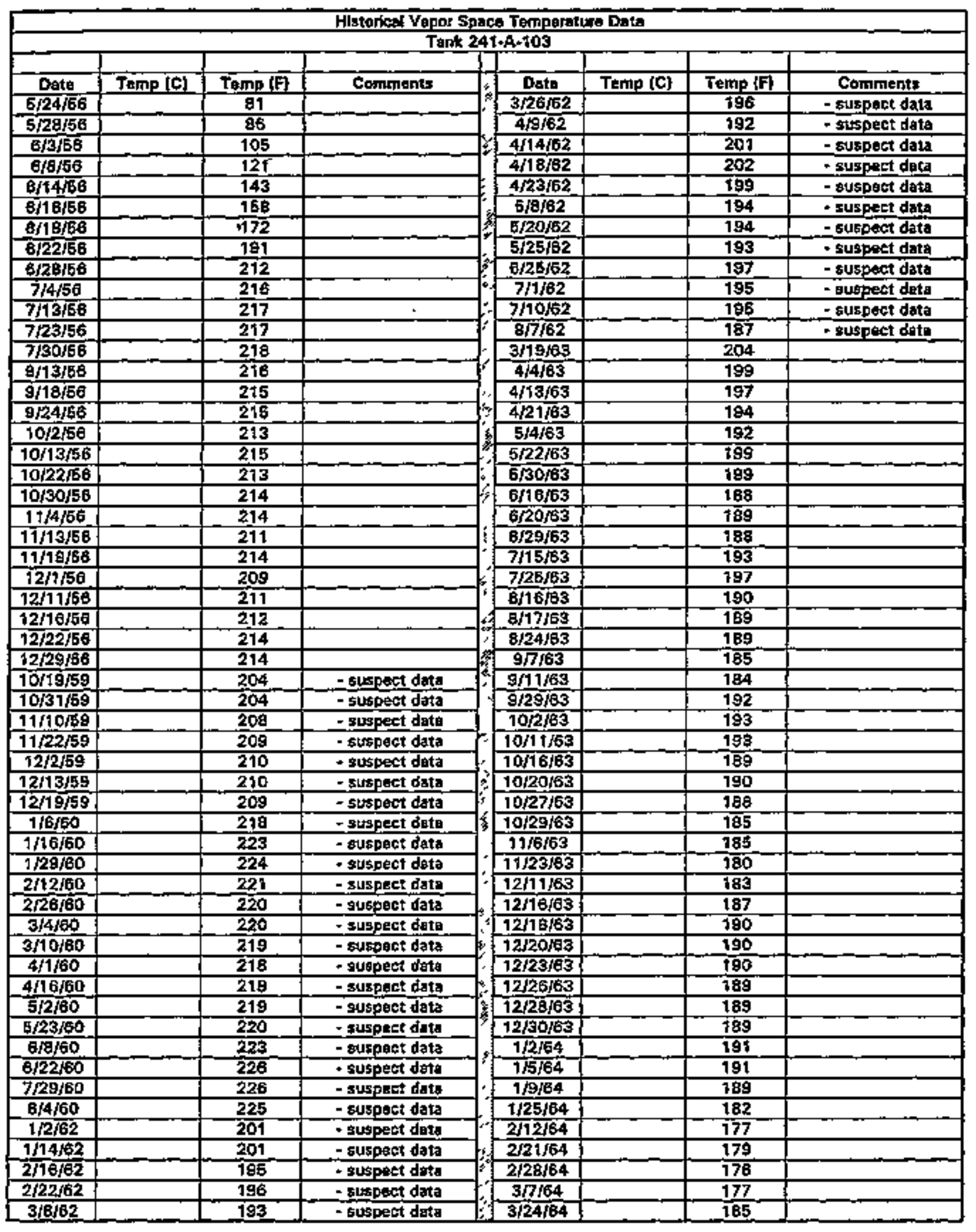

Data obtained from Documemt RHO-CD-1172 and Waste Status Surnmary Reports. 


\begin{tabular}{|c|c|c|c|c|c|c|c|}
\hline \multicolumn{8}{|c|}{ Hestorias Vapor Space Temperatura Data } \\
\hline & & & Tank 2 & $241+A \cdot 103$ & & & \\
\hline & & & & & & & \\
\hline Dote & Temp (C) & Temp (F) & Cornmerta & Date & Temp $\langle\mathrm{C}|$ & Tamp $\{F\}$ & Cotrments \\
\hline $3 / 28 / 64$ & & 783 & & $7 / 26 / 65$ & & 102 & \\
\hline $4 / 9 / 64$ & & 188 & & $7 / 30 / 65$ & & 701 & \\
\hline $4 / 13 / 64$ & & 187 & & $8 / 8 / 65$ & & 102 & \\
\hline $4 / 20 / 64$ & & 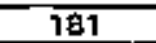 & & $6 / 21 / 65$ & & 107 & \\
\hline $4 / 25 / 64$ & & 181. & & eta/5s & & 109 & \\
\hline $5 / 6 / 64$ & & 165 & & $9 / 21 / 65$ & & 107 & \\
\hline $5 / 11 / 64$ & & $\sqrt{162}$ & & $9 / 28 / 65$ & & 108 & \\
\hline $5 / 17 / 64$ & & 165 & & $10 / 6 / 65$ & & 105 & \\
\hline $5 / 25 / 64$ & & 138 & & $10 / 23 / 65$ & & 905 & \\
\hline $8 / 9 / 54$ & & $T E B$ & & $10 / 30 / 65$ & & 107 & \\
\hline $6 / 14 / 64$ & & 139 & & $11 / 6 / 65$ & & 109 & \\
\hline $6 / 23 / 64$ & & $\overline{899}$ & & $1 1 \longdiv { 1 7 6 5 }$ & & 106 & \\
\hline $6 / 28 / 04$ & & 135 & & $11 / 30 / 35$ & & $\operatorname{tog}$ & \\
\hline $7 / 21 / 64$ & & 149 & & $12 / 9 / 65$ & & 100 & \\
\hline $7 / 30 / 64$ & & $\overline{143}$ & & $1 2 \longdiv { 1 5 / 8 5 }$ & & $f \overline{0 g}$ & \\
\hline $8 / 1 / 64$ & & 143 & & $12 / 24 / 65$ & & 109 & \\
\hline $9 / 8 / 64$ & & $\overline{131}$ & & $1 \sqrt{3} / 66$ & & 709 & \\
\hline $8 / 9 / 64$ & & 119 & & $1 / 9 / 66$ & & 108 & \\
\hline $8 / 16 / 64$ & & 114 & & $1 / 17 / 66$ & & 110 & \\
\hline 8/19/64 & & 119 & & $1 / 23 / 88$ & & 170 & \\
\hline$B / 23 / 64$ & & 124 & & $2 / 10 / 68$ & & 108 & \\
\hline 9/30/64 & & $\overline{344}$ & & $2 / 18 / 6 b^{2}$ & & 109 & \\
\hline $9 / 23 / 64$ & & 112 & & $2 / 20 / 50$ & & 110 & \\
\hline $10 / 3 / 64$ & & $\sqrt{3} 30$ & & $3 / 5 / 6 B$ & & 111 & \\
\hline $10 / 8 / 64$ & & 310 & & J/10/6 & & 111 & \\
\hline $10 / 19 / 64$ & & 104 & & $3 / 19 / 66$ & & 114 & \\
\hline $10 / 26 / 64$ & & 721 & & 3/26/B6 & & 175 & \\
\hline $10 / 29 / 64$ & & 132 & & $4 / 4 / 66$ & & 108 & \\
\hline $11 / 2 / 64$ & & 735 & & $4 / 14 / 68$ & & 111 & \\
\hline 11/26 64 & & $\overline{132}$ & & $4 / 22 / 66$ & & 91 & \\
\hline $11 / 191 / 64$ & & 131 & & $5 / 5 / 66$ & & 91 & \\
\hline $11 / 14 / 64$ & & 129 & & $5 / 19 / 66$ & & 100 & \\
\hline $11 / 16 / 64$ & & 123 & & $5 / 24 / 60$ & & 100 & \\
\hline $11 / 20 / 64$ & & 118 & & $6 / 3 / 66$ & & 105 & \\
\hline $11 / 22 \sqrt{64}$ & & 110 & & 6/12/6त & & $\overline{1 O 5}$ & \\
\hline $11 / 22 / 64$ & & 107 & & $6 \longdiv { 2 2 / 6 6 }$ & & 108 & \\
\hline $11 / 29 / 64$ & & 109 & & $7 \pi / 66$ & & 109 & \\
\hline $12 / 7 / 64$ & & 110 & & $7 / 15 / 66$ & & 110 & \\
\hline $12 / 11 / 64$ & & 110 & & $7 / 27 / 66$ & & 109 & \\
\hline $12 / 26 / 44$ & & 91 & & $8 / 3 / 66$ & & 106 & \\
\hline $1 / 31 / 65$ & & 110 & & $8 / 11 / 66$ & & 109 & \\
\hline $2 / 13 / 65$ & & 115 & & $8 / 17 / 66$ & & 109 & \\
\hline $2 / 22 / 65$ & & 105 & & $8 \overline{2} 6 / 6 \overline{6}$ & & 109 & \\
\hline $3 / 11 / 65$ & & 96 & & $8 / 31 / 65$ & & 111 & \\
\hline $3 / 47 / 65$ & & 99 & & $9 / 14 / 56$ & & 113 & \\
\hline $4 / 17 / 65$ & & 100 & & $9 / 21 / 66$ & & 109 & \\
\hline $4 / 24 / 65$ & & 99 & & $10 / 8 / 66$ & & 109 & \\
\hline $4 \sqrt{30} 0 / 65$ & & 98 & & $10 / 18 / 68$ & & 107 & \\
\hline $5 / 6 / 65$ & & 96 & & 11/1/ba & & 109 & \\
\hline $5 / 12 / 65$ & & 94 & & $11 / 1 \mathrm{~s} / 6 \mathrm{~B}$ & & 103 & \\
\hline $5 / 20 / 65$ & & 97 & & $11 / 30166$ & & $\overline{102}$ & \\
\hline $6 / 11 / 65$ & & 98 & & $12 / 5 / 66$ & & 108 & \\
\hline $6 / 21 / 65$ & & 103 & & $12 / 20 / 66$ & & 100 & \\
\hline $7 / 1 / 65$ & & 102 & & $1 / 5 / 67$ & & 97 & \\
\hline $7 / 20 / 65$ & & 103 & & $1 / 12 / 67$ & & 95 & \\
\hline
\end{tabular}

Data obtained ftam Dotument foto-CD-1172 and Waste Stgtus Summary Reports. 


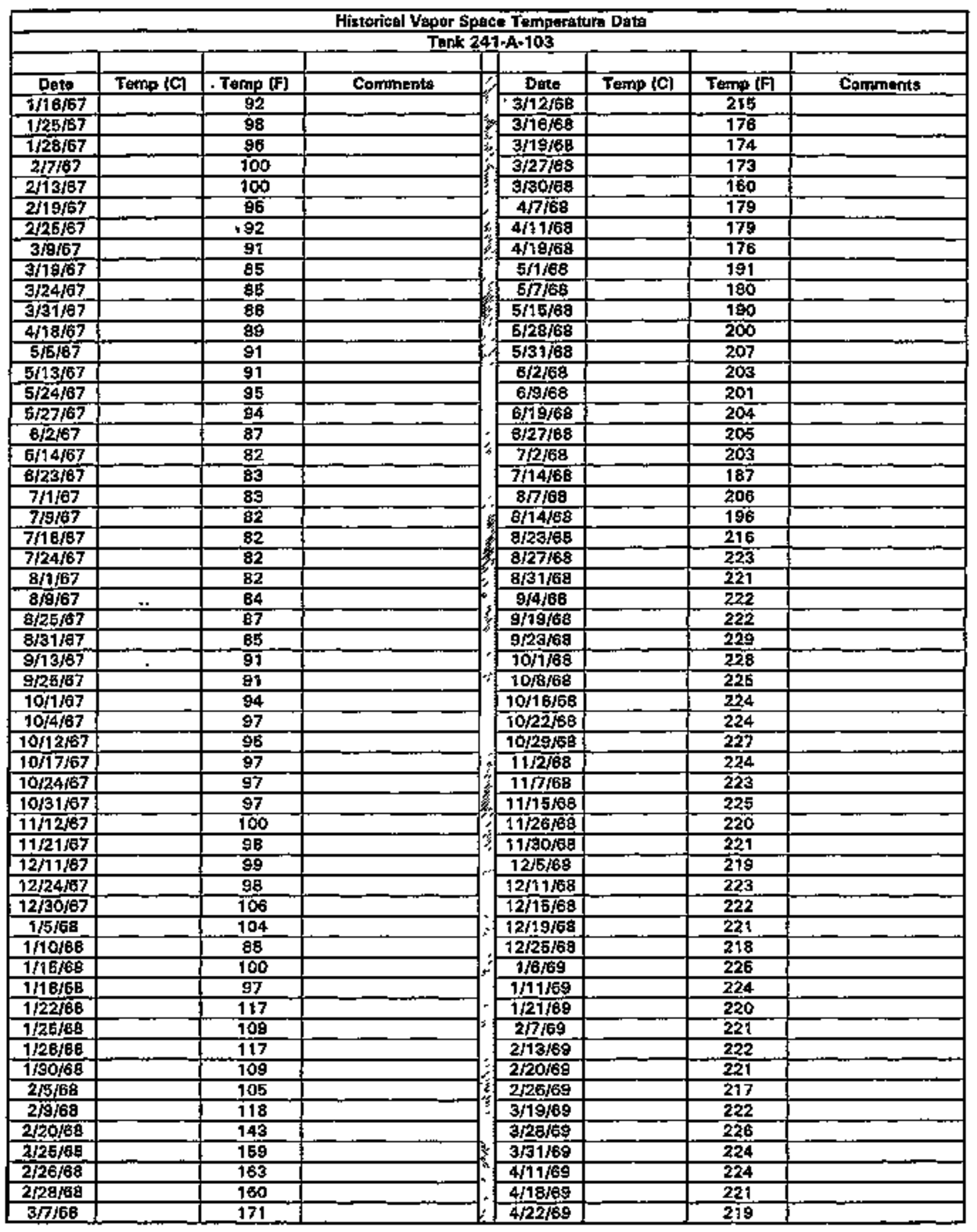

Data obtained from Doeument RHO-CD-\$172 and Wasto \$tetus Summary Reports. 


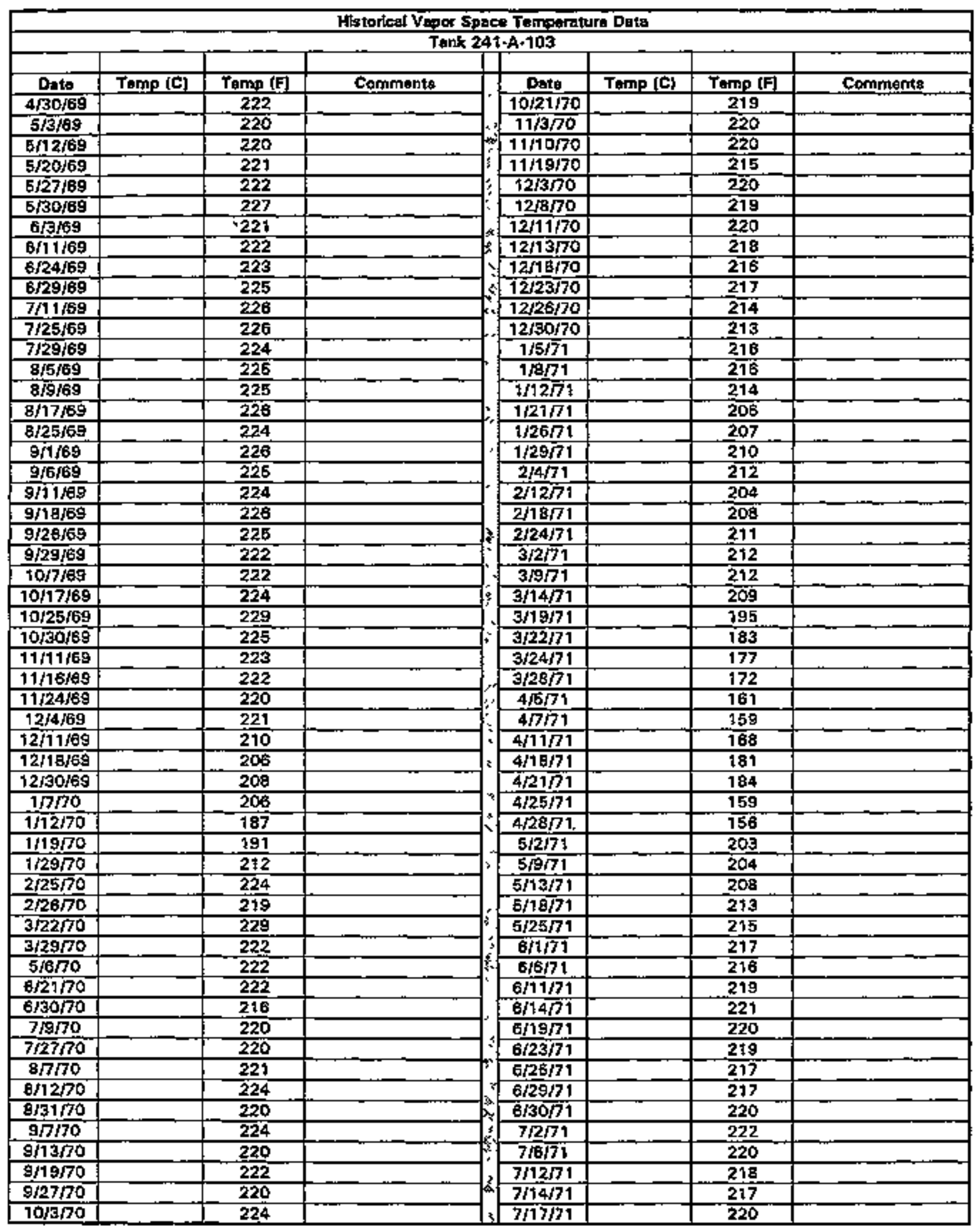

Data obtainad from Document gato-CD-1172 and Waste Stetu\& Summary Reports. 
WHC-SO-WM-ER-308, Rov, 0

\begin{tabular}{|c|c|c|c|c|c|c|c|c|}
\hline \multicolumn{9}{|c|}{ Historical Vepor Space Temperatura Data } \\
\hline \multicolumn{9}{|c|}{ Tank 241+A-103 } \\
\hline & & & & & & & & \\
\hline Date & Temp (c) & Tamp [F] & Comments & 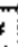 & Dats & Temp $\{\mathrm{C}\rceil$ & Tomp (F) & Comptents \\
\hline $7 / 19 / 71$ & & 224 & & : & & & & \\
\hline $7 / 24 / 71$ & & 226 & & $\xi$ & & & & \\
\hline $7 / 27 \sqrt{71}$ & & 224 & & $\xi$ & & & & \\
\hline $8 / 2 \sqrt{71}$ & & 222 & & 3 & & & & \\
\hline $8 / 6 / 71$ & & 224 & & > & & & & \\
\hline$B / 9 / 71$ & & 220 & & 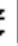 & & & & \\
\hline $8 / 13 / 71$ & & 224 & & . & & & & \\
\hline$B / 16 / 71$ & & 222 & & 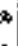 & & & & \\
\hline 6/21 71 & & 223 & & 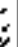 & & & & \\
\hline $8 \sqrt{27 / 7}$ & & 224 & & , & & & & \\
\hline $9 / 1 / 79$ & & 223 & & & & & & \\
\hline $9 / 4 / 71$ & & 222 & & & & & & \\
\hline o/11/71 & & 223 & & & & & & \\
\hline
\end{tabular}




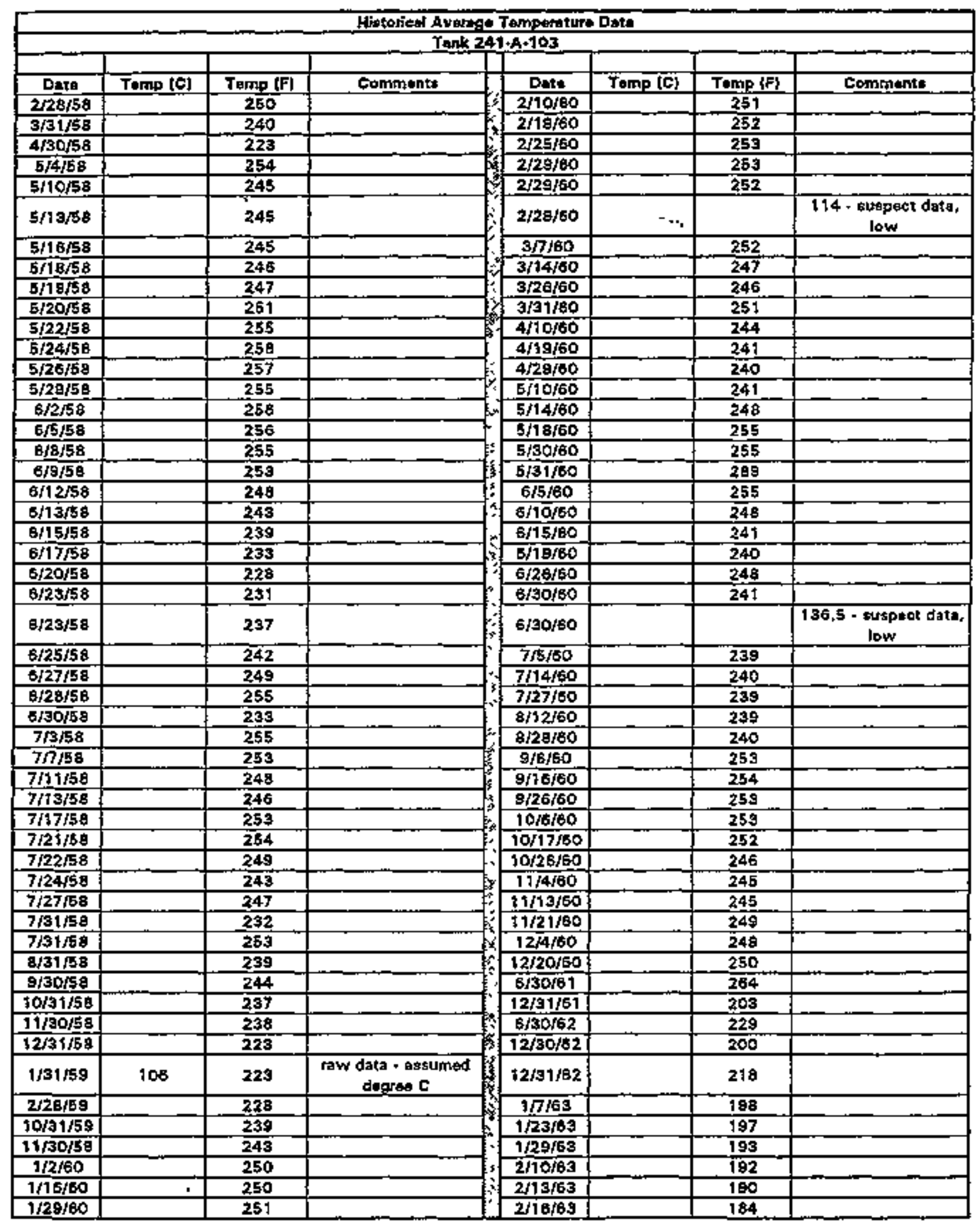

Data obtained from Ducument RH+O-CD+1172 and Waste Status Summary fieports. 


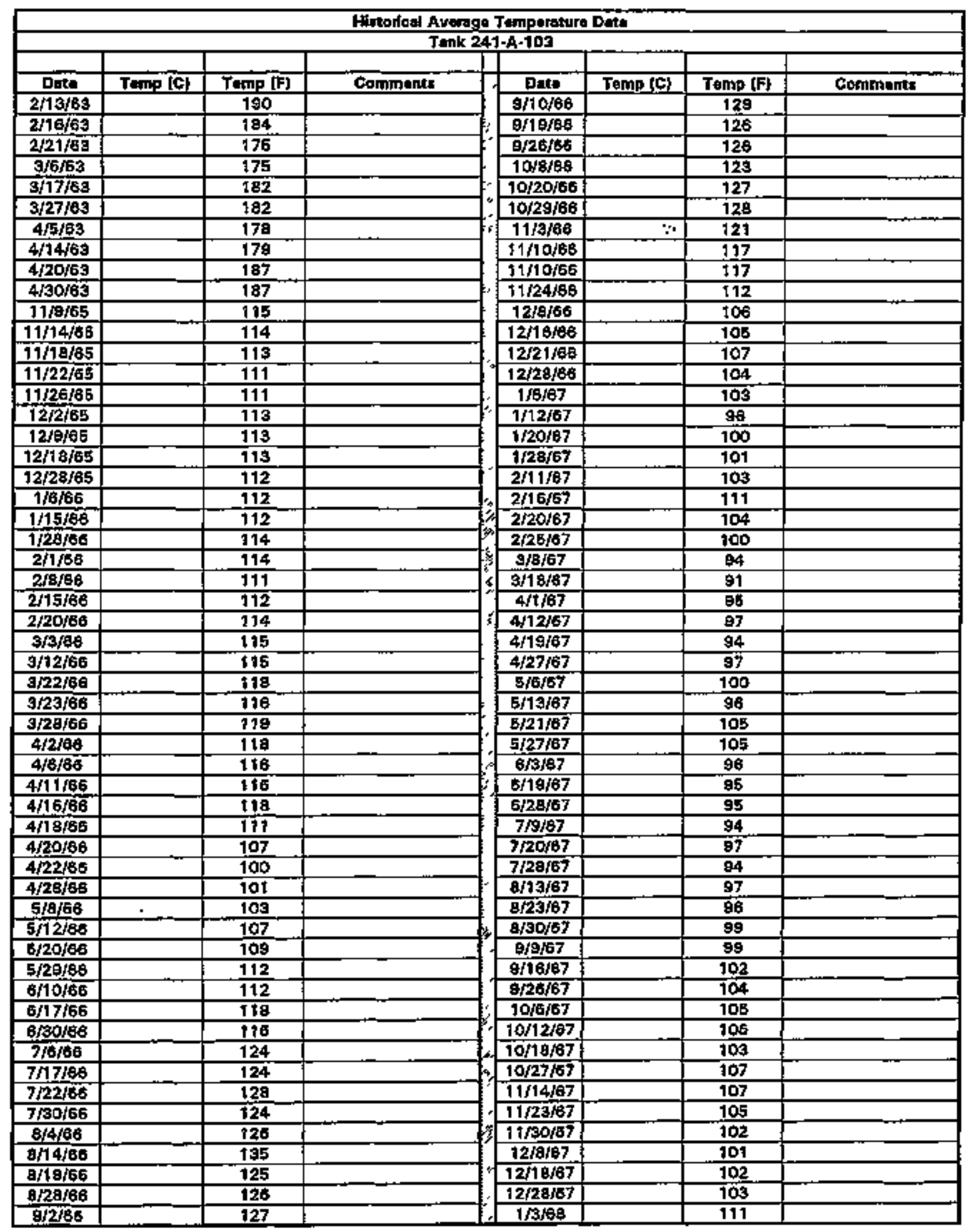

Data abtained fram Document RHO-CD+1172 and Wasto Status Sumrary fleports. 
WHC-SO-WM-ER-30B, REV, O

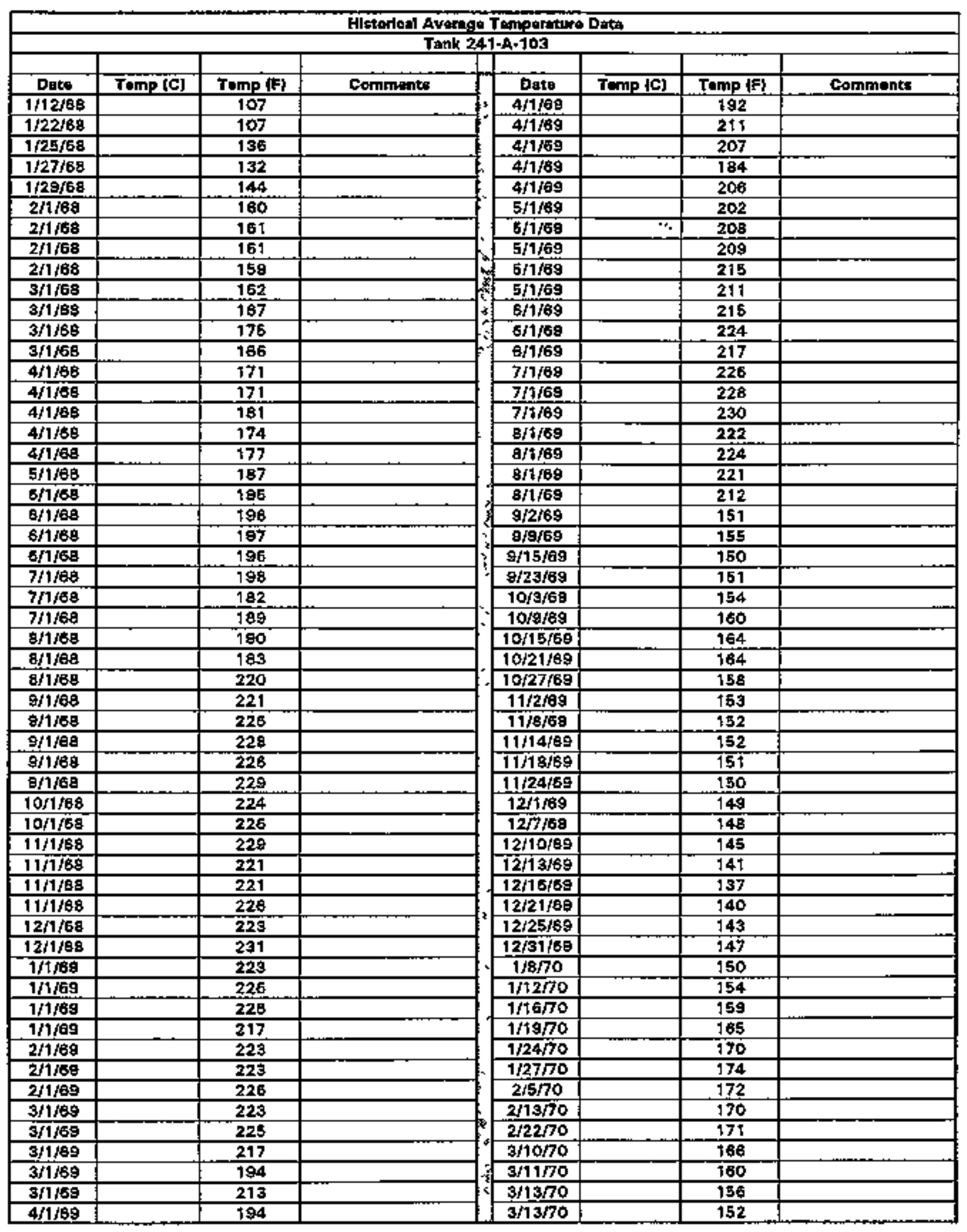

Dats obtained from Documert RHO-CO-1 172 and Waste Statts Summary Reperts. 
WHC-SD-WAI-ER+30O, REV, O

\begin{tabular}{|c|c|c|c|c|c|c|c|c|}
\hline \multicolumn{9}{|c|}{ Hratodoal Average Temperrature Dete } \\
\hline \multirow{2}{*}{\multicolumn{9}{|c|}{ Tenk $24 t+A+103$}} \\
\hline & & & & & & & & \\
\hline Dote & Tomp (C) & Tertp $\{F\}$ & Comment: & 格 & Dote & Tamp [t] & Tomp (f) & Comment: \\
\hline $3 / 15 / 70$ & & 146 & & t: & & & & \\
\hline $3 / 16 / 70$ & & 142 & & & & & & \\
\hline $4 / 4 / 70$ & & 139 & & & & & & \\
\hline $4 / 10 / 70$ & & 145 & & & & & & \\
\hline $4 / 13 / 70$ & & 141 & & & & & & \\
\hline $4 / 16 / 70$ & & 142 & & 1 & & & & \\
\hline $4 / 20 / 70$ & & 145 & & 7 & & $r_{*}$ & & \\
\hline $4 \sqrt{26 / 70}$ & & 149 & & & & & & \\
\hline
\end{tabular}

Dats obtained fom Dacument AHO-co-1172 and Wasto Status Summary Feports. 


\begin{tabular}{|c|c|c|c|c|c|c|c|}
\hline \multicolumn{8}{|c|}{ 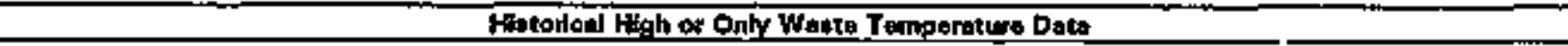 } \\
\hline & & & Tank? & A+A-103 & & & \\
\hline Date & $T=m[C]$ & Temp \{fF & Comrmants: & Dato & Temp (C) & $\operatorname{Tamp} \overline{[F]}$ & Comments \\
\hline $2 / 28 / 5 a$ & & 270 & & 2/29/60 & & & $\begin{array}{c}122 \text { - suspect data, } \\
\text { low }\end{array}$ \\
\hline $3 / 31 / 58$ & & 255 & & $2 / 29 / 60$ & & 277 & \\
\hline $4 / 30 / 58$ & & 235 & & $3 / B / 60$ & & 271 & \\
\hline $5 / 31 / 58$ & & 286 & & $3 / 10 / 60$ & & 273 & \\
\hline $7 / 31 / 58$ & & $23 \overline{2}$ & & 3/17/60 & & 277 & \\
\hline $8 / 1 / 58$ & & 254 & & $3 / 21 / 80$ & 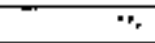 & $27 \bar{B}$ & \\
\hline $8 / 9 / 5 B$ & & 250 & & $3 / 26 / 50$ & & 283 & \\
\hline$9 \longdiv { 1 4 \sqrt { 5 8 } }$ & & 253 & & $3 / 2 a / 60$ & & $285^{-}$ & \\
\hline $9 / 10 / 58$ & & 254 & & $3 / 3 t / 80$ & & 280 & \\
\hline $8 / 21 / 58$ & & 250 & & $4 / 3 / 60$ & & 278 & \\
\hline $8 / 26 / \sqrt{58}$ & & 251 & & $4 / 5 / 60$ & & 273 & \\
\hline $9 / 30 / 58$ & & 251 & & 4/10/BO & & 285 & \\
\hline $8 / 31 / 58$ & & 253 & & $4 / 13 / 60$ & & 287 & \\
\hline $9 / 5 / 58$ & & 247 & & $5 / 160$ & & 281 & \\
\hline $9 / 8 / 58$ & & 244 & & $5 / 18 / 60$ & & 292 & \\
\hline 8/12/5B & & 239 & & $5 / 25 / 60$ & & 279 & \\
\hline $9 / 16 / 58$ & & 242 & & $5 / 30 / 60$ & & 284 & \\
\hline $9 / 19 / 58$ & & 247 & & $5 / 31 / 50$ & & 289 & \\
\hline $9 / 25 / 58$ & & 249 & & $8 / 8 / 60$ & & 279 & \\
\hline $9 / 30 / 58$ & & 282 & & $6 / \overline{14 / 90}$ & & 904 & \\
\hline $10 / 9 / 58$ & & 238 & & $6 / 20 / 50$ & & 288 & \\
\hline $10 / 17 / 68$ & & 251 & & $6 / 26 / 60$ & & 281 & \\
\hline $10 / 19 / 58$ & & 255 & & $6 / 30 / 60$ & 145 & 293 & $\begin{array}{c}\text { raw data - assumed } \\
\text { dagras C }\end{array}$ \\
\hline $10 / 21 / 58$ & & 251 & & $7 / 5 / 60$ & & 280 & \\
\hline $10 / 25 / 58$ & & 244 & & $7 / 12 / 80$ & & 292 & \\
\hline $10 / 39 / 58$ & & 244 & & $7 / 17 / 180$ & & 286 & \\
\hline $11 / 13 / 58$ & & 244 & & $7 / 22 / 60$ & & $2 \mathrm{ae}$ & \\
\hline $11 / 24 / 59$ & & 249 & & $8 / 1 / 60$ & & 289 & \\
\hline $11 / 30 / 58$ & & 250 & & $6 / 8 / 60$ & & $28 \theta$ & \\
\hline $11 / 30 \sqrt{58}$ & & 251 & & $8 / 18 / 90$ & & 291 & \\
\hline $12 / 3 / 58$ & & 250 & & $8 / 22 / 60$ & & 288 & \\
\hline $12 / 10 / 58$ & & $25 \overline{3}$ & & $8 / 28 / 60$ & & 289 & \\
\hline $22 / 13 / 58$ & & 250 & & $8 / 2 / 60$ & & 299 & \\
\hline $12 / 19 / 58$ & & 230 & & $9 / 5 / 60$ & & 298 & \\
\hline$1 2 / 3 1 \longdiv { 5 8 }$ & & 223 & & $9 / 13 / 50$ & & 290 & \\
\hline $1 / 31 / 59$ & 108 & 223 & $\begin{array}{c}\text { raw data - eastumbed } \\
\text { degroe C }\end{array}$ & $9 / 18 / 60$ & & 292 & \\
\hline $2 / 28 / 59$ & & 228 & & $9 / 24 / 60$ & & 298 & \\
\hline $10 / 31 / 5 s$ & & 243 & & $10 / 4 / 80$ & & 293 & \\
\hline $11 / 30 / \mathrm{fg}$ & & 243 & & to/7/BO & & 293 & \\
\hline $1 / 3 / 60$ & & 278 & & $10 / 11 / 60$ & & 281 & \\
\hline $1 / 8 / 60$ & & 276 & & $10 / 17 / 60$ & & 299 & \\
\hline $1 / 19 / 60$ & & 275 & & $10 / 22 / 60$ & & 281 & \\
\hline $1 / 15 / 60$ & & 276 & & $10 / 25 / 60$ & & 287 & \\
\hline $1 / 18 / 60$ & & 276 & & $10 / 31 / 60$ & & 290 & \\
\hline $1 / 22 / 60$ & & 274 & & $11 / 11 / 60$ & & 300 & \\
\hline $1 / 24 / 60$ & & 273 & & $11 / 19 / 60$ & & 304 & \\
\hline $1 / 28 / 60$ & & 275 & & $11 / 28 / 60$ & & 303 & \\
\hline $2 / 1 / 1 / 0$ & & 275 & & $12 / 2 / 50$ & & $28 \mathrm{~g}$ & \\
\hline $2 / 6 / 60$ & & 272 & & $125 / 60$ & & 296 & \\
\hline $2 / 12 / 50$ & & 272 & & $12 / 11 / 80$ & & 298 & \\
\hline $2 / 19 / 80$ & & 273 & & $12 / 14 / \sqrt{50}$ & & 298 & \\
\hline $2 / 28 / 60$ & & 279 & & $4 \longdiv { 1 2 / 1 8 / 8 0 }$ & & 297 & \\
\hline
\end{tabular}

Data obtained from Document FHO-CD-1172 and Waste Status Summary Peporte. 


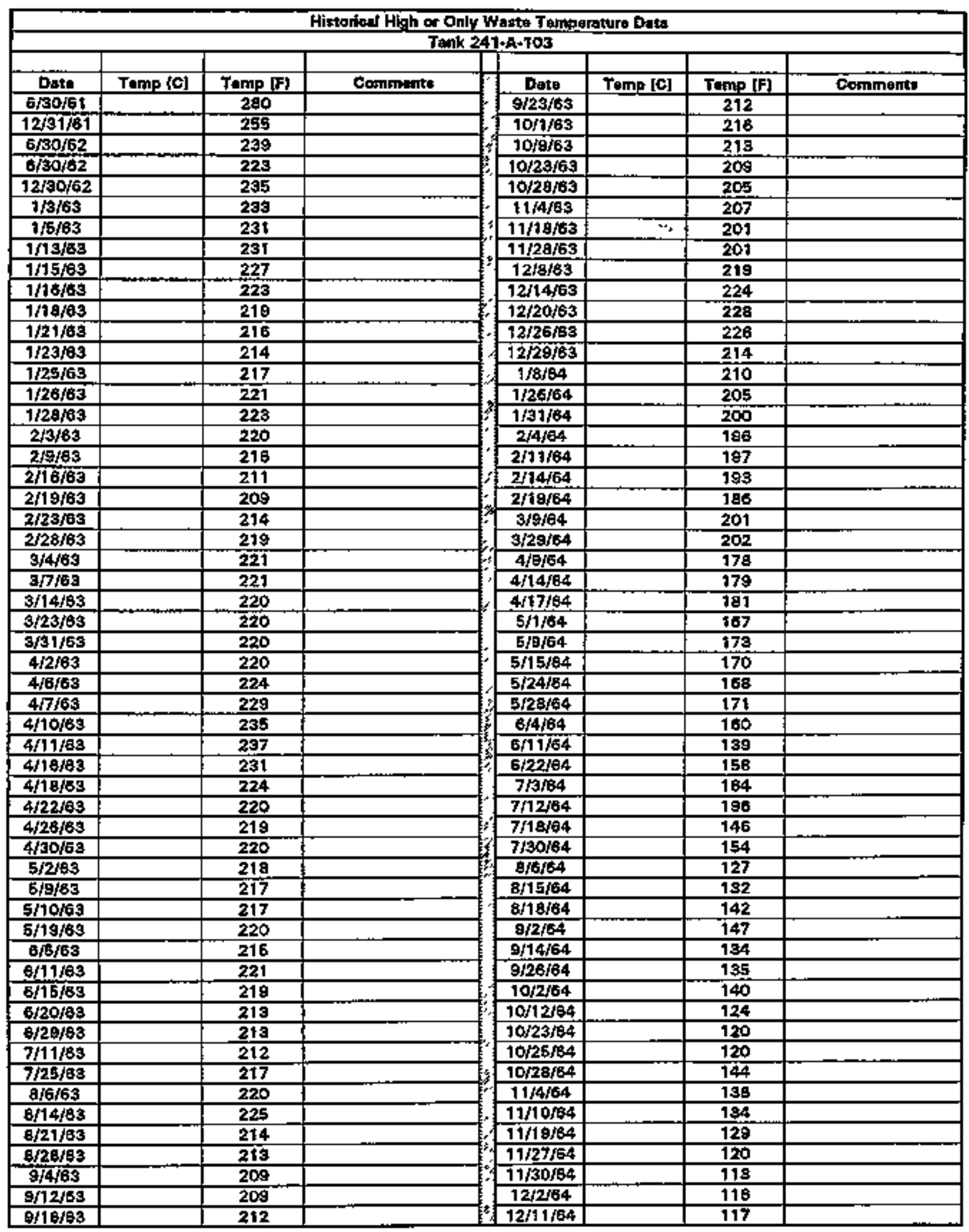

Date obtitied from Datumẹnt RHO-CD-1172 and Waste Status Summery Reporte. 


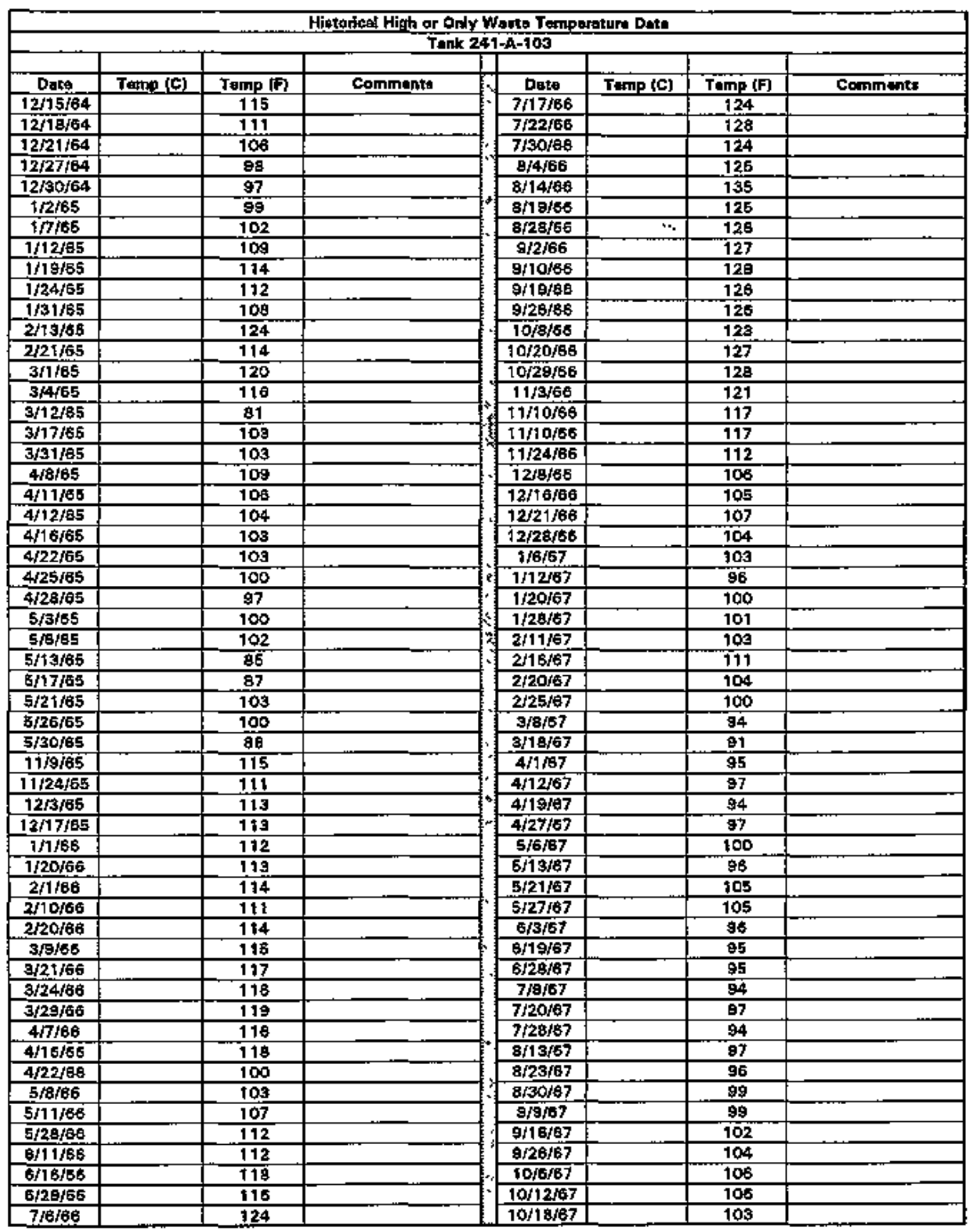

Dota abtained from Dotument RHO-CD-1172 and Waste Status Summary Reports. 


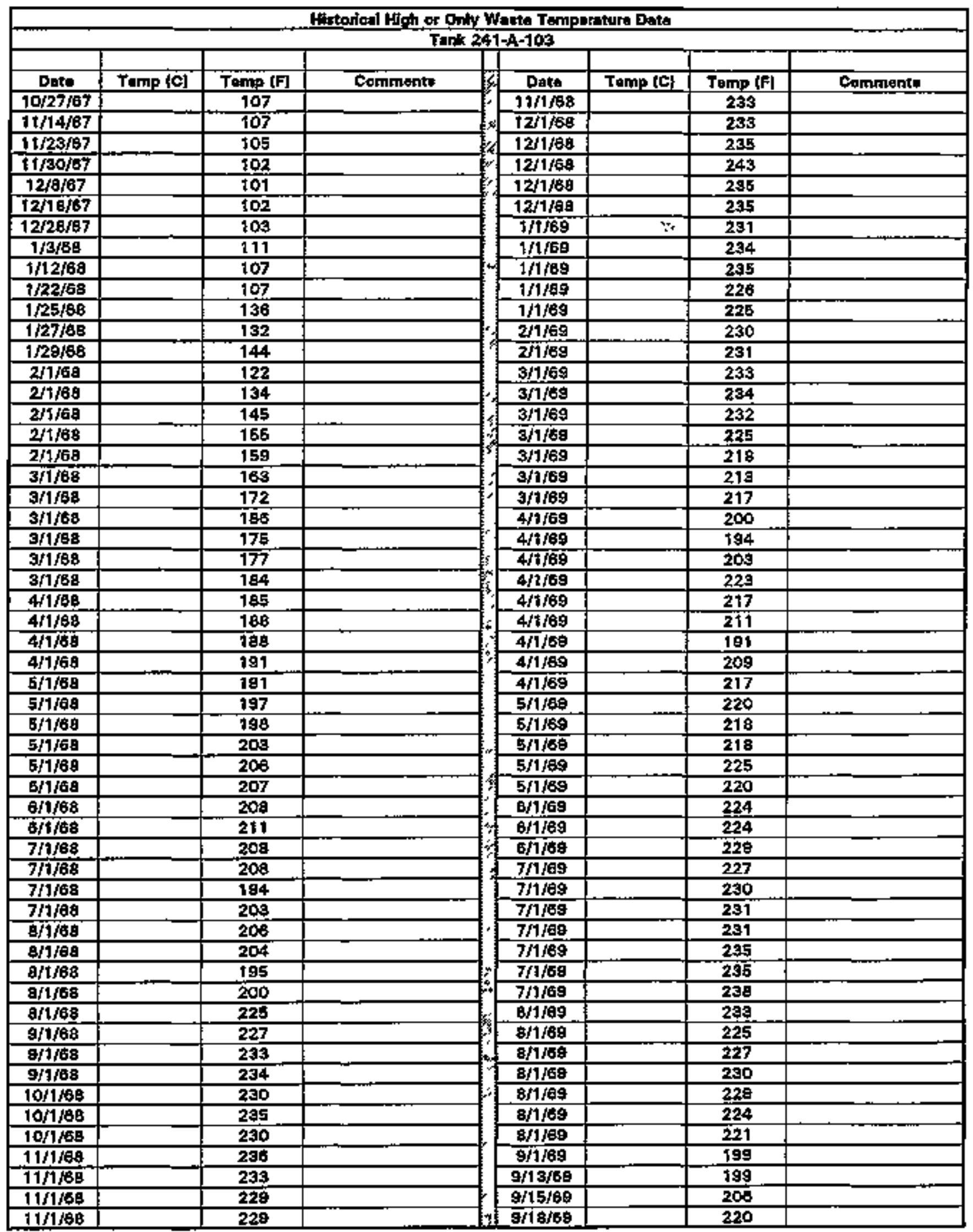

Data obteined from Document FHO-CD-1172 and Wasto Statua Summary Reports. 


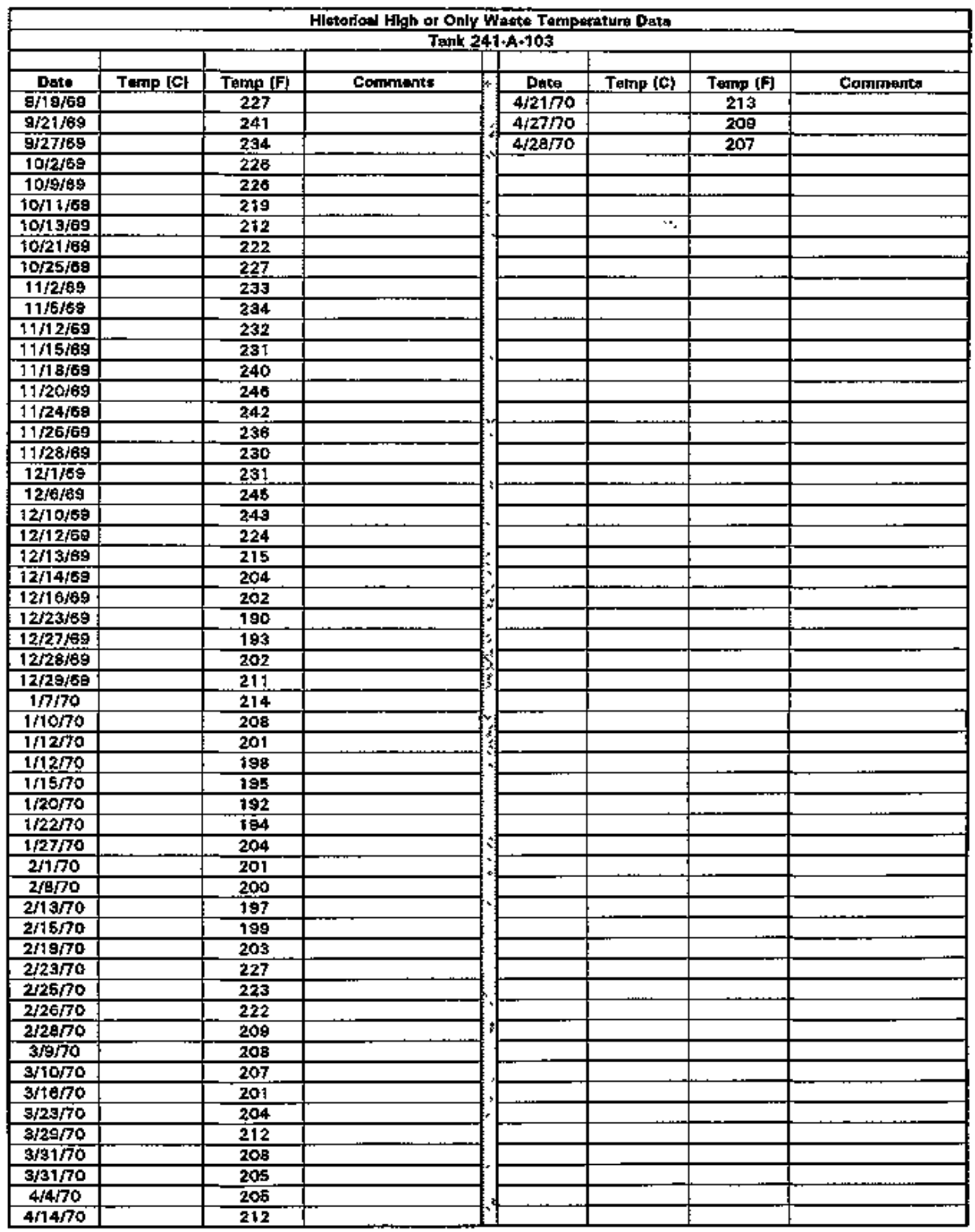

Date obtained from Doctument RHO-CD-1 172 and Waste Stetus Summery Raports. 


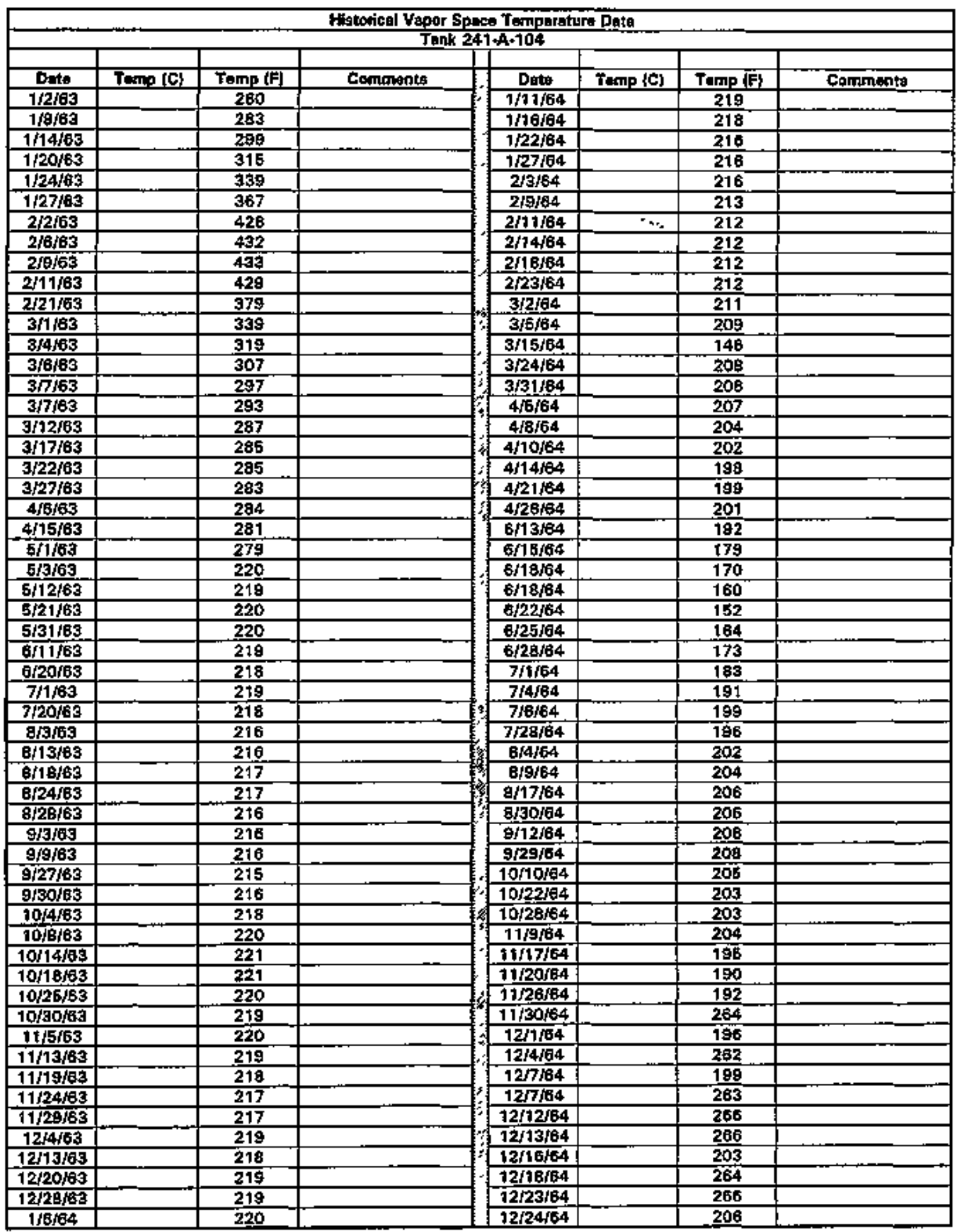

Data obtaired from Document RHO-CO-1172 and Wasto Status Summary Reporss. 


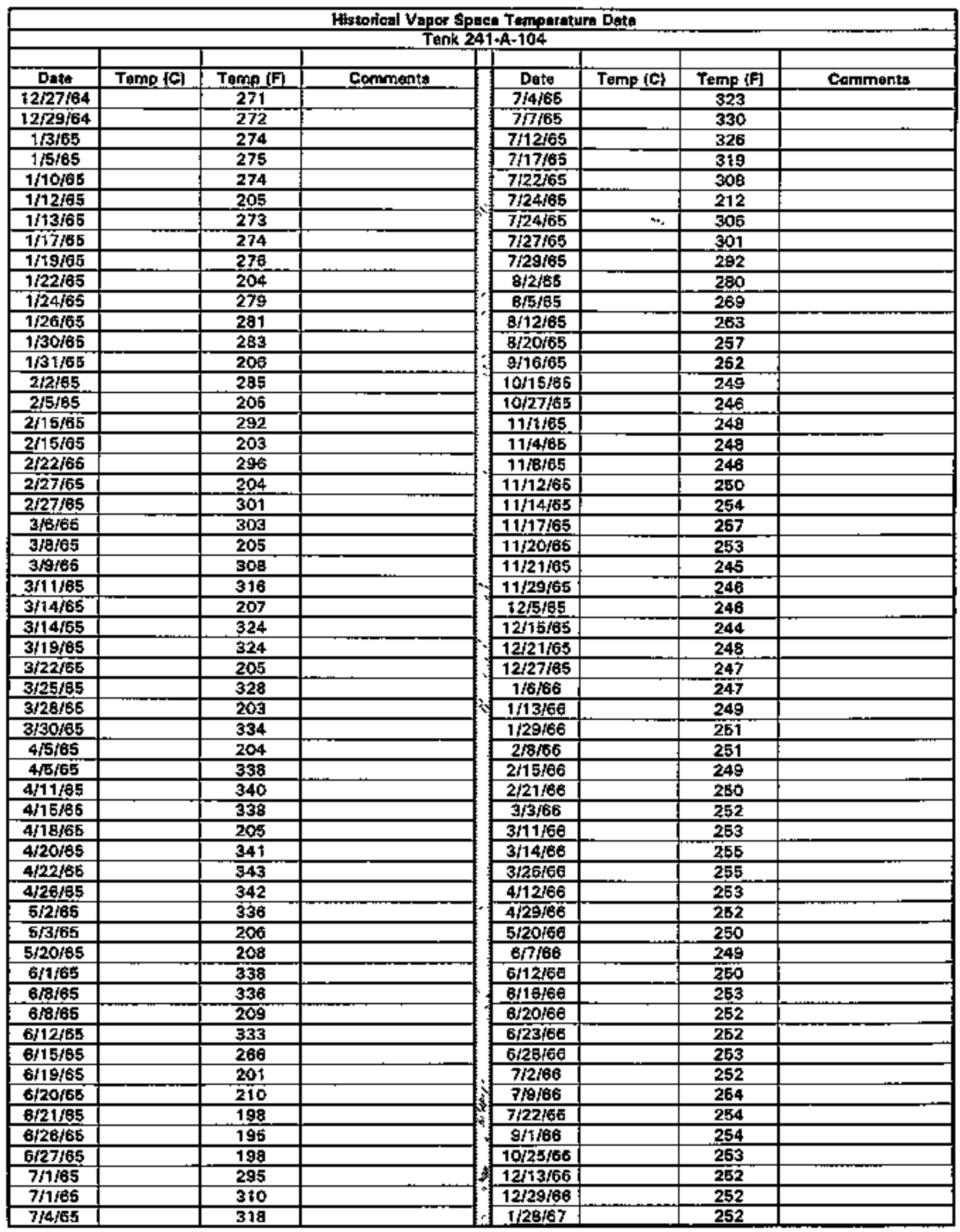

Data obtained from Dooument RHO-CD-1 172 and Weste Steturs Summery Reports. 


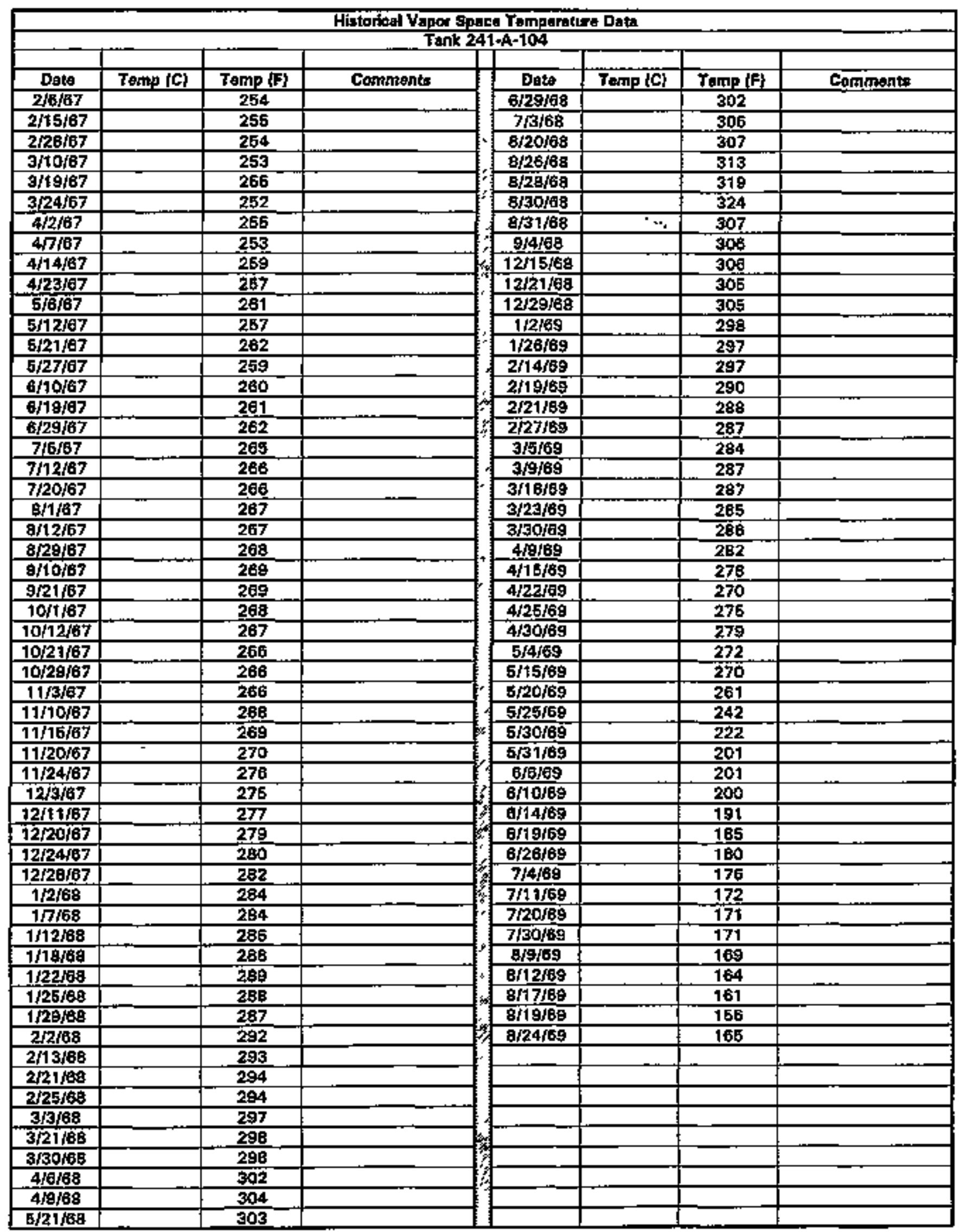

Date obtained from Doeument RHO-CD-1172 and Waste Status Stummary Reports. 


\begin{tabular}{|c|c|c|c|c|c|c|c|}
\hline \multicolumn{8}{|c|}{ Hlctorioal Averago Wosto Tamperaturi Oate } \\
\hline \multicolumn{8}{|c|}{$\operatorname{Tank} 241+\mathrm{A}+\operatorname{to4}$} \\
\hline Dete & Tomp [C] & $\overline{T e m p}\{F\}$ & Comments & Date & Temp (C) & Tum ta & Cortermente \\
\hline $3 / 31 / 59$ & & 172 & & $7 / 28 / 68$ & & 221 & \\
\hline 4/30/59 & & 179 & & $7 / 29 / 68$ & & 226 & \\
\hline 10/31/49 & & 207 & & $8 / 2 / 68$ & & 264 & \\
\hline $\pm 1 / 30 / 59$ & & 207 & & $8 / 20 / 68$ & & 251 & \\
\hline $2 / 29 / 60$ & 104 & $21 \mathrm{~s}$ & $\begin{array}{c}\text { raw data - assumed } \\
\text { degroe C }\end{array}$ & $8 / 27 / 68$ & & 224 & \\
\hline $3 / 31 / 80$ & & 215 & & $9 / 20 / 68$ & "r & 249 & \\
\hline $6 / 30 / 61$ & & 225 & & $9 / 28 / 68$ & & 221 & \\
\hline $12 / 31 / 81$ & & 225 & & $10 / 1 / 68$ & & 201 & \\
\hline $8 / 30 / 62$ & & 232 & & $105 / 68$ & & 186 & \\
\hline $12 / 31 / 62$ & & 325 & & $12 / 8 / 68$ & & 249 & \\
\hline $12 / 31 / 24$ & & 297 & & $12 / 9 / 68$ & & 236 & \\
\hline $3 / 23 / 65$ & & 282 & & $12 / 12 / 8$ & & 222 & \\
\hline $4 / 20 / 65$ & & 290 & & $12 / 19 / 8 g^{2}$ & & 228 & \\
\hline $5 / 8 / 65$ & & 285 & & $12 / 24 / 68$ & & 233 & \\
\hline $7 / 1 / 65$ & & 297 & & $12 / 28 / 68$ & & 233 & \\
\hline $8 / 20 / 65$ & & 280 & & $1 / 5 / 69$ & & 203 & \\
\hline $10 / 13 / 55$ & & $2 B 2$ & & 4/15/as & & 204 & \\
\hline $11 / 1 / B 5$ & & 264 & & $4 / 21 / 63$ & & 204 & \\
\hline 11 f17/6s & & 249 & & $4 / 24 / 69$ & & 218 & \\
\hline $12 / 18 / 65$ & & 273 & & $4 / 2 B / B \Rightarrow$ & & 234 & \\
\hline $1 / 5 / 66$ & & 273 & & & & & \\
\hline $2 / 11 / 66$ & & 259 & & & & & \\
\hline $5 / 21 / 66$ & & 282 & & & & & \\
\hline $5 / 31 / 86$ & & 265 & & & & & \\
\hline $7 / 1 / 66$ & & 267 & & & & & \\
\hline $2 / 3 / 67$ & & 289 & & & & & \\
\hline $2 / 26 / 57$ & & 285 & & & & & \\
\hline $4 / 1 / 67$ & & 279 & & & & & \\
\hline $6 / 28 / 67$ & & 287 & & & & & \\
\hline $7 / 4 / 67$ & & 291 & & & & & \\
\hline $7 / 18 / 67$ & & 286 & & & & & \\
\hline $7 / 24 / 67$ & & 281 & & & & & \\
\hline $8 / 31 / 67$ & & 290 & & & & & \\
\hline $10 / 43 / 67$ & & 292 & & & & & \\
\hline $11 / 19 / 67$ & & 293 & & & & & \\
\hline $12 / 29 / 67$ & & 310 & & & & & \\
\hline $2 / 4 / 68$ & & 220 & & & & & \\
\hline $2 / 9 / 68$ & & 227 & & & & & \\
\hline $2 / 21 / 68$ & & 223 & & & & & \\
\hline $2 / 27 / 58$ & & 227 & & & & & \\
\hline $3 / 7 / 68$ & & 231 & & & & & \\
\hline $3 / 14 / 88$ & & 227 & & & & & \\
\hline $4 / 3 / 68$ & & 219 & & & & & \\
\hline $4 / 13 / 69$ & & 214 & & & & & \\
\hline $4 / 19 / 68$ & & 214 & & & & & \\
\hline $5 / 8 / 68$ & & 214 & & & & & \\
\hline $5 / 26 / 68$ & & 214 & & & & & \\
\hline $5 / 30 / 68$ & & 218 & & & & & \\
\hline $6 / 5 / 28$ & & 222 & & & & & \\
\hline $6 / 13 / 58$ & & 222 & & & & & \\
\hline$\theta / 20 / 6 \mathrm{a}$ & & 222 & & & & & \\
\hline $8 / 23 / 68$ & & 218 & & & & & \\
\hline $6 / 28 / 69$ & & 213 & & & & & \\
\hline $7 / 22 / 68$ & & 209 & & & & & \\
\hline
\end{tabular}

Data obtained from Document RHO-CO-1172 and Waste Status Summary Reports. 


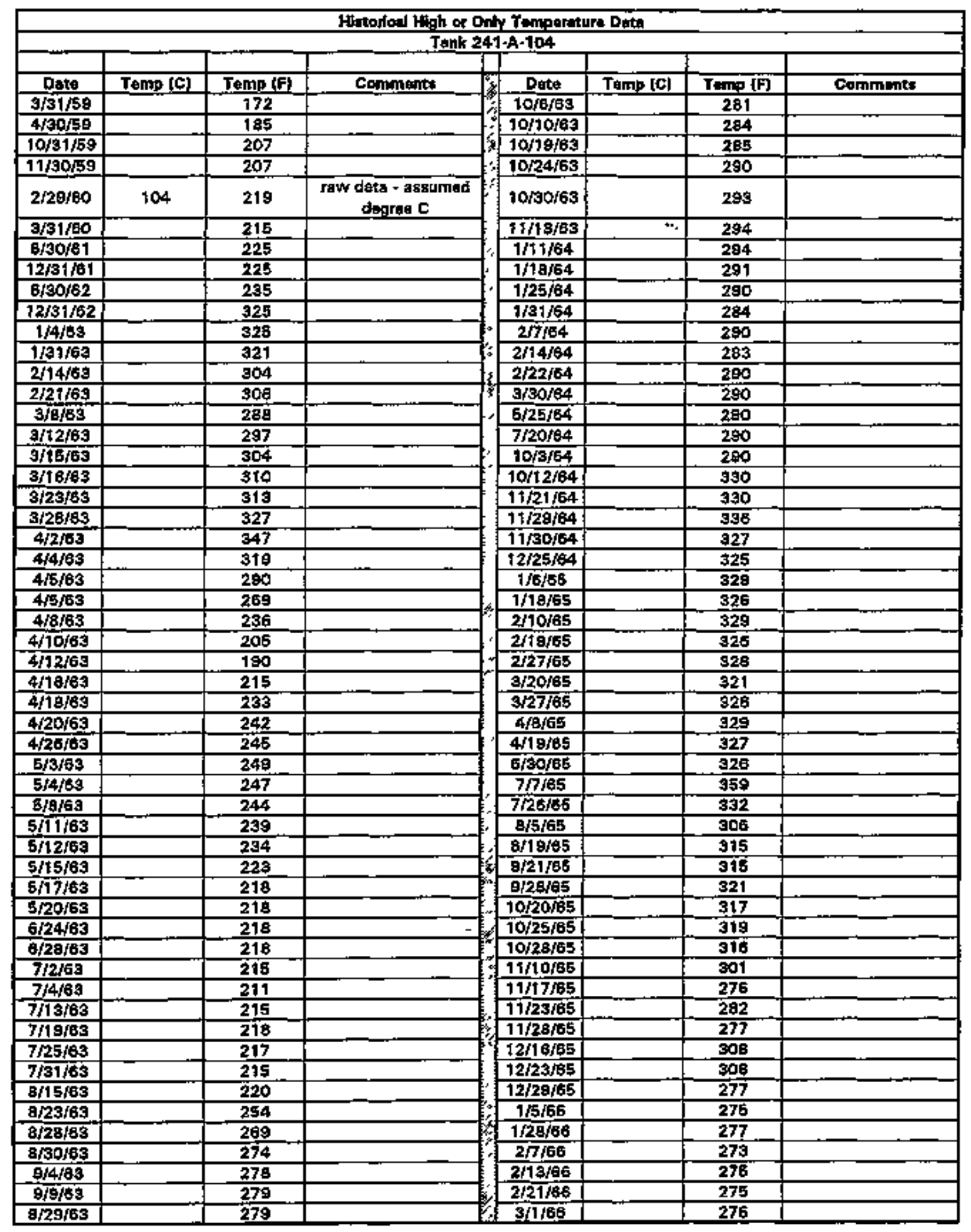

Date obtainad from Document RHO-CD-1172 and Waste Status Summary Raports. 


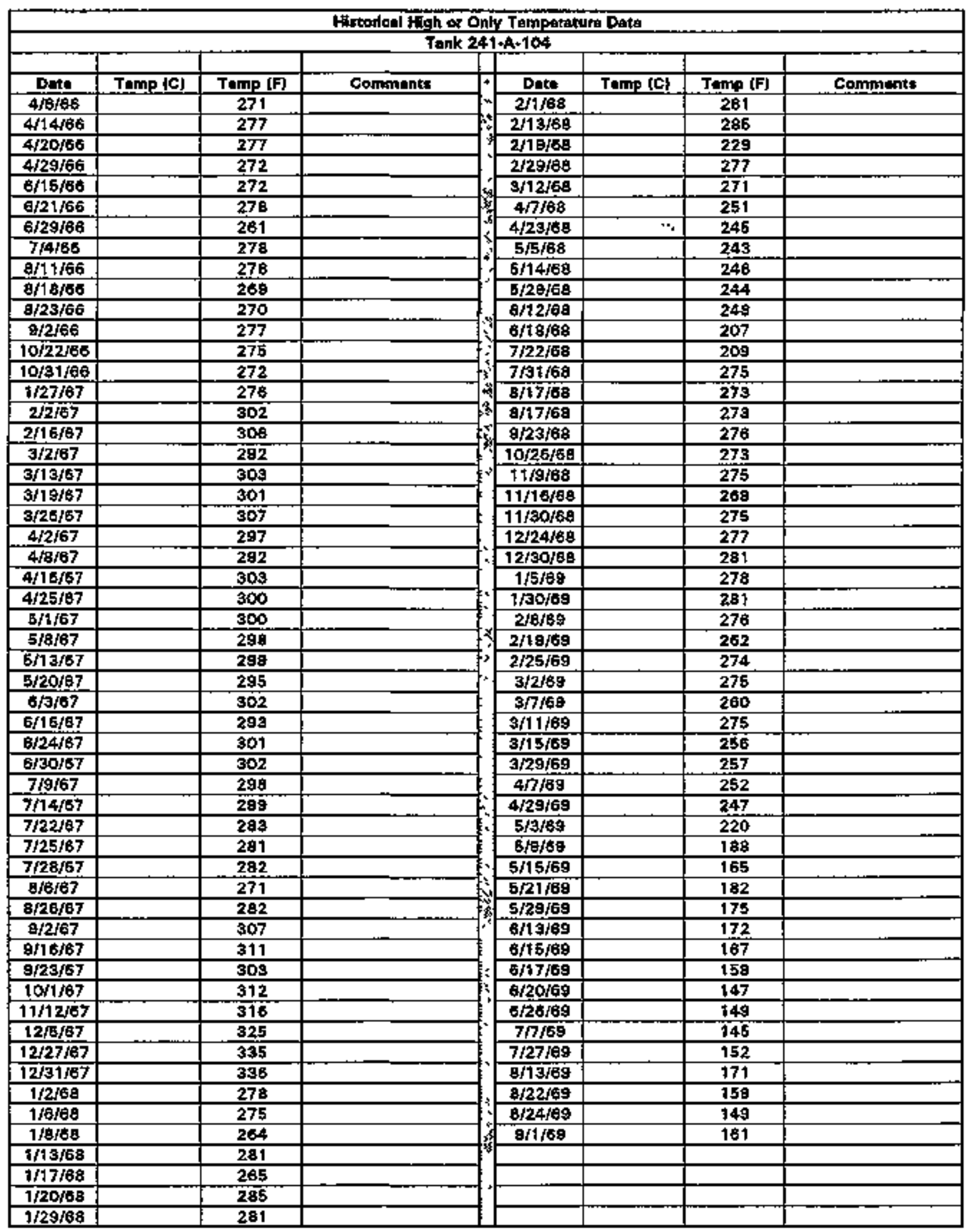

Data obteined from Dotument RHO-CO-1172 and Waste status Summery Reparte. 


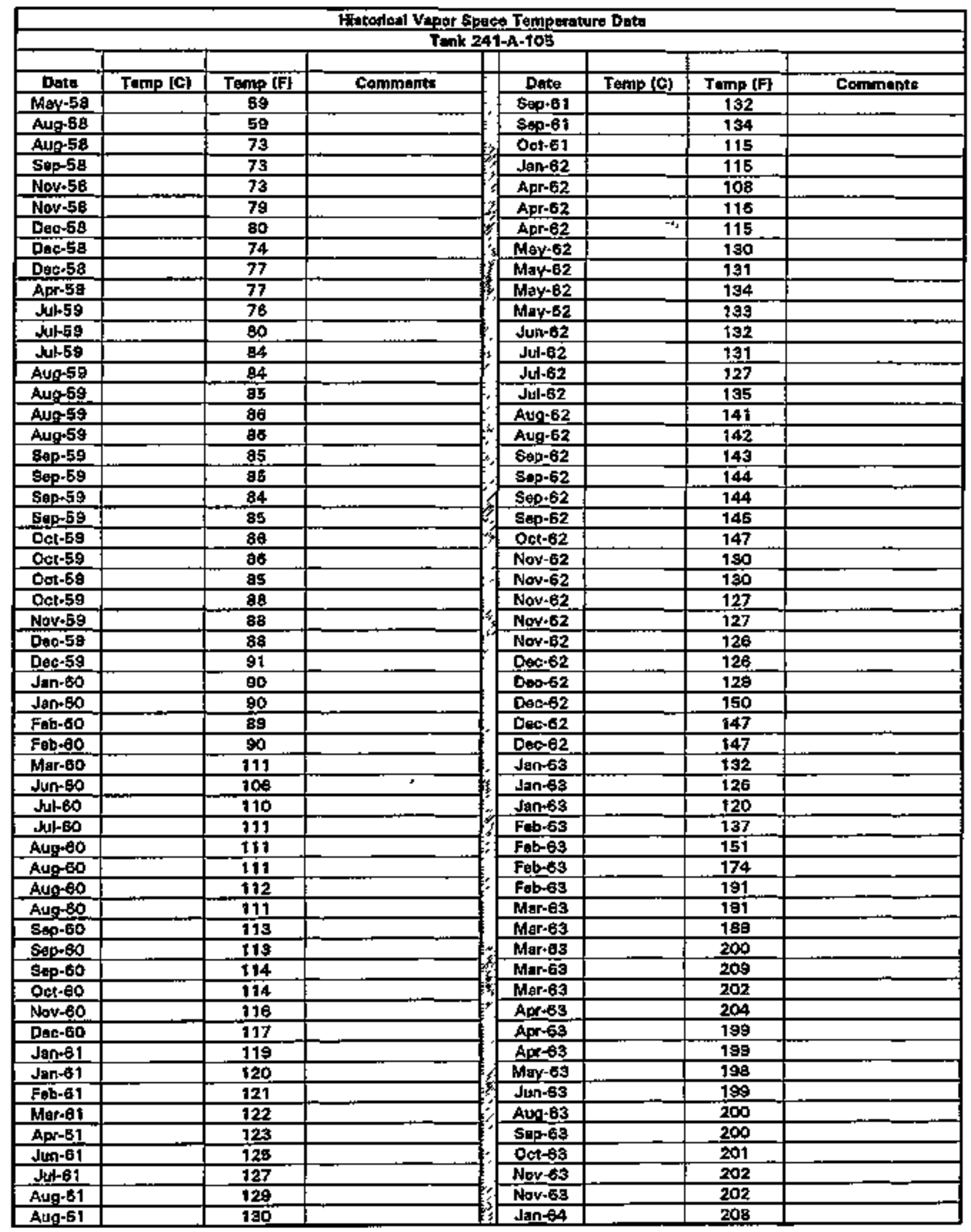

Data obteined from Dogument RHO-CD-1172 and Waste Status Summary Raports. 


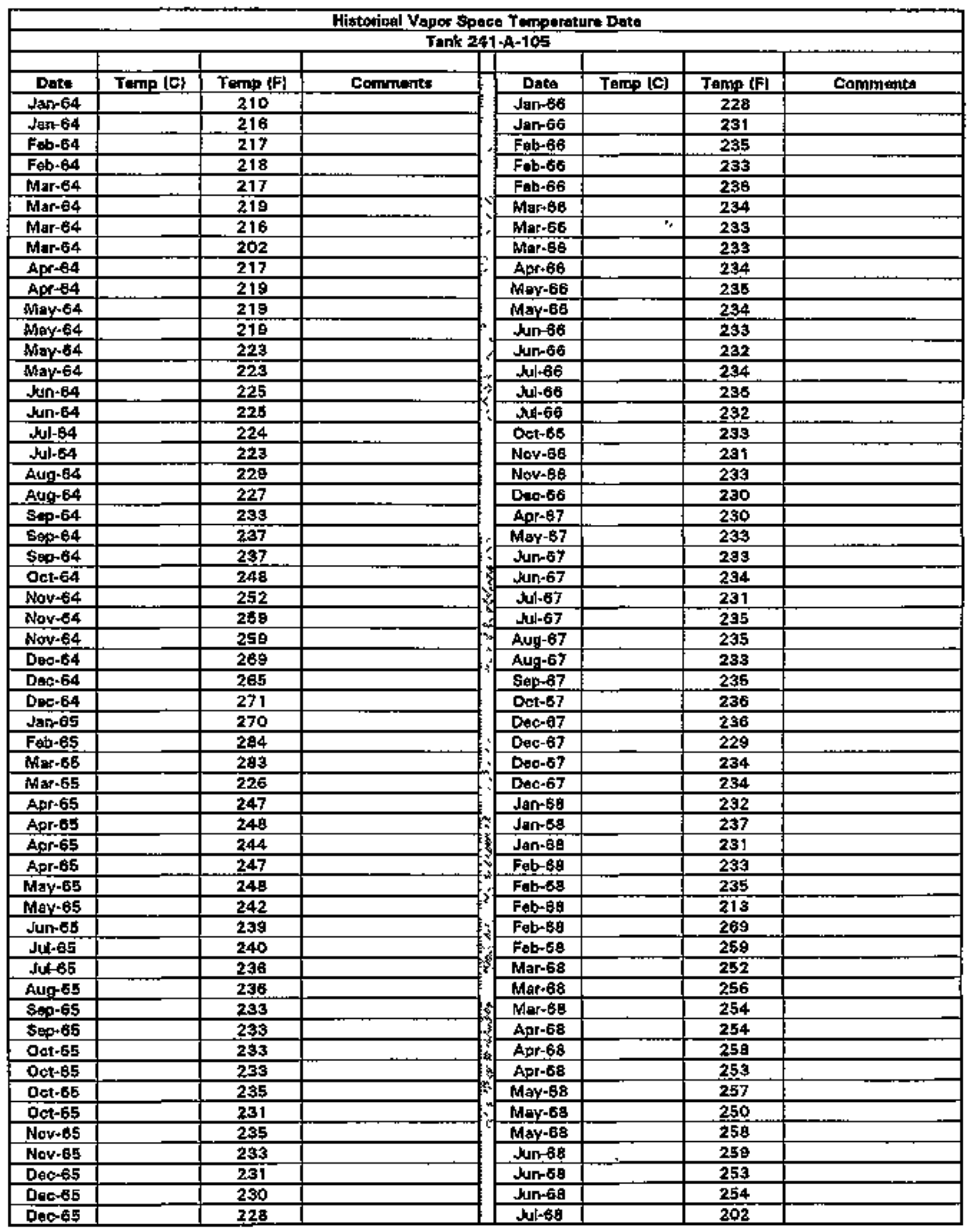

Data obtained from Dooument RHO-CD-1172 and Waste Stetus Summar fleports. 


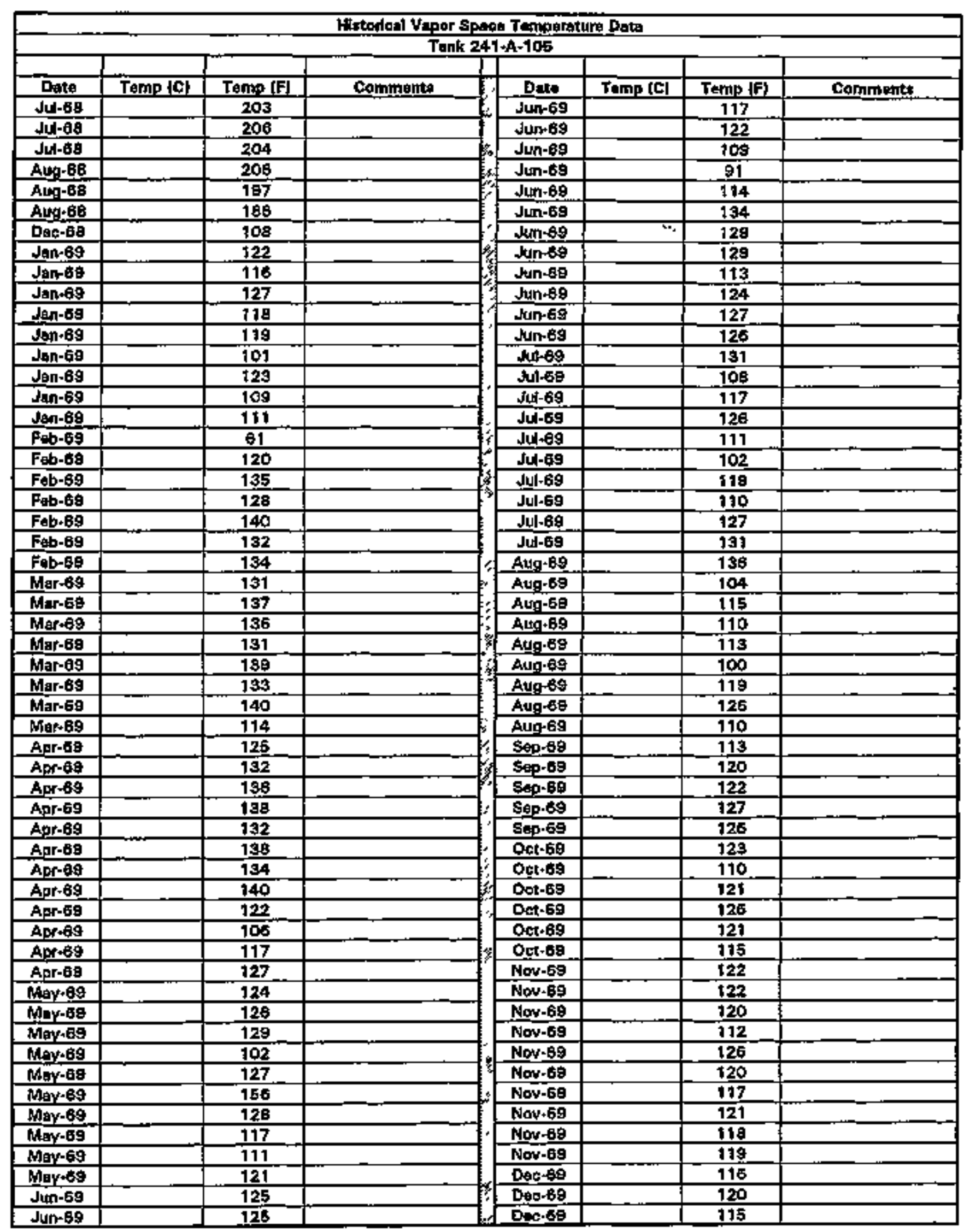

Data obtained from Oocument RHO-CO.1 172 and Waste Status Summay fleports. 


\begin{tabular}{|c|c|c|c|c|c|c|c|}
\hline \multicolumn{8}{|c|}{ Hiztolited Vapor Epace Temperaturs Date } \\
\hline \multicolumn{8}{|c|}{ Tank 241-A-105 } \\
\hline & & & & & & & \\
\hline Dits. & Temp [C] & Temp $\{F\}$ & Commants & Date & Temp (C) & Temp [F] & Comments \\
\hline Dec-69 & & 108 & & & & & \\
\hline $\operatorname{Jan}-70$ & & 112 & & & & & \\
\hline $\operatorname{Jan}-70$ & & 113 & & & & & \\
\hline $\operatorname{Jan} 70$ & & $9 B$ & & & & & \\
\hline $\operatorname{Jan} 70$ & & 106 & & & & & \\
\hline $\operatorname{Jan}-70$ & & 97 & & & & & \\
\hline $\operatorname{Jan} \cdot 70$ & & 115 & & & & & \\
\hline $\operatorname{Jan}-70$ & & 102 & & & & & \\
\hline Feb-70 & & 120 & & & & & \\
\hline Feb-70 & & 113 & & & & & \\
\hline Fab.70 & & 107 & & & & & \\
\hline Feb-70 & & 121 & & & & & \\
\hline Fab-70 & & 127 & & & & & \\
\hline Mar-70 & & 114 & & & & & \\
\hline Mar+70 & & 119 & & & & & \\
\hline Mar-70 & & 117 & & & & & \\
\hline Mar-70 & & 121 & & & & & \\
\hline Mar-70 & & 114 & & & & & \\
\hline Mar-70 & & 120 & & & & & \\
\hline App +70 & & 118 & & & & & \\
\hline Apr.70 & & 121 & & & & & \\
\hline Apr $=70$ & & 117 & & & & & \\
\hline Aptrit 70 & & 120 & & & & & \\
\hline Apr.70 & & 120 & & & & & \\
\hline Apr -70 & & $\$ 16$ & & & & & \\
\hline May-70 & & 121 & & & & & \\
\hline Mar-70 & & 120 & & & & & \\
\hline Mar-70 & & 121 & & & & & \\
\hline May-70 & & 113 & & & & & \\
\hline May-70 & & 120 & & & & & \\
\hline May-70 & & $\$ 23$ & & & & & \\
\hline May-70 & & t18 & & & & & \\
\hline May-70 & & 121 & & & & & \\
\hline Sun-70 & & 115 & & & & & \\
\hline Jurr 70 & & 120 & & & & & \\
\hline Jun 70 & & $\$ 19$ & & & & & \\
\hline
\end{tabular}




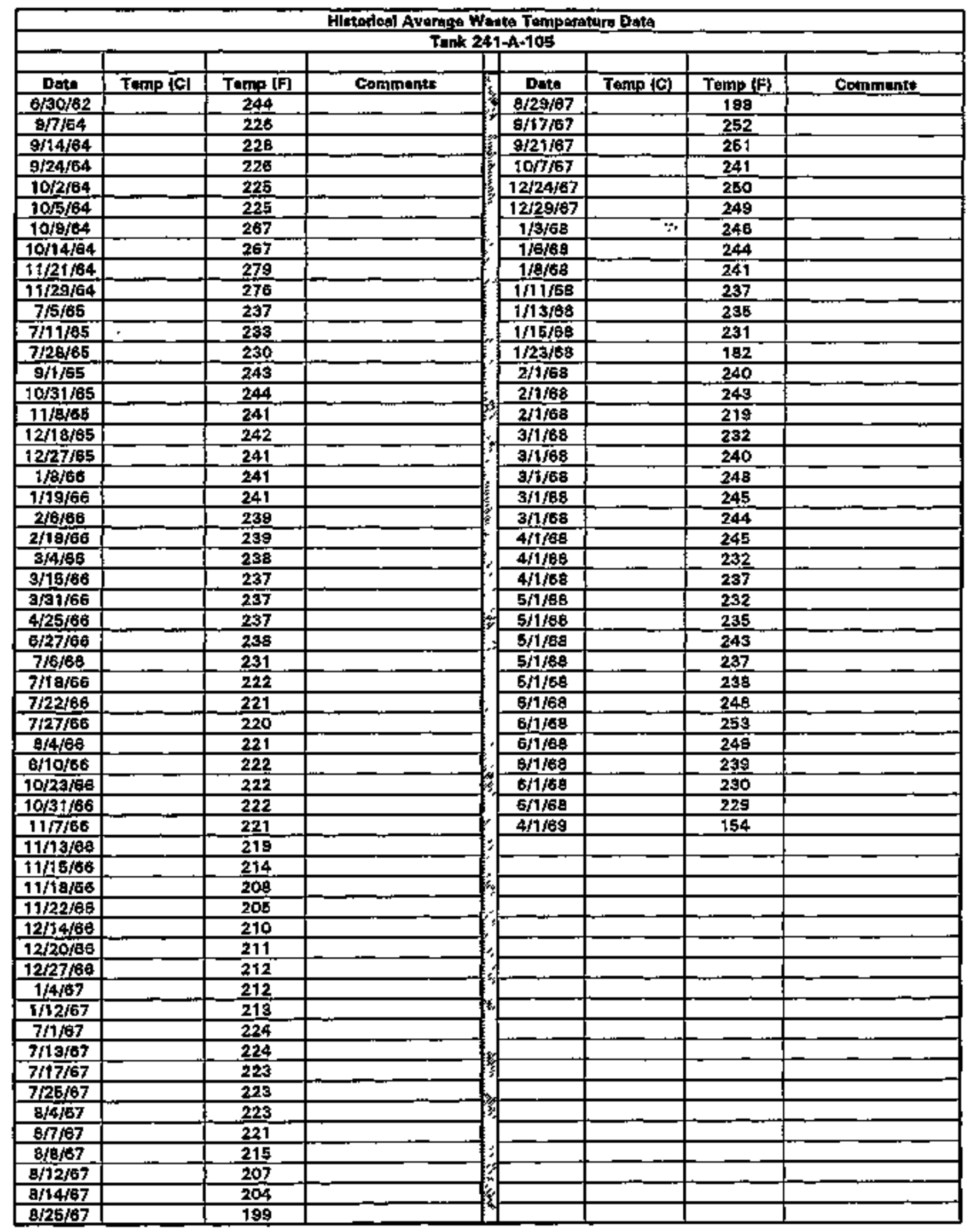

Data obtaitad from Document R1SO-CD-1172 and Wasta Etatus Sumrary feports. 


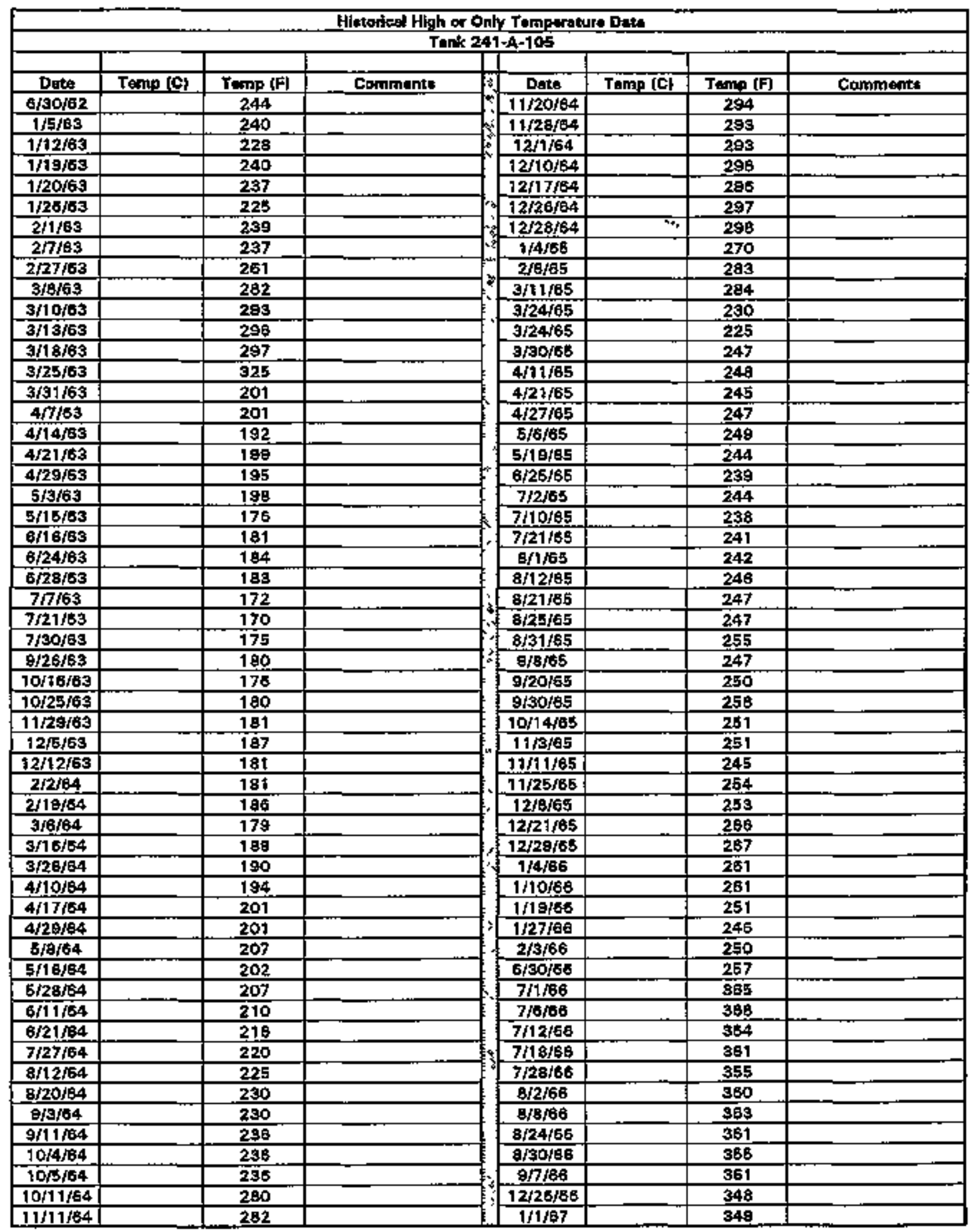

Data obtsined from Docurnent RHO-CD-1172 and Waste \$tatus Sumrinary Raports. 


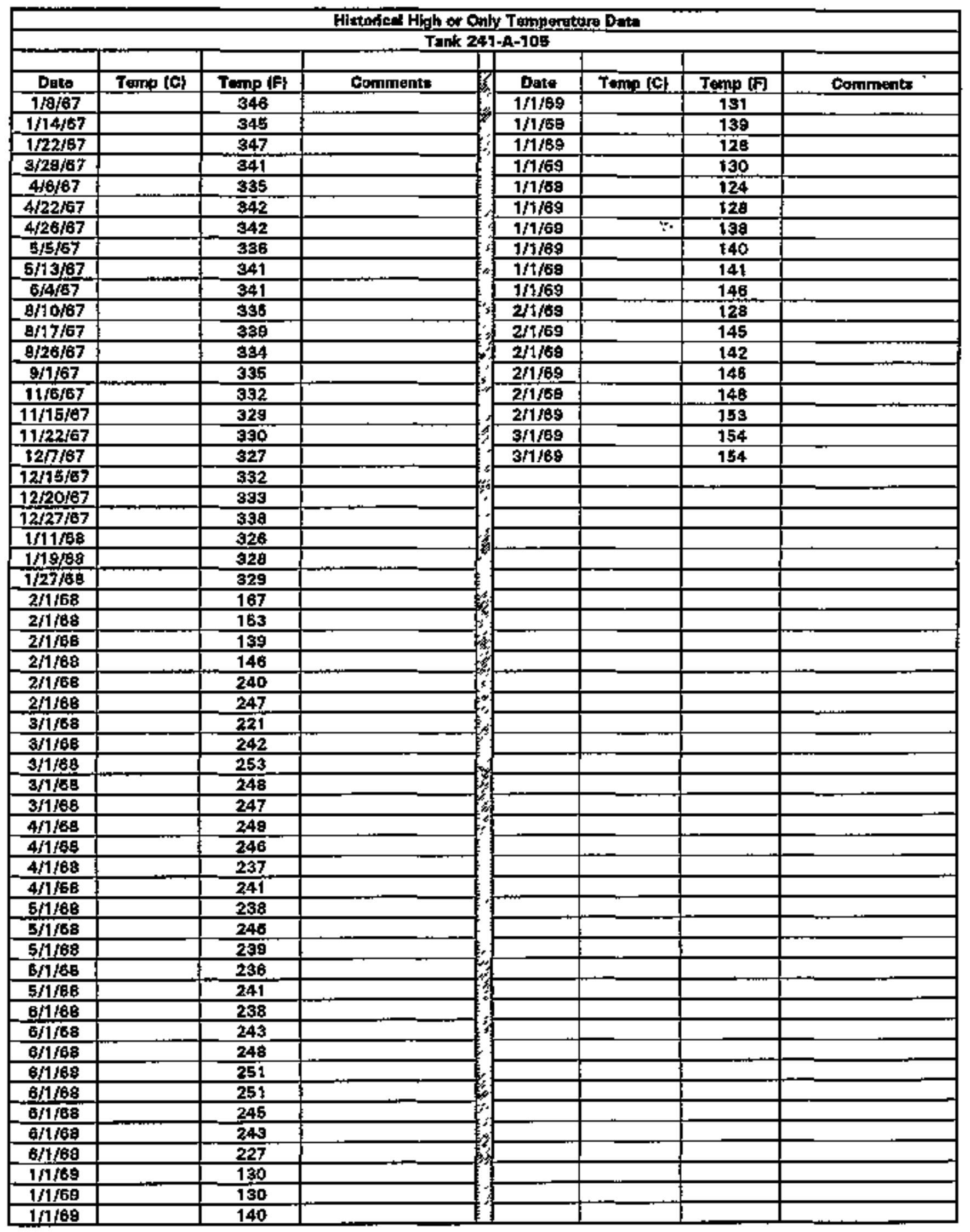

Data obtained frem Dooument Fuo-CD-1172 and Waste Status Summary Reports. 


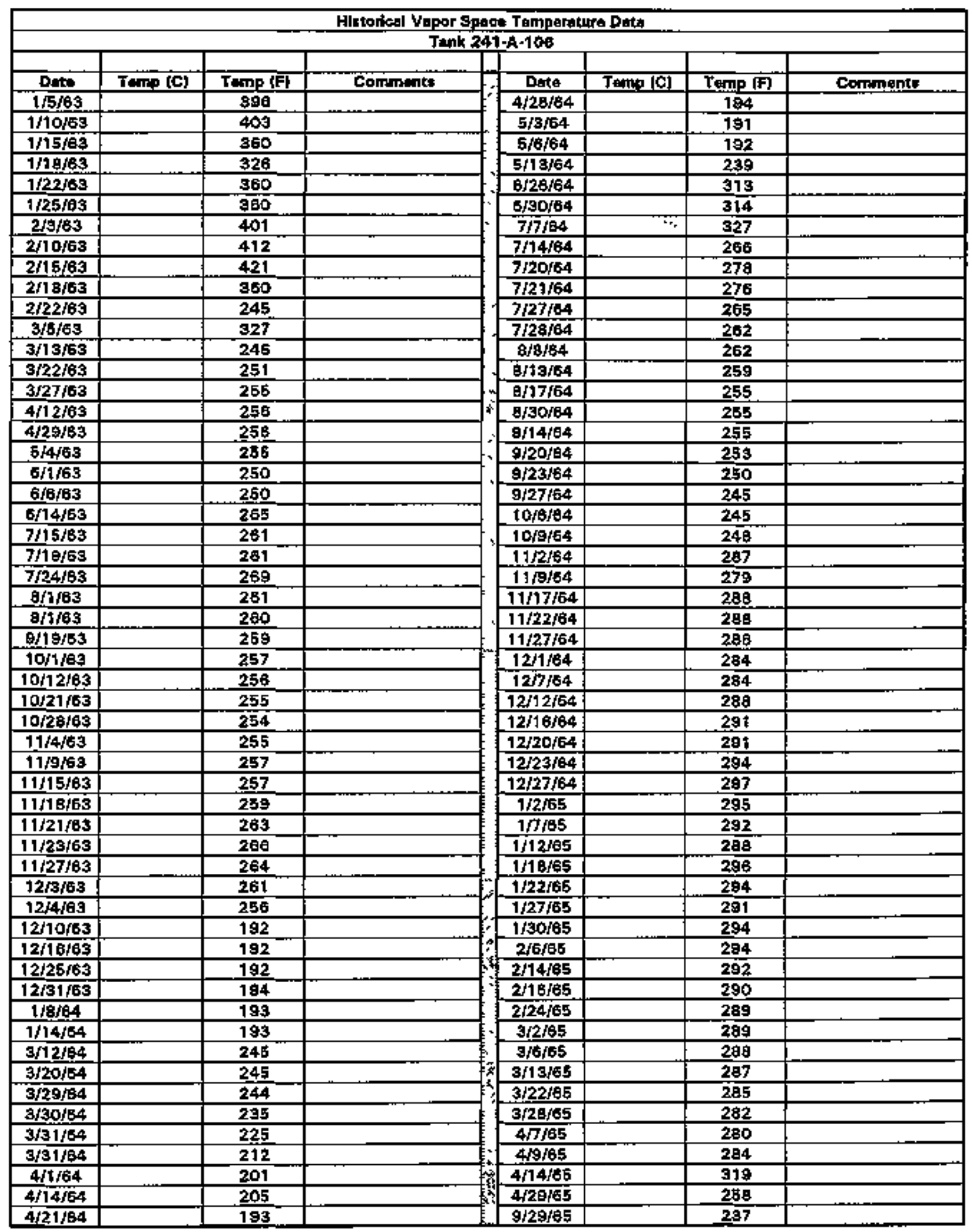

Data obtained from Document RHO-CO-1172 and Weste States Stummary Reports, 


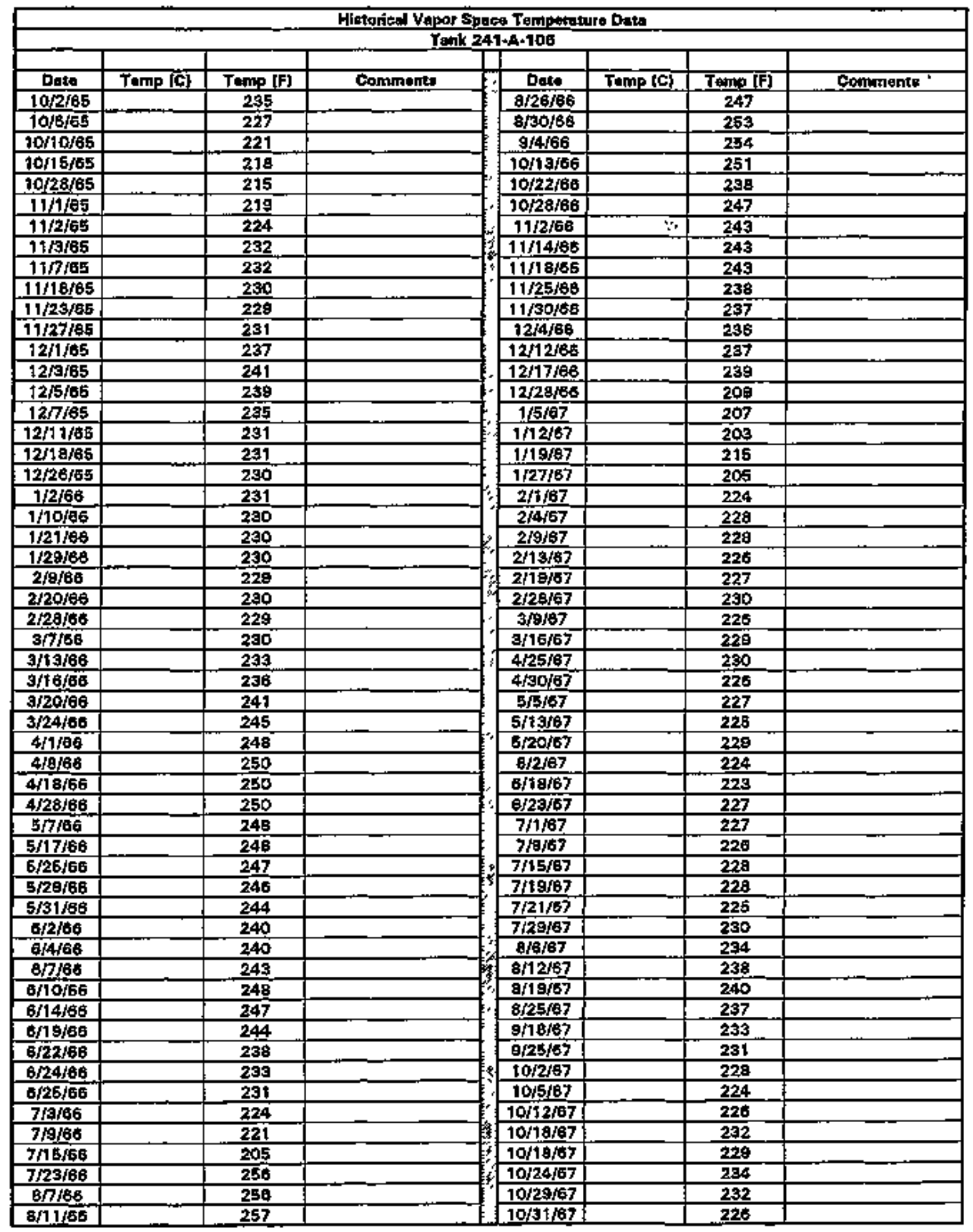

Data obtaind from Document RHO-CD-1172 and Waste Status Stammary Reports. 


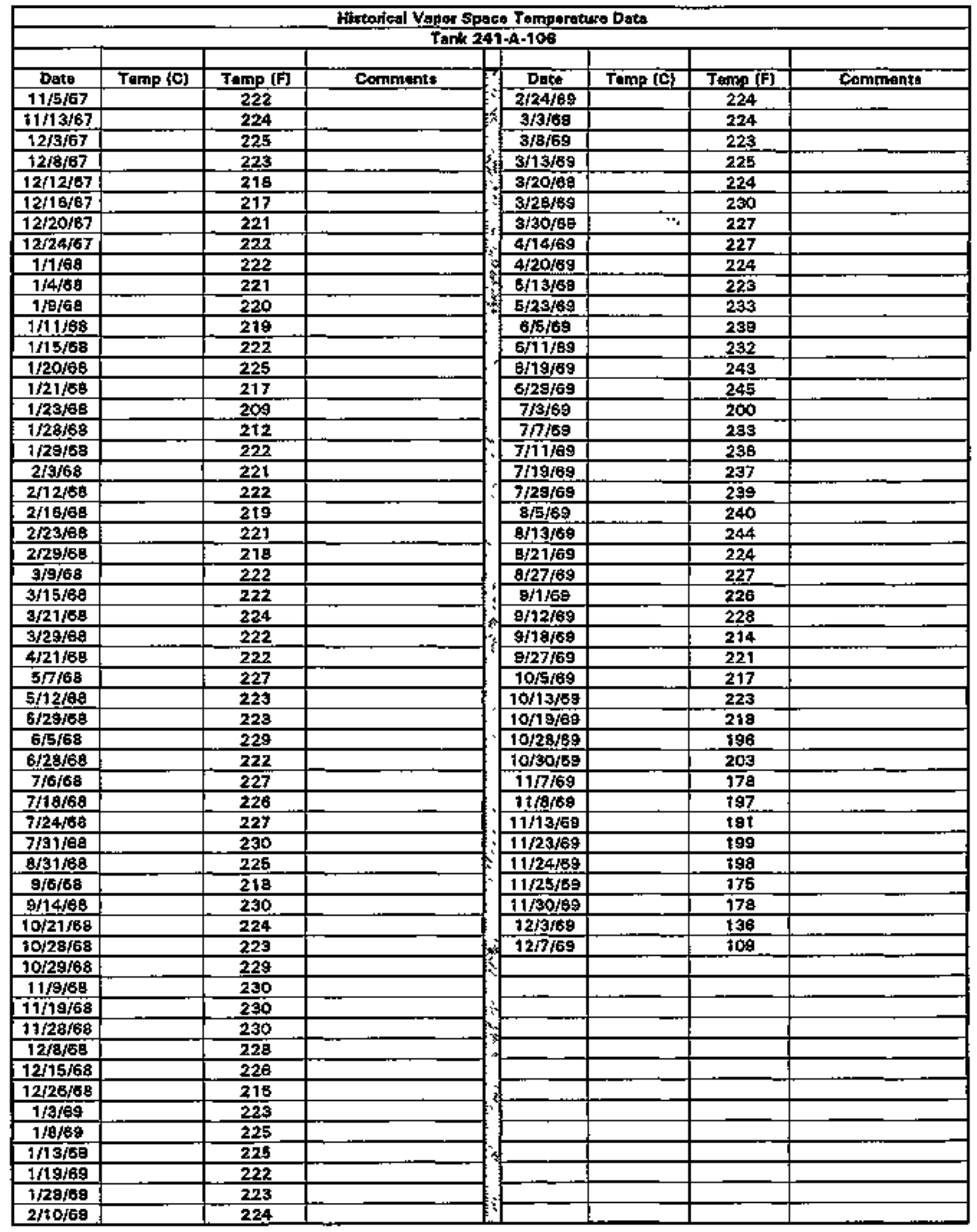

Data bbtained from Dosument RHO-CO-\$172 and Waste Status Summery Reporta. 


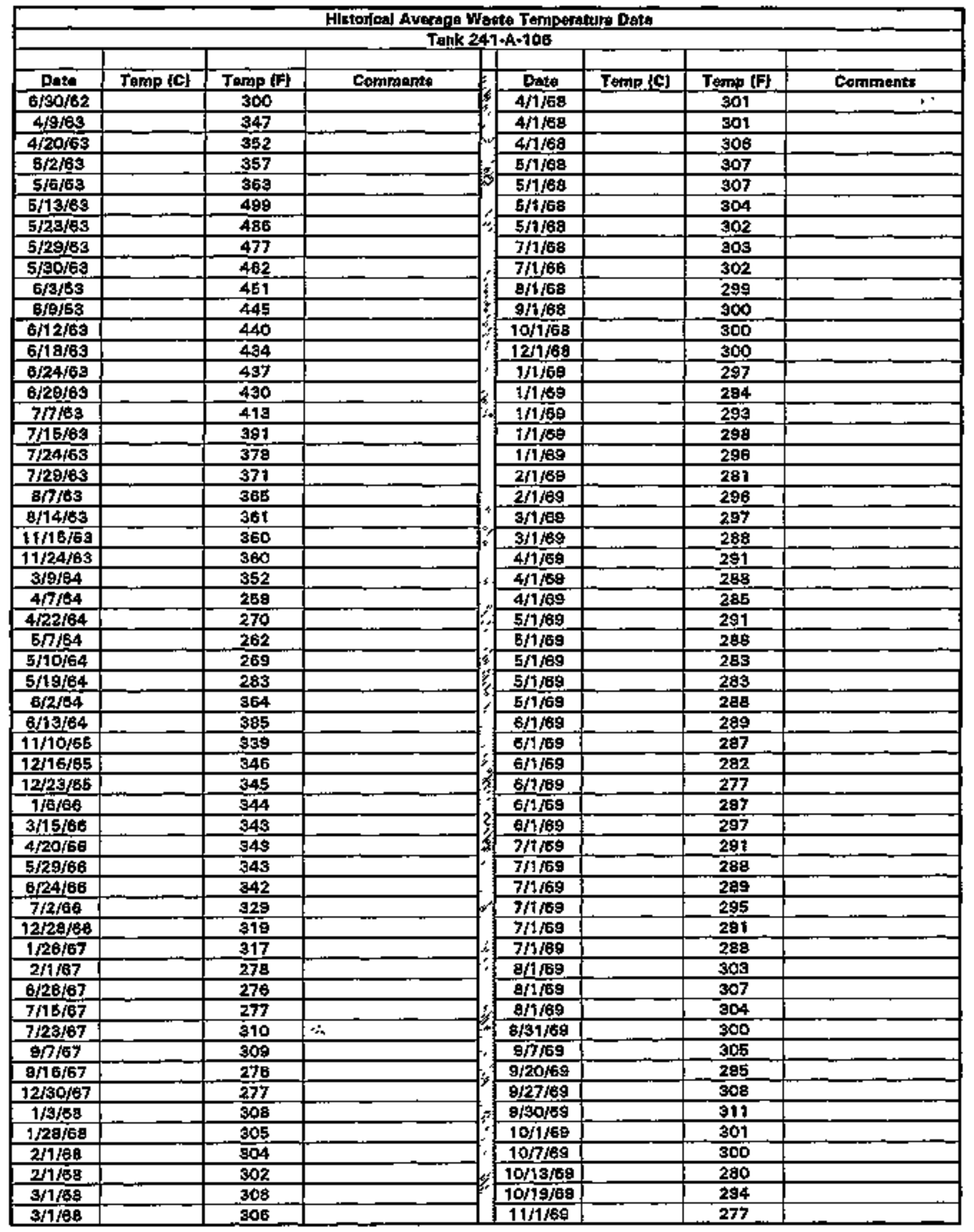

Date obtained fram Dooument BHC-CO-j172 ond Waste Status \$lummary Feporta. 


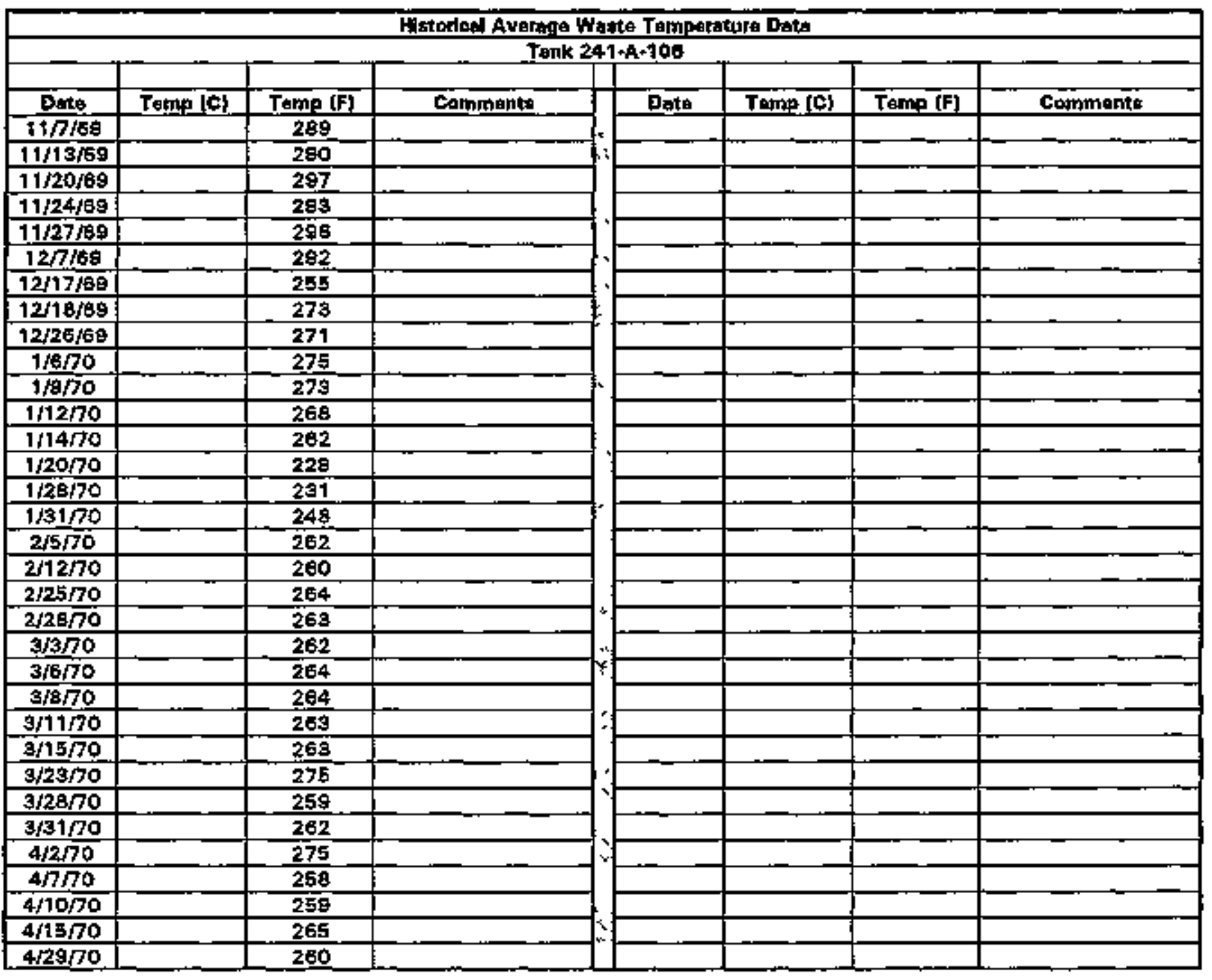




\begin{tabular}{|c|c|c|c|c|c|c|c|c|}
\hline \multicolumn{9}{|c|}{ Hetarich Hiph or Only Temperiture Dote } \\
\hline & & & Tonk 2 & 241 & $+A-100$ & & & \\
\hline Dat!l & $\operatorname{Tamp}|c|$ & Tomp \{fr| & Commant: & & Date & Temp (C) & Temp [F] & Commente \\
\hline $6 / 30 / 02$ & & $\mathbf{3 2 7}$ & & & $6 / 15 / 64$ & & 456 & \\
\hline $12 / 31 / 82$ & & 390 & & & $0 / 22 / 64$ & & 475 & \\
\hline $1 / 8 / 83$ & & 329 & & : & $6 / 29 / 64$ & & 489 & \\
\hline $1 / 12 / 63$ & & 337 & & tis & $7 / 7 / 24$ & & $4 \mathrm{AS}$ & \\
\hline $1 / 20 / 63$ & & 329 & & 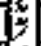 & $7 / 10 / 64$ & & 427 & \\
\hline $1 / 24 / 83$ & & 328 & & {$[x]$} & $7 / 12 / 64$ & & 407 & \\
\hline $2 / 8 / 69$ & & $\overline{\mathbf{3 2 2}}$ & & $=1$ & $7 / 13 / 64$ & & 374 & \\
\hline $3 / 27 / 63$ & & 321 & & . & $7 / 15 / 64$ & & 330 & \\
\hline $4 / 3 / 63$ & & 328 & & 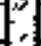 & $7 / 21 / 64$ & & 278 & \\
\hline $4 / 10 / 6 \mathrm{~s}$ & & 374 & & 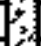 & $7 / 24 / 64$ & & 328 & \\
\hline $4 / 17 / 63$ & & 378 & & 行 & $7 / 27 / 64$ & & 376 & \\
\hline $4 / 21 / 63$ & & 379 & & ${ }^{3}$ & $7 / 29 / 64$ & & $\overline{413}$ & \\
\hline $5 / 1 / 63$ & & $3 B B$ & & 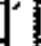 & $8 / 1 / 64$ & & 440 & \\
\hline $5 / 2 / 63$ & & 389 & &. & $8 / 11 / 64$ & & 459 & \\
\hline $5 / 6 / 63$ & & 460 & & s. & $8 / 15 / 64$ & & 457 & \\
\hline $5 / 8 / 83$ & & 518 & & st & $8 / 24 / 64$ & & 469 & \\
\hline $5 / 14 / 63$ & & 583 & & , & $8 / 30 / 64$ & & 467 & \\
\hline $5 / 25 / 63$ & & $\$ 70$ & & \& & $9 / 13 / 34$ & & 482 & \\
\hline $5 / 30 / 63$ & & 53도 & & 8 & $9 / 18 / 64$ & & 494 & \\
\hline $5 / 31 / 63$ & & 517 & & - & $10 / 2 / 64$ & & 480 & \\
\hline $6 / 4 / 63$ & & 500 & & 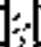 & $10 / 10 / 64$ & & 473 & \\
\hline B/18/63 & & 483 & & 28 & $10 / 23 / 64$ & & 470 & \\
\hline $\mathrm{g} / \mathrm{s} / \mathrm{0} / 3$ & & 446 & & 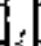 & $11 / 6 / 64$ & & 456 & \\
\hline $8 / 15 / 63$ & & $\overline{444}$ & & 3 & $11 / 15 / 04$ & & $4 \$ 1$ & \\
\hline $8 / 25 / 63$ & & 440 & & & $11 / 22 / 84$ & & 456 & \\
\hline $11 / 22 / 63$ & & 432 & & & $11 / 27 / 64$ & & 456 & \\
\hline $12 / 1 / 83$ & & 434 & & 6 & $32 / 2 / 64$ & & 457 & \\
\hline $12 / 9 / 63$ & & 408 & & 8 & $12 / 26 / 04$ & & 456 & \\
\hline $12 / 16 / 63$ & & 427 & & 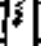 & $1 / 1 / 25$ & & 451 & \\
\hline $12 / 22 / 83$ & & 426 & & . & t/to/65 & & 466 & \\
\hline $12 / 28 / 63$ & & 417 & & 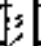 & \$/30/65 & & 443 & \\
\hline$t / 6 / 64$ & & 419 & & & $2 / B / 65$ & & 490 & \\
\hline $1 / 10 / 0^{4}$ & & 419 & & 8 & $2 / 13 / 65$ & & 430 & \\
\hline $1 / 20 / 24$ & & 413 & & 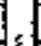 & $2 / 23 / 65$ & & 421 & \\
\hline $1 / 28 / 84$ & & 417 & & , & $3 / 1 / 65$ & & 434 & \\
\hline $2 / 13 / 64$ & & 410 & & & $4 \sqrt{7 / 65}$ & & 421 & \\
\hline $2 / 20 / B 4$ & & 411 & & $m$ & $4 / 27 / 65$ & & $\overline{419}$ & \\
\hline $3 / 1 / 64$ & & 422 & & & $5 / 4 / 65$ & & 411 & \\
\hline $3 / 17 / 64$ & & 422 & & $t$ & $5 / 26 / 65$ & & 417 & \\
\hline $3 / 16 / 64$ & & 426 & & 4 & $6 / 2 B / 65$ & & 411 & \\
\hline $3 / 20 / 64$ & & 400 & & 8 & $7 / 21 / 65$ & & 384 & \\
\hline $3 / 24 / 84$ & & 370 & & $\cdots$ & $7 / 24 / 65$ & & 386 & \\
\hline $3 / 28 / 64$ & & 348 & & 5 & $6 / 4 / 65$ & & 390 & \\
\hline $3 / 30 / 04$ & & 323 & & 3 & $8 / 11 / 65$ & & 400 & \\
\hline $4 / 7 / 64$ & & 309 & & & a/20/65 & & 413 & \\
\hline $4 / 13 / 64$ & & 302 & $i$ & 5 & $9 / 14 / 6 \mathrm{E}$ & & 410 & \\
\hline $4 / 20 / 64$ & & 282 & & 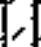 & 9/20/B5 & & 393 & \\
\hline $5 / 3 / 64$ & & 282 & & . & $9 / 28 / 65$ & & 398 & \\
\hline $5 / 18 / 64$ & & 300 & & 8 & $10 / 6 / 68$ & & 393 & \\
\hline $5 / 23 / 64$ & & 359 & & $x$ & $10 / 25 / 65$ & & 392 & \\
\hline $5 / 28 / 64$ & & 420 & & & $19 / 9 / 65$ & & 39] & \\
\hline $5 / 30 / 64$ & & 431 & & 多 & $11 / 9 / 65$ & & $\overline{990}$ & \\
\hline $6 / 2 / 84$ & & 498 & & $=$ & $11 / 16 / 65$ & & 362 & \\
\hline $6 / 7 / 64$ & & 446 & & 1 & $11 / 23 / 65$ & & 371 & \\
\hline $6 / 11 / 94$ & & 452 & & a & $11 / 28 / 85$ & & 366 & \\
\hline
\end{tabular}

Data obtalnad from Document FHO-CD-1172 and Waste Statue Summary Reporte. 


\begin{tabular}{|c|c|c|c|c|c|c|c|c|}
\hline \multicolumn{9}{|c|}{ 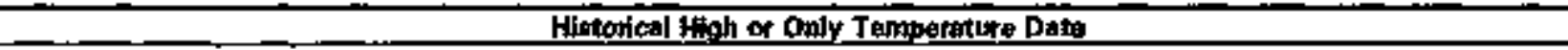 } \\
\hline \multicolumn{9}{|c|}{ Tank 241-A.100 } \\
\hline Dat: & Tomp (C) & $\operatorname{Temp}(F)$ & Comiments & 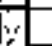 & Dato & Termp $\{c \mid$ & Temp [Fy & Cotmments \\
\hline $12 / 8 / 65$ & & 385 & & & $3 / 1 / 68$ & & 330 & \\
\hline $12 / 14 / 65$ & & 3989 & & t. & $3 / 1 / 68$ & & 327 & \\
\hline $1 / 2 / 66$ & & 378 & & 1 & $3 / 1 / 68$ & & 327 & \\
\hline $1 / 9 / 66$ & & 393 & & $:$ & $3 / 1 / 68$ & & 329 & \\
\hline $1 / 16 / 66$ & & 391 & & : & $4 / 1 / 68$ & & 327 & \\
\hline $1 / 2 \overline{4 / 66}$ & & 386 & & & $4 / 1 / \overline{68}$ & & 926 & \\
\hline $2 / 2 / 6 \theta$ & & 380 & & 1 & $5 / 1 / 68$ & & 330 & \\
\hline $2 / 7 / B B$ & & 380 & & 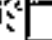 & $5 / 1 / 6 \mathrm{~B}$ & & 330 & \\
\hline $2 / 28 / 66$ & & 375 & & & $5 / 1 / 68$ & & $\overline{3} 25$ & \\
\hline $3 / 21 / 86$ & & 375 & & & $6 / 1 / B \mathrm{~B}$ & & 325 & \\
\hline 3/29/65 & & 372 & & 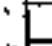 & $7 / 1 / 68$ & & 322 & \\
\hline $4 / 24 / 68$ & & 370 & & 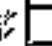 & $7 / 1 / 68$ & & 325 & \\
\hline $5 / 26 / 66$ & & 368 & & & $7 / 1 / 68$ & & 325 & \\
\hline $6 / 3 / 106$ & & 359 & & 1 & $8 / 1 / \bar{\beta}$ & & $\overline{321}$ & \\
\hline 6.f $\$ 4 / 66$ & & $3 \mathrm{B2}$ & & $\therefore$ & $10 / 1 / 68$ & & 321 & \\
\hline $6 \sqrt{21 / 66}$ & & 364 & & 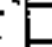 & $1017 \sqrt{68}$ & & $\overline{302}$ & \\
\hline $7 / 1 / 6 \mathrm{~s}$ & & 365 & & 8 & $11 / 1 / \mathrm{s}^{\mathrm{g}}$ & & 303 & \\
\hline $7 / 6 / 6 B$ & & 368 & & 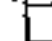 & $32 / 1 / 69$ & & 303 & \\
\hline $7 / 1 \overline{2 / 66}$ & & 364 & & $\therefore$ & $12 / 1 / 60$ & & $\overline{303}$ & \\
\hline $7 / 18 / 66$ & & 961 & & 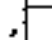 & $12 / 1 / 8 \mathrm{~g}$ & & 304 & \\
\hline $7 / 28 / 66$ & & 355 & & 1 & $12 / 1 / 88$ & & $\overline{313}$ & \\
\hline $8 / 2 / 66$ & & 360 & & 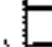 & $1 / 1 / 69$ & & 317 & \\
\hline$\theta / g / \dot{B}$ & & 363 & & s. & $1 / 1 / 69$ & & 303 & \\
\hline $8 / 24 / 66$ & & 361 & & 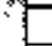 & $2 / 1 / 69$ & & 316 & \\
\hline $8 / 30 / 66$ & & 355 & & 9 & $3 / 1 / 69$ & & 313 & \\
\hline $9 / 7 / 66$ & & 361 & & & $4 \sqrt{6 / 69}$ & & 316 & \\
\hline $12 / 26 / 56$ & & 348 & & 1 & $4 / 1 / 69$ & & 317 & \\
\hline $1 / 1 / 87$ & & 349 & & $\cdot$ & $4 / 1 / 69$ & & 313 & \\
\hline $1 / 8 / 67$ & & 346 & & & $4 / 1 / \sqrt{6}$ & & 318 & \\
\hline $1 / 14 / 67$ & & 345 & & 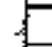 & $5 / 1 / 69$ & & 918 & \\
\hline $1 / 22 / 67$ & & 947 & & $\because$ & $5 / 1 / 69$ & & 313 & \\
\hline $3 / 29 / 67$ & & 347 & & 4 & $5 / / / 69$ & & 312 & \\
\hline $4 / 6 / 67$ & & 935 & & 1 & $5 / 1 / 69$ & & 318 & \\
\hline $4 / 22 / 67$ & & 342 & & 1 & $6 / 5 / 69$ & & 317 & \\
\hline $4 / 26 / 87$ & & 342 & & & $6 / 1 / 69$ & & 293 & \\
\hline $5 / 5 / 67$ & & 336 & & 象 & $8 / 1 / 69$ & & 301 & \\
\hline $5 / 13 / 67$ & & 341 & & & $6 / 1 / 69$ & & 313 & \\
\hline $6 / 4 / 67$ & & 341 & & s. & $B / 1 / 69$ & & 307 & \\
\hline $0 / 10 / 67$ & & 335 & & & $7 / 1 / 69$ & & 300 & \\
\hline $8 / 17 / 67$ & & 339 & & tet & $7 / 1 / 69$ & & 316 & \\
\hline $8 / 26 / 67$ & & 334 & & 㩁 & $7 / t / 69$ & & 317 & \\
\hline $9 / 1 / 67$ & & 335 & & 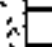 & $9 / 1 / 69$ & & 328 & \\
\hline $11 / 9 / 47$ & & 332 & & & $8 / t / 69$ & & $\overline{328}$ & \\
\hline $19 / 15 / 67$ & & 329 & & $\because$ & $8 / 1 / 69$ & & 333 & \\
\hline $11 / 22 / 67$ & & S30 & & & $8 / 2 / 69$ & & 331 & \\
\hline $12 / 7 / 67$ & & 327 & $\because$ & & $8 / 1 / 69$ & & $\overline{326}$ & \\
\hline $12 / 15 / 67$ & & 332 & & & $8131 / 99$ & & 328 & \\
\hline $12 / 20 / 67$ & & 333 & & : & $9 / 30 / 69$ & & 324 & \\
\hline $12 / 27 / 67$ & & 338 & & 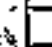 & $10 / 5 / 89$ & & 320 & \\
\hline $1 / 11 / 68$ & & $\$ 26$ & & & $10 / 9 / 69$ & & 315 & \\
\hline $1 / 10 / 68$ & & 328 & & is & 10/12/8 & & 305 & \\
\hline $1 / 27 / 68$ & & 329 & & 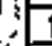 & $10 / 18 / 69$ & & 322 & \\
\hline $2 / 1 \mathrm{AB}$ & & 326 & & $i=1$ & $10 / 31 / 8$ 9 & & 304. & \\
\hline $2 / 1 / 68$ & & 327 & & $=$ & $11 / \overline{6} / 69$ & & $31 T$ & \\
\hline $3 / 1 / \$ B$ & & 329 & & 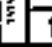 & $11 / 15 / 69$ & & 315 & \\
\hline
\end{tabular}

Osta obtained from Document RHO-CD-1172 and Waste Status Summary Reports. 


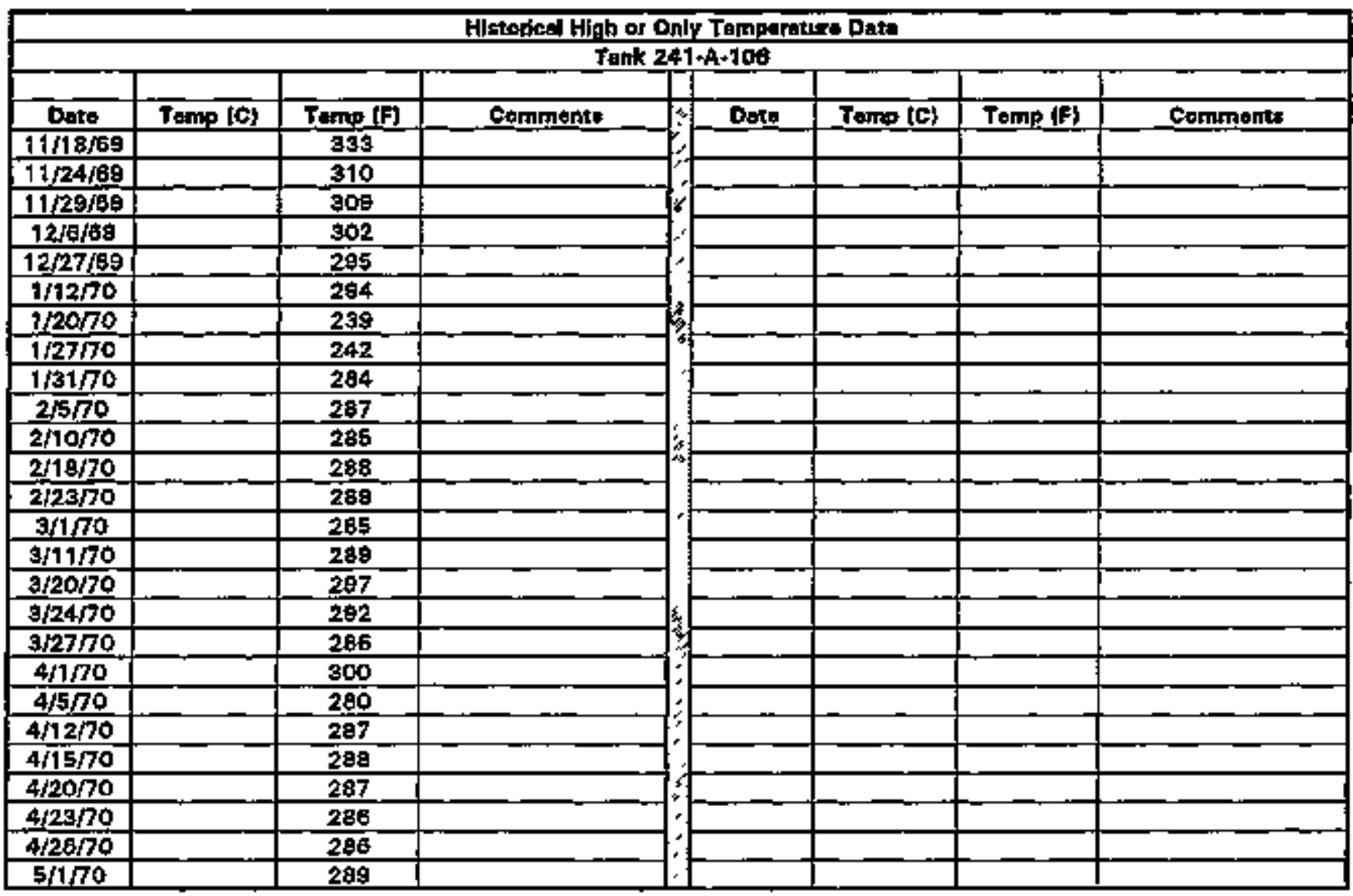

$\therefore:$

Data obtained from Document RHO-CD.1172 and Woste Status Summary Reporta. 


\begin{tabular}{|c|c|c|c|c|c|c|c|}
\hline \multirow{2}{*}{\multicolumn{8}{|c|}{$\begin{array}{l}\text { TAHK .441.A-101 } \\
\text { THEFMOCOWXIE DATA }\end{array}$}} \\
\hline & & & & & & & \\
\hline TAEE $?$ & DATE & THEjatio \# & TAEE SET \# & TEMPC & TEMPF F & $Y=M A \overline{X E M M P}$ & COMMEATS \\
\hline 1 & 2012478 & 1 & 1 & & 78 & $\mathrm{~N}$ & \\
\hline 1 & $10 / 24 / 76$ & 2 & 1 & & $7 a$ & $\mathbf{N}$ & \\
\hline 1 & $\mid 0 / 24 / 76$ & 3 & 1 & & 78 & N & \\
\hline 1 & $10 / 24 / 78$ & 4 & 1 & & 79 & $\mathrm{~N}$ & \\
\hline 1 & to/24/70 & 5 & 1 & & B4 & $\mathbf{N}$ & \\
\hline 7 & 10,2476 & 8 & 7 & & s1 & $\bar{N}$ & \\
\hline 1 & $10 / 24 / 7$ & 7 & 1 & & 82 & $Y$ & \\
\hline$i$ & $30 / 24 / 78$ & $\mathrm{E}$ & 1 & & 92 & $\mathbf{N}$ & \\
\hline 1 & $10124 / 78$ & 宣 & 1 & & 92 & $\mathbf{N}$ & \\
\hline 1 & $10 / 2477$ & 10 & 1 & & 8 息 & $\mathbf{N}$ & \\
\hline 1 & $10 / 24 \sqrt{70}$ & 11 & 1 & & 97 & $\mathrm{~N}$ & \\
\hline 1 & 1012470 & 12 & 1 & & 97 & $\mathbf{N}$ & \\
\hline 1 & $11 / 2070$ & 1 & 1 & & E2 & $\mathbf{N}$ & \\
\hline $\mathrm{i}$ & $11 / 20 / 70$ & 2 & $i$ & & 82 & $N$ & \\
\hline 1 & $11 / 2070$ & 3 & 1 & & 02 & N. & \\
\hline 1 & $11 / 2070$ & 4 & $\frac{7}{2}$ & & 84 & $\mathrm{~N}$ & \\
\hline 1 & $11 / 20 / 70$ & 5 & 1 & & $9 \theta$ & $\mathbf{N}$ & \\
\hline 1 & $11 / 20 / 76$ & 8 & 1 & & a9 & $\mathbf{N}$ & \\
\hline 1 & $11 / 20 / 78$ & $\overline{8}$ & 1 & & 95 & N & \\
\hline 1 & $11 / 20176$ & 皇 & 1. & & 98 & $\dot{y}$ & \\
\hline 1 & $11 / 20 / 70$ & 10 & 1 & & BB & i & \\
\hline 1 & $11 / 20 / 76$ & 91 & 1 & & 95 & N & \\
\hline 1 & 11,20070 & 12 & 1 & & 85 & $\mathbf{N}$ & \\
\hline 1 & $1 / 11777$ & 1 & 1 & & 86 & $\mathbf{N}$ & \\
\hline 1 & $1 / 11 / 77$ & 2 & 1 & & $8 \mathrm{ge}$ & $N$ & \\
\hline 1 & $1 / 11 / 77$ & $\$$ & 1 & & 97 & $Y$ & \\
\hline 1 & $1 / 11 / 72$ & 4 & 1 & & 96 & N & \\
\hline 1 & $1 / 11 / 77$ & 5 & 1 & & 84 & $\mathbf{N}$ & \\
\hline 1 & $1 / 11 / 77$ & B & 1 & & 79 & $\mathbf{N}$ & \\
\hline 9 & $1 / 11 / 77$ & 8 & 1 & & 79 & N & \\
\hline 1 & $1 / 11 / 77$ & $\theta$ & 1 & & 78 & N & \\
\hline$t$ & $1 / 71 / 77$ & 10 & 1 & & 70 & $N$ & \\
\hline 1 & $1 / 1 / 77$ & 17 & 1 & & 79 & $\mathbf{N}$ & \\
\hline 1 & $1 / t 1 / 77$ & 12 & 1 & & 79 & $\mathbf{N}$ & \\
\hline 1 & $2 / 4 / 77$ & 1 & 1 & & $8 \dot{82}$ & N & \\
\hline 1 & $2 / 23 / 77$ & 2 & 1 & & 90 & $\bar{Y}$ & \\
\hline 1 & $2 / 3 / 77$ & 3 & 1 & & $\$$ & $N$ & \\
\hline 1 & $2 \pi+3 \pi 7$ & 4 & 1 & & 50 & $\mathrm{~N}$ & \\
\hline 1 & $2 \pi 372$ & 5 & 1 & & 90 & $\bar{N}$ & \\
\hline 1 & $2 / 18 / 77$ & $\theta$ & 1 & & so & $N$ & \\
\hline 1 & $2 / 13 / 77$ & 8 & 1 & & 90 & $N$ & \\
\hline 1 & $2 n a / 7$ & 3 & 1 & & 80 & $\mathbf{N}$ & \\
\hline 1 & $2 / 13 / 77$ & 10 & 1 & & 89 & $\mathbf{N}$ & \\
\hline$i$ & $2 / 13 / 77$ & 11 & 1 & & 89 & $\mathbf{N}$ & \\
\hline 1 & $2 / 13 / 77$ & 12 & 1 & & 89 & N & \\
\hline 1 & $3 / 26 / 77$ & 1 & 1 & & 92 & $\mathbf{N}$ & \\
\hline 1 & $9 / 25 / 77$ & 2 & 1 & & 103 & $Y$ & \\
\hline 1 & $3 / 25 / 7 / 7$ & 3 & 1 & & 103 & $\mathbf{N}$ & \\
\hline 1 & $3 / 25 / 77$ & 4 & 1 & & 103 & $\mathrm{M}$ & \\
\hline 1 & $3 / 25 / 77$ & 5 & 1 & & 103 & $\bar{n}$ & \\
\hline 1 & $3 / 25 / 77$ & 8 & 1 & & 102 & $\bar{N}$ & \\
\hline 1 & $3 / 25 / 77$ & 7 & 1. & & 102 & $\mathbf{N}$ & \\
\hline 1 & $3 / 25 / 77$ & 8 & 1 & & 102 & $\mathbf{N}$ & \\
\hline 1 & $3 / 25 / 77$ & 10 & 1 & & 102 & $\mathbf{N}$ & \\
\hline 1 & $3 / 26 / 77$ & 17 & 1 & & 102 & N & \\
\hline 1 & $3 / 25 / 77$ & 12 & 1 & & 89 & $\bar{N}$ & \\
\hline 1 & $3125 \mathrm{~m} / \mathrm{h}$ & 13 & 1 & & 98 & $\mathbf{N}$ & \\
\hline 1 & $3 / 25 / 77$ & 14 & 1 & & s8 & $\mathbf{N}$ & \\
\hline 1 & $3 / 26 / 77$ & 15 & 1 & & 97 & N & \\
\hline 1 & $3 / 25 / 77$ & 16 & 1 & & 97 & $\mathbf{N}$ & \\
\hline 1 & $4 / 6 / 77$ & 1 & 1 & & 104 & $\bar{Y}$ & \\
\hline$i$ & $4 / 6 / 77$ & 2 & 1 & & 104 & $\mathbf{N}$ & \\
\hline 1 & $4 / 6 / 77$ & 3 & 1 & & 104 & $N$ & \\
\hline
\end{tabular}

Data obtained fton WHC Surveillence Analysis Computer System (SACS), July १, 1993. 


\begin{tabular}{|c|c|c|c|c|c|c|c|}
\hline \multicolumn{8}{|c|}{ TANIK $245 A-t 01$} \\
\hline & & & & THE & IDCOUPI & DATA & \\
\hline TAEE * & DATE & THERAMO \# & THEE GET \# & TEMP C & TEMP F & $Y=$ haX TIMF & COMMEHT: \\
\hline 1 & $A+B \cap 7$ & 4 & 1 & & 104 & $\mathbf{N}$ & \\
\hline 1 & $4 / 6 / 77$ & E & 1. & & 104 & N & \\
\hline 1 & $4 / 6 n 77$ & 6 & 1 & & 104 & $\mathbf{N}$ & \\
\hline 1 & $4 / 6 / 77$ & 8 & 1 & & 704 & $\mathbf{N}$ & \\
\hline 1 & $4 / 6 / 77$ & 8 & 1 & & tas & N & \\
\hline 1 & $4 / 1 / 77$ & 10 & 1 & & 99 & N & \\
\hline 1 & $4 / 6 \sqrt{7}$ & 31 & 3 & & 留 & $N$ & \\
\hline 1 & $4 / 9 / 77$ & 12 & 1 & & 是 & $\mathbf{N}$ & \\
\hline 1 & $4 / 0 / 77$ & 13 & 1 & & 89 & $\mathbf{N}$ & \\
\hline 1 & $4 / 6 / 77$ & 74 & 1 & & 99 & $\mathbf{N}$ & \\
\hline 1 & $4 / 0 / 77$ & 15 & 1 & & 99 & $\mathbf{N}$ & \\
\hline 1 & A/6/7 & 10 & 1 & & 99 & $\mathbf{N}$ & \\
\hline 1 & $5 / 2877$ & 1 & 1 & & 119 & $\mathbf{N}$ & \\
\hline 1 & $5 \longdiv { 2 9 7 7 }$ & 2 & 1 & & 125 & $\mathbf{N}$ & \\
\hline 1 & $5 / 28 / 77$ & 3 & 1 & & 130 & $\bar{Y}$ & \\
\hline 1 & $3 / 29 \sqrt{77}$ & 4 & 1 & & 130 & $\mathbf{N}$ & \\
\hline 1 & $5 / 29 / 77$ & 5 & 1 & & 128 & $\overline{\mathbf{N}}$ & \\
\hline 1 & E/29/77 & (a) & 1 & & 128 & $\mathbf{N}$ & \\
\hline 1 & 612877 & B & 1 & & $\$ 28$ & $\mathbf{N}$ & \\
\hline 1 & $6 \sqrt{29 / 77}$ & 9 & 1 & & 127 & $\mathbf{N}$ & \\
\hline 1 & $5 \sqrt{28,77}$ & 10 & 1 & & 114 & $\mathbf{N}$ & \\
\hline 1 & $5 / 28 / 7$ & 71 & $\boldsymbol{T}$ & & 99 & W & \\
\hline 1 & $6 / 28 \sqrt{77}$ & $\sqrt{2}$ & 1 & & 99 & $\mathbf{N}$ & \\
\hline 1 & $5 \sqrt{28 \sqrt{77}}$ & t5 & 1 & & 99 & $\mathbf{N}$ & \\
\hline 1 & $6 / 217$ & 14 & $\overline{1}$ & & 99 & $\mathbf{N}$ & \\
\hline 1 & $5 / 28 \sqrt{77}$ & 15 & 1 & & 白 & $\mathbf{N}$ & \\
\hline 1 & $5 / 28 \sqrt{77}$ & 70 & 1 & & 99 & $\mathbf{M}$ & \\
\hline 1 & a/10/7 & 1 & 1 & & 121 & $\mathbf{N}$ & \\
\hline 1 & drol7 & 2 & $j$ & & 126 & $\mathbf{N}$ & \\
\hline$t$ & $0 / 1077$ & 3 & 1 & & 130 & $\mathbf{N}$ & \\
\hline 1 & $0 / 10 / 7$ & 4 & 1 & & 131 & $Y$ & \\
\hline 7 & 9/10/7 & B & 1 & & 131 & $N$ & \\
\hline 1 & $8 / 10 \sqrt{7}$ & 6 & $\mathbf{T}$ & & 131 & $\mathbf{N}$ & \\
\hline 1 & Byph & 8 & 1 & & 130 & $\mathbf{N}$ & \\
\hline 1 & Q15017 & $\underline{9}$ & 1 & & 131 & $N$ & \\
\hline 1 & $6 / 1077$ & 10 & 1 & & 129 & $\mathbf{N}$ & \\
\hline 1 & $6 / 1077$ & 11 & 1 & & 110 & $\mathbf{N}$ & \\
\hline 1 & $9 / 10 / 77$ & 12 & 1 & & 102 & $\mathbf{N}$ & \\
\hline 1 & $8 / 10 / 77$ & 13 & 1 & & 102 & $\mathbf{N}$ & \\
\hline 1 & 8 Mon & 14 & 1 & & 102 & $\mathbf{N}$ & \\
\hline i & 71077 & 1 & 1 & & 123 & $\mathbf{N}$ & \\
\hline 1 & $7 / 10 / 77$ & 2 & 1 & & 127 & $\mathbf{N}$ & \\
\hline 1 & $7 / 10 / 77$ & 3 & 1 & & 130 & $\mathbf{N}$ & \\
\hline 1 & $7 / 18 \% 7$ & 4. & 1 & & 137 & $\bar{y}$ & \\
\hline 1 & $7 \pi 677$ & $\overline{6}$ & 1 & & 130 & $\mathbf{N}$ & \\
\hline 1 & 719077 & $\theta$ & 1 & & 129 & $\mathbf{N}$ & \\
\hline 1 & $7 / 16 / 77$ & 8 & $T$ & & 128 & $\overline{\mathbf{N}}$ & \\
\hline 1 & $7 / 16 / 77$ & 9 & 1 & & 129 & $\mathbf{N}$ & \\
\hline 7 & $7 \pi \operatorname{\theta i77}$ & $\overline{10}$ & 1 & & 129 & $\mathbf{N}$ & \\
\hline 1 & $7 / 6 \sqrt{7}$ & 11 & 1 & & 127 & $\mathbf{N}$ & \\
\hline 1 & $7 / 10 / 77$ & 12 & 1 & & 127 & $\mathbf{N}$ & \\
\hline 1 & $7 / 18 / 7$ & 13 & 2 & & 125 & $\mathbf{N}$ & \\
\hline 1 & $7 \pi 677$ & 14 & 1 & & 121 & $\mathrm{~N}$ & \\
\hline 1 & $7 / 22 \sqrt{7}$ & 1 & 1 & & 120 & $N$ & \\
\hline 1 & $7 / 22 / 77$ & 2 & 1 & & 124 & $\mathbf{N}$ & \\
\hline 1 & $7 / 2277$ & 3 & 1 & & 127 & $\mathbf{N}$ & \\
\hline 1 & $7 / 22 / 77$ & 4 & 1 & & 129 & $\mathbf{Y}$ & \\
\hline 1 & $7 / 22 / 77$ & 5 & 1 & & 128 & $N$ & \\
\hline 1 & $7 / 22 / 77$ & 8 & 1 & & $12 \overline{6}$ & $\mathbf{N}$ & \\
\hline 1 & $7 / 22 / 77$ & $\mathbf{8}$ & 1 & & 125 & $\mathbf{N}$ & \\
\hline 1 & $3 / 22 / 77$ & 9 & 1 & & 125 & $N$ & \\
\hline 1 & $7 / 2 / 77$ & 10 & 1 & & 125 & $\mathbf{N}$ & \\
\hline 1 & $7 / 22 / 77$ & 11 & 1 & & 125 & $N$ & \\
\hline
\end{tabular}

Data ohtained from WHC Surveillance Analysis Computer System (SACS), July 1, 1993. 


\begin{tabular}{|c|c|c|c|c|c|c|c|}
\hline \multicolumn{8}{|c|}{ TANO 261-A-101 } \\
\hline TrRE & DATE & THEANO : & TREE \&ET & TEMP C & TERP F & $Y=$ MAX TESAF & COMMENTS \\
\hline 1 & $7 \sqrt{22} 27$ & 12 & $1=$ & & 725 & $\mathbf{N}$ & \\
\hline$t$ & $7 / 22 / 77$ & 13 & 1 & & 123 & $\mathbf{N}$ & \\
\hline 1 & $7 / 22 / 77$ & 14 & 1 & & 121 & $\mathbf{N}$ & \\
\hline 1 & $7 / 29 / 77$ & 1 & 1 & & 124 & $\mathbf{N}$ & \\
\hline 1 & 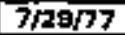 & $\overline{2}$ & 1 & & 928 & $\mathbf{N}$ & \\
\hline 1 & $729 \sqrt{7}$ & 8 & 1 & & 139 & $\mathbf{N}$ & \\
\hline 1 & $7 / 29 / 77$ & 4 & 1 & & 132 & $\gamma$ & \\
\hline 1 & 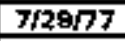 & 5 & $i$ & & 131 & $\mathbf{N}$ & \\
\hline 1 & $7 / 29 \sqrt[77]{7}$ & 8 & I & & 128 & $\mathbf{N}$ & \\
\hline 1 & $729 \sqrt{77}$ & 8 & 5 & & 129 & $\mathbf{N}$ & \\
\hline 1 & 712977 & 9 & 1 & & 129 & W. & \\
\hline 1 & $7 / 29 / 77$ & 10 & 1 & & 129 & $\mathbf{N}$ & \\
\hline 1 & $7 / 28 / 77$ & 11 & 1 & & 120 & N & \\
\hline 1 & $7 / 29 / 77$ & 12 & $\mathbf{1}$ & & 138 & $\mathbf{N}$ & \\
\hline J & $7 / 2 \mathrm{en} 7$ & 13 & 1 & & 120 & $\bar{N}$ & \\
\hline 1 & $7 / 29 / 77$ & 14 & 1 & & $12 \overline{4}$ & $N$ & \\
\hline 1 & 배택ㄱ & 1 & 1 & & 113 & $\mathbf{N}$ & \\
\hline 1 & erter77 & $\underline{2}$ & 1 & & $1 \overline{17}$ & $\mathbf{N}$ & \\
\hline 3 & g/1387 & 3 & 1 & & $12 \overline{3}$ & $\mathbf{H}$ & \\
\hline 1 & 9/18177 & 4 & 1 & & 126 & $\gamma$ & \\
\hline 1 & E/18/7 & 5 & 1 & & 123 & $\mathbf{N}$ & \\
\hline 1 & E/1E/77 & $\theta$ & 1 & & 122 & $\mathbf{N}$ & \\
\hline 1 & a/va/7 & 7 & 1 & & 10日 & $\mathbf{N}$ & \\
\hline ב & g/า8户 & $\mathbf{g}$ & 1 & & 122 & $\mathbf{N}$ & \\
\hline 1 & 9/1\%77 & $g$ & 1 & & 121 & $\mathbf{N}$ & \\
\hline 1 & g/18/77 & 10 & 1 & & 121 & $\mathbf{N}$ & \\
\hline 1 & 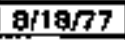 & $\$ 1$ & 1 & & 148 & $\mathbf{N}$ & \\
\hline$i$ & $8 / 28 / 77$ & 12 & 1 & & 118 & $\mathbf{N}$ & \\
\hline 1 & 8/38/77 & 13 & 1 & & 117 & $\mathbf{N}$ & \\
\hline 1 & 9/18/7? & 14 & 1 & & 119 & $\mathbf{N}$ & \\
\hline 1 & $8 / 18 / 77$ & 15 & 1 & & $10 \overline{4}$ & $\bar{N}$ & \\
\hline 1 & $8 / 19 \sqrt{77}$ & 36 & 1 & & 91 & $\mathbf{N}$ & \\
\hline 1 & 9/3077 & 1 & 1. & & 130 & $\mathbf{N}$ & \\
\hline 1 & Bssor7 & 2 & 1 & & 134 & $\mathbf{N}$ & \\
\hline 1 & $8 / 30 / 77$ & 3 & 1 & & 136 & $\bar{\gamma}$ & \\
\hline 1 & $8 / 30 / 77$ & 4 & 1 & & 130 & $\mathbf{N}$ & \\
\hline 1 & 9/30/7 & 5 & 1 & & 135 & $\mathbf{N}$ & \\
\hline 1 & 9/3077 & $\theta$ & 1 & & 135 & $\mathbf{N}$ & \\
\hline 1 & Brapof7 & 8 & 1 & & 134 & $\mathbf{N}$ & \\
\hline 1 & asol7 & $\theta$ & 1 & & 134 & $\mathbf{N}$ & \\
\hline 1 & a/sob7 & 10 & 1 & & 133 & N & \\
\hline 1 & assor7 & 11 & 1 & & 133 & $\mathbf{N}$ & \\
\hline 1 & $8 / 30 / 77$ & 12 & 1 & & 133 & N & \\
\hline 1 & $8 / 30 / 77$ & 13 & 1 & & 132 & $\mathbf{N}$ & \\
\hline 1 & a/sor7 7 & 14 & 1 & & 127 & $\mathbf{N}$ & \\
\hline 1 & 9/30/77 & 15 & 1 & & 118 & $\mathbf{N}$ & \\
\hline 1 & a/30/77 & 26 & 1 & & 105 & $\mathbf{N}$ & \\
\hline 1. & ent7 & 1 & 1 & & 132 & $\mathbf{N}$ & \\
\hline$\$$ & gin? & 2 & 1 & & 136 & $\mathbf{N}$ & \\
\hline 1 & $8 / 7 / 77$ & 3 & 1 & & 137 & $\mathbf{Y}$ & \\
\hline 1 & $97 / 77$ & 4 & 1 & & 136 & $\mathbf{N}$ & \\
\hline$\pi$ & e/m7 & 5 & d: & & 136 & $\mathbf{N}$ & \\
\hline 1. & $917 / 77$ & 0 & 1 & & 136 & $\mathbf{N}$ & \\
\hline 1 & g/7/77 & 息 & 1 & & 135 & $N$ & \\
\hline 5 & $9 / 7 / 77$ & 9 & 1 & & 134 & $\mathbf{N}$ & \\
\hline 1 & $8 / 7 / 77$ & 10 & 1 & & 134 & $\mathbf{M}$ & \\
\hline 1 & $9 / 7 / 77$ & 11 & 1 & & 134 & $\mathbf{N}$ & \\
\hline 1 & spin & 12 & 1 & & 132 & $N$ & \\
\hline 7 & 9 7177 & 13 & 1 & & 128 & $N$ & \\
\hline 1 & $8 / 7 / 7$ & 14 & 1 & & 118 & $\mathbf{N}$ & \\
\hline 1 & $9 / 7 / 73$ & 15. & 1 & & 104 & $\mathbf{N}$ & \\
\hline 1 & $9 / / 77$ & 16 & 1 & & 104 & $\mathbf{N}$ & \\
\hline 1 & $9 / 12 / 77$ & 1 & 1 & & 130 & $\mathbf{N}$ & \\
\hline
\end{tabular}

Data obtained from WHC Surveillance Analysis Computer SYstem (SACS), knity 1, 1993. 


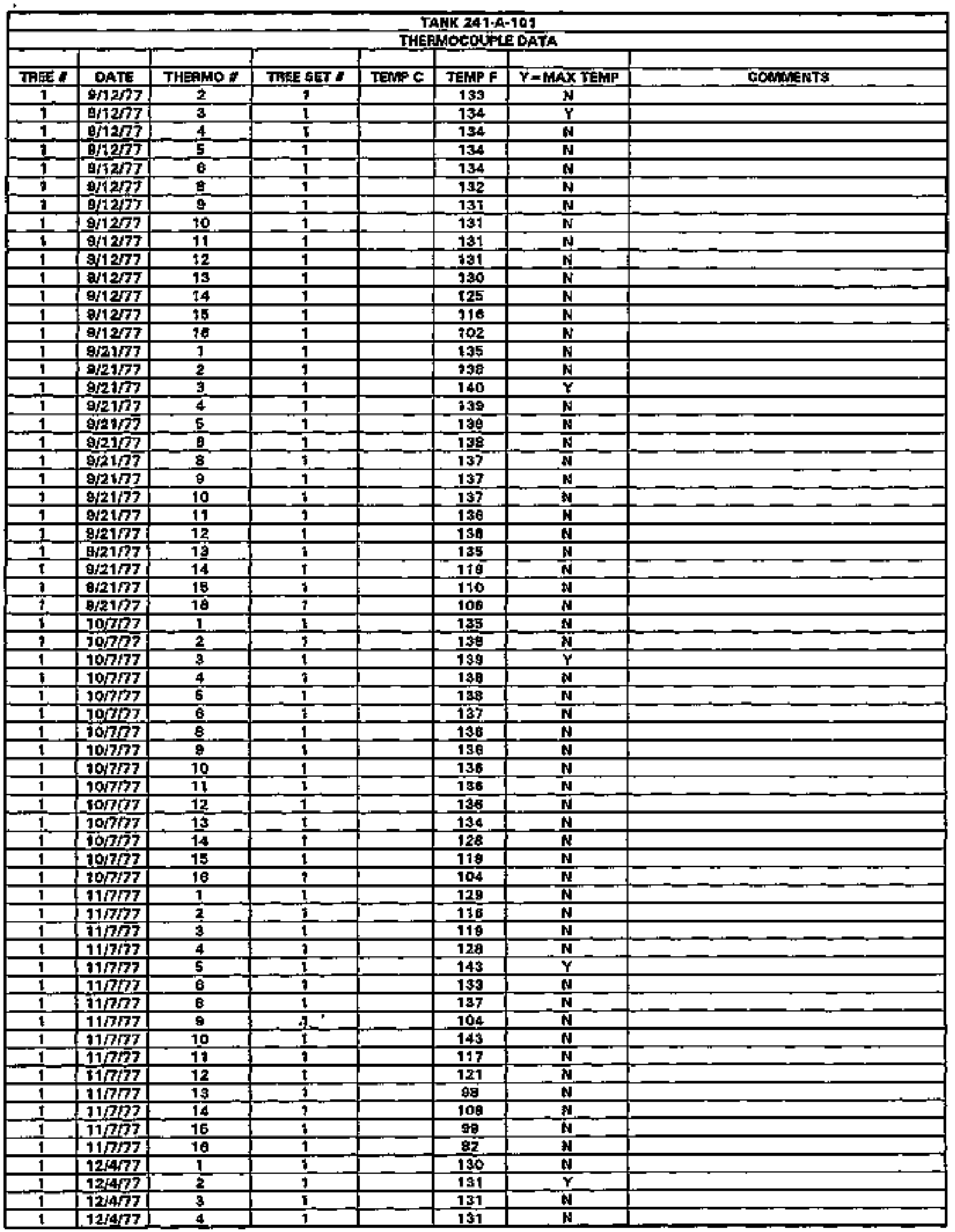

Data obtelned from WHC Surveillance Analysis Computer System [SACS), Juk 1. 1993. 


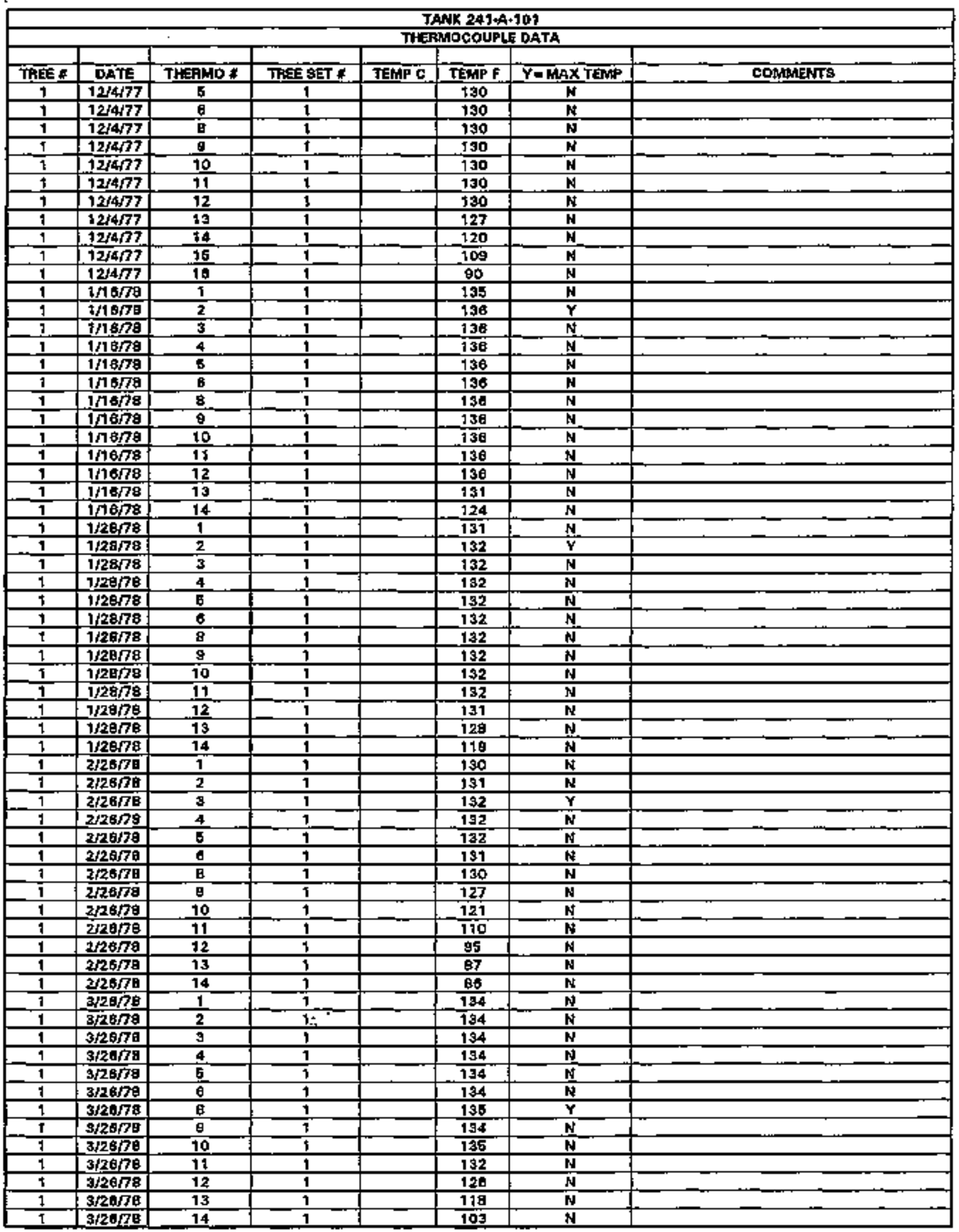

Data obtained from WHC Surveillance Analysis Computer System (SACS), July 1, 1993. 


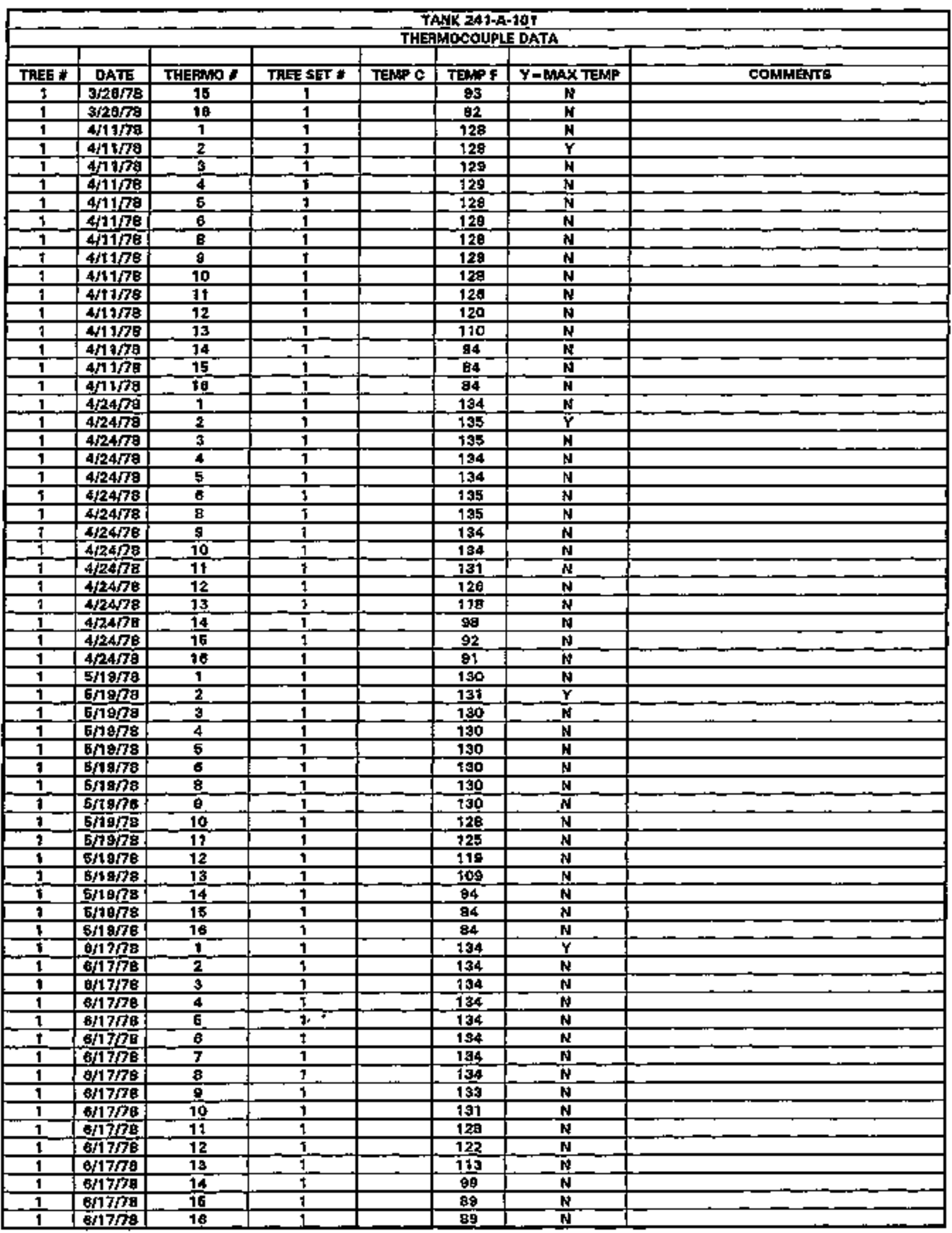

Data obtained from WHC Surveillance Analysis Computer System (SACSI, July 1, 1993.

D-115 


\begin{tabular}{|c|c|c|c|c|c|c|c|}
\hline \multicolumn{8}{|c|}{ TANK 241+A+101 } \\
\hline & & & & THE & & & \\
\hline TREE 8 & DATE & THEFAMO \# & TAEE \&ET * & TEMP C & TaMPF & $Y=M A X T B M P$ & COMMVIFNTA \\
\hline 1 & $375 / 78$ & 1 & 1 & & 130 & $\mathrm{k}$ & \\
\hline 1 & $7 / 15 / 78$ & 2 & 1 & & $13 t$ & $Y$ & \\
\hline 1 & $7 / 15 \%$ & E & 1 & & 131 & N & \\
\hline$\overline{7}$ & $715 / 7 \mathrm{E}$ & g & $\overline{1}$ & & 130 & $\bar{N}$ & \\
\hline $1^{2}$ & $7 / 5 \pi \mathrm{e}$ & 10 & 1 & & $\overline{28}$ & 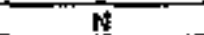 & \\
\hline$T$ & $7 / 15 / 79$ & 11 & 1 & & 104 & $\mathbf{N}$ & \\
\hline$\overline{1}$ & $7 / 15 / 7 \theta$ & 12 & $t$ & & 918 & $\overline{\mathbf{H}}$ & \\
\hline 1 & $7 1 6 \longdiv { 7 8 }$ & 13 & 1 & & 110 & $\mathbf{N}$ & \\
\hline$t$ & $7115 / 79$ & 14 & 7 & & 연 & N & \\
\hline 1 & $7 / 15 / 79$ & 15 & 1 & & 喝 & $\mathbf{N}$ & \\
\hline 1 & $7 / 15 / 78$ & 18 & 1 & & 99 & $\mathbf{N}$ & \\
\hline $\boldsymbol{t}$ & $8 / 13 / 78$ & 1 & $\dagger$ & & 130 & $\mathbf{Y}$ & \\
\hline 1 & $8 / 1379$ & 2 & 1 & & 130 & N & \\
\hline 1 & $8 / 13 / 79$ & 3 & 1 & & $\sqrt{30}$ & $\bar{H}$ & \\
\hline 1 & $8 / 13 / 79$ & 4 & 1 & & 130 & $\mathbf{N}$ & \\
\hline 1 & 87138 & 5 & 9 & & 130 & $\mathbf{W}$ & \\
\hline 1 & $8 / 1378$ & E & $\frac{1}{1}$ & & 129 & $\overline{\mathbf{N}}$ & \\
\hline 1 & $8 / 1378$ & 白 & 1 & & $\overline{12 \theta}$ & $\mathbf{N}$ & \\
\hline 1 & $6 / 13 / 78$ & e & $\$$ & & $\overline{125}$ & $N$ & \\
\hline 1 & a/138 & 10 & 1 & & 118 & $\mathbf{N}$ & \\
\hline 1 & $8 / 1378$ & 51 & 1 & & 111 & $\mathbf{H}$ & \\
\hline 1 & $8 / 1 3 \longdiv { 7 8 }$ & 12 & 1 & & 102 & $\mathbf{N}$ & \\
\hline 1 & $6 / 1378$ & 13 & 7 & & 53 & $\mathbf{N}$ & \\
\hline 1 & $613 / 78$ & 14 & $\frac{1}{1}$ & & $\overline{93}$ & N & \\
\hline 1 & B/13/78 & 15 & 1 & & 93 & $\overline{\mathbf{N}}$ & \\
\hline 1 & 9/13n & 16 & 1 & & 83 & $\overline{\mathbf{N}}$ & \\
\hline 1 & g/g/7 & 1 & 1 & & 139 & $\overline{\mathbf{Y}}$ & \\
\hline 1 & $9 / 9 / 78$ & 2 & 1 & & 139 & $N$ & \\
\hline 1 & $9 / 9 / 78$ & $\mathbf{s}$ & 1 & & 139 & $N$ & \\
\hline 1 & 9lape & 4 & 1 & & 130 & $N$ & \\
\hline$i$ & 9/977 & 5 & 1 & & 135 & $N$ & \\
\hline$\$$ & gratg & 8 & 1 & & 128 & $\mathbf{N}$ & \\
\hline 1 & $9 \sqrt{9 / 78}$ & e & 1 & & 107 & $\mathrm{~N}$ & \\
\hline 1 & $9 / 9 \sqrt{79}$ & 9 & 1 & & 103 & $\mathrm{~N}$ & \\
\hline 1 & 9н부그. & 10 & 1 & & 105 & $\mathbf{N}$ & \\
\hline$\$$ & gignes & 11 & 7 & & 103 & $\mathbf{N}$ & \\
\hline 1 & $9 / 9 \sqrt{79}$ & 12 & 1. & & $\overline{103}$ & $N$ & \\
\hline 1 & 9/9/79 & 13 & 1 & & 103 & $N$ & \\
\hline 1 & $9 / 9 / 78$ & 14 & 1 & & $8 \theta$ & N & \\
\hline 1 & $9+979$ & 15 & 1 & & 99 & k & \\
\hline 1 & 91978 & 18 & 1 & & 99 & $N$ & \\
\hline \pm & 10ק $7 \mathrm{~B}$ & 1 & 1 & & 132 & N & \\
\hline 1 & 108779 & 2 & 1 & & 133 & $Y$ & \\
\hline 1 & $10 / 8 / 78$ & 3 & 1 & & 133 & $\mathbf{N}$ & \\
\hline 1 & 10/a/70 & 4 & 1 & & 130 & $\mathbf{N}$ & \\
\hline$t$ & torif & 5 & 1 & & 128 & N & \\
\hline 1 & $108 / 7)^{2}$ & 6 & 1 & & 118 & $\mathbf{N}$ & \\
\hline 1 & to/B/78 & B & 1 & & 96 & N & \\
\hline 7 & 10/8/7 & 9 & 1 & & 99 & $N$ & \\
\hline 1 & $10 \mathrm{~B} / \mathrm{B}$ & 10 & 1 & & E9. & $N$ & \\
\hline 1 & tora/78 & 11 & 1 & & 89 & $\mathrm{~N}$ & \\
\hline$?$ & 10/a/7a & 12 & is & & ag & $N$ & \\
\hline$i$ & $1087 \overline{78}$ & 13 & 1. & & 89 & N. & \\
\hline 7 & 10泉7 & 14 & 1 & & 89 & $N$ & \\
\hline 1 & $10 / 2 / 78$ & 16 & 1 & & 80 & $\mathbf{N}$ & \\
\hline 1 & 10/a/7B & 16 & 1 & & 89 & $N$ & \\
\hline 1 & $11 / \mathrm{a} / \mathrm{B}$ & 1 & 1 & & 139 & N & \\
\hline 1 & $11 / \overline{78}$ & 2 & 1 & & 158 & $\mathbf{H}$ & \\
\hline 7 & $11 / \mathrm{A} 7 \mathrm{~B}$ & 3 & 1 & & 137 & $Y$ & \\
\hline 1 & $11 \mathrm{ra} / \mathrm{B}$ & 4 & 1 & & 134 & $\mathbf{N}$ & \\
\hline 1 & $11 / 6 / 78$ & 5 & 1 & & 128 & $\mathbf{N}$ & \\
\hline 3 & $11 / \mathrm{H} / \mathrm{Ta}$ & 5 & 1 & & 122 & $\mathbf{N}$ & \\
\hline 1 & $11 / \mathrm{d} / 8$ & a & 1 & & 90 & $\mathbf{N}$ & \\
\hline
\end{tabular}

Data obtained from WHC Surveillance Analysis Computer System [SACS\}, July 1, 1993. 


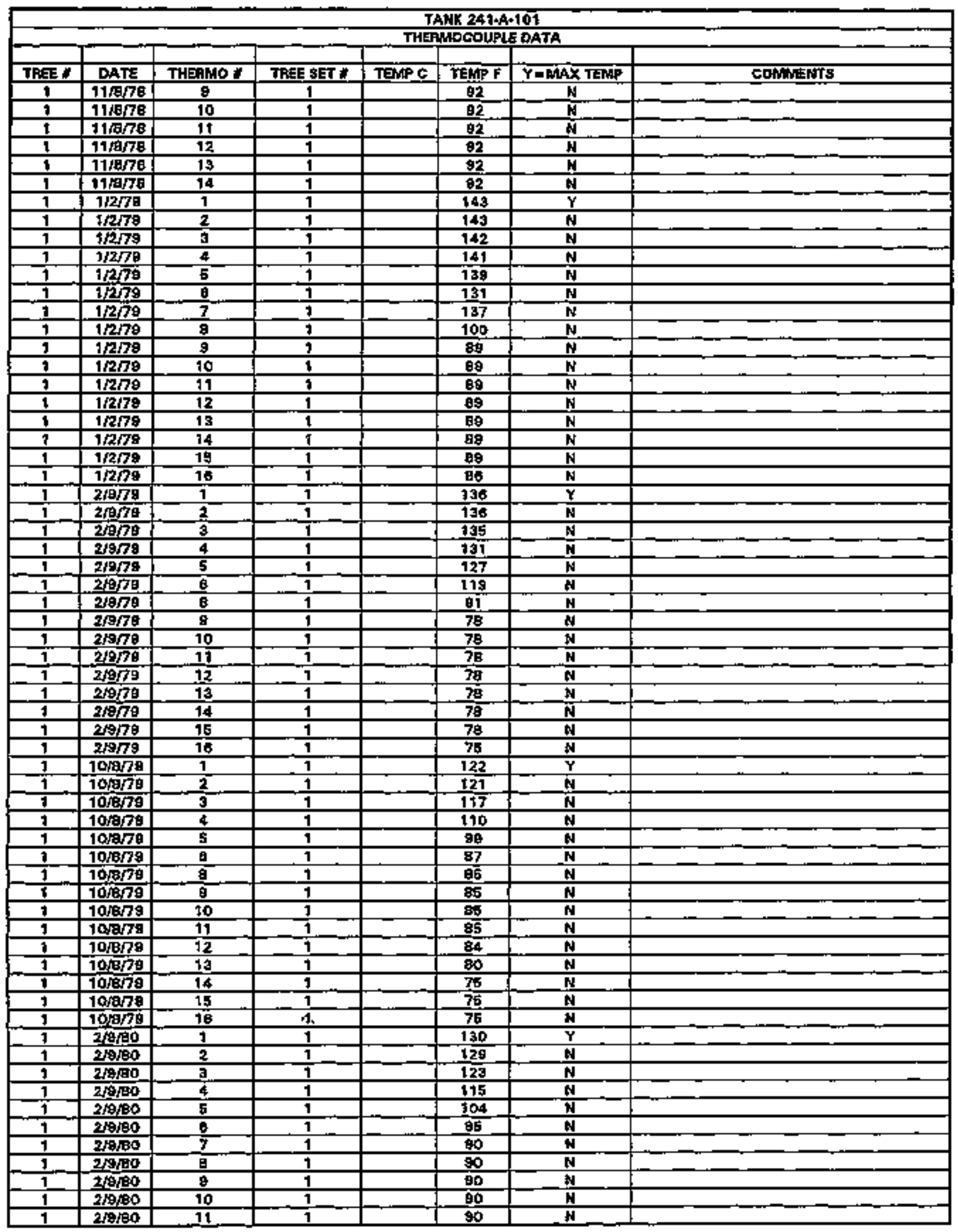

Data obtained from WHC Strveillance Analysis Computer System ISACSh, July 1, 1993. 
WHC-SD-WM-ER-308, REV, D

\begin{tabular}{|c|c|c|c|c|c|c|c|}
\hline \multicolumn{8}{|c|}{ TANM 247-A+101 } \\
\hline & & - & & THF & AOCOUPL & DATA & \\
\hline & & & & & & & \\
\hline & WALE & & & & & & \\
\hline 1 & $2 / 9190$ & 12 & 1 & & 90 & $N$ & \\
\hline 1 & 21980 & 13 & 1 & & 90 & $\mathbf{N}$ & \\
\hline 1 & $2 \mu 000$ & 14 & 1 & & 80 & $\overline{\mathbf{N}}$ & \\
\hline 1 & 29100 & 18 & 1 & & 90 & $\bar{N}$ & \\
\hline$\overline{1}$ & $2 / 90$ & 16 & $\overline{1}$ & & 90 & $\mathbf{N}$ & \\
\hline$\overline{1}$ & $10 / 26 / \mathrm{BO}$ & 1 & 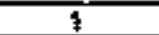 & & 123 & \$ & \\
\hline$\overline{\mathbf{T}}$ & $10 / 28 / 80$ & 2 & $\overline{1}$ & & 118 & $n_{*}^{*}$ & \\
\hline 1 & $10 / 28 \mathrm{sec}$ & 3 & 1 & & $12 \overline{7}$ & $\mathbf{N}$ & \\
\hline$\overline{1}$ & $10 / 28 / 80$ & 4 & 9 & & 124 & $\mathbf{N}$ & \\
\hline 1 & $10426 / 20$ & 5 & 1 & & 132 & N & \\
\hline 1 & 10/26/10 & $\theta$ & 7 & & 329 & $\mathbf{M}$ & \\
\hline 1 & $10 / 28 / 80$ & $\overline{7}$ & $\bar{T}$ & & 738 & $\bar{n}$ & \\
\hline 1 & $10 / 28 \pi 80$ & $\mathrm{~B}$ & 1 & & $13 \overline{8}$ & $N$ & \\
\hline 7 & $10 / 2 \mathrm{~s} / \mathrm{e}$ & 9 & 1 & & $14 \overline{8}$ & $\bar{N}$ & \\
\hline 1 & T0/26/80 & 10 & 1 & & 143 & $\mathbf{M}$ & \\
\hline 1 & $10 / 25 / 80$ & 11 & 7 & & 148 & $\mathbf{Y}$ & \\
\hline 1 & $10 / 28 \sqrt{-1} 0$ & 12. & 1 & & 743 & $\bar{N}$ & \\
\hline 1 & $10 / 26 / 30$ & 13 & 9 & & 148 & $\mathbf{N}$ & \\
\hline $\mathbf{I}$ & $10 / 26 / 80$ & 14 & $\mathbf{T}$ & & 130 & $\mathbf{N}$ & \\
\hline $\mathbf{1}$ & $10 / 26100$ & 15 & 1 & & 121 & $\mathbf{M}$ & $\bar{T}$ \\
\hline 1 & $70 / 28 / 60$ & 16 & 5 & & 117 & $\mathbf{n}$ & \\
\hline 1 & $1 / 18,81$ & 1 & 1 & & 226 & $\overline{\mathbf{N}}$ & \\
\hline 1 & 1/ara1 & 2 & 1 & & $13 \overline{3}$ & $\mathbf{N}$ & \\
\hline 1 & I/A/B1 & 3 & 1 & & $19 \bar{B}$ & $\bar{N}$ & \\
\hline 1 & $1 / 9 / 81$ & 4 & 1 & & 143 & M & \\
\hline 1 & $1 / 981$ & 5 & 1 & & $\$ 44$ & $\mathrm{~N}$ & \\
\hline 1 & $1 / 8 / 81$ & 6 & 5 & & 745 & $\bar{Y}$ & \\
\hline 1 & $1 / 9 / 31$ & 7 & 7 & & $74 \ddot{5}$ & $\mathbf{N}$ & \\
\hline 1 & $1 / 9 / 81$ & 8 & 1 & & 145 & H & \\
\hline 1 & $1 / 9 / 81$ & 9 & 1 & & 146 & H & \\
\hline 1 & $1 / 9 \sqrt{81}$ & 10 & 1 & & 146 & $\mathbf{M}$ & \\
\hline 1 & $1 / 9 / 81$ & 11 & 1 & & 144 & N & \\
\hline 1 & 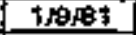 & 12 & 1 & & $14 \sqrt{3}$ & $\mathbf{N}$ & \\
\hline 1 & 1/9/8? & 13 & 1 & & $13 \overline{5}$ & $\bar{n}$ & \\
\hline 1 & $1 / 9 / 89$ & 14 & 1 & & 128 & $M$ & \\
\hline 1 & $1 / 9 / 81$ & 15 & 5 & & 106 & M & \\
\hline 1 & $1 / 9 / 81$ & 18 & 1 & & 90 & $\mathbf{M}$ & \\
\hline 1 & $2 / 8 / B !$ & 1 & 1 & & $12 \overline{8}$ & $\bar{N}$ & \\
\hline 1 & 26 et & 2 & 1 & & 133 & $\bar{N}$ & \\
\hline 1 & $2 / 8 / \sqrt{81}$ & 3 & 7 & & 138 & $\mathbf{M}$ & \\
\hline 1 & $2 / 8 / 81$ & 4 & 1 & & 141 & $\mathbf{M}$ & \\
\hline 1 & 3/6/8t & 5 & 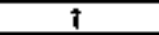 & & 142 & N & \\
\hline 1 & $2 / 6 / 61$ & T & 1 & & 142 & $\mathbf{N}$ & \\
\hline 1 & $2 / 8 \sqrt{81}$ & 7 & 1 & & 143 & $Y$ & \\
\hline 1 & $2 /$ 自右1 & 8 & 7 & & $\$ 4 \overline{2}$ & N & \\
\hline 1 & $2 / 9 / 81$ & 9 & 1 & & 143 & $\mathbf{N}$ & \\
\hline 1 & $2 / 6 / E)$ & 10 & 1 & & 142 & $\mathbf{N}$ & \\
\hline 1 & $2 / 5 / 81$ & 11 & 1 & & 140 & $\mathbf{N}$ & \\
\hline 1 & $2 / 8 \sqrt{81} 1$ & $\sqrt{12}$ & 7 & & $\overline{40}$ & 편 & \\
\hline 1 & $2 / B / 81$ & 13 & 1 & & 135 & N & \\
\hline 1 & $20 / 81$ & 14 & 1 & & 122 & $\mathbf{N}$ & \\
\hline 1 & $2 / 6 \sqrt{81}$ & 15 & ti & & 103 & $\mathbf{N}$ & \\
\hline 1 & $2 / \theta / 81$ & 16 & 1 & & 81 & $\mathbf{N}$ & \\
\hline 1 & $2 / 23 / 81$ & 1 & 1 & & 145 & $\mathbf{N}$ & \\
\hline$i$ & $2 / 23 \times 91$ & 2 & $\overline{1}$ & & 148 & $N$ & \\
\hline 1 & $2 / 23 / 81$ & 3 & 1 & & 153 & $\mathbf{N}$ & \\
\hline 1 & $2 / 23 / 81$ & 4 & 1 & & 156 & $\mathbf{Y}$ & \\
\hline 1 & $2 / 23 / 81$ & 5 & 1 & & $1 \overline{55}$ & $\mathbf{N}$ & \\
\hline 1 & $2 / 23 \bar{\theta}_{1}$ & $\bar{B}$ & $\overline{1}$ & & $1 \overline{6 B}$ & N & \\
\hline 1 & $2 / 23 / 91$ & 7 & 1 & & 15自 & $\mathbf{N}$ & \\
\hline 1 & $2 / 23 / 81$ & B & 1 & & $1 \overline{55}$ & $\mathbf{N}$ & \\
\hline 1 & $2 / 23 / 91$ & 9 & $\overline{1}$ & & $1 \overline{68}$ & $\mathbf{N}$ & \\
\hline t & $2 / 2391$ & 10 & 1 & & 158 & $\bar{N}$ & \\
\hline
\end{tabular}

Data obtained frotn WHC Surveillance Analysis Computer System (SACS), July 1, 1993. 


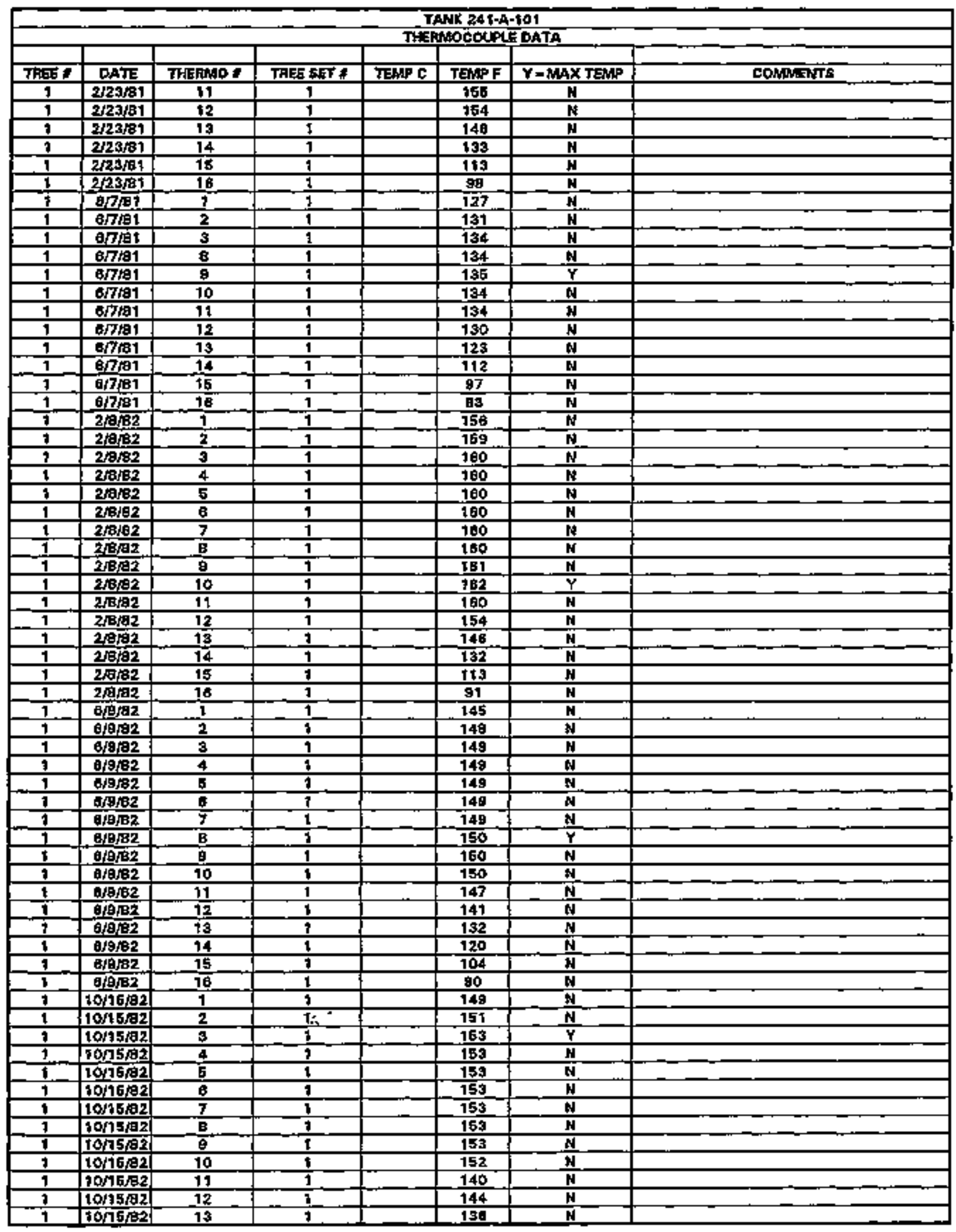

Date obtained from Whic Surveillance Analysis Computer System (SACS), July 1, 1993. 


\begin{tabular}{|c|c|c|c|c|c|c|c|}
\hline \multicolumn{8}{|c|}{ TAMK 241+A-101 } \\
\hline & & & & & & & \\
\hline TAEE & DATE & THERMO I & TAEE SET \& & TEMP C & TEMPP $F$ & $Y=$ MAX TEMP & COMNENTE \\
\hline 1 & $10 / 16 / 82$ & 14 & 1 & & 128 & $\mathrm{~N}$ & \\
\hline 1 & $10 / 16 / 82$ & 15 & 1 & & 113 & $\mathbf{N}$ & \\
\hline 1 & $10 / 15 / 92$ & 10 & 1 & & 100 & $\mathrm{~N}$ & \\
\hline 1 & 6jergs & $\$$ & $\mathbf{T}$ & & 151 & $\mathbf{N}$ & \\
\hline 1 & S/B/93 & 2 & 1 & & 153 & $\mathbf{N}$ & \\
\hline 1 & BABS3 & 3 & 1 & & 155 & $\mathbf{Y}$ & \\
\hline 1 & 6) & 4 & 1 & & 156 & $N$ & \\
\hline 1 & 6reras & E & 1 & & 155 & $N$ & \\
\hline 1 & B/9/83 & 8 & 1 & & 155 & $\mathbf{N}$ & \\
\hline 1 & B/R/83 & 7 & 1 & & 155 & N & \\
\hline 1 & Exps & $B$ & 1 & & 165 & $\mathbf{N}$ & \\
\hline 1 & 19/3 & 8 & 1 & & 155 & $\mathbf{N}$ & \\
\hline 1 & 6/2/83 & 10 & $\mathbf{T}$ & & 155 & $\mathbf{N}$ & \\
\hline $\bar{T}$ & 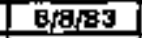 & $\overline{11}$ & 9 & & 152 & $\mathbf{N}$ & \\
\hline 1 & eta/83 & 12 & 1 & & 147 & $\mathbf{N}$ & \\
\hline 1 & 69:83 & 13 & 1 & & 139 & $\mathbf{N}$ & \\
\hline $\mathbf{1}$ & starga & 14 & 1 & & 128 & $\mathbf{N}$ & \\
\hline 1 & $679 / 83$ & 15 & 1 & & 114 & $\mathbf{N}$ & \\
\hline$y$ & $0 \% \sqrt{83}$ & $\overline{3}$ & 7 & & 707 & N & \\
\hline 1 & $12 / 22 / 93$ & 1 & 1 & & $t 52$ & $\mathbf{N}$ & \\
\hline 1 & $12 / 22 / 83$ & 2 & 1 & & 154 & $\mathbf{N}$ & \\
\hline 1 & $12 / 22 / 83$ & 3 & 1 & & 156 & $\mathbf{N}$ & \\
\hline 1 & $12 \sqrt{2} 2,83$ & 4 & 1 & & 157 & $y$ & \\
\hline 1 & $12 / 22193$ & 5 & 1 & & 157 & $N$ & \\
\hline 1 & 12/22/93 & 皇 & 1 & & 157 & $\mathbf{M}$ & \\
\hline 1. & $12 / 22,83$ & $\bar{y}$ & 1 & & 157 & $\mathbf{N}$ & \\
\hline 1 & $12 \overline{22 / 83}$ & $\bar{E}$ & $T$ & & 957 & $\mathbf{N}$ & \\
\hline 1 & $12 \sqrt{2283}$ & $\theta$ & 1 & & $15 \overline{7}$ & $\mathbf{N}$ & \\
\hline 1 & 12/22/a3 & 10 & 1 & & 157 & N & \\
\hline 1 & [12/22/89] & 11 & 1 & & 967 & $\mathbf{N}$ & \\
\hline 1 & $12 / 2,2 / 83$ & 32 & 1 & & 155 & $\mathbf{N}$ & \\
\hline 1 & {$[12 \sqrt{22193}]$} & $\overline{13}$ & 1 & & 145 & $\mathbf{N}$ & \\
\hline 1 & $12 \sqrt{22} 83$ & 14 & 1 & & 142 & $\mathbf{N}$ & \\
\hline 1 & $12 \sqrt{2} 2 \sqrt{83}$ & 15 & 1 & & 132 & $\mathbf{N}$ & \\
\hline 1 & $12 \sqrt{22} \mathrm{se}$ & 16 & 1 & & 103 & $N$ & \\
\hline 1 & $1 / 3$ Ha4 & 1 & 1 & & 972 & $\mathbf{N}$ & \\
\hline 1 & $1 \sqrt{364}$ & 2 & 1 & & 154 & $\mathbf{M}$ & \\
\hline 1 & $1 / 3 / 94$ & 3. & 1. & & 156 & $\mathbf{N}$ & \\
\hline 1 & $1 / 3 / 94$ & 4 & 1 & & $16 \overline{8}$ & $\mathrm{H}$ & \\
\hline 1 & $1 / 3 / 54$ & 5 & 1 & & 150 & $\mathbf{H}$ & \\
\hline 1 & $1 / 3 / 84$ & 6 & 1 & & $15 \mathrm{~A}$ & $\mathbf{N}$ & \\
\hline 1 & $1,13,84$ & $\overline{7}$ & 1 & & 158 & $\mathbf{N}$ & \\
\hline 1 & $1 / 3 / 94$ & 8 & 1 & & 158 & $\mathbf{N}$ & \\
\hline 7 & $1 / 3 / 24$ & 9 & 1 & & 157 & $\bar{Y}$ & \\
\hline 5 & $1 / 3 / 94$ & 10 & 1 & & 157 & $\mathbf{N}$ & \\
\hline 1 & $1 / 3 / 84$ & 11 & 1 & & 155 & $\mathbf{N}$ & \\
\hline$\$$ & $1 / 3 / 84$ & 12 & 1 & & 150 & $N$ & \\
\hline 1 & $1 / 1 / 94$ & 13 & 1 & & 142 & $\mathbf{M}$ & \\
\hline 7 & $1 / 3 / 84$ & 14 & 1 & & 132 & $\mathbf{N}$ & \\
\hline 1 & $\overline{1 / 3}$; 4 & 15 & 1 & & $7 \sqrt[7]{7}$ & N & \\
\hline 1 & $1 / 3 / 94$ & 10 & 1 & & $10 \bar{z}$ & $\mathbf{N}$ & \\
\hline 1 & $1 / 5 / 24$ & 1 & 11 & & $13 \mathrm{~g}$ & $\mathrm{~N}$ & \\
\hline 1 & $1 / \sqrt[5]{64}$ & 2 & 1 & & 141 & $\mathbf{H}$ & \\
\hline 1 & $1 \sqrt{6 / 94}$ & $\overline{3}$ & 1. & & 142 & $N$ & \\
\hline 1 & $1 / 6.94$ & 4 & 1 & & 143 & $Y$ & \\
\hline 1 & $1 / 6 / 34$ & 5 & 1 & & 143 & $\mathbf{N}$ & \\
\hline 1 & $1 / 5 / 24$ & 9 & 1 & & 143 & $\mathbf{N}$ & \\
\hline 1 & $9 \sqrt{5 / 64}$ & 7 & $\frac{1}{1}$ & & $14 \overline{3}$ & $\overline{\mathbf{N}}$ & \\
\hline 1 & $1 / 5,84$ & $\mathbf{g}$ & 1 & & $14 \overline{3}$ & $\overline{\mathbf{N}}$ & \\
\hline 1 & $\overline{1} / 5 \sqrt{6} 4$ & 白 & 1 & & 143 & N & \\
\hline 1 & 71584 & $\$ 0$ & 1 & & 143 & N & \\
\hline 1 & $1 / 5 / 34$ & 11 & 1 & & 141 & $\mathbf{N}$ & \\
\hline 1 & 1584 & 22 & 1 & & 138 & $\mathbf{N}$ & \\
\hline
\end{tabular}

Data obtained from WHC Survellance Anahyis Computer System (SACS). July 1, 1993. 


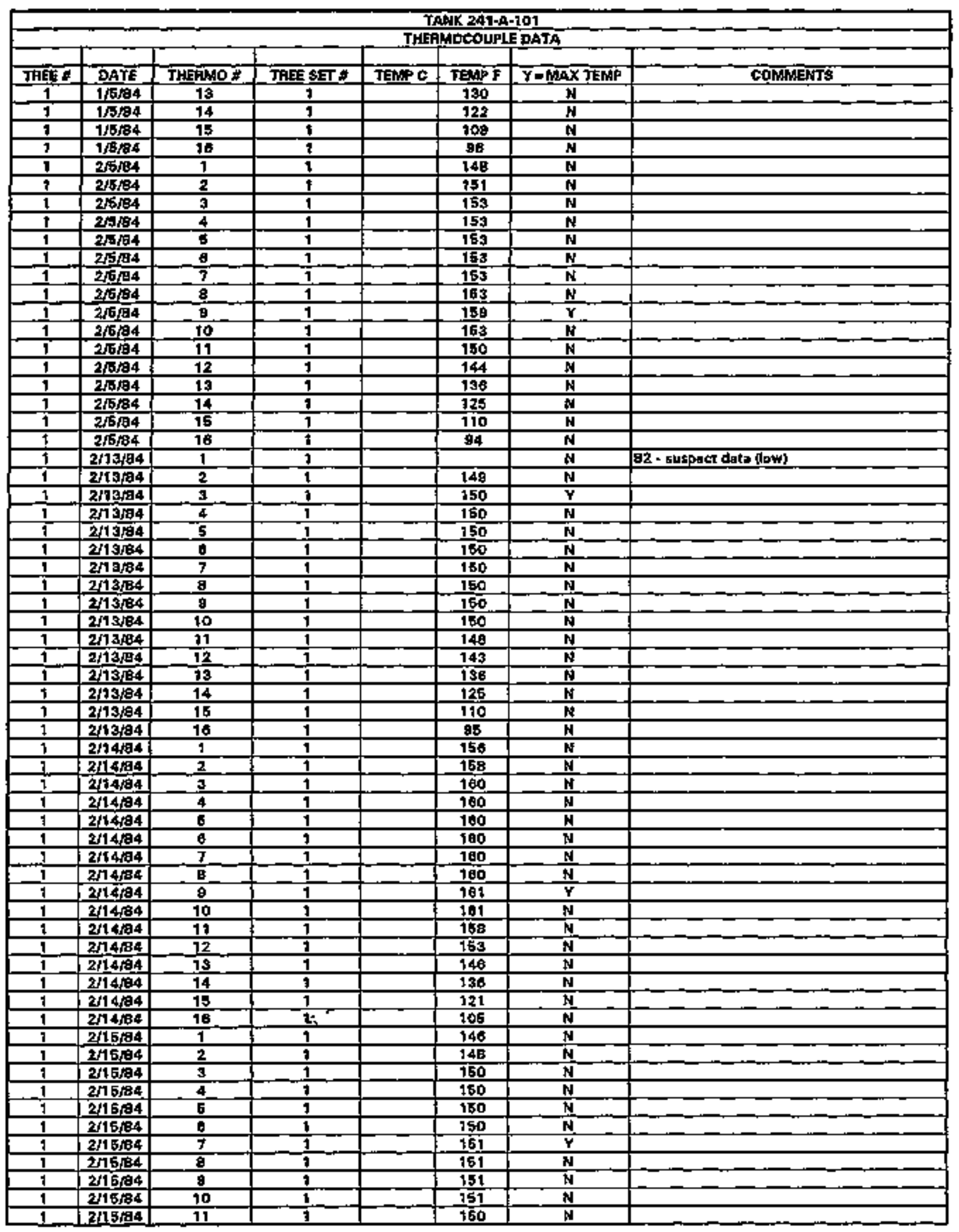

Date obtained from WHC Surveillance Analysis Computer \$ystem (SACS), July 1, 1993. 


\begin{tabular}{|c|c|c|c|c|c|c|c|}
\hline \multicolumn{8}{|c|}{ TAHK 241-A-101 } \\
\hline & & 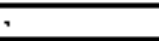 & & THFE & wocotupt & DATA & \\
\hline The & DATE & THA & THES SET & TEMA & TERPF & $\bar{\gamma}=5 \max$ & COOHUENTS \\
\hline 1 & $2 / 15 / 84$ & 12 & $\frac{1}{1}$ & & 144 & $N$ & \\
\hline 1 & $2715 / 84$ & 13 & 1 & & 137 & H & \\
\hline 1 & $2 / 15 / 94$ & 14 & 1 & & 120 & $\mathbf{N}$ & \\
\hline 1 & $2 / 15 / 24$ & 15 & 1 & & 111 & $\mathbf{N}$ & \\
\hline$\overline{1}$ & $2 / 15 / 84$ & 18 & 1 & & 96 & $\bar{N}$ & \\
\hline 1 & $2 / 18 / 84$ & 1 & 1 & & $12 \overline{0}$ & $\overline{\mathbf{N}}$ & \\
\hline 1 & $2 / 16 \mathrm{Ad}$ & 2 & 5 & & 130 & $N$ & \\
\hline 1 & $2 / 16 \sqrt{64}$ & 3 & 1 & & 132 & $N$ & \\
\hline 1 & $2 / 16 / 84$ & 4 & 1 & & 132 & $\mathbf{H}$ & \\
\hline 1 & $2 / 16 / 84$ & 5 & 1 & & 132 & $\overline{\mathbf{N}}$ & \\
\hline 7 & $2 / 18 / 04$ & 6 & 1 & & 132 & $N$ & \\
\hline$\$$ & $2 / 16 / 64$ & 7 & 5 & & 132 & $N$ & \\
\hline 7 & $2 / 16 / 94$ & E & 1 & & 132 & $\mathbf{N}$ & \\
\hline 1 & $2 / 16 / 84$ & 5 & 1 & & 133 & $\bar{Y}$ & \\
\hline$\overline{1}$ & $2 / 16 / 84$ & 10 & 1 & & $\overline{133}$ & $\mathbf{N}$ & \\
\hline 1 & $2 / 16 / 94$ & $\$ 1$ & 1 & & 130 & $\bar{N}$ & \\
\hline 1 & $2 / 1084$ & 12 & 1 & & 125 & तो & \\
\hline 1 & $2 / 10 / 94$ & 15 & 1 & & 119 & $N$ & \\
\hline 1 & $2 / 70 / 84$ & 14 & 1 & & 108 & $\mathbf{N}$ & \\
\hline 1 & $2 / 118 / 84$ & 16 & 1 & & $9 \overline{2}$ & $\mathbf{N}$ & \\
\hline 1 & 2116184 & 10 & i & & 76 & $N$ & \\
\hline$\overline{1}$ & $2 / 17 / 84$ & 1 & 1 & & 144 & $N$ & \\
\hline 1 & $2 / 17 / 94$ & 2 & 1 & & 147 & $M$ & \\
\hline$\overline{1}$ & 217784 & $\mathbf{3}$ & 1 & & 148 & $\mathbf{M}$ & \\
\hline 1 & 251784 & 4 & 1 & & 148 & $\mathbf{N}$ & \\
\hline 1 & $2 / 17 / 84$ & 5 & 1 & & 148 & $N$ & \\
\hline 1 & $2 / 17 / 84$ & 9 & 1 & & 748 & $\mathbf{N}$ & \\
\hline 1 & $2 / 17 / 84$ & 7 & 1 & & 148 & $\mathbf{N}$ & \\
\hline 1 & $2 / 1784$ & 8 & 1 & & 148 & $\mathbf{N}$ & \\
\hline 1 & $2 / 17 / 84$ & 皇 & 1 & & 145 & $\bar{N}$ & \\
\hline 1 & $2 / 17 / 4$ & 90 & 1 & & 149 & $Y$ & \\
\hline 1 & $2 / 17 / 84$ & 11 & 1 & & 246 & $\mathrm{~N}$ & \\
\hline 1 & $2 / 17 / 84$ & 12 & 1 & & 141 & $\mathbf{N}$ & \\
\hline 1 & $2 / 1784$ & 73 & $\overline{1}$ & & 134 & $\mathbf{N}$ & \\
\hline 1 & $2 / 17 / 64$ & 14 & 1 & & $1 \overline{24}$ & $\bar{N}$ & \\
\hline 1 & $2 / 17 / 94$ & 15 & 1 & & 109 & $\mathbf{N}$ & \\
\hline 1 & $2 / 17 / 84$ & 18 & 1 & & 93 & $\mathbf{N}$ & \\
\hline 1 & $2 \pi 8 / 94$ & 1 & 1 & & 141 & $\mathbf{N}$ & \\
\hline 1 & $2 / 18 \mathrm{~N} 4$ & 2 & 1 & & 144 & $\mathbf{N}$ & \\
\hline$\overline{1}$ & $2 \sqrt{18} 84$ & 3 & 1 & & 145 & $\mathbf{N}$ & \\
\hline 1 & $2 \sqrt{18 / 84}$ & 4 & 1 & & $\$ 45$ & $\mathbf{N}$ & \\
\hline 1 & $2 / 18 \sqrt{84}$ & 5 & 1 & & 345 & $\mathrm{~N}$ & \\
\hline 1 & 2/1ere 4 & 6 & 1 & & 146 & $\mathbf{N}$ & \\
\hline 1 & $2 / 18$ म 4 & 7 & 1 & & 145 & $\mathbf{N}$ & \\
\hline 3 & $2 / 18 / 94$ & 8 & 1 & & 145 & $\mathbf{N}$ & \\
\hline 1 & $2 / 18 / 4$ & $\boldsymbol{\theta}$ & 1 & & 187 & $\mathbf{N}$ & \\
\hline 1 & 2flef 4 & 10 & 1 & & $1 \overline{48}$ & $y$ & \\
\hline 1 & $2 / 18$ a & 11 & 1 & & 145 & $\mathbf{N}$ & \\
\hline 1 & 2/1어연 & 12 & 1 & & 740 & $\mathbf{N}$ & \\
\hline$\overline{3}$ & $2 / 16194$ & 13 & 1 & & 133 & $\mathbf{N}$ & \\
\hline 1 & $2 / 10 / 84$ & 14 & 1 & & $1 \overline{22}$ & $\mathbf{N}$ & \\
\hline 1 & $2 / 1 \overline{894}$ & 15 & $1 ;$ & & $1 \overline{08}$ & $\mathbf{N}$ & \\
\hline 3 & $2 / 1$ 白的 4 & 10 & 1 & & 92 & $\mathbf{N}$ & \\
\hline 7 & $2 / 19 / 94$ & 1 & 1 & & 144 & $\mathbf{N}$ & \\
\hline 1 & $2 / 19 / 84$ & 2 & 1 & & 146 & $\mathbf{N}$ & \\
\hline 7 & $2 / \sqrt{6 / 84}$ & 3 & 1 & & 147 & $\mathbf{N}$ & \\
\hline 1 & $2 / 19 / 4$ & 4 & 1 & & 148 & $\mathbf{Y}$ & \\
\hline 2 & $2 / 18 / 144$ & 5 & 1 & & 248 & $\overline{\mathbf{N}}$ & \\
\hline 1 & $2 / 18 / 84$ & B. & 1 & & 148 & $N$ & \\
\hline 1 & $2 / 19 / 84$ & 7 & 1 & & $14 B$ & $\mathbf{N}$ & \\
\hline 1 & 2/96 4 & 8 & 1 & & 148 & $\mathbf{N}$ & \\
\hline 1 & $2 / 19 / 94$ & 9 & 1 & & 348 & $\mathbf{N}$ & \\
\hline 1 & 2 219/94 & 70 & 1 & & $14 \mathrm{~B}$ & $\mathbf{N}$ & \\
\hline
\end{tabular}

Data obtained from WHC Surveillance Analysis Computer System (SACS), thly 1, 1993. 


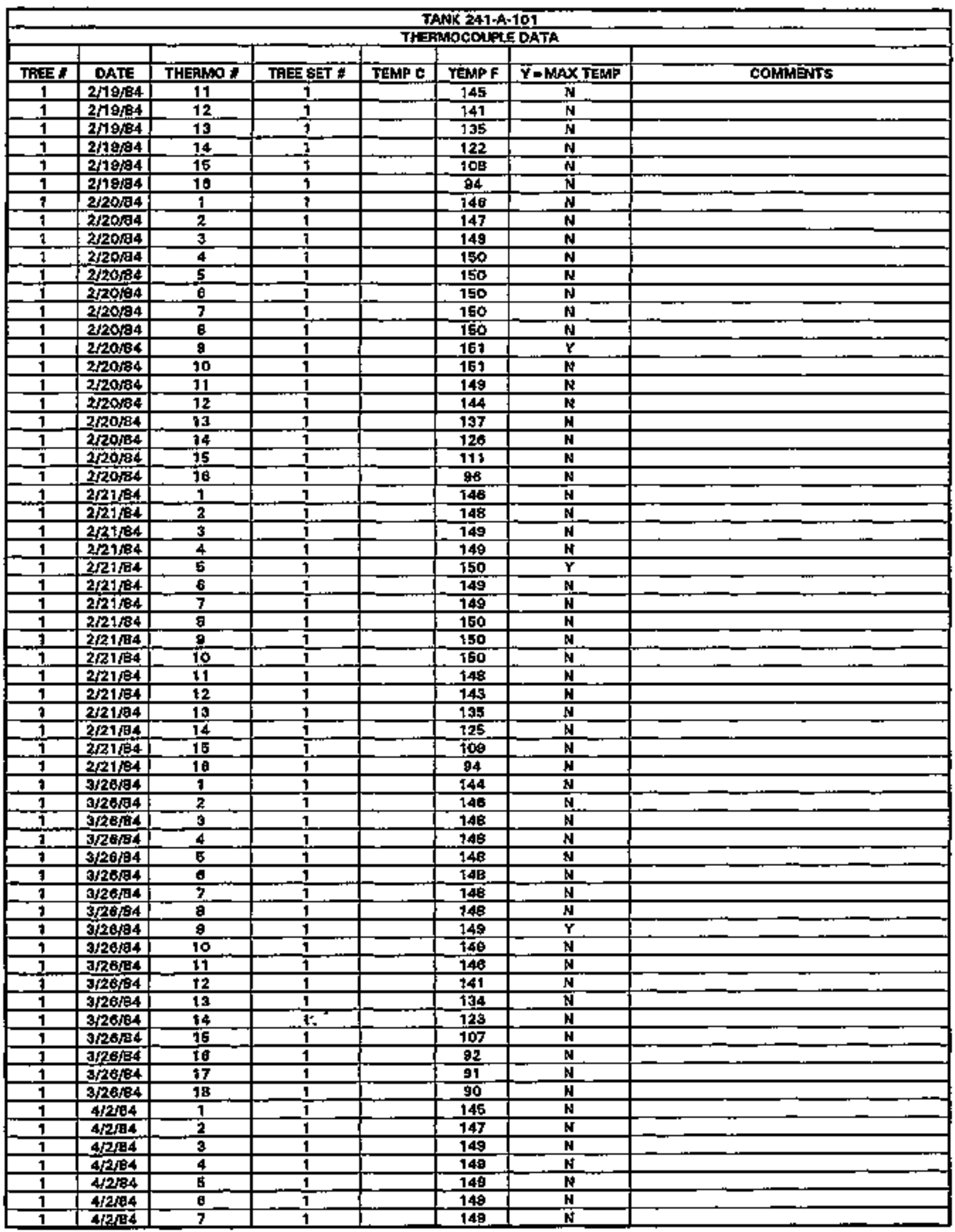

Data obtained from WhSC Surveillance Analysis Computer Systern (SACS1, July 1, 1993. 
WHC-SD-WM+ER-308, Rev. 0

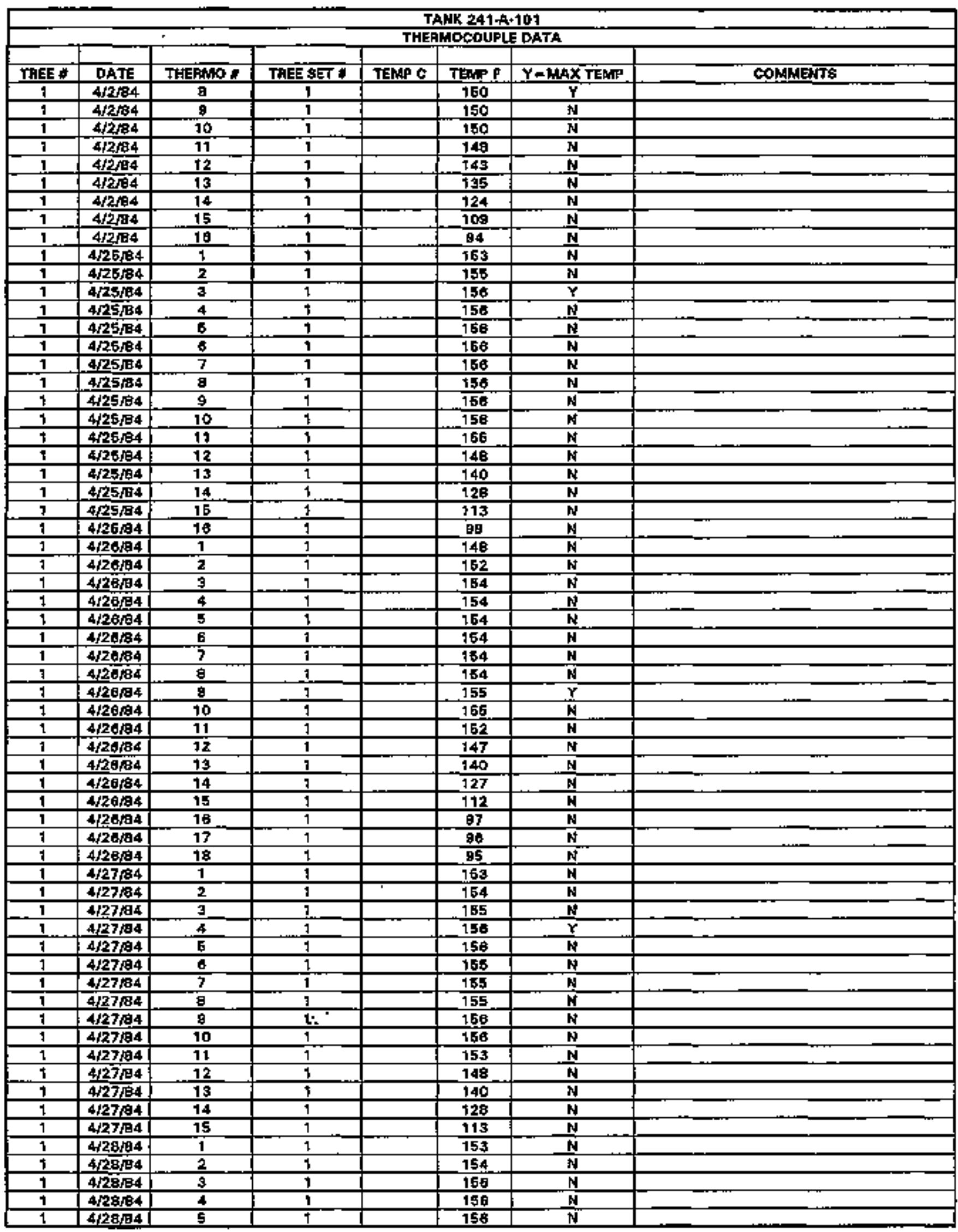

Data obtained frorn WHC Surveillance Analysis Computer System (SACS), July 1, 1993. 


\begin{tabular}{|c|c|c|c|c|c|c|c|}
\hline & & & & $\frac{T}{T H}$ & $\frac{\text { ax 24t-x }}{\text { socotipt }}$ & $\frac{101}{0 A T A}$ & \\
\hline & & & & & & & \\
\hline TREE * & DATE & FHERMO * & TAEE SET \# & TEMPC & TEMPE & $Y=M A X$ TEMPF & COMMENTS \\
\hline 1 & 4/28194 & $\theta$ & 1 & & 156 & $N$ & \\
\hline 1 & $4 / 29 / 84$ & 7 & 1 & & 150 & N & \\
\hline 1 & $4 \sqrt{2+184}$ & a & 1 & & 150 & $N$ & \\
\hline$i$ & $4 \longdiv { 2 8 / 3 4 }$ & $\frac{9}{9}$ & 1 & & $15 \%$ & $\mathbf{N}$ & \\
\hline$\sqrt{3}$ & $4 \sqrt{28 / 84}$ & 10 & 1 & & 169 & $\mathbf{Y}$ & \\
\hline 1 & $4 / 29 / 84$ & 11 & 1 & & 154 & $\mathbf{M}$ & \\
\hline 1 & 4/29/B4 & 12 & 1 & & 149 & N & \\
\hline 1 & $4 \sqrt{2 B N 4}$ & 13 & 1 & & 141 & $\mathbf{N}$ & \\
\hline $\mathbf{i}$ & 4/2814 & $\sqrt{14}$ & 1 & & 120 & $N$ & \\
\hline 1 & ARBE4 & 16 & 1 & & 114 & $\mathbf{N}$ & \\
\hline 1 & $4 / 29 / 84$ & 1 & 1. & & 161 & $\mathbf{N}$ & \\
\hline 1 & $4 / 29 / 84$ & 2 & 1 & & 152 & $\mathbf{N}$ & \\
\hline 1 & $4 / 29 / 94$ & 3 & 1 & & 154 & $\mathbf{N}$ & \\
\hline 1 & 4/29/94 & 4 & 1 & & 164 & $\mathbf{N}$ & \\
\hline 1 & $4 / 29 / 24$ & $\overline{5}$ & 1 & & 154 & $\mathbf{N}$ & \\
\hline 1 & 4/29/24 & 4 & 1 & & 154 & $\mathbf{N}$ & \\
\hline I. & $4 \sqrt{29}, 194$ & 7 & 1 & & 954 & H & \\
\hline 1 & 4/21:84 & $\mathbf{8}$ & 1 & & 154 & $\mathbf{N}$ & \\
\hline 1 & $4 \sqrt{29} 84$ & 量 & 1 & & 156 & $\bar{y}$ & \\
\hline 1 & $4 \longdiv { 2 9 6 4 }$ & 10 & 1 & & 155 & 1 & \\
\hline 1 & $4 \sqrt{29}$ & 11 & 1 & & 162 & $\mathbf{N}$ & \\
\hline 1 & $4 / 20 / 84$ & 12 & 1 & & 143 & N & \\
\hline 1 & $4 \sqrt{29} 4$ & 13 & 1 & & 739 & N & \\
\hline 1 & $4 / 20 / 84$ & 14 & 1 & & 328 & N & \\
\hline 1 & $4 / 29 / 84$ & 15 & 1 & & 112 & $N$ & \\
\hline 1 & $11,8 \sqrt{84}$ & 3 & 1 & & 149 & $\mathbf{N}$ & \\
\hline 1 & 11/8/8. & 2 & 1 & & 751 & $N$ & \\
\hline 1 & $11 / 2484$ & 3 & 1 & & is 1 & $N$ & \\
\hline 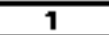 & 11/884 & 4 & 7 & & 152 & H & \\
\hline 1 & $11 B B{ }^{2}$ & 5 & $t$ & & 152 & $\mathrm{~N}$ & \\
\hline 1 & $11 / 8 \mathrm{~B}$ & 6 & 1 & & 152 & $\hat{N}$ & \\
\hline 1 & $11 / 8194$ & $\overline{7}$ & $t$ & & 152 & $N$ & \\
\hline 1 & $11 / 8 / 34$ & 8 & 1 & & 162 & $N$ & \\
\hline 1 & 11/烟4 & 5 & 1 & & 158 & $\mathbf{Y}$ & \\
\hline 1 & $19 / \mathrm{B} / 4$ & 10 & 1 & & 152 & $N$ & \\
\hline$\vec{t}$ & $11 \sqrt{8,84}$ & 11. & $\overline{1}$ & & 150 & $\overline{\mathbf{N}}$ & \\
\hline 1 & $17 / 8 / 84$ & 12 & 1 & & 149 & $\mathbf{N}$ & \\
\hline$t$ & 11964 & 13 & $t$ & & 142 & $N$ & \\
\hline 1 & $11 /$ B 194 & 14 & 1 & & 192 & $\mathbf{N}$ & \\
\hline 5 & $11 / 9 / 94$ & 15 & 1 & & 120 & $\mathbf{N}$ & \\
\hline 1 & T1/aka4 & 16 & 1 & & 107 & $\bar{N}$ & \\
\hline 1 & $12 / 14 / 84$ & 1 & 1 & & 148 & $\mathbf{N}$ & \\
\hline 1 & 121484 & 2 & 1 & & 153 & $\mathbf{N}$ & \\
\hline 1 & $1 / 2 / 14 / 6$ & 3 & 1 & & 153 & $\mathbf{N}$ & \\
\hline 1 & $12 / 44 / 94$ & 4 & $T$ & & 153 & $\bar{N}$ & \\
\hline 7 & $12 / 14 / 24$ & 6 & 1 & & 163 & $\mathbf{N}$ & \\
\hline 1 & $\sqrt{2 / t}+4 / \bar{a}$ & $\theta$ & 1 & & 153 & $\mathbf{N}$ & \\
\hline 1 & $12 / 14 / 94$ & 7 & 1 & & 153 & $\mathbf{N}$ & \\
\hline 1 & $12 / 94 / 84$ & $\mathbf{a}$ & 1 & & 163 & $\mathbf{N}$ & \\
\hline 1 & $12 / 14 / 84$ & 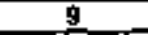 & 1 & & 164 & $Y$ & \\
\hline$\overline{1}$ & $12 / 74 / 84$ & 10 & 1 & & 154 & $N$ & \\
\hline 1 & $12 / 14 \bar{A}$ & 11 & Is & & 152 & $\mathbf{N}$ & \\
\hline 9 & $132 / 14 / 84$ & 12 & 1 & & 147 & $\bar{N}$ & \\
\hline 1 & $12 / 14 / 94$ & 13 & 1 & & 140 & $\mathbf{N}$ & \\
\hline 1 & $12 / 14 / 24$ & 14 & 1 & & 131 & $\mathbf{N}$ & \\
\hline 1 & $12 / 1484$ & 15 & 1 & & 11日 & $N$ & \\
\hline 1 & $12 / 14 / 84$ & 18 & 7 & & 106 & $\mathbf{N}$ & \\
\hline 7 & 1/28/95 & 1 & 1 & & 159 & $\mathbf{N}$ & \\
\hline 1 & 1126오 & 2 & 1 & & 181 & $\mathbf{N}$ & \\
\hline 1 & 1/26a6 & 3 & 1 & & 168 & $\mathbf{N}$ & \\
\hline 1 & $1 / 20 / 35$ & 4 & 1 & & 163 & $\mathbf{N}$ & \\
\hline 1 & $1 / 26 / 95$ & $E$ & 1 & & 10\$ & N & \\
\hline 1 & 12685 & 6 & 1 & & 163 & $\bar{N}$ & \\
\hline
\end{tabular}

Data obtained from Whe Surveillante Analysis Computer Systent iSACS1, Juły 1, 1993. 


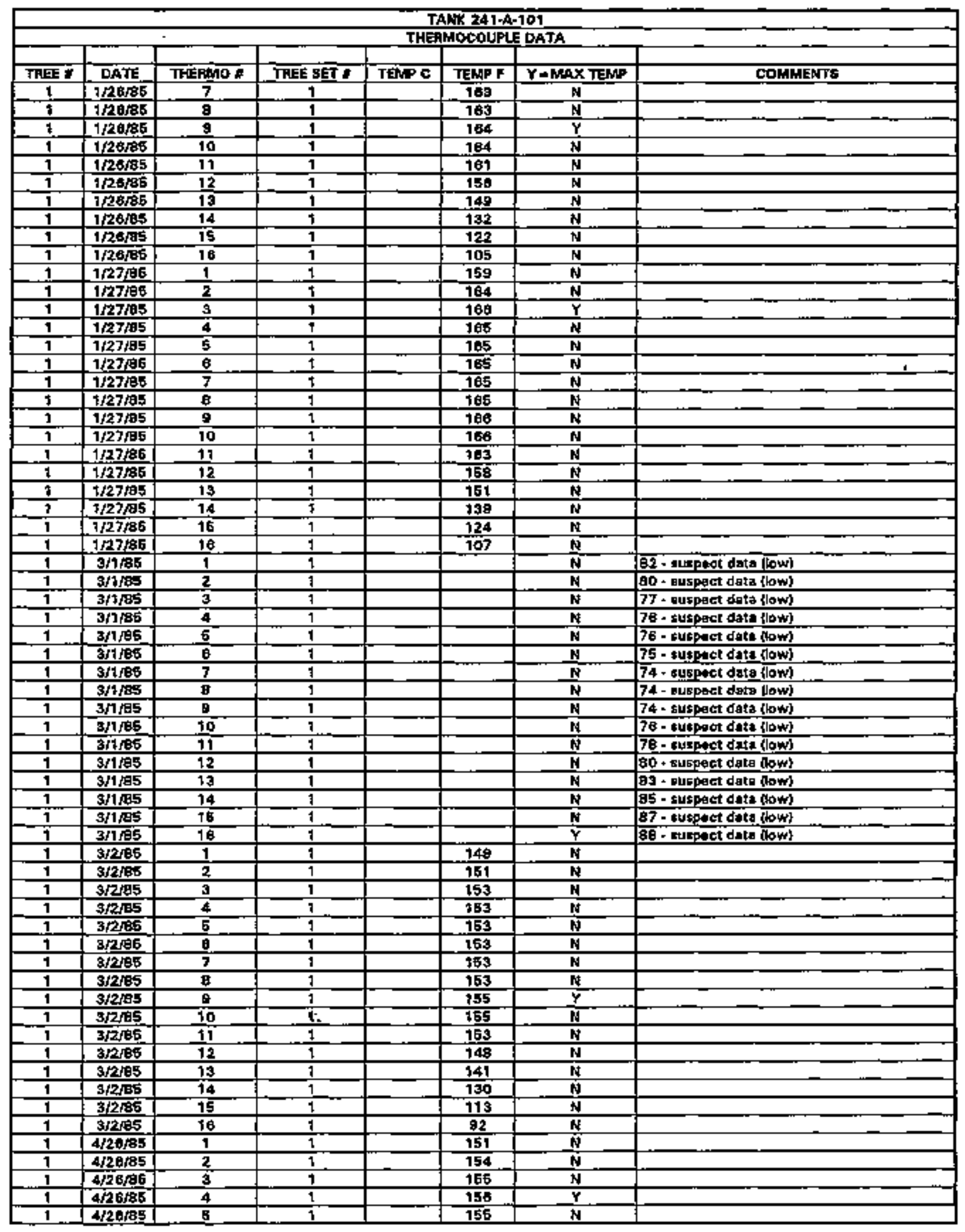

Data obtained tram WHC Surveitence Analysis Computer System [SACS\}, July 1, 1993. 


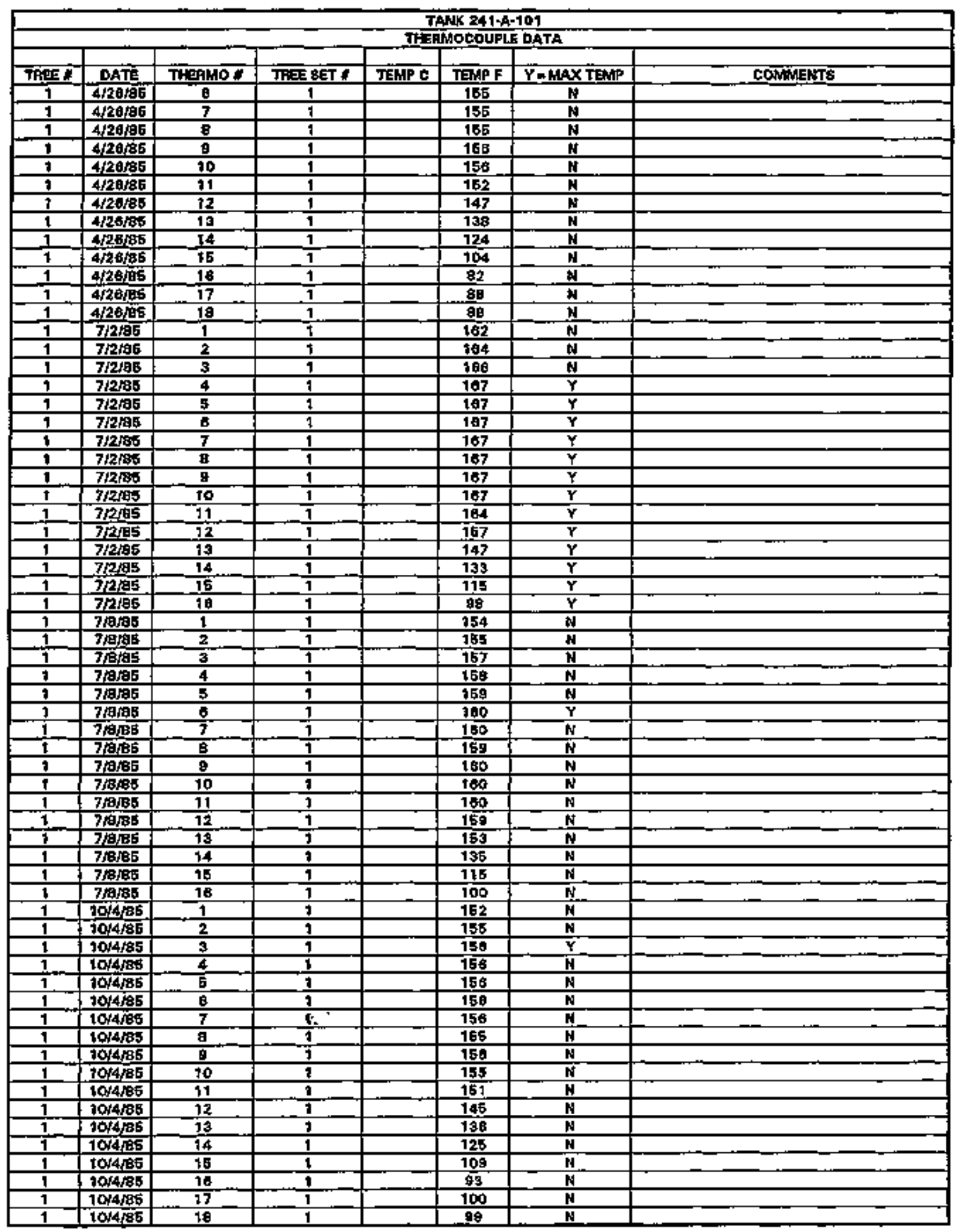

Data obtained from WHC Surveillance Analysis Computer System (SACS). July 1, 1993. 


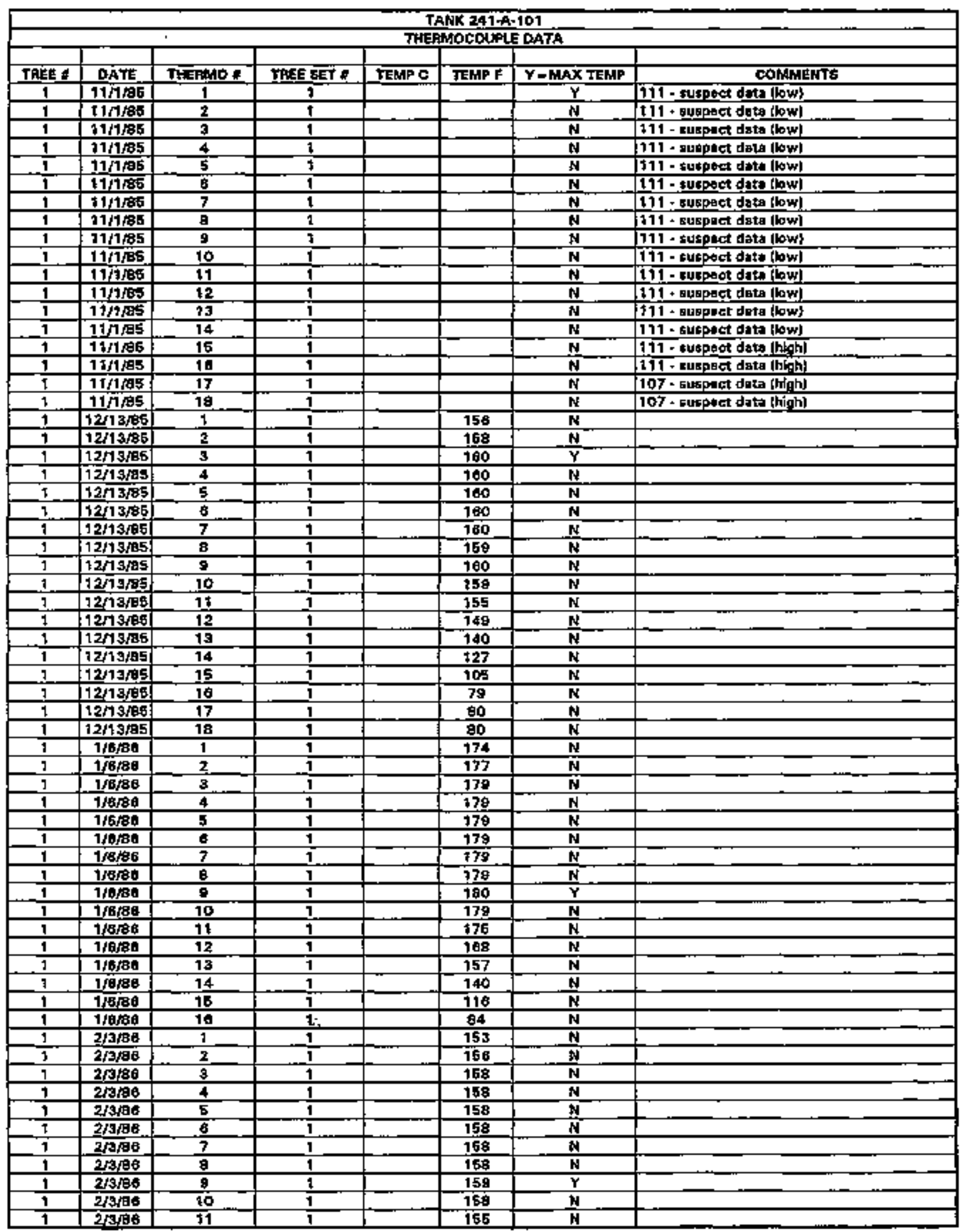

Data obtained from WHC Survellance Anslysis Computer System (SACS), July 1, 1993. 


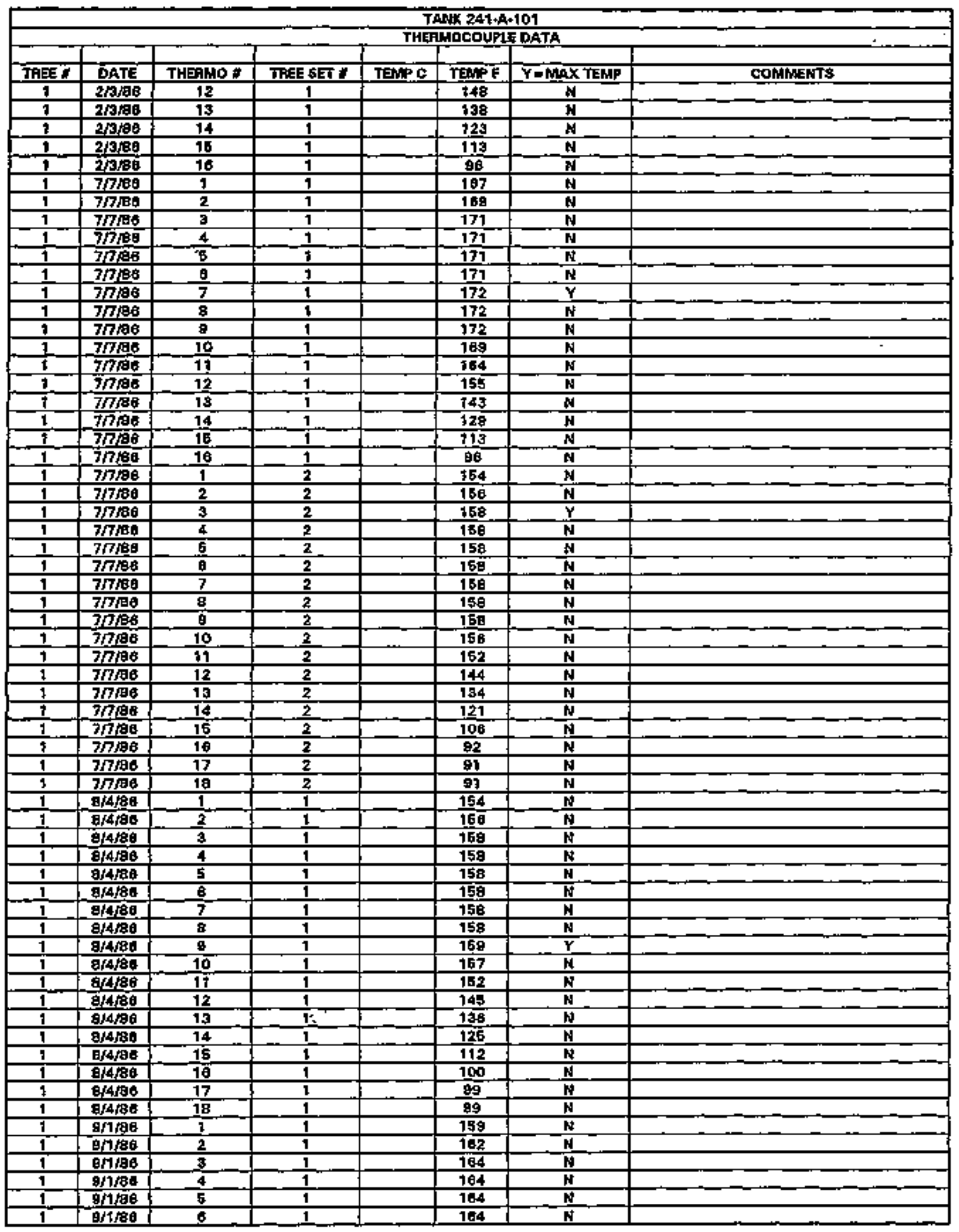

Data abtained from WhtC Surveillance Analysis Computer System [SACS], Hly 1, 1993. 
WHC-SD-WM-ER-308, Rev. D

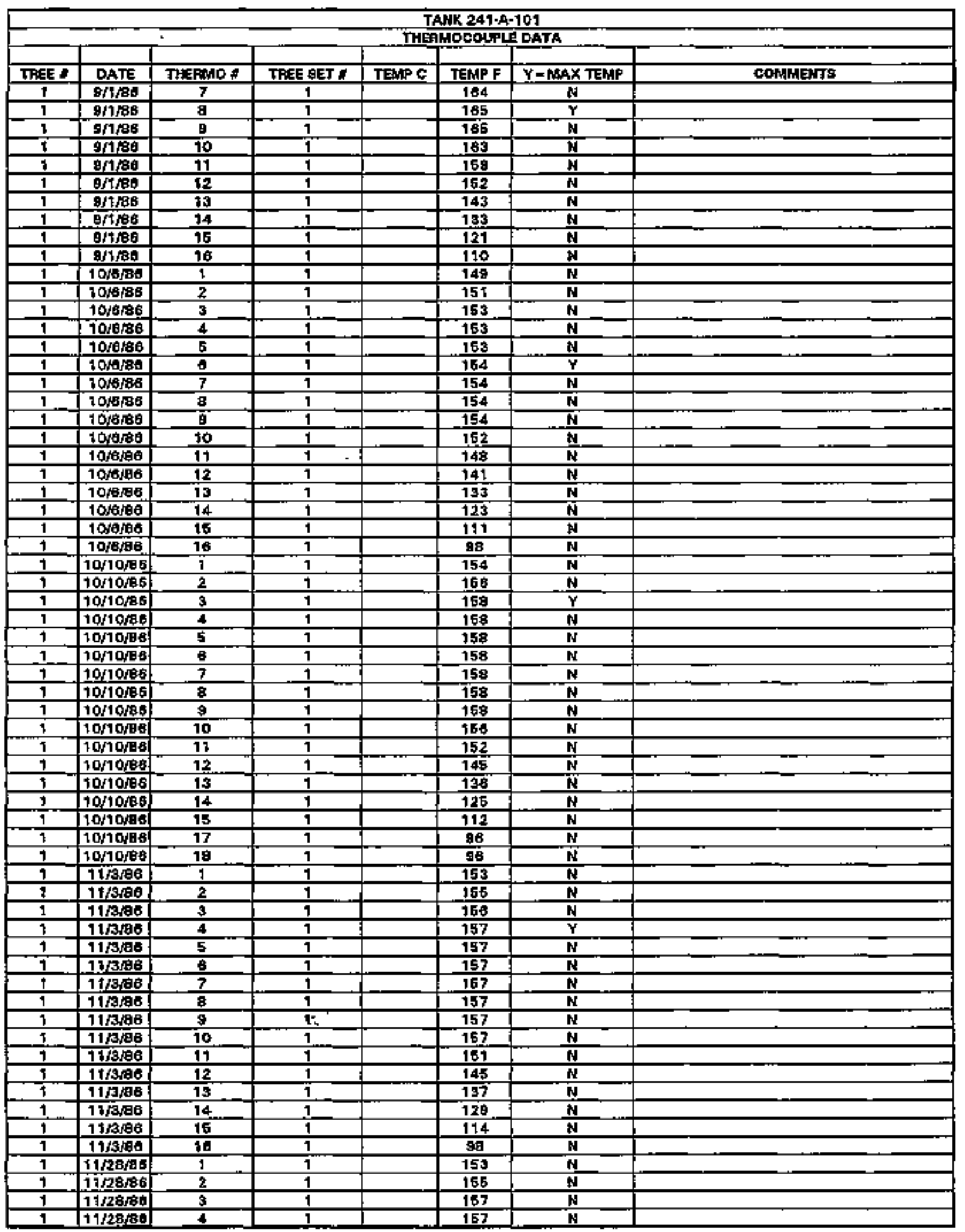

Data obtained from WHC Surveillance Aralysis Computer System (SACS), July 1, 1993. 


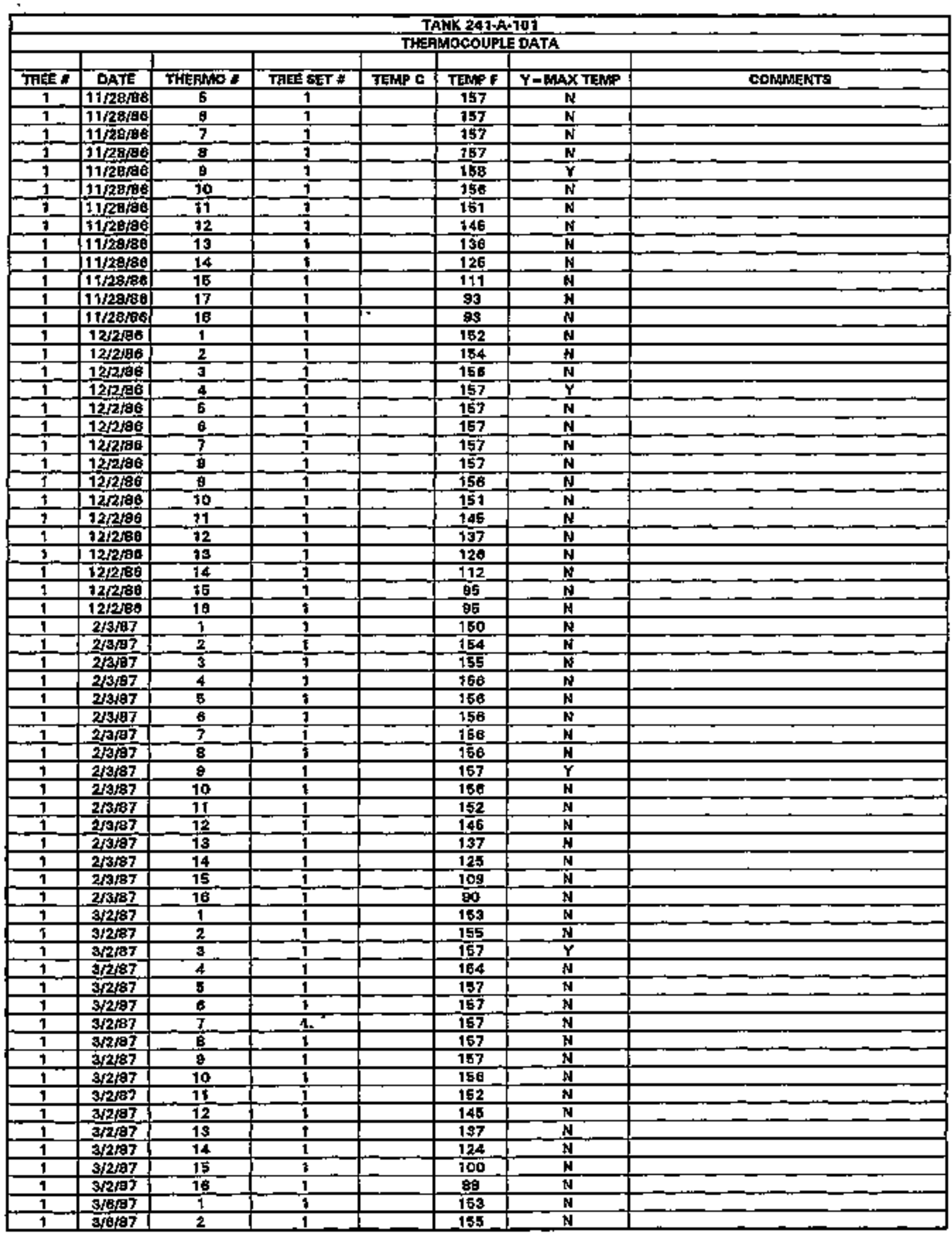

Dats obtained from WHC Survaillance Anahysis Computer System (SACS), July 1, 1993. 


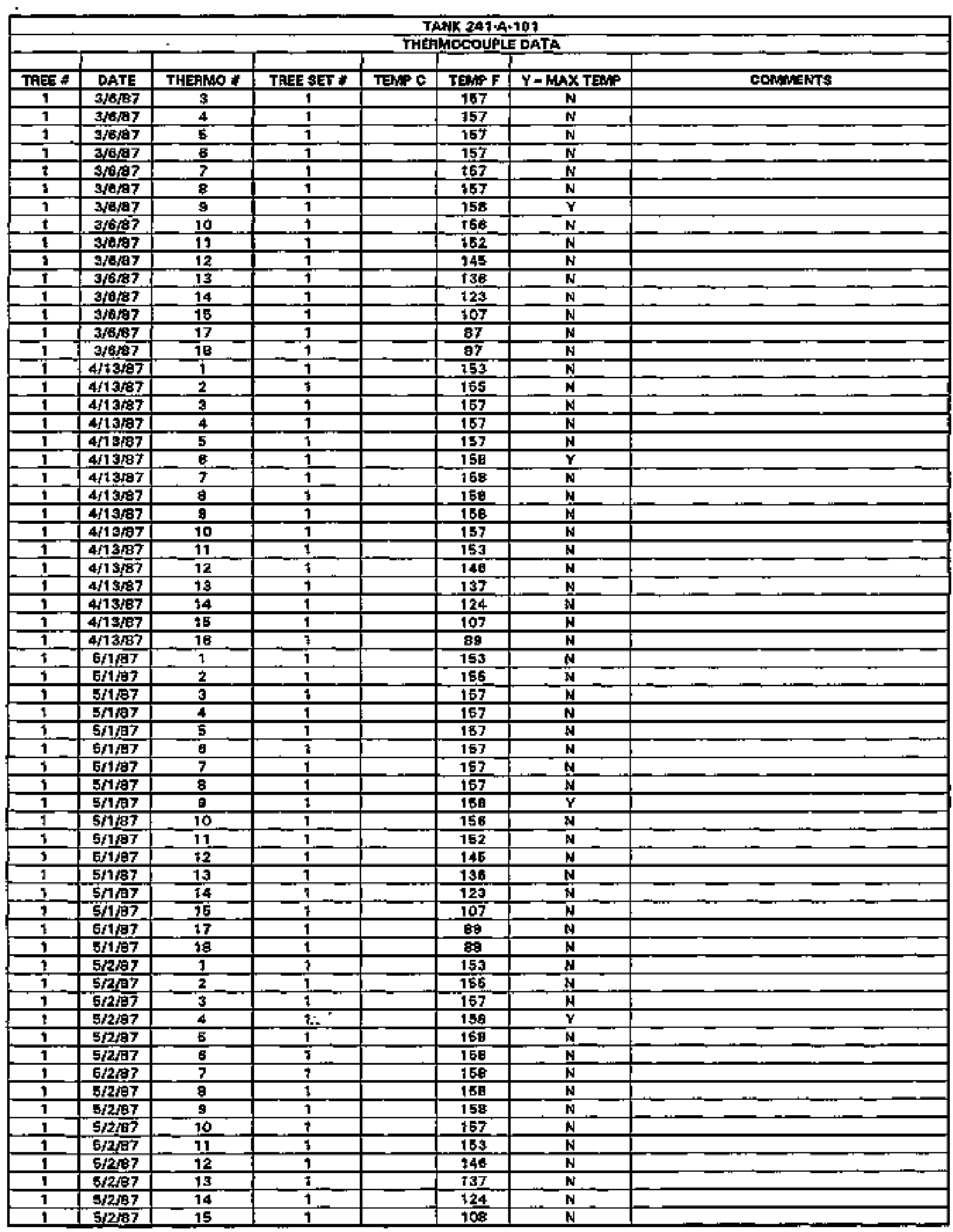

Data obtained from WHC Survellance Analysis Computer System (SACS), July 1, 1993. 


\begin{tabular}{|c|c|c|c|c|c|c|c|}
\hline \multicolumn{8}{|c|}{ TANK 241+A-10T } \\
\hline THEE * & DATE & THEFMO & TAFE SEF & TEMPC & TEAP F & $Y=M A X$ TEKA & COMMENTS \\
\hline 1 & $5 / 2 / 17$ & 19 & 1 & & 91 & $\mathbf{N}$ & \\
\hline 1 & 9187 & 7 & 1 & & 153 & $\mathbf{N}$ & \\
\hline 1 & $6 / 1 / 17$ & 2 & 2 & & 155 & $\mathbf{N}$ & \\
\hline 1 & 6787 & 3 & 1 & & 158 & $\mathbf{N}$ & \\
\hline 1 & $6 / 1 / 87$ & 4 & 1 & & 157 & $\overline{\mathbf{N}}$ & \\
\hline 1 & $6 / 1 / 97$ & $E$ & $I$ & & 157 & $\mathbf{N}$ & \\
\hline 1 & $6 / 1 / 87$ & e & 1 & & 157 & $\mathbf{N}$ & \\
\hline 1 & $6 / 1 / 87$ & 7 & 1 & & 167 & $N$ & \\
\hline 1 & $8 / 1 / 87$ & E & 1 & & 757 & $\mathbf{N}$ & \\
\hline 9 & of1/87 & 9 & 1 & & 158 & $\mathbf{Y}$ & \\
\hline 1 & 6/1/87 & 10 & 1 & & 958 & $\mathbf{N}$ & \\
\hline 1 & 6r1/37 & 11 & 1 & & 142 & H & \\
\hline 1 & 의1/87 & 12 & 1 & & 145 & $N$ & \\
\hline$t$ & 611/87 & 13 & 1 & & 196 & $\mathrm{~N}$ & \\
\hline 1 & 벼낙7 & 34 & 1 & & 124 & N & \\
\hline 1 & 아국 & 15 & 1 & & 109 & $N$ & \\
\hline 1 & $0 / 2 \longdiv { 8 7 }$ & 16 & 1 & & 93 & $\mathbf{N}$ & \\
\hline 1 & $7 / 0 / 87$ & 1 & 1 & & 144 & $N$ & \\
\hline 1 & $7 / 6 / 67$ & 2 & 1 & & 148 & $\mathbf{N}$ & \\
\hline 1 & 7 76/67 & 3 & 1 & & 149 & $\mathbf{N}$ & \\
\hline 1 & $7 / 6 / 97$ & 4 & 1 & & 149 & $\mathbf{N}$ & \\
\hline 1 & 7 Fo/a7 & 5 & 1 & & 149 & $\gamma$ & \\
\hline 1 & $7 / 8$ /a7 & 6 & 1 & & 149 & k & \\
\hline 1 & 7007 & 7 & 1 & & 149 & $\mathbf{N}$ & \\
\hline 1 & $7 \mathrm{P} / \mathrm{g} 7$ & B & 1 & & 149 & $\overline{\mathbf{N}}$ & \\
\hline 1 & 7 rera & 9 & 9 & & 149 & $\mathbf{N}$ & \\
\hline 1 & 7 Fefa 7 & 10 & 1 & & 147 & $\mathbf{N}$ & \\
\hline 1 & $7 \mathrm{Br} 87$ & 17 & 1 & & 143 & $\overline{\mathbf{N}}$ & \\
\hline 1 & $7 / 8,97$ & 12 & 9 & & 138 & $\mathbf{N}$ & \\
\hline 1 & 70/97 & 13 & 1 & & 128 & it & \\
\hline$\overline{1}$ & $7 / \mathrm{a} / \mathrm{B} 7$ & 14 & 1 & & I1B & $\mathbf{N}$ & \\
\hline 1 & 7 伯B? & 15 & 1 & & 104 & $\mathbf{N}$ & \\
\hline 1 & 7 ters & 18 & 1 & & $\mathbf{9 0}$ & $\dot{\mathbf{M}}$ & \\
\hline 1 & $9 / 3 / 87$ & 2 & 9 & & 143 & $\mathbf{N}$ & \\
\hline 1 & $8 / 3 / 87$ & 3 & 1 & & 144 & $\mathbf{N}$ & \\
\hline 1 & $8 / 8 / 87$ & $\overline{4}$ & 1 & & 144 & $\mathbf{N}$ & \\
\hline$t$ & g/3/B7 & $\bar{E}$ & 1 & & 144 & 利 & \\
\hline 1 & 813187 & 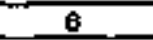 & 1 & & 144 & $\mathbf{N}$ & \\
\hline 1 & $8 / 3 / 87$ & 7 & 9 & & 144 & $\mathbf{N}$ & \\
\hline 1 & a/307 & $\theta$ & 1 & & 144 & $N$ & \\
\hline $\mathbf{T}$ & $9 / 3 / 87$ & $\mathbf{g}$ & 1 & & 245 & $\mathbf{Y}$ & \\
\hline 7 & G/3/87 & 10 & $t$ & & .74 & $\mathbb{N}$ & \\
\hline 1 & 91367 & 11 & 1 & & 130 & $\mathbf{N}$ & \\
\hline 1 & a/3/87 & 12 & 1 & & 135 & $N$ & \\
\hline 1 & atsen & 13 & 1 & & 12B & N & \\
\hline 1 & 9/3/67 & 14 & 1 & & $11 \frac{1}{8}$ & $\mathrm{~N}$ & \\
\hline 1 & $9 / 3 / 67$ & $\sqrt{16}$ & 1 & & 108 & $\mathbf{N}$ & \\
\hline 1 & abse? & 16 & 1 & & 96 & $N$ & \\
\hline 1 & $96 \sqrt{67}$ & 1 & $T$ & & 140 & $\mathbf{N}$ & \\
\hline 1 & B/6/97 & $\overline{2}$ & 7 & & 143 & $\mathbf{N}$ & \\
\hline 1 & 的它7 & 3 & 1 & & 144 & $\mathbf{N}$ & \\
\hline 1 & $9 / 6 / 67$ & 4 & $t$ & & 144 & $\mathbf{N}$ & \\
\hline 1 & B/9/67 & $\overline{\mathbf{E}}$ & 1 & & 144 & $\mathbf{N}$ & \\
\hline 1 & B/E & 8 & 1 & & 144 & $\mathbf{N}$ & \\
\hline$t$ & 1/6/87 & 7 & $t$ & & 144 & $\mathbf{N}$ & \\
\hline 1 & 910/87 & B & 7 & & 144 & $\mathbf{N}$ & \\
\hline 7 & E/9187 & g & 1 & & 145 & $\mathbf{Y}$ & \\
\hline$t$ & - & 10 & $i$ & & 144 & $N$ & \\
\hline 1 & Q/OJE7 & 11 & 1 & & 240 & Nㅔ & \\
\hline 1 & 6/0/87 & 12 & 1 & & 135 & $N$ & \\
\hline 7 & bora7 & 13 & 1 & & $12 \mathrm{~B}$ & $\mathbf{N}$ & \\
\hline $\bar{t}$ & - & 14 & 1 & & 120 & $\overrightarrow{\mathrm{N}}$ & \\
\hline 1 & 0/0/87 & 15 & 1 & & 110 & $\mathbf{N}$ & \\
\hline
\end{tabular}

Data obtained from WHC Survellance Anatysis Cornputer System [SACS], h5y 1, 1993. 


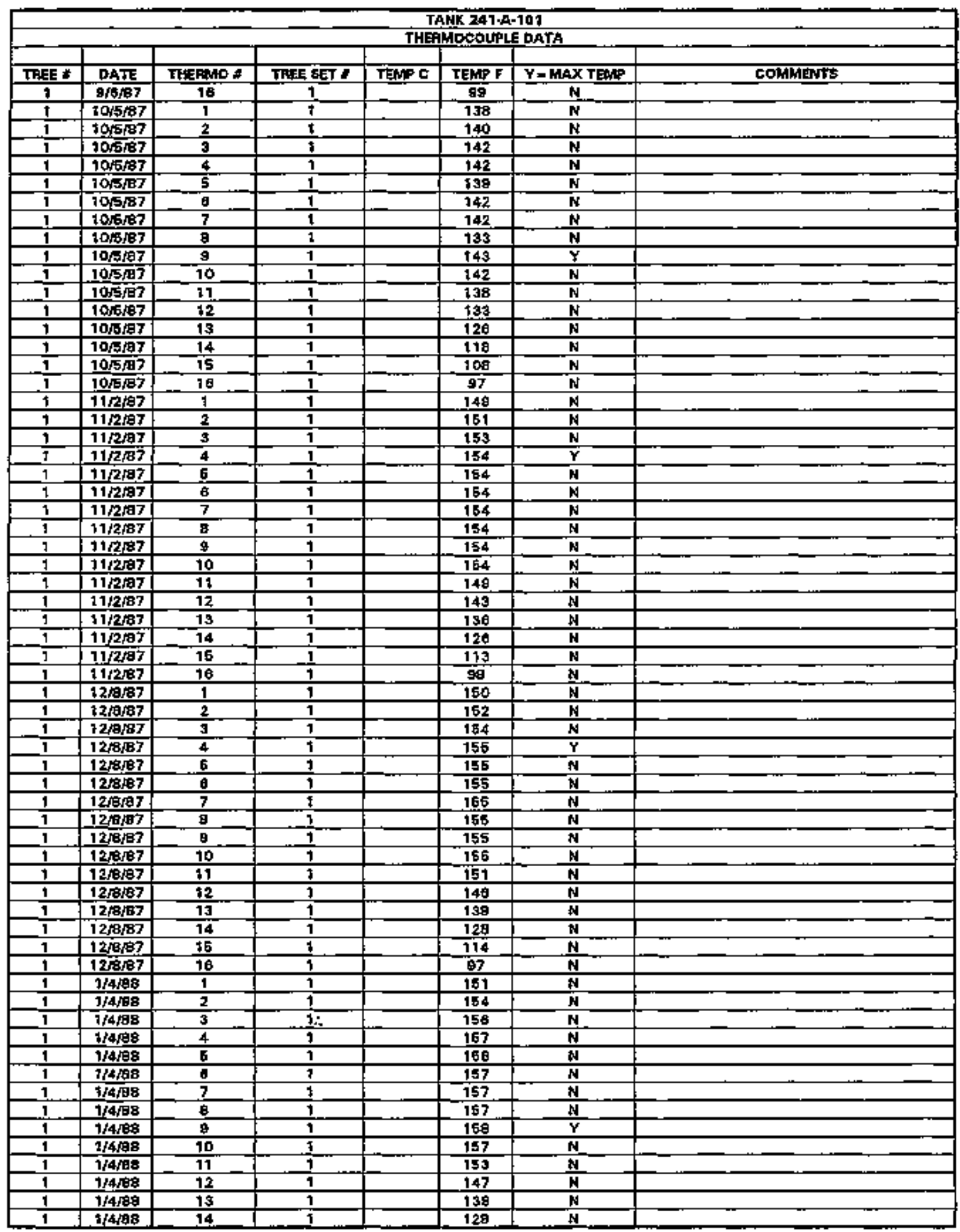

Data obtained from WHC Strveillance Analysis Computer System (SACS), July 1, 1993. 


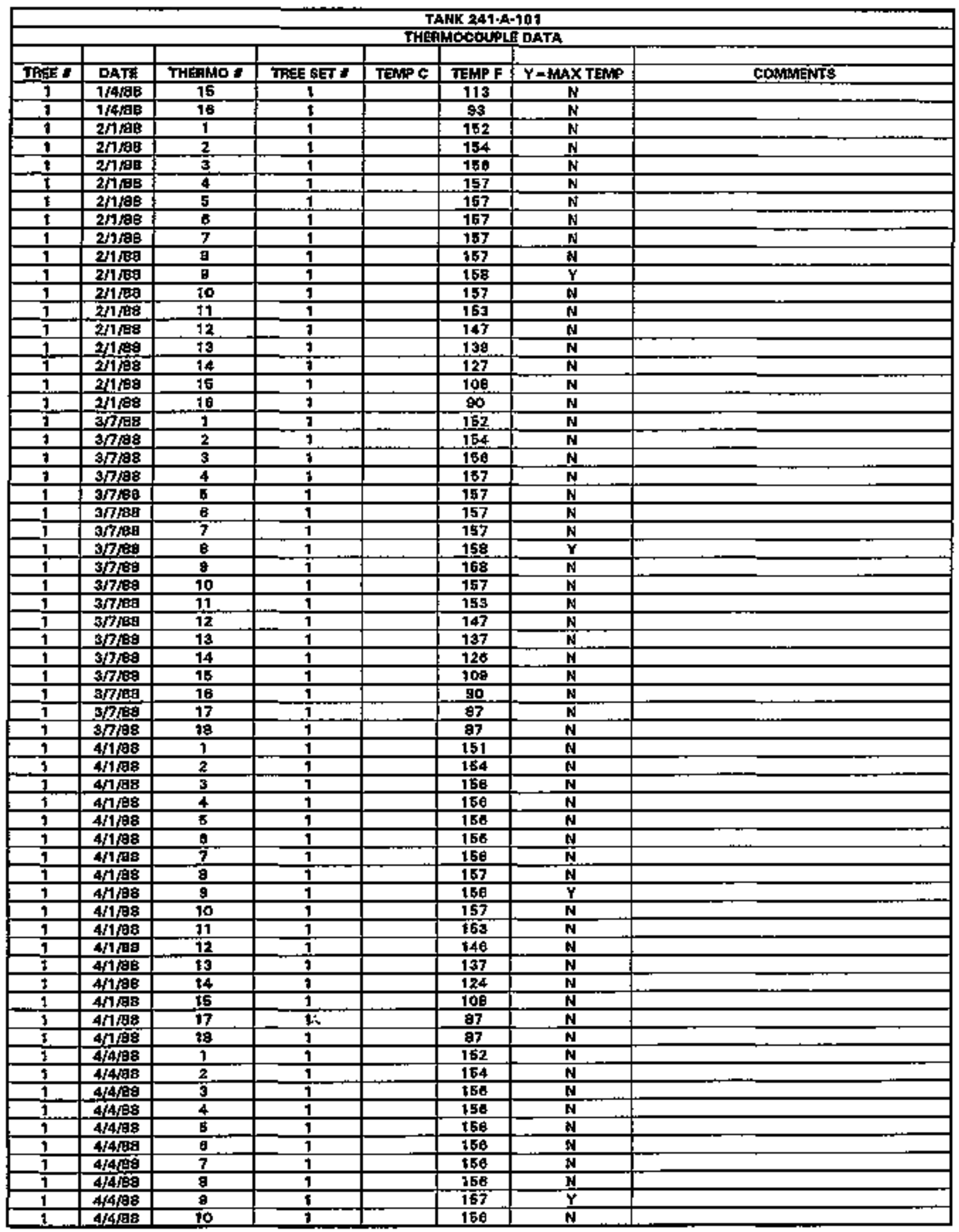

Data obtained from WHC Survellance Analysis Computer System iSACS1, July 1, 1993. 


\begin{tabular}{|c|c|c|c|c|c|c|c|}
\hline & & & & & MK 241A & 101 & \\
\hline & & & & FHE & Aocolph & DATA & \\
\hline & & & & & & & \\
\hline TREE O & DATE & THEFMD & TREE SET & TEMPC & TEMP P & $y=\operatorname{MAX} \times$ TEMP & COMWENTS \\
\hline 1 & 4/4ras & 11 & 1 & & 162 & $\mathbf{N}$ & \\
\hline 1 & $4 / 4 / 8 B$ & 12 & 1 & & 14\% & $N$ & \\
\hline 1 & 4/4/8B & 13 & 1 & & 137 & $\bar{N}$ & \\
\hline$\overline{1}$ & $4 / 48 \mathrm{rag}$ & 74 & 7 & & 125 & $\bar{N}$ & \\
\hline 1 & $4 / 4 / 98$ & 15 & 1 & & 109 & $\mathbf{M}$ & \\
\hline 1 & $4 / 4 / 83$ & 18 & 1 & & 82 & $\mathbf{N}$ & \\
\hline 1 & $4 / 4 / 3 a$ & 17 & 1 & & 90 & $\mathbf{N}$ & \\
\hline 1 & $4 / 4 / 80$ & 18 & 1 & & 90 & $N$ & \\
\hline 1 & $5 / 2 / 8$ & 1 & 1 & & $160^{\circ}$ & $N$ & \\
\hline 1 & $5 / 2 / 39$ & 2 & 1 & & 152 & $\mathbf{N}$ & \\
\hline 1 & $5 / 2 / 8 B$ & 3. & 2 & & 154 & $\mathbf{N}$ & \\
\hline$i$ & $6 / 2 / 88$ & 4 & 1 & & 154 & $\mathbf{N}$ & \\
\hline 1 & $5 / 2 / 98$ & B & 1 & & 155 & $\mathbf{N}$ & \\
\hline 1 & $5 / 2 \sqrt{68}$ & 5 & 1 & & 155 & N & \\
\hline$T$ & 5/2/98 & $\overline{7}$ & $\overline{1}$ & & 155 & $\mathbf{N}$ & \\
\hline 1 & $5 / 2$ & 量 & 1 & & 158 & $N$ & \\
\hline 1 & $5 / 2-8$ & $\theta$ & 1 & & 157 & $\bar{Y}$ & \\
\hline 1 & $\mathrm{~B} / 2 / \mathrm{B} 8$ & 10 & 1 & & $15 \mathrm{~B}$ & $N$ & \\
\hline 1 & $5 / 2 / \mathrm{E}^{\circ}$ & 11 & 1 & & 162 & $N$ & \\
\hline 1 & $5 / 2 \sqrt{18}$ & 12 & 1 & & 145 & $\mathbf{N}$ & \\
\hline 1 & $5 / 2 \sqrt{88}$ & 23 & 1 & & 138 & $\mathbf{N}$ & \\
\hline 1 & $5 / 2 \sqrt{98}$ & 14 & 1 & & 124 & $\mathbf{N}$ & \\
\hline 1 & B/2/es & 35 & 1 & & 110 & $N$ & \\
\hline $\mathbf{1}$ & $5 / 2 \sqrt{98}$ & 18 & 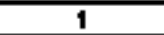 & & 84 & $N$ & \\
\hline 1 & $6 / 2 \sqrt{198}$ & 37 & 1 & & 92 & $\mathbf{N}$ & \\
\hline 1 & $5 / 288$ & 78 & 1 & & 82 & $\mathbf{N}$ & \\
\hline 1 & $5 / 3 / 9 \theta^{2}$ & 1 & 1 & & $160^{\circ}$ & $\mathbf{N}$ & \\
\hline 1 & $5 / 3 / 98$ & 2 & 1 & & 152 & $N$ & \\
\hline$\overline{1}$ & $5 / 3 / 98$ & 3 & $T$ & & 154 & $\bar{N}$ & \\
\hline 1 & $5 / 3 \longdiv { 9 8 }$ & 4 & $\overline{1}$ & & $\overline{154}$ & $\mathbf{N}$ & \\
\hline 1 & $5 / 3 \sqrt{18}$ & 5 & $\overline{1}$ & & 155 & $\mathbf{M}$ & \\
\hline 1 & $5 / 3 / 188$ & 8 & 1 & & 156 & $\overline{\mathbf{N}}$ & \\
\hline 1 & EAAB & 7 & 1 & & $150^{\circ}$ & $N$ & \\
\hline 1 & $5 / 3 / 8 \mathrm{~B}$ & 8 & 1 & & 150 & $\mathbf{N}$ & \\
\hline 1 & $5 / 398$ & 9 & 1 & & 157 & $\mathbf{Y}$ & \\
\hline 1 & $5 / 3 / 8 B$ & 10 & 1 & & 158 & $\mathbf{M}$ & \\
\hline 1 & $6 / 3$ 殒 & 11 & 1 & & 152 & $\mathbf{N}$ & \\
\hline 1 & 6/308 & 12 & 1 & & 145 & $N$ & \\
\hline 1 & $5 / 3 / 98$ & 13 & 1 & & 135 & $N$ & \\
\hline 1 & $5 / 3 / 8 \mathrm{~B}$ & 14 & 1 & & 724 & $\mathbf{M}$ & \\
\hline 1 & $6 / 3 \overline{8 B}$ & 15 & 1 & & 110 & $\bar{N}$ & \\
\hline 1 & $5 / 39 a^{2}$ & 10 & $\overline{1}$ & & 94 & $\mathbf{M}$ & \\
\hline 1 & 5/a/so & 1 & 1 & & 159 & $\mathbf{N}$ & \\
\hline 1 & $5 / 6 / 8 B$ & 2 & 1 & & 159 & $\mathbf{N}$ & \\
\hline$i$ & $5 / 87$ Bg & 3 & 1 & & 158 & $\mathbf{N}$ & \\
\hline 1 & Eforag & 4 & 1 & & 158 & $\dot{N}$ & \\
\hline 1 & $5 / 68 \mathrm{P}$ & 5 & 1 & & $\$ 50$ & $\mathbf{N}$ & \\
\hline 1 & $5 / 0 / B \beta$ & 6 & 1 & & 156 & $\mathbf{N}$ & \\
\hline 1 & $5 / 0 / 80$ & 3 & 1 & & 156 & $\mathbf{N}$ & \\
\hline 1 & 6/6/98 & 8 & 1 & & 157 & $\mathbf{N}$ & \\
\hline 1 & $5 / 0 / 98$ & 9 & 1 & & 158 & $\mathbf{Y}$ & \\
\hline 1 & 5/4as & 10 & 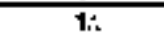 & & 150 & $\bar{N}$ & \\
\hline 1 & 5/8NB & 11 & 1 & & 152 & $\mathbf{N}$ & \\
\hline 1 & $6 / \theta / 98$ & 12 & 1 & & 148 & $\mathbf{N}$ & \\
\hline 1 & $5 / a A B$ & 13 & 1 & & 136 & $\vec{M}$ & \\
\hline 1 & 5/8/a8 & 14 & 1 & & 124 & $N$ & \\
\hline 1 & $5 / 8 \overline{98}$ & 15 & 1. & & 109 & $M$ & \\
\hline 1 & $5 / 89$ & 17 & 1 & & 91 & $\mathbf{A}$ & \\
\hline 1 & $5 / 6 / 88$ & 10 & 1 & & 91 & $\mathbf{N}$ & \\
\hline 1 & $6 / 6 / 98$ & $i$ & 1 & & 144 & $\mathbf{N}$ & \\
\hline 1 & 8/6/98 & 2 & 1 & & 146 & $\overline{\mathbf{N}}$ & \\
\hline 1 & $6 / 8 \sqrt{188}$ & 3 & 1 & & 148 & $\mathbf{M}$ & \\
\hline 1 & $6 / 6 \sqrt{88}$ & 4 & 1 & & 150 & $\bar{\gamma}$ & \\
\hline
\end{tabular}

Data obtained from WHC Survellance Anelysis Computer Systen (SACS), July 1, 1993. 


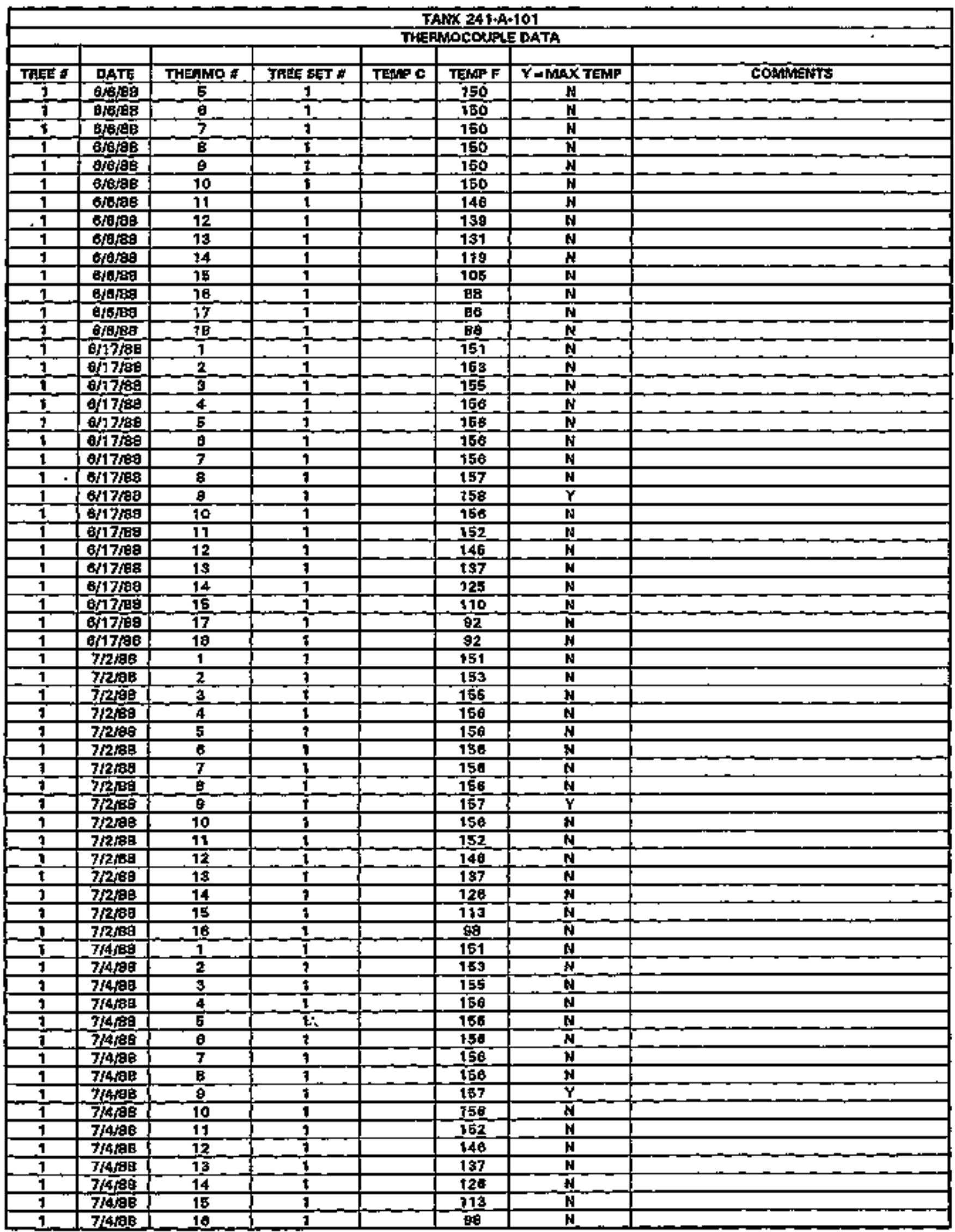

Deta obtained from WHC Surveillance Anałysis Computer Sץstem [SACS), July 1, 1993. 


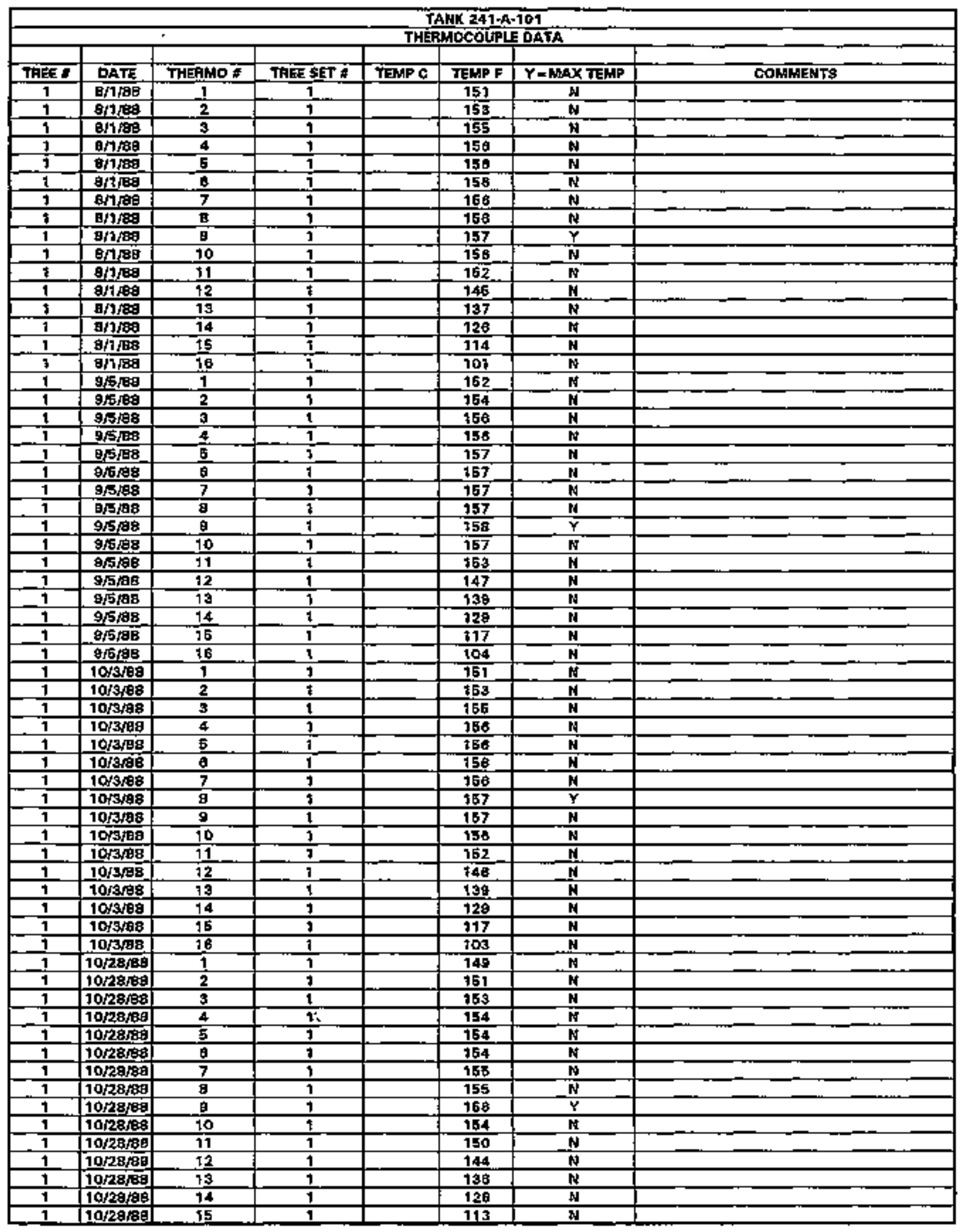

Data obtained from WHC Survefluance Analysis Computer System (SACS), July 1, 1993. 


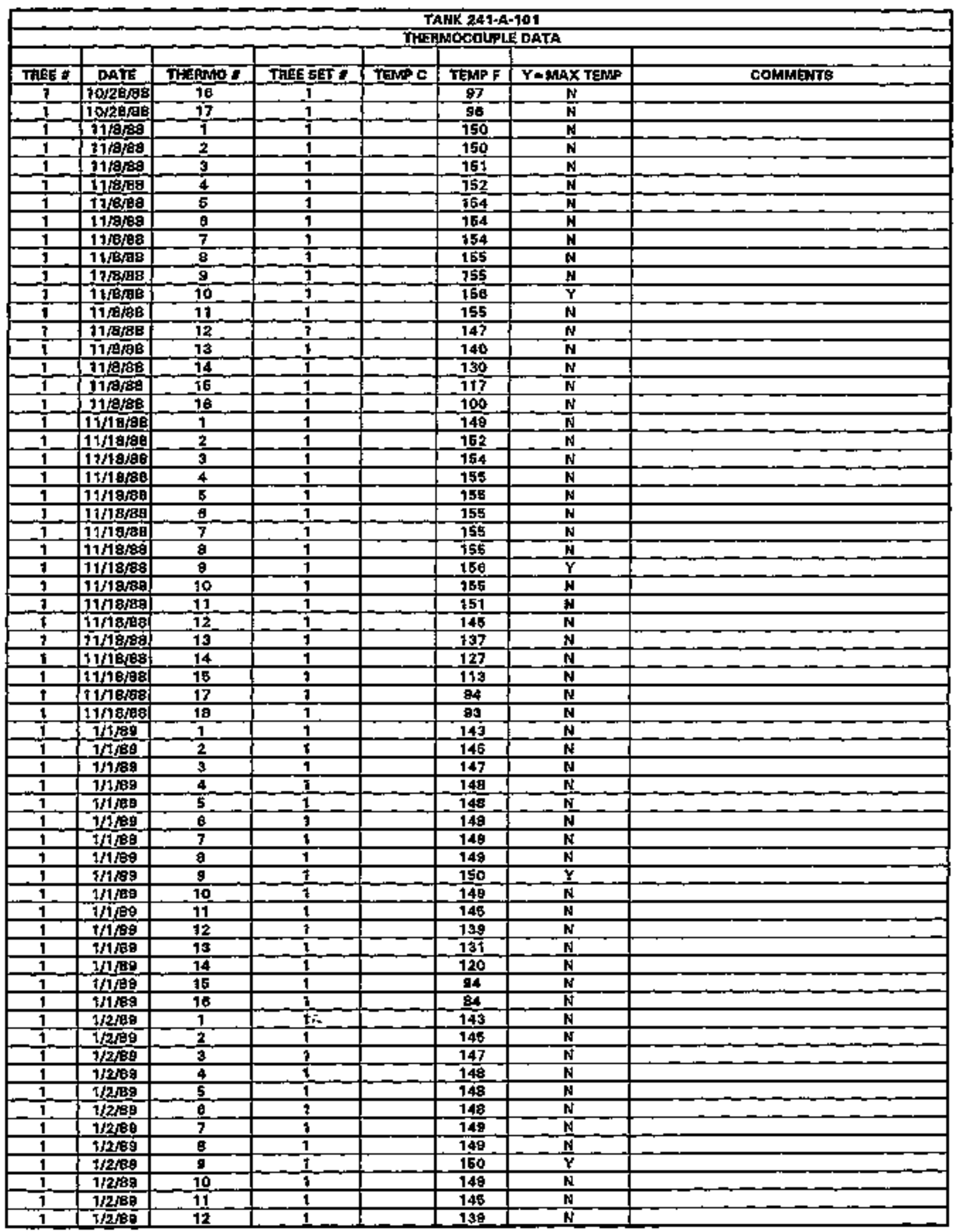

Data obtained trom WHic Survelliance Analysis Computer System (SACS), July 1, 1993. 


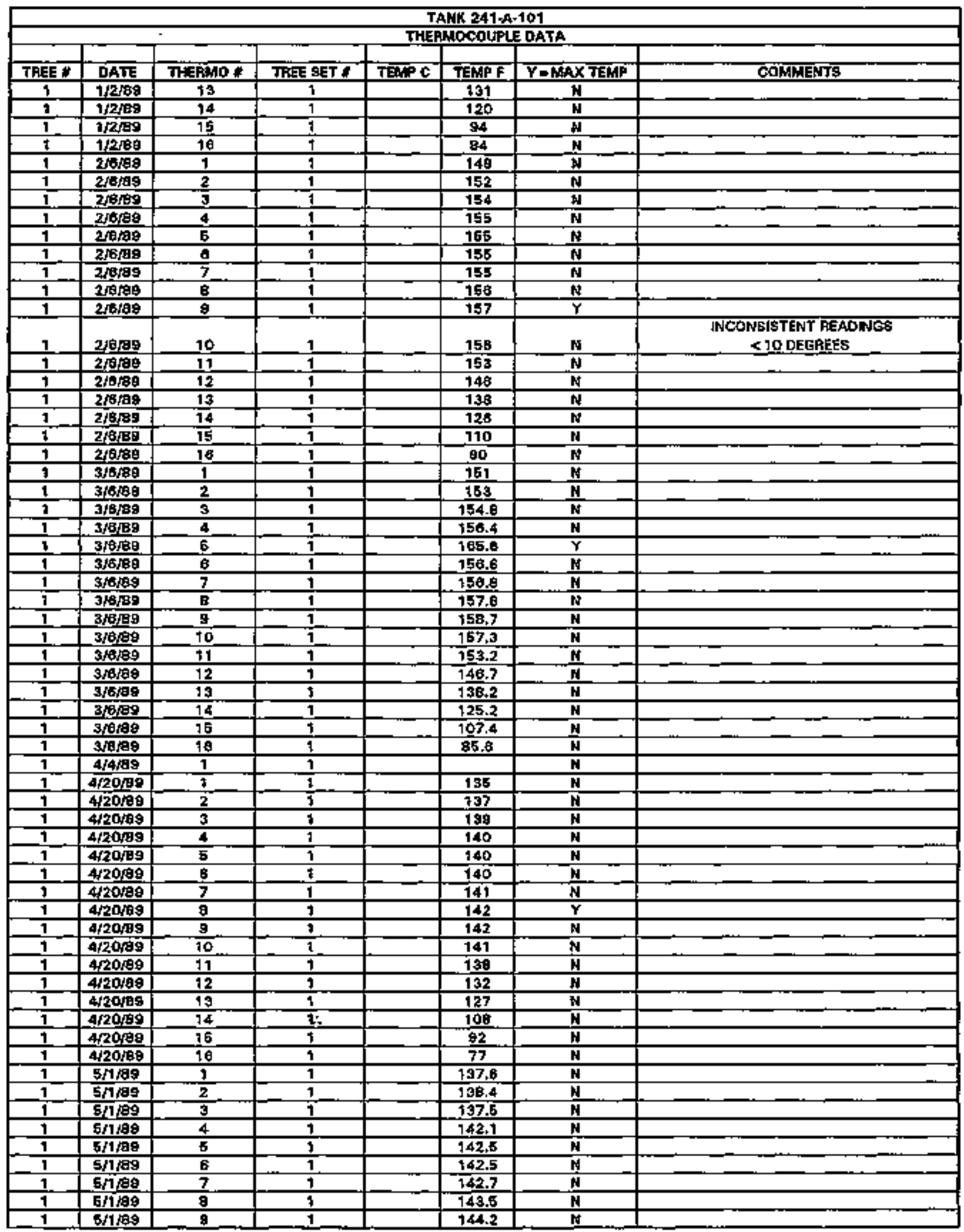

Data obtained from WHC Surveillance Analysis Computer System (SACSl, July 1. 1993. 


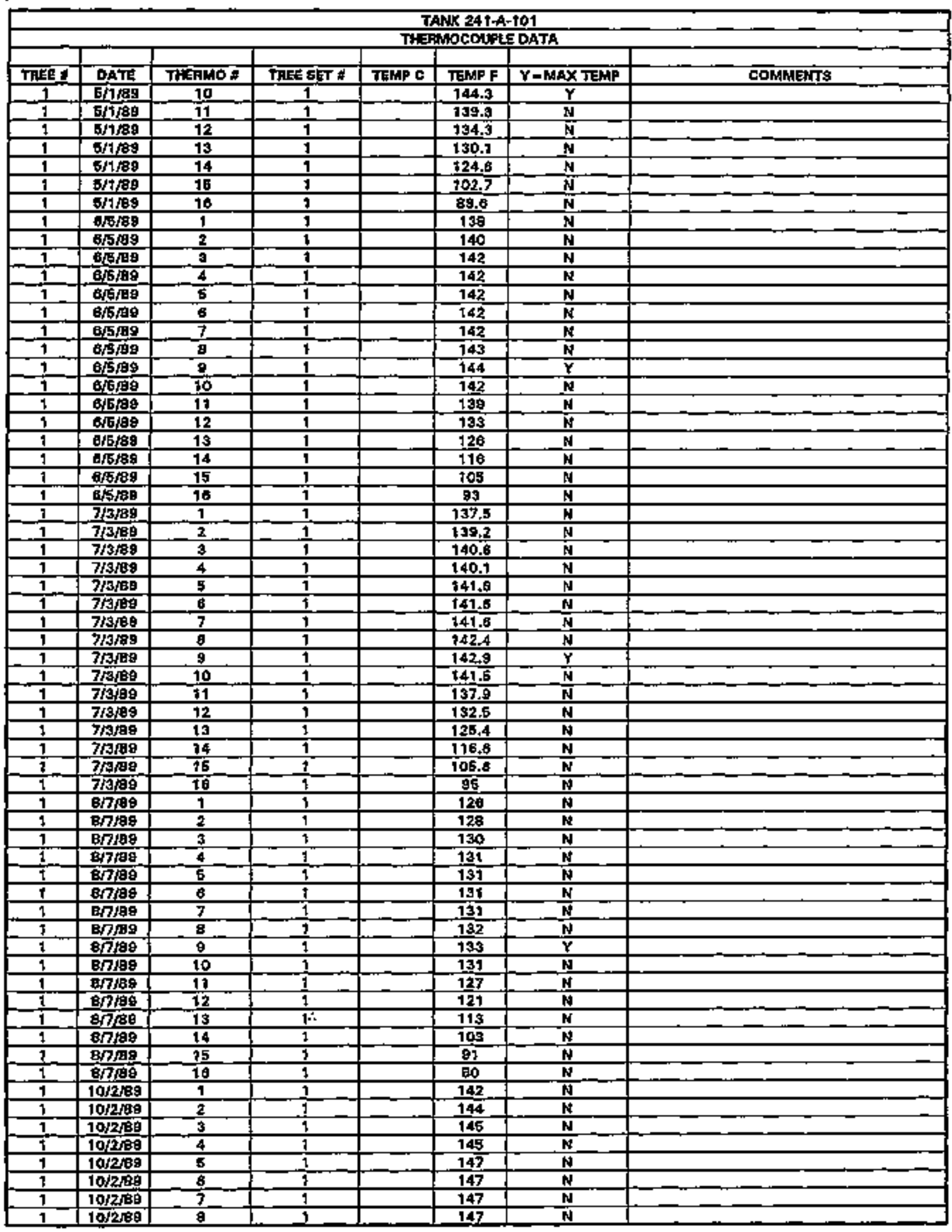

Data obtained from WHC Surveillance Analysis Computer System (SACS), July 1, 1993. 


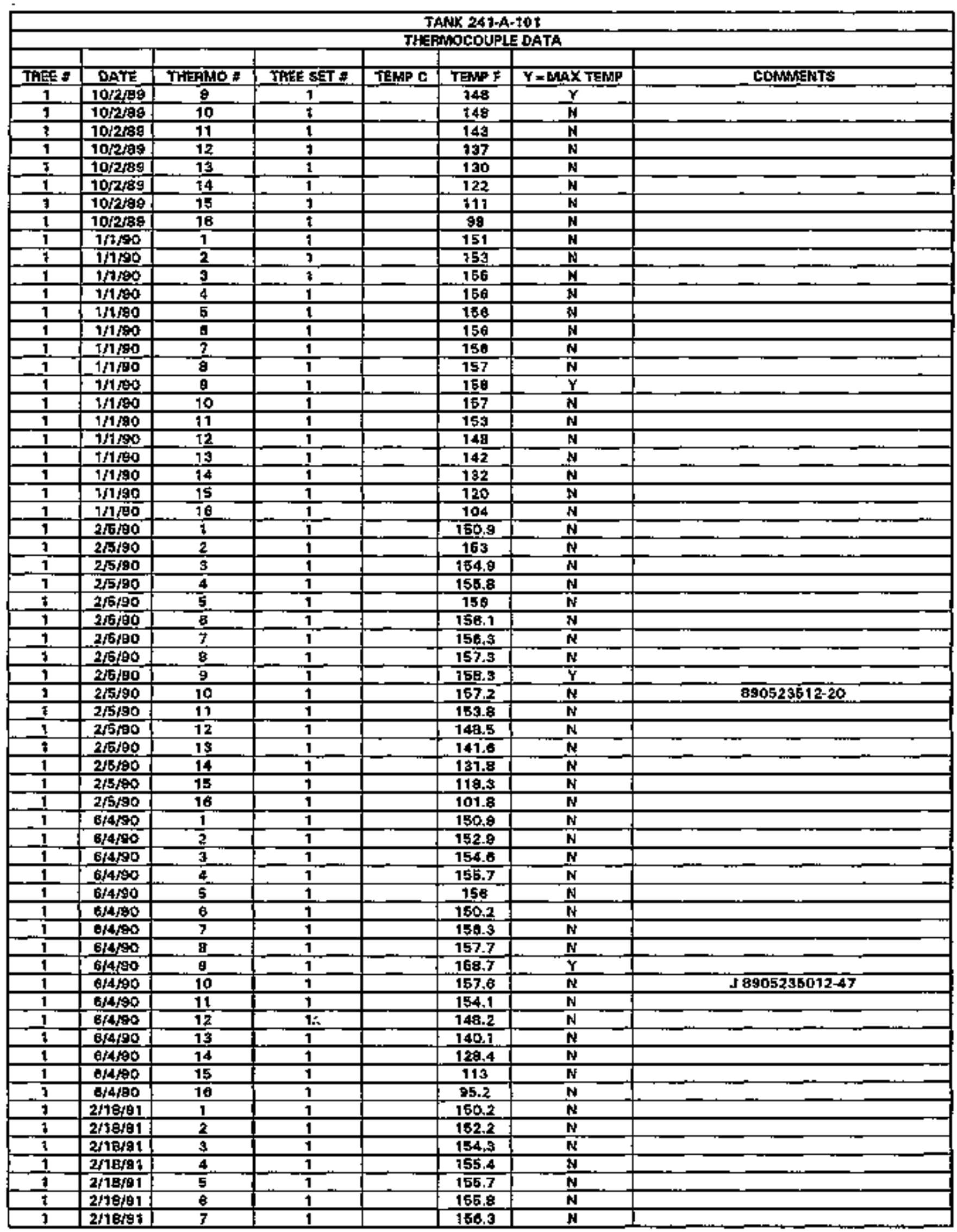

Dats obtained from WhC Surveilance Anglysis Computer System ISACS\}, July 1, 1993. 


\begin{tabular}{|c|c|c|c|c|c|c|c|}
\hline \multicolumn{8}{|c|}{ TAFK 241 A-101 } \\
\hline & & & & & & & \\
\hline R. & $\overline{D A T E}$ & Fifing & 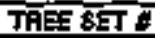 & FEMTC & TEMPF & $\bar{Y}=\mathrm{MAX}$ TST & COWMENTS \\
\hline 1 & $2 / 18 \% 91$ & $\mathrm{a}$ & 1 & & 167.8 & i & \\
\hline $\bar{t}$ & 2/18/91 & 9 & $\overline{1}$ & & 158 & $\bar{\gamma}$ & \\
\hline 1 & $2 / 8 / 91$ & 10 & $\overline{1}$ & & 157.5 & M & $2 E-0 P 021$ \\
\hline 1 & U18/at & 11 & 1 & & 153,4 & $\mathbf{N}$ & \\
\hline 1 & $2 / 18 / 85$ & 12 & 7 & & 145,5 & $\bar{N}$ & \\
\hline 1 & 20891 & 13 & 1 & & $137+5$ & H & \\
\hline 1 & 2/18/91 & 14 & 1 & & 123.7 & $\mathbf{N}$ & \\
\hline 1 & 2 f1E/9 & 15 & 1 & & 705.5 & $\mathbf{N}$ & \\
\hline 1 & $2 / 7 \mathrm{Br} 1$ & 16 & 1 & & B3.9 & $\mathbf{N}$ & \\
\hline 1 & $2 / 26 \overline{9}$ & 1 & $i$ & & 145 & N & \\
\hline$\overline{1}$ & $2 / 20 / 91$ & $\overline{2}$ & $\overline{1}$ & & 140.8 & H & \\
\hline$\overline{1}$ & $2 / 26 / 91$ & 5 & 1 & & 1486 & $N$ & \\
\hline 1 & $2 / 26 / 91$ & $\overline{4}$ & 1 & & 149.7 & $\mathbf{N}$ & \\
\hline 1 & $2 / 20 / 1$ & 5 & 1 & & 150 & $N$ & \\
\hline 1 & $2 / 26 / 91$ & 6 & $T$ & & 150,2 & $\overline{\mathbf{N}}$ & \\
\hline 1 & $2 / 26 / 91$ & 7 & 1 & & 150,4 & $\bar{N}$ & \\
\hline 1 & $2 / 20 / 91$ & 5 & 1 & & 152 & N & \\
\hline 1 & $2 / 26 / 91$ & 9 & 1 & & 152.8 & $\mathbf{Y}$ & \\
\hline $\mathbf{T}$ & $2 / 26 / 91$ & 10 & 1 & & 151.6 & N & $2=0 P \cdot 020$ \\
\hline 1 & $\overline{2 / 2}=\overline{91}$ & 11 & $\overline{1}$ & & 147.7 & $\mathbf{N}$ & \\
\hline 1 & 2/26/91 & 12 & 1 & & 141,3 & $\mathbf{N}$ & \\
\hline 7 & $2 / 26 / 91$ & 13 & 1 & & 1312 & N & \\
\hline$i$ & 2/20191 & 14 & $\overline{1}$ & & $\overline{178.6}$ & $\mathbf{N}$ & \\
\hline $\overrightarrow{1}$ & $2 / 26 / 91$ & 15 & $\overline{1}$ & & 101.1 & $\mathbf{N}$ & \\
\hline 1 & $2 / 26 / 91$ & 16 & 1 & & 60.6i & $\mathbf{N}$ & \\
\hline 1 & $3 / 5 / 91$ & 1 & 1 & & 149,1 & $\mathbf{N}$ & \\
\hline $\bar{F}$ & $3 / 5 / 91$ & $\overline{\mathbf{z}}$ & $T$ & & $1 \$ 1.1$ & N & \\
\hline 1 & $3 / 5 \sqrt{91}$ & 3 & $\overline{1}$ & & $\overline{152.9}$ & $\mathrm{~N}$ & \\
\hline$\overline{1}$ & $3 / 6 \sqrt{6 t}$ & 4 & $\overline{1}$ & & 754 & $N$ & \\
\hline 1 & $3 / 5 / 91$ & 5 & 1 & & 154,3 & $\mathbf{N}$ & \\
\hline 1 & $3 \sqrt{5 / 91}$ & $\overline{8}$ & 1 & & 154,4 & $\mathbf{N}$ & \\
\hline 1 & $36 / 81$ & 7 & 1 & & 154.6 & $\mathbf{N}$ & \\
\hline 1 & $3 \sqrt{5} \sqrt{81}$ & g & $\overline{1}$ & & 15.3. & $N$ & \\
\hline 1 & 367 & g一 & 1 & & $\overline{167.2}$ & $Y$ & \\
\hline 1 & 35但1 & 10 & 1 & & 155.8 & $\mathbf{N}$ & J $2 \mathrm{E}-\mathrm{OP}-021$ \\
\hline 1 & $3 \sqrt{5 / 31}$ & 11. & 1 & & T51.8 & $\mathbf{N}$ & \\
\hline 1 & $3 / 5,94$ & $\sqrt{12}$ & 1 & & 145.2 & N & \\
\hline 1 & $3 \sqrt{51}$ & 13 & 1 & & 136.4 & $\mathbf{N}$ & \\
\hline 1 & $3 \sqrt{61}$ & 14 & 1 & & 122.8 & $\mathbf{N}$ & \\
\hline 1 & $3 \sqrt{5 / 01}$ & 15 & 9 & & 104.9 & $\mathbf{N}$ & \\
\hline$\overline{1}$ & $3 \sqrt{5}, 91$ & 19 & $\overline{1}$ & & 83.8 & N & \\
\hline 1 & $3 / 10 / 91$ & 7 & 1 & & 149.7 & $N$ & \\
\hline 1 & 3r10rgi & 2 & 1 & & $15 t .0$ & $\mathbf{N}$ & \\
\hline 1 & $3+1009$ & 3 & 1 & & 159.6 & $\mathbf{N}$ & \\
\hline 1 & 370/91 & 4 & 1 & & 154.t. & $\mathbf{N}$ & \\
\hline 1 & $3 / 10 / 91$ & 5 & 1 & & I55 & N & \\
\hline 1 & 310/41 & 旁 & 1. & & 165.1 & $\mathbf{N}$ & \\
\hline 1 & 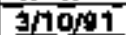 & 7 & 1 & & T5F. & $\mathbf{N}$ & \\
\hline$\overline{1}$ & अ10191 & a & 1 & & 157 & $\mathbf{N}$ & \\
\hline 1 & $3 / 10 / 91$ & 9 & 1 & & 157.9 & $\mathbf{Y}$ & \\
\hline 1 & $3 / 10 / 91$ & 10 & 1 & & 166.4 & $N$ & $\$ 890523512+20$ \\
\hline 1 & 30101 & 11 & 1: & & 152,4 & $\mathbf{N}$ & \\
\hline 1 & अव/ & 12 & 1 & & 145.8 & $\mathbf{N}$ & \\
\hline 1 & अ10/91 & 13 & 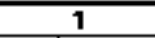 & & $\overline{1} 3 . \overline{9}$ & 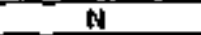 & \\
\hline 1 & अण्OS1 & $1 \overrightarrow{4}$ & 1 & & 123.2 & $\mathbf{N}$ & \\
\hline 1 & उ/काश & 75 & 1 & & 105.2 & $\mathbf{N}$ & \\
\hline 1 & \$/10/91 & 10 & 1 & & $\mathrm{~B} 4 . \overline{7}$ & $N$ & \\
\hline 1 & $3 / 7 / 91$ & 3 & 1 & & $149 . \bar{B}$ & $\mathbf{N}$ & \\
\hline 1 & अ17/91 & 2 & 1 & & 151.8 & N & \\
\hline 1 & अ/1791 & 3 & 1 & & 153,5 & $\mathbf{N}$ & \\
\hline 1 & $3 / 17 / 91$ & 4 & 1 & & 154,5 & $\mathbf{M}$ & \\
\hline 1 & आ17\%1 & 5 & 1 & & $164 . \dot{B}$ & $\mathbf{m}$ & \\
\hline 1 & आ17/91 & $\mathbf{8}$ & 1 & & 154.8 & N & \\
\hline
\end{tabular}

Data obtatned from WhC Survetiance Analysis Computer SYstam [SACSY, Juh 1, 1993.

D-143 


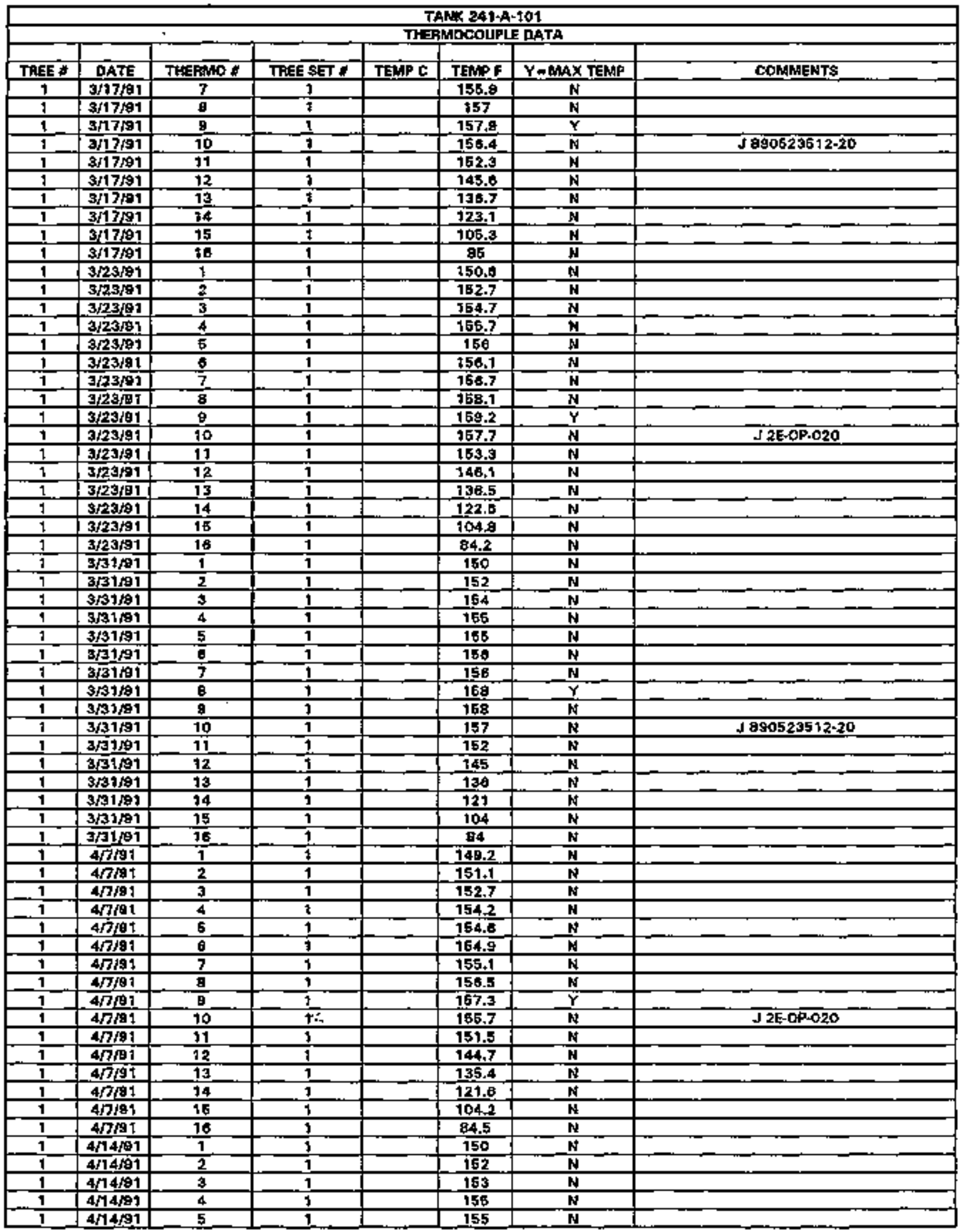

Datta obtained from WHC Surveillance Analysis Computer System [SACS], July 1, 1993. 


\begin{tabular}{|c|c|c|c|c|c|c|c|}
\hline \multicolumn{8}{|c|}{ TAMIK 241-A-101 } \\
\hline & & & & TrE & MOCOUP & DATA & \\
\hline TAEE & DATE & THEakT & THF 8 & TEMP C & YETip F & $Y=$ MAX YEHP & COHATENTS \\
\hline 1 & $4 / 24191$ & $\underline{6}$ & 1 & & 165 &. $\mathbf{N}$ & \\
\hline 1 & $4 / 14191$ & 7 & 1 & & 168 & $\mathbf{N}$ & \\
\hline 5 & $4 / 4497$ & 8 & $\mathbf{T}$ & & 157 & $\bar{N}$ & \\
\hline 1 & $4 / 14 / 81$ & 8 & 1 & & 158 & $\bar{Y}$ & \\
\hline 1 & $4 / 14 / 91$ & 10 & $t$ & & 156 & $\mathbf{N}$ & I2E+OP-O2O \\
\hline 1 & 4/14/91 & 11 & 1 & & 152 & $\mathbf{N}$ & \\
\hline 1 & $4 / 14 / 91$ & 12 & 1 & & 145 & $\mathbf{N}$ & \\
\hline 1 & 4/14/91 & 13 & 1 & & 136 & $\mathbf{N}$ & \\
\hline 1. & $4 / 14 / 91$ & 14 & 1 & & $12 \overline{2}$ & $\mathbf{N}$ & \\
\hline 1 & 4/14/01 & 15 & 1 & & 105 & $\mathbf{N}$ & \\
\hline 1 & $4 / 14 / 91$ & 10 & 1 & & 86 & $\mathrm{~N}$ & \\
\hline 1 & $4 / 21 / 81$ & 1 & 1 & & 148.8 & $\mathbf{N}$ & \\
\hline 1 & $4 / 21 / 91$ & 2 & 1 & & 151.8 & $\mathbf{N}$ & \\
\hline 1 & $4 / 21 / 1$ & 3 & 1 & & 158.4 & $\mathbf{N}$ & \\
\hline 1 & $4 / 21 / 91$ & 4 & 1 & & 154.4 & $\mathbf{N}$ & \\
\hline 1 & A21/ & 5 & 1 & & 154.8 & $\mathbf{N}$ & \\
\hline 1 & $4 / 21 / 91$ & $\$$ & 1 & & 154.6 & N & \\
\hline 1 & $4 / 21 / 91$ & 7 & 1 & & 155,4 & $\mathbf{N}$ & \\
\hline 1 & $4 / 21 / 80$ & 8 & 1 & & 155.9 & $\mathbf{N}$ & \\
\hline 1 & $4 / 21 / 91$ & $\theta$ & $t$ & & 167,9 & $\mathbf{Y}$ & \\
\hline 1 & $4 / 21 / 91$ & 10 & 3 & & 150 & N & $\mathrm{JHH}-\mathrm{gg} \mathrm{AN}=\mathrm{J}$ \\
\hline 1 & $4 \sqrt{21 / 91}$ & 11 & 1 & & 151,5 & $\mathbf{N}$ & \\
\hline 1 & $4 / 21 / 91$ & 12 & 1 & & 144.7 & $\mathbf{N}$ & \\
\hline 1 & $4 \sqrt{21 / 91}$ & 13 & 1 & & 135,4 & $\mathbf{N}$ & \\
\hline 1 & $4 / 21 / 91$ & 14 & 1 & & 1218 & $\mathbf{N}$ & \\
\hline 1 & $4 / 21 / 91$ & 15 & $t$ & & 104.6 & $\mathbf{N}$ & \\
\hline 1 & $4 / 23 / 91$ & 16 & 1 & & 80.1 & $\mathbf{N}$ & \\
\hline 1 & 4/29/91 & 1 & 1. & & 150 & $\mathbf{N}$ & \\
\hline 1 & $4 / 28 / 81$ & 2 & 1. & & 1619 & $\mathbf{N}$ & \\
\hline 1 & $4 / 29 / 91$ & 3 & 1 & & 153.7 & N & \\
\hline 1 & $4 / 28 / 91$ & 4 & 1 & & 154.8 & $\mathbf{N}$ & \\
\hline 1 & 4/28/91 & 5 & 1 & & 155 & $\mathbf{N}$ & \\
\hline 1 & d/28) & $\underline{6}$ & 1 & & 155.2 & $\mathbf{N}$ & \\
\hline 1 & $4 / 26 / 91$ & 7 & 1 & & $15 \underline{5.7}$ & $\mathbf{N}$ & \\
\hline 1 & $4 / 28$ det & 8 & 1 & & 157.1 & $\mathbf{N}$ & \\
\hline 1 & $4 / 28 / 91$ & $\mathbf{g}$ & 1 & & 157.9 & $\boldsymbol{Y}$ & \\
\hline 1 & 4/2E/31 & 10 & 1 & & 158.2 & $\mathbf{N}$ & J990523512.20 \\
\hline 1 & $4 / 26 / 31$ & 11 & 1 & & 151.6 & N & \\
\hline 1 & $4 \longdiv { 2 9 }$ & 12 & 1 & & 144.9 & $\mathbf{N}$ & \\
\hline 1 & $4 / 29 / 91$ & 13 & 1 & & 135.6 & $\mathbf{N}$ & \\
\hline 1 & 4/28/91 & 14 & 1 & & 122 & N & \\
\hline 1 & 4/20/91 & 15 & 1 & & 10.1 & $\mathbf{N}$ & \\
\hline 1 & $4 / 2,6 / 91$ & $1 \overline{6}$ & 1 & & 80.9 & $N$ & \\
\hline 1 & 5/4/91 & 1 & 1 & & 148.1 & $\mathrm{~N}$ & \\
\hline 1 & $5 / 4 / 41$ & 2 & 1 & & 180.1 & $\mathbf{N}$ & \\
\hline 1 & $\$ 4 / 91$ & $\mathbf{3}$ & 1 & & 161.8 & $\mathbf{N}$ & \\
\hline 1 & $5 / 4 / 91$ & 4 & 1 & & 15.2 .9 & H & \\
\hline 1 & $5 / 4 / 91$ & 5 & 1 & & 153.2 & $\mathrm{~N}$ & \\
\hline 1 & W/4/91 & 5 & 1 & & 163,4 & N & \\
\hline 1 & $5 / 4 / 91$ & 7 & 1 & & 153.8 & $\mathbf{N}$ & \\
\hline 1 & $5 / 4 \% 1$ & 8 & 1 & & 155.2 & $\mathrm{~N}$ & \\
\hline 1 & $5 / 4 / 31$ & 8 & W' & & 155.9 & $Y$ & \\
\hline 1 & 5/4/91 & 10 & 1. & & 154.2 & $N$ & $J 890523512+20$ \\
\hline 1 & $5 / 4 / 91$ & 11 & 1 & & 148.8 & $\mathbf{N}$ & \\
\hline 1 & $5 / 4 / 91$ & 12 & 1 & & 142,7 & N & \\
\hline 1 & $5 / 4 / 91$ & 13 & 3 & & 733.7. & $\mathbf{N}$ & \\
\hline 1 & $5 / 4 / 91$ & 14 & 1. & & 119.9 & $\mathbf{N}$ & \\
\hline 1 & $5 / 4 / 91$ & 15 & 1 & & $\$ 03.7$ & $\mathbf{N}$ & \\
\hline 1 & $5 / 4 / 91$ & 10 & 1 & & 96,4 & $\mathbf{N}$ & \\
\hline 1 & 5/24/91 & 1 & 1 & & 747.8 & N & \\
\hline 1 & $5 / 24 / 91$ & 2 & 1 & & 149.8. & $N$ & \\
\hline 1 & $5 / 24 / 91$ & 3 & 1 & & 151.4 & $\mathrm{~N}$ & \\
\hline 1 & $5 / 24 / 91$ & 4 & 1 & & 152.4 & $\mathbf{N}$ & \\
\hline
\end{tabular}

Data obtained from WHC Surveillanice Aralysis Computer System (SACS), July 1, 1993. 
WHC-SO-WM-ER-308, REv. 0

\begin{tabular}{|c|c|c|c|c|c|c|c|}
\hline \multicolumn{8}{|c|}{ TANK 241-A-101 } \\
\hline & & & & TSE & DOCONP & DATA & \\
\hline TREE & DATE & THEAMO & TAEE SET \# & TEMAP C & TEMPF & $Y=M A X T E M P$ & CDNWWENTS \\
\hline 1 & $5 / 24 / 91$ & 5 & 1 & & 152.7 & $\mathbf{N}$ & \\
\hline$\overline{1}$ & $5 / 2491$ & e & 1 & & 152.9 & $\mathbf{N}$ & \\
\hline 1 & $5 / 24 / 81$ & 7 & $t$ & & 153.3 & $\mathbf{N}$ & \\
\hline 1 & $5 / 24 / 91$ & 8 & 1 & & $15 \overline{4} . \overline{7}$ & $\mathbf{M}$ & \\
\hline 7 & $5 / 24 / 91$ & 8 & 1 & & 155.3 & $\bar{Y}$ & \\
\hline $\bar{t}$ & $5 / 24 / 91$ & 10 & 1 & & 153.5 & $\mathbf{N}$ & $4890523512-20$ \\
\hline 1 & $\overline{8 / 24 / 91}$ & 11 & 1 & & 148.9 & $\boldsymbol{N}$ & \\
\hline 1 & $\mathrm{~B} / 24 / 91$ & $\sqrt{12}$ & 1 & & 741.8 & $\bar{N}$ & \\
\hline 1 & $5 / 24 / 91$ & 13 & 1 & & 132,2 & $\mathbf{N}$ & \\
\hline 1 & 5/24/91 & 74 & 1 & & 110.2 & $\mathbf{N}$ & \\
\hline 1 & $5 / 24 / 91$ & 15 & 1 & & 103.6 & $N$ & \\
\hline$\dot{i}$ & $6 / 24 / 91$ & 18 & 1 & & 87,2 & $\mathbf{N}$ & \\
\hline 1 & $5 / 25 / 91$ & 1 & $\$$ & & 147.8 & $\mathbf{N}$ & \\
\hline 1 & $5 / 26 / 91$ & 2 & 1 & & 148.5 & $\mathbf{N}$ & \\
\hline 1 & $5 / 20181$ & 3 & 1 & & 151.2 & $\mathbf{N}$ & \\
\hline $\mathbf{1}$ & $5 / 20 / 91$ & 4 & $\overline{1}$ & & 152.2 & $\mathbf{N}$ & \\
\hline$\overline{1}$ & $5 \overline{20} / 91$ & 5 & 7 & & $\overline{152.5}$ & $\bar{N}$ & \\
\hline 1 & $5 / 25 / 97$ & 白 & 1 & & 152.6 & $\overline{\mathbf{N}}$ & \\
\hline 1 & $5 / 28 / 91$ & 7 & 1 & & 163 & $\mathbf{N}$ & \\
\hline 1 & $5 / 20 / 91$ & B & 1 & & 154.5 & $N$ & \\
\hline 1 & $5 / 2691$ & $\mathbf{\theta}$ & 1 & & 155,1 & $\mathbf{Y}$ & \\
\hline 1 & 5/28/91 & 10 & 1 & & 153.3 & $\mathbf{N}$ & $\$ 2 E$ 2EP-02末 \\
\hline 1 & $5 / 26 / 89$ & 11 & 1 & & 148.7 & $\bar{N}$ & \\
\hline 1 & $5 / 26 / 91$ & 12 & 1 & & 141.6 & $N$ & \\
\hline 1 & $5 / 26 / 91$ & 13 & $\mathbf{1}$ & & 132 & $\mathbf{N}$ & \\
\hline 1 & $6 / 20,93$ & 14 & 1 & & 118.1 & $\mathbf{N}$ & \\
\hline 1 & 5/2erla1 & 15 & 1 & & 103.5 & $\mathbf{N}$ & \\
\hline 1 & $\$ / 26 / 99$ & 10 & 1 & & 87.2 & N & \\
\hline 1 & $6 / 1 / 91$ & 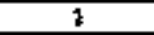 & 1 & & 147.9 & $\mathbf{N}$ & \\
\hline 1 & $6 / 1,91$ & 2 & 1 & & 149.9 & $\mathbf{N}$ & \\
\hline 1 & $6 / 1 \% 91$ & 3 & 1 & & 151.0 & $\mathbf{N}$ & \\
\hline 3 & Eก11 & 4 & 1 & & 152.6 & $\mathbf{M}$ & \\
\hline 1 & $8 / 1 / 91$ & 5 & 1 & & 152.E & $\mathbf{N}$ & \\
\hline$\$$ & 6f1/91 & 6 & 1 & & 153.6 & $\mathbf{N}$ & \\
\hline 1 & 0/t/91 & 7 & 1 & & 153.6 & $\mathbf{N}$ & \\
\hline 1 & B/T/91 & 8 & 1 & & 155 & $\mathbf{N}$ & \\
\hline .5 & $8 / 1 / 91$ & $\theta$ & 1 & & 158.5 & $\bar{Y}$ & \\
\hline 1 & ef191 & 10 & 1 & & 153.6 & $\mathbf{N}$ & J 990523512.20 \\
\hline 1 & $6 / 1 / 91$ & 11 & 1 & & 149.1 & $\mathbf{N}$ & \\
\hline 1 & W/91 & 12 & 1 & & 141.8 & $M$ & \\
\hline 1 & 1/1/91 & 13 & 1 & & 132.3 & $\mathbf{N}$ & \\
\hline$\overline{1}$ & 0/101 & 14 & 1 & & 119.0 & $\mathbf{N}$ & \\
\hline $\mathbf{1}$ & $6 / 1 / 91$ & 15 & 1 & & 104,2 & $\mathbf{M}$ & \\
\hline 1 & $6 / 1 / 91$ & 16 & 1 & & $86+2$ & $\mathbf{N}$ & \\
\hline 1 & $6 / 0 / 91$ & 1 & 1 & & 148.9 & $\mathbf{N}$ & \\
\hline 1 & 6/8/91 & 2 & 1 & & 160.8 & $\mathbf{N}$ & \\
\hline 1 & 6/9/91 & 3 & 1 & & 152.4 & $\mathbf{M}$ & \\
\hline 1 & $6 / 9 / 91$ & 4 & 1 & & 653,9 & $N$ & \\
\hline 7 & e/8/91 & 5 & 1 & & 154 & $\overline{\mathbf{N}}$ & \\
\hline 1 & 6/8/91 & 6 & 1 & & 154.2 & $\mathbf{N}$ & \\
\hline 1 & g/e/91 & 7 & 1 & & 154.3 & $\mathbf{M}$ & \\
\hline 1 & 0/9/91 & $\mathrm{g}$ & $1 ;$ & & $\$ 55.9$ & $\mathbf{H}$ & \\
\hline 9 & 6/8/91 & $\mathrm{g}$ & 1 & & 158.3 & $\bar{Y}$ & \\
\hline$t$ & $6 / 9 / 91$ & 10 & 1 & & 154.4 & $\mathbf{N}$ & J $890523512-20$ \\
\hline 1 & 0/0/91 & 11 & 1 & & 180 & $\mathbf{N}$ & \\
\hline 1 & $6 / 9 / 91$ & 12 & 1 & & 143.1 & $\mathbf{N}$ & \\
\hline 1 & BFengt & 13 & 1 & & $\pm \$ 3.6$ & $\mathbf{N}$ & \\
\hline 7 & e/e/91 & 14 & 1 & & 120.7 & $\mathbf{N}$ & \\
\hline 1 & H/91 & 15 & 1 & & $10 \% .4$ & $\overline{\mathbf{N}}$ & \\
\hline 1 & $6 / 9 / 91$ & 16 & 1 & & 89.3 & $\overline{\mathbf{N}}$ & \\
\hline$i$ & B/18/91 & 1 & 1 & & 147.8 & $\mathbf{N}$ & \\
\hline 1 & Bhero1 & 2 & 1 & & 149.7 & $\mathbf{N}$ & \\
\hline 1 & ofte/g1 & 3. & 1 & & 151,3 & $\mathbf{N}$ & \\
\hline
\end{tabular}

Data obtained from WHC Surveillence Analysis Computer System (SACS), July 1, 1993. 


\begin{tabular}{|c|c|c|c|c|c|c|c|}
\hline \multicolumn{8}{|c|}{ TANR 241+A-10' } \\
\hline & & & & & & & \\
\hline 被 & DATE & THEFMO & TREE SET & TEAP C & TEMP F & $Y=M A X$ TEMP & COMMENTS \\
\hline$t$ & E106191 & 4 & 1 & & $152+3$ & $\mathbf{N}$ & \\
\hline 1 & 6160t & 5 & 1 & & 152.7 & $\mathbf{N}$ & \\
\hline 7 & odiore? & $b$ & 1 & & 152.9 & $\bar{M}$ & \\
\hline 1 & O/19/1 & 7 & 1 & & 153.1 & $\overline{\mathbf{N}}$ & \\
\hline 1 & $0 / 16 / 01$ & B & 1 & & 154.8 & $\mathbf{N}$ & \\
\hline 9 & OAdogt & 8 & 1 & & 155 & $\bar{Y}$ & \\
\hline 1 & artara1 & 10 & 1 & & 153.1 & $\mathbf{N}$ & J 2E000247. \\
\hline 1 & ortejg1 & 11 & 1 & & 148.5 & $\mathbf{N}$ & \\
\hline 1 & oridg1 & 12 & 1 & & 147.5 & $\mathbf{N}$ & \\
\hline 1 & 6/10/91 & 13 & 1 & & 131.9 & $\bar{N}$ & \\
\hline 1 & $6 / 19 / 91$ & 14 & 1 & & 110.4 & $\mathbf{H}$ & \\
\hline 1 & 6/19/91 & 15 & 1 & & 104,6 & $\mathbf{n}$ & \\
\hline 1 & 6/10/91 & 28 & 1 & & aE,9 & $\mathbf{N}$ & \\
\hline 1 & $6 / 2391$ & 1 & 9 & & 147.9 & $\mathbf{M}$ & \\
\hline 1 & $6 \sqrt{23 / 91}$ & 2 & 1 & & 149,8 & $\mathbf{M}$ & \\
\hline$i$ & $6 \sqrt{2} 31$ & 3 & 1 & & 151,5 & $N$ & \\
\hline 1 & $6 / 231$ & 4 & 1 & & $\overline{152.5}$ & $\overline{\mathbf{N}}$ & \\
\hline 1 & $6 / 2+1$ & 5 & 1 & & 152.9 & N & \\
\hline 1 & 0/23/ & 6 & 1 & & 153 & $N$ & \\
\hline 1 & O/23/2t & 7 & 1 & & 153.2 & $N$ & \\
\hline 1 & $0 / 23 / 87$ & 8 & 1 & & 154.6 & $\mathbf{N}$ & \\
\hline 1 & 0/23/91 & $\theta$ & 1 & & 185.1 & $Y$ & \\
\hline 1 & $5 / 23 / 91$ & 10 & 1 & & 163.2 & $\mathbf{N}$ & $J 2 E 000247$ \\
\hline 1 & W/23/91 & 12 & 1 & & 148,6 & $\mathbf{N}$ & \\
\hline 1 & $8 \longdiv { 2 3 1 9 1 }$ & 12 & 1 & & 141.8 & $\mathbf{N}$ & \\
\hline 1 & 6/23日1 & 13 & 1 & & 131.9 & $\overline{\mathbf{N}}$ & \\
\hline 1 & 0/2301 & 14 & 1 & & 110.6 & $\mathbf{N}$ & \\
\hline 1 & 6/23/91 & 15 & 1 & & 106 & $\mathbf{N}$ & \\
\hline 1. & 6/23/81 & 16 & 1 & & 69.7 & $\mathbf{k}$ & \\
\hline 1 & $8 / 20191$ & 1 & 1 & & 140.8 & $\overline{\mathbf{N}}$ & \\
\hline 1 & 6/28/91 & 2 & 1 & & 161.8 & $\mathbf{N}$ & \\
\hline 1 & 6/29/91 & 3 & 1 & & 163,5 & $\mathbf{N}$ & \\
\hline 1 & 6/29/1 & 4 & 1 & & 154.5 & $N$ & \\
\hline 1 & $6 / 29 / 91$ & 5 & 1 & & 154.8 & $\mathbf{H}$ & \\
\hline 1 & $82 \overline{901}$ & 皇 & 1 & & I65 & $\bar{N}$ & \\
\hline 1 & $62 \overline{8191}$ & 7 & 1 & & $\overline{155.3}$ & $\mathbf{N}$ & \\
\hline 1 & 6/20/91 & 9 & 1 & & 166.6 & N & \\
\hline 1 & $6 / 20 / 91$ & 8 & 1 & & 167.1 & $\mathbf{Y}$ & \\
\hline 1 & $6 / 29 / 91$ & 10 & 1 & & 155.2 & $\mathbf{M}$ & J $910126143 \cdot 64$ \\
\hline 1 & 6/20/91 & 11 & 1 & & 750.7 & $\bar{N}$ & \\
\hline 1 & $6 / 20 / 91$ & 12 & 1 & & 143.0 & $\mathbf{N}$ & \\
\hline 1 & 6/29/91 & 13 & 1 & & 134.4 & $\mathbf{N}$ & \\
\hline 1 & $0 / 20 / 91$ & 14 & 1 & & 124,9 & $\mathbf{N}$ & \\
\hline T & $0 / 29 / 91$ & 75 & 1 & & 107.2 & $\overline{\mathbf{N}}$ & \\
\hline i & e/29/91 & 18 & 1 & & 92.2 & $\mathbf{N}$ & \\
\hline$i$ & $710 / 41$ & 1 & 1 & & 149.5 & $\mathbf{N}$ & \\
\hline 1 & $7 / 6$ & 2 & 1 & & 151.4 & $\mathbf{N}$ & \\
\hline 1 & THEA & 3 & 1 & & 153 & $\mathbf{H}$ & \\
\hline 1 & $7 / B / 91$ & 4 & 1 & & 154 & M & \\
\hline 1 & $7 / 8 / 91$ & 5 & 1 & & 154.3 & $\mathbf{N}$ & \\
\hline 1 & 78 ba & $\theta$ & 1 & & 154,4 & $\mathbf{N}$ & \\
\hline 1 & $7 / 6 / 91$ & 7 & Fi, & & 154.8 & $\mathbf{N}$ & \\
\hline 1 & 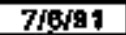 & $\mathbf{g}$ & 1 & & 750.2 & $\mathbf{N}$ & \\
\hline 1 & $70 \mathrm{se}$ & g & 1 & & 156.0 & $\mathbf{Y}$ & \\
\hline 1 & 7 Wab1 & 10 & 1 & & 154.7 & $\overline{\mathbf{H}}$ & \\
\hline 1 & $7 / 6 / 97$ & 11 & $\overline{1}$ & & 160.2 & $\mathbf{N}$ & \\
\hline 1 & $7 / 8 / 81$ & 12 & 1 & & 143.1 & $\mathbf{N}$ & J890523612-20 \\
\hline 1 & $7 / 6 / 91$ & 13 & 1 & & 134 & $\mathbf{M}$ & \\
\hline 1 & $7 / 0 / 21$ & 14 & 1 & & 121.7 & $\mathbf{H}$ & \\
\hline 1 & $7 / 0491$ & 15 & 1 & & 107.3 & $\bar{N}$ & \\
\hline 1 & 7hare1 & 18. & 1 & & 2.0 & $\mathbf{N}$ & \\
\hline 1 & $7 / 4 / 91$ & 1 & 1 & & 146,2 & $\mathbf{M}$ & \\
\hline 1 & $7 / 14 / 91$ & 2 & 1. & & 148.1 & 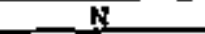 & \\
\hline
\end{tabular}

Data obtained from WHC Surveinance Anshyis Computer SYstem (SACS), Jhy 1, 1993. 
WHC-SO-WM-ER-308, Rev. 0

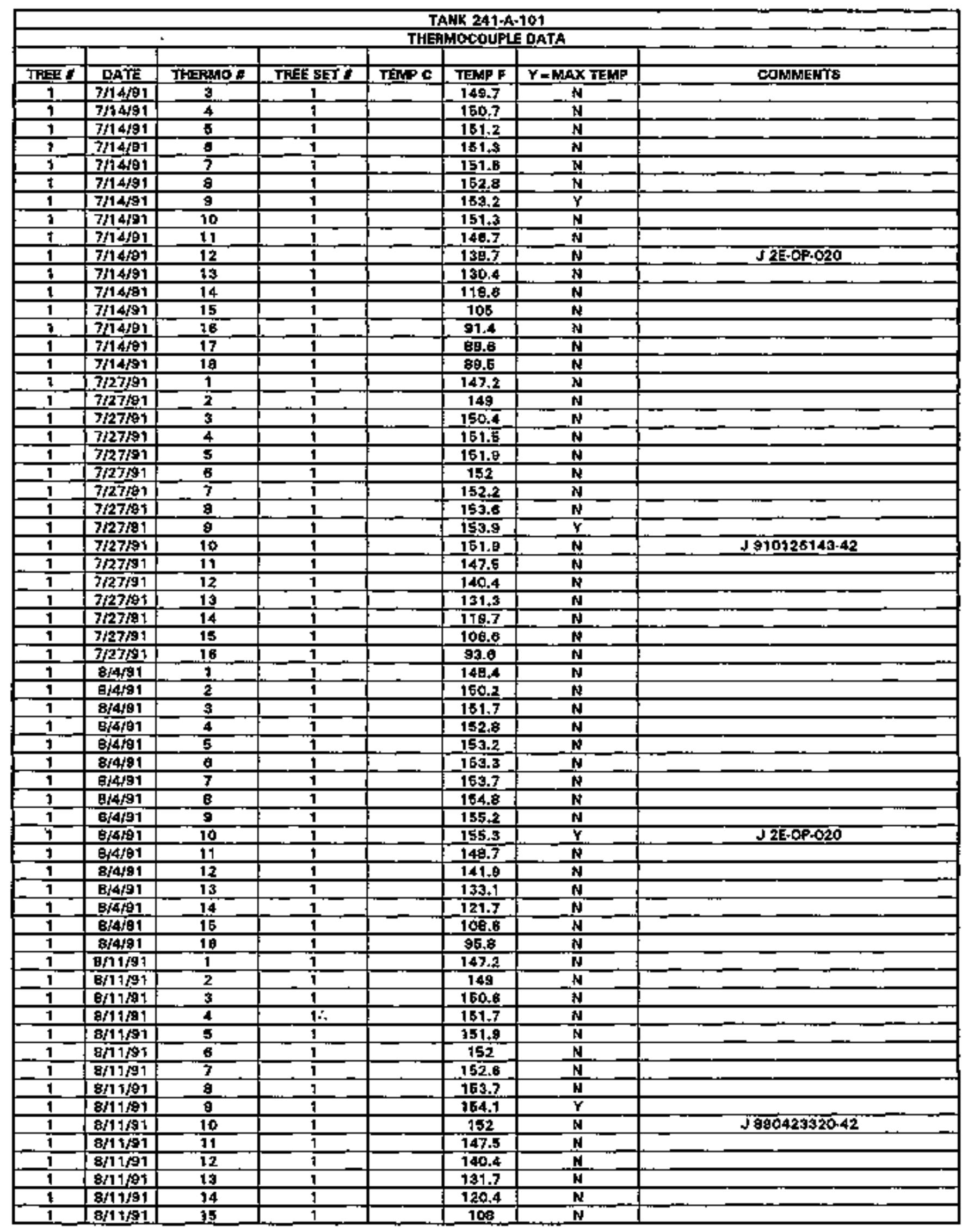

Data obtained from WHC Survellance Analysis Computer System ISACS), July 1, 1993. 


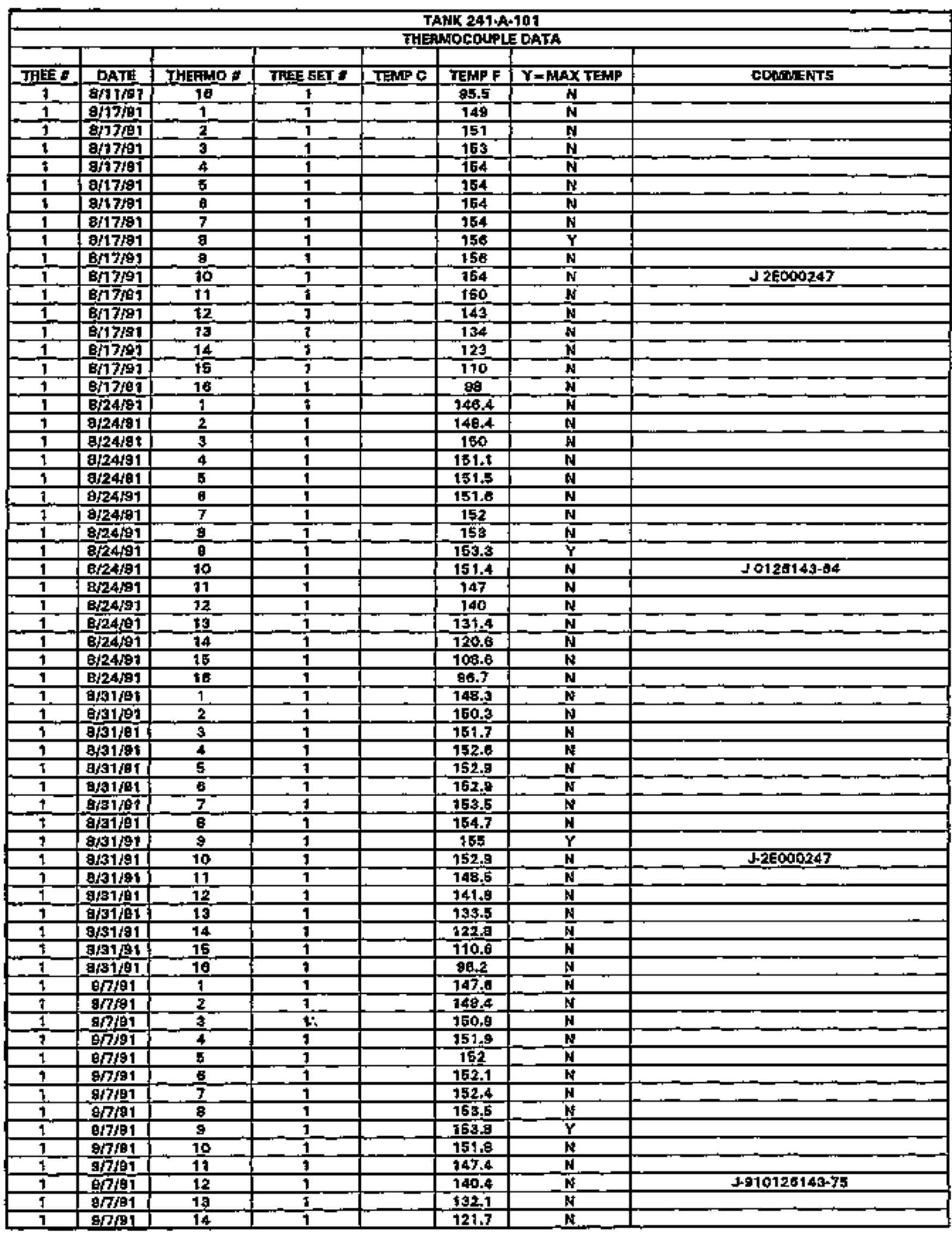

Data obtained from WHC Surveillance Analysis Computer System ISACSI, July 1, 1993. 


\begin{tabular}{|c|c|c|c|c|c|c|c|}
\hline \multirow{2}{*}{\multicolumn{8}{|c|}{ TANK 241 $+A=101$}} \\
\hline & & & & THEF & & DATA & \\
\hline TAEE & DATE & TFEFAMO & TAEE SET & TEM C & TEMP F & $\vec{y}=$ MAX TEMP & COMMENTS \\
\hline 1 & $9 / 7 / 81$ & 15 & 1 & & 109.8 & $\mathbf{N}$ & \\
\hline 1 & $9 / 7 / 9 t$ & 16 & 1 & & $97, \mathrm{~B}$ & $\mathbf{N}$ & \\
\hline 1 & $9 / 14 / 91$ & 1 & 1 & & 148.2 & N & \\
\hline 1 & $9 / 4 / 91$ & 2 & 1 & & 150,1 & $\mathbf{N}$ & \\
\hline$\overline{1}$ & $9 / 14 / 81$ & 3 & 1 & & $151+5$ & $\mathbf{N}$ & \\
\hline 1. & g/14/gti & 4 & 1 & & $1 \mathrm{625}$ & N & \\
\hline 1 & $8 / 14 / 8 t$ & 5 & 1 & & 152.0 & $N$ & \\
\hline 1 & $9 / 14 / 91$ & 6 & 1 & & 163.1 & $N$ & \\
\hline 1 & $9 / 14 / 91$ & 7 & 1 & & 153,2 & $\mathbf{N}$ & \\
\hline 1 & $9 / 14 / 97$ & 8 & 1 & & 154,3 & $N$ & \\
\hline 1 & 9/4 4/91 & 9 & 1 & & 154.6 & $Y$ & \\
\hline 1 & $9 / 1491$ & 10 & 1 & & 162.6 & $\mathbf{N}$ & \\
\hline 1 & $0 / 14 / 81$ & 11 & 1 & & 149.3 & $N$ & \\
\hline ? & $\theta / 1901$ & 12 & 1 & & 141.8 & $M$ & $12 E 000247$ \\
\hline 1 & g/4 49: & 13 & 1 & & $\overline{133.7}$ & $\bar{N}$ & \\
\hline$\$$ & $9 / 14 / 91$ & 14 & 1 & & 123.1 & $N$ & \\
\hline 1 & $9 / 74 / 91$ & 15 & $T$ & & 111.2 & $N$ & \\
\hline 1 & $9 / 14 / 91$ & 16 & 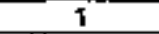 & & 98,5 & iq & \\
\hline 1 & $9 / 14 / 91$ & 17 & 1 & & 98.6 & N & \\
\hline 7 & $9 / 44 / 91$ & 18 & 1 & & 98.8 & $\mathbf{N}$ & \\
\hline 1 & $9 / 22 / 91$ & 1 & 1 & & 146.7 & $N$ & \\
\hline 1 & $9 / 22 / 91$ & 2 & $\mathrm{I}$ & & 147.8 & $N$ & \\
\hline 1 & 9/22/81 & 3 & $i$ & & 147.9 & is & \\
\hline 1 & $0 / 22 / 91$ & 4 & 1 & & 150.3 & $\mathbf{N}$ & \\
\hline 1 & $9 / 22 / 91$ & 5 & 1 & & 160.7 & $\mathbf{N}$ & \\
\hline 1 & $9 / 22 / 91$ & 8 & 1 & & 150.0 & N & \\
\hline 1 & $9 \longdiv { 2 2 9 1 }$ & 7 & 5 & & $15 \hat{R}$ & $\mathbf{N}$ & \\
\hline 1 & 9/22/91 & 8 & 1 & & 152.3 & $\bar{N}$ & \\
\hline 1 & $8 / 22 / 91$ & 9 & 1 & & 152.5 & $Y$ & \\
\hline 1 & $9 / 22 / 91$ & 10 & 1 & & 150.5 & $\mathbf{H}$ & $5-880523512,47$ \\
\hline 1 & $8 / 22 / 81$ & 11 & 1 & & 146.2 & N & \\
\hline 1 & $9 / 22 / 91$ & 12 & $\bar{z}$ & & 139.7 & $\mathbf{N}$ & \\
\hline 1 & $9 / 22 / 91$ & 13 & 1. & & 131.6 & N & \\
\hline 1 & $9 / 22 / 91$ & 14 & 1 & & 121.3 & $M$ & \\
\hline 1 & $9 / 22 / 91$ & 16 & 1 & & 109.7 & $\mathbf{N}$ & \\
\hline 1 & $8 / 22 / 91$ & 10 & 1 & & 07.1 & $\mathbf{N}$ & \\
\hline 1 & $9 / 28 / 81$ & 1 & 7 & & 148.1 & $\dot{N}$ & \\
\hline 1 & $9 / 28 / 91$ & 2 & 1 & & 148. & N & \\
\hline 1 & $9 / 28 / 91$ & 3 & 1 & & 151.6 & $\mathbf{M}$ & \\
\hline 1 & s/28/91 & 4 & 1 & & 152.5 & $\mathbf{N}$ & \\
\hline 1 & e/28/91 & 5 & 1 & & 152.日 & $\mathbf{N}$ & \\
\hline 1 & e/26197 & 8 & 1 & & 152.8 & $\overline{\mathbf{N}}$ & \\
\hline 1 & $8 / 28 / 81$ & 7 & 1 & & 153.3 & N & \\
\hline 1 & $8 / 28 / 91$ & $\mathbf{g}$ & 1 & & 154.3 & M & \\
\hline 1 & $9 / 28491$ & 9 & 1 & & 154.0 & $\bar{Y}$ & \\
\hline $\mathrm{T}$ & B/2E/S1 & 10 & 1 & & 152,7 & $\mathbf{N}$ & $1.890423320-42$ \\
\hline$\frac{t}{t}$ & g/26/95 & 11 & 1 & & $\uparrow 48.3$ & $\mathbf{N}$ & \\
\hline 1 & 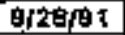 & 12 & 1 & & 141.9 & $\mathbf{N}$ & \\
\hline 1 & g/2B/g1 & 13 & 1 & & 134 & $M$ & \\
\hline 1 & $9 / 28 / 91$ & 14 & 1 & & 123.7 & $\mathbf{M}$ & \\
\hline $\mathbf{3}$ & E/2B/91 & 16 & 1 & & 111.3 & $\mathbf{n}$ & \\
\hline 1 & 0/28 & 10 & t: & & 88.0 & H & \\
\hline 1 & 10/5/9t & 1 & 1 & & $\$ 40.1$ & $\mathbf{N}$ & \\
\hline 1 & $105 \sqrt{94}$ & 2 & 7 & & 148 & $\mathbf{N}$ & \\
\hline 1 & $10 / 5 / 91$ & 3 & 1. & & 149.7 & N & \\
\hline 1 & $10 / 5 / 91$ & 4 & 1 & & 160.6 & $\mathbf{N}$ & \\
\hline 1 & $10 \mathrm{~b} / \mathrm{A} 1$ & 5 & 1 & & 150.8 & N & \\
\hline 1 & $10 / 9 / 91$ & 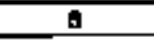 & 1 & & 150.8 & $\mathbf{N}$ & \\
\hline 1 & $105 / 91$ & 7 & 1 & & 151.5 & N & \\
\hline 1 & 105/81 & B & $t$ & & 152.5 & $\mathbf{N}$ & \\
\hline 1 & $10 / 5 / 91$ & 9 & 1 & & 162.8 & $\mathbf{Y}$ & \\
\hline 1 & $10 / 5 / 91$ & 10 & 1 & & 150.8 & $\mathbf{N}$ & 小H+99A.J \\
\hline 1 & $10 / 5 / 91$ & 11 & 1 & & 140,4 & $\mathbf{N}$ & \\
\hline
\end{tabular}

Data obtained from WHC Surveillance Analysis Computer System 1SACSI, July 1, 993. 


\begin{tabular}{|c|c|c|c|c|c|c|c|}
\hline \multicolumn{8}{|c|}{ TANK 24t-A-to1 } \\
\hline & & & & & Whenthe & DAIA & \\
\hline THEX & DATE & THEFWO : & THEE SET * & TEMP C & TEMPF & $Y=$ MAX TEHP & COMWLINTS \\
\hline 1 & $10 / 5 / 91$ & 12 & 1 & & 139.8 & . N & \\
\hline 1 & $10 / 3 / 81$ & 13 & 1. & & 132 & $\mathbf{N}$ & \\
\hline 1 & $10 / 5 / 91$ & 14 & 1 & & 122 & $\mathbf{N}$ & \\
\hline 1 & $10 / 5 / 11$ & $1 \overline{5}$ & 1 & & 110.5 & W & \\
\hline$i$ & $10 / 5191$ & 16 & 1 & & $0 \overline{\mathrm{B} 8}$ & $N$ & \\
\hline 1 & $10 / 139$ & 1 & \pm & & 144.5 & $N$ & \\
\hline 1 & 10, 301 & 2 & 1 & & 146,1 & N & \\
\hline 1 & 1아년1 & 3 & 1 & & 147.7 & $\mathbf{N}$ & \\
\hline 1 & $10+1391$ & 4 & 1 & & 146.4 & ri & \\
\hline 1 & $10 / 1301$ & E & 1 & & 148,8 & N & \\
\hline 1 & $1013 / 91$ & 0 & 1 & & 149.6 & N & \\
\hline 1 & 10y134at & 7 & 1 & & $149+9$ & N & \\
\hline 1 & $10 / 13 / 91$ & a & 1 & & 150.1 & $\mathbf{N}$ & \\
\hline 1 & 10/13/91 & 4 & 1 & & 150,3 & $Y$ & \\
\hline 1 & $10 / 3 / 91$ & 10 & 1 & & 148.3 & $\mathbf{N}$ & $J-190523512-47$ \\
\hline 1 & $10 / 13 / 91$ & 11 & 1 & & 144.1 & $\mathbf{N}$ & \\
\hline 1 & $10 / 13 / 91$ & 12 & 1 & & 137.0 & $\mathrm{~N}$ & \\
\hline 1 & 10/13re1 & 13 & 1 & & 129.8 & N & \\
\hline 1 & $10 / 13 \sqrt{6}$ & 14 & 1 & & $\$ 19.9$ & $N$ & \\
\hline 1 & $1013 \mathrm{f}=1$ & 15 & 1. & & 108.6 & $\mathbf{N}$ & \\
\hline 1 & $1013 \sqrt{2}$ & 16 & 1 & & 97.7 & $N$ & \\
\hline 1 & 10/18/ t & 1 & 1 & & 146 & $N$ & \\
\hline 1 & 10/0/91 & 2 & 1 & & 148,8 & $N$ & \\
\hline 1 & 10j58/91 & 3 & 1 & & 161.2 & $N$ & \\
\hline 1 & 10/18/91 & 4 & 1 & & 162.5 & N & \\
\hline 1 & 10/1091 & E & 1 & & 153.1 & 트 & \\
\hline 1. & $10 / 19$ 年1 & $\theta$ & 1 & & 153.5 & N & \\
\hline 1 & 10/10/91 & 7 & 1 & & 154.2 & $N$ & \\
\hline 1 & $10 / 19 / 91$ & 8 & 1 & & 155.4 & $M$ & \\
\hline 1 & $10 / 19$ & $D$ & 1 & & 155.9 & $Y$ & \\
\hline 1 & $10 / 19 / 91$ & to & 1 & & 164.2 & $\mathrm{~N}$ & Jegos23617.47 \\
\hline 1 & 10/18/91 & 11 & 1 & & 148.8 & M & \\
\hline 1 & 10/19/91 & 12 & 1 & & 143.8 & $\mathbf{N}$ & \\
\hline 1 & 10419/91 & 13 & 1 & & 136.1 & $\mathbf{N}$ & \\
\hline 1 & $70 / 1018$ & 14 & 1 & & 128.4 & $\mathbf{N}$ & \\
\hline 1 & toldegt & 15 & 1 & & 115.6 & N & \\
\hline 1 & tolderst & 10 & 1 & & 104.3 & $N$ & \\
\hline 1 & $10 / 28 j a 1$ & 1 & 1 & & 145.7 & N & \\
\hline 1 & $10 / 20191$ & 2 & 1 & & $147+6$ & $\mathbf{N}$ & \\
\hline 1 & 102에이 & 3 & 1 & & 149.3 & \$ & \\
\hline 1 & 10/2BSE & 4 & 1 & & 150.1 & s. & \\
\hline 1 & 10/26/81 & $E$ & 1 & & 150.3 & a & \\
\hline 1 & 10 - 201 & 6 & 1 & & 150.5 & N & \\
\hline 1 & 10/20/61 & 7 & 1 & & 150.9 & $\mathbf{N}$ & \\
\hline 1 & 1025191 & $B$ & 1 & & 352 & N & \\
\hline 1 & 10/2ata1 & 9 & 1 & & +52.2 & $\dot{y}$ & \\
\hline 1 & 10/26191 & 10 & 1 & & $150+2$ & $\mathbf{N}$ & \\
\hline 1 & 10/2a/91 & 11 & 1 & & 141 & $\mathbf{A}$ & \\
\hline 1 & 1020181 & 12 & 1 & & 139.7 & $N$ & $1-97012614376$ \\
\hline 1 & 10/29,81 & 13 & $t$ & & 132 & $N$ & \\
\hline 1. & 1026\%1 & 14 & 1 & & 122.3 & 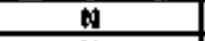 & \\
\hline 1. & $10 / 20191$ & 16 & 12 & & 111.4 & it & \\
\hline 1 & 10/20/a1 & 16 & 1 & & 99.5 & $\mathbf{N}$ & \\
\hline 1 & $11 / 3 / 91$ & 1 & I & & 146.7 & $N$ & \\
\hline 1 & $11 B 91$ & $\underline{z}$ & 1 & & 147.5 & $\mathbf{N}$ & \\
\hline 1 & $11 / 3 / 91$ & 3 & 1 & & 149.2 & it & \\
\hline 1 & 11/3/91 & 4 & 1 & & 150 & $\mathbf{N}$ & \\
\hline 1 & $11 / 3 / 81$ & $\underline{5}$ & 2 & & 360.3 & 4 & \\
\hline 1 & $11 / 3 / 91$ & $\overline{8}$ & 5 & & 150.4 & $\mathbf{N}$ & \\
\hline 1 & $11 / 3 / 91$ & 7 & 1 & & 150.9 & k & \\
\hline 1 & $11 / 3 / 91$ & 8 & $i$ & & 151.9 & N & \\
\hline 1 & $11 / 3 / 91$ & 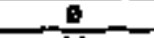 & 1 & & 152.2 & $\overline{\mathbf{y}}$ & \\
\hline 1 & 11/3191 & 10 & 1 & & 150.2 & $N$ & \\
\hline
\end{tabular}

Data obtained from WHC Surveillance Atralysis Computer System (SACS), July 1, 1993. 


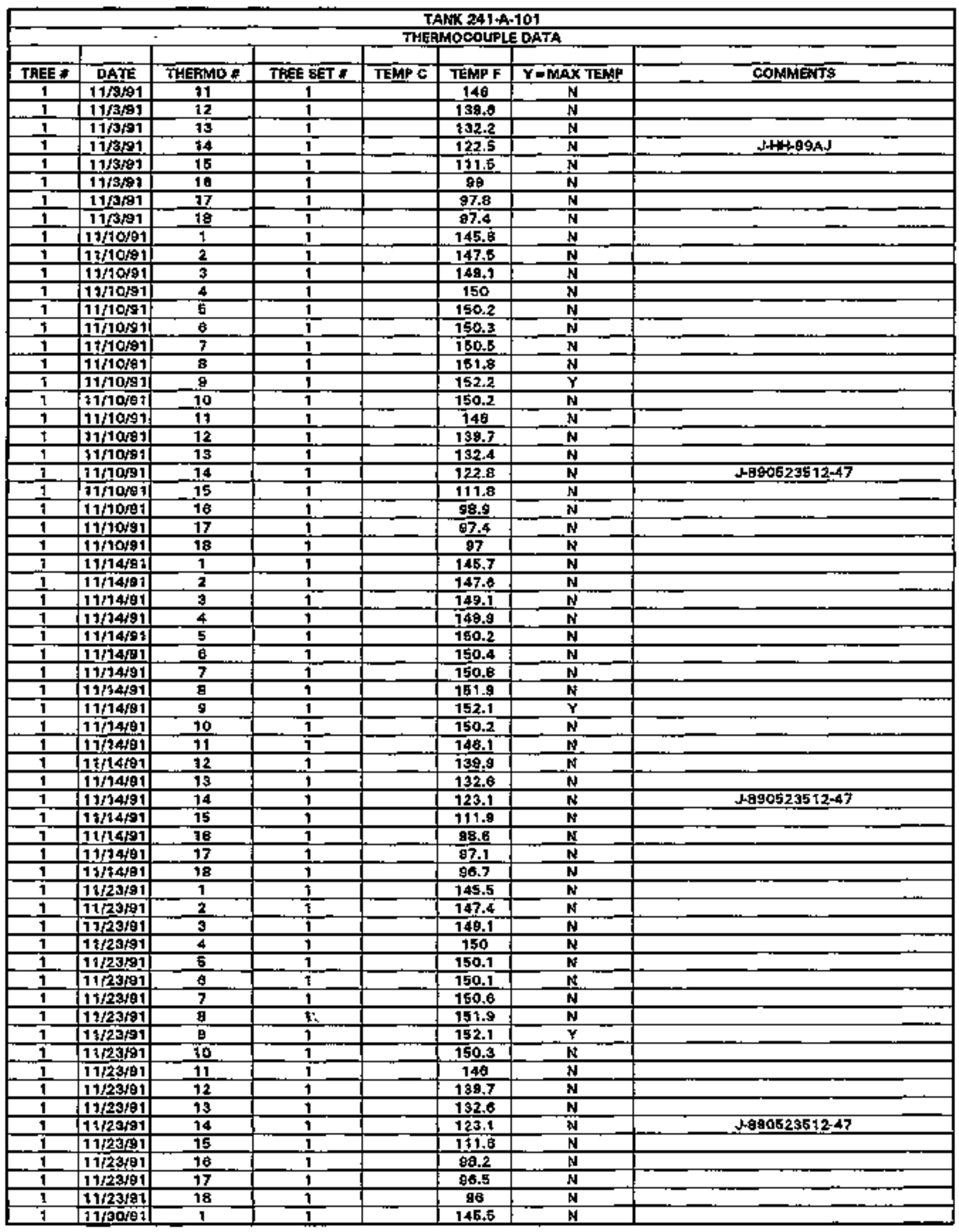

Data obtained from WHC Surveillance Analysis Computer System (SACS), July 1, 1993. 
WHC-SO-WM-ER-308, Rev. 0

\begin{tabular}{|c|c|c|c|c|c|c|c|}
\hline & & & & $\frac{T}{\text { THE }}$ & $\frac{\text { Not } 2414}{\text { Mocoter }}$ & 101 & \\
\hline & & & & & 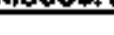 & conin & \\
\hline VPEE : & DATE & THEFitio = & TREE SET: & TEMP C & TENPF & $Y=M A X$ TEMP & COHWEHTS \\
\hline 1 & J1/10/91 & 2 & 1 & & 147.3 & $\mathbf{N}$ & \\
\hline 1 & $31 / 1004$ & 3 & 1 & & 149.1 & $\mathbf{N}$ & \\
\hline$t$ & $11 / 30 / 1$ & A & 1 & & 149.8 & $\mathbf{N}$ & \\
\hline$t$ & 11/1001 & 5 & 1 & & 150.1 & $\mathbf{N}$ & \\
\hline 1 & $11 / 30 / 1$ & 6 & 1 & & 750.2 & $N$ & \\
\hline$t$ & 11/aged & 7 & 1 & & 150.7 & $N$ & \\
\hline 1 & $11 / 30191$ & 8 & 1 & & 951.7 & $\mathbf{N}$ & \\
\hline 1 & 11/30191 & 9 & 1 & & 152,1 & $\mathbf{Y}$ & \\
\hline 1 & 11 so/91 & 10 & 1 & & 160,2 & $\mathbf{N}$ & \\
\hline 1 & 11/30191 & 11 & 1 & & 140 & $\mathbf{N}$ & \\
\hline 1 & $11 / 30 / 91$ & 12 & 1 & & 199,8 & $M$ & \\
\hline 1 & $19 / 30101$ & 13 & 1 & & 192,6 & $\mathbf{N}$ & \\
\hline 1 & 11/30이 & 14 & 1 & & 123.7 & $\mathbf{N}$ & J-HH-99+AV \\
\hline 1 & $17 / 30 / 91$ & 76 & J & & 71.4 & $M$ & \\
\hline 1 & $11 / 30491$ & 18 & 1 & & 97.6 & N & \\
\hline 1 & 11/30191 & 17 & 1 & & 95.8 & $\mathrm{~N}$ & \\
\hline 1 & 19/30/91 & 18 & 1 & & 95.3 & $\mathbf{N}$ & \\
\hline 1 & 12/5/91 & 1 & $t$ & & 145.8 & $\mathbf{N}$ & \\
\hline 1 & $12 / 6 / 97$ & 2 & 1 & & 147.8 & $\mathbf{N}$ & \\
\hline 1 & $12 / 5 / 91$ & 3 & 1 & & 149,4 & $\mathbf{N}$ & \\
\hline 1 & $12 / 8 / 8)$ & 4 & 1 & & 150.1 & $\mathbf{N}$ & \\
\hline 1 & $125 / 61$ & 5 & 1 & & 150.5 & $\mathbf{N}$ & \\
\hline 1 & $12 \sqrt{5 / 93}$ & 6 & 1 & & 150,6 & $\mathbf{N}$ & \\
\hline 1 & $12 \sqrt{61}$ & $\overline{7}$ & 7 & & 150.9 & $\mathbf{N}$ & \\
\hline 1 & $125 / 91$ & a & 1 & & 152 & $\mathbf{N}$ & \\
\hline 1 & $12 \sqrt{5 / 91}$ & 8 & 1 & & 162.4 & $\gamma$ & \\
\hline 1 & $125 / 64$ & 10 & 1 & & 150.5 & $\mathbf{N}$ & \\
\hline 1 & $12 \sqrt{5 / 95}$ & 11 & ? & & 149,4 & $\mathbf{N}$ & \\
\hline 1 & $12 \sqrt{6}$ & 72 & 7 & & 140.3 & $\mathbf{N}$ & $5.890623512-47 \mathrm{HH}-99 \mathrm{~A}-\mathrm{J}$ \\
\hline 1 & $12 / 5 / 91$ & 13 & 1 & & 133.2 & $\mathbf{N}$ & \\
\hline 1 & $12 \sqrt{5 / 101}$ & 14 & 1 & & 123.6 & $N$ & \\
\hline 1 & $12 / 6 / 91$ & 15 & t & & 111.8 & $\mathbf{M}$ & \\
\hline 1 & $12 \sqrt[5 / 94]{ }$ & 18 & 3 & & 97,9 & $\mathbf{N}$ & \\
\hline 1 & I2JB/91 & 17 & 1 & & Be & N & \\
\hline 1 & 12/6/91 & 18 & 1 & & 95.7 & $\mathbf{N}$ & \\
\hline 1 & 12/16/91 & 1 & 1 & & 145.4 & $\mathbf{N}$ & \\
\hline 1 & $12 / 76 / 91$ & 2 & 1 & & 147,3 & $\mathbf{N}$ & \\
\hline 1 & $12 / 15 / 91$ & 3 & 1 & & $14 \mathrm{~S}$ & $\mathbf{N}$ & \\
\hline 1 & $12 \mathrm{ft} 5 \mathrm{Al}$ & 4 & 1 & & 149.8 & N & \\
\hline 1 & 12/1GA1 & 5 & 1 & & 150 & $\mathbf{N}$ & \\
\hline 1 & 1245f & $\theta$ & 1 & & 160.1 & $\mathbf{N}$ & \\
\hline 1 & $12 / 16 / 91$ & 7 & 1 & & 1500 & $\mathbf{N}$ & \\
\hline 1 & $12 / 15 / 91$ & B & 1 & & 151.日 & $\mathbf{N}$ & \\
\hline 1 & $12 / 15 / 91$ & 9 & 1 & & 162 & $\mathbf{Y}$ & \\
\hline 1 & 12715地 & 10 & 1 & & 150.2 & $\mathbf{N}$ & Jeto126:43-14 \\
\hline 1 & $12 / 15 / 91$ & 11 & 1 & & 146.1 & $\mathbf{N}$ & \\
\hline 1 & $12 / 15 / 21$ & 12. & 1 & & 140 & $\mathbf{N}$ & \\
\hline 1 & 12/25:日 & 13 & $\overline{1}$ & & 132.8 & $\mathbf{N}$ & \\
\hline 1 & $12 / 15 / 91$ & 14 & 1 & & 123.1 & $\mathbf{N}$ & \\
\hline 1 & $12 / 15 / 91$ & 15 & 1 & & 111.1 & $\mathbf{N}$ & \\
\hline 1 & $12 / 15 / 91$ & 16 & 1 & & 98.6 & $N$ & \\
\hline 1 & $12 / 1901$ & 1 & $1 .:$ & & 148,5 & $\mathbf{N}$ & \\
\hline 1 & 12/t9/91 & 2 & 1 & & 147.4 & N & \\
\hline 1 & $12 / 19 / 91$ & 3 & 1 & & 149 & $\mathbf{N}$ & \\
\hline 1 & 12 forgth & 4 & $i$ & & 149.8 & $\mathbf{N}$ & \\
\hline 1 & 12 19/91 & 5 & 1 & & 150 & $\mathbf{N}$ & \\
\hline 1 & 12/19/91 & 6 & 1 & & 150.1 & $\mathbf{N}$ & \\
\hline 1 & $112 / 19 / 91$ & 7 & 1 & & 150.7 & N & \\
\hline 1 & $12 / 19 / 94$ & 8 & $\bar{I}$ & & 161.8 & $\mathbf{N}$ & \\
\hline 1 & L2nass & 皇 & $\bar{j}$ & & 152.1 & $\mathbf{Y}$ & \\
\hline 1 & 12 risel & 10 & 1 & & 150.3 & $\mathbf{N}$ & \\
\hline 1 & $12 / 19 / 8+$ & 11 & 1 & & $14 \dot{B} .2$ & $\mathbf{N}$ & \\
\hline 1 & $12 / 19 / 91$ & 12 & $\overline{1}$ & & 140.1 & $\mathbf{N}$ & $5.910126143-60$ \\
\hline
\end{tabular}

Data obtained from WHC Surveillance Analysis Computer System (SACS), July 1, 1993.

D.153 
WHC-SD-WM-ER-308, Rev. O

\begin{tabular}{|c|c|c|c|c|c|c|c|}
\hline \multicolumn{8}{|c|}{ TANK 241+A+101 } \\
\hline & & . & & THEF & OOCOUP & DATA & \\
\hline TREE * & DATE & THEAMO : & TREEE SET $a$ & TENP 6 & TEKAP F & $Y=M A X$ TEMP & COMMENTS \\
\hline 1 & $12 / 19 / 91$ & 13 & 1 & & 133,1 & $\mathbf{N}$ & \\
\hline 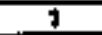 & $12 / 19 / 41$ & 14 & 1 & & 123.4 & $\mathbf{N}$ & \\
\hline$t$ & $12 / 19 / 91$ & 15 & 1 & & 111.2 & $\mathbf{N}$ & \\
\hline 1 & 12/19/91 & 10 & 1 & & 90.3 & $\mathbf{M}$ & \\
\hline 7 & 12126191 & 1 & 1 & & 14E.6 & $\mathbf{N}$ & \\
\hline 1 & 22126/91 & 2 & 1 & & 947,4 & $\mathbf{N}$ & \\
\hline 1 & $52 / 2691$ & 3 & 1 & & 14E. .9 & $\mathbf{N}$ & \\
\hline 1 & t2/26/191 & 4 & 1 & & 149.8 & $\mathbf{N}$ & \\
\hline 1 & $12 / 20 / 91$ & 車 & 1 & & 160 & $\mathbf{M}$ & \\
\hline 1 & $12 / 26 / 91$ & a & 1 & & $150+1$ & $\mathbf{N}$ & \\
\hline 1 & $12 / 26,91$ & 7 & 1 & & 150.3 & $\mathbf{N}$ & \\
\hline 1 & $12 / 261$ & g & 1 & & 181.7 & $\mathbf{W}$ & \\
\hline 1 & $12 / 20 / \theta_{1}$ & 皇 & 1 & & 152 & $\mathbf{Y}$ & \\
\hline 1 & $12 / 20 / 91$ & 10 & 1 & & 150.2 & $\mathbf{N}$ & \\
\hline 1 & $12 / 26 / 91$ & 11 & 1 & & 146.3 & $\mathbf{N}$ & \\
\hline 1 & $12 / 26 / 91$ & 12 & 1 & & 140.3 & $\mathbf{N}$ & \\
\hline 1 & 12/20/91 & 13 & 1 & & 933.2 & $\mathbf{N}$ & \\
\hline 1 & $12 / 26 / 91$ & 14 & 1 & & 123,4 & N. & $910121143 \cdot 40$ \\
\hline 1 & $12 / 26 / 91$ & 15 & 1 & & $1 \pi 1+1$ & $\mathbf{N}$ & \\
\hline 1 & $12 / 26 / 91$ & 16 & 1 & & 93,4 & $N$ & \\
\hline 1 & $1226+91$ & 17 & 1 & & 94.4 & $\mathbf{N}$ & \\
\hline 1 & $12 / 20 / 91$ & 10 & 1 & & 94 & $\mathbf{N}$ & \\
\hline 1 & $1 / 2 / 92$ & 1 & 1 & & 147.4 & $\mathbf{N}$ & \\
\hline 1 & $1 / 2 \sqrt{92}$ & 2 & 1 & & 149.1 & $\mathbf{N}$ & \\
\hline 1. & $1 / 2 / 92$ & 3 & 1 & & 150.7 & $N$ & \\
\hline 1 & $1 / 2 / \theta_{2}$ & 4 & 1 & & 161.7 & $\mathbf{M}$ & \\
\hline 1 & $1 / 2 / 82$ & 5 & 1 & & 152 & N & \\
\hline 1 & $1 / 2 / \mathrm{A2}$ & 9 & 1 & & 152.1 & $N$ & \\
\hline 1 & $1 / 2 / 32$ & 7 & 1 & & 152.3 & $\mathbf{N}$ & \\
\hline 1 & $1 / 2 / 92$ & $\mathbf{g}$ & 1 & & 153.6 & $N$ & \\
\hline 1 & $1 / 2 / 92$ & $\underline{\theta}$ & 1 & & 183.9 & $\mathbf{Y}$ & \\
\hline 1 & $1 / 2 / \theta 2$ & 10 & 1 & & 152.1 & $N$ & \\
\hline 1 & $1 / 2 / 92$ & 14 & 1 & & 149.1 & N & \\
\hline 1 & 1/2/92 & 12 & 1 & & 142.2 & $\mathbf{N}$ & \\
\hline 1 & $1 / 2 / 92$ & 13 & 1 & & 134.8 & $\mathbf{N}$ & \\
\hline 1 & $1 / 2 / 92$ & 14 & 1 & & 124.9 & $\mathbf{M}$ & J $010126143 \cdot 32$ \\
\hline 1 & $1 / 2 \% 2$ & 15 & 1 & & 112.8 & $\mathbf{N}$ & \\
\hline 1 & $1 / 2 / 102$ & 18 & 1 & & 97 & N & \\
\hline 1 & $1 / 2 / 62$ & 17 & 1 & & 94.9 & $N$ & \\
\hline 1 & $1 / 2 / 92$ & 18 & 1 & & 84.6 & $\mathbf{N}$ & \\
\hline 3 & $1 / 12 / 92$ & 1 & 1 & & 140 & $N$ & \\
\hline 1 & $1 / 12 / 92$ & 2 & 1 & & 148 & $\mathbf{N}$ & \\
\hline 1 & $1 / 12 / 92$ & 3 & 1 & & 149.7 & $\mathbf{N}$ & \\
\hline 1 & $1 / 12 / 2$ & 4 & 1 & & 150.6 & N & \\
\hline 1 & $1 / 12 / 92$ & 5 & 1 & & 160.8 & N & \\
\hline 1 & $1 / 12 / 92$ & 6 & 1 & & 150,8 & $\mathbf{N}$ & \\
\hline 1 & $1 / 12 / 92$ & 7 & 1 & & 154.4 & $\mathbf{N}$ & \\
\hline 1 & $1 / 12 / 82$ & B & 1 & & 152,4 & M & \\
\hline 1 & $1 / 12 / 92$ & 9 & 1 & & 152.5 & $\mathbf{Y}$ & \\
\hline 1 & $1 / 12 / 92$ & 10 & 1 & & 162.2 & $\mathbf{N}$ & \\
\hline 1 & $1 / 12 / 92$ & 14 & 1 & & 147.3 & $\mathbf{N}$ & \\
\hline 1 & $1 / 12 / 92$ & 12 & F & & 141.3 & $\mathbf{N}$ & +910128143.14 \\
\hline 1 & $1 / 12 / 92$ & 13 & 1 & & $1 \$ 1.4$ & $\mathbf{N}$ & \\
\hline 1 & $1 / 12 / \theta_{2}$ & 14 & 1 & & 124.2 & $\mathbf{N}$ & \\
\hline 1 & $1 / 12 / 92$ & 15 & 1 & & 111.4 & $\mathbf{N}$ & \\
\hline 1 & $1 / 12 / 92$ & 18 & 1 & & 98.1 & $\mathbf{N}$ & \\
\hline 1 & $1 / 12 / 92$ & 17 & 1 & & 103.1 & $\mathbf{N}$ & \\
\hline 1 & $1 / 12 / 92$ & 18 & 1 & & 102.4 & $\mathbf{N}$ & \\
\hline 1 & $1 / 10 / 92$ & 1 & 1 & & 140.2 & $\mathbf{N}$ & \\
\hline 1 & $1 / 16 / 92$ & 2 & 1 & & 13E.1 & $\mathbf{N}$ & \\
\hline 1 & 1/18/g2 & 3 & 1 & & 149,0 & $\mathbf{N}$ & \\
\hline 1 & 1716rg & 4 & 1 & & 130.7 & $\mathbf{N}$ & \\
\hline 1 & 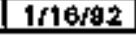 & 6 & 1 & & $150+9$ & N & \\
\hline
\end{tabular}

Data obtained from WHC Surveillance Analysis Computer System (SACS). July 1, 1993. 
WHC-SD-WMHER-309, Rev. 0

\begin{tabular}{|c|c|c|c|c|c|c|c|}
\hline \multicolumn{8}{|c|}{ TANK 247+A-101 } \\
\hline TREE $=$ & DATE & THEFMO & 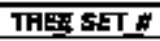 & TEMP C & TESPF F & $Y=$ MaX TEMP & Comprests \\
\hline 1 & $\sqrt[1]{16 / \overline{3}}$ & 6 & 1 & & 167.1 & $\mathrm{~N}$ & \\
\hline 1 & 1/10/92 & 7 & $\overline{1}$ & & 151 & $\mathbf{N}$ & \\
\hline$i$ & $1 / 16 / 92$ & $\mathbf{B}$ & 1 & & 152.5 & $\mathbf{N}$ & \\
\hline 1 & $1 / 16 / 92$ & 9 & 1 & & 753 & $\mathbf{Y}$ & \\
\hline 1 & $1 / 16 / 92$ & 10 & 1 & & +61.3 & $\mathbf{N}$ & $\begin{array}{l}\text { ACCESS RES TAICTED } 1 / 28+2 / 22 \text { DUE TO } \\
\text { BATTERY PUME INCIDENT }\end{array}$ \\
\hline 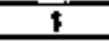 & 1/16/92 & 15 & 1 & & 947,5 & $\mathbf{N}$ & $1.890 \$ 23512-47$ \\
\hline 1 & 1月692 & 12 & 1 & & 141.6 & $\mathbf{N}$ & \\
\hline 1 & $1 / 16 / 92$ & 13 & 1 & & $\$ 34.3$ & $\mathbf{N}$ & \\
\hline 1 & $1 / 10 / 92$ & 14 & 1 & & 124.3 & N & \\
\hline 1 & $1 / 16 / 62$ & $\mathbf{T F}$ & 1 & & 116,4 & $\mathbf{N}$ & \\
\hline 1 & $1 / 402$ & 16 & 1 & & 90.1 & $N$ & \\
\hline 1 & $1 / 23 / 92$ & 1 & 1 & & 145 & $\mathbf{N}$ & \\
\hline 1 & $1 / 29 / 92$ & 2 & 1 & & 147 & $\mathbf{N}$ & \\
\hline 1 & $1 / 2,6 / 92$ & 3 & 1 & & 149 & $\mathbf{N}$ & \\
\hline 1 & $1 / 23 / 192$ & 4 & 1 & & 148 & $\mathbf{N}$ & \\
\hline 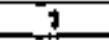 & $1 / 23 / 92$ & 5 & 1 & & 160 & N & \\
\hline$i$ & $5 / 23192$ & $\theta$ & 1 & & 150 & $N$ & \\
\hline 1 & $1 / 23 / 92$ & 7 & 1 & & 150 & $N$ & \\
\hline 1 & $1 / 23 / 92$ & 8 & 1 & & $15 t$ & $N$ & \\
\hline 1 & $\$ / 23 / 92$ & 9 & 1 & & 162 & $Y$ & \\
\hline 1 & $1 / 23 / 92$ & 10 & 1 & & 160 & $\mathbf{M}$ & \\
\hline 1 & $1 / 23 / 92$ & 11 & 1 & & 146 & $N$ & \$919126143.32 \\
\hline 1 & $1 / 23 / \mathrm{a}_{2}$ & 12 & 1 & & 140 & N & \\
\hline 1 & $1 / 23 / 92$ & 13 & $T$ & & 133 & $\mathbf{N}$ & \\
\hline 1 & $1 / 2 \sqrt{92}$ & $1 \mathrm{~A}$ & 7 & & $\sqrt{123}$ & $\overline{6}$ & \\
\hline 1 & $1 / 23 / 92$ & 15 & 1 & & 110 & $\mathrm{H}$ & \\
\hline 1 & $1 / 23 / 92$ & 16 & 1 & & 95 & $\mathbf{N}$ & \\
\hline 1 & $1 / 26 / 02$ & 1 & $\mathbf{J}$ & & & $\mathbf{n}$ & \\
\hline 1 & $1 / 31 / 12$ & 1 & 1 & & $1 \overline{45.1}$ & $N$ & \\
\hline 1 & $1 / 31 / 92$ & 2 & 1 & & 140.8 & M & \\
\hline 1 & $1 / 81 / 92$ & 3 & 1 & & 148,5 & $\mathbf{N}$ & \\
\hline 1 & $1 / 31 / 92$ & 4 & 1 & & 149.5 & $\bar{N}$ & \\
\hline 1 & $1 / 31 / 02$ & 5 & 1 & & $\$ 49.8$ & $N$ & \\
\hline 1 & $1 / 31 / 92$ & 6 & 1 & & 149.8 & $\mathbf{N}$ & \\
\hline 1 & $1 / 31 / 92$ & 7 & 1 & & $+50,1$ & N & \\
\hline 1 & $1 / 31 / 92$ & E & 1 & & 151.3 & $\mathbf{H}$ & \\
\hline 1 & $1 / 31 / 92$ & $\mathbf{9}$ & 1 & & $15 t .8$ & $\mathbf{Y}$ & \\
\hline 1 & $1 / 31 / 92$ & 10 & 1 & & 150,1 & $\mathbf{N}$ & $5870126143-32$ \\
\hline 1 & $\overline{1 / 31 / 92}$ & 11 & $\overline{\mathbf{T}}$ & & $1 \overline{48.2}$ & $N$ & \\
\hline 7 & $1 / 3 1 \longdiv { 1 2 }$ & 12 & $i$ & & 140.3 & $N$ & \\
\hline 1 & $1 / 31 / 92$ & 13 & 1 & & $+33,1$ & $N$ & \\
\hline 1 & 1/37/92 & 14 & 1 & & 122.9 & $\mathbf{N}$ & \\
\hline 1 & $1 / 91 / 92$ & 15 & 1 & & 109,0 & N. & \\
\hline 1 & $\overline{1 / 31 / 22}$ & $1 \overline{8}$ & 1 & & 94.5 & $N$ & \\
\hline$t$ & $2 \sqrt{23 \sqrt{82}}$ & 1 & t & & 140 & $\mathbf{N}$ & \\
\hline 7 & $2 / 23 / 92$ & 2 & 1 & & 148 & $\bar{N}$ & \\
\hline $\mathrm{T}$ & 2J23482 & $\mathbf{3}$ & $\mathbf{T}$ & & 140 & $\bar{N}$ & \\
\hline$t$ & $2 / 23 / 92$ & 4 & 1 & & 150 & $N$ & \\
\hline 1 & $2 / 23 / 92$ & 5 & $i$ & & 151 & N & \\
\hline 1 & $2 / 23 / 92$ & D & 1 & & 151 & $\mathbf{N}$ & \\
\hline 1 & $2 / 23 / 92$ & 7 & 15 & & 151 & $\mathbf{N}$ & \\
\hline 1 & $2 / 23 / 92$ & $\mathbf{g}$ & 1 & & 152 & $N$ & \\
\hline 1 & $2 / 23 / 92$ & 5 & $y$ & & 163 & $\mathbf{Y}$ & \\
\hline 1 & $2 / 23 / 92$ & 10 & 2 & & 351 & $\bar{n}$ & J 870126143.48 \\
\hline 1 & $2 / 23 / 92$ & 11 & $\pi$ & & 148 & $N$ & \\
\hline 1 & $2 / 23 / 02$ & $1 \overline{2}$ & 1 & & 142 & $\mathbf{N}$ & \\
\hline 7 & $2 / 23 / 92$ & 13 & 1 & & 134 & $N$ & \\
\hline 1 & $2 / 23 / 92$ & 14 & 3 & & 124 & $\mathbf{N}$ & \\
\hline 1 & $2 / 23 / 92$ & 15. & 7 & & 721 & $N$ & \\
\hline$T$ & $2 / 23 / 92$ & $1 \overline{6}$ & $\$$ & & 95 & $\mathbf{N}$ & \\
\hline 1 & $2 / 2702$ & 1 & 1 & & 146.5 & $\mathbf{M}$ & \\
\hline 1 & $2 / 27 / 02$ & 2 & 1 & & 148,2 & $\overline{\mathbf{N}}$ & \\
\hline
\end{tabular}

Oata obtained from WHC Surveillance Analysis Computor System (SACS)، July 1, 1993. 
WHC-SD-WM-ER-308, Rev. $O$

\begin{tabular}{|c|c|c|c|c|c|c|c|}
\hline \multicolumn{8}{|c|}{ TAMK 241+A-101 } \\
\hline & & & & THE & HOCOLF & DATA & \\
\hline TREX : & DATE & THERMD : & TAEE SET \# & TEMAP $\bar{c}$ & FEMP F & Y Y MAXX TEMLF & Commatis \\
\hline 1 & $2 \sqrt{27} \mathrm{~B}$ & 3 & 1 & & 149.8 & $N$ & \\
\hline 1 & $2 / 27 / 92$ & 4 & 1 & & 150.7 & $N$ & \\
\hline 1 & $2 / 27 / 92$ & 5 & 1 & & $151+1$ & $N$ & \\
\hline 1 & $2 / 27 / 92$ & $\bar{B}$ & 1 & & $151+2$ & $\mathbf{N}$ & \\
\hline 1 & $2 / 27 / 92$ & 7 & 1 & & 1E1.2 & $\mathbf{N}$ & \\
\hline 1 & $2 \sqrt{2}$ & 8 & 1 & & 162.4 & $N$ & \\
\hline 1 & $2 / 27 / 2$ & 9 & 1 & & 153.1 & $\bar{Y}$ & \\
\hline 1 & $2 / 27 / 92$ & 10 & 1 & & 151.8 & $\mathbf{N}$ & J 900925741.78 \\
\hline 1 & $2 / 27 / 92$ & 11 & 1 & & 147.8 & $\mathbf{N}$ & \\
\hline$t$ & $2 \longdiv { 2 7 0 2 }$ & 12 & 1 & & 141.8 & $\overline{\mathbf{N}}$ & \\
\hline 1 & $2 / 27 / 92$ & 13 & 1 & & 134.3 & $\bar{N}$ & \\
\hline 1 & $2 / 27 / 92$ & 14 & 1 & & 123,8 & $\mathbf{N}$ & \\
\hline 1 & $2 / 27 / 92$ & 15 & 1 & & 110.5 & $\mathbf{N}$ & \\
\hline 1 & $2 / 27 / 92$ & 18 & 1 & & 95.2 & $\mathbf{N}$ & \\
\hline 1 & अ/7日2 & 1 & $t$ & & 145.2 & $\mathbf{N}$ & \\
\hline 1 & $3 / 7 / 92$ & 2 & 1 & & 149.8 & $\mathbf{N}$ & \\
\hline 1 & 9/7/92 & 3 & 1 & & 151.3 & $\mathbf{N}$ & \\
\hline$\overline{1}$ & $3 / 7 / 92$ & 4 & 1 & & 157 & $\mathbf{N}$ & \\
\hline$\overline{1}$ & $3 / 192$ & 5 & $\overline{1}$ & & 152.4 & $\mathbf{M}$ & \\
\hline 1 & $3 / 792$ & 6 & 1 & & 162.8 & $\mathbf{N}$ & \\
\hline 1 & $3 / 7 / 92$ & 7 & 1 & & 162.8 & $\mathbf{N}$ & \\
\hline 1 & $37 / 92$ & E & 1 & & 154.1 & 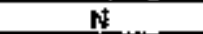 & \\
\hline 1 & $3 / 7 / 92$ & $\mathbf{g}$ & 1 & & 154.d & $\gamma$ & \\
\hline 1 & $3 / 7 / 92$ & 10 & 1 & & 153 & $\mathbf{N}$ & JSN91012B14332Tr03B \\
\hline 1 & 3 hrez & 11 & 1 & & $\$ 49.2$ & $\mathbf{N}$ & \\
\hline 1 & $3 \sqrt{7} / 22$ & 12 & 1 & & 143.3 & $\mathbf{N}$ & \\
\hline 1 & $3 / 7 / 92$ & 13 & 1 & & 136.6 & $\mathbf{N}$ & \\
\hline 1 & $3 / 7 / 92$ & 14 & 1 & & 124.8 & $\mathbf{N}$ & \\
\hline $\mathbf{1}$ & $3 / 7 / 92$ & 15 & 1 & & 111.4 & $\mathbf{N}$ & \\
\hline 1 & $37 / 92$ & 16 & 1 & & 85.8 & $\mathbf{N}$ & \\
\hline 1 & $3 / 2 / 92$ & 1 & 1 & & $\$ 46 . \mathrm{g}^{-1}$ & $\bar{N}$ & \\
\hline 1 & $3 / 12 / 82$ & 2 & 1 & & 147,0 & $\mathbf{M}$ & \\
\hline 1 & $3 / 12 / 92$ & $\mathbf{3}$ & 1 & & 148 & $\mathbf{N}$ & \\
\hline$i$ & $3 / 52 / 92$ & 4 & 1 & & 150 & $\mathbf{N}$ & \\
\hline 1 & $312 \times 2$ & E & 1 & & 160.2 & $\mathbf{N}$ & \\
\hline 1 & $3 / 12 / 92$ & $\theta$ & 1 & & 150.7 & $\mathbf{N}$ & \\
\hline 1 & $3 / 12 / 92$ & 7 & 1 & & 151,9 & $\mathbf{Y}$ & \\
\hline 1 & $3 / 12 / 92$ & $\mathbf{8}$ & 1 & & 151.8 & $\mathbf{N}$ & \\
\hline 1 & $3 / 12 / 2$ & 9 & 1 & & 152.3 & $\mathbf{N}$ & \\
\hline$\$$ & $3 / 1+2 / 2$ & 10 & 1 & & 150,7 & $\mathbf{N}$ & \\
\hline 1 & $3 / 42 / 92$ & 11 & 1 & & 147,2 & $\mathbf{N}$ & \\
\hline 1 & $3 / 12 / 92$ & 12 & 1 & & 141.3 & $N$ & \\
\hline 1 & $3 / 32 / 92$ & 3 & $1:$ & & 133.6 & $\mathbf{N}$ & \\
\hline 1 & $3 / 1 2 \longdiv { 9 2 }$ & 14 & 1 & & 123 & $\mathbf{N}$ & \\
\hline 1 & $3 / 12 / 92$ & 15 & 1 & & $109+9$ & $\mathbf{N}$ & \\
\hline 1 & $3 / 12 / 92$ & 10 & 1 & & 94,0 & $\mathbf{N}$ & \\
\hline 1 & $3 / 19 / 92$ & $\mathbf{1}$ & 1 & & 149 & $\mathbf{N}$ & \\
\hline 1 & $3 / 19 / 92$ & 2 & 1 & & 149.0 & $\mathbf{N}$ & \\
\hline 1 & 3/19/92 & 3 & 1 & & 181.4 & $\mathbf{N}$ & \\
\hline 1 & $3 / 19 / 92$ & 4 & 1 & & 152,3 & $\bar{N}$ & \\
\hline 1 & $3 / 19 / 92$ & 5 & 1 & & $1 E 2.8$ & $N$ & \\
\hline 1 & $3 / 19 / 92$ & 息. & I. & & 162.8 & $N$ & \\
\hline 1 & $3 / 9 / 92$ & 7 & 1 & & 153.2 & $\mathbf{N}$ & \\
\hline $\boldsymbol{I}$ & $3 / 59 / 92$ & 8 & 1 & & 154,4 & N & \\
\hline 1 & $3 / 49 / 92$ & 9 & 1 & & 155 & $\mathbf{Y}$ & \\
\hline $\mathbf{T}$ & $3 / 19 / 92$ & 10 & 1 & & 153.2 & $\mathbf{N}$ & \\
\hline 3 & $3 / 19 / 92$ & 11 & 1 & & 149.5 & N & \\
\hline 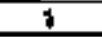 & $3 / 19 / 92$ & 12 & 1 & & 143.5 & $\mathbf{N}$ & J $910126143-48$ \\
\hline$t$ & 3/9/g2 & 13 & 1 & & 135.7 & $\mathbf{N}$ & \\
\hline$T$ & $3 / 18 / 92$ & 14 & 1 & & 125.1 & $\mathbf{N}$ & \\
\hline 1 & $3 / 19 / 42$ & 75 & 7 & & 311.5 & $N$ & \\
\hline 1 & $3 / 59 / 92$ & 16 & 1 & & 星 & $\mathbf{N}$ & \\
\hline 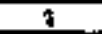 & $3 / 26 / 92$ & 1 & 1 & & 346.1 & $\mathbf{N}$ & \\
\hline
\end{tabular}

Data obtained from WHC Surveillance Apatysis Camputer System (SACS). July 1, 1993. 


\begin{tabular}{|c|c|c|c|c|c|c|c|}
\hline \multicolumn{8}{|c|}{ TANK 241-A-101 } \\
\hline & & & & THEF & AOCOLP & DATA & \\
\hline TFE? & DATE & ThEAHO \# & TAEE SET A & TEMF C & TEMPF & $Y=$ MAX TEMP & COMME़्NTS \\
\hline 1 & $3 / 20 / 92$ & 2 & 1 & & 148,2 & $\boldsymbol{N}$ & \\
\hline 1 & $3 / 20 / 92$ & 3 & $\frac{1}{1}$ & & 148 & $\omega$ & \\
\hline 1 & $3 / 20 / 92$ & 4 & 7 & & 149.0 & 束 & \\
\hline 1 & $3 / 20 / 92$ & 5 & 1 & & 950.9 & N & \\
\hline 1 & $3 / 20 / 92$ & B & 1 & & 950,7 & $\mathbf{N}$ & \\
\hline 1 & $3 / 20 / 92$ & 7 & 1 & & 150.8 & 세 & \\
\hline 1 & $3 / 26 / 92$ & a & 1 & & $151+\$$ & if & \\
\hline 1 & $5 / 26 / 92$ & $\overline{8}$ & 1 & & 152,8 & $\overline{\mathbf{Y}}$ & \\
\hline 1 & $3 / 2642$ & T6 & 1 & & $\overline{161,5}$ & it & \\
\hline 1 & $3 / 26 / 82$ & 11 & 1. & & 147,8 & $\mathbf{N}$ & \\
\hline 1 & $3 / 26 / 32$ & $\overline{12}$ & $\overline{1}$ & & 141,9 & $\bar{H}$ & \\
\hline 1 & $3 / 20 / 92$ & 13 & 1 & & 134.3 & $\mathbf{N}$ & \\
\hline 1 & 32682 & 14 & 1 & & 123.8 & $\mathrm{H}$ & J890623562800 \\
\hline 1 & $3 / 26 / 82$ & 75 & 1 & & $1 \pm 0.6$ & $\mathbf{N}$ & \\
\hline 1 & $3 / 26 / 92$ & 10 & 1 & & 95.0 & $\mathbf{N}$ & \\
\hline 1 & $3 / 26 / 92$ & 17 & 1 & & 93.2 & $\mathbf{N}$ & \\
\hline 1 & $3 / 20 / 92$ & 18 & 1 & & 93,2 & N & \\
\hline 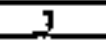 & $4 \sqrt{2,192}$ & 1 & 1 & & 146 & $\mathbf{N}$ & \\
\hline 1 & $4 / 2 / 92$ & 2 & 1 & & 147.8 & $\mathbf{N}$ & \\
\hline 1 & 42192 & 3 & $i$ & & 149,2 & $\mathbf{N}$ & \\
\hline 1 & $4 / 2 / 92$ & 4 & 2 & & 350 & $\mathbf{N}$ & \\
\hline 1 & $4 / 2 / 92$ & $E$ & 1 & & 150.3 & $\mathrm{~N}$ & \\
\hline 1 & $4 / 2192$ & 6 & $t$ & & 150.5 & $N$ & \\
\hline 1 & $4 / 2192$ & 7 & 1 & & 950,7 & N & \\
\hline 1 & $4 / 2 / 92$ & 8 & 1 & & 152.2 & N & \\
\hline 1 & $4 / 282$ & $\mathbf{g}$ & 1 & & $152+9$ & $Y$ & \\
\hline 1 & $4 / 2 \sqrt{2}$ & 10 & $i$ & & 151,2 & $M$ & \\
\hline 1 & $4 / 2 / 92$ & 11 & 1 & & 147.4 & it & \\
\hline 1 & $4 / 2 / 92$ & 12 & 1 & & 741.6 & $\mathbf{N}$ & $J 91012614349$ \\
\hline 1 & $4 / 2 / 62$ & 19 & 1 & & 933.8 & $\mathbf{N}$ & \\
\hline 1 & $4 / 2 / 92$ & 14 & 1. & & 123,3 & $\mathbf{N}$ & \\
\hline 1 & 4/2fen & 15 & 1 & & 110,1 & $\bar{y}$ & \\
\hline$i$ & $4 / 2 / 92$ & 10 & 1 & & 95.3 & N & \\
\hline$?$ & $4 / 2 / 92$ & 17 & 1 & & 83.2 & $\mathbf{N}$ & \\
\hline 7 & $4 / 2 / 92$ & 19 & 1 & & 92,9 & $\mathbf{N}$ & \\
\hline 1 & $4 / 9 / 92$ & 1 & 1 & & $147, \mathrm{~B}$ & $\mathbf{N}$ & \\
\hline 1 & $4 \sqrt{19} 92$ & $\overline{2}$ & 1 & & 148.5 & $\mathbf{N}$ & \\
\hline 1 & 4 伯的 & 3 & 1 & & 161.1 & N. & \\
\hline 1 & A/9/92 & 4 & 1 & & 152 & $N$ & \\
\hline t & $4 / 9 / 92$ & 5 & 1 & & 152.2 & D & \\
\hline 1 & $4 / 9 / 92$ & B & 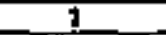 & & 152,4 & $\mathbf{M}$ & \\
\hline 1 & $4 / 2 / 92$ & 7 & 1 & & 162.5 & $N$ & \\
\hline 1 & $4 / 0 / 92$ & $\mathrm{E}$ & 1 & & 153.0 & $N$ & \\
\hline 1 & $4 / 9 / 92$ & 9 & $i$ & & 154.0 & $Y$ & $+910128143-64$ \\
\hline 1 & $4 / 6 / 92$ & 10 & 1 & & 153 & $\bar{N}$ & \\
\hline 1 & $4 / 9 / 82$ & 12 & 2 & & 249,1 & $N$ & \\
\hline 1 & $4 / 9 \sin ^{2}$ & 12 & 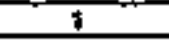 & & 145.2 & $\mathbf{N}$ & \\
\hline 1 & A/9/e2 & 13 & 1 & & 135,5 & $\mathrm{~N}$ & \\
\hline 1 & 4/a/42 & 14 & 1 & & 124.9 & $\mathbf{N}$ & \\
\hline 1 & $4 / 9$ & 16 & $t$ & & 111.8 & $\mathrm{~N}$ & \\
\hline 1 & $4 / 9 \sqrt{02}$ & 16 & 1 & & 97.1 & $\mathbf{M}$ & \\
\hline 1 & $410 / 92$ & 1 & $E$ & & $145+8$ & $\mathbf{N}$ & \\
\hline 1 & 4r16raz & 2 & $t$ & & 147.7 & N & \\
\hline 1 & 41602 & 3 & 7 & & 249.2 & $\mathrm{~N}$ & \\
\hline 1 & $4 / 16 / 82$ & 4 & 1 & & 150,1 & $\mathbf{N}$ & \\
\hline 1 & $4 / 16 / 92$ & 5 & 1 & & 150.4 & $\mathbf{N}$ & \\
\hline 1 & $4 \sqrt{16} \cdot 62$ & a & 1 & & 150.6 & N & \\
\hline 1 & 416192 & 7 & i & & 160.9 & $\mathbf{N}$ & \\
\hline 1 & $4 / 16 \theta 2$ & 8 & 7 & & 152.1 & $\mathrm{~N}$ & \\
\hline 1 & A/J6/O2 & $\theta$ & 1 & & 1527 & $y$ & \\
\hline 1 & $4 / 16 \sqrt{92}$ & 10 & 1 & & 1512 & N & \\
\hline 1 & $4 / 16 / 92$ & 11 & 1 & & $\$ 47.4$ & $\mathbf{N}$ & $9910126143-49$ \\
\hline 1 & $4 / 16 / 2$ & $\$ 2$ & 1 & & 141,4 & $\mathbf{N}$ & \\
\hline
\end{tabular}

Data obteined from WHC Surveillance Analysis Computer System (SACS), July 1, 1993. 


\begin{tabular}{|c|c|c|c|c|c|c|c|}
\hline \multicolumn{8}{|c|}{ TAMK 241-A-101 } \\
\hline & & - & & THE & AOcolp & DATA & \\
\hline & & & & & & & \\
\hline SREE & DATE & 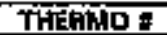 & TAE 8 这 & JEMP C & TEMP F & $Y=$ MAXX TEWP & COMMENTS \\
\hline 1 & $416 / 82$ & 13 & 1 & & 133.7 & $M$ & \\
\hline 1 & $4+6 / \theta 2$ & 14 & 1 & & 123.3 & $\mathbf{N}$ & \\
\hline 7 & $4 d 10 / 02$ & 15 & 1 & & 110.2 & $\mathbf{N}$ & \\
\hline 1 & $4 / 23 / 92$ & 1 & 1 & & $146+9$ & $\mathbf{N}$ & \\
\hline $\mathbf{1}$ & $4 / 2,3 / 92$ & 2 & ] & & 148 & $\mathbf{N}$ & \\
\hline$T$ & $4 / 23 / 92$ & 3 & $\$$ & & 148.7 & $\mathbf{N}$ & \\
\hline 1 & $4 / 23 / 92$ & 4 & \pm & & 150.6 & $N$ & \\
\hline 1 & $4 / 23 / 92$ & 5 & 1 & & 160.8 & Nㅗ & \\
\hline 1 & $4 / 23 / 92$ & $\theta$ & 1 & & 181 & $\mathbf{N}$ & \\
\hline 1 & 4 /2araz & 7 & 1 & & 1515 & $\mathbf{N}$ & \\
\hline 1 & $4 / 23 / 82$ & 皇 & $\$$ & & 152.0 & $\mathbf{N}$ & \\
\hline 1 & $4 / 23 / 92$ & $\theta$ & 1 & & 153.2 & $\mathbf{Y}$ & \\
\hline 1 & $4 \sqrt{2} 3 \sqrt{92}$ & 10 & 1 & & 151.7 & $N$ & \\
\hline$T$ & $4 / 23 / 92$ & โ1 & 1 & & 147.9 & $\overline{\mathbf{N}}$ & \\
\hline$\overline{1}$ & $4 / 23 \sqrt{92}$ & 12 & 1 & & 141.9 & $\mathbf{N}$ & 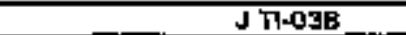 \\
\hline 1 & $4 / 23 / 92$ & 13 & $\hbar$ & & 134.7 & $\mathbf{N}$ & \\
\hline 1 & $4 / 23 / 92$ & 14 & 1 & & 123.6 & $N$ & \\
\hline 1 & $4 / 25 / 42$ & 15 & $\mathbf{1}$ & & 110.5 & $\mathbf{N}$ & \\
\hline 1 & $4 \longdiv { 2 3 / 8 2 }$ & 16 & 1 & & 55.8 & $\mathbf{N}$ & \\
\hline 1 & $4 / 23 / 42$ & 17 & 1 & & 94 & $\mathbf{N}$ & \\
\hline 1 & $4 / 23 / 2$ & 18 & 1 & & 93.6 & $\mathbf{N}$ & \\
\hline 1 & 4/30/9? & 1 & 1 & & 146.7 & $\mathbf{N}$ & \\
\hline 1 & $4 / 30 / 92$ & 2 & 1 & & 147,5 & $N$ & \\
\hline 1 & $4 / 30 / 92$ & 3 & 1 & & 148.1 & $\mathbf{N}$ & \\
\hline 1 & $4 / 30 / 22$ & 4 & 1 & & 150 & $\mathbf{N}$ & \\
\hline $\bar{T}$ & $4 / 30 \mathrm{rg} 2$ & $\overline{5}$ & $T$ & & 160.2 & $\mathbf{N}$ & \\
\hline 5 & $4 / 30 / 92$ & 5 & 1 & & 150,4 & $\mathbf{N}$ & \\
\hline 1 & $4 / 30 / 92$ & 7 & $T$ & & 150.6 & $\overline{\mathbf{N}}$ & \\
\hline 1 & $4 / 30 / 02$ & 8 & 1 & & 752 & $\mathbf{N}$ & \\
\hline $\mathbf{1}$ & 4/30일ㄹ & 9 & 1 & & 152.8 & $\gamma$ & \\
\hline 1 & $4 / 30192$ & 10 & 1 & & 151.1 & $\mathbf{N}$ & \\
\hline 1 & $4 / 30 / 92$ & 11 & 1 & & 147.4 & $\mathbf{N}$ & \\
\hline $\mathbf{3}$ & 430192 & $\sqrt{2}$ & 1 & & 142.4 & $\mathbf{N}$ & $J 91012614348$ \\
\hline$t$ & $4 / 3$ 앙ㄹ & 13 & 1 & & 133,6 & $\mathbf{N}$ & \\
\hline 1 & $4 / 30 / 92$ & 14 & 1 & & 123.2 & $\mathbf{N}$ & \\
\hline 1 & $4 / 30 / 92$ & 15 & 1 & & 110.2 & $\mathbf{N}$ & \\
\hline 1 & $4 / 30192$ & 16 & 1 & & 95.9 & $\mathbf{N}$ & \\
\hline 1 & $5 \pi / 92$ & 1 & 1 & & 147.2 & $\mathbf{N}$ & \\
\hline 1 & $5 / 7 \% 2$ & 2 & 1 & & 149.1 & $\mathbf{N}$ & \\
\hline 1 & $5 / 7 / 92$ & 3 & 1 & & 150,4 & $\mathbf{N}$ & \\
\hline 1 & $5 / 7 / 92$ & 4 & 1 & & 151.5 & $\mathbf{N}$ & \\
\hline 1 & $5 D P S 2$ & 5 & 1 & & 151.8 & $\mathbf{N}$ & \\
\hline$T$ & 8 S/F 2 & 6 & 1 & & 161.8 & $\mathrm{~N}$ & \\
\hline$\overline{1}$ & $6 / 7 / 92$ & 7 & 1 & & 152,1 & $\mathbf{N}$ & \\
\hline 1 & $5 / 7 / 92$ & E & 1 & & 153,4 & $\overline{\mathbf{Y}}$ & \\
\hline 1 & $5 / 7 / 92$ & 9 & 1 & & 154.2 & $\bar{N}$ & \\
\hline 1 & $5 / 7 / 92$ & 10 & 1 & & 152.5 & $\mathbf{N}$ & \\
\hline 1 & $5 / 7 / 92$ & 17 & 1 & & 148.7 & $\mathbf{N}$ & \\
\hline 1 & $5 / 7 / 92$ & 12 & 1 & & 142.7 & $\mathbf{N}$ & \\
\hline 1 & $5 / 7 / 42$ & 13 & 1 & & 134.A & $\mathbf{N}$ & \\
\hline 1 & $67 / 92$ & 14 & 1 & & 124.2 & $\mathbf{N}$ & $189052 \$ 612.47$ \\
\hline 1 & $6 / 7 / 92$ & 16 & $1 \%$ & & 111.2 & $\mathbf{N}$ & \\
\hline 1 & 3/782 & 16 & 1 & & 98.0 & $\mathbf{M}$ & \\
\hline 1 & $5 / 7 / 92$ & 17 & 1 & & 94.9 & $\mathbf{N}$ & \\
\hline 1 & $5 / / 792$ & 18 & 1 & & 94.4 & $N$ & \\
\hline 1 & $5 / 74 / 62$ & 1 & 1 & & 145.7 & $\mathbf{N}$ & \\
\hline $\mathbf{T}$ & $5 / 14 / 92$ & 2 & 1 & & 147.7 & $\mathbf{N}$ & \\
\hline 1 & $5 / 14 / 92$ & 3 & 1 & & 149.1 & $\mathbf{N}$ & \\
\hline 1 & $5 / 14 / 92$ & 4 & 1 & & 149.8 & $\mathbf{N}$ & \\
\hline 1 & $5 / 1412$ & 8 & 1 & & 150.2 & $\mathbf{N}$ & \\
\hline 1 & $5 / 14 / 92$ & $\theta$ & 1 & & 150.3 & $\mathbf{N}$ & \\
\hline 1 & $5 / 14 / 82$ & 7 & 1 & & 150.7 & $N$ & \\
\hline$\overline{1}$ & $5 / 14 / 92$ & 8 & 1 & & 152 & $N$ & \\
\hline
\end{tabular}

Data obtained from WhiC Surveillance Analysis Computer Systern ISACSI, July 1, 1993. 


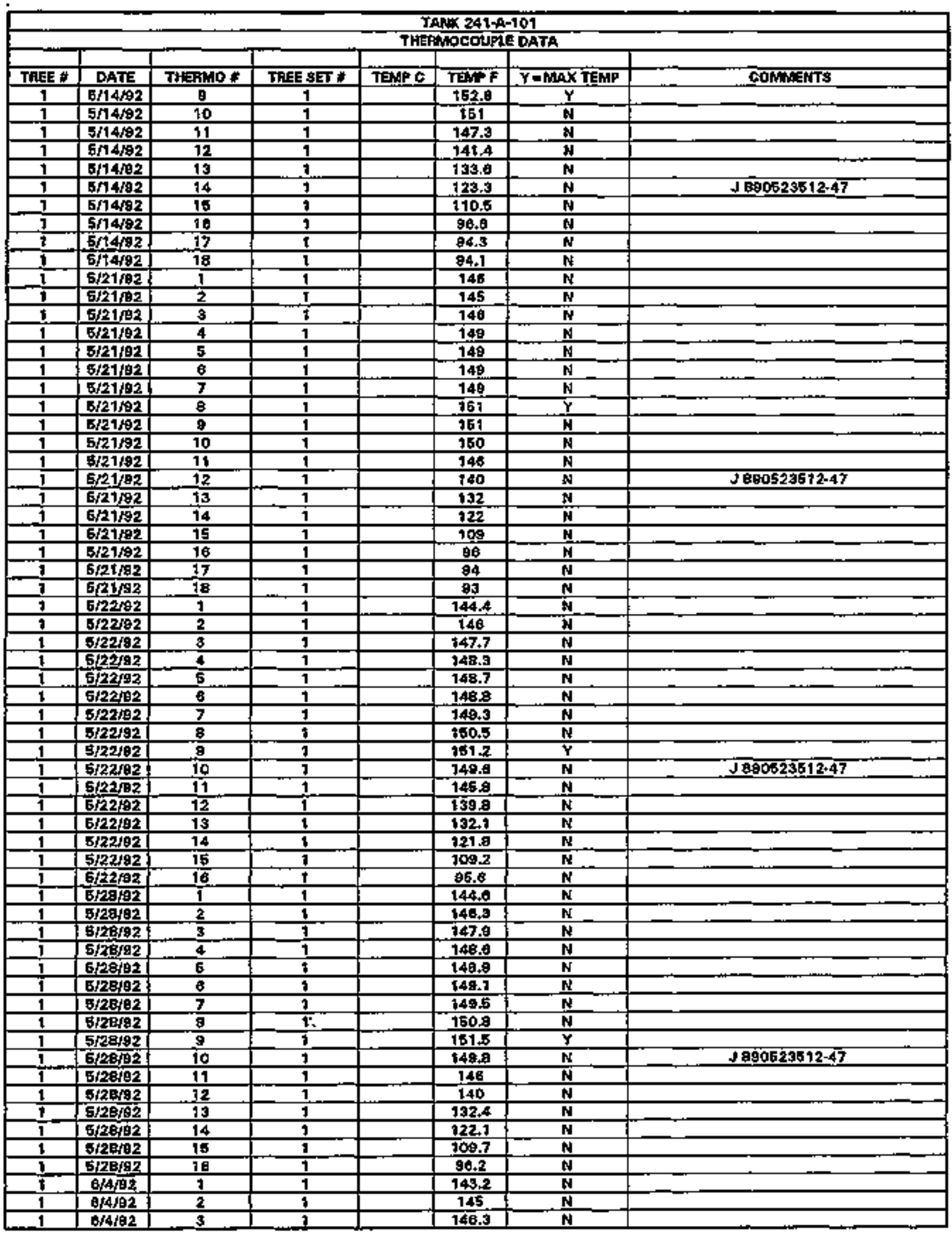

Data obtained from WHC Surveillenco Analysis Computer System [SACS\}, July 1, 1993. 


\begin{tabular}{|c|c|c|c|c|c|c|c|}
\hline \multicolumn{8}{|c|}{ rAkK 241-A-101 } \\
\hline & & & & & & & \\
\hline Tr:王 8 & DATE & THEAMO * & THEE SET & TEMP C & TEMP F & $Y=M A X$ TEMP & COMMENTS \\
\hline 1 & $6 \sqrt{4 / 92}$ & 4 & 1 & & 147.4 & $\mathbf{N}$ & \\
\hline 1 & 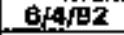 & 6 & 1 & & 747.8 & $\mathbf{N}$ & \\
\hline 1 & $6 / 4 / 92$ & 6 & 1 & & $947+9$ & $\mathbf{N}$ & \\
\hline 1 & $6 / 4 / 92$ & 7 & 7 & & 948,1 & $\mathbf{N}$ & \\
\hline 1 & $6 / 4 / 92$ & B & 1 & & 948,4 & $\mathbf{N}$ & \\
\hline I & $6 / 4 / 92$ & 8 & 1 & & 148.9 & $\mathbf{N}$ & \\
\hline 1 & ef/4 2 & 10 & $t$ & & 148.2 & $\mathbf{N}$ & \\
\hline 1 & $6 / 4 / 92$ & 11 & $\frac{1}{1}$ & & $\$ 44,7$ & $\mathbf{N}$ & \\
\hline 1 & $6 / 4 / 92$ & 12 & 1 & & 138.t. & $\mathbf{N}$ & J $1900523512-47$ \\
\hline 1 & $8 / 4 / 92$ & 13 & 1 & & 130.8 & $\mathbf{N}$ & \\
\hline 1 & 6/4/62 & 14 & 1 & & 120.7 & 소 & \\
\hline 1 & B/4/2 & 16 & 1 & & 108.4 & $N$ & \\
\hline 1 & $6 / 4 / 92$ & 16 & 1 & & 05.3 & $N$ & \\
\hline 2 & ef11/a2 & 1 & 2 & & 1427 & $\mathbf{N}$ & \\
\hline 1 & G/1.1/A2 & 2 & 1 & & 144.3 & $\mathbf{N}$ & \\
\hline 3 & $6(17 / 92$ & 3 & 1 & & 148.1 & N & \\
\hline 1 & 6/11/92 & 4 & 1 & & 747.2 & 杽 & \\
\hline 1 & $0 / 11 / 2$ & 5 & 1 & & 947.5 & 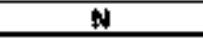 & \\
\hline 1 & $9 / 11 / 92$ & $B$ & 1 & & 147,6 & $\mathbf{N}$ & \\
\hline 1 & $\$ / 11 / 92$ & 7 & 1 & & $147+9$ & $\mathbf{N}$ & \\
\hline 1 & $6 / 11 / 22$ & $\mathbf{B}$ & 1 & & 14E.8 & $\mathbf{N}$ & \\
\hline 1 & $6 / 71 / 92$ & e & 1 & & 149.7 & $\mathbf{Y}$ & \\
\hline 1 & $6 / 11 / 92$ & 10 & 1 & & $148, t$ & $N$ & \\
\hline 1 & $6 / 11 / 92$ & 11 & 1 & & 144.8 & $\mathbf{N}$ & \\
\hline 1 & $6 / 11 / 92$ & 12 & 1 & & $13 \theta_{13}$ & $\mathbf{N}$ & 」010126143.28 \\
\hline 1 & $8 / 1+M 2$ & 13 & 1 & & 130.7 & $\mathbf{N}$ & \\
\hline 1 & d/11/92 & 14 & 1 & & 120.6 & $\bar{N}$ & \\
\hline 1 & $6 / 1+/ 92$ & 15 & 1 & & 108.5 & $\mathbf{N}$ & \\
\hline 1 & $6 / 1$ t/92 & 10 & 1 & & 95.7 & N & \\
\hline 1 & $8711 / 92$ & $1 \overline{7}$ & 1 & & 94 & $\mathbf{N}$ & \\
\hline 1 & Q/11/2 & 18 & 1 & & 93.6 & $\mathbf{N}$ & \\
\hline 1 & $0 / 27 / 42$ & 1 & 1 & & 142.7 & $\mathbf{N}$ & \\
\hline 1 & $6 / 27 / 92$ & $\underline{2}$ & 1 & & 144.8 & $N$ & \\
\hline 1 & $d / 27 / 92$ & 3 & 1 & & 146 & $\mathbf{N}$ & \\
\hline 1 & $6 / 27 / 92$ & 4 & 1 & & 147.1 & I & \\
\hline 1 & 6/27/92 & 5 & 1 & & 147.5 & N & \\
\hline 1 & $0 / 27 / 82$ & $a$ & 1 & & 147.0 & $\mathbf{N}$ & \\
\hline 1 & $6 / 27 / 92$ & 7 & 1 & & 148 & $\mathbf{N}$ & \\
\hline 1 & $6 / 27 / 92$ & $\mathbf{8}$ & 1 & & 149.2 & it & \\
\hline 1 & $0 / 27 / 92$ & $\mathbf{9}$ & 1 & & 148.8 & $Y$ & \\
\hline 1 & $0 / 27 / 92$ & 10 & 1 & & 149.2 & N & f010120143-29 \\
\hline 1 & $0 / 27 / 92$ & 11 & 1 & & 144.7 & N & \\
\hline 1 & $0 / 27 / 92$ & 12 & 1 & & $139+6$ & $\mathbf{N}$ & \\
\hline 1 & $6 / 27 / 92$ & 13 & 1 & & 1312 & $\mathbf{N}$ & \\
\hline 1. & e/27/92 & 14 & 1 & & 121.3 & $N$ & \\
\hline 1 & $6 / 27 / 92$ & 15 & 1 & & 109.5 & $N$ & \\
\hline 1 & or27/92 & 10 & 1 & & 97,2 & N & \\
\hline 1 & $7 / 3 / 92$ & 1 & 1 & & 146.8 & $\mathbf{N}$ & \\
\hline 7 & $7 \sqrt{3} 92$ & 2 & 1 & & 149.7 & W & \\
\hline 1 & $7 \mathrm{~s} / \mathrm{g} 2$ & 3 & 1 & & $150+1$ & $N$ & \\
\hline 1 & $7 / 3 / 82$ & 4 & 1 & & $961+1$ & H & \\
\hline 1 & $7 / 3 / 92$ & 5 & $1 ;$ & & 151.4 & $\mathbf{N}$ & \\
\hline 1 & $7 / 3 / 92$ & $\mathbf{B}$ & 1 & & 151.5 & $\mathbf{N}$ & \\
\hline 1 & $7 / 3 / 92$ & 7 & 1 & & 151.8 & $\mathbf{N}$ & \\
\hline 1 & 71302 & 8 & 1 & & $153+3$ & $\mathbf{N}$ & \\
\hline 1 & $7 / 3 / 92$ & 9 & 1 & & $15 \$ .8$ & $\mathbf{Y}$ & \\
\hline 1 & $7 / \$ / 92$ & 10 & 1 & & 152.2 & $\mathbf{N}$ & \\
\hline 1 & $7 / 3 / 92$ & 11 & .1 & & $14 \mathrm{~B}, 4$ & $N$ & \\
\hline 1 & $7 / 3 / 92$ & 12 & 1 & & 9425 & $\mathbf{N}$ & $1930126143-42$ \\
\hline 1 & $7 / 3 / 42$ & 13 & 1 & & 134.0 & $\mathbf{N}$ & \\
\hline 1 & $7 / 3 / 92$ & 14 & 1 & & 125 & $\bar{N}$ & \\
\hline 1 & $713 / 92$ & 15 & 1 & & 113.2 & $\mathbf{N}$ & \\
\hline 1 & $7 / 3 / 92$ & 16 & 1 & & 100,7 & $\mathbf{N}$ & \\
\hline
\end{tabular}

Dats obtained from WhC Survellance Anelysis Computer System (SACSI, July 1, 1993. 


\begin{tabular}{|c|c|c|c|c|c|c|c|}
\hline \multicolumn{8}{|c|}{ TAHK 241-A+101 } \\
\hline & & & & & & & \\
\hline TRE形 & DATE & THERMO & TRËE SET $\bar{y}$ & TETPC & TEMPF & $Y=M A X$ TEMP & COMW EN \\
\hline 1 & $7 / 8 / 92$ & 1 & 1 & & 140.5 & $\mathbf{N}$ & \\
\hline 1 & $7 / 8 / 92$ & 2 & 1 & & 146,4 & $\mathbf{N}$ & \\
\hline 1 & $7 / 9 / 92$ & 3 & 1 & & 148.9 & $\mathbf{N}$ & \\
\hline 1 & 7 79/92 & 4 & 1 & & 150.7 & $\mathbf{N}$ & \\
\hline$\overline{1}$ & $7 / 0 / 92$ & $\overline{5}$ & 1 & & 151,2 & $\mathbf{N}$ & \\
\hline 1 & $7 \operatorname{los} 2$ & 0 & 1 & & 151.4 & N & \\
\hline 1 & $7 / 9 / 92$ & 7 & 1 & & 151.7 & $\mathbf{N}$ & \\
\hline 1 & $7 / 9 / 92$ & $\mathbf{g}$ & 1 & & 152.8 & $\mathbf{N}$ & \\
\hline 1 & $7 / 9 / 92$ & 9 & 1 & & 153.5 & $Y$ & \\
\hline 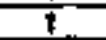 & $7 / 2 / 92$ & 10 & 1 & & 152 & $\mathbf{M}$ & \\
\hline 1 & $7 / 9 / 82$ & 11 & 1 & & 148.2 & $\mathbf{N}$ & \\
\hline$i$ & 3/902 & 12 & 1 & & 142.3 & $\mathbf{N}$ & J $910128143-32$ \\
\hline 1 & $7 / 2$ & 13 & \pm & & 134.6 & $\mathbf{H}$ & \\
\hline 1 & $7 / 9 \sqrt{2}$ & 14 & 1 & & 134.8 & $N$ & \\
\hline 1 & $7 / 9 / 92$ & 16 & 1 & & $\$ 13+2$ & $\mathbf{N}$ & \\
\hline 1 & 7/9/e2 & 16 & 1 & & 100.9 & $\mathbf{N}$ & \\
\hline 1 & 799192 & 17 & 1 & & 99.2 & $\mathrm{H}$ & \\
\hline 1 & $7 / 8 / 92$ & 18 & 1 & & 9日.8 & $N$ & \\
\hline 1 & $7 f 17 / 92$ & 1 & 1 & & 148 & A & \\
\hline$\overline{1}$ & $7 / 17 / 92$ & 2 & 1 & & 148 & $\mathbf{N}$ & \\
\hline 1 & $7 n 7 / 92$ & 3 & 1 & & 149.3 & $\mathbf{N}$ & \\
\hline 1 & $7 t 1702$ & 4 & 1 & & 150.2 & M & \\
\hline 1 & $7 / 7 / 192$ & 5 & 1 & & 150.8 & D & \\
\hline 1 & 711792 & 6 & 1 & & 150.7 & $\mathbf{N}$ & \\
\hline 1 & $7 / 17192$ & 7 & 1 & & 161 & $\mathbf{N}$ & \\
\hline 7 & $7117 / 92$ & 吾 & 1 & & 152,4 & $\mathbf{N}$ & \\
\hline 1 & $7 / 17122$ & 9 & 1 & & 152.9 & $Y$ & \\
\hline$t$ & $7 / 7 \% 2$ & 10 & 1 & & 151.5 & i & J $91 \overline{0+12614 \overline{3-29}}$ \\
\hline 1 & 719702 & 11 & 1 & & 147.8 & $\mathbf{N}$ & \\
\hline 1 & $7 / 17 / 42$ & 12 & 1 & & 141.8 & $\mathbf{N}$ & \\
\hline 1 & 7117192 & 13 & 1 & & 134,4 & $\mathbf{N}$ & \\
\hline 1 & $7 / 17 / 92$ & 14 & $\overline{1}$ & & 124.7 & $M$ & \\
\hline 1 & $747 a_{2}$ & 15 & 1 & & 113.2 & $N$ & \\
\hline 1 & $7 / 1792$ & 10 & 1 & & 101.1 & N & \\
\hline 1 & $7 / 23 / 92$ & 1 & 1 & & 141.5 & $N$ & \\
\hline 1 & $7 / 2392$ & 2 & 1 & & 149.3 & $\mathbf{N}$ & \\
\hline 1 & $7 / 2.392$ & 3 & 1 & & 150.8 & $\mathbf{N}$ & \\
\hline 1 & $7 / 25 / 92$ & 4 & 1 & & 151.8 & N & \\
\hline$\overline{1}$ & $7 / 2 \sqrt{12}$ & 5 & $\bar{T}$ & & $\overline{152.1}$ & $\bar{N}$ & \\
\hline 1 & $7 / 23 / 02$ & 6 & $\bar{T}$ & & 152.3 & $\mathbf{N}$ & \\
\hline 1 & ThS raz & 7 & 1 & & 152.7 & $\bar{N}$ & \\
\hline 1 & 7/23/82 & $\vec{B}$ & 1 & & 1638 & $\mathbf{N}$ & \\
\hline 1 & $7 / 2 \overline{3} / 2$ & 9 & 1 & & 154,5 & $\mathbf{Y}$ & \\
\hline 1 & $7 / 23192$ & 10 & $\overline{1}$ & & 153 & $\overline{\mathbf{N}}$ & \\
\hline 1 & $7 / 23 / 92$ & 11 & 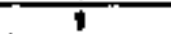 & & 149.2 & $\mathbf{N}$ & \\
\hline 1 & $723 \sqrt{22}$ & 12 & J & & 143.2 & $\mathrm{H}$ & J 810126143,42 \\
\hline 1 & $7 / 23 \sqrt{12}$ & 13 & 1 & & 135,1 & $\mathbf{N}$ & \\
\hline 1 & $7 / 23 / 92$ & 14 & 1 & & 125.8 & N & \\
\hline 1 & $7 / 23 / 92$ & 15 & 1 & & 114.0 & $\mathbf{N}$ & \\
\hline 1 & $7 / 23 / 92$ & 16 & 1 & & 102,0 & $\mathbf{N}$ & \\
\hline$i$ & $7 / 30192$ & 1 & 1 & & $\$ 46,0$ & $N$ & \\
\hline 1 & $7 / 30102$ & 2 & 1 & & 148.5 & $\mathbf{N}$ & \\
\hline$\overline{1}$ & $7 / 30 / 92$ & 3 & 1 & & 149.8 & $\mathbf{H}$ & \\
\hline 1 & $7 \sqrt{30 / 82}$ & 4 & 7 & & 150,9 & $\mathbf{N}$ & \\
\hline 1 & 730102 & E & 1 & & $15 t .3$ & $\mathbf{N}$ & \\
\hline 1 & $7 / 30192$ & 6 & 1 & & 167.4 & $N$ & \\
\hline 1 & $7 \mathrm{rop} / 92$ & 7 & $j$ & & $\operatorname{t51} \theta$ & $\mathbf{N}$ & \\
\hline 1 & $7 / 30192$ & $B$ & 1 & & 153.1 & $\mathbf{N}$ & \\
\hline 1 & $7 / 30 / 92$ & 9 & 1 & & 153.8 & $\bar{\gamma}$ & \\
\hline 1 & $7 / 30102$ & 10 & 1 & & 152.1 & $\mathbf{N}$ & \\
\hline 1 & $7 / 30192$ & 11 & 1 & & 148,4 & $\mathbf{n}$ & \\
\hline 1 & $7 / 90 / 92$ & 12 & 1 & & 142,5 & $\mathbf{N}$ & \\
\hline$\frac{1}{1}$ & $7 / 30182$ & 13 & 1 & & 135.1 & $\mathrm{~N}$ & \\
\hline
\end{tabular}

Data obtgined from WHC Surveillance Analysis Computer System (SACS), July 1, 1993. 


\begin{tabular}{|c|c|c|c|c|c|c|c|}
\hline & & & & & 1K 241-A & 101 & \\
\hline & & - & & THE & ADCole' & DATA & \\
\hline & & & & & & & \\
\hline THEE : & DATE & THEFWA : & THEE \$ET \# & TEMPC & TEMP F & $Y=$ MAX TEHP & COHAEKTS \\
\hline 1 & $7130 / 92$ & 14 & 1 & & 126.8 & $\mathbf{N}$ & J $910+26143.42$ \\
\hline 1 & $7 / 30 / 92$ & 15 & 1 & & 1140 & $N$ & \\
\hline 1 & $7 / 30 / 92$ & 10 & 1 & & 102.7 & $N$ & \\
\hline 1 & $7 / 30 / 92$ & 17 & 1 & & 101,1 & N & \\
\hline 1 & $7 / 30 / 92$ & 18 & 1 & & 100.9 & N & \\
\hline 1 & erefen & $t$ & 1 & & 146 & $\mathbf{N}$ & \\
\hline 1 & B/ota2 & 2 & 1 & & 149 & $N$ & \\
\hline 1 & 0/0192 & 3 & 1 & & 140 & $\mathbf{N}$ & \\
\hline 1 & 6/6192 & 4 & 1 & & 150 & $\mathbf{N}$ & \\
\hline$i$ & 6/6/92 & 5 & 1 & & 150 & $\mathbf{N}$ & \\
\hline 1 & 9/0y2 & 6 & 1 & & 165 & $N$ & \\
\hline 1 & $8 / 6 / 92$ & 7 & 1 & & 161 & $N$ & \\
\hline 1 & 8/9/92 & 8 & 1 & & 162 & $N$ & \\
\hline 1 & s/ost & g & 1 & & 753 & $Y$ & \\
\hline 1 & $8 / 6 / 62$ & 10 & 1 & & 151 & $\mathbf{N}$ & \\
\hline 1 & $8 / 6 / 92$ & 11 & 1 & & 148 & $N$ & \\
\hline 1 & $8 / 6 / 92$ & 12 & 1 & & 142 & N & J91012643442 \\
\hline 1 & $8 / 5 / 92$ & 13 & 1 & & 135 & $\mathbf{N}$ & \\
\hline 1 & 8/62 & 14 & 1 & & 125 & $\mathbf{N}$ & \\
\hline 1 & $\$ / 6 / 92$ & 15 & 1 & & 114 & $\mathbf{N}$ & \\
\hline 1 & $8 / 6 / 92$ & 16 & 1 & & 103 & $\mathbf{N}$ & \\
\hline 1 & $8 / 8 / 82$ & 17 & 1 & & 101 & $\mathbf{N}$ & \\
\hline 1 & 8fortz & $1 \mathrm{~B}$ & 1 & & 101 & $\mathbf{N}$ & \\
\hline 1 & 8/13/92 & 1 & 1 & & $\$ 46.2$ & $\mathbf{N}$ & \\
\hline 1 & 어13요 2 & 2 & 1 & & 148.2 & $\mathbf{N}$ & \\
\hline 1 & 머13/82 & 3 & 1 & & 149.6 & N & \\
\hline 1 & 요f(13/42 & 4 & 1 & & 150,3 & $\mathrm{~N}$ & \\
\hline 1 & $8113 / 92$ & 6 & 1 & & 150.6 & $\mathbf{N}$ & \\
\hline 1 & 아낼 2 & $\theta$ & 1 & & 150.5 & $\mathbf{N}$ & \\
\hline 1 & $8 / 13 / 92$ & 7 & 1 & & 151.6 & $\mathbf{N}$ & \\
\hline 1 & 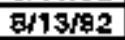 & a & 1 & & +52.8 & $\mathbf{N}$ & \\
\hline 1 & 택13 & 9 & 1 & & 153.4 & $\bar{Y}$ & \\
\hline 1 & $8 / 13 / 92$ & 10 & 1 & & 151.8 & N & IHH-9SA J \\
\hline 1 & 8/13/92 & 11 & 1 & & 148 & N & \\
\hline$i$ & $8 / 13 / 92$ & 72 & 1 & & 142.1 & M & \\
\hline 1 & aft3 & 13 & 1 & & 1352 & $\mathbf{N}$ & \\
\hline 1 & a/13/92 & 14 & 1 & & 125.9 & $\mathrm{H}$ & \\
\hline 1 & a/23/92 & 15 & 1 & & 116.1 & $\mathbf{M}$ & \\
\hline 1 & $\mathrm{~g} / 13 / \mathrm{A2}$ & 76 & 1 & & 108,6 & $\mathbf{N}$ & \\
\hline 1 & g/21/92 & 7 & 1 & & 146,2 & $\mathbf{N}$ & \\
\hline 1 & g/21/1/2 & 2 & 1 & & 140 & $\mathbf{N}$ & \\
\hline 1 & $8 / 21 / 62$ & 3 & 1 & & 149.5 & $M$ & \\
\hline$i$ & $9 / 21 / 82$ & 4 & 1 & & 750.4 & $\mathbf{N}$ & \\
\hline 7 & a/21/92 & 5 & 1 & & 1508 & $\mathbf{N}$ & \\
\hline 3 & $8 / 21 / 92$ & 8 & $\overline{1}$ & & 951,1 & $\mathbf{M}$ & \\
\hline 1 & 8/21/92 & 7 & 1 & & 151.8 & $\mathbf{N}$ & \\
\hline 1 & $8121 / 2$ & 8 & 1 & & 152.5 & $\mathbf{N}$ & \\
\hline 7 & $8 / 21 / 82$ & 9 & $T$ & & t53.2 & $\bar{\gamma}$ & \\
\hline 1 & 배21/92 & 10 & 1 & & t518 & $\mathbf{N}$ & $5910126143-32$ \\
\hline T & $8 / 21 / 92$ & $T 1$ & 1 & & 148 & $\mathbf{N}$ & \\
\hline$\overline{1}$ & $9 / 21 / 92$ & 72 & 1 & & $\$ 42.3$ & $\mathbf{N}$ & \\
\hline 1 & $8 / 21 / 92$ & 13 & $\mathrm{~F}:$ & & 135 & $\mathbf{M}$ & \\
\hline 1 & $8 / 2+/ 82$ & 14 & 1 & & 125.8 & $\overline{\mathbf{N}}$ & \\
\hline 1 & $8 / 24 / 92$ & 15 & $\mathbf{T}$ & & 115,1 & $\overline{\mathbf{N}}$ & \\
\hline 1 & B/2 $/ / 92$ & 16 & $T$ & & 102.E & $\mathbf{N}$ & \\
\hline 1 & Ev27192 & 1 & 1 & & 140.3 & $\mathbf{N}$ & \\
\hline 1 & B/27/92 & 2 & 1 & & 149.1 & $\mathbf{N}$ & \\
\hline 1 & $6 / 27 / 82$ & 3 & 3 & & 144.5 & $\mathbf{N}$ & \\
\hline 1 & ex/27/92 & 4 & 1 & & 150.4 & $\mathbf{N}$ & \\
\hline 1 & 6/27/92 & $\mathbf{5}$ & 1 & & 160.6 & $\mathbf{M}$ & \\
\hline 1 & $8 / 27 / 92$ & 6 & 1 & & 151,1 & $\mathbf{M}$ & \\
\hline 1 & B $27 / 92$ & 7 & 3 & & 151.5 & $\mathbf{N}$ & \\
\hline 1 & $8 / 27 / 92$ & 官 & 1 & & 152.5 & $\mathbf{N}$ & \\
\hline
\end{tabular}

Data obtained from WHC Surveillance Analysls Computer System (SACS1, Jư 1, 1993. 


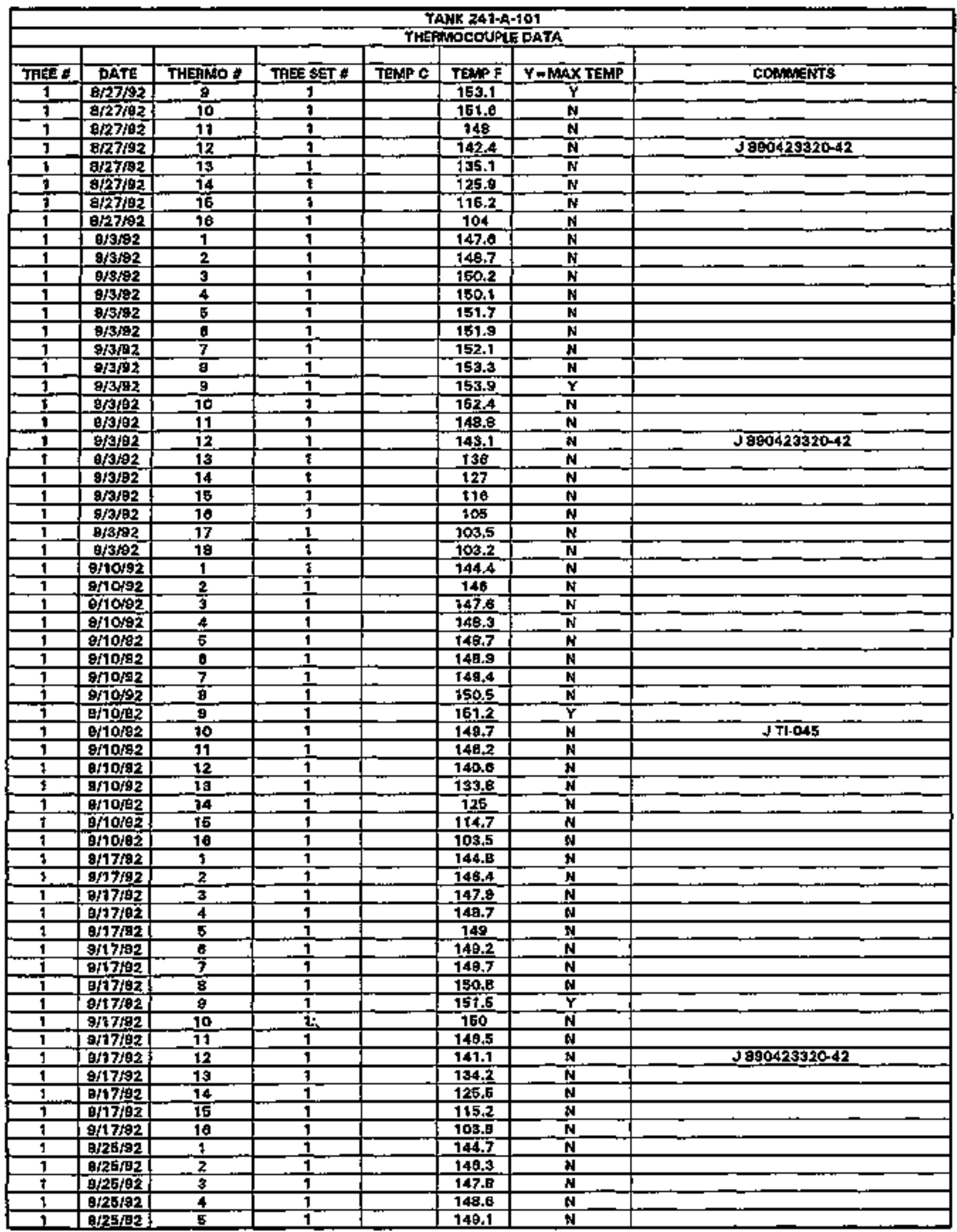

Datta obtained from WHC Survellance Anahyis Computer System (SACS1, thly 1, 1993. 


\begin{tabular}{|c|c|c|c|c|c|c|c|}
\hline \multicolumn{8}{|c|}{ TANK 241+A+107 } \\
\hline & & & & THEF & MOCOUPL & DATA & \\
\hline TREE I & DATE & THFiMO * & TREE SET A & TEMP C & TEMP F & $\bar{Y}=\mathrm{MAX}$ TEMP & COMMENTS \\
\hline 1 & $9 / 25 / 92$ & 6 & 1 & & 149,2 & $\mathbf{N}$ & \\
\hline 1 & $9 / 25 / 92$ & 7 & 1 & & 149.7 & $\mathbf{N}$ & \\
\hline 1 & - $125 / 92$ & $\mathbf{8}$ & 1 & & 150.E & $\mathbf{N}$ & \\
\hline 1. & $9 / 26 / 92$ & 皇 & 1 & & 151.5 & $\gamma$ & \\
\hline 1 & $0 / 28 / 92$ & 10 & 1 & & 150 & 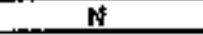 & \\
\hline 1 & $9 / 25 / 92$ & 71 & 1 & & 148.6 & $\mathbf{N}$ & \\
\hline 1 & $9 / 25 / 92$ & 12 & 1 & & $14 t .2$ & $\mathbf{N}$ & \\
\hline 1 & 9/2t/92 & 33 & 1 & & 134.4 & $\mathbf{N}$ & \\
\hline 1 & $\theta / 25 / 92$ & $\$ 4$ & 1 & & $\$ 25.7$ & $\vec{N}$ & $J 910120143-40$ \\
\hline 1 & $9 / 25 / 92$ & 15 & 1 & & 115.4 & $\overline{\mathrm{N}}$ & \\
\hline 1 & $9 / 25 / 32$ & 10 & 1 & & 103.9 & $\mathbf{N}$ & \\
\hline 1 & $9 \sqrt{25} 92$ & 17 & 1 & & 102.3 & $\mathbf{N}$ & \\
\hline 1 & $9 / 26 / 92$ & 18 & 1 & & 101.8 & $\mathbf{N}$ & \\
\hline 1 & $10 / 1 / 92$ & 1 & 1 & & 144.8 & $\mathbf{N}$ & \\
\hline 1 & 10/1/92 & 2 & 1 & & 146.5 & $\mathbf{N}$ & \\
\hline 1 & $10 / 1 / 92$ & $\mathbf{3}$ & 1 & & 148 & $\mathbf{H}$ & \\
\hline 1 & $10 / 1 / 92$ & 4 & 1 & & 148.8 & $\mathbf{N}$ & \\
\hline 1 & $10 / 1 / 92$ & 5 & 1 & & 149.2 & N & \\
\hline 1 & 10/1/02 & 8 & 1 & & 149,4 & $\mathbf{N}$ & \\
\hline 1 & $10 / 1 / 92$ & 7 & 1 & & 148.8 & $\mathbf{N}$ & \\
\hline 1 & $10 / 1 / 92$ & B & $t$ & & 160.9 & $N$ & \\
\hline 1 & $10 / 1 / 92$ & 9 & 1 & & 151.7 & $\mathbf{Y}$ & \\
\hline 3 & $1001 / 192$ & 10 & 1 & & 150.2 & $\mathbf{N}$ & \\
\hline 1 & tor1/92 & 11 & $t$ & & 146.7 & $\mathrm{~N}$ & \\
\hline 3 & $10 / 1 / 92$ & 12 & 1 & & 141.5 & $\mathbf{N}$ & \\
\hline $\mathbf{T}$ & $10 / 1 / 92$ & 13 & 1 & & 134.7 & $\mathbf{N}$ & \\
\hline 1 & $10 / 1 / 02$ & 14 & 1 & & 128 & $\mathbf{N}$ & $J 89062$ \\
\hline 1 & $10 / 1 / 92$ & 16 & 1 & & 116.8 & N & \\
\hline 1 & $10 / 1 / 92$ & 16 & 1 & & 104.2 & N & \\
\hline 1 & $10 / 1 / 92$ & 17 & 1 & & 102.6 & $N$ & \\
\hline 1 & $10,1 / 92$ & 18 & 1 & & 102,1 & $\mathbf{N}$ & \\
\hline 1 & $10 \times 13 / 92$ & 1 & 1 & & 145.3 & $\mathbf{N}$ & \\
\hline 1 & $10113 / 2$ & 2 & 1 & & 147.2 & $\overline{\mathbf{N}}$ & \\
\hline 1 & $10 / 13 / 92$ & 3 & $\frac{1}{1}$ & & 149.6 & $\mathbf{N}$ & \\
\hline 1 & $10 / 13 / 92$ & 4 & 1 & & 149.6 & $\mathbf{N}$ & \\
\hline 1 & $10 / 13 / 92$ & 5 & 1 & & 149.9 & $\mathrm{~N}$ & \\
\hline 1 & $1013 / 92$ & 6 & 1 & & 150.7 & $N$ & \\
\hline 1 & $1013 / 92$ & 7 & 1 & & 150.4 & N & \\
\hline 1 & 10/1 3/92 & 8 & 1 & & 151,7 & $\mathbf{N}$ & \\
\hline 1 & $10 / 13 / 92$ & 9 & 1 & & 152.4 & $Y$ & \\
\hline 1 & $10 / 13 / 92$ & 10 & 1 & & 151.1 & $\mathbf{N}$ & \\
\hline 1 & $10 / 18 / 92$ & $\pi$ & 1 & & 147.7 & $N$ & \\
\hline 1 & $10 / 13 / 92$ & 52 & 1 & & 142.3 & $N$ & J $09042,3320.42$ \\
\hline 1 & $10 / 13 / 92$ & 13 & 1 & & 138.7 & $\mathbf{N}$ & \\
\hline 1 & $10 / 13 / 92$ & 14 & 1 & & 127.1 & $\mathbf{N}$ & \\
\hline 1 & $10 / 13 / 92$ & 16 & 1 & & 116.6 & N & \\
\hline 1 & $10 / 13 / 92$ & 16 & 1 & & 104.7 & N & \\
\hline 1 & $10 / 13 / 92$ & 17 & 1 & & 103 & $N$ & \\
\hline 1 & $10 / 13 / 92$ & 10 & 1 & & 107.6 & $\mathbf{N}$ & \\
\hline 1 & $10 / 15 / 92$ & 1 & 1 & & 147.4 & N & \\
\hline 1 & $10 / 16 / 92$ & 2 & 1 & & 149.1 & $\mathbf{N}$ & \\
\hline 1 & $10 / 15 / 92$ & 3 & 5.2 & & 150.5 & $\mathbf{M}$ & \\
\hline 1 & $10 / 5 / 92$ & 4 & 1 & & 151.5 & $\mathbf{N}$ & \\
\hline 1 & $10 / 15 / 92$ & 5 & 1 & & 151.9 & $N$ & \\
\hline 1 & $10 / 15 / 92$ & 8 & 1. & & $162 . ?$ & $N$ & \\
\hline 1 & 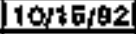 & 7 & 1 & & 152,3 & $\mathbf{N}$ & \\
\hline 1 & $10 / 5 / 92$ & B & $T$ & & 153,5 & $\mathrm{~N}$ & \\
\hline 1 & $10 / 75 / 92$ & 8 & 1 & & 154.4 & $N$ & \\
\hline 1 & $10 / 15 / 92$ & 10 & 1 & & 152.9 & $\mathbf{N}$ & $J$ 19042332042 \\
\hline 1 & 10/15/92 & 11 & 1 & & 149.5 & $N$ & \\
\hline 1 & $10 / 15 / 92$ & 12 & $\frac{1}{1}$ & & 144.2 & $\overline{\mathbf{N}}$ & \\
\hline 1 & $10 / 15 / 92$ & 13 & 7 & & 137.5 & $\mathbf{N}$ & \\
\hline 1 & $10116 / 02$ & 14 & 1 & & 128.7 & $\mathbf{N}$ & \\
\hline
\end{tabular}

Data obtained from WHC Surveillance Analysis Computer System ISACS), July 1, 1993. 


\begin{tabular}{|c|c|c|c|c|c|c|c|}
\hline \multicolumn{8}{|c|}{ TANK 241-A-101 } \\
\hline & & & & Tht: & AOCDUP & DATA & \\
\hline TREE & DATE & THEBMD & Thite SET : & TEMP $\bar{c}$ & TESAPF & $\bar{Y}=\mathrm{MAX}$ T this & conhteits's \\
\hline 1 & 10/75/22 & 16 & 7 & & $11 \mathrm{~B} .1$ & $\mathrm{~N}$ & \\
\hline$i$ & 10 ífó & 10 & 1 & & 105,9 & $\mathrm{~N}$ & \\
\hline 1 & $10 / 22 / 92$ & 1 & 1 & & 144.5 & $\mathbf{N}$ & \\
\hline 1 & $19 \sqrt{22192}$ & 2 & 1 & & 146.7 & $\mathbf{N}$ & \\
\hline \pm & $10 / 22 / 02$ & 3 & 1 & & $147 . \overline{7}$ & N & \\
\hline 1 & $10 / 22 / 92$ & 4 & 1 & & 149.5 & $\mathbf{N}$ & \\
\hline 1 & $10 / 22 / 92$ & 5 & 1 & & 149.9 & $\mathbf{N}$ & \\
\hline 1 & $10 / 22 / 92$ & $\mathbf{6}$ & 1 & & 149.1 & $\mathbf{N}$ & \\
\hline 1 & $10 / 22 / 92$ & 7 & 1 & & 149,4 & N & \\
\hline 1 & $10 / 22 / 82$ & $g$ & 1 & & 150.6 & $N$ & \\
\hline 1 & $10 / 22 / 92$ & 9 & 1 & & 153.4 & $Y$ & \\
\hline 1 & $10 / 2219$ & 10 & 1. & & 150 & $\mathbf{N}$ & \\
\hline 1 & $10 / 22 / 92$ & 11 & 1. & & 146.6 & $\mathbf{N}$ & \\
\hline 7 & $10 / 22 / 92$ & 12 & 1 & & $141 . \overline{5}$ & $\mathbf{N}$ & J910126143-48 \\
\hline 1. & $10 / 2 / 2 / 92$ & 13 & 1 & & 134.8 & $\mathbf{N}$ & \\
\hline 7 & 10122182 & 14 & 1 & & 126.1 & $\bar{N}$ & \\
\hline$t$ & $10 / 22 / 82$ & 15 & 1 & & 115.0 & $N$ & \\
\hline 1 & $10 / 22 / 92$ & 10 & 1 & & 103.4 & $\mathbf{N}$ & \\
\hline 1 & $10 / 28 / 82$ & 1 & 1 & & 143.9 & $\mathbf{N}$ & \\
\hline 1 & $10 / 29 / 92$ & 2 & $\$$ & & 145.6 & $\mathbf{N}$ & \\
\hline 1 & 10/29/92 & 3 & 1 & & $147+3$ & N & \\
\hline 1 & 10/29/92 & 4 & 1 & & 24, 1 & $\bar{N}$ & \\
\hline 1 & $10 / 29 / 92$ & 5 & 7 & & 146,4 & $\bar{N}$ & \\
\hline 1 & 10/29/92 & G & 1 & & 149,6 & $\mathbf{N}$ & \\
\hline 1 & 10/20/92 & 7 & 1 & & 148 & $\mathbf{N}$ & \\
\hline 1 & $10 / 29 / 92$ & 早 & 1 & & 160.2 & N & \\
\hline 1 & $10 / 29 / 42$ & 9 & 1 & & 151.1 & $\bar{Y}$ & \\
\hline 1 & $10 / 28 / 32$ & to & 1 & & 149.7 & $\mathbf{N}$ & \\
\hline 1 & $10 / 29 / 92$ & 71 & 1 & & 146.2 & $\mathbf{N}$ & \\
\hline 1 & $10 / 20 / 92$ & 12 & 1 & & 141,1 & $\mathrm{M}$ & J $914126143-48$ \\
\hline 9 & 10/20/a2 & 13 & 1 & & 134,6 & $N$ & \\
\hline 1 & $10 / 29 / 92$ & 14 & 1 & & 125,8 & $\mathrm{~N}$ & \\
\hline 1 & to/28/az & 15 & 1 & & 115,3 & $\mathbf{N}$ & \\
\hline 1 & $70 / 29 \times 32$ & 10 & 1 & & 102.8 & $\mathbf{N}$ & \\
\hline 1 & $10 / 2 \sin 2$ & 17 & 1 & & 101.1 & $\mathbf{N}$ & \\
\hline$t$ & $10 / 29 / 92$ & 19 & 1 & & 100.7 & $\mathbf{N}$ & \\
\hline 1 & 11/0/az & 7 & 1 & & 145,5 & $\mathbf{N}$ & \\
\hline 1 & $11 / 9 / \mathrm{Pz}$ & 2 & 1 & & 147.3 & $\mathbf{N}$ & \\
\hline 1 & D1/18 & 3 & 1 & & 148.9 & $\mathbf{N}$ & \\
\hline 1 & T10/6z & 4 & 1 & & 149.7 & $\mathbf{N}$ & \\
\hline 1 & 11/19292 & 5 & 1 & & 150 & $\mathbf{N}$ & \\
\hline$T$ & $11 / 9 / 92$ & 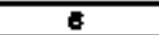 & 7 & & 150,2 & $\overline{\mathbf{N}}$ & \\
\hline 1 & $11 / 9 / 22$ & 7 & 1 & & 160.8 & $N$ & \\
\hline $\mathrm{j}$ & 11ब192 & B & 1 & & 151.8 & $\mathbf{N}$ & \\
\hline 1 & $\pm 1 / \theta / \theta 2$ & 8 & 1 & & 1520 & $\mathbf{Y}$ & \\
\hline 1 & $11 / 0 / 92$ & 10 & 1 & & 151,3 & $\mathbf{N}$ & \\
\hline 1 & $11 / 0 / 92$ & 11 & 1 & & $14 \mathrm{~B}$ & $\mathbf{N}$ & \\
\hline 1 & $21 / 8 / 92$ & 12 & 1 & & 142.6 & $\mathbf{N}$ & J \\
\hline 1 & I1/Bte2 & 13 & 1 & & 136.2 & $\mathbf{M}$ & \\
\hline 1 & $11 / 0 / 92$ & 14 & 1 & & 127.5 & $\mathbf{N}$ & \\
\hline 1 & $11 / d / 92$ & 15 & 1. & & 116.6 & $\mathbf{N}$ & \\
\hline 1 & $11 / 0 / 92$ & 16 & $1 x_{x}$ & & 103.6 & $\mathbf{N}$ & \\
\hline$i$ & $11 / 13 / \theta 2$ & 1 & 1 & & 145,9 & $\mathbf{N}$ & \\
\hline 1 & $11 / 3 / 92$ & 2 & 1 & & 147.3 & $\mathbf{N}$ & \\
\hline 1 & $11 / 13 / 82$ & 3 & 1 & & 148.6 & $\mathbf{N}$ & \\
\hline 1 & $11 / 13 / 2$ & 4 & 1 & & 149.8 & $\mathbf{N}$ & \\
\hline 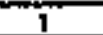 & $11 / 13 / 02$ & 5 & $T$ & & 150.1 & $\mathbf{N}$ & \\
\hline 1 & $11 / 13 / 92$ & 8 & 1 & & 150.2 & $\mathbf{N}$ & \\
\hline 1 & $11 / 13 / 92$ & 7 & 7 & & 160.7 & $\mathbf{N}$ & \\
\hline 1 & 11/13/92 & $B$ & 1 & & 151. & $\mathbf{N}$ & \\
\hline 1 & $11 / 13 / 02$ & 8 & 1 & & 152.7 & $\mathbf{Y}$ & \\
\hline 1 & $11 / 13 / 92$ & 10 & 1 & & 151.5 & $\mathbf{N}$ & \\
\hline 1 & $11 / 23 / 82$ & 11 & 1 & & $14 \overline{8}$ & $\mathbf{N}$ & \\
\hline
\end{tabular}

Data obtained from WHC Survelnance Anzhysis Computer System (SACS], July 1, 1993. 


\begin{tabular}{|c|c|c|c|c|c|c|c|}
\hline \multicolumn{8}{|c|}{ TANK 241-A101 } \\
\hline & & & & THE & HOCOUPL & DATA & \\
\hline THEE \# & DATE & THE:RAO A & TRA: SET R & TEMPC & TEMP F & $Y=$ MAX TEMP & CDMMENTS \\
\hline 1 & ijriaraz & 72 & -1 & & 142.8 & $\bar{N}$ & \\
\hline$T$ & $11 / 3 / 92$ & 13 & 1 & & 130.3 & $\mathbf{N}$ & $J 990523512-20$ \\
\hline 1 & $11 / 13 / 92$ & 14 & $T$ & & 127.6 & $\mathbf{N}$ & \\
\hline 1 & $11 / 13 / 92$ & 15 & 1 & & $110+5$ & N & \\
\hline 1 & $11 / 13 / 92$ & 10 & 9 & & 103,2 & $\mathbf{N}$ & \\
\hline 1 & {$[\overline{17 / 13 / 92}$} & 37 & $\frac{1}{1}$ & & 101.5 & $\mathbf{N}$ & \\
\hline 1 & $12 \sqrt{13 / 92}$ & 18 & 1 & & $101+1$ & $\mathbf{N}$ & \\
\hline$T$ & $1 \$ 19 / 92$ & 1 & $\frac{1}{1}$ & & 945.4 & $\mathbf{N}$ & \\
\hline$\overline{1}$ & $11 / 19 / 92$ & 2 & $T$ & & 147.3 & $\mathbf{N}$ & \\
\hline 1 & $11 \sqrt{19} 92$ & 3 & 7 & & 148.7 & $\bar{N}$ & \\
\hline 1 & $11119 / 92$ & 4 & 1 & & 149.7 & $\mathbf{N}$ & \\
\hline 1 & $11 / 19 / 92$ & 5 & 1 & & 150 & $\mathbf{N}$ & \\
\hline$\overline{1}$ & $11 / 19 / 92$ & 9 & 1 & & 150,2 & $N$ & \\
\hline$\overline{1}$ & 11/19/82 & 7 & $\mathbf{1}$ & & 150.5 & $\mathbf{N}$ & \\
\hline 1 & $\$ 1 / 19 / 2$ & 8 & 1 & & 151.9 & $\mathbf{N}$ & \\
\hline 1 & $71 / 19 / 82$ & 9 & i & & 162.6 & $\dot{\mathbf{Y}}$ & \\
\hline 1 & $11 / 19 / 92$ & 10 & 1 & & 151,4 & $\mathbf{N}$ & \\
\hline 1 & $11 / 19 / 92$ & 11 & 1 & & 148 & $\mathbf{N}$ & \\
\hline$\overline{1}$ & $11 / 19 / 92$ & 12 & $\mathbf{1}$ & & 142.8 & $\mathbf{N}$ & \\
\hline 1 & $11 / 1$ s & 13 & 1 & & 136.4 & $\mathbf{N}$ & $J B 905235+2-20 \overline{T C F B}, 5,7$ BAD \\
\hline 1 & $111 / 19 / 92$ & 14 & 1 & & 127.7 & 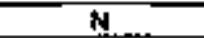 & \\
\hline $\mathbf{1}$ & $11 / 19 / 92$ & 19 & 1 & & 119,4 & $\mathbf{N}$ & \\
\hline 1 & $11 / 19 / 92$ & 17 & 1 & & 101 & $\mathbf{N}$ & \\
\hline 1 & 11119/92] & 18 & $\mathbf{1}$ & & 100.8 & $\mathbf{N}$ & \\
\hline 1 & $11 / 26 / 92$ & 1 & 1 & & 147,3 & $\mathbf{N}$ & \\
\hline$\overline{3}$ & $11 / 26 / 92$ & $\overline{2}$ & 1 & & 149.8 & $\mathbf{N}$ & \\
\hline 1 & $11 / 26192$ & 3 & 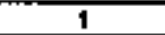 & & 150.5 & $\mathbf{N}$ & \\
\hline ] & $11 / 26 / 32$ & 4 & $\mathbf{1}$ & & 151.6 & $\mathbf{N}$ & \\
\hline 1 & $71 / 28+192$ & 5 & i & & 151.9 & $\mathbf{N}$ & \\
\hline 1 & $11 / 26 / 02$ & 6 & 1 & & 152 & N & \\
\hline$t$ & $11 / 28 / 62$ & 7 & $\mathbf{1}$ & & 152.3 & $\mathbf{N}$ & \\
\hline 1 & $11 / 20 / 92$ & E & 1 & & 153.7 & $\mathbf{N}$ & \\
\hline 1 & $11 / 20 / 02$ & 9 & 1 & & 154.8 & $\bar{Y}$ & \\
\hline 7 & $11 / 20192$ & 10 & 1 & & 163.3 & $\mathbf{N}$ & \\
\hline 1 & $11 / 26 / 92$ & 11 & 1 & & 150 & $\mathbf{N}$ & JE90S2 \\
\hline 1 & $11 / 28 \mathrm{~d} 02$ & 12 & 1 & & 144.8 & $N$ & \\
\hline 1 & $11 / 26 / 92$ & 13 & 1 & & 139.1 & $\mathbf{N}$ & \\
\hline 1 & 11120 ed & 14 & 1 & & 120.2 & $N$ & \\
\hline 1 & $111 / 20 / 92$ & 16 & 1 & & 117.7 & $N$ & \\
\hline 1 & $11 / 20 / 92$ & 18 & 1 & & 103,6 & $\mathbf{N}$ & \\
\hline 1 & $11 / 20 / 92$ & 17 & 1 & & 101,8 & $\mathbf{N}$ & \\
\hline 7 & $11 / 26 / 92$ & 18 & 1 & & 101.4 & $N$ & \\
\hline 1 & \begin{tabular}{|l|l}
$7 / 3 / 92$ \\
\end{tabular} & 1 & 1 & & 147.8 & $N$ & \\
\hline 1 & $12 / 3 / 92$ & 2 & 1 & & 140.1 & $\mathbf{N}$ & \\
\hline 1 & $12 / 3 / 92$ & 4 & 1 & & 151.6 & $\mathbf{N}$ & \\
\hline 1 & $12 / 3 / 92$ & 6 & 1 & & 152.3 & $N$ & \\
\hline 1 & $12 / 3 / 92$ & $B$ & 1 & & 153.5 & $N$ & \\
\hline 1 & $72 / 3 / 92$ & 9 & 1 & & 154.4 & $y$ & \\
\hline 1 & $1 / 2 / 3 f 2$ & 10 & 1 & & 153.1 & $\mathbf{N}$ & \\
\hline 1 & $12 / 3 / 92$ & 19 & 1 & & 149.8 & $\mathbf{N}$ & \\
\hline 1 & $12 / 3 / 92$ & 12 & 1 & & 144,8 & $N$ & \\
\hline$\$$ & 1240192 & 13 & $\ldots$ & & $13 \frac{1}{1.1}$ & $N$ & \\
\hline 1 & $12 / 3 / 02$ & 14 & 1 & & $12 \theta, 8$ & $\mathbf{N}$ & $3 \pi-042$ \\
\hline 1 & $12 / 3 / 92$ & 15 & 1 & & 198.8 & $\mathbf{N}$ & \\
\hline 1 & $12 / 3 / 92$ & 16 & 1 & & 102.2 & $\mathrm{~N}$ & \\
\hline 1 & $12 / 3 / 92$ & 17 & 1 & & 100.3 & $\mathbf{N}$ & \\
\hline 1 & $12 / 3 / 92$ & 19 & 1 & & $100+1$ & $\mathbf{N}$ & \\
\hline 1 & $12 / 10 / 82$ & 1 & 1 & & 147.3 & N & \\
\hline 1 & $12 / 10 / 92$ & 2 & 1 & & 148.8 & N & \\
\hline 1 & $12 / 10 / 92$ & 3 & 1 & & 160.8 & $N$ & \\
\hline 1 & $12 / 10 / 92$ & 4. & 1 & & 130,5 & $\mathbf{N}$ & \\
\hline 1 & $12 / 10 \mathrm{de2}$ & 5 & 1 & & 152 & $\mathbf{N}$ & \\
\hline 1 & 12л0/9? & t & 1 & & 152.1 & $\mathrm{~N}$ & \\
\hline
\end{tabular}

Date obtained from WHC Surveillance Anelysis Computer System \{SACS\}, July 1, 1993. 


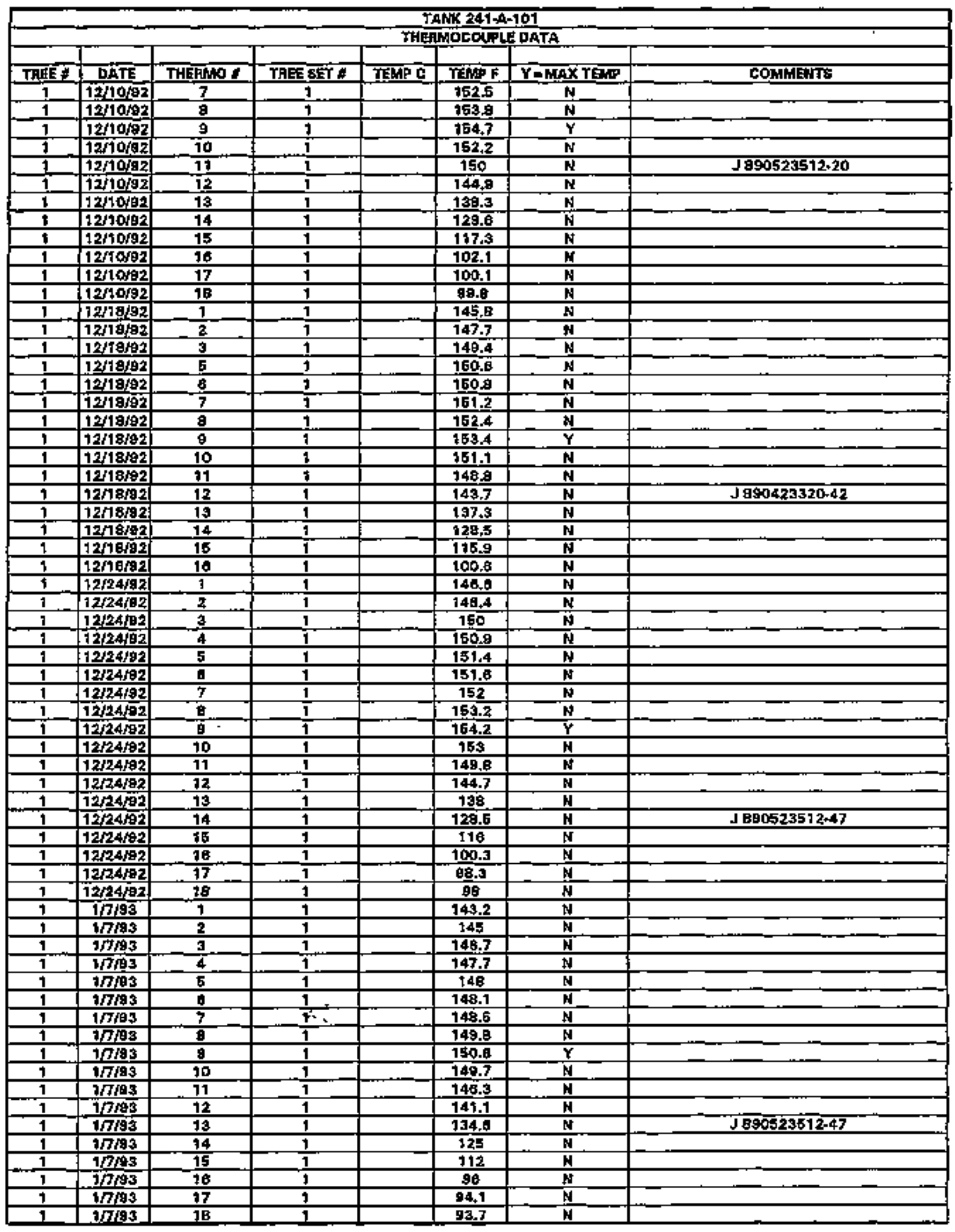

Data obteined from WHC Surveillance Analysis Computer System (SACS), July $7,1993$. 


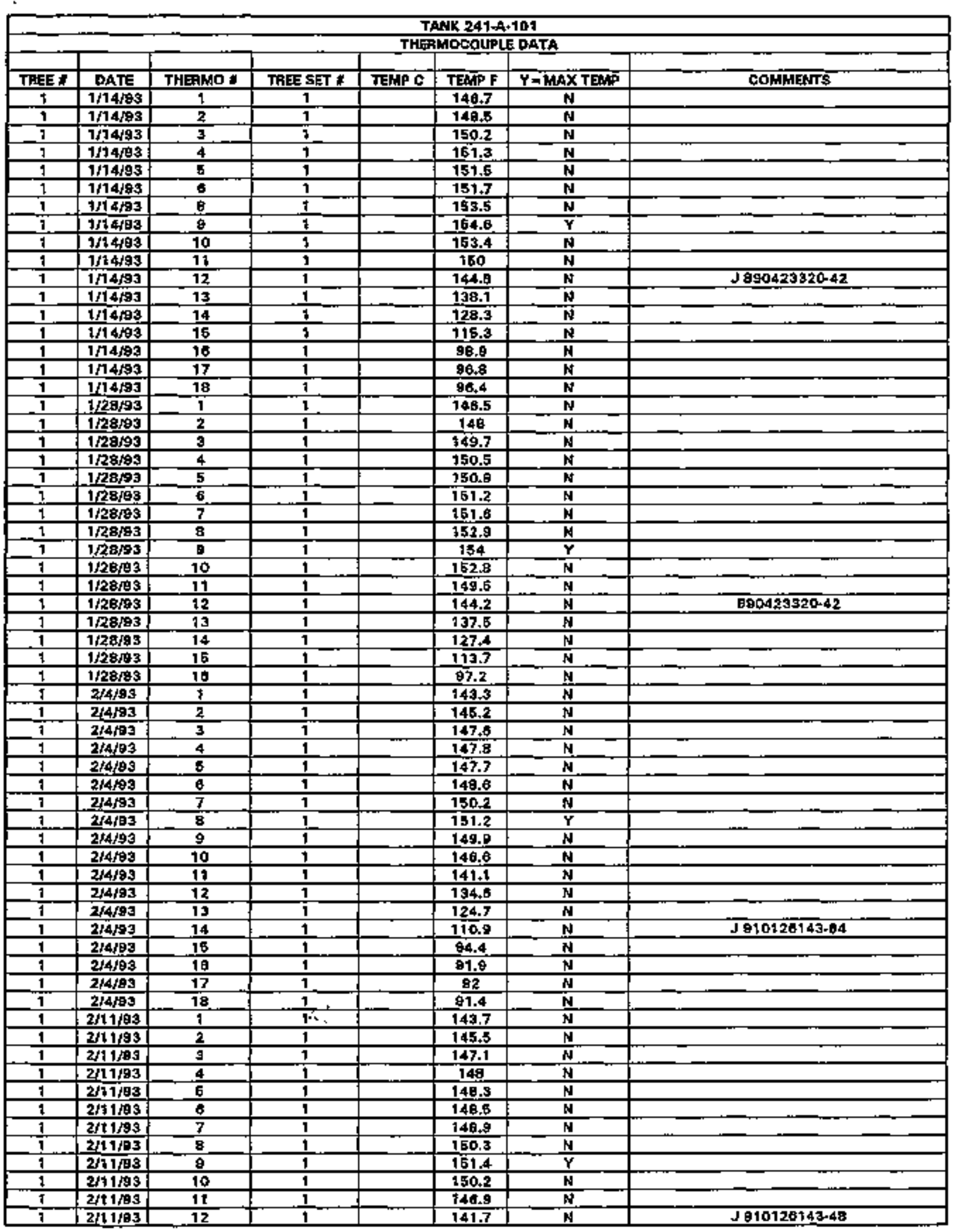

Dats obtained from WhC Surveillance Aratysis Computer System ISACSI, July 1, 1993. 


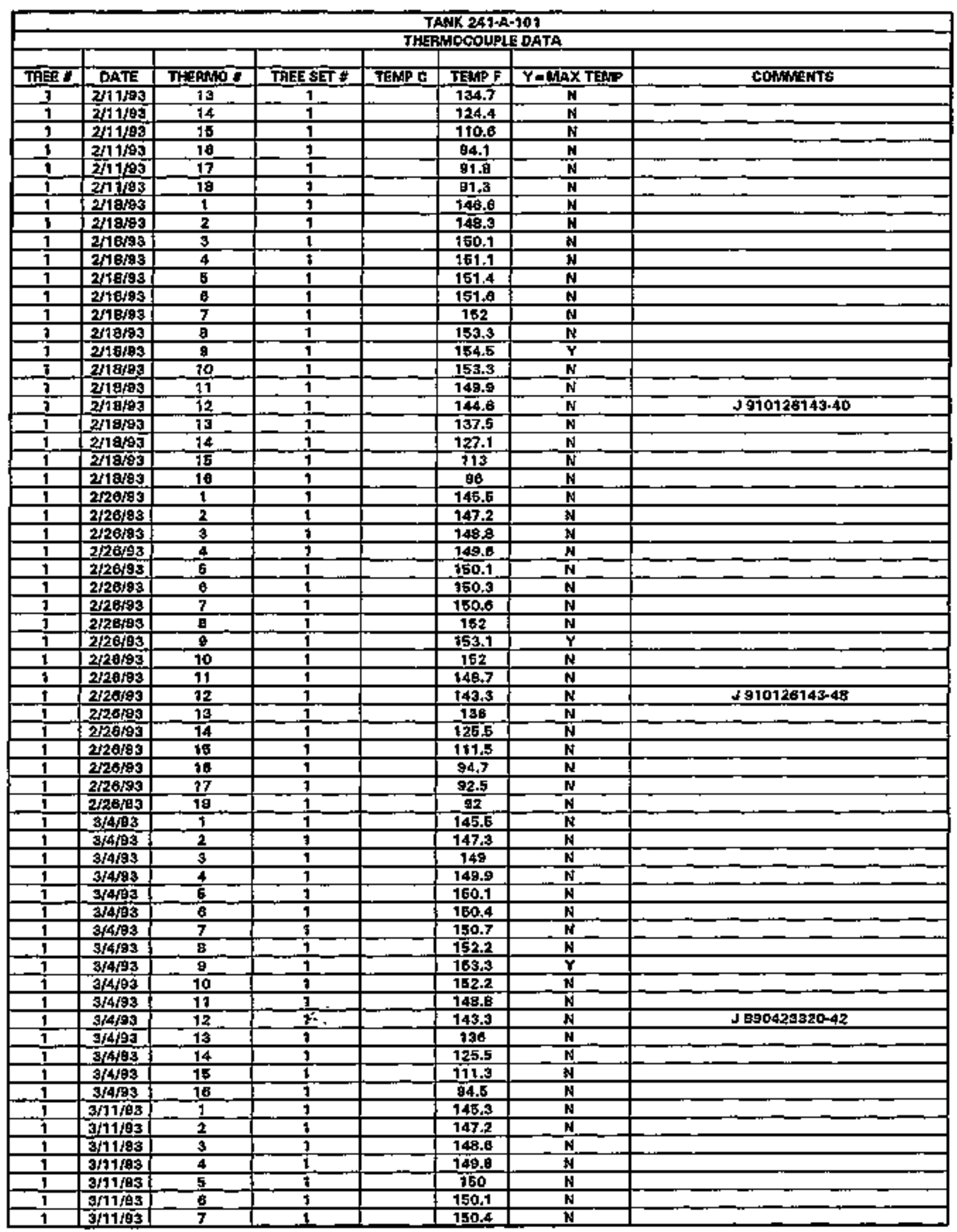

Data obtained from WHC Surveillance Analysis Computer Svstem (SACS), wuy 1, 1993. 


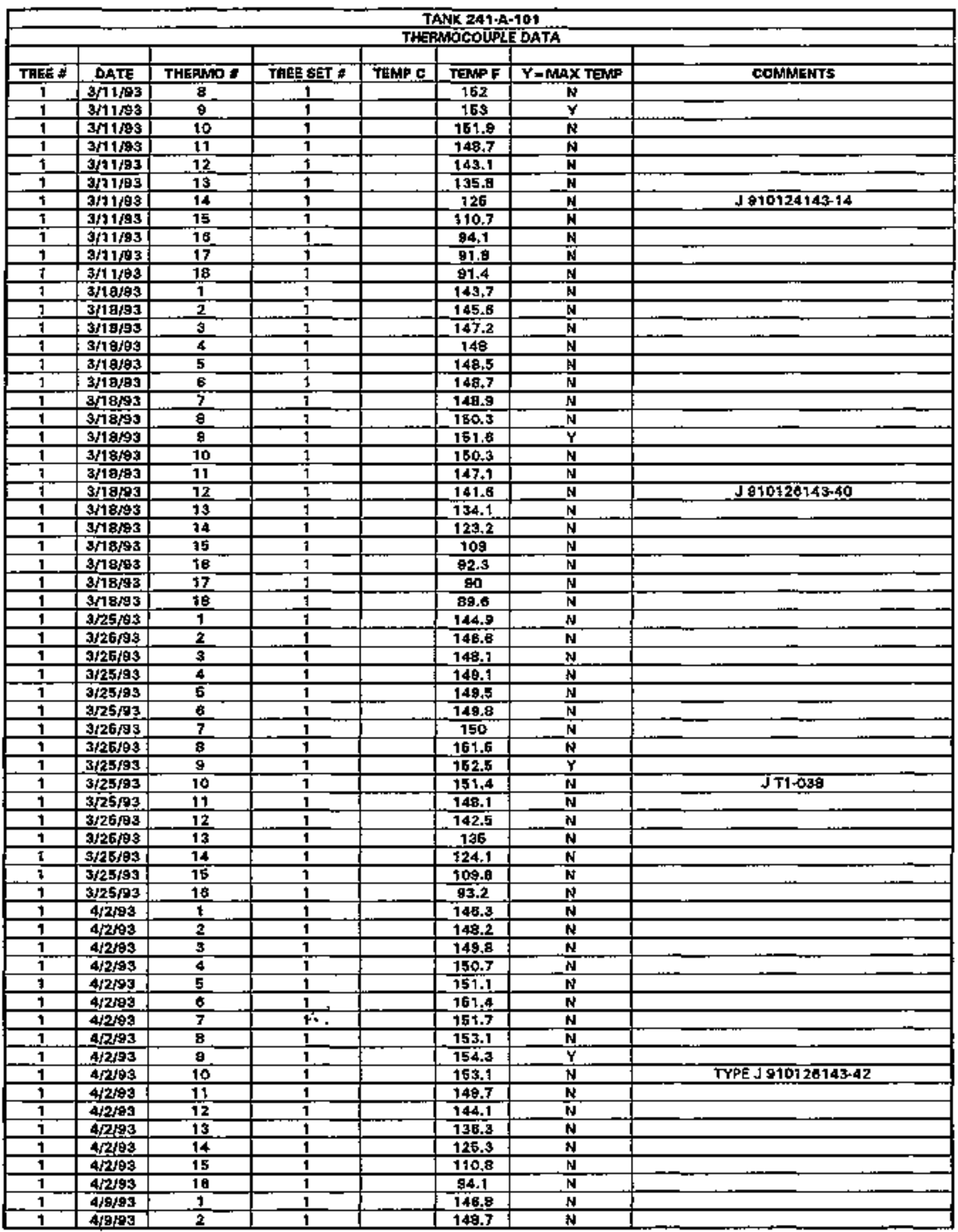

Data obtained from WHC Survellance Analysis Computer System (SACSI, July 1, 1993. 


\begin{tabular}{|c|c|c|c|c|c|c|c|}
\hline \multicolumn{8}{|c|}{ TANK 241+A+101 } \\
\hline & & & & & & & \\
\hline TAEE : & DATE & THEAMO & TAEE SET $A$ & TEMAP C & TEMP F & $Y=$ HAX TEMP & CÖLWENTS \\
\hline i & $4 / 949$ & $\mathbf{a}$ & 1 & & 130.2 & $\mathbf{N}$ & \\
\hline 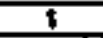 & $4 / 9 / 93$ & 4 & 1 & & 151.2 & $N$ & \\
\hline 1 & $4 / 9 \sqrt{93}$ & 5 & 1 & & 151.8 & $\overline{\mathbf{H}}$ & \\
\hline 1 & $4 / 9 \sqrt{03}$ & 8 & $t$ & & 152 & $\mathbf{N}$ & \\
\hline 1 & 489,93 & $\overline{7}$ & $\overline{1}$ & & $152+1$ & $\mathbf{N}$ & \\
\hline 1 & 4 4ta3 & E & 1 & & 153.7 & $\mathbf{N}$ & \\
\hline 1 & A/9193 & 9 & 1 & & 154.8 & $Y$ & \\
\hline 1 & $4 \sqrt{2} / 93$ & 10 & 1 & & 153.6 & $\mathbf{N}$ & J910726143.50 \\
\hline 1 & $4 / 9 / 93$ & 11 & 1 & & 160.5 & N & \\
\hline 1 & $4 / 9 / 93$ & 12 & 1 & & 144.6 & N & \\
\hline 1 & $4 / 9 / 93$ & 13 & 1 & & 130.6 & $\mathbf{N}$ & \\
\hline 1 & $4 / 9 / 93$ & 14 & 1 & & 125.5 & $\mathbf{H}$ & \\
\hline 1 & $4 / 9 / 93$ & 15 & 1 & & 110.8 & N & \\
\hline 1 & $4 / 9 / 93$ & 16 & 1 & & 94.4 & N & \\
\hline$i$ & $4 / 15 / 93$ & 1 & 1. & & 148.2 & $\mathbf{N}$ & \\
\hline 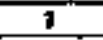 & $4 / 15 / 93$ & 2 & 1 & & 148 & $\mathbf{N}$ & \\
\hline 1 & 4/5/193 & 3 & 1. & & 140.6 & $\mathbf{N}$ & \\
\hline 1 & $4 / 15 / 93$ & 4 & 1 & & 150.6 & $\mathbf{H}$ & \\
\hline 1 & $4 / 18 / 93$ & 5 & 1 & & 151.1 & $\mathbf{N}$ & \\
\hline 1 & $4 / 16 / 03$ & 8 & 1 & & 150.3 & $\mathbf{N}$ & \\
\hline 1 & $4 / 1$ is 3 & 7 & 1 & & 761.6 & $\mathbf{N}$ & \\
\hline 1 & $4 / 15 / 93$ & a & 1 & & 162.9 & $\mathbf{N}$ & \\
\hline 1 & $4 / 15 / 93$ & 9 & 1 & & 154.1 & $\bar{Y}$ & \\
\hline 1 & $4 / 15 / 93$ & 10 & 1 & & 152.8 & $\mathbf{N}$ & $J 910126143464$ \\
\hline 1 & $4 / 15 / 23$ & 11 & 1 & & 948.4 & $\mathbf{N}$ & \\
\hline 1 & $4 / 15 / 93$ & $\sqrt{2}$ & 1 & & 143.6 & $\boldsymbol{N}$ & \\
\hline 1 & $4 / 76 / 93$ & 13 & 1 & & 135.8 & N & \\
\hline 1 & $4718 / 83$ & 14 & 1 & & 124,8 & $\mathbf{N}$ & \\
\hline 1 & $4 / 15 / 93$ & 15 & 1 & & 110 & N & \\
\hline 1 & $4 / 1 \overline{5 / 3}$ & 16 & 1 & & 93.6 & $\mathbf{N}$ & \\
\hline 1 & $4 / 2393$ & 1 & 1 & & $746 . \overline{2}$ & 신. & \\
\hline 1 & $4 / 29 / 93$ & 2 & 1 & & 148,1 & $N$ & \\
\hline 1 & $4 / 23 / 93$ & 3 & 1 & & $14 g_{+} \theta$ & $\mathbf{N}$ & \\
\hline 1 & $4 / 23 / 93$ & 4 & 1 & & $150+6$ & N & \\
\hline 1 & $4 / 25 / 95$ & 5 & 1 & & 151 & $N$ & \\
\hline 1 & $4 / 23 / 183$ & $\overline{8}$ & $\overline{1}$ & & $151 . \overline{3}$ & $\mathbf{N}$ & \\
\hline 1 & $A / 23 \sin ^{3}$ & 7 & 1 & & $151+6$ & $\mathbf{N}$ & \\
\hline 1 & $4 / 23 / 93$ & a & 1 & & 153 & $\mathbf{N}$ & \\
\hline 1 & $4 / 23 / 93$ & 9 & 1 & & 154,5 & $\mathbf{Y}$ & \\
\hline 1 & $4 / 23 / 93$ & 10 & 1 & & 1528 & $N$ & $\mathbf{J}$ \\
\hline 1 & $4 / 23 / 93$ & 11 & 1 & & 749.4 & $N$ & \\
\hline 1 & $4 / 29 \mathrm{~B}^{3}$ & 12 & 1 & & 143,6 & $N$ & \\
\hline 1 & A/23103 & 13 & 1 & & 135,6 & $\mathbf{R}$ & \\
\hline 1 & $4 / 23 / 63$ & 14 & 1 & & 124.5 & $\mathbf{N}$ & \\
\hline 1 & $4 / 23 / 93$ & 15 & 1 & & $110^{-}$ & $\mathbf{H}$ & \\
\hline $\bar{T}$ & $4 / 23 / 93$ & 16 & 1 & & 942 & $\mathbf{N}$ & \\
\hline 1 & $4120 / 93$ & 1 & 1 & & 140.5 & $\mathbf{N}$ & \\
\hline 1 & 4/29/03 & 2 & 1 & & 148.3 & 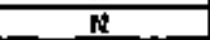 & \\
\hline 1 & $4 / 28 / 93$ & 3 & 1 & & 160.9 & $N$ & \\
\hline 1 & $4 / 29 / 95$ & 4 & 1 & & 150.9 & $\mathbf{N}$ & \\
\hline$\overline{1}$ & $4 / 29 / 83$ & 5 & 1 & & 151.4 & $\bar{M}$ & \\
\hline$\overline{1}$ & $4 / 29 / 93$ & 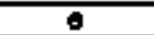 & F. & & $151 . \overline{7}$ & $\mathbf{N}$ & \\
\hline 1 & $4 / 28 / 93$ & 7 & 1 & & 151.5 & $N$ & \\
\hline 1 & $4 / 29 \sqrt{3} 3$ & $\mathrm{a}$ & 1 & & 153.3 & $\mathrm{~N}$ & \\
\hline 1 & $4 / 20 / 03$ & 早 & 1 & & 1544 & $\mathbf{Y}$ & \\
\hline 1 & $4 / 28 / 93$ & 10 & 1 & & 153,2 & $N$ & \\
\hline 1 & $4 / 29 / 3^{3}$ & 11 & 1 & & $148+9$ & $\mathbf{N}$ & \\
\hline 1 & 412918 & 12 & 1 & & 143.9 & $\mathbf{N}$ & J91012814,3+32 \\
\hline 1 & $4 / 29 / 93$ & 13 & 1 & & $\$ \$ 90$ & $N$ & \\
\hline 1 & $4 / 29 / 93$ & 14 & 1 & & 124.0 & $\mathbf{N}$ & \\
\hline 1 & $4 / 29 / 93$ & 46 & 1 & & 910.9 & W & \\
\hline 1 & $4 / 2 \theta / 93$ & 76 & 1 & & 55.1 & $\mathbf{N}$ & \\
\hline 1 & $5 / 8$ ras & 1 & 1 & & 147 & $\mathbf{N}$ & \\
\hline
\end{tabular}

Data obtained from WHC Surveillance Anshysis Computer System (SACS), July 1, 1 \$93. 
WHC-SD-WM-ER-308, Rev. O

\begin{tabular}{|c|c|c|c|c|c|c|c|}
\hline \multicolumn{8}{|c|}{$\frac{\text { TAMH 241+A+101 }}{\text { THERMOCOLPE DATA }}$} \\
\hline & & & & & & & \\
\hline TAF卧 8 & DATE & TH白hO : & THE王 SET \# & TEMP C & TEMPF F & $Y=$ MAX TEMP & COMWERTS \\
\hline 1 & 6/但3 & 2 & 1 & & 149 & N & \\
\hline 1 & $5 / 903$ & 3 & 1 & & 147 & $\mathbf{N}$ & \\
\hline 1 & $5 / 0 / 93$ & 4 & 1 & & 152 & $\mathbf{N}$ & \\
\hline 1 & $5 / 6 / 93$ & 5 & 1. & & 152 & $\mathbf{N}$ & \\
\hline 1 & 5, & 6 & 1 & & 152 & $\mathbf{N}$ & \\
\hline 1 & $6 / 9 / 93$ & 7 & 1 & & 152 & $\mathbf{N}$ & \\
\hline 1 & $5 / 5 / 93$ & E & 1 & & 154 & $\mathbf{N}$ & \\
\hline 1 & 5/B/93 & 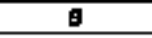 & 1 & & 155 & $\mathbf{Y}$ & \\
\hline 1 & $5 / 6193$ & 10 & 1 & & 154 & $\mathbf{N}$ & \\
\hline 1 & $5 / 6 / 63$ & 11 & $\overline{1}$ & & 160 & $\mathbf{N}$ & \\
\hline 1 & $5 / 6 / 93$ & 12 & 1 & & 144 & $N$ & \\
\hline 1 & 5/6/93 & 13 & 1 & & 139 & $N$ & \\
\hline 1 & F/6/03 & 14 & 1 & & 125 & $N$ & J s10128143.64 \\
\hline 1 & $5 / 8103$ & 15 & 1 & & 171 & $\mathbf{N}$ & \\
\hline 1 & 5003 & 10 & 1 & & 86 & $\mathbf{N}$ & \\
\hline 1 & $6 / 0 / 93$ & 17 & 1 & & 84 & $\mathbf{N}$ & \\
\hline 1 & $5 / 6 / 93$ & 18 & 1 & & 83 & $N$ & \\
\hline 1 & $5 / 16 / 93$ & j & 1 & & 147.2 & $\mathbf{N}$ & \\
\hline 1 & $5 / 1 \mathrm{~B} / 93$ & 2 & 1 & & $14 \mathrm{~B}, \mathrm{~B}$ & $\mathbf{N}$ & \\
\hline 1 & 5ก5/93 & 3 & 1 & & 147 & $N$ & \\
\hline 1 & $5 / 15 / 93$ & 4 & 1 & & 151.4 & $N$ & \\
\hline 1 & $5 / 15 / 93$ & 5 & 1 & & 151.8 & $\mathbf{N}$ & \\
\hline 1 & $6 / 55 \mathrm{gs}$ & 章 & 1 & & 152 & $\mathbf{N}$ & \\
\hline 1 & $5 / 15 / 93$ & 7 & 1 & & 162.1 & $N$ & \\
\hline 1 & $8 / 15 / 93$ & 8 & 1 & & 183.7 & $\mathbf{N}$ & \\
\hline 1 & $5 / 15 / 03$ & 9 & 1 & & 154.7 & $Y$ & \\
\hline 7 & $5 / 15 / 93$ & 10 & 1 & & 153.5 & $\mathbf{N}$ & \\
\hline 1 & 5/15/es & 11 & 1 & & 150 & $\mathbf{N}$ & \\
\hline 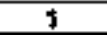 & 5/5/93 & 12 & 1 & & 144.2 & $\mathbf{N}$ & \\
\hline$y$ & $5 / 15 / 93$ & 13 & 1 & & 138.8 & $\mathbf{N}$ & \\
\hline 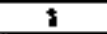 & $5 / 15 / 93$ & 14 & 1 & & 124.8 & $\mathbf{N}$ & J $090523512-20$ \\
\hline$I$ & $5 / 15 / 03$ & 15 & 1 & & 110.8 & $\mathbf{N}$ & \\
\hline 1 & $5 / 16 / 93$ & 16 & 1 & & 96.8 & $\mathbf{N}$ & \\
\hline 1 & $6 / 15 / 93$ & 17 & 1 & & 83.5 & $\mathbf{N}$ & \\
\hline 1 & $5 / 15 / 93$ & 18 & 1 & & 93.1 & $\mathbf{N}$ & \\
\hline 1 & $5 / 23 / 93$ & 1 & 1 & & 140.2 & $\mathbf{N}$ & \\
\hline$I$ & $5 / 23 / 93$ & 2 & 1. & & 148 & $\mathbf{N}$ & \\
\hline 1 & $5 / 23 / 93$ & 3 & 1 & & 146.2 & $\mathbf{N}$ & \\
\hline 1 & $5 / 23 / 93$ & 4 & 1 & & 150.4 & $\mathbf{N}$ & \\
\hline 1 & $5 / 23 / 93$ & 5 & 1 & & 150.8 & $\mathbf{N}$ & \\
\hline 1 & $5 / 23 / 43$ & 6 & 1 & & 151.1 & $\mathbf{N}$ & \\
\hline 1 & $5 / 23 / 93$ & 7 & 1 & & 151.4 & $\mathbf{N}$ & \\
\hline 1 & $5 / 23 / 93$ & 8 & 1 & & 152.8 & $\mathbf{N}$ & \\
\hline 1 & $5 / 23 / 93$ & 9 & 1 & & 163.0 & $Y$ & \\
\hline 1 & $5 / 23 / 93$ & 10 & 1 & & 152.0 & $\mathbf{N}$ & \\
\hline 1 & $5 / 29 / 83$ & 11 & 1 & & 148.1 & $\mathbf{N}$ & \\
\hline 1 & $5 / 23 / 973$ & 12 & 1 & & 143.2 & $\mathbf{N}$ & JT1.022 \\
\hline 1 & $5 \sqrt{23} / 43$ & 13 & 1 & & 135.2 & I & \\
\hline 1 & $5 / 23 / 43$ & 14 & 1 & & 124,3 & N & \\
\hline 1 & $5 / 23 / 93$ & 15 & 1 & & 110.3 & $\mathbf{N}$ & \\
\hline 1 & $5 / 23 / 93$ & 18 & 1 & & O8 & $\mathbf{N}$ & \\
\hline 1 & $5 / 23 / 83$ & 17 & i. & & 94.2 & $\mathbf{M}$ & \\
\hline 1 & $5 / 29 / 93$ & to & 1 & & 93,7 & $\mathbf{N}$ & \\
\hline 1 & $5 / 27 / 93$ & 1 & 1 & & 146.8 & $\mathbf{N}$ & \\
\hline 1 & $5 / 27 / 93$ & 2 & 1 & & 148.8 & $\mathbf{N}$ & \\
\hline$i$ & Б/27/93. & 3 & 1. & & 148.7 & $\mathbf{N}$ & \\
\hline 1 & $5 / 27 / 03$ & 4 & 1 & & 180.9 & $\mathbf{N}$ & \\
\hline 1 & $6 / 27 / 93$ & 5 & 1 & & 151.5 & $N$ & \\
\hline 1 & $5 / 27 / 93$ & 8 & 1 & & 151.7 & $\mathbf{N}$ & \\
\hline 1 & $5 / 27 / 93$ & 7 & 1 & & $7 \overline{51.9}$ & $N$ & \\
\hline 1 & $5 / 27 / 83$ & 8 & 1 & & 163,3 & $N$ & \\
\hline 1 & $5 / 27193$ & 9 & 1 & & 154,3 & $\mathbf{Y}$ & \\
\hline 9 & $5 / 27 / 93$ & 10 & 1 & & 153 & $\mathbf{N}$ & \\
\hline
\end{tabular}

Data obtained from WhiC Surveillance Analysis Computer System (SACS), July 3, 1993. 


\begin{tabular}{|c|c|c|c|c|c|c|c|}
\hline \multicolumn{8}{|c|}{ TANK 241-A+101 } \\
\hline & & & & 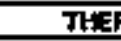 & SOCOUPL & DATA & \\
\hline TAEE & DATE & THEAMHO & THEE SET & JEHP $\mathrm{C}$ & TENP F & $Y=$ MAX TEMP & COFkEN's \\
\hline 1 & $6 / 27 / 93$ & 11 & 1 & & 140.8 & $\mathrm{~N}$ & \\
\hline 1 & $6 / 27 / 83$ & 12 & 1 & & 143.7 & 柿 & TYPE J $010120143-48$ \\
\hline$T$ & $5 / 27 / 93$ & 13 & 1 & & $135+3$ & $\mathbf{N}$ & \\
\hline 1 & 6/27/93 & 14 & 1 & & 124.7 & $N$ & \\
\hline 1 & $5 / 27 / 93$ & 15 & 1 & & 111 & N & \\
\hline 1 & $5 / 27 / 93$ & 18 & 3 & & 90,3 & $\mathbf{N}$ & \\
\hline 1 & E/a/93. & 1 & 1 & & 140.2 & No & \\
\hline 2 & का/34a & 2. & $\overline{1}$ & & 140,1 & $\mathbf{N}$ & \\
\hline$\overline{1}$ & 自/ & 3 & 1 & & 140,2 & $\mathbf{w}$ & \\
\hline$t$ & a/siges & 4 & 7 & & 150,4 & $\mathbf{F}$ & \\
\hline 1 & 6/3iso & 5. & 1 & & $150 . \overline{1}$ & $\mathbf{N}$ & \\
\hline 1 & $6 / 3 \sqrt{93}$ & $\overline{6}$ & $\overline{1}$ & & $15 t .2$ & $\mathbf{M}$ & \\
\hline 1 & $0 / 3 / 93$ & 7 & 1 & & 161.4 & $\mathbf{N}$ & \\
\hline 1 & $6 / 3103$ & 9 & 1 & & $152 \overline{8}$ & $\mathbf{M}$ & \\
\hline 1 & a/s/93 & 8 & 1 & & 168. & $\bar{Y}$ & \\
\hline 1 & $6 / 3 / 93$ & 10 & 1 & & 152.5 & N & \\
\hline 1 & $0 / 3 / 93$ & 11 & 1 & & 148.0 & $\mathbf{N}$ & \\
\hline 1 & 6/3/93 & 12 & 1 & & 143,1 & $\mathbf{N}$ & \\
\hline 1 & 6/9/93 & 13 & 1 & & +36.1 & $\mathbf{N}$ & \\
\hline 1 & 6/3193 & 14 & 1 & & 324,4 & $\mathbf{N}$ & $\begin{array}{c}J \text { BSOS235 12-20 ADES TAKEN FFOA E. } \\
\text { AAEA UIO. LGVE, REPORT }\end{array}$ \\
\hline 7 & 6/9/93 & 15 & 1 & & 111 & N & \\
\hline 1 & $6 / 9 / 93$ & 16 & 7 & & 98.8 & it & \\
\hline j & $6 \sqrt{3 / 92}$ & 17 & 1 & & $94, \overline{9}$ & $\sqrt{4}$ & \\
\hline 1 & $0 \longdiv { 3 / 9 3 }$ & 18 & 1 & & 94.5 & $\mathrm{~N}$ & \\
\hline 1 & $6 / 10193$ & 1 & 1 & & 140.2 & $N$ & \\
\hline 1 & 6/0/3a & 2 & 1 & & 148 & $\mathbf{N}$ & \\
\hline 1 & 6/10/93 & 3 & 1 & & 140.1 & if & \\
\hline 1 & 이무요 & 4 & 1 & & 150.4 & $\mathbf{N}$ & \\
\hline 1 & $6 / 10 / 93$ & 5 & 1 & & 150.8 & $\bar{N}$ & \\
\hline 1 & $6 / 10 / 93$ & 4 & 1 & & 151.2 & $\mathbf{N}$ & \\
\hline 1 & $6 / 10 / 83$ & 7 & 1 & & 151,3 & $\mathbf{N}$ & \\
\hline 1 & 6/10/93 & $\underline{\mathrm{g}}$ & 1 & & 152,7 & $\mathbf{N}$ & \\
\hline 1 & e/poras & 9 & 1 & & $\uparrow 63.7$ & $r$ & \\
\hline 1 & doforas & 10 & 1 & & 152.3 & $\mathbf{N}$ & \\
\hline 7 & D/1003 & 11 & 1 & & $14 \mathrm{~B}, \mathrm{~g}$ & N & \\
\hline 1 & 6/10) & 12 & 1 & & 142.0 & $\mathbf{N}$ & \\
\hline 1 & O/1 of & 13 & 1 & & 134.8 & NN & \\
\hline 1 & ojtoas & 14 & 1 & & 123.9 & $\mathbf{N}$ & J980126143.14 \\
\hline 1 & No/93 & 15 & 1 & & 110,7 & $\mathbf{H}$ & \\
\hline 1 & D/10/e3 & 10 & 1 & & $80 . \overline{5}$ & 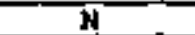 & \\
\hline T & $8 / 10193$ & 17 & 1 & & 94.9 & $\mathbf{N}$ & \\
\hline 2 & 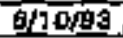 & 19 & 1 & & 94.5 & $\mathbf{N}$ & \\
\hline 1 & $0 / 97 / 03$ & $\mathbf{I}$ & 1 & & 147,1 & $\mathbf{N}$ & \\
\hline$t$ & $4+7 / 93$ & 2 & 1 & & 148,0 & N & \\
\hline 1 & T/7/83 & $\underline{3}$ & 1 & & 148.6 & $\underline{N}$ & \\
\hline$i$ & $6 / 7203$ & 4 & 1 & & $150, B$ & $\mathbf{N}$ & \\
\hline$i$ & e/7/70; & 5 & 1 & & 151.4 & $\mathbf{N}$ & \\
\hline 1 & $0 / 1789$ & 8 & 1 & & 151,5 & $\underline{N}$ & \\
\hline 1 & e/17/93 & 7 & 1 & & 1517 & $N$ & \\
\hline 1 & $617 / 93$ & 8 & 1 & & 153.2 & $\mathrm{~N}$ & \\
\hline 1 & 614703 & 9 & $1=$ & & 154.2 & $\underline{Y}$ & \\
\hline 1 & W17/93 & 10 & 1 & & 1628 & $N$ & \\
\hline 1 & Q/47/03 & 11 & $\overline{1}$ & & 148.2 & $\mathbf{N}$ & \\
\hline 1 & Bบ179a & 12 & $\mathbf{1}$ & & 143,3 & $\bar{M}$ & JTt-042 \\
\hline 7 & $0 / 7 / 93$ & 13 & 1 & & 135.2 & $N$ & \\
\hline 1 & a/17/93 & 14 & 1 & & 124.8 & $\mathbf{N}$ & \\
\hline$t$ & $6 / 17 / 93$ & 15 & 1 & & 171.0 & $N$ & \\
\hline 1 & 6/17903 & 16 & 1 & & 97.9 & $\mathbf{M}$ & \\
\hline$\overline{1}$ & arit/93 & 17 & 5 & & 96 & $\mathrm{~N}$ & \\
\hline 1 & a/17/93 & 18 & 1 & & 95.5 & $N$ & \\
\hline 1 & 6/20/93 & 1 & $i$ & & 144.8 & $\mathbf{M}$ & \\
\hline 1 & e/20/63 & $\mathbf{2}$ & 1 & & $14 \mathrm{~B} .6$ & $\mathbf{N}$ & \\
\hline
\end{tabular}

Data obtained from WHC Surveillance Analysis Computer System (SACS), July 1, 1993. 


\begin{tabular}{|c|c|c|c|c|c|c|c|}
\hline \multirow{2}{*}{\multicolumn{8}{|c|}{$\begin{array}{l}\text { TANK 241.A-101 } \\
\text { TTEZFTHOCOUPAE DATA }\end{array}$}} \\
\hline & & & & & & & \\
\hline TAEE & DATE & THERWO H & TAEE SET * & TEMP C & TEkP F & $Y=$ MAX TEMP & CONWEKTS \\
\hline 1 & $0 / 26 / 99$ & 3 & 1 & & $\$ 44.7$ & N & \\
\hline$\frac{1}{1}$ & $8 / 26 / 93$ & 4 & 1 & & 748.9 & $\mathbf{N}$ & \\
\hline 1 & $6 \longdiv { 2 0 / 6 3 }$ & 5 & 5 & & 149.4 & $\mathbf{N}$ & \\
\hline 1 & $6 / 26 / 93$ & 6 & 1 & & 14,8 & $\mathbf{N}$ & \\
\hline 1 & $0 / 26 / 93$ & .7 & $i$ & & 149.8 & $\mathbf{M}$ & \\
\hline 1 & B/26/93 & 8 & 1 & & 151.3 & $\mathbf{N}$ & \\
\hline 3 & $0 / 28 / 93$ & 9 & $i$ & & 152.1 & $\bar{Y}$ & \\
\hline 1 & 6/26/e3 & 10 & 1 & & $\overline{150.8}$ & $N$ & \\
\hline 1 & $0 / 20 / 93$ & 13 & 1 & & 147.4 & $\mathbf{N}$ & \\
\hline 1 & $0 / 20 / 93$ & 12 & 1 & & 141.9 & $\mathbf{N}$ & TYPE J $910126143-50$ \\
\hline 1 & 8/26/93 & 13 & 1 & & 133.8 & $\mathbf{N}$ & \\
\hline 1 & $8 / 20,03$ & 14 & 1 & & 123.2 & N & \\
\hline 1 & a/20/93 & 15 & 1 & & 910.8 & N & \\
\hline 1 & N20/93 & 16 & 1 & & $97+9$ & N & \\
\hline 1 & $7 / 2 / 93$ & 1 & 1 & & 149.1 & $\mathbf{N}$ & \\
\hline 1 & $7 / 2 / 93$ & $\overline{\mathbf{z}}$ & 1 & & 151 & N & \\
\hline 1 & $7 / 2 / \mathrm{pas}^{3}$ & 3 & 1 & & 149.1 & N & \\
\hline 1 & $7 / 2 / 93$ & 4 & 1 & & 153,4 & $N$ & \\
\hline 1 & $7 / 2 / 99$ & 5 & 1 & & 153.8 & $\mathbf{N}$ & \\
\hline 1 & $7 / 2 / 93$ & $\theta$ & 1 & & 154 & $N$ & \\
\hline 1 & 7121993 & 7 & $i$ & & 184.3 & $\mathbf{N}$ & \\
\hline 1 & $7 / 293$ & B & 1 & & 155.7 & $\mathbf{N}$ & \\
\hline 1 & $7 / 2 / 83$ & $\mathbf{g}$ & 1 & & 154.6 & $\bar{Y}$ & \\
\hline 1 & $7 / 2 / 93$ & 10 & 1 & & 155.3 & $\mathbf{n}$ & \\
\hline 1 & $7 / 2 \mathrm{~m} 3$ & \pm 1 & 1 & & 151.7 & N & \\
\hline 1 & $7 / 2 / 9$ & $\sqrt{2}$ & 1 & & 145.6 & N & \\
\hline 1 & $7 / 2 \sqrt{93}$ & $\mathbf{1 3}$ & 1 & & 137,6 & $\mathbf{N}$ & \\
\hline 1 & $7 / 2 / 93$ & 74 & 1 & & 124.8 & N & J $1010126143-42$ \\
\hline 1 & $7 / 2 / 93$ & $\sqrt{15}$ & 1 & & 114.1 & $\mathbf{A}$ & \\
\hline 1 & $7 / 2 / 43$ & 10 & 1 & & 100.6 & $\pi$ & \\
\hline 1 & $7 / 2 / 93$ & 17 & 1 & & 98.9 & $\mathbf{N}$ & \\
\hline 1 & $7 / 2 / 83$ & 18 & 1 & & 98.7 & $\mathrm{~N}$ & \\
\hline 1 & 718193 & 1 & 1 & & 145.6 & $N$ & \\
\hline 1 & 7 716/93 & 2 & 1 & & $1 \overline{47,8}$ & $N$ & \\
\hline 1 & $7 / 18 / 93$ & 3 & 1 & & 145.5 & $\mathbf{N}$ & \\
\hline 1 & 7/18/99 & 4 & 1 & & 149.8 & $N$ & \\
\hline 1 & $7 / 18 / 93$ & $\overline{6}$ & 1 & & 150.1 & $\mathbf{N}$ & \\
\hline 1 & 7/18/93 & $\overline{6}$ & 1 & & 150.6 & $\bar{N}$ & \\
\hline 1 & 7fl18 & 7 & $i$ & & 150.2 & $\bar{N}$ & \\
\hline 1 & $7 / 16 / 93$ & $B$ & 1 & & 132.2 & $\mathbf{N}$ & \\
\hline 1 & $7 / 18 / 93$ & 9 & 1 & & 153 & $\bar{Y}$ & \\
\hline 1 & 7/18/999 & 10 & 1 & & $151 . \mathrm{B}$ & N & \\
\hline 5 & $7 / 18 / 93$ & 11 & 1 & & 148 & H & \\
\hline 1 & 7 718/93 & 12 & 1 & & 142.4 & $\mathbf{H}$ & J910120143-50 \\
\hline 1 & 711603 & 13 & 1 & & 134.4 & $\mathbf{N}$ & \\
\hline 1 & $7 / 16 \mathrm{~kg}$ & 14 & 1 & & 124 & $\mathbf{N}$ & \\
\hline 1 & $7 / 18 / 93$ & 15 & 1 & & 111.8 & $\mathbf{N}$ & \\
\hline 1 & $7 / 18 / 93$ & 16 & 1 & & 98.4 & $\mathrm{~N}$ & \\
\hline 5 & Tr1Bres & 17 & 1 & & 87.7 & $\mathrm{~N}$ & \\
\hline 1 & $7 / 11$ ed 3 & 19 & 1 & & 87.5 & $\mathbf{N}$ & \\
\hline 1 & $7 / 23 / 99$ & 7 & 1 & & 148.2 & $\mathbf{N}$ & \\
\hline$\overline{1}$ & $7 / 23 / 93$ & 2 & $7^{2}+$ & & 148.1 & $\mathbf{N}$ & \\
\hline 1 & $7 \sqrt{2393}$ & 3 & 1 & & 140.2 & $\mathbf{N}$ & \\
\hline 1 & $7 / 2393$ & 4 & 1 & & 130.4 & $N$ & \\
\hline 1 & $7 / 23 / 83$ & 5 & 1 & & 150.9 & $N$ & \\
\hline 1 & $7 / 23 / 83$ & 8 & 1 & & 151.7 & $N$ & \\
\hline 1 & $7 / 23 / 93$ & 7 & 1 & & 151,3 & N & \\
\hline 1 & $7 / 23 / 93$ & 量 & 1 & & 152.7 & $N$ & \\
\hline 1 & $7 / 23 / 93$ & $\theta$ & 1 & & 153.7 & $\bar{\gamma}$ & \\
\hline 1 & $7 / 2,3 / 93$ & 10 & 1 & & 762.3 & $M$ & \\
\hline 1 & $7 / 23 / 93$ & 11 & 1 & & 148.0 & $\bar{N}$ & \\
\hline 1 & $7 / 23 / 93$ & 12 & 1 & & 942.7 & $\mathbf{N}$ & J010126143.39 \\
\hline 1 & $7 / 23 / 93$ & 13 & 1 & & 135 & $N$ & \\
\hline
\end{tabular}

Data obtained from WhC Suryeillance Anblysis Computer System (SACS), July 1, 1993. 


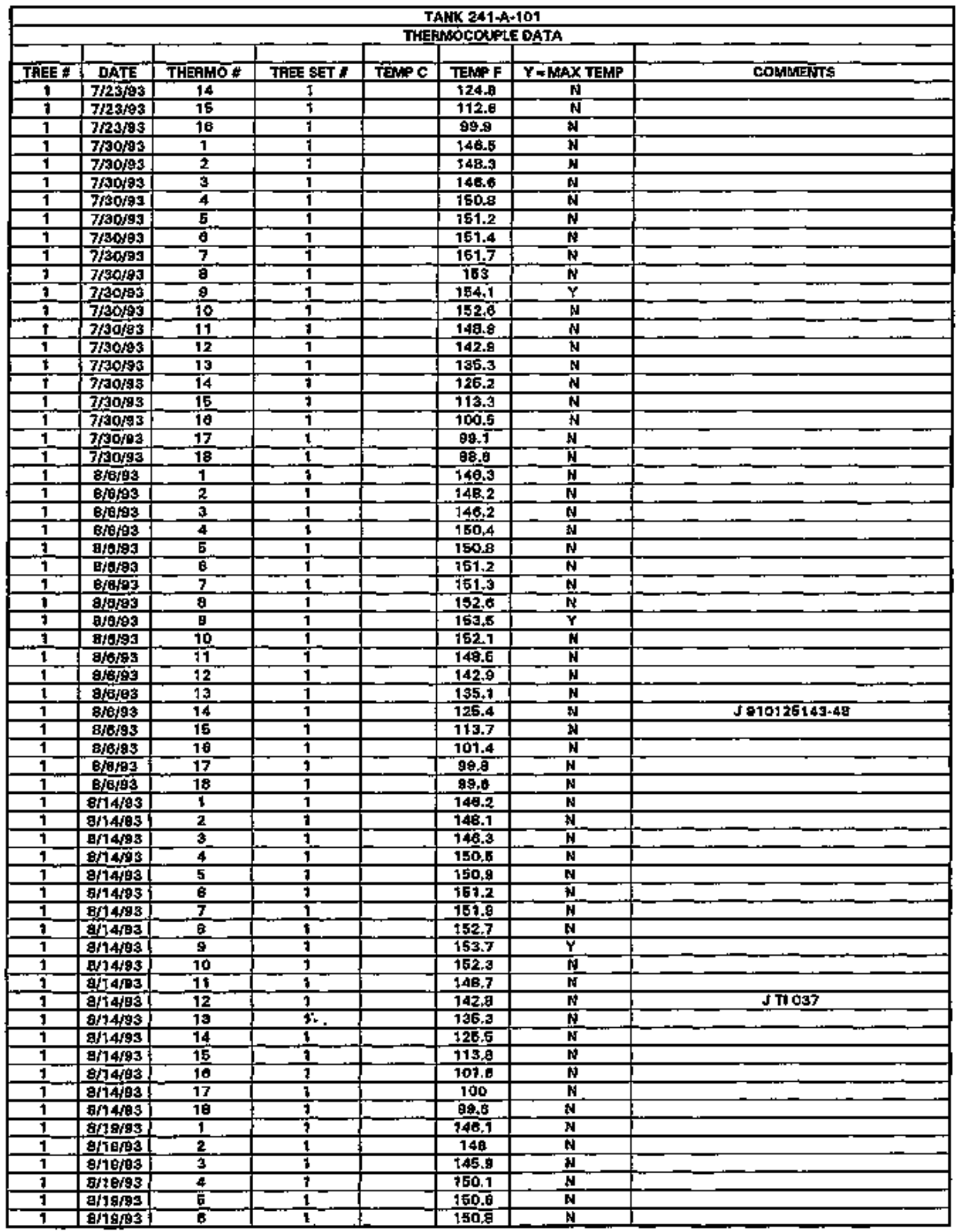

Data obtatned from WhiC Surveillance Anglysis Computer System [SACS], July 1, 1993. 
WHC-SD-WM-ER-308, Rev. 0

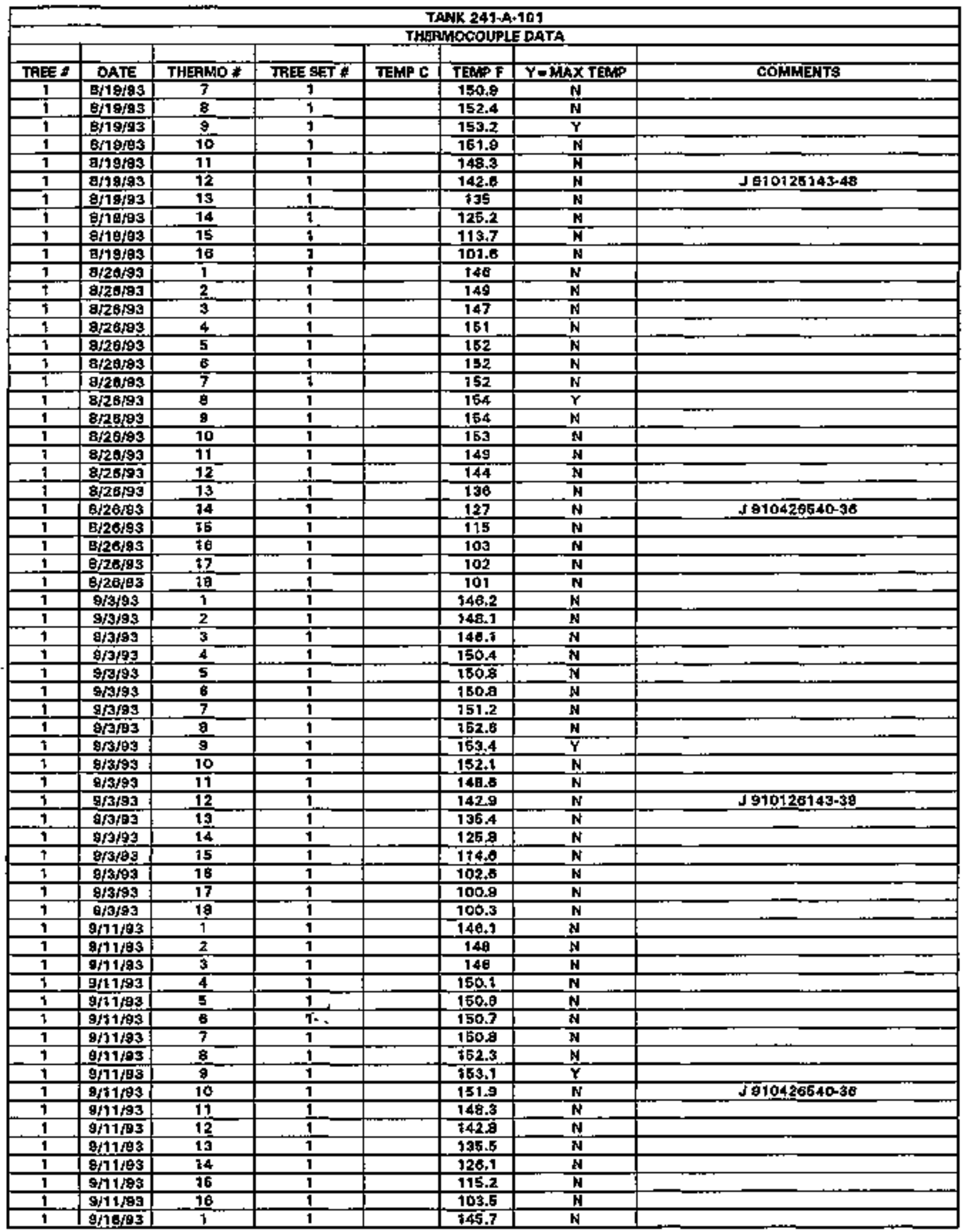

Date obtatned from WhiC Surveillance Analysis Camputer System (SACSI, July 1, 1993. 


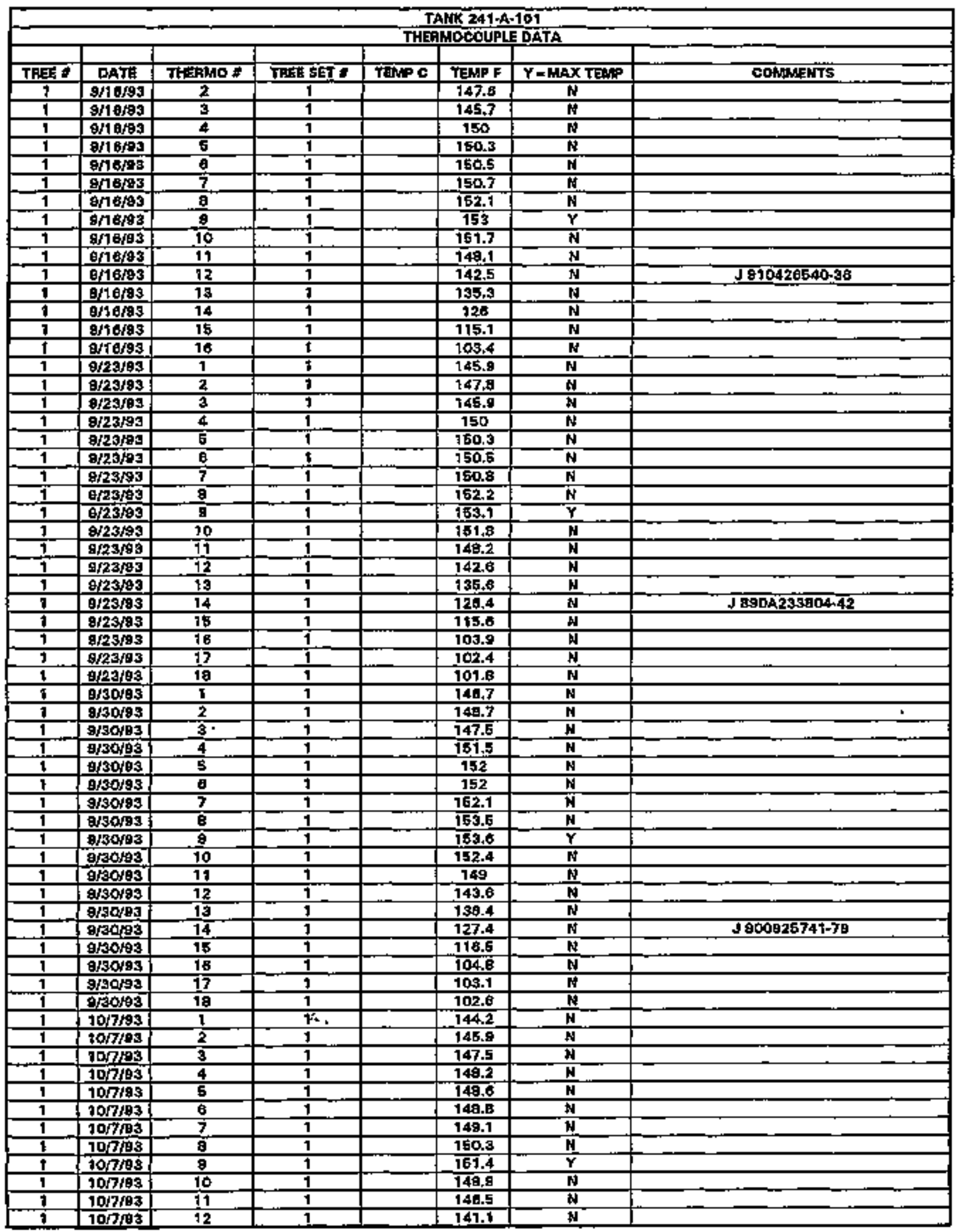

Data obtained from WHC Strveillance Analysls Computer System ISACS\}, July 1, 1993. 
WHC-SD-WM-ER-308, Rev. 0

\begin{tabular}{|c|c|c|c|c|c|c|c|}
\hline \multicolumn{8}{|c|}{ IANK 241-A.101 } \\
\hline & & & & $\mid \mathrm{In} x$ & AOLOWI & DATA & \\
\hline TiEE & DATtE & THEFMD & TREEEST & TENPC & TEMPF F & $Y=\operatorname{MAX}$ TEMP & COWMENTS \\
\hline 1. & $10 / 7 / 93$ & 13 & 1 & & 134.1 & $\mathbf{N}$ & \\
\hline 1 & $107 / 93$ & $\$ 4$ & 1 & & 125 & $\mathbf{N}$ & J 8904233200-42 \\
\hline 1 & $10 / 7 / 93$ & 15 & 1 & & 114.3 & $\mathbf{N}$ & \\
\hline 1 & $10 / 7 / 93$ & 10 & 1 & & 102.3 & $\mathbf{N}$ & \\
\hline 1 & 10079 & 17 & 1 & & 1008 & $\mathbf{N}$ & \\
\hline 1 & $10 / 7 / 93$ & 19 & 1 & & 100,9 & $\mathbf{N}$ & \\
\hline 1 & $10 / 21 / 83$ & 1 & 1 & & 147 & N & \\
\hline 1 & $10 / 21 / 99$ & 2 & 1 & & 149 & $\mathbf{N}$ & \\
\hline 1 & $10 / 2, / 9 a$ & 3 & 1 & & 150 & $\mathbf{N}$ & \\
\hline 1 & $10 / 21 / 43$ & $\overline{4}$ & 1 & & 151 & $\mathbf{N}$ & \\
\hline 1 & $10 / 2 \div / 93$ & 5 & 1 & & 152 & $\mathbf{N}$ & \\
\hline 1 & $10 / 21 / 93$ & 6 & 1 & & 152 & $\mathbf{N}$ & \\
\hline 1 & $10 \sqrt{2}, \sqrt{9} 3$ & 7 & 1 & & 152 & $\mathbf{N}$ & \\
\hline 1 & $10 / 27 / 9 a$ & 8 & 1 & & 163 & $\mathbf{N}$ & \\
\hline 1 & $10 / 21 / 83$ & 9 & 1 & & 154 & $\mathbf{Y}$ & \\
\hline 1 & $10 / 27 / 93$ & 10 & 1 & & 153 & $\mathbf{N}$ & \\
\hline 1 & $10 / 2$ 小 3 & 11 & 1 & & 148 & $\mathbf{N}$ & \\
\hline 1 & $10 / 27 / 93$ & 12 & 1 & & 144 & $\mathbf{N}$ & J010128143.60 \\
\hline 1 & $10 / 24 / 93$ & 13 & 1 & & 137 & $\mathbf{N}$ & \\
\hline 1 & $10 / 21 / 83$ & 14 & 1 & & 128 & $\mathbf{N}$ & \\
\hline 1 & 10/21/93 & 15 & 1 & & 177 & $\mathbf{N}$ & \\
\hline 1 & $10 / 21, / 93$ & 16 & 1 & & 105 & $\mathbf{N}$ & \\
\hline 1 & $10 / 21 / 03$ & 17 & 1 & & 103 & $\mathbf{N}$ & \\
\hline 1 & $10 / 21 / 93$ & 19 & 1. & & 103 & $\mathbf{N}$ & \\
\hline 1 & $10 / 26 / 8.3$ & 2 & 1 & & 143.8 & $\mathbf{N}$ & \\
\hline 1 & 10/26/39 & 2 & 1 & & 145,5 & $\mathbf{N}$ & \\
\hline 1 & $10 / 28 / 93$ & 3 & 1. & & 147.3 & $\mathrm{~N}$ & \\
\hline 1 & $10 / 26 / 13$ & 4 & 1 & & 148 & $\mathbf{N}$ & \\
\hline 1 & $10 / 26 / 9$ & 5 & 1 & & 146.3 & $\mathbf{N}$ & \\
\hline 1 & 10N20103 & 6 & 1 & & 148,3 & $\mathbf{N}$ & \\
\hline 1 & $10 \mathrm{H} 26 \mathrm{das}$ & 7 & 1 & & 148.7 & $\mathbf{N}$ & \\
\hline 1 & 10/26jas & 8 & 1 & & $150+1$ & $\mathbf{N}$ & \\
\hline 1 & $10 / 26+93$ & 9 & 1 & & 151 & $\mathbf{Y}$ & \\
\hline 1 & 10/28/e3 & 10 & 1 & & $1 \mathrm{Ag}, 8$ & $\mathbf{N}$ & \\
\hline 1 & torzeres & 11 & 1 & & 146.3 & $\mathbf{N}$ & \\
\hline $\mathbf{I}$ & 10/20jas & 12 & 1 & & 140.8 & $\mathbf{N}$ & $\sqrt{890423320-40}$ \\
\hline 5 & $10 / 20 / 93$ & 13 & 1 & & 134,1 & $\mathbf{N}$ & \\
\hline 1 & 60126193 & 14 & 1 & & $125+1$ & $\mathbf{N}$ & \\
\hline 1 & to/2e/e3 & 16 & 1 & & 114.2 & $\mathbf{N}$ & \\
\hline 1 & $70 / 20103$ & 18 & 1 & & 101.8 & $\mathbf{N}$ & \\
\hline 1 & $\$ 0 / 28 / 93$ & 1 & 1 & & 140.4 & $\mathbf{N}$ & \\
\hline 1 & $10 / 28 \mathrm{~d} 93$ & 2 & 1 & & 146.3 & $\mathbf{N}$ & \\
\hline$y$ & 10/28/93 & 3 & 1 & & 149.8 & $\mathbf{N}$ & \\
\hline 1 & $10 / 28 / 93$ & 4 & 1 & & 150.8 & $\mathbf{N}$ & \\
\hline 1 & $10 / 2 \mathrm{es}$ & $\mathbf{5}$ & 1 & & $151+1$ & $\mathrm{~N}$ & \\
\hline 1 & $10 / 28 / 8$ & 6 & 1 & & 151,4 & $\mathbf{N}$ & \\
\hline 1 & $10 \mathrm{~d} 28 / 93$ & 7 & 1 & & $151+7$ & $\mathbf{N}$ & \\
\hline 1 & $10 \mathrm{~d} 28 \mathrm{~d} / \mathrm{s}$ & 8 & 1 & & 153 & $\mathbf{N}$ & \\
\hline 1 & $10 / 2 B / 93$ & $\dot{\theta}$ & 1 & & 153.0 & $\gamma$ & \\
\hline 1 & $10 / 28 \mathrm{~s}$ & 10 & 1 & & 151,0 & $\mathbf{N}$ & \\
\hline 1 & $10 / 28 / 9$ & 11 & 1 & & $149+1$ & $\mathbf{N}$ & \\
\hline 1 & 10/26/93 & 12 & 7.. & & 343.0 & $\mathbf{N}$ & \\
\hline 1 & $10 / 28 / 93$ & 13 & 1 & & 138.7 & $\mathbf{N}$ & \\
\hline 1 & $10 / 28 / 9$ & 14 & 1 & & +27.7 & N. & J 由аOA23320-42 \\
\hline 1 & $10 / 28 / 93$ & 15 & 1 & & 116.5 & $\mathbf{N}$ & \\
\hline 1 & $10 / 28 / 83$ & 10 & 1 & & 104 & $\mathbf{N}$ & \\
\hline 1 & 10/28/93: & 17 & 1 & & 102.3 & N & \\
\hline 1 & $10 / 28 / 93$ & 18 & 1 & & 102 & $\mathbf{N}$ & \\
\hline 1 & $11019 \overline{3}$ & 1 & 1 & & 143.9 & N & \\
\hline 1 & $11 / 5 / 93$ & 2 & 1 & & 145.7 & $\mathbf{N}$ & \\
\hline 1 & $11 / 5 / 93$ & 3 & 1 & & 147.1 & $\mathbf{N}$ & \\
\hline 1 & $11 / 5 / 83$ & 4 & 1 & & +48 & $\mathbf{N}$ & \\
\hline 1 & 11 店93 & 5 & 1 & & 148,4 & $\mathbf{N}$ & \\
\hline
\end{tabular}

Data obtoined from WHC Surveillance Analysis Computer System (SACS), July 1. 1993. 


\begin{tabular}{|c|c|c|c|c|c|c|c|}
\hline \multicolumn{8}{|c|}{ TAPK $249-1-101$} \\
\hline & & & & THEF & ACDUP: & DATA & \\
\hline TREE " & DATE & THFAMO \# & TREE SET * & TEMP C & TEMP F & $Y=$ MAX TEHFP & COHANENTS \\
\hline 1 & 11/5/as & 6 & 1 & & $148.0^{\circ}$ & $\mathrm{N}=$ & \\
\hline 1 & $11 / 6 / 93$ & 7 & 1 & & 149.5 & $\mathbf{N}$ & \\
\hline 1 & $11 / 5 / 93$ & $\bar{B}$ & 7 & & 150 & $\mathbf{N}$ & \\
\hline 1 & $11 / \mathrm{B}$ & 8 & 1 & & 150.9 & $Y$ & \\
\hline $\bar{t}$ & $11+5 / 93$ & 10 & 1 & & $\{49.6$ & $\bar{N}$ & \\
\hline 1 & $11 / 6 / 93$ & 11 & 1 & & 146.2 & $\mathbf{M}$ & \\
\hline $\bar{t}$ & $11 / 5 / 93$ & 12 & 1 & & 141.2 & N & $5890423320-42$ \\
\hline 1 & $11 / 5 / 93$ & 13 & 1 & & 734.2 & 复 & \\
\hline 1 & $11 / 5 / 93$ & 14 & 1 & & 125.2 & A & \\
\hline 1 & 11500 & 75 & $\pi$ & & 114,3 & $\mathbf{H}$ & \\
\hline 1 & t1/5/ga & 10 & 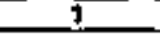 & & 101.6 & Ii & \\
\hline 1 & $11 / 5 / 93$ & 17 & 1 & & 99.0 & $N$ & \\
\hline 1 & 11: 193 & 19 & 1 & & 99.7 & $\mathbf{N}$ & \\
\hline 1 & 11/ & 1 & 1 & & $143 . \overline{6}$ & $\mathbf{N}$ & \\
\hline 1 & 15/Bdes & $\underline{z}$ & 1 & & 145,5 & $\mathbf{N}$ & \\
\hline 1 & $11 / \mathrm{B} / 93$ & 3 & 1 & & 148,4 & $\mathbf{N}$ & \\
\hline 1 & $17 / 8 / 93$ & 4 & 1 & & 143.9 & $\mathbf{N}$ & \\
\hline 1 & $11 \mathrm{~B} / 93$ & 5 & 1 & & 149.7 & $\mathbf{M}$ & \\
\hline 1 & $11 / \mathrm{B} / 93$ & 6 & 1 & & 149.3 & $\mathbf{N}$ & \\
\hline 1 & 11 i & 7 & 1 & & 148.6 & $\mathbf{N}$ & \\
\hline 1 & $11 / 2 / 93$ & B & 1 & & 150 & $\mathbf{N}$ & \\
\hline 1 & $118 / 93$ & 8 & 1 & & 160.8 & $\mathbf{Y}$ & \\
\hline 1 & 11talaz & 10 & 1 & & 149.7 & $\mathbf{N}$ & \\
\hline 1 & 11, para & 11 & 1 & & 146.3 & $N$ & \\
\hline 1 & 11 taves & 12 & 1 & & $740 \overline{8}$ & N & $\sqrt{800423320-42}$ \\
\hline 1 & 11/4/93 & 13 & 1 & & 134.3 & $\mathbf{N}$ & \\
\hline 1 & $11 / 9 / 83$ & TA & 1 & & 1259 & $\mathbf{K}$ & \\
\hline 1 & $11 / 2 / 83$ & 16 & $\overline{1}$ & & $\$ 14.9$ & $\mathbf{N}$ & \\
\hline 1 & $11 / 9 / 93$ & 16 & 1 & & 501.3 & $\mathbf{N}$ & \\
\hline 1 & 11/11/93 & 1 & 1 & & 143,4 & $\mathbf{N}$ & \\
\hline 1 & $11 / 1 / 93$ & 2 & 1 & & $\$ 452$ & $N$ & \\
\hline 7 & $11 / 11 / 93$ & 3 & $y$ & & 146.7 & N & \\
\hline 1 & $11 / 11 / 93$ & 4 & 1 & & 147.7 & $\mathbf{N}$ & \\
\hline 1 & $11 / 11 / 93$ & 5 & 1 & & 148 & $\mathbf{N}$ & \\
\hline 1 & $11 / 11 / 93$ & $b$ & 1 & & 149,1 & $\mathbf{N}$ & \\
\hline 1 & 11/11/93 & 7 & $t$ & & 149.3 & $\mathbf{N}$ & \\
\hline 1 & $11 / 11, \sqrt{93}$ & 8 & 1 & & 149.7 & N & \\
\hline 1 & $51 / 11 / 93$ & $\theta$ & 1 & & 150.7 & $\mathbf{Y}$ & \\
\hline 1 & $71 / 19193$ & 10 & 1 & & 149.6 & $N$ & \\
\hline 1 & $11 / 11 / 93$ & 11 & 1 & & $14 \%$ & $\mathbf{N}$ & \\
\hline 1 & $11 / 11 / 93$ & 12 & 1 & & 140,6 & $\mathbf{N}$ & \\
\hline 1 & $11 / 11 / 93$ & 13 & 1 & & 134.1 & $\mathbf{N}$ & \\
\hline 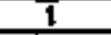 & $11 / 17 / 8$ & 14 & 1 & & 125.1 & $\mathbf{N}$ & $J 99042320-42$ \\
\hline$t$ & $11 / 11 / 93$ & 15 & 1 & & 174,1 & $N$ & \\
\hline 1 & $11 / 11 / 93$ & 16 & 1 & & 101.1 & $\mathbf{N}$ & \\
\hline 1 & $11 / 17 / 93$ & 17 & 1 & & 89.5 & N & \\
\hline 1 & $11 / 11 / 93$ & 19 & 1 & & $99 . \overline{1}$ & $\mathbf{N}$ & \\
\hline 1 & $11 / 16 / 93$ & 1 & 1 & & 143 & $\mathbf{N}$ & \\
\hline 1 & $11 / 15 / 93$ & 2 & 1 & + & 144.9 & $\mathbf{N}$ & \\
\hline 1 & $11 / 45 / 93$ & 3 & 1 & & 148.4 & N & \\
\hline 1 & $11 / 16 / 93$ & 4 & $T$ & & 147,4 & $\mathbf{N}$ & \\
\hline 1 & $11 / 15 \mathrm{gs}$ & 5 & $1 *$ & & 147.7 & $\mathbf{M}$ & \\
\hline 1 & $11 / 19 / 93$ & 8 & 1. & & 147.8 & N & \\
\hline 1 & $11 / 15 / 93$ & 7 & 1 & & $14 \overline{8}$ & $\mathbf{N}$ & \\
\hline 1. & $11 / 16 / 93$ & 8 & 1 & & 148.4 & $\mathbf{N}$ & \\
\hline 1 & 11/1E/e3 & $\mathbf{g}$ & 1 & & 160.2 & $\mathbf{Y}$ & \\
\hline 1 & $11 / 15 / 93$ & 10 & 1 & & 148.1 & $\mathbf{N}$ & \\
\hline$t$ & $1,1 / 15 / 93$ & 11 & 1 & & 145,7 & $\mathbf{N}$ & \\
\hline 1 & J1/16/ & 12 & 1 & & 140.2 & $\mathbf{N}$ & \\
\hline 1 & 11/15/43 & 13 & 1 & & 139.7 & $\overline{\mathbf{N}}$ & \\
\hline 7 & $11 / 15 / 93$ & 14 & 1 & & 124.7 & N & $J 910128143-50$ \\
\hline$t$ & $11 / 16 / 93$ & 15 & 1 & & 113,5 & $\mathbf{N}$ & \\
\hline 1 & $11 / 15 / 93$ & 10 & 1 & & 100.2 & $N$ & \\
\hline
\end{tabular}

Data obtained trom WHC Surveillance Analysis Computer System (SACS), Jhty 1, 1993. 
WHC-SD-WM-ER-3OB, Rev. 0

\begin{tabular}{|c|c|c|c|c|c|c|c|}
\hline \multicolumn{8}{|c|}{ TAHK 241A+101 } \\
\hline & & & & THE & AOCOLPA & DATA & \\
\hline THEE \# & DATE & THEARO & THEE SEY & 臽的C & $\overline{T E M P F}$ & $Y=$ MAX TEMP & COMMNENTS \\
\hline 1 & $11115 / 93$ & 17 & 1 & & 99.7 & $N$ & \\
\hline 1 & $11 / 15 / 93$ & 拍 & 1 & & 98,4 & $\mathbf{N}$ & \\
\hline 1 & $11 / 22 / 93$ & $i$ & 1 & & 142 & $\mathbf{N}$ & \\
\hline 1. & $10 / 22 / 93$ & 2 & 1 & & 144 & $\mathbf{N}$ & \\
\hline 1 & $11 / 22 / 93$ & $\mathbf{s}$ & 1 & & 145 & $\mathbf{N}$ & \\
\hline 1 & $11 / 2-2 / 93$ & 4 & 1 & & 146 & $\mathbf{N}$ & \\
\hline 1 & 11/22/93] & $\mathbf{5}$ & 1 & & 149 & N & \\
\hline 1 & $11 / 22 / 93$ & 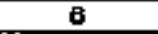 & 1 & & 147 & $\mathbf{M}$ & \\
\hline 1 & $11 / 22 / 83$ & 7 & 1 & & 147 & $\mathbf{N}$ & \\
\hline 1 & $11 / 2293$ & $\dot{\mathbf{B}}$ & 1 & & 148 & $\mathbf{N}$ & \\
\hline 1 & $11 / 22 / 93$ & 9 & 1 & & 149 & $\mathbf{Y}$ & \\
\hline 1 & 11/22/as & 10 & 1 & & 148 & $\mathbf{N}$ & \\
\hline 7 & $11 / 22 / 93$ & 11 & 1 & & 144 & $\mathbf{N}$ & \\
\hline 1 & $11 / 2$ / & 12 & 1 & & 130 & $\mathbf{N}$ & \\
\hline 1 & $11 / 22 / 03$ & 13 & 1 & & 132 & $\mathbf{N}$ & \\
\hline $\mathbf{I}$ & $11 / 22 / 93$ & 14 & 1 & & 123 & $\mathbf{N}$ & TYPE \\
\hline 1 & $11 / 22 / 93$ & 15 & 7 & & 112 & $\mathbf{N}$ & \\
\hline$T$ & $11 / 22 / 93$ & 16 & 7 & & 99 & $\overline{\mathbf{N}}$ & \\
\hline 1 & $11 / 22 / 93$ & 17 & $i$ & & 97 & $\mathbf{N}$ & \\
\hline 1 & $11 / 22 / 93$ & TB & 1 & & 97 & $\mathbf{N}$ & \\
\hline 1 & $11 / 25 / 93$ & 1 & 7 & & 146 & $\mathbf{N}$ & \\
\hline 1 & $11 / 25 / 93$ & 2 & 1 & & 148 & $\mathbf{N}$ & \\
\hline 1 & $11 / 25 / 93$ & 3 & 1 & & 149.6 & $\mathbf{M}$ & \\
\hline 1 & $11 / 25 / 93$ & 4 & 1 & & 150.4 & $\mathbf{N}$ & \\
\hline 1 & $11 / 25 / 93$ & 5 & 7 & & 150.7 & $\mathbf{N}$ & \\
\hline 1 & $11 / 25 / 93$ & 8 & 1 & & 150.9 & $\mathbf{N}$ & \\
\hline 1 & $11 / 25 / 93$ & 7 & 1 & & $15 t .1$ & $\mathbf{N}$ & \\
\hline 1 & $11 / 26 / 93$ & 8 & 1 & & 162.0 & $\mathbf{N}$ & \\
\hline 1 & $11 / 25 / 93$ & 9 & 1 & & t53.6 & $y$ & \\
\hline 1 & $11 / 25 / 93$ & 10 & 1 & & 152,3 & $N$ & \\
\hline 1 & $11 / 25 / 93$ & 11 & 1 & & T48.9 & $\mathbf{N}$ & \\
\hline 1 & $11 / 25 / 93$ & 12 & $\bar{T}$ & & 143.5 & $\mathbf{N}$ & \\
\hline 1 & $11 / 26 / 93$ & 13 & 1 & & t36.0 & $\mathbf{N}$ & \\
\hline 1 & $11 / 26 / 93$ & 14 & 1 & & $\$ 27.5$ & $\mathbf{M}$ & TYFE J \\
\hline 1 & $11 / 25 / 93$ & 75 & 1 & & 115.8 & $\mathbf{N}$ & \\
\hline 1 & $11 / 25 / 93$ & $\sqrt{6}$ & 1 & & 102 & $\mathbf{N}$ & \\
\hline 1 & $11 / 25 \mathrm{NS3}$ & 17 & 1 & & 100.3 & $N$ & \\
\hline 1 & $11 / 26 / 93$ & 19 & 1. & & 100 & $\mathbf{N}$ & \\
\hline 1 & $11 / 20 / 93$ & 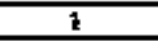 & 1 & & 146.3 & $\mathbf{N}$ & \\
\hline $\mathbf{T}$ & $11 / 29 / 93$ & 2 & 1 & & $14 \mathrm{B.1}$ & $\mathbf{N}$ & \\
\hline 1 & $11 / 29 / 43$ & 3 & 1 & & 149.9 & $\mathbf{N}$ & \\
\hline$\$$ & $11 / 29 / 53$ & 4 & 1 & & 150.6 & 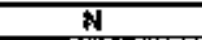 & \\
\hline 1 & $11 / 29 / 93$ & 5 & 1 & & 160.9 & N & \\
\hline 1 & $11 / 29 / 93$ & $\theta$ & 1 & & 151.2 & $\mathbf{N}$ & \\
\hline 1 & $11 / 20 / 93$ & 7 & 1 & & 951,3 & $\mathbf{N}$ & \\
\hline 7 & $11 / 29 / 93$ & $\overline{8}$ & $\overline{1}$ & & 152.9 & $\mathbf{N}$ & \\
\hline 1 & $11 / 20 / 93$ & $\bar{\theta}$ & $T$ & & 153.8 & $\bar{Y}$ & \\
\hline 1 & $11 / 29 / 93$ & 10 & 1 & & 152.5 & $N$ & \\
\hline 1 & $19 / 28 / 93$ & 11 & 1 & & 149.1 & $\mathbf{N}$ & \\
\hline 1 & $11 / 29 / 93$ & 12 & $T$ & & 143.7 & 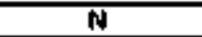 & J91012\$24350 \\
\hline 1 & $11 / 29 / 93$ & 13 & 1 & & 136,9 & $N$ & \\
\hline 1 & $19 / 2 \theta / 93$ & 14 & $F$ & & 127.7 & $\mathbf{N}$ & \\
\hline 1 & $11 / 29 / 93$ & 16 & 1 & & 115.9 & $\mathbf{N}$ & \\
\hline$\overline{1}$ & $11 / 29 / 93$ & 96 & 1 & & $101 . \dot{0}$ & $\mathbf{N}$ & \\
\hline 1 & $11 / 29 / 93$ & 97 & 1 & & 100 & $\mathbf{N}$ & \\
\hline$\overline{1}$ & $\overline{11 / 20 / 93}$ & 18 & 1 & & 99.9 & $\mathbf{N}$ & \\
\hline 1 & $12 / 2 / 93$ & 1 & 1 & & 146,1 & $\mathbf{N}$ & \\
\hline 1 & $12 / 2 / 43$ & 2 & 1 & & 148 & $\mathbf{N}$ & \\
\hline 1 & $12 / 2 / 93$ & 3 & 1 & & 149.8 & $\mathbf{N}$ & \\
\hline 1 & $12 / 2 / 93$ & 4 & 1 & & 160.4 & $\mathbf{N}$ & \\
\hline 1 & $12 / 2 / 93$ & 5 & 1 & & 150.8 & $\mathbf{N}$ & \\
\hline 1 & $12 / 2 / 93$ & B & 1 & & 150.9 & $\mathbf{N}$ & \\
\hline 1 & $12 / 2 / 93$ & $\overline{7}$ & 7 & & 151.1 & $\mathbf{N}$ & \\
\hline
\end{tabular}

Data obtained trom WHC Surveidance Analysis Computer System (SAC5), July 1, 1993. 


\begin{tabular}{|c|c|c|c|c|c|c|c|}
\hline \multicolumn{8}{|c|}{ TANK 241.A-101 } \\
\hline & & & & THE & Pogul & DATA & \\
\hline TAEE & DATE & THEAMO & TAEE \$ET \# & TEMP C & TEMP F & $Y-M A X T E A F$ & COMMAjts \\
\hline 1 & $12 / 2 / 93$ & g & 1 & & 152.0 & N & \\
\hline$\overline{1}$ & $12 / 2 / 93$ & g & $\mathbf{T}$ & & 153.0 & $\mathbf{Y}$ & \\
\hline 7 & $12 / 2 / 93$ & 10 & 1 & & 152.3 & $\mathbf{N}$ & \\
\hline 1 & $12 / 2 / 93$ & $\$ 1$ & 1 & & $148 . \mathrm{B}$ & $\mathbf{N}$ & \\
\hline 7 & $12 / 2 / 93$ & 52 & 1 & & 143.6 & $\mathbf{N}$ & $ل$ gt0129143-50 \\
\hline 1 & $12 / 2 / 93$ & 13 & 1 & & 136.7 & $\mathbf{H}$ & \\
\hline 1 & $12 / 2 / 93$ & 14 & 1 & & 127.5 & $\mathbf{N}$ & \\
\hline 1 & $12 / 2193$ & 括 & 7 & & 115.6 & $\mathbf{N}$ & \\
\hline 1 & $12 / 249$ & 10 & $t$ & & 101.4 & $\mathbf{N}$ & \\
\hline 1 & $12 / 8 / 93$ & 1 & $t$ & & $146, \bar{B}$ & $\mathbf{N}$ & \\
\hline 1 & $12 \sqrt{6} \overline{3}$ & 2 & $\dot{T}$ & & 148,5 & $\mathbf{N}$ & \\
\hline 1 & 12 (6) & 3 & 1 & & 150 & $\mathbf{N}$ & \\
\hline 1 & 12/3/e3 & 4 & 1 & & 150,9 & $\mathbf{N}$ & \\
\hline $\mathbf{1}$ & $12 / 0$ re3 & 5 & 1 & & $151, \overline{3}$ & $\mathbb{N}$ & \\
\hline 1 & $12 / 63$ & E & 1 & & 1515 & $\mathbf{N}$ & \\
\hline 1 & $12 / 6 / 93$ & 7 & 1 & & 251.0 & $\mathbf{N}$ & \\
\hline 1 & 12 reas & B & 1 & & 153.2 & $\mathbf{N}$ & \\
\hline 1 & 12 / $/ 93$ & 安 & 1 & & 154.1 & $\bar{Y}$ & \\
\hline 1 & 12 Howas & 10 & 1 & & 1528 & $\mathbf{N}$ & \\
\hline 1 & $12 / 6 / 93$ & 11 & 1 & & 149.5 & $\mathbf{N}$ & \\
\hline 1 & $12 / 9 / 93$ & 12 & 1 & & 144.1 & $\mathbf{N}$ & \\
\hline$t$ & $12 / 0 / 93$ & 13 & 1 & & 137.4 & $\mathbf{N}$ & \\
\hline 1 & 12 / & 74 & 1 & & 128 & $\mathbf{N}$ & J $810126143 \cdot 45$ \\
\hline 1 & $52 / 6 / 93$ & 95 & 1 & & 116 & $\mathbf{H}$ & \\
\hline 1 & $12 / \overline{9} 9 \theta^{2}$ & 16 & 1 & & $101, \overline{6}$ & $\mathrm{~N}$ & \\
\hline 1 & $12 / 6 / 93$ & 17 & 1 & & 99.1 & $\mathbf{N}$ & \\
\hline 1 & $12 / 6 / 93$ & 19 & 1 & & 99.5 & $\mathbf{M}$ & \\
\hline 1 & $12 / 19 / 93$ & 1 & 1 & & $\overline{148 . \bar{B}}$ & $\mathbf{N}$ & \\
\hline 1 & $12 / 18 \sqrt{93}$ & 2 & 1 & & $148, \bar{B}$ & $\bar{N}$ & \\
\hline 1 & $12 / 18 / 43$ & 3 & 7 & & 150.2 & $\mathbf{M}$ & \\
\hline 1 & $12 / 18 \% 3$ & 4 & 1 & & $15 \$ .2$ & $\mathbf{N}$ & \\
\hline 1 & $1208 / 93$ & 5 & 7 & & 151.5 & $\mathbf{N}$ & \\
\hline 1 & $12 / 18 / 93$ & 6 & 1 & & 151.6 & $\mathbf{M}$ & \\
\hline 1 & $12 / 18 / 93$ & 7 & 1 & & 151.8 & $\mathbf{N}$ & \\
\hline 1 & 12 /16/93 & $\bar{E}$ & $\hbar$ & & $\$ 53.2$ & $\bar{N}$ & \\
\hline$t$ & T2/1E/93 & 9 & 1 & & $154 . \overline{4}$ & $Y$ & \\
\hline 1 & $1208 / 93$ & 10 & 1 & & 153,1 & N & \\
\hline 1 & 12 ineses & 11 & 1 & & $\$ 48.7$ & $\mathbf{N}$ & \\
\hline 1 & 12/16/9s & 12 & $\mathbf{t}$ & & 344.3 & $\mathbf{N}$ & \\
\hline $\mathbf{j}$ & $12 / 1 \mathrm{~B} / 93$ & 13 & 1 & & 137.5 & $\mathbf{N}$ & \\
\hline 1 & $12 / 16 / 93$ & 14 & 1 & & 128 & $\mathbf{N}$ & \\
\hline 1 & $12 / 18 / 93$ & 15 & $i$ & & 715.4 & $N$ & \\
\hline 1 & 12/79/93 & 16 & 1 & & 100.2 & $\mathbf{N}$ & \\
\hline 1 & 12/10/93 & 17 & 1 & & 99.5 & $\mathbf{N}$ & \\
\hline 1 & $12 / 40 / 93$ & 10 & 1 & & 80.3 & N & \\
\hline 7 & $12 / 20 / 93$ & 1 & 1 & & $140^{\circ}$ & $\mathbf{N}$ & \\
\hline$T$ & $12 / 20183$ & 2 & 1 & & 149 & $\mathbf{N}$ & \\
\hline 1 & $12 / 2003$ & 3 & 1 & & 149.5 & $\mathbf{N}$ & \\
\hline 7 & 12120193 & 4 & 7 & & 750.3 & $\mathbf{N}$ & \\
\hline 1 & $12 / 20,93$ & 6 & 1 & & 150.9 & $\mathbf{N}$ & \\
\hline 9 & $12 k 0 \% 93$ & 6 & 1 & & 150.9 & $N$ & \\
\hline$T$ & 12/20183 & 7 & 7 & & 750.8 & $\mathbf{N}$ & \\
\hline 1 & $1 / 2 / 20 / 93$ & E & 1 & & 1525 & $\mathbf{N}$ & \\
\hline 1 & $12 / 20 / 93$ & 8 & 1 & & 153.5 & $\mathbf{Y}$ & \\
\hline \pm & $12 / 20 / 90$ & 10 & 1 & & 152,2 & $\bar{N}$ & \\
\hline 1 & $12 / 20193$ & 11 & 1 & & 148.8 & $\mathbf{N}$ & \\
\hline$T$ & $12 / 20193$ & 12 & 7 & & 743.5 & $\mathbf{N}$ & \\
\hline $\bar{t}$ & $12 / 20 / 93$ & 13 & $t$ & & 136.7 & $\mathbf{N}$ & \\
\hline$\frac{1}{1}$ & $12 / 20 y^{3}$ & 14 & 1 & & $127+3$ & $\mathbf{N}$ & \\
\hline 1 & $12 / 20 \mathrm{da}$ & 16 & 1 & & $\$ 14.7$ & $\mathbf{N}$ & \\
\hline$T$ & $12 / 20$ pes & 18 & 1 & & $69 . \overline{7}$ & $\mathbf{N}$ & \\
\hline $\bar{y}$ & $12 / 20 / 83$ & 17 & 1 & & 97.9 & $\mathbf{N}$ & \\
\hline 1 & $12 / 20 \mathrm{de}$ & IB & 1 & & 97.7 & $\mathbf{N}$ & \\
\hline
\end{tabular}

Data obtained trom Wic Surveillance Analysls Computer System (SACS), Juhy 1, 1993. 


\begin{tabular}{|c|c|c|c|c|c|c|c|}
\hline \multicolumn{8}{|c|}{$\begin{array}{l}\text { TANK 241-A-102 } \\
\text { THERTMOCOT FLE DATA }\end{array}$} \\
\hline TREE : & DATE & THERMO : & TAEE SET \# & TEMP' C & TEMPF & $Y=$ MAX TुAMP & COMAENTS \\
\hline 1 & $3 / 25 / 77$ & 1 & 1 & & 99 & $\mathbf{N}$ & \\
\hline 1 & $3 / 26 / 77$ & 2 & 1 & & 90 & $N$ & \\
\hline 1 & $3 / 25 / 77$ & 3 & 1 & & 99 & $\mathbf{N}$ & \\
\hline 1 & $3 / 25 / 77$ & 4 & 1 & & 99 & $\mathbf{N}$ & \\
\hline 1 & $3 / 25 / 77$ & 5 & 1 & & 99 & $\mathbf{N}$ & \\
\hline 1 & $3 / 25 / 77$ & 7 & 1 & & 100 & $\bar{Y}$ & \\
\hline$T$ & $3 / 25 / 77$ & 8 & 1 & & 99 & $\mathbf{N}$ & \\
\hline$\overline{1}$ & $3 / 25 / 77$ & 9 & 1 & & 99 & N & \\
\hline 1 & $3125 / 77$ & 10 & 1 & & 99 & $\mathbf{N}$ & \\
\hline 1 & $3 / 25 / 77$ & 11 & $\overline{1}$ & & 99 & $\mathbf{N}$ & \\
\hline 1 & $3 / 25 / 77$ & 12 & 1 & & 90 & $\mathbf{N}$ & \\
\hline 1 & $4 / 6177$ & 1 & 1 & & 98 & $\mathbf{N}$ & \\
\hline 1 & $4 / 6 / 77$ & 2 & 1 & & 98 & $\mathbf{N}$ & \\
\hline 1 & 4 4BT77 & 3 & 7 & & 98 & $\mathbf{N}$ & \\
\hline 1 & $40 / 77$ & 4 & 1 & & 98 & $\mathbf{N}$ & \\
\hline 1 & $4 \sqrt{6 n}$ & 5 & 1 & & $\mathbf{g 8}$ & $\mathbf{N}$ & \\
\hline 1 & $46 / 77$ & 7 & 1 & & 98 & $Y$ & \\
\hline 1 & 46177 & $\mathbf{g}$ & $T$ & & 89 & $\mathbf{H}$ & \\
\hline 1 & 4 rer7 & in & $i$ & & 98 & $\overline{\mathbf{N}}$ & \\
\hline$\overline{1}$ & 4rent 7 & 10 & 1 & & S⿳⺈ & $\mathbf{H}$ & \\
\hline 1 & $4 \sqrt{6 / 7}$ & 11 & 1 & & 95 & $\overline{\mathrm{H}}$ & \\
\hline 1 & $4 / 6 \sqrt{7}$ & 12 & 1 & & 96 & $\overline{\mathbf{N}}$ & \\
\hline $\bar{T}$ & $4 / 6 / 77$ & 13 & 1 & & 96 & N & \\
\hline 5 & $4 \sqrt{6} 77$ & 14 & $T$ & & 96 & $\bar{N}$ & \\
\hline $\bar{t}$ & $46 b^{2}$ & 15 & 1 & & 84 & $\mathbf{N}$ & \\
\hline$\overline{1}$ & 4 / 9777 & 19 & 1 & & 84 & $\mathrm{~N}$ & \\
\hline 1 & $5119 \pi 7$ & 1 & 1 & & 9日8 & $\mathbf{N}$ & \\
\hline$\overline{\mathbf{a}}$ & $519 / 77$ & 2 & 1 & & $\overline{8}$ & $\dot{\mathbf{N}}$ & \\
\hline$y$ & 5/19/7 & 3 & 1 & & 100 & $\overline{\mathbf{N}}$ & \\
\hline $\bar{T}$ & $519 / 77$ & 4 & 1 & & 100 & $\mathbf{N}$ & \\
\hline$\$$ & अ19/77 & 5 & 1 & & 100 & $\overline{\mathbf{N}}$ & \\
\hline 3 & 5小19/77 & 6 & 1 & & $10 t$ & $\bar{Y}$ & \\
\hline 1 & $519 / 7$ & 7 & 1 & & 901 & $\mathbf{N}$ & \\
\hline 1 & $5 / 9 n 7$ & 8 & 1 & & $10 t$ & $\bar{N}$ & \\
\hline$\overline{1}$ & $5 / 19 / 77$ & 9 & 1 & & 101 & $\bar{N}$ & \\
\hline 1 & Sh10/7 & 10 & 1 & & 100 & $N$ & \\
\hline$\overline{1}$ & $5 / 19 / 77$ & 11 & 1 & & 100 & $\mathbf{N}$ & \\
\hline 1 & s/40/7 & 12 & 1 & & g9 & $\vec{N}$ & \\
\hline 1 & $5 / 19 / 77$ & 13 & 1 & & 8d & $\mathbf{N}$ & \\
\hline 1 & 510/77 & 14 & 1 & & 90 & W & \\
\hline 1 & हhoग & 1 & 1 & & 103 & $\bar{Y}$ & \\
\hline$T$ & - 1007 & 2 & 1 & & $\overline{\mathrm{gg}}$ & $\mathrm{N}$ & \\
\hline 1 & 6no/7 & 3 & 1 & & 93 & $\bar{H}$ & \\
\hline 1 & 61077 & 4 & 1 & & gg & $\mathbf{N}$ & \\
\hline 1 & $010 / 77$ & 5 & 1 & & 99 & $\mathbf{H}$ & \\
\hline 1 & ereor7 & 7 & $\overline{1}$ & & 98 & H & \\
\hline 1 & 6/3077 & $\bar{B}$ & 1 & & 93 & $\mathbf{H}$ & \\
\hline$T$ & Bdjor77 & $\bar{\theta}$ & $T$ & & 86 & $\overline{\mathbf{N}}$ & \\
\hline$\overline{1}$ & 619077 & 30 & 1 & & 86 & $\mathbf{N}$ & \\
\hline 1 & 81077 & 91 & 1 & & 88 & $\mathbf{N}$ & \\
\hline 1 & 61077 & $\overline{12}$ & 1 & & 88 & $\bar{N}$ & \\
\hline 1 & Bijol77 & 13 & 1 & & 88 & $\mathbf{N}$ & \\
\hline$\overline{1}$ & $6 / 10 / 77$ & 14 & .14 & & 68 & $\mathbf{N}$ & \\
\hline 1 & 61077 & 15 & 1 & & 68 & $\mathbf{H}$ & \\
\hline$\overline{1}$ & G10/7 & 18 & 1 & & 88 & $\bar{N}$ & \\
\hline 1 & 87877 & 1 & 1 & & 94 & $\bar{Y}$ & \\
\hline 1 & $8 / \mathrm{s} b 77$ & 2 & $T$ & & $\theta 6$ & Ti & \\
\hline 1 & 845677 & 3 & 1 & & 8 & $\bar{H}$ & \\
\hline 1 & 87977 & 4 & 1 & & 68 & $\mathbf{N}$ & \\
\hline 1 & अ19/7 & 5 & 1 & & 68 & $\bar{N}$ & \\
\hline 1 & $8 \sqrt{19} 77$ & a & 1 & & 67 & $\mathbf{N}$ & \\
\hline 7 & B/19/77 & 3 & 1 & & 67 & N & \\
\hline 1 & $8 / 18 \sqrt{77}$ & a & 1 & & $\overline{E B}$ & $N$ & \\
\hline 9 & $8 / 1877$ & $g$ & 7 & & 74 & $N$ & \\
\hline 1 & $8 / 18 / 77$ & 10 & 1 & & 75 & $\bar{N}$ & \\
\hline
\end{tabular}

Data obtainad from WHC Surveillence Analyais Computer System (SACSI, June 2,1993. 


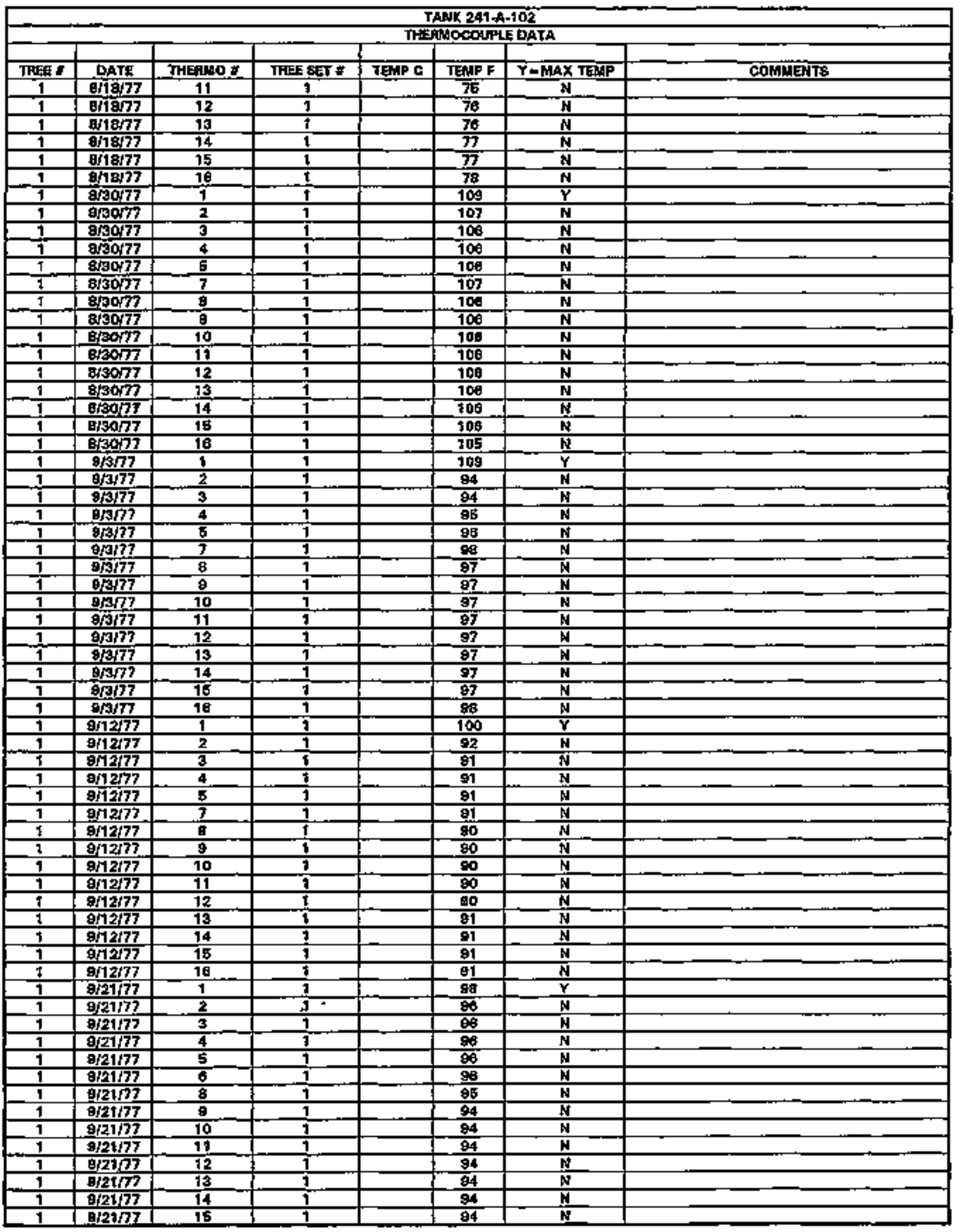

Data abtained from WHC Surveillance Analyals Computer System (SACS), June 2,1993. 


\begin{tabular}{|c|c|c|c|c|c|c|c|}
\hline \multicolumn{8}{|c|}{ TANK 241-A-102 } \\
\hline & & & & & & & \\
\hline TRE: & DATE & TH世中心 & TAEE SET F & TENP C & TEAPF F & $\bar{Y}=\operatorname{MAX}$ TEFAP & COUNENTS \\
\hline 1 & $9 / 21 / 7$ & 18 & 1 & & 84 & N & \\
\hline$\overline{1}$ & $10 \mathrm{~h} / 7$ & 1 & $\overline{1}$ & & 97 & $\overline{\mathbf{Y}}$ & \\
\hline 1 & $10 / 7 / 77$ & 2 & 1 & & 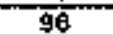 & $\mathbf{M}$ & \\
\hline$T$ & $10+777$ & 3 & $\overline{1}$ & & 95 & $\mathbf{N}$ & \\
\hline 1 & $10 / 7 / 77$ & 4 & 1 & & 95 & it & \\
\hline 1 & 10in? & E & $\overline{1}$ & & 94 & $\mathbf{P}$ & \\
\hline 1 & $10 / 7 / 77$ & $\mathbf{7}$ & 1 & & 93 & M & \\
\hline $\bar{T}$ & $10 / 7 / 7$ & $\bar{\theta}$ & 7 & & 92 & $\mathbf{N}$ & \\
\hline $\bar{\xi}$ & $10 / 7 / 77$ & $\bar{B}$ & 1 & & 92 & $\mathbf{N}$ & \\
\hline$y$ & $10 / 7177$ & 10 & 1 & & 02 & $\mathbf{N}$ & \\
\hline $\mathbf{t}$ & $10 / 7 / 77$ & 11 & 1 & & 92 & $\mathbf{N}$ & \\
\hline 1 & $10 / 7 / 77$ & 12 & 1 & & $\mathbf{0 2}$ & $\mathbf{M}$ & \\
\hline 1 & 10 DI7 & 13 & 1 & & 92 & $\mathbf{H}$ & \\
\hline 1 & $10 / 777$ & 14 & $i$ & & $\overline{\mathbf{6 2}}$ & N & \\
\hline 1 & $70 / 177$ & 15 & 1 & & 92 & $\mathbf{N}$ & \\
\hline 1 & $10 / 7 / 77$ & 16 & 1 & & $\overline{92}$ & $\overline{\mathbf{N}}$ & \\
\hline $\mathbf{I}$ & Jo/177 & 1 & 2 & & & $Y$ & 137 - atipect dita lhight \\
\hline 7 & $90 / 7 / 7$ & 2 & 2 & & 104 & N & \\
\hline 7 & 70 in & 3 & 2 & & 119 & N & \\
\hline$t$ & 100177 & 4 & $\frac{1}{2}$ & & 114 & $\mathbf{N}$ & \\
\hline 1 & $10 / 177$ & 5 & 2 & & 94 & $N$ & \\
\hline$i$ & $10 / 7 / 7$ & 7 & 2 & & $10^{\prime}$ & $\mathbf{N}$ & \\
\hline$t$ & 70017 & 8 & $\bar{z}$ & & 113 & $\mathbf{N}$ & \\
\hline 1 & 101777 & 9 & $\sqrt{2}$ & & 132 & $\bar{N}$ & \\
\hline 1 & laibt & 10 & 2 & & 112 & NN & \\
\hline 1 & $10 / 7 / 77$ & 11 & $\overline{2}$ & & 80 & $\bar{N}$ & \\
\hline 1 & 10577 & 12 & 2 & & 108 & N & \\
\hline 1 & $1017 / 77$ & 13 & 2 & & $\mathrm{Q} b$ & $N$ & \\
\hline 1 & 10n77 & 14 & 2 & & 93 & $\mathbf{N}$ & \\
\hline 1 & $10 / 7 / 77$ & 15 & 2 & & $\overline{83}$ & N & \\
\hline 1 & $10 / 777$ & 16 & 2 & & 95 & $\mathbf{N}$ & \\
\hline 1 & $12 / 4 / 77$ & 1 & 1 & & 100 & $\gamma$ & \\
\hline 1 & $12 / 4 / 77$ & $\overline{2}$ & 1 & & 85 & $\mathbf{N}$ & \\
\hline 1 & $12 / 4 / 7 / 7$ & 3 & 1 & & 71 & N & \\
\hline 1 & $12 / 4 / 77$ & 4 & 1 & & 83 & $\mathbf{N}$ & \\
\hline 1 & $12 / 4 / 77$ & $\overline{\text { 市 }}$ & 1 & & 71 & $N$ & \\
\hline 1 & $12 / 4 / 77$ & 7 & 1 & & 74 & $\mathbf{N}$ & \\
\hline 1 & $12 / 4 / 77$ & 8 & 1 & & 73 & $\mathbf{N}$ & \\
\hline 1 & $12 / 4 / 77$ & 9 & 1 & & 74 & $\bar{N}$ & \\
\hline 1 & $12 / 4 \sqrt{77}$ & 10 & 1 & & 74 & $N$ & \\
\hline 1 & $12 / 4 / 77$ & 11 & 1 & & 74 & $\mathbf{N}$ & \\
\hline 1 & $12 / 4 \sqrt{73}$ & 12 & 1 & & 74 & $\mathbf{N}$ & \\
\hline 1 & $12 / 4 / 77$ & 13 & 1 & & 7 & $N$ & \\
\hline 7 & $12 / 477$ & 14 & 1 & & 75 & $\mathrm{~N}$ & \\
\hline $\mathbf{1}$ & $12 / 4 / 73$ & 15 & 1 & & 75 & N & \\
\hline 1 & $12 / 4 / 77$ & 16 & 1 & & 75 & $\mathbf{N}$ & \\
\hline 1 & $1 / 16 / 7 \theta$ & 1 & 1 & & 101 & $\bar{Y}$ & \\
\hline 1 & 1/16/7e & 2 & 1 & & 96 & $\bar{N}$ & \\
\hline 1 & $1 / 16 / 79$ & $\overline{3}$ & 1 & & 92 & $\mathrm{~N}$ & \\
\hline 1 & $1 / 1678$ & 4 & 1 & & 89 & $N$ & \\
\hline 1 & $1 / 16 / 78$ & 5 & 1 & & 90 & $\mathbf{M}$ & \\
\hline 1 & 1/76/7 & 7 & $\overline{1}$ & & 8 & $N$ & \\
\hline 1 & $1 / 16 / 78$ & 8 & .1 & & 89 & $\mathbf{N}$ & \\
\hline$i$ & $1 / 7678$ & 9 & 1 & & 88 & $N$ & \\
\hline 1 & $1 / 16 / 78$ & 10 & 1 & & BB & $\mathbf{N}$ & \\
\hline 1 & $1 / 9678$ & 11 & 1 & & 88 & $N$ & \\
\hline 1 & $1 / 16 / 79$ & 12 & 1 & & 88 & $\mathbf{N}$ & \\
\hline 1 & $1 / 16 / 78$ & 13 & $\overline{1}$ & & 83 & $\mathbf{H}$ & \\
\hline 1 & $1 / 16 / 78$ & 14 & 1 & & 89 & $\mathbf{N}$ & \\
\hline 1 & $1 / 28 / 78$ & 1 & 1 & & 103 & $Y$ & \\
\hline 1 & 1/26/78 & 2 & 1 & & 98 & $N$ & \\
\hline 1 & $1 / 2 \mathrm{~B} / \mathrm{B}$ & 3 & 1 & & 83 & N & \\
\hline 1 & $1 / 2$ B) & 4 & 1 & & 92 & $\mathbf{N}$ & \\
\hline 1 & $7 / 28 / 78$ & 5 & 1 & & 01 & N & \\
\hline 1 & $1 / 26 / 78$ & 7 & 1 & & 93 & $\bar{N}$ & \\
\hline
\end{tabular}

Date obtained from WHC Survaifence Analysis Computer Syatem ISACSI, JunE 2،1993. 


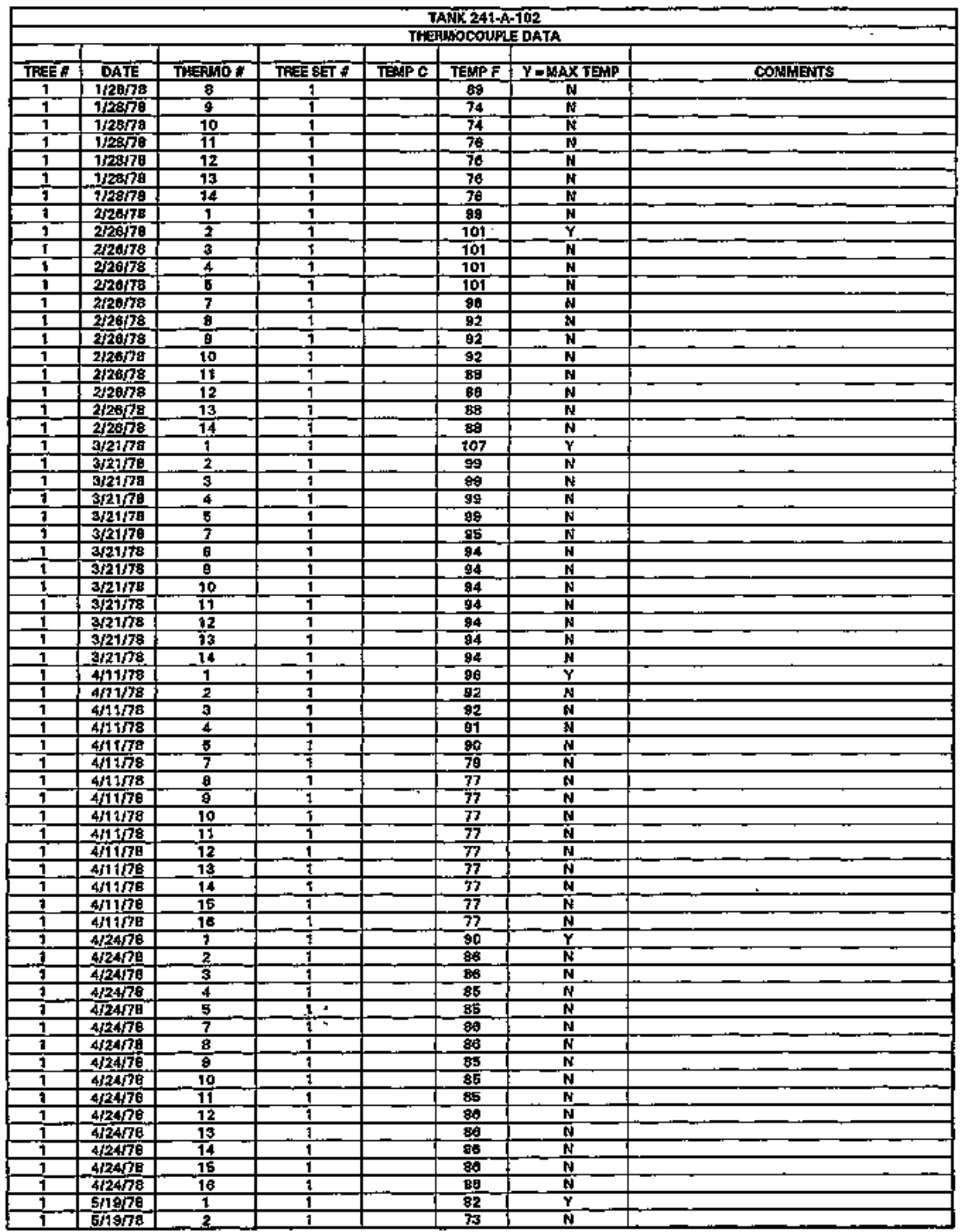

Data obtainad from WHC Sturvedlaned Analvais Computer Svstem [SACS], Juna 2,1993. 


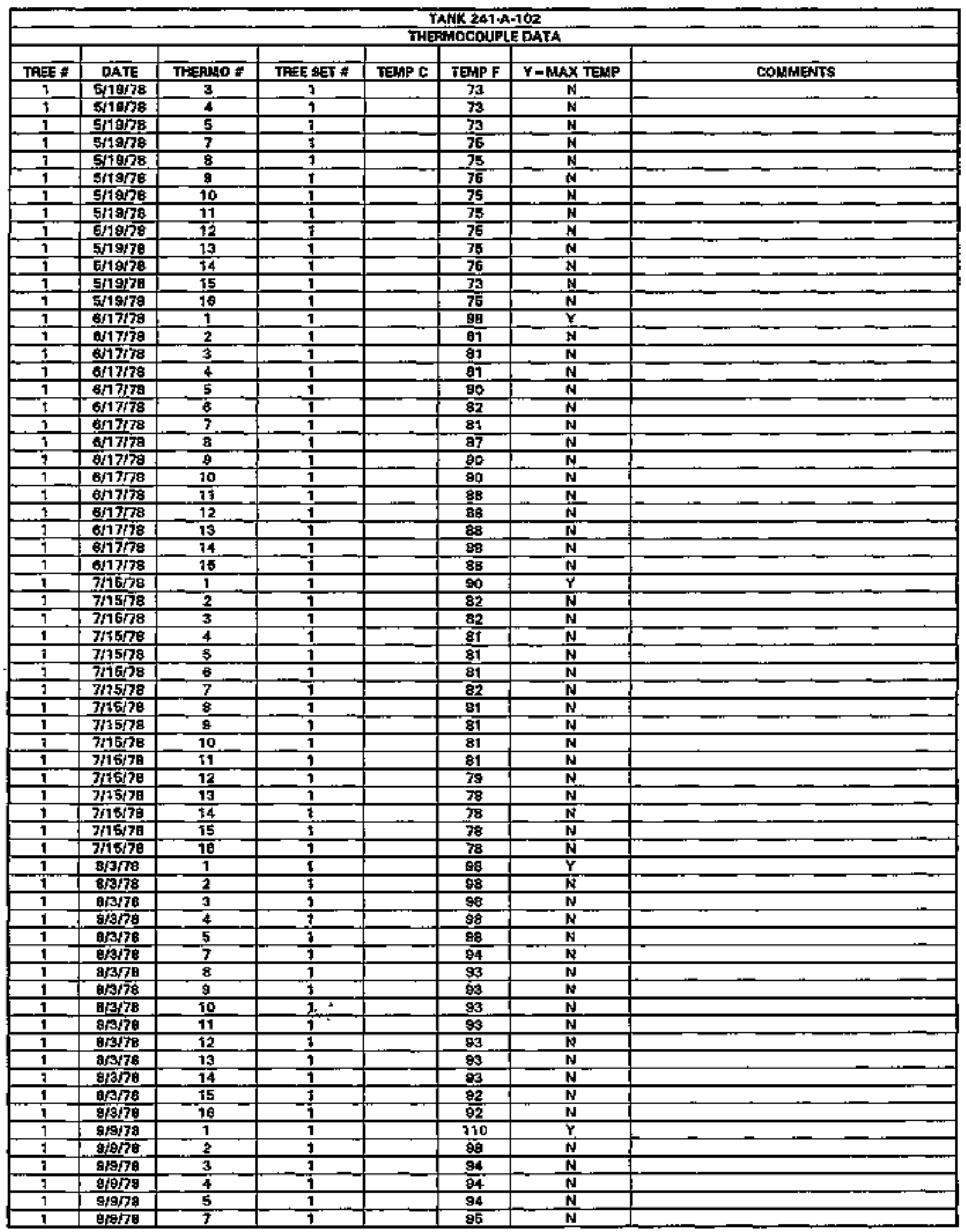

Data obtained from WHC Surveillance Analyaia Computer System (SACS). June 2,1993. 


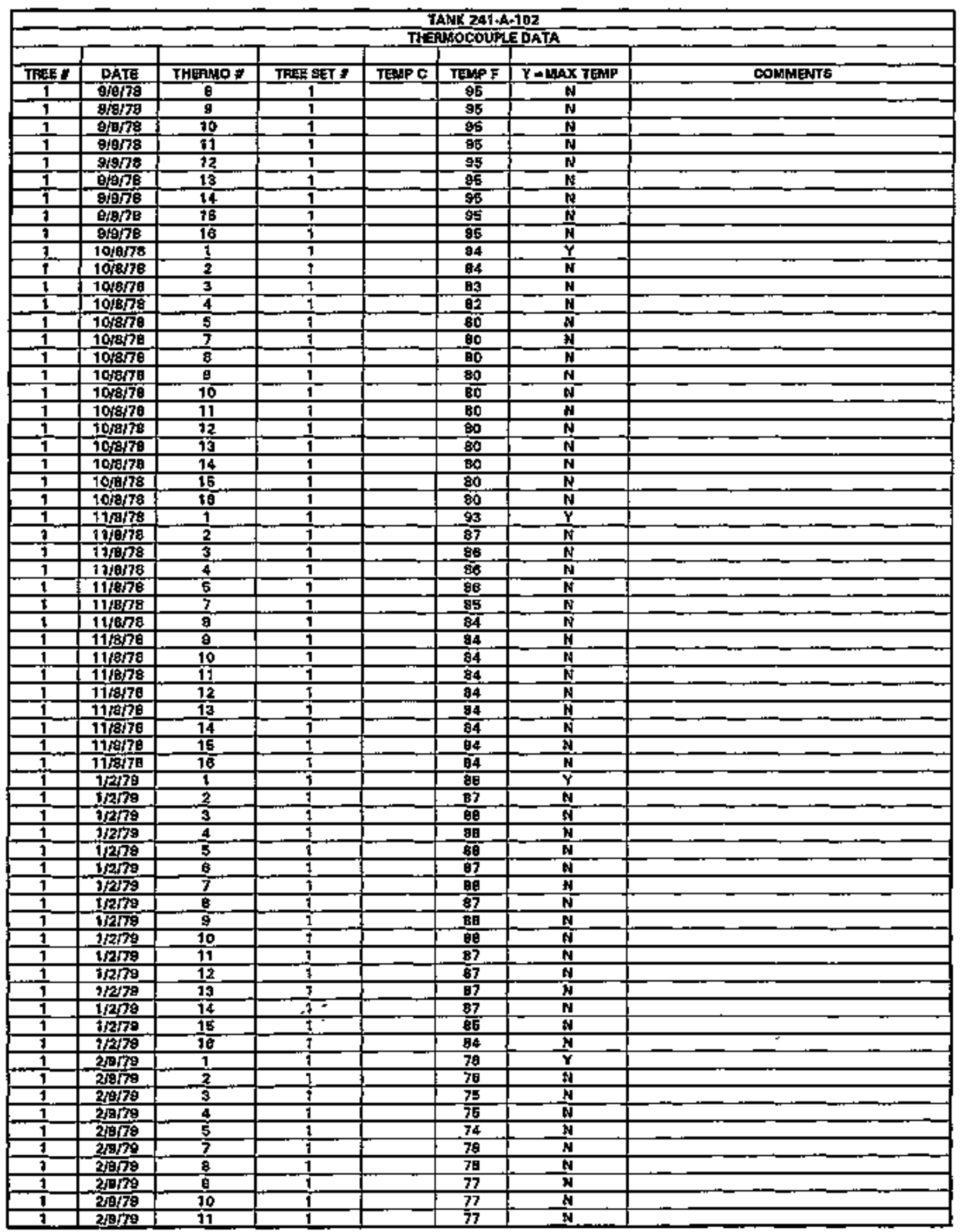

Dete obtained from WHC Surveillance Anelysis Computer System [SAC5\}, June 2,1983. 


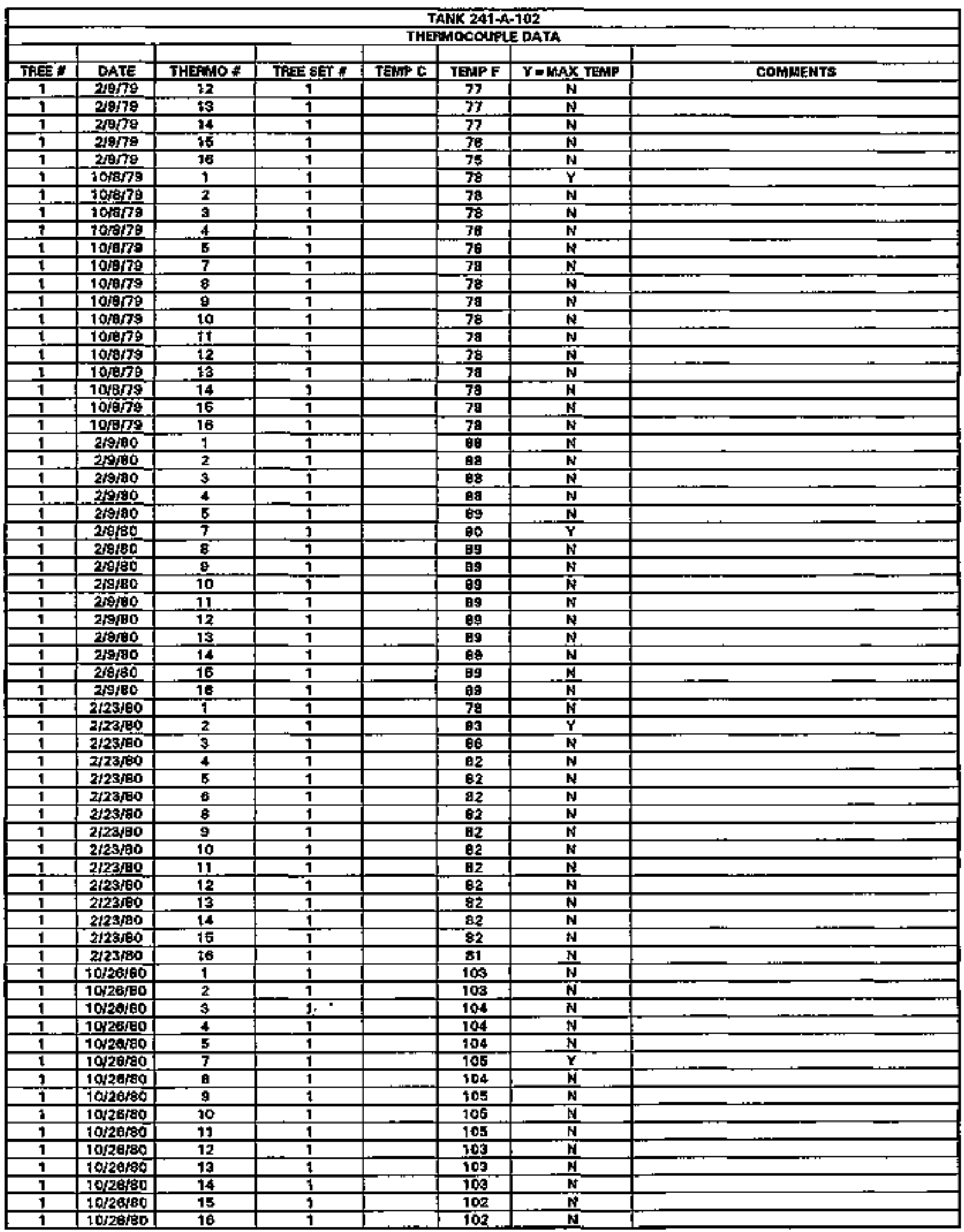

Data obteined from WHC Survellance Anelysis Computer Syatem (SACS), Juan 2,1993. 


\begin{tabular}{|c|c|c|c|c|c|c|c|}
\hline \multirow{2}{*}{\multicolumn{8}{|c|}{ 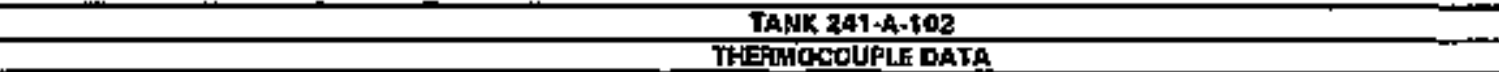 }} \\
\hline & & & & & & & \\
\hline TRE开 I & DAYE & 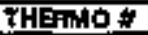 & TALE BET & TEMP C & TEUP F & $Y=$ HAX TJAP & COLANENTS \\
\hline 1 & 1NA1 & 1 & 1 & & 94 & $\gamma$ & \\
\hline 1 & $1 / 9 / 91$ & 2 & 1 & & 88 & $\mathbf{N}$ & \\
\hline 1 & 1, Bre1 & 3 & 1 & & 86 & $\mathbf{N}$ & \\
\hline 1 & 1/9/61 & 4 & 1 & & 85 & $\mathrm{H}$ & \\
\hline$t$ & 1/981 & 5 & 1 & & 95 & N & \\
\hline$t$ & 1 peje1 & 7 & 1 & & 皇自 & $\mathbf{N}$ & \\
\hline 1 & 1/9/a1 & 8 & 1 & & e日 & N & \\
\hline 1 & 1, perat & E & 1 & & BA & $\mathbf{M}$ & \\
\hline 1 & 190191 & 10 & $i$ & & 6a & $N$ & \\
\hline 1 & 10日9 1 & 11 & 1. & & B日 & $\mathbf{N}$ & \\
\hline 1 & $1 / 6 / 81$ & 12 & $\mathrm{I}$ & & 86 & $\mathrm{~N}$ & \\
\hline 1 & 1/8187 & 13 & 1 & & Bg & $\mathbf{N}$ & \\
\hline 1 & J997 & 14 & 1 & & 88 & $\mathbf{N}$ & \\
\hline 1 & 1583 & 15 & 1 & & 60 & $\mathbf{N}$ & \\
\hline 1 & $1 / 8 / 81$ & 28 & 1 & & 88 & $\mathbf{N}$ & \\
\hline 1 & $2 / 6 / 8 t$ & 1 & 1 & & 88 & $\mathbf{Y}$ & \\
\hline 1 & $2 \mathrm{MPN}$ & 2 & 1 & & 82 & $\mathbf{N}$ & \\
\hline 1 & $2 \sqrt{68}$ & 3 & 1 & & 79 & $\mathbf{N}$ & \\
\hline 3 & $2 \sqrt{0 / 81}$ & 4 & 1 & & 76 & $\mathbf{N}$ & \\
\hline 1 & $2 / 4 / 81$ & 5 & 1 & & 79 & $\mathbf{N}$ & \\
\hline 7 & $2 / 6 / 81$ & 7 & 1 & & 80 & $N$ & \\
\hline 1 & 2/3/B1 & g & 1 & & 79 & N & \\
\hline 1 & $2 / 0 / 81$ & 白 & 1 & & 70 & $\$$ & \\
\hline 1 & 2거잉 81 & 10 & 1 & & 79 & $\mathbf{N}$ & \\
\hline 1 & 26181 & 11 & 1 & & 78 & $\mathbf{N}$ & \\
\hline 1. & $26 / 61$ & 12 & 1 & & 78 & $\mathbf{N}$ & \\
\hline$\hat{i}$ & $2 / a / 81$ & $\sqrt{3}$ & 1 & & 78 & $\mathbf{N}$ & \\
\hline 1 & 2/B;B1 & 14 & 1 & & 78 & $\mathbf{N}$ & \\
\hline 1 & 2Be1 & 15 & 1 & & 79 & $\mathbf{N}$ & \\
\hline 1 & 2H拍 1 & 18 & 1 & & 78 & $\mathbf{N}$ & \\
\hline 1 & $2 \sqrt{23} 81$ & 7 & 1 & & 78 & N & \\
\hline 1 & 2/23/1 & $\mathbf{Z}$ & 1 & & Dg & $\mathbf{Y}$ & \\
\hline 1 & $2 / 23501$ & 3 & 1 & & 60 & $\mathbf{N}$ & \\
\hline 1 & 2/2018 & 4 & 1. & & 息 & $\mathbf{N}$ & \\
\hline 1 & $2 / 23481$ & $\underline{5}$ & 1 & & 82 & N & \\
\hline 1 & 2/231 & e & 1. & & 童 & $\mathbf{N}$ & \\
\hline 1 & 2/23181 & 8 & 1 & & B2 & $\mathbf{N}$ & \\
\hline 1 & $2 / 23 / 91$ & 8 & 1 & & - & $\mathbf{N}$ & \\
\hline 1 & $2 / 23 / 31$ & 10 & 1 & & 82 & $\mathbf{N}$ & \\
\hline 1 & $2 / 23191$ & 11 & 1 & & 82 & $\mathbf{N}$ & \\
\hline 1 & 2/2341 & 12 & 1 & & 82 & N & \\
\hline 1 & $2 / 23 / 81$ & 13 & 1 & & 82 & $\sqrt{1}$ & \\
\hline 1 & 2/23/B1 & 14 & \pm & & 9 & $\mathbf{N}$ & \\
\hline 1 & $2 / 23181$ & 15 & 1 & & 82 & N & \\
\hline$\$$ & $2 / 23 / 81$ & 10: & 1 & & 87 & $\mathbf{N}$ & \\
\hline 1 & $2 / 27 / 81$ & 1 & 1 & $\mathbf{3 5}$ & 86 & $\mathbf{Y}$ & degres C/TWIN's sonfim \\
\hline 1 & $2 / 27 / B 1$ & 2 & 1 & $3 y$ & 87.8 & $\bar{N}$ & dertos CTMilis confilm \\
\hline 1 & $2 / 27 / 81$ & 3 & 1 & 29 & 94.2 & $\mathbf{N}$ & 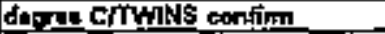 \\
\hline 1 & $2[2781$ & 4 & \pm & 29 & 34.2 & $\mathbf{M}$ & dorres CJTWhS conflam \\
\hline$t$ & $2 / 27 / 81$ & 8 & 1 & 29 & 84.2 & N & deroo CJTWINS enfitm \\
\hline 1 & $2 / 27 / 81$ & 7 & 1 & 29 & 84,2 & N & degren CTTHINS contilm \\
\hline 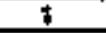 & $2 / 27 / 81$ & $\theta$ & \pm & 29 & 04.2 & N & 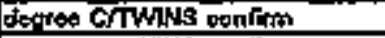 \\
\hline 1 & $2 / 27 / 81$ & $\bar{\theta}$ & $a^{-}$ & 29 & 84.2 & $\mathbf{N}$ & 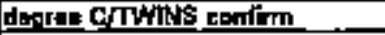 \\
\hline 1 & $2 / 27 / 81$ & 10 & 1 & 20 & 84.2 & N & depas CTMINB opnirm \\
\hline 1 & $2 / 27 / 91$ & 11 & 1 & 29 & 84.2 & $\mathbf{N}$ & 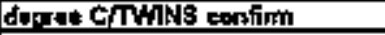 \\
\hline 1 & 2/27/B1 & 12 & 1 & 29 & 84.2 & M & degren CNTWLS conflem \\
\hline 1 & $2 / 27 / 191$ & 13 & 1 & 29. & 84.2 & $\mathbf{N}$ & 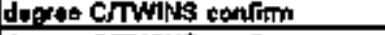 \\
\hline 1 & 2/27/61 & 14. & 1 & 29 & 84,2 & $\mathbf{N}$ & depres CTTWINS enring \\
\hline 1 & $2 / 27 / 61$ & 15 & $\underline{z}$ & 29 & 94.2 & $\mathbf{M}$ & doncos CTMNIN bonfirm \\
\hline 1 & 2/27/91 & 18 & 1 & 29 & 84,2 & $\mathbf{M}$ & 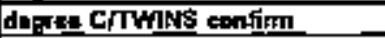 \\
\hline$T$ & aypraj & 1 & 1 & & 02 & $\overline{\mathbf{Y}}$ & \\
\hline 1 & 3 39ta1 & 2 & 1 & & os & $\mathbf{N}$ & \\
\hline 1 & $3 / 9 / 31$ & 3 & 1 & & 83 & $\mathbf{N}$ & \\
\hline 1 & 3 39tan & 4 & 1 & & 3 & $\mathbf{N}$ & \\
\hline 1 & 3981 & $\underline{\xi}$ & 1 & & 69 & 柱 & \\
\hline
\end{tabular}

Date obtelned from WHC Surveillance Analysis Computer Systam ISACS), JUne 2,1993. 


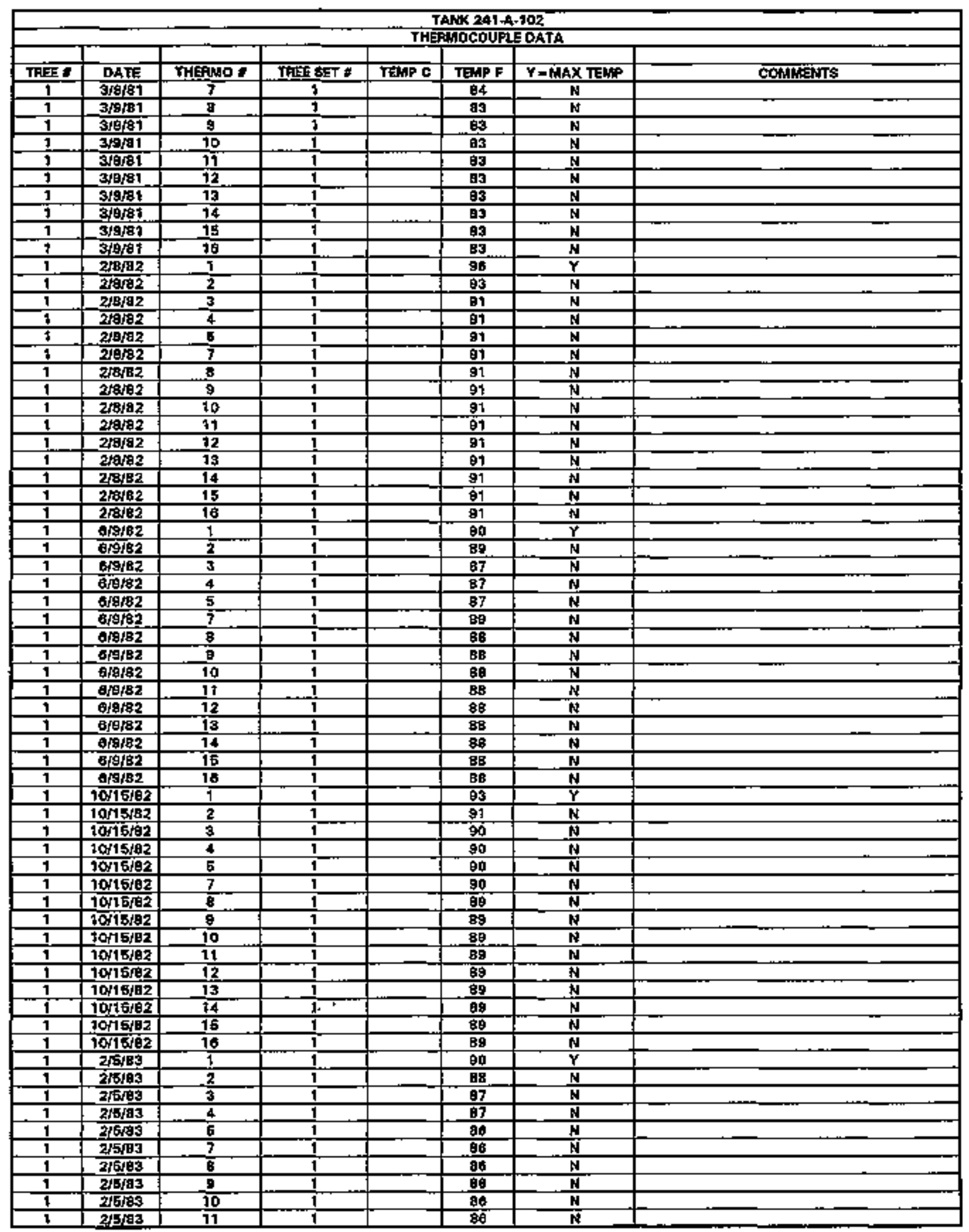

Data obtained from WHC Surveillance Aratysts Computer System \$SACS1, June 2,1983. 


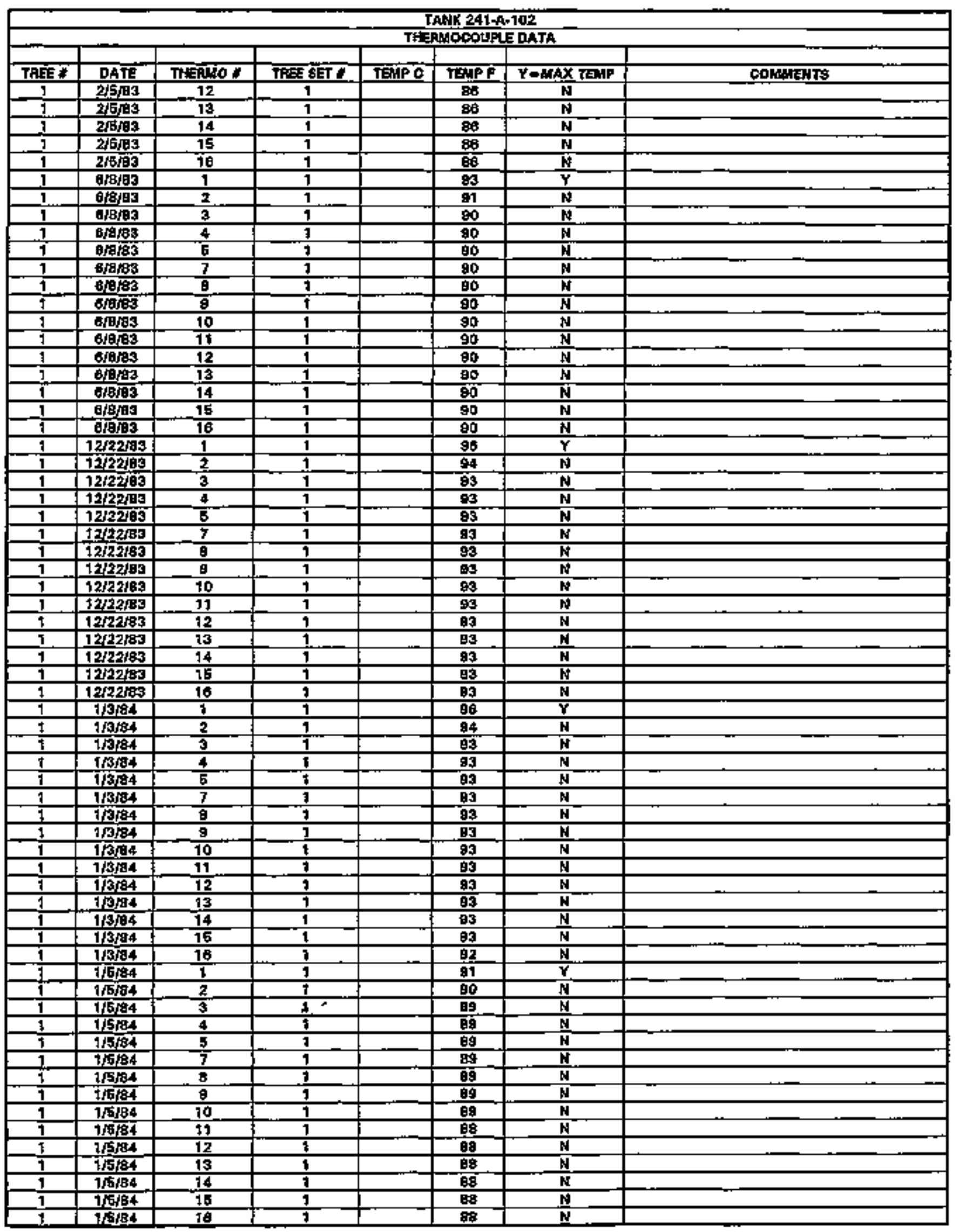

Deta obtained from WHC Surveillance Analysto Camputer System (SACS), bune 2,1993. 


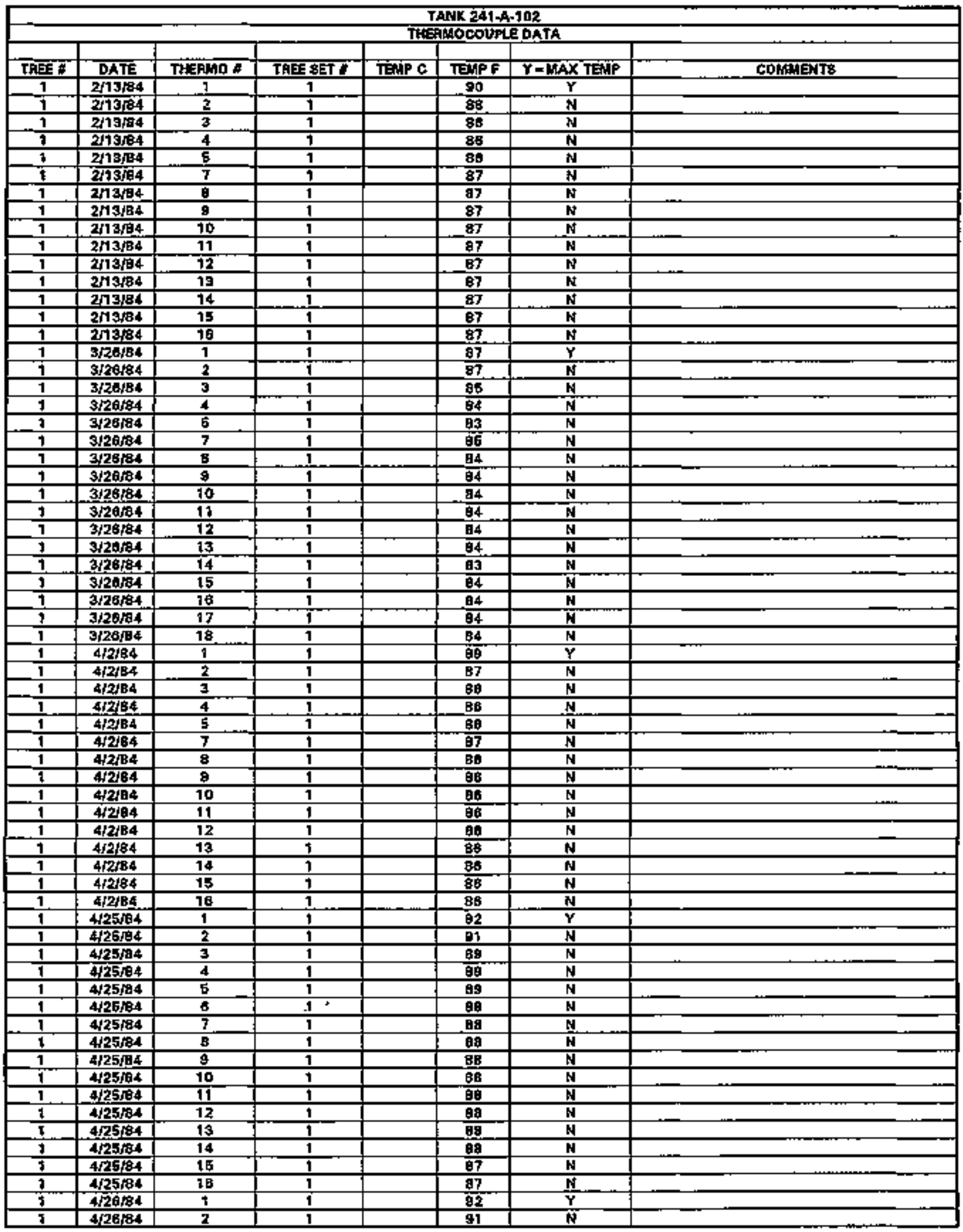

Data obtained from WHC Surveillence Analysic Computer Syatem (SAC\$), June 2,1993. 


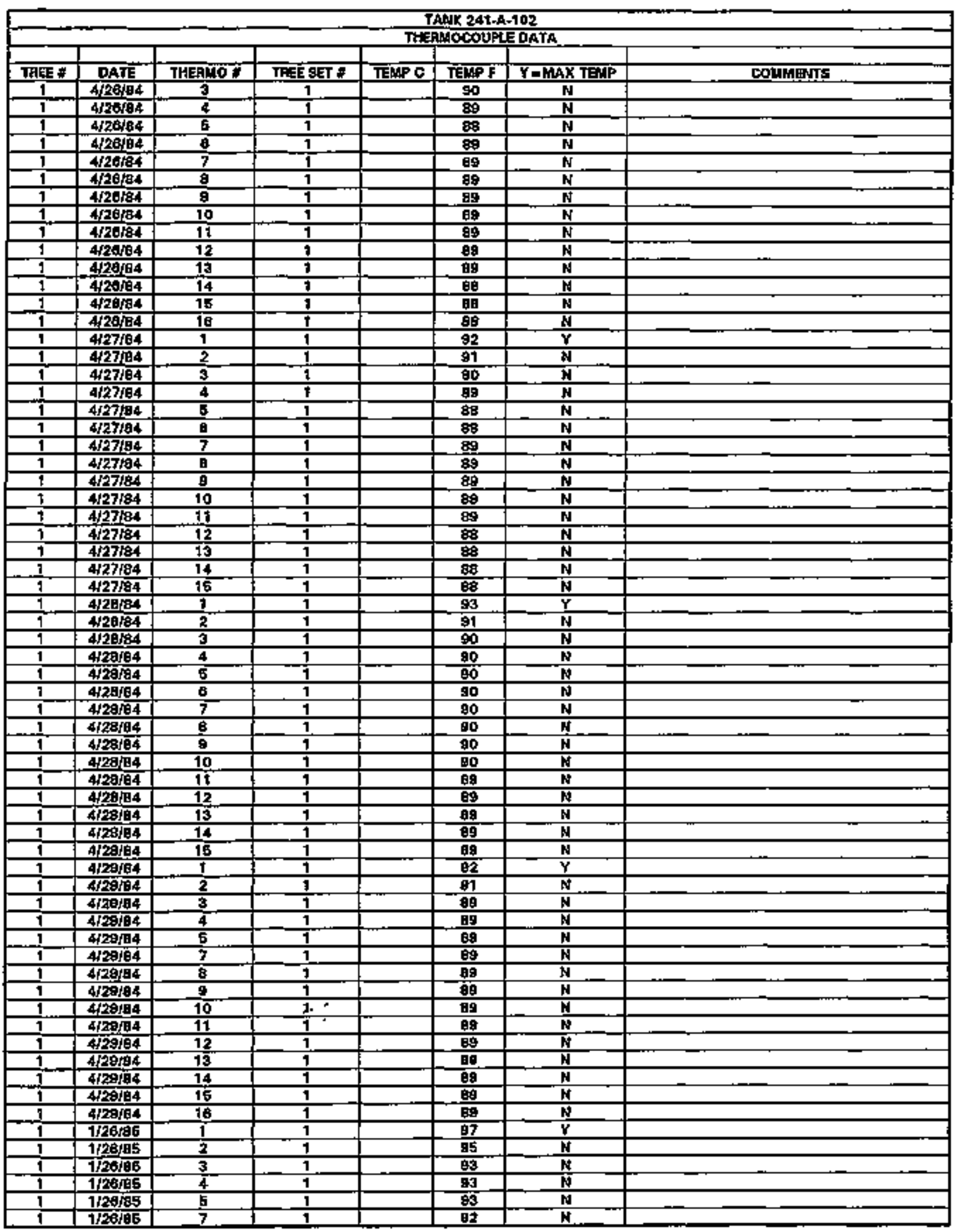

Data obtained from WHC Surveillence Analyate Computer SY\$tem \{SACSI. June 2, 1993. 


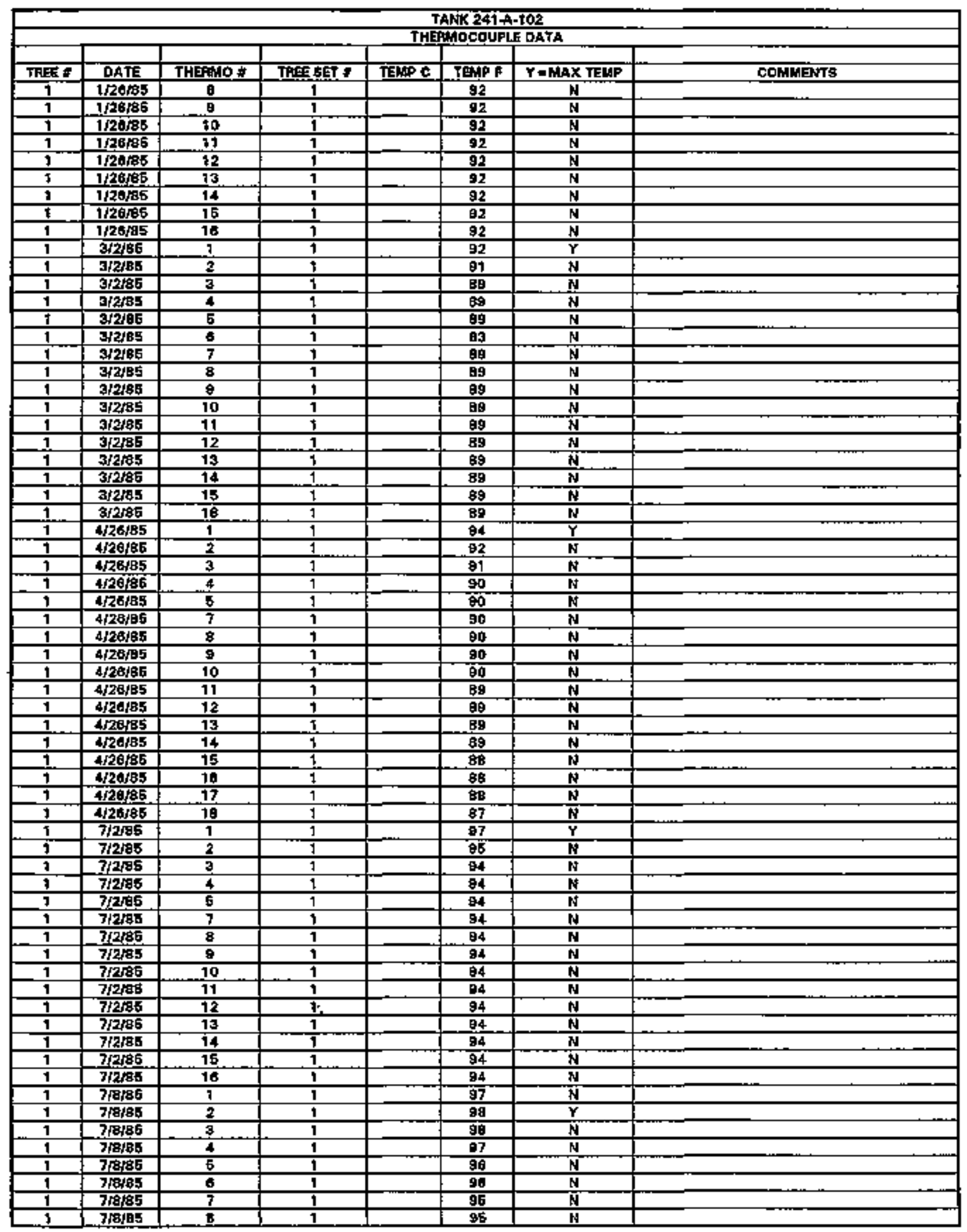

Data obtained from WHC Surveillance Analyzis Combuter Systers (SACS), June 2, 1993. 


\begin{tabular}{|c|c|c|c|c|c|c|c|}
\hline \multicolumn{8}{|c|}{$\frac{\text { TANKK 241.A-102 }}{\text { THEFYMOCOUPLE DATA }}$} \\
\hline TAEE * & DATE & THERMD * & TAEE SIT & TEMP G & TEHP F & Y WHAX TEGIF & COMWESTS \\
\hline 1 & 7/8 & $\theta$ & 1 & & 95 & $\mathbf{N}$ & \\
\hline 1 & 7 78:B55 & 10 & 1 & & 56 & N & \\
\hline 1 & 7 Fers & 11 & 3 & & 85 & \$ & \\
\hline 1 & $7 \mathrm{rer}$ & 12 & 9 & & 86 & $N$ & \\
\hline 1 & 2F/日as & 13. & 1 & & 99 & $\mathbf{N}$ & \\
\hline 1 & $76 / 85$ & 74 & 1 & & $8 \mathbf{B}$ & $\mathbf{N}$ & \\
\hline 1 & 7 Thes5 & 15 & 1 & & $\overline{\theta 0}$ & $\boldsymbol{N}$ & \\
\hline 1 & $7 \mathrm{AN5}$ & 18 & 1 & & 8 & $\mathbf{N}$ & \\
\hline 1 & $10 / 4 / 85$ & 1 & 1 & & 99 & $\mathbf{Y}$ & \\
\hline 1 & 10/4/as & 2 & 1 & & 97 & N & \\
\hline 7 & $10 / 4 / 85$ & 3 & 1 & & 98 & $\mathbf{N}$ & \\
\hline 1 & $10 / 4 / 85$ & 4 & 1 & & 90 & in & \\
\hline 1 & $10 / 4 / 85$ & 5 & 1 & & 98 & is & \\
\hline 7 & $10 / 4 / 8 \%$ & 7 & 1 & & 80 & $\mathbf{N}$ & \\
\hline 1 & $10 / 4 / 65$ & $\mathbf{B}$ & 1 & & 96 & $\mathbf{N}$ & \\
\hline$t$ & $10 / 485$ & 9 & 1 & & 95 & $\mathbf{N}$ & \\
\hline$t$ & 10/4/as & 10 & 1 & & 95 & $\vec{H}$ & \\
\hline $\mathbf{I}$ & $10 / 4 \sqrt{85}$ & 11 & 1 & & 95 & $\mathbf{N}$ & \\
\hline 1 & $1014 / 85$ & 12 & 1 & & 94 & $\mathbf{n}$ & \\
\hline 1 & 10448 & 13 & 1 & & 94 & $\mathbf{N}$ & \\
\hline 1 & $104 \sqrt{05}$ & 14 & 1 & & 84 & $\mathbf{N}$ & \\
\hline 1 & $10 / 4 / 65$ & 15 & 1 & & 93 & $\mathbf{N}$ & \\
\hline$\overline{1}$ & 10/4/15 & 18 & 1 & & 93 & H & \\
\hline 1 & $10 \mathrm{~d} / \mathrm{j}$-5 & 17 & $T$ & & B2 & $\mathbf{N}$ & \\
\hline 1 & 10485 & 18 & 1 & & 91 & $\mathbb{N}$ & \\
\hline 1 & 12/1365 & 1 & 1 & & B6 & $\mathbf{Y}$ & \\
\hline 1 & 121385 & 2 & 1 & & 84 & $\mathbf{N}$ & \\
\hline 1 & $1213 / 85$ & 3 & 1 & & 본 & $\mathbf{N}$ & \\
\hline 1 & 1213135 & 4 & 1 & & 82 & $N$ & \\
\hline 1 & $1 2 \longdiv { 1 3 9 5 }$ & 5 & 7 & & 92 & $\mathbf{N}$ & \\
\hline 1 & $12 / 1385$ & 7 & 1 & & D2 & N & \\
\hline 1 & $1213 / 35$ & B & 1 & & 92 & $\mathbf{N}$ & \\
\hline 7 & 1213185 & 9 & $\overline{1}$ & & 是 & $\mathbf{N}$ & \\
\hline$t$ & 12/13B5 & I0 & 1 & & 97 & M & \\
\hline 1 & $12 / 13 / 85$ & 17 & 1 & & 87 & $\mathbf{N}$ & \\
\hline 1 & 12/toles & 12 & 1 & & $8 t$ & $\mathbf{N}$ & \\
\hline 1 & $12 / 13 / 85$ & 13 & 1 & & 81 & N & \\
\hline 1 & 1211365 & 14 & 1 & & so & $\mathbf{N}$ & \\
\hline 1 & $12113 / 65$ & 18 & 1 & & 80 & $\mathbf{N}$ & \\
\hline 1 & 12h3画 & 18 & 1 & & 89 & $\mathbf{N}$ & \\
\hline 1 & $12113 \sqrt{6}$ & 17 & 1 & & 89 & $\sqrt{N}$ & \\
\hline 1 & J2月3-65 & 18 & $\overline{1}$ & & 68 & $\mathbf{N}$ & \\
\hline 1 & IBbad & 1 & 1 & & 106 & $\bar{Y}$ & \\
\hline 1 & 10 1989 & 2 & 1 & & 104 & $\mathbf{N}$ & \\
\hline 1 & 1/868 & 3 & 1 & & 103 & $\mathbf{N}$ & \\
\hline 1 & 1 Bese & 4 & 1 & & 103 & $\mathbf{N}$ & \\
\hline 1 & 1, & 5 & 1 & & 102 & $\mathbf{N}$ & \\
\hline 1 & $1 / 0100$ & 7 & 1 & & 103 & $\mathbf{N}$ & \\
\hline 1 & 18B日e & 8 & 1 & & 102 & N & \\
\hline 1 & 16186 & 9 & 1 & & 103 & $\mathbf{N}$ & \\
\hline 1 & 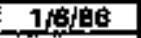 & 10 & 1 & & 103 & $\mathrm{H}$ & \\
\hline 1 & $16 \sqrt{60}$ & 11 & 1 & & 103 & $\mathbf{N}$ & \\
\hline 1 & $1 \mathrm{~B}-\mathrm{AB}$ & 12 & $1.0^{\circ}$ & & 102 & $\mathbf{N}$ & \\
\hline 1 & 1/B & 13 & 1 & & 102 & $\mathbf{H}$ & \\
\hline 1 & $10 \mathrm{BOB}$ & 14 & 1 & & 102 & $\mathbf{N}$ & \\
\hline 1 & 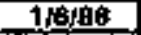 & 15 & 1 & & 102 & $\mathbf{H}$ & \\
\hline 1 & 16100 & 16 & 1 & & 102 & $\mathbf{N}$ & \\
\hline 1 & $2 / 3 / 18$ & $t$ & 1 & & 94 & $Y$ & \\
\hline 1 & $2 / 3190$ & 2 & 1 & & 92 & $\mathbf{N}$ & \\
\hline 1 & $2 \sqrt{2} \mathrm{se \theta}$ & 3 & 1 & & 91 & $\mathrm{~N}$ & \\
\hline 1 & $2 / 3 / 8 B$ & 4 & 1 & & 만 & $\mathbf{N}$ & \\
\hline 1 & $28 / 86$ & 5 & 1 & & 80 & $\mathbf{N}$ & \\
\hline 1 & $2 / 3 / B E$ & 7 & 1 & & 80 & $\mathbf{N}$ & \\
\hline 1 & $2 / 3106$ & 8 & 1 & & 90 & $\mathbf{N}$ & \\
\hline 1 & $213 \sqrt{106}$ & 8 & 1 & & 90 & $\mathbf{N}$ & \\
\hline
\end{tabular}

Date obtained from WHC Surveilance Anelyeis Compiuter Syetern (SACS), June 2,1993. 


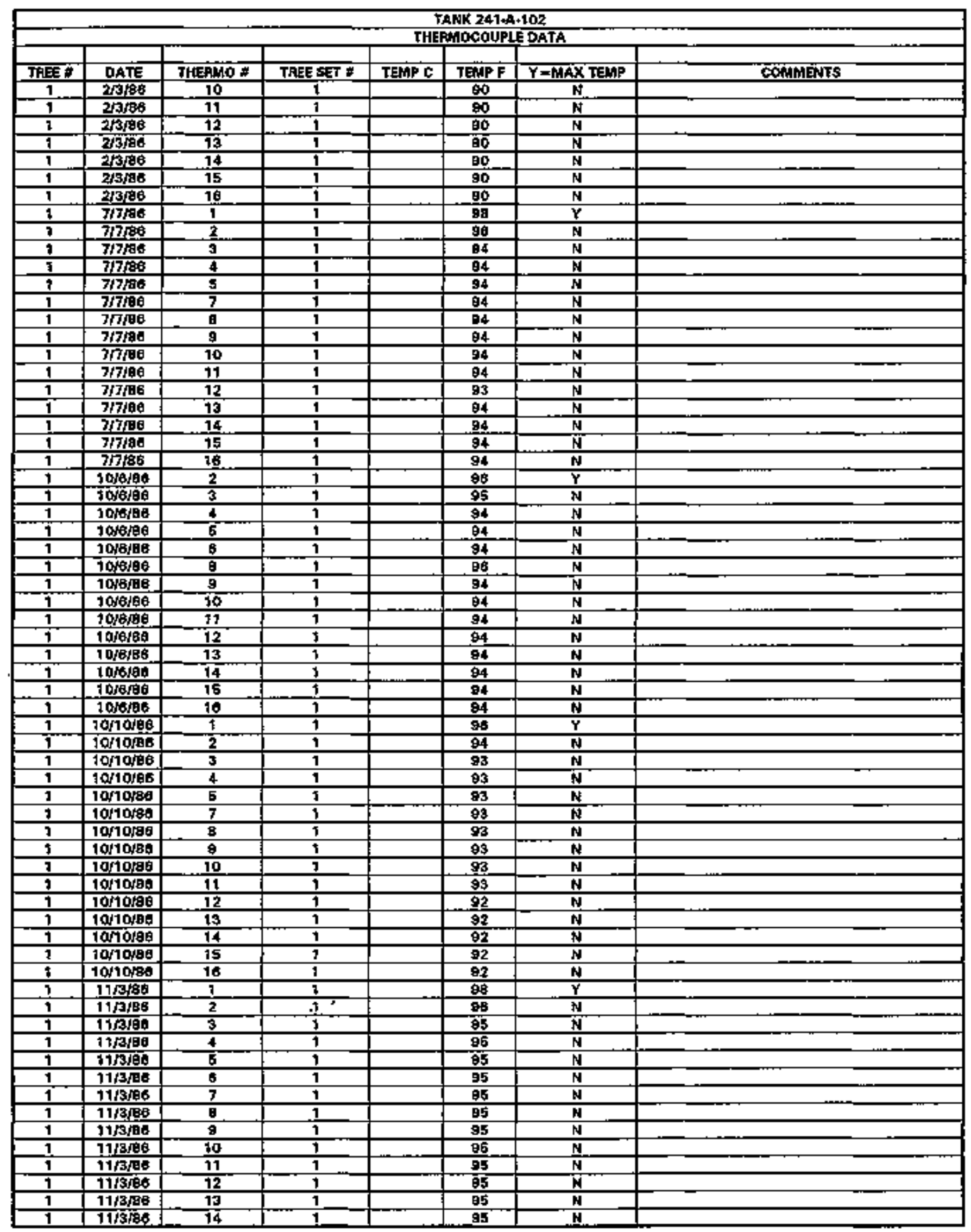

Data obtained from WHC Survelliance Analyefa Camputer System (SACS), June 2,1993. 


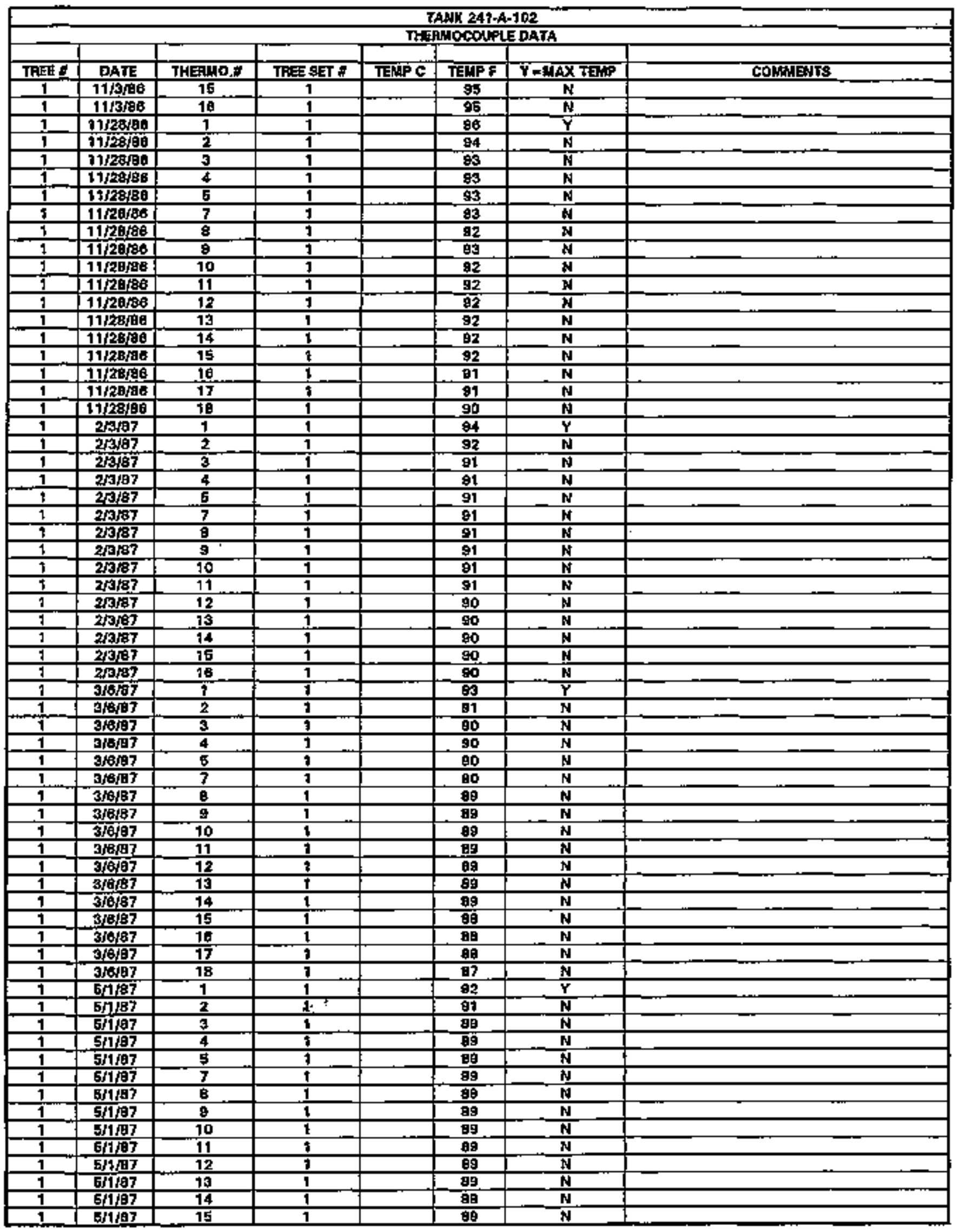

Data obtained from WHC Surveillance Anolyela Computer Systam (SACS), June 2,1993. 


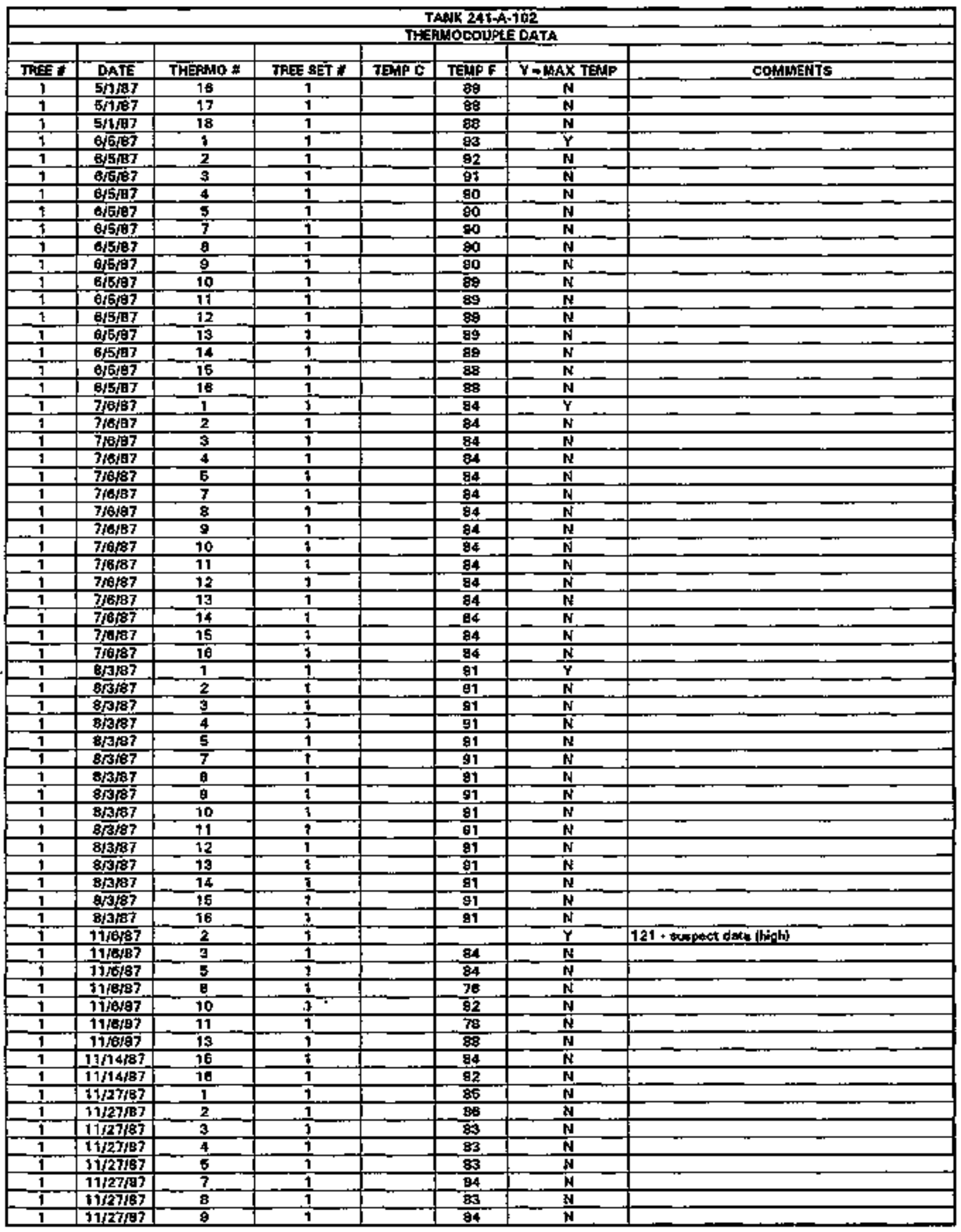

Dets abtained fram WHC Survellance Andyois Computer System (SACS), June 2,1993. 


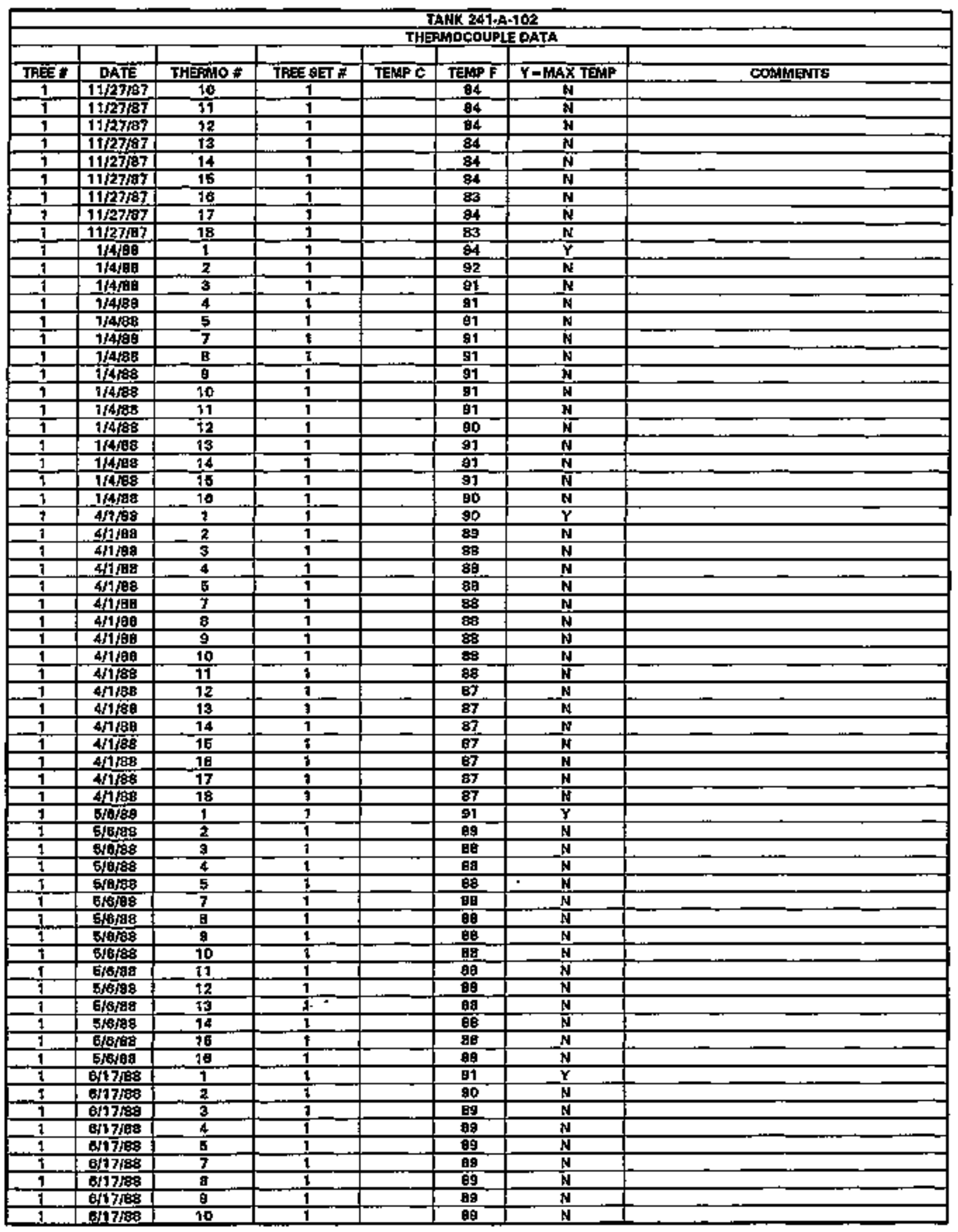

Date obtained from WHC Surveillance Anelyais Computer System [SACS], whne 2,1993. 


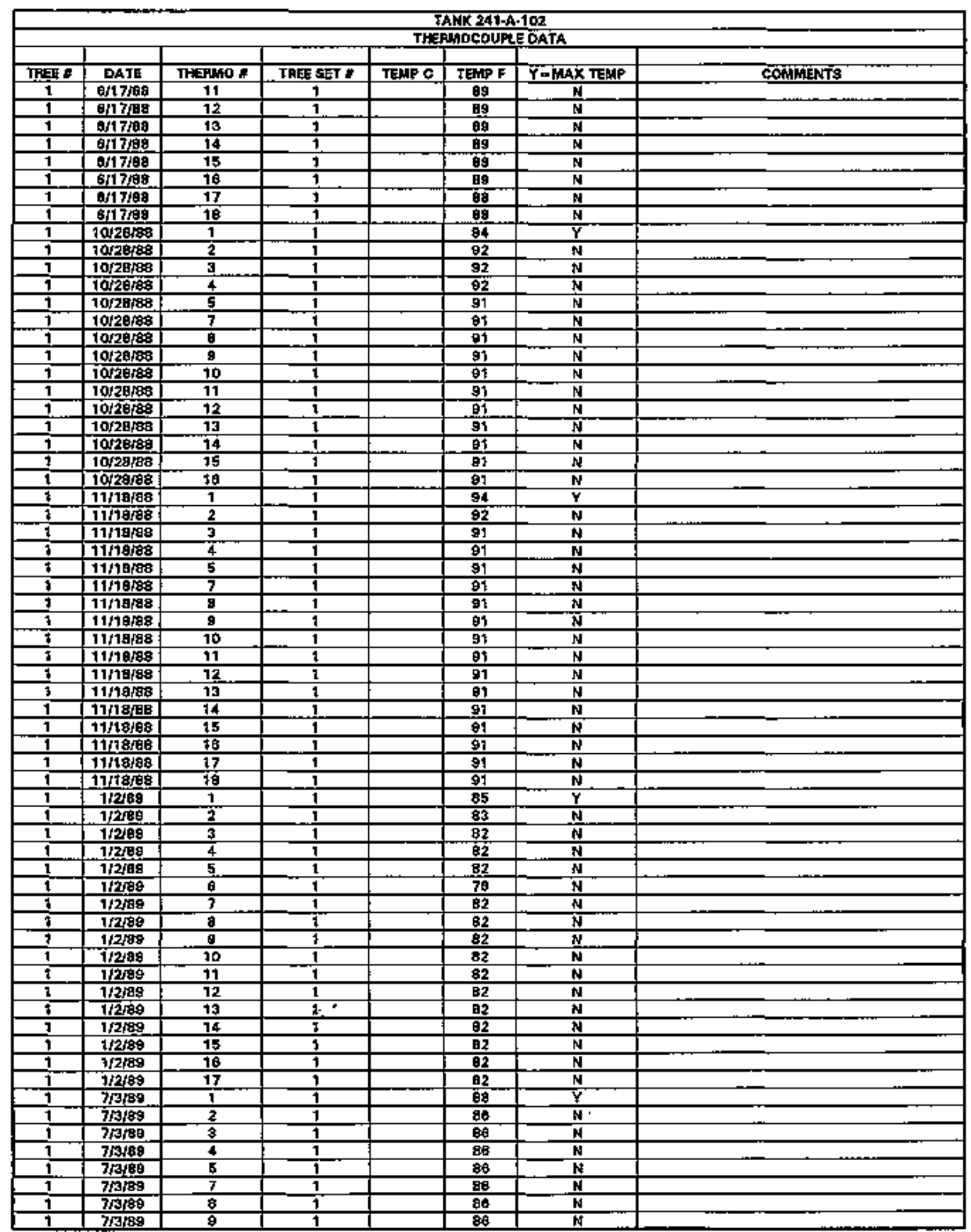

Dats obtained from WHC Survaillence Anelysis Computer Spatem ISACSI, June 2, 993. 


\begin{tabular}{|c|c|c|c|c|c|c|c|}
\hline \multicolumn{8}{|c|}{ TAMK 247-A.102 } \\
\hline & & & & JHE & 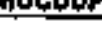 & DATA & \\
\hline ZHEE 䄅 & DATE & THEFIN A & ThEE SET * & 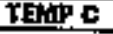 & TEMPF F & $Y=$ MAX TEMP & COMHENTS \\
\hline 1 & 713 wa9 & 10 & 1 & & $\overline{\mathrm{ag}}$ & $\mathbf{N}$ & \\
\hline 1 & $7 / 3 / 10$ & 11 & 1 & & BB & $\mathbf{N}$ & \\
\hline 1 & 7 73a日 & 12 & 1 & & 要官 & $\mathbf{N}$ & \\
\hline 1 & $7 / 3 / 69$ & 13 & 1 & & B6 & $\mathbf{N}$ & \\
\hline 1. & $7 / 389$ & 14 & 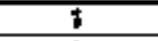 & & B8 & N & \\
\hline 1 & $7 / 3 / 89$ & 15 & 1 & & 88 & is & \\
\hline 1 & $7 / 3 / 89$ & 10 & 1 & & 86 & it & \\
\hline 1 & $1 / 1 / 90$ & 1 & 1 & & 92 & $\bar{Y}$ & \\
\hline 1 & $1 / 1 / 90$ & 2 & 1 & & 89 & $N$ & \\
\hline 1 & $1 / 1 / 90$ & 3 & 1 & & 99 & $\mathbf{N}$ & \\
\hline 1 & $1 / 1 / 90$ & 4 & 1 & & 90 & क्षे & \\
\hline 1 & $1 / 1 / 1 / 00$ & E & 1 & & 99 & $N$ & \\
\hline 1 & 1/1/90 & 7 & 1 & & 昰 & N & \\
\hline 1 & $111 / 90$ & $\mathbf{B}$ & 1 & & 89 & $\mathbf{N}$ & \\
\hline 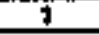 & $1 / 1 / \mathrm{g}^{2}$ & $\theta$ & 1 & & 的 & $\mathbf{N}$ & \\
\hline 1 & $1 / 1 / 90$ & 10 & 1 & & 99 & $\mathbf{N}$ & \\
\hline 1 & $1 / 1 / 1 / 90$ & 11 & 1 & & 99 & $\mathbf{N}$ & \\
\hline 1 & $1 / 1 / 90$ & 12 & 1 & & 60 & N & \\
\hline 1 & 1/N/go & 13 & 1 & & 69 & $\mathbf{N}$ & \\
\hline 1 & $1 / 1 / 100$ & 14 & 1. & & Bg & $\mathbf{N}$ & \\
\hline 1 & 1/1/90 & 15 & 1 & & 89 & $\mathbf{N}$ & \\
\hline 1 & $1 / 1 / \theta_{0}$ & 16 & 1 & & B9 & $\mathbf{N}$ & J-930523512-47 TC 15 ND GODO \\
\hline 1 & $11 / 12 / 81$ & 1 & 1 & & 91.5 & $\mathbf{Y}$ & \\
\hline 1 & $11 / 12 / 91$ & 2 & 1 & & 89.5 & $\mathbf{N}$ & \\
\hline 1 & $11 / 12191$ & 3 & 1 & & $\theta, \hat{B}$ & $\mathbf{N}$ & \\
\hline 1 & j) 12年1. & 4 & 1 & & 89.5 & $\mathbf{n + 2}$ & \\
\hline 1 & $11 / 12 / 91$ & 5 & 4 & & 69.5 & $\mathbf{N}$ & \\
\hline 1 & $51 / 2197$ & 7 & 5 & & 89.8 & N & \\
\hline 1 & 7\%/12/91 & 8 & 1 & & 99.4 & N & \\
\hline 1 & 1W1219 & 9 & 9 & & 98,5 & $\mathbf{N}$ & \\
\hline 1 & ttf129) & 10 & 1 & & E9.5 & $\mathbf{N}$ & \\
\hline 1 & $11 / 12 / 91$ & 11 & 1 & & 89,4 & is & \\
\hline 1 & $51 / 1290$ & 12 & 1 & & 白. & it & \\
\hline 1 & $6 \% / 12 \% 9$ & 13 & 1 & & 69,4 & H & \\
\hline 1 & 12/297 & 14 & 1 & & Bg.4.4 & ii & \\
\hline 1 & $1 / 1 / 1291$ & 16 & 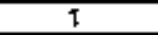 & & 89,3 & $\mathbf{N}$ & \\
\hline 1 & 1th219t & 10 & 1 & & 百.2.2 & $\mathbf{N}$ & \\
\hline 1 & $11 / 12 \%$ & 17 & 1 & & 89,3 & $\mathbf{N}$ & \\
\hline 1 & 11/269 & 18 & 9 & & 89.2 & $\mathbf{M}$ & J.610126143-29 TC \#A a/5 \\
\hline 1 & $1 / 1 / 92$ & 1 & 1 & & 81.8 & $\mathbf{Y}$ & \\
\hline 1 & $1 / 1 / 92$ & 2 & 1 & & 69.2 & $\mathbf{H}$ & \\
\hline $\mathbf{1}$ & 1/1/A2 & 3 & 1 & & 69,4 & $\mathbf{N}$ & \\
\hline 1 & 1/102 & 4 & $i$ & & 89.3 & $\mathbf{N}$ & \\
\hline 1 & $1 / 2 / 92$ & 5 & 1 & & 89.2 & $\mathbf{N}$ & \\
\hline 1 & $1 / 2 \mathrm{e} 2$ & 7 & 1 & & 89.2 & $\mathbf{N}$ & \\
\hline 1 & $1 / 1 / 92$ & 旦 & 1 & & 89.7 & $\mathbf{N}$ & \\
\hline 7 & 1 h/en & 9 & 1 & & 89.2 & $\mathbf{N}$ & \\
\hline 7 & $1 / 9 / 92$ & 10 & 1 & & 99.2 & $\mathbf{N}$ & \\
\hline 1 & $1 / 2182$ & 11 & 1 & & 89.1 & N & \\
\hline $\bar{t}$ & $1 / 2+2$ & $\sqrt{2}$ & 1 & & 88.8 & $\mathbf{N}$ & \\
\hline$i$ & $1 / 3 / 92$ & 13 & 1 & & 89.1 & $\mathbf{N}$ & \\
\hline \pm & $1 / 2 \sqrt{92}$ & $\$ 4$ & 1 & & 99 & $\mathbf{N}$ & \\
\hline 1 & $1 / 2 / 92$ & I5 & 1 & & 㿟 & $\mathbf{N}$ & \\
\hline$T$ & $1 / 1 / 92$ & 19 & 1 & & 98 & N & \\
\hline 7 & $10 / 92$ & 17 & 1 & & ab.9. & $\mathbf{N}$ & \\
\hline 7 & $1 / 1 / 92$ & 10 & 1 & & 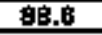 & $N$ & J $890023528-80$ \\
\hline$\overline{1}$ & $4 / 902$ & 1 & 9 & & 90,0 & $\bar{\gamma}$ & \\
\hline$\overline{1}$ & $49 \sqrt{92}$ & 2 & 1 & & 89.7 & $\overline{\mathbf{N}}$ & \\
\hline$\overline{1}$ & $4 \sqrt{9 \sqrt{62}}$ & $\mathbf{a}$ & 1 & & 明.1 & $\mathbf{N}$ & \\
\hline 1 & 4/2/a2 & 4 & 1 & & 86.1 & $\mathbf{N}$ & \\
\hline 1 & $4 / 9 / 92$ & 5 & 1 & & 88 & $\mathbf{H}$ & \\
\hline 1 & $4 /$ fen $_{2}$ & 7 & 1 & & Ba.1 & $\mathbf{n}$ & \\
\hline 1 & $4 \sqrt{0 / 42}$ & 息 & 1 & & 89 & $\mathbf{N}$ & \\
\hline 1 & $4 / 9 / 92$ & 9 & 1 & & 88 & $\mathbf{N}$ & \\
\hline 1 & 4062 & 10 & $\bar{y}$ & & 88 & $\mathbf{H}$ & \\
\hline
\end{tabular}

Data obtained from WHC Survallanca Analvsis Computer SYctem (SACS), June 2,1993. 


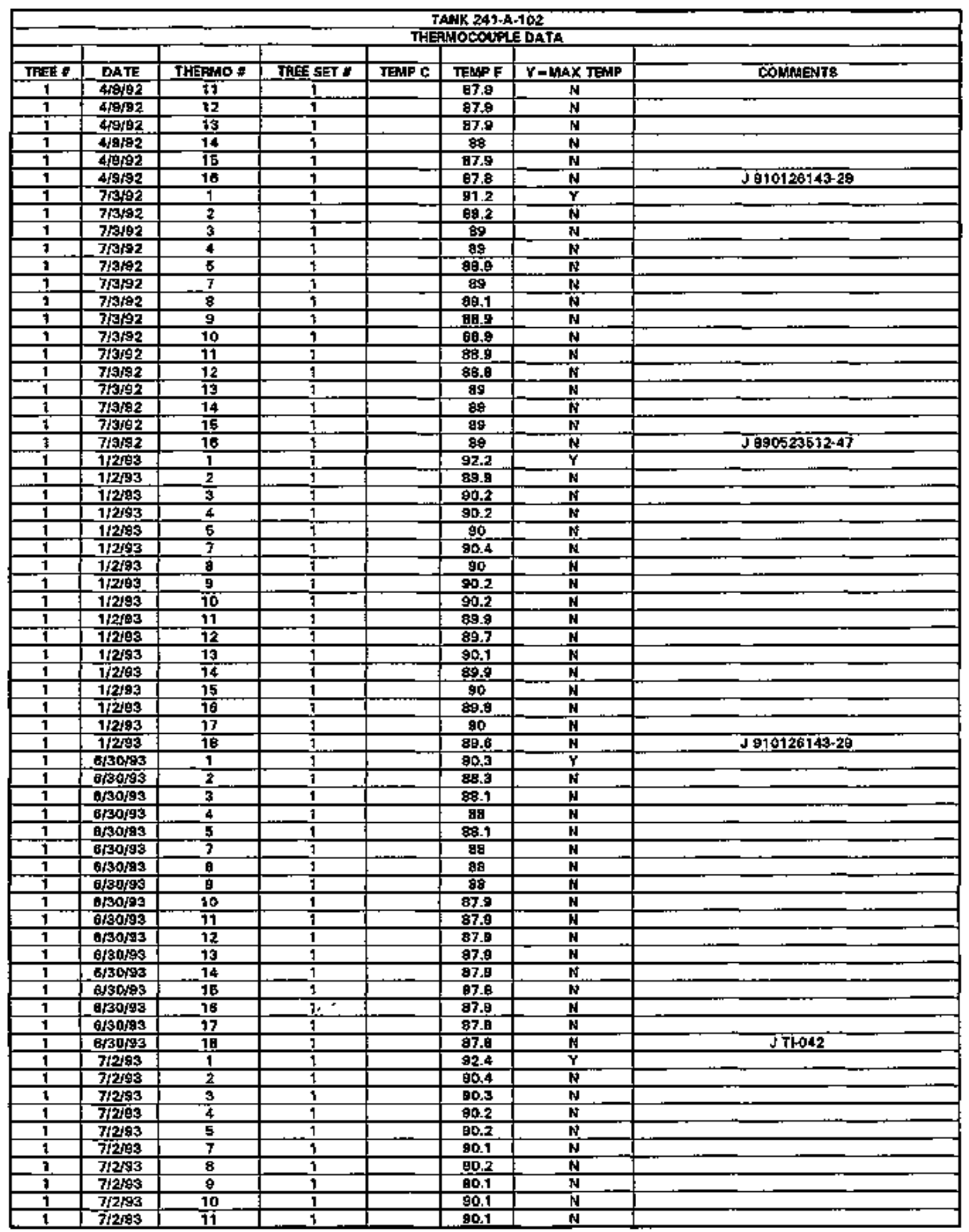

Data obtainạd from WHC Survaillance Analysis Computer Syatam (SACSI, June 2,1993. 
WHC-SD-WM-ER-30B, Rav. O

\begin{tabular}{|c|c|c|c|c|c|c|c|}
\hline & & & & & NK 247. & 102 & \\
\hline & & & & THE & HOCOUP & DATA & \\
\hline Thi丶t & DATE & EHFANO * & TREE SET \# & TEHP G & TENP F & $Y=$ MAX TEMP & COAMENTS \\
\hline 1 & 72193 & 12 & 1 & & so & $N$ & \\
\hline 1 & $7 / 2 / 93$ & $1 \overline{3}$ & $\overline{1}$ & & 80 & $\mathbf{N}$ & \\
\hline$\overline{1}$ & $7 / 2 / 03$ & 14 & 1 & & 00.1 & $\mathbf{N}$ & \\
\hline 1 & $7 / 2 / 3$ & $1 \overline{5}$ & 7 & & 69.8 & $\overline{\mathrm{M}}$ & \\
\hline 2 & $7 / 2493$ & 10 & 5 & & 00 & $\mathbf{N}$ & \\
\hline 1 & $\overline{7 / 2 / 93}$ & 17 & $\overline{1}$ & & 89.9 & $\mathbf{N}$ & \\
\hline 1 & $7 / 2 / 93$ & 18 & 1 & & 899 & $\mathbf{N}$ & \\
\hline & & & & & & & \\
\hline & & & & & & & \\
\hline
\end{tabular}

$\therefore$

Date obtained from WHC Survellente Afralyais Computer Syetem (SACS, June 2,1993,

D-203 


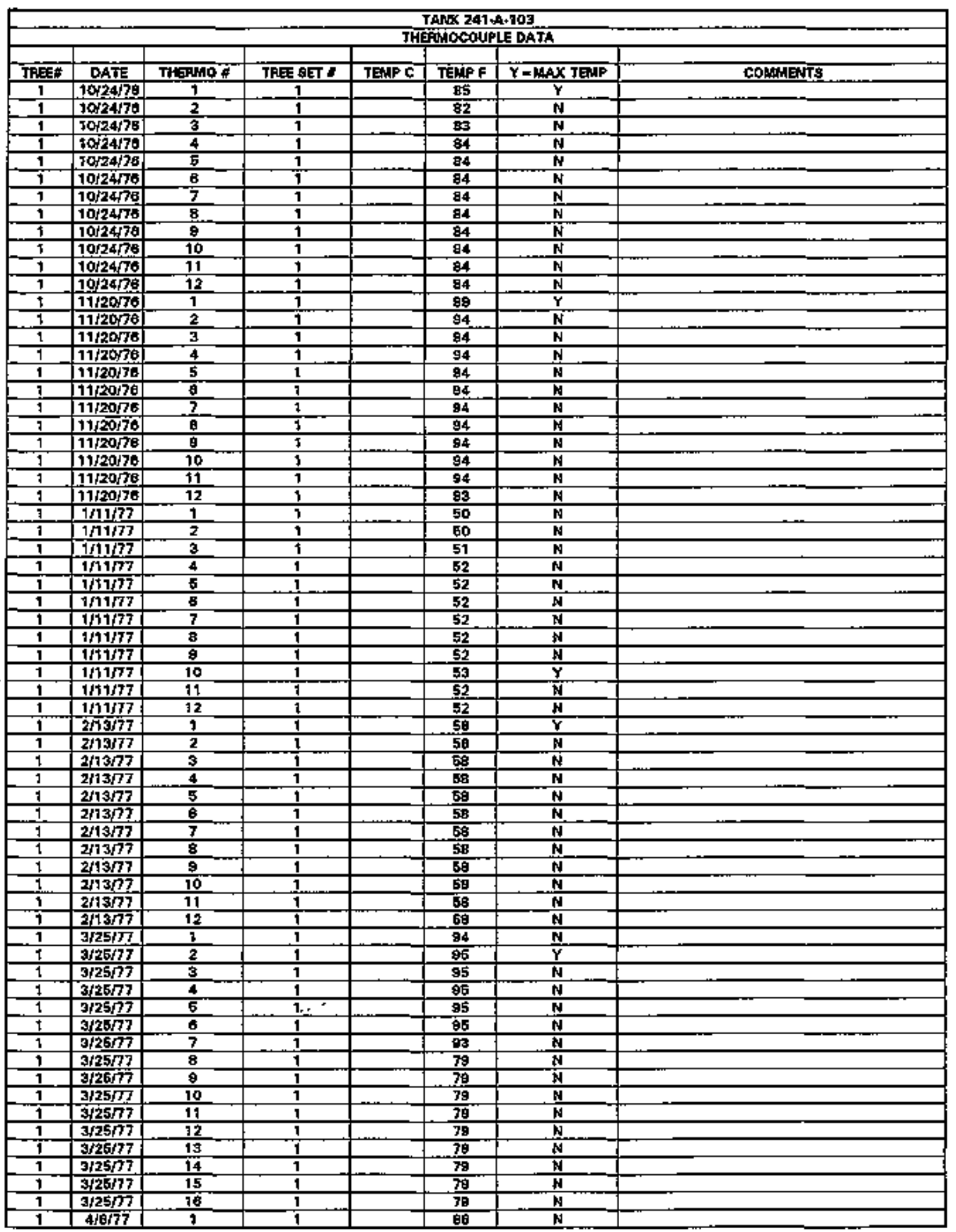

Data obtained from WHC Surveillence Analyais Computer System (SACS), June 2,199:3. 


\begin{tabular}{|c|c|c|c|c|c|c|c|}
\hline \multicolumn{8}{|c|}{ TANK 241-A-103 } \\
\hline & & & & & & & \\
\hline ThExt & DATE & THEFLO & TREE EET & Tethe & TEMPF F & $Y=\| A X$ TEAP & COMNENTS \\
\hline 1 & sib77 & 2 & 1 & & 87 & $Y$ & \\
\hline 1 & $4 / 977$ & 3 & 1 & & 67 & $\bar{N}$ & \\
\hline 1 & 4 (16/77 & 4 & 1 & & 97 & H & \\
\hline 1 & $4 / 007$ & 6 & 1 & & 日7 & $\mathbf{N}$ & \\
\hline 1 & $4 \sqrt{77}$ & 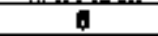 & 1 & & 67 & N & \\
\hline 1 & 4lom & 7 & 1 & & B日9 & $\mathbf{N}$ & \\
\hline 1 & $4 \sqrt{77}$ & a & 1 & & 72 & N & \\
\hline 1 & $4 \sqrt{27}$ & 8 & 1 & & 71 & $N$ & \\
\hline 1 & $4 \sqrt{0} 77$ & 70 & 1 & & 71 & $\bar{N}$ & \\
\hline 1 & $4 / \sqrt{6 / 7}$ & 11 & 1 & & 71 & $\mathrm{~N}$ & \\
\hline 1 & $410 \%$ & 52 & 1 & & 71 & $\mathbf{N}$ & \\
\hline 1 & $4 / 0 / 77$ & 13 & 1 & & 71 & 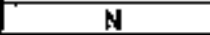 & \\
\hline 1 & 4 4lin & 14 & 1 & & 71 & $\mathrm{~N}$ & \\
\hline 1 & $4 0 \longdiv { 7 7 }$ & 15 & 1 & & 71 & $\mathbf{N}$ & \\
\hline 1 & $4 / 6 / 27$ & 18 & 1 & & 77 & $\mathbf{N}$ & \\
\hline$j$ & El2977 & 1 & 1 & & 80 & $\overline{\mathbf{Y}}$ & \\
\hline 1 & $523 \sqrt{77}$ & 2 & 1 & & 72 & $N$ & \\
\hline 1 & $5 / 28477$ & 3 & 1 & & 73 & $N$ & \\
\hline 7 & $5 / 2877$ & 4 & $\overline{1}$ & & 73 & $N$ & \\
\hline 1 & $5 / 2877$ & 5 & 1 & & 73 & $N$ & \\
\hline 7 & 528177 & $\bar{b}$ & $\pi$ & & 73 & $N$ & \\
\hline 1 & $5 \sqrt{28} / 77$ & 7 & 1 & & 73 & $\mathbf{N}$ & \\
\hline 1 & $526 / 77$ & 8 & \pm & & 74 & $\bar{M}$ & \\
\hline 1 & $521 / 77$ & 8 & 1 & & 74 & N & \\
\hline 1 & $5 / 26177$ & 10 & 1 & & 74 & $\mathbf{N}$ & \\
\hline 1 & S/2日/77 & 11 & 1 & & 74 & $\bar{N}$ & \\
\hline 1 & $5 / 2007$ & 12 & 1 & & 74 & $\mathbf{N}$ & \\
\hline 1 & $6 / 28 n 7$ & 13 & $T$ & & 74 & $\mathbf{N}$ & \\
\hline 1 & 512877 & 14 & 1 & & 74 & N & \\
\hline 1 & Ehon7 & 1 & 1 & & 78 & $Y$ & \\
\hline 1 & Mar7 & 2 & 1 & & 74 & $\bar{N}$ & \\
\hline 1 & Ghor7 & 3 & 1 & & 74 & $\mathbf{N}$ & \\
\hline 1 & E1077 & 4 & $i$ & & 74 & $\bar{N}$ & \\
\hline 1 & Ehloh77 & 5 & 1 & & 74 & $\mathbf{N}$ & \\
\hline 1 & प1077 & $\dot{\theta}$ & 1 & & 74 & T & \\
\hline 1 & 6hor7 & $\overline{7}$ & 7 & & 74 & $\mathbf{N}$ & \\
\hline 1 & 日1077 & $\overline{8}$ & 1 & & 74 & $\mathrm{~N}$ & \\
\hline 7 & $6 / 1007$ & g & 1 & & 74 & I & \\
\hline 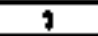 & $0 / 10 \overline{77}$ & 10 & 1 & & 74 & W & \\
\hline 7 & o/10ry & 11 & $t$ & & 74 & $N$ & \\
\hline 1 & 01077 & 12 & 1 & & 74 & $\mathbf{N}$ & \\
\hline 7 & 061007 & 13 & 1 & & 74 & $\bar{N}$ & \\
\hline$T$ & $6 / 177$ & 14 & 1 & & 74 & $\mathbf{N}$ & \\
\hline 1 & $810 \mathrm{ph}$ & 15 & 1 & & 74 & $\mathbf{N}$ & \\
\hline$T$ & dian & 70 & 1 & & 74 & $\mathbf{N}$ & \\
\hline 1 & $8 / 11 / 77$ & 1 & 1 & & 118 & $N$ & \\
\hline 1 & a/11/77 & 2 & 1 & & 118 & $\mathrm{~N}$ & \\
\hline 1 & $9 / 11 / 7$ & 3 & 1 & & 118 & $\mathbf{N}$ & \\
\hline$T$ & มั11/77 & 4 & 1 & & 123 & $\mathbf{N}$ & \\
\hline 1 & gil1m7 & 5 & $i$ & & 125 & $\bar{Y}$ & \\
\hline 1 & $8[11 / 77$ & 8 & 1 & & 125 & $\mathbf{N}$ & \\
\hline 1 & a/1/77 & 7 & 1 & & 121 & $\bar{N}$ & \\
\hline 1 & gilin & 百 & $1 ., \cdot$ & & 109 & $\mathrm{~N}$ & \\
\hline 1 & ar11/77 & $\bar{a}$ & 1 . & & 81 & $\mathbf{N}$ & \\
\hline 1 & $8 / 1177$ & 70 & 1 & & 90 & $\bar{N}$ & \\
\hline 1 & $8 / 11 / 77$ & 11 & 1 & & 90 & $\mathbf{N}$ & \\
\hline 1 & $911 \overline{77}$ & 12 & 1 & & 80 & $N$ & \\
\hline 1 & 9/11/72 & 13 & 1 & & 80 & $\mathrm{~N}$ & \\
\hline 1 & M/1/77 & 14 & 1 & & 6 & $\bar{N}$ & \\
\hline 1 & $8 / 1 \notin 77$ & 1 & 1 & & 111 & $\mathbf{N}$ & \\
\hline 1 & 1/18/77 & 2 & 1 & & 112 & $\mathrm{~N}$ & \\
\hline 1 & 91977 & 3 & 1 & & 114 & $\mathrm{~N}$ & \\
\hline 1 & 8918:77 & 4 & 1 & & 117 & W & \\
\hline 1 & $8 / 1977$ & 5 & 1 & & 118 & $Y$ & + \\
\hline 1 & gile7n & $\bar{B}$ & 1 & & 118 & $\mathrm{~N}$ & \\
\hline
\end{tabular}

Data abteined fram WHC Survaillance Analyaí Computer Systent (SACS\}. Juna 2,1999. 


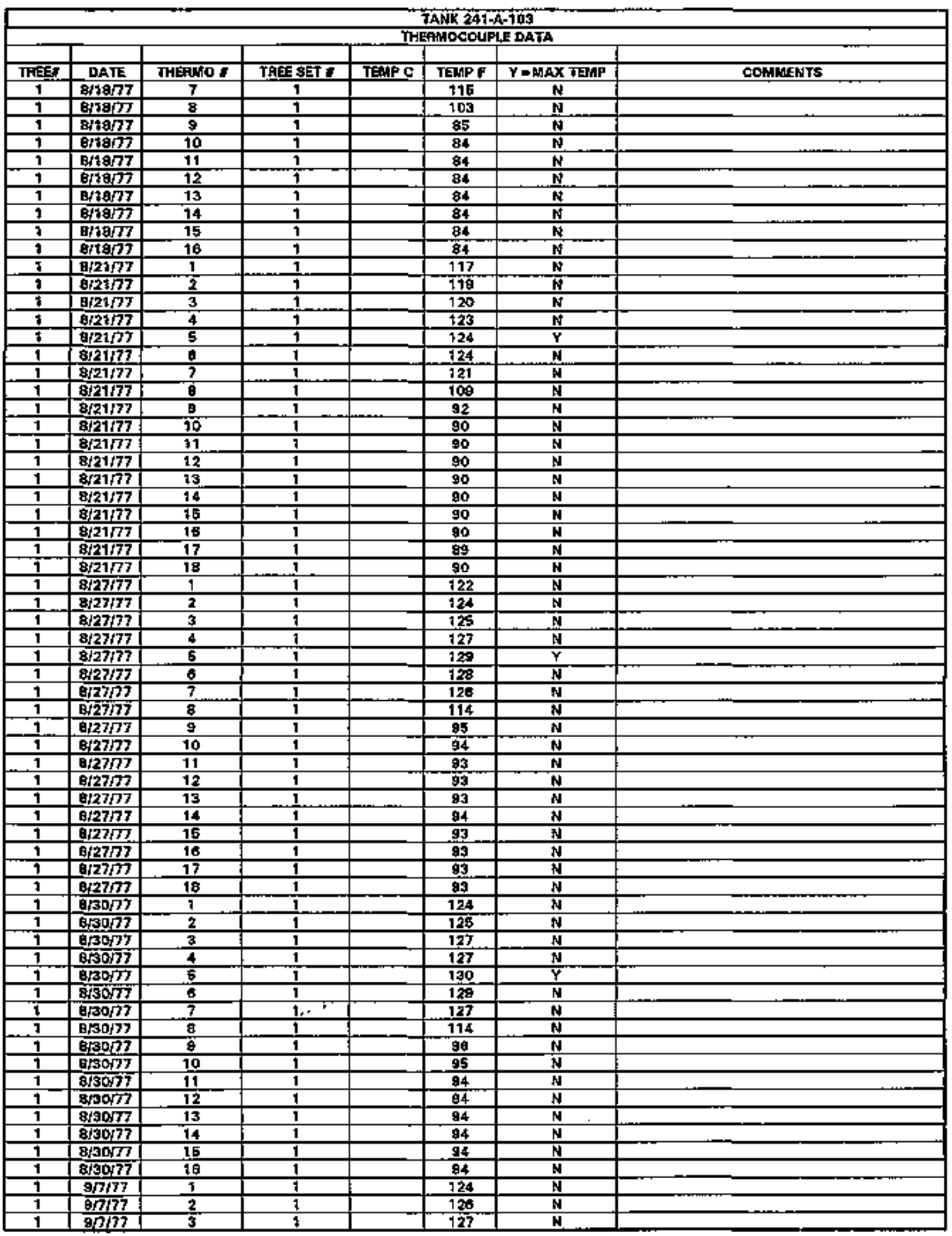

Date abtalned from WHC Surveillance Anelysis Computer System (SACS), June 2,1093. 


\begin{tabular}{|c|c|c|c|c|c|c|c|}
\hline \multicolumn{8}{|c|}{ 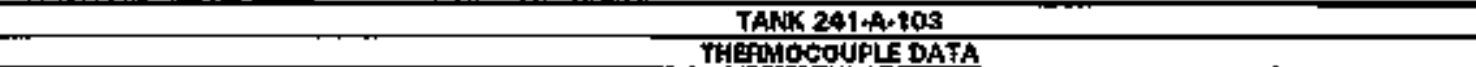 } \\
\hline & & & & & & & \\
\hline TFEET: & DATE & 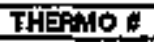 & TREA EET $n$ & TEMPC & TENPF & $Y$-HAX TEMP & COALISITS \\
\hline 1 & $8 / 7 / 77$ & 4 & $t$ & & 128 & $\mathrm{Y}$ & \\
\hline$\frac{1}{1}$ & bi7/77 & 5 & 1 & & 120 & $N$ & \\
\hline 1 & $8 / 777$ & $\theta$ & 1 & & $\$ 27$ & $\bar{N}$ & \\
\hline 1 & $8 / 7 \sqrt{77}$ & 7 & 1 & & $t 27$ & $\mathrm{~N}$ & \\
\hline 1 & 9/7/7 & 8 & $\frac{1}{1}$ & & 114 & $\mathrm{~N}$ & \\
\hline 1 & $9 / 7 / 77$ & 9 & 1 & & 86 & $\bar{N}$ & \\
\hline 1 & $9 / 7 / 77$ & 10 & 1 & & 94 & $\mathbf{N}$ & \\
\hline 1 & $9 \sqrt{7 / 7}$ & 11 & 1 & & 84 & $\pi$ & \\
\hline 1 & 9/7/7 & 12 & 1 & & 94 & $\mathbf{N}$ & \\
\hline 1 & $9 / 7 / 77$ & 13 & 1 & & 94 & $\mathbf{M}$ & \\
\hline 1 & 9/7m & 14 & 1 & & 94 & N & \\
\hline 1 & 9/7h & 15 & 1 & & 94 & N & \\
\hline 1 & $9 / 7 / 7$ & $1 \overline{\mathrm{e}}$ & 1 & & 94 & $\bar{N}$ & \\
\hline 1 & $9 / 12 / 77$ & 1 & 1 & & 122 & H & \\
\hline 1 & $0 / 1277$ & $\frac{1}{2}$ & $i$ & & 124 & $\mathrm{~N}$ & \\
\hline 1 & $0 / 1277$ & 3 & 1 & & 125 & $\mathbf{N}$ & \\
\hline 9 & $0 / 12 / 77$ & 4 & $i$ & & 126 & $\bar{Y}$ & \\
\hline 1 & $9 / 1277$ & 5 & 1 & & 124 & $\bar{N}$ & \\
\hline 1 & $9 / 12 / 7)$ & 6 & 5 & & 124 & $\mathbf{N}$ & \\
\hline 1 & $0 / 12 / 77$ & 7 & 7 & & 124 & $\mathbf{N}$ & \\
\hline 9 & $9 / 1277$ & B & 1 & & 124 & $\mathbf{N}$ & \\
\hline 1 & 91207 & 8 & \pm & & 111 & $\mathbf{N}$ & \\
\hline 1 & $9 / 1277$ & 10 & 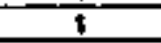 & & 94 & $N$ & \\
\hline 1 & 배래 & 11 & $t$ & & 92 & $\mathbf{N}$ & \\
\hline 1 & $9 / 12 / 77$ & $1 \overline{2}$ & 1 & & 92 & $\mathbf{k}$ & \\
\hline 1 & 9h12m & 13 & 1 & & 92 & $*$ & \\
\hline 1 & 9/12/77 & 14 & 1 & & 92 & $\mathrm{~N}$ & \\
\hline 1 & $9 / 12 / 77$ & 15 & 1 & & 82 & $\mathrm{n}$ & \\
\hline 1 & $9 / 12 / 77$ & 18 & 1 & & 92 & $N$ & \\
\hline 1 & $9 / 21 / 77$ & 1 & 1 & & 127 & 4 & \\
\hline 1 & $9 / 21 / 7$ & $\overline{2}$ & 1 & & 128 & $N$ & \\
\hline 1 & $9 / 21177$ & 3 & 1 & & 129 & $\mathrm{~N}$ & \\
\hline 1 & $9 / 21 / 77$ & 4 & 1 & & 130 & $\overline{1}$ & \\
\hline 1 & $9 / 21 / 77$ & $E$ & 1 & & 129 & $\bar{N}$ & \\
\hline 1 & $9 / 21 / 77$ & $\bar{\theta}$ & 1 & & 137 & 7 & \\
\hline 7 & $9 / 21 / D 7$ & $z$ & 1 & & 127 & $\mathbf{N}$ & \\
\hline 1 & 9/21/77 & 8 & 1 & & 114 & M & \\
\hline 1 & $9 / 21 / 07$ & 8 & 1 & & 87 & $\mathbb{N}$ & \\
\hline 1 & 8/21/77 & 10 & 1 & & 95 & A & \\
\hline 1 & $9 / 21 / 07$ & $1 \overline{1}$ & 1 & & 85 & $\bar{N}$ & \\
\hline 1 & $9 / 21 / 77$ & 12 & 1 & & 95 & $\mathbf{M}$ & \\
\hline 1 & e/21/77 & $1 \overline{3}$ & 1 & & 85 & M & \\
\hline 1 & $9 / 21 / 77$ & 14 & 1 & & 05 & $\mathbf{N}$ & \\
\hline 1 & $9 / 21 / 7$ & 15 & 1 & & 98 & $\mathbf{N}$ & \\
\hline 1 & s/21/T & 18 & 1 & & 86 & $\bar{N}$ & \\
\hline$i$ & $10 / 7 / 7$ & 1 & 1 & & 127 & $\mathbf{N}$ & \\
\hline 3 & $10 / 207$ & 2 & 1 & & 128 & $N$ & \\
\hline 1 & $10 / 77$ & 3 & 1 & & 129 & $Y$ & \\
\hline 1 & $10 / 707$ & 4 & 1 & & 128 & N & \\
\hline 1 & $1017 / 77$ & 5 & 1 & & 127 & $\mathbf{N}$ & \\
\hline 1 & $10 / 7 / 7$ & 6 & 1 & & 125 & $\mathrm{~N}$ & \\
\hline 1 & $1017 \% 7$ & 7 & 1 & & 125 & $N$ & \\
\hline 1 & $107 / 77$ & 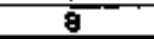 & $1, \ldots$ & & 112 & $\mathrm{~N}$ & \\
\hline 1 & $10 \% \pi$ & 8 & 1 & & 88 & N & \\
\hline 1 & $107 / 77$ & 10 & 1 & & 93 & $\mathbf{N}$ & \\
\hline 1 & $10 y / 7 ?$ & 11 & 1 & & $\overline{93}$ & $\bar{N}$ & \\
\hline 1 & 100777 & 12 & 1 & & 83 & N & \\
\hline 1 & $10 \% / 77$ & 13 & 1 & & 93 & N & \\
\hline 1 & $1017 / 7$ & 14 & 1 & & $\frac{63}{3}$ & N & \\
\hline 1 & 1 ah/77 & 15 & 1 & & 83 & $\mathbf{N}$ & \\
\hline 1 & I07/7 & 16 & 1 & & 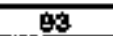 & $\bar{M}$ & \\
\hline 1 & 110177 & $t$ & 1 & & 123 & $\mathrm{~N}$ & \\
\hline 1 & $17 / / 77$ & 2 & 1 & & 125 & $\bar{Y}$ & \\
\hline 1 & 19/7/77 & 8 & 1 & & 122 & $\mathrm{~N}$ & \\
\hline 1 & $170 / 77$ & 4 & 1 & & 120 & $\bar{N}$ & \\
\hline
\end{tabular}

Date obtained from WHC Surveillence Anblyala Computer System (SACSI, Juno 2, 1993. 


\begin{tabular}{|c|c|c|c|c|c|c|c|}
\hline \multicolumn{8}{|c|}{ TANK 241A-703 } \\
\hline \multicolumn{8}{|c|}{ THEFWOCOUPLE DATA } \\
\hline TFED: & DATE & THEFATO F & TPEX SET E & TEMF C & TEAP F & $Y=$ MAX TENP & COMNENTS \\
\hline 1 & $11 / 7 / 77$ & 5 & 1 & & 107 & $\mathbf{N}$ & \\
\hline 1 & 1 1/377 & $\mathbf{B}$ & 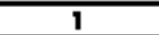 & & 97 & $\mathbf{N}$ & \\
\hline 1 & $1+1 / 777$ & 7 & 1 & & 92 & $\overline{\mathbf{N}}$ & \\
\hline 1 & $11 / 7 / 77$ & 旦 & 1 & & 89 & $\mathbf{N}$ & \\
\hline 1 & $11 / 7 / 77$ & a & 1 & & 9日9 & $\mathbf{N}$ & \\
\hline 1 & 110177 & 70 & 1 & & $\mathbf{8 9}$ & $\mathbf{N}$ & \\
\hline 1 & $11 / 7 / 77$ & 11 & 1 & & ag & $\mathbf{N}$ & \\
\hline 1 & $11 / 7 / 7$ & $\sqrt{2}$ & 1 & & $\mathrm{gB}$ & $\bar{N}$ & \\
\hline$\overline{1}$ & 1 1/77 & 13 & $\overline{1}$ & & 89 & $\mathbf{N}$ & \\
\hline 1 & $11 / 7 / 77$ & 14 & 1 & & 百 & $\mathbf{N}$ & \\
\hline $\mathbf{T}$ & $11 \cap N 7$ & 75 & 1 & & 89 & $\mathbf{N}$ & \\
\hline 1 & $11 / 7 / 77$ & 16 & 1 & & 88 & $\mathbf{N}$ & \\
\hline 7 & $12 / 4 / 77$ & $\mathbf{T}$ & 1 & & 124 & $\bar{Y}$ & \\
\hline 1 & $12 / 4 \pi 7$ & 2 & 1 & & 121 & $\mathbf{N}$ & \\
\hline 7 & $12 / 4 / 77$ & 3 & 1 & & 119 & $\mathbf{M}$ & \\
\hline 1 & $1244 \pi 7$ & 4 & 1 & & 110 & $\mathbf{N}$ & \\
\hline 1 & $12 / 4 / T$ & $\overline{\mathbf{5}}$ & 1 & & 119 & $\mathbf{N}$ & \\
\hline 1 & $12 \sqrt{477}$ & $\theta$ & 1 & & 118 & $\bar{N}$ & \\
\hline$\overline{1}$ & $12 / 4 / 77$ & 7 & $\overline{1}$ & & 115 & $\mathbf{N}$ & \\
\hline$\overline{1}$ & $12 / 4 / 77$ & 8 & $\overline{1}$ & & त11 & $\mathbf{N}$ & \\
\hline 1 & $12 \mathrm{~A} / 7$ & 音 & 1 & & 107 & $N$ & \\
\hline 1 & $12 / 4 / 77$ & 10 & 1 & & $\theta 9$ & $\mathbf{N}$ & \\
\hline 1 & $12 / 4 m$ & 11 & 1 & & 82 & $\mathbf{N}$ & \\
\hline 7 & $12 / 4 / 77$ & 12 & 1 & & 81 & $\mathbf{N}$ & \\
\hline 1 & $124 \sqrt{7}$ & 13 & 1 & & 92 & $\bar{N}$ & \\
\hline 1 & $12 / 4 / 3$ & 74 & 1. & & 82 & $\mathbf{N}$ & \\
\hline 1 & $12 / 4 n 7$ & 16 & 1 & & 82 & $\bar{N}$ & \\
\hline 1 & $12 / 4 \sqrt{77}$ & 18 & 1 & & 82 & $\mathbf{N}$ & \\
\hline 1 & $1 / 16478$ & 1 & 1 & & 127 & $\bar{Y}$ & \\
\hline 7 & 1/16/78 & 2 & 1 & & 125 & $\mathbf{N}$ & \\
\hline i & 1/167白 & $\overline{3}$ & $i$ & & 124 & $\bar{N}$ & \\
\hline 1 & $1 / 16479$ & 4 & 1 & & 124 & $\mathbf{M}$ & \\
\hline 1 & 1/A7t & $\overline{5}$ & 1 & & 124 & $\mathbf{N}$ & \\
\hline 9 & $1 / 16178$ & 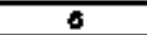 & 1 & & 124 & $\mathbf{N}$ & \\
\hline 1 & $1 / 16 / 78$ & 7 & 1 & & 110 & $\mathbf{N}$ & \\
\hline 7 & $1 / 16 / 78$ & 8 & 1 & & 109 & $\mathbf{N}$ & \\
\hline 1 & $1 / 16 / 78$ & g & 1 & & 105 & $\mathbf{N}$ & \\
\hline 1 & $1 / 18 / 78$ & 70 & 1 & & 98 & $\mathbf{N}$ & \\
\hline 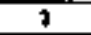 & $1 / 16 / 78$ & 11 & 1 & & 78 & $\mathbf{N}$ & \\
\hline 7 & $1 / 1878$ & $\sqrt{2}$ & $\overline{1}$ & & 77 & $\mathbf{N}$ & \\
\hline $\mathbf{T}$ & $1 / 19 / 78$ & 13 & 1 & & 77 & $\mathbf{N}$ & \\
\hline 1 & 1/67自 & 14 & 1 & & 7 & $\mathrm{~N}$ & \\
\hline 1 & $1 / 28178$ & 1 & 1 & & 128 & $\bar{Y}$ & \\
\hline 1 & $1 / 2878$ & 2 & 1 & & 123 & $\bar{N}$ & \\
\hline 1 & $1 / 28178$ & 3 & 1 & & 122 & $\mathbf{N}$ & \\
\hline$\overline{1}$ & $1 / 2878$ & 4 & $\overline{1}$ & & 122 & $\bar{N}$ & \\
\hline 1 & $1 / 28 \sqrt{78}$ & 5 & 1 & & 122 & N & \\
\hline $\bar{t}$ & $1 / 23 / 78$ & $B$ & 1 & & 120 & $N$ & \\
\hline $\mathbf{J}$ & $1 / 2878$ & 7 & 1 & & 115 & $\mathbf{N}$ & \\
\hline$\$$ & $1 / 29 / 78$ & 严 & 1 & & $70 B$ & $\mathbf{N}$ & \\
\hline 1 & $1 / 28 / 78$ & 9 & 1 & & 502 & $\mathbf{N}$ & \\
\hline 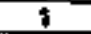 & 129078 & 10 & 1 & & 92 & $\mathbf{N}$ & \\
\hline 7 & $1 / 28 / 78$ & 71 & $1 .-$ & & 76 & $\mathbf{N}$ & \\
\hline 7 & $1 / 28 / 78$ & 12 & 1 & & 75 & $\mathbf{N}$ & \\
\hline 1 & $1 / 28 / 78$ & 73 & 1 & & 75 & $\mathbf{N}$ & \\
\hline 1 & $1 / 29 / 78$ & 14 & 1 & & 75 & $\mathbf{N}$ & \\
\hline$\hbar$ & $2 / 2678$ & 1 & 1 & & 122 & $Y$ & \\
\hline 7 & $2126 \sqrt{78}$ & 2 & 1 & & 119 & $\mathbf{N}$ & \\
\hline 1 & $2 / 2678$ & 3 & 1 & & $\$ 10$ & $\mathbf{N}$ & \\
\hline 1 & $2 / 20 / 78$ & 4 & 1 & & 116 & $\mathbf{N}$ & \\
\hline 1 & 21268 & 5 & 1 & & 110 & $N$ & \\
\hline 1 & $2 / 20 / A B$ & B & 1 & & 112 & $\mathbf{N}$ & \\
\hline 1 & 2/20its & 7 & 1 & & 108 & $N$ & \\
\hline$\overline{1}$ & $2 / 2 / 78$ & 官 & 1 & & 106 & N & \\
\hline 1 & $2 \sqrt{20} 79$ & 8 & 1 & & 104 & $\mathbf{H}$ & \\
\hline
\end{tabular}

Data obtained from WHC Surveillance Analysis Computer System (SACS), June 2, 1993. 


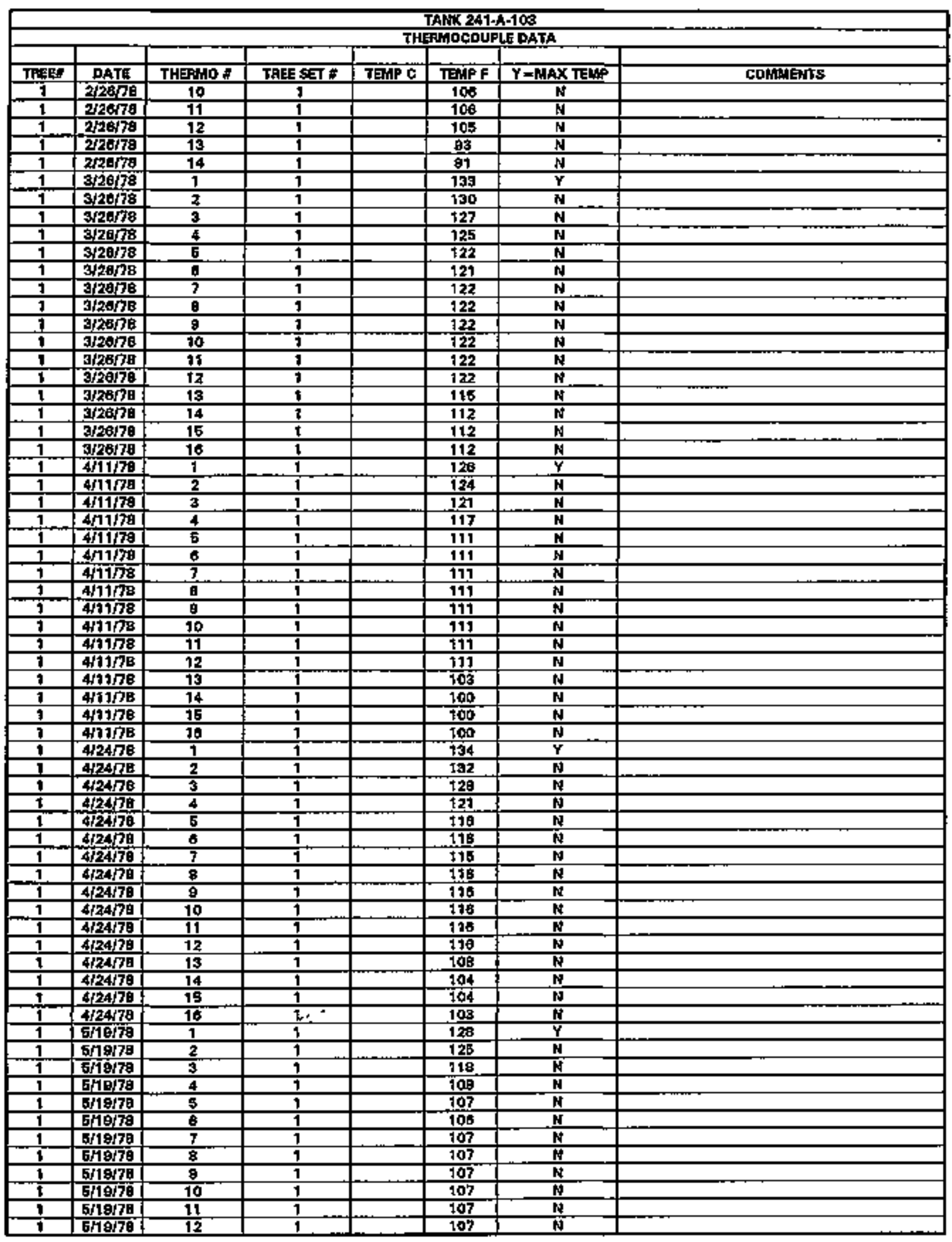

Dats abtained from WHC Survaillanes Analysis Computer System (SACS), June 2,1993. 


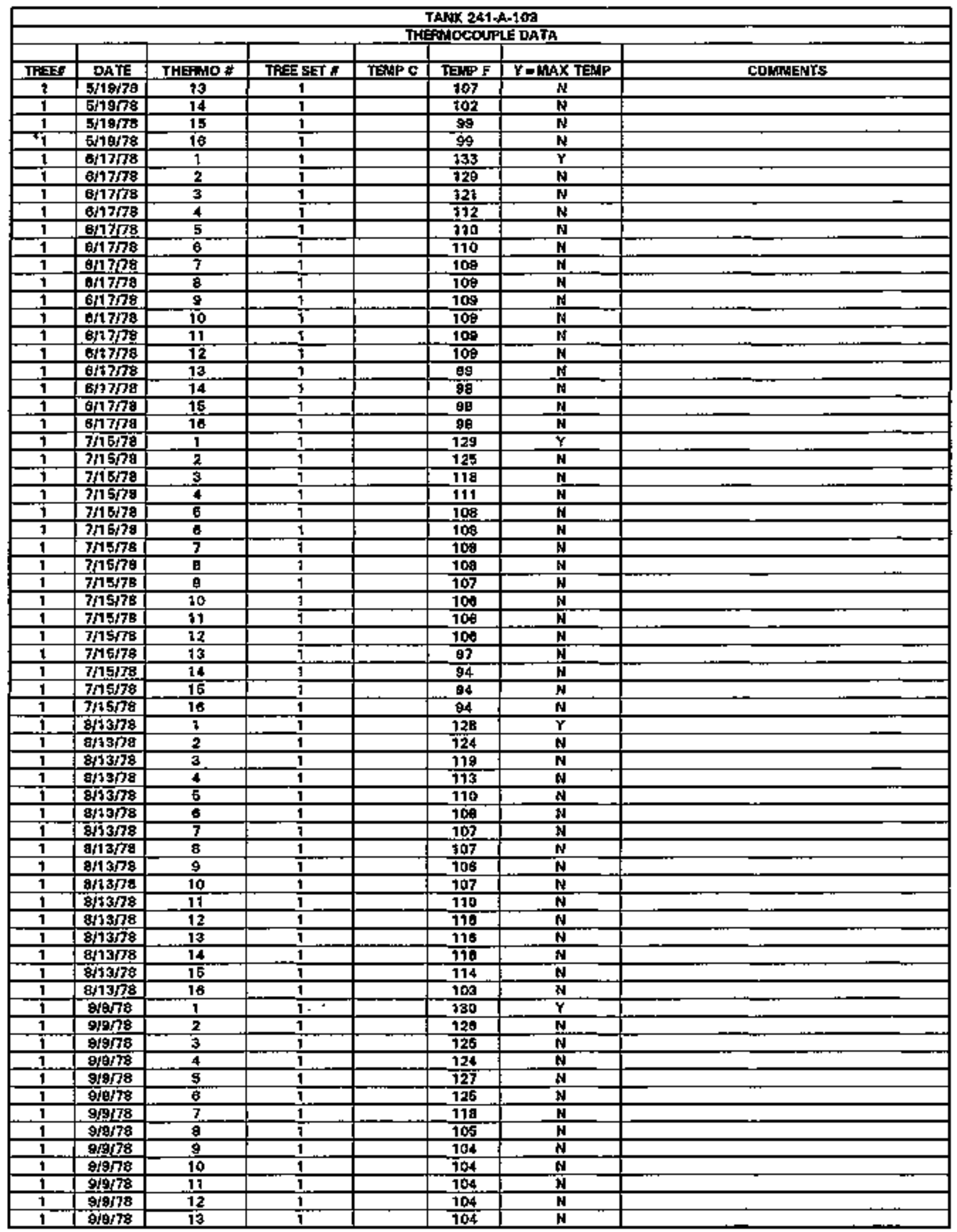

Date obtained fram WHC Surveillankt Analysis Comptstor Systom (SACS), June 2,1093. 


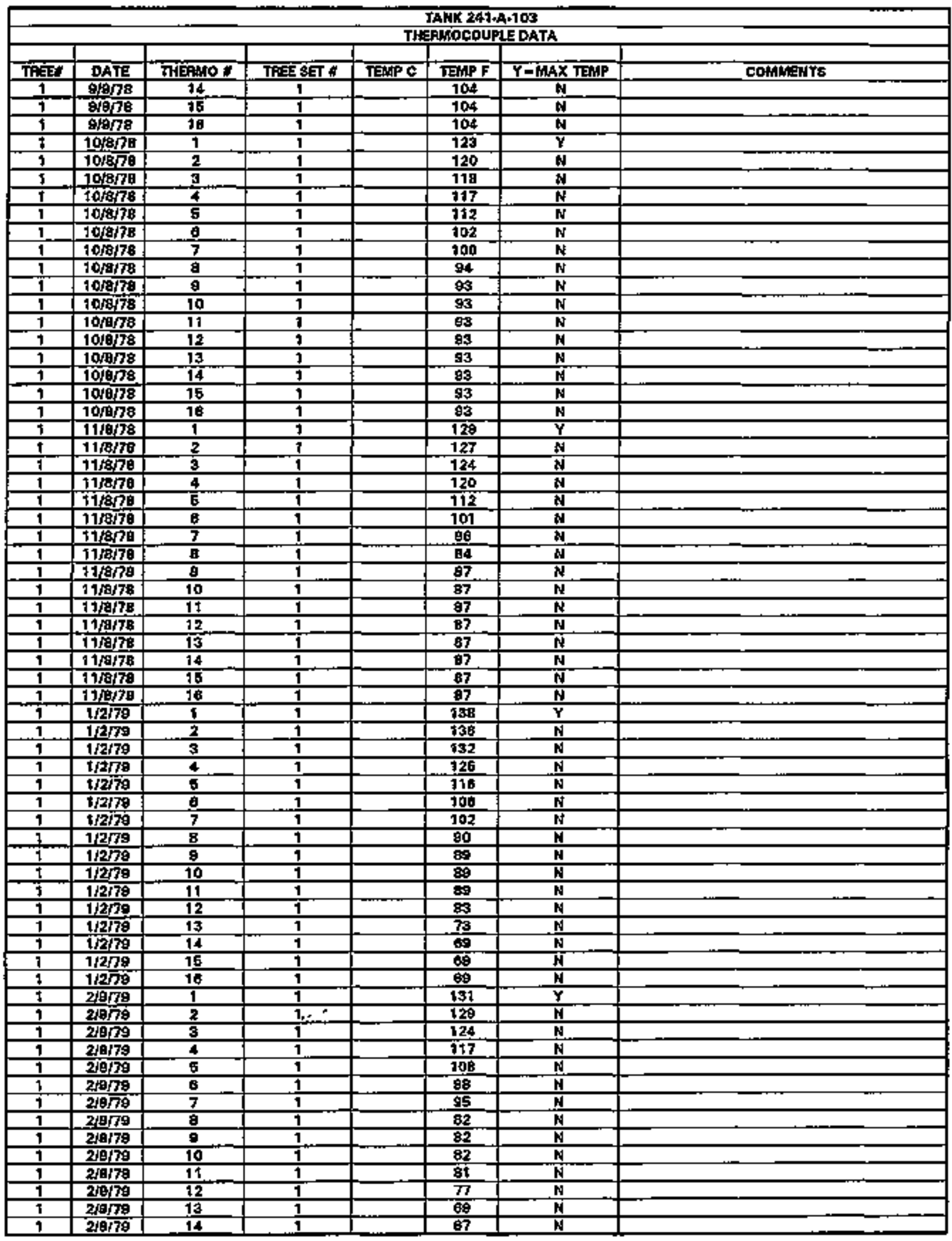

Date obtained from WHC Survaillenos Analysis Compttar Syatem (SACS), June 2,1993. 


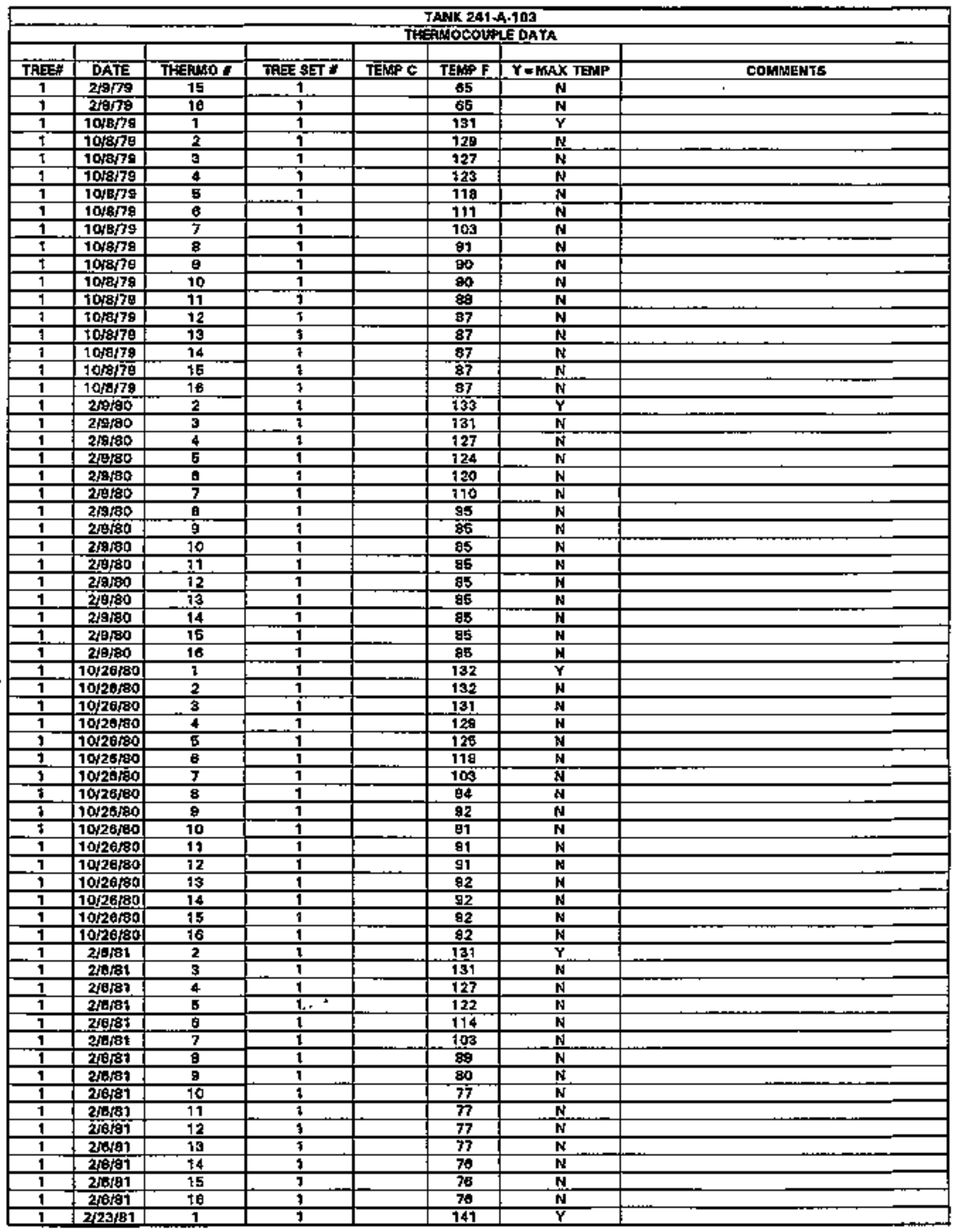

Data obtained from WHC Surveillance Anglysis Computer Syatem (SACS\}, Junn 2,1993. 


\begin{tabular}{|c|c|c|c|c|c|c|c|}
\hline \multicolumn{8}{|c|}{$\begin{array}{c}\text { TAKK 241+A-105 } \\
\text { THEPIAOCOUPLE DATA }\end{array}$} \\
\hline 仴的: & DATE & THEFLO I & THE st & TElP G & TENAP F & $Y \approx M A X$ TENP & COHWENTS \\
\hline 1 & $273 / 81$ & 2 & 1 & & 141 & $\mathbf{N}$ & \\
\hline 1 & $2123 / 81$ & 3 & 1 & & 137 & $\mathbf{N}$ & \\
\hline 1 & $2 / 23 / 81$ & 4 & 1 & & 1391 & $\mathbf{N}$ & \\
\hline 1 & $2123 \sqrt{81}$ & E & 1 & & 122 & $\mathbf{N}$ & \\
\hline 1 & $2 / 23 / 91$ & E & 1 & & 111 & $\mathbf{N}$ & \\
\hline 1 & 2/23/91 & 7 & 1 & & 93 & $\mathbf{N}$ & \\
\hline 1 & $2 / 23 / 01$ & E & 1 & & 82 & $\bar{N}$ & \\
\hline 1 & $2 / 231$ & $\theta$ & $t$ & & 78 & $\mathbf{N}$ & \\
\hline 1 & 2/23/月1 & 10 & 1 & & 78 & $\mathbf{N}$ & \\
\hline$T$ & $2 / 23 / 91$ & 11 & 1 & & 78 & $\mathbf{N}$ & \\
\hline 9 & $2 / 23 / 91$ & 12 & 1 & & 78 & $\mathbf{N}$ & \\
\hline 1 & $2 / 23 / 91$ & 13 & 1 & & 78 & $\mathbf{N}$ & \\
\hline 1 & 2/28一1 & 14 & 1 & & 78 & $\mathbf{N}$ & \\
\hline 1 & $2 / 23 / 01$ & 15 & 1 & & 落 & iN & \\
\hline 1 & $2 / 23 / 91$ & 16 & 1 & & $\pi$ & $\mathbf{N}$ & \\
\hline 1 & $2 \mathrm{bar}$ & 1 & 1 & & 137 & $\bar{Y}$ & \\
\hline 1 & $2 / 8192$ & 2 & 1 & & 134 & N & \\
\hline 1 & $2 / 8182$ & 3 & 1 & & 130 & $\mathbf{N}$ & \\
\hline 1 & $2 / 8 / 92$ & 4 & 1 & & 124 & $N$ & \\
\hline 1 & 2/8182 & 5 & 1 & & 117 & $\mathbf{N}$ & \\
\hline 1 & 2实自2 & 6 & 1 & & 107 & $\mathbf{N}$ & \\
\hline 1 & $2 / 8382$ & 7 & 1 & & 99 & $\mathbf{N}$ & \\
\hline 1 & 2 Bra2 & 8 & 1 & & 旬 & $\mathbf{N}$ & \\
\hline 1 & $2 \sqrt{B}+32$ & 9 & 1 & & 93 & $\mathbf{N}$ & \\
\hline 1 & $2 / 3 / 82$ & 10 & 1 & & 93 & $\mathbf{N}$ & \\
\hline 1 & $2 \sqrt{8 / 2}$ & 11 & 1 & & $\overline{\theta 2}$ & $\bar{N}$ & \\
\hline 1 & 2 vers2 & 12 & 1 & & 02 & $N$ & \\
\hline 1 & $2 / \mathrm{B} / \mathrm{B} 2$ & 13 & 1 & & 多 & $\mathbf{N}$ & \\
\hline 1 & $2 \sqrt{8} 82$ & 14 & 1 & & 配 & $\mathbf{N}$ & \\
\hline 1. & $2 \mathrm{~N} / 8 \mathrm{B2}$ & 15 & 1 & & 92 & $\mathbf{N}$ & \\
\hline 1 & 26182 & 10 & 1 & & $\overline{92}$ & $\mathbf{N}$ & \\
\hline 1 & B] 182 & 1 & 1 & & 129 & $\mathbf{Y}$ & \\
\hline 1 & 619/82 & 2 & 1 & & 128 & $\mathbf{N}$ & \\
\hline 7 & 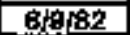 & $\overline{3}$ & $\overline{1}$ & & 122 & $\mathbf{N}$ & \\
\hline 1 & ajeroz & 4 & 1 & & 116 & 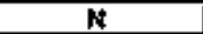 & \\
\hline 1 & (6/9/A2 & 5 & 1 & & 108 & $N^{*}$ & \\
\hline 1 & $\theta / 9 \sqrt{182}$ & $\theta$ & 1 & & 97 & $\mathbf{N}$ & \\
\hline 1 & 9/9/1 & 7 & $i$ & & 89 & $N$ & \\
\hline 1 & 6rorez & $\theta$ & 1 & & 89 & $N$ & \\
\hline 1 & व/9/ez & 皇 & 1 & & 88 & $\bar{N}$ & \\
\hline 1 & 6 projez & 10 & 1 & & 87 & $\boldsymbol{N}$ & \\
\hline 1 & a/prez & 11 & 1 & & 88 & $\mathrm{k}$ & \\
\hline 1 & 0/9jez & 12 & $\$$ & & 88 & $\mathrm{~N}$ & \\
\hline 1 & $0 / 9 / 62$ & $\sqrt{3}$ & 1 & & 88 & $N$ & \\
\hline 1 & 9/9jez & 14 & $t$ & & 88 & $N$ & \\
\hline 1 & 6/9/62 & 16 & 1 & & 88 & $N$ & \\
\hline 1 & G/9j-az & 18 & 1 & & 88 & $N$ & \\
\hline 1 & 1017日ez & 1 & 7 & & 113 & $\bar{Y}$ & \\
\hline 1 & 10419/E2 & 2 & 7 & & 100 & $N$ & \\
\hline$\frac{1}{1}$ & Torteje2 & $\overline{3}$ & 1 & & 1I1 & $\mathbf{N}$ & \\
\hline 1 & 10/10jez & 4 & 1 & & 110 & $\mathbf{N}$ & \\
\hline 1 & 10/20/82 & 5 & 1 & & 203 & $\mathbf{N}$ & \\
\hline 1 & 70yteriz & $\theta$ & $t_{r}:$ & & 98 & $\mathbf{N}$ & \\
\hline 1 & $1019 \sqrt{182}$ & 7 & 1 & & 88 & $\mathbf{N}$ & \\
\hline 1 & 10늘 & $\theta$ & 1 & & 88 & $\mathbf{H}$ & \\
\hline 1 & 10/5遮2 & 夏 & 1 & & 87 & $\mathbf{N}$ & \\
\hline 1 & $\overline{10 / 79 / 82}$ & 10 & $y$ & & 88 & is & \\
\hline$i$ & 10/ & 11 & 1 & & 86 & $\mathbf{N}$ & \\
\hline 1 & 10/18j82 & 12 & $\$$ & & 86 & $N$ & \\
\hline 1 & 10 10)2 & 13 & 1 & & 86 & $N$ & \\
\hline 1 & 10/7bB2 & 14 & 1 & & 86 & $\mathbf{N}$ & \\
\hline 1 & 10179je2 & 15 & 7 & & 80 & $\mathbf{n}$ & \\
\hline 1 & $10 / 1982$ & 10 & 1 & & 85 & $\mathbf{N}$ & \\
\hline 1 & $2 / 0 / 33$ & 1 & 1 & & 518 & $\mathbf{Y}$ & \\
\hline 1 & 2 5 & 2 & 1 & & 116 & $\mathbf{N}$ & \\
\hline
\end{tabular}

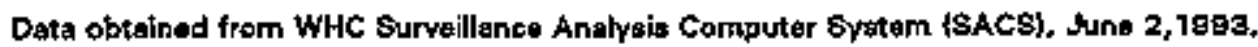




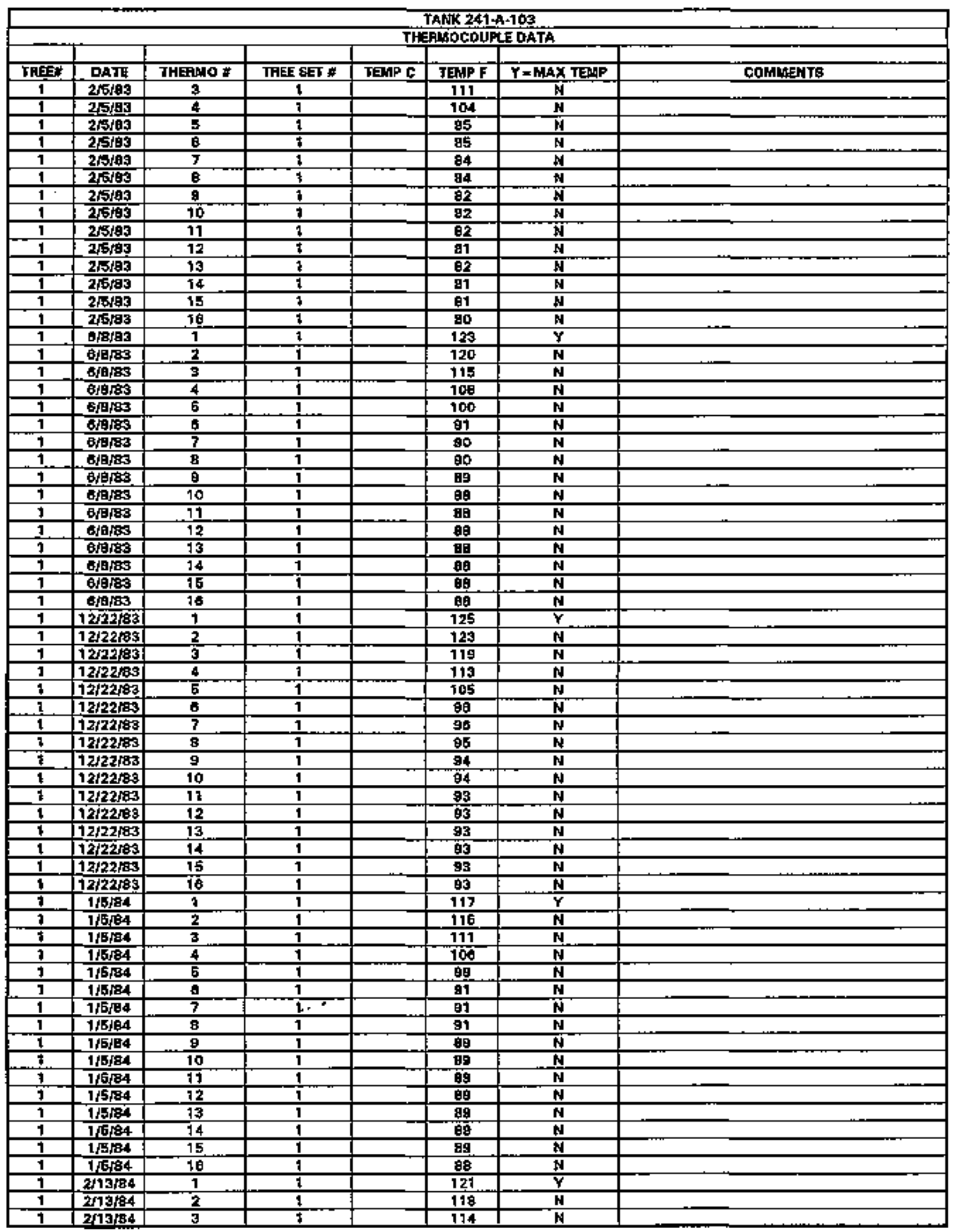

Data obtained from WHC Strveillence Anelysis Computar SYstem \{SACS\}, June Z,1 1 g93. 


\begin{tabular}{|c|c|c|c|c|c|c|c|}
\hline \multicolumn{8}{|c|}{$\begin{array}{c}\text { TAKK 241+A+108 } \\
\text { THEFMOCOUPLE DATA }\end{array}$} \\
\hline TREAT & DATE & THFALOY & TFEE SEF & TEMP C & TENFF F & $Y=M A X$ TEMP & COMHERTS \\
\hline 1 & 2/13/84 & 4 & 1 & & 100 & $\mathrm{~N}$ & \\
\hline 1 & $2 \pi 3 / 84$ & 5 & 1 & & 100 & $\mathbf{N}$ & \\
\hline 1 & $2 / 13 / 84$ & 6 & 1 & & $\theta 0$ & iN & \\
\hline 1 & $2 / 13 \sqrt{4}$ & 7 & 1 & & 89 & $\mathbf{H}$ & \\
\hline 1 & $313 \sqrt{14}$ & 皇 & $\overline{1}$ & & 99 & N & \\
\hline 1 & $2 / 13 / 64$ & 8 & 1 & & en & N & \\
\hline 1 & $2 / 13 \sqrt{14}$ & 10 & 1 & & 97 & $\mathbf{N}$ & \\
\hline 1 & $2 / 13 / 94$ & it & 1 & & 67 & $\mathbf{N}$ & \\
\hline 1 & $2113 \sqrt{4}$ & 12 & 1 & & 67 & N & \\
\hline 1 & $2 / 13 / 94$ & $\sqrt{3}$ & 1 & & 白7 & $\mathbf{N}$ & \\
\hline 1 & $2 / 13 \sqrt{14}$ & 14 & 1 & & 67 & $N$ & \\
\hline 1 & $2 / 13 / 64$ & 15 & 1 & & 67 & $\mathbf{N}$ & \\
\hline 1 & $2 / 13 / 84$ & 10 & 1 & & 97 & $\mathbf{N}$ & \\
\hline 1 & 3/20j84 & 1 & 1 & & 118 & $\bar{Y}$ & \\
\hline 1 & $3 / 26 / 84$ & 2 & 1 & & 117 & $\mathbf{N}$ & \\
\hline 1 & $3 / 20 / 84$ & 3 & 1 & & $\$ 12$ & $\mathbf{N}$ & \\
\hline 1 & $3 / 28 / 84$ & 4 & 1. & & 208 & $\mathbf{N}$ & \\
\hline 1 & अ20184 & E & 1 & & 97 & $\mathbf{N}$ & \\
\hline 1 & $3 / 20 / 44$ & 6 & 1 & & 87 & $\mathbf{N}$ & \\
\hline 1 & $3 / 26 / 84$ & 7 & 1 & & 87 & N. & \\
\hline 1 & $3 / 20 / 34$ & 8 & 1 & & 87 & $\mathbf{N}$ & \\
\hline 1 & $3 / 2984$ & G & 1 & & 85 & $\mathbf{N}$ & \\
\hline 1 & $3 / 26 / 04$ & 10 & 1 & & 85 & $\mathbf{f t}$ & \\
\hline 1 & $3 / 25 / 84$ & 11 & 1 & & 95 & $\mathbf{N}$ & \\
\hline 1 & $3 / 28 / 94$ & 12 & 1 & & 85 & $\mathbf{N}$ & \\
\hline 1 & $3 / 25 / 94$ & 13 & 1 & & 85 & $\mathbf{N}$ & \\
\hline 1 & $3 / 26 \sqrt{84}$ & $\sqrt{A}$ & 1 & & 85 & $\mathrm{~N}$ & \\
\hline 1 & $3 / 26 / 94$ & 15 & 1 & & 84 & M & \\
\hline 1 & $3 / 26 \sqrt{64}$ & 18 & 1 & & 84 & $\mathbf{N}$ & \\
\hline 1 & $3 / 26+14$ & 17 & 1 & & 84 & N & \\
\hline 1 & $3 / 26184$ & 18 & 1 & & 64 & $\overline{\mathbf{N}}$ & \\
\hline 1 & 40184 & 1 & 1 & & 122 & $\bar{Y}$ & \\
\hline 1 & $4 / 7 / 94$ & 2 & 1 & & 120 & $\mathbf{N}$ & \\
\hline 1 & $40+94$ & 3 & 1 & & 115 & $\mathbf{N}$ & \\
\hline 1 & $4 / 7 / B 4$ & 4 & $\mathbf{T}$ & & 109 & $\mathbf{N}$ & \\
\hline 1 & $A \sqrt{2184}$ & 5 & 1 & & 100 & $\mathbf{N}$ & \\
\hline 1 & $4 \sqrt{7 / 34}$ & 6 & 1 & & 80 & $\mathbf{N}$ & \\
\hline 1 & $4 \sqrt{2184}$ & 7 & 1 & & 69 & $\mathbf{N}$ & \\
\hline 1 & $4 \sqrt{784}$ & 8 & 1 & & 99 & $N$ & \\
\hline 1 & ADt84 & 9 & 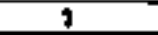 & & 80 & $\overline{\mathbf{N}}$ & \\
\hline 1 & $4 / 7 / 84$ & 10 & 1 & & घ晖 & $N$ & \\
\hline 1 & $4 / 7 / 84$ & 11 & 1 & & 百 & $\mathbf{N}$ & \\
\hline 1 & $4 / 7 / 84$ & 12 & 1 & & 旦 & $\mathbf{N}$ & \\
\hline 1 & $4 / 7 / 84$ & 12 & 1 & & 99 & $\mathbf{N}$ & \\
\hline 1 & $4 \sqrt{784}$ & 14 & 1 & & 8B & $\mathbf{N}$ & \\
\hline 1 & $4 \sqrt{184}$ & 16 & 1 & & 夏 & $N$ & \\
\hline 1 & $47 \sqrt{124}$ & 16 & 7 & & 皕 & $\mathbf{N}$ & \\
\hline 1 & $4 / 25 / 84$ & 1 & 1 & & 122 & $\mathbf{Y}$ & \\
\hline 1 & $4 / 25 / 94$ & 2 & 5 & & 121 & $\mathbf{N}$ & \\
\hline 1 & $4 / 25 / 84$ & 3 & 7 & & 116 & $\mathbf{N}$ & \\
\hline 1 & $4 / 2684$ & 4 & 1 & & 110 & 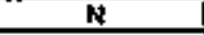 & \\
\hline 1 & $4125 / 84$ & 5 & 1 & & 100 & $M$ & \\
\hline 1 & $4 / 26 / 84$ & 8 & $1 \%$ & & 90 & $\mathbf{N}$ & \\
\hline 1 & $4 / 25 / 84$ & 7 & 1 & & 80 & $\mathbf{N}$ & \\
\hline 1 & $4 / 26 / 84$ & B. & 1 & & 奛 & $\mathbf{N}$ & \\
\hline 1 & $4 / 25 / 84$ & 9 & $i$ & & 百 & $N$ & \\
\hline 1 & 42584 & 10 & 7 & & 97 & $N$ & \\
\hline 1 & $4 / 25 / 34$ & 11 & 1 & & $\theta 7$ & $\mathbb{N}$ & \\
\hline 1 & 4/25IBA & 12 & 1 & & B7 & $\mathbf{N}$ & \\
\hline 1 & $4 / 29 / 84$ & 12 & 7 & & 97 & $\mathbf{N}$ & \\
\hline 1 & $4 / 2 \mathrm{E} / 84$ & 14 & 1 & & 百 & $\mathbf{N}$ & \\
\hline 1 & $4 / 25 / 84$ & 16 & 1 & & 97 & $\bar{N}$ & \\
\hline 1 & $4 / 25 / 84$ & 16 & 1 & & 87 & $\mathbf{N}$ & \\
\hline 1 & $4 / 26 / 84$ & 1 & 1 & & 124 & $Y$ & \\
\hline 1 & 42aks & 2 & $t$ & & 123 & $\mathbf{N}$ & \\
\hline
\end{tabular}

Data obtained from WHC Surveälance Analysis Comptete System (SACS), June 2, 9993 . 


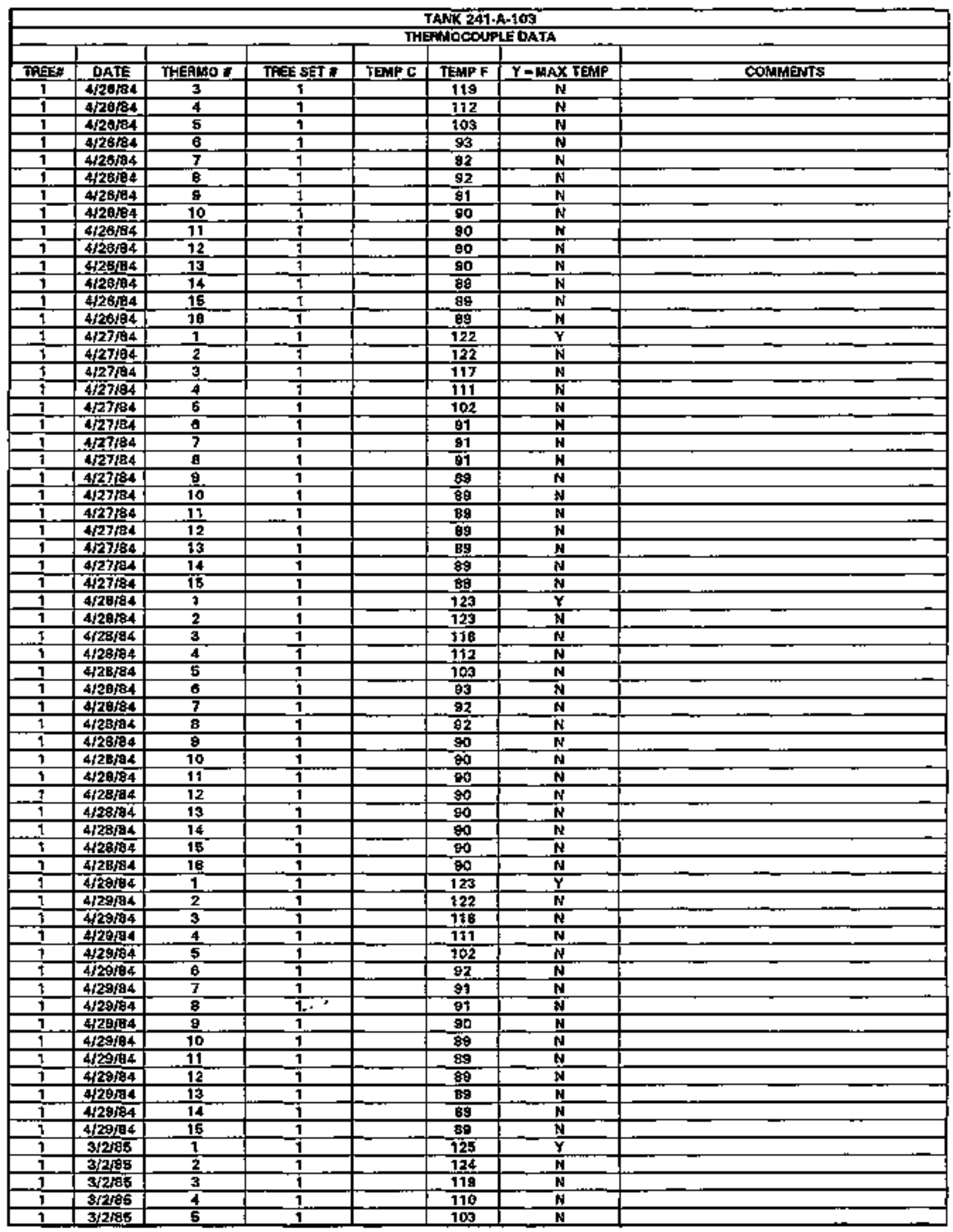

Date obtained from WHC Surveillance Analysis Computer Syetern ISACSH, June 2,1993. 


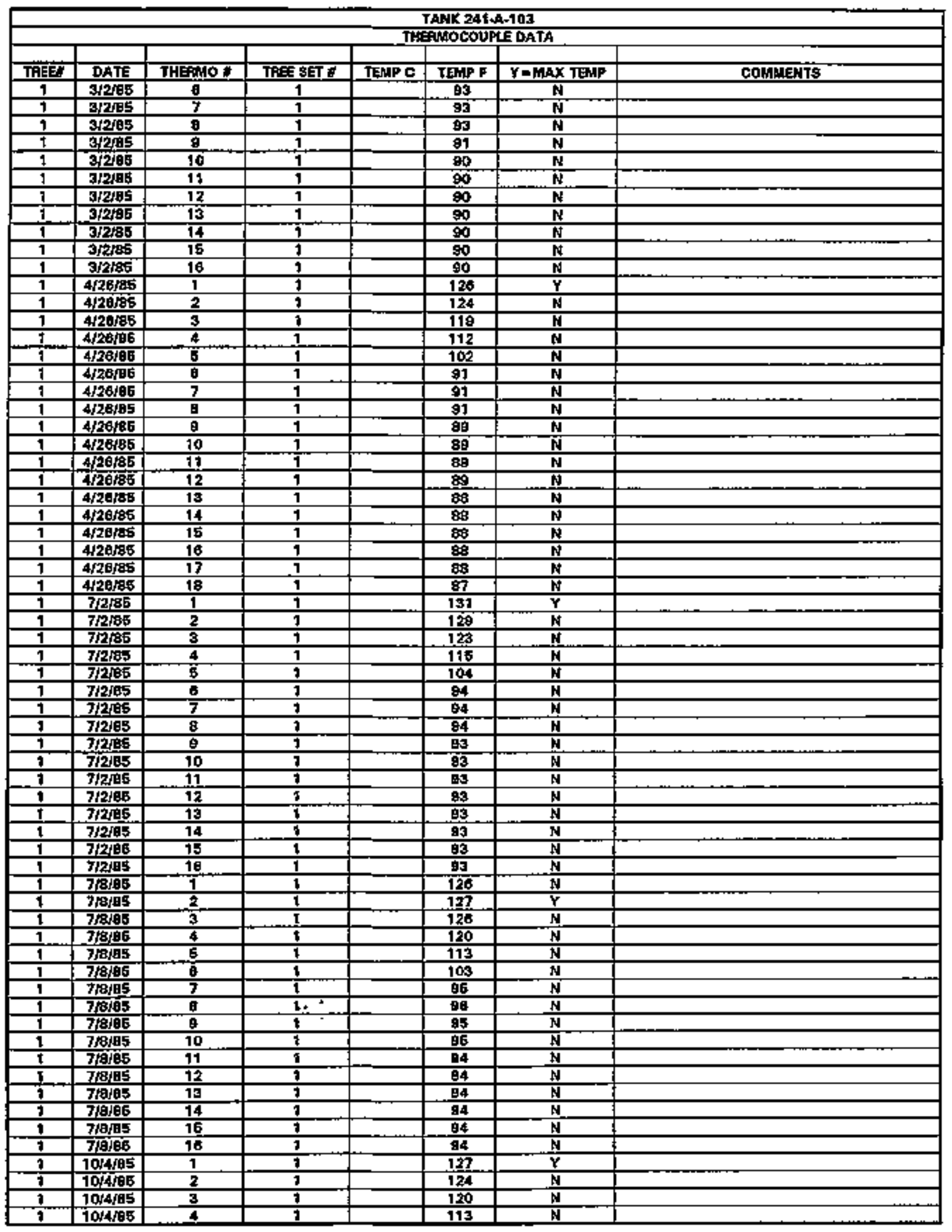

Data obtained from WhC Surveillance Anslysis Computer Sygtom (SACS), Juno 2,1903. 


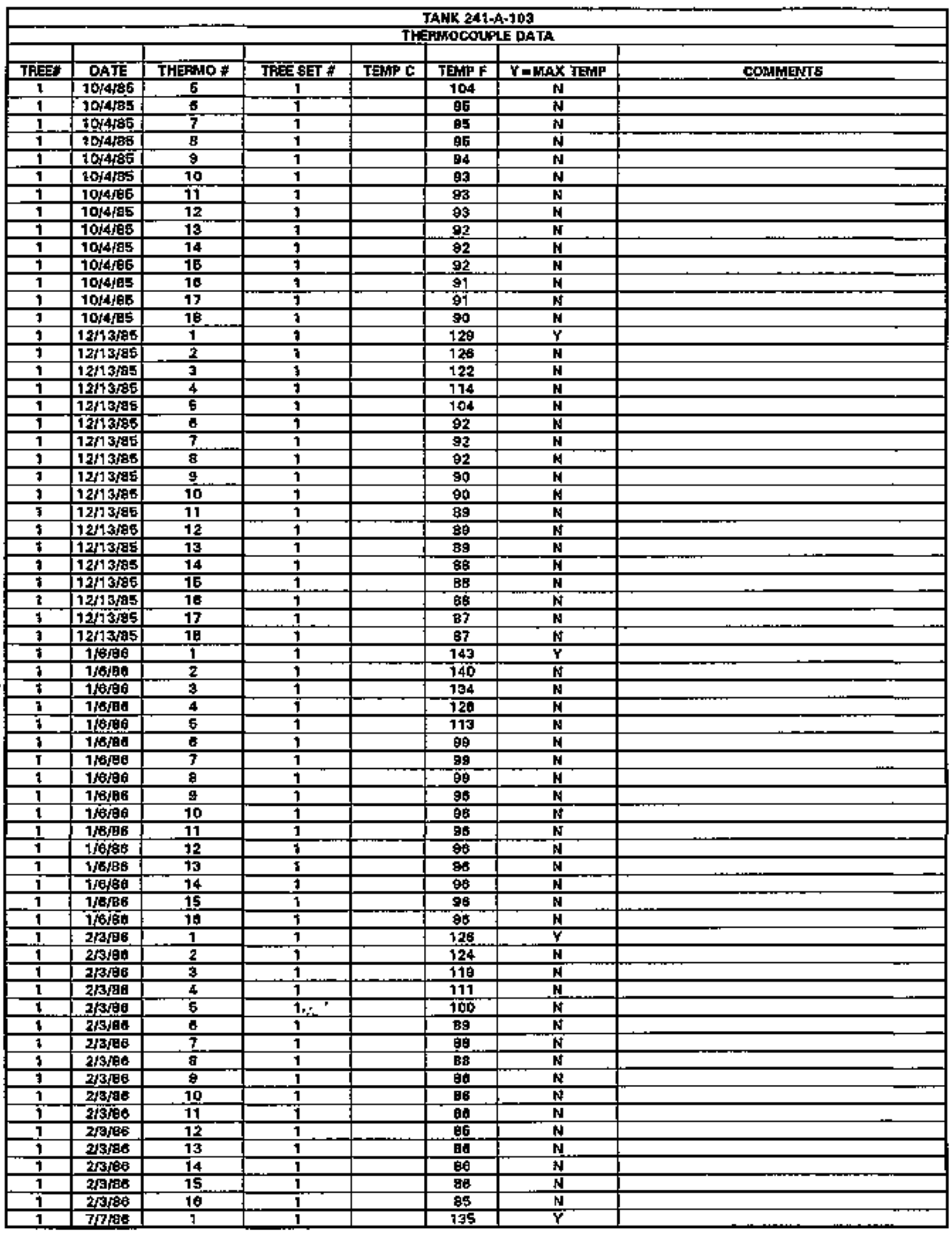

Deta obteined from WHC Surveillence Analysia Computer System [SACS), Juns 2,1903. 


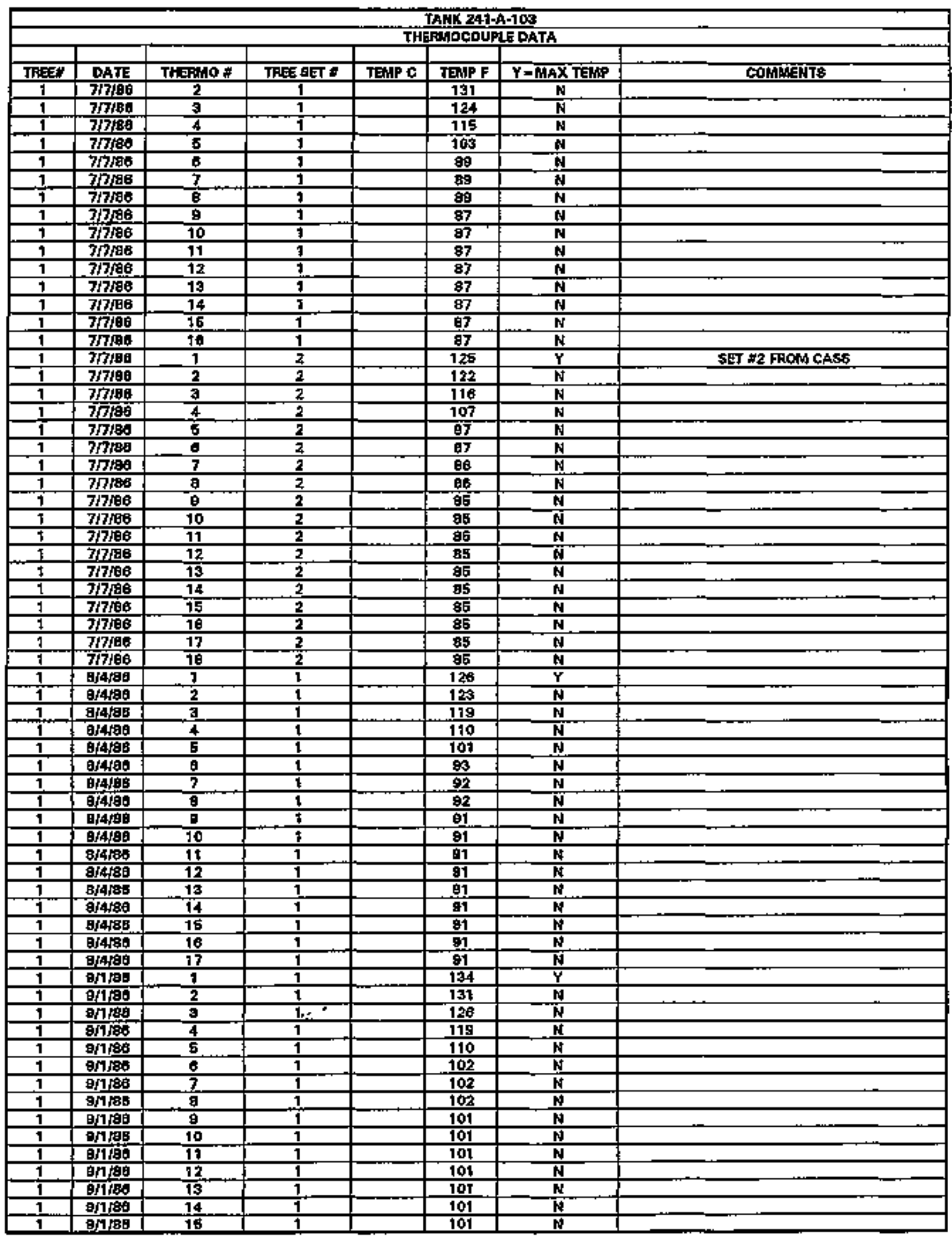

Date obtained from WhC Burveillance Ansysie Computer Syotam \{SACS\}, June 2,1993. 


\begin{tabular}{|c|c|c|c|c|c|c|c|}
\hline \multicolumn{8}{|c|}{ 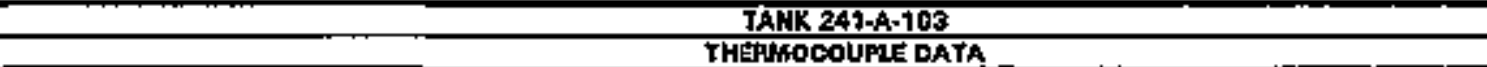 } \\
\hline TfF & DATE & THERLO * & If & Feind $\mathrm{c}$ & TEMPF & $Y-M A X$ TEMP & CoMMENTS \\
\hline 1 & s/1/86 & 16 & 1 & & 101 & $\mathbf{N}$ & \\
\hline 7 & 10 10jee & 1 & 7 & & 123 & $\bar{Y}$ & \\
\hline 1 & $10 \%$ /月6 & 2 & 1 & & 120 & $\mathbf{H}$ & \\
\hline$i$ & $10 / 8 j$ en & 3 & 1 & & 115 & $\mathbf{N}$ & \\
\hline$i$ & $10 / 8 \sqrt{10}$ & 4 & 1 & & 100 & N & \\
\hline 1 & 1006160 & 5 & 1 & & 100 & $\mathbf{N}$ & \\
\hline 1 & 10/0his & 5 & 1 & & 92 & $N$ & \\
\hline 1 & $10 / a r s$ & 7 & 1 & & 92 & $\mathrm{~N}$ & \\
\hline 1 & 10/0/Bs & 8 & 1 & & $\theta 2$ & $N$ & \\
\hline 1 & $100 / 80$ & $\theta$ & 1 & & $9 !$ & $\mathbf{N}$ & \\
\hline 1 & $10 / 6 / 88$ & 10 & 1 & & gt & N & \\
\hline$i$ & $10 / 0 / 186$ & 11 & 1 & & gt & N & \\
\hline 1 & $10 / 6 / 88$ & 12 & 1 & & 91 & $n$ & \\
\hline 1 & $10 / 8 / 186$ & 13 & $i$ & & 9t & N & \\
\hline 1 & $10 \mathrm{FG} B \mathrm{~B}$ & 14 & 1 & & 81 & $\mathbf{N}$ & \\
\hline 1 & 10/5jat & 13 & 1 & & $9 t$ & N & \\
\hline 1 & $10 / 9160$ & 18 & 1 & & 99 & $\mathbf{N}$ & \\
\hline 1 & $1010 / 098$ & 1 & 1 & & 120 & $\bar{\gamma}$ & \\
\hline 7 & 101080 & 2 & 1 & & 122 & $\mathrm{~N}$ & \\
\hline 1 & 10 t $10 / 86$ & 3 & $t$ & & 117 & 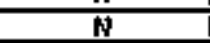 & \\
\hline 5 & $10,+1080$ & 4 & 1 & & 110 & $\mathrm{~N}$ & \\
\hline 1 & $10 / 10 / 86$ & 5 & $i$ & & 107 & N & \\
\hline 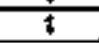 & $10 y+0 / 28$ & $\theta$ & 1 & & 92 & $\mathrm{~N}$ & \\
\hline 1 & $10 \% 10 / 86$ & 7 & 1 & & $\theta 2$ & $N$ & \\
\hline 7 & $1010 / 88$ & 8 & 1 & & 52 & $\mathrm{~A}$ & \\
\hline 1 & $10 / 10 / 86$ & 9 & $i$ & & 91 & $\frac{1+}{N}$ & \\
\hline 1 & $10 \%$ tor 8 & 10 & 1 & & so & $\mathrm{nt}$ & \\
\hline 1 & 10 10 108 & 11 & $y$ & & 80 & $N$ & \\
\hline$i$ & $10+\omega^{2}=0$ & 12 & 1 & & 90 & $\mathrm{~N}$ & \\
\hline$i$ & $10 \%$ toras & 13 & 1 & & $\theta$ & $\mathbf{N}$ & \\
\hline 7 & $10 \% 1086$ & 14 & 1 & & 90 & $\mathrm{~N}$ & \\
\hline 1 & $10 / 10 / 98$ & 15 & $i$ & & 90 & it & \\
\hline 1 & $1010 / 86$ & 16 & $t$ & & 69 & $\mathrm{~N}$ & \\
\hline 1 & $1010 / 80$ & 17 & 1 & & 69 & in & \\
\hline 1 & tortoras & 18 & 1 & & 89 & N & \\
\hline 1 & $11 / 3 / 38$ & 1 & 1 & & 124 & $Y$ & \\
\hline 1 & $11 / 3190$ & 2 & 1 & & 127 & $\mathrm{~N}$ & \\
\hline 1 & $11 / 3 / 18$ & 3 & 1 & & 117 & $\mathrm{~m}$ & \\
\hline 1 & $11 / 3 / 1 \mathrm{ge}$ & 4 & 1 & & 110 & iv & \\
\hline 1 & $11 / \mathrm{s} / \mathrm{Bg}$ & 5 & 1 & & 103 & N & \\
\hline 1 & $11 / 3 / 46$ & 8 & $i$ & & 3 & $\mathbf{N}$ & \\
\hline 1 & $51 / 300$ & 7 & 1 & & 92 & N & \\
\hline 1 & 11/3/86 & $\overline{8}$ & 1 & & 92 & N & \\
\hline 1 & $11 / 9180$ & $\theta$ & 1 & & 91 & II & \\
\hline 1 & $11 / \mathrm{B} / \mathrm{s}$ & 10 & $\frac{1}{1}$ & & 91 & 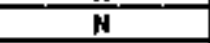 & \\
\hline 1 & $11 / \mathrm{A} / \mathrm{B} B$ & 11 & 1 & & 90 & $N$ & \\
\hline 1 & $11 / 3 / 83$ & 12 & $\frac{1}{1}$ & & 90 & $\mathbf{N}$ & \\
\hline 1 & 119/8B & 13 & 9 & & 80 & $\mathrm{~N}$ & \\
\hline 1 & $1+\mathrm{T} / \mathrm{gB}$ & 74 & 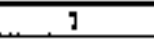 & & 90 & II & \\
\hline 1 & 11/3/as & 15 & 1 & & 90 & $\mathrm{~N}$ & \\
\hline 1 & $11 / 3 / B \theta$ & 10 & 9 & & 90 & II & \\
\hline 1 & $12 / 2 / \mathrm{k}$ & 1 & $\frac{1}{1}$ & & 123 & $\frac{11}{Y}$ & \\
\hline 1 & $12 / 2 / 90$ & 2 & 9 & & 121 & $M$ & \\
\hline 1 & 1212196 & 3 & 1 & & 197 & I & \\
\hline 1 & 1212100 & 4 & $\frac{1}{1}$ & & 176 & $\frac{1}{N}$ & \\
\hline$i$ & 12/2/218 & 5 & 1 & & 107 & $n$ & \\
\hline 1 & $1212 / 186$ & 6 & $i$ & & 92 & i & \\
\hline 3 & $12\{2\} 86$ & 7 & 1 & & 91 & $n$ & \\
\hline 1 & $12 / 2 / 86$ & 8 & 7 & & ot & $N$ & \\
\hline 1 & $12\left\{\left.2\right|^{8} 8\right.$ & 9 & 1 & & 89 & $\mathbf{N}$ & \\
\hline 1 & $12 / 2 / 86$ & 10 & 5 & & 89 & N & \\
\hline 1 & $12 / 2 \mathrm{j} / \mathrm{s}$ & 11. & 1 & & 89 & H & \\
\hline 1 & $12 / 21 / 6 \theta$ & 12 & 1 & & 自 & N & \\
\hline 7 & $12\{2 / 88$ & 13 & 1 & & go & $\mathbf{N}$ & \\
\hline 1 & 1212186 & 14 & 1 & & 29 & $\mathbf{N}$ & \\
\hline
\end{tabular}

Data obtained from Whic Surveillance Analysia Comptret System [SACS\}. June 2,1993. 


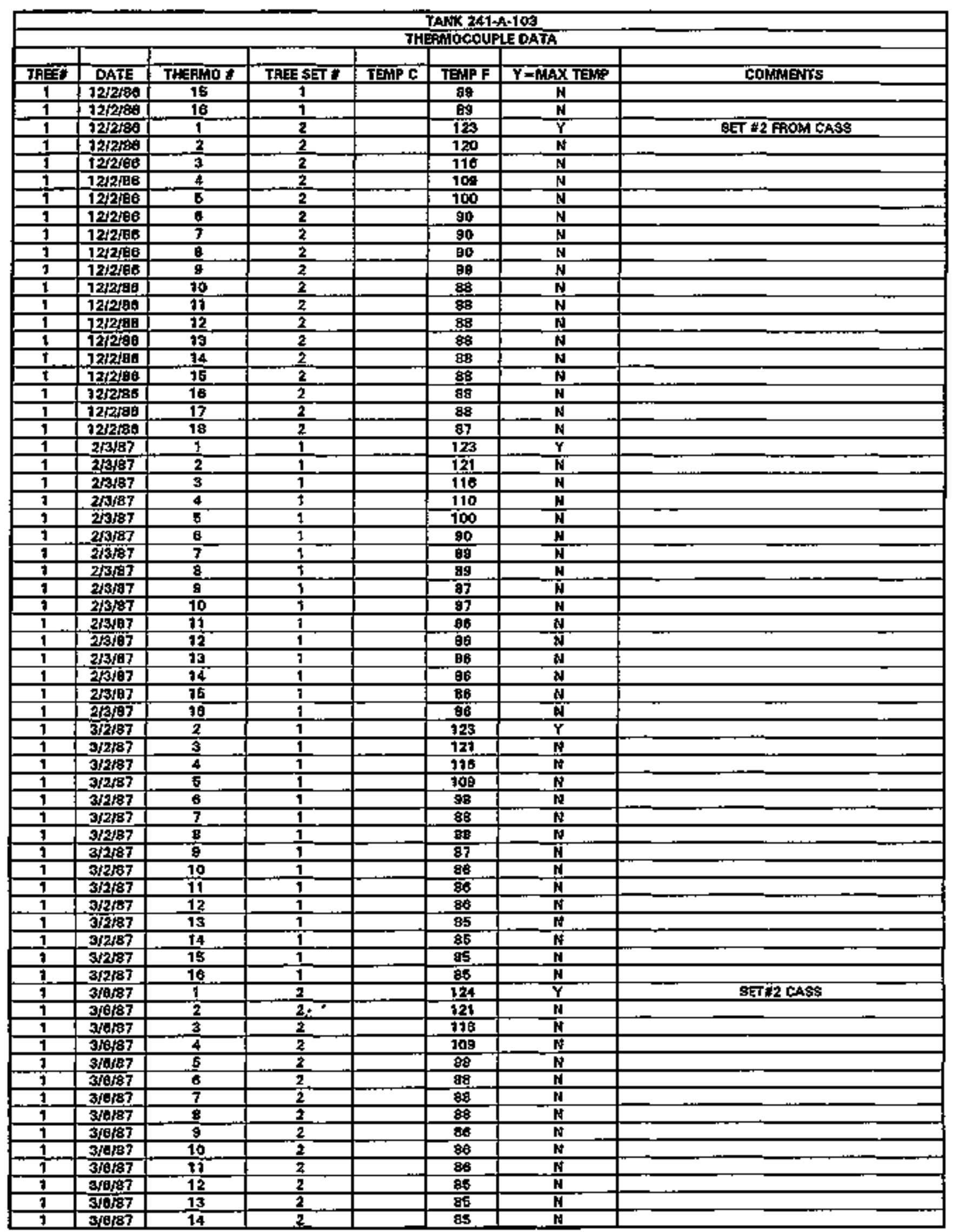

Dete obtained from WHC Survellansa Analysis Computer System (SACS), wune 2,1993. 


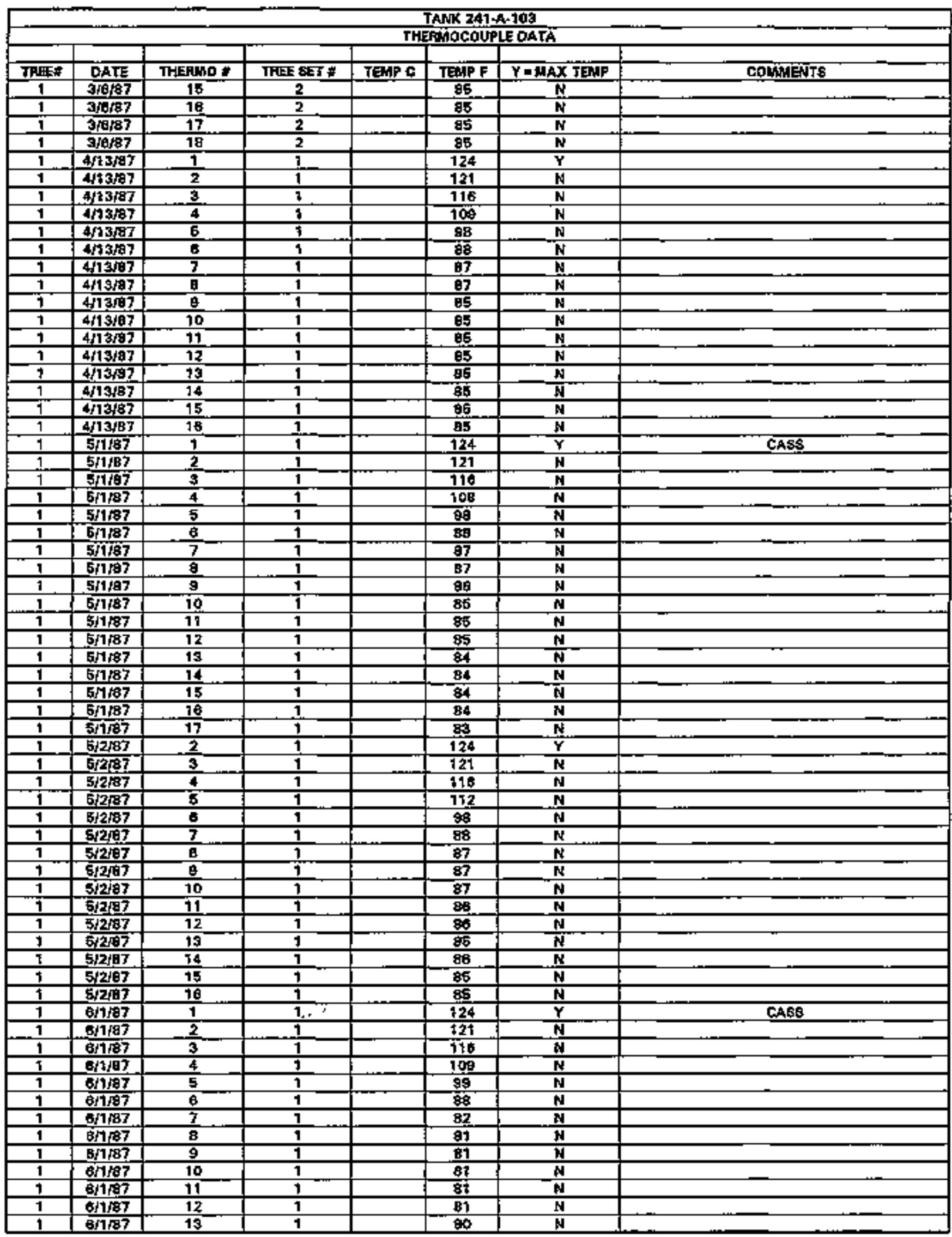

Dota obtained from WHC Surveillant: Analysis Computer System (SACS), June 2,1993. 


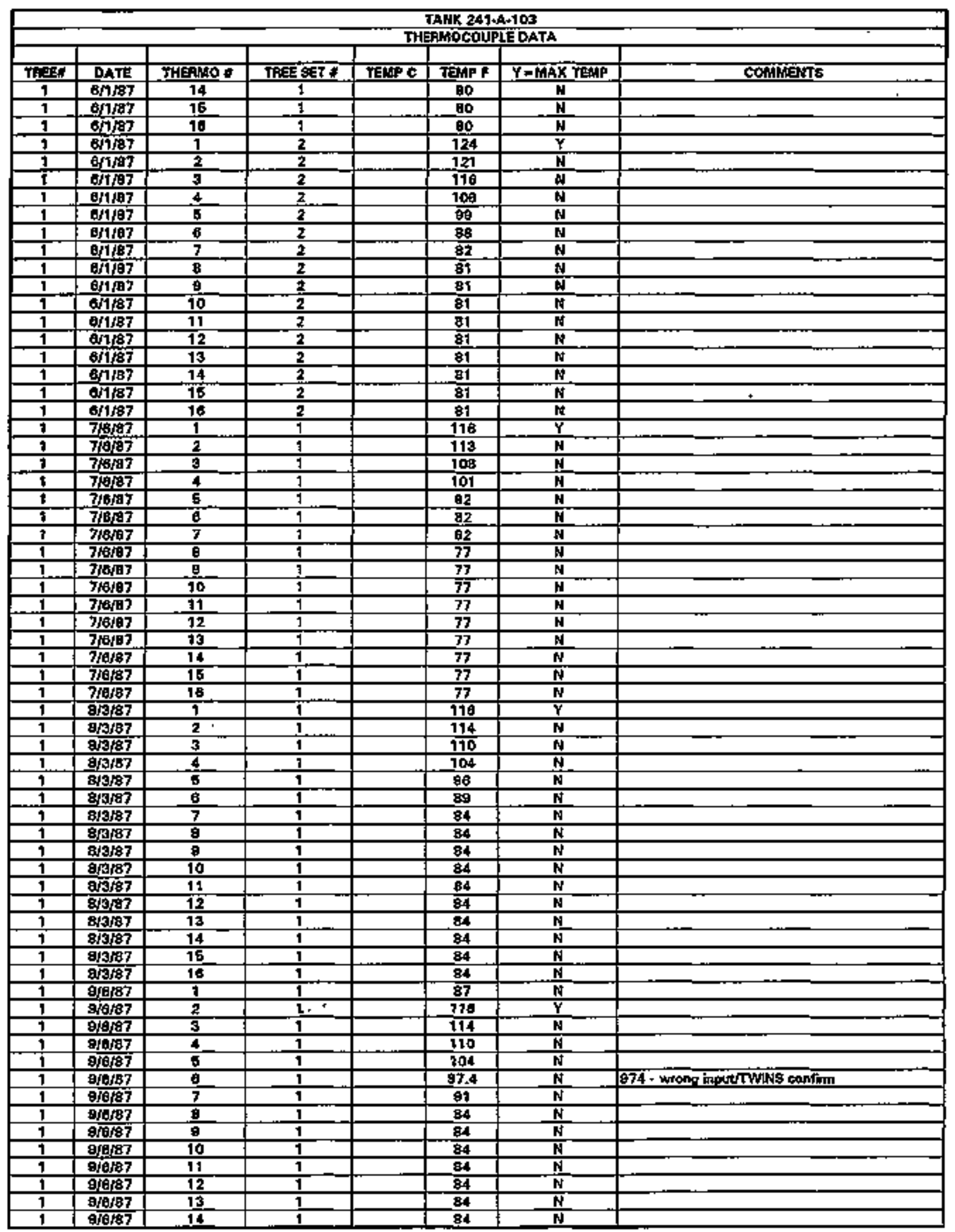

Data obtainad from WHC Survoilance Analysis Computar System \{SACS\}, June 2, 1993. 


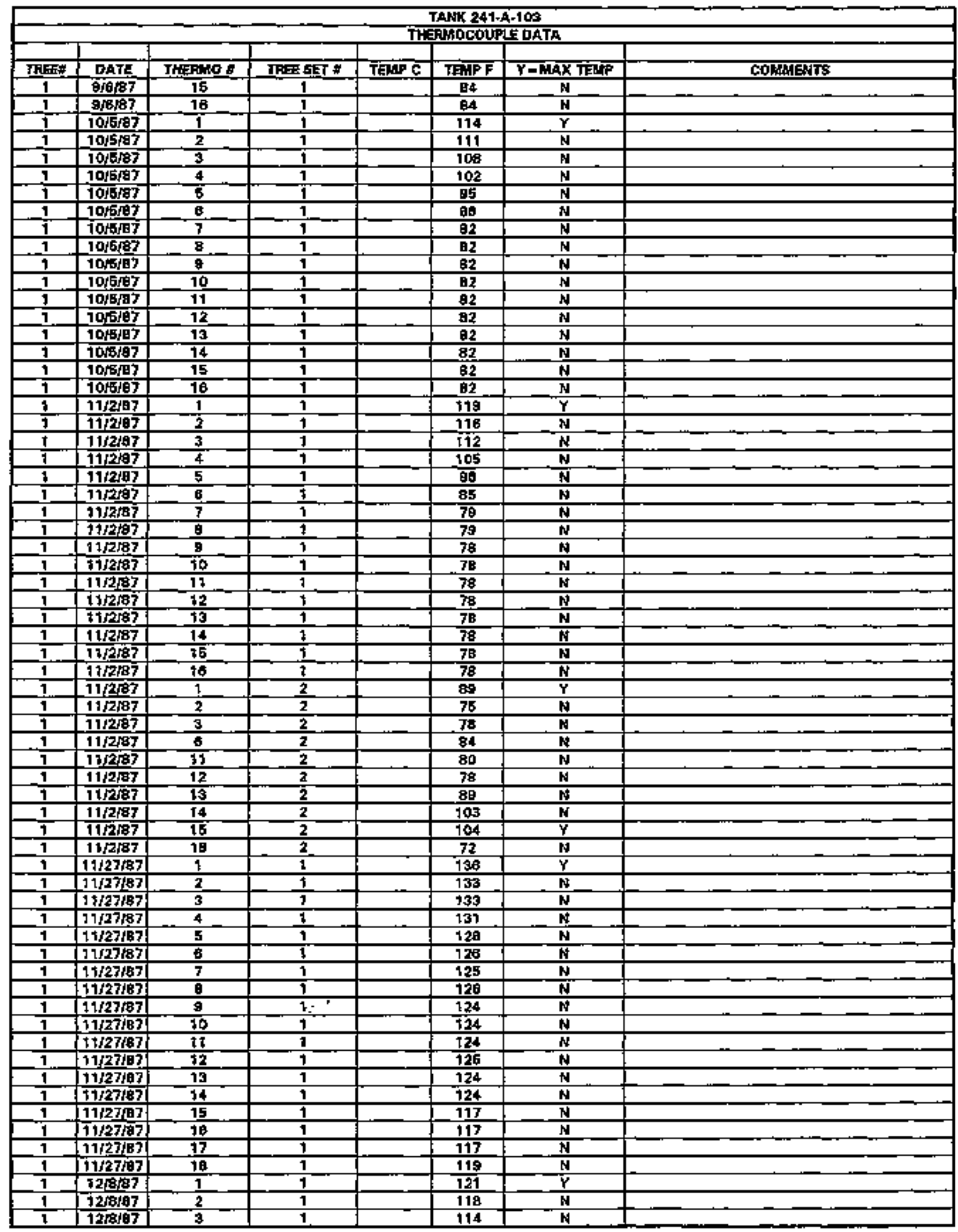

Date ohtained from WHC Survetilance Analysis Computer Syetem (SACS), June 2,1993. 


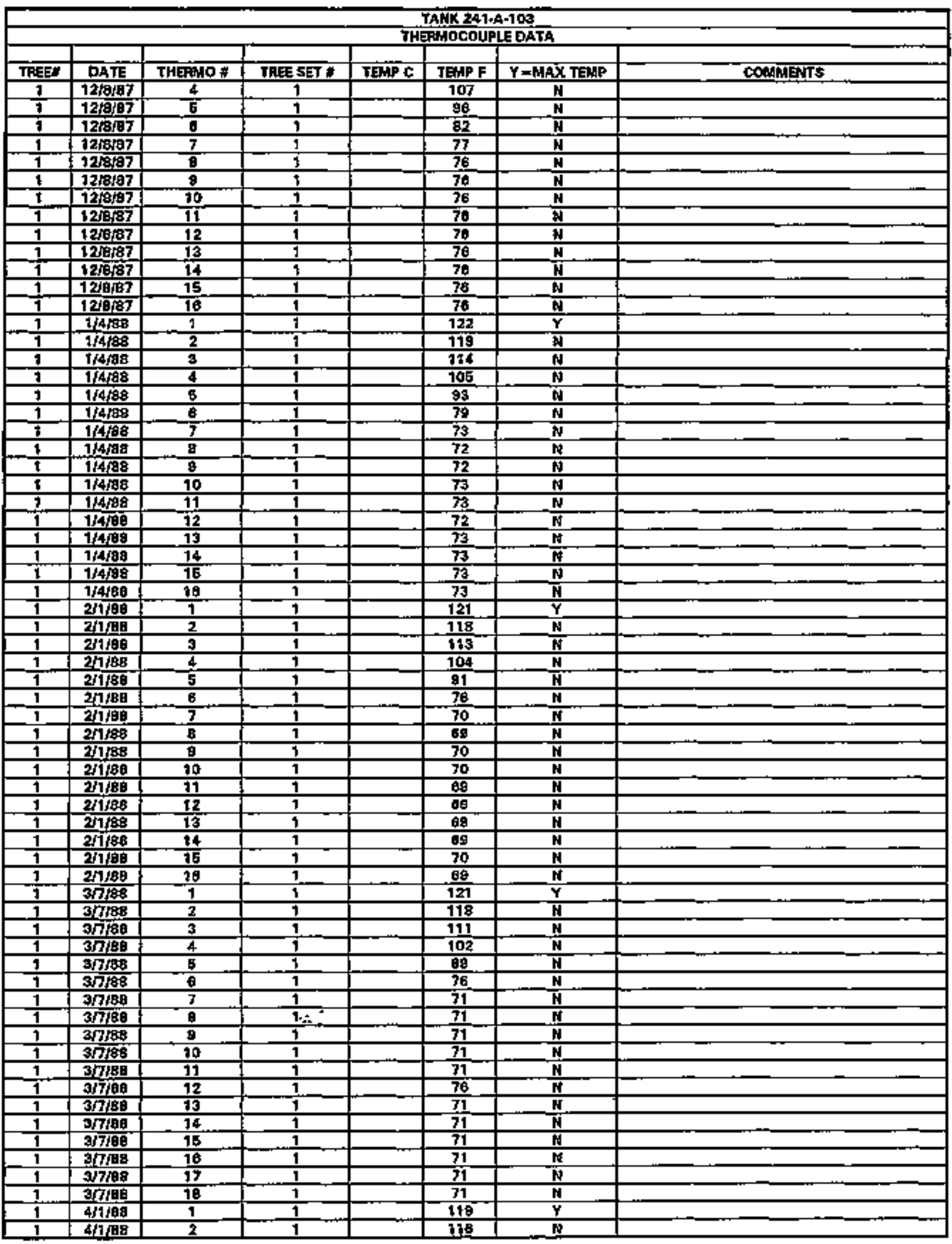

Date obteined from WHC Survaillence Anatysia Computer System (SACS, June 2, 1993, 


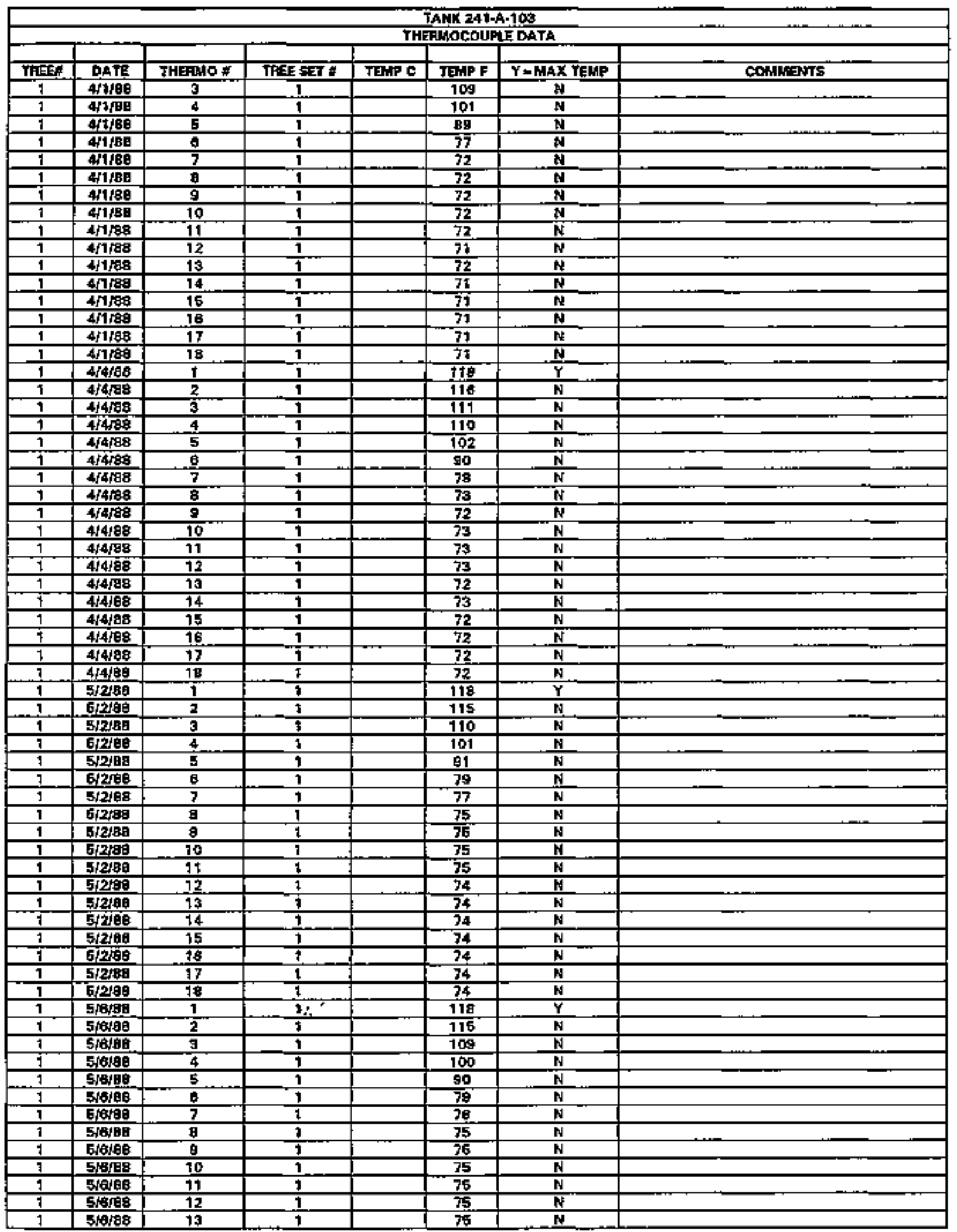

Data obtainad from WHC Survaillance Analysis Computer Srstem [SACS), June 2,1993. 


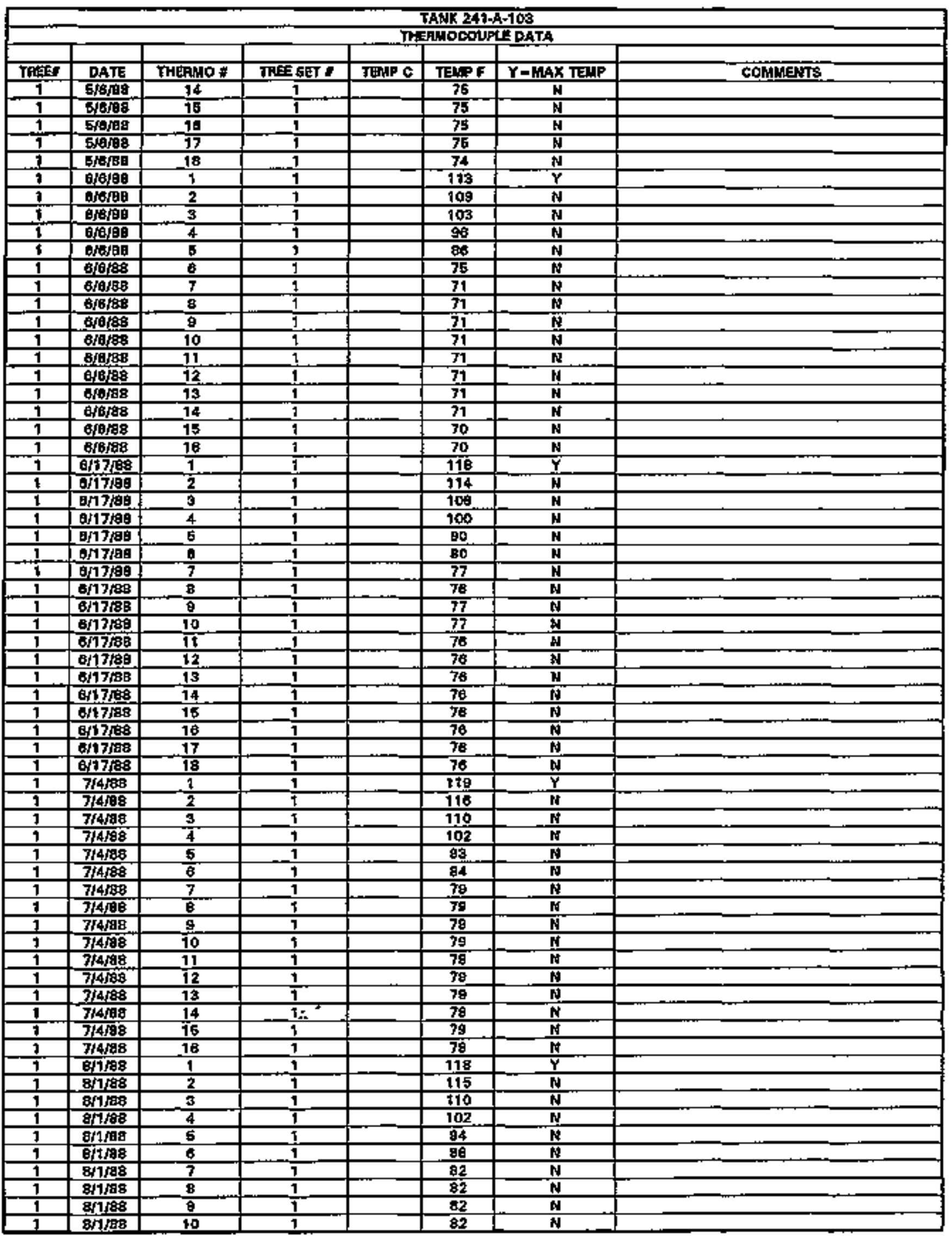

Date obtaired from WHC Survailiance Anolysis Camputar Srstam 13AC St, June 2,1993. 


\begin{tabular}{|c|c|c|c|c|c|c|c|}
\hline \multicolumn{8}{|c|}{ TANK 241-A-103 } \\
\hline & & & & & ANOCOU & E DATA & \\
\hline TREF & DATE & 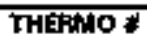 & TAEE SET \# & TEMPC & TEMPF & $Y=M A X$ TEMP & CONHENTE \\
\hline 1 & g/1, & $\$ 1$ & 3 & & $\mathbf{B 2}$ & $\mathbf{N}$ & \\
\hline 1. & gros & 12 & 1 & & 82 & $N$ & \\
\hline 1 & $B / 1 / 8 B$ & 13 & 1 & & 82 & $\mathbf{N}$ & \\
\hline 1 & $8 / 188$ & 14 & 1 & & 82 & $N^{*}$ & \\
\hline 1 & $8 / 1 / 3 B$ & 15 & 1 & & 82 & N & \\
\hline 1 & 87188 & 16 & 1 & & 82 & $\mathbf{N}$ & \\
\hline 1 & O/S/AB & 1 & 1 & & $1 \overline{19}$ & $Y$ & \\
\hline$\overline{1}$ & O/5/B8 & 2 & 7 & & 176 & $N$ & \\
\hline 1 & 5/5/18 & 3 & 1 & & 111 & N & \\
\hline 1 & 9/5/98 & 4 & 1 & & 104 & $\mathbf{N}$ & \\
\hline 1 & $9 / 5 / 88$ & 5 & 1 & & 86 & $\mathbf{N}$ & \\
\hline 1 & 9/5/68 & 8 & $\overline{1}$ & & 69 & M & \\
\hline 1 & g158 & $\bar{T}$ & 1 & & 85 & $\mathbf{N}$ & \\
\hline 1 & 9/5 & 8 & 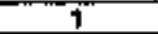 & & 65 & $\mathbf{M}$ & \\
\hline 1 & $9 / 6 \sqrt{88}$ & 8 & 1 & & 白5 & M & \\
\hline 1 & 9/568 & 10 & 1 & & 65 & H & \\
\hline$T$ & $9 / 68$ & 11 & 1 & & 85 & N & \\
\hline 1 & $9 / 5 / 68$ & 12 & 1 & & 65 & $\mathrm{~N}$ & \\
\hline 1 & g/6的8 & 13 & $\overline{1}$ & & e5 & $\bar{N}$ & \\
\hline 1 & 9/3/98 & 14 & 1 & & $\bar{E} 5$ & $N$ & \\
\hline 1 & g/6店 & 15 & 1 & & 85 & $\mathbf{N}$ & \\
\hline 3 & 9/5/8 & 16 & 1 & & 6 & $\mathbf{N}$ & \\
\hline $\bar{z}$ & 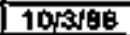 & 1 & 1 & & 118 & 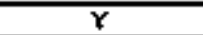 & \\
\hline 1 & 10/38E & 2 & 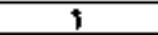 & & 116 & $\mathrm{~N}$ & \\
\hline 1 & 10 & 3 & 1 & & 111 & $\mathbf{N}$ & \\
\hline 1 & 10/3/Ba & 4 & 1 & & $10 \%$ & $\mathrm{~N}$ & \\
\hline$\overline{1}$ & torjes & 5 & $T$ & & 97 & $\mathbf{N}$ & \\
\hline 1 & $10 / 3 / 98$ & $\theta$ & 1 & & 89 & $\mathbf{N}$ & \\
\hline 1 & $10 / 3 / 88$ & 7 & 1 & & 85 & $\mathbf{N}$ & \\
\hline 1 & 10/3/98 & $\mathbf{B}$ & 1 & & 84 & $\mathbf{N}$ & \\
\hline 1 & $10 / 3 / 88$ & 9 & 1 & & 84 & $\mathbf{N}$ & \\
\hline 1 & $10 / 3 / 68$ & 10 & 1 & & 84 & $\mathbf{N}$ & \\
\hline 1 & $10 / 3 / 98$ & $1 \bar{t}$ & 1 & & 84 & $\mathbf{N}$ & \\
\hline 1 & $10 / 3 / 88$ & 12 & 1 & & 84 & $\mathbf{N}$ & \\
\hline 1 & 10لjshes & 13 & $\overline{1}$ & & 84 & $\mathbf{N}$ & \\
\hline 1 & $10 / 3+88$ & 14 & 1 & & 84 & $\mathbf{N}$ & \\
\hline 1 & 10어교 & 16 & 1 & & 84 & N & \\
\hline 1 & 10.388 & 16 & 1 & & 84 & $\mathbf{N}$ & \\
\hline 1 & $10 \mathrm{~d} 2 \mathrm{a} / \mathrm{s} 8$ & 9 & 1 & & T18 & $\bar{Y}$ & \\
\hline 1 & 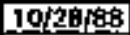 & 2 & 1 & & 113 & $\mathbf{N}$ & \\
\hline 1 & 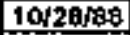 & 3 & 1 & & 700 & N & \\
\hline 1 & $10 / 28 \pi 8$ & 4 & 1 & & 702 & $\mathbf{N}$ & \\
\hline 1 & $10 / 26 / 98$ & B & 1 & & 94 & $\mathbf{N}$ & \\
\hline 1 & $10 / 2188$ & 8 & 1 & & 85 & $\mathbf{N}$ & \\
\hline 1 & 10/2B/88 & 7 & 1 & & 80 & $\mathbf{N}$ & \\
\hline 1 & 10/20/8s & 8 & 1 & & 70 & $\mathbf{N}$ & \\
\hline 1 & 1042B/88 & 9 & 1 & & 79 & $N$ & \\
\hline 1 & $10 / 26 / 89$ & 10 & 1 & & 79 & $\mathbf{N}$ & \\
\hline 1 & 10/2bras & 11 & 1 & & 79 & $\mathbf{N}$ & \\
\hline 1 & $10 \mathrm{rer} 8 \mathrm{~s}$ & 12 & 1 & & 79 & $\mathbf{M}$ & \\
\hline 1 & 10/26/8 & 13 & 1 & & 79 & $N$ & \\
\hline 1 & $10 \mathrm{f} 2 \mathrm{a} / \mathrm{s}$ & 14 & 1 & & 79 & $\mathbf{N}$ & \\
\hline 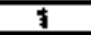 & 10/28: & 15 & $1 t^{\prime}$ & & 79 & $\mathbf{M}$ & \\
\hline 1 & 10,28ja日 & 10 & 1 & & 79 & $\mathbf{N}$ & \\
\hline 1 & 10강s & 17 & 1 & & 79 & $\mathbf{N}$ & \\
\hline 1 & 10/2B/as & 18 & 1 & & 79 & $\mathbf{N}$ & \\
\hline 1 & 11 pes & 1 & $\overline{1}$ & & 118 & $\bar{Y}$ & \\
\hline 1 & 11, & 2 & 1 & & 113 & $\mathbf{N}$ & \\
\hline$\overline{1}$ & 11 rides & 3 & 1 & & 912 & 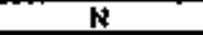 & \\
\hline 1 & 11/4/88 & 4 & 1 & & 105 & $\mathbf{N}$ & \\
\hline 1 & $11 / 8188$ & $\overline{8}$ & 1 & & 88 & $\mathbf{M}$ & \\
\hline 1 & 11/9 gres & 8 & $T$ & & $8 \mathrm{~B}$ & $\bar{N}$ & \\
\hline 1 & 11 19/8 & 7 & 1 & & $\mathbf{8 5}$ & $\mathbf{N}$ & \\
\hline 1 & 11/4 Afg & B & 1 & & 81 & $\mathbf{N}$ & \\
\hline 1 & 11, & $\bar{\theta}$ & 1 & & 81 & N & \\
\hline
\end{tabular}

Data obteingd fram WhC Sturvillance Analyaí Computer Syatem (SACS), June 2، 1993. 


\begin{tabular}{|c|c|c|c|c|c|c|c|}
\hline \multirow{2}{*}{\multicolumn{8}{|c|}{ 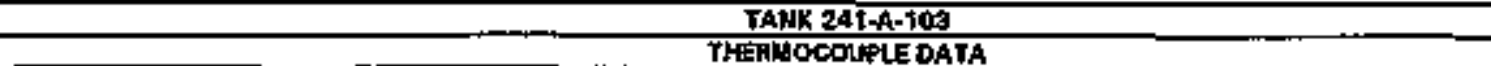 }} \\
\hline & & & & & & EDATA & \\
\hline TADEF & DATE & THERMO * & TREA SET 辛 & TEMP C & TEMP F & $Y=M A X$ THP & CDWHANES \\
\hline 1 & 11/gres & 10 & 1 & & 80 & $\mathbf{N}$ & \\
\hline 1 & 11B BNA & 11 & 1 & & 80 & $N$ & \\
\hline 1 & 11/8g & 12 & 1 & & 80 & $\mathbf{n}$ & \\
\hline 1 & $15 / 1 / 18 B$ & 13 & 1 & & 80 & $\mathbf{H}$ & \\
\hline 1 & $15 \sqrt{1 / 88}$ & 14 & 1 & & 80 & $\boldsymbol{N}$ & \\
\hline 1 & $11 / 8 / 88$ & 16 & 1 & & 80 & $\mathbf{N}$ & \\
\hline 1 & $11 \sqrt{8 / 888}$ & 16 & 1 & & 80 & H & \\
\hline 1 & 1 J16 & 1 & 1 & & 116 & $Y$ & \\
\hline 1 & 17 /18kg & 2 & 1 & & 113 & $\mathbf{N}$ & \\
\hline 1 & $17 / 18 / 89$ & 3 & 1 & & 108 & $\mathrm{H}$ & \\
\hline 1 & 11/1/Bg & 4 & 1 & & 102 & $\mathbf{N}$ & \\
\hline 1 & 11/18/88 & 5 & 7 & & 93 & $\mathbf{N}$ & \\
\hline 1 & 11/19/88 & i & 1 & & 03 & $N$ & \\
\hline 1 & 11/1 & 7 & 1 & & 78 & $\mathbf{N}$ & \\
\hline$t$ & $1148 \mathrm{BB}$ & 自 & 1 & & 78 & $\mathbf{N}$ & \\
\hline 1 & $11 / 18 / 8$ & $\bar{\theta}$ & 1 & & 78 & $\bar{N}$ & \\
\hline 1 & 11/19res & 10 & 1 & & 78 & N & \\
\hline 1 & $11 / 10 / 28$ & 11 & 1 & & 77 & $\mathrm{~N}$ & \\
\hline 1 & 11/1 B/g8 & 12 & 1 & & 77 & $\mathbf{N}$ & \\
\hline 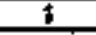 & $11 / 49 / 88$ & 13 & 1 & & 77 & $\mathbf{N}$ & \\
\hline 1 & I1/18400 & 14 & 1 & & 77 & $\mathrm{H}$ & \\
\hline 1 & I1/18ig的 & 15 & 1 & & 77 & $\mathbf{N}$ & \\
\hline 1 & $11 / 18 \mathrm{Ag}$ & 16 & 1 & & 77 & 소 & \\
\hline 1 & 11/18셔 & 17 & 1 & & 77 & $\mathbf{N}$ & \\
\hline 1 & $11 / 18 \mathrm{He}$ & 18 & 1 & & 77 & $\mathbf{N}$ & \\
\hline 1 & 12586 & 1 & 1 & & & $Y$ & (189- tuspoot dato (high) \\
\hline 1 & $12 / 6 / 8 B$ & $\overline{2}$ & 1 & & & $\overline{\mathbf{N}}$ & A \\
\hline 1 & $12 \sqrt{5}, 88$ & 3 & 1 & & & $\mathbf{N}$ & I81 - suspect date (hiph) \\
\hline 1 & $12 / 5 / 88$ & 4 & 1 & & & $\mathbf{N}$ & (174- nupasot d to (hioh) \\
\hline$\tau$ & 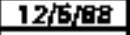 & 5 & 1 & & & $\mathbf{N}$ & 164 - stupect dats [hight \\
\hline 1 & 12/5/ag & $\theta$ & 1 & & & $\mathbf{N}$ & 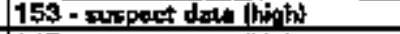 \\
\hline 1 & $12 / 6$. & 7 & 1 & & & $\mathbf{N}$ & 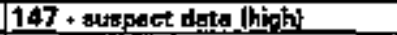 \\
\hline 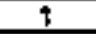 & 12/5/ag & B & $I$ & & & $\mathbf{N}$ & 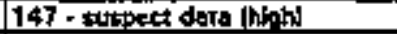 \\
\hline 1 & 12/6/日9 & g & 5 & & & $M$ & 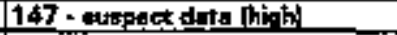 \\
\hline 1 & 12/3196 & 90 & 7 & & & $N$ & 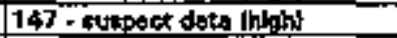 \\
\hline 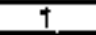 & $1215 \mathrm{E}$ & 11 & $t$ & & & $\mathbf{N}$ & 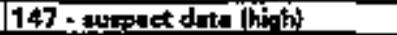 \\
\hline 1 & 12/5iag & 12 & 1 & & & $\mathbf{N}$ & 147- eutpect dets lhight \\
\hline 1 & $12 / 6 \mathrm{Be}$ & 13 & $\$$ & & & $\mathbf{N}$ & 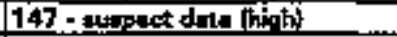 \\
\hline 1 & 12/50a & 74 & $?$ & & & $\mathbf{N}$ & 147 + entupart data thioly \\
\hline 1 & 12568 & 15 & 1 & & & N & 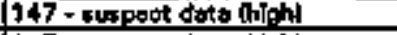 \\
\hline 1 & $12 / 5 / B B$ & 16 & 1 & & & N & 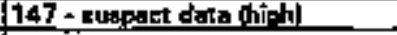 \\
\hline 1 & $1 / 2 / 89$ & 1 & 1 & & 120 & $\mathbf{Y}$ & \\
\hline 1 & $1 / 2 / 89$ & 2 & 1 & & 167 & N & \\
\hline 1 & $1 / 2 / 89$ & 当 & 1 & & 103 & $\mathbf{N}$ & \\
\hline 1 & $1 / 2 / 39$ & 4 & 1 & & 20 & $N$ & \\
\hline 1 & $9 / 289$ & 5 & 1 & & 88 & $\mathbf{N}$ & \\
\hline 1 & $1 / 2 / 89$ & $a$ & 1 & & 74 & N & \\
\hline 1 & $1 / 2 / 89$ & 7 & 1 & & B5 & N & \\
\hline 1. & $1 / 2 / 89$ & g & 1 & & 67 & $\mathrm{~N}$ & \\
\hline 1 & $1 / 2 / 88$ & 8 & 1 & & 67 & $\mathrm{~N}$ & \\
\hline 1 & $7 / 2 / 89$ & 10 & 1 & & 87 & $\mathbf{N}$ & \\
\hline 1 & $1 / 2 / 89$ & 11 & 1 & & 67 & $\mathbf{N}$ & \\
\hline 1 & $1 / 2 / 89$ & 12 & $k_{4}$ & & 67 & $\mathbf{N}$ & \\
\hline 1 & $1 / 2 / 189$ & 13 & $i$ & & 67 & $\mathbf{N}$ & \\
\hline 1 & $1 / 2169$ & 14 & 1 & & 67 & $N$ & \\
\hline 1 & 12100 & 16 & 1 & & 57 & $\mathbf{N}$ & \\
\hline$i$ & 1/2/8B & 16 & 1 & & 67 & $\mathbf{N}$ & \\
\hline 1 & $2 / 0 / 89$ & 7 & 1 & & 117 & $\mathbf{Y}$ & \\
\hline 1 & 2/prog & 2 & 1 & & $\$ 15$ & $\sqrt{N}$ & \\
\hline 1 & $2 / 0189$ & 5 & 1 & & tto & $M$ & \\
\hline 1 & 216189 & $\pi$ & 1 & & tos & $\mathbf{N}$ & \\
\hline 1 & $250 / 29$ & 5 & 1 & & 82 & $\overline{\mathbf{N}}$ & \\
\hline 1 & 28089 & $\theta$ & 1 & & 80 & $N$ & \\
\hline 1 & 25/199 & 7 & 1 & & 72 & $\mathbf{N}$ & \\
\hline 1 & $2 / 189$ & $\overline{8}$ & 1 & & $7 t$ & $\mathbf{N}$ & \\
\hline
\end{tabular}

Data cbtained from WHC Surveilenea Anolysis Computer Syatem \{SACS\}, Jume 2, 1993. 


\begin{tabular}{|c|c|c|c|c|c|c|c|}
\hline \multicolumn{8}{|c|}{ TAMK 241A-103 } \\
\hline \multicolumn{8}{|c|}{ THEAOOCOLFIE DATA } \\
\hline TFEF & DATE & THEFPHO \# & TREG GET & TEMP C & TEAP F & $Y=$ MAX TEAP & COMMENTS \\
\hline$i$ & 20780 & - & $i$ & & 72 & $N$ & \\
\hline 1 & $2 / 8 / 89$ & 10 & 1 & & 71 & $\mathbf{N}$ & \\
\hline 1 & 218189 & 11 & $i$ & & $7 t$ & $\mathbf{N}$ & \\
\hline$T$ & $2 \sqrt{6 / 39}$ & 12 & 1 & & 71 & $\mathbf{N}$ & \\
\hline 1 & $2 / 6 / 89$ & 13 & 1 & & 71 & $\mathbf{N}$ & \\
\hline 1 & $2 / 0189$ & 14 & 1 & & $7 t$ & $\mathbf{N}$ & \\
\hline 1 & $2 / 4 / 89$ & 15 & 1 & & $7 t$ & $\mathbf{N}$ & \\
\hline 1 & $2 / 6 / 89$ & 18 & 1 & & $7 t$ & $N$ & \\
\hline 1 & अणj89 & 1 & 1 & & 117.2 & $\overline{\mathbf{Y}}$ & \\
\hline 1 & $3 \sqrt{6 / 89}$ & 2 & $\mathbf{1}$ & & 114.2 & $\mathbf{N}$ & \\
\hline 1 & 36/as & $\mathrm{s}$ & 1 & & $50 \overline{10}$ & N & \\
\hline$\overline{1}$ & 36/89 & 4 & 1 & & 101.1 & $\mathbf{N}$ & \\
\hline 1 & आ0/39 & 5 & 1 & & 90.1 & $\mathbf{M}$ & \\
\hline 1 & 3989 & 6 & 1 & & 77 & $\mathbf{N}$ & \\
\hline 1 & S/O/B9 & 7 & 1 & & 70.5 & $\mathbf{N}$ & \\
\hline 1 & $3 / 6 / 89$ & 8 & 1 & & 70.2 & $\mathbf{N}$ & \\
\hline$\overline{1}$ & $3 / 5 / 69$ & 9 & $\mathbf{1}$ & & 70.2 & $\bar{N}$ & \\
\hline$\overline{1}$ & अ & 10 & 1 & & 70.1 & if & \\
\hline 1 & 3\$/9/9 & 11 & 1 & & 0.8 & M & \\
\hline$\overline{1}$ & अं6198 & 12 & 1 & & 6.9 & $\bar{N}$ & \\
\hline$\underline{i}$ & 3/8/8요 & 13 & 1 & & 69,8 & $\mathbf{R}$ & \\
\hline$\$$ & Storas & 14 & 1 & & 69.8 & N & \\
\hline 1 & अ & 15 & 1 & & 69.8 & $\mathbf{N}$ & \\
\hline 1 & 3\%/98 & 16 & 1 & & 09.6 & $N$ & \\
\hline 1 & $44 \sqrt{89}$ & 1 & 1 & & & $\mathbf{N}$ & 3) - eubace dita llowl hemp imurgel \\
\hline 1 & 4/4/998 & 2 & 1 & & & $\mathbf{N}$ & 41 - tuspect dete Howl (temp havarad \\
\hline 1 & $\$ / 4 / 99$ & 3 & 1 & & & 숭 & 48 - nuapeg dota llowl] [tegre invervel] \\
\hline 1 & $4 / 4 / 9 \mathrm{~S}$ & 4 & 1 & & & M & 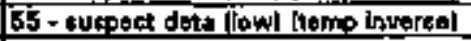 \\
\hline 1 & $4 / 4 / 98$ & 5 & 1 & & & $\mathbf{A}$ & 65 - wapect dita fowl [temp irversel] \\
\hline$I$ & 4/4/89. & E & 1 & & & 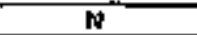 & 77. suepece deta (themp imueres) \\
\hline$\overline{5}$ & $4 / 4 / 28$ & 7 & 1 & & & $\mathbf{H}$ & B1 - Burpect data (high) (temp invernet) \\
\hline 1 & 4/4/99 & E & 1 & & & $\mathbf{N}$ & 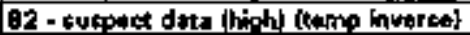 \\
\hline 1 & $4 / 4 / 89$ & 9 & 1 & & & $\mathbf{N}$ & 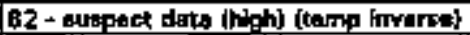 \\
\hline 1 & $4 / 4 / 39$ & 10 & 1 & & & $\mathbf{M}$ & 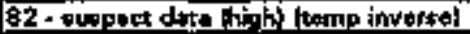 \\
\hline 1 & 4/4/8g & 11 & 1 & & & $\mathbf{N}$ & 82 + Elypact dets thegh ltemp inversed \\
\hline 1 & $4 / 4 / 69$ & 12 & 1 & & & $\overline{\mathbf{H}}$ & 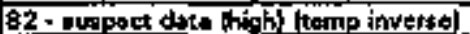 \\
\hline $\mathbf{T}$ & 4/4/189 & 13 & 1 & & & $\mathbf{N}$ & 82 - cubpart dats high ltamp Imverael \\
\hline 1 & $4 / 4 / 98$ & 14 & 1 & & & 포 & 82 - puspect dro thigh (temp invorsel \\
\hline $\mathbf{1}$ & 4/4/6S & 15 & 1 & & & $\mathbf{N}$ & 82 - eturpent date thlsh) (temp inversel \\
\hline 7 & $4 / 4 / 98$ & 16 & $\overline{1}$ & & & $\overline{\mathbf{Y}}$ & 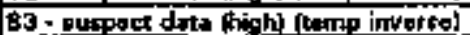 \\
\hline $\bar{T}$ & $4 / 20 / 69$ & 1 & $\mathbf{1}$ & & 103 & $\mathbf{Y}$ & \\
\hline T & $4 \sqrt{2}$ 애요 & 2 & i & & 98 & $\bar{N}$ & \\
\hline 1 & $4 / 20 / 89$ & 3 & 1 & & 93 & $\mathbf{N}$ & \\
\hline 1 & $4 / 20199$ & 4 & $\overline{1}$ & & 85 & $\bar{N}$ & \\
\hline 1 & $4 / 20 / 99$ & 5 & 1 & & $\boldsymbol{T}$ & $\mathbf{n}$ & \\
\hline$\overline{1}$ & $4 \sqrt{20} \mathrm{dag}$ & $\overline{\mathbf{B}}$ & 1 & & 票 & i & \\
\hline 1 & $4 / 20 / 99$ & 7 & 1 & & 69 & A & \\
\hline 1 & $4 / 20 / 99$ & 8 & 1 & & 60 & $\mathbf{N}$ & \\
\hline 1 & $4 \sqrt{20}$ 잉 & 9 & 1 & & 60 & $\mathbf{N}$ & \\
\hline 7 & $4 / 20 / 99$ & 10 & 1 & & 60 & $\mathbf{N}$ & \\
\hline 1 & $4 / 20 / 99$ & 11 & $\mathrm{I}$ & & 80 & H & \\
\hline 1 & $4 / 20 / 19$ & 12 & 1 & & 60 & $\mathbf{N}$ & \\
\hline 1 & $4 / 20 / 99$ & 13 & $1 i^{5}$ & & 60 & $\mathbf{N}$ & \\
\hline$T$ & $4 / 20 / 98$ & 14 & 1 & & 60 & $\mathbf{N}$ & \\
\hline$\$$ & $4 / 20 \mathrm{~d}=9$ & 16 & 1 & & 60 & $N$ & \\
\hline $\mathbf{T}$ & $4 / 20 / 89$ & 10 & 1 & & so & N & \\
\hline 1 & $5 / 499$ & 1 & 1 & & 109.7 & $\mathbf{Y}$ & \\
\hline 1 & 57/89 & 2 & 1 & & 100.2 & N & \\
\hline 1 & $5 / 1 / 89$ & 3 & 1 & & 103.2 & $\mathbf{N}$ & \\
\hline 7 & Whas & 4 & $i$ & & 85.7 & $\mathbf{N}$ & \\
\hline 1 & 51/99 & 5 & 1 & & 87.7 & $\mathbf{N}$ & \\
\hline 1 & W1/189 & 6 & 1 & & 79.6 & $\mathbf{N}$ & \\
\hline 1 & $51 / 89$ & 7 & 1 & & 76.8 & $\psi$ & \\
\hline 1 & S1/29 & B & 1 & & 75.6 & $\mathbf{N}$ & \\
\hline 1 & $6 / 1889$ & 9 & $\overline{1}$ & & 75.3 & $\mathbf{H}$ & \\
\hline
\end{tabular}

Date obtained from WHC Sturveillanos Analvals Comptsief System (SACS), June 2, 5993. 


\begin{tabular}{|c|c|c|c|c|c|c|c|}
\hline \multicolumn{8}{|c|}{ TANK 24E-A-103 } \\
\hline & & & & & Anocou & FDATA & \\
\hline TARE & DATE & THEALEO & TFEE EST $E$ & TEMP C & TEMAP F & $Y=$ MAX TENP & COOHLEATS \\
\hline 1 & 551899 & 10 & 1 & & 75.2 & $\mathbf{N}$ & \\
\hline 1. & 5/189 & 11 & 1 & & 75,1 & $\mathbf{N}$ & \\
\hline 1 & 5 (1) & 12 & 1 & & 75 & $\mathbf{N}$ & \\
\hline 1 & \$1199 & 13 & 1 & & 74.9 & $\mathbf{N}$ & \\
\hline 1 & 5/1/99 & 14 & 1 & & 75 & $\mathbf{N}$ & \\
\hline 1 & $5 / 1 / 80$ & 16 & 1 & & 75 & $\mathbf{N}$ & \\
\hline 1 & 학/198 & 18 & 1 & & 74.8 & $\mathbf{M}$ & \\
\hline$\$$ & 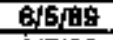 & 1 & 1 & & 109 & $\mathbf{Y}$ & \\
\hline 1 & 6/508 & 2 & 1 & & $50 B$ & $N$ & \\
\hline 1 & 배혀 & 3 & 1 & & 102 & $\mathbf{N}$ & \\
\hline 1 & 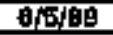 & 4 & 1 & & 96 & $N$ & \\
\hline 1 & EFWag & E & 1 & & 80 & $\mathbf{M}$ & \\
\hline 1 & GEВ & 皇 & 1 & & 82 & $\mathrm{~N}$ & \\
\hline 1 & 65더요 & 7 & 1 & & 78 & $N$ & \\
\hline 1 & A & 8 & 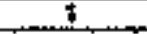 & & 28 & $\mathbf{N}$ & \\
\hline 1 & $6 / 6 / 89$ & 8 & $i$ & & 70 & N & \\
\hline 1 & $0 / 5 / 89$ & 10 & 1 & & 78 & $\mathbf{N}$ & \\
\hline 1 & $6 \sqrt{5 / 89}$ & 11 & 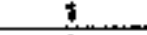 & & 78 & N & \\
\hline 1 & $6 / 6 / 89$ & 12 & 1 & & 78 & $\mathbf{N}$ & \\
\hline 1 & 0/5/89 & 13 & $\mathbf{I}$ & & 77 & $\mathbf{N}$ & \\
\hline 1 & Otgreg & 14 & $t$ & & 77 & $N$ & \\
\hline 1 & $6 / 1589$ & 15 & 1 & & 77 & $\mathbf{N}$ & \\
\hline 1 & $8 / 6 / 89$ & 18 & $\$$ & & i & $\mathbf{N}$ & \\
\hline 1 & $7 / 3 / 89$ & 1 & $\mathbf{I}$ & & 108,3 & $\mathbf{Y}$ & \\
\hline 1 & $7 / 2 / 89$ & 2 & 1 & & 105,8 & $\boldsymbol{N}$ & \\
\hline 1 & 7/3/29 & 3 & 1 & & 101.6 & $\mathbf{N}$ & \\
\hline 1 & $7 \times 189$ & 4 & 1 & & 98 & $N$ & \\
\hline 1 & $713 / 89$ & 5 & 1 & & 99.5 & $N$ & \\
\hline 1 & $7 / 3 / 89$ & B & 1 & & 82.8 & $\mathbf{N}$ & \\
\hline 1 & $7 / 3 / 89$ & 7 & 1 & & 79.1 & $\mathbf{N}$ & \\
\hline$\$$ & $7 / 3 / 89$ & $\overline{8}$ & 1 & & 79 & 시 & \\
\hline 1 & $7 / 3 / 69$ & $\mathbf{A}$ & 1 & & 79.7 & $\mathbf{N}$ & \\
\hline 1 & $7 / 369$ & 10 & 1 & & 78.7 & $\mathbf{N}$ & \\
\hline 1 & 73 Hag & 11 & 1 & & 78.7 & $\mathbf{N}$ & \\
\hline 1 & $7 / 3 \sqrt{100}$ & 12 & 1 & & 78.7 & $\mathbf{N}$ & \\
\hline 1 & 7/3/89 & 13 & 1 & & $7 \mathrm{~A} . \mathrm{E}$ & $N$ & \\
\hline 1 & $7 / 3 / 80$ & 14 & 1 & & 70.6 & $\mathbf{N}$ & \\
\hline 1 & 7/398 & 15 & 1 & & 78.6 & $\mathbf{N}$ & \\
\hline 1 & $7 / 399$ & 10 & 1 & & 78.0 & N & \\
\hline 1 & giz/sis & 1 & 1 & & 91 & $\bar{Y}$ & \\
\hline 1 & arfiga & 2 & 1 & & 88 & $\mathbf{N}$ & \\
\hline 1 & 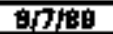 & 3 & 1 & & 84 & $\mathbf{N}$ & \\
\hline 1 & By/89 & 4 & 1 & & 78 & $\mathbf{N}$ & \\
\hline 1 & entra & 5 & 1 & & 71 & N & \\
\hline 1 & 877199 & G & 1 & & 64 & $N$ & \\
\hline 1 & B/7/89 & 7 & 1 & & 59 & $\mathbf{N}$ & \\
\hline 1 & $8 / 7 / 90$ & B & 1 & & 59 & N & \\
\hline 1 & 敫侮息 & 8 & 1 & & 89 & $\mathbf{M}$ & \\
\hline 1 & $8 \longdiv { 7 / 8 9 }$ & 10 & 1 & & 59 & $\mathbf{N}$ & \\
\hline 1 & 8778 & 11 & 1 & & 58 & $\mathbf{N}$ & \\
\hline 1 & $9 \sqrt{7 / 89}$ & 12 & 1 & & 5 & $\mathbf{N}$ & \\
\hline 1 & 917789 & 13 & 1 & & 59 & N & \\
\hline 1 & er789 & 74 & $10^{\prime}$ & & 58 & $\mathbf{N}$ & \\
\hline 1 & B/7ros & 15 & 1 & & 58 & N & \\
\hline 1 & 自硯的 & 16 & 1 & & 58 & $\mathbf{N}$ & \\
\hline 1 & 10 20/89 & 1 & 1 & & 107 & $\mathbf{Y}$ & \\
\hline 1 & 7022109 & 2 & 1 & & 100 & $\mathbf{N}$ & \\
\hline 1 & 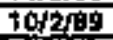 & 3 & 1 & & 101 & $\mathbf{N}$ & \\
\hline 1 & Tor2tog & 4 & 1 & & 97 & $N$ & \\
\hline$i$ & 102西白 & E & 1 & & 92 & $\mathbf{M}$ & \\
\hline 1 & 1012ies & 6 & 1 & & 84 & $\mathbf{N}$ & \\
\hline 1 & 10 2西白 & 7 & 1 & & 20 & $\mathbf{N}$ & \\
\hline 1 & 10내영 & B & 1 & & 79 & $N$ & \\
\hline 1 & 10 2189 & 9 & 1 & & 79 & $\mathbf{N}$ & \\
\hline 1 & 1012月8 & 10 & 1 & & 79 & $\mathbf{N}$ & \\
\hline
\end{tabular}

Data obtained from WHC Surveillence Aneryste Computer Syctem \{SACS\}, Juno 2, 1993. 


\begin{tabular}{|c|c|c|c|c|c|c|c|}
\hline \multicolumn{8}{|c|}{ TANK 247. } \\
\hline & & & & & inocou & EDATA & \\
\hline & & & & & & & \\
\hline ThEx: & DATE & THËFTAO है & TRES SET \# & T征 c & TENPF & $Y=\operatorname{MAX}$ TEloP & COMHENTS \\
\hline 1 & $10 / 2 / 89$ & 11 & 1 & & 79 & $\mathbf{N}$ & \\
\hline 1 & 10 2/89 & $\overline{12}$ & $i$ & & 79 & $\bar{N}$ & \\
\hline 1 & 10나녕 & 13 & 1 & & 78 & $\mathbf{H}$ & \\
\hline 1 & 10/2狍 & 14 & 1 & & 79 & $N$ & \\
\hline 1 & 10/2/89 & 15 & 7 & & 79 & $\mathbf{N}$ & \\
\hline 1 & 10/2/89 & 18 & $i$ & & 78 & $\mathbf{N}$ & \\
\hline 1 & Iת100 & 1 & 1 & & 118 & $\mathbf{Y}$ & \\
\hline 1 & $1 / 1 / 90$ & 2 & 1 & & $11 \overline{6}$ & $\bar{N}$ & \\
\hline 1 & $7 / 1 / 80$ & 3 & 1 & & 112 & $\mathbf{N}$ & \\
\hline 1 & $1 / 1 / 90$ & 4 & 1 & & 108 & $\mathbf{N}$ & \\
\hline 7 & $11 / 100$ & 5 & $i$ & & 101 & $\bar{N}$ & \\
\hline 1 & $1 / 5 / 90$ & a & $T$ & & 92 & $\mathbf{N}$ & \\
\hline 1 & 1/tego & $\overline{7}$ & 1 & & 自7 & $\bar{N}$ & \\
\hline 1 & $1 / 1 / 90$ & $\mathbf{a}$ & 1 & & 67 & $\mathbf{N}$ & \\
\hline 1 & 11190 & g & 1 & & 96 & N & \\
\hline 1 & $1 / 1 / 90$ & 10 & 1 & & 60 & M & \\
\hline 1 & $1 / 190$ & 11 & 1 & & 68 & $\mathbf{N}$ & \\
\hline $\boldsymbol{j}$ & $1 / 1 / 90$ & $\$ 2$ & 1 & & 80 & $N$ & \\
\hline 7 & $1 / 1 / 90$ & $\sqrt{13}$ & 1 & & 66 & $\mathbf{N}$ & \\
\hline 1 & $1 / 1 / 90$ & 14 & 1 & & 80 & $\mathrm{~N}$ & \\
\hline 1 & $1 / 1 / 90$ & 15 & 1 & & 86 & $\mathbf{N}$ & \\
\hline 1 & $1 / 1 / 90$ & 10 & 1 & & Bq̣ & $\mathbf{N}$ & \\
\hline 1 & $2 \sqrt{500}$ & 1 & 1 & & 118.4 & $\bar{Y}$ & \\
\hline 1 & $2 / 5 / 90$ & 2 & 1 & & 118,3 & $\mathbf{N}$ & \\
\hline 1 & 2560 & 交 & 1 & & 112.9 & $\mathbf{N}$ & \\
\hline 1 & $2 \sqrt{5 / 90}$ & 4 & 1 & & 107.7 & $\mathbf{N}$ & \\
\hline 1 & $2 \sqrt{5 / 90}$ & E & 1 & & 100,4 & $\mathbf{N}$ & \\
\hline 1 & $2 \sqrt{5} 90$ & 8 & 1 & & 91,1 & $N$ & \\
\hline 1 & $2 / 5 / 90$ & 7 & 1 & & 85.4 & $N$ & \\
\hline 1. & $2 / 5 / 90$ & 8 & 1 & & 95,2 & $\mathbf{N}$ & \\
\hline 1 & 25150 & 8 & 1 & & 85 & $N$ & \\
\hline 1 & 26590 & 10 & $\overline{1}$ & & 64.9 & $\mathbf{N}$ & \\
\hline 1 & $215 / 90$ & 11 & 1 & & 84,8 & $\mathbf{N}$ & \\
\hline 1 & 2650 & 12 & 1 & & 84.8 & $\mathbf{N}$ & \\
\hline 1 & $2 / 5 / 90$ & 13 & 1 & & 94.8 & $\mathbf{N}$ & \\
\hline 1 & 21690 & 14 & 1 & & 84.7 & $\mathbf{N}$ & \\
\hline 1 & $2 \sqrt{5}, 0$ & 15 & 1 & & 84,6 & $\mathbf{N}$ & \\
\hline 1 & 25190 & 16 & 1 & & 84.5 & $N$ & \\
\hline 1 & $8 / 4 / 90$ & 1 & 1 & & 119.4 & $\mathbf{Y}$ & \\
\hline 1 & $\$ / 4 / 90$ & 2 & 1 & & 117 & $N$ & \\
\hline 1 & $8 / 4 / 90$ & 3 & 1 & & 1128 & $\mathbf{N}$ & \\
\hline 1 & $6 / 4 / 80$ & 4 & 1 & & 100.3 & N & \\
\hline$\overline{1}$ & $6 / 4 / 90$ & 5 & 1 & & 07.9 & N & \\
\hline 1 & $8 / 4 / 90$ & 6 & 1 & & 88,3 & $\mathbf{N}$ & \\
\hline 1 & 6/4/90 & 7 & 1 & & 83.3 & $\mathbf{N}$ & \\
\hline 1 & $5 / 4 / 90$ & 8 & 1 & & 83.5 & $\mathbf{N}$ & \\
\hline 1 & $6 / 4 / 90$ & 9 & $\overline{1}$ & & 63.3 & $\mathbf{N}$ & \\
\hline 1 & $5 / 4 / 90$ & 10 & 1 & & 83.5 & $\mathbf{N}$ & \\
\hline 1 & $8 / 4 / 90$ & 11 & 1 & & 93,4 & $\mathbf{N}$ & \\
\hline 1 & $6 / 4 / 90$ & 12 & 1 & & 83,4 & $\mathbf{N}$ & \\
\hline 1 & $6 / 4 / 90$ & 13 & 1 & & 83,4 & N & \\
\hline 1 & $6 / 4 / 90$ & 14 & 1 & & 83,3 & $N$ & \\
\hline 1 & $6 / 4 / 90$ & 16 & $1 i^{\prime \prime}$ & & 63.3 & NN & \\
\hline 1 & $8 / 4 / \% 0$ & 16 & 1 & & 83.3 & $N$ & \\
\hline 1 & $11 / 12 / 91$ & 1 & 1 & & 114,8 & $Y$ & J-6905 2361247 \\
\hline$\overline{1}$ & $17 / 12 \%]$ & $\overline{2}$ & $\overline{1}$ & & 112.7 & तN & \\
\hline 1 & $11 / 12 / 91$ & 3 & 1 & & 109,3 & $\mathbf{N}$ & \\
\hline 1 & 71,1291 & 4 & 1 & & 104.5 & $\mathbf{N}$ & \\
\hline 1 & 11/12/91 & 5 & 1 & & $\$ 8,3$ & $N$ & \\
\hline 1 & 171201 & 6 & 1 & & 91.7 & $\mathbf{N}$ & \\
\hline 1 & $11 / 12 / 91$ & 7 & 1 & & 98.8 & $\mathbf{N}$ & \\
\hline 1 & $11 / 12 / 91$ & 8 & 1 & & 86.7 & $\mathbf{N}$ & \\
\hline 1 & 11/12/91 & $\mathbf{9}$ & 1 & & 86.4 & $N$ & \\
\hline 1 & 11/12191 & 10 & 1 & & 86.3 & $\mathbf{N}$ & \\
\hline 1 & $71 / 12 \%$ & 11 & 1 & & 86.2 & $\mathbf{N}$ & \\
\hline
\end{tabular}

Data obtainad from WHC Surveillanke Anglybis Computer System (SACS), J(the 2,1993. 


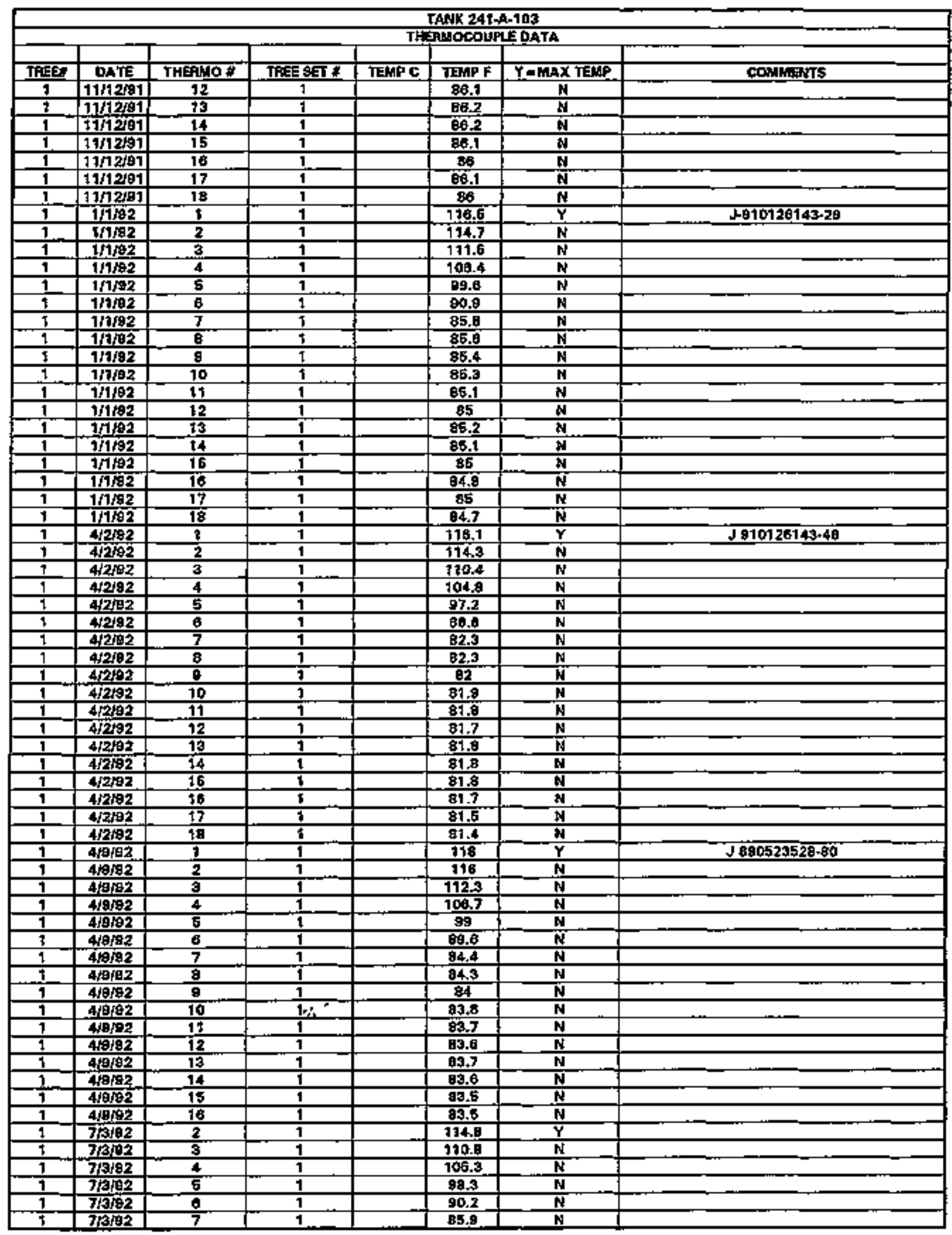

Data obtained from WHC Survaillanos Anatysts Computer System (SACS1, June 2,1993. 


\begin{tabular}{|c|c|c|c|c|c|c|c|}
\hline \multicolumn{8}{|c|}{ TANK 241.A-103 } \\
\hline TFEF & DATE & THEFFHA : & TAR GET P & TENPC & TENP F & $\ddot{Y}=$ MAX TKI & COMTMENTS \\
\hline 1 & $7 / 3 / 92$ & 8 & 1 & & $\mathbf{8 6}$ & $\mathbf{N}$ & \\
\hline$\overline{1}$ & $7 / 3 / 92$ & 9 & $\overline{1}$ & & 95.6 & $\mathbf{N}$ & \\
\hline 1 & $7 / 2 / s 2$ & 10 & 1 & & 85.5 & $\mathbf{N}$ & \\
\hline$T$ & $7 / 3 / 92$ & 11 & 1 & & 86.6 & $\mathbf{N}$ & \\
\hline 1 & $7 / 3 / 92$ & 12 & 1 & & 85,4 & $\mathbf{N}$ & \\
\hline 1 & $7 / 3 / 92$ & 13 & 1 & & 86.5 & $\mathbf{N}$ & \\
\hline 1 & 7 Irg2 & 14 & 7 & & 85.5 & $\mathbf{N}$ & \\
\hline 1 & $7 / 392$ & 15 & 1 & & 85,4 & $\mathbf{A}$ & \\
\hline 1 & $7 / 3 / 92$ & 16 & 1 & & 85.4 & $\mathbf{N}$ & \\
\hline 1 & $1 / 2 / 93$ & 2 & 1 & & 177.5 & $\bar{Y}$ & \\
\hline 1 & $1 / 2 / 93$ & 3 & 7 & & 115.9 & $\mathbf{M}$ & \\
\hline 9 & $1 / 2 / 93$ & 4 & $\bar{t}$ & & 113,4 & $\mathbf{N}$ & \\
\hline 9 & $1 / 2 / 3$ & 5 & 1 & & 108,2 & $\mathbf{N}$ & \\
\hline$\overline{7}$ & $1 / 2 / 93$ & 6 & $\overline{3}$ & & 101.3 & $\mathbf{N}$ & \\
\hline 1 & $1 / 2 / 93$ & 7 & 9 & & 82.2 & N & \\
\hline 1 & $1 / 2 / 83$ & 百 & 9 & & 88.5 & $M$ & \\
\hline 1 & $1 / 2193$ & 9 & 1 & & 86.1 & $\mathbf{H}$ & \\
\hline 1 & $1 / 2003$ & 10 & 7 & & 88.2 & $\bar{M}$ & \\
\hline$\frac{1}{1}$ & $1 / 2 / 93$ & 11 & 1 & & 86.7 & N & \\
\hline 9 & $1 / 2 / \theta^{3}$ & 12 & 1 & & 85.0 & $\mathbf{N}$ & \\
\hline 7 & $1 / 2193$ & 13 & 1 & & 85.3 & $\mathbf{N}$ & \\
\hline 1 & $1 / 2 / 93$ & 74 & 1 & & 80 & $\mathbf{N}$ & \\
\hline 7 & $1 / 2 / 93$ & 15 & 7 & & 85.9 & N & \\
\hline 1 & $1 / 2 / 93$ & To & 1 & & 85.0 & M & \\
\hline 7 & $1 / 293$ & 17 & 7 & & 85.8 & N & \\
\hline 1 & $1 / 2 / 99$ & 78 & 1 & & 65.8 & $N$ & \\
\hline 1 & $6 \sqrt{30}$ 워 3 & 2 & 1 & & 115 & $\mathbf{Y}$ & $J 910126149-20$ \\
\hline 1 & $8 / 30 \mathbf{p}^{\circ} 3$ & $\mathbf{s}$ & 1 & & 110.8 & $N$ & \\
\hline 1 & $8 / 30193$ & 4 & 7 & & 105 & $\mathbf{N}$ & \\
\hline 1 & $6 \sqrt{30} 0 \sqrt{3}$ & 5 & 7 & & 97.8 & $\mathrm{~N}$ & \\
\hline 1 & $6 / 30193$ & 6 & 1 & & 69,2 & $N$ & \\
\hline 1 & $6 \sqrt{30993}$ & 7 & 1 & & 643 & $N$ & \\
\hline$\overline{1}$ & 6/30jes & 8 & 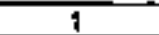 & & 64.2 & $N$ & \\
\hline$\overline{1}$ & $6 \sqrt{30} 9 \sqrt{93}$ & 9 & 1 & & 93.9 & $N$ & \\
\hline 1 & o/30ys & 10 & 1 & & 6.8 & iN & \\
\hline$\overline{1}$ & 5130193 & 71 & 1 & & 93. & $N$ & \\
\hline 1 & edsars & $\sqrt{2}$ & 1 & & 33.8 & $N$ & \\
\hline 1 & 450193 & 13 & 1 & & 93.7 & $\mathbf{N}$ & \\
\hline 1 & $0 / 30 / 83$ & 14 & 1 & & 83.7 & N & \\
\hline 1 & arsorg 3 & 16 & 1 & & 63,6 & $\mathbf{N}$ & \\
\hline 7 & $6 / 30 / 93$ & 16 & 1 & & 83.7 & $N$ & \\
\hline 1 & $6 / 30 / 93$ & 17 & 1 & & B3.5 & $\mathbf{N}$ & \\
\hline 1 & 6romas & 18 & 1 & & 03.5 & $\mathbf{N}$ & \\
\hline$T$ & $7 / 2 \sqrt{83}$ & 1 & 1 & & 118.6 & $\overline{\mathbf{Y}}$ & JTh042 \\
\hline 1 & 7/2/O3 & 2 & 1 & & 117,4 & $\mathbf{N}$ & \\
\hline 1 & $7 / 2183$ & 3 & 1 & & 113.2 & $\mathbf{N}$ & \\
\hline 1 & $7 / 2 \sqrt{93}$ & 4 & 1 & & 107.3 & $\mathbf{N}$ & \\
\hline 1 & $7 / 2 / 93$ & 5 & 1 & & 99,7 & $\bar{N}$ & \\
\hline 1 & $7 / 2 \mathrm{Fes}$ & 6 & 1 & & $\overline{91.4}$ & $\mathbf{N}$ & \\
\hline 1 & $7 / 2 / 93$ & 7 & 1 & & 86. & $\mathbf{N}$ & \\
\hline 1 & 72 ras & 8 & 1 & & 6al 1 & $M$ & \\
\hline$i$ & $7 / 2 / 93$ & 9 & 1 & & 8 审 & $N$ & \\
\hline 1 & $7 / 2 \sqrt{3}$ & 10 & $\overline{1:}$ & & 95.9 & $\mathbf{N}$ & \\
\hline 1 & $7 / 2 / 93$ & 17 & 1 & & 帛5.8 & N & \\
\hline 1 & $7 / 2193$ & 12 & 1 & & 95.8 & $N$ & \\
\hline 1 & $7 / 2193$ & 13 & 1 & & 86.8 & $M$ & \\
\hline 1 & $7 / 2 / 93$ & 14 & 1 & & 85.7 & $\bar{N}$ & \\
\hline 1 & $7 / 2$ pas & 16 & 1 & & 86.7 & $\mathrm{~N}$ & \\
\hline 1 & $7 / 2 / 93$ & 18 & 1 & & 85.7 & $N$ & \\
\hline 1 & $7 / 2 \sqrt{93}$ & 17 & 1 & & 95.6 & $\mathbf{N}$ & \\
\hline 1 & $7 / 2193$ & 18 & 1 & & 65.5 & $N$ & \\
\hline
\end{tabular}

Data abteined tram WHC Survailiance Analyals Computer System (SACSI, June 2,1993. 
WHC-SO-WH-ER-309, Rey. 0

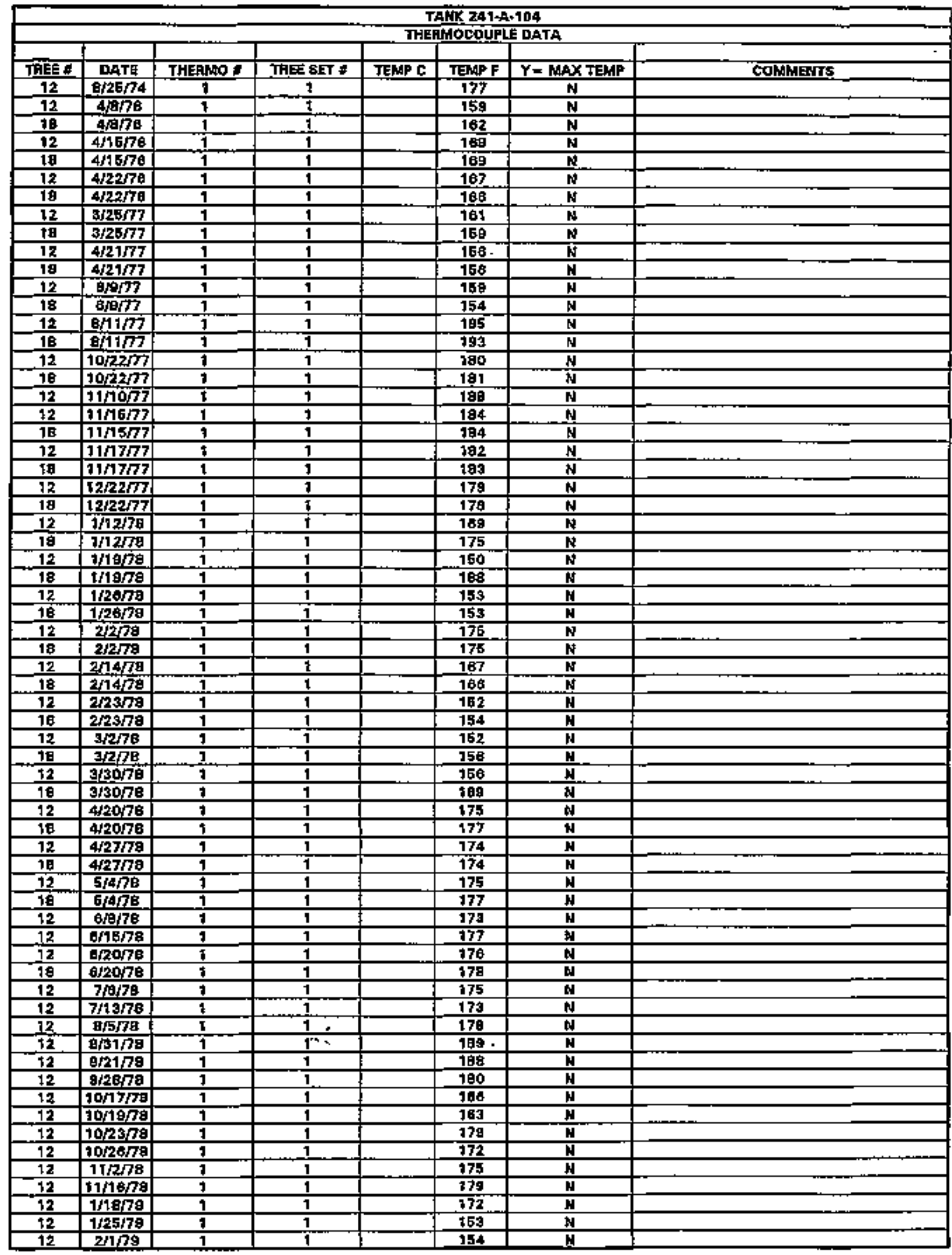

Data obtaingd from WhC Surva|lantB Anelyside Cothputer (SACS), June 2, 1993. 
WHC-\$D-WM-EF-308, Rev. O

\begin{tabular}{|c|c|c|c|c|c|c|c|}
\hline \multicolumn{8}{|c|}{ 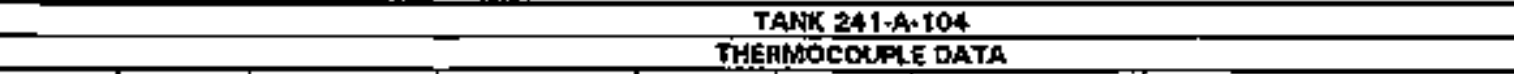 } \\
\hline THEE 7 & DATE & THFAHO \# & TAFF SET \# & TEMP C & TEMAP F & $Y=$ MAX TEHP & COMMENTS \\
\hline$\overline{12}$ & $2 / 8,79$ & $I$ & 1 & & 169 & $\mathbf{N}$ & \\
\hline 12 & $2 / 1678$ & 1 & 1 & & 157 & $\mathbf{N}$ & \\
\hline 12 & $3 / 6 / 79$ & 1 & 1 & & 155 & $\mathbf{N}$ & \\
\hline 12 & $3 / 15 / 78$ & 1 & 1 & & 157 & $\mathbf{N}$ & \\
\hline 12 & $3 / 22 / 79$ & 1 & 1 & & 145 & $\mathbf{N}$ & \\
\hline 12 & $4 \sqrt{779}$ & 1 & 1 & & $1 \overline{52}$ & $\mathbf{N}$ & \\
\hline 12 & $4 / 2 / 79$ & 1 & 1 & & 15帛 & $N$ & \\
\hline 12 & $4 / 20 / 70$ & 1 & $?$ & & 146 & $\mathbf{N}$ & \\
\hline 12 & $4 / 2877$ & 1 & 1 & & 753 & $\mathbf{N}$ & \\
\hline 12 & $5 / 4 / 79$ & 1 & 1 & & 131 & $\mathbf{M}$ & \\
\hline .12 & $10 / 4 \sqrt{79}$ & 1 & 1 & & 348 & $\mathbf{N}$ & \\
\hline$\overline{\mathfrak{1}}$ & $10 / 4 / 70$ & 1 & 1 & & 124 & $\mathbf{H}$ & \\
\hline 镍 & $3 / 26 / 9 t$ & 1 & 1 & & 155 & $N$ & \\
\hline 10 & 4/3ka1 & 1 & 1 & & 567 & $\boldsymbol{N}$ & \\
\hline 19 & 4/B/a1 & 1 & 1 & & 150 & $\mathbf{N}$ & \\
\hline 21으. & 4ftedi & 1 & 1 & & 152 & $\mathbf{N}$ & \\
\hline $1 \overline{18}$ & 4/30/61 & 1 & 1 & & $1 \overline{104}$ & $\mathbf{N}$ & \\
\hline 18 & 5/7a1 & 1 & 1 & & 183 & $\mathbf{N}$ & \\
\hline 12 & $5 / 21 / 91$ & 1 & 1 & & $\$ 76$ & $\mathbf{N}$ & \\
\hline$\overline{18}$ & $5 / 21 / 81$ & 1 & 1 & & tog & $\mathbf{N}$ & \\
\hline 18 & $6 / 1981$ & $\overline{1}$ & 1 & & 183 & $\mathbf{N}$ & \\
\hline 18 & 710 血1 & 5 & 1. & & 150 & $\mathbf{N}$ & \\
\hline 12 & 7/10/a1 & 1 & 1 & & 184 & $\mathbf{N}$ & \\
\hline 78 & 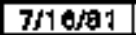 & $\mathbf{1}$ & 1 & & 179 & $\mathbf{N}$ & \\
\hline 12 & 7130 a1 & 1 & 1 & & & $\mathbf{N}$ & 109 - suspetct date (low) \\
\hline 18 & $7 / 30 / 97$ & 1 & $\mathbf{1}$ & & 147 & $\mathbf{N}$ & \\
\hline 12 & 8/1381. & 1 & 1 & & 159 & $\mathbf{N}$ & \\
\hline 18 & EN13/97 & 1 & 1 & & 160 & $\mathbf{N}$ & \\
\hline 12 & E/2001 & 1 & 1 & & 160 & $N$ & \\
\hline 18 & a/20/a1 & 1 & 1 & & 162 & $N$ & \\
\hline 12. & $8 / 27 / 8]$ & 1 & 1 & & 151 & $\mathbf{N}$ & \\
\hline 16 & $8 / 27 / 81$ & 1 & 1 & & 153 & $\mathbf{N}$ & \\
\hline 12 & E/S/B1 & 1 & 1 & & 186 & $\mathbf{N}$ & \\
\hline 18 & $9 / 381$ & 1 & 1 & & 183 & $N$ & \\
\hline$\overline{12}$ & $970 / 81$ & 1. & 1 & & 179 & $\mathbf{N}$ & \\
\hline 18 & $9 / 1081$ & 1 & 1 & & 179 & $\mathrm{~N}$ & \\
\hline 12 & 10/22a1 & 1 & 1 & & 171 & N & \\
\hline 18 & 10/22/81 & 1 & 1 & & 160 & N & \\
\hline 12 & 10/29/a1 & 1 & 1 & & 168 & $N$ & \\
\hline 18 & 10129/81 & $\overline{\mathbf{i}}$ & 1 & & 175 & $\mathbf{N}$ & \\
\hline$\overline{18}$ & 1261801 & $i$ & 1 & & 187 & $\mathbf{N}$ & \\
\hline 12 & 2/the2 & 1 & 1 & & 170 & $\mathbf{N}$ & \\
\hline 18 & $2 / / / B 2$ & 1 & 1 & & $180^{\circ}$ & $\mathbf{N}$ & \\
\hline 12 & $1 / 2 / 94$ & 1 & 1 & & 179 & $\mathbf{N}$ & \\
\hline 18 & $1 / 2 / 94$ & 1 & $i$ & & 184 & $\mathbf{N}$ & \\
\hline$\sqrt{2}$ & $1 \sqrt{3} \sqrt{84}$ & 1 & 1 & & 175 & $\mathbf{N}$ & \\
\hline te & $1 / 3 \sqrt{84}$ & 1 & 1 & & $1 \overline{\mathrm{BE}}$ & $\mathbf{N}$ & \\
\hline 18 & $1 / 4 / 84$ & 1 & 1 & & 182 & $\mathbf{N}$ & \\
\hline 12 & $1 / 5 / 84$ & 1 & 1 & & 202 & $\mathbf{N}$ & \\
\hline 18 & $1 / 4 \sqrt{84}$ & 1 & 1 & & 182 & $\mathbf{N}$ & \\
\hline 12 & $1 / 9 / 84$ & 1 & 1 & & 207 & $\mathbf{N}$ & \\
\hline 12 & $1 / 10 / 84$ & 1 & 1 & & 200 & $\mathbf{N}$ & \\
\hline 18 & $1 / 10184$ & $t$ & 1. & & 205 & $\mathbf{N}$ & \\
\hline$\overline{12}$ & $1 / 11 / 84$ & 7 & $i^{\prime}=$ & & 202 & $\mathbf{N}$ & \\
\hline 18 & $1 / 11 / 94$ & 1 & 1 & & 206 & $\mathbf{N}$ & \\
\hline 12 & $1 / 12 / 84$ & 1 & 1 & & 210 & $N$ & \\
\hline 18 & $1 / 12184$ & 1 & 1 & & 209 & $\mathbf{N}$ & \\
\hline 12 & $1 / 98 / 84$ & 1 & 1 & & 197 & A & \\
\hline 18 & $1 / 19 / 84$ & $i$ & 1 & & 229 & $\mathbf{N}$ & \\
\hline 12 & $1 / 21 / 84$ & 1 & 1 & & $18 \mathrm{~B}$ & $N$ & \\
\hline 78 & $1 / 21 / 94$ & 1 & 1 & & 165 & $\mathbf{M}$ & \\
\hline 12 & $1 / 2284$ & 1 & 1 & & 165 & $\mathbf{N}$ & \\
\hline 18 & $1 / 22 / 94$ & 1 & 1. & & 206 & N & \\
\hline 12 & $1 / 24 / 64$ & 1. & 1 & & 208 & $\mathrm{~N}$ & \\
\hline 18 & $1 / 24 / 84$ & 1 & 1 & & 2008 & $\mathbf{N}$ & \\
\hline
\end{tabular}

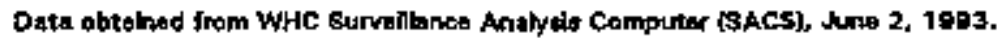


WHC-SD-WM-ER-300, Rev, 0

\begin{tabular}{|c|c|c|c|c|c|c|c|}
\hline \multicolumn{8}{|c|}{$\begin{array}{l}\text { TAHK 241-A-104 } \\
\text { THEAWOCOUFHE OATA }\end{array}$} \\
\hline & & & & & & & \\
\hline 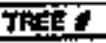 & DAT & 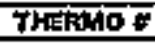 & 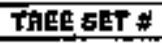 & TENAPC & TEPPE & Y= MAX TFMP & COAMENT: \\
\hline 12 & $1 / 25 / 84$ & 1 & $i$ & & 204 & $\mathrm{~N}$ & \\
\hline 18 & $1 / 25 / E A$ & 1 & 1 & & 203 & $\mathbf{N}$ & \\
\hline 12 & $1 / 26 / 84$ & 1 & 1 & & 201 & $\mathbf{N}$ & \\
\hline 12 & $1 / 28 / 64$ & 1 & 1 & & 203 & $\mathbf{M}$ & \\
\hline 12 & $1 / 20 / E^{2}$ & $i$ & $i$ & & 204 & 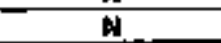 & \\
\hline 18 & $1 / 20184$ & 1 & 1 & & $2 \sqrt{4}$ & $\mathbf{N}$ & \\
\hline 12 & 1/30, & 1 & 1 & & 200 & W & \\
\hline 18 & $1 / 30 / 84$ & 1 & 1 & & 212 & N & \\
\hline 12 & $2 / 4 / 94$ & 1 & 1 & & 199 & n & \\
\hline 钴 & $2 / 4 \sqrt{194}$ & 1 & 1 & & 204 & in & \\
\hline 12 & 2064 & 1 & 1 & & 206 & 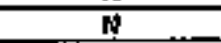 & \\
\hline 18 & $2 / 964$ & 1 & 1 & & 200 & $\mathbf{N}$ & \\
\hline 10 & $2 \pi{ }^{4} 4$ & 1 & 1 & & 204 & $M$ & \\
\hline 12 & $2 / 15 / 94$ & 1 & 1 & & 200 & $\mathrm{~N}$ & \\
\hline 918 & $2 / 15 / 94$ & 1 & 1 & & 190 & 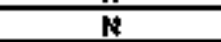 & \\
\hline 12 & $2 / 16 / 84$ & 1 & 1 & & 185 & $\mathbf{N}$ & \\
\hline 18 & 2/0/84 & 1 & 1 & & 190 & $\mathbf{N}$ & \\
\hline$\sqrt{12}$ & $2 / 1584$ & 1 & 1 & & 194 & $\mathbf{N}$ & \\
\hline 19 & $2 / 78 / 84$ & 1 & 1 & & 196 & $\mathbf{N}$ & \\
\hline 12 & $2 / 19 / 84$ & $\frac{1}{1}$ & $\overline{1}$ & & 192 & $\mathbf{N}$ & \\
\hline 18 & $240 \times 84$ & 1. & 1 & & 193 & $\mathbf{N}$ & \\
\hline$\sqrt{12}$ & $2 / 20+94$ & 1 & 1 & & 196 & $\mathbf{N}$ & \\
\hline 18 & $2 / 20 / 84$ & 1 & 1 & & 100 & $\mathbf{N}$ & \\
\hline 12 & $2 / 2 1 \longdiv { 8 4 }$ & 9 & 1 & & 198 & $\bar{N}$ & \\
\hline 18 & $2 / 21 / 84$ & 1 & 1 & & 204 & $\mathbf{N}$ & \\
\hline 12 & $2 / 22 / 84$ & 1 & 1 & & 102 & NN & \\
\hline 18 & $2 / 22 / 84$ & 1 & 1 & & 190 & $\mathrm{~N}$ & \\
\hline 12 & $2 / 23$ Ne4 $^{2}$ & 1 & 1 & & 194 & N & \\
\hline 18 & $2 / 23 / 64$ & 7 & 1 & & 193 & $\mathbf{N}$ & \\
\hline 12 & $2 / 24 / 04$ & $t$ & $t$ & & 102 & $\mathrm{~N}$ & \\
\hline 18 & $2 / 24 / 94$ & 1 & 1 & & 193 & $\mathbf{N}$ & \\
\hline 12 & $2127 / 184$ & 1 & 7 & & 198 & $\bar{N}$ & \\
\hline 18 & $2127 / 24$ & 1 & 1 & & 100 & $N$ & \\
\hline 12 & $2 / 28 / 04$ & 1 & 1 & & 100 & $\bar{N}$ & \\
\hline 18 & $21 / 28 \mathrm{~g} 94$ & 1 & 1 & & 190 & $\mathbf{N}$ & \\
\hline 12 & $2 / 29 / 84$ & 1 & 1 & & 190 & $\mathbf{N}$ & \\
\hline 18 & $2 / 28 / 84$ & 1 & 1 & & 189 & $\mathbf{N}$ & \\
\hline 12 & $3 / 1 / 84$ & 1 & 1 & & 184 & $\overline{\mathbf{N}}$ & \\
\hline 19 & $3 / 1 / 84$ & 1 & $i$ & & 190 & $\mathrm{~N}$ & \\
\hline 12 & $3 / 2 / 84$ & 1 & 1 & & 189 & N & \\
\hline 18 & $3 / 2 / 84$ & 1 & 1 & & 181 & $\mathbf{N}$ & \\
\hline 12 & $3 / 3 / 84$ & 1 & 1 & & 202 & $\mathrm{~N}$ & \\
\hline 19 & 3rapa4 & 1 & 1 & & 210 & $\mathrm{~N}$ & \\
\hline 12 & $3 / 4 / 94$ & 1 & 1 & & 199 & $N$ & \\
\hline 19 & $3 / 4 \sqrt{84}$ & 1 & 1 & & 183 & $\mathbf{N}$ & \\
\hline 12 & $3 / 184$ & 1 & 1 & & 198 & $\mathbf{N}$ & \\
\hline 19 & $3 / 8 / 84$ & 1 & 1 & & 199 & $\overline{\mathbf{N}}$ & \\
\hline 12 & 3 3hora 4 & $\frac{1}{1}$ & 1 & & 102 & $\mathrm{~N}$ & \\
\hline 18 & 3 Tho 4 & 1 & 1 & & 155 & $\mathbf{N}$ & \\
\hline 12 & $3 / 11 / 84$ & 1 & 1 & & 104 & $\bar{N}$ & \\
\hline 18 & $9 / 11 / 94$ & 1 & 1 & & 197 & $\mathrm{~N}$ & \\
\hline 12 & $9 / 12 / 94$ & 1 & 1 & & 186 & $\mathbf{N}$ & \\
\hline 19 & $3 / 12 / 94$ & 1 & 1. & & 109 & $\mathbf{N}$ & \\
\hline $1 \sqrt{2}$ & $3 / 13 / 94$ & 1 & 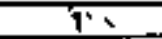 & & 187 & it & \\
\hline 19 & 9/23/34 & 1 & 1 & & 100 & $\mathbf{N}$ & \\
\hline 12 & $3 / 16 / 94$ & 1 & 1 & & 189 & $\mathrm{~N}$ & \\
\hline 18 & 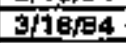 & 1 & 1 & & 193 & $\mathrm{~N}$ & \\
\hline$\$ 2$ & $3 / 1784$ & 1 & 1 & & 180 & $N$ & \\
\hline 19 & $9 / 6>794$ & 1 & $i$ & & 180 & $\mathbf{N}$ & \\
\hline 12 & 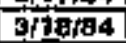 & 1 & 1 & & 190 & $\mathbf{N}$ & \\
\hline 18 & $3 / 7894$ & 1 & 1 & & 192 & N & \\
\hline 12 & $3 / 7964$ & 1 & 1 & & 191 & N & \\
\hline 19 & $9 / 19 / 24$ & 1 & 1 & & 184 & $\mathbf{M}$ & \\
\hline 12 & $3 / 26 / 84$ & 1 & 1 & & 189 & $\bar{N}$ & \\
\hline 18 & $3 / 26,4$ & 1 & 1 & & 100 & $N$ & \\
\hline
\end{tabular}

Dats obtsined from WHC Esrvellance Analysis Computer (SACS), Jung 2, 1993. 


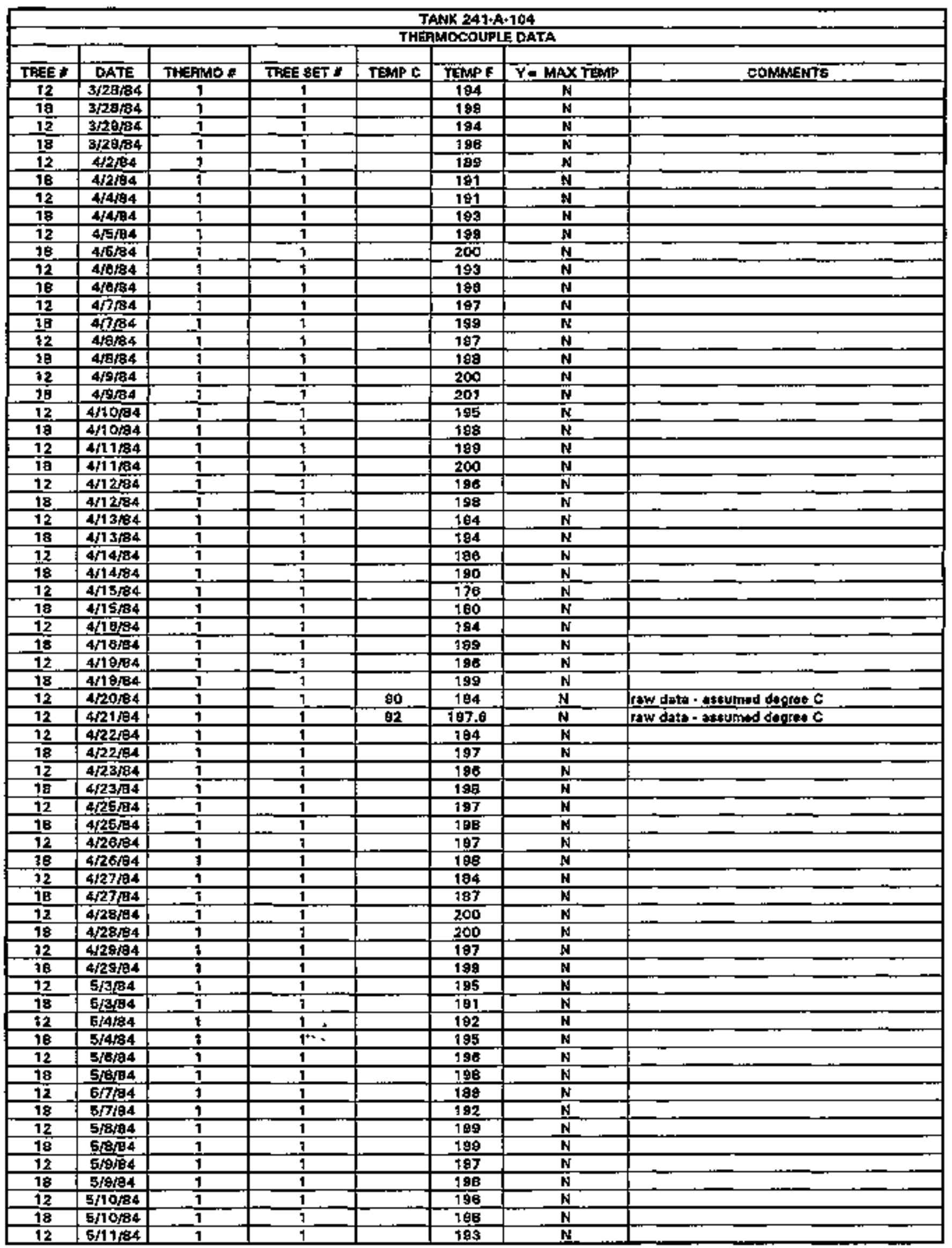

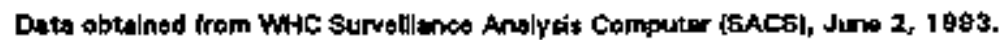




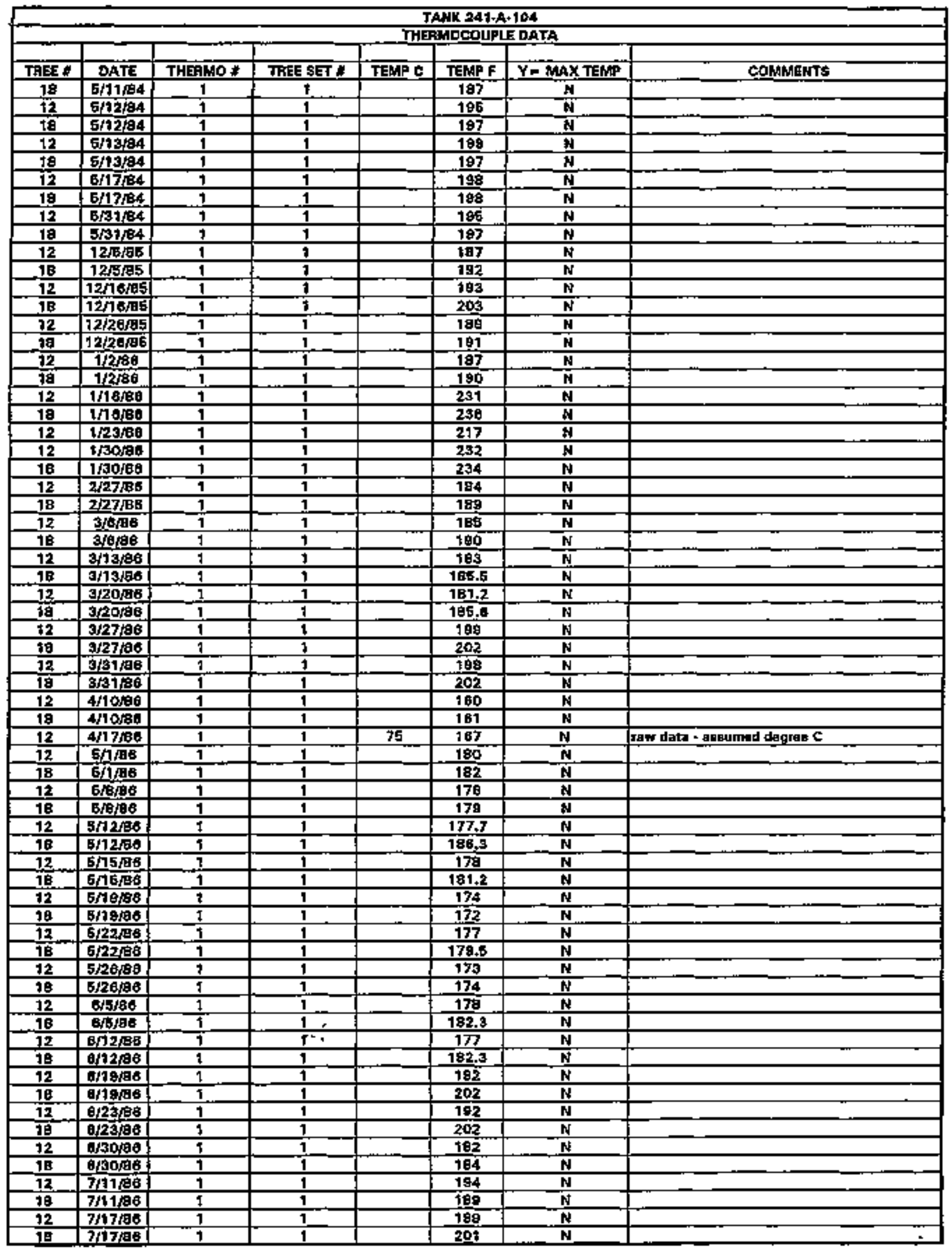

Dato obtained frem Whic arrellance Andireid Computef (SACS), Junt 2, 1993. 
WHC-5D-WM-ER-308, Rov, O

\begin{tabular}{|c|c|c|c|c|c|c|c|}
\hline \multicolumn{8}{|c|}{ TANIK 241-A-T04 } \\
\hline THEE * & DATE & THERHO \# & TREE BET & TEMP C & TEMP F & $Y=M A X$ JEMP & colihtents \\
\hline 12 & $7 / 24,196$ & 1 & 1 & & 183.6 & $N$ & \\
\hline Iạ & $7 / 24 / 96$ & 1 & 1 & & 184,8 & $\mathbf{N}$ & \\
\hline 12 & $7 / 31 / 8 \theta$ & 1 & 1 & & 182.5 & $\mathbf{N}$ & \\
\hline 18 & $7 / 31,88$ & 1 & 1 & & 183.6 & $\mathbf{N}$ & \\
\hline 12 & $1 / 2 / 87$ & 1 & 1 & & 173. & $\dot{\gamma}$ & \\
\hline 18 & 1/287 & 1 & 1 & & 173.8 & $\mathbf{N}$ & \\
\hline 12 & $1 / 287$ & 1 & 1 & & $18 \%$ & $\mathbf{N}$ & \\
\hline 18 & $1 / \mathrm{a} / 87$ & 1 & 1 & & 187 & $\mathbf{Y}$ & \\
\hline $1 \overline{2}$ & $1 / 15 / 97$ & 1 & 1 & & 160 & $\bar{Y}$ & \\
\hline 18 & $1 / 16 / 87$ & 1 & 1 & & 162 & $\mathbf{N}$ & \\
\hline 12 & $1 / 22 / 87$ & 1 & 1 & & 162,2 & $\mathbf{N}$ & \\
\hline 16 & $1 / 22 / 87$ & 1 & 1 & & 164.8 & $\bar{Y}$ & \\
\hline $1 \overline{2}$ & $1 / 29 / 87$ & 1 & 3 & & 1的 & $\mathbf{N}$ & A.C8 Wetr geuge 34 \\
\hline 18 & $1 / 29 / 87$ & 1 & 7 & & 169 & $\mathbf{Y}$ & \\
\hline 12 & $2 / 2 \sqrt{6}$ & 1 & 1 & & 182 & $\mathbf{N}$ & \\
\hline 18 & $2 / 2 / 97$ & 1 & 1 & & 182 & $\mathbf{N}$ & \\
\hline 12 & $2 / 12 / 87$ & 1 & 1 & & 179 & $\mathbf{M}$ & \\
\hline 18 & $2 \sqrt{12 \sqrt{87}}$ & 1 & 7 & & 185 & $\mathbf{Y}$ & \\
\hline 12 & $2 / 19 / 87$ & 1 & 1 & & 1B: & $\mathbf{N}$ & \\
\hline 18 & $2 / 19 / 87$ & 1 & 1 & & 184 & $r$ & \\
\hline 12 & $3 \sqrt{5 / 97}$ & 1 & 1 & & 169 & $\mathbf{N}$ & \\
\hline 18 & $3 / 5 / 27$ & 1 & 1 & & 168 & $Y$ & \\
\hline 12 & $3 / 13 / 87$ & 1 & 1 & & 178 & $\mathbf{N}$ & \\
\hline 18 & $3 / 13 / 83$ & 1. & 1 & & 178 & $\mathbf{Y}$ & \\
\hline 12 & $3 / 19 / 97$ & 1 & 1 & & 184 & $\underline{Y}$ & \\
\hline 19 & $3 / 19 / 97$ & 1 & 1 & & 182 & $\mathrm{~N}$ & \\
\hline $1 \overline{2}$ & $4 / 4 / 27$ & $\overline{1}$ & 1 & & 193.g & $\overline{\mathbf{N}}$ & \\
\hline 18 & $4 / 4 / 87$ & 7 & 1 & & 105.6 & $\mathbf{Y}$ & \\
\hline 12 & 41687 & 1 & 1 & & 184.5 & $\mathbf{N}$ & \\
\hline 18 & $4 / 9 / 87$ & 1 & 1 & & 187.6 & $Y$ & \\
\hline 12 & 4/30197 & 1 & 1 & & 165 & $\mathbf{N}$ & \\
\hline 19 & $4 / 30$ taj & 1 & 1 & & 186 & $\bar{Y}$ & \\
\hline 12 & $5 / 74 / 97$ & 1 & 1 & & 167.5 & $\mathbf{N}$ & \\
\hline 18 & $5 / 4487$ & 1 & 1 & & 169.3 & $\mathbf{Y}$ & \\
\hline 12 & 5/20/97 & 1 & 1 & & 187 & k & \\
\hline 19 & $5 / 20 / 47$ & 1 & 1 & & 170 & $\mathbf{Y}$ & \\
\hline 12 & $6 / 3 \sqrt{67}$ & $t$ & 1 & & 167 & $Y$ & \\
\hline 18 & $0 / 3 \sqrt{87}$ & 1 & 1 & & 107 & $Y$ & \\
\hline 12 & $6 / 19 / 87$ & $i$ & 1 & & 179.8 & $\overline{\mathbf{N}}$ & \\
\hline $1 \overline{8}$ & $8 / 19 / 87$ & 3 & 1 & & 174.2 & $\overline{\mathbf{Y}}$ & \\
\hline 12 & $8 / 25 / 87$ & $\exists$ & $T$ & & 172 & $\mathbf{N}$ & \\
\hline 18 & 6/26/97 & 1 & 1 & & 178,0 & $Y$ & \\
\hline 12 & $7 / 2 / 87$ & 1 & 1 & & 107.2 & $\mathbf{Y}$ & \\
\hline 18 & $7 / 2 / 87$ & 1 & 1 & & 165.7 & $\mathbf{N}$ & \\
\hline $1 \overline{2}$ & 7Nok立 & 1 & 1 & & 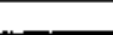 & $\mathbf{N}$ & ofs \\
\hline 19 & $7 / 167$ & 1 & 1 & & 172 & $\mathbf{Y}$ & \\
\hline 12 & $7 / 2307$ & 1 & 1 & & & $\mathbf{N}$ & O/S \\
\hline 18 & $7 / 23 / 97$ & 1 & 1 & & 171 & $Y$ & \\
\hline 12 & $7 / 30 / 97$ & 1 & 1 & & & $\mathbf{N}$ & o/s \\
\hline 18 & $7 / 30 / 87$ & 1 & 1 & & 174 & $Y$ & \\
\hline 12 & 日/BJB7 & 1 & 1 & & 100 & $\mathbf{Y}$ & \\
\hline 18 & 9/A/B7 & 1 & 1 & & 155 & $\mathbf{N}$ & \\
\hline 12 & $8 / 13 / 97$ & 1 & 1. & & 170 & $\mathbf{N}$ & \\
\hline 18 & $8 / 13 / 87$ & $\overline{1}$ & 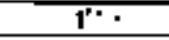 & & 171 & $\mathbf{Y}$ & \\
\hline 12 & $3 / 20 / 97$ & 1 & $1 \ldots$ & & & $\mathbf{N}$ & o/s \\
\hline 12 & $8 / 27 / 87$ & 1 & 1 & & $t 08$ & $Y$ & \\
\hline 19 & e/27/A7 & 1 & 1 & & 169 & $\mathbf{k}$ & \\
\hline 12 & $9 / 21 / 177$ & 1 & 1 & & 172.1 & $\mathbf{N}$ & \\
\hline $2 \bar{B}$ & $9 / 21 / 47$ & 1. & 1 & & 173.5 & $\mathbf{N}$ & \\
\hline 12 & $9 / 24 / 7$ & 1 & 1 & & 170 & $\mathbf{N}$ & \\
\hline 18 & g/24/67 & 1 & 1 & & 171 & $Y$ & \\
\hline 12 & $10 / 1 / 87$ & 1 & 1 & & 170 & $\mathbf{N}$ & \\
\hline 18 & 10/1/87 & 1 & 1 & & $17 i$ & $Y$ & \\
\hline 12 & 10/9/87 & 1 & 1 & & 171 & $Y$ & \\
\hline 18 & $10 / 9 / 87$ & 2 & 1 & & 170 & $\mathbf{N}$ & \\
\hline
\end{tabular}

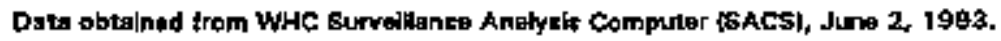


WHC-SD-WH.EA-3OS, AOV. 0

\begin{tabular}{|c|c|c|c|c|c|c|c|}
\hline \multicolumn{8}{|c|}{ TANK 241-A-104 } \\
\hline & & & & THE & MDcouP & DATA & \\
\hline & & & & & & & $\mathrm{T}$ \\
\hline TRe日l: & DATE & THERMO H & TFER SET & TEMP C & TEMAF F & $Y=$ RAAX TENP & COMAMENTS \\
\hline 12 & 10r18/87 & 1 & 1 & & 108 & $N$ & \\
\hline 18 & 10/1, & 1 & 1 & & 170 & $\bar{Y}$ & \\
\hline 12 & $1 0 \longdiv { 2 2 / 9 7 }$ & 1 & 1 & & $16 \theta_{12}$ & $\mathbf{N}$ & \\
\hline 18 & 1022197 & 1 & 1 & & 189,2 & $Y$ & \\
\hline 12 & $10 / 29 / 97$ & 1 & 1 & & 171.3 & $\mathbf{N}$ & \\
\hline 18 & $10 / 29 / 97$ & 1 & 1 & & 171.5 & $\mathbf{N}$ & \\
\hline 12 & 116/8? & 1 & 1 & & 189 & $\mathbf{N}$ & \\
\hline 18 & $11 / 5 / 87$ & 1 & 1 & & 189 & $N$ & \\
\hline 12 & $11 / 19 / 87$ & 1 & 1 & & 167.7 & N & \\
\hline 18 & $11 / 19 / 87$ & 1 & 1 & & 169.8 & $\mathbf{Y}$ & \\
\hline 12 & $11 / 26 / 87$ & 1 & 1 & & 109 & $\mathbf{N}$ & \\
\hline 10 & $11 / 20 / 87$ & 1 & 1 & & 169.6 & $\mathbf{Y}$ & \\
\hline 12 & $12 / 3,187$ & 1 & 1 & & 187 & $\mathbf{N}$ & \\
\hline $1 \mathrm{E}$ & $12 / 3187$ & 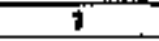 & 1 & & 193 & $\mathbf{Y}$ & \\
\hline 12 & 12/10/87 & 1 & 1 & & 168 & $\mathbf{N}$ & \\
\hline 18 & 12110097 & $i$ & 1 & & 189 & $Y$ & \\
\hline 12 & $12 / 17 / 97$ & 1 & 1 & & 190.8 & $\mathbf{N}$ & \\
\hline 18 & $12 / 17,97$ & 1 & $t$ & & 195.5 & $\mathbf{Y}$ & \\
\hline 12 & $12 / 24 / 67$ & 1 & 1 & & 192.3 & $\mathbf{N}$ & \\
\hline 18 & $12 / 24 / 87$ & 1 & 1 & & 192.8 & $\mathbf{Y}$ & \\
\hline 12 & $12 / 31 / 87$ & 1 & $i$ & & 199 & $\mathrm{~N}$ & \\
\hline 18 & $12 / 31 / 87$ & 1 & 1 & & $\$ 91$ & $Y$ & \\
\hline 12 & $1 / 7 \mathrm{Neg}$ & 1 & 1 & & $18 \mathrm{E}, 4$ & $N$ & \\
\hline 18 & w/reg & 1 & 1 & & 190.2 & $Y$ & \\
\hline 12 & $1 / 14 / 8 ; 9$ & 1 & 1 & & $18 B, 4$ & $\mathbf{N}$ & \\
\hline 18 & $1 / 14 / 8 d$ & 1 & 1 & & 1908 & $\mathbf{Y}$ & \\
\hline 12 & $1 / 21 / 89$ & 1 & 1 & & 189 & $\bar{N}$ & \\
\hline 18 & $1 / 21 / 80$ & 1 & 1 & & 902.5 & $Y$ & \\
\hline 12 & 1/2area & 1 & 1 & & 164,8 & $\mathbf{N}$ & \\
\hline 隹 & $1 / 2 \overline{8 / B S}$ & 1 & $\overline{1}$ & & 168 & $\bar{Y}$ & \\
\hline 12 & $2 / 4,8 B$ & 1 & 1 & & 186.8 & $\mathbf{N}$ & \\
\hline 18 & $2 / 4 / 98$ & 1 & 1 & & 189.3 & $Y$ & \\
\hline 12 & $2 / 11 / 88$ & 1 & 1 & & 185 & $\mathbf{N}$ & \\
\hline 18 & $2 / 11 / 68$ & 1 & 1 & & 168 & $\mathbf{Y}$ & \\
\hline 12 & $2 / 18 / 88$ & 1 & 1 & & 185.3 & $\mathbf{N}$ & \\
\hline 18 & $2 / 18 / 48$ & 1 & 1 & & 186.7 & $\mathbf{Y}$ & \\
\hline 12 & $2 / 25 / 88$ & 1 & 1 & & 180.8 & $N$ & \\
\hline te & $2 / 25 / 8$ & $t$ & 1 & & 190.5 & $Y$ & \\
\hline 12 & $3 \mathrm{APas}$ & 1 & 1 & & 187.1 & $\mathbf{N}$ & \\
\hline 18 & $3 / 2 / 96$ & 1 & 1 & & 187.9 & $Y$ & \\
\hline 12 & $3 / 10 / 18$ & 1 & 1 & & 186 & $\bar{N}$ & \\
\hline 18 & $310 \mathrm{pg}$ & 1 & 1 & & 189.5 & $Y$ & \\
\hline 12 & $3 / 17$ 它8 & 1 & 1 & & 1B4 & $\mathbf{N}$ & \\
\hline $\mathrm{jB}$ & $3 / 17 / 68$ & 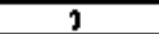 & 1 & & 194 & $\bar{Y}$ & \\
\hline 12 & $3 / 24 / a \mathrm{aB}$ & 1 & 1 & & 1 198 & $\mathbf{N}$ & \\
\hline 18 & $3 / 24 / 98$ & 1 & 1 & & 189 & $y$ & \\
\hline 12 & $3 / 31 / 8 B$ & $t$ & 1 & & 184 & $\mathbf{N}$ & \\
\hline 18 & $3 / 31 / 98$ & 1 & 1 & & 188 & $Y$ & \\
\hline 12 & $47 \mathrm{ras}$ & 1 & 1 & & 1的 & N & \\
\hline 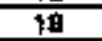 & $4 / 7$ a & 1 & 1 & & 181 & $Y$ & \\
\hline$\sqrt{2}$ & $4 / 74 / 8$ & 1 & 1 & & 1Es & $\mathbf{N}$ & \\
\hline 18 & $4 / 14 \mathrm{deB}$ & $T$ & 1 & & 196 & $\mathbf{Y}$ & \\
\hline 12 & $4 / 21 / 188$ & 1 & 1. & & 187 & $N$ & \\
\hline 18 & $4121 / \mathrm{Ag}$ & 1 & $t^{2} \%$ & & 189 & $\bar{Y}$ & - \\
\hline 12 & $4 / 28 / 68$ & 1 & 1 & & 188.6 & $\mathbf{N}$ & \\
\hline 18 & $4 / 28 / 88$ & 1 & 1 & & 189.6 & $\mathbf{N}$ & \\
\hline 12 & $5 \sqrt{36}$ & 1 & 1 & & 167 & $\mathbf{N}$ & \\
\hline 18 & $6 \sqrt{6 / 8}$ & 1 & 1 & & 169 & $\mathbf{N}$ & \\
\hline 12 & 5/19/8 & 1 & 1 & & 184 & $N$ & \\
\hline 18 & 5/18/98 & 1 & 1 & & 195 & $\mathbf{N}$ & \\
\hline 12 & G/26/88 & 7 & 1 & & 183 & $\mathbf{N}$ & \\
\hline 18 & 6/26/8g & 1 & 1 & & 106 & $\overline{\mathbf{H}}$ & \\
\hline 12 & $6 / 2169$ & 1 & 1 & & 1的 & $\mathbf{N}$ & \\
\hline 18 & $6 / 2 / 6 a$ & 1 & 1 & & 168 & $\mathbf{N}$ & \\
\hline 12 & $6 / 16 / 89$ & 1 & 1 & & 176 & $\mathbf{N}$ & \\
\hline
\end{tabular}

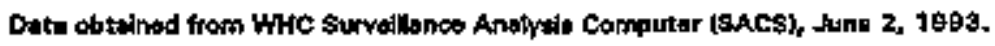


WHC-SO-WM-ER-308, Rav. 0

\begin{tabular}{|c|c|c|c|c|c|c|c|}
\hline \multicolumn{8}{|c|}{ TAHK 241-A=104 } \\
\hline & & & & & & & \\
\hline TAEE $:$ & DATE & THEA & TRE SET I & TEMP C & TElup F & $Y=$ MAX TEMPF & COMUNENTS \\
\hline 19. & B/16/8B & 1 & 5 & & 175 & $\mathbf{N}$ & \\
\hline 12 & 6/23/98 & 1 & $t$ & & 184 & $\mathbf{N}$ & \\
\hline 18 & $6 / 25 / 80$ & 1 & 1 & & 184 & $\mathbf{N}$ & \\
\hline 12 & Drsoras & 1 & 1 & & 782 & $\mathbf{N}$ & \\
\hline 18 & $5 / 30 / 89$ & 1 & $i$ & & 100 & $\mathbf{M}$ & \\
\hline 12 & $7 / 7 / 88$ & $T$ & 1 & & 182 & $\mathbf{H}$ & \\
\hline 18 & $7 / 7 / \mathrm{s}$ & 1. & 1 & & 185 & $\mathbf{N}$ & \\
\hline 12 & $7 / 14 \sqrt{89}$ & 1 & 1 & & 183 & $\mathbf{N}$ & \\
\hline $1 \mathrm{~B}$ & $7 / 14 / 88$ & 1 & 1 & & 180 & $N$ & \\
\hline 12 & $7 / 21 / 88$ & $\bar{i}$ & 1 & & 185 & $\mathbf{N}$ & \\
\hline 58 & $7 / 21 / 88$ & 1 & 1 & & 186 & $\mathbf{N}$ & \\
\hline 12 & $7 / 2609$ & 1 & 1 & & 185 & $N$ & \\
\hline 12 & B/4/98 & 1 & 1 & & 183 & $N$ & \\
\hline 1E & B/4/A8 & 1 & 1 & & 184 & $N$ & \\
\hline 12 & 自/11/88 & 1 & i & & 170 & $\mathbf{N}$ & \\
\hline 18 & 6/11俩8 & 1 & 1 & & 171 & $\bar{N}$ & \\
\hline 12 & 9/18/39 & 1 & 1 & & 185 & $\mathbf{N}$ & \\
\hline 18 & G/1E/E8 & 1 & 1 & & 180 & $M$ & \\
\hline 12 & 百/25/98 & 1 & $T$ & & 185 & $\mathrm{~N}$ & \\
\hline 19 & a/25res & 1 & 1 & & $i \overline{8} 6$ & $\mathbf{N}$ & \\
\hline 12 & $\theta / 1 / 8$ & 1 & 1 & & $169, \hat{9}$ & $\mathbf{N}$ & \\
\hline 19 & $9 / 1 / 8 B$ & 1 & 1 & & $171+3$ & $N$ & \\
\hline 12 & gfafBa & 1 & 1 & & 185 & $N$ & \\
\hline 18 & g/9/8B & 1 & 1 & & 189 & $\mathbf{N}$ & \\
\hline 12 & $9 / 158 \mathrm{~B}$ & 1 & 1 & & 188 & $\mathbf{N}$ & \\
\hline 18 & $9 \pi 15 / 98$ & 1 & 1 & & 189 & $\mathbf{N}$ & \\
\hline 12 & $9 / 22$ as & 1 & 1 & & 165 & $\mathbf{N}$ & \\
\hline 18 & $9 / 22 / B B$ & 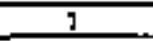 & 1 & & 161 & $\mathbf{N}$ & \\
\hline 12 & 9/2日8B & 1 & 1 & & 182 & $\mathbf{N}$ & \\
\hline 19 & e/29/as & 1 & 1 & & $\overline{187}$ & $\mathbf{N}$ & \\
\hline 12 & 10/aras & 1 & 1 & & $18 \%$ & $\mathbf{N}$ & \\
\hline 18 & $10 / \mathrm{b} / \mathrm{B}$ G & 1 & 1 & & 181 & $\mathbf{N}$ & \\
\hline 12 & $10 / 1388$ & 1 & 1 & & 188 & $\mathbf{N}$ & \\
\hline 18 & $10 / 13 / 8 \mathrm{~B}$ & 1 & 1 & & 167 & $\mathbf{N}$ & \\
\hline 12 & $10 / 20 / 88$ & 1 & 1 & & T68 & $\mathbf{N}$ & \\
\hline 18 & $10 / 20 / 80$ & 1 & 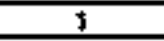 & & $\overline{100}$ & $\tilde{\mathbf{N}}$ & \\
\hline 12 & $11 / 3 / 39$ & 1 & 1 & & 796 & $\mathbf{N}$ & \\
\hline 1B & $11 / 3 / \mathrm{BA}$ & 1 & 1 & & 168 & $\mathbf{N}$ & \\
\hline 12 & $1 \mathrm{NOWBB}$ & 3 & 1 & & 185 & $\mathbf{N}$ & \\
\hline 1ह & 1 1 10 ose & 1 & 1 & & 187 & $\mathbf{N}$ & \\
\hline 12 & $11 / 17 / 8 \mathrm{~s}^{2}$ & 1 & 1 & & TE5 & $\mathbf{M}$ & \\
\hline 18 & $1 / 117 / 89$ & 1 & 1 & & tes & $\mathbf{N}$ & \\
\hline 12 & $11 / 24 / 8 \mathrm{~A}$ & 1 & 1 & & 213 & $\mathbf{N}$ & \\
\hline 18 & $11 / 24 / 89$ & 1 & 1 & & 187 & $\overline{\mathbf{N}}$ & \\
\hline$\$ 2$ & $12 / 8 / 89$ & 1 & $i$ & & 163 & $\mathbf{N}$ & \\
\hline 18 & $12 \mathrm{rrs}$ & 1 & 1 & & 106 & $\mathbf{N}$ & \\
\hline $1 \mathrm{~B}$ & $12 / 18 / 38$ & 1 & 1 & & 163 & $\mathbf{N}$ & \\
\hline 12 & 12/228B & 1 & 1 & & 160 & $\mathbf{N}$ & \\
\hline 18 & $12 / 2 \mathrm{Rs}$ & 1 & 1 & & 162 & $\mathbf{N}$ & \\
\hline 12 & 1/5/e9 & 1 & 1 & & 9Q5 & $\mathbf{N}$ & \\
\hline 18 & $3 / 5 / 80$ & 1 & 1 & & 197 & $\mathbf{N}$ & \\
\hline 12 & 1/12/B9 & 1 & 1 & & 192 & $\mathbf{N}$ & \\
\hline $1 \overline{8}$ & $1 / 12 \mathrm{Bg}$ & 1 & $7=$ & & 183 & $\mathbf{N}$ & \\
\hline 12 & 1/19/8B & 1 & \$’! & & 160 & $\mathbf{N}$ & \\
\hline 18 & 1/ & 1 & 1 & & 905 & $\mathbf{N}$ & \\
\hline 12 & $1 / 26 / 899$ & 1 & 1 & & 185 & $\mathbf{N}$ & \\
\hline 18 & $t / 28 / 89$ & 1 & 1 & & 166 & $\mathbf{N}$ & \\
\hline 12 & $2 / 2 / 80$ & 1 & 1 & & 179 & $\mathbf{N}$ & \\
\hline 18 & $2 / 2 / 89$ & 1 & 1 & & 102 & $\mathbf{N}$ & \\
\hline 12 & $2 / 8 / 89$ & 1 & 1 & & 179 & $N$ & \\
\hline $1 \mathrm{~B}$ & $2 / 5 / 93$ & 1 & 1 & & 184 & $\mathbf{N}$ & \\
\hline 12 & 2 27 Bdeg & 1 & 1 & & 178 & $\mathbf{N}$ & \\
\hline 1E & $2 / 18 / 89$ & 1 & 1 & & 160 & $\mathbf{N}$ & \\
\hline 12 & $2 / 23$ ka9 & 1 & 1 & & 179 & $\mathbf{N}$ & \\
\hline $1 \overline{8}$ & $2 / 23 / 89$ & 7 & 1 & & 182 & $\mathbf{N}$ & \\
\hline
\end{tabular}

Date obtsined from WHC Surveillemce Analycit Comptrter [SACSI, June 2, 1993. 
WHC-Sb-MM-ER-308، Rev. O

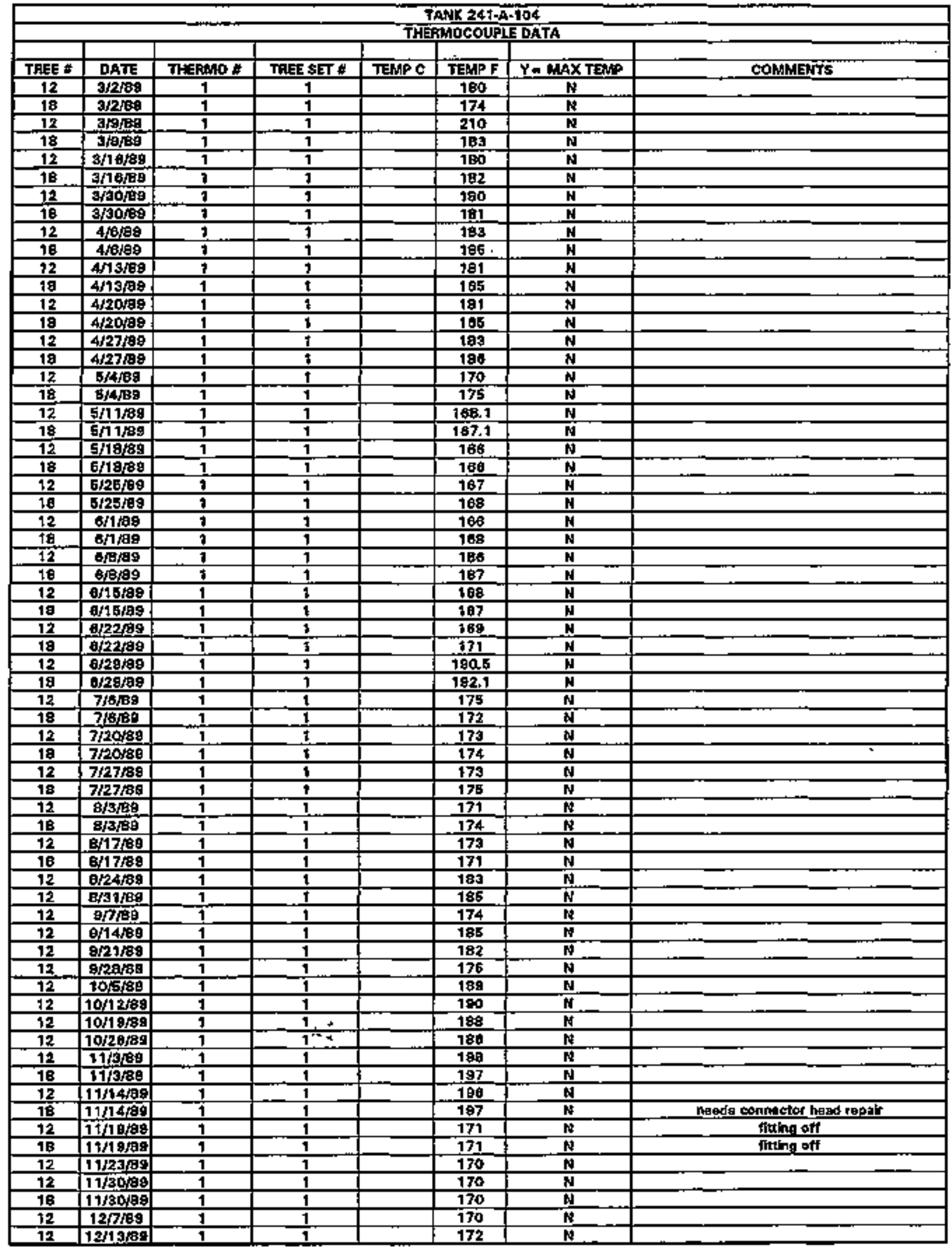

Date obteinad from WHC Survallanco Analycis Computer (SACS), Juna 2, 1993. 
WHCP.SO-WM-ER-3OE, AOY, O

\begin{tabular}{|c|c|c|c|c|c|c|c|}
\hline \multicolumn{8}{|c|}{ TANK 24 $\$+A+194$} \\
\hline \multicolumn{8}{|c|}{ TFEPMWCOUPLE DATA } \\
\hline TAEE ? & DATE & THEAMO * & TREE SET * & TEMF C & TEMP F & $Y=$ MAX TEMP & COMMENTS \\
\hline 18 & $12 / 13 / \mathrm{ES}$ & 1 & 1 & & 174 & $\mathrm{~N}$ & \\
\hline 12 & $12 / 21 / 8$ & 1 & 1 & & 199 & $\mathbf{N}$ & \\
\hline 12 & $12 / 28 / 69$ & 1 & 1 & & 199 & $\mathbf{N}$ & \\
\hline 18 & $12 / 2 \sin 3$ & 1 & 1 & & 190 & $\mathbf{N}$ & se pluphin is off TC wite. \\
\hline 12 & $1 / 11 / 00$ & 1 & 1 & & 198 & $\mathbf{N}$ & \\
\hline 18 & $1 / 11 / 90$ & 1 & 1 & & 190 & $\mathbf{N}$ & connectar hoad broke off \\
\hline 12 & $1 / 18 / 90$ & 1 & 1 & & 198 & $\mathbf{N}$ & \\
\hline 18 & $1 / 18 / 90$ & 1 & 1 & & 195 & $\mathbf{N}$ & tonnector hoded still broken \\
\hline 12 & $1 / 25 / 90$ & 1 & 1 & & 197 & $\mathbf{N}$ & \\
\hline 12 & $2 / 1 / 90$ & 1 & 1 & & 187 & $\mathbf{N}$ & \\
\hline 18 & $2 / 1 / 90$ & 1 & 1 & & 189 & $\mathbf{N}$ & \\
\hline 12 & $2 \sqrt{8}$ & 1 & 1. & & 178 & $\mathbf{N}$ & \\
\hline 18 & $2 \mathrm{r}$ & 1 & 1 & & 174 & $\mathbf{N}$ & \\
\hline 12 & $2 / 15 / 90$ & 1 & 1 & & 192 & $\mathbf{N}$ & \\
\hline 12 & $2 \sqrt{22} / 90$ & 1 & 1 & & 194 & $\mathbf{N}$ & \\
\hline 12 & $3 / 1 / 90$ & 1 & 1 & & 193 & $\mathbf{N}$ & \\
\hline 12 & 3a & 1 & $\mathbf{1}$ & & 193 & $\mathbf{N}$ & \\
\hline $1 \mathrm{\theta}$ & $3 / \sqrt{1900}$ & 1 & $\mathbf{T}$ & & 196 & $\mathbf{N}$ & \\
\hline 12 & 3/16/00 & 1 & 1 & & 192 & $\mathbf{N}$ & \\
\hline+2 & $3 / 22 / 90$ & 1 & 1 & & 169 & $\mathbf{N}$ & \\
\hline 12 & $3 / 29 / 90$ & 1 & 1 & & $\$ 91$ & $\mathbf{N}$ & \\
\hline 1日 & $3 / 29 / 90$ & 1 & 1 & & 393 & $\mathbf{N}$ & \\
\hline 12 & $4 \sqrt{6}, 190$ & 1 & 1 & & 168 & $\mathbf{N}$ & \\
\hline 18 & $4 / 5 / 60$ & 1 & 1 & & 170 & N & \\
\hline 12 & $4 / 12 / 90$ & 1 & 1 & & 106 & N & \\
\hline 18 & $4 / 12 / 90$ & 1 & 1 & & 168 & $\mathbf{N}$ & \\
\hline 12. & $4 / 19 / 00$ & 1 & 1 & & 169 & $\mathbf{N}$ & \\
\hline t白 & $4 / 19190$ & 1 & 1 & & 171 & $\mathbf{N}$ & \\
\hline 12 & $4 / 20 / 00$ & 1 & 1 & & 168 & $\mathbf{N}$ & \\
\hline 12 & $5 / 3 / 90$ & 1 & 1 & & 180 & $\mathbf{N}$ & \\
\hline 16 & $5 / 3 / 90$ & 1 & 1 & & 191 & $\mathrm{~N}$ & \\
\hline 12 & $5 / 10 / 90$ & 1 & 1 & & 168 & $\mathbf{N}$ & \\
\hline 18 & 5/1아이 & 1 & 1 & & 178 & $\mathbf{N}$ & \\
\hline 12 & 3/7150 & 1 & 1 & & 1088 & $\mathbf{N}$ & \\
\hline 32 & Shaнo & 1 & 1 & & 107.6 & $\mathbf{N}$ & \\
\hline 12 & $5 / 24 / 90$ & 1 & $\$$ & & 107.1 & $\mathbf{N}$ & \\
\hline 12 & 5/31/90 & 1 & 1 & & 199 & $\mathbf{N}$ & \\
\hline 12 & ONN90 & 1 & 1 & & 168 & $\mathbf{N}$ & \\
\hline 18 & $0 / 150$ & 1 & 5 & & 170 & $\mathbf{N}$ & \\
\hline 12 & $8[14 / 90$ & 1 & 1 & & 188 & $\mathbf{N}$ & \\
\hline 12 & $\mathrm{~B} / 2 \mathrm{t} / \mathrm{gQ}$ & 1 & 1 & & 172 & $\mathbf{N}$ & \\
\hline 18 & $6 / 29 / 90$ & 1 & 1 & & 173 & $\mathbf{N}$ & \\
\hline 12 & $6 \sqrt{26 \sqrt{90}}$ & 1 & 1 & & 173 & $\mathbf{N}$ & \\
\hline 18 & $6 / 28 / 90$ & 1 & 1 & & 191 & $\mathbf{H}$ & \\
\hline 12 & $7 / 5 \mathrm{SO}$ & 1 & 1 & & 172 & $\mathbf{N}$ & \\
\hline 18 & $7 / 5 / 80$ & 1 & 1 & & 173 & $\mathbf{N}$ & \\
\hline 18 & 7 poreo & 1 & 1 & & 769.4 & $\mathbf{N}$ & \\
\hline 12 & $7 / 12 / 80$ & 1 & 1 & & 174 & $\mathbf{N}$ & \\
\hline 12 & $7 / 13 / 20$ & 1 & 1 & & 180 & $\mathbf{H}$ & \\
\hline 12 & 719R0 & 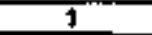 & 7 & & 187 & $\bar{N}$ & \\
\hline 12 & $3 / 26 / 90$ & 1 & 1 & & 186.1 & N & \\
\hline 19 & $7 / 26 / 90$ & 1 & 1 & & 190.5 & W & \\
\hline 12 & $8 / 2 / 90$ & 1 & 1. & & 201 & $\mathbf{N}$ & \\
\hline 12 & $8 / 9 / 20$ & 1 & $t^{2}$ & & 199.3 & $\mathbf{N}$ & \\
\hline 18 & 81960 & 1 & 1 & & 172.8 & $\mathbf{N}$ & \\
\hline 12 & $8 / 18 / 90$ & 1 & 1 & & 797.5 & $\mathbf{N}$ & \\
\hline 19 & a/to/oo & 1 & 1 & & 190.1 & $\mathbf{N}$ & \\
\hline 12 & a/23/so & 1 & 1 & & 188.1 & $\mathbf{N}$ & \\
\hline 18 & $9 / 23 / 90$ & 1 & 1 & & 190.8 & $\mathbf{N}$ & \\
\hline 12 & E/BOpo & 1 & 3 & & 168 & $\mathbf{N}$ & \\
\hline 18 & a/soleo & 1 & $t$ & & 173 & $\mathbf{N}$ & \\
\hline 12 & $0 / 7 / 80$ & 1 & 1 & & 199.2 & $\mathbf{N}$ & \\
\hline 19 & $9 / 7 / 90$ & 1 & 1 & & 192.1 & $\mathbf{N}$ & \\
\hline 12 & $8 / 13 / \%$ & 1. & 1 & & 103.9 & N & \\
\hline 18 & $9 / 3 / 90$ & 1 & 1 & & 106,5 & $\mathbf{N}$ & \\
\hline
\end{tabular}

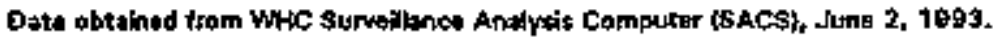




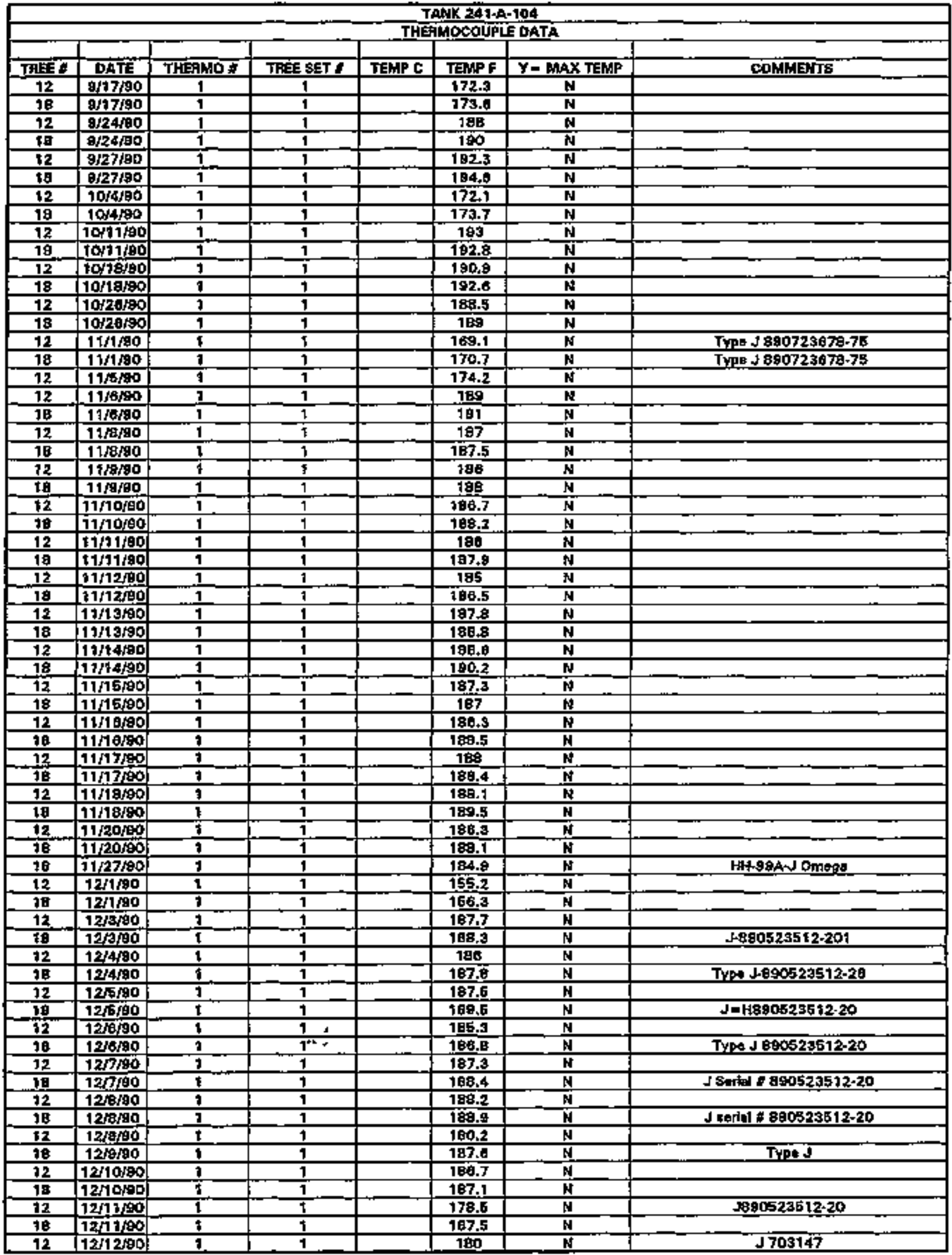

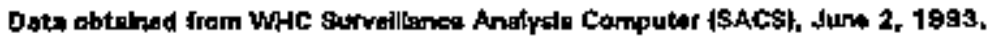


WHC-SD-WM-ER-308, Rov, O

\begin{tabular}{|c|c|c|c|c|c|c|c|}
\hline \multicolumn{8}{|c|}{ TAMK $241 . \bar{A}=104$} \\
\hline \multicolumn{8}{|c|}{ IHERTOCOUPLE DATA } \\
\hline TREE $\boldsymbol{*}$ & DATE & THETAHO : & THEE SET A & TESMP C & FEMP F & $Y=\operatorname{MAX} \times \mathrm{TEMP}$ & COHAENTS \\
\hline 1E & $12 / 12 / 80$ & 1 & 1 & & 189 & $N$ & \\
\hline 12 & $12 / 13 / 00$ & 1 & 1 & & 189 & $\mathbf{N}$ & Je90 423320.11 \\
\hline 18 & $12 / 13 / 90$ & 1 & 1 & & 199.3 & $\mathbf{N}$ & \\
\hline 12 & $12 / 14 / 90$ & 1 & 1 & & 189 & $\mathbf{N}$ & $E 7 0 3 \longdiv { 4 7 }$ \\
\hline 18 & $12 / 14 / 90$ & 1 & 1 & & 187,5 & $\mathbf{N}$ & \\
\hline 12 & $12 / 15 / 00$ & 1 & $\bar{t}$ & & 185.8 & $\mathbf{N}$ & \\
\hline 18 & $12 / 6 / 00$ & 1 & 1 & & 187.8 & $\mathbf{N}$ & \\
\hline 12 & $12 / 10190$ & 1 & 1 & & 184.6 & $\mathbf{N}$ & Fomale pluo and broken otf \\
\hline 1a & $12 / 16 / 90$ & 1 & 1 & & 187,2 & $\mathbf{N}$ & $E-703147$ \\
\hline 18 & $12 / 3 / 90$ & 1 & 1 & & 186.7 & $\mathbf{N}$ & HH-79g自等 \\
\hline 12 & $12 / 1890$ & 1 & 1 & & 184.7 & $\bar{N}$ & \\
\hline 18 & 12 r1a/90 & 1 & 1 & & 186.5 & $\mathbf{N}$ & J.990623512-20 \\
\hline 18 & $12 / 19 / 90$ & 1 & $\mathbf{1}$ & & 188.3 & $\mathbf{N}$ & \\
\hline 15 & $12 \sqrt{20190}$ & 1 & 1 & & 197 & $\mathbf{N}$ & Jaso523512-20 omega \\
\hline $1 \mathrm{~B}$ & $12 / 24 / 90$ & 1 & 1 & & 187.2 & $\mathbf{N}$ & \\
\hline 18 & $12 / 27 / 90$ & 1 & 1 & & 148.6 & $\mathbf{N}$ & 日90523512-20 TYPE J \\
\hline 12 & $12 / 31 / 90$ & 1 & 1 & & 183.6 & $\mathbf{N}$ & TYPE J 990423320.11 \\
\hline 18 & $12 / 31 / 90$ & 1 & 1 & & 185.5 & $\mathrm{~N}$ & \\
\hline 18 & $1 / 2 / 91$ & 1 & 1 & & 183.7 & $\mathbf{N}$ & $\$ 690523512-20$ \\
\hline 18 & $1 / 3 / 27$ & $t$ & 1 & & 181.7 & $\mathbf{N}$ & $890523512-20$ \\
\hline 18 & 1/4/A1 & 1 & 1 & & 164.6 & $\mathbf{N}$ & \\
\hline 18 & $1 / 5 / 91$ & $I$ & 1 & & 158.6 & $\mathbf{N}$ & \\
\hline 3日 & $1 / 6 / 91$ & 1 & 1 & & $18 B .6$ & $\mathbf{N}$ & 890523512,20 Typo J \\
\hline $7 \mathrm{I}_{\mathrm{C}}$ & 1/7/B1 & $\tau$ & 1 & & 185 & $\mathbf{N}$ & \\
\hline 12 & $1 \mathrm{fa/91}$ & 1 & 1 & & 783.4 & $\mathbf{N}$ & \\
\hline 18 & $1 \mathrm{~m} / \mathrm{A}_{1} 1$ & 1 & 1 & & 786.日 & $\mathbf{N}$ & \\
\hline 18 & $1 / 17 / 91$ & 1 & 1 & & 185 & $\mathbf{N}$ & 2890523512.20 \\
\hline 18 & $1 / 22 / 91$ & 1 & 1 & & 104,5 & $\mathbf{M}$ & J-990423320-11 \\
\hline 12 & $1 / 24 / 91$ & 1. & 1 & & 281.4 & $\mathbf{N}$ & \\
\hline 18 & $1 / 24 / 91$ & 1 & 1 & & 192.0 & $\mathbf{M}$ & \\
\hline 12 & $1 / 25 / 91$ & 1 & 1 & & 191.9 & $\mathbf{N}$ & \\
\hline 18 & 1/25/91 & 1 & 1 & & 193,0 & $N$ & $\mathrm{HH}$ \\
\hline 12 & $1 / 28 / 91$ & $T$ & 1 & & 182,4 & N & \\
\hline 18 & $1 / 26 / 91$ & 1 & 1 & & 184.4 & $\mathrm{~N}$ & HH-99A-E, E90723678-75 \\
\hline 12 & $1 / 27 / 91$ & 1 & 1 & & 192.7 & $\mathrm{~N}$ & HH.99A-E B90723678-75 \\
\hline 18 & $1 / 27 / 91$ & 1 & 1 & & 194.5 & $\mathbf{M}$ & \\
\hline 12 & 1/28/81 & 1 & 1 & & 182 & $\mathbf{N}$ & HHQQQd.JPQOOA23320-11 \\
\hline 18 & $1 / 28 / 91$ & 1 & $\mathbf{1}$ & & 184 & $\mathrm{~N}$ & \\
\hline 12 & $1 / 29 / 91$ & 1 & t & & 182.4 & $\mathbf{N}$ & \\
\hline 18 & $1 / 29 \%$ & 1 & 1 & & 184,3 & $\mathbf{M}$ & \\
\hline 12 & $1 / 31 / 91$ & 1 & 1 & & 182.1 & $\mathbf{N}$ & \\
\hline 18 & $1 / 31 / 81$ & 1 & 1 & & 183,8 & $\mathbf{N}$ & E-890723078.76 \\
\hline 12 & $2 / 4 / 97$ & 1 & 1 & & $181+8$ & $\mathbf{N}$ & \\
\hline 18 & $2 / 4 / T^{2}$ & 1 & 1 & & 183.4 & $\overline{\mathbf{M}}$ & Typ E 890723578-22 \\
\hline 12 & 2/5/et & 1 & 9 & & 181,3 & $\bar{N}$ & \\
\hline 16 & 2/5/at & 1 & 1 & & 183 & $\mathbf{N}$ & \\
\hline 12 & $2 / 7 / 91$ & 1 & 1 & & $187+7$ & $\mathbf{N}$ & \\
\hline 18 & $2 \pi / 93$ & 1 & 1 & & 159.9 & $\mathbf{N}$ & \\
\hline 12 & $2 / 11 / 9 t$ & 1 & 1 & & 189 & $\mathbf{N}$ & $E[890728678-22]$ \\
\hline 18 & $2 / 11 / 9 t$ & 1 & 1 & & 199,0 & $\mathbf{N}$ & \\
\hline 12 & $2 / 12 / 91$ & 1 & 1 & & $187+9$ & $\mathbf{N}$ & \\
\hline $1 \mathrm{~B}$ & $2 / 2 / 81$ & 1 & 1 & & 189.5 & $\mathbf{N}$ & LTYpot Bops 23512-20 \\
\hline 12 & $2 / 4401$ & 1 & 1. & & 187.7 & $\mathbf{N}$ & \\
\hline 18 & $2 / 44 / 91$ & 1 & r'. & & $\$ 69,00$ & $\mathbf{N}$ & Tyре J HH:SA- $/ \mathrm{BPO} 523512,20$ \\
\hline 12 & 2/20/a & 1 & 1 & & 186.6 & $\mathbf{M}$ & Trpe J \\
\hline IB & $2 / 20 / 9 ?$ & 1 & 1 & & 169.6 & $\mathbf{N}$ & \\
\hline 12 & $2 / 21 / 91$ & $\mathbf{1}$ & 1 & & 187,4 & $\mathbf{N}$ & $\$ 89023512.20$ \\
\hline 18 & $2 / 21 / 91$ & 1 & 1 & & 189,3 & $\mathbf{Y}$ & \\
\hline 12 & $2 / 25 / 81$ & 1 & 1 & & 186.2 & $\mathbf{N}$ & 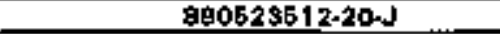 \\
\hline 18 & $2 / 25 / 91$ & 1 & 1. & & 189.1 & $\bar{Y}$ & \\
\hline 12 & $2 / 28 / 91$ & 1 & 1 & & $187+1$ & $\mathbf{N}$ & \\
\hline 18 & $2 / 28 / 91$ & 1 & 1 & & 18B.8 & $\mathbf{N}$ & J Typa $890523512+20$ \\
\hline 12 & $3 / 4 / 97$ & 1 & 1 & & 183 & $\mathbf{N}$ & $3 \# 2 E-0 P .021$ \\
\hline 18 & $3 / 4 / 91$ & 1 & 1 & & 185 & $\mathbf{N}$ & \\
\hline 12 & 37891 & 1 & 1 & & 190.7 & $N$ & ZE:0P-020 \\
\hline
\end{tabular}

Data obtained from WHC Survellanoe Anahyels Compuler (SACSI, Juna 2 1983. 
WHG-SD-WM-EA-30S, Aav. 0

\begin{tabular}{|c|c|c|c|c|c|c|c|}
\hline \multicolumn{8}{|c|}{ TANK 241-A-104 } \\
\hline \multicolumn{8}{|c|}{ THEFINOCOXPLE DATA } \\
\hline TREE $\boldsymbol{y}$ & DATE & THERMO & TREE ELE & thMP $\mathrm{c}$ & TEMP F & Y= MAX TEMP & COMHENTS \\
\hline 18 & $3 \pi / 91$ & 1 & 1 & & 182 & $\mathbf{Y}$ & \\
\hline 12 & $3 / 24 / 91$ & 1. & 1 & & $1 \mathrm{~B}+9$ & A & \\
\hline 18 & $3 / 14 / 91$ & 1 & 1 & & 185.6 & $\mathbf{Y}$ & \\
\hline 12 & $3 \sqrt{21 / 81}$ & 1 & 1 & & 186.1 & $\mathbf{N}$ & 890523512.20 type J \\
\hline 18 & 321/91 & 1 & 1 & & 187.1 & $\boldsymbol{\gamma}$ & \\
\hline 12 & $3 / 28 / 6 t$ & 1 & 1 & & j88.1 & $\mathbf{N}$ & 3-28+a1: Jtype figags23512-20 \\
\hline 18 & $3 / 26 / 97$ & 1 & 1 & & $19 \mathrm{~g}$ & $Y$ & \\
\hline 12 & 4/1/91 & 1 & 1 & & 182 & $N$ & TYPE! \\
\hline t且 & 4/1/21 & 1 & 1 & & 193.7 & $N$ & \\
\hline 12 & $4 / 4 / 81$ & 1 & 1 & & 182 & $\mathbf{N}$ & \\
\hline 18 & $4 / 4 / 91$ & 1 & 1 & & 184 & $\mathbf{N}$ & Ompga 450 AET TYpeE \\
\hline 12 & 4/6/61 & 1 & 1 & & 182,9 & $\mathbf{N}$ & Trpa J \\
\hline $1 \dot{8}$ & 481 & 1 & 1 & & 194.7 & $\mathbf{N}$ & \\
\hline 12 & $4 / 11 / 91$ & 1 & 1 & & 183 & $\mathbf{N}$ & Irpa d \\
\hline 18 & 4/11/91 & 1 & 1 & & 185 & $\mathbf{N}$ & \\
\hline 12 & $4 / 45 / 91$ & 1 & 1 & & 182.2 & $\mathbf{N}$ & \\
\hline 18 & 4/t5/91 & 1 & 1 & & 184.2 & $\mathbf{N}$ & HH-B9 A.J BSO23512-20 \\
\hline 12 & 4/te/91 & 1 & 1 & & 181.8 & $\mathbf{N}$ & \\
\hline 18 & 4/19/91 & 1 & 1 & & 183.6 & $\mathbf{M}$ & TYPEJ \\
\hline 12 & 4/25/91 & 1 & 1 & & 182 & $\mathrm{M}$ & \\
\hline 18 & $4 / 25 / 91$ & 1 & 1 & & 184 & $\mathbf{N}$ & Type $\$$ \\
\hline 12 & $6 / 9 / 91$ & 1 & 1 & & 182,7 & $\mathrm{H}$ & J 2E-OP-D20 \\
\hline 19 & $5 / 0 / 91$ & 1 & 1 & & 185.2 & $\mathbf{N}$ & \\
\hline 12 & $6 / 13 / 91$ & 1 & 1 & & $1 \mathrm{Bz} \cdot \dot{\mathrm{B}}$ & $\mathbf{N}$ & J日90583512.20 \\
\hline 18 & $6 / 13 / J_{1}$ & 1 & 1. & & 184.7 & $\bar{Y}$ & \\
\hline 12 & 6/16/O1 & 1 & 1 & & 182.7 & $\mathbf{N}$ & $\pm 890523512-20$ \\
\hline 18 & B/16/9t & 1 & 1 & & 184.7 & $\mathbf{y}$ & \\
\hline 12 & $5 / 20 / 91$ & 1 & 1 & & 180.7 & $\mathbf{M}$ & \pm 890523512.20 \\
\hline 18 & 5laten & 1 & $\overline{1}$ & & 182.6 & $\gamma$ & \\
\hline 12 & $5 / 23 \sqrt{91}$ & 1 & 1 & & 182 & $\mathrm{~N}$ & Es0523512-20 \\
\hline 18 & E/23/10 & 1 & 1 & & 194.2 & $\boldsymbol{Y}$ & \\
\hline 12 & 5/30/67 & 1 & 1 & & 193,2 & $\mathbf{N}$ & $299052312-20$ \\
\hline 98 & 5/30/e? & 1 & 1 & & $1 \mathrm{104.5}$ & $\gamma$ & \\
\hline 12 & ENA1 & 1 & 1 & & 192.8 & $\boldsymbol{Y}$ & الد. \\
\hline 18 & OPA1 & 1. & 1 & & 194.7 & $\mathbf{Y}$ & $J$ \\
\hline 12 & e/a/e1 & 1 & 1 & & 184 & $\mathbf{N}$ & ل \\
\hline 19 & toldsi & 1 & 1 & & 187 & $\gamma$ & J \\
\hline 12 & o/as/a1 & 1 & $\mathbf{1}$ & & 181.8 & $\mathbf{N}$ & JaD0523512-20 \\
\hline 18 & 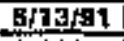 & 1 & 1 & & 183.5 & $\mathbf{Y}$ & \\
\hline 12 & B/20181 & 1 & 1 & & t9.3.8 & $\mathbf{N}$ & Oming H H \\
\hline 18 & O/20/101 & 1 & 1 & & 196,2 & $\mathbf{Y}$ & \\
\hline 12 & a/27/ 1 & 1 & 1 & & 180.7 & $\mathbf{Y}$ & $\mathbf{J}$ \\
\hline 19 & 0/27/91 & 1 & 1 & & 184,7 & $\mathbf{N}$ & \\
\hline 12 & $7 / 1 / 91$ & 1 & 7 & & & $\mathbf{N}$ & 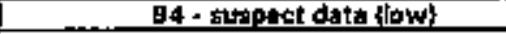 \\
\hline 12 & $7 / 4 / 91$ & 1 & 1 & & 184.7 & $\mathrm{~N}$ & $\jmath$ \\
\hline 18 & $7 / 4 / 91$ & 1 & 1 & & 196,3 & $Y$ & \\
\hline 12 & $7 / 11 / 91$ & 1 & 1 & & 184.7 & $\mathbf{N}$ & J-2E-OP-021 Nend rad labels. \\
\hline 18 & $7 / 11 / 91$ & 1 & 1 & & 186.4 & $\bar{Y}$ & Noed roal books \\
\hline 12 & 715891 & 1 & 1 & & 185 & $\mathbf{N}$ & Ja72 \\
\hline $1 \dot{\theta}$ & $7 / 15 / 91$ & 1 & 1 & & 196 & $\mathbf{Y}$ & \\
\hline 12 & 7 18/91 & 1 & 1 & & 184.9 & $\mathbf{N}$ & J-2E-09-021 \\
\hline 12 & $7 / 20$ fa1 & 1 & $t$ & & 185 & $\mathbf{M}$ & $\sqrt{ }$ \\
\hline 19 & $7 \sqrt{20} 91$ & 1 & 12 & & 186.8 & $\mathbf{Y}$ & \\
\hline 12 & $7 / 2519$ & $i$ & $1 \%$ & & 185.5 & $\mathbf{N}$ & \\
\hline tg & $7 / 25 / 61$ & 1 & 1 & & 187,3 & $\bar{Y}$ & \\
\hline 12 & eft/B1 & 1 & 1 & & 185.2 & $\mathbf{N}$ & E $89073267 \mathrm{~B}+\mathrm{B5}$ \\
\hline ig & $8 / 191$ & 1 & 1 & & 189.9 & $\mathbf{Y}$ & \\
\hline$\sqrt{2}$ & exta1 & 1 & 1 & & 180 & $\mathbf{N}$ & $\mathbf{J}$ \\
\hline 18 & Bis/a1 & 1 & 1 & & 189 & $\mathbf{Y}$ & \\
\hline 12 & G/A/O1 & 1 & 1 & & 185 & $\mathbf{N}$ & Tres $\downarrow$ \\
\hline 28 & 白官为1 & 1 & $i$ & & 187 & $Y$ & Trpe J \\
\hline 12 & 8)/12/ & 1 & 1 & & 184.7 & $\mathbf{N}$ & Tratd \\
\hline 19 & B12/121 & 1 & 1 & & tge.8 & $y$ & Typo J \\
\hline 12 & EflBret & 1 & 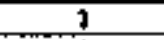 & & 86.9 & $\mathbf{N}$ & Trpe J-2E-DPA2O \\
\hline 19 & E/1E/91 & 1 & 1 & & 597.4 & $\mathbf{r}$ & \\
\hline
\end{tabular}

Data obtained from WHC Survellance Anelyas Computer (SACS), June 21093. 
WHE-SD-WM-ER-306, Rev. 0

\begin{tabular}{|c|c|c|c|c|c|c|c|}
\hline \multicolumn{8}{|c|}{$\begin{array}{l}\text { TANA 241+A-104 } \\
\text { THERMOCOUPLE DATA }\end{array}$} \\
\hline THEE W & DATE & THERAMO : & TAEE SEY : & TEMP E & TEMPF & $Y=$ MAX TEMP & ConMANTs \\
\hline 12 & a/22/81 & $\ddagger$ & 1 & & 180 & $N$ & \\
\hline 18 & $8 / 22 / 91$ & 1 & $\mathbf{1}$ & & 189 & $\mathbf{Y}$ & \\
\hline$\overline{12}$ & $8 / 29 / 81$ & 1 & 1 & & 181 & $\mathbf{Y}$ & J-2E-OP-020 \\
\hline 18 & $8 / 29 / 91$ & 1 & 1 & & 177 & $\mathbf{N}$ & \\
\hline 12 & 9/5/a1 & 1 & 1 & & & $\mathbf{N}$ & O/5 Work Ordar written (Bad Connectar) \\
\hline 18 & $0 / 5 / 91$ & 1 & 1 & & & $\mathbf{N}$ & \\
\hline 12 & $9 / 7 / 81$ & 1 & 1 & & 186.8 & $Y$ & \\
\hline 18 & $9 / 7 / 91$ & 1 & 1 & & 170.6 & $\mathbf{N}$ & \\
\hline$\overline{12}$ & $9 / 2 / 91$ & $1:$ & 1 & & 187.7 & $\mathbf{N}$ & $J-2 E 000247$ \\
\hline 18 & 9/12/91 & 1 & 1 & & 189.8 & $\mathbf{Y}$ & \\
\hline 12 & s/19/91 & 1 & 1 & & 184,6 & $\mathbf{N}$ & E-891222429-104 \\
\hline 18 & $9 / 19 / 91$ & $\mathbf{1}$ & 1 & & 189,4 & $\bar{Y}$ & \\
\hline 12 & $9 / 28+91$ & 1 & 1 & & $\overline{186.4}$ & W & $\mathbf{J}$ \\
\hline 18 & 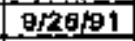 & 1 & 1 & & 189.1 & $y$ & \\
\hline 12 & $0 / 27 / 91$ & 1 & 1 & & 188.4 & $\mathbf{N}$ & J.89005235. $22470 \mathrm{mega}$ \\
\hline 18 & $0 / 27 / 91$ & 1 & 1 & & 188.2 & $\gamma$ & \\
\hline 12 & $10 / 4 / 91$ & 1 & 1 & & 186.7 & $M$ & 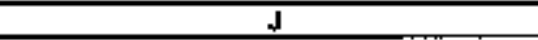 \\
\hline 18 & $10 / 4 / 91$ & 1 & 1 & & 168 & $\gamma$ & \\
\hline$\overline{12}$ & $1070+91$ & $\mathbf{T}$ & $\mathbf{T}$ & & 187.7 & $\bar{M}$ & $5-810128143-75$ \\
\hline 18 & 10/toㄹ 1 & 1 & 1 & & 189.1 & $\boldsymbol{\gamma}$ & \\
\hline 12 & $10 / 13 / 91$ & 1 & 1 & & 186.8 & $N$ & $E-891222428 \cdot 104$ \\
\hline 18 & $10 / 13 / 91$ & 1 & 1 & & 180.3 & $Y$ & \\
\hline 12 & $10 / 14 / 91$ & 1 & 1 & & 186.2 & $\mathbf{N}$ & \\
\hline 18 & $10 / 14 / 91$ & 1 & 1 & & 188.2 & $\mathbf{N}$ & \\
\hline 12 & $10 / 17 / 91$ & 1 & 1 & & 186.8 & $r$ & $J 1980423320-42$ \\
\hline FE & $10 / 17 / 91$ & 1 & 1 & & 186.8 & $\mathbf{N}$ & \\
\hline 12 & $10 \sqrt{21 / 91}$ & 1 & 1 & & 198.8 & $\mathbf{N}$ & Jomepa \\
\hline 10 & $10 / 21 / 91$ & 1 & 1 & & 190.5 & $\mathbf{Y}$ & \\
\hline 12 & $10 / 3 \times 91$ & 1 & 1 & & 189.2 & $\mathbf{Y}$ & TREE U19 ONS \\
\hline 19 & $11 / 1 / 91$ & 1 & 1 & & & $\mathbf{N}$ & TAEEN18 Out of SQRutho \\
\hline 12 & $11 / 6 / 91$ & 1 & 1 & & 189.7 & $\mathbf{N}$ & \\
\hline 18 & $11 / \mathrm{e} / \mathrm{a} 1$ & 1 & 1 & & 101.3 & $\gamma$ & \\
\hline 12 & $11 / 7 / 91$ & 1 & 1 & & 189.2 & $\mathbf{N}$ & \\
\hline 18 & $11 / R \mathrm{a}$ & 1 & 1 & & 180.5 & $\mathbf{Y}$ & \\
\hline 12 & $21 / 10 / 81$ & 1 & 1 & & 191.6 & $\mathbf{N}$ & TREE \#19 O/8 WIU FETEST $11 / 13 / 91$ \\
\hline 12 & 11/13/91 & 1 & 1 & & 190 & $\mathbf{N}$ & 1. $190523512-47$ \\
\hline 18 & $51 / 13 / 81$ & 1 & 1 & & 101 & $\mathbf{Y}$ & \\
\hline 12 & 11/1B/81 & 1 & 1 & & 168.7 & $\mathbf{N}$ & E-900325184-57 \\
\hline 19 & $\$ 1 / 18 / 81$ & 1 & 1 & & 180.3 & $\mathbf{Y}$ & \\
\hline 12 & $11 / 21 / 91$ & $\mathbf{1}$ & 1 & & 189.8 & $\overline{\mathbf{N}}$ & \\
\hline 18 & $11 / 21 / 91$ & $\overline{1}$ & 1 & & 197.8 & $\overline{\mathbf{Y}}$ & \\
\hline 12 & $11 / 2 B / 81$ & 1 & 1 & & 189.6 & $\mathbf{N}$ & \\
\hline 19 & $11 / 28 / 81$ & 1 & 1 & & 191.1 & $Y$ & \\
\hline 12 & $12 / 2 / 9$ & 1 & 1 & & 189.3 & H & $1-890523512 \cdot 47$ \\
\hline 19 & $12 / 2 / 99$ & 1 & 1 & & 190.3 & $\mathbf{Y}$ & \\
\hline 12 & $12 / 5 / 91$ & 1 & 1 & & $1 \mathrm{BS} .7$ & $\bar{N}$ & 890523512.47 \\
\hline 18 & $175 / 91$ & 1 & 1 & & 191.1 & $\overline{\mathbf{Y}}$ & \\
\hline 12 & $12 / 9 / \mathrm{Al}$ & 1 & 1 & & 169.5 & $\mathbf{N}$ & \\
\hline 19 & $12 \mathrm{gat}$ & 1 & 1 & & I 1 & $Y$ & \\
\hline 12 & $12 / 12 / 91$ & 1 & 1 & & 168.8 & $\mathbf{N}$ & \\
\hline 19 & $12 / 12 / 91$ & 1 & 1 & & 990.5 & $\mathbf{Y}$ & \\
\hline 12 & $12 / 19 / 91$ & 1 & 1 & & 198.5 & $\bar{N}$ & \\
\hline 19 & $12 / 10 / 91$ & 1 & $1+$ & & 189.8 & $\gamma$ & \\
\hline 12 & $12 / 31 / 91$ & 1 & $T^{2} x$ & & 181.5 & $\mathbf{N}$ & \\
\hline 19 & $12 / 31 / 81$ & 1 & 1 & & 192.5 & $\mathbf{Y}$ & \\
\hline 12 & $1 / 2 / \mathrm{Bz}$ & 1 & 7 & & 189.2 & $N$ & \\
\hline 19 & $1 / 2 / 02$ & 1 & 1 & & 190.6 & $y$ & \\
\hline 12 & $1 / 9 / 92$ & 1 & 1 & & t90 & $\mathbf{N}$ & $\mathrm{J}$ \\
\hline 18 & $1 / 9 / 92$ & 1 & 1 & & 191 & $Y$ & J \\
\hline 12 & $1 / 18$ rez & 1 & 1 & & Teg & $\mathbf{N}$ & \\
\hline 18 & $1 / 16 / 92$ & 1 & 9 & & 185 & $\mathbf{Y}$ & \\
\hline 12 & $1 / 23 / 92$ & 1 & 1 & & 109 & 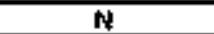 & \\
\hline 19 & $1 / 23 / 92$ & 1 & 1 & & 180 & $Y$ & \\
\hline 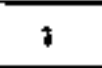 & $1 / 2$ Brez & 1 & 1 & & & $\mathbf{N}$ & $\begin{array}{l}\text { ACCESS RESTRICTED } 1 / 2 \mathrm{~B}-2 / 22 \text { DUE TO } \\
\text { BATIERY FUME WUCLESTT }\end{array}$ \\
\hline
\end{tabular}

Oots obteined trom WHC surveillance Anelyels Compuler [SACS), stune 2, 1993. 


\begin{tabular}{|c|c|c|c|c|c|c|c|}
\hline \multicolumn{8}{|c|}{ 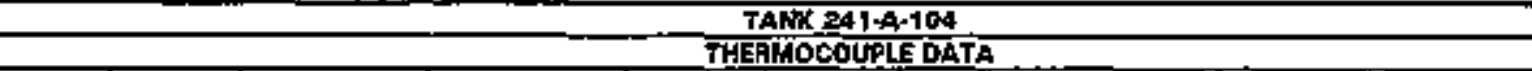 } \\
\hline 㶽馬 & DATE & THEFMOA & TAEE SET & TEMP C & TEMPF & $Y-M A X$ TEKAP & COTHENTS \\
\hline 12 & 1/30/92 & 1 & 2 & & 190 & $N$ & $1.9101230143-32$ \\
\hline 19 & $1 / 30$ of & 1 & $\frac{T}{1}$ & & 182 & $\bar{r}$ & \\
\hline$\overline{12}$ & $2 \longdiv { 8 / 9 2 }$ & 1 & $t$ & & 19B & N & 1910126143+32 \\
\hline 19 & $2 / 1 / 92$ & 1 & 1 & & 169,7 & $\bar{Y}$ & \\
\hline 12 & $2 / 11 / 92$ & 1 & $I$ & & $19 \sqrt{1+5}$ & $N$ & \\
\hline 18 & $2 \sqrt{1 / 92}$ & $i$ & 1 & & $191+8$ & $\bar{Y}$ & \\
\hline 12 & 2120192 & \pm & 7 & & 199, & $\mathbf{N}$ & $5.910126143 \cdot 32$ \\
\hline 18 & $2 / 20092$ & 1 & 1 & & 192 & $\mathbf{Y}$ & \\
\hline 12 & $2 / 22 / 92$ & 7 & 1 & & 190 & $N$ & $5.910128143-32$ \\
\hline 28 & $2 / 22 / 92$ & 1 & 1 & & 191 & $Y$ & \\
\hline 12 & $2 / 27 / 82$ & 1 & 1 & & 189.8 & $\mathbf{N}$ & \\
\hline 98 & $2 / 27 / 92$ & 1 & 1 & & 191 & $Y$ & \\
\hline 12 & 3/52 & 1 & 1 & & $181, \overline{8}$ & $\bar{Y}$ & J910126143-32 \\
\hline 18 & अ/0/92 & 1 & 1 & & 191,5 & $\mathbf{M}$ & \\
\hline 12 & $3 \longdiv { 1 2 / 9 2 }$ & 1 & 1 & & 1896.7 & $\mathbf{N}$ & $\sqrt{1900925741.79}$ \\
\hline If & $3 / 12 / 92$ & 1 & 1 & & 189.8 & $\bar{Y}$ & \\
\hline 12 & $3 \sqrt{24 / 92}$ & 1 & 1 & & 189.9 & $\mathbf{N}$ & J 896972,367日B \\
\hline 19 & $3124 / 22$ & 1 & 1 & & 188.9 & $Y$ & \\
\hline 12 & $3 / 26 / 2$ & 1 & 1 & & $189 . \overline{6}$ & $\mathbb{N}$ & j91012614366 \\
\hline 18 & 3/2e/s2 & 1 & 1 & & 180 & $Y$ & \\
\hline 12 & $4 / 2 / 92$ & 1 & 1 & & 189.5 & $\mathbf{N}$ & $\mathrm{J} 010128143-68$ \\
\hline 18 & $4 / 2192$ & 1 & 1 & & 190 & $\mathbf{Y}$ & \\
\hline 12 & 4 /9/92 & 1 & 1 & & 192.1 & $\mathbf{N}$ & $9990523612-20$ \\
\hline 18 & $4 / 9 / 92$ & 1 & 1 & & 193.5 & $\bar{Y}$ & \\
\hline 12 & 4/10/92 & 1 & 1 & & 199.3 & N & E 900932515447 \\
\hline 18 & $4 / 18 / 32$ & $\underline{1}$ & 1 & & $191+2$ & $\bar{Y}$ & \\
\hline 12 & $4 / 23 / 92$ & $\overline{3}$ & 1 & & 109.8 & $\mathrm{~N}$ & $5910128143-64$ \\
\hline 18 & $4 / 23 / 92$ & $\overline{1}$ & 9 & & 191 & $r$ & \\
\hline 12 & $5 / 3 / 82$ & 1 & 1 & & 191.4 & $\mathbf{N}$ & 9810120143.48 \\
\hline to & $5 / 9 / 92$ & 1 & 1 & & 102.8 & $Y$ & \\
\hline 12 & $5 \overline{792}$ & 1 & 1 & & t91 & $\mathbf{N}$ & $\sqrt{11-022}$ \\
\hline 18 & 57702 & \pm & 1 & & 192.6 & $\bar{Y}$ & \\
\hline 12 & E/14/92 & 1 & 1 & & 109.6 & $\mathbf{N}$ & $1910126143-49$ \\
\hline 18 & 5/14/92 & 1 & 1 & & 191.2 & $\bar{Y}$ & \\
\hline 12 & $5 / 21 / 92$ & 1 & 1 & & 189 & $\mathrm{M}$ & J $590523512-47$ \\
\hline 18 & $561 / 92$ & 1 & 1 & & 989.5 & $\vec{Y}$ & \\
\hline 12 & $6 \longdiv { 2 8 1 9 2 }$ & 1 & 1 & & 198,4 & N & \\
\hline 19 & 5/2每/ & 1 & 1 & & 100.7 & $Y$ & \\
\hline 12 & N4102 & 1 & $T$ & & 187.9 & $\mathbf{M}$ & J 690523512,47 \\
\hline 19 & $0 / 4 / 92$ & 1 & 1 & & 150 & $\bar{Y}$ & \\
\hline 12 & en $11 / 92$ & 1 & 1 & & 187.8 & $\mathbf{N}$ & J800523512-47 \\
\hline 18 & $6 / 11 / 92$ & 1 & 1 & & 189.3 & $Y$ & Ja905235512.47 \\
\hline 12 & $6 j 18 / 22$ & 1 & 1 & & 189,2 & $\mathbf{N}$ & J日Fo5 $23512-07$ \\
\hline 19 & a/46/92 & 1 & 1 & & 190 & $\mathbf{Y}$ & \\
\hline 12 & 0/25/92 & 1 & 1 & & 101.8 & $\overline{\mathbf{N}}$ & 与 \\
\hline 18 & $6 \longdiv { 2 5 / 9 2 }$ & 1 & 1 & & 193.2 & $Y$ & \pm \\
\hline 12 & $6 \sqrt{27192}$ & 1 & 1 & & 189.7 & $\mathbf{N}$ & I \\
\hline 19 & $0 / 27 / 92$ & 1 & 1 & & 190.4 & $\bar{Y}$ & J \\
\hline 12 & $7 / 2$ t92 & 1 & 1 & & 197,3 & in & $\mathrm{J}$ \\
\hline 18 & $72 / 92$ & 1 & 1 & & 103 & $\bar{Y}$ & 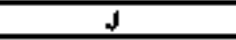 \\
\hline 12 & $7 / 92$ & 1 & 1 & & 193.3 & $\mathbf{N}$ & $9910128143-29$ \\
\hline 19 & 70,02 & 1 & 1 & & 195 & $\bar{Y}$ & \\
\hline 12 & $7 / 10 / 92$ & 1 & 15 & & $10 \overline{3.6}$ & $Y$ & 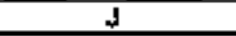 \\
\hline 18 & $7 / 76 / 92$ & 1 & $i=$ & & 195.8 & $\bar{Y}$ & $\mathrm{~J}$ \\
\hline 12 & $7 \sqrt{2392}$ & 1 & 1 & & 195.7 & $\mathbf{N}$ & \\
\hline 18 & $7 / 23 / 92$ & 1 & 1 & & 187.4 & $Y$ & \\
\hline 12 & $7 / 30 / 92$ & 1 & 1 & & 193.5 & H & 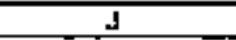 \\
\hline 18 & $7 / 30 / 92$ & 1 & 1 & & 195 & $\bar{Y}$ & \\
\hline 12 & 8/3192 & 1 & 1 & & 194.3 & $\mathbf{N}$ & \\
\hline 18 & B/3/az & 1 & 1 & & 105.3 & $Y$ & \\
\hline 12 & $8 / 6 / 92$ & 1 & 1 & & 184 & $\mathbf{N}$ & \rfloor \\
\hline 18 & $8 / 6 / 92$ & $i$ & 1 & & 195 & $Y$ & \\
\hline 12 & 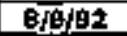 & 1 & 1 & & 193 & $\mathbf{N}$ & $J 910126143-20$ \\
\hline 18 & B药的2 & 1 & 1 & & 194.4 & $\bar{Y}$ & \\
\hline 12 & $8 / 13 / 92$ & 1 & 1 & & $1 \overline{94}$ & $N$ & Jo1012a143-42 \\
\hline
\end{tabular}

Date obtained from WHC Strvellance Analysit Computer (SACS), thane 2, 1 Bg3. 
WHC-SO-WM-EF-3DE, ROW, O

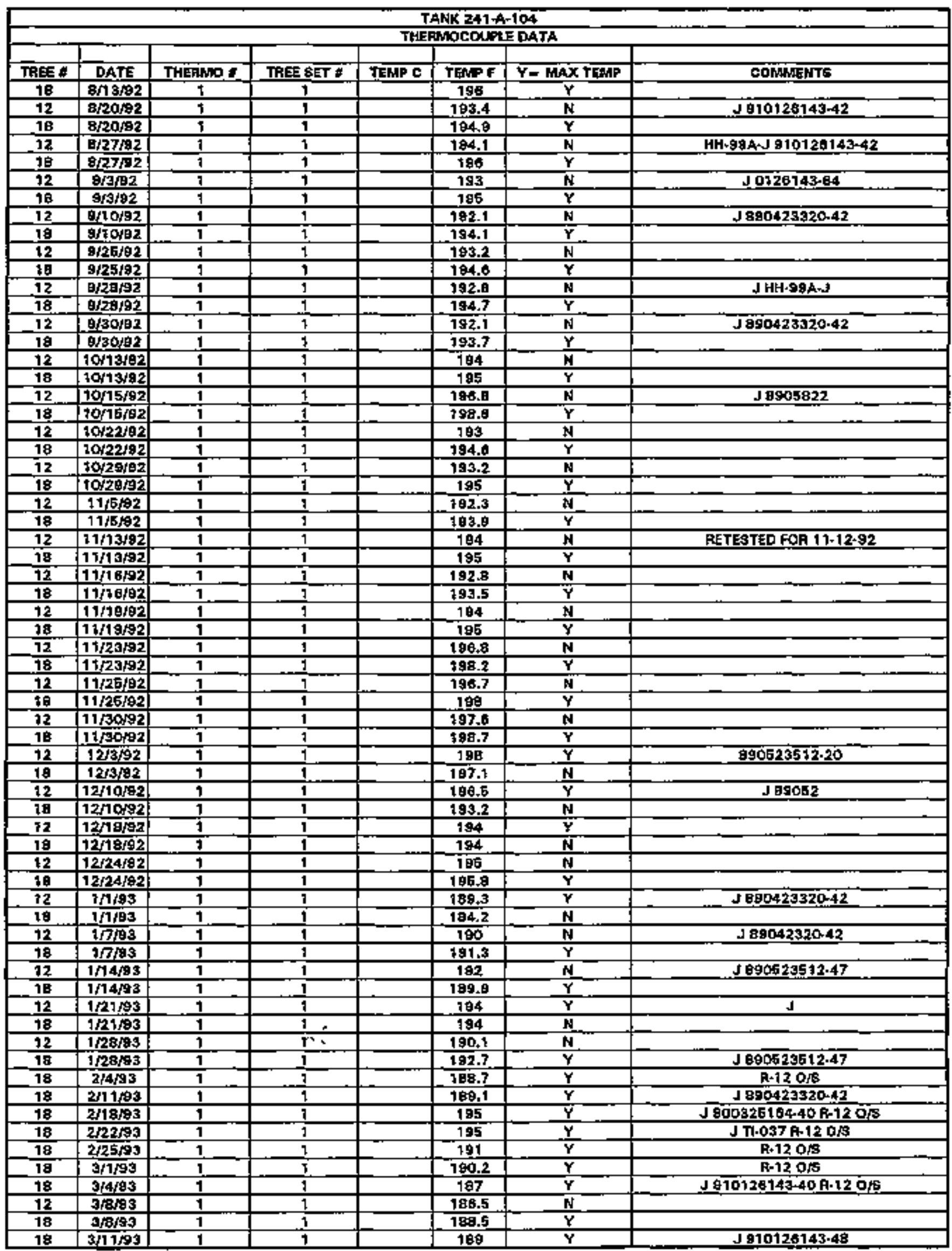

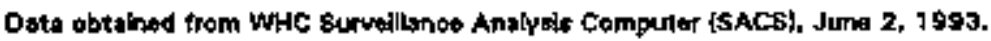


WHC-SO-WM-ER-309, Rav. 0

\begin{tabular}{|c|c|c|c|c|c|c|c|}
\hline & & & & 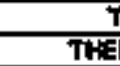 & $\frac{\text { NK 2414 }}{\text { WOCOUA }}$ & $\frac{104}{0 A T A}$ & \\
\hline & & & & & & & \\
\hline TAEE A & DATET & 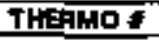 & FREE $5 E 7$ & T地 & TEMF F & $Y=\operatorname{MAX} T E M P$ & COMMENTS \\
\hline 18 & $3 / 18 \mathrm{~s} 3$ & 1 & 1 & & 189 & $\frac{\gamma}{\gamma}$ & J B90423320-42 R-12 O/S \\
\hline 18 & $3 / 22 / 93$ & 1 & 1 & & 180.1 & $\mathbf{Y}$ & J $10120143+40$ \\
\hline 12 & $3 / 25 / 93$ & 1 & 1 & & & $\mathbf{M}$ & DUT OF SERUICE, J $910-12,9-143-14$ \\
\hline 18 & $3 / 25 / 93$ & 1 & 1 & & 18g & $N$ & OUT OF SEFVICE, J $910-12 B-143-34$ \\
\hline IB & 4/1/93 & 1 & 1 & & $18 \mathrm{~g} .7$ & $\mathbf{r}$ & J日tol20143-40 \\
\hline 12 & 4/5/83 & 1 & 1 & & 189.7 & $\mathbf{N}$ & -5 \\
\hline 18 & $4 / 6 / 83$ & 1 & 1 & & 1 160 & $\mathbf{Y}$ & \\
\hline 18 & $4 / 3 / 93$ & 1 & 1 & & 191.8 & $\mathbf{Y}$ & TYPE J TREE $/ 12$ OR \\
\hline 12 & $4 / 15 / 93$ & 1 & 1 & & 168.8 & $\mathbf{N}$ & $J 010129143-42$ \\
\hline 19 & $4 / 5 / 93$ & 1 & 1 & & 189.5 & $\mathbf{Y}$ & J $910120143-42$ \\
\hline 12 & $4 / 9 / 93$ & 1 & 1 & & & N & OUT OF SEFVICE \\
\hline 18 & $4 / 16 / 9 a$ & $I$ & $\overline{1}$ & & 590.2 & $\mathbf{Y}$ & TYPE J \\
\hline 18 & $4 / 25 \sqrt{93}$ & 1 & 1 & & \$90.五 & $\mathbf{Y}$ & IFAfE $120 / 5$, TYPE J \\
\hline 18 & $4 / 26 / 93$ & $T$ & $\xi$ & & 189.9 & $\bar{Y}$ & TREE 12 0/8 \\
\hline $1 \mathrm{~B}$ & 6/0/A3 & 1 & 1. & & 199.8 & $\mathbf{Y}$ & \$51072614329 TREEA120/5 \\
\hline 1 & $5 / 13 / 13$ & 12 & 1 & & & $\mathbf{N}$ & ors \\
\hline 2 & $6713 \mathrm{gm}$ & 18 & 1 & & 199.8 & $\bar{Y}$ & \\
\hline 18 & 5/17/83 & 1 & 1 & & 191.4 & $\bar{Y}$ & J910128143-50,TR\#12 o/s \\
\hline 12 & $5 / 20 / 93$ & 1 & 1 & & 189.4 & $\mathbf{N}$ & $g=1$ \\
\hline 10 & 5/20/133 & 1 & 1 & & $191+6$ & $\mathbf{Y}$ & \\
\hline 18 & $5 / 24 / 93$ & 1 & 1 & & 190,2 & $\bar{Y}$ & J 910126143.42 TAEE $120 \%$ \\
\hline 1 & $5 / 27 / 93$ & 18 & 1 & & 191 & $\mathbf{Y}$ & J E905 235 12-20, TREE 12 DS \\
\hline 19 & 6roto3 & 1 & 1 & & $190+1$ & $\mathbf{Y}$ & J-T1-022 TREE $120 / 3$ \\
\hline 19 & $8 / 10 / 93$ & 1 & 1 & & 101.1 & $\boldsymbol{\gamma}$ & $\begin{array}{c}J \text { St0126143.48 A.12 O/S. J.3 WAS } \\
\text { WFITEN. }\end{array}$ \\
\hline 12 & 8/1793 & 1 & 1 & & 190 & $\mathbf{N}$ & J 090529512,20 \\
\hline 18 & 5/17993 & 1. & 1 & & 192 & $\bar{Y}$ & \\
\hline 12 & $6 / 21 / 83$ & 1 & 7 & & 1B日.g & N & J日10126143-48 \\
\hline 18 & $6 / 21 / 93$ & 1 & $\$$ & & $19 t .0$ & $\mathbf{Y}$ & \\
\hline 12 & $6 / 24 / 93$ & 1 & 1 & & 189.3 & $\mathbf{N}$ & J 010126143449 \\
\hline 18 & $8 \sqrt{24 / 93}$ & 1 & 1 & & 189.9 & $\mathbf{Y}$ & \\
\hline 12 & 21/193 & 1 & 1 & & 189.B & $\mathbf{N}$ & 571041 \\
\hline 78 & $7 / 1 \sqrt{13}$ & 7 & 1 & & 191 & $\mathbf{Y}$ & \\
\hline 12 & 7/e/93 & 1 & 1 & & 181 & $\mathbf{N}$ & J $900925141-78$ \\
\hline 18 & $7 / 6 / 93$ & 1 & 1 & & 103 & $\mathbf{Y}$ & \\
\hline 12 & $7 / 12 \mathrm{as}$ & 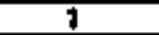 & 1 & & 增4 & $\bar{N}$ & \\
\hline 78 & $7 / 12 \sqrt{93}$ & 1 & 1 & & 195 & $\bar{Y}$ & \\
\hline$\sqrt{2}$ & $7 / 24 \sqrt{13}$ & $i$ & 1 & & 194,2 & $\mathbf{N}$ & 5 WH 910120914360 \\
\hline 18 & $7 / 24 / 93$ & 1 & $I$ & & 195.8 & $\overline{\mathbf{Y}}$ & \\
\hline 12 & $7 / 26 / 93$ & 1 & 1 & & 188.9 & $\bar{N}$ & TYPE JNO SEFYAL NUMBËS GVEN \\
\hline 18 & $7 / 26 / 93$ & 7 & 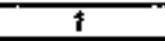 & & 160.7 & $\bar{r}$ & \\
\hline 12 & Etabas & 1 & 1 & & 194 & $\underline{N}$ & JTF042910126 143006 \\
\hline 18 & 8/2/93 & 1 & 7 & & 195 & $\bar{Y}$ & $\mathrm{~s}_{2}$ \\
\hline$\overline{12}$ & (6) & 1 & 1 & & 190 & $N$ & J910126143-39 \\
\hline$\overline{18}$ & Bfefaz & 1 & 1 & & 192 & $\mathbf{Y}$ & \\
\hline 12 & 8/23/93 & 1 & 1 & & 190 & $\bar{Y}$ & $J 91012 \mathrm{~g} 143.30$ \\
\hline 19 & a/23/93 & 1 & 1 & & 1918 & 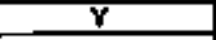 & $\mathrm{j} 910120143-38$ \\
\hline 12 & $8 / 30 / 93$ & $\overline{1}$ & $\overline{1}$ & & 192 & $\overline{\mathbf{Y}}$ & $J 90092 B 741.79$ \\
\hline 12 & $0 / 2 \sqrt{3} 3$ & 1 & 1 & & 191 & $\overline{\mathbf{Y}}$ & J TYPE \\
\hline 18 & $0 / 2 / 83$ & 1 & 1 & & & $\mathbf{N}$ & os \\
\hline 12 & o/der & 1 & 1 & & 100.7 & $\mathbf{Y}$ & J TYPE \\
\hline 19 & ERgas. & 1 & 1 & & & $\mathbf{N}$ & OFs \\
\hline 12 & 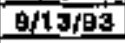 & 1 & 1. & & 192 & $Y$ & JT1097 \\
\hline 19 & 0/1393 & 1 & $y^{2}=$ & & & $\mathbf{N}$ & $0 / \mathrm{B}$ \\
\hline 12 & o/1dya & 1 & 1 & & 191 & $\mathbf{Y}$ & J $910125243-38$ \\
\hline 19 & B/10/83. & $\overline{1}$ & 1 & & & $\mathbf{N}$ & os \\
\hline$\overline{12}$ & 9/20193 & 1 & 1 & & $101+8$ & $Y$ & J $910423540+30$ \\
\hline 19 & 0/20/93 & $\mathbf{1}$ & 1 & & & $\mathbf{N}$ & 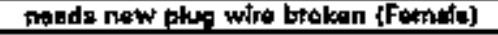 \\
\hline 12 & $9 / 27 / 93$ & 1 & 1 & & 1904 & $\mathbf{Y}$ & J91012自143-38R-180/S \\
\hline 12 & 6/30/93 & 1 & 1 & & 189 & $\mathbf{N}$ & JTYPE \\
\hline 19 & 0/30/93 & 1 & 7 & & 190 & $\boldsymbol{\gamma}$ & \\
\hline 12 & $10 / 4193$ & I & 7 & & 191 & $\mathbf{N}$ & J930126143-39 \\
\hline 18 & $10 / 4 \sqrt{93}$ & 7 & 1 & & 193 & $\boldsymbol{y}$ & \\
\hline 12 & 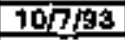 & $\overline{1}$ & 1 & & 189.6 & $\mathbf{N}$ & JE的0423380.42 \\
\hline 18 & $10 / 7 \mathrm{e}^{3}$ & 1 & 1 & & 181.3 & $Y$ & \\
\hline
\end{tabular}

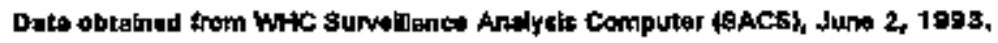


WFCC-\$D-WM-ER-309, FDy. O

\begin{tabular}{|c|c|c|c|c|c|c|c|}
\hline \multicolumn{8}{|c|}{ IANX 241-A-104 } \\
\hline \multicolumn{8}{|c|}{ THEAMOCDUPLE DATA } \\
\hline TAEE $=$ & DATE & THEAMO : & TREE SET \# & TEMP C & TEMP F & $Y=$ MAXX TENP & COHARENG \\
\hline 12 & $10 / 1 \mathrm{k} / 93$ & 1 & 1 & & 189.0 & $\mathrm{~N}$ & 4890423330.42 \\
\hline 18 & $10 / 1+/ 93$ & 1 & 1 & & 191,3 & $y$ & \\
\hline$\overline{12}$ & $10 / 14 / 93$ & 1 & $\overline{1}$ & & 191 & $M$ & TYPE \\
\hline 18 & $10 / 14 / 93$ & $T$ & $\overline{1}$ & & 192 & $\bar{Y}$ & TYPEJ \\
\hline 12 & $10 / 18 / 93$ & $\mathbf{T}$ & 7 & & $19 \bar{B}$ & $\bar{\gamma}$ & J 90092574179 \\
\hline 18 & $10 / 18 / 83$ & $\bar{T}$ & 5 & & 196 & $\bar{N}$ & $J 90082574179$ \\
\hline 12 & $10 / 26 / 93$ & 1 & 1 & & 192 & $\dot{\mathbf{N}}$ & J T1-037 \\
\hline TB & $10 / 26 / 93$ & 1 & $\mathbf{1}$ & & 195 & $\mathbf{Y}$ & J $7 \cdot 037$ \\
\hline $1 \tilde{2}$ & $10 / 28 / 43$ & $\mathbf{1}$ & 1 & & 193.3 & $\mathbf{N}$ & J $610126143-60$ \\
\hline 18 & $10 / 2 \mathrm{~g} / 93$ & 1 & 1 & & 195 & $\bar{Y}$ & $\sqrt{91012014350}$ \\
\hline 22 & $11 / 1 / 93$ & 1 & 2 & & 189.1 & $N$ & $J 7-045 ; 890423320-42$ \\
\hline 19 & $11 / 1 / 93$ & 1 & 1 & & 197.5 & $\mathbf{Y}$ & 3TR-045; 890423320-42 \\
\hline 12 & $11 / 6 / 93$ & 1 & 1 & & $1 \mathrm{Bg} .4$ & $\mathbf{N}$ & ITYPE \\
\hline$\overline{18}$ & $11 / 5 / 5 a$ & 1 & $T$ & & 180.8 & $\mathbf{Y}$ & JTYPE \\
\hline 12 & $71,8 / 93$ & 1 & 1 & & 189.2 & $\mathbf{N}$ & J $1000423320-42$ \\
\hline 18 & $11 / 8 / 93$ & 1 & 1 & & 180.3 & $\dot{Y}$ & J $69042,3320-42$ \\
\hline 12 & $11 / 11 / 93$ & 1 & 1 & & 1日B.9 & $\mathbf{N}$ & 3 TYPE \\
\hline 18 & $11 / 11 / 93$ & 1 & 1 & & 189.5 & $\mathbf{Y}$ & JTYPE \\
\hline 12 & $11 / 1 \mathrm{~B} / 93$ & $i$ & 1 & & $18 \% .2$ & $\mathbf{M}$ & JTYPE \\
\hline 18 & $11 / 15 / 93$ & 1 & 1 & & 189.7 & $\mathbf{Y}$ & ITYPE \\
\hline 12 & $11 / 22 / 93$ & 1 & 1 & & 188.5 & $\mathbf{N}$ & TTYPE \\
\hline 18 & $11 / 22 / 93$ & 1 & 1 & & 189.5 & $\mathbf{Y}$ & J TYPE \\
\hline 12 & $11 / 25 / 93$ & 1 & 1 & & 183 & $\bar{Y}$ & J970126143-50 \\
\hline 18 & $11 / 25 / 93$ & 1 & 1 & & 190 & $\overline{\mathbf{N}}$ & \\
\hline 12 & $1+/ 29 / 93$ & 7 & 1 & & 195.4 & $\mathbf{Y}$ & TYPE J \\
\hline 18 & $11 / 2 \theta / 93$ & 1 & 1 & & 192.8 & $\mathbf{N}$ & \\
\hline 12 & $12 / 2 / 93$ & 1 & 1 & & 18 审 & $\mathbf{A}$ & $J \dot{01012 B 143.50}$ \\
\hline 18 & 121213 & 9 & 1 & & 188 & $\bar{Y}$ & TYPE J \\
\hline 12 & $12 / 8$ 迏 & 1 & 1 & & 191,7 & $\mathbf{N}$ & 581012514350 \\
\hline 18 & $12 / 0 / 03$ & 1 & 1 & & 192.4 & $Y$ & TrPE J \\
\hline 12 & $12 / 9 / 93$ & 1. & 1 & & 189.2 & N & J $8904-23320-42$ \\
\hline 18 & $12 / 9 / 93$ & 1 & 1 & & \$98.3 & $\mathbf{Y}$ & TYPE J \\
\hline 12 & $12 / 13 / 93$ & 1 & 1 & & 587.日 & $\mathbf{N}$ & TrFE J \\
\hline $1 \mathrm{~B}$ & $12 / 13 / 93$ & 1 & 1 & & 798.2 & $\mathbf{Y}$ & TrPE J \\
\hline
\end{tabular}

$$
\therefore:
$$




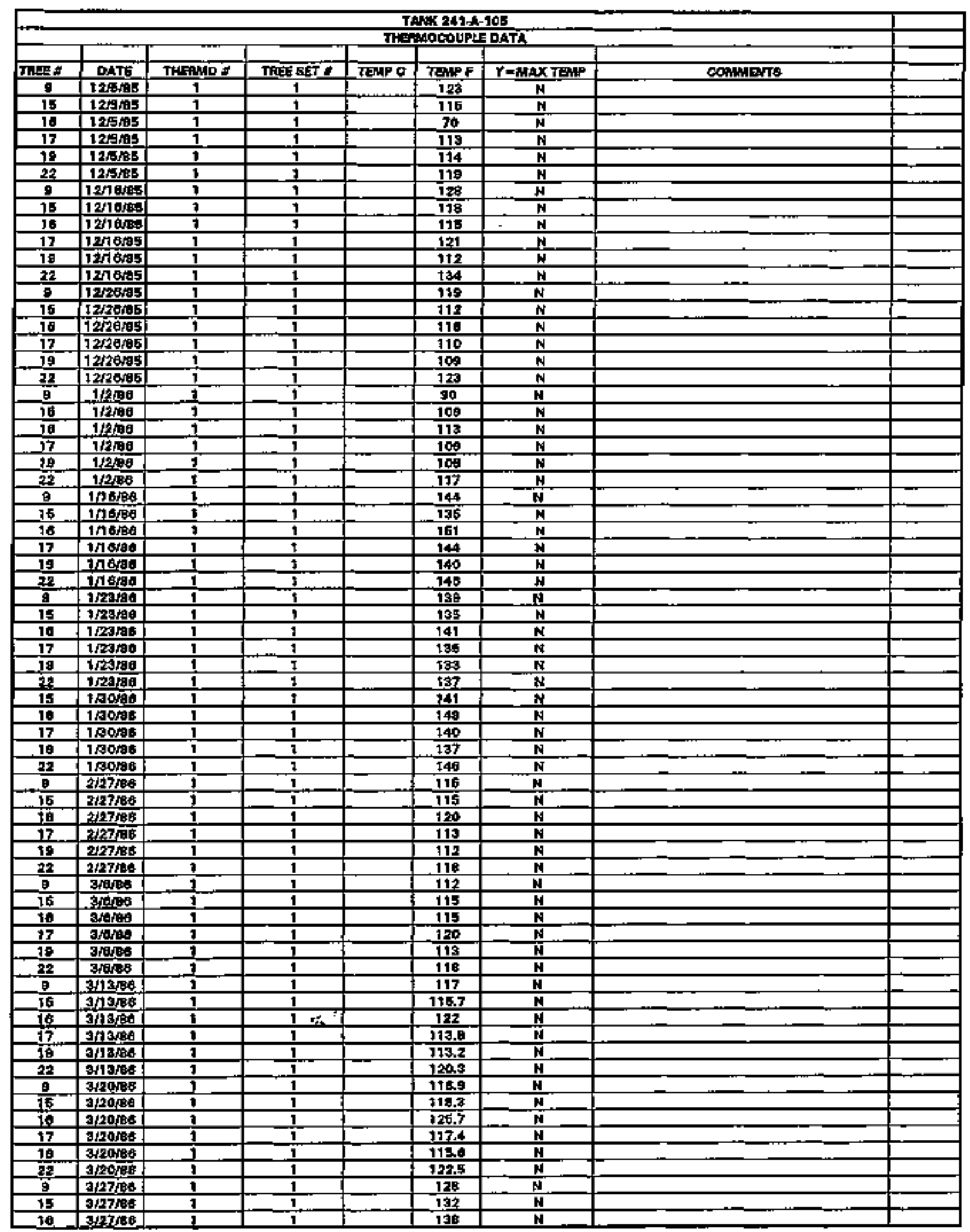




\begin{tabular}{|c|c|c|c|c|c|c|c|c|}
\hline & & & & & NXK11-A & & & \\
\hline & & & & THEF & tocour & DAtA & & \\
\hline & & & & & & & & \\
\hline TAFE \# & DATE & THERMA \# & TAEE 的 \# & Teip $\mathrm{c}$ & TEMPF & $Y=\operatorname{MAX}$ TEIP & COMMENT & \\
\hline 17 & 3/27an & 1 & 1 & & 129 & $\mathbf{N}$ & & \\
\hline 19 & $3 / 27 / 90$ & 1 & 1 & & 220 & $\bar{N}$ & & \\
\hline 27 & $3 / 27 / 95$ & 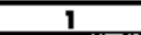 & 1 & & 731 & $\mathbf{N}$ & & \\
\hline 9 & $3 \times 1 / 106$ & $T$ & 1 & & 730 & $\mathbf{N}$ & & \\
\hline 15 & $\$ N 1 / B B$ & 1 & 1 & & $\$ 38$ & $\mathbf{N}$ & & \\
\hline 10 & 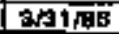 & 1 & 1 & & t98 & N & & \\
\hline 17 & अa1d5 & 1 & 1 & & 130 & $\mathrm{~N}$ & & \\
\hline 19 & 301/9s & 1 & 1 & & 130 & $\bar{N}$ & & \\
\hline 22 & $\$ \$ 31 / 96$ & 1 & 1 & & 731 & $\bar{N}$ & & \\
\hline 16 & 410 며 & 1 & 1 & & 107 & N & & \\
\hline 16 & $410 \mathrm{det}$ & 1 & 1 & & 108 & $\mathbf{N}$ & & \\
\hline 17 & 4/10/es & 1 & 1 & & 107 & $\mathbf{N}$ & & \\
\hline 10 & $4 / 10$ is & $\overline{1}$ & $\overline{1}$ & & 100 & $N$ & & \\
\hline 15 & $4 / 1 5 \longdiv { 1 0 6 }$ & $T$ & 1 & & 72 & $\mathbf{N}$ & & \\
\hline 18 & $4 / 17 \overline{86}$ & 1 & 1 & & 73 & $\mathbf{N}$ & & \\
\hline 17 & 413 & 1 & 1 & & 72.4 & $\mathbf{N}$ & & \\
\hline 10 & 4/17Bg & 1 & 1 & & 73 & N & & \\
\hline 22 & $4 / 17 / 6 \mathrm{~s}$ & 1 & 1 & & 72.2 & $\mathbf{N}$ & & \\
\hline$\theta$ & 42400 & 1 & 7 & & 62.8 & $n$ & & \\
\hline 15 & $4 \sqrt{24 / 86}$ & 5 & 1 & & 119,7 & $\mathbf{N}$ & & \\
\hline 18 & $4 / 24 \sqrt{80}$ & 1 & 1 & & 93,2 & $\mathbf{H}$ & & \\
\hline 17 & $4 / 24 / 80$ & $\dot{i}$ & 1 & & 110.9 & $N$ & & \\
\hline 19 & $4 / 24 / 60$ & $\$$ & 1 & & 110.4 & $\dot{\mathbf{N}}$ & & \\
\hline 22 & $4 / 24 / 26$ & 1 & 1 & & 117 & $\dot{N}$ & & \\
\hline $\mathbf{q}$ & E/Ase & 1 & 1 & & 70 & $\mathbf{N}$ & & \\
\hline 17 & Endros & 7 & 1 & & 127 & $\mathbf{M}$ & & \\
\hline 17 & $\sin \overline{8} \theta$ & $t$ & 1 & & 127 & $\mathbf{N}$ & & \\
\hline 19 & 5 MIBA & $i$ & 1 & & $11 \mathrm{~B}$ & $\mathbf{N}$ & & \\
\hline 22 & Shlose & 1 & 1 & & 126 & $\mathrm{~N}$ & & \\
\hline $\mathbf{g}$ & 다B:日ed & 1 & 1 & & 72 & $\mathbf{N}$ & & \\
\hline 16 & 6rEte6 & 1 & 1 & & 127 & $\mathbf{N}$ & & \\
\hline 10 & $5 \mathrm{~s}+16$ & 7 & 1 & & 128 & $\bar{N}$ & & \\
\hline 17 & 5 sotgo & 1 & 1 & & 120 & $\mathbf{N}$ & & \\
\hline 19 & 5 thenta & 1 & 1 & & $11 B$ & $\mathbf{N}$ & & \\
\hline 27 & ENetas & 1 & $\overline{1}$ & & 110 & $\mathbf{N}$ & & \\
\hline 9 & 5n2/56 & 1 & 1 & & $\theta 6$ & $\boldsymbol{M}$ & & \\
\hline 16 & 5n12) & 1 & $T$ & & 113 & $\mathbf{H}$ & & \\
\hline 10 & जयू日 & 1 & 1 & & 181.8 & $\mathrm{H}$ & & \\
\hline 17 & 51290 & 1 & 1 & & 121.5 & $\mathbf{N}$ & & \\
\hline 10 & F/12Bst & 1 & 1 & & 157.1 & $\bar{N}$ & & \\
\hline 22 & SIIRE & 1 & 1 & & BE & $\mathbf{N}$ & & \\
\hline 9 & 하15isos & 1 & 1 & & 71.2 & $N$ & & \\
\hline 15 & Silsib & 1 & i & & 1203 & $\bar{M}$ & & \\
\hline 16 & SN15Bs & 1 & 1 & & 121 & $\bar{N}$ & & \\
\hline 17 & S小15施它 & 1 & 1 & & 119.3 & $\$$ & & \\
\hline 10 & EnBSBE & 1 & 1 & & 118.3 & N & & \\
\hline 22 & 5N15/BE & 1 & 1 & & 124 & $N$ & & \\
\hline 오 & Extloss & 1 & 1. & & 120 & $\mathbf{M}$ & & \\
\hline 15 & Snd & 1. & 1 & & 124 & $\mathrm{~N}$ & & \\
\hline 16 & कnossts & 1 & 1 & & 92 & $\mathbf{N}$ & & \\
\hline 17 & \begin{tabular}{|l|l|} 
SIOUSE \\
\end{tabular} & 1 & 1 & & 86 & $\mathbf{N}$ & & \\
\hline 18 & Flipas & 1 & 1 & & 69 & $\mathbf{N}$ & & \\
\hline 22 & \begin{tabular}{|l|l|}
$5 / 1968$ \\
\end{tabular} & 1 & 1 & & $12 \%$ & $\mathbf{N}$ & & \\
\hline 9 & 5/2248g & 1 & 1 & & & $\mathbf{N}$ & 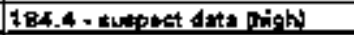 & \\
\hline 15 & B22 & 1 & 1 & & 121.5 & $A$ & & \\
\hline 78 & FH22E: & 1 &. & & 92.5 & $\mathbf{N}$ & & \\
\hline 10 & 5/22158 & 1 & 1 & & $11 \mathrm{BA}$ & 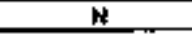 & & \\
\hline 22 & EL22 & 1 & 1 & & 127.3 & $\mathbf{N}$ & & \\
\hline 童 & $5 \sqrt{20} 186$ & 1 & 1. & & 115 & $N$ & & \\
\hline 15 & 52Ad祭 & 1 & 1 & & 120 & $\mathbf{N}$ & & \\
\hline 15 & Stangs & 1 & 1 & & 49 & $\mathbf{N}$ & & \\
\hline 17 & Whougts & 1 & 1 & & 70 & $\mathbf{N}$ & & \\
\hline 10 & Wo/kst & 1 & 1 & & 80 & $\mathbf{N}$ & & \\
\hline 32. & ENस6rbs & 1 & 1 & & 132 & $\bar{n}$ & & \\
\hline 8 & ersis & $i$ & 1 & & as.7 & $\bar{N}$ & & \\
\hline$\$ 5$ & $1 5 \longdiv { B 8 }$ & 1 & 1 & & 125.3 & $\bar{F}$ & & \\
\hline 76 & Affiteg & 1 & 1 & & 132.3 & $\mathbf{H}$ & & \\
\hline 17 & d5/ES & 1 & 1 & & 1757 & $\mathbf{H}$ & & \\
\hline
\end{tabular}




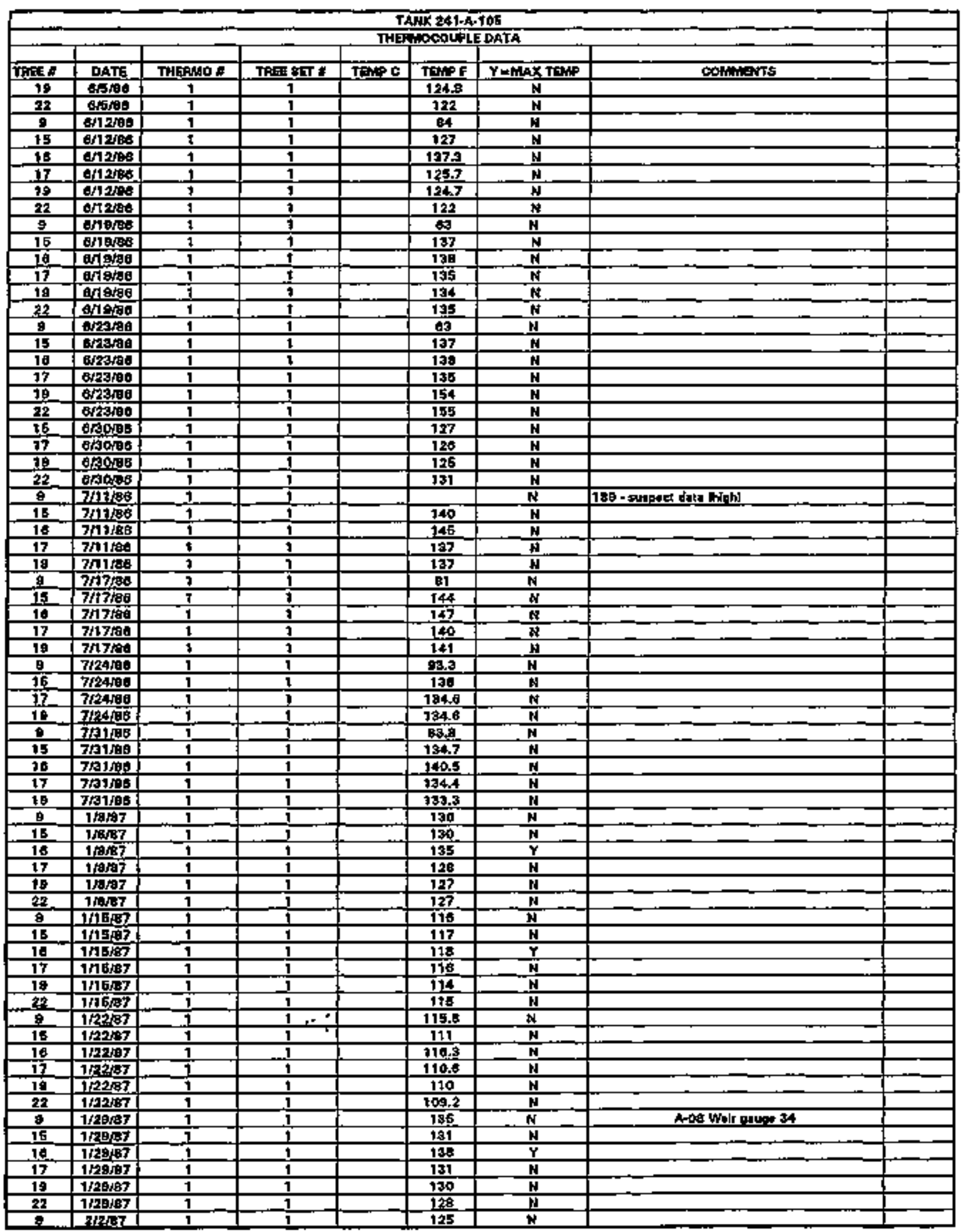




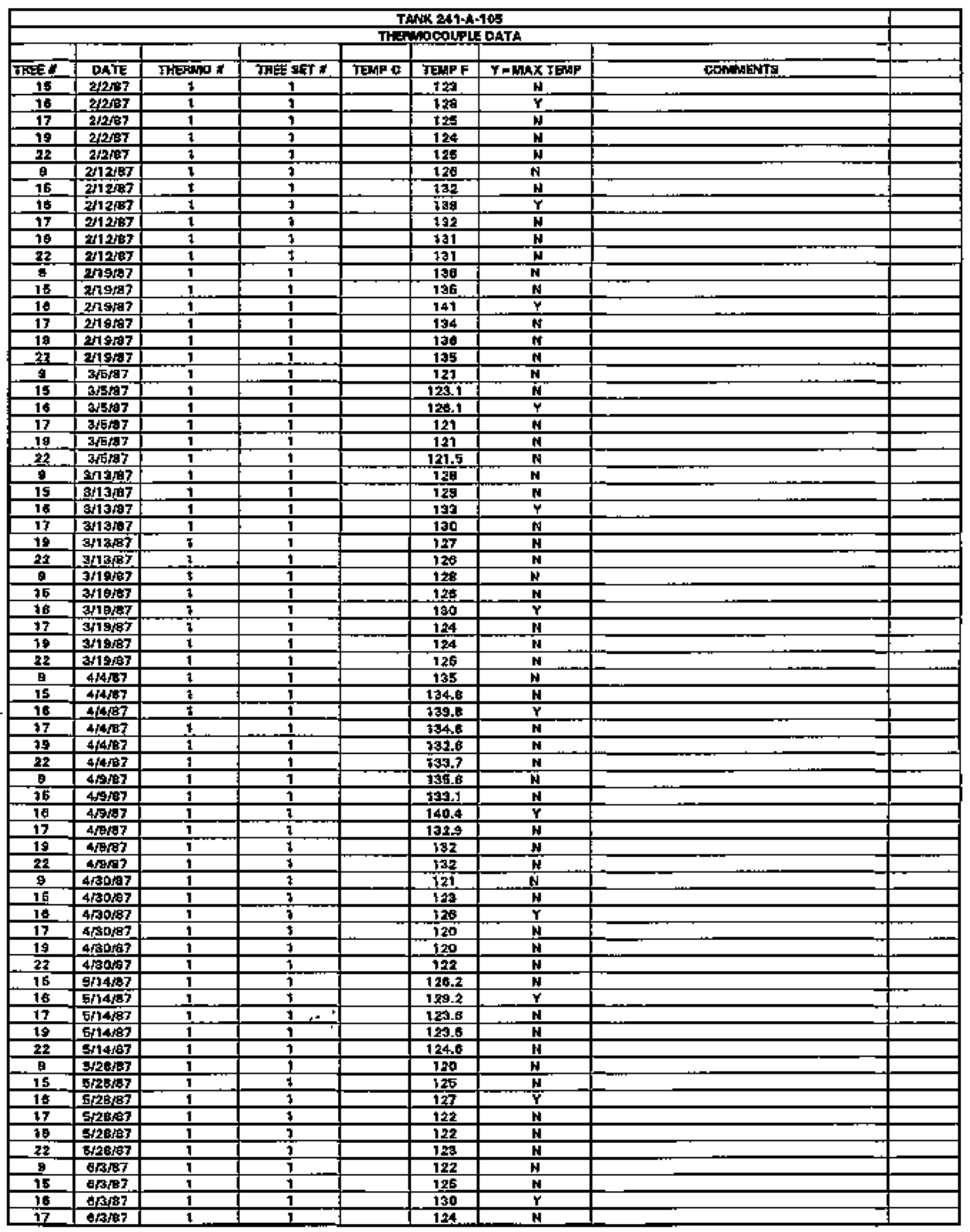




\begin{tabular}{|c|c|c|c|c|c|c|c|c|}
\hline \multicolumn{9}{|c|}{ TANK 241-A-t05 } \\
\hline & & & & THF & DOCONPL & DATA & & \\
\hline Trix & DATE & THEFiMO & 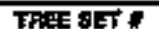 & TERPC C & Exp F & $Y=A A X$ TIP & COMHATS & \\
\hline 10 & $0 \sqrt{387}$ & 1 & $t$ & & 124 & $M$ & & \\
\hline 72 & ABR & $t$ & 1 & & 127 & $\mathbf{N}$ & & \\
\hline 8 & artide & 1 & 1 & & 120.3 & N & 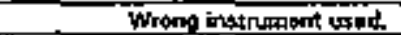 & \\
\hline E & et1日a & 1 & 1 & & 124.2 & E & Whong instrument used & \\
\hline 10 & fill $1 / 97$ & 1 & 1 & & 130.7 & $\bar{Y}$ & Wrong hot rurfent usud. & \\
\hline 17 & 6ी1187 & 1 & 1 & & .734 .2 & $\mathbf{M}$ & 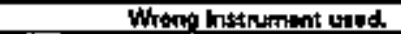 & \\
\hline 19 & Onlw & 1 & 1 & & 123.4 & in & Wrope instruffemt ured. & \\
\hline $2 \overline{23}$ & B11/57 & 1 & 1 & & 125.6 & W & 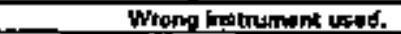 & \\
\hline$g$ & \$19/67 & 1. & 1 & & \pm 25 & $\mathbf{N}$ & & \\
\hline 15 & $6719 / B 7$ & $\overline{1}$ & 1 & & $12 \mathrm{~B} .8$ & $\overline{\mathbf{N}}$ & & \\
\hline 16 & t/19/B7 & 1 & 1 & & 133,4 & $\bar{Y}$ & & \\
\hline 17 & G19B7 & 1 & 1 & & 128 & $\mathbf{M}$ & & \\
\hline 10 & ON19s7 & 1 & 1 & & 127.3 & $\mathbf{M}$ & & \\
\hline 27 & angar & 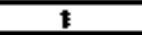 & 1 & & 127.2 & $\mathbf{N}$ & & \\
\hline 9 & 마당ㄱ & 1 & 1 & & 125 & $\mathbf{M}$ & & \\
\hline TE & EDESET & 1 & 1 & & 130 & $\mathbf{N}$ & & \\
\hline 18 & OAPEST & 1 & I & & 139 & $\bar{y}$ & & \\
\hline I7 & B/25887 & 7 & $\mathbf{t}$ & & 130 & N & & \\
\hline 8 & $6 / 2687$ & 7 & $t$ & & 125,4 & N & & \\
\hline 22 & 6/2B:7 & 1 & i & & 124.7 & N & & \\
\hline 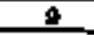 & $72 \pi 97$ & 1 & 1 & & 116 & $\mathbf{F}$ & & \\
\hline 15 & $7 / 2 k 97$ & 1 & $\overline{1}$ & & 119.4 & 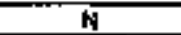 & & \\
\hline 17 & 72497 & 1 & 1 & & 116.4 & i & & \\
\hline 19 & $7 / 2 h 7$ & 1 & 1 & & $11 \overline{7.2}$ & in & & \\
\hline 22 & 7 7era 7 & 1 & 1 & & 120 & $y$ & & \\
\hline 9 & 710/97 & 1 & 1 & & 122 & $N$ & & \\
\hline 15 & 7/10/97 & 1 & 1 & & 124 & $\mathbf{H}$ & & \\
\hline 10 & $7 / 10 / 97$ & 1 & 1 & & 128 & $\mathbf{N}$ & & \\
\hline 17 & $710 \mathrm{~g}$ & 1 & 1 & & $12 \overline{33}$ & N & & \\
\hline 19 & 710/柏. & 1 & J_ & & 123 & N & & \\
\hline 22 & 7/10/87 & 1 & 1 & & 124 & W & & \\
\hline 15 & $7 / 23 / \mathrm{BP}$ & 1 & 1 & & 124 & $\mathbf{N}$ & & \\
\hline 10 & $7 / 23 / 45$ & 1 & 1 & & 130 & $Y$ & & \\
\hline 12 & $7 / 23 / 87$ & 1 & 1 & & 124 & $\mathbf{N}$ & & \\
\hline 18 & 7/23187 & 1 & 1 & & $1 \overline{23}$ & $\bar{N}$ & & \\
\hline 22 & $7 / 2 \mathrm{~s} / \mathrm{ar}$ & $t$ & 1 & & \pm 2 & N & & \\
\hline 15 & 7 sowil7 & 1 & 1 & & 128 & N & & \\
\hline 10 & 7 30067 & 1 & 5 & & 134 & $\mathbf{Y}$ & & \\
\hline 17 & 7 \%оH? & 1 & 1 & & 129 & $M$ & & \\
\hline 19 & 28B97 & 1 & 1 & & 129 & $M$ & & \\
\hline 22 & 780.7 & $\frac{1}{1}$ & 1 & & 130 & $N$ & & \\
\hline 9 & 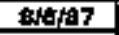 & 1 & 1 & & 100 & $\mathbf{N}$ & & \\
\hline 15 & Beta7 & 1 & 1 & & 109 & $N$ & & \\
\hline 10 & areta7 & 1 & 1 & & 120 & 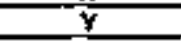 & & \\
\hline 17 & Affrta7 & 1 & 7 & & 115 & $N$ & & \\
\hline 19 & 98B:87 & 1 & \pm & & 115 & $N$ & & \\
\hline 22 & SNBt97 & 1 & 5 & & 110 & $\mathbf{N}$ & & \\
\hline$\theta$ & 6113a7 & 1 & 1 & & 123 & $\mathbf{N}$ & & \\
\hline 15 & Biata7 & 1 & 1 & & 125 & $\mathbf{M}$ & & \\
\hline 17 & Bл13/97, & 1 & 1 & & 196 & $\mathbf{N}$ & & \\
\hline 19 & M13ts? & 1 & 1 & & 124 & $\mathbf{N}$ & & \\
\hline 22 & B/13/a7 & 1 & 1 & & 120 & $r$ & & \\
\hline 15 & Emojer & 1 & 1 & & 123 & $\bar{N}$ & & \\
\hline 10 & Btzorat & 1 & 1 & & 128 & $Y$ & & \\
\hline 17 & B120197 & 1 & 1 & & 122 & $\mathbf{N}$ & & \\
\hline 18 & BRoba & 1 & $1, x^{2}$ & & 122 & $\mathbf{N}$ & & \\
\hline 22 & Brata 7 & 1 & 1 & & 123 & $\mathbf{N}$ & & \\
\hline 9 & 6/27/97 & 1 & 1 & & 135 & K & & \\
\hline 16 & B/27/07 & 1 & 1 & & 128 & $N$ & & \\
\hline 16 & Bat27s? & 1 & 1 & & 131 & $\mathbf{Y}$ & & \\
\hline 17 & B[?]伯? & 1 & 1 & & 125 & N & & \\
\hline 19 & в/27/a7 & 1 & 1 & & 125 & $\mathbf{N}$ & & \\
\hline 22 & Bn:a7 & 1 & 1 & & 126 & N & & \\
\hline 9 & $0 / 21 / 97$ & 1 & 7 & & 137.2 & $\mathbf{H}$ & & \\
\hline 16 & Bk1/97 & $\sqrt{1}$ & 1 & & 125.0 & $\mathbf{N}$ & & \\
\hline 10 & 바눅 & 1 & 1 & & 131.5 & $\mathbf{Y}$ & & \\
\hline 17 & $0 / 21 / 97$ & 1 & 1 & & 120.4 & $\overrightarrow{\mathbf{M}}$ & & \\
\hline 10 & $9 / 21 / 97$ & 1 & 1 & & $12 \overline{4.1}$ & $N$ & & \\
\hline
\end{tabular}




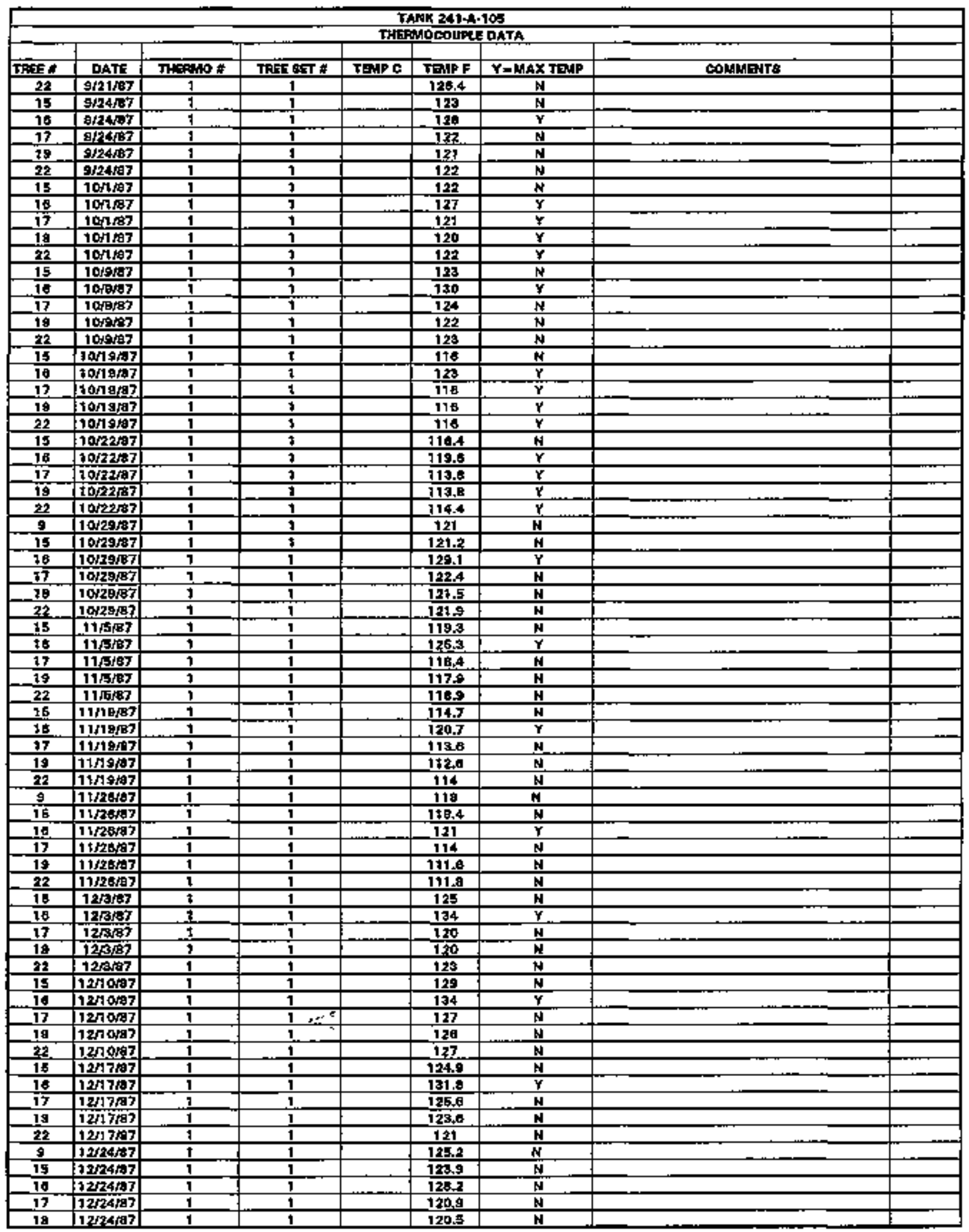




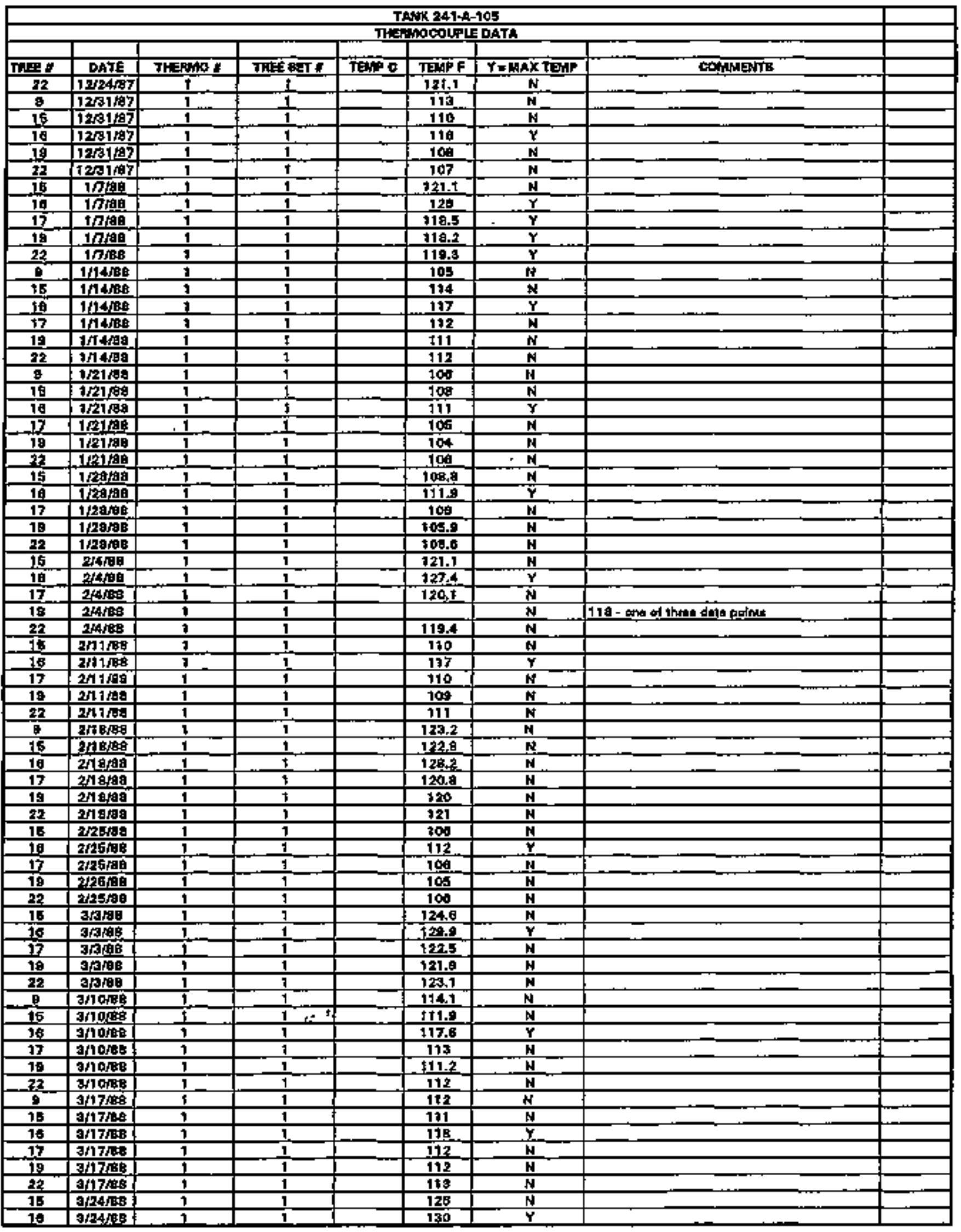

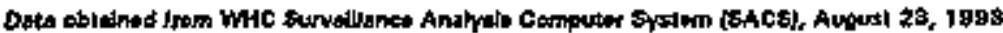




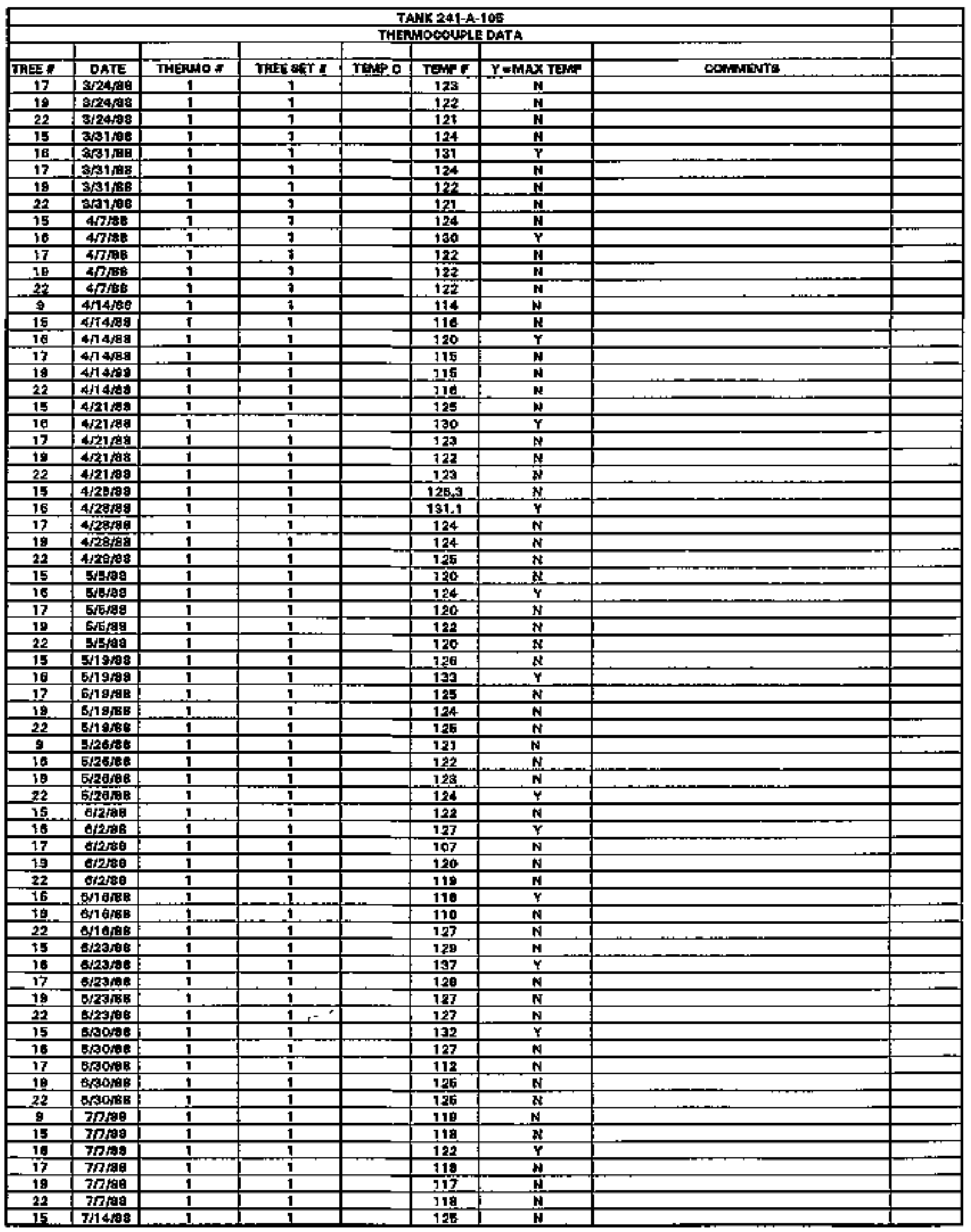




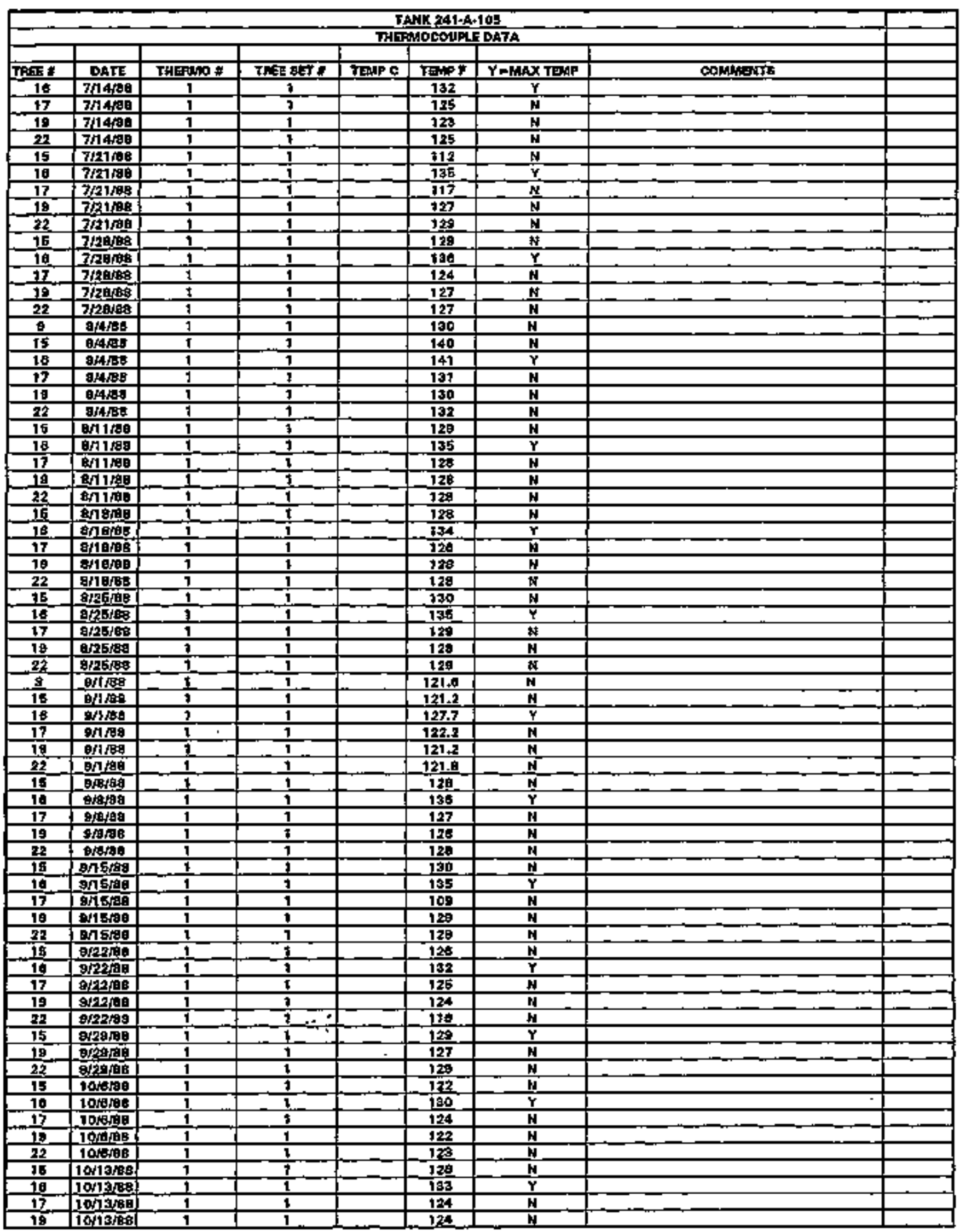




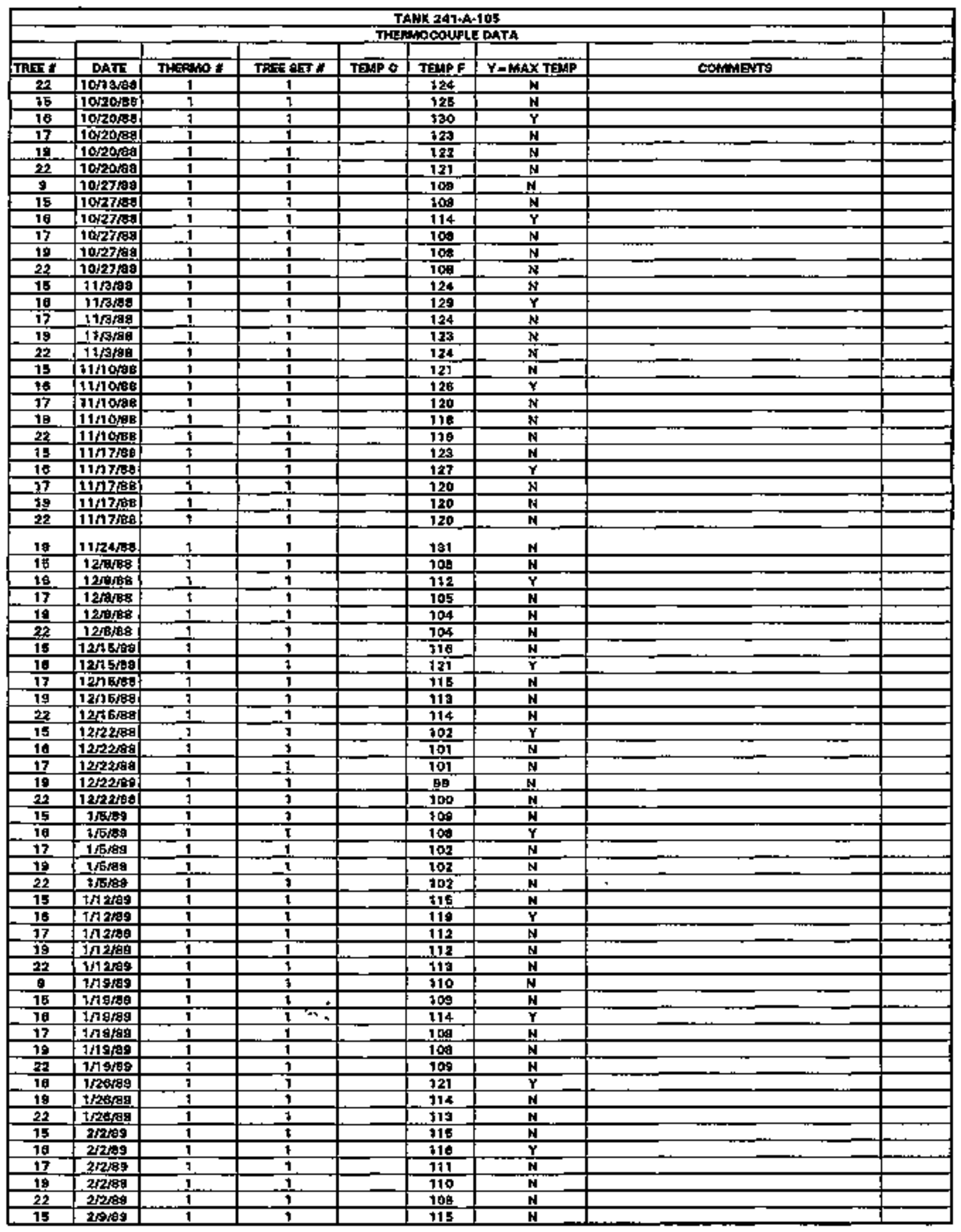




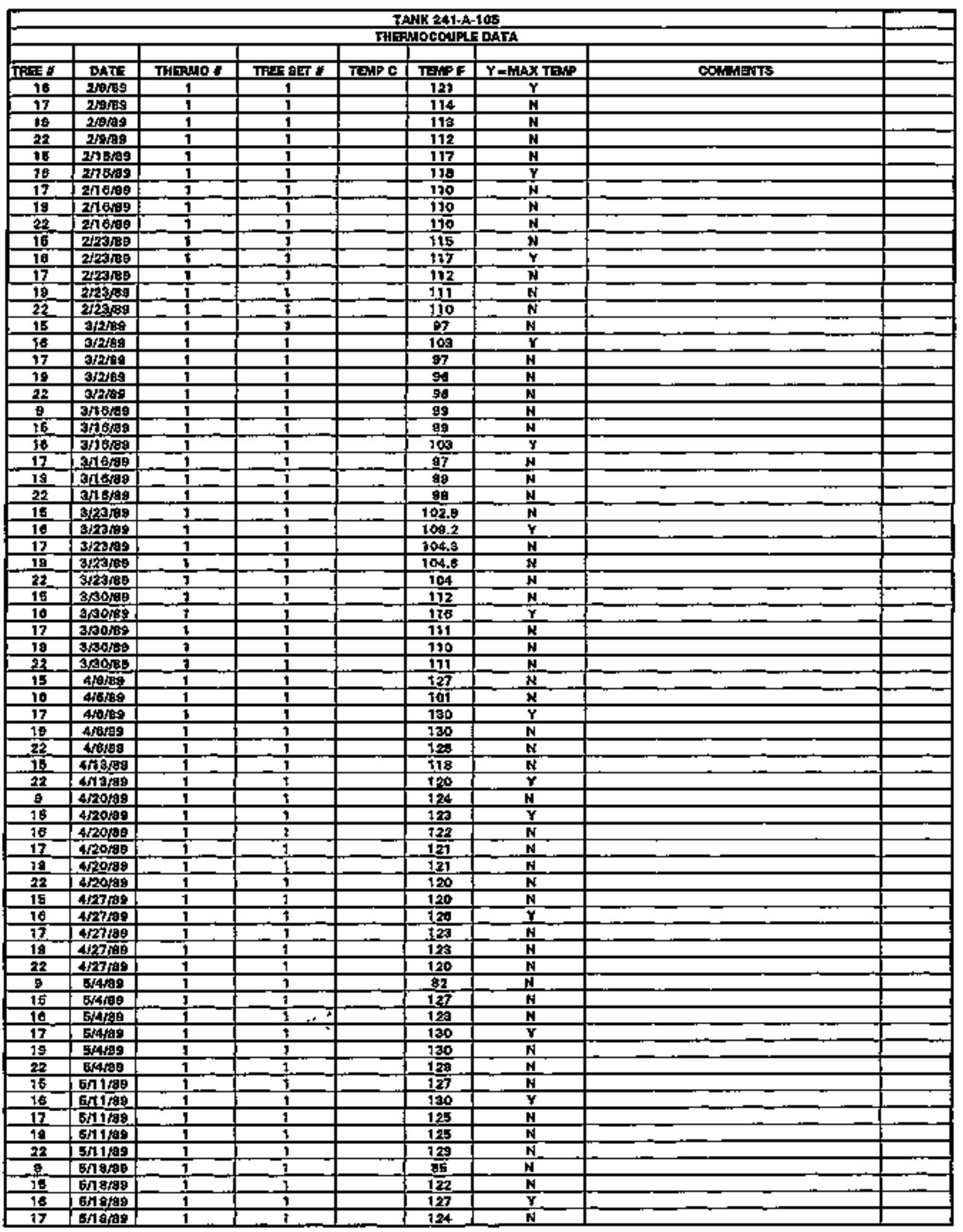

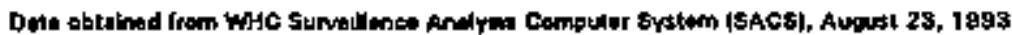




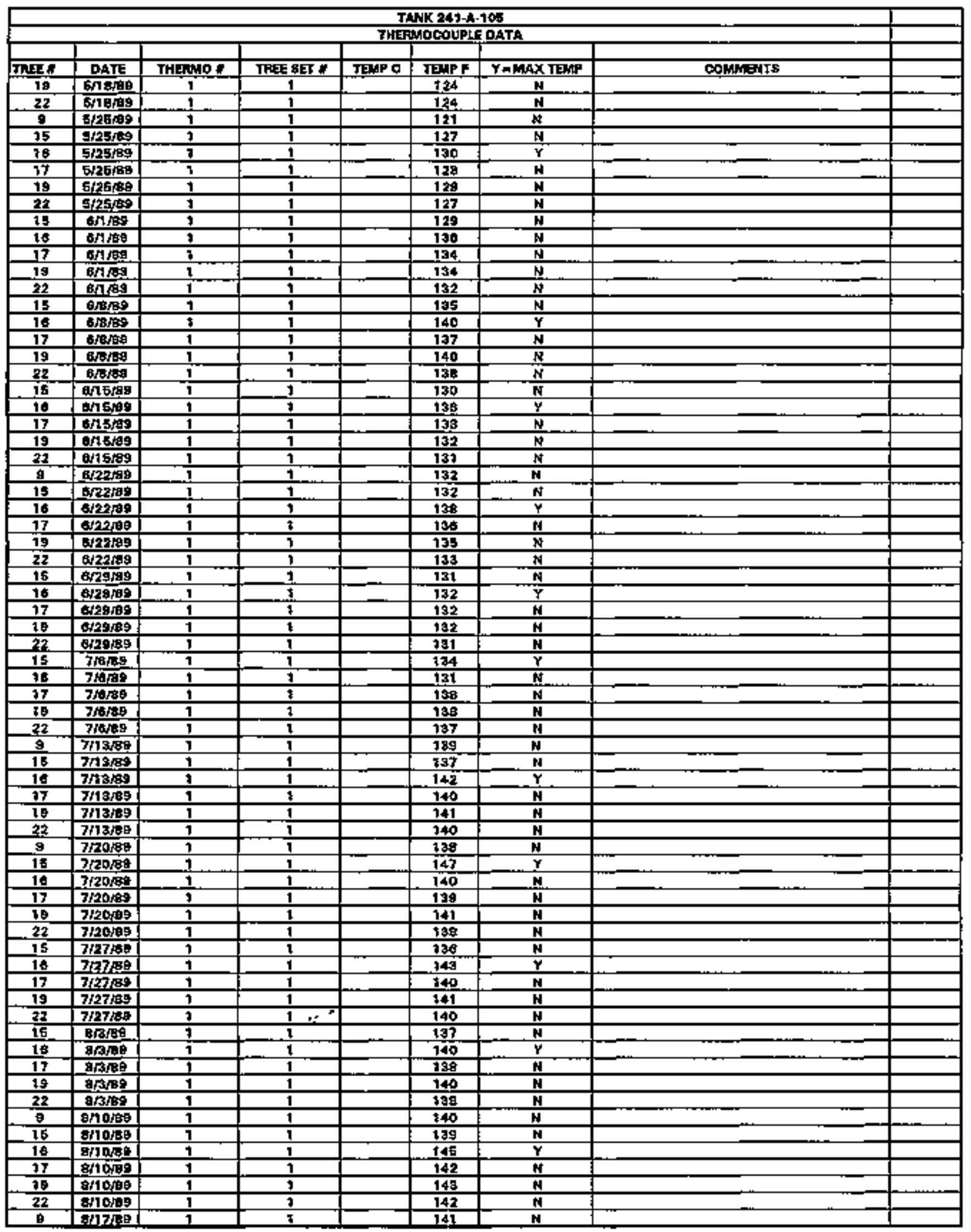




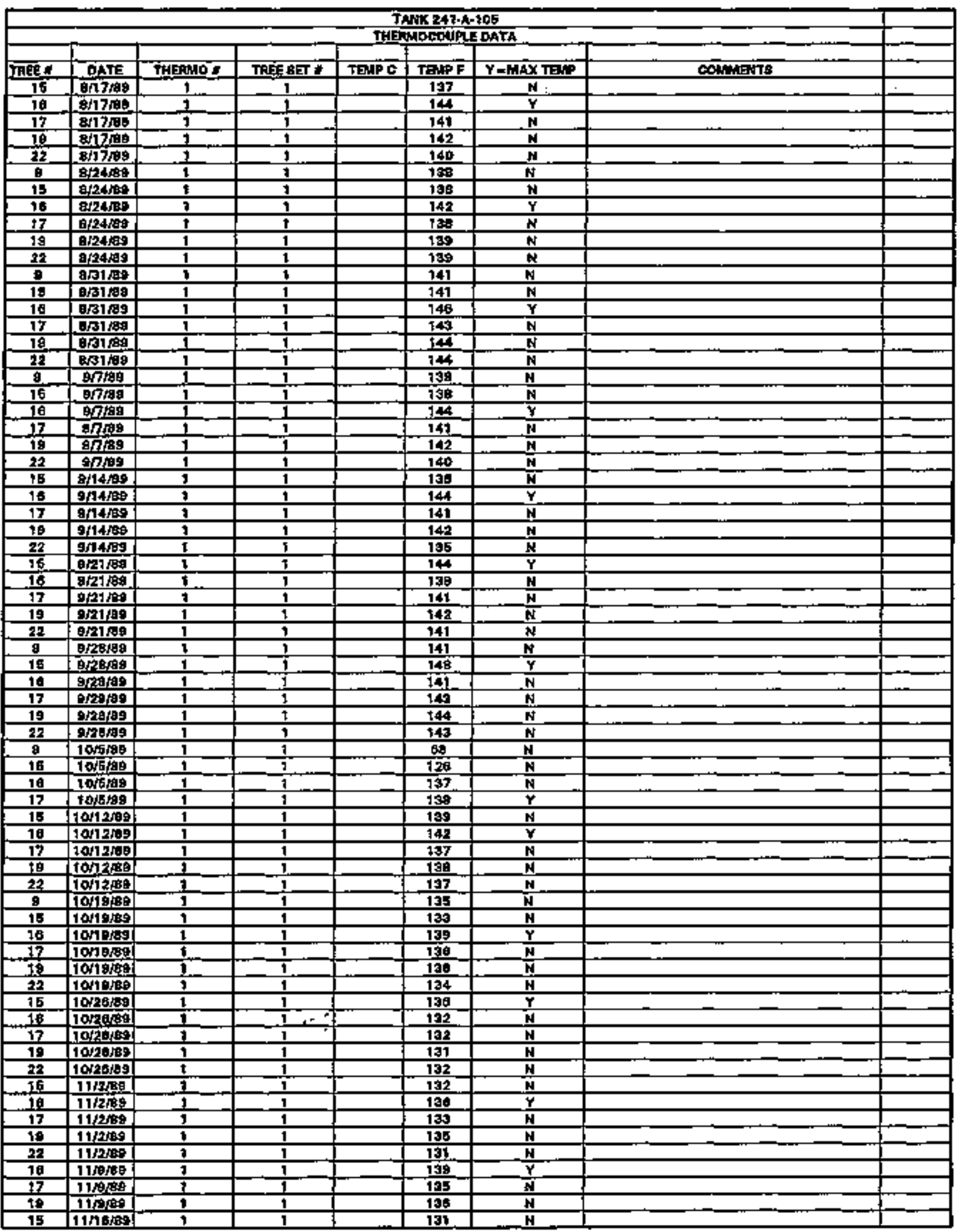




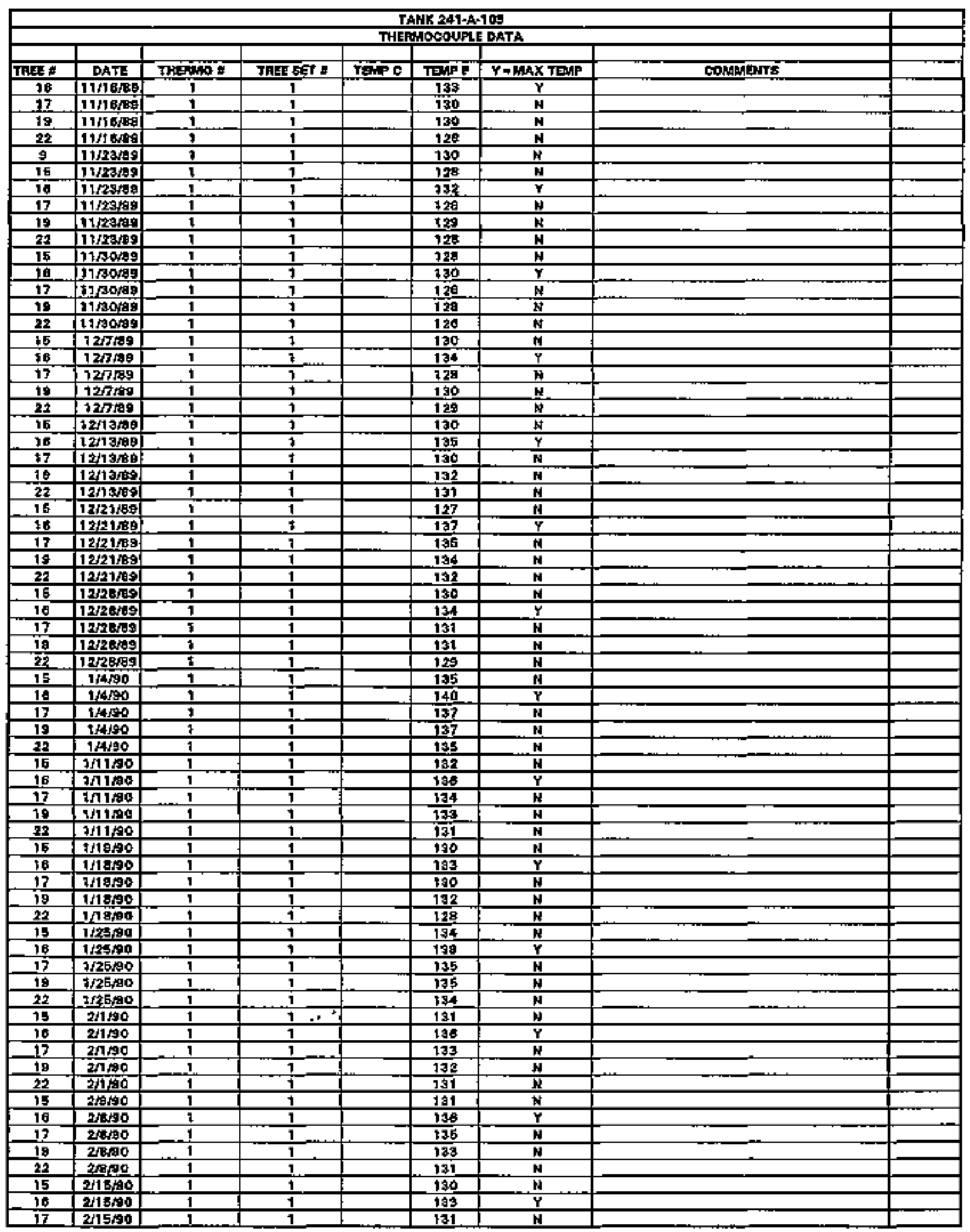




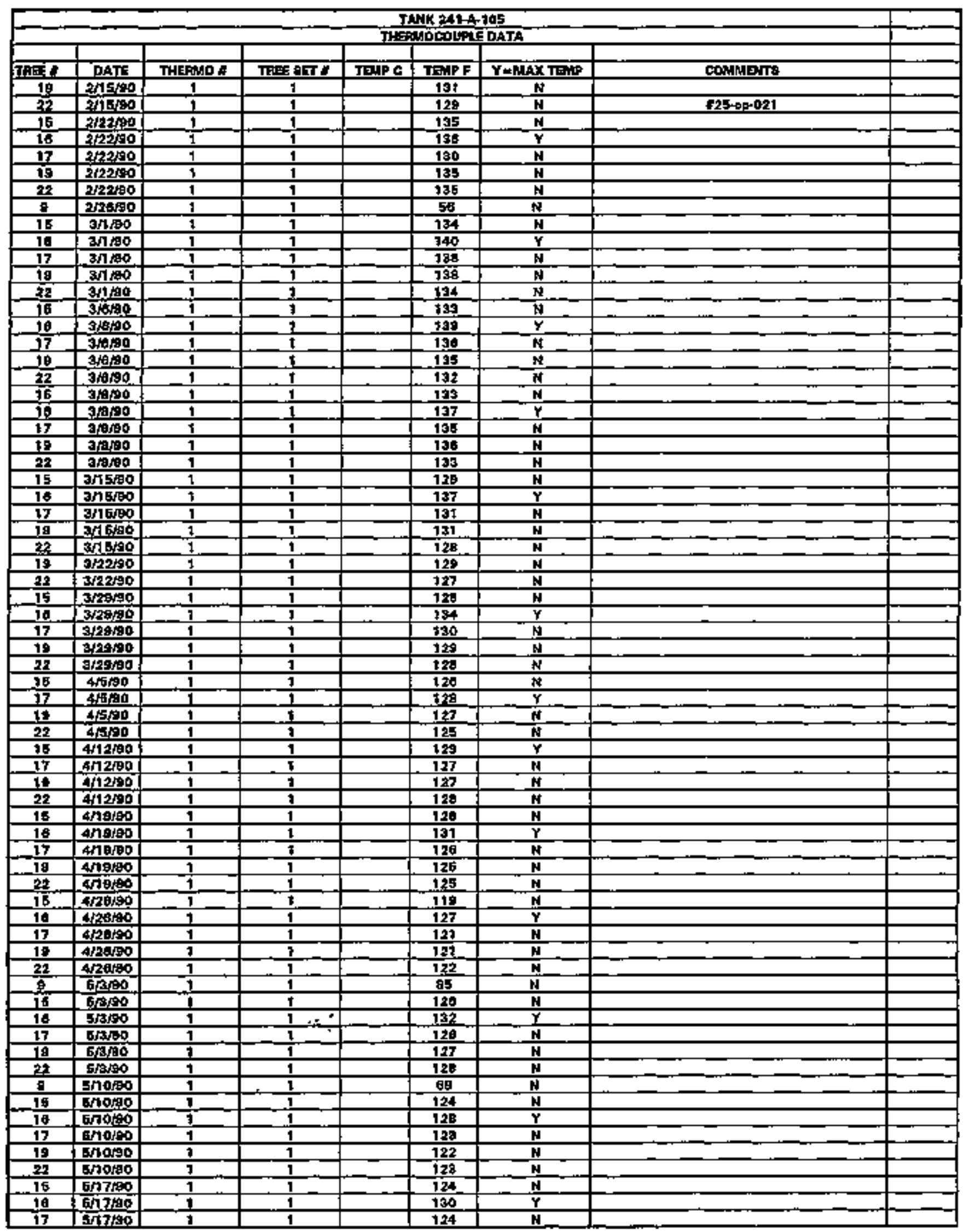




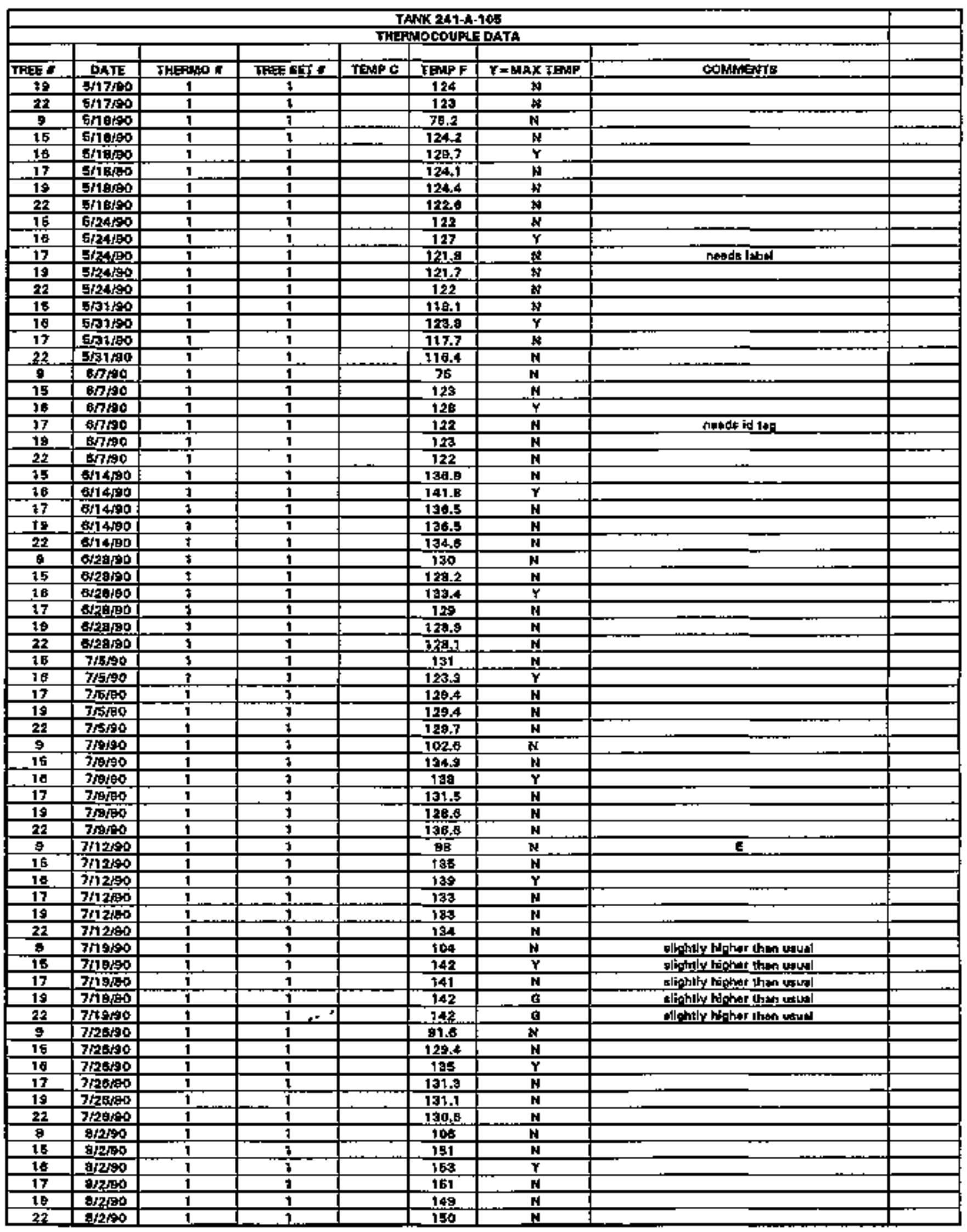




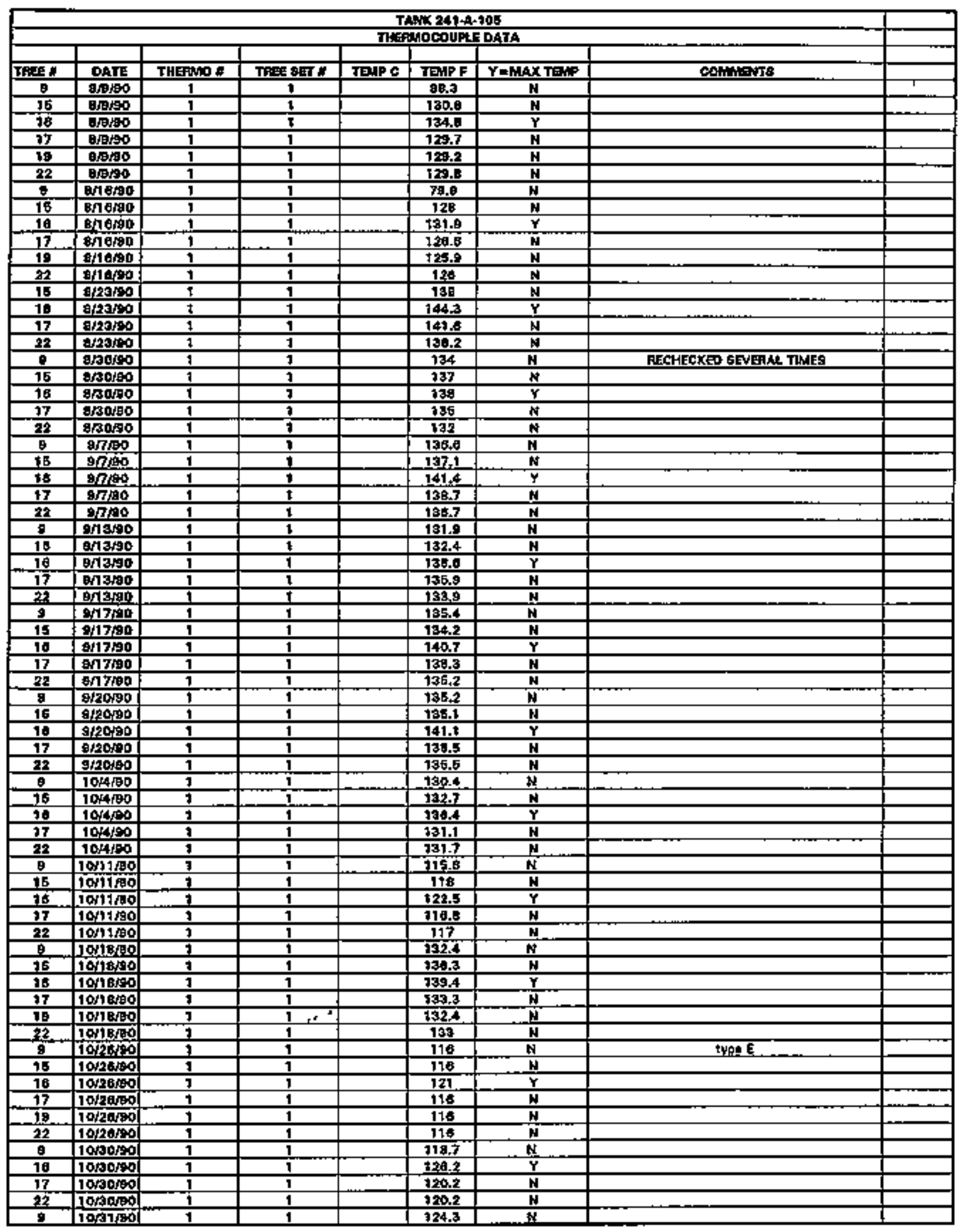




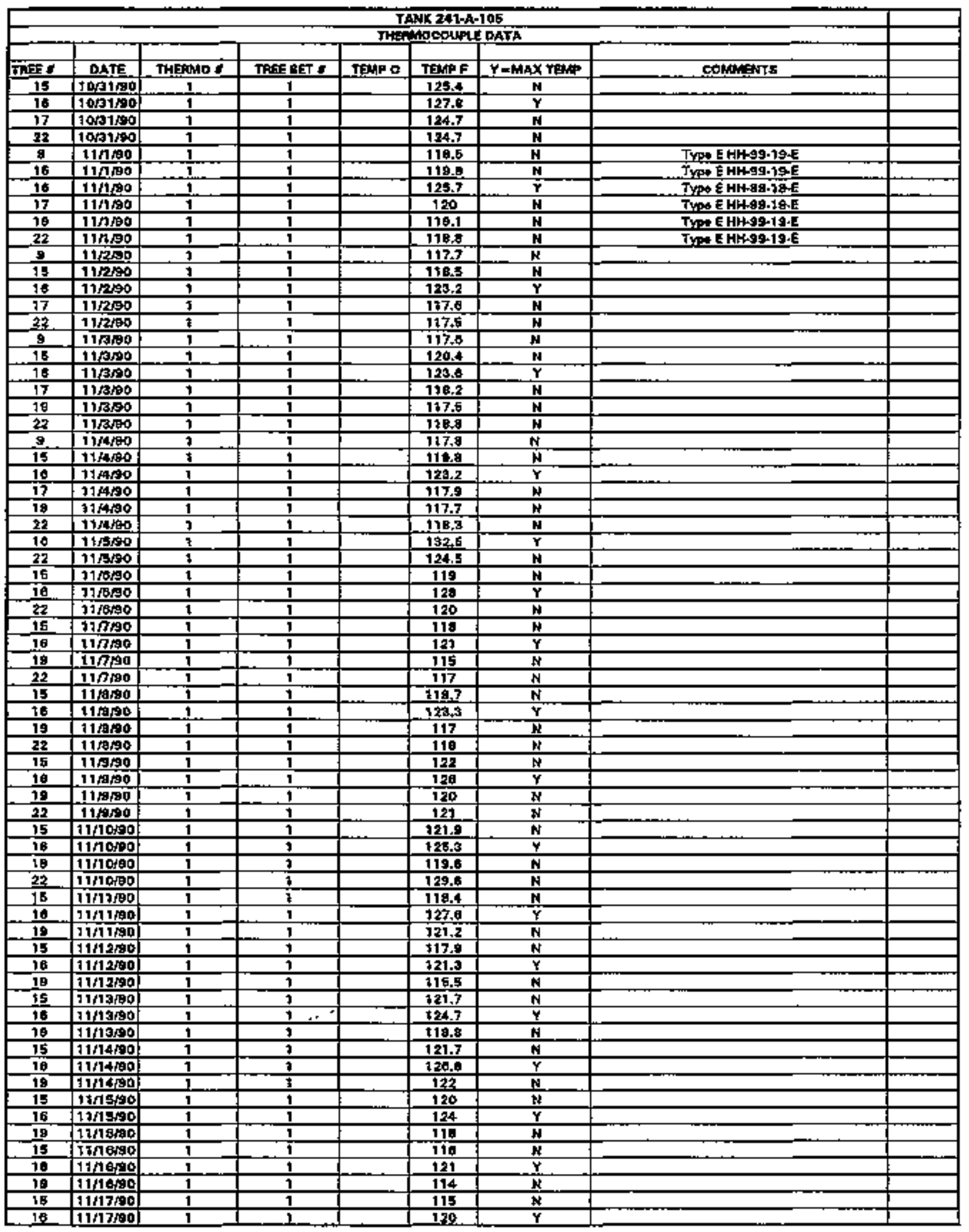




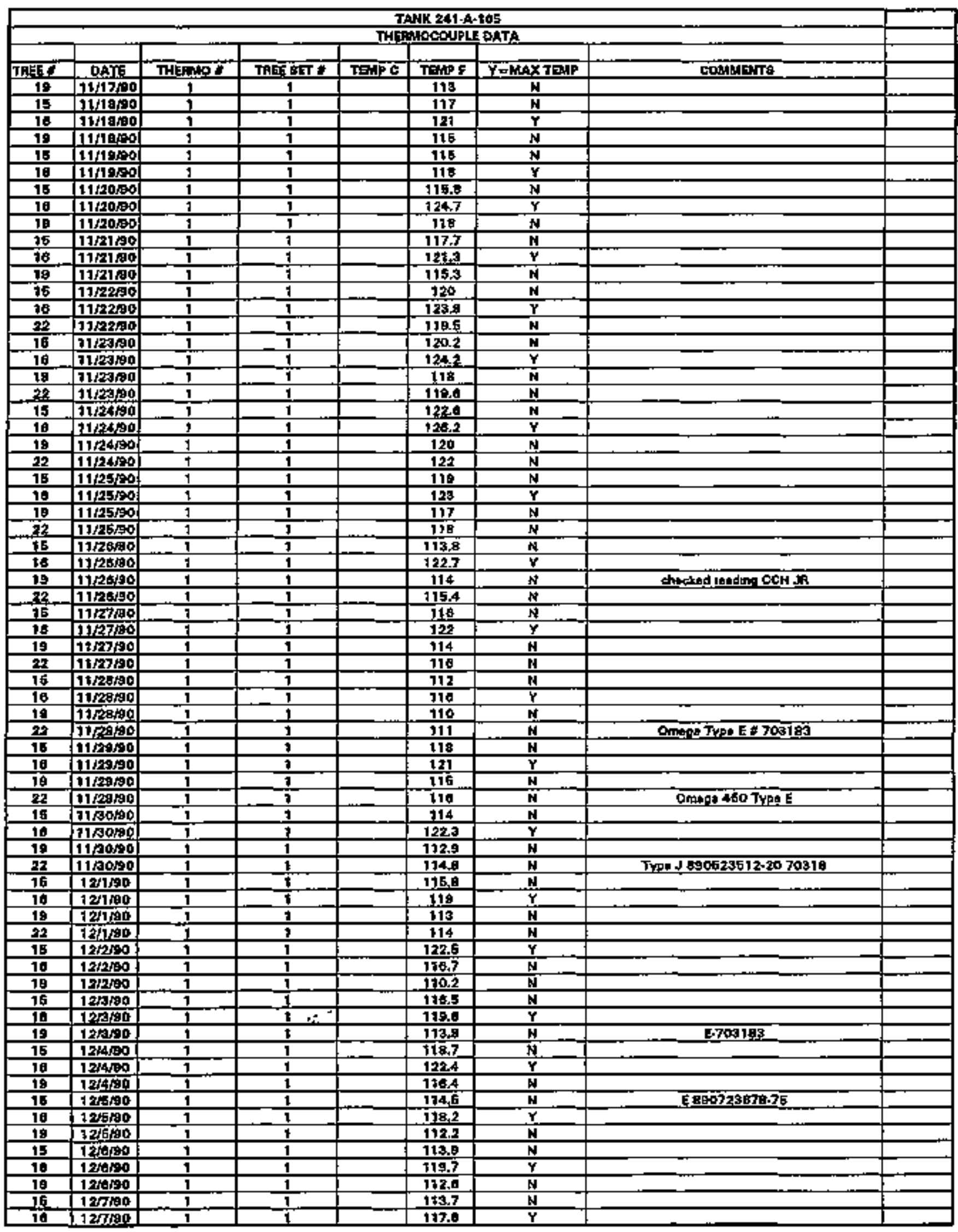




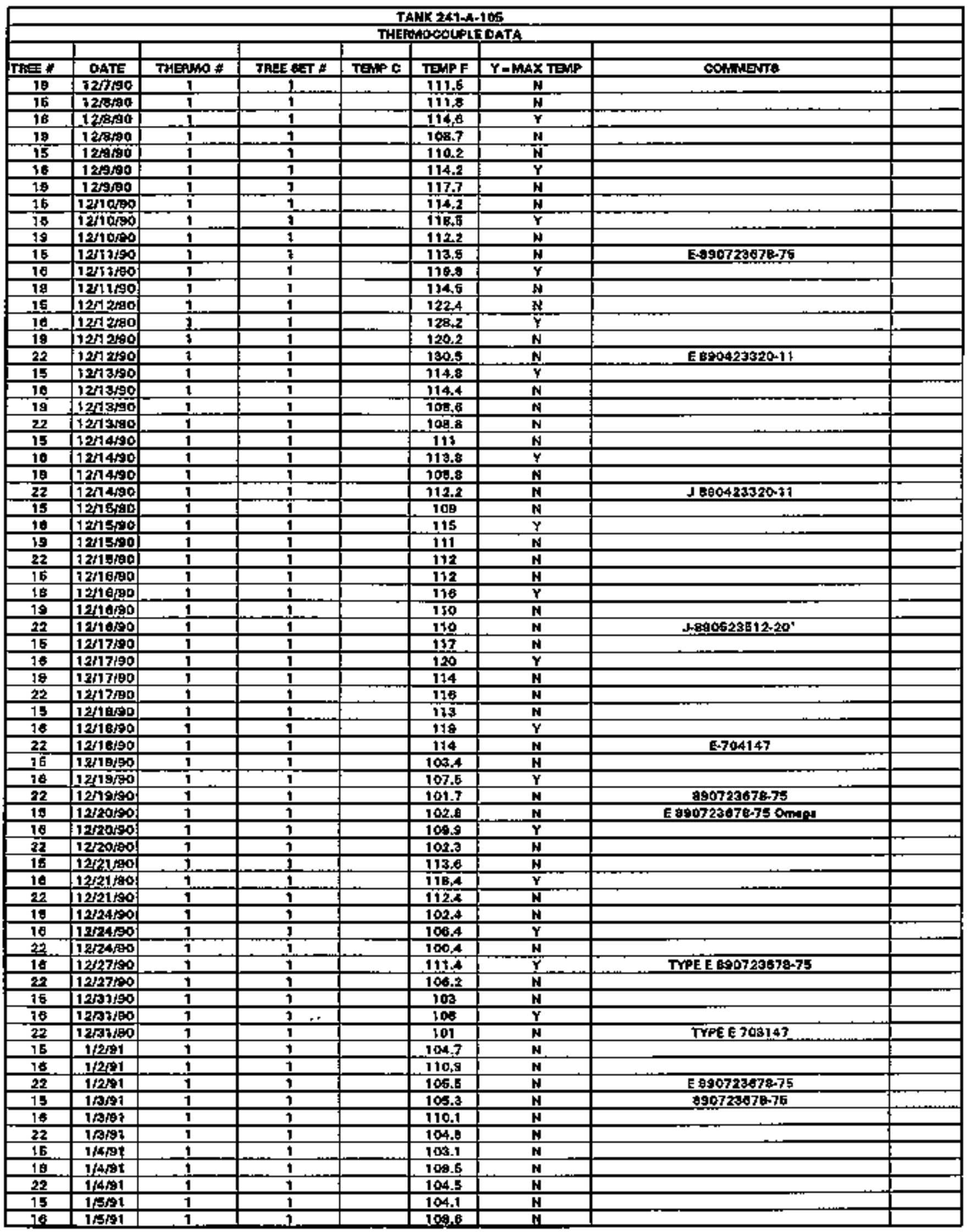




\begin{tabular}{|c|c|c|c|c|c|c|c|c|}
\hline \multicolumn{9}{|c|}{ TANK 241-A.105 } \\
\hline & & & & FHA & PCoplot & DATA & & \\
\hline TAEE & $\overline{\mathrm{DATE}}$ & 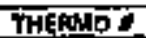 & TAEF的早 & Tento 0 & TEAPEF & $Y=\overline{M A} \times$ TEAN & DONDAgarts & \\
\hline 22 & $\sqrt{6 / 21}$ & 1 & 1 & & 1002 & N & & \\
\hline 16 & t商的 & 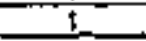 & 1 & & $\log z$ & $N$ & Bgo723670.25 Type E & \\
\hline 10 & 1 1HE1 & 7 & 1 & & 105.9 & $N$ & & \\
\hline 32 & 1001 & $i$ & 1 & & $10 t 5$ & i & & \\
\hline 15 & $1 \longdiv { 7 N 9 1 }$ & $i$ & 1 & & 104,7 & in & & \\
\hline 15 & $1 \overline{D Q 1}$ & 1 & 1 & & 1074 & $\mathbf{N}$ & & \\
\hline 22 & 1) & 1 & 1 & & 103.4 & $\mathrm{~N}$ & & \\
\hline 15 & Ded & 1 & 1 & & 104.1 & N & & \\
\hline 16 & $1 / B+1$ & 1 & 1 & & 107.9 & $N$ & & \\
\hline 23 & 1 kte1 & 1 & 1 & & 1024 & $\mathbf{N}$ & & \\
\hline 8 & Thop! & 1 & 1 & & 137 & $\mathbf{N}$ & & \\
\hline 15 & 1תap1 & 1 & 1. & & $105 . \theta$ & $\mathbf{N}$ & & \\
\hline 10 & I/AOA1 & 1 & 1 & & 110.2 & $\mathbf{N}$ & & \\
\hline 18 & hotg1 & 1 & 1 & & & $\mathbf{N}$ & 164.9 - and of thret points & \\
\hline 19 & 10091 & 1 & 1 & & 115 & $\bar{N}$ & & \\
\hline 23 & 1मOब1 & 1 & 1 & & 104.7 & $\bar{N}$ & & \\
\hline 15 & 10493 & 1 & 1 & & 100.2 & N & & \\
\hline 16 & 1/4hat & 1 & 1 & & 112.4 & 쇼 & & \\
\hline 노 & 1/14tent & 1 & 1 & & 1073 & N & & \\
\hline 15 & 1/fifs? & 1 & 1 & & 1118 & $\mathrm{~N}$ & & \\
\hline 10 & 1/1fie1 & $t$ & 1 & & 115.4 & $N$ & & \\
\hline 22 & $1 / 15 m$ & 1 & 1 & & 110.7 & $\mathbf{N}$ & & \\
\hline 10 & 1/10/91 & 3 & 1 & & 111.7 & $N$ & & \\
\hline 22 & W1191 & 7 & $\mathbf{t}$ & & 108.7 & $\mathbf{N}$ & Ea905mast 2+20 Typo d & \\
\hline 75 & 1/17/B1 & 1 & 3 & & 107 & $\mathbf{N}$ & & \\
\hline 78 & 11721 & 1 & $\bar{T}$ & & 110.7 & $\mathbf{N}$ & & \\
\hline 22 & 1/7781 & 1 & 1 & & 100 & $\mathbf{N}$ & E-990723678-75 & \\
\hline 16 & $1 / 22 \times 91$ & 1 & 1 & & 105.5 & $\mathbf{N}$ & & \\
\hline 16 & $1 / 22=1$ & 1 & 1 & & 150.1 & $\bar{n}$ & & \\
\hline 22 & $1 / 2291$ & 1 & 1 & & 104.0 & $\mathbf{N}$ & E-990072 $3679-75$ & \\
\hline G & 1/mas & 1 & 1 & & 104.2 & $\mathbf{n}$ & HH-OH-A-E SN 900723079-75 & \\
\hline 15 & $1 / \sqrt{23} / 91$ & 1 & 1 & & 105.8 & $\mathbf{N}$ & & \\
\hline 10 & $1 / 2351$ & 1 & 1 & & 710.5 & $\mathbf{N}$ & & \\
\hline 17 & 1/23:1 & 1 & 1 & & tos.1 & $\mathbf{N}$ & & \\
\hline 19 & 1/29at & 1 & 1 & & 104.8 & $\mathbf{N}$ & & \\
\hline 22 & 1/2001 & $\overline{1}$ & 1 & & 105.7 & 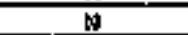 & & \\
\hline$\theta$ & 1/24,81 & 1 & 1 & & 102.9 & $\mathbf{N}$ & & \\
\hline 15 & 1/24/O1 & 1 & 1 & & 1048 & $\mathbf{N}$ & & \\
\hline 18 & $1 \longdiv { 2 4 5 1 }$ & 1 & 1 & & $10 \mathrm{~B}, \mathrm{9}$ & $\mathbf{N}$ & & \\
\hline 17 & $1 / 2491$ & 1 & 1 & & 108,5 & $\bar{a}$ & & \\
\hline 1s & 1/24/B1 & 1 & 1 & & 102.3 & w & & \\
\hline 22 & $1 / 24+91$ & 1 & $\bar{T}$ & & 1042 & $\mathbf{N}$ & HWSGACE ago723670.75 & \\
\hline$\$$ & W25191 & $t$ & 7 & & 103.8 & N & & \\
\hline $1 \overline{5}$ & 1/26501 & 1 & 1 & & 104,1 & $\bar{\pi}$ & & \\
\hline 10 & 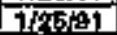 & 1 & $\mathbf{I}$ & & 108.1 & in & & \\
\hline 17 & 1/25/91 & 1 & 1 & & 103.9 & $\mathbf{N}$ & & \\
\hline 18 & $1 / 25491$ & 7 & 1 & & 108.9 & $\mathbf{M}$ & & \\
\hline 22 & 1/26/a1 & $\bar{T}$ & i. & & 1043 & $\bar{n}$ & 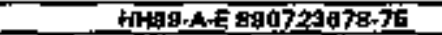 & \\
\hline $\mathbf{g}$ & $1 / 28 f_{1} 1$ & 1 & $i$ & & 101.9 & $\bar{N}$ & 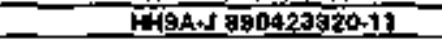 & \\
\hline 16 & $1125+91$ & $t$ & 1 & & 104.5 & $\mathbf{H}$ & & \\
\hline 10 & 120191 & 1 & $i$ & & 107.4 & $\mathbf{N}$ & & \\
\hline 17 & 1/20t91 & 1 & T & & 102.2 & $\mathbf{N}$ & & \\
\hline 19 & 1mats1 & 1 & 7 & & 101,7 & $\mathbf{N}$ & & \\
\hline 22 & I/20, & $t$ & 7 & & 109.3 & $\mathbf{N}$ & & \\
\hline $\mathbf{g}$ & 7/27/91 & 1 & 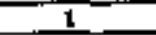 & & 101 & $\mathbf{N}$ & 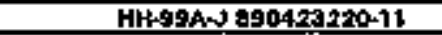 & \\
\hline 16 & 1/7/91 & 1 & $t, A^{2}$ & & 102.8 & $\mathbf{N}$ & & \\
\hline 19 & $1 / 27+91$ & 1 & 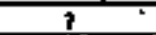 & & 108.6 & N & & \\
\hline 17 & $1 / 27 / 93$ & 1 & 1 & & 101. & $\mathbf{M}$ & & \\
\hline 19 & $1 / 27 / 41$ & 1 & $t$ & & & $\mathbf{N}$ & ofATC pertin off & \\
\hline $2 \pm$ & 1/27/91 & 1 & $\overline{1}$ & & 102.3 & $\mathbf{N}$ & & \\
\hline 9 & Imapa & 1 & 1 & & 101.5 & $\mathbf{W}$ & 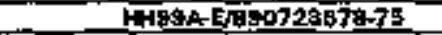 & \\
\hline 15 & 1/PBV1 & 1 & $i$ & & $10<0$ & $N$ & & \\
\hline 10 & 1/28sis & $t$ & $t$ & & 10e.s. & $\mathrm{N}$ & & \\
\hline 17 & 30891 & 1 & 1 & & 102.1 & $\mathbf{N}$ & & \\
\hline 22 & Ireas & 1 & 1 & & 202.6 & $\mathbf{N}$ & & \\
\hline 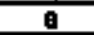 & $1 \mathrm{man1}$ & 1 & 1 & & 100.6 & $\mathbf{N}$ & & \\
\hline 15 & W/29/9 & 1 & 1 & & 100.8 & $N$ & & \\
\hline 10 & 1resta & 1 & 1 & & $109 . \overline{5}$ & $\mathbf{N}$ & & \\
\hline
\end{tabular}




\begin{tabular}{|c|c|c|c|c|c|c|c|c|c|}
\hline & & & & & 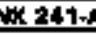 & & & & \\
\hline & & & & Th & Nocolup & DATA & & & \\
\hline THEF * & DATE & THEISTO & TREX SET & TIMPC & TEMPF & $Y=$ MAX TENP & 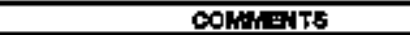 & & \\
\hline 17 & 1/20\%1 & 1 & .1 & & $101+5$ & $\mathbf{N}$ & & & \\
\hline 27 & $1 / 2991$ & i & 1 & & 102.3 & $\bar{N}$ & & & \\
\hline 요 & fow & 1 & 1 & & 1029 & $N$ & & & \\
\hline 15 & 19191 & 1 & 1 & & 105,5 & $\mu$ & & & \\
\hline 10 & $1 / 0101$ & 1 & 1 & & 1000 & $N$ & & & \\
\hline 17 & $1 / 3191$ & 1 & 1 & & 104 & $N$ & & & \\
\hline$\overline{22}$ & 18101 & 1 & 1 & & 104,3 & $N$ & J-990423320-1t & & \\
\hline $\mathbf{8}$ & $2(4 \sqrt{91}$ & $\mathbf{I}$ & 1 & & 107 & $\mathbf{N}$ & & & \\
\hline 15 & $2 / 4 / 91$ & 1 & 1 & & 110 & Nㅗ & & & \\
\hline 10 & $24 / 91$ & 1 & 1 & & 114 & N & & & \\
\hline 17 & $34 \sqrt{91}$ & 1 & 1 & & 109 & $\mathrm{~N}$ & & & \\
\hline 22 & $2 / 4 \sqrt{91}$ & 1 & $\overline{1}$ & & 100 & $N$ & TYp 189052351247 & & \\
\hline 8 & 2 bol & 1 & 1 & & 118.4 & $\mathbf{N}$ & & & \\
\hline 16 & 2019 & 1 & 1 & & 126.9 & $\mathbf{N}$ & & & \\
\hline 17 & $2 / 51$ & 1 & 1 & & 108.5 & $\mathbf{N}$ & & & \\
\hline$\theta$ & $2 \pi+91$ & 1 & 1 & & 100 & $\mathbf{N}$ & & & \\
\hline 15 & $2 \pi+91$ & 1 & 1 & & 110 & $\mathbf{N}$ & & & \\
\hline 16 & 2hta1 & 1 & 1 & & 116 & $N$ & & & \\
\hline 17 & $2 \overline{291}$ & $i$ & $\overline{1}$ & & 110 & $\mathbf{N}$ & & & \\
\hline 22 & $2 \pi / 91$ & 1 & 1 & & 110 & $\mathbf{N}$ & & & \\
\hline 9 & 2/1191 & 1 & 1 & & 105 & $\mathbf{N}$ & & & \\
\hline t5 & 211191 & 1 & 1 & & 111 & N & & & \\
\hline 15 & $2 / 1191$ & 1 & 1 & & 117 & $\mathbf{N}$ & & & \\
\hline 17 & All1tet & 1 & 1 & & 107 & $\mathbf{N}$ & & & \\
\hline 22 & 2月1191 & 1 & 1 & & 10 & $\mathbf{N}$ & & & \\
\hline 5 & 2n12N1 & $\$$ & 1 & & 100 & $\mathbf{N}$ & & & \\
\hline 15 & $2 / 12 \sqrt{91}$ & 7 & 1 & & 110.9 & $N$ & & & \\
\hline 10 & $2 / 1201$ & \pm & 1 & & 114,8 & $\bar{N}$ & & & \\
\hline 17 & $2 / 1291$ & 1 & 1 & & 108.7 & $\mathbf{N}$ & & & \\
\hline 22 & $2 n 1291$ & 3 & 1 & & 100.3 & $\mathbf{N}$ & E-Tyon B:00723679-78 & & \\
\hline 9 & $2 / 1391$ & 1 & 1 & & 110 & $\mathbf{N}$ & & & \\
\hline 75 & $2 \sqrt{391}$ & 1 & 1 & & 115.9 & $\mathbf{N}$ & & & \\
\hline 16 & 2h13/191 & 1 & 1 & & 118,4 & $\mathbf{N}$ & & & \\
\hline 22 & 2)1391. & 1 & 1 & & 110.1 & $\mathbf{N}$ & $5990723673-75$ & & \\
\hline 요 & 2/14/81 & 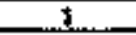 & $\mathbf{1}$ & & 113 & $\mathbf{N}$ & & & \\
\hline 15 & $2 / 1491$ & $t$ & 1 & & 113.9 & $\mathbf{N}$ & & & \\
\hline 18 & $2 / 14 / 91$ & 1 & 1 & & 112.4 & $\mathbf{N}$ & & & \\
\hline 17 & $2 / 14 / 91$ & 1 & 1 & & 112 & N & & & \\
\hline 22 & $2 / 1481$ & 1 & 1 & & 112.2 & is & 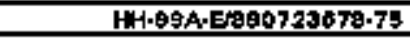 & & \\
\hline 8 & 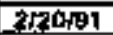 & $\$$ & $T$ & & 106.1 & $\mathbf{N}$ & Type E & & \\
\hline 16 & 2/20/O1 & 1 & 1 & & 110.1 & $N$ & & & \\
\hline 10 & $2 \sqrt{2001}$ & $E$ & 1 & & 114.2 & $\mathbf{N}$ & & & \\
\hline 22 & $2 / 2001$ & $t$ & 1 & & 706.B & $\mathbf{N}$ & & & \\
\hline 9 & $2 / 2191$ & 1 & 1 & & 1078 & $\overline{\mathbf{N}}$ & E.890723\$78.5E & & \\
\hline 15 & $2 / 2+181$ & $t$ & 1 & & $10 \mathrm{~g}$ & $\mathbf{N}$ & & & \\
\hline 18 & 22381 & 1 & 1 & & 114.5 & $\mathbf{Y}$ & & & \\
\hline 22 & 2/23/81 & 1 & 1 & & 109.1 & $\mathbf{N}$ & & & \\
\hline $\mathbf{8}$ & 2/25/91 & $\overline{7}$ & 1 & & 100.8 & $\mathbf{N}$ & BBO723879.85E & & \\
\hline 15 & 2सकाब1 & $\$$ & 1 & & 100 & $\mathbf{N}$ & & & \\
\hline 18 & $2 / 25 / 91$ & 1. & 1. & & 118.5 & $\overline{\mathbf{N}}$ & & & \\
\hline 22 & 212551 & $t$ & 1 & & 109.8 & $\mathbf{N}$ & & & \\
\hline 9 & 2120\%1 & 1 & 1 & & 10.9 & $\mathbf{N}$ & & & \\
\hline 16 & ZHABO1 & 1 & 1 & & 107.8 & $\mathbf{N}$ & & & \\
\hline 10 & 2/28/91 & 1 & 1 & & 113.1 & $\mathbf{N}$ & & & \\
\hline 22 & 2212001 & 1 & 1 & & 100,9 & $\mathbf{N}$ & Then E 890723679-68. & & \\
\hline 15 & $3 / 4 / 21$ & 1 & $1 \ldots$ & & 1104 & $\mathbf{N}$ & & & \\
\hline 16 & $3 / 4 / 91$ & 1 & 1 & & $11 \$ .2$ & $N$ & & & \\
\hline 22 & $3 / 4 / 91$ & 1 & 1 & & 100.8 & $N$ & E. 700147 & & \\
\hline $1 \mathrm{E}$. & आ14/91 & 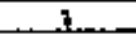 & 1. & & 108,6 & $\mathbf{N}$ & & & \\
\hline 16 & 311491 & \pm & 1 & & 117,0 & Nㅗ & & & \\
\hline 15 & अ2191 & 1 & 1 & & $1 \pm 2.5$ & $N$ & & & \\
\hline 10 & 3/21/91 & 1 & 1 & & 120,0 & $Y$ & & & \\
\hline 22 & अ2191 & 1 & 1 & & 12B,6 & 소 & & & \\
\hline 15 & अ2901 & 1 & 1 & & 713 & $N$ & & & \\
\hline 16 & 32ales & 1 & 1 & & 118.1 & $\mathbf{Y}$ & & & \\
\hline 22 & अ28/91 & 1 & 1 & & 131.7 & $\mathbf{N}$ & & & \\
\hline 15 & $4 / 1 / 91$ & 1 & 1 & & 117 & $\mathbf{N}$ & TYPEE & & \\
\hline IB & 41191 & 1 & 1 & & 325 & $\mathbf{N}$ & & & \\
\hline
\end{tabular}




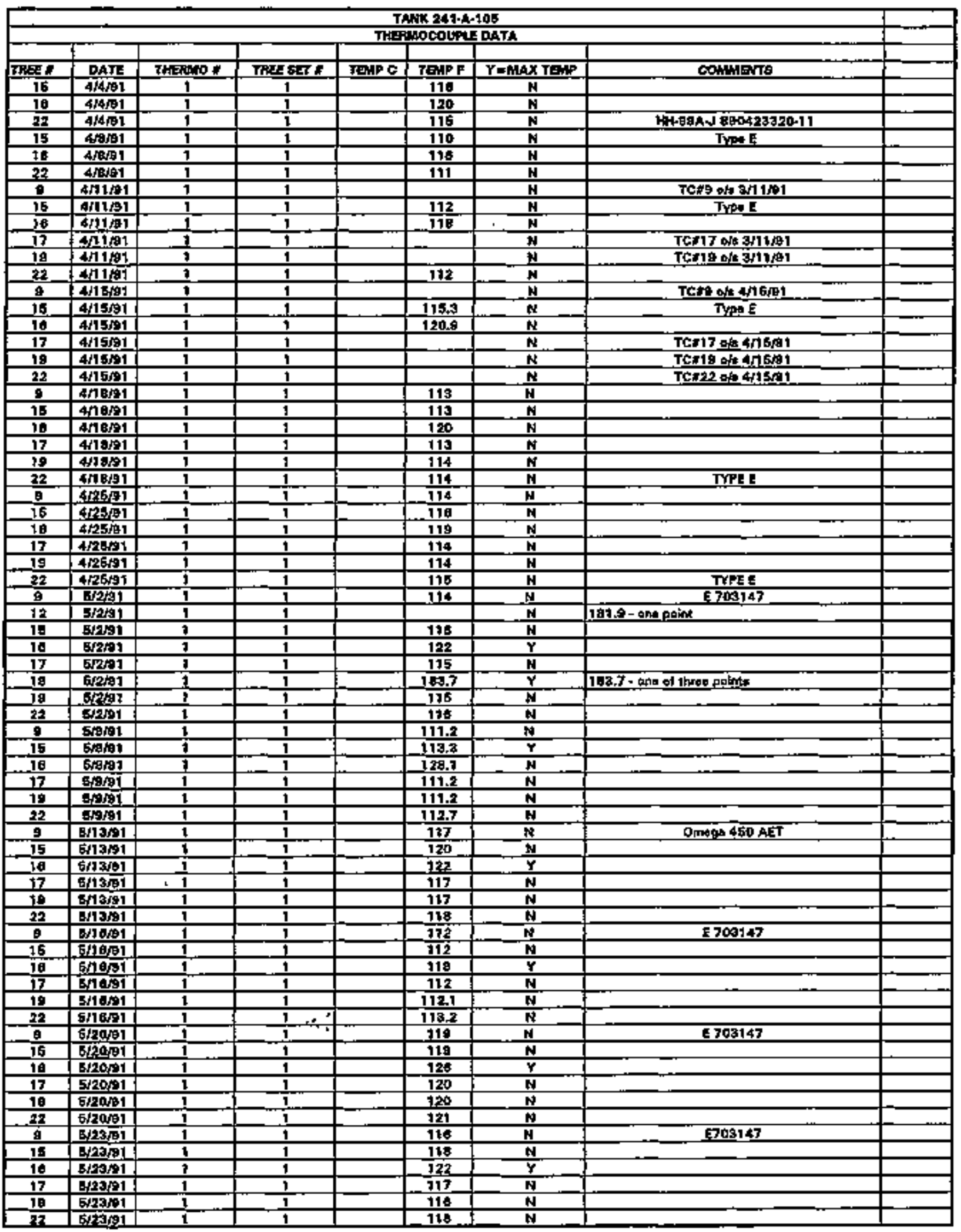




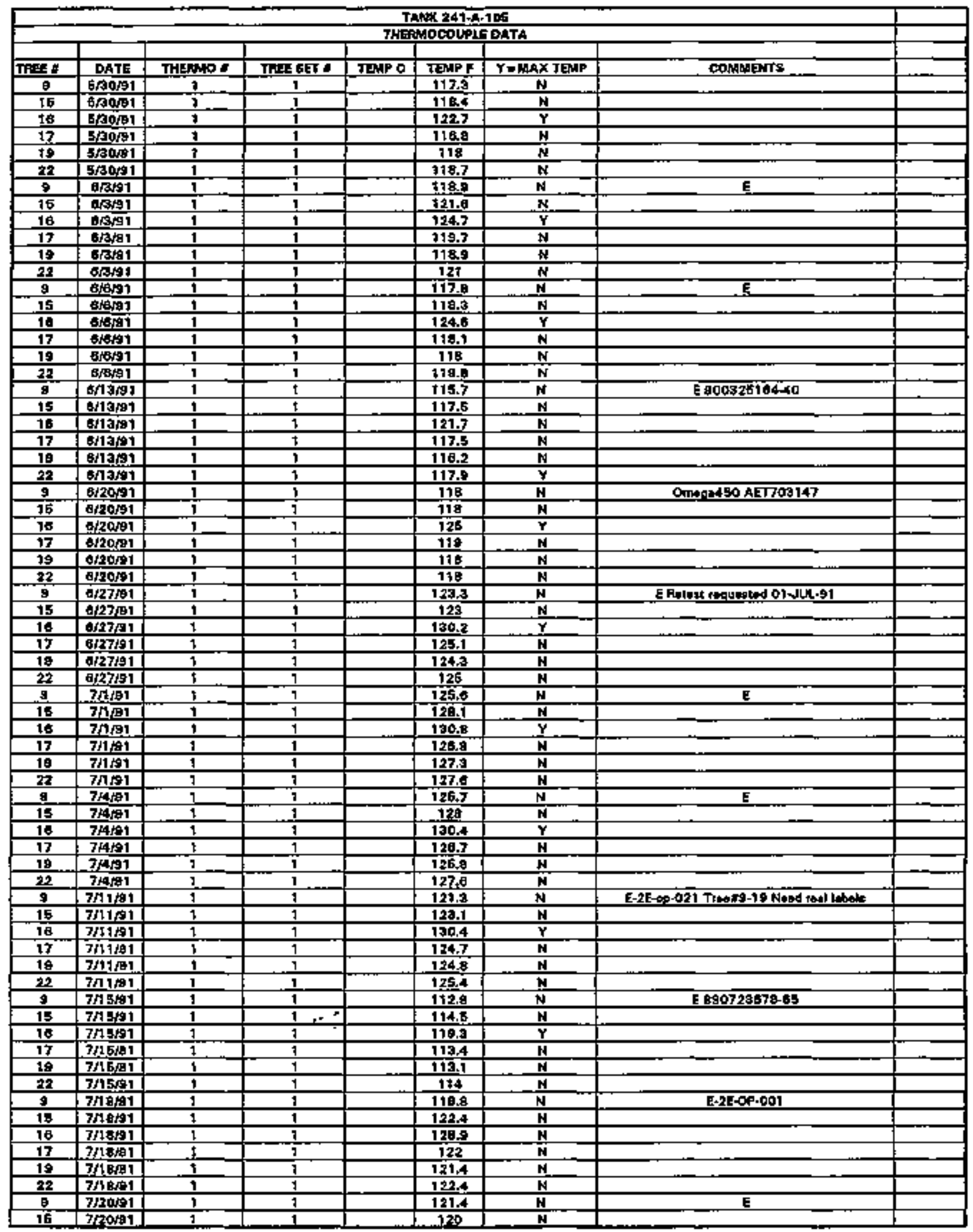




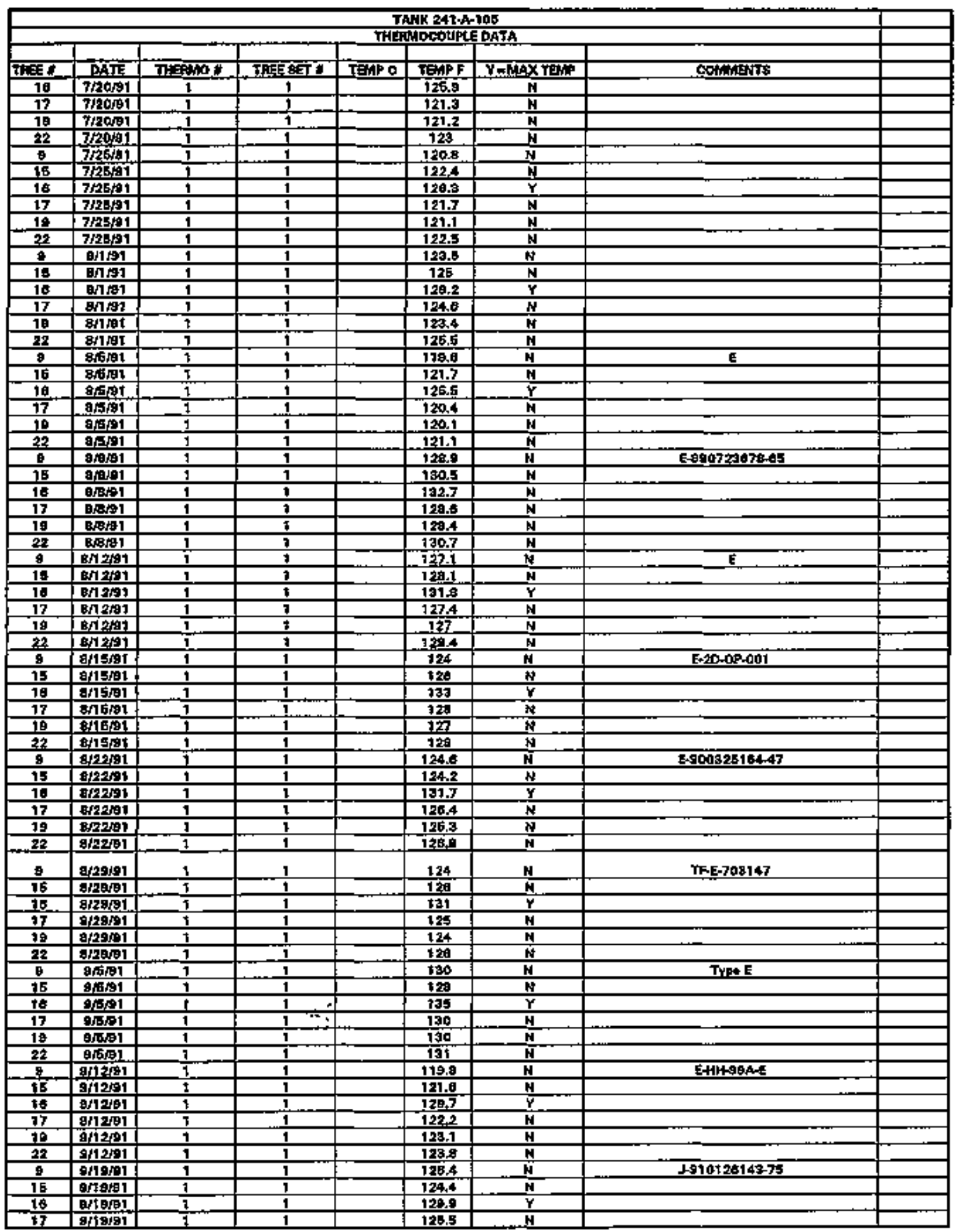




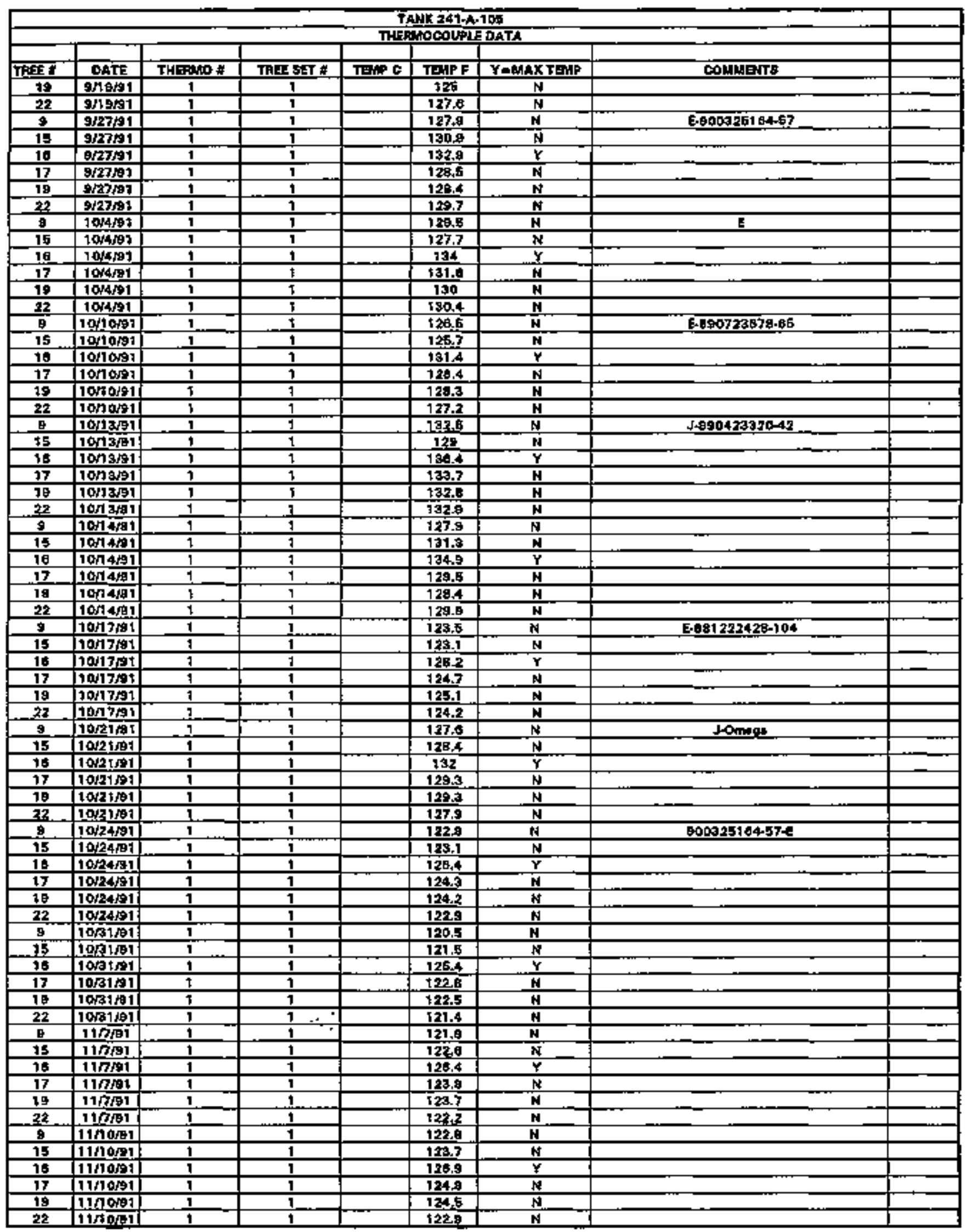




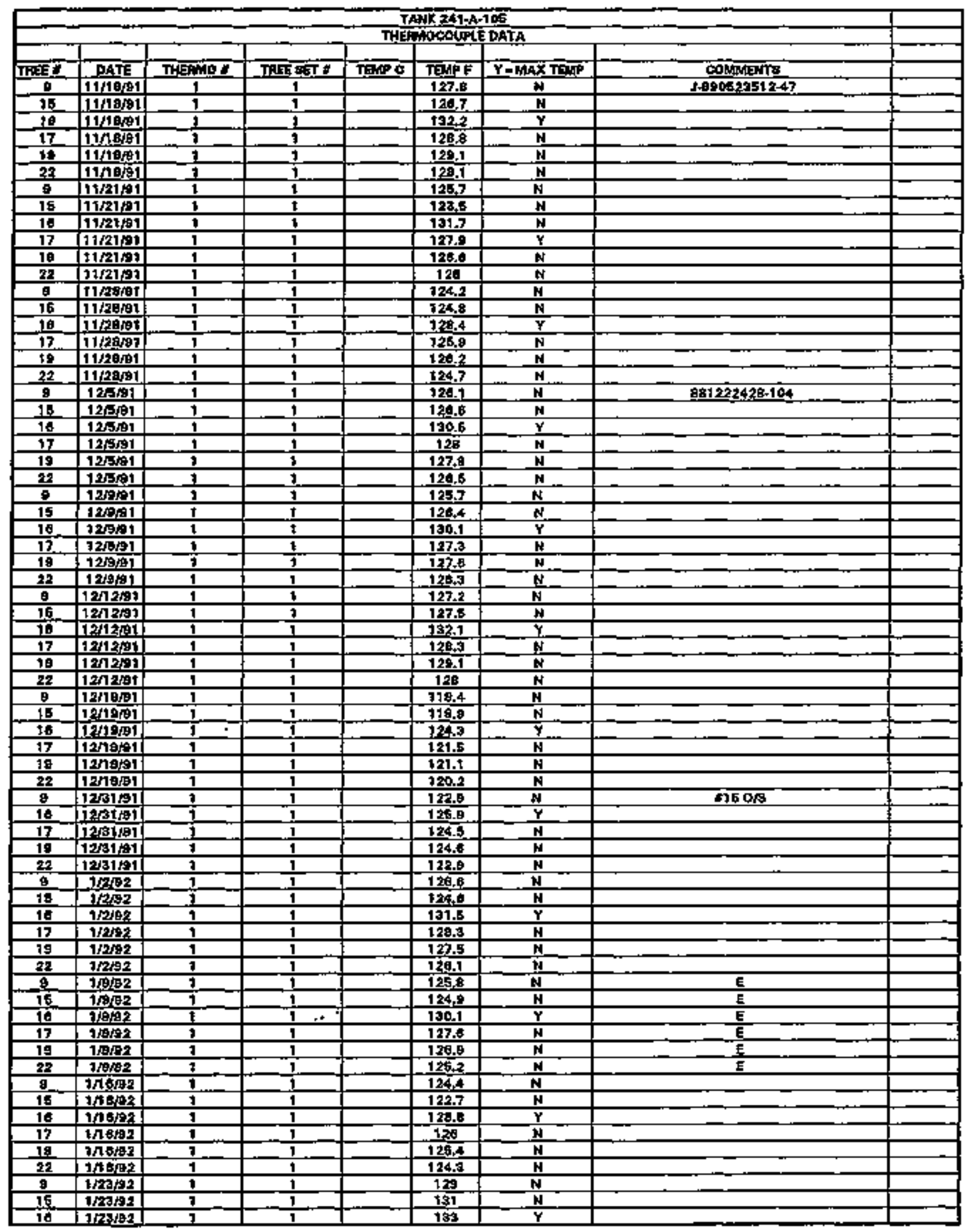




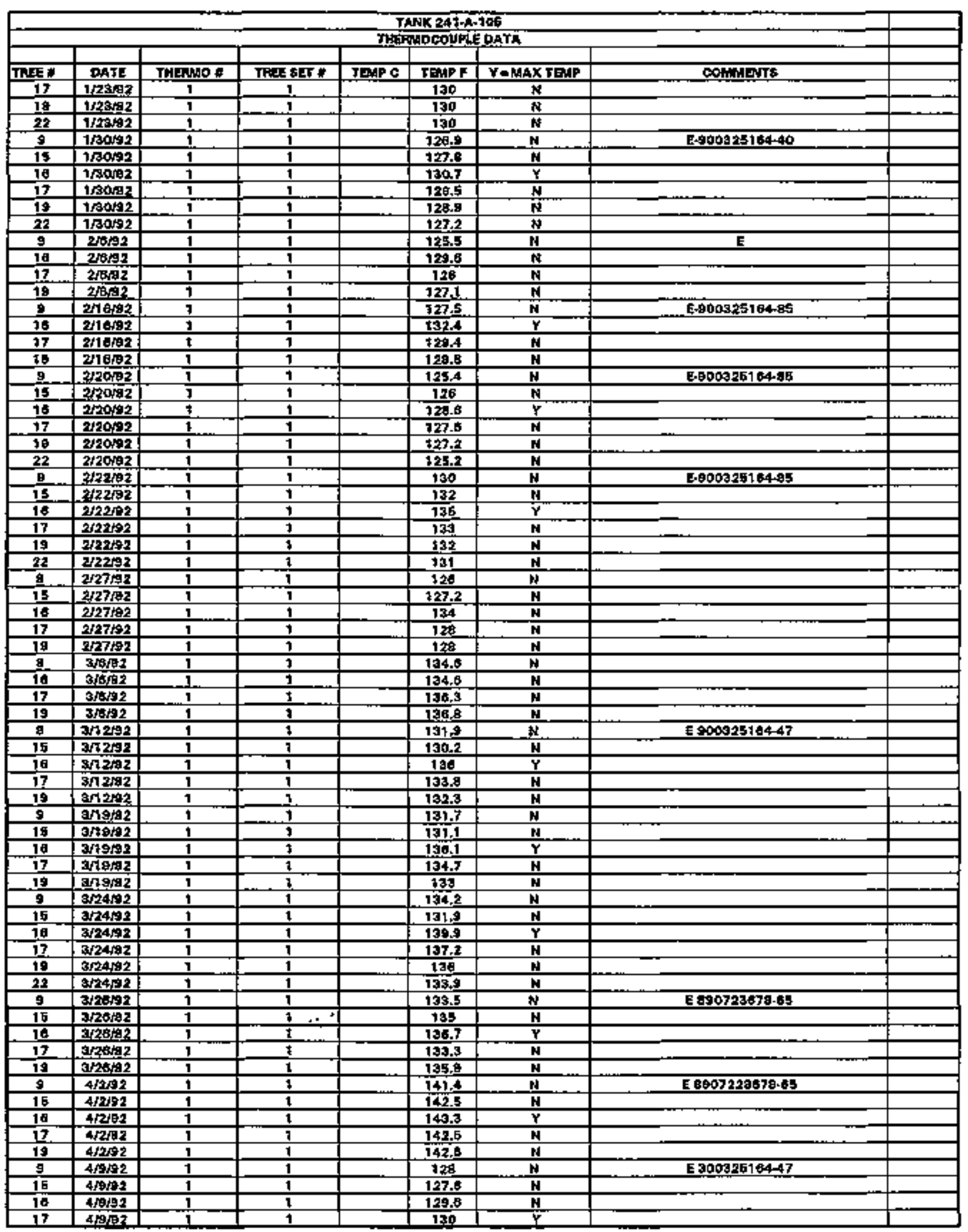




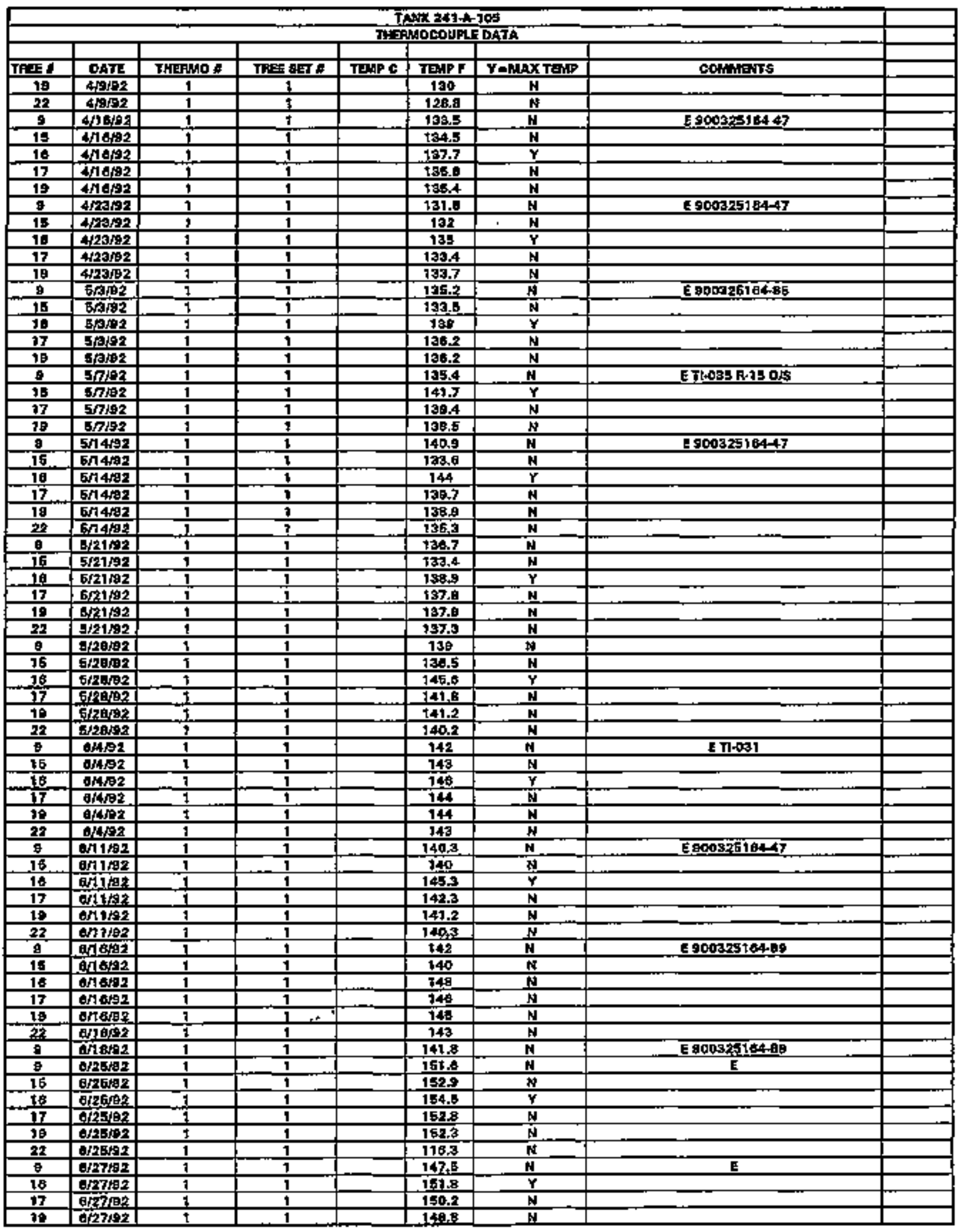




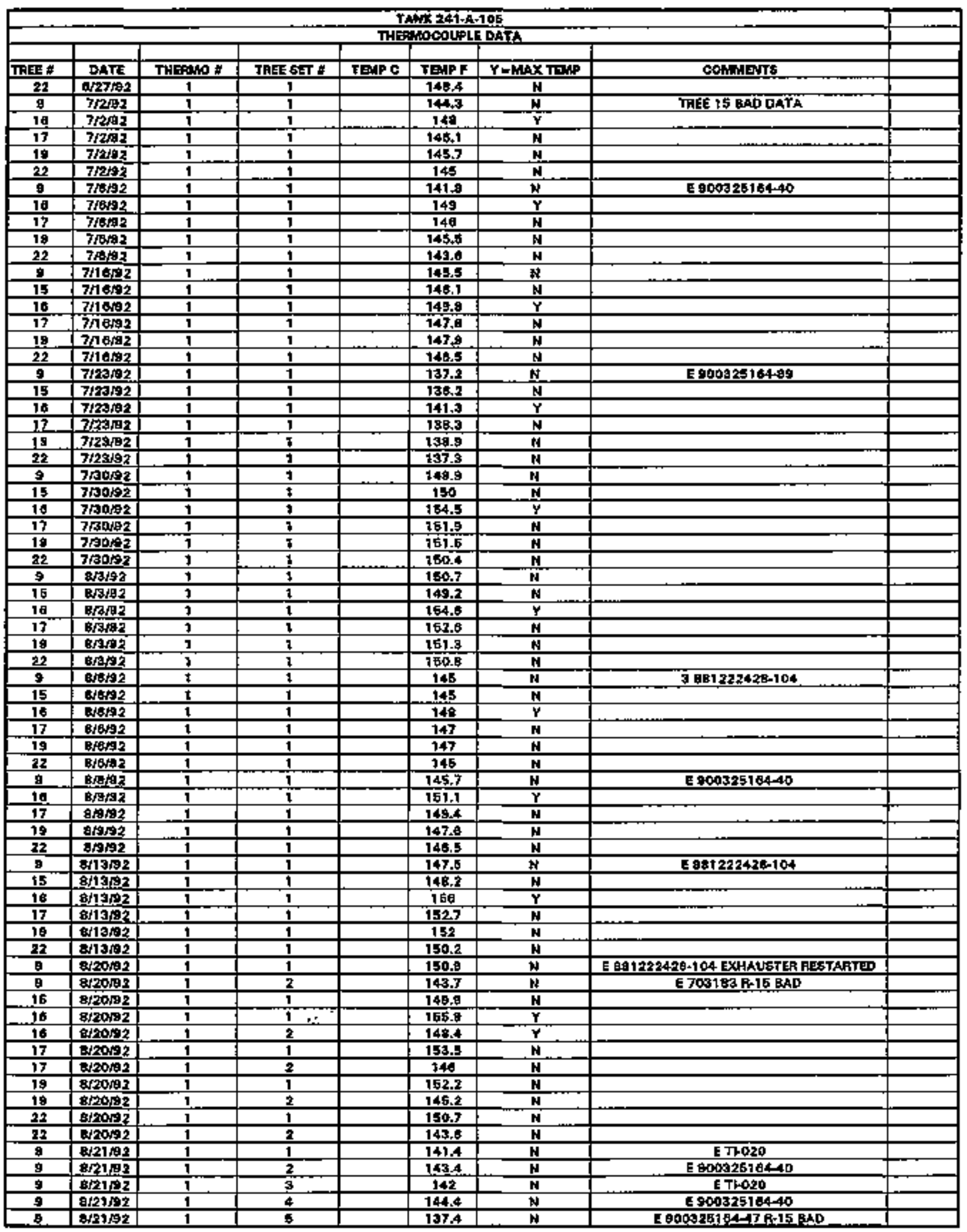




\begin{tabular}{|c|c|c|c|c|c|c|c|c|}
\hline \multicolumn{9}{|c|}{ TANK 24TR-TO5 } \\
\hline & & & & THE & AOCOAIf: & DATA & & \\
\hline Tf: & DATE & THEF 10 \% & TAFFAT A & TF네 C & $T \cdot \bar{F} F$ & $Y=$ MAX TT.MP & Eodimgirs & \\
\hline 9 & Sh102 & 3 & 6 & & 144 & $\mathbf{N}$ & E $\angle F=0$ P -009 R-15 $\mathrm{BAD}$ & \\
\hline 15 & BN21192 & 3 & 1 & & 142.8 & $\mathbf{H}$ & & \\
\hline 16 & 핵192 & 1 & 2 & & 1A4.B & $\mathbf{H}$ & & \\
\hline 15 & 812192 & 1 & 3 & & 143.5 & N & & \\
\hline 16 & 백1192 & 1 & 4 & & $145 . B$ & $\mathbf{H}$ & & \\
\hline 10 & $912+12$ & 1 & 1 & & 140,2 & $\bar{Y}$ & & \\
\hline 10 & a/21/02 & 1 & 2 & & 146.1 & $\mathbf{Y}$ & & \\
\hline 16 & $9 / 21 / 22$ & 1 & 3 & & 146.9 & $\mathbf{Y}$ & & \\
\hline 16 & 9/21/02 & 1 & 4 & & 150.2 & $\bar{Y}$ & & \\
\hline 16 & 8/21/22 & 1 & E & & 142.5 & $\mathbf{Y}$ & & \\
\hline 56 & 8/23/92 & 1 & B & & t & $Y$ & & \\
\hline 17 & $8 / 3902$ & 1 & 1 & & 144.2 & $\underline{x}$ & & \\
\hline 17 & 8/2162 & 1 & 2 & & 146.2 & $\mathbf{N}$ & & \\
\hline 17 & E/21/92 & 1 & 3 & & 144.9 & $\mathbf{N}$ & & \\
\hline 17 & 21k2 & 1 & 4 & & 147.6 & $\mathbf{N}$ & & \\
\hline 17 & 1/21, & 1 & 5 & & 140.4 & N & & \\
\hline 17 & grikg & 1 & 흐 & & 1鎴 & $\mathbf{N}$ & & \\
\hline 19 & - $\sqrt{21 / 92}$ & 1 & 1 & & 143.1 & $\mathbf{N}$ & & \\
\hline 19 & Bí21 $\mathrm{kg}$ & 1 & 2 & & 145.4 & $N$ & & \\
\hline 10 & 821/92 & 1 & 3 & & 143.6 & $\mathbf{N}$ & & \\
\hline 10. & 8 & 1 & 4 & & \pm 49.7 & $\mathbf{N}$ & & \\
\hline 19 & $81 / 92$ & 1 & $E$ & & 139.3 & $\mathbf{N}$ & & \\
\hline 10 & 8tz192 & 1 & a & & 147 & $\mathbf{N}$ & & \\
\hline 22 & $8 / 21 / 92$ & 1 & 1 & & 140.18 & $\mathbf{N}$ & & \\
\hline 22 & - $121 / 92$ & 1 & 2 & & 143 & $\mathbf{N}$ & & \\
\hline 23 & - $/ 21 / 82$ & $\mathbf{2}$ & $\mathbf{3}$ & & $2+1,3$ & N. & & \\
\hline 22 & 9/21/22 & 1 & 4 & & 140.0 & $\mathbf{N}$ & & \\
\hline 22 & 9pila2 & 1 & 5 & & 130.8 & N & & \\
\hline 23 & 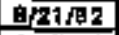 & 1 & a & & 144 & N & & \\
\hline 8 & ay 27152 & 1 & 1 & & 145,6 & $\bar{N}$ & 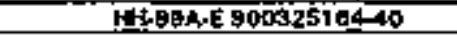 & \\
\hline 15 & 9/27/42 & 1 & 1 & & 1442 & $\mathbf{N}$ & & \\
\hline 10 & 9/27/22 & 1 & 1 & & 181.5 & $\mathbf{Y}$ & & \\
\hline 17 & g/27有2 & 1 & 1 & & 149.9 & $N$ & & \\
\hline 19 & Befta2 & 1 & 1 & & 24 & $\mathbf{N}$ & & \\
\hline 22 & B/7.92 & 1 & 1 & & $14 \overline{9,5}$ & A & & \\
\hline 9 & 9tata2 & 1 & 1 & & 150 & H & E 70319a & \\
\hline 16 & 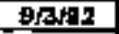 & 1 & $T$ & & 748 & $\mathbf{N}$ & & \\
\hline 16 & 03782 & 1. & $t$ & & 155 & $y$ & & \\
\hline 17 & $9 / 3+92$ & 1 & 1 & & $15 \overline{2}$ & N & & \\
\hline 19 & 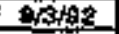 & 1 & $\$$ & & 150 & $\mathbf{N}$ & & \\
\hline 9 & 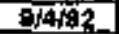 & 1 & 1 & & 142 & $\mathbf{N}$ & E EN S0032518499 RETEST HE & \\
\hline 15 & BHet & 1 & 1 & & 142.0 & $\mathbf{N}$ & & \\
\hline 10 & S4492 & 1 & 1 & & 146 & $\mathbf{Y}$ & & \\
\hline 17 & 9/4/92 & 1 & 1 & & $14 \overline{3} 7$ & $\mathbf{N}$ & & \\
\hline 10 & $8 / 4 / 92$ & 1 & 7 & & 143.8 & $\mathbf{N}$ & & \\
\hline $\mathbf{g}$ & $0 \overline{10 \% 2}$ & 1 & 1 & & 143.6 & $\mathbf{N}$ & E9000325:84-4 & \\
\hline E & sog? & 1 & 1 & & 141.7 & $\mathbf{N}$ & & \\
\hline 10 & $5 / 10 / 92$ & 1 & 1 & & 147.4 & $\bar{Y}$ & & \\
\hline 17 & $8 / 10 / 92$ & 1 & 1 & & 145.0 & $\mathbf{N}$ & & \\
\hline 10 & " $/ 10592$ & 1 & 1 & & 146.2 & $\mathbf{N}$ & & \\
\hline 8 & $3 \longdiv { 7 0 2 }$ & 1 & 1 & & $14 \mathrm{~B}$ & $\mathbf{N}$ & E2E-OP-003 & \\
\hline 15 & $9 / 17 / 22$ & 1 & 1 & & 144 & $\mathbf{N}$ & & \\
\hline 10 & Br1792 & 1 & 7 & & 150 & $\overline{\mathbf{Y}}$ & & \\
\hline 17 & E/1702 & 1 & 1 & & 167 & $\overline{\mathbf{N}}$ & & \\
\hline 18 & $9 / 17 / 92$ & 1 & 1 & & $14 \mathrm{~B}$ & $\mathbf{N}$ & & \\
\hline 8 & $9 \overline{2692}$ & 1 & $1 \mathrm{in}$ & & 142.3 & $\mathbf{N}$ & & \\
\hline 15 & $9 / 2582$ & 1 & 1 & & 140.3 & $\mathbf{N}$ & & \\
\hline 10 & $5 / 25 / 92$ & 1 & 1 & & 147.4 & $\mathbf{Y}$ & & \\
\hline 17 & S45ر92 & 1 & 1 & & 143.7 & N & & \\
\hline 18 & Erf5je2 & 1 & 1 & & \pm 43.7 & $N$ & & \\
\hline$\theta$ & Afarez & 1 & 1 & & 145 & N & & \\
\hline 15 & $9 \sqrt{28 / 92}$ & 1 & 1 & & 142 & $\mathbf{N}$ & & \\
\hline 10 & 7/29/92 & 1 & 1 & & 148 & $Y$ & & \\
\hline 17 & shaje2 & 1. & 1 & & 146 & $\mathbf{N}$ & & \\
\hline $1 \dot{\theta}$ & $09 / 92$ & 1 & 1 & & $14 \mathrm{~B}$ & $\mathbf{N}$ & & \\
\hline 童 & 요말ㄹ & 1 & 1 & & 14.9 & $N$ & & \\
\hline 15 & D/atag 2 & 1 & 1 & & $\$ 16.7$ & $\mathbf{N}$ & & \\
\hline 10 & adachen & 1 & 1 & & 153.5 & $r$ & & \\
\hline
\end{tabular}




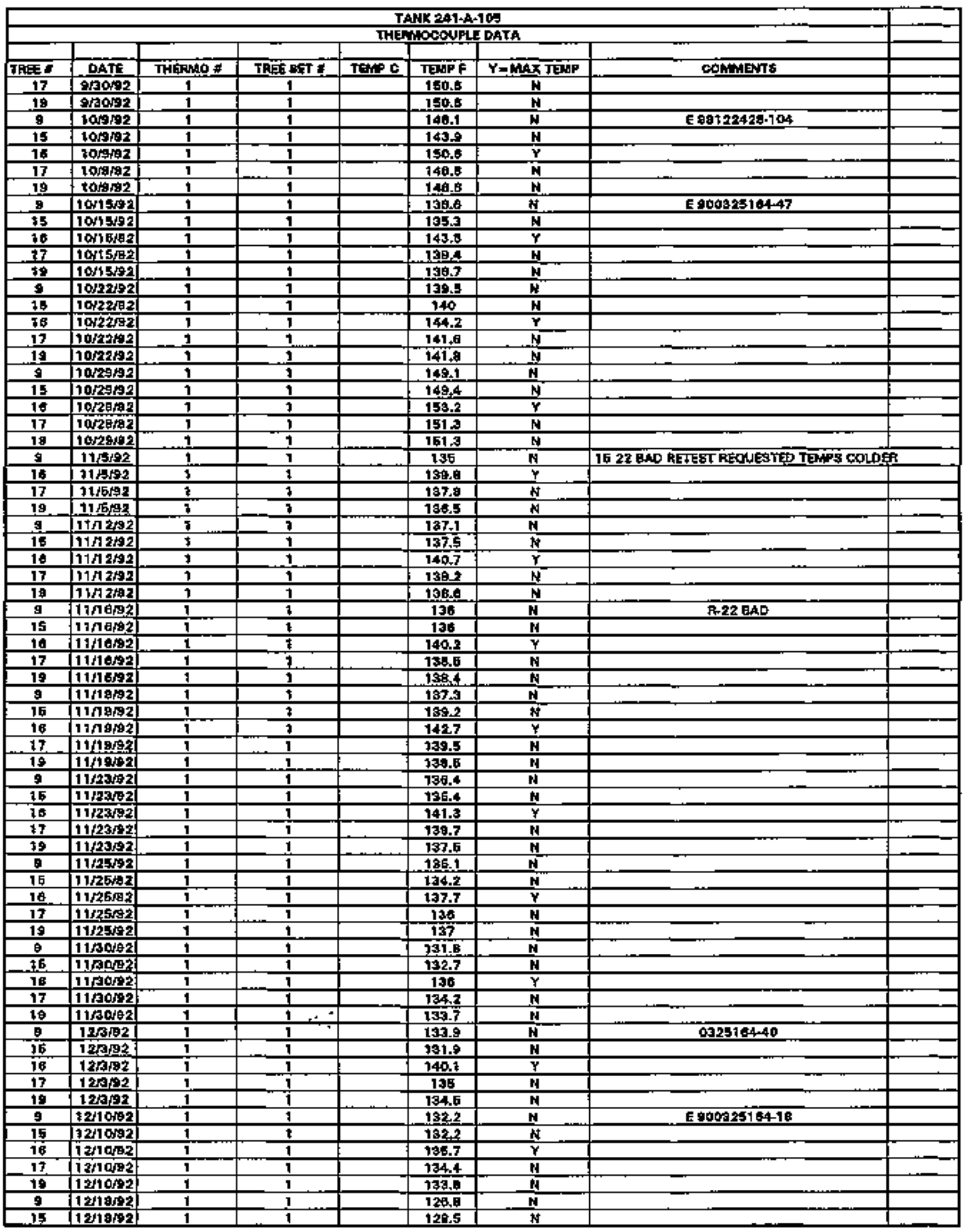




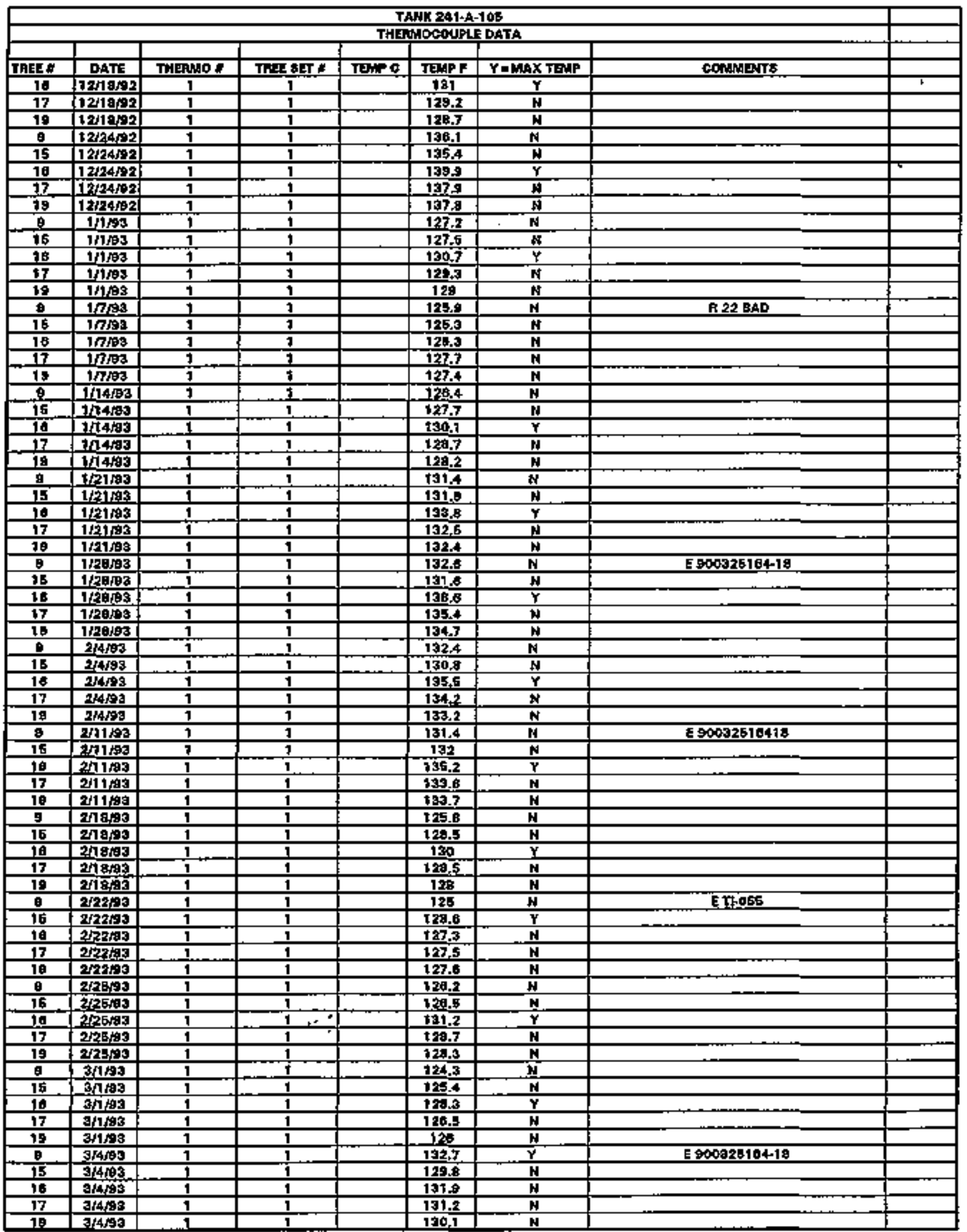




\begin{tabular}{|c|c|c|c|c|c|c|c|c|}
\hline & & & & & N2*1-A & & & \\
\hline & & & & THE & Nocolpti & EATA & & \\
\hline Tri=1* & DATTI & 7मdग10 & TiJ) 8ET : & TPup 1 & TOMPE & YamaX TJPP & Cothuts: & \\
\hline 8 & $3 \sqrt{8+2}$ & $-\frac{1}{1}$ & 1 & & 133 & $\frac{N}{N}$ & 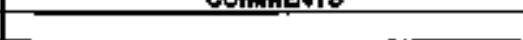 & \\
\hline 18 & $3 / 18 / 43$ & T & 1 & & 13 & $\mathbf{N}$ & & \\
\hline 1E & 3 sista & 1 & 1 & & 136,2 & $\mathbf{Y}$ & & \\
\hline 17 & IBBS3 & $\overline{1}$ & 1 & & 134,7 & $\bar{N}$ & & \\
\hline 19 & $3 / 203$ & 1 & 1 & & 134.7 & $\mathbf{N}$ & & \\
\hline$\theta$ & $3 / 193$ & $\overline{1}$ & 1 & & 132.7 & $\bar{N}$ & E 900325104-16 & \\
\hline 16 & MMIra & $T$ & 1 & & 139.8 & $\mathbf{N}$ & & \\
\hline 18 & 3m1ras & 1 & 1 & & 138 & $\overline{\mathbf{Y}}$ & & \\
\hline 17 & 3A1 993 & 1 & 1 & & 135.2 & $\mathbf{N}$ & & \\
\hline 19 & 3ก11 & 1 & 1 & & $13 \overline{3.6}$ & $\mathbf{N}$ & & \\
\hline 5 & 3 M8:93 & 1 & 1 & & 133.1 & $\mathbf{N}$ & E $900325104-4$ R+22 ELSPECT & \\
\hline 15 & $319 / 93$ & $\overline{1}$ & $\overline{1}$ & & 131.5 & $\bar{N}$ & & \\
\hline 16 & $3 / 18 / 93$ & $T$ & 1 & & 137.7 & $Y$ & & \\
\hline 13 & 31:/9/93 & 1 & 1 & & 136.1 & $N$ & & \\
\hline 19 & $3 \sqrt{9 \sqrt{83}}$ & 1 & 1 & & 184,4 & $\mathbf{N}$ & & \\
\hline 9 & 3/2nis & 1 & 1 & & 131,9 & $\mathbf{N}$ & E S003251144-77 R-22 SUEPECT & \\
\hline 15 & $3+22 \% 3$ & $\overline{1}$ & 1 & & 1348 & $\mathbf{N}$ & & \\
\hline 16 & $3 / 2 / 3 / 3$ & $\overline{1}$ & 1 & & 1379 & $\bar{Y}$ & & \\
\hline 17 & 322193 & $T$ & 1 & & 134.0 & N & & \\
\hline 19 & सम2203 & $\overline{1}$ & T & & 134.3 & N & & \\
\hline 8 & \$2 & 1 & 1 & & 133 & $\mathbf{N}$ & E $90035 \overline{2}=16440$ & \\
\hline 15 & W/2509 & 1 & 1 & & $137+9$ & $\mathbf{N}$ & & \\
\hline 16 & $325 / 93$ & $\overline{1}$ & $\mathbf{1}$ & & 130 & $\bar{Y}$ & & \\
\hline 17 & $3 / 25 / 93$ & $\overline{1}$ & $\overline{1}$ & & 135.4 & $\bar{N}$ & & \\
\hline 19 & 3/2503 & $\bar{T}$ & 1 & & 133.9 & N & & \\
\hline 22 & 3426 ing & 1 & 1 & & 133,9 & $\bar{N}$ & & \\
\hline 9 & 4/1/93 & 1 & 1 & & 137,3 & $\mathbf{N}$ & E900376t164-40 & \\
\hline 15 & $4 / 1 / 193$ & 1 & 1 & & $736 . \mathrm{B}$ & $\mathbf{N}$ & & \\
\hline 10 & $4 \sqrt{1 / 93}$ & 1 & 1 & & 140.7 & $\mathbf{Y}$ & & \\
\hline 97 & $4 / 793$ & 1 & $\overline{1}$ & & 1398 & $\mathbf{N}$ & & \\
\hline נig & $4 / 183$ & 1 & $\mathbf{T}$ & & 730.8 & $\bar{N}$ & & \\
\hline 22 & $4 \pi \sqrt{93}$ & 1 & 1 & & & $\mathbf{N}$ & 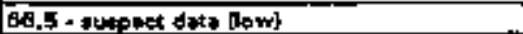 & \\
\hline 9 & $4 \sqrt{6 / 43}$ & 1 & 1 & & $\$ 34.6$ & $\mathbf{M}$ & 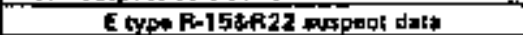 & \\
\hline 15 & 45 & 1 & 1. & & 340.4 & $y$ & & \\
\hline 17 & $4 \sqrt{5} \operatorname{sg}$ & 1 & 1 & & 337,4 & $\mathbf{N}$ & & \\
\hline 18 & $4 \sqrt{493}$ & $\overline{1}$ & $T$ & & 135.6 & $\mathbf{N}$ & & \\
\hline 22 & $4 \sqrt{593}$ & 1 & 1 & & & $\mathbf{N}$ & 59,2 + aweet dote fowl & \\
\hline$\overline{\bar{\varepsilon}}$ & $4 \sqrt{18}$ & 1 & 1 & & 132.9 & $\mathbf{N}$ & 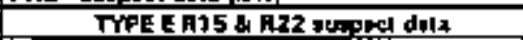 & \\
\hline 18 & $4 / 8 \sqrt{6}$ & 1 & 1 & & $\$ 36.3$ & $\bar{Y}$ & TYPEE & \\
\hline 17 & $4 \sqrt{8}$ & 1 & 1 & & 135.4 & $\bar{N}$ & TRPEE & \\
\hline 18 & $4 P^{3} \mathrm{SB}$ & 1 & 1 & & 134,7 & $\mathbf{N}$ & TNFEE & \\
\hline 32 & $4 / 8$ 每3 & 1 & 1 & & & $\mathbf{N}$ & 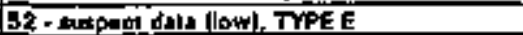 & \\
\hline$\theta$ & 415 sig & 1 & 1 & & 734.9 & $\mathbf{N}$ & 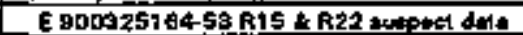 & \\
\hline 18 & $4 \sqrt{1593}$ & 1 & $\overline{1}$ & & 139,6 & $\mathbf{Y}$ & & \\
\hline 17 & $4 \pi 159^{3}$ & 1 & $\$$ & & 137.4 & $\mathbf{N}$ & & \\
\hline 19 & $4 / 1593$ & 1 & $\overline{1}$ & & 139.8 & $\mathbf{N}$ & & \\
\hline 27. & 4 41593 & 1 & $\bar{T}$ & & & $\mathbf{N}$ & 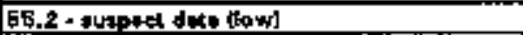 & \\
\hline 8 & $4 \sqrt{19 / 93}$ & 1 & 7 & & 136.1 & $\mathbf{N}$ & 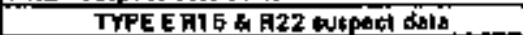 & \\
\hline 10 & $4 \sqrt{18 / 93}$ & 1 & 1 & & 141.9 & $\mathbf{Y}$ & & \\
\hline 17 & $4 \sqrt{10}+93$ & 1 & $\overline{1}$ & & 739.9 & $\mathbf{H}$ & & \\
\hline 19 & $4 / 40193$ & 1 & 1 & & t37.6 & $\mathbf{N}$ & & \\
\hline 27 & $4 / 993$ & $\overline{1}$ & 1 & & & $\mathbf{H}$ & 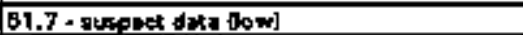 & \\
\hline 8 & $4 / 22693$ & 1 & $\overline{1}$ & & $t 39.2$ & $\overline{\mathbf{N}}$ & E E000326744.63 & \\
\hline 13 & $4 / 22 \pi 93$ & 1. & 1. & & 739.7 & $\mathbf{N}$ & & \\
\hline 18 & $4 / 22 / 93$ & $\overrightarrow{1}$ & 1 & & 542.1 & $Y$ & & \\
\hline 17 & $4 \sqrt{2253}$ & 1 & $T$ & & 1398 & $\bar{N}$ & & \\
\hline 79 & $4 \sqrt{22 / 93}$ & 1 & T & & 140.2 & $\bar{N}$ & & \\
\hline 22 & $4 \sqrt{22+93}$ & 1 & $\overline{\mathbf{i}}$ & & & N & 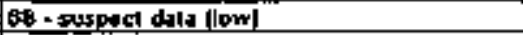 & \\
\hline 8 & $429 / 93$ & 1 & 1 & & 133.5 & $\mathbf{N}$ & E9000325te4477 & \\
\hline 15 & $4 \sqrt{29 / 93}$ & 1. & \pm & & 133.6 & $\mathbf{N}$ & & \\
\hline 10 & 4/29/33 & 1 & 7 & & 138.6 & $\mathbf{Y}$ & & \\
\hline 17 & $4 / 29 / 93$ & 1 & $\overline{7}$ & & 195.7 & $\mathbf{M}$ & & \\
\hline 78 & $4 / 25 / 93$ & 1. & 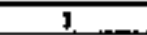 & & 135.8 & $\mathbf{N}$ & & \\
\hline 22 & $4 / 29 / 93$ & 1 & 1 & & & $\mathbf{N}$ & 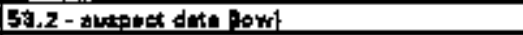 & \\
\hline 9 & sites & 1 & 1 & & 140 & $\mathbf{H}$ & E900325164.5 & \\
\hline 15 & 5 5.93 & 1 & 1 & & $\$ 40.3$ & $\mathbf{N}$ & & \\
\hline 96 & 5 Hets & 1 & 1 & & 143.8 & $y$ & & \\
\hline 17 & ENot 3 & 1 & 1 & & 141,9 & $\mathbf{N}$ & & \\
\hline
\end{tabular}




\begin{tabular}{|c|c|c|c|c|c|c|c|c|}
\hline \multicolumn{8}{|c|}{ 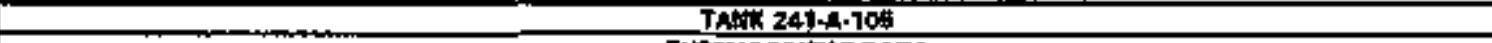 } & \\
\hline 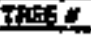 & DATE & 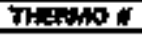 & 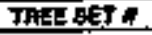 & Tetapd a & T्रूसाF & $Y=M A X$ TDMP & COMAMEITs & \\
\hline 19 & 50/193 & 1 & 1 & & 141,0 & N & & \\
\hline 22 & 5/0/03 & 1 & $\overline{7}$ & & & $\mathbf{N}$ & 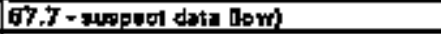 & \\
\hline 9 & घ1309 & 1 & 1 & & 140. & N & & \\
\hline $\mathbf{E}$ & 6/13/53 & 1 & 1 & & 140.3 & N & & \\
\hline 16 & $5 \mu 3 / 3$ & 1 & 1 & & $14 \overline{7} . \overline{1}$ & $y$ & E300325184.98 & \\
\hline 17 & $6 / 4393$ & 1 & $\overline{1}$ & & 144.7 & $\mathbf{N}$ & & \\
\hline 18 & 6hy & 1 & 1 & & 143.4 & $\mathbf{N}$ & & \\
\hline 22 & \$1/3:93 & 1 & 1 & & & $\mathbf{N}$ & 76 - fuspont dids low & \\
\hline 8 & 617263 & 1 & 1 & & 1418 & $\mathbf{N}$ & Eg00025184-59 & \\
\hline 15 & $5 / 2793$ & 1. & 1 & & 140.7 & $\mathbf{N}$ & & \\
\hline 18 & 512落 & 1 & 1 & & 149.3 & $\mathbf{Y}$ & & \\
\hline 17 & 5MI:03 & 1 & 1 & & 144.6 & N & & \\
\hline 19 & MHPA & 1 & 1 & & 143.8 & $\mathbf{H}$ & & \\
\hline 22 & E17293 & 1 & 1 & & & $\mathbf{N}$ & e0 - wapes dite lowh & \\
\hline 9 & 5mapos & 1 & 1 & & 740.2 & $\mathbf{H}$ & $E$ & \\
\hline 16 & Erapa & 1 & 1 & & 740.8 & $\mathbf{N}$ & & \\
\hline 10 & Erioner & 1 & 1 & & 744.5 & $\mathbf{Y}$ & & \\
\hline 17 & $6 / 20 / 93$ & 1 & 1 & & 143 & $\mathbf{N}$ & & \\
\hline 19 & 6/20/93 & 7 & $\mathbf{1}$ & & 142.2 & $\mathbf{N}$ & & \\
\hline 22 & $6 / 20 / 3$ & 1 & 1 & & & $\mathbf{N}$ & 70,8 - aupect dels low] & \\
\hline $\mathbf{\theta}$ & $8 / 24 / 83$ & 1 & 1 & & 142.9 & N & $\begin{array}{r}\text { E037516440 } \\
\end{array}$ & \\
\hline 16 & $8 / 24 \sqrt{13}$ & 1 & 1 & & 143.7 & $\mathbf{N}$ & & \\
\hline 18 & $524 \sqrt{103}$ & 7 & 1 & & 148,4 & $\mathbf{Y}$ & & \\
\hline 17 & 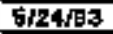 & 1 & 1 & & $14 \$, 1$ & $\mathbf{N}$ & & \\
\hline to & $5 / 24 / 93$ & 1 & 7 & & 145.6 & $\mathbf{N}$ & & \\
\hline 2 & 6t248 & $T$ & \pm & & & $N$ & 78.9 SUSPECT DATA [law & \\
\hline 소 & $6 / 27 / 93$ & 1 & 1. & & 1400 & N & E 0003251645 & \\
\hline 18 & 512703 & 1 & \pm & & 141.4 & * & & \\
\hline 18 & $5 / 27 / 93$ & 1 & $\underline{1}$ & & 144 & $\mathbf{r}$ & & \\
\hline 17 & E/27/93 & 1 & $\overline{1}$ & & j42.18 & $\mathbf{N}$ & & \\
\hline 19 & Б/27/93 & 1 & \pm & & $7,2,4$ & $\mathbf{N}$ & & \\
\hline 22 & 5/27/93 & 1 & 1 & & & $\mathbf{N}$ & 70.5 - SUSPECT DATA laril & \\
\hline 9 & 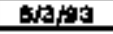 & 1 & 1 & & 139.9 & $\mathbf{N}$ & EThos1 & \\
\hline 15 & $80 / 93$ & 1 & 1 & & 130 & $\mathbf{N}$ & & \\
\hline 10 & 810/93 & 1 & 1. & & 144.0 & $\mathbf{Y}$ & & \\
\hline 17 & $8 \beta$ & $i$ & 1. & & 742.8 & N & & \\
\hline 18 & AMAB & 1 & 1. & & 141.8 & N & & \\
\hline 22 & ARA3 & $i$ & 1 & & 0.8 & $\mathbf{N}$ & 69.8 - avepect dell lowl & \\
\hline 9 & A110 & $t$ & 1 & & 140.3 & $\mathbf{N}$ & $\mathbf{E}$ & \\
\hline 15 & anojes & $i$ & 1 & & 146.5 & $\mathbf{N}$ & & \\
\hline 16 & Anora & $\tau$ & 1 & & 147.8 & $\mathbf{N}$ & & \\
\hline 17 & 自酶方 & 1. & 1 & & 149.5 & $\mathbf{N}$ & & \\
\hline 19 & etosis & 1 & 1 & & 149.1 & $\mathbf{Y}$ & & \\
\hline 22 & etcos & 1 & 1 & & & $\mathbf{N}$ & 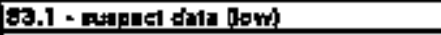 & \\
\hline 旦 & Antidsa & 1 & 1 & & 143.7 & $\mathbf{Y}$ & E Q0032518485 & \\
\hline 15 & OM7/93 & 1 & 1 & & 142.2 & $\mathbf{N}$ & & \\
\hline 16 & arita3 & 1 & $i$ & & 151.2 & $\mathbf{Y}$ & & \\
\hline 12 & artikg3 & 1 & 1 & & 147.9 & $\mathbf{N}$ & & \\
\hline 19 & en7row & 1 & 1 & & 147,4 & $\mathbf{N}$ & & \\
\hline 22 & Milita & 1 & 1 & & $141+8$ & $\mathbf{N}$ & & \\
\hline $\mathrm{g}$ & arziss & 1 & 7 & & 140,7 & $N$ & $\mathbf{E}$ & \\
\hline 16 & 6/21/93 & 1 & 7 & & 141.0 & N & & \\
\hline 10 & 0/21/93 & 1 & 1 & & 144.0 & $\mathbf{Y}$ & & \\
\hline 17 & D/1/93 & 1 & 2 & & 143 & $N$ & & \\
\hline 19 & ar/193 & 1 & $\overline{1}$ & & $142 . \mathrm{B}$ & $N$ & & \\
\hline 22 & e/2nos & 9 & $1, \ldots$ & & 144.5 & 样 & & \\
\hline$\theta$ & O124183 & 1 & $1 \div$ & & 144.8 & $\underline{M}$ & E0101267434-48 & \\
\hline 16 & $6 / 24 / 58$ & 1 & 1 & & 746 & $\mathbf{N}$ & & \\
\hline 18 & $0 / 24 / 98$ & 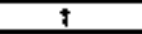 & 1 & & .145 & $N$ & & \\
\hline 17 & d/34/e3 & 1 & 1 & & $14 \mathbf{7}^{2}$ & $\mathbf{r}$ & & \\
\hline ts & O/2A/B & $t$ & 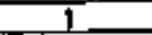 & & 140.3 & $\mathbf{N}$ & & \\
\hline 22 & $2 / 24 \sqrt{63}$ & 1 & 1 & & 148.1 & $\mathbf{N}$ & & \\
\hline 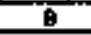 & Thes & $i$ & 1 & & 144.1 & 6 & ET1.019 & \\
\hline 15 & 7nNes & 1 & 1 & & 745 & $\mathbf{H}$ & & \\
\hline 18 & $74 \omega^{3}$ & 7 & 1 & & 1467 & $\mathbf{Y}$ & & \\
\hline 17 & $7 / 1 / 93$ & 7 & $\overline{1}$ & & 145.9 & $N$ & & \\
\hline 18 & $7 / 1 / 93$ & 1 & 1 & & 148 & $\mathbf{N}$ & & \\
\hline 22 & $7 \mathrm{TPA}$ & 1 & 1 & & 164. & N & & \\
\hline
\end{tabular}




\begin{tabular}{|c|c|c|c|c|c|c|c|c|}
\hline & & & & & 1) $241-A$ & 105 & & \\
\hline & & & & TF- & लOCOWD & DATA & & \\
\hline 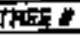 & DATE & THFFHO E & TRET \&ET & TEM 6 & TEMF & $Y=$ MAX TEM & COLWhINTS & \\
\hline 9 & 7 Weses & 1 & 1 & & 147.9 & $\mathbf{N}$ & E $9191320426-104$ & \\
\hline 15 & 7Nojes & 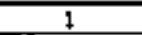 & 7 & & 145.2 & $\mathbf{M}$ & & \\
\hline 78 & $7 \sqrt{1098}$ & \pm & 1 & & 157.7 & $y$ & & \\
\hline 17 & 7/193 & 8 & 1 & & 150,5 & $\mathbf{N}$ & & \\
\hline 19 & 7 thos & 5 & 1 & & 150.2 & $\mathbf{N}$ & & \\
\hline 22 & 7 sisas & 1 & 1 & & & $\bar{N}$ & 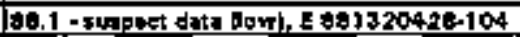 & \\
\hline 9 & $7 / 2 \times 3$ & 1 & $\mathbf{1}$ & & 143,6 & $\mathbf{H}$ & & \\
\hline $\mathbf{3 5}$ & 7/22ta3 & 1 & 1 & & 142.3 & $\mathbf{H}$ & & \\
\hline 78 & Мाля & 7 & 1 & & $148, \theta$ & $\mathbf{Y}$ & & \\
\hline 17 & 71293 & 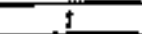 & $\overline{1}$ & & 145.9 & $\mathbf{M}$ & & \\
\hline 19 & $7 / 2993$ & 1 & $\mathbf{1}$ & & 148.3 & $\mathbf{N}$ & & \\
\hline 22 & $7 / 2 / 93$ & 1 & 1 & & & $\bar{N}$ & 75.3 - guapect dats dam & \\
\hline F & $7 / 24 / 93$ & 7. & 1 & & 146,4 & $\mathbf{N}$ & 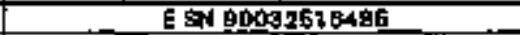 & \\
\hline 15 & 7 724 A & 1 & 1 & & 144,1 & $N$ & & \\
\hline 16 & $7 / 24 / 93$ & 1 & $\overline{1}$ & & $15 \mathrm{Lt} 1$ & $\mathbf{Y}$ & & \\
\hline 17 & $7 / 24 / 93$ & $\overline{1}$ & $\overline{1}$ & & 149 & *ो & & \\
\hline 18 & $7 / 24 / 93$ & $\overline{1}$ & 1 & & 147,2 & N & & \\
\hline 2 & $7 / 24 / 93$ & 1 & 1 & & & $\bar{N}$ & 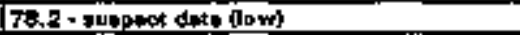 & \\
\hline 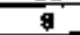 & 720193 & i & 1 & & 149,7 & $\bar{N}$ & TYPEE & \\
\hline 15 & 7MANo & 1 & 1 & & 140.8 & $\mathbf{N}$ & & \\
\hline 16 & $3 / 26 j 93$ & $\mathbf{1}$ & $\overline{1}$ & & 1522 & $\mathbf{Y}$ & & \\
\hline 17 & $7 / 25 / 93$ & 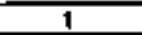 & $T$ & & 151,3 & $\bar{N}$ & & \\
\hline 18 & $7 / 20 / 93$ & $T$ & 1 & & 151.5 & $\bar{N}$ & & \\
\hline 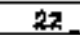 & 720193 & 1 & 1 & & & N & 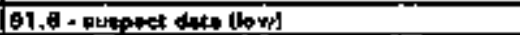 & \\
\hline $\mathbf{g}$ & $8 \mathrm{rapa}$ & $\mathbf{i}$ & 1 & & 151.0 & $\mathbf{N}$ & TH001E & \\
\hline 15 & 8.953 & 1 & 1 & & 148.0 & N & & \\
\hline 16 & $\operatorname{arsis} 3$ & 1 & 1 & & 154.7 & $Y$ & & \\
\hline 17 & 8/9/93 & 1 & 1 & & 153,0 & $\mathbf{N}$ & & \\
\hline 10 & $8 / 9 / 93$ & $\mathbf{1}$ & 1 & & 152.2 & $\mathbf{N}$ & & \\
\hline s & 8/1बलs & $T$ & i & & $\mathbf{1 4 1 . 4}$ & $\mathbf{N}$ & 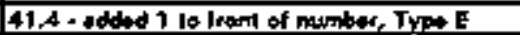 & \\
\hline 15 & 9hans & $\mathbf{T}$ & 1 & & 143.2 & $\mathbf{N}$ & $49.2=$ added 1 to trom of number & \\
\hline 18 & anto/s3 & 1 & $t$ & & 145.4 & $\Psi$ & & \\
\hline 17 & attos 3 & 1 & 1 & & $t+3.0$ & $\mathbf{N}$ & & \\
\hline 19 & ansis3 & 1 & $\overline{1}$ & & 743,4 & $\mathbf{N}$ & & \\
\hline 22 & Ghas & 1 & 1 & & & $\mathbf{H}$ & 12.2 - sureged doll llow| & \\
\hline 8 & 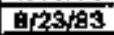 & 1 & $\bar{t}$ & & 743.5 & $\mathbf{N}$ & E 90032516447 & \\
\hline 15 & $8 / 2303$ & 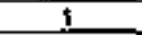 & \pm & & 143.4 & $\mathbf{N}$ & & \\
\hline 18 & $8 / 22 / 93$ & 1 & 1 & & LAOA & $y$ & & \\
\hline 17 & $8 / 2393$ & 1 & 1 & & 145.0 & $\mathbf{N}$ & & \\
\hline 19 & 9/2353 & 1 & 1 & & 148.9 & $\mathbf{N}$ & & \\
\hline $2 \overline{2}$ & $6 \sqrt{23 / 93}$ & 1 & $\overline{1}$ & & & $\mathbf{N}$ & 70,8 - wapeet deth lowl & \\
\hline 8 & E/30193 & 7 & 7 & & 148.5 & $\mathbf{N}$ & E30032516410 & \\
\hline 13 & 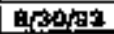 & $t$ & 1 & & 147.3 & $\bar{H}$ & & \\
\hline 16 & anatas & 1 & $i$ & & $\sqrt{52.8}$ & $\bar{Y}$ & & \\
\hline 17 & 9/30093 & 1 & $\overline{1}$ & & 149.7 & $\mathbf{N}$ & & \\
\hline 19 & 630193 & 1 & 1 & & 140.2 & $\mathbf{N}$ & & \\
\hline $2 z$ & Brofias & 1 & 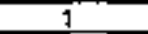 & & & $\mathbf{N}$ & 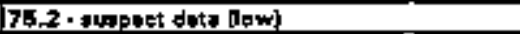 & \\
\hline 動 & $9 / 2+83$ & 1 & 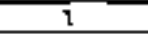 & & 150 & $\mathbf{N}$ & $\begin{array}{r}\text { ETYPE } \\
\end{array}$ & \\
\hline 13 & $9 \operatorname{ses} 2$ & 1 & 1 & & .342 .2 & $\mathbf{N}$ & & \\
\hline 16 & $9 / 293$ & $\overline{1}$ & $\overline{1}$ & & 193.0 & $\dot{\gamma}$ & & \\
\hline i) & $9 / 29$ & $\frac{1}{1}$ & $\overline{1}$ & & 151.5 & $\mathbf{H}$ & & \\
\hline 19 & $9 / 2+83$ & 1 & $\frac{1}{1}$ & & 151.8 & $\mathbf{N}$ & & \\
\hline 22 & $9 \sqrt[2]{203}$ & 1 & $t$ & & 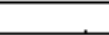 & N & 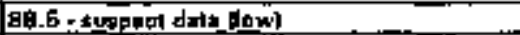 & \\
\hline औै & 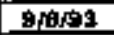 & 1 & $t$ & & 149 & $\overline{\mathbf{N}}$ & TYPEE & \\
\hline 15 & $9 / 5193$ & 1 & 1 & & 145.0 & $\mathbf{N}$ & & \\
\hline 10 & $6 / 0+93$ & 1 &. & & 153.7 & $\bar{Y}$ & & \\
\hline 17 & $9 / 899$ & b. & 7. & & 151 & $\mathbf{H}$ & & \\
\hline 19 & 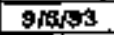 & 1 & 1 & & 151.3 & $\mathbf{N}$ & & \\
\hline 22 & gitis & 1 & $t$ & & & $\mathbf{N}$ & 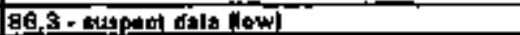 & \\
\hline$\theta$ & $9 / 13 / 93$ & 1 & 1 & & $\$ 44.2$ & $\mathbf{N}$ & ETR-O49 & \\
\hline 15 & 97. & 1 & 7 & & 141.3 & $\mathbf{H}$ & & \\
\hline 18 & 9лस & $\overline{1}$ & $\overline{1}$ & & 149,8 & $\bar{Y}$ & & \\
\hline 17 & 9/3E3 & 1 & $\overline{1}$ & & 146.9 & $\bar{n}$ & & \\
\hline 10 & g/t\$s & 1 & 1 & & 148 & $\mathbf{N}$ & & \\
\hline 22 & $9 / 13 / 93$ & 1 & 1 & & & $\mathbf{N}$ & 76.3 - enspact duts llowl & \\
\hline 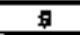 & Motas & 1 & 1 & & 144.4 & $\mathbf{N}$ & $E T 001$ & \\
\hline 15 & 976 & 5 & $T$ & & 143.3 & $\mathbf{N}$ & & \\
\hline 10 & 0rster & $\frac{1}{1}$ & $\overline{1}$ & & 148.6 & $Y$ & & \\
\hline
\end{tabular}




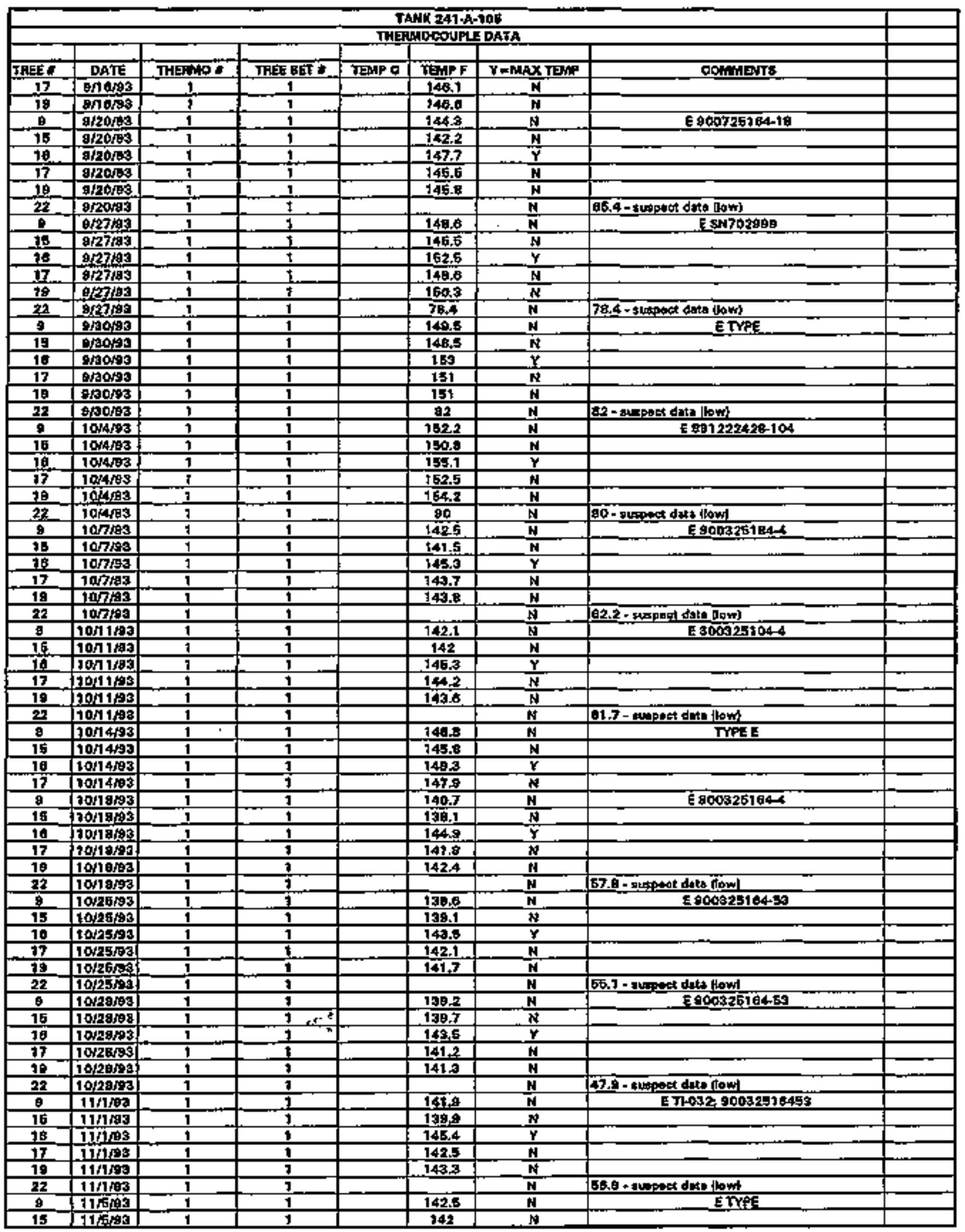




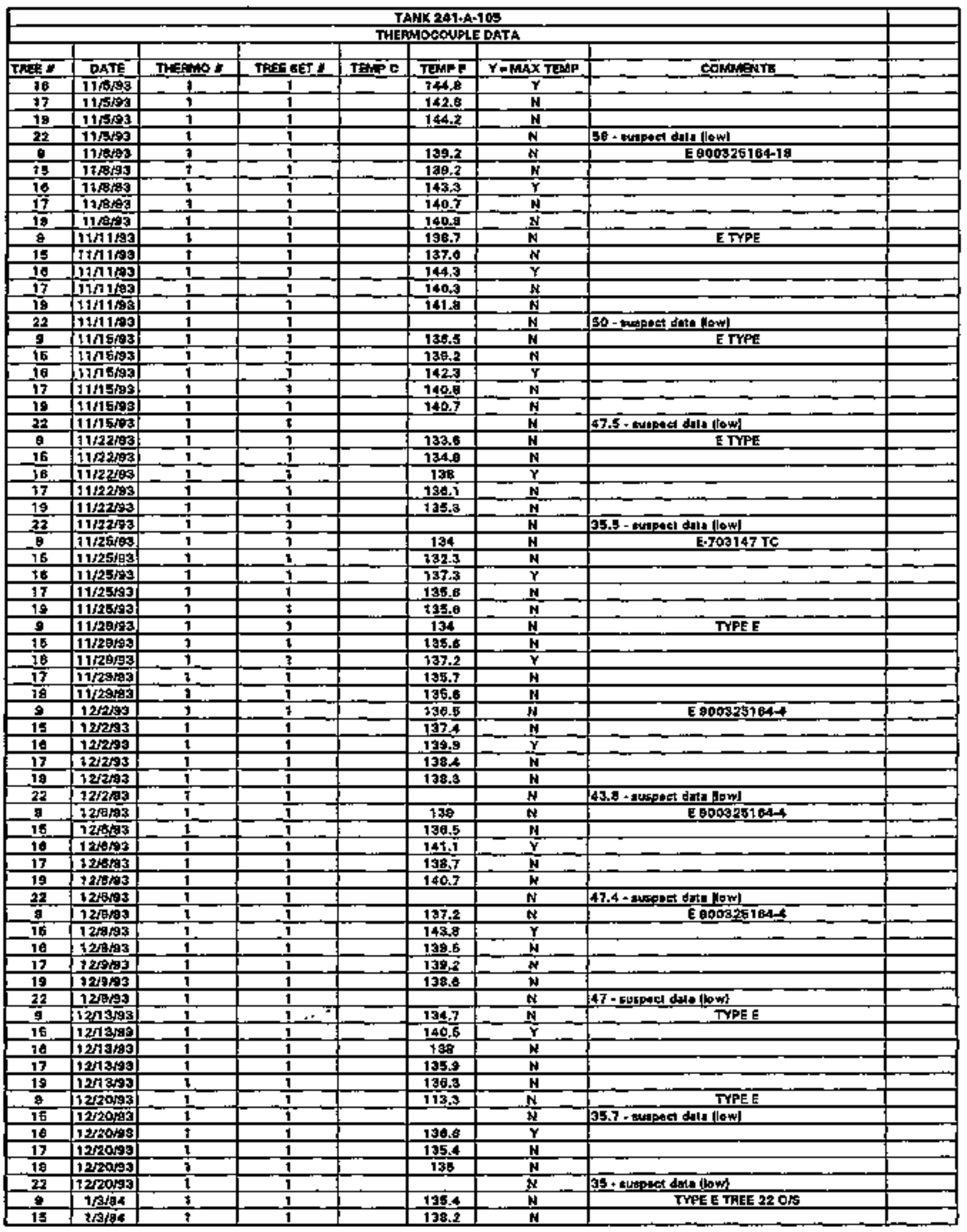




\begin{tabular}{|c|c|c|c|c|c|c|c|c|}
\hline \multirow{2}{*}{\multicolumn{9}{|c|}{ JWNK 241-A-305 }} \\
\hline & & & & & & & & \\
\hline Titer & D隹 & Thesen & 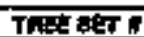 & TINP 0 & TLAPF & $\gamma=$ MAX TEAP & COMMANTE & \\
\hline 10 & $\sqrt{1254}$ & 1 & 1 & & I的息 & $y$ & & \\
\hline 17 & 104 & 1 & 1 & & $130 \mathrm{~B}$ & $\mathbf{N}$ & & \\
\hline 18 & 1004 & 1 & i & & 137.2 & $\overline{\mathbf{N}}$ & & \\
\hline 72 & 1064 & 1 & $t$ & & & $\mathbf{N}$ & & \\
\hline
\end{tabular}

$$
\therefore
$$




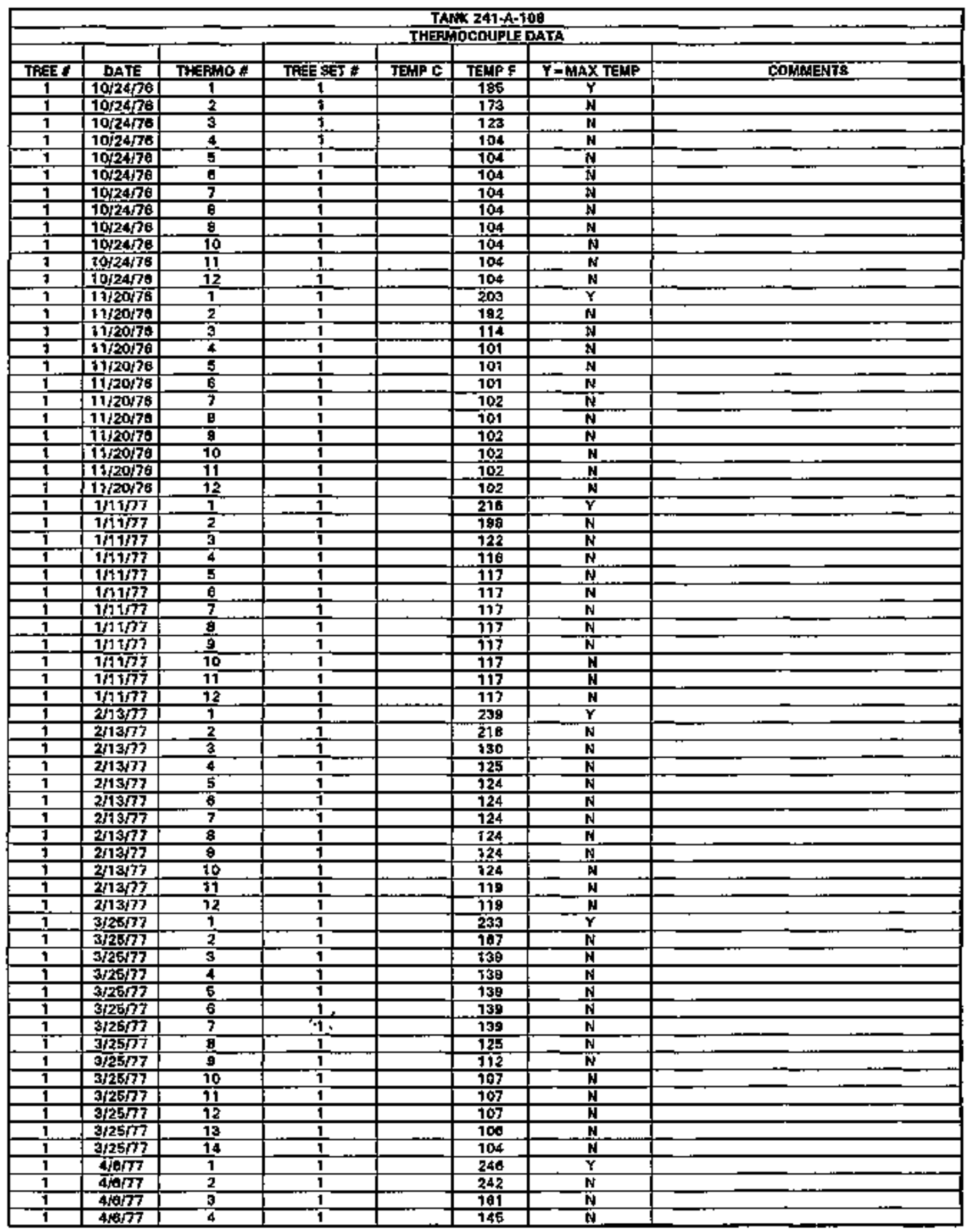




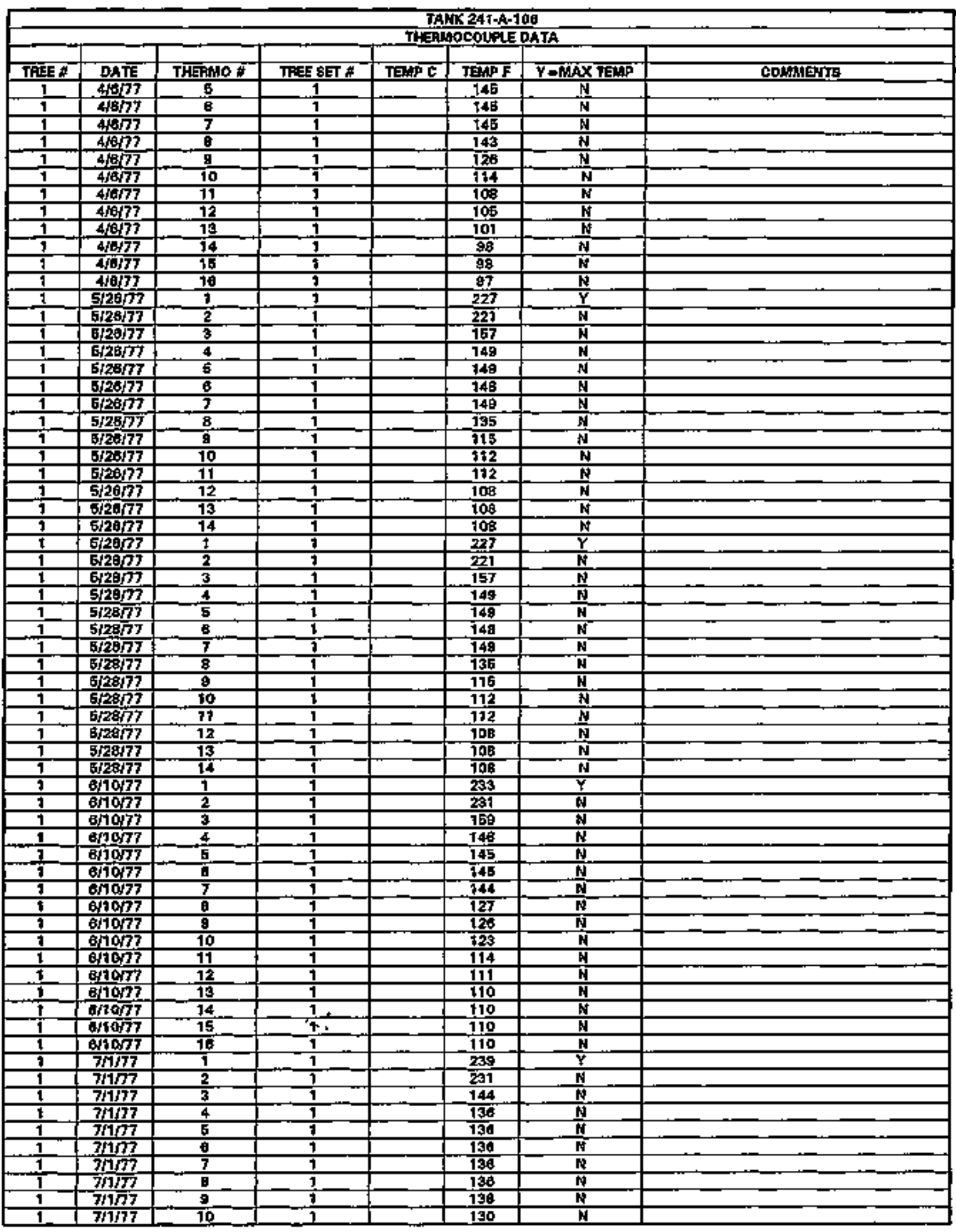

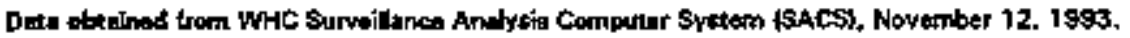




\begin{tabular}{|c|c|c|c|c|c|c|c|}
\hline \multicolumn{8}{|c|}{ 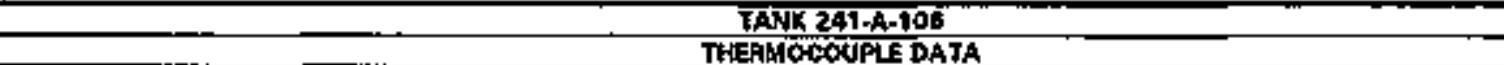 } \\
\hline & & & & & & & \\
\hline ThEE \# & DATE & THEFAO \# & TARE SET \& & TEMPC & TEMP F & Y = MAX YEMP & COMDENTS \\
\hline-1 & 7 Wh7 & t1 & 1 & & 129 & $\mathrm{~N}$ & \\
\hline 9 & $7 / 77$ & 92 & 1 & & 125 & $\mathbf{N}$ & \\
\hline$t$ & $7 / 177$ & 13 & 1 & & $\sqrt{28}$ & $\mathbf{N}$ & \\
\hline 1 & $7 / 1 / 77$ & 14 & $T$ & & 128 & $\overline{\mathbf{N}}$ & \\
\hline 1 & $71 \% 7$ & T5 & 1 & & 128 & $\mathbf{N}$ & \\
\hline 9 & $7 / 1 / 7$ & 9 & 1 & & 128 & $\mathbf{N}$ & \\
\hline 9 & $7 / 277$ & 17 & $\overline{1}$ & & 127 & $\mathbf{N}$ & \\
\hline$T$ & $7+\sqrt{7}$ & Tg & 1 & & 127 & $M$ & \\
\hline 1 & $7114 \sqrt{7}$ & 1 & 1 & & 241 & $Y$ & \\
\hline 1 & $7 / 14 / 77$ & 2 & 1 & & 227 & $N$ & \\
\hline 1 & $7 / 14 / 73$ & 3 & 1 & & 144 & $\mathbf{N}$ & \\
\hline 1 & $7 \pi 4 / 7$ & 4 & $\overline{1}$ & & 137 & $\bar{N}$ & \\
\hline 1 & $7 / 14 / 77$ & $\mathbf{5}$ & 1 & & 137 & $\mathbf{N}$ & \\
\hline$T$ & $7 / 14 / 77$ & 6 & 1 & & 137 & $N$ & \\
\hline 1 & $7114 / 77$ & 7 & 1 & & 137 & N & \\
\hline 1 & $7 / 14 / 7$ & 8 & 1 & & 137 & $\mathbf{N}$ & \\
\hline 1 & $714 / 77$ & 9 & 1 & & 137 & $\mathbf{N}$ & \\
\hline 1 & $7 \sqrt{1477}$ & 10 & 1 & & 132 & $\bar{M}$ & \\
\hline 1 & $714 / 77$ & 11 & 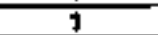 & & 131 & M & \\
\hline 1 & 71477 & 12 & 1 & & 131 & $\mathbf{N}$ & \\
\hline 1 & $714 \sqrt{77}$ & 13 & $\frac{1}{1}$ & & 137 & $\mathrm{~N}$ & \\
\hline 1 & $7 / 14 / 77$ & 14 & 1 & & 191 & $\mathbf{N}$ & \\
\hline 1 & $7 / 14 / 77$ & 16 & 1 & & 131 & $\mathbf{N}$ & \\
\hline$\overline{1}$ & $7 / 14 / 77$ & 16 & 1 & & 130 & $\mathbf{M}$ & \\
\hline 1 & $7 / 14 / 77$ & 17 & 1 & & 130 & $N$ & \\
\hline 7 & $7 / 14 / 77$ & 18 & 3 & & 129 & $\bar{N}$ & \\
\hline 1 & $7 / 14 / 77$ & 1 & $\frac{2}{2}$ & & 238 & $\bar{Y}$ & TF ping. \\
\hline 1 & $7 / 14 / 77$ & 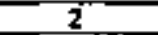 & $\overline{2}$ & & 224 & $\bar{N}$ & \\
\hline 1 & $7 / 14 \sqrt{7}$ & 3 & $\overline{2}$ & & 135 & $\mathbf{N}$ & \\
\hline 1 & 711407 & 4 & 2 & & 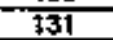 & $\mathbf{N}$ & \\
\hline 1 & $7 / 6477$ & 5 & 2 & & 131 & $\bar{N}$ & \\
\hline 7 & $714 \sqrt{7}$ & 8 & 2 & & 131 & $\mathbf{N}$ & \\
\hline 9 & $7 \sqrt{4 \sqrt{7}}$ & 7 & 2 & & 133 & $\mathbf{N}$ & \\
\hline 9 & $7114 \sqrt{7}$ & $\bar{\theta}$ & 2 & & 131 & $\mathbf{N}$ & \\
\hline$\$$ & $7 / 14 / 77$ & $\theta$ & 2 & & 131 & 产 & \\
\hline 1 & $7 / 34 \sqrt{7}$ & 10 & 2 & & 728 & $N$ & \\
\hline 1 & $7 / 14 / 77$ & 11 & 2 & & 528 & $N$ & \\
\hline 1 & $7 / 4 \sqrt{7}$ & 12 & 2 & & 728 & $\mathbf{N}$ & \\
\hline 1 & $714 \sqrt{7}$ & 13 & $\overline{2}$ & & 126 & $\mathbf{N}$ & \\
\hline 1 & $714 \sqrt{7}$ & 14 & $Z$ & & 126 & $\mathbf{N}$ & \\
\hline$T$ & $714 / 77$ & 16 & 2 & & 126 & $\mathbf{N}$ & \\
\hline 1 & $7 / 44 \sqrt{7}$ & 16 & 2 & & 126 & $N$ & \\
\hline 1 & $7 / 4 \sqrt{7}$ & 17 & $\overline{2}$ & & 128 & $\mathbf{N}$ & \\
\hline 1 & $714 \sqrt{7}$ & 16 & 2 & & 122 & $\mathrm{~N}$ & \\
\hline 1 & BilBip) & 2 & 1 & & 214 & $\mathbf{Y}$ & \\
\hline 1 & в,18/7 & 2 & 1 & & 207 & $\mathbf{N}$ & \\
\hline 1 & बing & 3 & $T$ & & 131 & $\bar{N}$ & \\
\hline 1 & a/18/77 & 4 & $\overline{1}$ & & 106 & $N$ & \\
\hline$\overline{1}$ & 9/8h7 & $\frac{5}{8}$ & 1 & & 105 & $\mathbf{N}$ & \\
\hline 1 & 9/8/7 & 6 & 1 & & 101 & $\mathbf{N}$ & \\
\hline 1 & entibs & 7 & 1 & & 201 & $\mathbf{N}$ & \\
\hline 1 & (m8h) & 8 & 1 & & 501 & N & \\
\hline 1 & 배그 & 9 & 1 & & 101 & $\mathbf{N}$ & \\
\hline 1 & a/1B/7) & 10 & 1. & & 101 & $N$ & \\
\hline 1 & - & J1 & 1. & & 101 & $\bar{N}$ & \\
\hline 1 & G/18/7) & 22 & 1 & & 101 & $N$ & \\
\hline$T$ & EMBh7 & 13 & 1 & & 101 & $\bar{N}$ & \\
\hline$\overline{1}$ & EN18/7 & 14 & 1 & & 101 & $N$ & \\
\hline 1 & 6/18/7 & 15 & 1 & & 101 & $\mathbf{N}$ & \\
\hline 1 & $0 / 1877$ & 90 & 1 & & 101 & $\bar{N}$ & \\
\hline 7 & 813077 & 1 & 1 & & 225 & $\bar{Y}$ & \\
\hline 7 & $8+3077$ & 2 & 1 & & $22 t$ & $\overline{\mathbf{N}}$ & \\
\hline 1 & $8 \mathrm{~s} 30 \mathrm{7} 77$ & 3 & 1 & & 534 & $\mathbf{N}$ & \\
\hline 7 & s3ort7 & 4 & 1 & & 211 & $N$ & \\
\hline 7 & 8/sort7 & 5 & 1 & & $\$ 11$ & $\mathrm{~N}$ & \\
\hline 1 & अ30ㄱ7 & 9 & 1 & & $\$ 11$ & $\mathbf{N}$ & \\
\hline
\end{tabular}




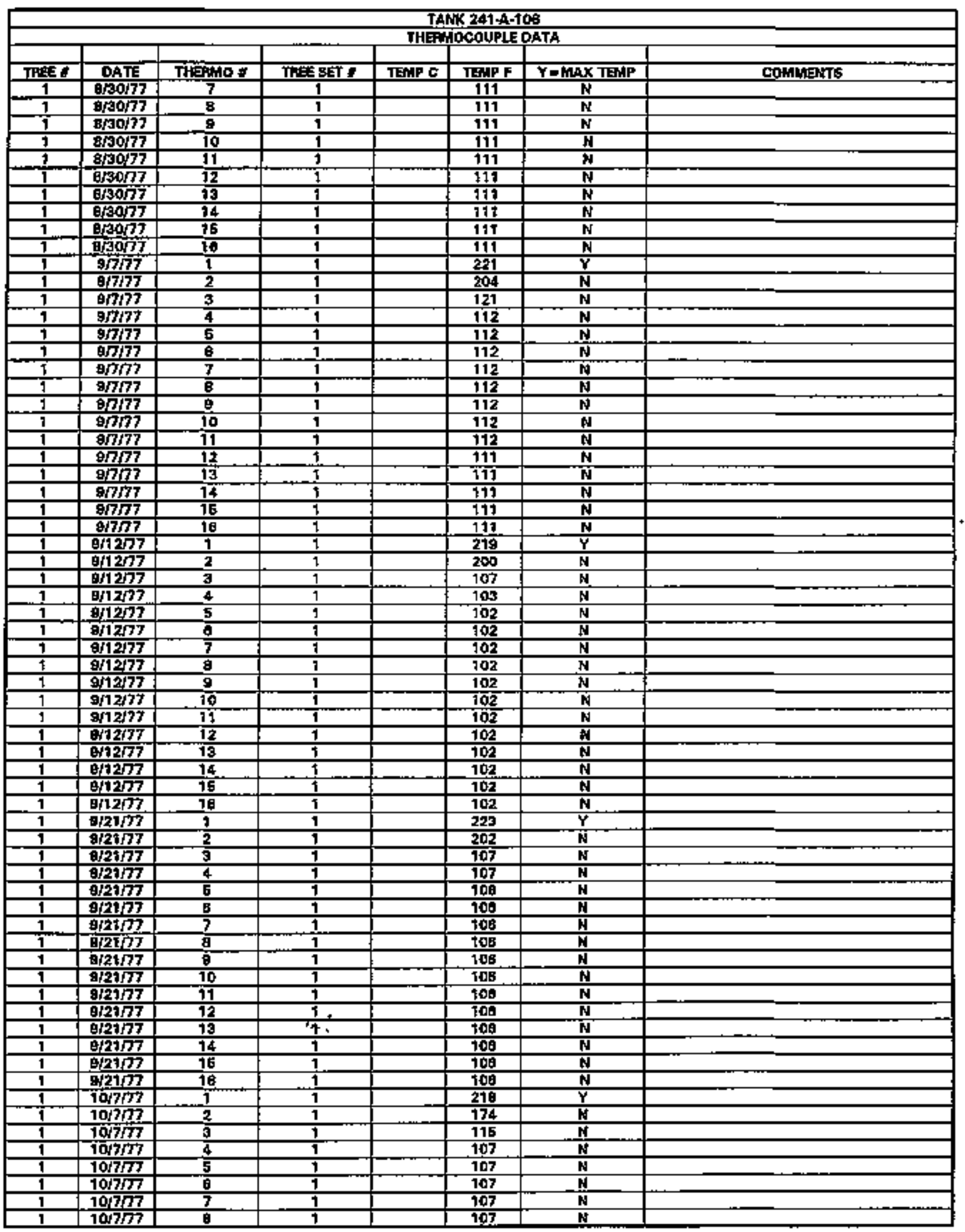

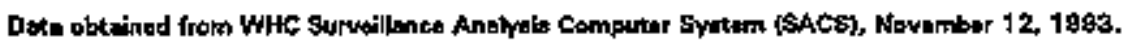




\begin{tabular}{|c|c|c|c|c|c|c|c|}
\hline \multirow{2}{*}{\multicolumn{8}{|c|}{ TANR 241-A-100 }} \\
\hline & & & & & ocolpis & AtA & \\
\hline TAEE * & BATE & THERMO : & TRE SET * & TENFC & TEUFF & $Y=M A X T E M P$ & COWHENTS \\
\hline 1 & $10 / 7 / 7$ & $\theta$ & 1 & & 107 & $-\mathbf{N}$ & \\
\hline j & $10 / 7 / 7$ & 10 & $\overline{7}$ & & 107 & $\mathbf{N}$ & \\
\hline 1 & $10 / 777$ & $\overline{11}$ & $\overline{1}$ & & $\overline{108}$ & $\bar{N}$ & \\
\hline 1 & $10 \% / 77$ & 12 & 1 & & $10 \mathrm{~B}$ & $N$ & \\
\hline 1 & $10 / 3 / 7$ & 13 & 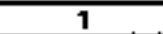 & & 108 & $\mathbf{N}$ & \\
\hline 1 & $10 / 7 / 77$ & 14 & 1 & & 100 & $N$ & \\
\hline 1 & $10 / 7 \pi 7$ & 15 & 1 & & 108 & $\mathbf{N}$ & \\
\hline 1 & $1017 / 77$ & 18 & 1 & & 108 & $\mathbf{N}$ & \\
\hline 1 & $11 / 7 \pi 7$ & 1 & $i$ & & 206 & 7 & \\
\hline$\overline{1}$ & $11 / 7 / 77$ & 2 & $\bar{\imath}$ & & 169 & $\bar{N}$ & \\
\hline$\overline{1}$ & $11 / 7 / 7$ & $\frac{5}{3}$ & $i$ & & 88 & $N$ & \\
\hline 1 & $11 / 777$ & 4 & 1 & & 96 & $\mathbf{N}$ & \\
\hline 1 & $11 \cap D 7$ & 5 & 7 & & 95 & $\mathbf{N}$ & \\
\hline 1 & $11 / 7 \% 7$ & 8 & 1 & & 95 & $\mathbf{N}$ & \\
\hline$\overline{1}$ & $11 / 7 / 77$ & 7 & $\overline{1}$ & & 96 & $N$ & \\
\hline$\overline{1}$ & $11 / 7 / 77$ & 8 & 1 & & 86 & $\mathbf{N}$ & \\
\hline 1 & $11 / 7 / 77$ & $\theta$ & 7 & & 86 & N & \\
\hline 1 & $11 / 707$ & 10 & $\overline{7}$ & & 96 & $N$ & \\
\hline 1 & 111107 & 11 & $\mathbf{j}$ & & 98 & $N$ & \\
\hline $\mathbf{1}$ & $11 / 7 / 77$ & 12 & 1 & & 95 & $N$ & \\
\hline 1 & $11 / \pi / 7$ & 13 & $T$ & & 90 & $\bar{N}$ & \\
\hline j & $91 / 7 / 77$ & 14 & 1 & & 95 & $\bar{N}$ & \\
\hline 7 & $11 / 7 / 77$ & 15 & 1 & & E5 & 졍 & \\
\hline 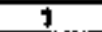 & $11 m 7$ & 16 & 1 & & 96 & $\mathbf{N}$ & \\
\hline$\$$ & $72 / 477$ & 1 & 1 & & 198 & $\mathbf{Y}$ & \\
\hline 1 & $12 / 477$ & $\overline{2}$ & 1 & & 151 & $\sqrt{n t}$ & \\
\hline 3 & $12 / 4 \sqrt{77}$ & 3 & 1 & & 93 & N & \\
\hline$i$ & $12 \sqrt{47}$ & 4 & $T$ & & 99 & $N$ & \\
\hline$i$ & $12 \sqrt{4 / 7}$ & 5 & 1 & & 89 & N & \\
\hline $\bar{t}$ & $12 \sqrt{47}$ & $\bar{\theta}$ & 1 & & 90 & $\mathbf{N}$ & \\
\hline 7 & 124477 & 7 & 1 & & 89 & to & \\
\hline 7 & $12 / 4 n ?$ & 要 & 1 & & $\mathrm{gg}$ & $\mathbf{n}$ & \\
\hline 7 & $12 / 4 h 7$ & 蒠 & 1 & & 89 & is & \\
\hline 1 & $12 / 4 / 77$ & 10 & i & & $\mathbf{B 9}$ & $\bar{N}$ & \\
\hline 1 & $12 / 4 / 77$ & 11 & 1 & & 69 & N & \\
\hline 1 & $12 \sqrt{4 / 7}$ & 12 & 1 & & 69 & $\bar{F}$ & \\
\hline 1 & $12 / 4 / 77$ & 13 & $T$ & & 89 & $\bar{N}$ & \\
\hline$\overline{1}$ & $12 \sqrt{4 / 77}$ & 14 & 1 & & 8 & $\bar{N}$ & \\
\hline$\overline{1}$ & 124477 & 15 & 1 & & 8 & $\mathbf{N}$ & \\
\hline 1 & $12 / 477$ & 18 & 1 & & $\mathbf{B D}$ & $\mathbf{N}$ & \\
\hline 1 & $1 / 16 / 78$ & 1 & 1 & & 183 & $\bar{Y}$ & \\
\hline 1 & 1/16/7B & 2 & $T$ & & 342 & $\bar{H}$ & \\
\hline 1 & 1Ha/TB & 3 & 1 & & 95 & $\mathbf{H}$ & \\
\hline 1 & $1 \pi 978$ & 4 & 1 & & 96 & IN & \\
\hline$\overline{1}$ & $1 / 16 / 78$ & 5 & 1 & & 94 & $\bar{N}$ & \\
\hline 1 & $1 / 16 / 70$ & $\theta$ & 1 & & 84 & $N$ & \\
\hline 1 & $1 / 2 \theta / 7 \theta$ & 7 & 1 & & 84 & N & \\
\hline 7 & 1/ti/78 & 8 & 1 & & 84 & Tín & \\
\hline 1 & $1 / 16 / 78$ & $\theta$ & 1 & & 84 & $\mathbf{N}$ & \\
\hline 1 & 1/A678 & 10 & $T$ & & 94 & H & \\
\hline$\overline{1}$ & $1 / 1678$ & 11 & 1 & & 94 & N & \\
\hline 1 & $1 / 30 / 78$ & 12 & 1 & & 04 & H & \\
\hline 1 & 1月6/78 & 13 & 1 & & 94 & $\bar{n}$ & \\
\hline 1 & $1 / 16 / 78$ & 14 & 1. & & 血 & $\mathbf{N}$ & \\
\hline 1 & $1 / 26 / 78$ & 1 & $7=$ & & 173 & $\mathbf{Y}$ & \\
\hline 1 & $1 / 29 / 7 \mathrm{~s}$ & 2 & 1 & & $13 B$ & $\mathbf{N}$ & \\
\hline 1 & $1 / 29 / 7 \mathrm{~B}$ & 3 & 1 & & 93 & $\mathbf{N}$ & \\
\hline 1 & $9 / 28 / 78$ & 4 & 1 & & 92 & $\mathbf{M}$ & \\
\hline 1 & $9 \sqrt{2} 8 / 76$ & 5 & 1 & & 92 & in & \\
\hline 7 & $1 / 28 / 78$ & 5 & 1 & & 92 & $N$ & \\
\hline 1 & $1 / 26 / 78$ & 7 & 7 & & Q2 & $\mathbf{N}$ & \\
\hline 1 & $1 / 26 / 78$ & 8 & 1 & & 81 & $\mathbf{N}$ & \\
\hline 1 & $1 / 29 / 78$ & P & 1 & & 91 & $\mathbf{N}$ & \\
\hline 1 & $1 / 2 \mathrm{a} / 7 \mathrm{~B}$ & 10 & 1 & & 91 & $\mathbf{N}$ & \\
\hline 1 & $1 / 2 a / 75$ & 11 & $T$ & & 91 & $\bar{M}$ & \\
\hline 1 & 1/za/TB & 12 & 1 & & 91 & $\bar{a}$ & \\
\hline
\end{tabular}




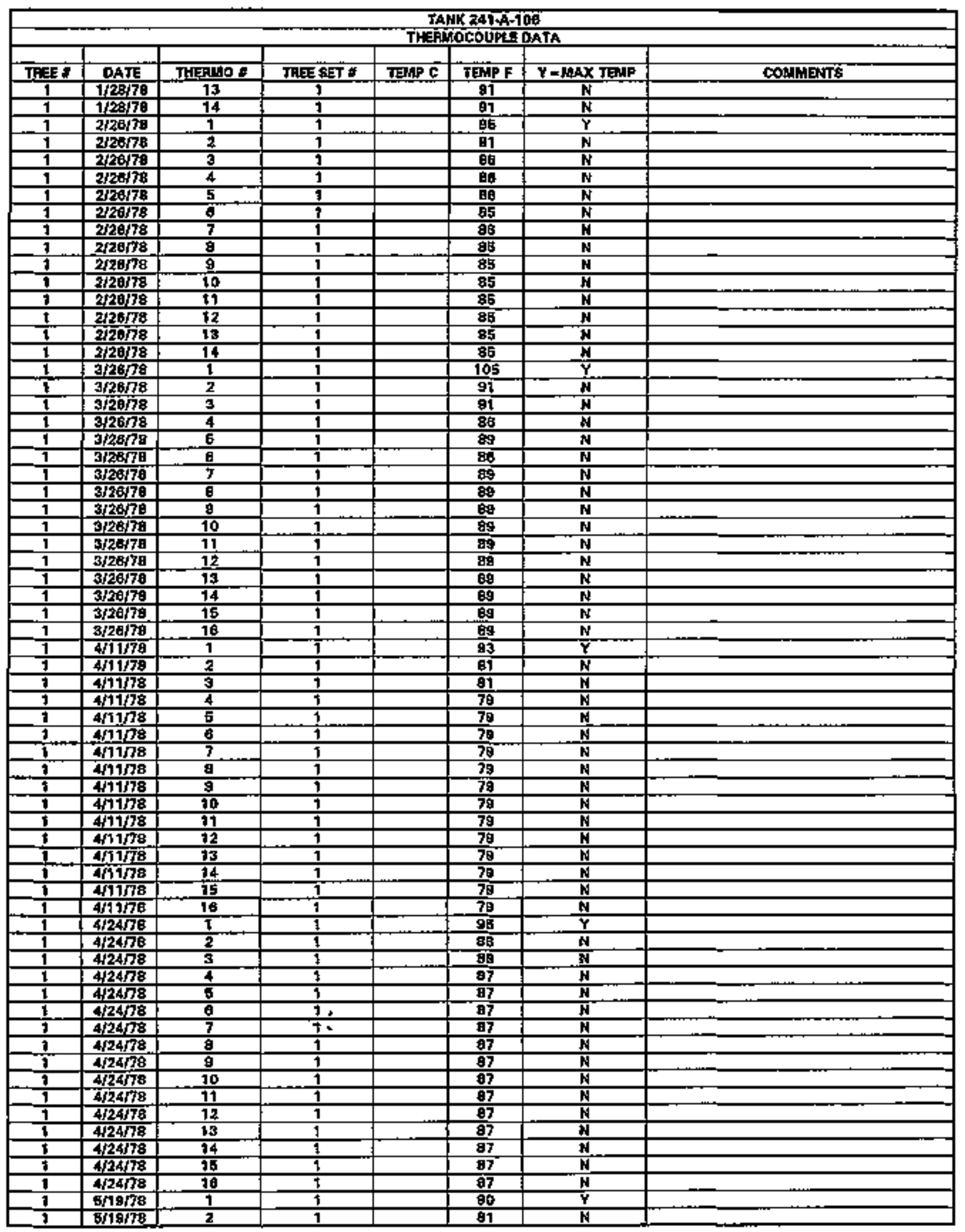

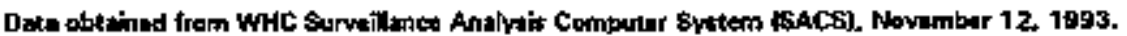




\begin{tabular}{|c|c|c|c|c|c|c|c|}
\hline \multicolumn{8}{|c|}{ TANA 241-A.100 } \\
\hline & & & & & & & \\
\hline IFE & DATE. & TH邹UO & TREE SET & FEAPC & TENPF & $Y=$ WAX 愐P & CDLWHENTS \\
\hline$i$ & $5119 / 78$ & 3 & 1 & & 79 & $\mathrm{~N}$ & \\
\hline$\overline{9}$ & \$19/78 & 4 & 1 & & 78 & $\mathbf{N}$ & \\
\hline I & B/46/78 & 5 & 1 & & $7 \theta^{2}$ & $\mathbf{N}$ & \\
\hline 1 & 510न78 & a & 1 & & $7 \theta$ & $\mathbf{N}$ & \\
\hline $\mathbf{1}$ & $5 / 19 / 78$ & 7 & $\bar{J}$ & & 79 & $\mathbf{N}$ & \\
\hline 1 & $5197 \mathrm{~B}$ & 8 & 1 & & 79 & $\mathbf{H}$ & \\
\hline $\mathbf{1}$ & $5 / 19 / 78$ & 9 & 1 & & 79 & $N$ & \\
\hline I & E/19/7日 & 10 & 1 & & 79 & $\mathbf{N}$ & \\
\hline 1 & 5ा97\% & 11 & 1 & & 79 & $\mathbf{N}$ & \\
\hline$\overline{1}$ & \$1976 & 12 & i & & 79 & $\mathbf{N}$ & \\
\hline 1 & 51970 & 13 & 1 & & 79 & $\overline{\mathbf{N}}$ & \\
\hline$\overline{1}$ & हलभife & 14 & 9 & & 79 & $\bar{N}$ & \\
\hline$T$ & E/19/78 & 15 & $\frac{9}{9}$ & & 79 & $\bar{N}$ & \\
\hline 1 & 61978 & 16 & 1 & & 79 & $N$ & \\
\hline 1 & 617778 & 1 & $\bar{J}$ & & $\mathbf{9 8}$ & $\bar{Y}$ & \\
\hline 1 & $0 / 17 / 7 a$ & 2 & 1 & & 87 & $\mathbf{N}$ & \\
\hline 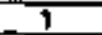 & 6ring & 3 & 1 & & 96 & $\overline{\mathbf{N}}$ & \\
\hline 1 & \$91778 & 4 & T & & 65 & $\overline{\mathbf{M}}$ & \\
\hline 1 & $817 / 70$ & 5 & 1 & & 86 & $\mathbf{N}$ & \\
\hline 1 & $6 / 17 / 79$ & 皇 & 1 & & es & N & \\
\hline 1 & क517/79 & 7 & 1 & & 65 & $\bar{N}$ & \\
\hline 1 & 811778 & $\bar{B}$ & 1 & & 前 & $\mathbf{N}$ & \\
\hline 1 & B/17/7g & 蒠 & 1 & & 85 & $\mathbf{N}$ & \\
\hline 1 & $6 / 17 / 78$ & 10 & 1 & & 85 & $\bar{N}$ & \\
\hline 1 & 6 617/7a & 11 & 1 & & B5 & $\mathbf{M}$ & \\
\hline T & 67176 & 12 & $T$ & & 85 & $\mathbf{N}$ & \\
\hline 1 & $6 / 17 / 79$ & 13 & 1 & & ES & $\mathbf{N}$ & \\
\hline $\bar{t}$ & $6+7 / 78$ & 14 & 1 & & 86 & $\mathbf{N}$ & \\
\hline 9 & 917/8 & 15 & 1 & & 皇5 & $\mathbf{N}$ & \\
\hline 7 & 봬강 & 18 & 1 & & 95 & $\mathbf{N}$ & \\
\hline 1 & $715 / 78$ & 1 & 1 & & 94 & $\gamma$ & \\
\hline$T$ & 715178 & 2 & 1 & & 的 & $\bar{N}$ & \\
\hline 1 & 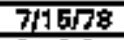 & 3 & 1 & & 8B & N & \\
\hline J & 7nG78 & 4 & 1 & & 晒 & $\mathbf{M}$ & \\
\hline 1 & MБГ8 & 5 & 1 & & e日8 & $\mathbf{N}$ & \\
\hline 1 & TI1578 & 8 & 1 & & 80 & $\mathbf{N}$ & \\
\hline 1 & 71578 & 7 & 1 & & $\theta$ & N & \\
\hline 1 & $7 / 1578$ & 8 & 1 & & 昌 & $\mathrm{N}$ & \\
\hline 1 & $715 \sqrt{78}$ & 白 & 1 & & 80. & $\mathbf{N}$ & \\
\hline 1 & 7115교 & 10 & 1 & & 86 & $\mathbf{N}$ & \\
\hline 1 & $7 / 15 / 78$ & 11 & 1 & & 可 & $\mathbf{N}$ & \\
\hline 1 & 715न7 & 12 & 1 & & 80 & $N$ & \\
\hline$T$ & /15/78 & 13 & 1 & & 96 & N & \\
\hline 1 & W1578 & 14 & 1 & & B8 & $\mathbf{N}$ & \\
\hline 1 & /1578 & 76 & 1 & & Bo & $\mathbf{N}$ & \\
\hline 1 & भIDE & 78 & 1 & & A6 & $\mathbf{N}$ & \\
\hline i & 9i1378 & 1 & $i$ & & 103 & $\mathbf{Y}$ & \\
\hline$\overline{1}$ & ब1338 & 2 & 1 & & 82 & $\mathbf{N}$ & \\
\hline 1 & 배3 & $\sqrt[3]{3}$ & 1 & & $\mathbf{9 2}$ & $\bar{N}$ & \\
\hline 1 & 6/1378 & 4 & 1 & & 81 & $\mathbf{N}$ & \\
\hline 1 & 9M37a & 5 & 1 & & 81 & $\mathbf{N}$ & \\
\hline 1 & QVI/7/7 & 6 & 1 & & 81 & $\mathbf{N}$ & \\
\hline 1 & $8 / 1379$ & 7 & 1 & & 82 & $\mathbf{N}$ & \\
\hline 1 & ब/3\% & 8 & 1. & & 82 & N & \\
\hline 1 & gy/317 & 9 & 7. & & g2 & $\bar{N}$ & \\
\hline 1 & 9rim7e & 90 & 1 & & 82 & $\bar{N}$ & \\
\hline 1 & drił7 & 11 & 7 & & 82 & $\bar{N}$ & \\
\hline 1 & - & $\sqrt{2}$ & 1 & & 皇2 & $\mathbf{N}$ & \\
\hline 1 & 배13교 & 73 & 1 & & 92 & $\mathbf{N}$ & \\
\hline 1 & 배अ & 74 & 1 & & B2 & $\mathbf{N}$ & \\
\hline 1 & 夏/3/78 & 15 & 1 & & $\overline{82}$ & $\mathbf{N}$ & \\
\hline 1 & 8/1378 & 18 & 1 & & 82 & $\bar{N}$ & \\
\hline 1 & 9 997a & 1 & 1 & & 108 & $\mathbf{Y}$ & \\
\hline 1 & $9 / 79$ & 2 & $\overline{1}$ & & $\theta 2$ & $\mathbf{N}$ & \\
\hline 1 & $9 / 9 / 78$ & 3 & 1 & & 92 & $\mathbf{N}$ & \\
\hline 1 & g/ont & 4 & $t$ & & 90 & $\mathbf{N}$ & \\
\hline
\end{tabular}

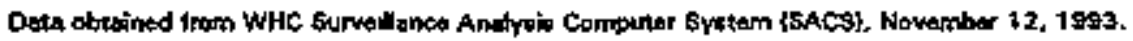




\begin{tabular}{|c|c|c|c|c|c|c|c|}
\hline \multicolumn{8}{|c|}{$\begin{array}{l}\text { TAKK 241 A-70B } \\
\text { THEFAMOCOSFLE DATA }\end{array}$} \\
\hline & & & & & & & \\
\hline 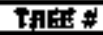 & DATE & THFFitho \& & MEE SAT & FENPC & TEMPF & $Y=$ MAX TEUP & COMNENTS \\
\hline 1 & Syl78 & 5 & 1 & & 50 & $N$ & \\
\hline 1 & 6/9/7B & 8 & 1 & & So & $N$ & \\
\hline 7 & 9/9/78 & 7 & 1 & & 80 & $N$ & \\
\hline 1 & glone & 8 & $i$ & & 90 & $\mathbf{N}$ & \\
\hline 1 & sylts & 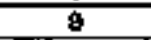 & 1 & & 90 & $\mathbf{N}$ & \\
\hline 1 & 90178 & 10 & $\mathbf{I}$ & & 90 & $\mathbf{N}$ & \\
\hline$i$ & $8 / 0 / 78$ & 17 & 1 & & 80 & $N$ & \\
\hline 1 & gpant & 12 & 1 & & gg & N & \\
\hline 1 & 01978 & 13 & 1 & & 90 & k & \\
\hline 1 & Bp/70 & 14 & 1 & & 68 & A & \\
\hline 1 & 9/9/7 & 15 & 1 & & 80 & if & \\
\hline 1 & OHA & 18 & 1 & & 80 & $\mathbf{N}$ & \\
\hline 1 & $10 \sqrt{8} 78$ & 1 & 1 & & 01 & $\bar{Y}$ & \\
\hline 1 & $10 / 8 / 7 \mathrm{~B}$ & 2 & 1 & & 日1 & N & \\
\hline 1 & $10 \mathrm{~g} / 7 \mathrm{~g}$ & 3 & 1 & & B1 & $\mathbf{N}$ & \\
\hline 7 & $1010 / 78$ & 4 & 3 & & 87 & $\overline{\mathbf{N}}$ & \\
\hline 7 & 10 jors & 5 & i & & 81 & N & \\
\hline I & 10 - & 6 & 1 & & 81 & $\mathbf{N}$ & \\
\hline 1 & $10 \mathrm{~m} / \mathrm{B}$ & 7 & 1 & & B1 & $\mathbf{N}$ & \\
\hline 1 & 101878 & 8 & 1 & & 81 & $N$ & \\
\hline 1 & $10 / 878$ & 8 & 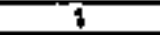 & & 82 & N & \\
\hline 1 & $104 \mathrm{p} 78$ & 70 & 7 & & 82 & $\mathbf{N}$ & \\
\hline 1 & $10 \%$ 月 & 11 & 1 & & 82 & $\mathbf{N}$ & \\
\hline 1 & $10 \mathrm{~B} / 70$ & $\overline{12}$ & 1 & & 82 & $\mathbf{N}$ & \\
\hline$T$ & 906870 & 13 & 1 & & $\overline{62}$ & N & \\
\hline$T$ & 10,877 & $\overline{14}$ & $T$ & & 82 & 10 & \\
\hline 1 & 101877 & 15 & 1 & & 92 & $\$$ & \\
\hline 1 & 70/8/78 & 18 & $T$ & & 82 & i & \\
\hline 1 & $1 / 2 / 7 \mathrm{a}$ & 1 & 1 & & 95 & $\bar{Y}$ & \\
\hline $\bar{T}$ & W/27 & $\overline{2}$ & 1 & & 70 & $\bar{N}$ & \\
\hline 7 & w279 & $\overline{3}$ & $i$ & & 75 & $\mathbf{N}$ & \\
\hline 1 & $1 / 2 / 79$ & 4 & 1 & & 75 & 部 & \\
\hline$\overline{7}$ & $1 / 279$ & 5 & $T$ & & 76 & H & \\
\hline 1 & 1279 & E & 1 & & 70 & $\mathbf{H}$ & \\
\hline 3 & $7 / 2 / 78$ & 7 & 1 & & 70 & N & \\
\hline 1 & $1 / 2 / 79$ & 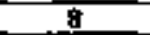 & 1 & & 36 & $N$ & \\
\hline 1 & 1/2/79 & 9 & 1 & & 77 & $\mathbf{N}$ & \\
\hline$\frac{1}{1}$ & $1 / 2 / 79$ & To & $\overline{\mathbf{1}}$ & & 77 & $\mathbf{H}$ & \\
\hline 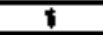 & $5 / 2 / 79$ & 11 & 1 & & 77 & $\mathbf{H}$ & \\
\hline 7 & $1 / 279$ & 12 & 1 & & 77 & H & \\
\hline 1 & $1 / 2779$ & 13 & 7 & & 77 & $\mathbf{N}$ & \\
\hline 1 & $1 / 2 / 78$ & 14 & 1 & & $n$ & $N$ & \\
\hline$\overline{1}$ & $1 / 2 / 78$ & 15 & 1 & & 77 & $\mathbf{N}$ & \\
\hline $\bar{t}$ & $1 / 2 / 79$ & 16 & $\overline{1}$ & & 77 & $\mathbf{N}$ & \\
\hline 1 & 25909 & 1 & 1 & & 87 & $Y$ & \\
\hline 1 & 25079 & 2 & 1 & & 73 & $\bar{N}$ & \\
\hline 1 & 2श79 & 3 & 1 & & 64 & $N$ & \\
\hline 1 & एका79 & 4 & 7 & & 84 & $N$ & \\
\hline$T$ & $219 / 79$ & 5 & 5 & & 63 & $\mathbf{N}$ & \\
\hline 1 & 29070 & 8 & $i$ & & 64 & $\mathbf{N}$ & \\
\hline 1 & $2 / 970$ & 7 & $\overline{1}$ & & 65 & $\mathbf{N}$ & \\
\hline 1 & 219079 & 8 & 1 & & 85 & $\mathbf{N}$ & \\
\hline 1 & 27278 & 8 & 1 & & 65 & $\bar{M}$ & \\
\hline 1 & $2 / 978$ & 10 & 1, & & 65 & $\bar{N}$ & \\
\hline 1 & 26979 & 71 & 70 & & 65 & $N$ & \\
\hline$\frac{1}{1}$ & $29 \sqrt{78}$ & $\sqrt{2}$ & 1 & & 65 & $\mathbf{N}$ & $\because$ \\
\hline 1 & 21979 & 73 & 1 & & 65 & N & \\
\hline$\overline{1}$ & 2 1979 & 14 & $\overline{7}$ & & 65 & $\bar{N}$ & \\
\hline$\overline{1}$ & $219 / 78$ & TE & $\overline{7}$ & & 65 & $\mathbf{N}$ & \\
\hline 1 & 29179 & 79 & $T$ & & 65 & $\mathbf{N}$ & \\
\hline 1 & 10,878 & 1 & 1 & & 88 & $\bar{Y}$ & \\
\hline 1 & T0/\&79 & $\frac{2}{2}$ & $\overline{1}$ & & 91 & $\bar{N}$ & \\
\hline 1 & $10 \mathrm{~g} / 70$ & 5 & 9 & & 78 & $N$ & \\
\hline 1 & 100879 & 4 & 7 & & 76 & $\mathbf{N}$ & \\
\hline 1 & $10+80$ & 5 & 1 & & 76 & $\overline{\mathbf{N}}$ & \\
\hline 1 & 10,879 & 6 & 1 & & 77 & $\bar{N}$ & \\
\hline
\end{tabular}

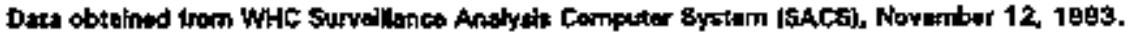




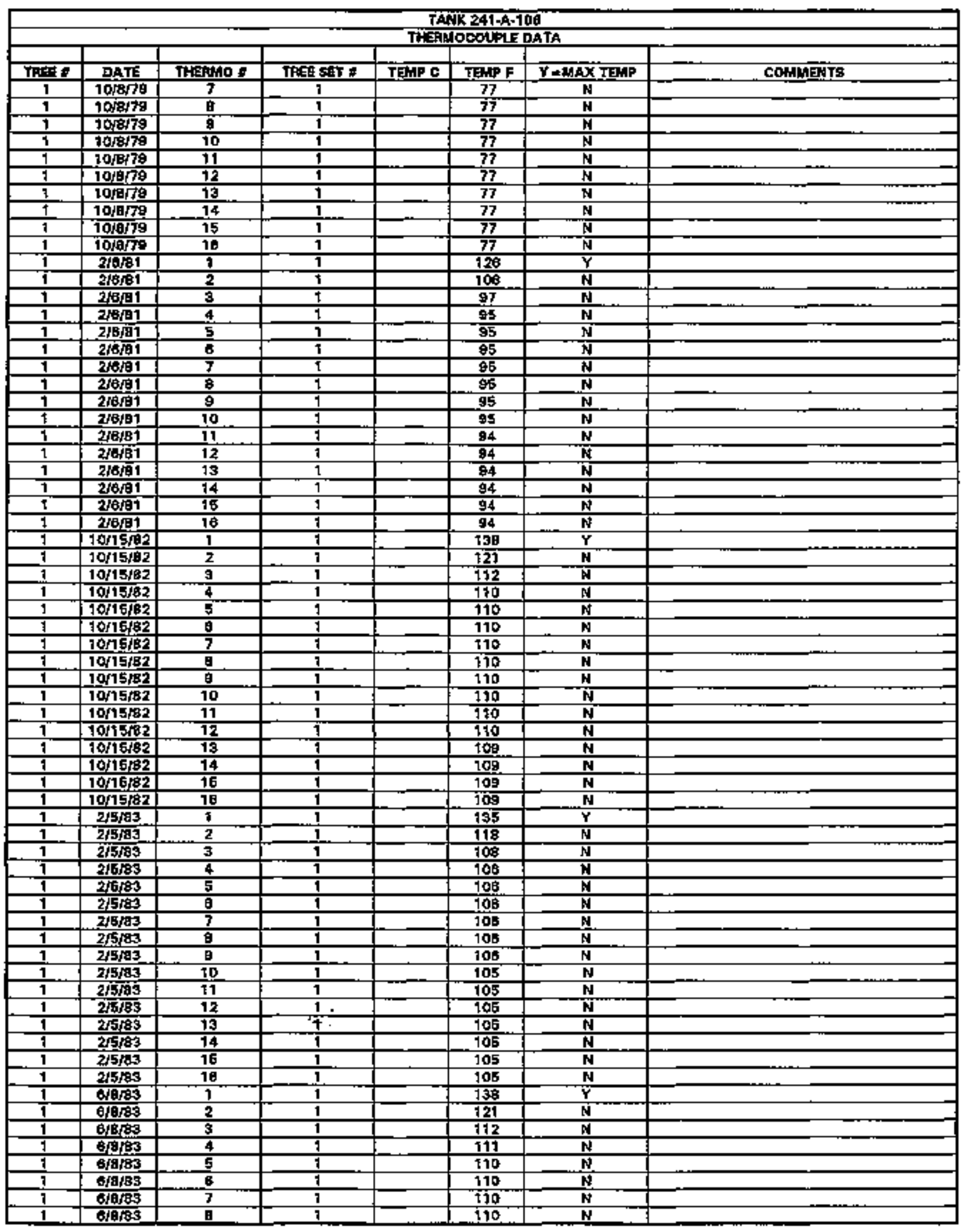

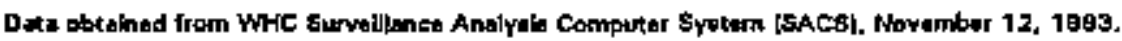




\begin{tabular}{|c|c|c|c|c|c|c|c|}
\hline \multicolumn{8}{|c|}{$\begin{array}{c}\text { TANK 241+A-TOB } \\
\text { THETHCOUPLE bATA }\end{array}$} \\
\hline & & & & & & & \\
\hline Trifift & DA7t & 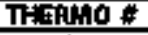 & TRAEE EAT : & THUPC & TSUPF & $Y=h A X$ TESP & COLWENTS \\
\hline 1 & 6/6/83 & 白 & 1 & & 110 & $\mathrm{~N}$ & \\
\hline$\frac{1}{1}$ & sares & 10 & 1 & & 110 & $\bar{N}$ & \\
\hline 1 & $6 \sqrt{63}$ & 11 & 1 & & 110 & $\mathbf{N}$ & \\
\hline 1 & $8 \sqrt{6 / 83}$ & 12 & 1 & & 110 & $\mathbf{N}$ & \\
\hline 1 & 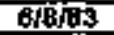 & 19 & 1 & & 110 & $\mathbf{H}$ & \\
\hline$\frac{1}{1}$ & eraria & 94 & 1 & & 110 & $\overline{\mathbf{N}}$ & \\
\hline $\bar{y}$ & Geres & IE & 1 & & 110 & $\mathbf{N}$ & \\
\hline 1 & discas & 76 & $t$ & & 110 & $N$ & \\
\hline 1 & $4 \sqrt{26184}$ & 1 & 1 & & $13 \mathbb{B}$ & $Y$ & \\
\hline 1 & $4 / 283^{4}$ & 2 & 9 & & 121 & $\mathrm{~N}$ & \\
\hline 1 & 426104 & $\overline{3}$ & 1 & & 111 & $\mathbf{N}$ & \\
\hline 1 & $4 / 26194$ & 4 & 1 & & 108 & $\mathbf{N}$ & \\
\hline 1 & $4 \sqrt{26 / 24}$ & 5 & 1 & & 109 & $\dot{\mathbf{M}}$ & \\
\hline 1 & $4 / 26 \sqrt{34}$ & 8 & 1 & & 109 & N & \\
\hline 1 & $4 / 26484$ & $\overline{7}$ & $\bar{T}$ & & 709 & $\bar{N}$ & \\
\hline 1 & $4 / 26$, & 官 & 1 & & 100 & N & \\
\hline 1 & 4/20Na4 & 9 & 1 & & 108 & $\mathbf{N}$ & \\
\hline 1 & 4/20194 & 10 & 1 & & 106 & $\mathbf{N}$ & \\
\hline$t$ & $4 \longdiv { 2 0 1 8 4 }$ & 11 & 1 & & 100 & $\mathbf{N}$ & \\
\hline$T$ & 4726184 & 12 & 1 & & 108 & $\mathbf{N}$ & \\
\hline 1 & 4 2aka & 13 & 1 & & 108 & $\mathbf{N}$ & \\
\hline 7 & $4 / 20 / 84$ & 14 & 1 & & 108 & $\mathbf{N}$ & \\
\hline 1 & 42的度4 & 16 & 1 & & 108 & $\mathbf{N}$ & \\
\hline 7 & $4 \sqrt{29 \sqrt{84}}$ & $\overline{18}$ & 1 & & 108 & $\overline{\mathbf{N}}$ & \\
\hline 1 & 4127184 & T & $i$ & & 122 & $\bar{Y}$ & \\
\hline 1 & $427 \sqrt{64}$ & 2 & 1 & & 120 & $\mathbf{N}$ & \\
\hline 1 & 427164 & 3 & 1 & & 115 & $\mathbf{M}$ & \\
\hline 1 & $427 e^{4}$ & 4 & 1 & & 113 & $\mathbf{N}$ & \\
\hline 1 & $4 / 27 / 84$ & 5 & 1 & & 112 & $n$ & \\
\hline 1 & $4 / 27 / 64$ & 6 & 1 & & 110 & $\bar{k}$ & \\
\hline $\overrightarrow{1}$ & $4 \sqrt{27 / 64}$ & $\overline{7}$ & 1 & & 108 & $\mathbf{N}$ & \\
\hline 1 & $4 \sqrt{27184}$ & 8 & 1 & & 108 & $\mathbf{N}$ & \\
\hline 1 & $4 / 27 / 94$ & 8 & 7 & & 104 & $\mathbf{N}$ & \\
\hline$\overline{1}$ & 42764 & 10 & 7 & & 103 & $\mathbf{N}$ & \\
\hline 1 & $4 \sqrt{27 / 84}$ & 11 & $i$ & & 101 & $\bar{N}$ & \\
\hline 1 & $4 / 27184$ & 12 & $j$ & & $\overline{100}$ & N & \\
\hline $\bar{T}$ & $4 \sqrt{27 / 34}$ & 15 & 5 & & 99 & $\mathbf{H}$ & \\
\hline$T$ & $4 / 27 / 84$ & 14 & $i$ & & 98 & N & \\
\hline 1 & $4 \sqrt{27 / 84}$ & 15 & $\overline{1}$ & & 97 & H & \\
\hline 1 & 1/20185 & 1 & 1 & & 135 & $Y$ & \\
\hline 7 & $1 / 2685$ & 2 & 5 & & 177 & $\mathbf{N}$ & \\
\hline 1 & 1/28/35 & 3 & 3 & & 108 & $\mathbf{N}$ & \\
\hline 1 & $1 / 20785$ & 4 & ] & & 105 & N & \\
\hline 7 & 1/2085 & 5 & $i$ & & 605 & $\overline{\mathbf{N}}$ & \\
\hline 1 & 1/20/85 & 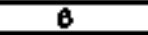 & 7 & & 105 & $\mathbf{N}$ & \\
\hline 7 & $1 / 20 / 85$ & 7 & 1 & & 105 & $\mathbf{N}$ & \\
\hline$t$ & 120165 & E & $t$ & & tab & $\bar{N}$ & \\
\hline 1 & $1 / 26 \% 5$ & 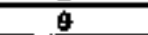 & 1 & & 105 & $\mathbf{N}$ & \\
\hline 7 & $1 / 2985$ & 10 & 7 & & 705 & $\mathbf{N}$ & \\
\hline 1 & $1 / 20 / 85$ & 11 & 7 & & 505 & $\mathbf{N}$ & \\
\hline$T$ & 1/2a/B5 & 12 & $i$ & & 105 & $\mathbf{N}$ & \\
\hline 7 & 1/2018S & 13 & $i$ & & 105 & $\mathbf{N}$ & \\
\hline 7 & 1/2a/85 & 14 & 7 & & 705 & $N$ & \\
\hline$i$ & 1/20/85 & 15 & $i$ & & 105 & $\mathbf{N}$ & \\
\hline 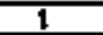 & $1 / 20 / 85$ & 18 & $7 x$ & & 105 & $\mathrm{~N}$ & \\
\hline$\hbar$ & $4 / 20185$ & 1 & 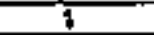 & & 135 & $\mathbf{Y}$ & \\
\hline 1 & 4/20/85 & 2 & 7 & & 185 & $\bar{N}$ & \\
\hline 1 & 4 H201E5 & 3 & $\overline{1}$ & & 104 & N & \\
\hline$t$ & $4 / 20 / 85$ & 4 & 1 & & 103 & N & \\
\hline 1 & $4 / 2$ बrB5 & 6 & $\pi$ & & 109 & $\overline{\mathbf{N}}$ & \\
\hline j & $4 / 2085$ & 8 & 7 & & 102 & $\mathbf{N}$ & \\
\hline f & $4 / 20105$ & 7 & 1 & & 103 & $\mathbb{N}$ & \\
\hline 1 & $420 \sqrt{85}$ & $\mathrm{~g}$ & 1 & & 103 & $N$ & \\
\hline 1 & 4/20/85 & 9 & 1 & & 103 & $\mathbf{N}$ & \\
\hline 1 & 4126185 & 10 & $\overline{\mathbf{1}}$ & & 102 & $N$ & \\
\hline 1 & 42的它 & 11 & 1 & & 102 & $N$ & \\
\hline
\end{tabular}

Date abtained from WHC Survaldence Andysid Carnpter Syrium ISACSI, November 12, 1993. 


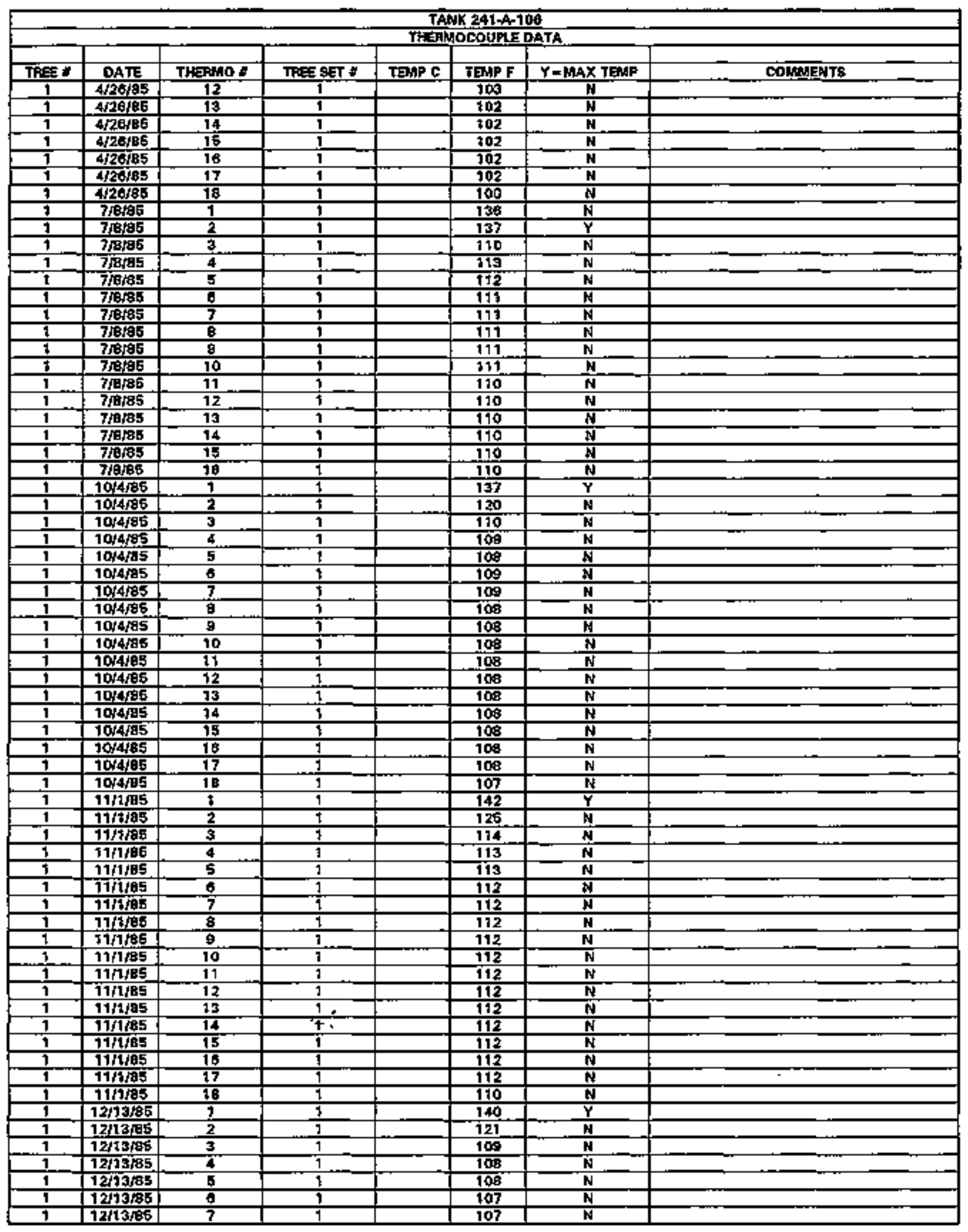

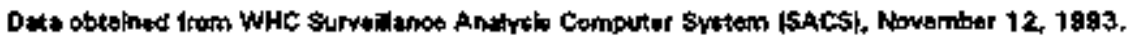




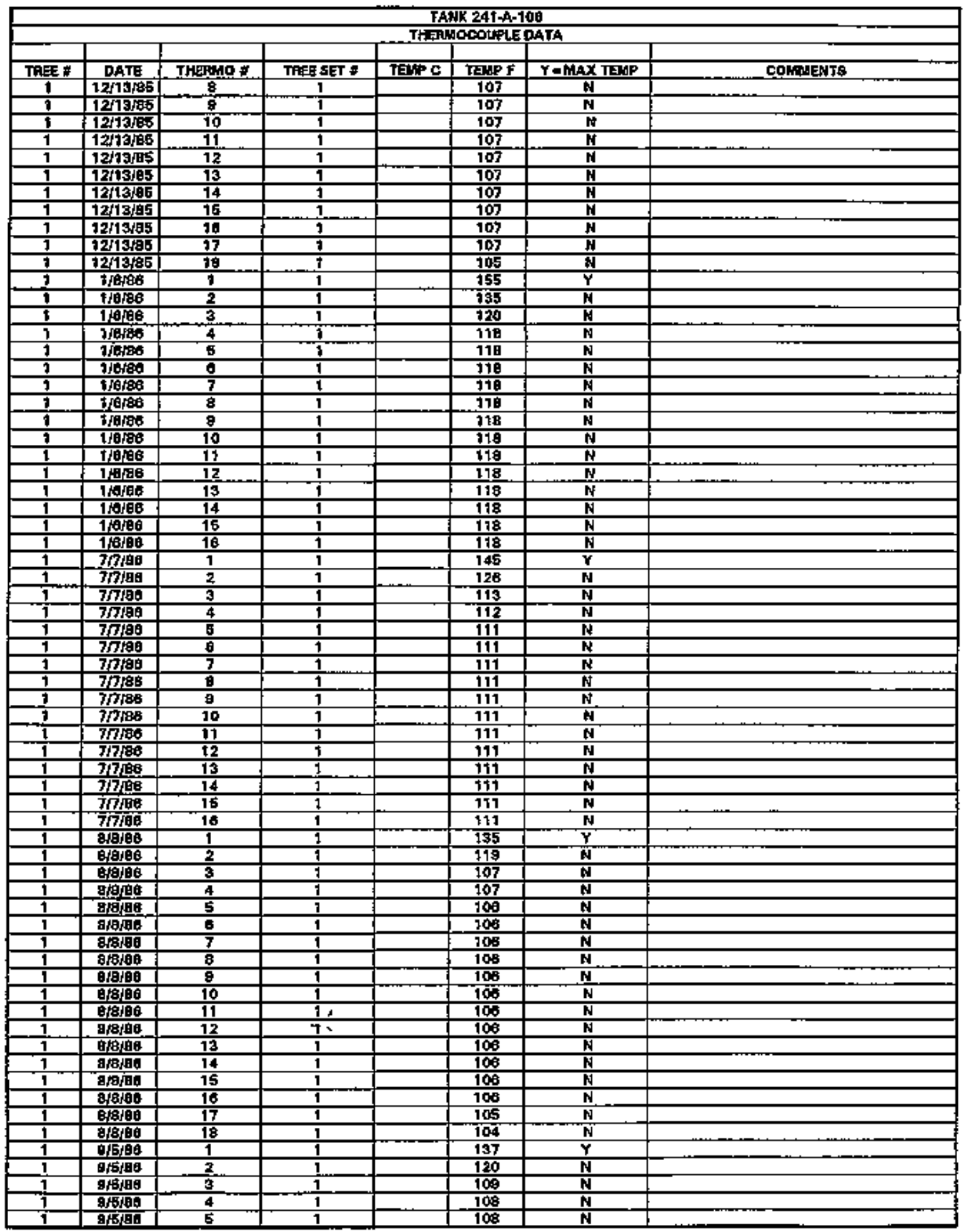

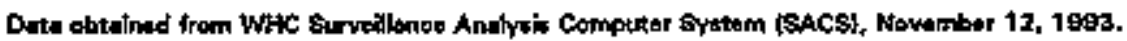


WHC-SD.WMHER-306, RAY, O

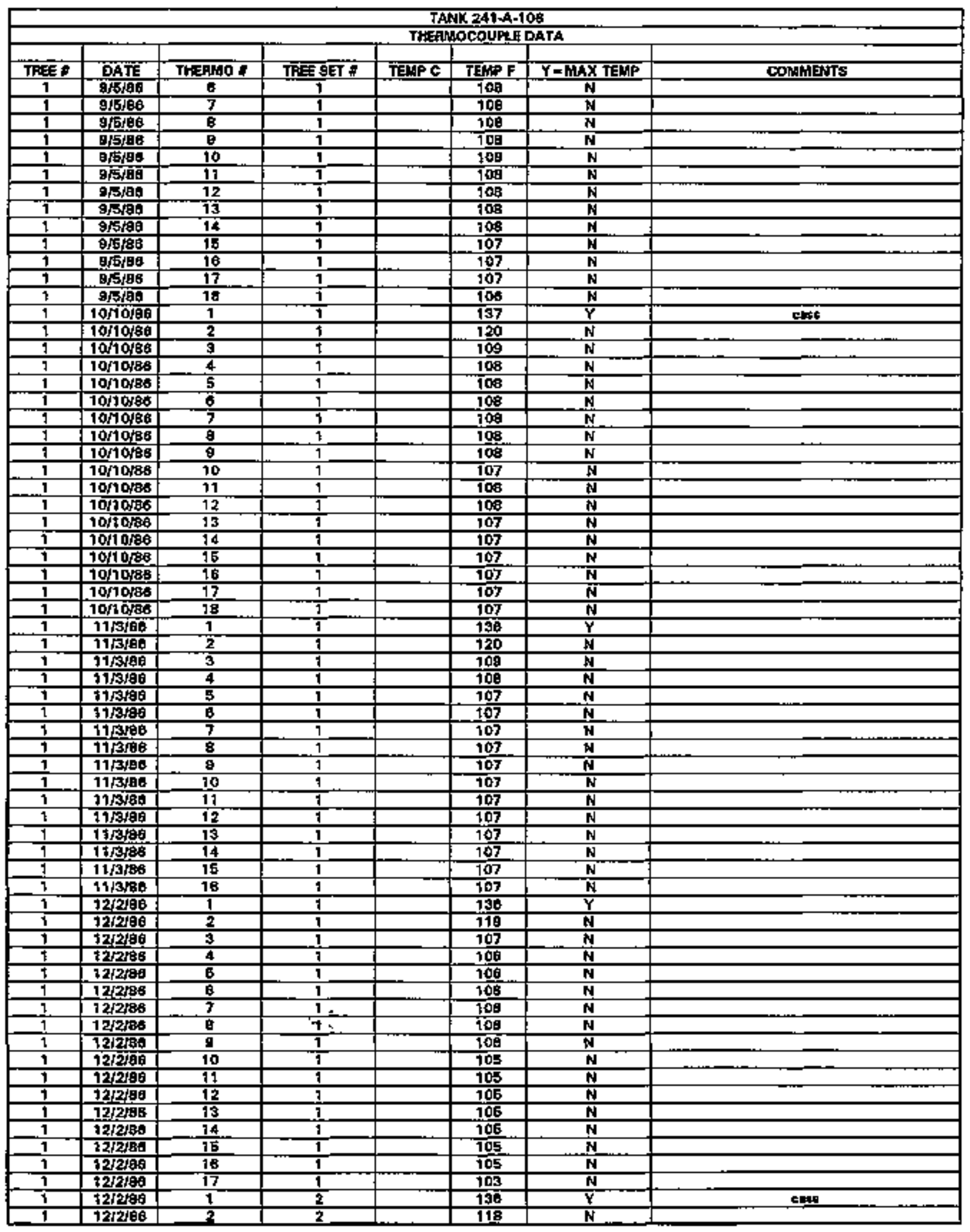

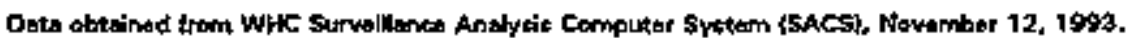




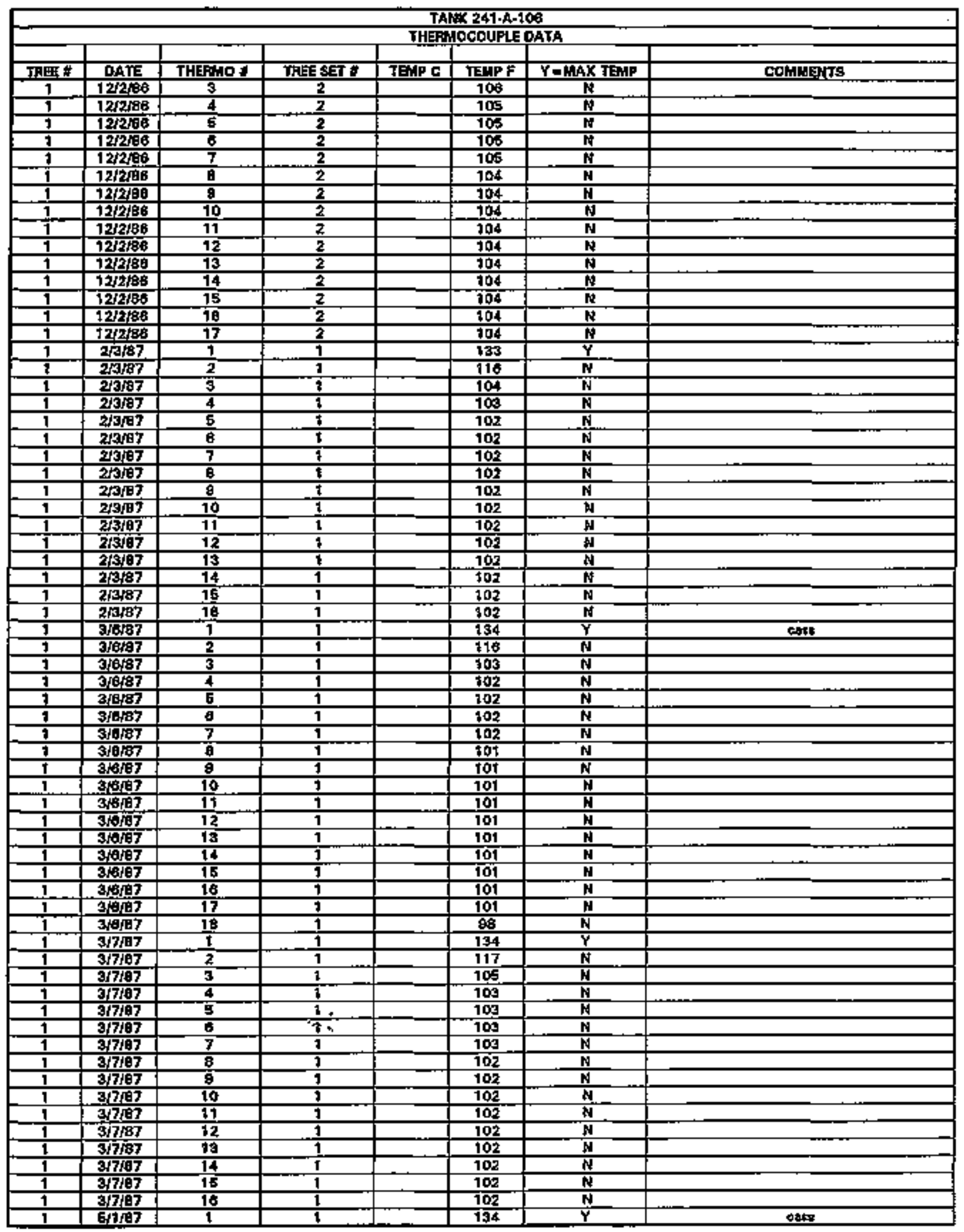

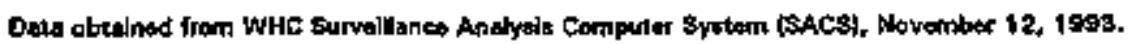




\begin{tabular}{|c|c|c|c|c|c|c|c|}
\hline \multicolumn{8}{|c|}{ TANK 241-A-10B } \\
\hline \multicolumn{8}{|c|}{ THERMOCOUPLE DATA } \\
\hline & & & & & & & 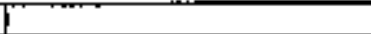 \\
\hline TREE : & DATE & THEAYO & THEE EET 5 & TEMP C & TELPF F & $Y=\operatorname{MKX} \overline{T E M P}$ & COLANENTS \\
\hline 1 & $5 \sqrt{187}$ & 2 & 1 & & 110 & $N$ & \\
\hline 1 & B/1, & 3 & 1 & & 102 & त्ञ & \\
\hline$i$ & $5 / 187$ & 4 & 1 & & 102 & W & \\
\hline 1 & $5 / 187$ & $\overline{5}$ & $i$ & & 101 & $\bar{N}$ & \\
\hline 1 & $5 / 1 / 87$ & $\bar{a}$ & 1 & & 101 & $\bar{N}$ & \\
\hline 1 & $5 / 1 / 87$ & 7 & 1 & & 101 & $\mathbf{N}$ & \\
\hline 1 & $5 \sqrt{17}$ & 8 & $\overline{1}$ & & 101 & $\bar{N}$ & \\
\hline 1 & \$187 & 9 & 1 & & 101 & $\mathrm{~N}$ & \\
\hline 1 & $5 / 187$ & 70 & $T$ & & 101 & $\mathbf{N}$ & \\
\hline$T$ & $8 / 187$ & 11 & 7 & & 101 & N & \\
\hline 1 & $5 / 7 \times 3$ & 12 & $\Phi$ & & 101 & $\mathbf{N}$ & \\
\hline 1 & $5 / 1 / 197$ & 13 & 1 & & 101 & $\mathbf{N}$ & \\
\hline 1 & 5/1/B? & 14 & 1 & & 101 & $\overline{\mathbf{N}}$ & \\
\hline 1 & 5/1/187 & IE & 1 & & ID1 & $\mathbf{N}$ & \\
\hline$\overline{1}$ & 5M/A7 & $\overline{6}$ & $\overline{1}$ & & 100 & N & \\
\hline 1 & $5 / 1 / 97$ & 17 & 1 & & 100 & N & \\
\hline$\overline{1}$ & 5/1/67 & 18 & $\overline{1}$ & & 的 & $\overline{\mathbf{N}}$ & \\
\hline 1 & 615107 & 1 & j & & 134 & $\bar{Y}$ & cotply \\
\hline 1 & 6/5的7 & 2 & 1 & & 718 & $\mathbf{N}$ & \\
\hline 1 & 6 & 3 & $\frac{1}{1}$ & & 103 & $\mathbf{N}$ & \\
\hline 1 & $6 / 5 / 87$ & 4 & $\overline{7}$ & & $\overline{102}$ & $\overline{\mathbf{N}}$ & \\
\hline 1 & A/5ل/67 & 5 & 1 & & 102 & $\mathbf{N}$ & \\
\hline 1 & $0 / 5 / 97$ & $\overline{8}$ & 1 & & 102 & $\mathbf{N}$ & \\
\hline 1 & $9 \sqrt{6197}$ & 7 & 1 & & 102 & $\mathbf{N}$ & \\
\hline 1 & 6Б5 & 8 & 1 & & 102 & $\mathbf{N}$ & \\
\hline 1 & $6 \sqrt{6} 19$ & 6 & 9 & & 102 & $\mathbf{N}$ & \\
\hline 1 & $6 \sqrt{587}$ & 10 & 1 & & 102 & $\mathbf{N}$ & \\
\hline 1 & $8 / 5 / 87$ & 11 & 1 & & 102 & $N$ & \\
\hline 1 & $8 / 5 / 87$ & 12 & 1 & & 102 & $\mathrm{~N}$ & \\
\hline 1 & $6 \sqrt{3+57}$ & 13 & 1 & & 102 & $\bar{N}$ & \\
\hline 1 & $6 \sqrt{6 / 87}$ & 14 & 1 & & 102 & $\mathbf{N}$ & \\
\hline 1 & $6 / 5 / 87$ & 15 & 1 & & 102 & $\mathbf{N}$ & \\
\hline 1 & $6 / 5 / 87$ & 16 & 1 & & 101 & $\mathbf{N}$ & \\
\hline 1 & $6 \sqrt{5} 87$ & 17 & 1 & & 101 & $\overline{\mathrm{N}}$ & \\
\hline 1 & 61587 & 19 & 1 & & 100 & 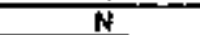 & \\
\hline 1 & 8/dore7 & 2 & 1 & & m & $\mathbf{N}$ & \\
\hline 1 & $9 / 8 / 87$ & 3 & 1 & & 71 & $\mathbf{N}$ & \\
\hline 1 & 9/8/87 & 4 & 1 & & 71 & $\mathbf{N}$ & \\
\hline 1 & 9/8/87 & $\overline{5}$ & 1 & & 72 & $\mathbf{Y}$ & \\
\hline 1 & $9 / 6 \sqrt{157}$ & 6 & 1 & & 72 & $\bar{N}$ & \\
\hline 1 & $9 / 8 / 87$ & 7 & 1 & & 72 & $\bar{N}$ & \\
\hline 1 & g/8ra7 & $\bar{a}$ & 1 & & 72 & $N$ & \\
\hline 1 & 早唒7 & 9 & $i$ & & 72 & $\mathbf{N}$ & \\
\hline$\overline{1}$ & $0 / 8 \sqrt{17} 7$ & 10 & 1 & & $\overline{7 z}$ & $\mathbf{N}$ & \\
\hline 1 & $2 / 6 \sqrt{107}$ & 11 & 1 & & $\overline{72}$ & $\mathbf{N}$ & \\
\hline 1 & $9 / 6 / 97$ & 12 & 1 & & $\overline{72}$ & $\mathbf{N}$ & \\
\hline 1 & $9 / 6 \sqrt{87}$ & 13 & $\mathbf{i}$ & & $\overline{72}$ & $\overline{\mathbf{N}}$ & \\
\hline 1 & 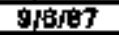 & 74 & 1 & & 72 & $\mathbf{N}$ & \\
\hline 7 & 9/8ja7 & 15 & 1 & & $\overline{72}$ & $\mathbf{N}$ & \\
\hline 1 & 9 9/B/A7 & 16 & 1 & & 72 & $\mathbf{N}$ & \\
\hline 1 & 10.5197 & 1 & 1 & & & $\mathbf{N}$ & 20 - bispect data lowl \\
\hline 1 & 10,5je7 & $\mathbf{2}$ & 1 & & & $\mathbf{N}$ & 35 - etspect dita (low) \\
\hline 1 & 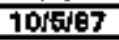 & $\overline{3}$ & 1 & & & $\mathbf{N}$ & 40 - tulpeot date $\{\mathrm{law}\}$ \\
\hline 1 & 10학 7 & 4 & 1. & & & $\mathbf{N}$ & 47- Uapold datta law! \\
\hline$\overline{1}$ & 10/6/97 & $\overline{5}$ & 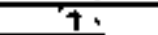 & & & $\mathbf{N}$ & 47 - [espert dota [ow] \\
\hline 1 & $10 / 5 / 8 ?$ & 6 & 1 & & & $\pi$ & 47 - gropect dath fow] \\
\hline 1 & $10 / 5 / 97$ & 7 & 1 & & & $\mathbf{N}$ & 47 - ampect dita low] \\
\hline 1 & 10,5/a7 & 8 & 1 & & & $\mathbf{N}$ & 47 - wepect deta (bw) \\
\hline 1 & 10:\$ด7 & 9 & 1 & & & $\mathbf{N}$ & 47 - ordepect data flowl \\
\hline 1 & 101507 & 10 & 1 & & & $\mathbf{N}$ & 47 + otrpoct data (low) \\
\hline$T$ & 10/567 & 11 & $T$ & & & $\bar{n}$ & 47 + ENepect date (low) \\
\hline 1 & 10 bje7 & 12 & 1 & & & $\mathbf{N}$ & 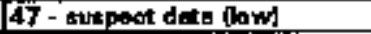 \\
\hline$\overline{1}$ & $10 \mathrm{j} 6 \overline{87}$ & 13 & 1 & & & $\overline{\mathbf{H}}$ & 47 - tutpoot dath dow \\
\hline 1 & 10 6) & 14 & 1 & & & $\overline{\mathbf{N}}$ & $47-$ twopot dats (apw) \\
\hline 1 & 105ㅏㅈㄱ & 26 & 1 & & & $\mathbf{Y}$ & 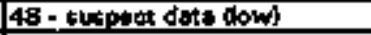 \\
\hline 1 & 10/567 & 16 & 1 & & & $\bar{N}$ & 48 - nugpert deto llow \\
\hline
\end{tabular}

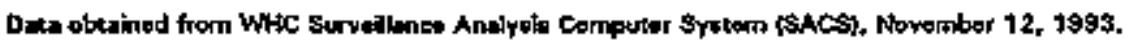




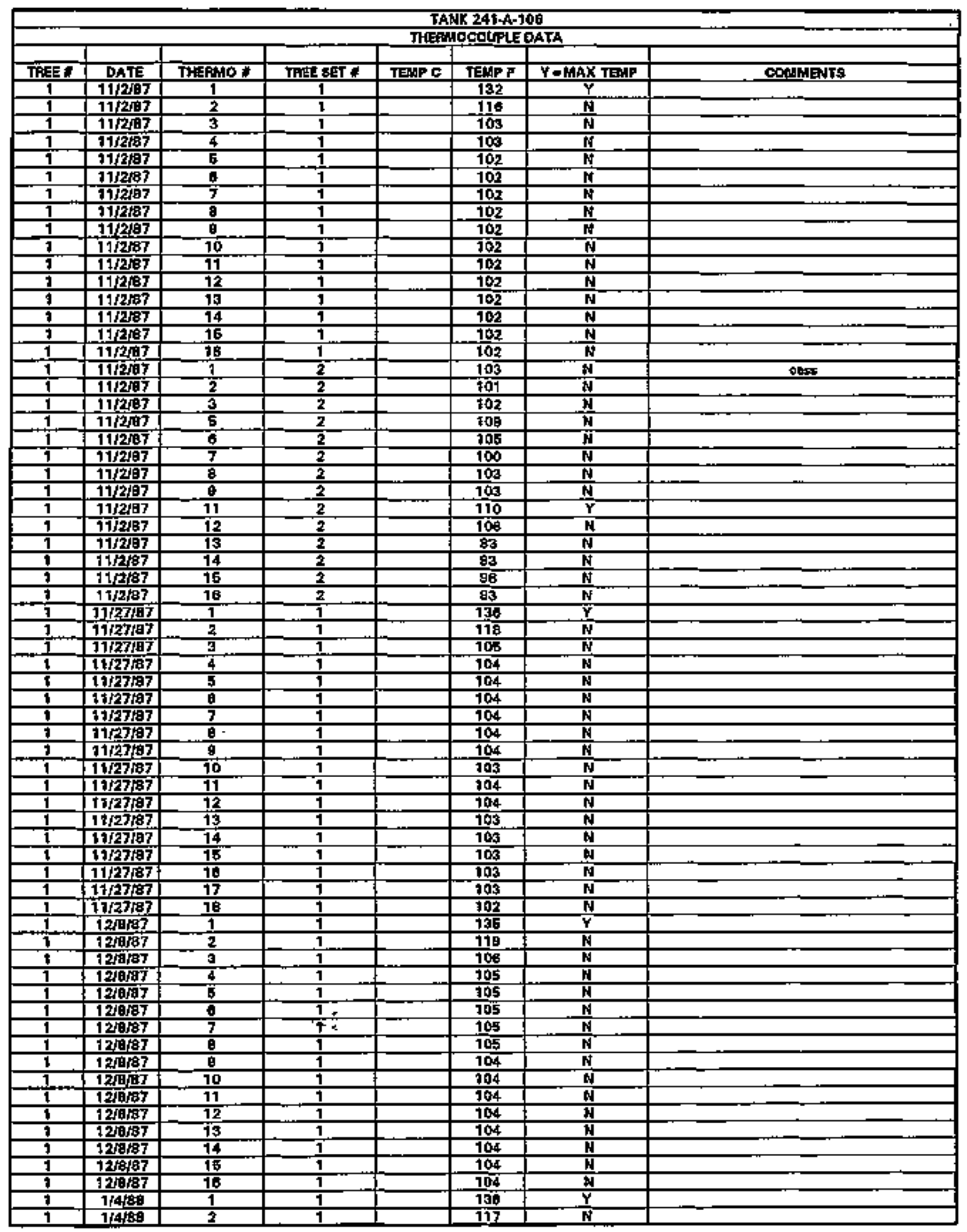

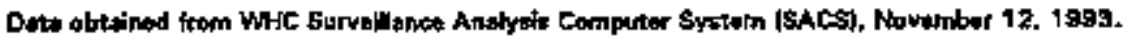




\begin{tabular}{|c|c|c|c|c|c|c|c|}
\hline \multicolumn{8}{|c|}{ TANK 2\$1A-10S } \\
\hline & & & & THEFi: & DCOUPLE & ATA & \\
\hline THEA & DATE & THEFAO & Th: Eef * & TEMP & TEMFF & $Y=$ MAX TEMP & COMMTENTS \\
\hline 1 & $1 / 488$ & 3 & 1 & & 104 & $\frac{N}{N}$ & \\
\hline 1 & $1 / 4 \sqrt{88}$ & 4 & 1 & & 101 & $\mathbf{N}$ & \\
\hline 1 & $1 / 4 / 88$ & 호 & 1 & & 102 & $\mathbf{N}$ & \\
\hline 1 & $1 / 4 \sqrt{88}$ & 8 & 1 & & $10 \overline{2}$ & $\mathbf{N}$ & \\
\hline 1 & 1/4/B8 & 7 & 1 & & 102 & $\mathbf{N}$ & \\
\hline 1 & $1 / 4 \sqrt{18}$ & 8 & 1 & & 102 & $N$ & \\
\hline 1 & $1 / 4 \sqrt{68}$ & 9 & $T$ & & 102 & $\mathbf{N}$ & \\
\hline 1 & 1/AJAB & 10 & 1 & & 102 & $\mathbf{N}$ & \\
\hline 1 & $1 / 4 / \mathrm{Ag}^{2}$ & 11 & 1 & & 102 & N & \\
\hline 1 & 1/4/4a & 12 & 1 & & 102 & $\mathbf{N}$ & \\
\hline 1 & $1 / 496$ & 13 & 1 & & 102 & $\overline{\mathbf{N}}$ & \\
\hline 1 & i/4/a & 14 & 1 & & 102 & $\mathbf{N}$ & \\
\hline 1 & $1 / 4 / 98$ & 18 & 1 & & 102 & $\mathbf{N}$ & \\
\hline 1 & $1 / 4 / 98$ & 16 & 1 & & 102 & $\mathbf{N}$ & \\
\hline 1. & $21 / 89$ & 1. & 1 & & 135.3 & $\vec{Y}$ & \\
\hline$\vec{\jmath}$ & 21889 & 2 & 1 & & 101 & $\mathbf{N}$ & \\
\hline 1 & M/BB & 3 & 1 & & 101.7 & $\mathbf{N}$ & \\
\hline 1 & $2 \sqrt{188}$ & 4 & 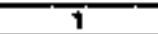 & & 101 & N & \\
\hline 1 & 2 ก18 & 5 & 1 & & 101 & $\mathbf{N}$ & \\
\hline 1 & $2 \sqrt{188}$ & 皇 & 1 & & 101 & $\mathbf{N}$ & \\
\hline 1 & $2,1 / 88$ & $\overline{7}$ & 1 & & 101 & $\mathbf{N}$ & \\
\hline 1 & $2 \sqrt{1 / 88}$ & 8 & 1 & & 101 & $\mathbf{N}$ & \\
\hline 1 & $2 / 1 / 88$ & 9 & 1 & & 101 & $\mathbf{N}$ & \\
\hline 1 & $2 / 1 / 8 B$ & 10 & 1 & & 901 & N & \\
\hline 1 & आाहB & 11 & 1 & & 907 & $\bar{N}$ & \\
\hline$T$ & $2 \sqrt{1 / 88}$ & 12 & 1 & & 100 & $N$ & \\
\hline 1 & $2 / 1 / 68$ & 13 & 1 & & $10 \mathrm{I}$ & $\mathbf{N}$ & \\
\hline 1 & $2 / 1 / 88$ & 14 & 1 & & 100 & $\mathbf{N}$ & \\
\hline 1 & $2 / 1 / B B$ & 15 & 7 & & 100 & $\mathbf{N}$ & \\
\hline 1 & 219 Jas & 10 & $\overline{\mathbf{t}}$ & & 100 & $\mathbf{N}$ & \\
\hline 1 & अ7/8B & 2 & $T$ & & & $\bar{Y}$ & 198- suppect dita thight \\
\hline 1 & 3788 & $\mathbf{3}$ & 1 & & & $\bar{N}$ & 51 - Etropect doto llowel \\
\hline 1 & आ7/68 & 4 & $\hbar$ & & & $\bar{N}$ & 52 - rogpect drto tow? \\
\hline 1. & आ768 & 5 & 1 & & & N & 52 - Eunpect dita Row \\
\hline$\overline{1}$ & $3 n \pi 8$ & 6 & 1 & & & $\mathbf{N}$ & 52 - ztupport data frawh \\
\hline 1 & $3 \pi / 88$ & 7 & 7 & & & $\mathbf{N}$ & [52- strapert data Maw] \\
\hline 1. & $3 / 7 / 88$ & 8 & 1 & & & $\mathbf{N}$ & 52 + tuspent data Pow \\
\hline 7 & $3 / 7 / 68$ & 9 & 1 & & & N & $\overline{5} \overline{2}+$ tedspent data llow| \\
\hline 1 & 3/7/E8 & 10 & $i$ & & & N & B2 - entepeot data llowl \\
\hline$\overline{1}$ & $3 / 768$ & 11 & $\overline{1}$ & & & $\mathbf{N}$ & 52 - nlupert doto tow \\
\hline$\overline{1}$ & 37/68 & 92 & 1 & & & $\bar{N}$ & 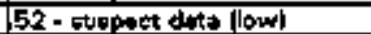 \\
\hline 1 & 317.es & 13 & $\frac{1}{1}$ & & & $\mathbf{N}$ & 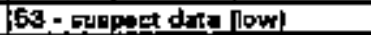 \\
\hline 5 & $3 / 7 / 88$ & 14 & 1 & & & $N$ & 53 - sulpect data llows \\
\hline 1 & 3/7/8 & 15 & 1 & & & $\bar{N}$ & 53 + stispont data Now] \\
\hline 1 & M7/p: & 16 & $\bar{i}$ & & & $N$ & 53 - sutpost data llow \\
\hline 1 & $3 / 788$ & 17 & 1 & & & $\bar{N}$ & 53 - cutspeot dota (low) \\
\hline 1 & $3 / 7 / 68$ & 18 & 1 & & & N & 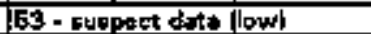 \\
\hline 7 & $4 / 1 / \sqrt{8}$ & 1 & 7 & & 133 & $\overline{\mathbf{Y}}$ & \\
\hline 1 & $4 / 1 / 189$ & 2 & 1 & & 115 & $\mathbf{N}$ & \\
\hline 1 & 4 ing & 3 & 1 & & 100 & $N$ & \\
\hline 1 & 4/1/89 & 4 & 1 & & 98 & $\mathbf{N}$ & \\
\hline 1 & $41 /$ ing & 5 & 1 & & 99 & $\mathbf{N}$ & \\
\hline 1 & $4 / 1 / \sqrt{\text { 日a }}$ & E & 1 & & 89 & $\mathbf{N}$ & \\
\hline$T$ & $4 / 1 / 68$ & 7 & 1. & & 99 & N & \\
\hline 1 & $4 / 1 / 68$ & 8 & +7 & & 99 & $\overline{\mathbf{N}}$ & \\
\hline 1 & $4 / 1 / 88$ & 9 & 1 & & 99 & N & \\
\hline 1 & $4 \pi / \sqrt{68}$ & 70 & 1 & & 99 & $N$ & \\
\hline 1 & 42189 & 11 & 7 & & 99 & $\mathbf{N}$ & \\
\hline $\mathbf{i}$ & $4 \sqrt{68}$ & 12 & 1 & & 9 & $\mathbf{N}$ & \\
\hline 1 & $4 \sqrt{168}$ & 93 & 1 & & 99 & $\mathbf{N}$ & \\
\hline 1 & $4 / 7 \sqrt{68}$ & 14 & 1 & & 99 & $\overline{\mathbf{N}}$ & \\
\hline 1 & $4 / 788$ & 15 & 1 & & 89 & $\mathbf{N}$ & \\
\hline 1 & $4 / 188$ & 16 & 1 & & 9日 & $\mathbf{N}$ & \\
\hline 1 & 4/1/E8 & 17 & 1 & & 98 & $\mathbf{N}$ & \\
\hline 1 & 4148 & 18 & 1 & & 99 & $\mathbf{N}$ & \\
\hline 1 & $44 / 88$ & 1 & 1 & & & $\mathrm{~N}$ & 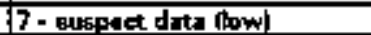 \\
\hline
\end{tabular}




\begin{tabular}{|c|c|c|c|c|c|c|c|}
\hline \multicolumn{8}{|c|}{ TAN" 241-A-10B } \\
\hline \multicolumn{8}{|c|}{ THERMOCONPLE DATA } \\
\hline Triet & DATE & THEFAO \# & TREF SET : & TENP $\mathrm{C}$ & TEMP F & Y.MAX TEMP & COMLATS \\
\hline 1 & $4 / 489$ & 2 & 1 & & & $\mathbf{N}$ & 26 - gucpect dita (low) \\
\hline 1 & $4 / 4 / 88$ & 3 & 1 & 41 & 10.8 & $\mathbf{N}$ & raw data + exsumed degres C \\
\hline 5 & 4/4/88 & 4 & 1 & 42 & 107.4 & $\mathbf{N}$ & raw deta + atsumed depoes C \\
\hline 1 & 4/4/EB & $\mathbf{5}$ & 1 & 43 & 109.4 & $\mathbf{N}$ & 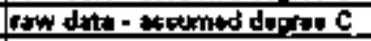 \\
\hline 1 & 4/4/Ee & 8 & 1 & 43 & 109.4 & $\mathbf{N}$ & raw doto - somomed degat C \\
\hline 1 & $4 / 4 / 6$ & 7 & 1 & 43 & $\$ 09.4$ & $\mathbf{N}$ & 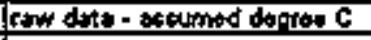 \\
\hline 1. & 4/4/B百 & $\mathbf{E}$ & 1 & 49 & 109.4 & $\mathbf{N}$ & raw dita - aleoplaned degrea C \\
\hline 1 & 4/4/89 & $\underline{\theta}$ & $\overline{1}$ & $\sqrt{3}$ & 109.4 & $\bar{x}$ & 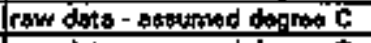 \\
\hline 1 & $44 \sqrt{68}$ & 10 & 1 & $\mathbf{A 3}$ & 109,4 & i & 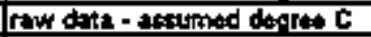 \\
\hline 1 & $4 / 4 / 80$ & 11 & 1 & 13 & 1094 & $\mathbf{N}$ & 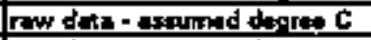 \\
\hline 1 & A/4/88 & 12 & 1. & 43 & 1094 & $\mathbf{N}$ & 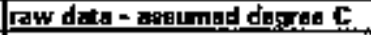 \\
\hline 1 & $4 / 4,18 B$ & 73 & 1 & 43 & 1094 & $N$ & rew deta - essumed deren $\mathrm{C}$ \\
\hline 1 & $4 / 4,188$ & 14 & 1 & 43 & 100.4 & N & 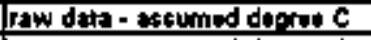 \\
\hline 1 & $4 / 4 \sqrt{88}$ & 95 & 1 & 4 & 100.4 & $N$ & 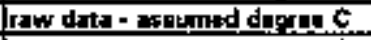 \\
\hline 1 & $4 / 4,38$ & 16 & 1 & 43 & $10 \mathrm{~g}_{4}^{4}$ & $\mathbb{N}$ & raw data - asalumed dapraqu $C$ \\
\hline 1. & $4 / 488$ & 17 & 1 & 47 & 109,4 & $\mathbf{N}$ & Iraw dota - astumad degreat $\mathrm{C}$ \\
\hline 1 & $4 / 4 / 8 B$ & 18 & 1 & 49 & 114,8 & $\gamma$ & 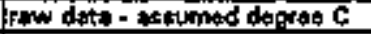 \\
\hline 1 & $5 / 2 / p_{B}$ & $\frac{2}{2}$ & 1 & & & $\mathbf{N}$ & 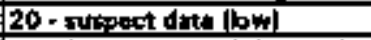 \\
\hline 1 & $5 / 2 / 88$ & 3 & 1 & 34 & 93.2 & $\mathbf{N}$ & raw data - apolumed deron C \\
\hline 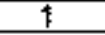 & 5/2jegt & 4 & 1 & 35 & $9 \dot{0}$ & N & 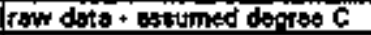 \\
\hline 1 & $\$ / 2 / 68$ & 5 & 1 & 35 & 96 & $\mathbf{N}$ & 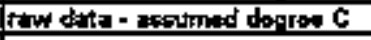 \\
\hline 1 & B/268 & e & 1 & 36 & 88.9 & $\mathbf{Y}$ & raw data - soschined dograe C \\
\hline 1 & E24 & 7 & 1 & 3a & 86.9 & $\mathbf{N}$ & raw date - atsumed dogros C \\
\hline 1 & 5pas & E & 1 & 3 & 86.8 & $\mathbf{N}$ & 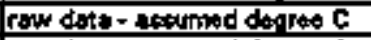 \\
\hline 1 & W21日3 & 9 & 1 & 36 & 86.8 & $\mathbf{H}$ & 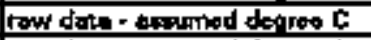 \\
\hline 1 & $5 / 290$ & 10 & 1 & 38 & 98.9 & $\mathbf{N}$ & row difte- alfourted derote C \\
\hline 1 & $5 / 2+98$ & 11 & 1 & 35 & 99.8 & $\mathbf{N}$ & row dath - aboumed degrat C \\
\hline 1 & 6/2自要 & 12 & 1 & 36 & 9B,8 & $\overline{\mathbf{N}}$ & raw data + aboulned dogreo C \\
\hline 1 & 6/2的 & 13 & 1 & 3 & 86,8 & $\overline{\mathrm{N}}$ & renw data- sobumed degres C \\
\hline 1 & EL28B & 14 & 1 & $\mathbf{3 0}$ & 96.8 & $\mathbf{N}$ & raw datb - Aselumed degina C \\
\hline 1 & Б/2/BB & 16 & 1 & 50 & 86.8 & $\mathbf{N}$ & row dato- ateumind dograto C \\
\hline$\overline{1}$ & 5kafBR & 16 & 9 & 36 & 98.8 & $\mathbf{H}$ & law data - istumed dogtob C \\
\hline 1 & 5/2k86 & 17 & 1 & 36 & 96.8 & $\mathbf{N}$ & 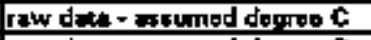 \\
\hline 1 & 5/BB & 918 & $t$ & 36 & 06.8 & N & 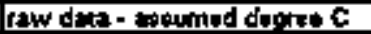 \\
\hline 1 & $50 / 38$ & $t$ & 1 & & 133 & $\bar{Y}$ & \\
\hline 1 & $5 / 6 \%$ & 2 & 1 & & 175 & $N$ & \\
\hline 1 & 5/aph & $\mathbf{3}$ & $\bar{q}$ & & 102 & N & \\
\hline 1 & Bafas & 4 & 1 & & Tot & $\mathbf{N}$ & \\
\hline 7 & Waras & $\mathbf{5}$ & 1 & & $10 \mathrm{t}$ & $\mathbf{N}$ & \\
\hline 9 & FOAB & $\mathbf{B}$ & 1 & & 101 & $\mathbf{N}$ & \\
\hline 1 & Ed & 7 & 1 & & 101 & $N$ & \\
\hline 1 & $5 \mathrm{~g}$ & 8 & 1 & & 100 & $N$ & \\
\hline 1 & Bतs & $\mathrm{a}$ & 1 & & 100 & $\mathbf{N}$ & \\
\hline 1 & 5/408 & 10 & 1 & & 100 & $\mathbf{N}$ & \\
\hline$\overline{1}$ & Fbras & 17 & 1 & & 100 & $\bar{k}$ & \\
\hline 1 & 5/9E8 & 12 & 1 & & 100 & N & 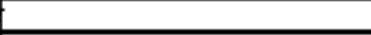 \\
\hline 1 & $5 / 018$ & 13 & 1 & & 100 & N & \\
\hline 1 & Elem & 14 & 1 & & 100 & it & \\
\hline 1 & 5/9j88 & 15 & 1 & & 100 & $\mathbf{H}$ & \\
\hline 1 & slofreg & 16. & 1 & & 100 & $N$ & \\
\hline$\overline{1}$ & 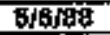 & 17 & 1 & & 100 & $\mathbf{N}$ & \\
\hline 9 & Bdos & 19 & $i$ & & $\mathbf{8 B}$ & $\mathbf{N}$ & \\
\hline 7 & 6rojes & 3 & $T$ & 43 & 10044 & $\mathbf{N}$ & raw drta - sosimed dereal C \\
\hline 1 & Gokas & 4 & 10 & 44 & 211.2 & $\mathbf{N}$ & 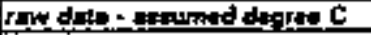 \\
\hline 1 & E/8/89 & 5 & $1+$ & 44 & 177.2 & $\sqrt{H}$ & Frw data - abunned degrad C \\
\hline 1 & e/680 & $\bar{\theta}$ & 1 & 44 & $\$ 11.2$ & i & $\operatorname{rew}$ dots - sueurned degrae C \\
\hline 1 & GEB & 7 & 1 & 44 & 191.2 & $\mathrm{~N}$ & 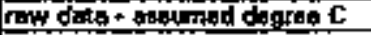 \\
\hline 1 & 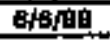 & E & 1 & 44 & $\$ 11.2$ & $\mathbf{k}$ & raw dato - atoumed degat C \\
\hline 1 & 68一丽 & g & $\overline{1}$ & 45 & 113 & it & 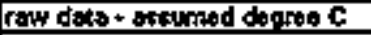 \\
\hline 1 & yoja & 10 & 1 & 45 & 113 & N & row doto - allonded dentaph C. \\
\hline 1 & Fify & 11 & 1 & 45 & 113 & i & 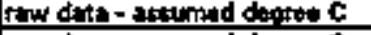 \\
\hline 1 & Q/9/60 & 12 & 1 & 45 & 113 & $N$ & 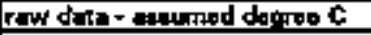 \\
\hline 1 & Expas & 13 & 1 & 45 & 113 & $\mathbf{N}$ & 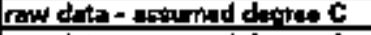 \\
\hline 1 & ojares & 14 & 1 & 45 & 113 & $\mathbf{N}$ & 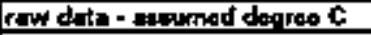 \\
\hline 1 & $6 / 8 / 88$ & 15 & 1 & 45 & 113 & $\mathbf{N}$ & 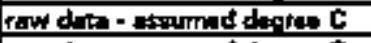 \\
\hline 1 & 69رAB & 16 & 1 & 45 & 113 & $\bar{N}$ & 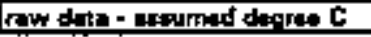 \\
\hline
\end{tabular}

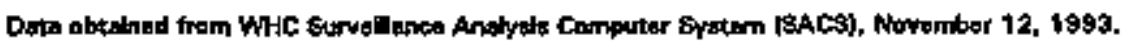


TANK 241-A-TOB

\begin{tabular}{|c|c|c|c|c|c|c|c|}
\hline \multicolumn{8}{|c|}{ THERHOCOLPLE DATA } \\
\hline Thi: I & DATE & THERTO * & TARE 551 & TEMP $\mathbf{c}$ & TFMP F & $Y=M A X$ TIAP & CO \\
\hline 1 & $6 / 6 / 88$ & 17 & 1 & 46 & 113 & $N$ & raw data - andmed degres $\overline{\mathrm{C}}$ \\
\hline 1 & $6 \sqrt{6 / 28 B}$ & To & $T$ & 48 & 114.8 & $\mathbf{Y}$ & row deta - atetrmad degrat C \\
\hline 1 & \$17/88 & $\pi$ & $\bar{t}$ & & 134 & $\bar{Y}$ & \\
\hline 1 & 6/778 & 2 & 1 & & 116 & N & \\
\hline 1 & $6 / 77 \mathrm{r} 8$ & 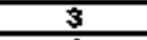 & 1 & & 102 & N & \\
\hline $\mathbf{1}$ & 47788 & 4 & $\overline{1}$ & & 101 & $\mathbf{N}$ & \\
\hline 1 & 바같ㅇ & 5 & 1 & & 101 & $\mathbf{N}$ & \\
\hline 1 & 타가조 & 8 & 1 & & 101 & $\mathbf{N}$ & \\
\hline 1 & Fi7BB & 7 & 1 & & 101 & $N$ & \\
\hline 1 & intoe & 8 & 1 & & 101 & $N$ & \\
\hline 1 & OH17'te & $\theta$ & 1 & & 101 & N & \\
\hline 1 & 6/7/8B & 70 & 1 & & 100 & $\mathbf{N}$ & \\
\hline 1 & E/ITAR & 17 & 1 & & $\overline{100}$ & $\mathbf{N}$ & \\
\hline 1. & B/17/AE & 12 & 1 & & 100 & N & \\
\hline$\tau$ & 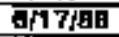 & $\mathbf{1 3}$ & 1 & & 100 & $\mathbf{M}$ & \\
\hline$\tau$ & 9ी179ạ & 14 & 1 & & 100 & $M$ & \\
\hline 1 & of17a8 & 15 & 1 & & 100 & $\bar{N}$ & \\
\hline 7 & ब/7/ & 16 & 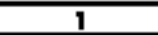 & & 100 & $\bar{N}$ & \\
\hline 1 & 6h17k9 & 17 & 1 & & 100 & $\mathbf{N}$ & \\
\hline 1 & Ef17ra & $1 \mathrm{~B}$ & 1 & & 98 & $\mathbf{N}$ & \\
\hline 1 & $7 / 4 \mathrm{Bg}$ & 1 & 1 & & 134 & $\mathbf{Y}$ & \\
\hline 1 & 7/4/BE & 2 & 1 & & $15 \overline{1}$ & $\bar{N}$ & \\
\hline 1 & $7 / 4 / B B$ & 3 & 1 & & 905 & N & \\
\hline 1 & $7 / 4 / 88$ & 4 & $\mathbf{i}$ & & 104 & N & \\
\hline 1 & $7 / 4 / 88$ & $\mathbf{5}$ & 1 & & 104 & $\mathbf{N}$ & \\
\hline$T$ & $7 / 4 / 88$ & g & $\overline{1}$ & & 103 & $\mathbf{N}$ & \\
\hline 1 & $7 / 4 / 98$ & 7 & 1 & & 103 & $\mathbf{N}$ & \\
\hline 1 & $7 / 4 / 98$ & 8 & 1 & & 103 & N & \\
\hline 1 & $7 / 4 / 88$ & 9 & 1 & & 103 & N & \\
\hline 1 & $7 / 4 / 98$ & 10 & 1 & & 109 & $\mathbf{N}$ & \\
\hline 1 & $7 / 4 / 98$ & 11 & 1 & & 103 & $\mathbf{N}$ & \\
\hline$T$ & $7 / 4 \sqrt{18}$ & 12 & $\overline{1}$ & & 103 & $\mathbf{N}$ & \\
\hline 1 & $7 / 4 \sqrt{9}$ & $\sqrt{13}$ & 1 & & 103 & $\mathbf{N}$ & \\
\hline 1 & $7 / 4 / 98$ & 14 & 1 & & $10 \overline{3}$ & $\mathbf{N}$ & \\
\hline 1 & $7 / 4 / a 9$ & 15 & $\mathbf{T}$ & & 103 & $\mathbf{N}$ & \\
\hline 1 & $7 / 4 \sqrt{9 a}$ & $\sqrt{9}$ & 1 & & 100 & $\mathbf{N}$ & \\
\hline 1 & $8 / 1,18$ & 3 & 1 & & & $\mathbf{N}$ & 69 - aupact dnta how) \\
\hline$\overline{1}$ & GARE & 4 & 7 & & & N & Bo-eutpect daja (dow) \\
\hline 1 & 自/的息 & E & 1 & & & $\mathrm{~N}$ & 60 - ouspet dalo daw) \\
\hline 1 & B/1/A日 & 6 & $\mathbf{t}$ & & & $\mathbf{N}$ & 50 - sutpect dette dow) \\
\hline 1 & कीजि & 7 & 5 & & & 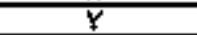 & Gt - suspent dite dow) \\
\hline 1 & EM/BE & 8 & 5 & & & $\mathbf{N}$ & [bil - gurpiact data (low] \\
\hline 1 & 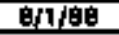 & $\mathrm{g}$ & 3 & & & $\mathbf{N}$ & 01 - euspact dala (dow) \\
\hline$\overline{1}$ & EMI/Bd & 10 & $\$$ & & & $\mathrm{~N}$ & B1 - curpect dala (Naw) \\
\hline 5 & B/1/BB & 11 & 1 & & & $N$ & [51-evipert data (low) \\
\hline 1 & B/1/Ba & 12 & 3 & & & N & [B1 - tutpeot dila (low) \\
\hline $\mathbf{T}$ & ल/10a & 13 & $\bar{T}$ & & & $\mathbf{N}$ & 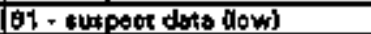 \\
\hline 1 & G1/Be & 14 & 1 & & & $\mathbf{N}$ & [B1 - Elospet dito dow) \\
\hline$\overline{1}$ & G/18B & 15 & 7 & & & $\mathbf{N}$ & [B1 - ctuspeut difa dow] \\
\hline$\overline{1}$ & OM/Bg & 16 & 1 & & & $\mathbf{N}$ & 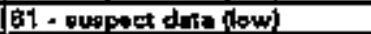 \\
\hline$\$$ & B/5B & 2 & $i$ & & & $N$ & 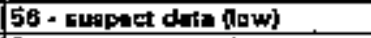 \\
\hline 1 & $9 / 5188$ & 3 & 1 & & & $\mathbf{N}$ & 71 - nuspost dota fow) \\
\hline $\mathbf{T}$ & 9/S/BB & 4 & 1 & & & N & (71 - vuapact date (dew) \\
\hline 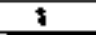 & 9/5/BB & 5 & 1. & & & $\mathbf{N}$ & [71 - eurpact data dow] \\
\hline$\overline{1}$ & $9 \sqrt{3} \mathrm{~B} 8 \mathrm{~B}$ & $\overline{9}$ & 4. & & & $\bar{N}$ & 77 - entpert dutp dow) \\
\hline$\overline{1}$ & OSABB & 7 & 1 & & & $\bar{N}$ & 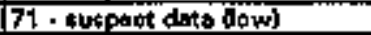 \\
\hline 1 & B/S/BB & 8 & 1 & & & $\mathbf{N}$ & [71 - cusposot dite dow] \\
\hline 1 & 9/5/BB & g & 1 & & & $\mathbf{N}$ & [71 - gugpont dito dow) \\
\hline 1 & ENEFa & 10 & 1 & & & $\mathbf{N}$ & $771 \cdot$ sutpost drate dow \\
\hline 1 & 9/5/日9 & 11 & $\mathbf{1}$ & & & $\bar{Y}$ & 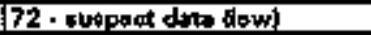 \\
\hline 1 & 95ist & 12 & 7 & & & $\mathbf{N}$ & 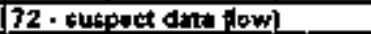 \\
\hline 1 & 9/5/6a & 13 & 1 & & & N & 71 - vurged date fowl \\
\hline$\overline{1}$ & 98190 & 14 & $T$ & & & $\mathbf{N}$ & 71 - eurgfinct data firw) \\
\hline$\overline{1}$ & $9 / \sqrt{60}$ & 15 & $\overline{1}$ & & & $\mathbf{N}$ & 71 - ausence date How] \\
\hline 1 & O/568 & 16 & 1 & & & $\mathbf{N}$ & $\sqrt{72}$ - guapeat data low] \\
\hline 1 & $10 / 3 \mathrm{rge}$ & 1 & 5 & & & $N$ & 42- tubpect date liowl \\
\hline
\end{tabular}

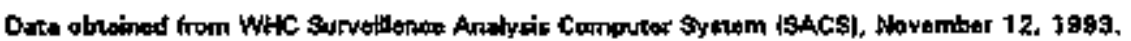




\begin{tabular}{|c|c|c|c|c|c|c|c|}
\hline \multicolumn{8}{|c|}{ 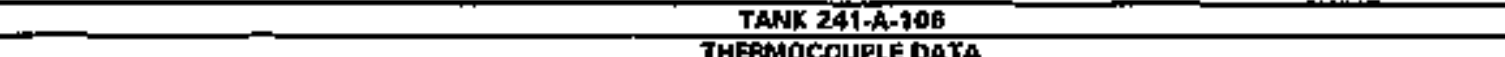 } \\
\hline & & & & & & & \\
\hline TREE $*$ & DATE & THFillo: & THEI SE & TENPC & TEMPF & $Y=$ FIAX TEMP & Conlinakis \\
\hline 1 & 202388 & 2 & 1 & & & $+\mathbf{N}$ & 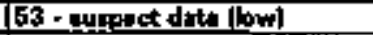 \\
\hline 1 & $10 / 388$ & 3 & 1 & & & $\bar{Y}$ & [54- auspege data lbw] \\
\hline 1 & $20 / 3 / 89$ & 4 & $\mathbf{j}$ & & & $\mathbf{N}$ & 54 - Eubpecs data (low] \\
\hline 7 & $10 / 3103$ & 5 & 1 & & & $\$$ & 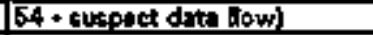 \\
\hline 1 & $10 / 3 / 88$ & 6 & 1 & & & $\mathbf{N}$ & 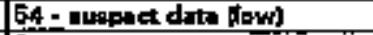 \\
\hline 1 & $10 / 3 / 88$ & 7 & 1 & & & $\mathbf{N}$ & 54 + cuspent data dowi \\
\hline 1 & $10 / 3 / 38$ & 8 & 1 & & & $\mathbf{N}$ & 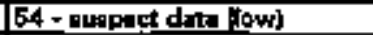 \\
\hline 1 & $10 \sqrt[3]{88}$ & 8 & 1 & & & $\mathbf{N}$ & [54- muapuct dita [ow] \\
\hline$t$ & 10,318 & 10 & 1 & & & N & 54 - turpart deto haw \\
\hline 1 & 10어뵤 & 11 & 1 & & & $\overline{\mathbf{N}}$ & 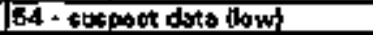 \\
\hline 1 & 10/390 & 12 & 1 & & & N & 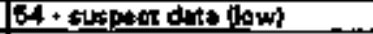 \\
\hline 1 & 10\%3日8 & 13 & 1 & & & N & F4 - atraxpent date (lowh \\
\hline 1 & 106389 & 14 & 1 & & & $\overline{\mathbf{M}}$ & 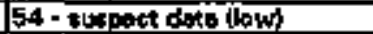 \\
\hline 1 & J0\%380 & 16 & 1 & & & N & 54 - ongest deto lowh \\
\hline 1 & $10 / 3 / 38$ & 18 & 1 & & & N & [64-aulepes data [low] \\
\hline 1 & 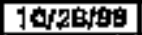 & 1 & $\mathbf{T}$ & & 135 & $\mathbf{Y}$ & दsest \\
\hline 1 & 10Kk IF & $\mathbf{3}$ & $i$ & & 104 & N & \\
\hline 1 & $10 / 2819 a$ & 4 & 1 & & 103 & $N$ & \\
\hline $\mathrm{I}$ & $10 / 2889$ & 5 & 1 & & 108 & W & \\
\hline 1 & Tor & b & 1. & & 102 & \$ & \\
\hline$t$ & $10 / 26 / 8$ & 7 & 1 & & 102 & $\overline{\mathbf{N}}$ & \\
\hline 1 & 1012बह & 8 & 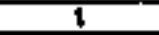 & & 102 & $\mathbf{N}$ & \\
\hline 1 & $10 / 28 / 38$ & 8 & 1 & & 102 & $M$ & \\
\hline 1 & 10/2B89 & 10 & \pm & & 102 & $\mathbf{N}$ & \\
\hline 1 & 10 pares & 5 & $t$ & & 102 & $\mathbf{N}$ & \\
\hline $\mathbf{1}$ & 10/2Bras & 12 & $i$ & & 102 & $\mathbf{M}$ & \\
\hline 1 & $10428 / 88$ & 13 & 1 & & 102 & $\mathbf{N}$ & \\
\hline 1 & 10129188 & 14 & 1 & & 102 & $\mathbf{N}$ & \\
\hline 1 & 1012808 & 15 & 1 & & 102 & $\mathbf{N}$ & \\
\hline 1 & $10 / 28 / 88$ & 16 & 1 & & $\overline{102}$ & N & \\
\hline 1 & 10/28/88 & 17 & 1 & & 102 & $\mathbf{N}$ & \\
\hline 1 & 10/21/88 & 18 & 1 & & 101 & N & \\
\hline 1 & T1/ariga & 1 & 1 & & $\overline{156}$ & $\overline{\mathbf{Y}}$ & \\
\hline 1 & 11\% & 2 & 1 & & 118 & H & \\
\hline 1 & 118\%a日 & $\overline{3}$ & 1 & & 118 & $\bar{N}$ & \\
\hline 1 & 11/8190 & 4 & 1 & & 110 & $\mathbf{N}$ & \\
\hline 1 & $11 / 8 / \mathrm{Bg}$ & 6 & 1 & & 1116 & $\mathbf{M}$ & \\
\hline$j$ & 11/8留 & $\theta$ & 1 & & 318 & N & \\
\hline 1 & 17/g/ag & 7 & 1 & & $\mathbf{t 1 8}$ & $\mathbf{N}$ & \\
\hline 7 & 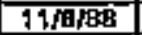 & $\bar{B}$ & $\bar{T}$ & & 318 & $\bar{D}$ & \\
\hline$i$ & $11 / 0 / 38$ & 9 & 1 & & 796 & H & \\
\hline 7 & $11 / 9 k 8$ & 10 & 1 & & 178 & $\mathbf{N}$ & \\
\hline 1 & 1tros & 11 & 1 & & 778 & $N$ & \\
\hline$i$ & 11 deta & 12 & 1 & & 778 & $\mathbf{N}$ & \\
\hline 1 & $11 / 8 / 98$ & 13 & 1 & & 118 & $\mathbf{N}$ & \\
\hline 1 & 11/बras & 14 & 1 & & $\overline{118}$ & $\mathbf{N}$ & \\
\hline 1 & 11 pes & 5 & 1 & & $\overline{118}$ & $\mathrm{~N}$ & \\
\hline 1 & $11 / 8 / 38$ & 58 & 1 & & 118 & $\mathbf{R}$ & \\
\hline 1 & tofleres & 1 & 1 & & 135 & $\bar{Y}$ & cthill \\
\hline 1 & $11 / 10 / 88$ & 2 & 1 & & 116 & $\mathbf{N}$ & \\
\hline 1 & $11 / 1688$ & 3 & 1 & & 103 & $\mathbf{N}$ & \\
\hline 1 & 15/16rso & 4 & 1 & & 102 & $\mathbf{N}$ & \\
\hline 1 & IN1타영 & 5 & 1 & & 102 & $\mathbf{N}$ & \\
\hline 1 & 11/16reg & 8 & 1. & & 102 & $N$ & \\
\hline 1 & ग2/18/8 & 7 & 7. & & 202 & N & \\
\hline$i$ & 11/1\% & 8 & 1 & & 102 & $\mathbf{N}$ & \\
\hline 1 & IW/AB & 9 & 1 & & 102 & $\mathbf{N}$ & \\
\hline 1 & tw1889 & 50 & 7 & & 101 & $N$ & \\
\hline 7 & 17/ & 17 & 1 & & $\overline{101}$ & 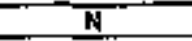 & \\
\hline 1 & 71/1日的息 & 12 & 1 & & to1 & R & \\
\hline$T$ & ग1 & 13 & 1. & & 101 & $\mathbf{N}$ & \\
\hline 1 & 1t/10/83 & 14 & 1 & & 101 & $\mathbf{N}$ & \\
\hline 1 & 11) & 15 & 1 & & 107 & N & \\
\hline 1 & 7 N & 19 & 1 & & 101 & $\mathbf{N}$ & \\
\hline 1 & 11/1889 & 17 & 1 & & 101 & $n$ & \\
\hline 1 & Jarkege & 18 & 1 & & 300 & $N$ & \\
\hline
\end{tabular}

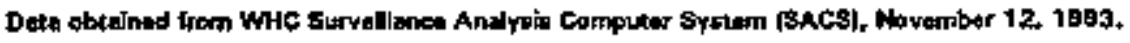




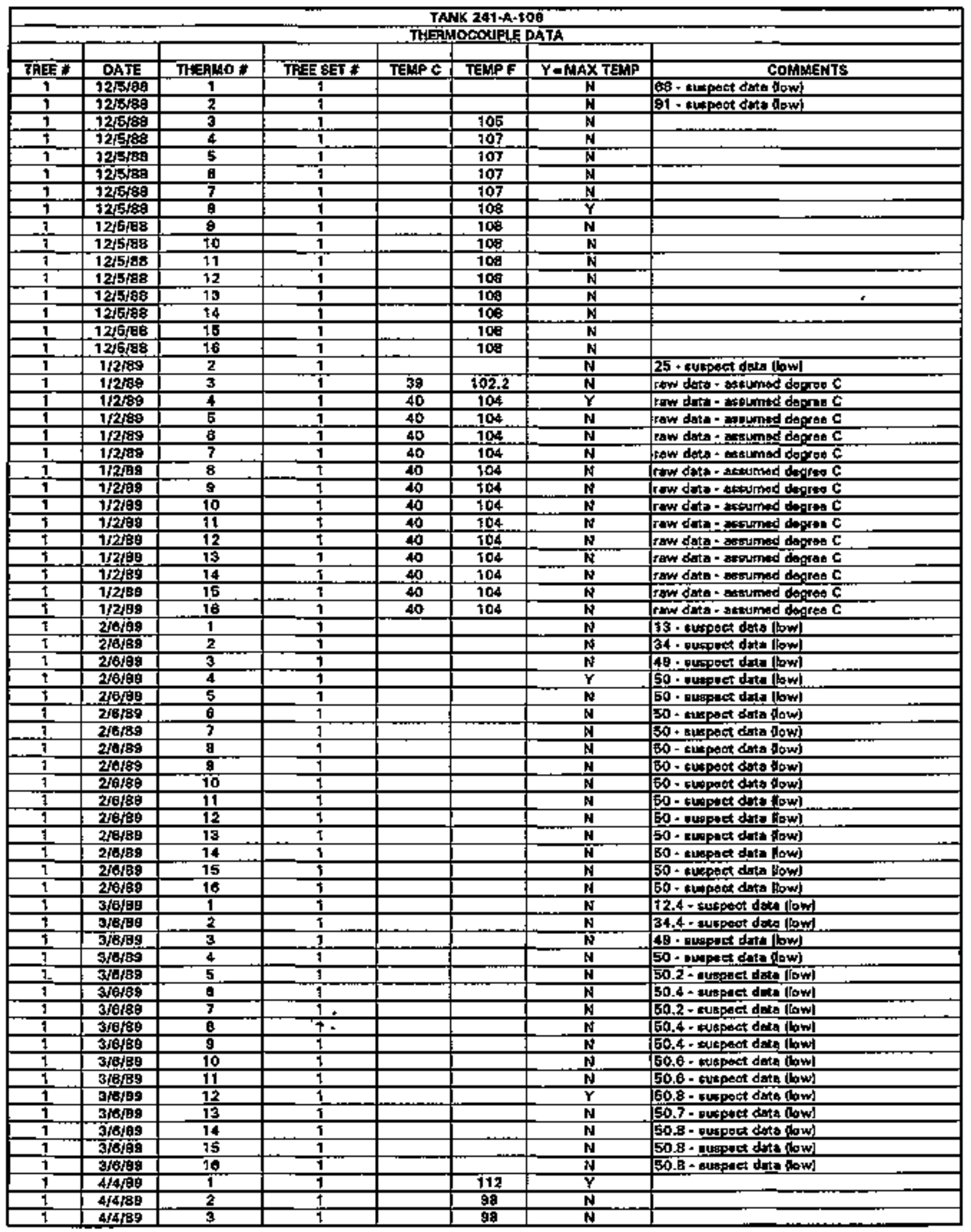




\begin{tabular}{|c|c|c|c|c|c|c|c|}
\hline \multicolumn{8}{|c|}{ 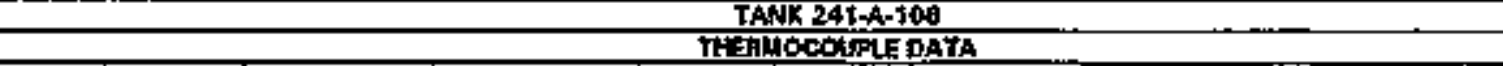 } \\
\hline TR过 E : & $\overline{\text { DATE }}$ & THEFAO * & 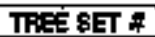 & TEMPC & TEMPF & $Y=M A X$ TEMP & COMLERTSS \\
\hline 1 & $4+4 / 99$ & 4 & 1 & & 98 & 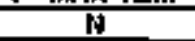 & \\
\hline 1 & $44 \mathrm{ABO}$ & 5 & 1 & & 67 & $\mathbf{N}$ & \\
\hline 1 & $4 / 4 / 99$ & $\underline{6}$ & 1 & & 97 & $\mathbf{N}$ & \\
\hline 1 & $4 / 4 / 99$ & $\overline{7}$ & 1 & & 97 & $\mathbf{M}$ & \\
\hline 1 & $4 / 4 \sqrt{68}$ & $\overline{8}$ & 1 & & 87 & $\mathbf{N}$ & \\
\hline 1 & $4 / 4 / \mathrm{g}^{9}$ & 8 & 1 & & 97 & $\mathrm{n}$ & \\
\hline 1 & 4मकब & 10 & 1 & & 97 & N & \\
\hline 1 & $4 \longdiv { 4 1 8 0 }$ & 11 & 1 & & 97 & $\mathbf{H}$ & \\
\hline 1 & $A / 4 B B$ & 12 & 1 & & 可 & $\mathbf{N}$ & \\
\hline 1 & $A / 4 / B B$ & 13 & 1 & & 97 & $\mathbf{N}$ & \\
\hline$i$ & $4 \longdiv { 4 1 3 9 }$ & 14 & 1 & & 97 & $\mathbf{N}$ & \\
\hline 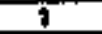 & $4 \sqrt[4 / 89]{4}$ & 15 & 1 & & 97 & $\mathbf{N}$ & \\
\hline 1 & $4 / 4 / 89$ & 16 & 1 & & 96 & H & \\
\hline \pm & $6 / 1 / 99$ & $t$ & $i$ & & & $\mathbf{N}$ & 39.4 - aubect date [low] \\
\hline 1 & $51 / 99$ & 2 & 1. & & & $\mathbf{N}$ & 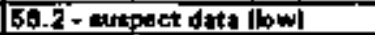 \\
\hline 1 & $5 \longdiv { 1 / 8 9 }$ & 3 & 1 & & & $N$ & 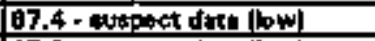 \\
\hline 1 & $5 \longdiv { 1 / 8 9 }$ & 4 & 1 & & & $\mathbf{N}$ & 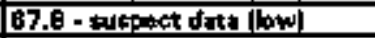 \\
\hline 1 & \$1/Be & 5 & 1 & & & $\mathbf{N}$ & 67.8 - con \\
\hline 1 & E1/99 & 6 & 1 & & & $N$ & 6H - nuipand data $(\mathrm{pow})$ \\
\hline 1 & 6708 & 7 & 1 & & & $\mathbf{N}$ & 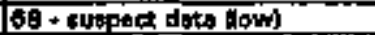 \\
\hline 1 & Wheg & 8 & 1 & & & $N$ & 89 - buspet dete low \\
\hline 1 & EN/BB & g & 1 & & & $\mathbf{N}$ & 68, ] - cuspact data [low] \\
\hline$\$$ & SHIBa & 10 & 1 & & & $\mathbf{N}$ & $\overline{68, \overline{2}}$ - tuspart dota (now) \\
\hline 1 & 51189 & $\overline{11}$ & 1 & & & $\sqrt{N}$ & 68.2 - ouspoct dita $(\mathrm{buw})$ \\
\hline 1 & w1/8g & 12 & 1 & & & N & 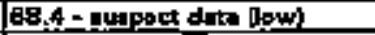 \\
\hline$\overline{1}$ & 51/89 & 13 & 1 & & & $\bar{N}$ & 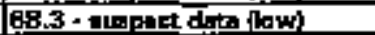 \\
\hline 1 & G/18 & 14 & 1 & & & is & 68,4 - ubspect data (low) \\
\hline 1 & 511899 & 16 & 1 & & & $\mathbf{N}$ & 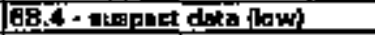 \\
\hline 1 & $5 / 1 / 89$ & 16 & $t$ & & & $\bar{Y}$ & BH.5 - atdopect deta lbw) \\
\hline 1 & B & $\tau$ & I & & & W & 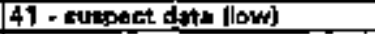 \\
\hline 1 & BWa99 & 2 & 1 & & & $\bar{N}$ & 58 - tuspect deto flom \\
\hline 1 & Bj5\%99 & $\overline{3}$ & 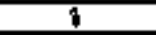 & & & W & Be - tuspoct dats llowl \\
\hline 1 & 65.99 & 4 & 1 & & & $\mathbf{Y}$ & 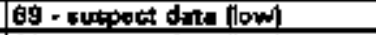 \\
\hline 1 & Bमf59 & 5 & 7 & & & $\bar{N}$ & 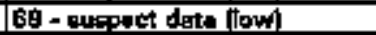 \\
\hline 1 & 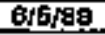 & B & $\$$ & & & $\mathbf{N}$ & Bg - ruspact data l|pwl \\
\hline 1 & G/5\%9 & 7 & $t$ & & & $\mathbf{N}$ & 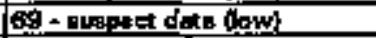 \\
\hline 1 & E. & 8 & 1 & & & $\mathbf{M}$ & 69- suppart dste dow \\
\hline 1 & a & $\mathrm{g}$ & 1 & & & $\mathbf{N}$ & 69-auppart dista dows \\
\hline 1 & 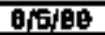 & 10 & $T$ & & & $\bar{N}$ & 66- tuspert diks dowh \\
\hline 1 & 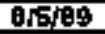 & 17 & 1 & & & $\mathbf{N}$ & 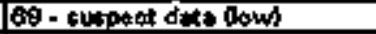 \\
\hline 1 & 야형 & 12 & 1 & & & $\mathbf{N}$ & 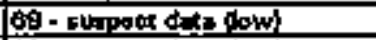 \\
\hline 1 & $6 / 5 / 98$ & 13 & 1 & & & $\mathbf{N}$ & 69 - nupact do form \\
\hline$i$ & Sha9 & 14 & 1 & & & $\mathbf{N}$ & 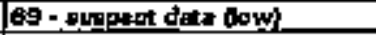 \\
\hline 1 & D5 & 15 & 1 & & & $\mathbf{N}$ & 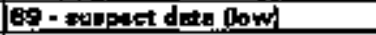 \\
\hline 1 & 9 ibj89 & 16 & 1 & & & $\mathbf{N}$ & 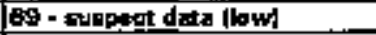 \\
\hline 1 & $7 / 399$ & 1 & 1 & & & $\mathbf{N}$ & 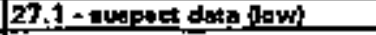 \\
\hline$T$ & $73 \mathrm{~kg}$ & $\overline{2}$ & $\overline{1}$ & & & $\mathbf{N}$ & 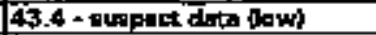 \\
\hline 1 & 7 及画 & 3 & 1 & & & $\mathbf{N}$ & 54.3 - tuppart data (0w) \\
\hline 7 & 7 अ & 4 & 1 & & & $\bar{N}$ & (34,6 + Euspart dota (daw) \\
\hline 1 & 7 73: & 6 & 1 & & & $\mathbf{N}$ & 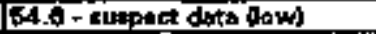 \\
\hline 1. & $7 \sqrt{1899}$ & E & 1 & & & $\mathbf{N}$ & 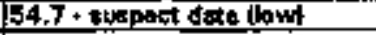 \\
\hline 1 & 73 & 7 & 1 & & & $\sqrt{N}$ & B4.8 - tispost data dawt \\
\hline 1 & $7 / 389$ & 早 & 1 & & & W & 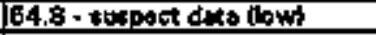 \\
\hline 1 & $7 / 3 \% 9$ & 9 & 1, & & & $\bar{M}$ & 54.9 - budpagt deto thot \\
\hline $\mathbf{I}$ & $7 / 399$ & 10 & 7. & & & $\mathbf{N}$ & 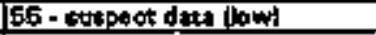 \\
\hline 1 & $7 / 3 \% 9$ & 11 & 1. & & & $\mathbf{N}$ & E5 - sutepot data lawi \\
\hline 1 & $7 \sqrt{3}+9$ & $\overline{12}$ & 1 & & & $\mathbf{N}$ & 55 - tacosot data low \\
\hline 1 & 7 然89 & 13 & 1 & & & N & 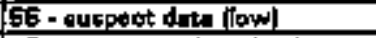 \\
\hline 1 & 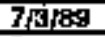 & 14 & 1 & & & $\mathbf{N}$ & 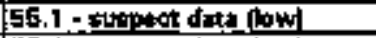 \\
\hline 1 & $7 /, 189$ & 15 & 1 & & & $\mathbf{Y}$ & 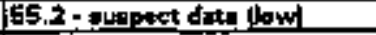 \\
\hline 1 & 73339 & 16 & 1 & & & $\mathbf{N}$ & 155.2 - augpest date llowt \\
\hline$I$ & a 7739 & 1 & 1 & & & $\mathbf{N}$ & 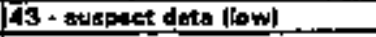 \\
\hline 1 & aribe & 2 & 1 & & & N & 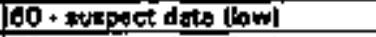 \\
\hline 1 & 90709 & 3 & 1. & & & $\mathbf{N}$ & 72 - tedspost dato lowl \\
\hline 7 & Bतilag & 4 & 1 & & & $\bar{Y}$ & 73 - twatpat date law \\
\hline 1 & 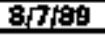 & 点 & 1 & & & N & 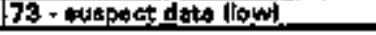 \\
\hline
\end{tabular}

Doto obteined fram WHC Survallance Analyeis Computar Syctem (SACB, Navamber 12, 1993. 


\begin{tabular}{|c|c|c|c|c|c|c|c|}
\hline \multicolumn{8}{|c|}{ TANK 241-A-T09 } \\
\hline & & & & THEFin & DCOUPLE & ATA & \\
\hline TFFE & DATE & 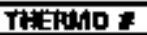 & TREA SET & TENP C & TEMP F & $Y=$ MAX TEUP & COHAENTS \\
\hline 1 & enfirs & 8 & 1 & & & $\mathbf{N}$ & 73 - traport disto low) \\
\hline 1. & B/7/Ag & 7 & 1 & & & N & 73 - sutpotet dits [ow \\
\hline 1 & B7/69 & 日 & 1 & & & $\mathbf{N}$ & 73 - tettegect dste dowl \\
\hline 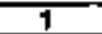 & Bनrleg & 9 & $\mathbf{T}$ & & & $\mathbf{N}$ & 73 - stspect date haw) \\
\hline 1 & 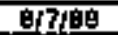 & 10 & 1 & & & $\mathbf{N}$ & 73 - sappect dota (law) \\
\hline 1 & Ellikg & 11 & 1 & & & $\mathbf{N}$ & 73 : suppet data lawh \\
\hline$\overline{1}$ & EMRB & 12 & 1 & & & $\mathbf{N}$ & 73 - sustepert dota (10w) \\
\hline 1 & E/7/Bg & 13 & 1 & & & $\mathbf{M}$ & 73 - eltipont dito dow) \\
\hline 1 & Bmis & 14 & 1. & & & $\bar{M}$ & $73+$ totspsent doto dow \\
\hline 7 & E/7/Ba & 15 & 1 & & & $\mathbf{N}$ & 73 - tutpot dato flow \\
\hline 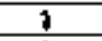 & $8 / 7 / 89$ & 16 & 1 & & & $\mathbf{N}$ & 73 - sulspot dita low \\
\hline 1 & 1D2196 & 1 & 1 & & & $\mathbf{N}$ & 24 - cuspect dita Dow \\
\hline$\frac{t}{t}$ & $10 \mathrm{j} 2 \mathrm{Bg}$ & $\overline{2}$ & 7 & 37 & 98.8 & $\mathbf{N}$ & 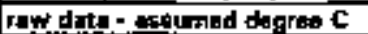 \\
\hline 1 & $10 \mathrm{HABg}$ & 3 & 1 & 37 & 08.5 & $\mathbf{N}$ & gat dita - gopguned degres C \\
\hline$\overline{1}$ & $10 \mathrm{~F} / \mathrm{Bg}$ & 4 & 1 & 37 & 98,8 & N & raw data - esurined degrea C \\
\hline 1 & 1012180 & $\mathbf{6}$ & 7 & 30 & 100.4 & $\mathbf{Y}$ & raw data - tateumed degres $C$ \\
\hline 1 & $1 0 \longdiv { 2 8 0 }$ & 6 & 1 & 38 & 100,4 & $\overline{\mathbf{N}}$ & 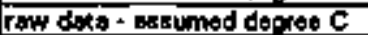 \\
\hline 1 & $1 0 \longdiv { 2 / 8 9 }$ & 7 & 1 & 37 & 98,6 & $\overline{\mathbf{N}}$ & row dsta - astumed dorogo C \\
\hline $\mathbf{i}$ & 10/2f & 8 & 1 & $\overline{38}$ & 100.4 & $\bar{N}$ & 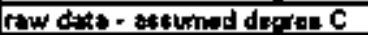 \\
\hline 1 & $10 / 2 / 89$ & 9 & $i$ & 38 & 100.4 & $\mathbf{N}$ & 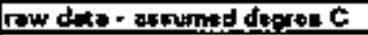 \\
\hline$T$ & $10 / 288$ & 10 & $T$ & 38 & 100.4 & $\overline{\mathbf{N}}$ & row dta - atzumedderan C \\
\hline 1 & $10 / 2,89$ & If & 1 & 38 & 100.4 & $\mathbf{N}$ & raw doxe : arumad degree C \\
\hline 1 & 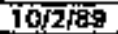 & 12 & 7 & 35 & 96,8 & $\overline{\mathbf{N}}$ & raw dSt - ansumed degrat C \\
\hline 1 & $10 \mathrm{~d} 2 \mathrm{ja}$ & 73 & 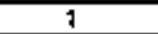 & 31 & 87.8 & $\overline{\mathbf{N}}$ & 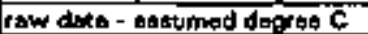 \\
\hline 1 & $10 / 2 / 89$ & $\sqrt{4}$ & 3 & & & $\bar{N}$ & 29 - sumpeck dita $\mid(b w)$ \\
\hline 1 & $10 / 2 / 39$ & $\mathbf{1 5}$ & $i$ & & & $\overline{\mathbf{N}}$ & 21) - surpent dita llowl \\
\hline 1 & $10 / 289$ & 10 & 5 & & & $\bar{N}$ & 18 - cusgend dota (low) \\
\hline 1 & $1 / 1 / 190$ & 2 & 1. & & & $\mathbf{N}$ & 29 - nugpact dota libw| \\
\hline 1 & $1 / 1 / \mathrm{g} 0$ & 3. & $t$ & 49 & 100,4 & $\mathbf{N}$ & raw data - otsumind degrae C \\
\hline 1 & $1 / 1 / 90^{\circ}$ & 4 & 1 & 45 & 100.4 & $\overline{\mathbf{N}}$ & 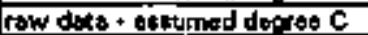 \\
\hline 1 & $1 / 1 / 90$ & $\overline{5}$ & 1 & 43 & 109.4 & $\overline{\mathbf{N}}$ & 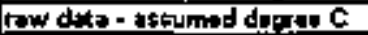 \\
\hline 1 & $1 / 1 / 90$ & 8 & 1 & 44 & 111,2 & $\mathbf{Y}$ & raw data - angulmed degren C \\
\hline 1 & 1 11190 & 7 & 1 & $4 \mathbf{4 3}$ & 109.4 & $\mathbf{N}$ & raw dote + nEBsumed degren C \\
\hline 1 & $1 / 1 / 90$ & 8 & 7 & 44 & 111,2 & N & raw data - eserumed dogrep $C$ \\
\hline 1 & $1 / 1 / 90$ & $\mathbf{s}$ & 1 & $4 \overline{4}$ & $\overline{111,2}$ & $\mathbf{N}$ & row date - itstumod dogrea C \\
\hline 1 & 17190 & 10 & 1 & 44 & 111.2 & $\overline{\mathbf{N}}$ & raw date - atiofmed dorse C \\
\hline 1 & 11190 & 11 & 1 & 44 & 111.2 & $\mathbf{N}$ & fow data - tatumet derog C \\
\hline 1 & $1 / 1 / 00$ & 12 & 1 & 44 & 1112 & $\mathbf{N}$ & raw date - stourned derou c \\
\hline 7 & $1 / 1 / 00$ & 13 & 1 & 44 & 111,2 & $\mathbf{N}$ & Irw data - nequimed deron c \\
\hline 7 & 11190 & 14 & 1 & $44^{-1}$ & 111.2 & $\mathbf{N}$ & rew data - sosumed deprop C. \\
\hline $\mathrm{J}$ & $1 / 1 / 90$ & 18 & 1 & 44 & 111.2 & $\mathbf{N}$ & 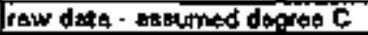 \\
\hline T & 1/190 & 16 & 1 & 44 & 111.2 & $\bar{N}$ & 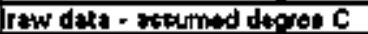 \\
\hline 9 & $1 / 1 / 00$ & 1 & 2 & & 139.8 & $\bar{Y}$ & \\
\hline $\mathbf{7}$ & ThHBD & 2 & $\overline{2}$ & & 120 & $\overline{\mathbf{N}}$ & \\
\hline 1 & $1 / 2 / 80$ & 3 & 2 & & 105.5 & $\mathbf{N}$ & \\
\hline 1 & $1 / 2 / 90$ & 4 & 2 & & 104.8 & $\overline{\mathbf{N}}$ & \\
\hline 1 & $1 / 3 / 90$ & 5 & $\overline{\mathbf{z}}$ & & 1048 & $\mathbf{N}$ & \\
\hline 1 & $1 / 1 / 90$ & E & 2 & & 104,6 & $\overline{\mathbf{N}}$ & \\
\hline 1 & $1 / 1 / 90$ & $\frac{7}{7}$ & 2 & & 104.7 & $\mathbf{\mathbf { H }}$ & \\
\hline $\mathbf{T}$ & $1 / 1900$ & 8 & $\overline{2}$ & & 104.6 & $\overline{\mathbf{N}}$ & \\
\hline 1 & $1 / 1 / 90$ & 9 & 2 & & 104.6 & $\mathbf{H}$ & \\
\hline 1 & $1 / 1 / 40$ & 10 & 2 & & 104.4 & $\mathbf{N}$ & \\
\hline 1 & $1 / 1 / 90$ & 11 & 2 & & 104.4 & $\mathbf{N}$ & \\
\hline 1 & $1 / 1 / 90$ & 12 & 2 & & 104,4 & $\mathbf{N}$ & \\
\hline 1 & $1 / 190$ & $\overline{\mathbf{1 3}}$ & 2 & & 104,4 & $\mathbf{N}$ & \\
\hline 1 & $1 / 560$ & $\overline{14}$ & 2 & & 104.3 & $\mathbf{m}$ & \\
\hline 1 & $1 / \mathrm{t} / 90$ & 16 & 2 & & 104.4 & $\mathbf{N}$ & \\
\hline 1 & $1 / 1 / 90$ & 16 & 2 & & 104.2 & $\mathbf{N}$ & \\
\hline 1 & $2+5 \sqrt{90}$ & 2 & 1 & & & $\mathbf{M}$ & 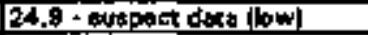 \\
\hline 1 & 221590 & 3 & 1 & 40.3 & 104.54 & $\mathbf{N}$ & raw dita - asturind da jon C \\
\hline 1 & गअक्षण & 4 & 1 & $40 . \overline{9}$ & 105,62 & $\mathbf{M}$ & cow deto + attumed deren C \\
\hline 1 & $2 \sqrt{190}$ & 5 & 1 & 41 & 105.8 & N & 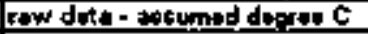 \\
\hline$\overline{1}$ & $2 \sqrt{6 j 80}$ & 6 & i & $41, \overline{1}$ & 10598 & $\mathbf{N}$ & 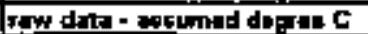 \\
\hline 1 & 2 25jo & 7 & 1 & 41 & 105.8 & $\mathbf{N}$ & 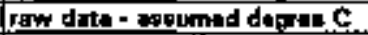 \\
\hline 1 & $2 / 6 j 90$ & 8 & 1 & 41,1 & 108.08 & $\mathbf{M}$ & raw dite - covomed degase C \\
\hline 1 & $2 / 5 / 90$ & $\bar{\theta}$ & $\mathbf{t}$ & 41,2 & 100.16 & $M$ & raw drta - alfumed degres C \\
\hline
\end{tabular}




\begin{tabular}{|c|c|c|c|c|c|c|c|}
\hline \multicolumn{8}{|c|}{ TAHK 244.A+106 } \\
\hline & & & & The & DCOUPLE & ATA & \\
\hline & & & & & & & \\
\hline TPGF & DATE & 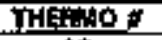 & 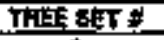 & TGｅ & 宝部 F & $Y=\mathrm{WAX}_{\mathrm{T}} \mathrm{THA}$ & COMHIENTS \\
\hline 1 & 2650 & 10 & 3 & 41.3 & 10834 & $\mathbf{N}$ & row deta - enfunind degrea C \\
\hline 1 & 2060 & $T+1$ & 1 & $41 . \overline{2}$ & 10,16 & $\mathbf{M}$ & 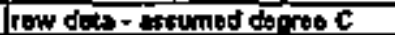 \\
\hline 1 & $2 / 5,90$ & 12 & 1 & 41.4 & 100.62 & N & 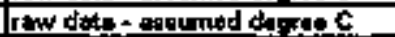 \\
\hline 1 & 25190 & 13 & 1 & $41 . \overline{3}$ & 106,34 & N & 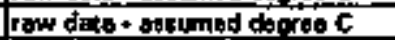 \\
\hline 1 & $2 \sqrt{100}$ & 14 & 1 & 41.4 & 100.52 & $\mathbf{N}$ & cow dace - salumed de coo c \\
\hline \pm & 25 & $\sqrt{5}$ & 1 & 41,4 & 106.52 & $\overline{\mathbf{N}}$ & 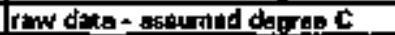 \\
\hline 1 & $2 \sqrt{15} \% 0$ & 18 & 1 & $41, \overline{5}$ & 104.7 & $\bar{Y}$ & raw date - adasmed degrect C \\
\hline 1 & 21590 & 2 & 2 & & & $\mathbf{N}$ & $\overline{21,3}+$ cuspost data lowh \\
\hline 1 & 2या590 & 3 & 2 & 36.1 & 晒.98 & N & rew dita - affumed degras C \\
\hline 1 & $2 / 5 / 90$ & 4 & 2 & 36.7 & $9 B .06$ & $\mathrm{~N}$ & raw data + astumed degrea C \\
\hline 1 & $216 / 90$ & 5 & 2 & 39.8 & 96.24 & $N$ & 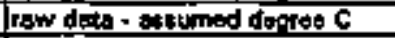 \\
\hline 1 & $26 \sqrt{90}$ & $\bar{a}$ & 2 & 39.9 & 708.02 & $\bar{Y}$ & raw dat - tspumed deresen C \\
\hline 1 & $2 F / 90$ & 7 & 2 & 30.0 & 98.42 & $\mathbf{N}$ & raw dita - arsourred degreal C \\
\hline 1 & $2 / 590$ & 白 & 2 & 37.1 & 98.76 & $\mathbf{N}$ & 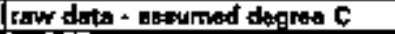 \\
\hline 1 & $2 \sqrt{300}$ & 9 & $\overline{2}$ & $\$ 7.1$ & 98.76 & $\mathbf{N}$ & 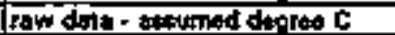 \\
\hline 1 & 2 (5) & 10 & 2 & 37.3 & 99.74 & $\mathbf{N}$ & loww dete - atsumad deques C \\
\hline 1 & $26 / 90$ & 11 & 2 & 37.2 & 98.86 & $\mathbf{N}$ & raw dato - vesarned degrea C \\
\hline 1 & $2 / 5 \%$ & 12 & 2 & 37,4 & 98,32 & $\mathbf{N}$ & 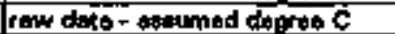 \\
\hline $\bar{T}$ & $2 / 6100$ & 13 & $\overline{2}$ & 37,4 & 98.32 & $\bar{N}$ & 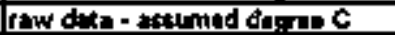 \\
\hline 1 & 25100 & 74 & 2 & 37.5 & 99.5 & II & 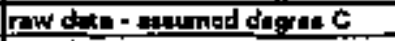 \\
\hline 1 & 2 aron & 75 & 2 & 37.5 & 99.5 & N & rew dofs + aturned deges C \\
\hline 1 & 25\%90 & 30 & 2 & 37.7 & 98,80 & $\mathbf{N}$ & 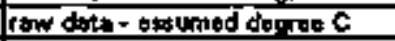 \\
\hline 1 & $8 / 4 \sqrt{90}$ & 1 & 1 & & 139.2 & $\bar{Y}$ & \\
\hline 1 & $6 / 4 / 90$ & 2 & 1 & & 118 & $\bar{N}$ & \\
\hline 1 & $B / 4 / 90$ & 3 & 1 & & 1033 & $\mathbf{N}$ & \\
\hline 1 & $6 / 4 / 90$ & 4 & 1 & & 102,8 & $\mathbf{N}$ & \\
\hline 1 & $6 / 4 / 90$ & 5 & 1 & & 1026 & $\mathbf{N}$ & \\
\hline 1 & G1490 & a & 1 & & $\$ 02.4$ & $N$ & \\
\hline 1 & $0 / 4 / 80$ & 7 & 1 & & 902.5 & $\mathbf{N}$ & \\
\hline 1 & $0 / 490$ & $\overline{8}$ & 1 & & 102.4 & $N$ & \\
\hline 1 & e/4100 & $\theta$ & 1 & & 102.4 & $\mathbf{N}$ & \\
\hline 1 & etateo & 10 & 1 & & 102.3 & $\mathbf{N}$ & \\
\hline 1 & 6/4100 & 11 & $\mathbf{1}$ & & 102.2 & $\mathbf{N}$ & \\
\hline 1 & 6/4/90 & 12 & $i$ & & 102.1 & $\mathbf{N}$ & \\
\hline 1 & D/AFO & 13 & $\mathbf{t}$ & & 902.1 & $\mathbf{N}$ & \\
\hline 1 & d/4/9a & 14 & 1 & & 102.1 & N & \\
\hline 1 & a/400 & 16 & 7 & & 102 & $N$ & \\
\hline 1 & $0 / 4 / 90$ & 16 & 7 & & 701.9 & $\mathbf{N}$ & \\
\hline 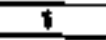 & 101291 & 1 & 1 & & 136 & $\mathbf{Y}$ & 5890523512.47 \\
\hline 3 & 14 & 2 & 1 & & 116,5 & $\mathbf{N}$ & \\
\hline i & 1ग1291 & 3 & 1 & & 105 & $\mathbf{N}$ & \\
\hline $\bar{T}$ & $19 \sqrt{1297}$ & 4 & $\overline{1}$ & & 104.9 & $\bar{H}$ & \\
\hline 1 & 31 12的 & E & 1 & & 104.5 & $\mathbf{N}$ & \\
\hline 1 & 1111291 & 8 & 1 & & 104.3 & $\mathbf{M}$ & \\
\hline$T$ & $11,12=1$ & 7 & $\overline{1}$ & & 104,4 & $\bar{y}$ & \\
\hline$T$ & 1t/218t & 8 & $\overline{1}$ & & 1043 & $\mathbf{N}$ & \\
\hline$t$ & 11 /219t & 9 & 1 & & 104,3 & $\mathbf{N}$ & \\
\hline 1 & $10 / 12190$ & 30 & 1 & & 104.1 & 州 & \\
\hline$t$ & $1+1249$ & 17 & 1 & & 104,2 & $\mathbf{N}$ & \\
\hline 1 & 11/12R1 & 12 & $\overline{1}$ & & 104.1 & $\mathbf{N}$ & \\
\hline 1 & 11/12k1 & 13 & 1 & & 104.2 & $\mathbf{N}$ & \\
\hline 1 & $1 1 \longdiv { 1 2 9 1 }$ & 14 & 1 & & 104.1 & $\mathbf{N}$ & \\
\hline 1 & T1/12/E & 15 & 1 & & 104.1 & $\mathbf{N}$ & \\
\hline 1 & $11 / 12 \mathrm{~g}$ & 5 & 1 & & 104 & $N$ & \\
\hline 1 & T1m291 & 17 & 1 & & 104 & $\mathbf{N}$ & \\
\hline 1 & 11/2/291 & 18 & 1 & & 102.3 & $\mathbf{N}$ & \\
\hline 1 & $1 / 1 / 92$ & 1 & 1 & & 135 & $\bar{Y}$ & $5-910128143 \cdot 29$ \\
\hline 1 & $1 / 1 / 92$ & 2 & 1 & & 116.8 & $N$ & \\
\hline 1 & t/1/92 & 3 & 1 & & 1045 & $\mathbf{N}$ & \\
\hline 1 & $1 / 2 / 2$ & 4 & 1 & & 104 & $\mathbf{N}$ & \\
\hline 1 & $1 / 1 / 92$ & 5 & 1 & & 100.8 & N & \\
\hline 1 & $1 / 1 / 92$ & 8 & 1 & & 108,6 & N & \\
\hline 1 & $1 / 1 / 92$ & 7 & 1 & & 108.8 & N & \\
\hline 1 & $1 / 1 / 92$ & 8 & 1 & & 103.7 & $\mathbf{N}$ & \\
\hline 1 & $1 / 1 / 92$ & 9 & 1 & & 103.7 & N & \\
\hline 1 & $1 / 1 / \theta 2$ & 10 & 1 & & 103.5 & $N$ & \\
\hline
\end{tabular}

Deta obtalned frem WHC Eurvelienes Andyele Computer Syttom ISACSI, Nowember 12, 1003. 


\begin{tabular}{|c|c|c|c|c|c|c|c|}
\hline \multicolumn{8}{|c|}{ TAMK 241.A+10B } \\
\hline & & & & THEF & OCOUPLE & ATA & \\
\hline TFAE : & DATEE & THEPAO \# & TAEE SET * & JEAP C & TEMP $\bar{F}$ & $Y=$ TAX TES & COMTENTS \\
\hline 1 & $1 / \sqrt{92}$ & 11 & 1 & & 103.18 & N & \\
\hline 1 & $1 / 3 / 62$ & 12 & 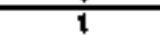 & & 708.4 & $\mathbf{N}$ & \\
\hline$\overline{1}$ & 1/1/s2 & 13 & $\$$ & & 108.8 & $\mathbf{N}$ & \\
\hline 1 & $1 / 1 / 92$ & 14 & 1 & & 103.5 & $\mathbf{N}$ & \\
\hline$\overline{1}$ & WTA2 & $\overline{15}$ & 1 & & 103.5 & $\bar{N}$ & \\
\hline 1 & 1/the & 16 & $T$ & & 103.3 & $\mathbf{N}$ & \\
\hline 1 & $1 / 1 / 82$ & 17 & 8 & & 101 & $\mathbf{N}$ & \\
\hline 1. & $1 / 1 \mathrm{faz}$ & 18 & 1 & & 100.8 & $\mathbf{M}$ & \\
\hline 1 & $11 / 492$ & 1 & 1 & & & & 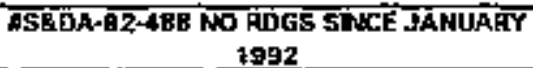 \\
\hline $\bar{T}$ & $11 / 6 / 92$ & $\overline{1}$ & 1 & & $\$ 40$ & $\bar{Y}$ & $\sqrt{77713-20-010}$ \\
\hline 7 & $11 / 5 / 92$ & 2 & 1 & & 132 & $\mathbf{N}$ & \\
\hline 1 & 115492 & 3 & 1 & & 110 & $\mathbf{N}$ & \\
\hline 7 & $11 / 6 / 92$ & 4 & 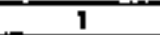 & & 209 & $M$ & \\
\hline$t$ & $17 \sqrt{5 / 92}$ & 5 & 1 & & 109 & $\mathbf{N}$ & \\
\hline 1 & $11 / 5192$ & s & 1 & & 709 & $N$ & \\
\hline 7 & $9 \sqrt{5 \sqrt{22}}$ & 7 & 1 & & 709 & $\mathbf{N}$ & \\
\hline$T$ & $11 / 5 / 2$ & $\overline{8}$ & 1 & & 700 & $\bar{N}$ & \\
\hline 1 & $1+\sqrt{642}$ & 9 & 1 & & 100 & $\mathbf{N}$ & \\
\hline$T$ & $11 / 5 / 92$ & 10 & 1 & & 109 & $\mathbf{N}$ & \\
\hline 1 & $11 / 5 / 92$ & 11 & 1 & & 109 & $\mathbf{N}$ & \\
\hline$T$ & $11 / 5 / 42$ & $\sqrt{12}$ & $\overline{1}$ & & 100 & $\overline{\mathbf{N}}$ & \\
\hline 1 & 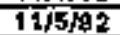 & 93 & 1 & & 500 & N & \\
\hline$T$ & 11 15az & 14 & 1 & & 109 & $\mathbf{N}$ & \\
\hline$\overline{1}$ & $11 / 5 / 22$ & 15 & 1 & & 109 & $\mathbf{N}$ & \\
\hline 1 & $11 / 6 / 92$ & 36 & 1 & & 109 & $\mathbf{N}$ & \\
\hline 1 & $11 / 6 / 92$ & 18 & 1 & & 107 & $N$ & \\
\hline 1 & $1 / 2 / 93$ & 2 & 1 & & 138.2 & $\bar{Y}$ & \\
\hline 1 & $1 / 2 / 293$ & 3 & 1 & & 118.9 & $\mathbf{N}$ & \\
\hline 1 & $1 / 2 \sqrt{93}$ & 4 & $\overline{1}$ & & 100 & $\bar{N}$ & \\
\hline 1 & $1 / 2 \sqrt{93}$ & 5 & 1 & & 106,4 & i & \\
\hline$T$ & $1 / 2 / 93$ & 8 & $T$ & & 105.2 & $\mathbf{N}$ & \\
\hline 7 & 1/293 & 7 & 7 & & 106.1 & $\mathbf{N}$ & \\
\hline 1 & $1 / 2 / 93$ & 8 & 1 & & $105 . \overline{3}$ & $\mathbf{N}$ & \\
\hline 1 & $1 / 2183$ & 9 & 1 & & $108, \overline{2}$ & $\mathbf{N}$ & \\
\hline $\mathrm{J}$ & $3 \sqrt[273]{93}$ & 10 & $\frac{1}{1}$ & & 100.1 & $\mathbf{N}$ & \\
\hline 1 & j2 & 19 & 1 & & 104.8 & N & \\
\hline 1 & 1/2193 & 12 & $T$ & & 105 & \$ & \\
\hline 1 & $5 / 2+93$ & 13 & T & & 104.6 & A & \\
\hline 7 & $1 / 2193$ & 14 & 1 & & 105 & $\mathbf{N}$ & \\
\hline $\bar{T}$ & $1 / 2 \sqrt{93}$ & 15 & 1 & & 104.8 & $\pi$ & \\
\hline$t$ & $t / 2 \longdiv { 9 3 }$ & 16 & 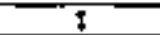 & & 105 & N & \\
\hline$\overline{1}$ & \$2193 & 17 & 7 & & 104,8 & $N$ & \\
\hline 1 & $1 / 2 \longdiv { 9 3 }$ & 18 & $\overline{1}$ & & 102.3 & $N$ & \\
\hline 1 & ersorss & 1 & 1 & & & & $\begin{array}{c}\text { J970† 261432g EAD OATA RECHECK } \\
\text { AEQUESTED }\end{array}$ \\
\hline$i$ & $7 / 2 \longdiv { 1 9 3 }$ & i & 1 & & 197.7 & $\overline{\mathbf{Y}}$ & $-\frac{1}{\sqrt{T 1} \cdot 0 \cdot \sqrt{2}}$ \\
\hline 2 & $7 / 2 \sqrt{30}$ & 2 & 7 & & 118,6 & $\overline{\mathbf{N}}$ & \\
\hline$\overline{1}$ & 72 re3 & 3 & 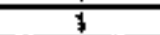 & & 706,8 & $N$ & \\
\hline 7 & 72,93 & 4 & 1 & & 108.2 & $\mathbf{M}$ & \\
\hline 9 & 72103 & 5 & 7 & & 106.1 & N & \\
\hline$t$ & $7 / 2 \% 03$ & 8 & 1 & & 108 & $\mathbf{N}$ & \\
\hline$\overline{1}$ & $7 / 2 \sqrt{9}$ & 7 & 1 & & $10 \%$ & $\mathbf{N}$ & \\
\hline 1 & $7 / 2 ; 93$ & 8 & 1. & & 1009 & $\mathbf{N}$ & \\
\hline 1 & 7279 & $\mathbf{g}$ & 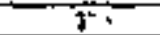 & & $105 . \overline{\mathrm{E}}$ & $\mathbf{N}$ & \\
\hline 1 & $7 / 2193$ & 10 & 1 & & 1057 & $\mathbf{N}$ & \\
\hline$T$ & 712193 & 11 & 1 & & 105.6 & $\overline{\mathbf{N}}$ & \\
\hline 9 & 72193 & 12 & 5 & & 105.6 & $\Phi$ & \\
\hline 1 & $7 / 2+93$ & 13 & 1 & & 105.5 & $\bar{N}$ & \\
\hline 1 & $712 \sqrt{93}$ & 14 & 1 & & 106.8 & $\mathbf{N}$ & \\
\hline 7 & $7 \sqrt{203}$ & 15 & 1 & & 1054 & H & \\
\hline$\overline{1}$ & $7 / 293$ & 18 & 1 & & 105,6 & $\overline{\mathbf{N}}$ & \\
\hline$\overline{1}$ & $7 / 2 / 93$ & 17 & $\overline{1}$ & & 105,3 & $\bar{N}$ & \\
\hline$\overline{9}$ & $7 / 2 / 93$ & $\overline{18}$ & 7 & & 100,4 & $\overline{\mathbf{N}}$ & \\
\hline & & & & & & & \\
\hline & & & & & & & \\
\hline
\end{tabular}

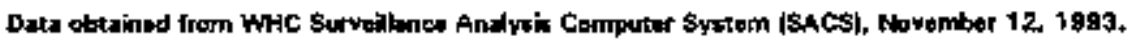


241-A-101

Surface Level Data

Manual Tapa

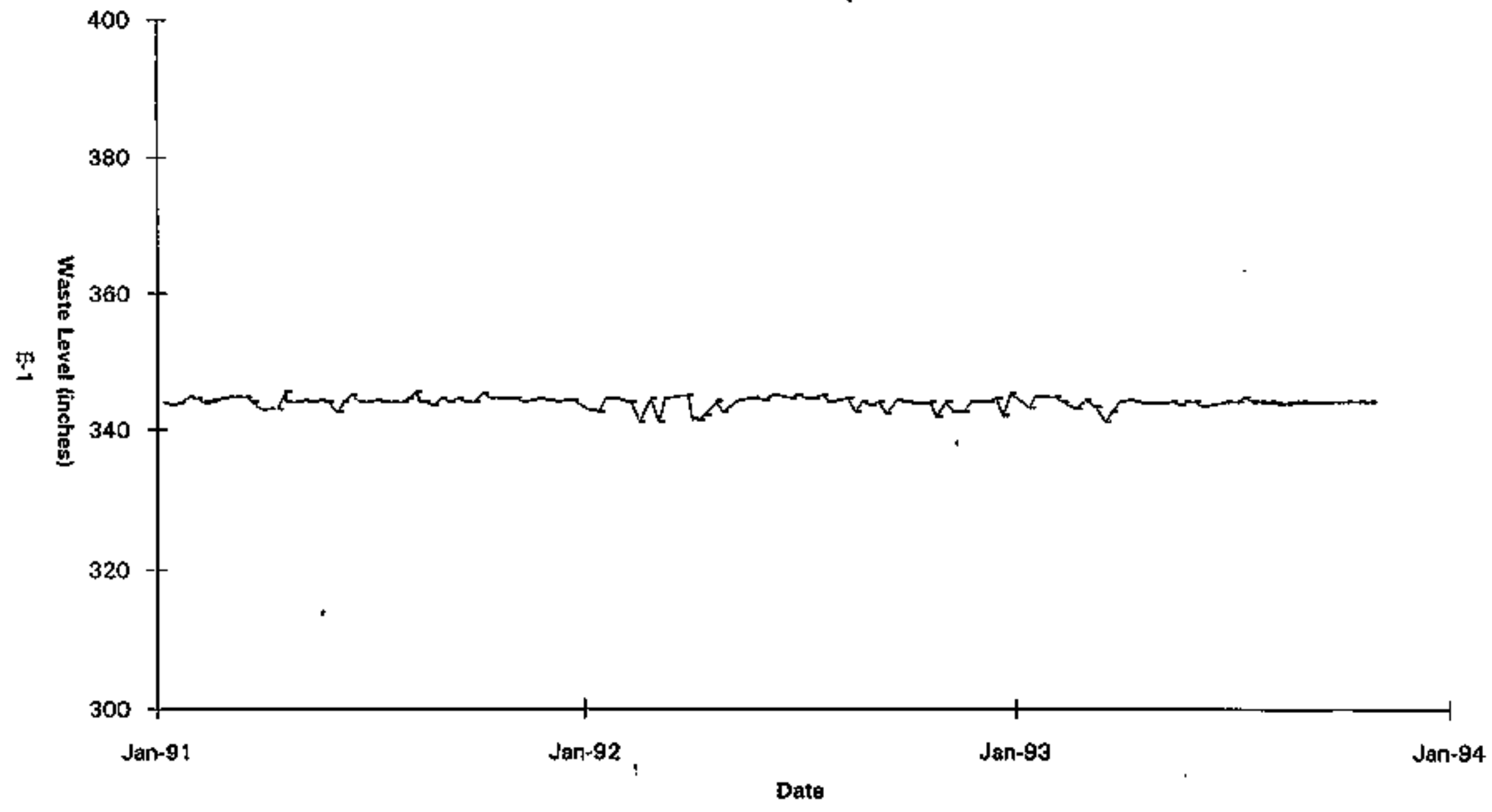

Data obtained from WHC Survetllance Analysis Computer System (SACS), November 16, 1993. 


\section{1-A-104}

\section{Surface Level Data}

\section{Manual Tape}

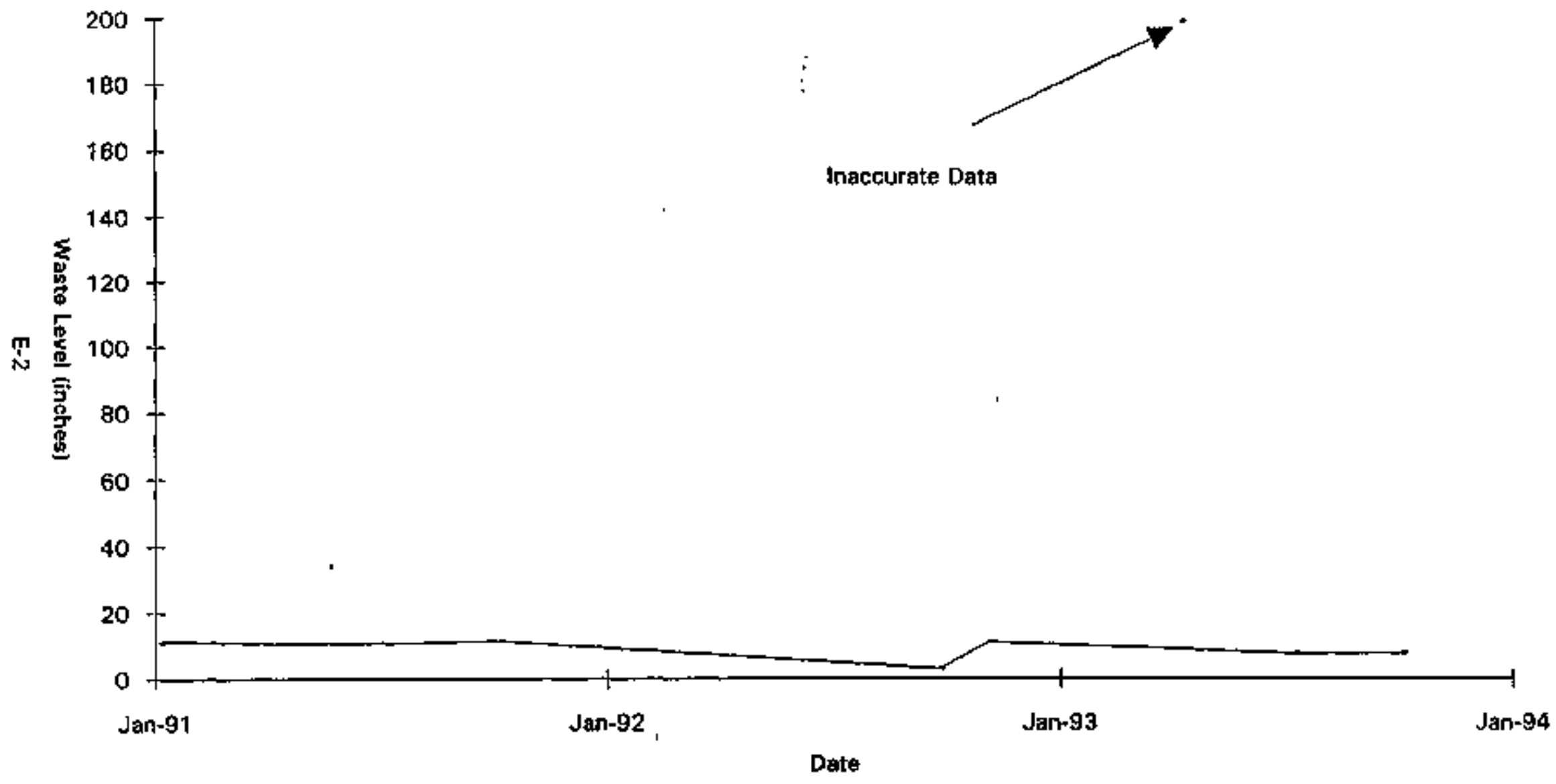

Data obtained from WHC Surveillance Anallysis Computer System (SACS), Navember 16, 1993. 


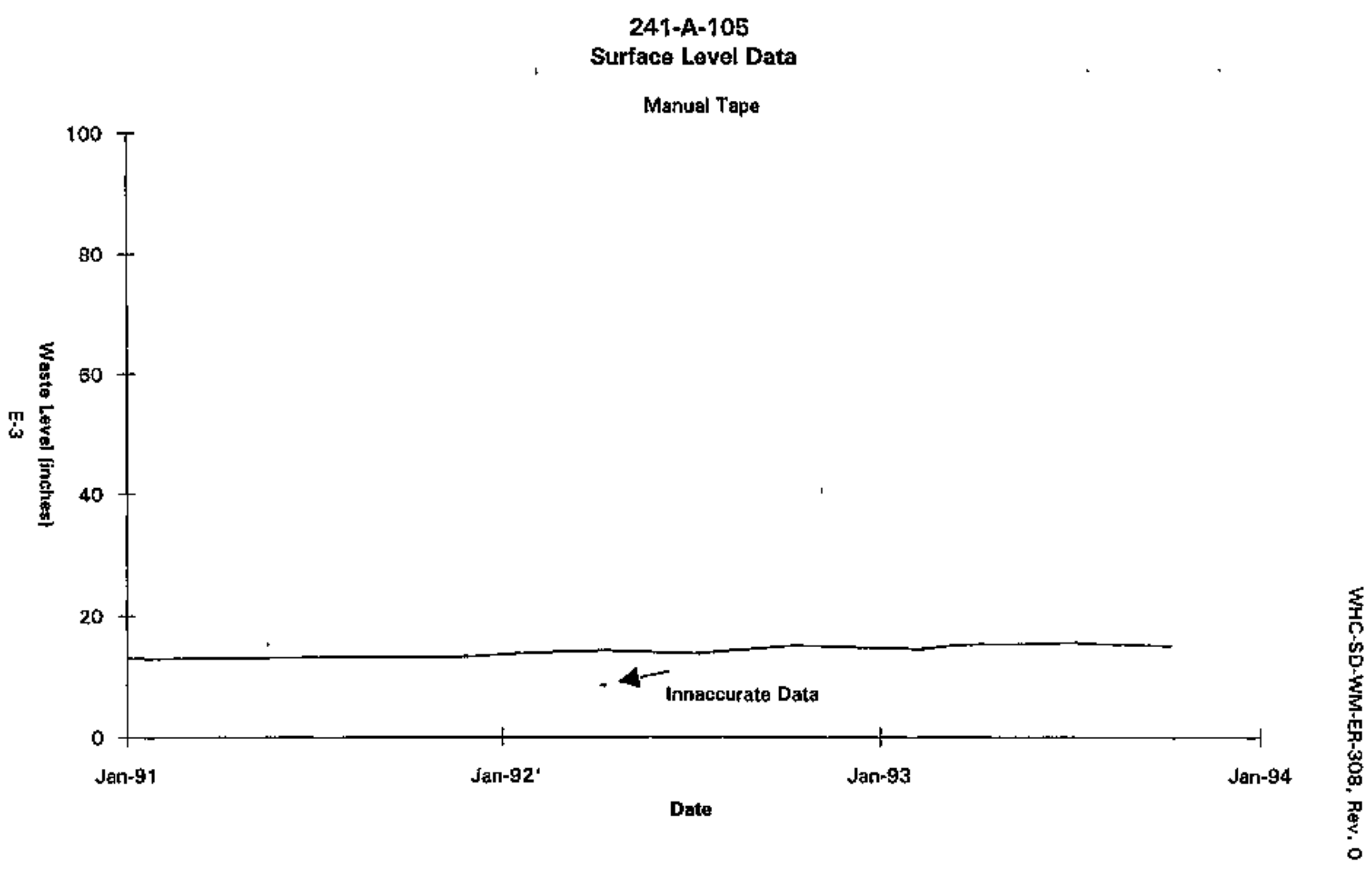

Data obtained from WHC Surveiltance Analysis Computer System (SAC\$), November 16, 1993. 


\begin{tabular}{|c|c|c|c|c|c|}
\hline \multicolumn{6}{|c|}{ Surface Level Data } \\
\hline & & Ma & & & \\
\hline Year & Month & Day & Hour & Minutes & Surface Leval \\
\hline 91 & 1 & 7 & 15 & 33 & 344 \\
\hline 91 & $\mathbf{i}$ & 14 & 17 & 26 & 343.6 \\
\hline 91 & 1 & 21 & 1 & 31 & 344 \\
\hline 91 & 7 & 29 & 17 & 39 & 344.75 \\
\hline 91 & 2 & 5 & 8 & 19 & 344.5 \\
\hline 91 & 2 & 11 & 8 & 19 & 344 \\
\hline 91 & 2 & 12 & 16 & 39 & 344 \\
\hline 91 & 2 & 18 & 8 & 19 & 344.25 \\
\hline 97 & 2 & 26 & 15 & 23 & $3 \overline{44.5}$ \\
\hline 91 & 3 & 5 & 16 & 22 & 344.75 \\
\hline 97 & 3 & 11 & 16 & 42 & 344.75 \\
\hline 97 & 3 & 19 & 17 & 55 & 344.75 \\
\hline 91 & 3 & 26 & 15 & 1 & 344 \\
\hline 91 & 4 & 1 & 15 & 2 & 343 \\
\hline 91 & 4 & 8 & 18 & 2 & 343 \\
\hline 91 & 4 & 15 & 15 & 23 & 343 \\
\hline 91 & 4 & 22 & 15 & 3 & 345.5 \\
\hline 91 & 4 & 22 & $\underline{22}$ & 37 & 344 \\
\hline 91 & 5 & 1 & 6 & 17 & 344 \\
\hline 91 & 5 & 7 & $\overline{13}$ & 24 & 344.25 \\
\hline 91 & 5 & 14 & 7 & 59 & 344 \\
\hline 91 & 5 & 20 & 14 & 57 & 344.25 \\
\hline 91 & 5 & 27 & 20 & 9 & 344 \\
\hline 91 & 5 & 28 & 18 & 41 & 344 \\
\hline 91 & 6 & 5 & 1 & 1 & 342.6 \\
\hline 91 & 6 & 10 & 14 & 55 & 344 \\
\hline 91 & 6 & 17 & 15 & 8 & 345 \\
\hline 91 & 6 & 24 & 17 & 53 & 344 \\
\hline 91 & 7 & 1 & 14 & 23 & 344 \\
\hline 91 & 7 & 8 & 14 & 23 & 344.25 \\
\hline 91 & 7 & 15 & 14 & 23 & 344 \\
\hline 91 & 7 & $\overline{22}$ & 21 & 50 & 344 \\
\hline 91 & 7 & 31 & 0 & 29 & 344 \\
\hline 91 & 8 & 5 & 18 & 12 & 344.5 \\
\hline 91 & 8 & 12 & 19 & 21 & 345.5 \\
\hline 91 & 8 & 14 & 19 & 21 & 344 \\
\hline 91 & 8 & 19 & 17 & 14 & 344 \\
\hline 91 & 8 & 26 & 19 & 21 & 343.5 \\
\hline 91 & 9 & 2 & 14 & 57- & $3 \overline{44.5}$ \\
\hline 91 & $\dot{9}$ & 9 & 14 & 57 & 344 \\
\hline 91 & 9 & 16 & 14 & 57 & 344.5 \\
\hline 91 & 9 & 23 & 14 & 57 & 344 \\
\hline 91 & 9 & 30 & 14 & 57 & 344 \\
\hline 91 & 10 & $\mathrm{~g}$ & 14 & 57 & 345.26 \\
\hline $9 \overline{1}$ & 10 & 14 & 14 & 57 & 344.5 \\
\hline 91 & 10 & 21 & 14 & 57 & 344.5 \\
\hline
\end{tabular}

Data obtained from WHC Surveillance Analysis Computer System [SACS], Novernber 16, 1993. 


\begin{tabular}{|c|c|c|c|c|c|}
\hline \multicolumn{6}{|c|}{ Manual Tape } \\
\hline Year & Month & Day & Hour & Minutes & Surface Level \\
\hline 91 & 10 & 28 & 14 & 57 & 344.6 \\
\hline 91 & 11 & 4 & 14 & 57 & 344.5 \\
\hline 91 & 11 & 11 & 14 & 57 & $34 \dot{4}$ \\
\hline 91 & 11 & 18 & 14 & $5 \overline{7}$ & 344.25 \\
\hline 91 & 11 & 25 & 14 & 57 & 344,5 \\
\hline 91 & 12 & 2 & 14 & $5 \overline{7}$ & 344.25 \\
\hline 91 & 12 & 9 & 14 & 57 & 344 \\
\hline 91 & 12 & 16 & $\overline{14}$ & 57 & 344.25 \\
\hline 91 & 12 & 23 & 14 & 57 & 344.26 \\
\hline$\overline{92}$ & 1 & 7 & 15 & $4 \overline{1}$ & 342.75 \\
\hline 92 & 1 & 14 & 15 & 41 & $34 \overline{2.5}$ \\
\hline$\overline{92}$ & 1 & 20 & 15 & $4 \overline{41}$ & 344.5 \\
\hline 92 & 1 & 27 & 15 & 41 & 344.5 \\
\hline$\overline{9} 2$ & 2 & 10 & 15 & 41 & 344 \\
\hline 92 & $\underline{2}$ & 18 & 16 & 15 & 341 \\
\hline 92 & 2 & 27 & 16 & 31 & 344.5 \\
\hline 92 & 3 & 5 & 4 & 42 & 341 \\
\hline 92 & 3 & 10 & 11 & $5 \overline{9}$ & 344.5 \\
\hline 92 & 3 & 30 & 15 & 33 & 345 \\
\hline$\overline{92}$ & 4 & 2 & 14 & 45 & $34 \overline{1} .5$ \\
\hline 92 & 4 & 8 & 9 & $\overline{15}$ & 341.26 \\
\hline 92 & 4 & 13 & 16 & 39 & 342 \\
\hline 92 & 4 & 23 & 8 & $\mathrm{~g}$ & $\overline{344}$ \\
\hline 92 & 4 & 23 & 8 & 28 & 344 \\
\hline 92 & 4 & 28 & 14 & 14 & 342.5 \\
\hline 82 & 5 & 12 & 15 & 26 & 344.25 \\
\hline 92 & $\underline{5}$ & 25 & $\overline{15}$ & $2 \overline{7}$ & 344.5 \\
\hline 92 & 6 & 2 & 7 & 32 & 344.25 \\
\hline .92 & 6 & 11 & 9 & 17 & 345 \\
\hline 92 & 6 & 17 & 8 & 1 & 344.75 \\
\hline 92 & 6 & 25 & 7 & 36 & 344.5 \\
\hline 92 & 6 & 30 & 15 & 35 & 345 \\
\hline 92 & 7 & 7 & 13 & 7 & 344.5 \\
\hline 92 & 7 & 14 & 14 & 36 & 344.5 \\
\hline$\overline{92}$ & 7 & 22 & 14 & 52 & 345 \\
\hline 92 & 7 & 28 & 14 & 31 & 344 \\
\hline 82 & B & 3 & 17 & 27 & 344.25 \\
\hline 92 & B & $1 \overline{4}$ & 8 & 33 & 344.5 \\
\hline 92 & 8 & 19 & 15 & 13 & 342.5 \\
\hline 92 & $\overline{8}$ & 24 & 15 & 14. & 344 \\
\hline 92 & 8 & 31 & 14 & 57 & 343.5 \\
\hline 92 & 9 & $B$ & 23 & 5 & 344 \\
\hline 92 & $\overline{\mathbf{9}}$ & 14 & 23 & 5 & 342.25 \\
\hline 92 & 9 & 23 & 14 & 20 & 344.25 \\
\hline 92 & $\overline{9}$ & 30 & 23 & 5 & $3 \overline{44}$ \\
\hline 92 & 10 & 1 & 8 & 4 & 344 \\
\hline 92 & 10 & 6 & 14 & 59 & $34 \overline{3} .7 \overline{5}$ \\
\hline
\end{tabular}

Data obtatned from WHC Survefllance Analysis Computer System (SACS), November 16, 1993. 


\begin{tabular}{|c|c|c|c|c|c|}
\hline \multicolumn{6}{|c|}{ Manual Tape } \\
\hline Year & Month & Day & Hour & Minutes & Surface Level \\
\hline 92 & 10 & 12 & 14 & 59 & 343.75 \\
\hline 92 & 10 & 19 & 14 & 30 & 343.75 \\
\hline 92 & 10 & 21 & 8 & 4 & 344 \\
\hline$\overline{92}$ & 10 & 26 & 15 & 40 & 341.75 \\
\hline 92 & 11 & 3 & 15 & 45 & 344 \\
\hline 92 & 11 & 9 & $\$ 5$ & 45 & 342.5 \\
\hline 92 & 11 & 18 & 15 & 45 & 342.5 \\
\hline 92 & 11 & 25 & 7 & 57 & 344 \\
\hline 92 & 12 & 1 & 14 & 43 & 344 \\
\hline$\overline{92}$ & 12 & $\overline{9}$ & 15 & 46 & 344 \\
\hline 92 & 12 & 36 & $\$ 5$ & 9 & $34 \overline{4.5}$ \\
\hline 92 & $\overline{12}$ & 22 & 21 & 22 & 342 \\
\hline 92 & 12 & 28 & 22 & 13 & 345.25 \\
\hline 93 & 1 & 13 & 15 & 2 & $34 \overline{3.1}$ \\
\hline 93 & 1 & 78 & 20 & 43 & 344.75 \\
\hline 93 & 1 & 25 & 22 & 57 & 344.75 \\
\hline$\overline{93}$ & $\overline{2}$ & 4 & 15 & 29 & 344.7 \\
\hline$\overline{93}$ & 2 & 10 & 9 & $5 \overline{5}$ & 344 \\
\hline 93 & 2 & 22 & 16 & 22 & 343 \\
\hline 93 & 3 & 1 & 17 & 22 & $344.2 \overline{5}$ \\
\hline 93 & 3 & 10 & $\mathrm{~B}$ & 36 & 343.25 \\
\hline 93 & 3 & 38 & 14 & 51 & 344 \\
\hline 93 & 3 & 23 & 18 & 35 & 342.5 \\
\hline 93 & 3 & 30 & 22 & 22 & 344 \\
\hline 93 & $\overline{4}$ & 7 & 8 & 4 & $\overline{3} 4 \overline{4.25}$ \\
\hline 93 & 4 & 24 & $T B$ & 39 & 343.75 \\
\hline 93 & 4 & 26 & 19 & 2 & $343.7 \overline{5}$ \\
\hline 93 & 5 & 13 & 14 & 33 & 344 \\
\hline 93 & 5 & 20 & $\overline{15}$ & 30 & 343.5 \\
\hline 83 & 5 & 24 & $1 \overline{4}$ & $1 \overline{4}$ & 344 \\
\hline 93 & 6 & 1 & 16 & 37 & 344 \\
\hline 93 & 6 & 7 & 17 & 0 & 343.25 \\
\hline 93 & 6 & 28 & 14 & $5 \overline{1}$ & 344 \\
\hline 93 & 7 & 7 & 12 & 16 & 344 \\
\hline 93 & 7 & 12 & 22 & 7 & 344.7 \\
\hline 93 & 7 & 20 & 17 & 44 & 344 \\
\hline 93 & 7 & 26 & 15 & $\overline{1}$ & 344 \\
\hline 93 & 8 & 4 & 7 & 54 & 344 \\
\hline 93 & 8 & 9 & 15 & 9 & 343.75 \\
\hline 93 & 8 & 18 & 16 & 54. & 343.75 \\
\hline 93 & 8 & 23 & 19 & 28 & 344 \\
\hline 93 & 8 & 30 & 10 & 59 & 344 \\
\hline 93 & 10 & 7 & 11 & 19 & 344 \\
\hline 93 & 10 & 18 & $1 \overline{4}$ & 50 & 344 \\
\hline 93 & 10 & 27 & 21 & 20 & 344 \\
\hline
\end{tabular}

Data obtained from WHC Surveillance Anslysis Computer System (SACS), November 16, 1993. 


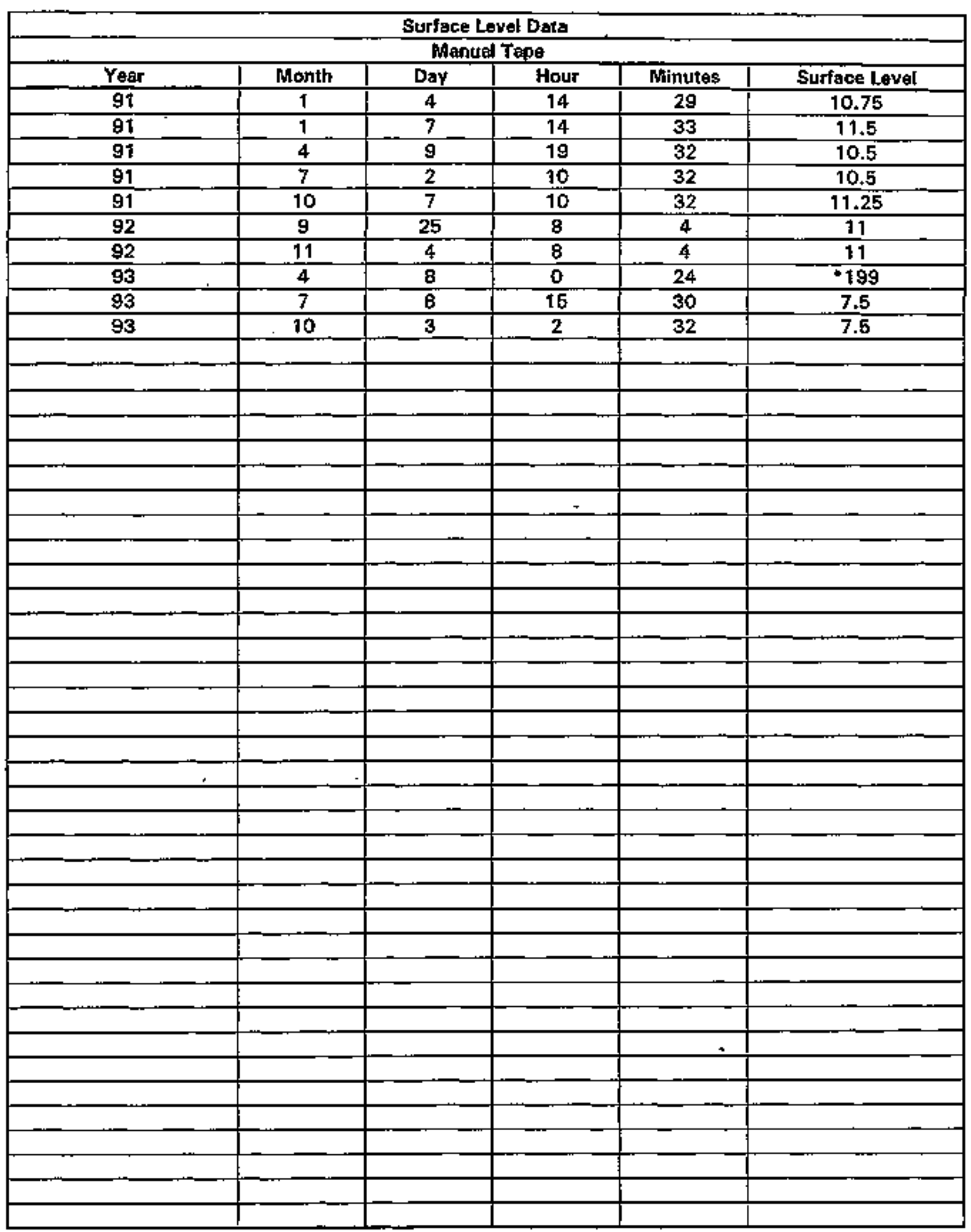

Dato obtained from WHC Surveillance Analysis Computer System (SACS), November 16, 1993. $\mathrm{E}+7$ 


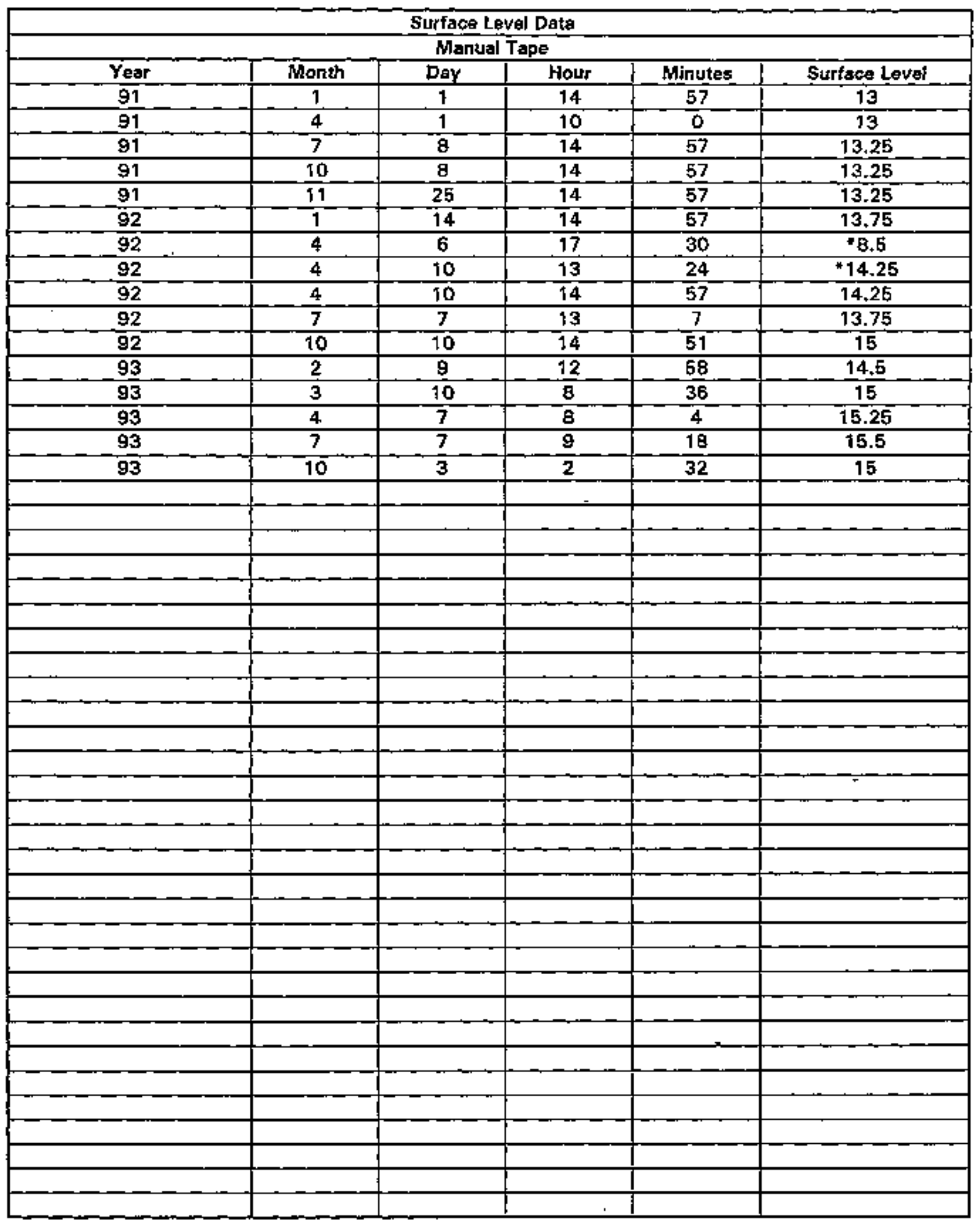

Data obtained from WHC Surveillance Analysis Computer System (SACS), November 16,1993. 
•0001
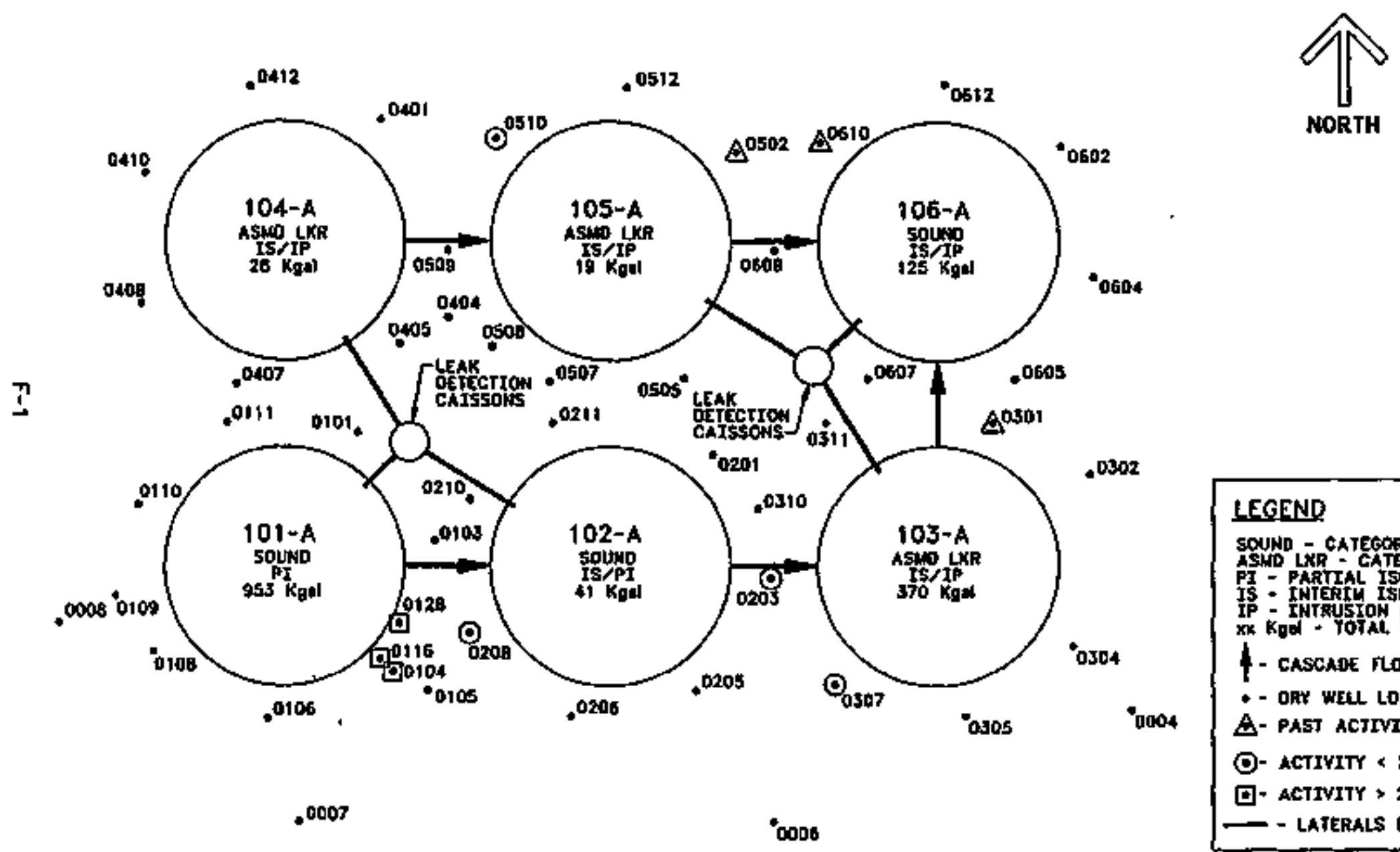

241-A FARM

\section{LEGEND}

SOUND - CATEGgRIRATYON

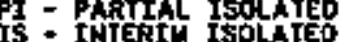

IP - INTRUSTON PREMLNTION

xKK Kgel - YOFAL, WASTE

4 - cascade rlom

- - ork well locartón

A- PAST ACTIVITY

O- ACtIVITY \& $200 \mathrm{c} / \mathrm{s}$

回- ActTVITY > $200 \mathrm{c} / 5$

- Laterals 13! 
241-A-101 Drywell Activity

Well \#10-01-04 Well Depth 125'

Scintillation Probe 1st Peak

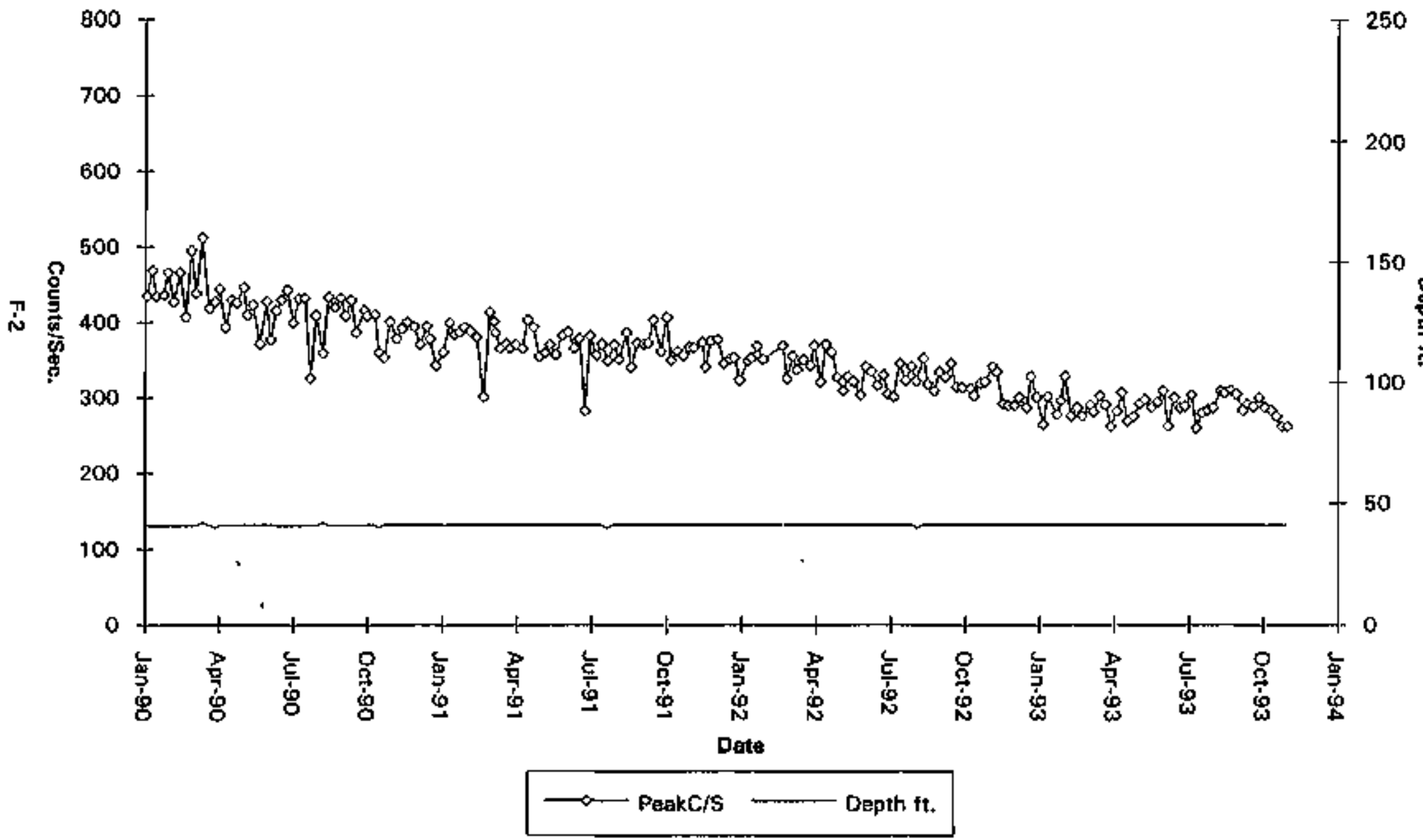

Data obtained from WhC Surveillance Data Acquisition (SDA), November 30, 1993. 
241-A-101 Drywell Activity

Well \#10-01-04 Well Depth 125'

Scintillation Probe 2nd Peak

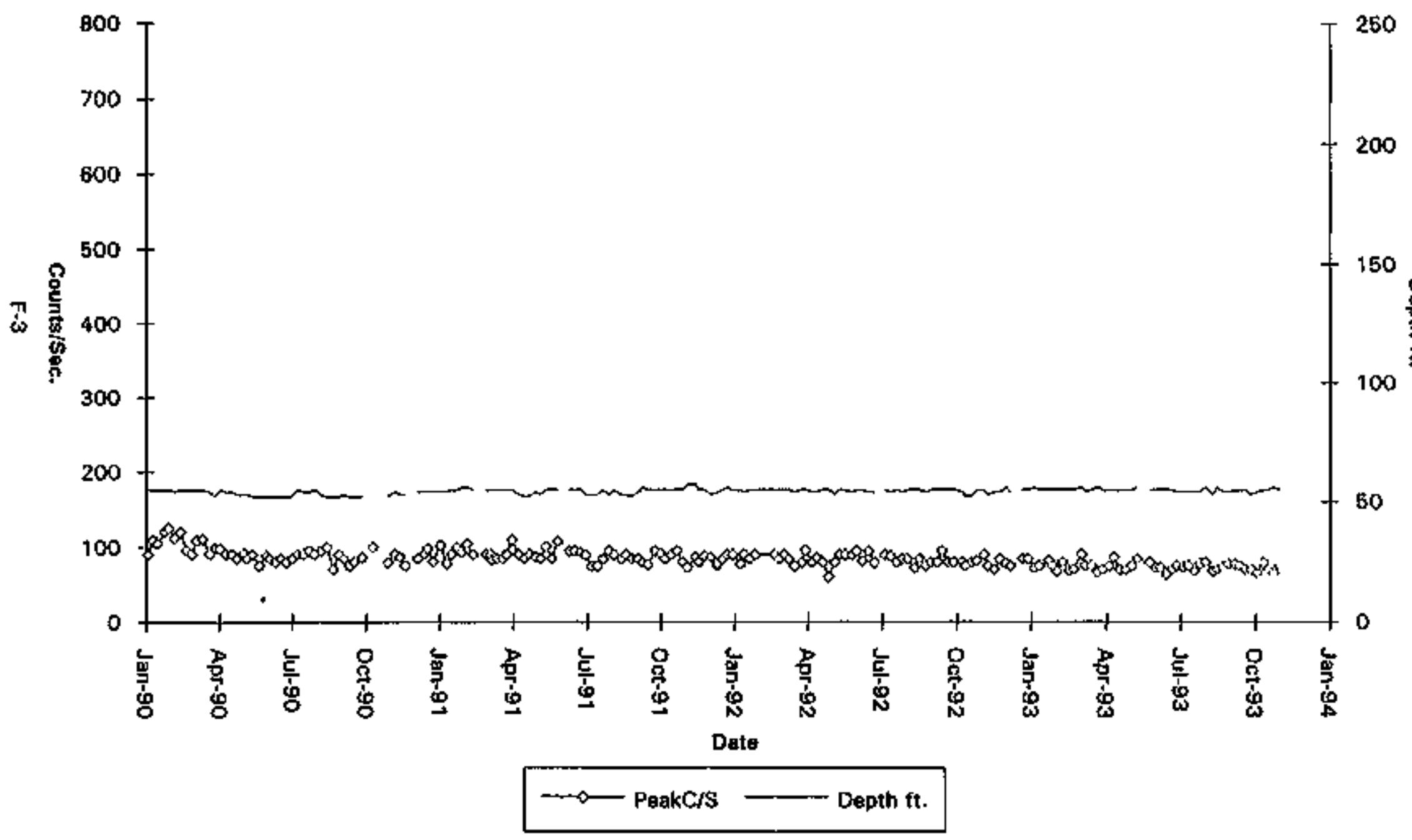

Data obtained from WHC Surveillance Data Acquisition (SDA), November 30, 1993. 
241-A-101 Drywell Activity

Well \#10-01-04 Well Depth 125'

Scintillation Probe 3rd Peak

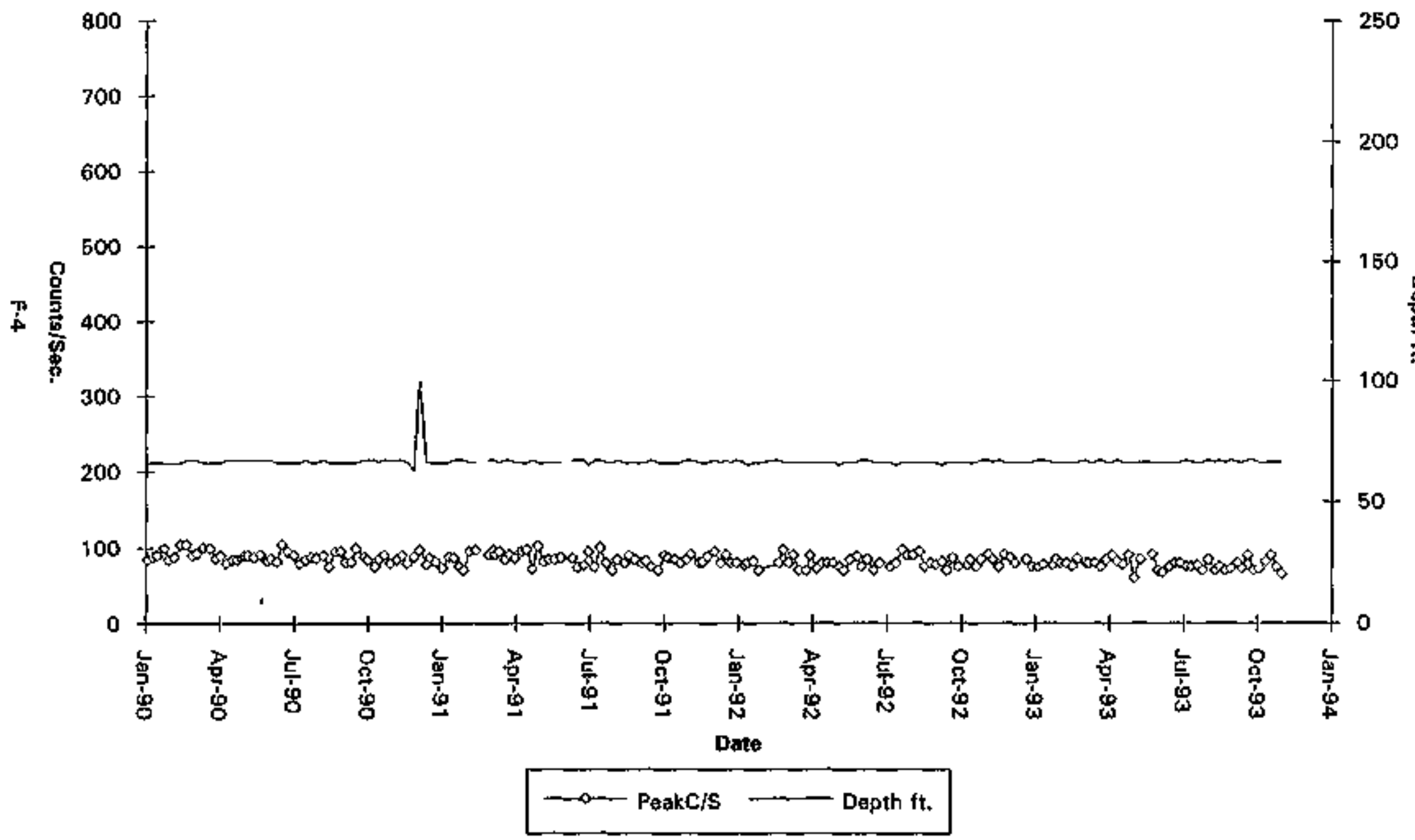


241-A-101 Drywell Activity

Well \# 10-01-16 Well Depth 50'

Scintillation Probe

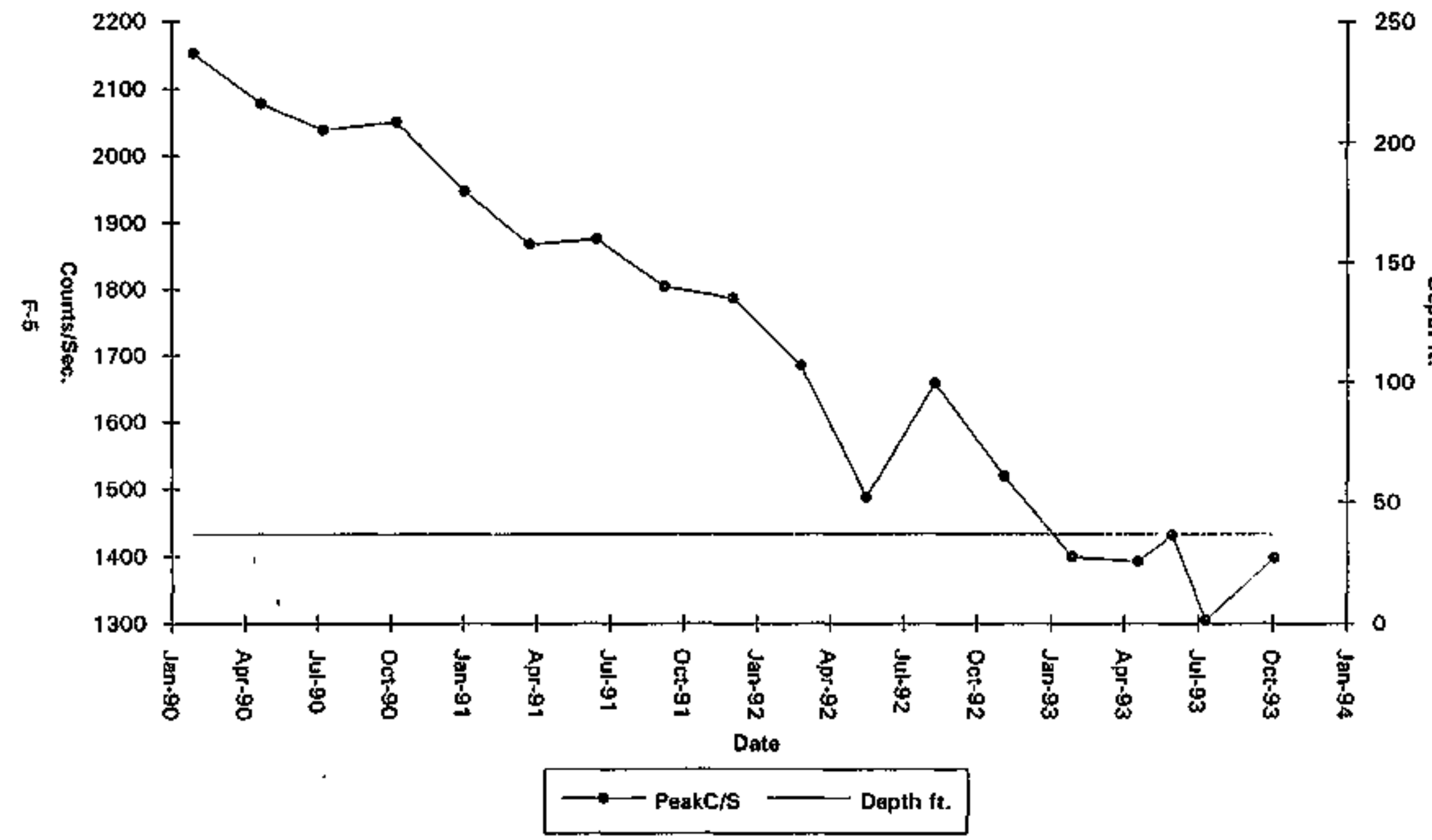


241-A-101 Drywell Activity

Well \#10-01-28 Well Depth 45'

Scintillation Probe
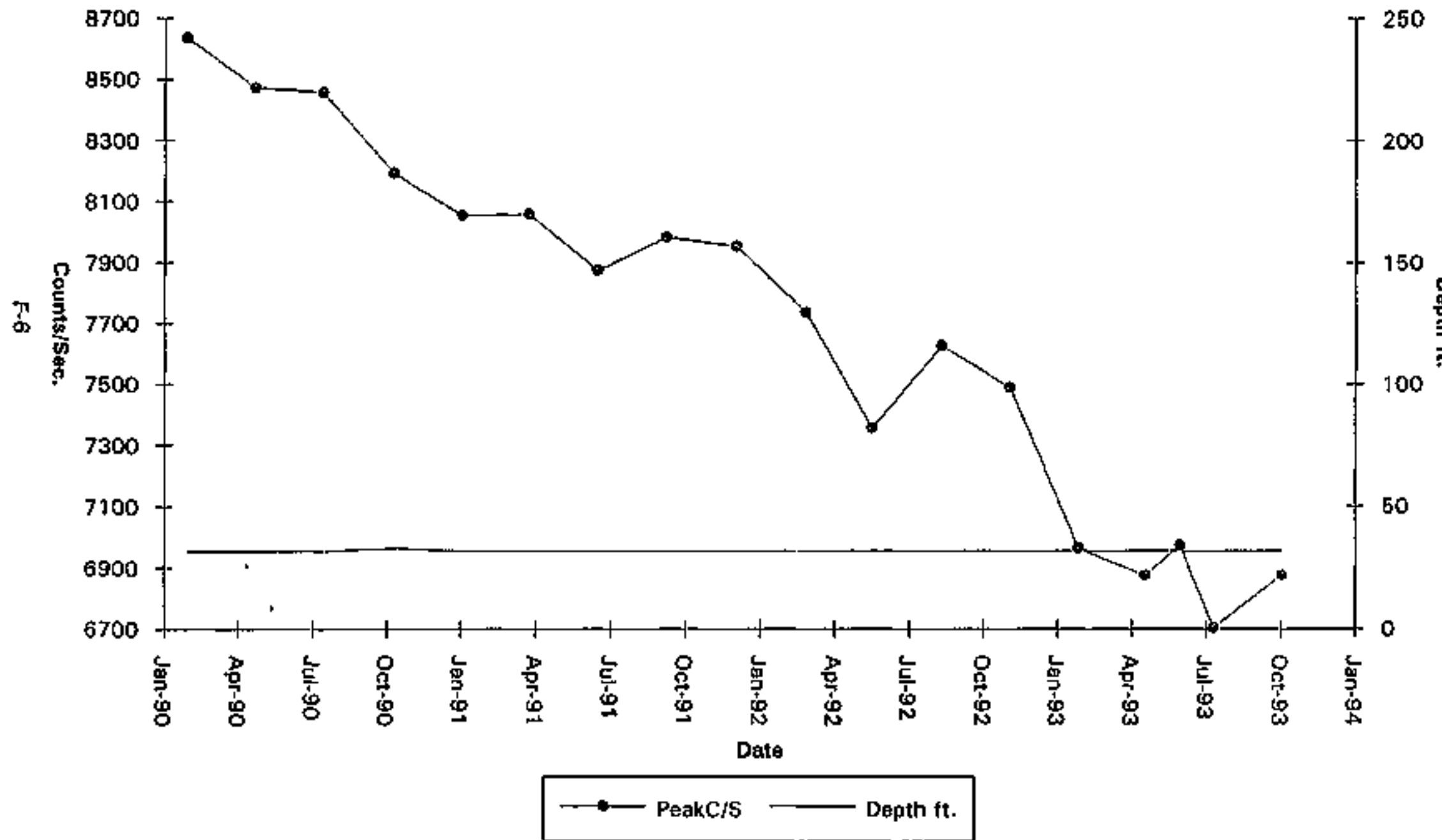

Oinph not on --ala mith Dtaws.

Data obtained from WHC Surveillance Data Acquisition (SDA), November 30, 1993. 


\section{1-A-102 Drywell Activity \\ Well \#10-02-03 Well Depth 125' \\ Sojntillation Probe}

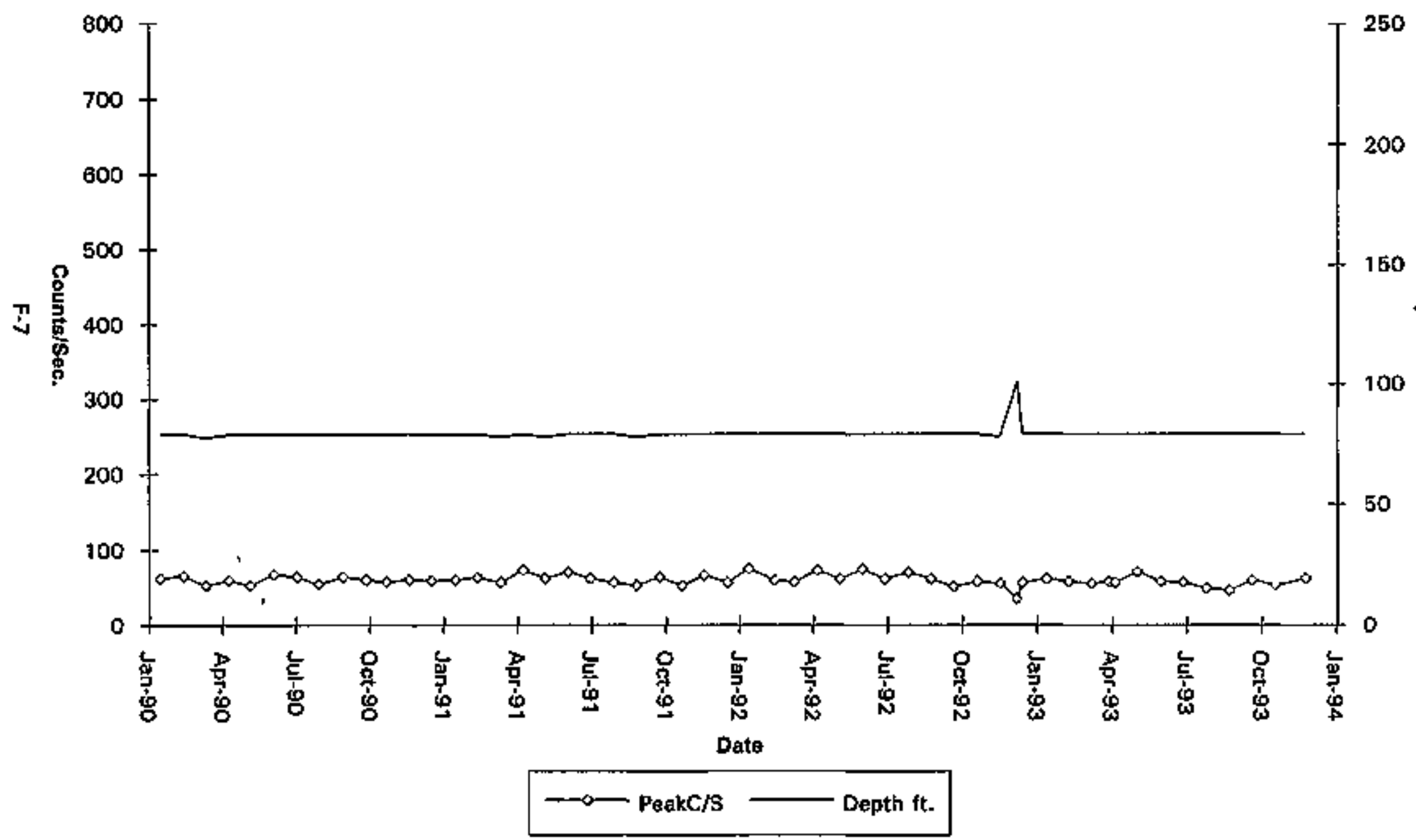


241-A-102 Drywell Activity

Well \#10-02-08 Well Depth 126'

Scintillation Probe

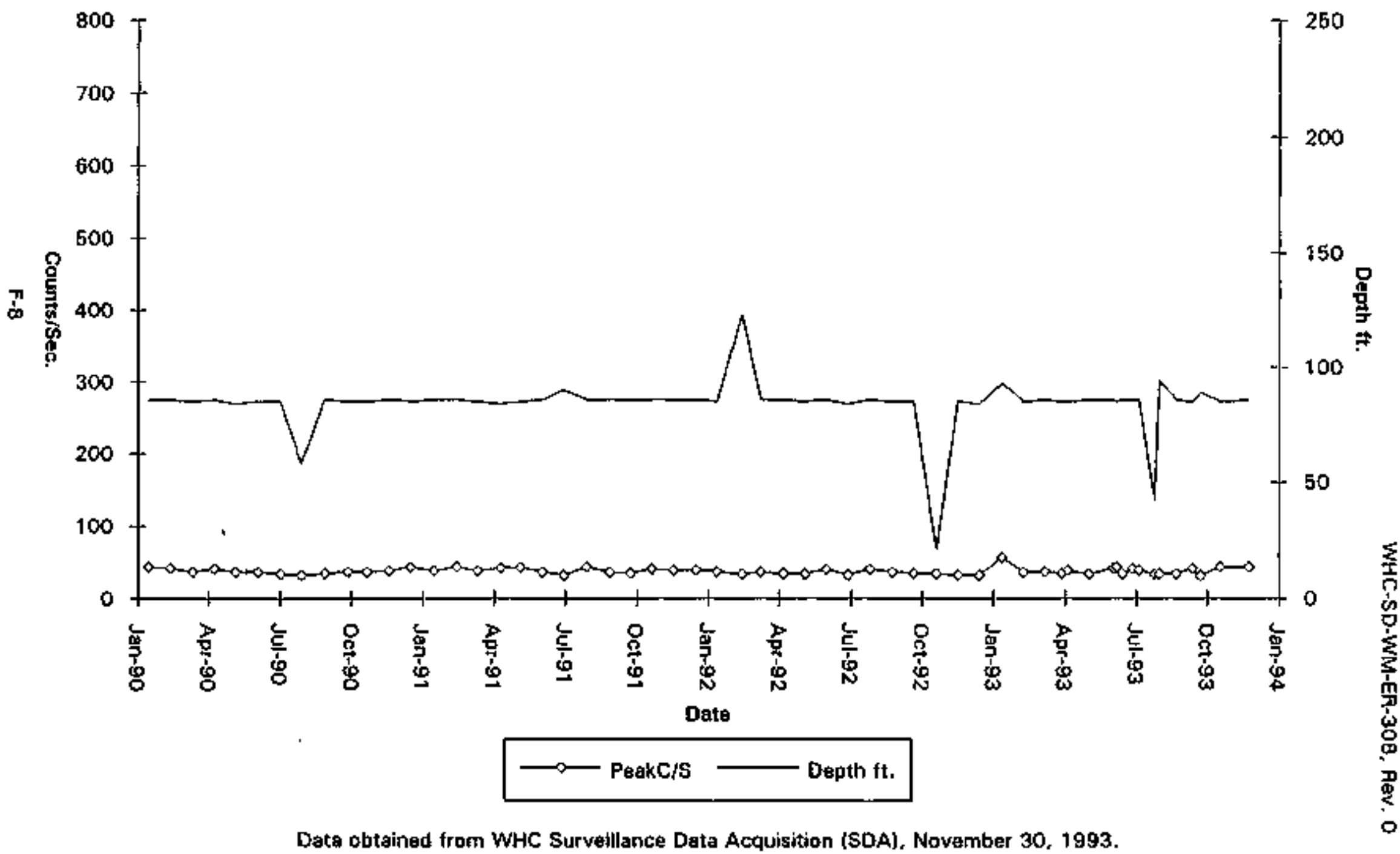


241-A-103 Drywell Activity

Well \#10-03-07 Well Depth 125'

Scintillation Probe 1st Peak

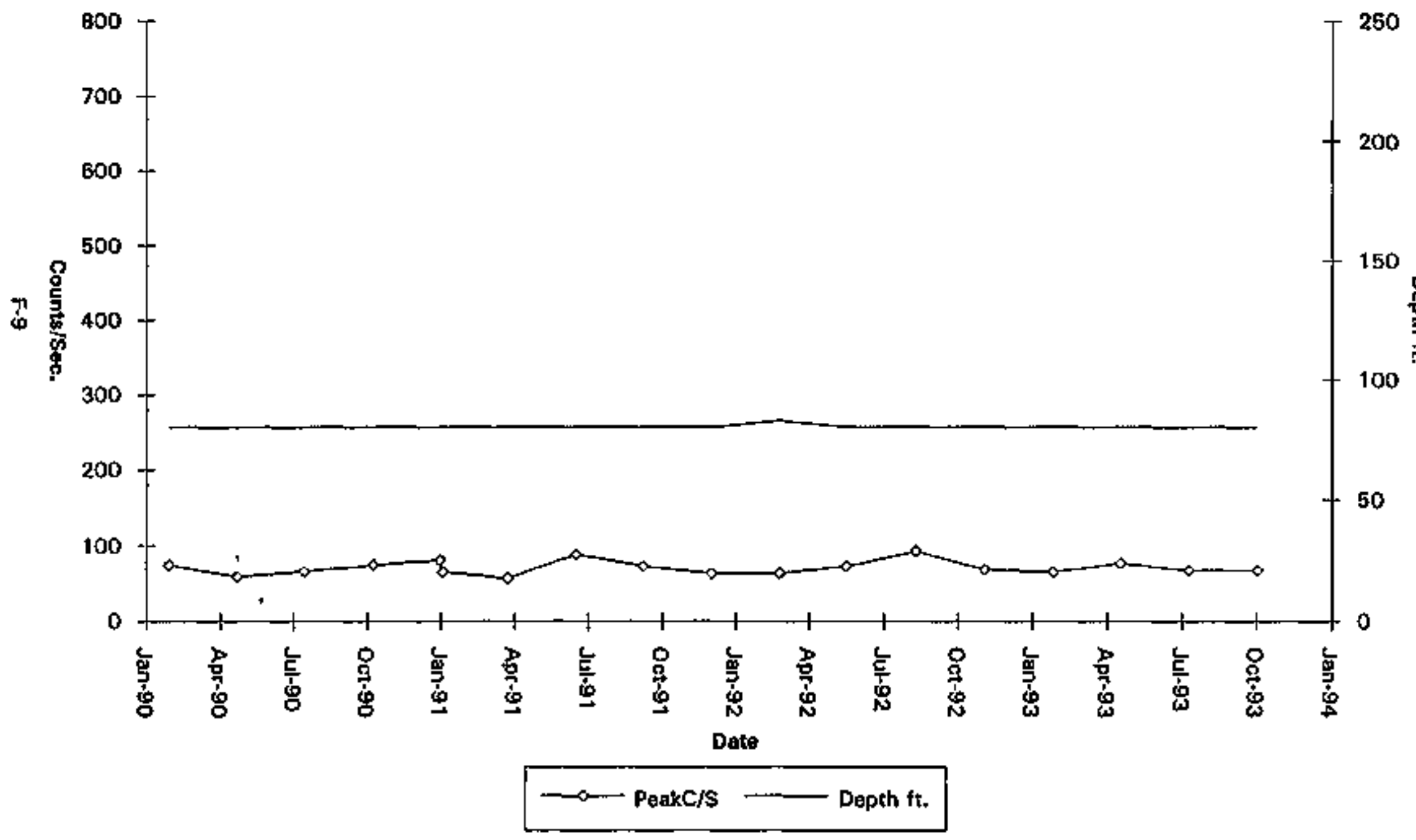

Data obtained from WHC Surveillance Data Acquisition (SDA), Novernber 30, 1993. 
241-A-103 Drywell Activity

Well \# 10-03-07 Well Depth 125*

Scintillation Probe 2nd Peak

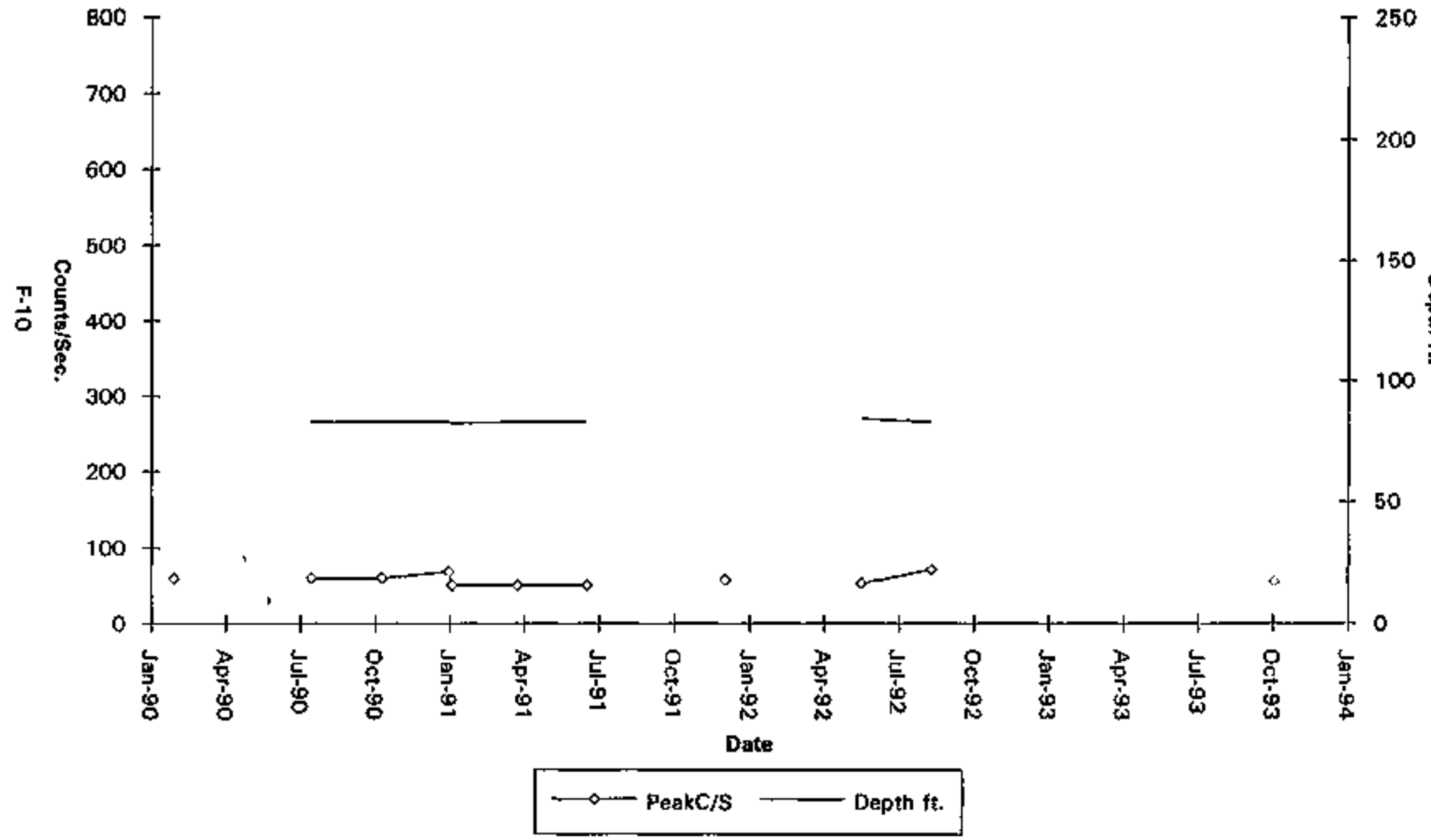


241-A-105 Drywell Activity

Well \#10-05-10 Well Depth 125'

Scintllation Probe 1st Peak

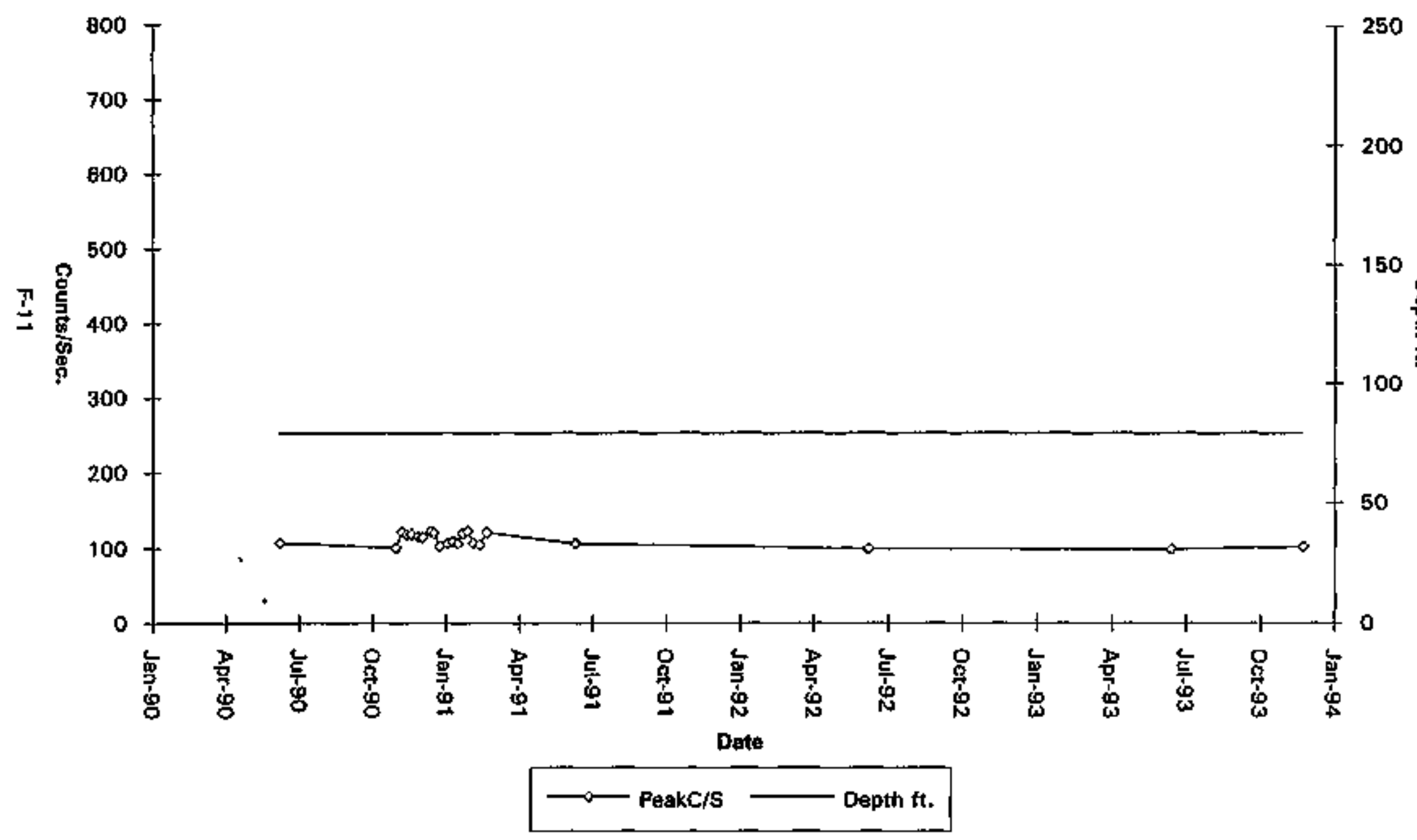

Deta obtained from WHC Survaillance Data Acquisition (SDA), November 30, 1993. 
241-A-105 Drywell Activity

Well \#10-05-10 Well Depth 125'

Scintillation Probe 2nd Peak

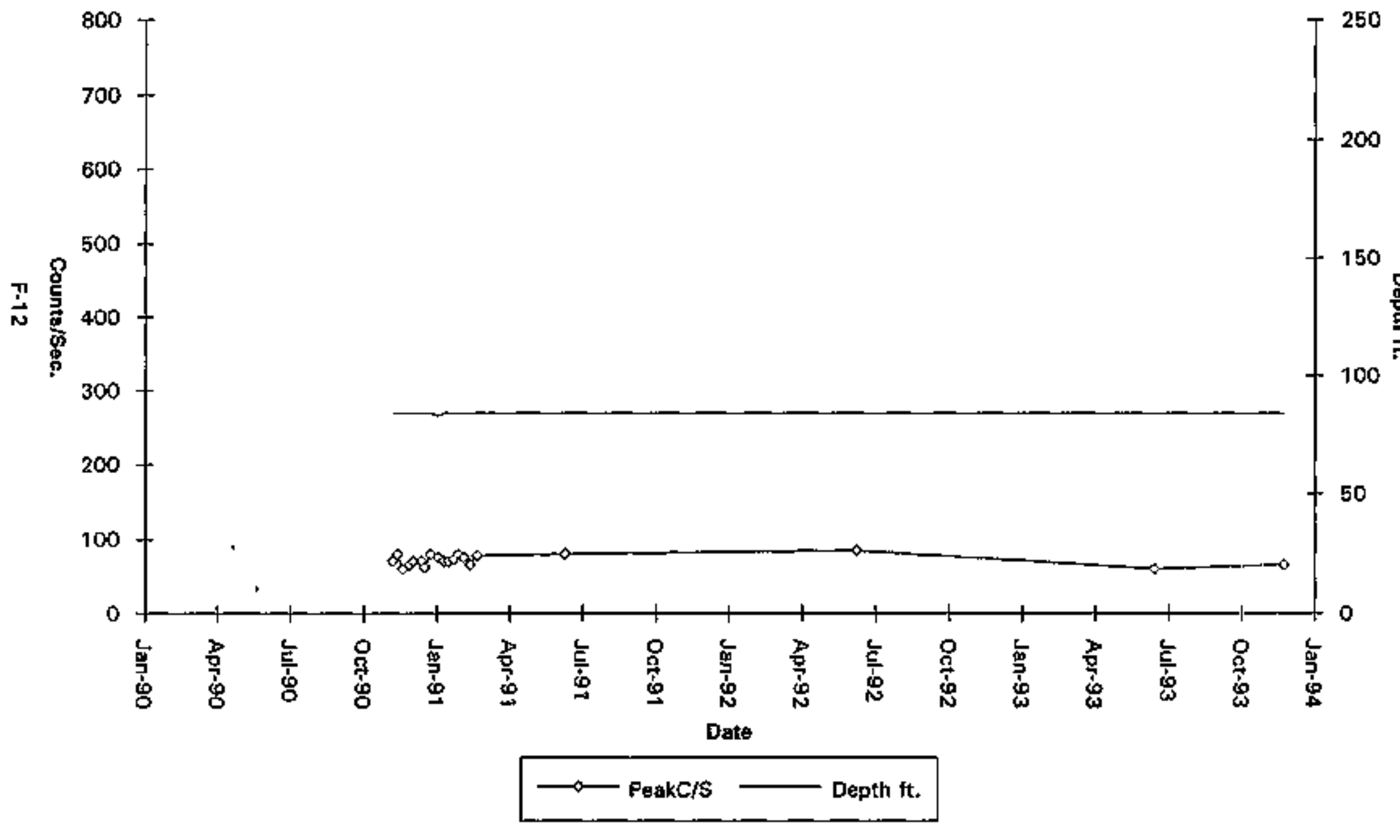

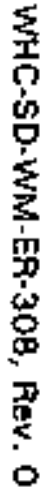

Data obtained from WHC Surveillance Data Acquisition (SDA), November 30, 1993. 


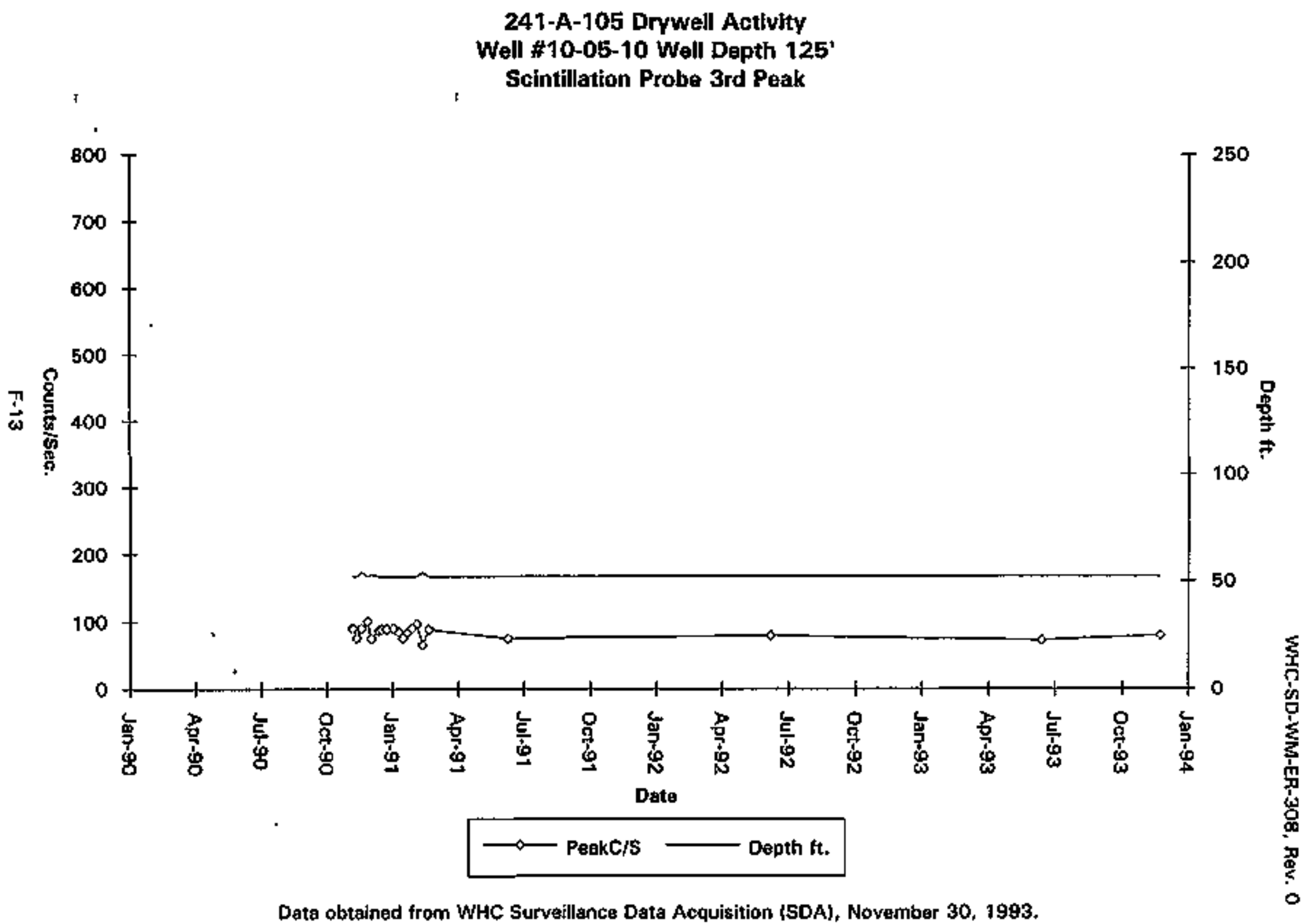




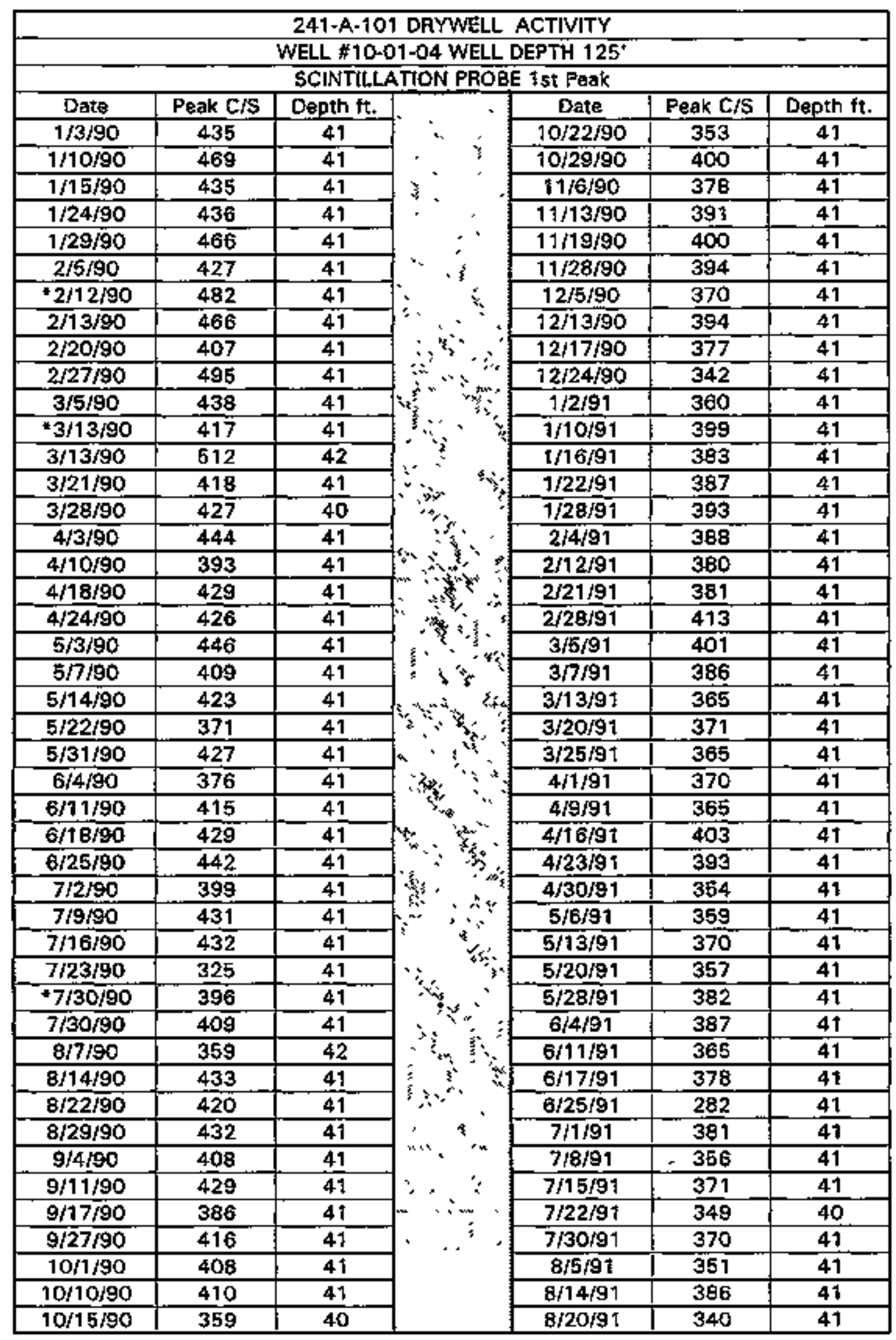

Data obtained from WHC Surveillance Data Acquisition (SDAI, November 30, 1993. 


\begin{tabular}{|c|c|c|c|c|c|c|}
\hline \multicolumn{7}{|c|}{ WELL \#10-01-04 WELL DEPTH 125' } \\
\hline \multicolumn{7}{|c|}{ SCINTILLATION PRŌB̈E 1st Peak } \\
\hline Date & Peak $\mathrm{C} / \mathrm{S}$ & Depth ft. & \multirow{3}{*}{ : } & Date & Peak C/S & Depth $\mathrm{ft}$. \\
\hline $8 / 27 / 91$ & 372 & 41 & & $8 / 3 / 92$ & 322 & 40 \\
\hline $9 / 4 / 91$ & 370 & 41 & & $8 / 13 / 92$ & 352 & 41 \\
\hline $9 / 9 / 91$ & $\overline{372}$ & 41 & & $8 / 17 / 92$ & 318 & 47 \\
\hline $9 / 16 / 91$ & 403 & 41 & & $8 / 26 / 92$ & 309 & 41 \\
\hline $9 / 25 / 91$ & 361 & 41 & & $8 / 31 / 92$ & 334 & 41 \\
\hline $10 / 2 / 91$ & 406 & 41 & & $9 / 8 / 92$ & 327 & 41 \\
\hline $10 / 7 / 97$ & 349 & 41 & & $9 / 14 / 92$ & 345 & $\overline{47}$ \\
\hline $10 / 15 / 9 t$ & 360 & 41 & & $9 / 21 / 92$ & 314 & 41 \\
\hline $10 / 22 / 91$ & $\overline{356}$ & $\overline{41}$ & & $9 / 28 / 92$ & 313 & 41 \\
\hline $10 / 29 / 9 J$ & 367 & 41 & & $10 / 8 / 92$ & 312 & 41 \\
\hline $11 / 4 / 91$ & 366 & $\overline{41}$ & & $10 / 12 / 92$ & 302 & 41 \\
\hline $11 / 14 / 91$ & 373 & 41 & & $10 / 20 / 92$ & 379 & 41 \\
\hline $11 / 19 / 9 t$ & 340 & 41 & & $10 / 26 / 92$ & 321 & 41 \\
\hline $11 / 25 / 9 t$ & 375 & 41 & & $11 / 4 / 92$ & 341 & 41 \\
\hline $12 / 3 / 91$ & 377 & 41 & & $11 / 9 / 92$ & 334 & 41 \\
\hline$\pi 12 / 10 / 91$ & 347 & 41 & & $11 / 17 / 92$ & 292 & 41 \\
\hline $12 / 1 / 191$ & 345 & 41 & & $11 / 23 / 92$ & 289 & 41 \\
\hline $12 / 17 / 91$ & 351 & 41 & & $12 / 1 / 92$ & 289 & 41 \\
\hline $12 / 23 / 91$ & 353 & 41 & & $12 / 7 / 92$ & 300 & 41 \\
\hline $12 / 30 / 91$ & 323 & 41 & & $+12 / 14 / 92$ & 965 & 56 \\
\hline $1 / 9 / 92$ & 348 & 41 & & $12 / 16 / 92$ & 286 & 41 \\
\hline $1 / 13 / 92$ & 352 & 41 & & $12 / 21 / 92$ & 328 & 41 \\
\hline $1 / 20 / 92$ & 368 & 41 & & $12 / 2 \mathrm{~B} / 92$ & 300 & 41 \\
\hline $1 / 27 / 92$ & 351 & 41 & & $1 / 6 / 93$ & 264 & 41 \\
\hline $2 / 20 / 92$ & 369 & 41 & & $1 / 11 / 93$ & 301 & 41 \\
\hline $2 / 26 / 92$ & $\overline{325}$ & 41 & & $1 / 22 / 93$ & 277 & 41 \\
\hline $3 / 3 / 92$ & 355 & 41 & & $1 / 27 / 93$ & 295 & 41 \\
\hline $3 / 9 / 92$ & 337 & 41 & & $2 / 1 / 93$ & 328 & 41 \\
\hline $3 / 16 / 92$ & 350 & $4 I$ & & $2 / 8 / 93$ & 275 & 41 \\
\hline $3 / 25 / 92$ & 342 & 41 & & $2 / 16 / 93$ & 286 & 41 \\
\hline $3 / 30 / 92$ & 369 & 41 & & $2 / 22 / 93$ & 276 & 41 \\
\hline $4 / 7 / 92$ & 321 & 47 & & $2 / 22 / 93$ & 30 & 92 \\
\hline $4 / 13 / 92$ & 370 & 41 & & $3 / 4 / 93$ & 290 & 41 \\
\hline $4 / 20 / 92$ & 380 & 41 & & $3 / 8 / 93$ & 281 & 41 \\
\hline $4 / 27 / 92$ & 327 & 41 & & $3 / 16 / 93$ & 302 & 41 \\
\hline $5 / 5 / 92$ & 309 & 41 & & $3 / 23 / 93$ & 290 & 41 \\
\hline $5 / 11 / 92$ & 328 & 41 & & $3 / 29 / 93$ & 261 & 41 \\
\hline $5 / 18 / 92$ & 321 & 41 & . & $4 / 6 / 93$ & 282 & 41 \\
\hline $5 / 26 / 92$ & 303 & 41 & & $4 / 12 / 93$ & $3 Q 6$ & 41 \\
\hline $6 / 1 / 92$ & 341 & 41 & & $4 / 19 / 93$ & .269 & 41 \\
\hline $6 / 8 / 92$ & 335 & 41 & & $4 / 26 / 93$ & 275 & 41 \\
\hline $6 / 16 / 92$ & 316 & 41 & $y^{2}:$ & $5 / 3 / 93$ & 292 & 41 \\
\hline $6 / 23 / 92$ & 330 & 41 & $F_{\infty}$, & $5 / 10 / 93$ & 297 & 41 \\
\hline $6 / 29 / 92$ & 304 & 41 & & $5 / 18 / 93$ & 287 & 41 \\
\hline $7 / 6 / 92$ & 301 & 41 & & $5 / 25 / 93$ & 294 & 41 \\
\hline $7 / 13 / 92$ & 345 & 41 & $\because$ & $6 / 1 / 93$ & 309 & 41 \\
\hline
\end{tabular}

Data obtatned from WHC Surveillence Date Acquisition (SDA), November 30, 1993. 


\begin{tabular}{|c|c|c|c|c|c|c|}
\hline \multicolumn{7}{|c|}{ WELE $\$ 10-01-04$ WELL DEPTH 125 } \\
\hline \multicolumn{7}{|c|}{ SCINTILLATION PROBE 1st Peak } \\
\hline Date & Peak C/S & Depth $\mathrm{ft}$. & \multirow[b]{2}{*}{ ' } & Date & Peak $\mathrm{C} / \mathrm{s}$ & Depth ft. \\
\hline $7 / 21 / 92$ & 322 & 41 & & $6 / 7 / 93$ & 262 & 41 \\
\hline $7 / 27 / 92$ & $34 \overline{1}$ & $\overline{41}$ & \multirow{2}{*}{$\because \cdots$} & $6 / 1 \overline{4 / 93}$ & 360 & 41 \\
\hline${ }^{*} 6 / 15 / 93$ & 933 & 41 & & & & \\
\hline${ }^{*} 6 / 15 / 93$ & 264 & 41 & \multirow{7}{*}{ i } & & & \\
\hline $6 / 35 / 93$ & 300 & $\overline{4 t}$ & & & & \\
\hline${ }^{*} 6 / 22 / 93$ & 261 & 41 & & & & \\
\hline $6 / 22 / 93$ & 286 & $4 i$ & & & & \\
\hline $6 / 26 / 93$ & 288 & 41 & & & & \\
\hline $7 / 6 / 93$ & 303 & 41 & & & & \\
\hline $7 / 12 / 93$ & 259 & 41 & & & & \\
\hline $7 / 19 / 93$ & $27 \overline{9}$ & 41 & \multirow[t]{2}{*}{4} & & & \\
\hline $7 / 26 / 93$ & 283 & 41 & & & & \\
\hline $8 / 2 / 93$ & 286 & 41 & \multirow{5}{*}{$\therefore 8$} & & & \\
\hline $8 / 11 / 93$ & 309 & 41 & & & & \\
\hline $8 / 16 / 93$ & 306 & 41 & & & & \\
\hline $8 / 23 / 93$ & 370 & 41 & & & & \\
\hline $8 / 30 / 93$ & 305 & $\overline{41}$ & & & & \\
\hline $9 / 7 / 93$ & 283 & 41 & \multirow[b]{5}{*}{$y^{3}$} & & & \\
\hline $9 / 13 / 93$ & 292 & 41 & & & & \\
\hline $9 / 20 / 93$ & $2 \overline{88}$ & 41 & & & & \\
\hline $9 / 27 / 93$ & 300 & 41 & & & & \\
\hline $10 / 4 / 93$ & 287 & 41 & & & & \\
\hline $10 / 12 / 93$ & 283 & 41 & \multirow{3}{*}{ "s. } & & & \\
\hline *10/18/93 & 270 & 41 & & & & \\
\hline $10 / 18 / 93$ & 275 & 41 & & & & \\
\hline $10 / 25 / 93$ & 262 & 41 & \multirow{6}{*}{ 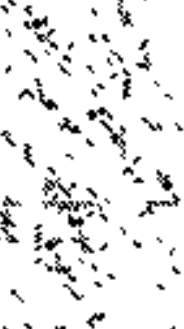 } & & & \\
\hline $11 / 1 / 93$ & $2 \overline{62}$ & 41 & & & & \\
\hline \multirow{2}{*}{-1} & & & & & & \\
\hline & & & & & & \\
\hline & & & & & & \\
\hline & & & & & & \\
\hline & & & $\because$ & & & \\
\hline & & & & & & \\
\hline & & & 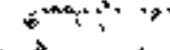 & & & \\
\hline & & & & & & \\
\hline & & & & & & \\
\hline & & & & & & \\
\hline & & & $\because$ & & . & \\
\hline & & & & & & \\
\hline & & & $\therefore$ & & & \\
\hline & & & 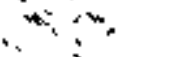 & & & \\
\hline & & & & & & \\
\hline & & &. & & & \\
\hline & & & & & & \\
\hline
\end{tabular}

Data obtained from WHC Surveillance Data Acquisition (SDA), November 30, 1993. 


\begin{tabular}{|c|c|c|c|c|c|c|}
\hline \multicolumn{7}{|c|}{ 241-A-10t DRYWELL ACTIVITY } \\
\hline \multicolumn{7}{|c|}{ WELL \#10-01-04 WELL DEPTH 125' } \\
\hline \multicolumn{7}{|c|}{ SCINTILLATION PROBE 2nd Peak } \\
\hline Date & Peak C/S & Depth ft. & \multirow{6}{*}{ 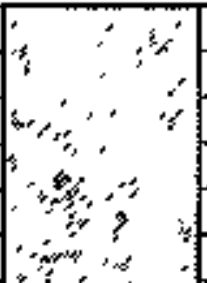 } & Dete & Peak C/S & Depth ft. \\
\hline $1 / 3 / 90$ & 90 & 55 & & $10 / 22 \sqrt{90}$ & & \\
\hline $1 / 10 / 90$ & 110 & 55 & & $10 / 29 / 90$ & 79 & 52 \\
\hline $1 / 15 / 90$ & 105 & 55 & & $11 / 6 / 90$ & 90 & 54 \\
\hline $1 / 24 / 90$ & 120 & 55 & & $11 / 13 / 90$ & 87 & $\overline{\mathbf{3}}$ \\
\hline $1 / 29 / 90$ & 125 & $\overline{55}$ & & $11 / 19 / 90$ & 75 & $\mathbf{5 3}$ \\
\hline $2 / 5 / 90$ & 112 & 54 & \multirow{7}{*}{ 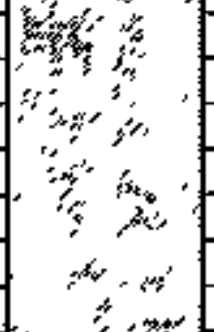 } & $11 / 28 / 90$ & & \\
\hline $2 / 12 / 90$ & 111 & 52 & & $12 / 6 / 90$ & 85 & 54 \\
\hline $2 / 13 / 90$ & 120 & 55 & & $12 / 13 / 90$ & 91 & 54 \\
\hline $2 / 20 / 90$ & 96 & 55 & & $12 / 17 / 90$ & 98 & 54 \\
\hline $2 / 27 / 90$ & 91 & 55 & & $12 / 24 / 90$ & $\mathrm{BD}$ & 54 \\
\hline $3 / 5 / 90$ & 109 & 55 & & $1 / 2 / 91$ & 102 & 54 \\
\hline *3/13/90 & 91 & 55 & & $1 / 10 / 9 t$ & 78 & 54 \\
\hline $3 / 13 / 90$ & 111 & 55 & 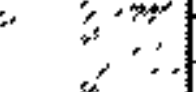 & $1 / 16 / 91$ & 90 & 55 \\
\hline $3 / 21 / 90$ & 91 & 54 & \multirow{2}{*}{$\Leftrightarrow$} & $1 / 22 / 91$ & 100 & 55 \\
\hline $3 / 28 / 90$ & 99 & 52 & & $1 / 28 / 91$ & 94 & 56 \\
\hline $4 / 3 / 90$ & 98 & 55 & \multirow{2}{*}{$\left(\begin{array}{lll}4 & \end{array}\right.$} & $2 / 4 / 91$ & 105 & 56 \\
\hline $4 / 10 / 90$ & 90 & 54 & & $2 / 12 / 91$ & 90 & 55 \\
\hline $4 / 18 / 90$ & 90 & 54 & \multirow{2}{*}{ 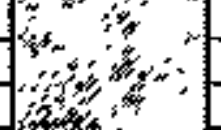 } & $2 / 21 / 91$ & & \\
\hline $4 / 24 / 90$ & 85 & 53 & & $2 / 28 / 91$ & 90 & 55 \\
\hline $5 / 3 / 90$ & 92 & 53 & \multirow{5}{*}{ 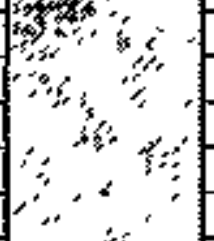 } & $3 / 5 / 91$ & 90 & 55 \\
\hline $5 / 7 / 90$ & 85 & 53 & & $3 / 7 / 91$ & 83 & $5 \overline{5}$ \\
\hline $5 / 14 / 90$ & 90 & 52 & & $3 / 13 / 91$ & 85 & 55 \\
\hline $5 / 22 / 90$ & 75 & 52 & & $3 / 20 / 91$ & 85 & 55 \\
\hline $5 / 31 / 90$ & 88 & 52 & & $3 / 25 / 91$ & 90 & 55 \\
\hline $6 / 4 / 90$ & 85 & 52 & \multirow{2}{*}{ 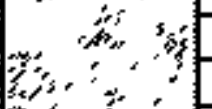 } & $4 / 1 / 91$ & 110 & 55 \\
\hline $6 / 11 / 90$ & 80 & 52 & & $4 / 9 / 91$ & 90 & 53 \\
\hline $6 / 18 / 90$ & 85 & 52 & \multirow{2}{*}{$\left(\begin{array}{l}3 \\
3\end{array}\right.$} & $4 / 16 / 91$ & B5 & 52 \\
\hline $6 / 25 / 90$ & 80 & 52 & & $4 / 23 / 91$ & 90 & 52 \\
\hline $7 / 2 / 90$ & 85 & 52 & \multirow{2}{*}{ 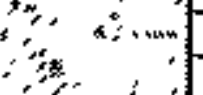 } & $4 / 30 / 91$ & 87 & 54 \\
\hline $7 / 9 / 90$ & 90 & 55 & & $5 / 6 / 91$ & 85 & 53 \\
\hline $7 / 16 / 90$ & 90 & 54 & \multirow{2}{*}{ 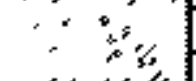 } & $5 / 13 / 91$ & 101 & 55 \\
\hline $7 / 23 / 90$ & 95 & 54 & & $5 / 20 / 91$ & 85 & 55 \\
\hline $7 / 30 / 90$ & 82 & 55 & 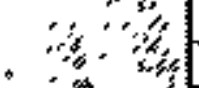 & $5 / 28 / 91$ & 108 & $5 \overline{5}$ \\
\hline $7 / 30 / 90$ & 90 & 55 & \multirow{2}{*}{ 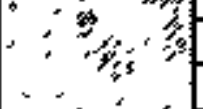 } & $6 / 4 / 91$ & & \\
\hline $8 / 7 / 90$ & 95 & 53 & & $6 / 11 / 91$ & 95 & 55 \\
\hline $8 / 14 / 90$ & 100 & 52 & \multirow{2}{*}{ 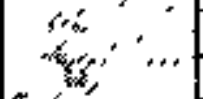 } & $6 / 17 / 91$ & 95 & 55 \\
\hline $8 / 22 / 90$ & 70 & 52 & & $6 / 25 / 91$ & 93 & $5 \overline{55}$ \\
\hline $8 / 29 / 90$ & 90 & 52 & \multirow{2}{*}{$\left(\begin{array}{lll}* & & \\
* & & *\end{array}\right.$} & $7 / 1 / 91$ & 90 & 53 \\
\hline $9 / 4 / 90$ & 85 & 53 & & $7 / 8 / 91$ & 75 & 53 \\
\hline $9 / 11 / 90$ & 75 & 52 & "争”, & $7 / 15 / 91$ & 75 & 53 \\
\hline $9 / 17 / 90$ & 80 & 52 & \multirow{5}{*}{ 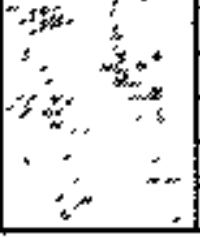 } & $7 / 22 / 91$ & 85 & 55 \\
\hline $9 / 27 / 90$ & 86 & 52 & & $7 / 30 / 91$ & 95 & 53 \\
\hline $10 / 1 / 90$ & & & & $3 / 5 / 91$ & 90 & 55 \\
\hline $10 / 10 / 90$ & 100 & 52 & & $8 / 14 / 91$ & 85 & 53 \\
\hline $10 / 15 / 90$ & & & & $8 / 20 / 91$ & 90 & 53 \\
\hline
\end{tabular}

Data obteined from WHC Surveillance Data Acquisition ISDAl. November 30, 1993. 


\begin{tabular}{|c|c|c|c|c|c|c|}
\hline \multicolumn{7}{|c|}{ WELL \#10-01-04 WELL DEP7"H 125* } \\
\hline \multicolumn{7}{|c|}{ SCINTILLATION PROBE 2nó Peak } \\
\hline Date & Peak C/S & Depth ft. & & Date & Peak C/S & $\overline{\text { Depth } \mathrm{ft} .}$ \\
\hline $8 / 27 / 91$ & 85 & 52 & - & $8 / 3 / 92$ & 85 & 55 \\
\hline $9 / 4 / 91$ & 85 & 54 & & $8 / 11 / 92$ & 72 & $5 \underline{5}$ \\
\hline $9 / 9 / 91$ & 80 & 56 & ' & $8 / 17 / 92$ & 85 & 55 \\
\hline $9 / 16 / 91$ & 77 & 55 & & $8 / 25 / 92$ & 75 & 54 \\
\hline $9 / 25 / 91$ & 95 & 55 & & $8 / 31 / 92$ & 80 & 55 \\
\hline $10 / 2 / 91$ & 92 & 55 & & $\overline{9 / 8 / 92}$ & 80 & 55 \\
\hline $10 / 7 / 91$ & 85 & 55 & & $9 / 14 / 92$ & 95 & 55 \\
\hline $10 / 16 / 91$ & 90 & 55 & & $9 / 21 / 92$ & 80 & 55 \\
\hline $30 / 22 / 91$ & 95 & 55 & & $9 / 28 / 92$ & 80 & 55 \\
\hline $10 / 29 / 91$ & 80 & 55 & & $10 / 8 / 92$ & 80 & 54 \\
\hline $11 / 4 / 91$ & 73 & 57 & & $10 / 12 / 92$ & 75 & 52 \\
\hline $11 / \overline{4 / 91}$ & $\overline{B 7}$ & 57 & & $10 / 20 / 92$ & 80 & 52 \\
\hline $11 / 18 / 91$ & BO & 55 & & $10 / 26 / 92$ & 82 & 55 \\
\hline $11 / 25 / 91$ & 88 & 55 & & $11 / 4 / 92$ & 90 & 56 \\
\hline $12 / \overline{3 / 91}$ & $\overline{87}$ & 53 & & $11 / 9 / 92$ & 75 & 53 \\
\hline $92 / 10 / 91$ & 76 & 55 & & $11 / 17 / 92$ & 70 & 64 \\
\hline $12 / 11 / 91$ & 77 & 54 & & $11 / 23 / 92$ & 85 & 54 \\
\hline $12 / 17 / 91$ & 85 & 55 & & $12 / 1 / 92$ & 73 & 56 \\
\hline $12 / 23 / 91$ & 90 & 56 & & $12 / 7 / 92$ & 75 & 54 \\
\hline $12 / 30 / 91$ & 90 & 55 & & $* 12 / 14 / 92$ & & \\
\hline $1 / 9 / 92$ & 78 & 55 & & $12 / 16 / 92$ & & \\
\hline $1 / 13 / 92$ & $\overline{91}$ & 54 & & $12 / 21 / 92$ & 85 & 55 \\
\hline $1 / 20 / 92$ & 85 & 55 & & $12 / 28 / 92$ & 85 & 55 \\
\hline $1 / 27 / 92$ & 90 & 55 & & $1 / 5 / 93$ & 72 & 56 \\
\hline $2 / 20 / 92$ & 90 & 55 & & $1 / 11 / 9 \overline{3}$ & 75 & 55 \\
\hline $2 / 26 / 92$ & 85 & 55 & & $1 / 22 / 93$ & 83 & 55 \\
\hline $3 / 3 / 92$ & 90 & 55 & & $1 / 27 / 93$ & 75 & 55 \\
\hline $3 / 9 / 92$ & 85 & 55 & & $2 / 1 / 93$ & 67 & 55 \\
\hline $3 / 26 / 92$ & 75 & 54 & & $2 / 8 / 93$ & 80 & 55 \\
\hline $3 / 25 / 92$ & $\overline{B O}$ & 55 & & $2 / 16 / 9 \overline{3}$ & 68 & 55 \\
\hline $3 / 30 / 92$ & 96 & 55 & & $2 / 22 / 93$ & 70 & 55 \\
\hline $4 / 7 / 92$ & $B O$ & 54 & & $2 / 22 / 93$ & & \\
\hline $4 / 13 / 92$ & 86 & 54 & & $3 / 4 / 93$ & 90 & 56 \\
\hline $4 / 20 / 92$ & 80 & 55 & & $3 / 8 / 93$ & 75 & 54 \\
\hline $4 / 27 / 92$ & 60 & 55 & & $3 / 16 / 93$ & 75 & 55 \\
\hline $5 / 5 / 92$ & 80 & 53 & & $3 / 23 / 93$ & 66 & 58 \\
\hline $5 / 11 / 92$ & 80 & 55 & & $3 / 29 / 93$ & 70 & 55 \\
\hline $5 / 18 / 92$ & 90 & 55 & & $4 / 6 / 93$ & 75 & 55 \\
\hline $6 / 26 / 92$ & 90 & 54 & & $4 / 12 / 93$ & 87 & 55 \\
\hline $6 / 1 / 92$ & 95 & 55 & $\therefore$ & $4 / 19 / 93$ & .70 & 55 \\
\hline $6 / 8 / 92$ & 82 & 55 & 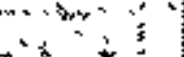 & $4 / 26 / 93$ & 70 & 55 \\
\hline $6 / 16 / 92$ & 95 & 54 & 0. & $5 / 3 / 93$ & 75 & 55 \\
\hline $6 / 23 / 92$ & 80 & 54 & $\operatorname{lon}_{2}=z_{2}$ & $5 / 10 / 93$ & 85 & 56 \\
\hline $6 / 29 / 92$ & & & & $5 / 18 / 93$ & & \\
\hline $7 / 6 / 92$ & 90 & $\overline{5} \overline{5}$ & & $5 / 25 / 93$ & 80 & 55 \\
\hline $7 / 13 / 92$ & 89 & 54 & & $6 / 1 / 93$ & 73 & 55 \\
\hline
\end{tabular}

Data obteined from WHC Survellance Data Acquisition (SOA), November 30, 1993. 


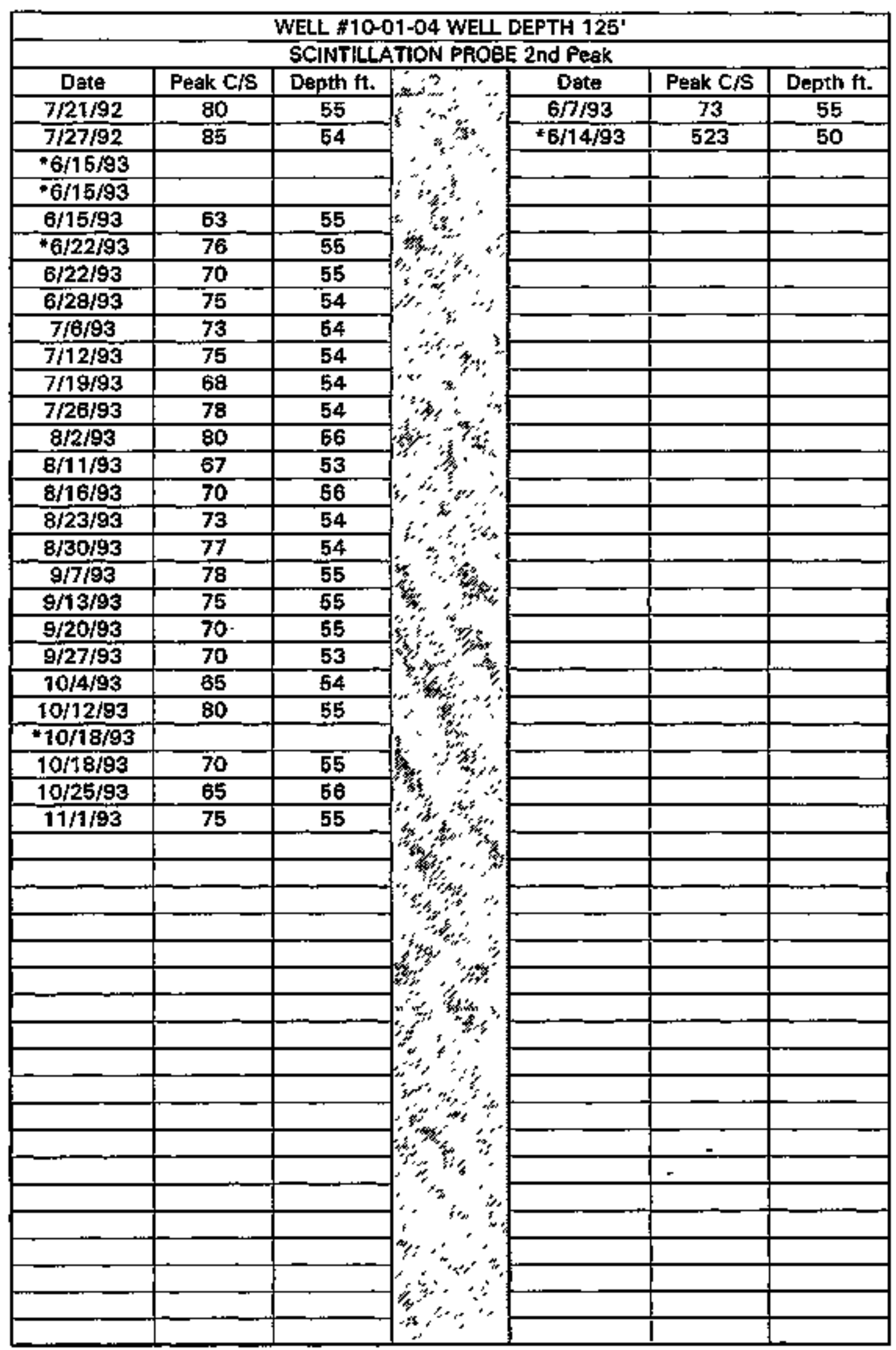

Data obtained from WHC Surveillence Data Acquisition (SDA), November 30, 1993. 


\begin{tabular}{|c|c|c|c|c|c|c|}
\hline \multicolumn{7}{|c|}{ 241-A-101 DRYWEU ACTVITY } \\
\hline \multicolumn{7}{|c|}{ WELL \# } \\
\hline \multicolumn{7}{|c|}{ SCINTELATION PFOBE 3rd Peak } \\
\hline Date & Peak C/S & Depth ft. & & Datse & Peak C/S & Depth ft. \\
\hline $1 / 3 / 90$ & 85 & 66 & $=$ & $10 / 22 / 90$ & 90 & 67 \\
\hline $1 / 10 / 90$ & 88 & 56 & & $10 / 29 / 90$ & $\mathbf{B O}$ & 67 \\
\hline $1 / 7 \overline{5 / 90}$ & 90 & 66 & & $11 / 6 / 90$ & 85 & 67 \\
\hline $1 / 24 / 90$ & $10 \overline{0}$ & 66 & & $11 / 13 / 90$ & 90 & 67 \\
\hline $1 / 29 / 90$ & $8 \overline{5}$ & 66 & & $11 / 19 / 90$ & $\mathrm{BO}$ & 66 \\
\hline $2 / 5 / 90$ & 88 & 66 & & $1 \overline{1 / 28 / 90}$ & 88 & 63 \\
\hline${ }^{*} 2 / 12 / 90$ & 105 & 66 & & $12 / 5 / 90$ & $\overline{97}$ & 100 \\
\hline $2 / 13 / 90$ & 105 & $B 6$ & & $12 / 13 / 90$ & 78 & 66 \\
\hline $2 / 20 / 90$ & 105 & 67 & & $\sqrt{2 / 17 / 90}$ & 87 & 66 \\
\hline $2 / 27 / 90$ & 90 & 67 & & $12 / 24 / 90$ & $\overline{82}$ & $\overline{6}$ \\
\hline $3 / 5 / 90$ & 93 & 67 & & $1 / 2 / 91$ & 73 & 66 \\
\hline $3 / 13 / 90$ & 80 & 66 & & $1 / 10 / 91$ & 88 & 66 \\
\hline $3 / 13 / 90$ & 101 & 66 & & $1 / 16 / 91$ & 87 & $6 \overline{7}$ \\
\hline $3 / 21 / 90$ & 100 & 66 & & $1 / 22 / 91$ & 76 & 67 \\
\hline $3 / 28 / 90$ & $\overline{87}$ & $\overline{66}$ & & $1 / 28 / 91$ & 70 & 67 \\
\hline $4 / 3 / 90$ & 90 & $\overline{66}$ & & $2 / 4 / \overline{91}$ & 95 & $\overline{66}$ \\
\hline $4 / 10 / 90$ & 80 & 67 & & $2 / 12 / 91$ & 97 & 66 \\
\hline $4 / 78 / 90$ & 85 & 67 & & $2 / 21 / 91$ & & \\
\hline $4 / 24 / 90$ & $8 \overline{5}$ & 67 & & $2 / 28 / 91$ & 91 & 67 \\
\hline $5 / 3 / 90$ & 90 & 67 & & $3 / 5 / 91$ & 97 & 67 \\
\hline $5 / 7 / 90$ & 90 & 67 & & $3 / 7 / 91$ & 91 & 67 \\
\hline $5 / 14 / 90$ & 88 & 67 & & $3 / 13 / 91$ & 95 & 66 \\
\hline $5 / 22 / 90$ & 92 & 67 & & $3 / 20 / 91$ & 35 & 67 \\
\hline $5 / 31 / 90$ & 83 & 67 & & $3 / 25 / 91$ & 91 & 67 \\
\hline $6 / 4 / 90$ & 86 & 67 & & $4 / 1 / 91$ & 86 & 66 \\
\hline $6 / 71 / 90$ & 82 & 66 & & $4 / \overline{9} / \overline{91}$ & 96 & 66 \\
\hline $6 / 18 / 90$ & 105 & 66 & & $4 / 16 / 97$ & 98 & 66 \\
\hline $6 / 25 / 90$ & 95 & 66 & & $4 / 23 / 99$ & 72 & 67 \\
\hline $7 / 2 / 90$ & $\overline{90}$ & 68 & & $4 / 30 / 91$ & 103 & 66 \\
\hline $7 / 9 / 90$ & 80 & 66 & & $5 / 6 / 97$ & 82 & 66 \\
\hline $7 / 16 / 90$ & $\overline{86}$ & 67 & & $5 / 13 / 91$ & 85 & $\overline{66}$ \\
\hline $7 / 23 / 90$ & 88 & 66 & & $5 / 20 / 91$ & 85 & 60 \\
\hline$+7 / 30 / 90$ & 100 & 66 & & $5 / 28 / 91$ & 87 & 65 \\
\hline $7 / 30 / 90$ & 87 & 66 & & $6 / 4 / \overline{91}$ & & \\
\hline $8 / 7 / 90$ & 90 & 67 & & $6 / 11 / 91$ & 97 & 67 \\
\hline $8 / 14 / 90$ & 75 & 66 & & $6 / 17 / 91$ & 74 & 67 \\
\hline $8 / 22 / 90$ & 95 & 66 & & $6 / 25 / 91$ & 77 & 67 \\
\hline $8 / 29 / 90$ & 95 & 66 & & $7 / 1 / 91$ & 95 & 65 \\
\hline $9 / 4 / 90$ & 81 & 66 & & $7 / 8 / 91$ & .75 & 67 \\
\hline $9 / 11 / 90$ & 82 & 66 & & $7 / 15 / 91$ & 100 & 67 \\
\hline $9 / 17 / 90$ & 99 & 66 & & $7 / 22 / 91$ & 30 & 66 \\
\hline $9 / 27 / 90$ & 89 & 67 & & $7 / 30 / 91$ & 70 & 66 \\
\hline $10 / 1 / 9 \overline{0}$ & $\overline{84}$ & 67 & & $8 / 5 / \overline{9} t$ & 85 & 67 \\
\hline $10 / 10 / 90$ & 75 & 67 & & $8 / 14 / 91$ & $\mathrm{BO}$ & 66 \\
\hline $10 / 15 / 90$ & 85 & 60 & & $8 / 20 / 91$ & 90 & 66 \\
\hline
\end{tabular}

Data obtained from WHC Surveillance Data Acquisition (SDAl, November 30, 1993. 


\begin{tabular}{|c|c|c|c|c|c|c|}
\hline \multicolumn{7}{|c|}{ WELL $\# 10-01-04$ WELL DEPTH 125 } \\
\hline \multicolumn{7}{|c|}{ SCINTLLLATION PROBE 3rd Pegk } \\
\hline Date & Peak CJS & Depth ft. & \multirow[b]{5}{*}{ 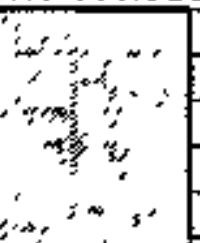 } & Date & Peak C/S & Depth ft. \\
\hline $8 / 27 / 91$ & 85 & 66 & & $8 / 3 / 92$ & 90 & 66 \\
\hline $9 / 4 / 91$ & 80 & 66 & & $8 / 11 / 92$ & 95 & $6 \overline{\mathrm{B}}$ \\
\hline $9 / 9 / 91$ & 83 & 66 & & $8 / 17 / 92$ & 75 & 66 \\
\hline $9 / 16 / 91$ & $\overline{75}$ & 67 & & $8 / 25 / 92$ & 80 & 66 \\
\hline $9 / 25 / 91$ & 70 & $\overline{66}$ & \multirow{4}{*}{ 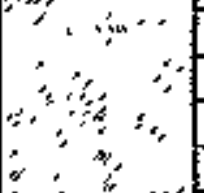 } & $8 / 31 / 92$ & 78 & $6 \overline{6}$ \\
\hline $10 / 2 / 91$ & 90 & 66 & & $9 / 8 / 92$ & 81 & 65 \\
\hline $10 / 7 / 91$ & 87 & 66 & & $9 / 14 / 92$ & 70 & 66 \\
\hline $10 / 15 / 91$ & 85 & 66 & & $9 / 21 / 92$ & 87 & 66 \\
\hline $10 / 22 / 91$ & 80 & 66 & \multirow{5}{*}{ 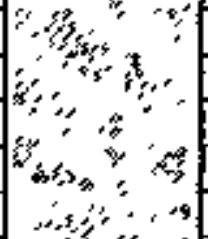 } & $9 / 28 / 92$ & 75 & 66 \\
\hline $10 / 29 / 91$ & 85 & 67 & & $10 / 8 / 92$ & $\overline{77}$ & 65 \\
\hline $11 / 4 / 91$ & 92 & 67 & & $10 / 12 / 92$ & 85 & 68 \\
\hline $11 / 14 / 91$ & 80 & 66 & & $10 / 20 / 92$ & 75 & $6 \overline{6}$ \\
\hline $11 / 18 / 91$ & 81 & 66 & & $10 / 26 / 92$ & 85 & 67 \\
\hline $11 / 25 / 91$ & 89 & 66 & \multirow{2}{*}{ 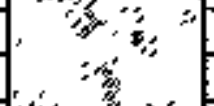 } & $11 / 4 / 92$ & 92 & 67 \\
\hline $12 / 3 / 91$ & 95 & 67 & & $11 / 9 / 92$ & 85 & 66 \\
\hline *12/10/91 & 95 & 67 & \multirow{4}{*}{ 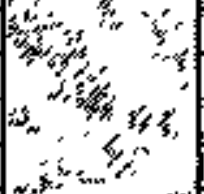 } & $11 / 17 / 92$ & 75 & 67 \\
\hline $12 / 11 / 91$ & 80 & 66 & & $11 / 23 / 92$ & 92 & 66 \\
\hline $12 / 17 / 91$ & 91 & 67 & & $12 / 1 / 92$ & 87 & $6 \overline{\mathrm{B}}$ \\
\hline $12 / 23 / 91$ & 80 & 66 & & $12 / 7 / 92$ & 80 & 68 \\
\hline $12 / 30 / 91$ & 80 & 67 & \multirow{2}{*}{\multicolumn{2}{|c|}{$\frac{* 12 / 14 / 92}{12 / 16 / 92}$}} & & \\
\hline $1 / 9 / 92$ & 77 & 66 & & & & \\
\hline $1 / 13 / 92$ & 90 & 65 & \multirow{5}{*}{ 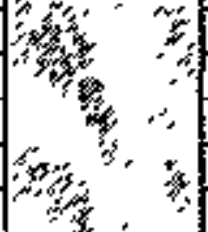 } & $12 / 21 / 92$ & 85 & 66 \\
\hline $1 / 20 / 92$ & 82 & 66 & & $12 / 28 / 92$ & 75 & 66 \\
\hline $1 / 27 / 92$ & 70 & 66 & & $1 / 5 / 93$ & 75 & 67 \\
\hline $2 / 20 / 92$ & 80 & 67 & & $1 / 11 / 93$ & 78 & 67 \\
\hline $2 / 26 / 92$ & 97 & 66 & & $1 / 22 / 93$ & 77 & $6 \overline{6}$ \\
\hline $3 / 3 / 92$ & 80 & 66 & \multirow{6}{*}{ 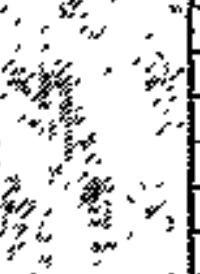 } & $1 / 27 / 93$ & 85 & 66 \\
\hline $3 / 9 / 92$ & 91 & 66 & & $2 / 1 / 93$ & 80 & 66 \\
\hline $3 / 16 / 92$ & 70 & 66 & & $2 / 8 / 93$ & 80 & 86 \\
\hline $3 / 25 / 92$ & 70 & $6 \overline{6}$ & & $2 / 16 / 93$ & 76 & $6 \overline{6}$ \\
\hline $3 / 30 / 92$ & 90 & $6 \theta$ & & $2 / 22 / 93$ & $B 6$ & 66 \\
\hline $4 / 7 / 92$ & 72 & 66 & & $2 / 22 / 93$ & & \\
\hline $4 / 13 / 92$ & 80 & 66 & \multirow{8}{*}{ 绻", } & $3 / 4 / 93$ & 80 & 67 \\
\hline $4 / 20 / 92$ & 80 & 66 & & $3 / 8 / 93$ & $\mathrm{BO}$ & 66 \\
\hline $4 / 27 / 92$ & 80 & 66 & & $3 / 16 / 93$ & 80 & 66 \\
\hline $5 / 5 / 92$ & 75 & 65 & & $3 / 23 / 93$ & 75 & 67 \\
\hline $5 / 11 / 92$ & 70 & $6 \overline{6}$ & & $3 / 29 / 93$ & 85 & 66 \\
\hline $5 / 18 / 92$ & 95 & 66 & & $4 / 6 / 93$ & 90 & $6 \overline{6}$ \\
\hline $5 / 26 / 92$ & 89 & 68 & & $4 / 12 / 93$ & 83 & 67 \\
\hline $6 / 1 / 92$ & 75 & 67 & & $4 / 19 / 93$ & 79 & 56 \\
\hline $6 / 8 / 92$ & 85 & 67 & \multirow{4}{*}{ 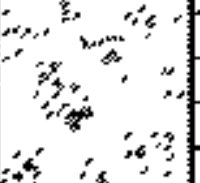 } & $4 / 26 / 93$ & 90 & $6 \overline{6}$ \\
\hline B/16/92 & 70 & $6 \overline{6}$ & & $5 / 3 / 93$ & 60 & $\overline{66}$ \\
\hline $6 / 23 / 92$ & 80 & 66 & & $5 / 10 / 93$ & 85 & $\overline{66}$ \\
\hline $6 / 29 / 92$ & & & & $5 / 18 / 93$ & & \\
\hline $7 / 6 / 92$ & 75 & 66 & \multirow[b]{2}{*}{ 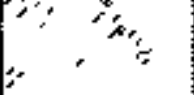 } & $5 / 25 / 93$ & 92 & 66 \\
\hline $7 / 13 / 92$ & 80 & $6 \overline{5}$ & & $6 / 1 / 93$ & 70 & 66 \\
\hline
\end{tabular}

Data obtained from WHC Surveillance Deta Acquisitlon (SDA), November 30, 1993. 


\begin{tabular}{|c|c|c|c|c|c|c|}
\hline \multicolumn{7}{|c|}{ WELL $\$ 10-01-04$ WELL DEPTH 125' } \\
\hline \multicolumn{7}{|c|}{ SC̄INTILLATIOÑ PR̄OBE 3rd Peak } \\
\hline Date & Peak C/S & Depth $\mathrm{ft}$. & & Date & Peak C/S & Depth ft. \\
\hline$\overline{7} / 2 \overline{1} / 9 \overline{2}$ & 97 & 66 & & $6 / 7 / 93$ & 67 & 66 \\
\hline $7 / 27 / 92$ & go & $\overline{66}$ & & $* 6 / 14 / 93$ & 80 & 66 \\
\hline \multicolumn{6}{|l|}{$6 / 15 / 93$} & \\
\hline \multicolumn{7}{|l|}{$6 / \overline{15 / 93}$} \\
\hline $6 / 15 / 93$ & 75 & 66 & \multirow{5}{*}{ 1..." } & & & \\
\hline$* 6 / 22 / 93$ & GB & 66 & & & & \\
\hline $6 / 22 / 9 \overline{3}$ & 80 & 66 & & & & \\
\hline $6 / 28 / 93$ & 80 & 66 & & & & \\
\hline $7 / 6193$ & 75 & $\overline{67}$ & & & & \\
\hline $7 / 12193$ & 75 & 66 & & & & \\
\hline $7 / 19 / 93$ & 77 & 66 & & & & \\
\hline $7 / 26 / 93$ & 70 & 66 & & & & \\
\hline $8 / 2 / 93$ & $\overline{85}$ & $\overline{67}$ & & & & \\
\hline $8 / 11 / 93$ & 70 & 68 & & & & \\
\hline $8 / 16 / 93$ & 75 & 67 & & & & \\
\hline $8 / 23 / 93$ & 70 & 66 & & & & \\
\hline $8 / 30 / 93$ & 72 & 67 & & & & \\
\hline$9 \longdiv { 7 / 9 3 }$ & 80 & 66 & & & & \\
\hline $9 / 13 / 93$ & 73 & 66 & & & & \\
\hline $9 / 20 / 93$ & 90 & 67 & & & & \\
\hline $9 / 27 / 93$ & 70 & 67 & & & & \\
\hline $10 / 4 / 93$ & 72 & 66 & & & & \\
\hline $10 / 12 / 93$ & 82 & 66 & & & & \\
\hline \multicolumn{7}{|l|}{$-10 / 19 / 93$} \\
\hline $10 / 18 / 93$ & 90 & 66 & & & & \\
\hline $10 / 25 / 93$ & 74 & 66 & $\therefore$ & & & \\
\hline $11 / 1 / 93$ & 65 & 66 & & & & \\
\hline & & & & & & \\
\hline & & & & & & \\
\hline & & & & & & \\
\hline & & & & & & \\
\hline & & & $\because \mathrm{n}^{n}$ & & & \\
\hline & & & 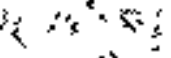 & & & \\
\hline & & & $\therefore y$ & & & \\
\hline & & & $4 x^{3}$ & & & \\
\hline & & & & & & \\
\hline & & & $\therefore \vdots$ & & & \\
\hline & & & $\because$ & & & \\
\hline & & & . & & . & \\
\hline & & & $\leq \leqslant$ & & & \\
\hline & & & $\because \div$ & & & \\
\hline & & & an $\ln ^{n+\infty}$ & & & \\
\hline & & & $\therefore \quad \therefore$ & & & \\
\hline & & & 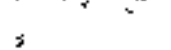 & & & \\
\hline & & & & & & \\
\hline
\end{tabular}

Data obtained from WHC Surveiliance Data Acquisition [SDA], November 30, 1993. 


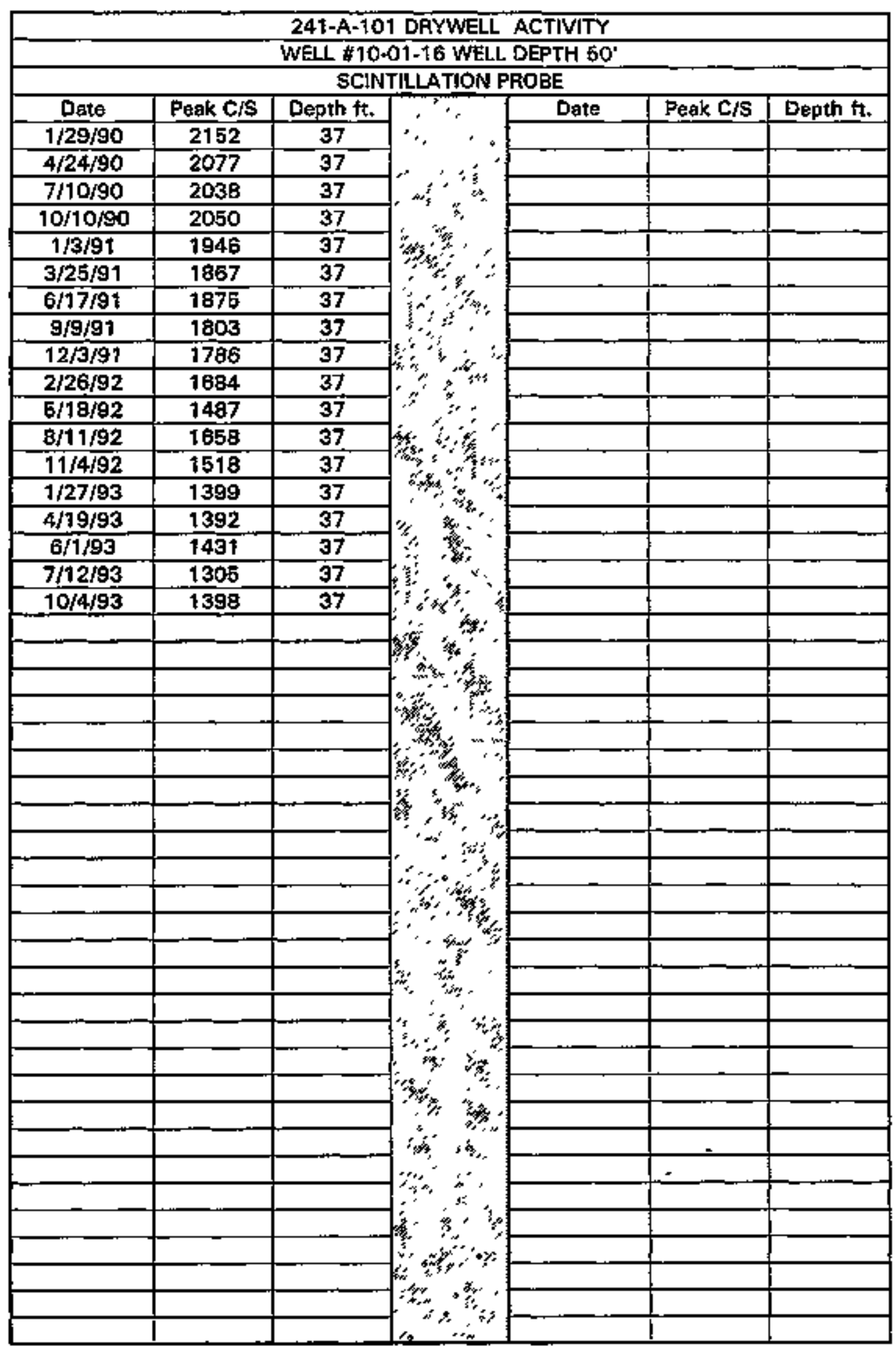

Data obtained from WHC Survellance Data Acquisition (SDA), November 30, 1993. 


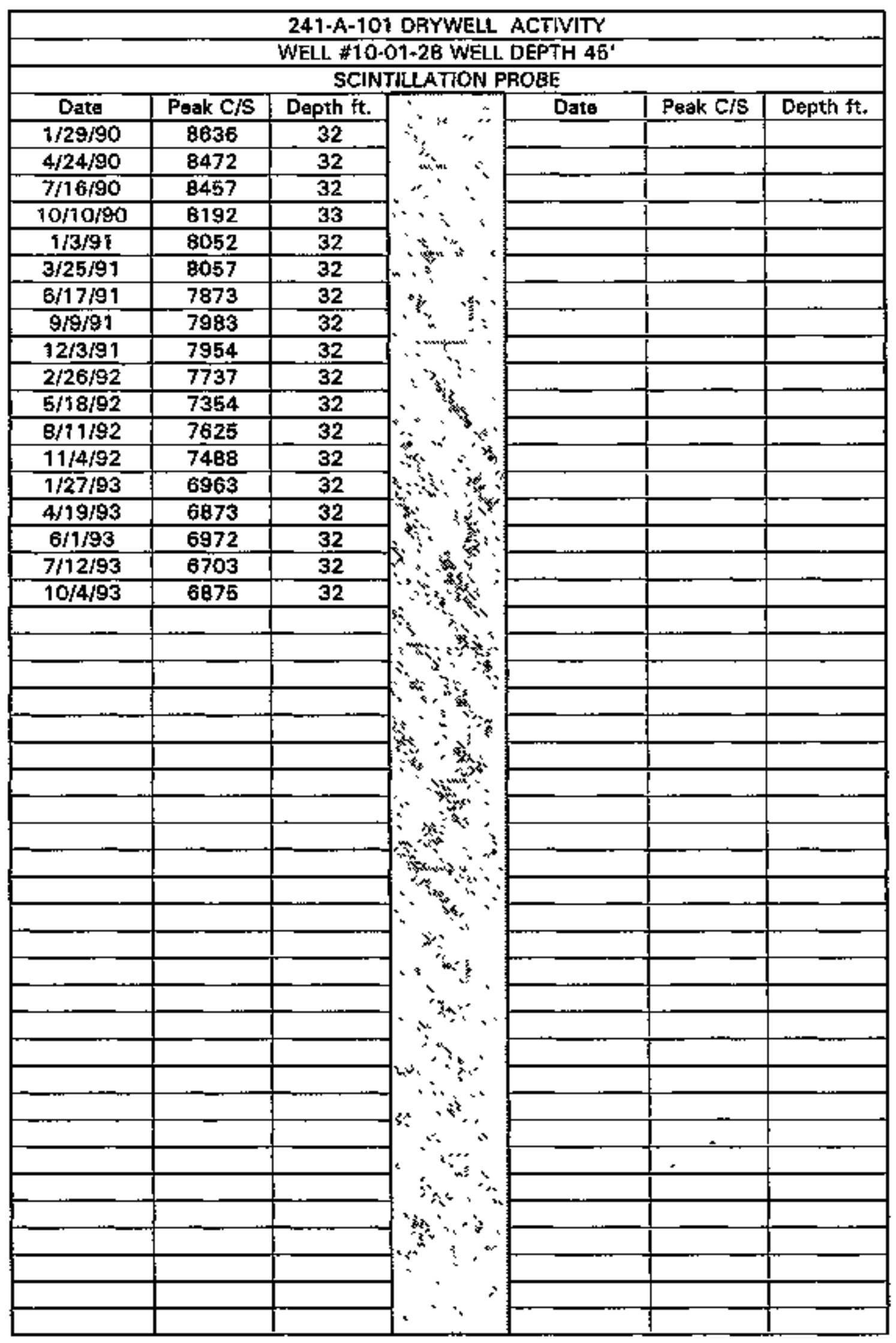

Data obtained from WHC Surveflance Data Acquistoton (SDA), November 30, 1993. 


\begin{tabular}{|c|c|c|c|c|c|c|}
\hline \multicolumn{7}{|c|}{ 241-A-102 DRYWELL ACTIVITY } \\
\hline \multicolumn{7}{|c|}{ WELL \#10-02-03 WELL DEPTH $125^{\circ}$} \\
\hline \multicolumn{7}{|c|}{ SCINTILLATION PROBE } \\
\hline Date & Peak C/S & Depth ft. & $7+4$ & Date & Peak CNS & Depth ft. \\
\hline $1 / 16 / 90$ & 62 & 79 & & $12 / 14 / 92$ & $5 \overline{6}$ & 79 \\
\hline $2 / 13 / 90$ & 65 & 79 & & $\pm 1 / 11 / 93$ & 70 & 69 \\
\hline $3 / 13 / 90$ & 57 & 78 & & $* 1 / 12 / 93$ & 83 & 79 \\
\hline $3 / 13 / 90$ & 53 & 78 & & $1 / 12 / 93$ & 61 & 79 \\
\hline$\overline{4 / 10 / 90}$ & 60 & 79 & & $2 / 8 / 93$ & $\mathbf{5} \overline{8}$ & $\overline{79}$ \\
\hline $5 / 7 / 90$ & 69 & $7 \theta$ & & $3 / 8 / 93$ & 65 & 79 \\
\hline $5 / 7 / 90$ & 53 & 79 & & $3 / 29 / 93$ & 58 & $\overline{79}$ \\
\hline $6 / 5 / 90$ & 67 & 79 & & $4 / 6 / 93$ & 56 & 79 \\
\hline $7 / 2 / 90$ & 77 & 79 & & $6 / 3 / 93$ & 71 & 79 \\
\hline $7 / 3 / 90$ & 73 & 79 & & $6 / 1 / 93$ & 58 & 79 \\
\hline $7 / 3 / 90$ & 64 & 79 & & $6 / 28 / 93$ & 57 & 79 \\
\hline $7 / 30 / 90$ & 54 & 79 & & $7 / 26 / 93$ & 49 & 79 \\
\hline $8 / 29 / 90$ & 64 & 79 & & $8 / 23 / 93$ & 46 & 79 \\
\hline $9 / 27 / 90$ & 60 & 79 & & $9 / 20 / 93$ & 59 & $\overline{79}$ \\
\hline $10 / 22 / 90$ & 57 & 79 & & $10 / 18 / 93$ & 53 & 79 \\
\hline $11 / 19 / 90$ & 60 & 79 & & $11 / 24 / 93$ & 62 & 79 \\
\hline $12 / 17 / 90$ & 59 & 79 & & & & \\
\hline $1 / 16 / 91$ & 60 & 79 & & & & \\
\hline $2 / 12 / 91$ & 63 & 79 & & & & \\
\hline $3 / 13 / 91$ & 56 & 78 & & & & \\
\hline $4 / 9 / 91$ & 73 & 79 & & & & \\
\hline $5 / 6 / 91$ & 62 & 78 & & & & \\
\hline $6 / 4 / 91$ & 70 & 79 & & & & \\
\hline $7 / 1 / 91$ & 62 & 79 & & & & \\
\hline $7 / 30 / 9 t$ & $\overrightarrow{56}$ & 79 & & & & \\
\hline $8 / 27 / 91$ & 53 & 78 & & & & \\
\hline $9 / 24 / 9 t$ & 64 & 79 & & & & \\
\hline $10 / 22 / 91$ & 52 & 79 & & & & \\
\hline $11 / 18 / 91$ & 66 & 79 & & & & \\
\hline $12 / 17 / 91$ & 56 & 79 & & & & \\
\hline $1 / 13 / 92$ & 74 & 79 & & & & \\
\hline$* 2 / 13 / 92$ & 149 & 122 & & & & \\
\hline $2 / 14 / 92$ & 59 & 79 & & & & \\
\hline $3 / 9 / 92$ & 57 & 79 & & & & \\
\hline $4 / 7 / 92$ & 72 & 79 & & & & \\
\hline $5 / 5 / 92$ & 61 & 79 & & & & \\
\hline $6 / 1 / 92$ & 1 & 122 & & & & \\
\hline $6 / 2 / 92$ & 74 & 79 & & & & \\
\hline $6 / 29 / 92$ & 61 & 79 & & & . & \\
\hline $7 / 27 / 92$ & 70 & 79 & & & & \\
\hline $8 / 24 / 92$ & 61 & 79 & & & & \\
\hline $9 / 21 / 92$ & $5 t$ & 79 & & & & \\
\hline $10 / 20 / 92$ & 58 & 79 & & & & \\
\hline $11 / 17 / 92$ & 55 & 78 & & & & \\
\hline $12 / 7 / 92$ & 35 & 101 & 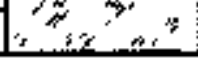 & & & \\
\hline
\end{tabular}

Data obtained from WHC Survellance Dete Acquisition (SDA). November 30, 1993. 


\begin{tabular}{|c|c|c|c|c|c|c|}
\hline \multicolumn{7}{|c|}{ 241-A-102 DAYWELL_ACTIVITY } \\
\hline \multicolumn{7}{|c|}{ WELL \$10-02-08 WELL DEPTH 125' } \\
\hline \multicolumn{7}{|c|}{ SCINTILLATION PROBE } \\
\hline Dete & Peak C/S & Depth ft. & & Date & Peak C/S & Depth ft. \\
\hline $1 / 15 / 90$ & 43 & 86 & & $12 / 14 / 92$ & 32 & 84 \\
\hline $2 / 12 / 90$ & 42 & $\overline{86}$ & & $1 / 11 / 93$ & $\overline{82}$ & 42 \\
\hline $3 / 13 / 90$ & 36 & 85 & & $1 / 12 / 93$ & 45 & 85 \\
\hline $4 / 10 / 90$ & 41 & 86 & & $1 / 12 / 93$ & 56 & 93 \\
\hline $6 / 7 / 90$ & $\overline{36}$ & 84 & & $2 / 8 / 93$ & 36 & 85 \\
\hline $6 / 5 / 90$ & 37 & 85 & & $3 / 8 / 93$ & $3 \overline{7}$ & $\overline{\mathrm{B}} 6$ \\
\hline $7 / 2 / 90$ & 37 & 86 & $\hat{\imath}$ & $3 / 29 / 93$ & 34 & 85 \\
\hline $7 / 3 / 90$ & $\overline{34}$ & 85 & & $4 / 6 / 93$ & 38 & 85 \\
\hline $7 / 30 / 90$ & 32 & 58 & & $5 / 3 / 93$ & 33 & 86 \\
\hline $8 / 29 / 90$ & 36 & 86 & & $-6 / 1 / 9 \overline{3}$ & 40 & 85 \\
\hline $9 / 27 / 90$ & 37 & 85 & & $+6 / 2 / 93$ & 175 & 96 \\
\hline $10 / 22 / 90$ & $\overline{37}$ & 85 & & $6 / 3 / 93$ & 41 & 86 \\
\hline $11 / 19 / 90$ & 38 & 86 & & $6 / 8 / 93$ & $4 \overline{3}$ & 85 \\
\hline $12 / 17 / 90$ & 43 & 85 & & $6 / 14 / 93$ & 112 & 92 \\
\hline $1 / 16 / \overline{9} 1$ & 38 & 86 & & $6 / 15 / 93$ & 100 & 45 \\
\hline $72 / 12 / 91$ & 41 & 85 & & $\overline{6 / 15 / 93}$ & $3 \overline{4}$ & 86 \\
\hline $2 / 14 / 91$ & 84 & 39 & & $6 / 28 / 93$ & 41 & 86 \\
\hline$-2 / 14 \sqrt{91}$ & 43 & $\overline{42}$ & & $7 / 6 / 93$ & 39 & 86 \\
\hline $2 / 14 / 91$ & 43 & 86 & & $7 / 26 / 93$ & $3 \overline{3}$ & 42 \\
\hline $3 / 13 / 91$ & 38 & 85 & & $8 / 2 / 93$ & 33 & 94 \\
\hline $4 / \overline{1} / \overline{91}$ & 42 & 84 & & $8 / 23 / 93$ & 34 & $\overline{86}$ \\
\hline $5 / 6 / 91$ & 43 & 85 & & $9 / 13 / 93$ & 41 & 85 \\
\hline$6 \longdiv { 4 / 9 1 }$ & 36 & 86 & & $9 / 23 / 93$ & 31 & 89 \\
\hline $7 / 1 / 91$ & 32 & 90 & & $10 / 1 \mathrm{~B} / 9 \mathrm{3}$ & 43 & 86 \\
\hline $7 / 30 / 91$ & 43 & 86 & & $11 / 24 / 93$ & 43 & 86 \\
\hline$-8 / 27 / 91$ & 58 & $10 \overline{3}$ & & & & \\
\hline $8 / 29 / 91$ & 36 & $\overline{86}$ & & & & \\
\hline $9 / 24 / 91$ & 35 & 8B & & & & \\
\hline $10 / 22 / 91$ & 40 & 86 & & & & \\
\hline $13 / 18 / 91$ & $\overline{39}$ & 86 & & & & \\
\hline $12 / 17 / 91$ & 39 & 86 & & & & \\
\hline $1 / 1 / 3 / 92$ & 145 & 111 & & & & \\
\hline $1 \sqrt{13 / 92}$ & 37 & 85 & & & & \\
\hline $2 / 13 / 92$ & 204 & 124 & & & & \\
\hline $2 / t 4 / 92$ & 33 & $\$ 23$ & & & & \\
\hline $3 / 9 / 92$ & 36 & 86 & & & & \\
\hline $4 \Pi / 92$ & 34 & 86 & & & & \\
\hline $5 / 5 / 92$ & 33 & 85 & & & & \\
\hline $6 / 1 / 92$ & 40 & 86 & & & & \\
\hline $6 / 29 / 92$ & 32 & 84 & & & & \\
\hline $7 / 27 / 92$ & 40 & 86 & $\therefore "$ & & & \\
\hline $8 / 25 / 92$ & 36 & 85 & $y_{1} \cdots$ & & & \\
\hline $9 / 21 / 92$ & 34 & 85 & & & & \\
\hline $1 \overline{0 / 20 / 92}$ & 34 & 21 & $5:$ & & & \\
\hline $11 / 17 / 92$ & 32 & 85 & 32 & & & \\
\hline
\end{tabular}

Data obtained from WHC Surveillance Data Acquisition (SOAl, November 30, 1993. 


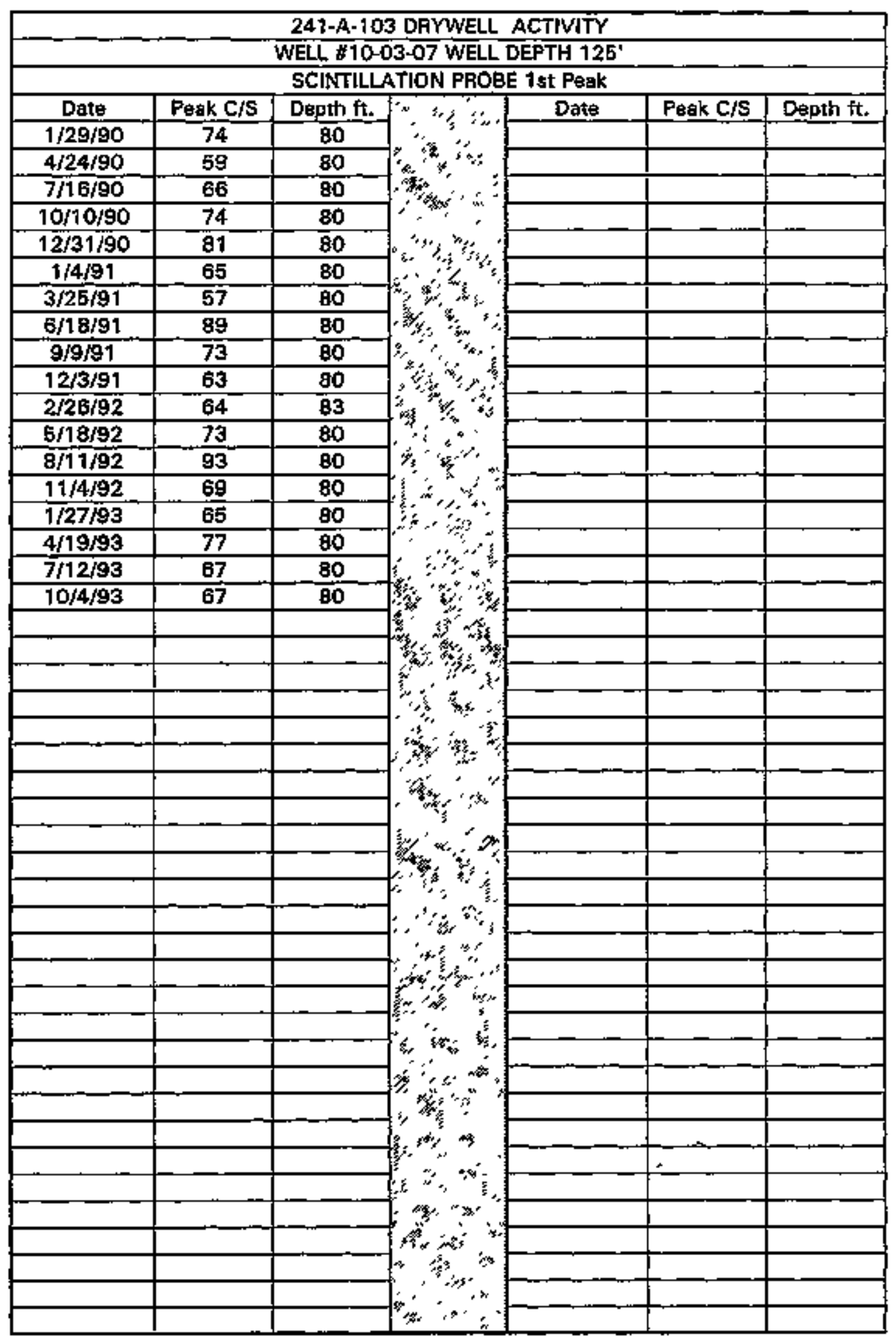

Data obtained from WHC Surveillance Data Acquitition (SDA), November 30, 1993. F-27 


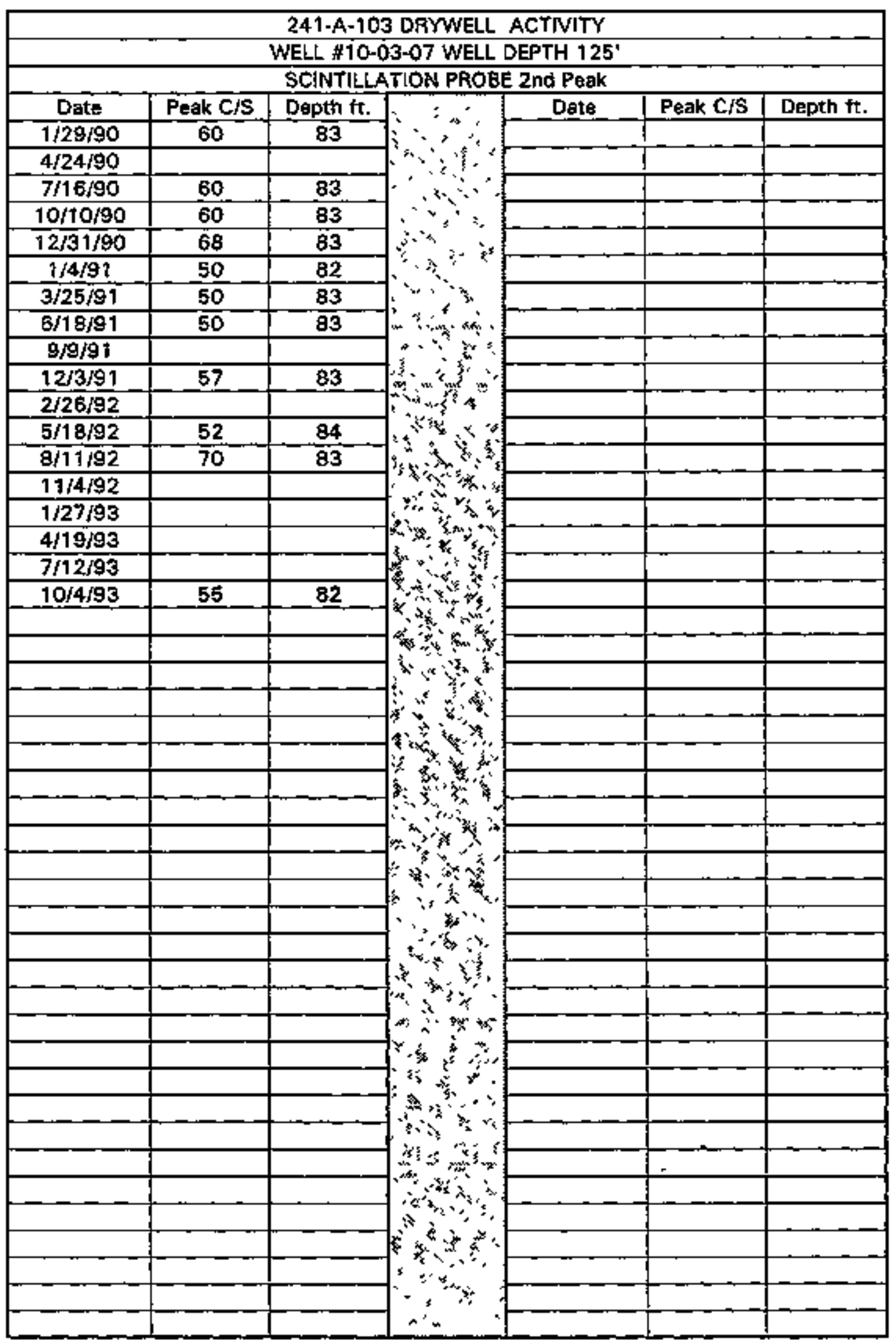

Data obtained from WHC Survejllance Data Acquisition (SDA), November 30, 1993. 


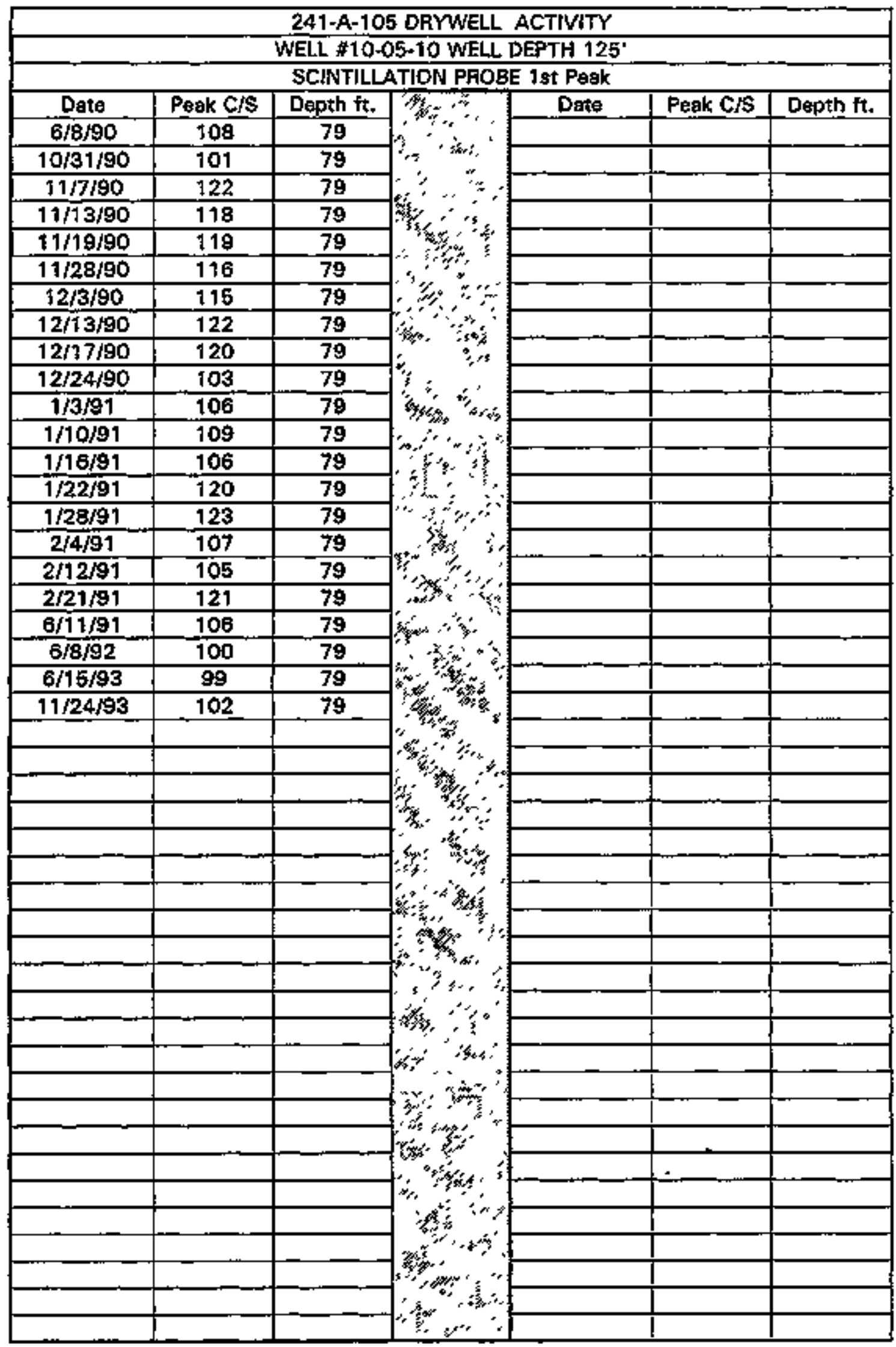

Date obtained from WHC surveiltance Data Acquisition (SDA), November 30, 1993. 


\begin{tabular}{|c|c|c|c|c|c|c|}
\hline \multicolumn{7}{|c|}{ 241-A-105 DRYWELL ACTIVITY } \\
\hline \multicolumn{7}{|c|}{ WELL $\$ 10-05-10$ WELL DEPTH $125^{\circ}$} \\
\hline \multicolumn{7}{|c|}{ SCINTILLATION PFOBE $2 \mathrm{nd}$ Pegk } \\
\hline Date & Peak C/S & Depth tt. & \multirow{3}{*}{ 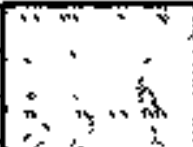 } & Date & Peak Crs & Depth ft. \\
\hline $6 / 8 / 90$ & & & & & & \\
\hline $10 \overline{31} \overline{190}$ & & & & & & \\
\hline $71 / 7 / 90$ & 70 & 84 & & & & \\
\hline $11 / 13 / 90$ & 80 & 84 & & & & \\
\hline $11 / 19 / 90$ & 60 & B4 & & & & \\
\hline $11 / 28 / 90$ & 65 & 84 & & & & \\
\hline $12 / 3 / 90$ & $\overline{70}$ & 84 & & & & \\
\hline $12 / 13 / 90$ & 70 & 84 & & & & \\
\hline $12 / 17 / 90$ & 62 & 84 & & & & \\
\hline $12 \overline{2} \overline{4 / 90}$ & 80 & 84 & & & & \\
\hline $1 / 3 / 91$ & 75 & 83 & & & & \\
\hline $1 / 10 / 91$ & 70 & 84 & & & & \\
\hline $1 / 16 / 91$ & $\overline{70}$ & $\overline{84}$ & & & & \\
\hline $1 / 22 / 91$ & 73 & 84 & & & & \\
\hline $1 / 28 / 91$ & 80 & 84 & & & & \\
\hline $2 / 4 / 91$ & 75 & 84 & & & & \\
\hline $2 / 12 / 91$ & $\ddot{6} 5$ & 84 & & & & \\
\hline $2 / 21 / 97$ & 78 & 84 & & & & \\
\hline $6 / 11 / 91$ & 80 & 84 & & & & \\
\hline $6 / 8 / 92$ & 85 & 84 & & & & \\
\hline $6 / 15 / 93$ & 60 & 84 & & & & \\
\hline $11 / 24 / 93$ & 65 & 64 & & & & \\
\hline & & & & & & \\
\hline & & & & & & \\
\hline & & & & & & \\
\hline & & & & & & \\
\hline & & & & & & \\
\hline & & & 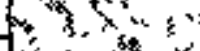 & & & \\
\hline & & & 13 & & & \\
\hline & & & & & & \\
\hline & & & $*$ & & & \\
\hline & & & & & & \\
\hline & & & & & & \\
\hline & & & & & & \\
\hline & & & ? 粱 & & & \\
\hline & & & $1=3$ & & & \\
\hline & & & & & & \\
\hline & & & & & & \\
\hline & & & $3 x_{s} x^{2}$ & & & \\
\hline & & & & & & \\
\hline & & & 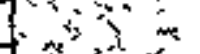 & & & \\
\hline & & & & & & \\
\hline & & & 10 & & & \\
\hline
\end{tabular}

Data obtained from WHC Survellance Data Acquistion (SDA), November 30, 1993. 


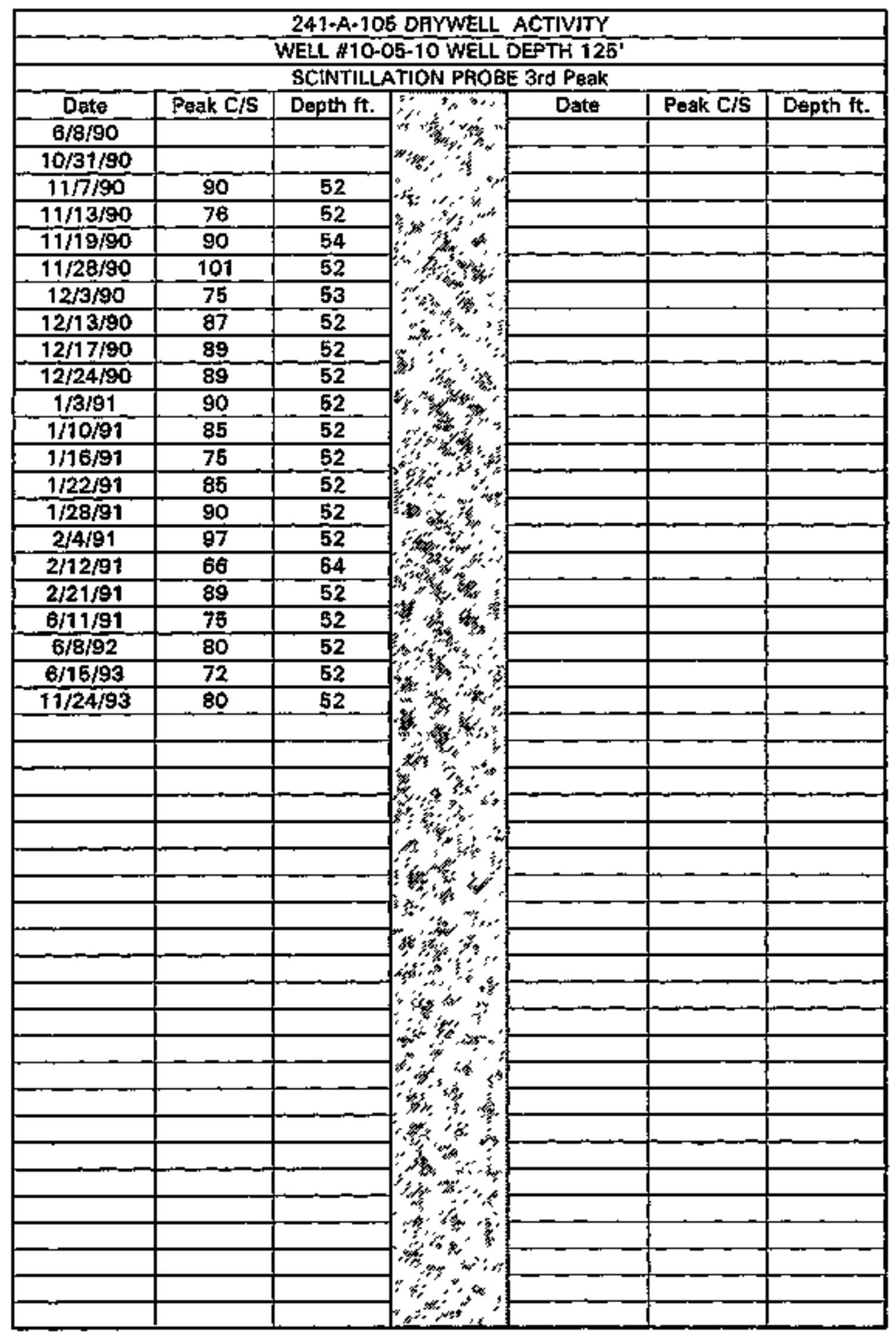

Data obtained from WHC Surveillance Data Acquisition (SDA), November \$D, 1993. 
WHC-SD-WM-ER-3OB， Rev , 0

THIS PAGE INTENTIONALLY LEFT BLANK

G-1 


\begin{tabular}{|c|c|c|}
\hline \multicolumn{3}{|r|}{$241-A-101$} \\
\hline No. & DIA. & DESCRIPTION AND COMMENTS \\
\hline 1 & $\overline{12 \pi}$ & AIR CIRCULATOR IIKE, CUT \& CAPPED \\
\hline 2 & 8" & EARLY THERMOCOUPLE, LEAD COVERED \\
\hline 3 & g" & DRYWELL WELDED TO BTM-HOT MOVAELE \\
\hline 4 & $1.2 "$ & SALTWELL SCREEN AND P-10 PUMP \\
\hline 5 & 4" & \\
\hline 6 & $\overline{4 "}$ & EIC, BM \\
\hline 7 & 4" & WC CRUST BRBARER \\
\hline 8 & 4" & (PIT DRAIN) \\
\hline 9 & $20 "$ & UNDERGROUND VENT LINE - BG \\
\hline 10 & 6" & SMP/FLANGE \\
\hline 11 & 4" & FROCESS FAST'E LINE - BG \\
\hline 12 & $4 "$ & TEMPERATYURE \\
\hline 13 & $42 "$ & SPARE \\
\hline 14 & 811 & TBX (INSIDE INSTRUMBNT BNCIOSURE) \\
\hline 15 & 4" & LIQUID LEVBL WELL "A" \\
\hline$\overline{16}$ & 181 & SPARE /OBSRV PORT (12* SPOOL) \\
\hline 17 & $18 "$ & WC \\
\hline 18 & $18^{n}$ & WC \\
\hline 19 & $4 "$ & $B-436$ LOW \\
\hline 20 & 4" & BREATHER FILTER, BM \\
\hline 21 & $12 \pi$ & $\overline{\mathrm{WC}}$ \\
\hline$\overline{22}$ & $12^{\prime \prime}$ & WC \\
\hline
\end{tabular}


WHC-SD-WA-ER-308, Rev. O

$241-A-101$

3.790,000 Liters

[1,000,000 Gailons]

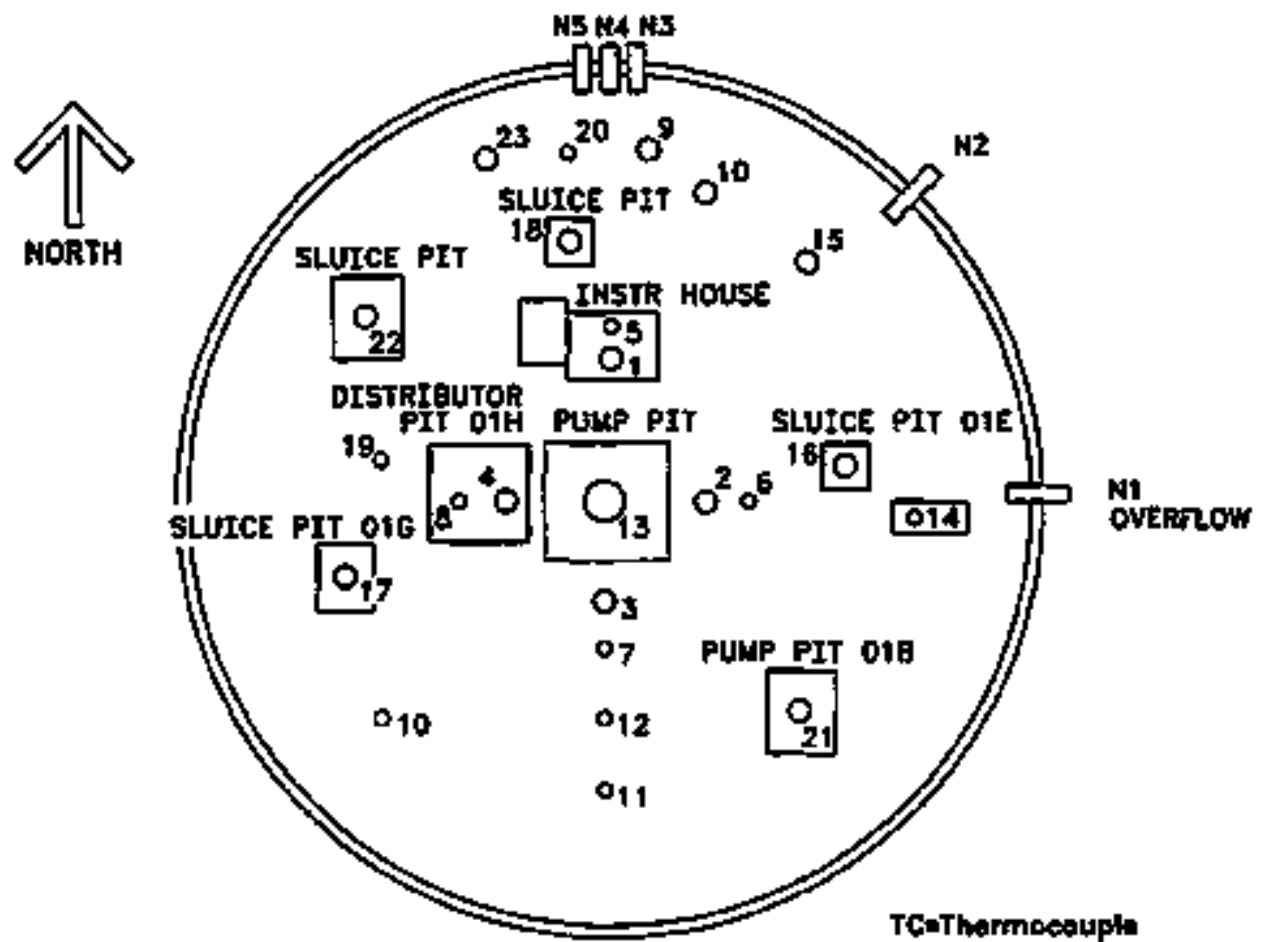

Ref: whe-50-RE-TI-053, Rav. 8 Whe-50-WM-TI-553, Rov. O 1+2-73390. Rew, $Z$

\section{TANK RISER LOCATION}

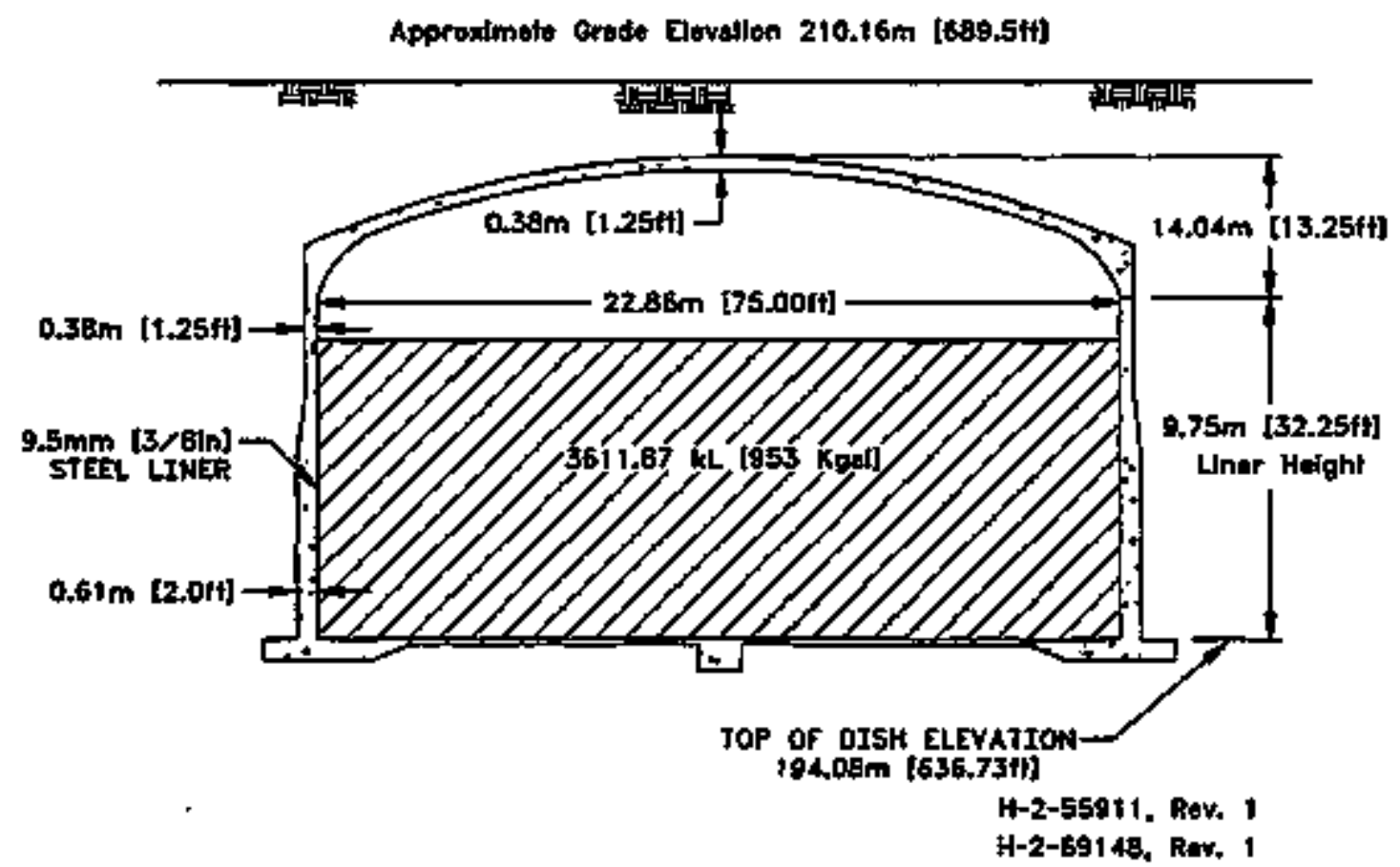


WHC-SO-WM-ER-308, REV, O

\begin{tabular}{|c|c|c|}
\hline \multicolumn{3}{|r|}{ 241-A-102 } \\
\hline No. & DIA. & DESCRIPTION AND COMMENTS \\
\hline 1 & $12^{\pi t}$ & AIR CIRCULATOR LINES \\
\hline 2 & 8" & FLANGSE, BM \\
\hline 3 & $8^{\prime \prime}$ & DRYWELL \\
\hline 4 & $12^{\prime \prime}$ & SUR, PUNP, FC \\
\hline 5 & $4 "$ & LIQUID IEVEL REEL \\
\hline$\overline{6}$ & $4 "$ & FIC, BM \\
\hline 7 & $4 "$ & TEMPERATURE \\
\hline$\overline{8}$ & $4 "$ & (PIT DRAIN) WC \\
\hline 9 & $20^{\pi}$ & U,G. VENT LINE - BG \\
\hline 10 & 4" & DRAIN - BG \\
\hline 11 & 4" & BG \\
\hline 13 & $42^{*}$ & $\overline{\mathrm{WC}}$ \\
\hline 14 & $6 "$ & DRAIN BG \\
\hline 15 & $6 " 11$ & $\mathrm{BG}$ \\
\hline 16 & $10 "$ & DRAIN BG \\
\hline 17 & $10 "$ & DRAIN BG \\
\hline 18 & $4 "$ & BREATHER FIZTER \\
\hline 19 & $12 "$ & B-222 OBSRV PORT, BM \\
\hline 22 & $12 "$ & WC \\
\hline 23 & $12 "$ & WC \\
\hline
\end{tabular}




\section{1-A-102}

3.790,000 Liters

[1,000,000 Gallons]

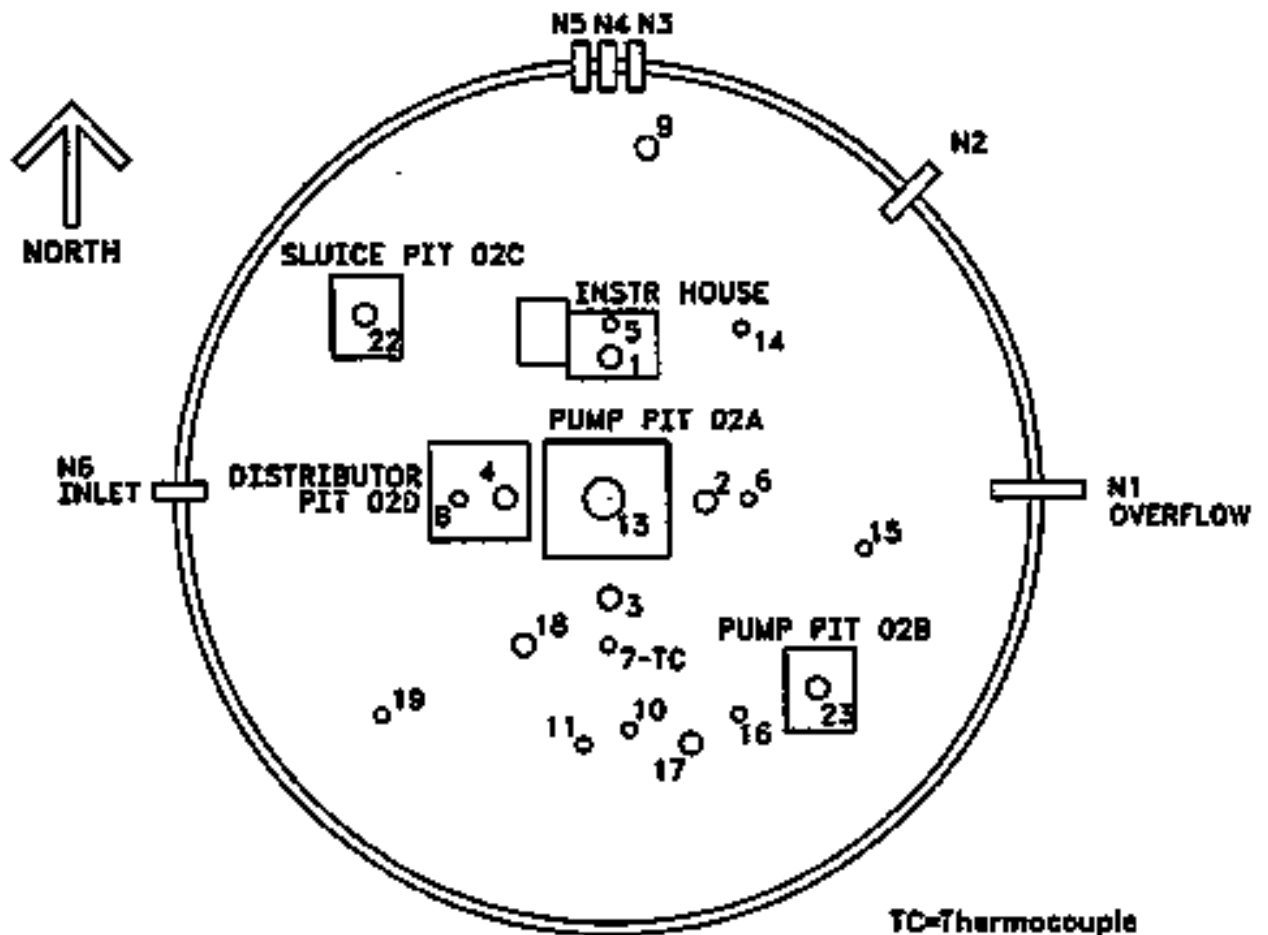

TC-Thurmotouple

Rol: WHC-SD-RE-TI-0S3, Rav, s WHC-50-WW-T1-553, Rew, 0

H-2-73390, Rav. 2

\section{TANK RISER LOCATION}

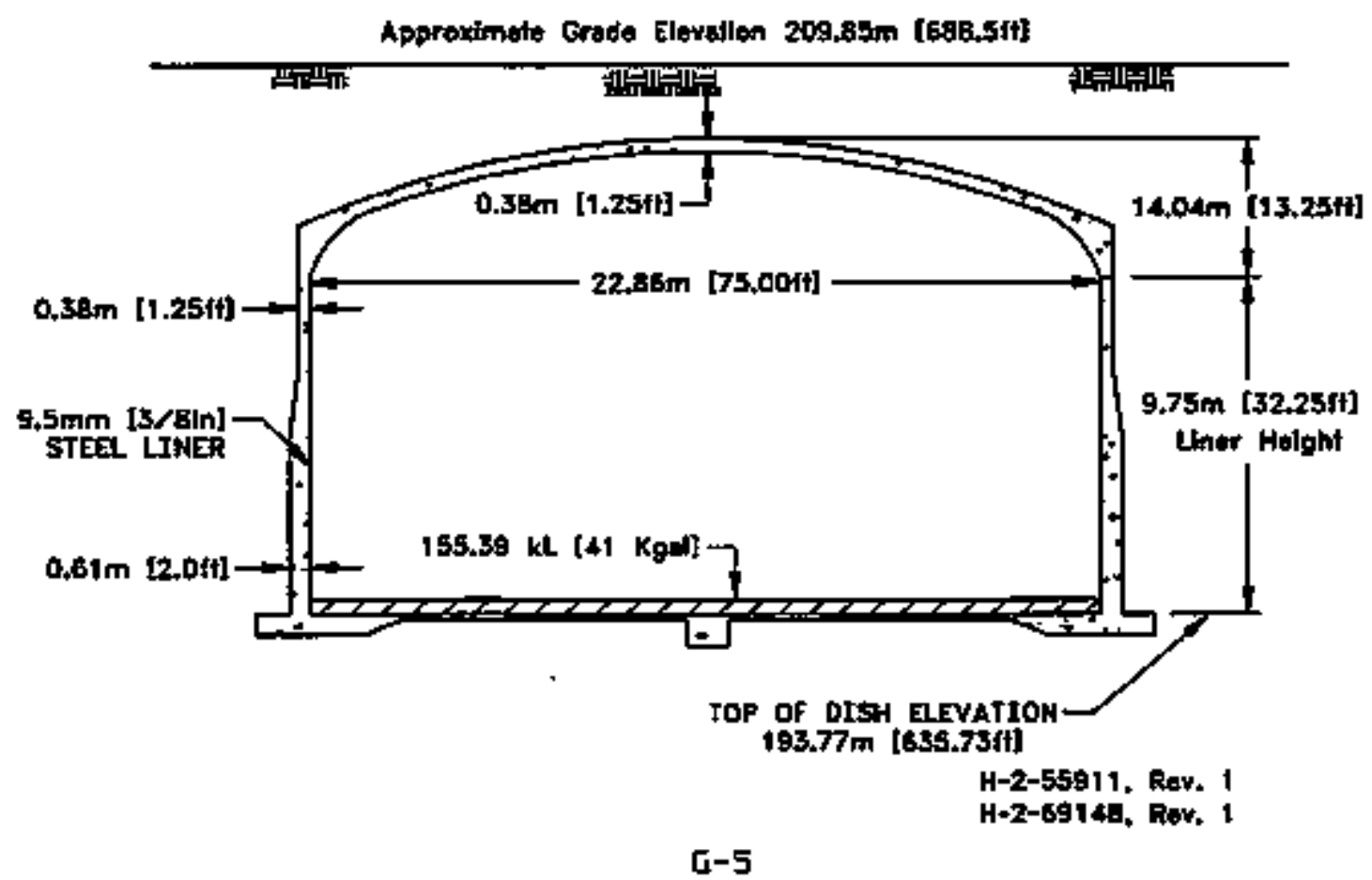




\begin{tabular}{|c|c|c|}
\hline \multicolumn{3}{|r|}{ 241-A-103 } \\
\hline No. & DIA. & DESCRIPTION AND COMMENTS \\
\hline 1 & $12^{\pi}$ & AIR CIRCOLATOR LINES \\
\hline$\overline{2}$ & $8^{n}$ & LB, BM \\
\hline 3 & $8 "$ & DRYWELL \\
\hline 4 & $12^{*}$ & (SLURRY DISTRIBUTOR) \\
\hline 5 & $4 "$ & FLANGE \\
\hline 6 & $4 \mathbf{4}^{n}$ & FIC \\
\hline 7 & 4" & WC \\
\hline 8 & 4" & (PIT DRAIN) \\
\hline 9 & $20^{\pi}$ & VAPOR MANIEOLD - BG \\
\hline 10 & $4 "$ & PROCBSS WASTE LINE - BG \\
\hline 11 & $8 "$ & LEAD CAP (BLANGE) \\
\hline 12 & $12^{n}$ & B-222 OBSRV PORT \\
\hline 13 & 42" & \\
\hline 14 & $12^{n}$ & WC, PUMP \\
\hline 15 & $4^{\text {tt }}$ & TEMPERATURE \\
\hline 16 & $22^{\circ}$ & WC \\
\hline 17 & $4^{4}$ & BRBATHER FILTER \\
\hline 19 & $4^{n}$ & B-436 IOW, BM \\
\hline 20 & & WC \\
\hline
\end{tabular}




\section{$241-A-103$}

$3,790,000$ Liters

[1,000,000 Galllons]
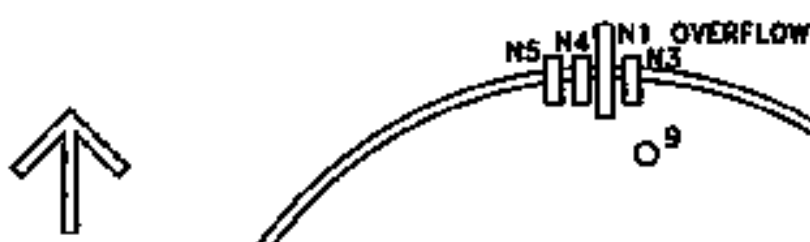

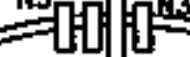

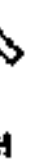

16

$\int$

Pump Pit 03C

Q

Oistributar

PH 03

$\underbrace{\sum_{0}^{0}}_{011} \underbrace{O_{12}}_{0_{7}}$

010

Pume Pit ozs

$0^{10} 0^{\circ}$

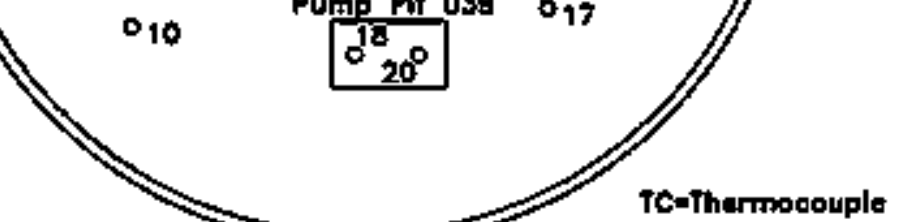

Roft WHC-5D-RE-TI-053, Rw. $B$ WhC-5D-Wu-tr-583, fiev, 0 H-2-75392, Rov. 4

\section{TANK RISER LOCATION}

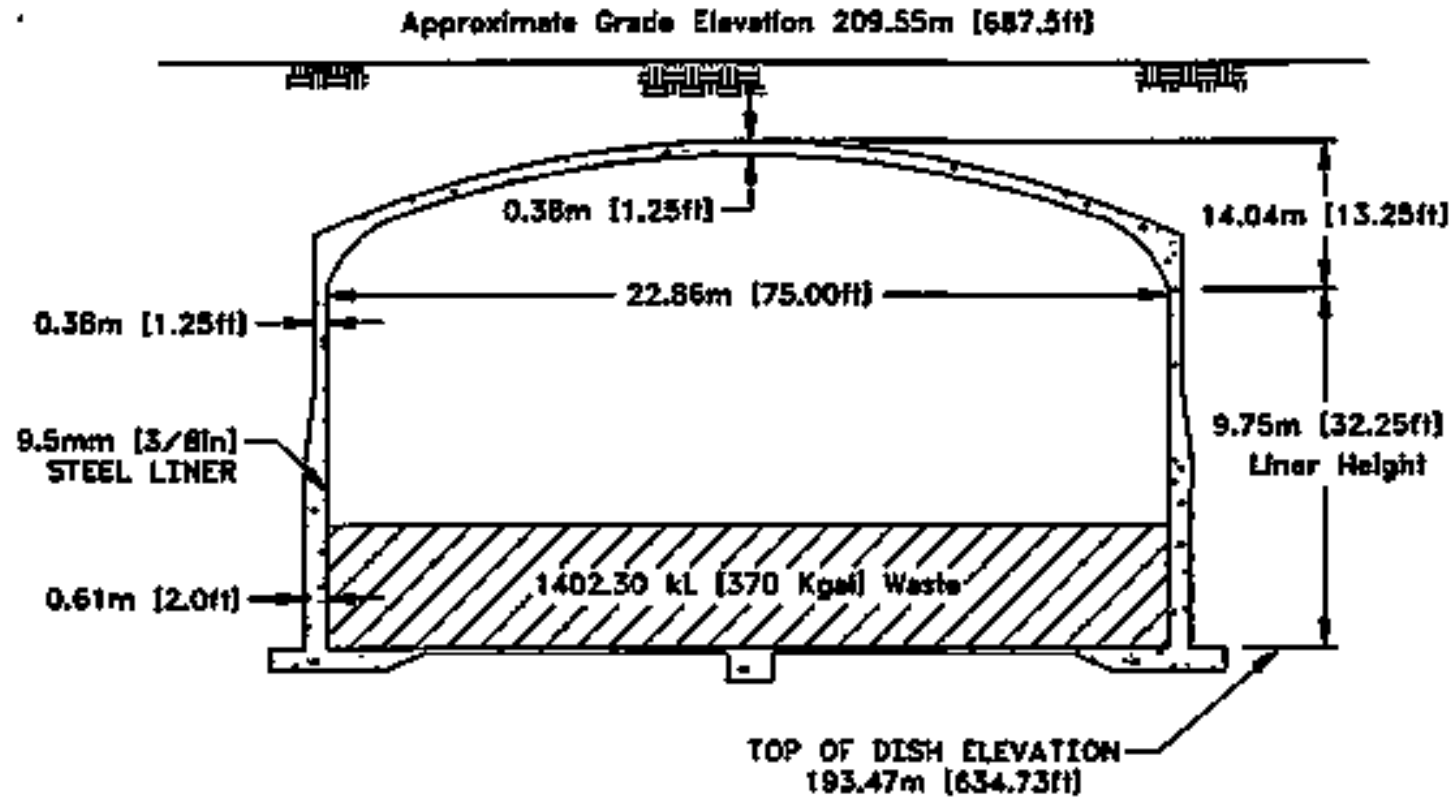

H-2-55911. Rov. I

H-2-6914B, Rar, 1 


\begin{tabular}{|c|c|c|}
\hline \multicolumn{3}{|r|}{ 241-A-104 } \\
\hline No. & DIA. & DESCRIPTION AND COMMENTS \\
\hline 1 & $8^{\prime \prime}$ & DRYWELL \\
\hline 2 & $8 "$ & IB \\
\hline 3 & $\overline{12 \pi}$ & AIR CIRCULATOR IINES \\
\hline 4 & $12^{\prime \prime}$ & FLANGE/B-222 OBSRV PORT \\
\hline 5 & 4" & HC - CRUST BREAKER \\
\hline 6 & 411 & BM， FLANGE IIQUID LEVEL REEL \\
\hline 7 & $4 "$ & BLANGE, LEAD CAP \\
\hline 8 & 4" & WC \\
\hline 9 & $20^{\prime \prime}$ & VAPOR MANIFOLD - BG \\
\hline 10 & 2.511 & SMP \\
\hline 11 & $6^{\prime \prime}$ & PLANGE， BM， ZIP CORD \\
\hline 12 & $2.5^{\prime \prime}$ & TEMPERATURE \\
\hline 13 & $42^{n}$ & PUMP WC \\
\hline 14 & $4 n$ & BRBATHER FILTER \\
\hline 15 & $4^{n}$ & PROCESS WRSTR LINE - BG \\
\hline 17 & $12^{\prime \prime}$ & FLANGE \\
\hline 18 & $4^{n}$ & TEMPERATURB \\
\hline 20 & $2.5^{n}$ & SMP \\
\hline 21 & $12^{n}$ & WC \\
\hline 22 & $12 "$ & (SLUICBR) WC \\
\hline 26 & $8 "$ & $\mathrm{TBX}$ \\
\hline
\end{tabular}




\section{1-A-104 \\ $3,790,000$ Liters \\ [1,000,000 Gaillons]}
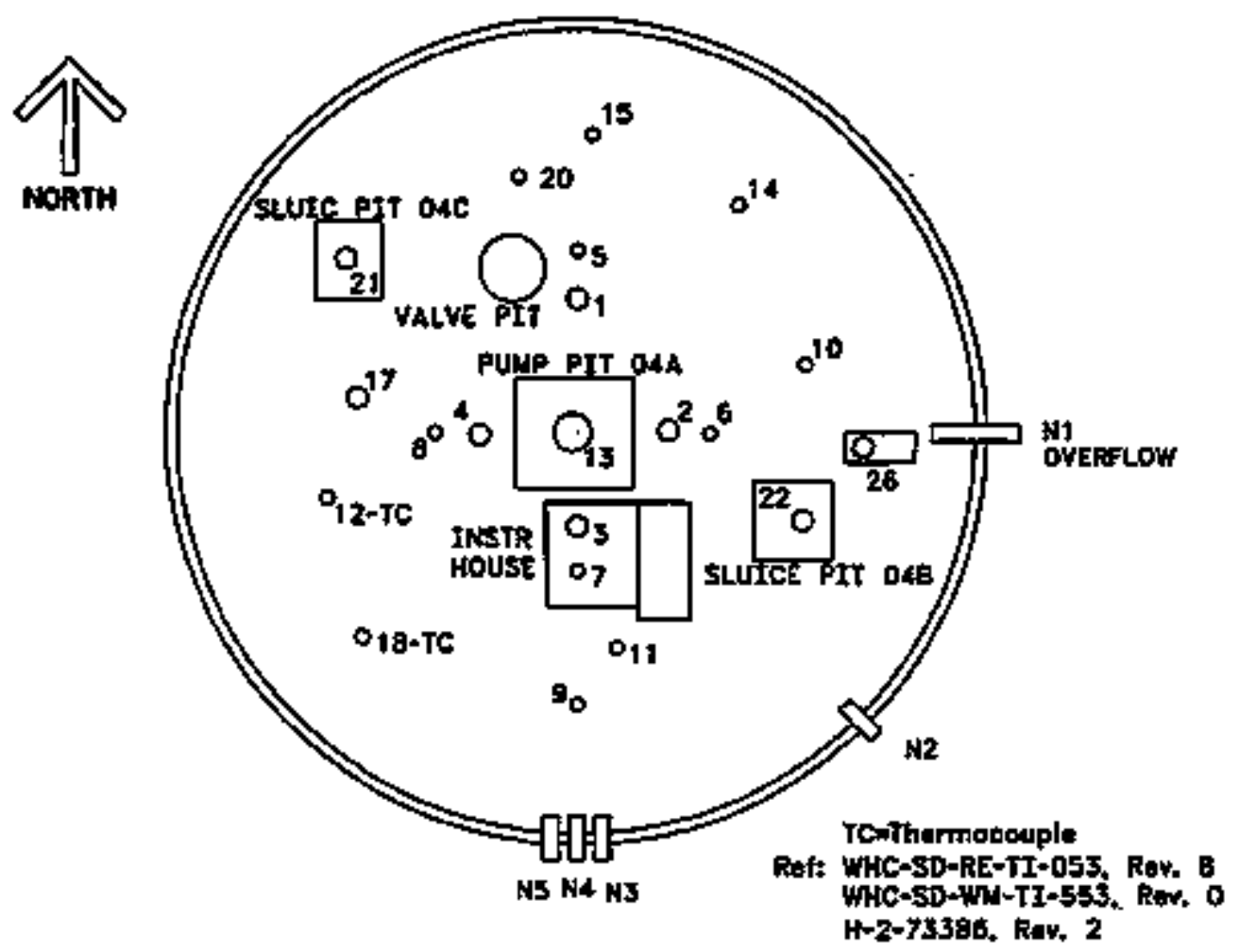

TANK RISER LOCATION

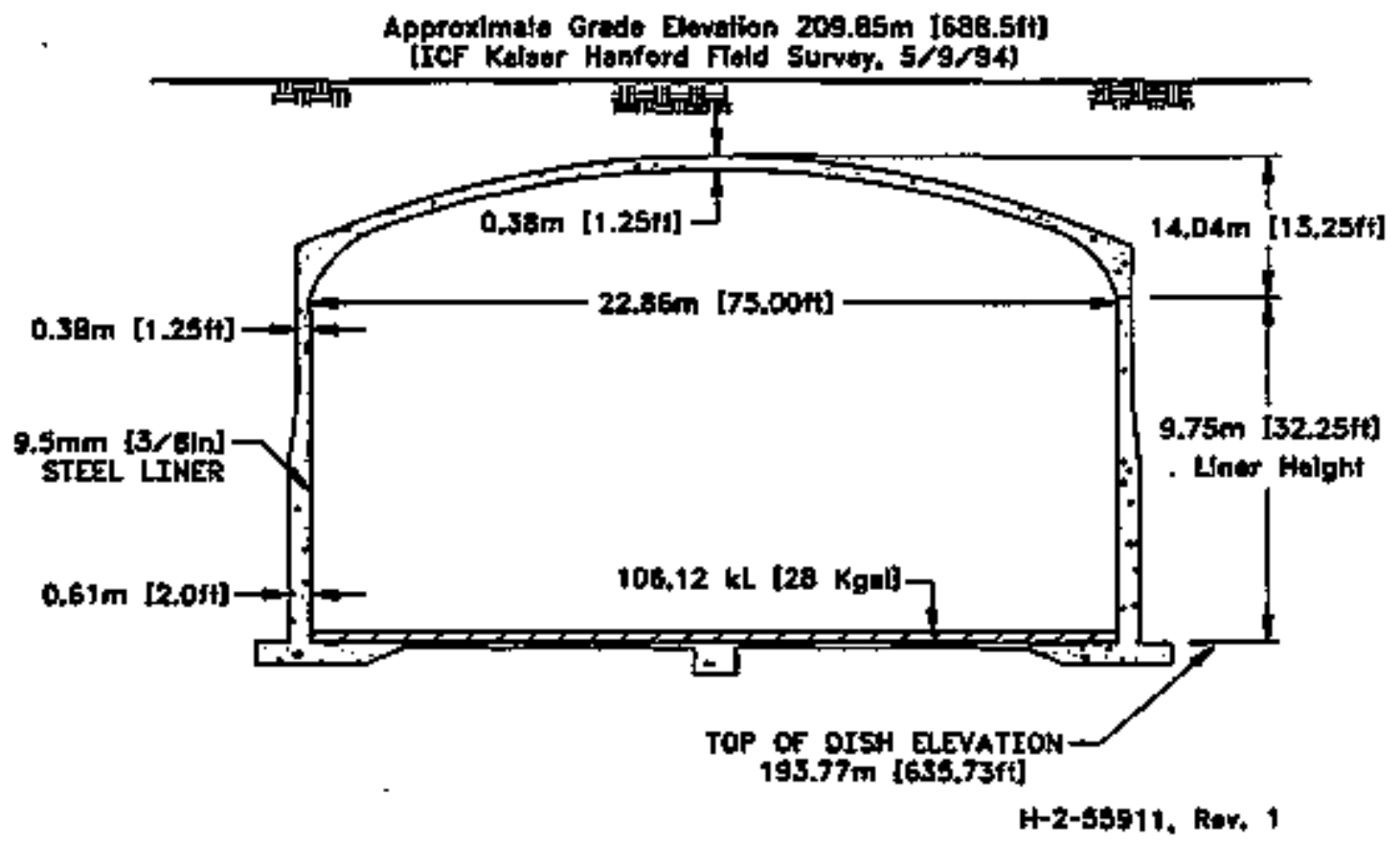




\begin{tabular}{|c|c|c|}
\hline \multicolumn{3}{|r|}{$241-A-105$} \\
\hline No. & DIA. & DESCRIPTION AND COMMENTS \\
\hline 1 & 8" & DRYWELL \\
\hline$\overline{2}$ & $8 "$ & EXHAUSTER PORT \\
\hline 3 & $12 "$ & AIR CIRCULAATOR \\
\hline 4 & $12 "$ & LEAD COVERED/B-222 OBSRV PORT \\
\hline 5 & $4 "$ & IIQUUID LEVEL REEL \\
\hline $\bar{\epsilon}$ & 4" & FLANGE - EXHAUST CONDENSATE, BM \\
\hline 7 & $4^{\text {th }}$ & BLIND FLANGE \\
\hline $\mathrm{g}$ & 4" & PRESSURE GAUGE, FLANGE \\
\hline 9 & $8^{\prime \prime}$ & TEMPERATURE WC AT BASE \\
\hline 10 & $22 "$ & VAPOR MANIFOLD - BG \\
\hline 31 & $4 "$ & PROCESS WASTE LINE - BG \\
\hline 13 & $42 \pi$ & (POMP) WC \\
\hline 14 & $4^{\pi}$ & FLANGE \\
\hline 15 & $4 \pi$ & TEMAPERATURE, FC AT BASE \\
\hline 16 & $4 "$ & TEMPERATORE, WC AT BASB \\
\hline 17 & $4^{n}$ & TEMPERATURE， WC AT BASE \\
\hline 18 & 411 & FILANGE \\
\hline 19 & 4" & TEMYERATURE, WC AT FLANGE \\
\hline 20 & $4 \pi$ & SPRINKLER, FALNGE \\
\hline 21 & $4 "$ & AIR INLET FILTER \\
\hline 22 & 4" & TEMPERATURE WC AT BASE \\
\hline 23 & $12 "$ & (SLUICER - NOT MOVABLE) WC \\
\hline 24 & $12 "$ & (SLUICER - WOT MOVABLE) WCC \\
\hline 25 & $12 "$ & (PUMP) WC \\
\hline 27 & $4 "$ & TBX - BG \\
\hline
\end{tabular}




\section{1-A-105 \\ $3,790,000$ Liters \\ [1,000,000 Gallons]}

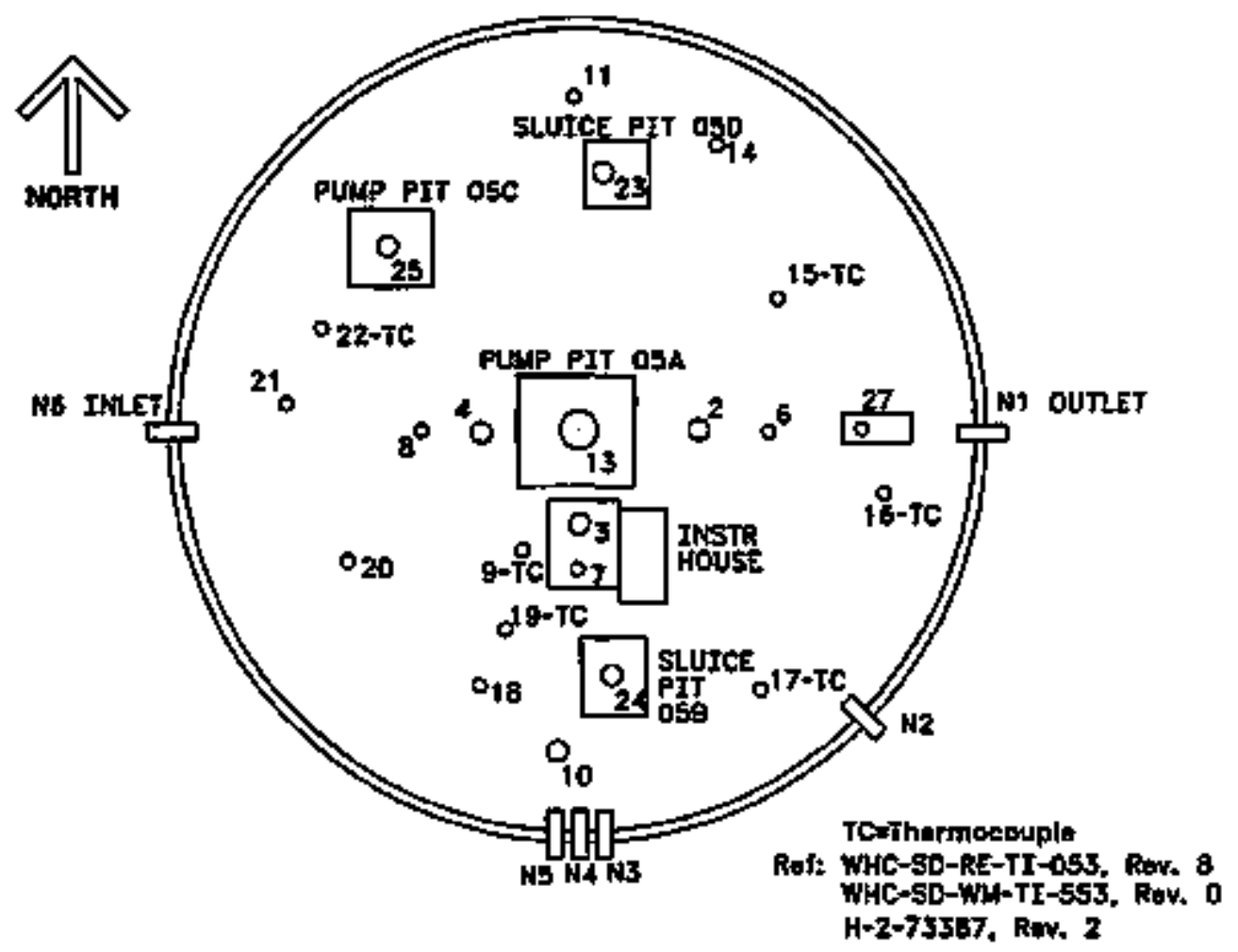

TANK RISER LOCATION

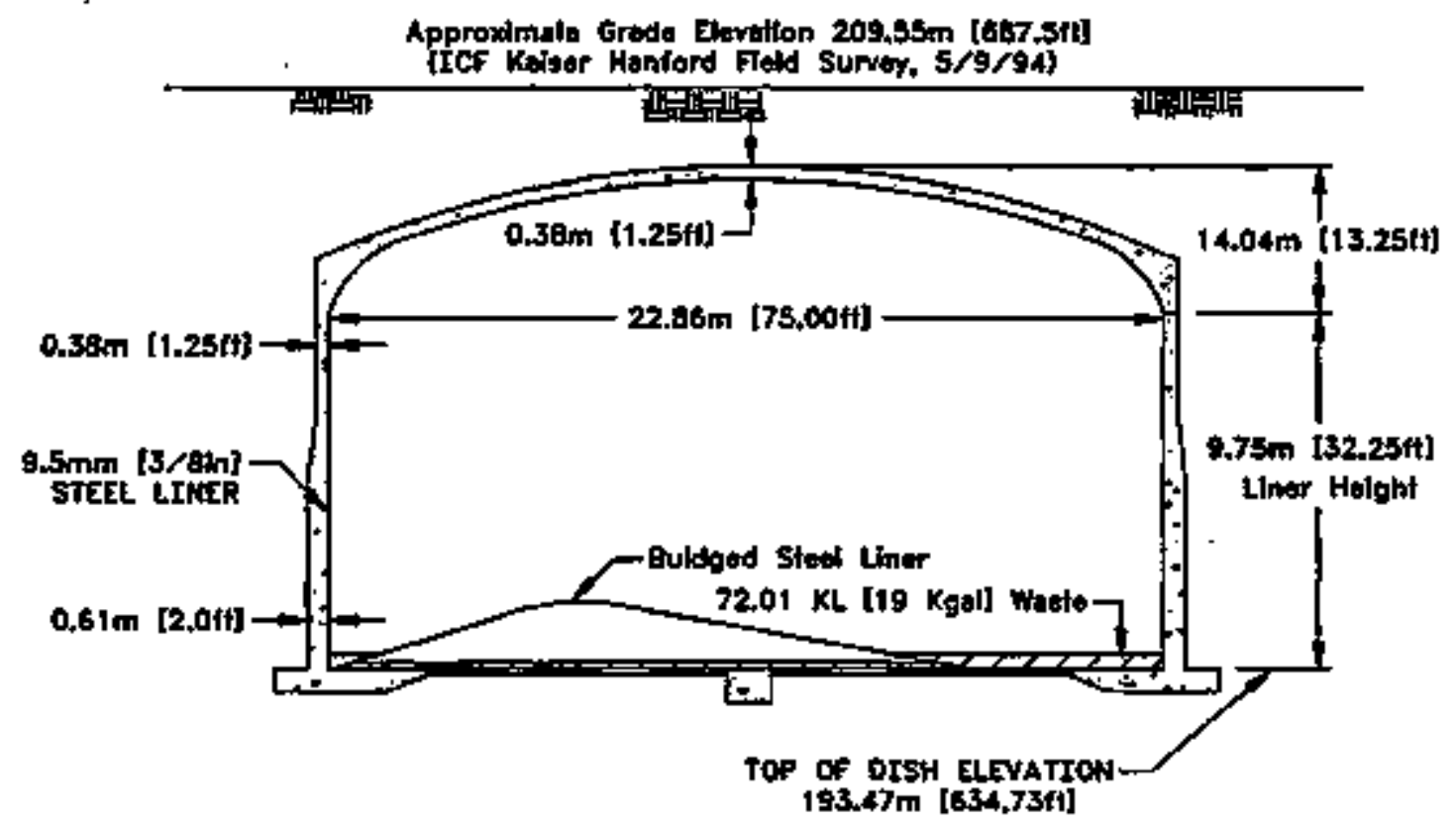

H-2-55911. Rov. 1 


\begin{tabular}{|c|c|c|}
\hline \multicolumn{3}{|r|}{ 241-A-106 } \\
\hline No. & DIA. & DESCRIPTION AND COMMEITS \\
\hline 1 & 8 8" & DRYWELA， BM \\
\hline 2 & 8" & LB \\
\hline 3 & $12 "$ & AIR CIRCULATOR LINES \\
\hline 4 & $12 "$ & FI_ANGE WC \\
\hline 5 & 41 & HC - CRUST BREAKER \\
\hline 6 & 4" & FIC, BM \\
\hline 7 & 4" & FLANGE (LEAD CAP) \\
\hline 8 & 4 & (RIT DRAIN) WC \\
\hline 9 & $20 "$ & U.G. VENT LINE - BG \\
\hline 10 & $6 "$ & SMP \\
\hline 11 & $6 "$ & SMP, BM， PRESSURE GAUGE \\
\hline 12 & 6" & SMP \\
\hline 13 & $42^{t 1}$ & (POMM) WC \\
\hline 14 & $4^{\pi}$ & TEMPERATURE \\
\hline 15 & $6^{n}$ & WT BACTOR DIP TUBE, WC \\
\hline 16 & $42 "$ & PUMP, WC \\
\hline 17 & $12^{\prime \prime}$ & FLANGE/B-222 OBSRV PORT \\
\hline 18 & 4" & BREATEER FILTER \\
\hline 20 & $6^{4}$ & FLANGB \\
\hline 21 & $12 "$ & (\$LUICE PUMP) \\
\hline 22 & $12^{\pi t}$ & (SLUICER) WC \\
\hline
\end{tabular}




\section{1-A-106}

$3,790,000$ Liters

[1,000,000 Galilons]

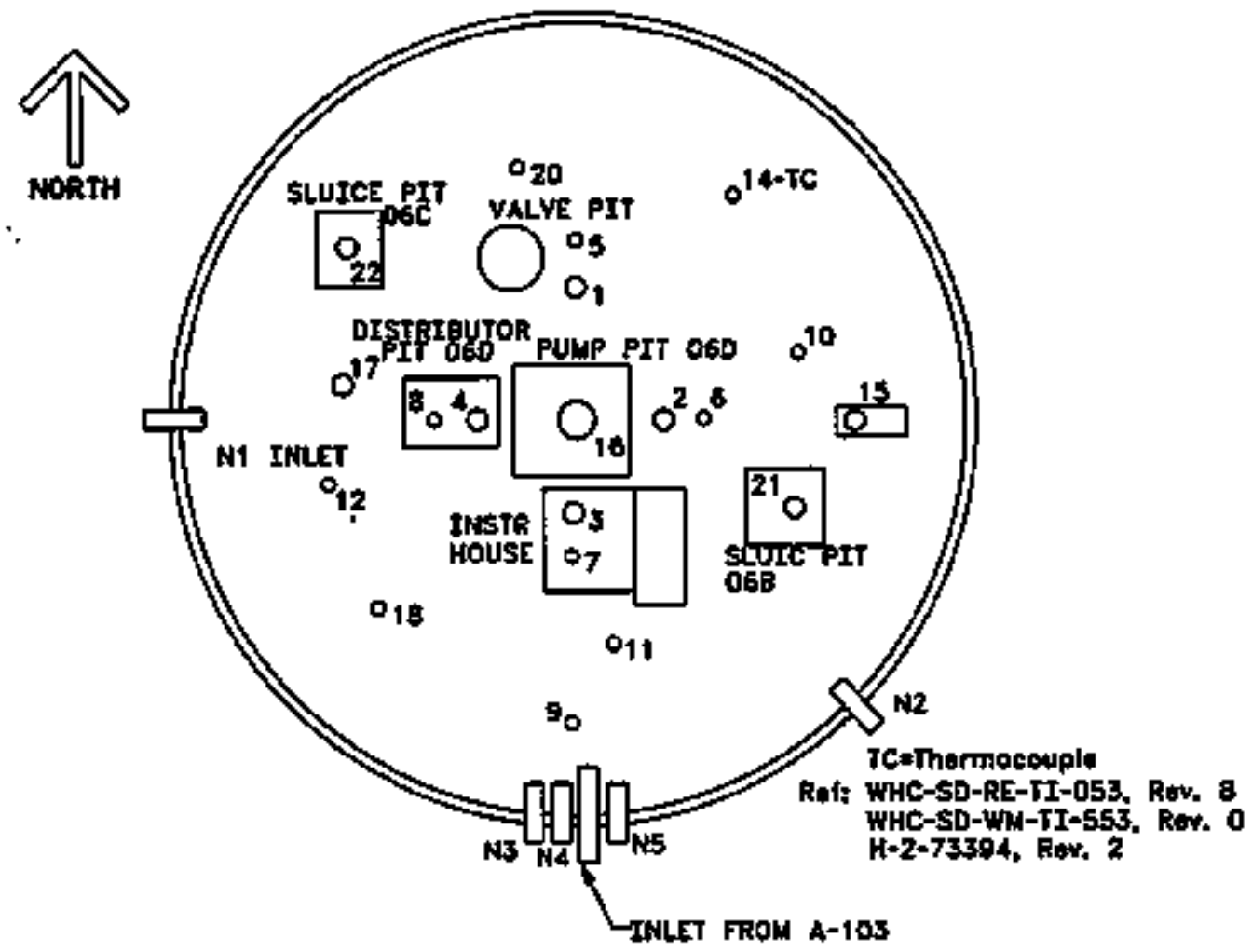

\section{TANK RISER LOCATION}

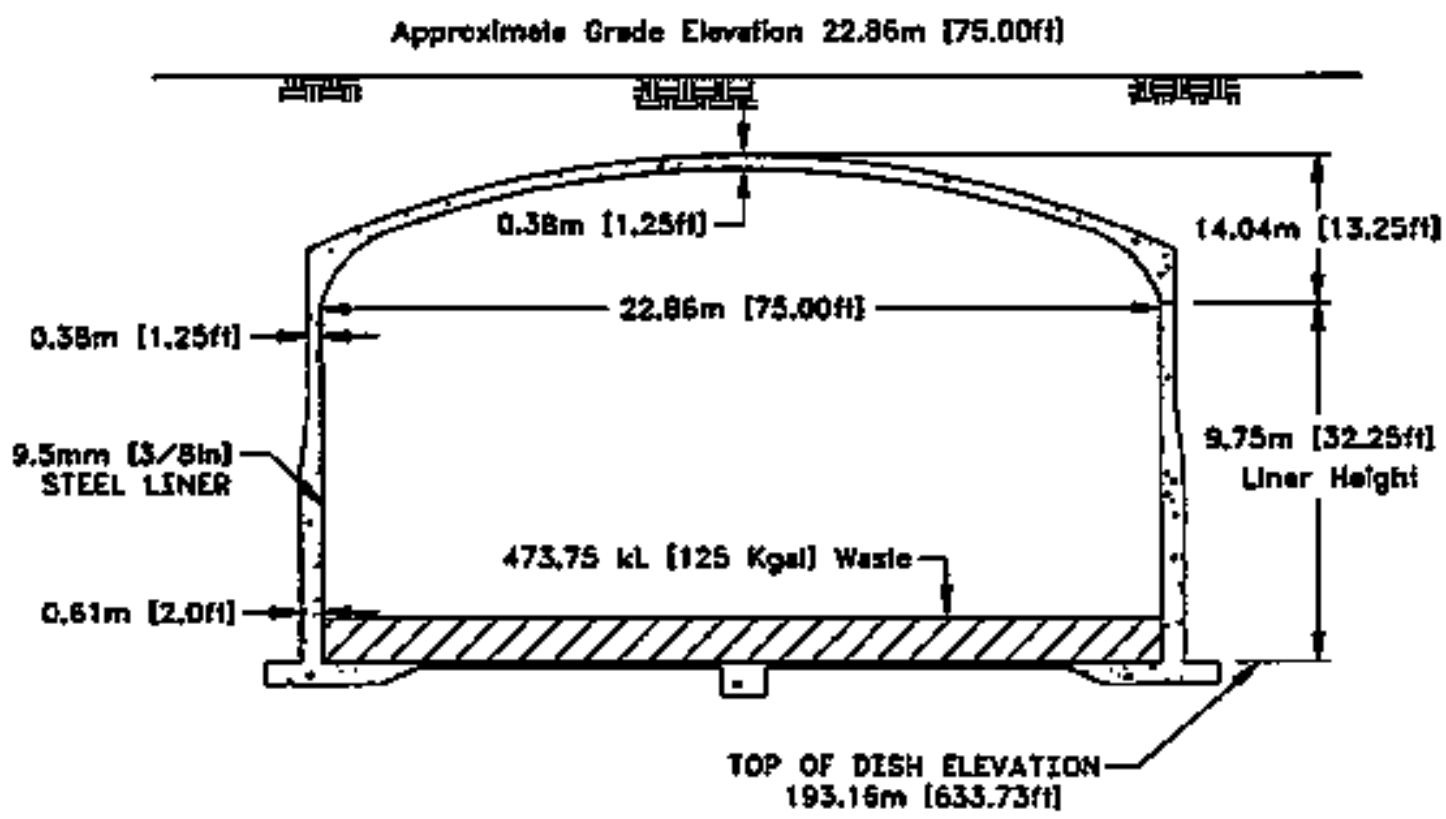

H-z-55911. Rav. 1 H-2-691 4B, Rev. 1 


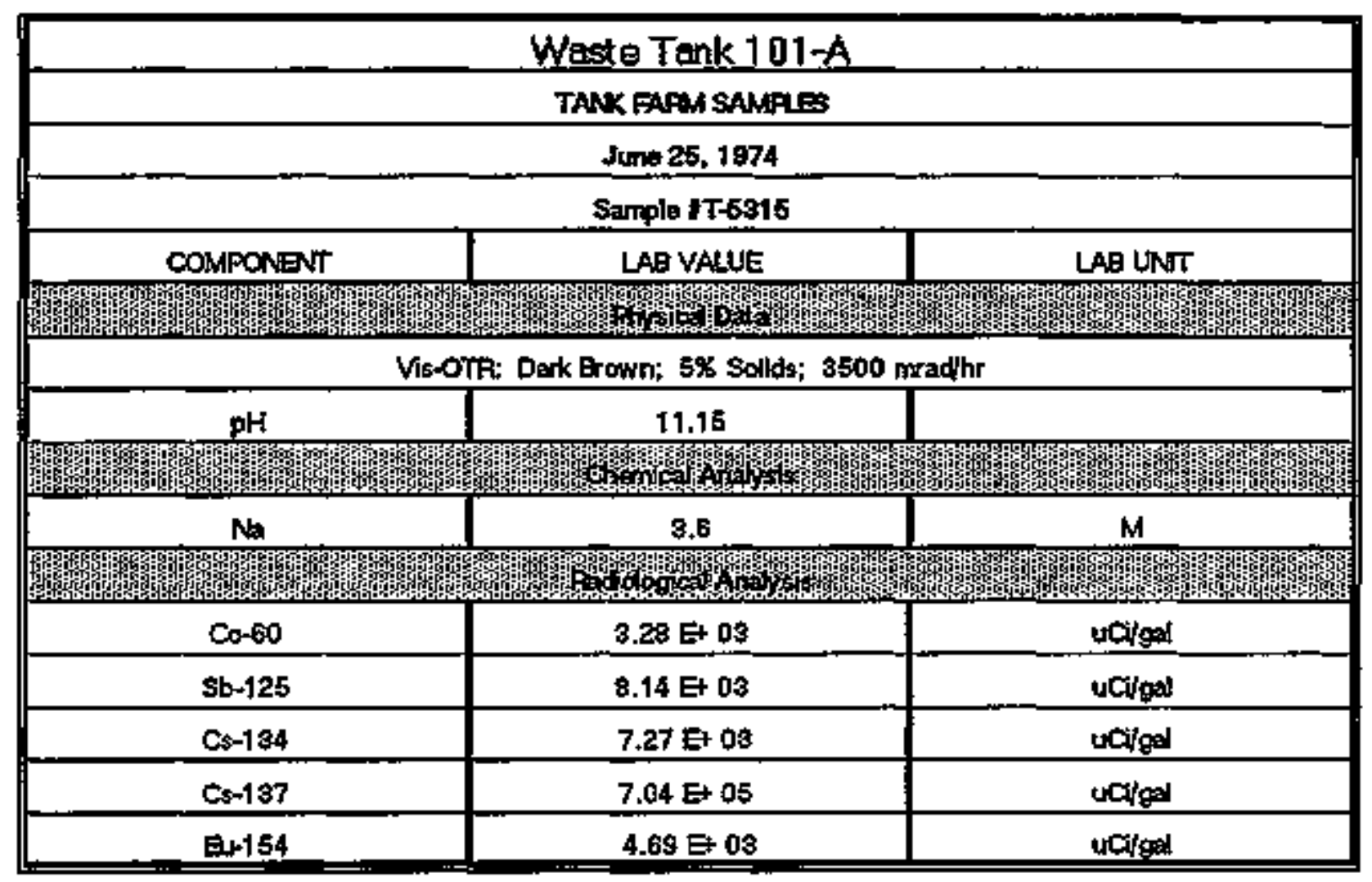




\begin{tabular}{|c|c|c|}
\hline \multicolumn{3}{|c|}{ Weste Tenk 101-A } \\
\hline \multicolumn{3}{|c|}{ ANALYSIS OFTANK FAPM SAMFES } \\
\hline \multicolumn{3}{|c|}{ SAMPE I T-8996 } \\
\hline \multicolumn{3}{|c|}{ Dectimber 17.1974 } \\
\hline \multicolumn{3}{|c|}{ Peoulyod: Octobur 14, 1974} \\
\hline COMPONENT & LAB VALLE & LAB UNVT \\
\hline \multicolumn{3}{|c|}{ 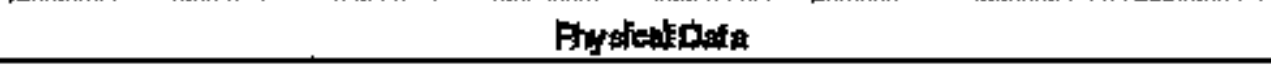 } \\
\hline \multicolumn{3}{|c|}{ Vts-OTR: Fooddish Brown; 5 Padthr } \\
\hline $\mathrm{pH}$ & $>9.7$ & \\
\hline $\mathrm{SpG}$ & 1.289 & \\
\hline $\mathrm{H} 2 \mathrm{O}$ & 69.31 & $\%$ \\
\hline \multicolumn{3}{|c|}{ Chontical Anatysth } \\
\hline OH & 0.526 & $M$ \\
\hline Al & $2.52 E-02$ & $\mathrm{M}$ \\
\hline $\mathrm{Na}$ & 2,4 & $\mathrm{M}$ \\
\hline NO2 & 0.252 & $M$ \\
\hline NOS & 2.86 & $M$ \\
\hline PO4 & $1.72 \mathrm{E} 02$ & $M$ \\
\hline $\mathbf{F}$ & $1.65 \mathrm{E}-0.9$ & $M$ \\
\hline $\cos$ & 0.402 & $M$ \\
\hline $\mathbf{N}-4$ & < 7,155-03 & $M$ \\
\hline \multicolumn{3}{|c|}{ Fuctiologinal Arthosis. } \\
\hline Pu & $1,65 \mathrm{E}-02$ & $\mathrm{gm} / \mathrm{geal}$ \\
\hline $\operatorname{cs}-134$ & $2.98 \mathrm{E}+03$ & uClgal \\
\hline$C \$-1 \% 7$ & 2.77E. 05 & بC flogal \\
\hline $5 r-89,90$ & $0.99 E \div 05$ & uolgal \\
\hline
\end{tabular}




\begin{tabular}{|c|c|c|}
\hline \multicolumn{3}{|c|}{ Wasie Tank 101-A } \\
\hline \multicolumn{3}{|c|}{ ANALYSIS OF I01-A SLUDGE } \\
\hline \multicolumn{3}{|c|}{ April 9.1976 } \\
\hline \multicolumn{3}{|c|}{ Sample recelved: Aptil 1, 1976 } \\
\hline \multicolumn{3}{|c|}{ SAMFL 18991} \\
\hline COMPONENT & LAB VALUE & LAB UNIT \\
\hline 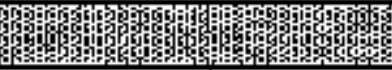 & 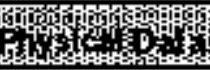 & 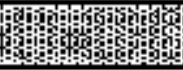 \\
\hline \multicolumn{3}{|c|}{ 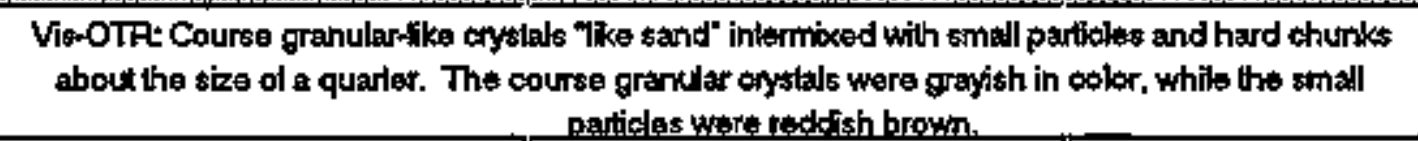 } \\
\hline$\% \mathrm{H} 2 \mathrm{O}$ & 9.10 & $\%$ \\
\hline \multicolumn{3}{|c|}{ 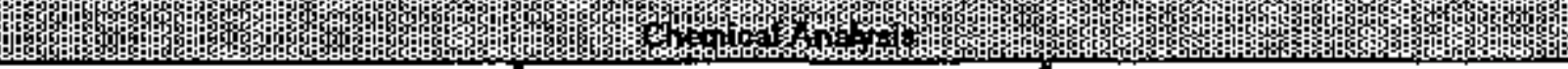 } \\
\hline Al & $\$ .95$ & moles/liter \\
\hline $\mathrm{Fe}$ & 0.5 & mol|msliter \\
\hline si & 3.95 & moles lltter \\
\hline \multicolumn{3}{|c|}{ 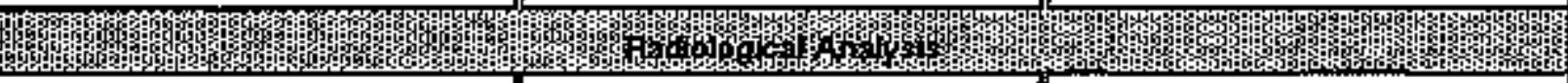 } \\
\hline $89+90 \mathrm{Sr}$ & 1.85 E+ 06 & uciliter \\
\hline $137 \mathrm{Cs}$ & $2.68 \mathrm{E}+05$ & uCiliter \\
\hline
\end{tabular}


WHC-SD-WM-ER-308, Rev, 0

\begin{tabular}{|c|c|c|c|c|c|c|}
\hline \multicolumn{7}{|c|}{ Waste Tank 101-A } \\
\hline \multicolumn{7}{|c|}{ ANALYSTS OF RESIDUAL SWDEES FNM TANK 101-A } \\
\hline \multicolumn{7}{|c|}{ April \$0, 1978} \\
\hline DOMPONENT & & & AB VALUE & & & LAB UNT \\
\hline \multicolumn{7}{|c|}{ 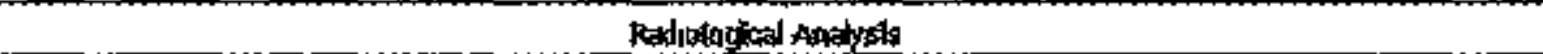 } \\
\hline $\begin{array}{c}\text { SOURCE SAMFLE } \\
\text { WUNEER }\end{array}$ & $\begin{array}{l}\text { REF } \\
\text { LETTER } \\
\text { SOO.1 }\end{array}$ & $\begin{array}{l}R 18 N \\
48 \% 5\end{array}$ & $\underset{1 / 865}{\text { SLUICE PIT }}$ & $\begin{array}{l}\text { R19 } \\
4554\end{array}$ & $\begin{array}{l}R 20 \\
45656\end{array}$ & \\
\hline $80+805 r$ & 186 & 20 & 23 & 69 & 156 & $\mathrm{CH}$ \\
\hline $137 \mathrm{Cs}$ & 022 & 031 & 095 & 0.22 & 026 & Coll \\
\hline
\end{tabular}




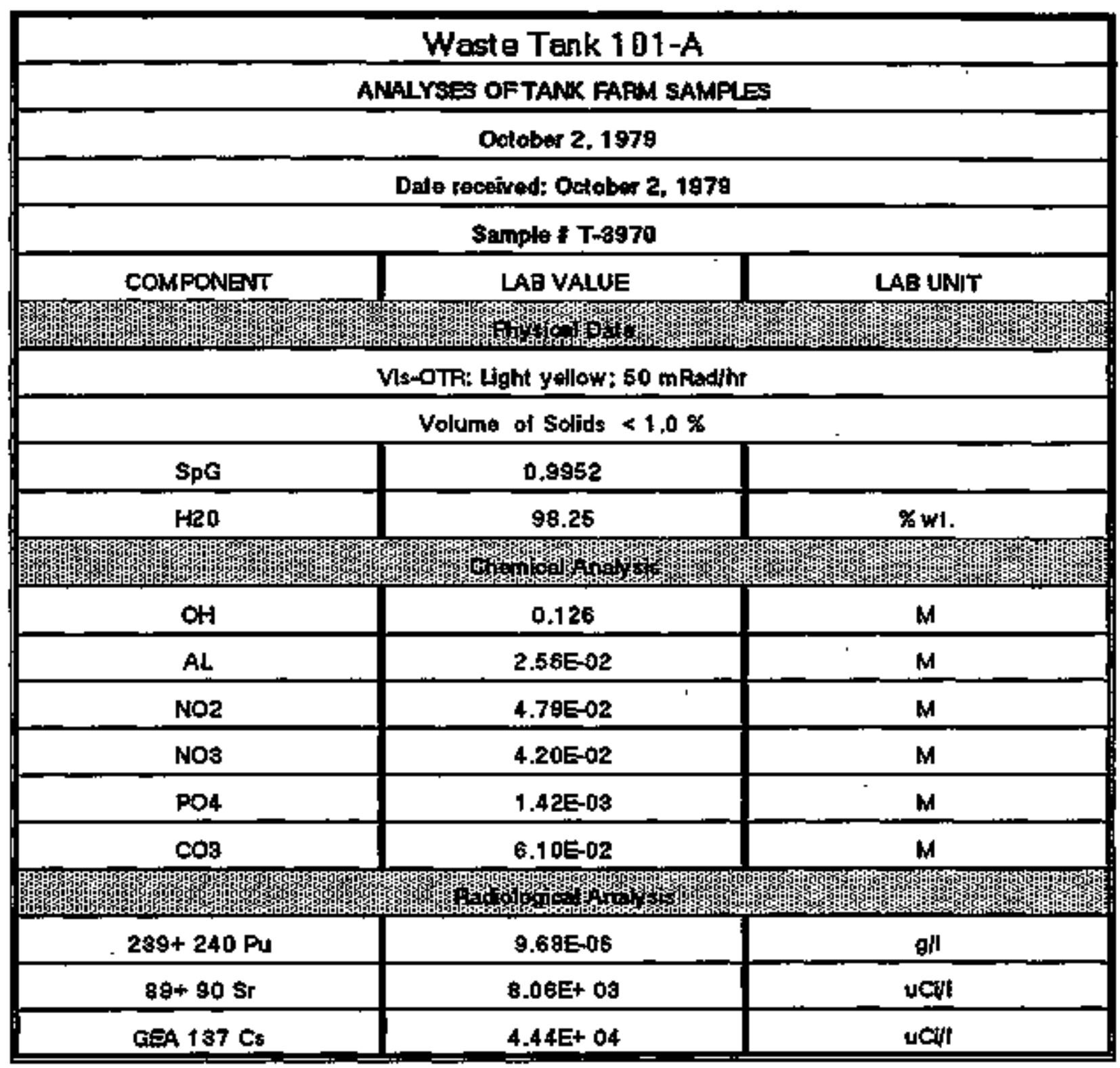




\begin{tabular}{|c|c|c|}
\hline \multicolumn{3}{|c|}{ Waste Tank $101-A$} \\
\hline \multicolumn{3}{|c|}{ ANALYSES OF TANK FAPM SAMPLES } \\
\hline \multicolumn{3}{|c|}{ Oefober 3,1979} \\
\hline \multicolumn{3}{|c|}{ Date received Odtober 3.1979 } \\
\hline \multicolumn{3}{|c|}{ Samplo T-9971 } \\
\hline COMPONENT & LAB VALUEE & LAB UNIT \\
\hline \multicolumn{3}{|c|}{ Faysyles: Dala } \\
\hline \multicolumn{3}{|c|}{ Vis-OTR: Light goen; a00 mRad/hr } \\
\hline \multicolumn{3}{|c|}{ Volume of Solids $<5.0 \%$} \\
\hline SpG & 1.052 & \\
\hline $\mathrm{H} 20$ & 98.7 & \% w1. \\
\hline \multicolumn{3}{|c|}{ Whemical Aralizheis } \\
\hline $\mathrm{OH}$ & 0.518 & $\mathbf{M}$ \\
\hline AL & 0.274 & $M$ \\
\hline $\mathrm{NO} 2$ & $7.72 \mathrm{E}-02$ & $M$ \\
\hline NOS & 0.421 & $M$ \\
\hline PO4 & 2.12E-09 & $M$ \\
\hline $\cos$ & $1.00 \mathrm{E}-00$ & $M$ \\
\hline \multicolumn{3}{|c|}{ 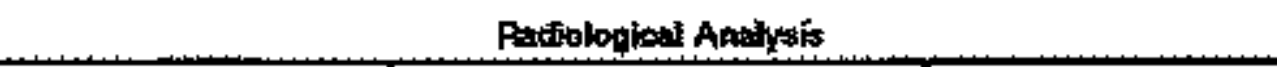 } \\
\hline $239+240 \mathrm{Fu}$ & $1.27 E-05$ & git \\
\hline $89+90 \mathrm{Sr}$ & 1.59 다 04 & uan \\
\hline GEA $137 \mathrm{Cs}$ & $2.08 \mathrm{E}+05$ & uCtr \\
\hline
\end{tabular}




\begin{tabular}{|c|c|c|c|c|}
\hline \multicolumn{5}{|c|}{ Waste Tank 101-A } \\
\hline \multicolumn{5}{|c|}{ ANALYSES OFFEID AND FPODUCT SLUFAY } \\
\hline \multicolumn{5}{|c|}{ November 2, 1979} \\
\hline \multicolumn{5}{|c|}{ thom } \\
\hline \multirow[t]{2}{*}{ COMPONENT } & $\begin{array}{l}\text { PEES } \\
\text { MOLABSTY }\end{array}$ & \multicolumn{2}{|c|}{ PRODUCT LOUOA } & $\begin{array}{c}\text { PRODUCT SOUD WEIGHT } \\
\text { PERCENT }\end{array}$ \\
\hline & & MOLAFTY & $\begin{array}{l}\text { WaGHT } \\
\text { PERCENT }\end{array}$ & \\
\hline SpG (d/m) & 1.052 & 1.978 & & \\
\hline & 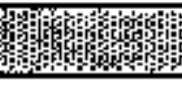 & 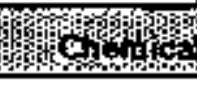 & 4 & 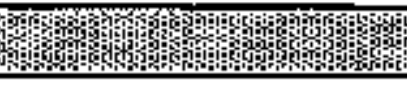 \\
\hline \multirow[t]{2}{*}{ COMPONENT } & $\begin{array}{l}\text { FEED } \\
\text { MOLAPTYY }\end{array}$ & \multicolumn{2}{|c|}{ PRODUCT HIOUOR } & PRODUCT SOUD WEIGHT \\
\hline & & MOLAFUTY & $\begin{array}{l}\text { WEISHT } \\
\text { PERCENT }\end{array}$ & \\
\hline NaAlO2 & 0.274 & 1.26 & 7.5 & o \\
\hline $\mathrm{NaOH}$ & 0.618 & 2.31 & 6.7 & $\mathbf{0}$ \\
\hline NaNo2 & 0.0772 & 1.56 & 7.82 & 104.5 \\
\hline NaNDS & 0.421 & 2.27 & 14 & $\mathbf{0}$ \\
\hline $\mathrm{Na2} C \mathrm{O}(\mathrm{H} 2 \mathrm{O})$ & 0.061 & 0.75 & 6.75 & $\mathbf{0}$ \\
\hline NasPQ $4(12 \mathrm{H} 2 \mathrm{O})$ & 0.02117 & 0.037 & 0.99 & 0 \\
\hline TOC (gll) & & 20 & 1.45 & 0 \\
\hline $\mathrm{HzO}$ (w/s) & 96.7 & & 50.25 & 0 \\
\hline TOTAL & & & $\$ 5.45$ & 104.5 \\
\hline & & - Total Or & io Carbon & \\
\hline
\end{tabular}


WHC-SD-WM-ER-30B, Rev. 0

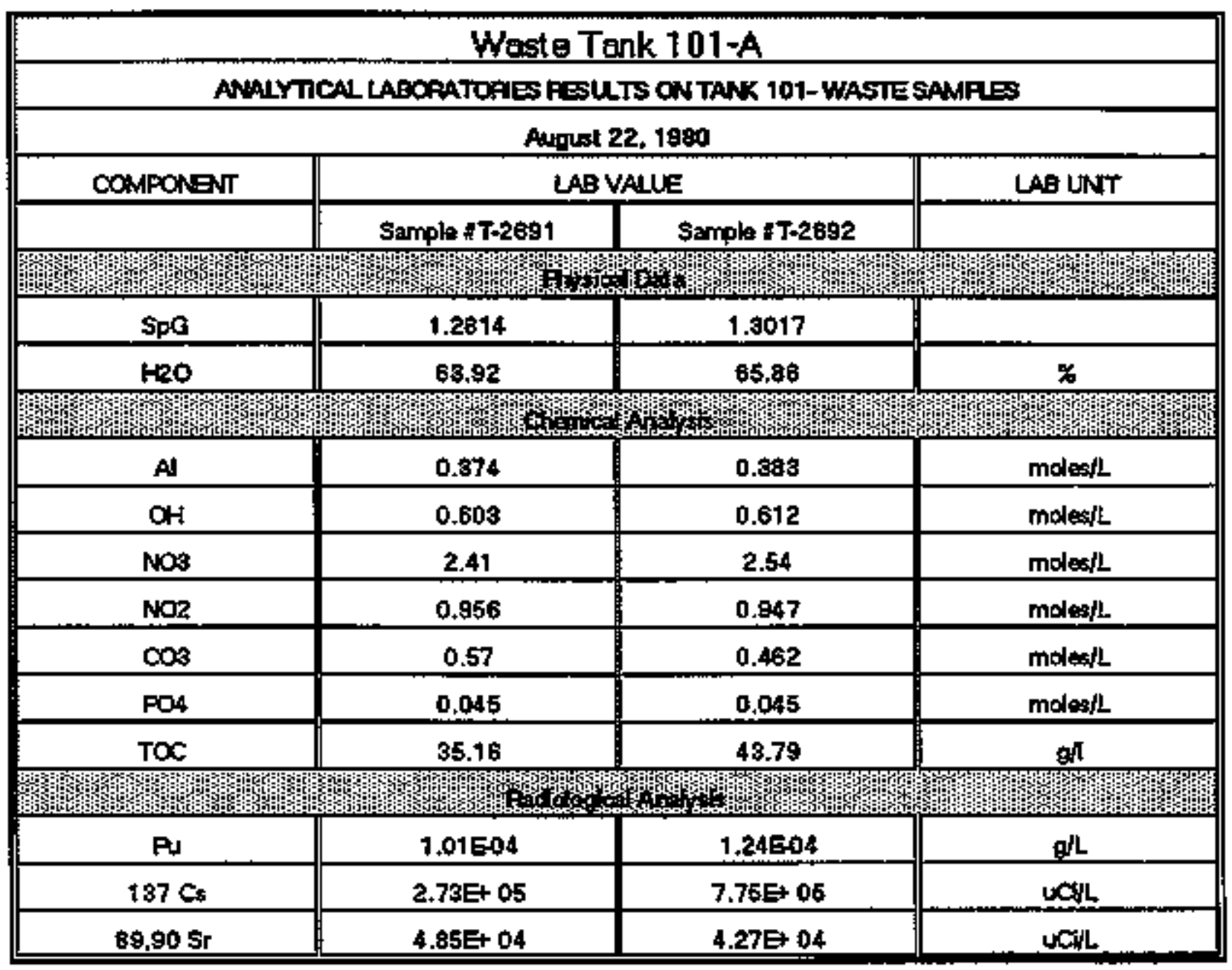


WHC-SD-WM-ER-309, Rev. 0

\begin{tabular}{|c|c|c|c|}
\hline \multicolumn{4}{|c|}{ Weste Tank 101-A } \\
\hline \multicolumn{4}{|c|}{$\begin{array}{l}\text { COMPOSITION OF TANK 101-A WASTE } \\
\text { NNEAR SURFACE SAMPLA) }\end{array}$} \\
\hline \multicolumn{4}{|c|}{ Siptember 22, 1990} \\
\hline \multicolumn{4}{|c|}{ SAMFE 14218} \\
\hline \multirow[t]{2}{*}{ COMPONDNT } & \multicolumn{2}{|c|}{ SUFENATANT } & souos \\
\hline & MOLAFTY & W.\% & Wh.\% \\
\hline & 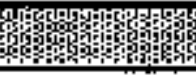 & 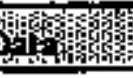 & 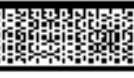 \\
\hline Spg & \multicolumn{2}{|c|}{1.344} & \\
\hline 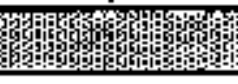 & \multicolumn{2}{|c|}{ 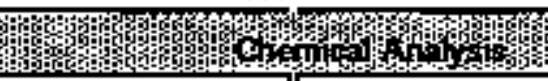 } & \\
\hline Al & 1.364 & 8.32 & $\mathbf{a}$ \\
\hline $\mathrm{OH}$ & 3.06 & 9.11 & 0 \\
\hline NO2 & 2.04 & 10.47 & b \\
\hline NOS & 2.72 & 17.2 & D \\
\hline $\cos$ & 0.98 & 7.72 & 68 \\
\hline PO4 & 0.016 & 0.2 & 9 \\
\hline Toc & $16.24 \mathrm{~g} / \mathrm{l}$ & 3.59 & 23 \\
\hline$\% \mathrm{H} 2 \mathrm{O}$ & & 50.56 & \\
\hline TOTAL & & 107.17 & 100 \\
\hline
\end{tabular}


WHC-SD-WM-ER-308，Rev. 0

\begin{tabular}{|c|c|c|c|}
\hline \multicolumn{4}{|c|}{ Waste Tank $101-A$} \\
\hline \multicolumn{4}{|c|}{$\begin{array}{c}\text { COMPOSITON OF TANK JGI-A WASTE } \\
\text { MNEAB SLLUDGE SAMPLE }\end{array}$} \\
\hline \multicolumn{4}{|c|}{ Suptamber 22, 1980} \\
\hline \multicolumn{4}{|c|}{ SAMPE $\$ 4378$} \\
\hline \multirow[t]{2}{*}{ COMPONENT } & \multicolumn{2}{|c|}{ SUPGNATANT } & SOLIDS \\
\hline & MOLAPITY & Wh. & W..\% \\
\hline \multicolumn{4}{|c|}{ Plopstor Doth } \\
\hline SpG & \multicolumn{2}{|c|}{1.144} & \\
\hline \multicolumn{4}{|c|}{ Chemical Dota } \\
\hline Al & 1.758 & 12.8 & 0 \\
\hline $\mathrm{OH}$ & $\$ .16$ & 11.01 & 0 \\
\hline NO2 & 2.565 & 15.47 & 0 \\
\hline NOS & 2.290 & 17.07 & 45 \\
\hline $\cos$ & 0.156 & 1.45 & 45 \\
\hline PO4 & 0.33 & 4.73 & 0 \\
\hline Toc & $10.14 \mathrm{~g} / \mathrm{L}$ & 2.69 & 10 \\
\hline$\% \mathrm{H} 20$ & & 41.56 & \\
\hline TOTAL & & 106.52 & 100 \\
\hline
\end{tabular}




\begin{tabular}{|c|c|c|c|}
\hline \multicolumn{4}{|c|}{ Waste Tank $101-A$} \\
\hline \multicolumn{4}{|c|}{$\begin{array}{c}\text { COMPOSITIDN OF TANK IOI-A WASTE } \\
\text { (SLUDGE SAMPUE) }\end{array}$} \\
\hline \multicolumn{4}{|c|}{ September 22, 1980} \\
\hline \multicolumn{4}{|c|}{ SAMFE 14499} \\
\hline \multirow[t]{2}{*}{ COMPONENE } & \multicolumn{2}{|c|}{ \$UPGINATANT } & SOLIDS \\
\hline & MOLARTY & Wh.s & W.\% \\
\hline 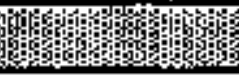 & 5 & 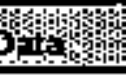 & - \\
\hline SpG & \multicolumn{2}{|c|}{1.449} & \\
\hline 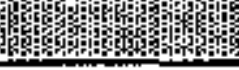 & \multicolumn{2}{|c|}{ 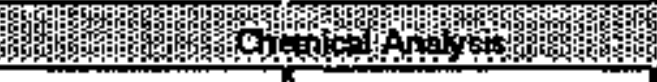 } & 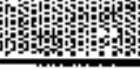 \\
\hline A & 1.36 & 7.69 & 14 \\
\hline $\mathrm{OH}$ & 9.974 & 10.7 & 0 \\
\hline $\mathrm{NO} 2$ & $1,4 \$ 8$ & 6.85 & $\mathbf{0}$ \\
\hline NOS & 2.53 & 14.84 & $\$ 3$ \\
\hline $\cos$ & 0.089 & 0.65 & 43 \\
\hline $\mathrm{PO4}$ & 0.125 & 1.42 & 0 \\
\hline Toc & $11.025 \mathrm{~g} / \mathrm{f}$ & 2.26 & 9 \\
\hline$\%$ H2O & & 42.34 & \\
\hline TOTAL & & 88.75 & 100 \\
\hline
\end{tabular}




\begin{tabular}{|c|c|c|c|}
\hline \multicolumn{4}{|c|}{ Weste Tank 101-A } \\
\hline \multicolumn{4}{|c|}{$\begin{array}{l}\text { COMPOSITION OF TANK 101-A WASTE } \\
\text { ONEFOOE BELOW SILAACE }\end{array}$} \\
\hline \multicolumn{4}{|c|}{ Odober 13, 1980} \\
\hline COMPONBNT & \multicolumn{2}{|c|}{ SUPBANATANT } & SOLIDs" \\
\hline & MOLAFITY & Wh. ע & $\mathbf{W}_{+} \%$ \\
\hline \multicolumn{4}{|c|}{ Pryrsiced pata } \\
\hline SpG & \multicolumn{2}{|c|}{1.306} & \\
\hline $\mathrm{HzO}$ & & 45.42 & \\
\hline \multicolumn{4}{|c|}{ Chamilaid Anolysts } \\
\hline $\mathrm{Na} \mathrm{AlOZ}$ & 2.54 & 15.95 & 0 \\
\hline $\mathrm{NaD} \$-1$ & 4.2 & 22.96 & $\mathbf{0}$ \\
\hline NaNO2 & 3.79 & 19.34 & $\mathbf{0}$ \\
\hline NaNos & 2.11 & 13.73 & 50.7 \\
\hline $\mathrm{Na2} \mathrm{CO} 3$ & 0.24 & 1.95 & 26.1 \\
\hline $\mathrm{NasPO}_{4}$ & $0.0 \mathrm{~B}$ & 1 & 23.4 \\
\hline TOC & $10.71 \mathrm{oL}$ & 2.44 & 0 \\
\hline $\begin{array}{l}\text { Analyical rosults } \\
\text { Complexants she }\end{array}$ & pitade, ho & $\begin{array}{l}\text { TOCII } \\
\text { asPO4/1 } \\
\text { he sofds } \\
\text { OC. }\end{array}$ & $\begin{array}{l}\text { gave whight } \\
\text { achustitad to }\end{array}$ \\
\hline
\end{tabular}




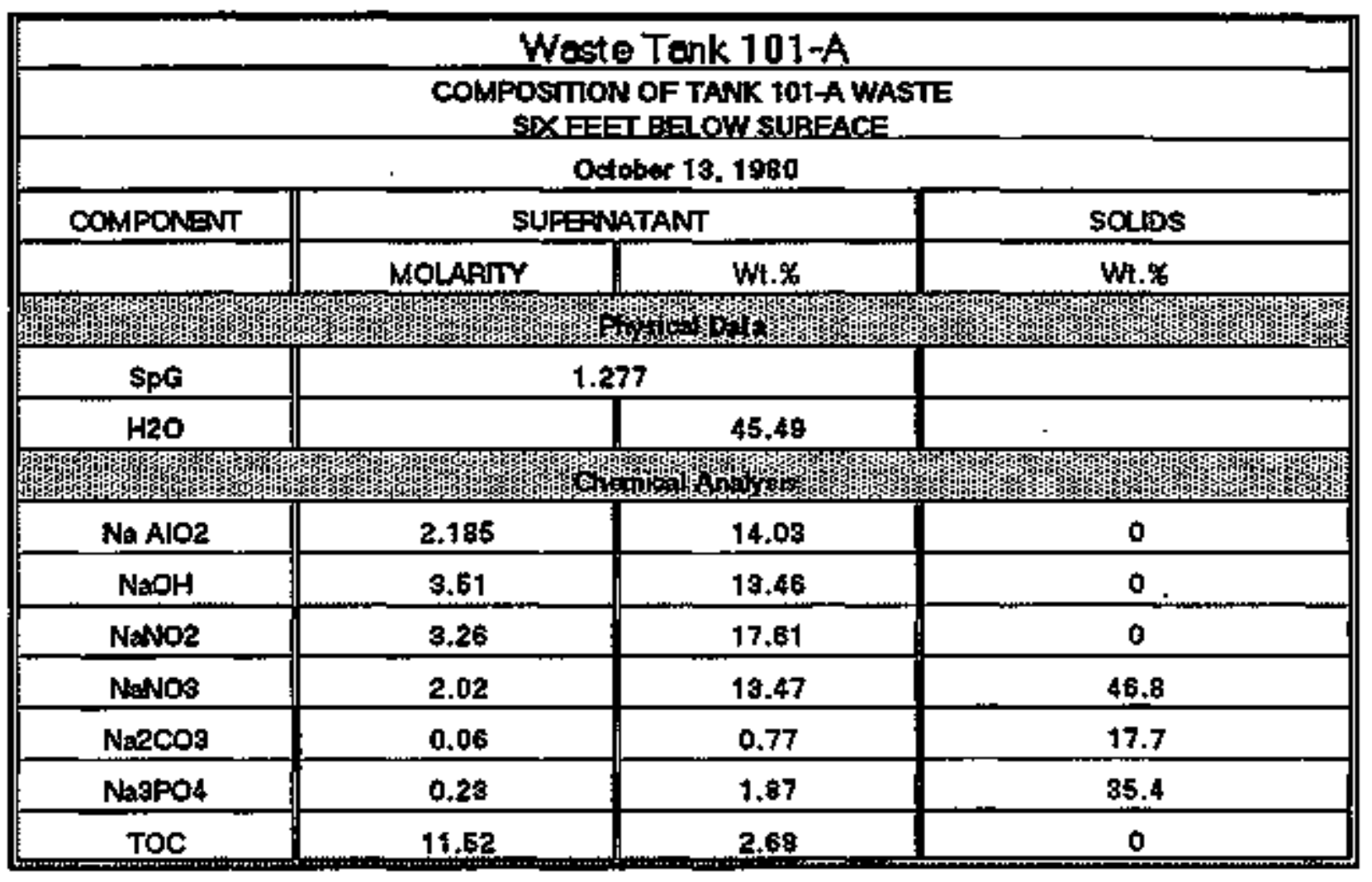


WHC-SD-MM-ER-308, Rev, 0

\begin{tabular}{|c|c|c|c|}
\hline \multicolumn{4}{|c|}{ Waste Tank 101-A } \\
\hline \multicolumn{4}{|c|}{ COMPOSTTION OF TANK 101-A WASTE } \\
\hline \multicolumn{4}{|c|}{ Oataber 22, 1980} \\
\hline COMPONENT & \multicolumn{2}{|c|}{ RLTRATE } & SOLIDS \\
\hline & MOLARTY & Wh. $\%$ & $W 1 . \%$ \\
\hline \multicolumn{4}{|c|}{ Pkysten: Data } \\
\hline SpG & \multicolumn{2}{|c|}{1.445} & \\
\hline \%H2O & & 46.10 & \\
\hline \multicolumn{4}{|c|}{ Chempical Adratysis } \\
\hline $\mathrm{Nha} \mathrm{AIO}$ & 1.53 & 8.68 & \\
\hline $\mathrm{NaOH}$ & 3.51 & 13,40 & 16 \\
\hline NaNO2 & 1.11 & 14.86 & 4 \\
\hline $\mathrm{NaNOS}$ & 1.89 & 11.12 & 52 \\
\hline $\mathrm{NazCOB}$ & 0.16 & 1.17 & 24 \\
\hline $\mathrm{Na3PO}_{4}$ & 0.05 & 0.57 & \\
\hline TOC & $19,10 \mathrm{~g}$ ㄴ. & 3.83 & \\
\hline
\end{tabular}




\begin{tabular}{|c|c|c|c|c|c|c|c|}
\hline \multicolumn{8}{|c|}{ Weste Tank $101-A$} \\
\hline \multicolumn{8}{|c|}{$\begin{array}{l}\text { COMPOSITION OF TANK IOT-A WASTE } \\
\text { TWO FOOT SAMPLE }\end{array}$} \\
\hline \multicolumn{8}{|c|}{ November 10. 1980} \\
\hline COMPONENT & \multicolumn{3}{|c|}{ TOTAL SLURRY= } & \multicolumn{3}{|c|}{ FLTAATE: } & \multirow{2}{*}{ SOLIDS } \\
\hline & $\mathbf{M}$ & Wh.\% & & $\mathbf{M}$ & W.. & & \\
\hline & 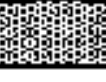 & 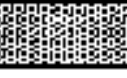 & 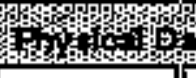 & 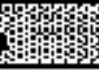 & 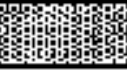 & & \\
\hline$x \mathrm{HzO}$ & & 42.37 & & & $4 B .26$ & & \\
\hline SpG & \multicolumn{3}{|c|}{1.58} & \multicolumn{3}{|c|}{1.43} & \\
\hline \multicolumn{8}{|c|}{ 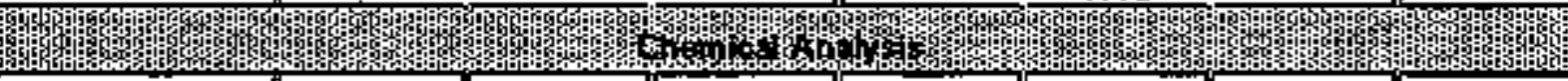 } \\
\hline $\mathrm{Na} \mathrm{AlO}_{2}$ & $1.5 t$ & 7.84 & & 1.21 & 6.96 & & 4 \\
\hline $\mathrm{NaOH}$ & 2.45 & 6.20 & & 2.31 & 6.48 & & \\
\hline NaNO2 & 2.22 & 9.69 & & 2.22 & 10.4 & & \\
\hline NaNO3 & 2.77 & 14.0 & & 2.57 & 15.3 & & \\
\hline Na2cos & 1.33 & 6.92 & & 0.31 & 2.3 & & 94 \\
\hline $\mathrm{Na3PO} 4$ & 0.08 & 0.83 & & 0.09 & 1,02 & & 1 \\
\hline $\mathrm{NazSO} 4$ & 0.13 & 1.17 & & 0.01 & 0.13 & & \\
\hline TOC $(g / L)$ & $\mathbf{3 . 9 8}$ & 0.75 & & 6.86 & 1.48 & & \\
\hline & 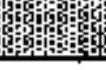 & 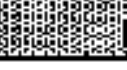 & $\mu_{10}$ & 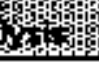 & 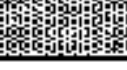 & 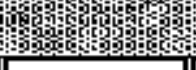 & 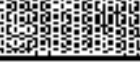 \\
\hline $137 \mathrm{Cs}$ (uCill) & & & $1.17 \mathrm{E}-0.5$ & & & 4.65E+0 & \\
\hline $90 \mathrm{sr}$ (uCin) & & & $1.23 \mathrm{E}+04$ & & & $4.28 E+03$ & \\
\hline$P u(g)$ & & & $5.67 E 05$ & & & 1.08E 05 & \\
\hline Wekght peroont & not & de 137 & $90 \mathrm{Sr}, 0$ & & & & \\
\hline
\end{tabular}


WHC-SD-WM-ER-308, Rev. 0

\begin{tabular}{|c|c|c|c|c|c|c|c|}
\hline \multicolumn{8}{|c|}{ Weste Tank 101-A } \\
\hline \multicolumn{8}{|c|}{$\begin{array}{l}\text { COMPOSITION OF TANK 101-A WASTE } \\
\text { FOUA FODT SAMPLE }\end{array}$} \\
\hline \multicolumn{8}{|c|}{ November 10, 1980} \\
\hline COMPONENT & \multicolumn{3}{|c|}{ TOTAL SLURAY' } & \multicolumn{3}{|c|}{ FLTPATE" } & Souns \\
\hline & $\mathbf{M}$ & Wt.\% & & M & $W 1 . x$ & & Wt.\% \\
\hline \multicolumn{8}{|c|}{ Phystical Dala } \\
\hline$\% \mathrm{H}_{2} \mathrm{O}$ & & 41.94 & & & 45.47 & & \\
\hline SpG & & 1,66 & & & 1.47 & & \\
\hline \multicolumn{8}{|c|}{ Chemingl Ahalysis: } \\
\hline Nat AIOZ & 1.59 & 7.9 & & 1.67 & $9+32$ & & \\
\hline $\mathrm{NaOH}$ & 2.46 & 5.94 & & 2.51 & 8.84 & & \\
\hline NaNO2 & 2.28 & 9.53 & & 2.4 & 71.26 & & \\
\hline NaNOS & 3.07 & 15.82 & & 3.24 & 18.71 & & \\
\hline $\mathrm{Na} 2 \mathrm{CO}$ & 1.5 & 9.04 & & 0.34 & 2.42 & & 90 \\
\hline Naspo4 & 0.09 & 0.89 & & 0.08 & 0.94 & & \\
\hline $\mathrm{Na}_{2} \mathrm{SO}_{4}$ & 0.15 & 1.29 & & & & & 8 \\
\hline TOC (gلي) & $\$ .51$ & $1.7 t$ & & $7.5 t$ & 1.52 & & \\
\hline \multicolumn{8}{|c|}{ 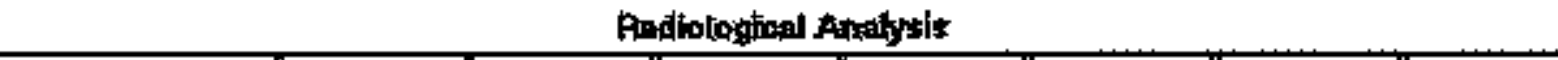 } \\
\hline $137 \mathrm{Co}$ (uCidt) & & & $5.19 E+05$ & & & $7.695+05$ & \\
\hline 90 St (uCiflL) & & & $1.97 E+04$ & & & $4,80 \mathrm{E}+0,8$ & \\
\hline Pu (oll) & & & 1.99E-04 & & & $1.34 \mathrm{E}-05$ & \\
\hline
\end{tabular}




\begin{tabular}{|c|c|c|c|c|c|c|c|}
\hline \multicolumn{8}{|c|}{ Waste Tank 101-A } \\
\hline \multicolumn{8}{|c|}{$\begin{array}{l}\text { COMPOSTTON OF TANK IOI-A WASTE } \\
\text { FOUR FOOT SAMPLE }\end{array}$} \\
\hline \multicolumn{8}{|c|}{ November 11, 1980} \\
\hline COMPONENT & \multicolumn{3}{|c|}{ TOTAL SLUARY* } & \multicolumn{3}{|c|}{ RLTFATE: } & SOLIDS \\
\hline & M & Wt.\% & & M & W. $\%$ & & Wt.\% \\
\hline & $f(x)=8$ & sichents & 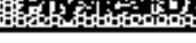 & & & & \\
\hline \multicolumn{8}{|c|}{ Vls-OTR: Quite visebus : tolids precipilate when allow ed to cool } \\
\hline$\% \mathrm{H} 2 \mathrm{O}$ & & 34.19 & & & 34.86 & & \\
\hline $\mathbf{\$ p} \mathbf{C}$ & \multicolumn{3}{|c|}{1,85} & & 1.45 & & \\
\hline \multicolumn{8}{|l|}{ 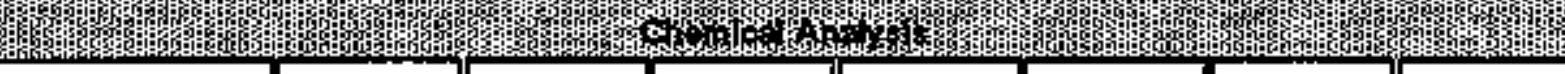 } \\
\hline $\mathrm{Na} \mathrm{AlO2}$ & 1.95 & B.64 & & $2.0 \mathrm{~B}$ & 51.8 & & \\
\hline NaOH & 3.51 & 7.59 & & 3.12 & 8.64 & & \\
\hline NaNo2 & 3.01 & 11.23 & & 2.94 & 14,02 & & \\
\hline NaNOS & 3.66 & 16.82 & & 3.48 & 20.45 & & \\
\hline $\mathrm{NazCOS}$ & 2.31 & 13.24 & & 0.13 & 0.95 & & 95 \\
\hline $\mathrm{NaBPO} 4$ & 0.12 & 1.06 & & 0.13 & 1.53 & & \\
\hline NazSO4 & \multicolumn{6}{|c|}{ ANALYSIS NOT AVAILABLE } & \\
\hline TOC (e凡) & 15.61 & 2.51 & & 9.94 & 2.04 & & \\
\hline \multicolumn{8}{|c|}{ 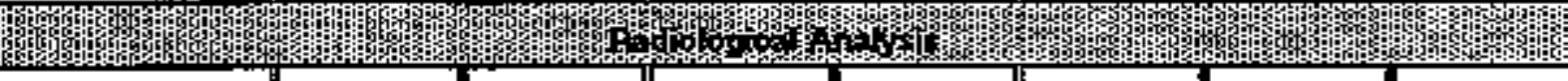 } \\
\hline $137 \mathrm{Cs}$ (uCill) & & & $5.96 \mathrm{E}+05$ & & & $6.22=+0.5$ & \\
\hline $90 \mathrm{Sr}(\mathrm{UCH})$ & & & $9.55 E+04$ & & & 4.19E+ 03 & \\
\hline $\mathrm{Pu}(g / L)$ & & & 5.19E-0.4 & & & $3.96 E-05$ & \\
\hline Weigh peroent & es nol & de 13? & .9D \$r, o & & & & \\
\hline
\end{tabular}


WHC-SD-WM-ER-309, Rev, 0

\begin{tabular}{|c|c|c|}
\hline \multicolumn{3}{|c|}{ Waste Tank 101-A } \\
\hline \multicolumn{3}{|c|}{ Januaxy 3. 1984} \\
\hline \multicolumn{3}{|c|}{ 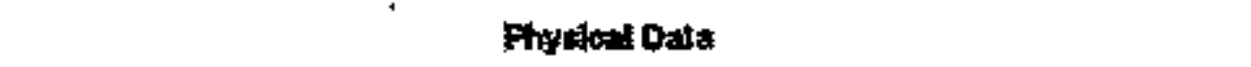 } \\
\hline SPECIFC GRAVITY : & 7879 & 17898 \\
\hline slurry & t.3597 & 1.6400 \\
\hline Supernalank & 1.3617 & 1.6847 \\
\hline Centrifuged Solids" & 1.3307 & 1.6253 \\
\hline
\end{tabular}




\begin{tabular}{|c|c|c|c|}
\hline \multicolumn{4}{|c|}{ Waste Tank 101-A } \\
\hline \multicolumn{4}{|c|}{ COMPOSITION OF TANK 101-A WASTE } \\
\hline \multicolumn{4}{|c|}{ January 8, 1904} \\
\hline \multicolumn{4}{|c|}{ SAMPLE \$7878 } \\
\hline CONAPONENT & \multicolumn{2}{|c|}{ CONCENTRATION } & SOLOS \\
\hline & SLUFAY $(M)$ & SUPGINATANT (M) & Wt.\% \\
\hline 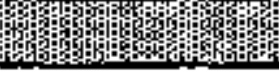 & \multicolumn{2}{|c|}{ 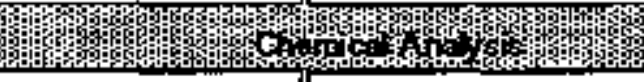 } & 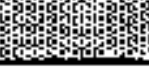 \\
\hline Al & 1,09 & 0.9 & 38 \\
\hline OH & \pm & 1.43 & \\
\hline $\mathrm{NO2}$ & 0.01 & 1.57 & \\
\hline Nos & 4.11 & 2.59 & \\
\hline $\cos$ & - & NA & \\
\hline TOC $\left(g^{\prime} L\right)$ & 7.02 & 5.23 & 44 \\
\hline $\mathrm{PO4}$ & 0.13 & 0.02 & \\
\hline $\mathrm{SO4}$ & 0.20 & 0.05 & 23 \\
\hline \multicolumn{4}{|c|}{ 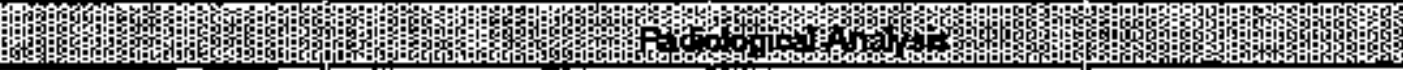 } \\
\hline $137 \mathrm{Cs}$ (UCAL) & 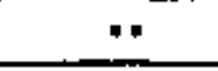 & $\because$ & \\
\hline $90 \mathrm{Sr}(\mathrm{UCIL})$ & $3.49^{\wedge} 4$ & $2.805+03$ & \\
\hline Pu (OR) & 1,88E-05 & 7.22E-05 & \\
\hline \multicolumn{4}{|c|}{ Dlsolved in Acid. } \\
\hline \multicolumn{2}{|c|}{ - Below detection limits. } & & \\
\hline NA-Not Available. & & & \\
\hline
\end{tabular}




\begin{tabular}{|c|c|c|c|}
\hline \multicolumn{4}{|c|}{ Waste Tonk $101-A$} \\
\hline \multicolumn{4}{|c|}{ COMPOSSTION OF TANK ID1A WASTE } \\
\hline \multicolumn{4}{|c|}{ Januay 3,1984} \\
\hline \multicolumn{4}{|c|}{ SAMPLE 77899} \\
\hline COMPONENT & \multicolumn{2}{|c|}{ CONCENTAATION } & SOLIDS \\
\hline & SLUFRY (M) & SUPERNATANT (M) & $W . \%$ \\
\hline \multicolumn{4}{|c|}{ Chenglod Analysis } \\
\hline Al & 1.59 & 2.27 & 27 \\
\hline $\mathrm{OH}$ & $\star$ & 3.86 & \\
\hline $\mathrm{NO2}$ & 0.01 & 2.53 & \\
\hline NO3 & 5.08 & 3.26 & 62 \\
\hline $\cos$ & $\cdot$ & NA & \\
\hline $\mathrm{PO4}$ & $\therefore$ & $\cdots$ & \\
\hline$\$ 04$ & 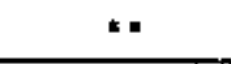 & 0.19 & \\
\hline $\operatorname{TOC}(g / t)$ & 9.78 & 11 & 11 \\
\hline \multicolumn{4}{|c|}{ 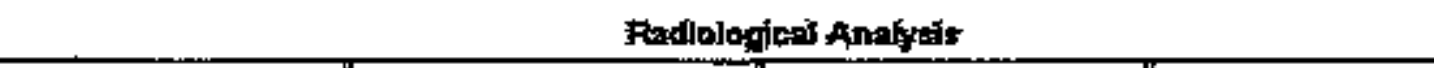 } \\
\hline $137 \mathrm{Cs}$ (uGilL) & $\because$ & $\because$ & \\
\hline 90 Sr (UCill) & $6.11 \mathrm{E}+04$ & $9.11 \mathrm{E}+04$ & \\
\hline Pu (ofl) & 6.52E-04 & NA & \\
\hline \multicolumn{4}{|c|}{ Dissolved in Acid. } \\
\hline \multicolumn{4}{|c|}{ * Below Detedion Limitt. } \\
\hline NA- Not Availablo & & & \\
\hline
\end{tabular}


WHC-SD-WM-ER-30B, Rev. 0

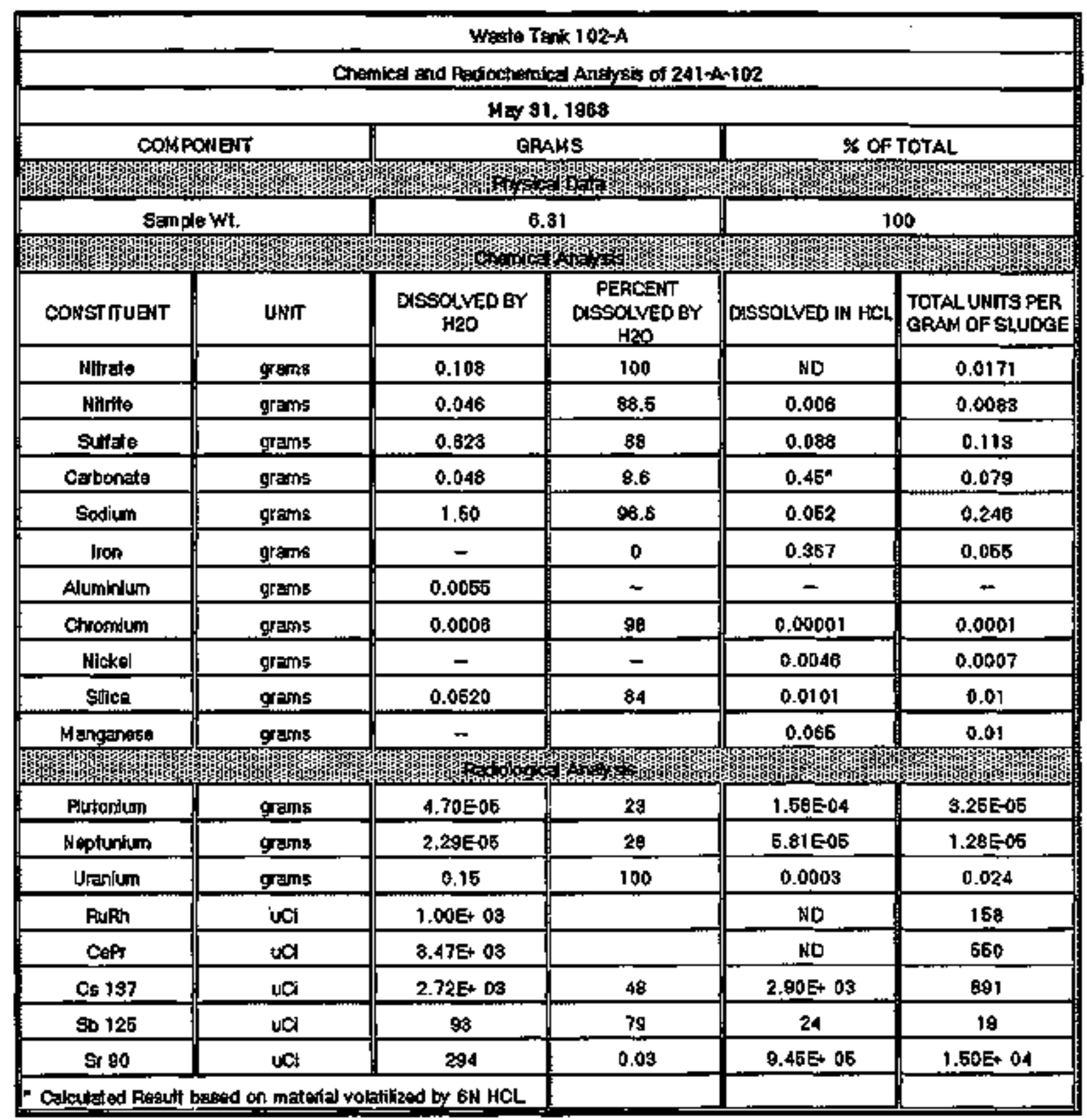


WHC-SD-WM-ER-308, Rev, O

\begin{tabular}{|c|c|c|}
\hline \multicolumn{3}{|c|}{ Wate Tank t02A } \\
\hline \multicolumn{3}{|c|}{ Charadetention a 102A STudo: } \\
\hline \multicolumn{3}{|c|}{ January 16, 1979 } \\
\hline COMFCNENT & LAB VALUE & LAB UNT \\
\hline \multicolumn{3}{|c|}{ 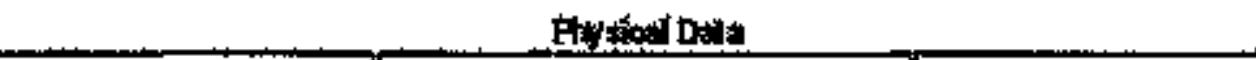 } \\
\hline wet SpG & 1.62 & \\
\hline dy spg & 0.54 & \\
\hline \multicolumn{3}{|c|}{ 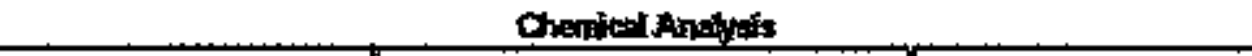 } \\
\hline$F$ & 0.13 & do \\
\hline A & $<2.32 E 02$ & $g$ \\
\hline \$i & $1.40 \mathrm{E}-02$ & gfo \\
\hline $\mathrm{Mn}$ & 3.SDE-02 & glg \\
\hline $\mathrm{Na}$ & $1.20 \mathrm{E}-01$ & $g$ \\
\hline \multicolumn{3}{|c|}{ 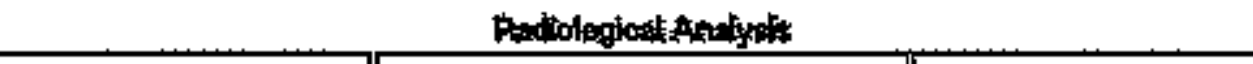 } \\
\hline $5 r \cdot 59 / 90$ & $2.94 E 02$ & clg \\
\hline C:-137 & $1.0: E=08$ & $\mathrm{Org}$ \\
\hline E-154 & $1,92 E-04$ & $\mathrm{CHg}$ \\
\hline $95-125$ & $3.07 E 04$ & CHg \\
\hline
\end{tabular}




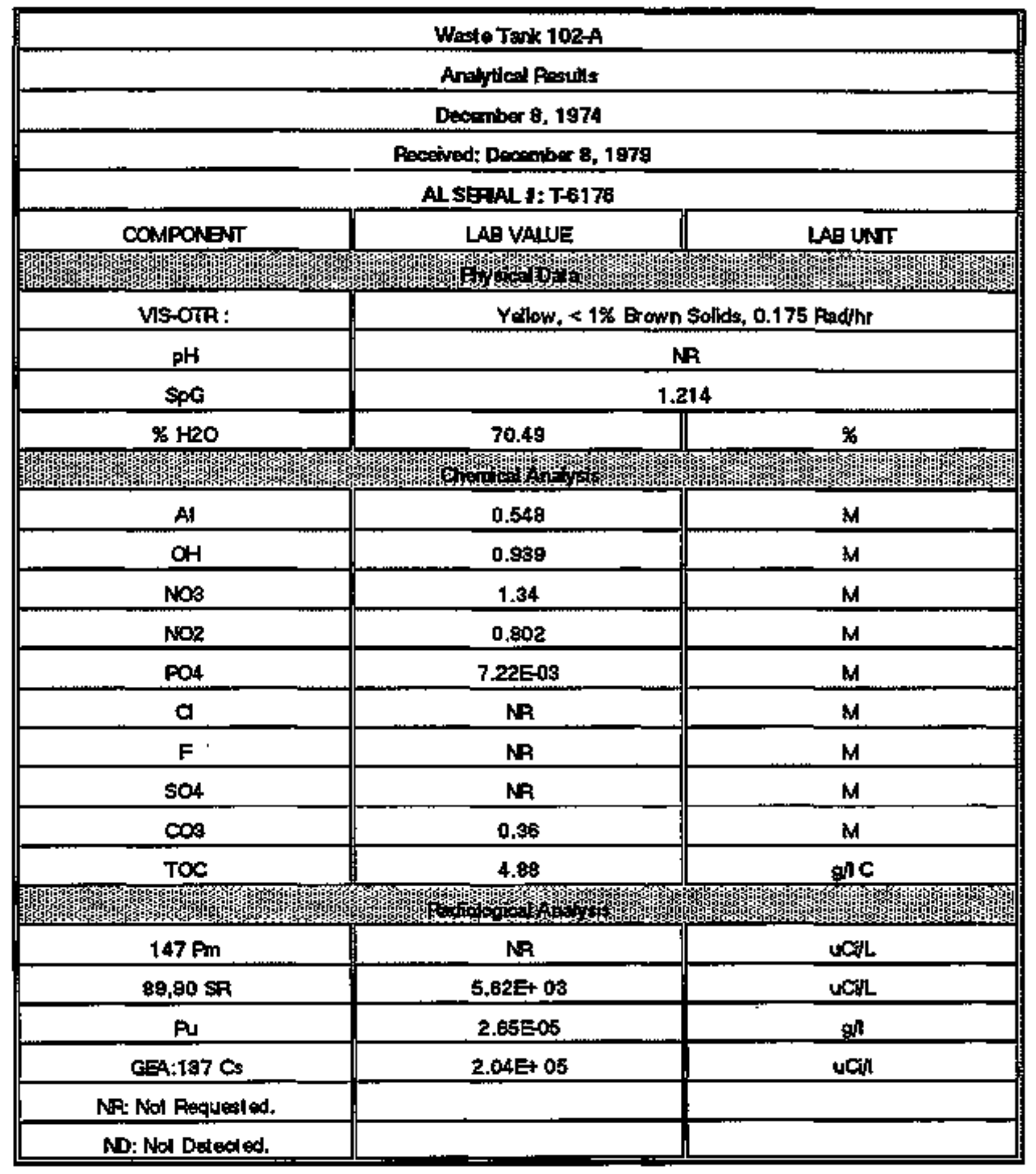




\begin{tabular}{|c|c|c|}
\hline \multicolumn{3}{|c|}{ Waste Tenk 102-A } \\
\hline \multicolumn{3}{|c|}{ ANALYSAS OFTANK FAPN SAMPES } \\
\hline \multicolumn{3}{|c|}{ October 20, 1975} \\
\hline \multicolumn{3}{|c|}{ Dade recetived: Auguet 19, 1975} \\
\hline \multicolumn{3}{|c|}{ Samplex T-7252 } \\
\hline COMPONENT & LAB VALUE & LAB LNT \\
\hline \multicolumn{3}{|c|}{ Frosiged bets } \\
\hline Vis-OTR & \multicolumn{2}{|c|}{ Erown, No solids, aso mradihr } \\
\hline $\mathrm{pH}$ & 10.3 & \\
\hline Spg & 1.087 & \\
\hline HeO & $88.9 t$ & $\%$ \\
\hline \multicolumn{3}{|c|}{ 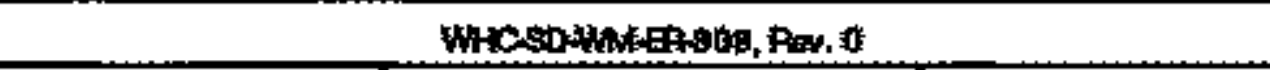 } \\
\hline $\mathrm{OH}$ & $\times 9.50503$ & $\underline{M}$ \\
\hline Al & $9.13 E-09$ & $M$ \\
\hline $\mathrm{Na}$ & 1.58 & $\underline{M}$ \\
\hline $\mathrm{NO2}$ & 0.112 & $\underline{M}$ \\
\hline Nos & 1.01 & $\underline{M}$ \\
\hline $\mathrm{SOH}$ & Cancelled & $\underline{M}$ \\
\hline $\mathrm{PO4}$ & 1.09E-02 & $\underline{M}$ \\
\hline$a$ & 5.10E 04 & $\underline{M}$ \\
\hline$F$ & $2.195-04$ & $\underline{M}$ \\
\hline$\infty$ & 0.175 & $\underline{M}$ \\
\hline \multicolumn{3}{|c|}{ 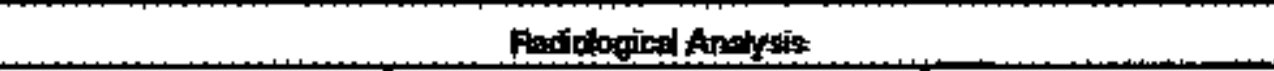 } \\
\hline Sr-98/90 & $4.73 E+05$ & uciog: \\
\hline Pu & $1.85 \mathrm{E} 0 \mathrm{~S}$ & uCilgal \\
\hline CEA: 60 Co & $9.18=02$ & uCigal \\
\hline $\mathrm{CEA}: 125 \mathrm{Sb}$ & 8.75라 $0 \mathrm{~s}$ & uCigal \\
\hline GEA: $C_{5} 137$ & $2,196+0.5$ & uCiffal \\
\hline CEA:Eu 154 & $2.95 \mathrm{E}+03$ & ucilpal \\
\hline CEA: Cs134 & $1.71 E+03$ & ucipad \\
\hline
\end{tabular}


WHC-SD-WM-ER-308, Rev, O

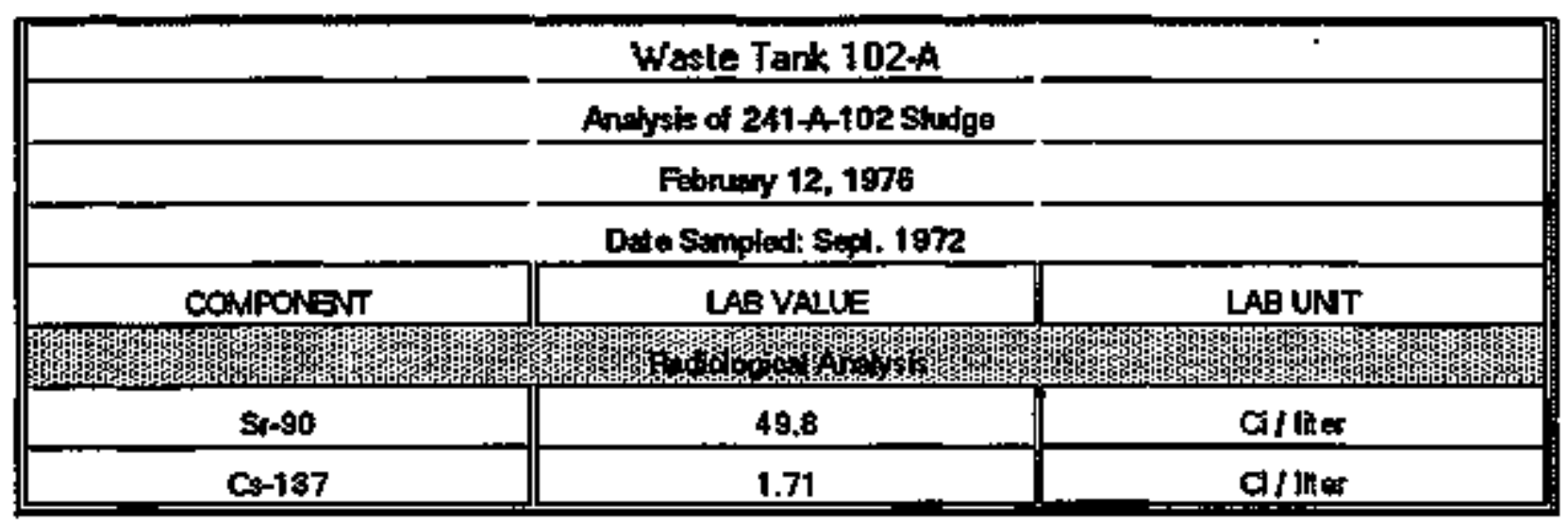


WHC-SD-WM-ER-308, Rev. 0

\begin{tabular}{|c|c|c|c|c|c|c|c|}
\hline \multicolumn{8}{|c|}{ WasteTak 102A } \\
\hline \multicolumn{8}{|c|}{ ANALYSES OFFENDUAL 102A TANK SANAE } \\
\hline \multicolumn{8}{|c|}{ Novitibu 1976} \\
\hline COMIRONENT & \multicolumn{6}{|c|}{ LAB VALLE } & LAB LNT \\
\hline Sample Number & 6038 & 6159 & 6828 & 6327 & 5698 & 7279 & \\
\hline \multicolumn{8}{|c|}{ 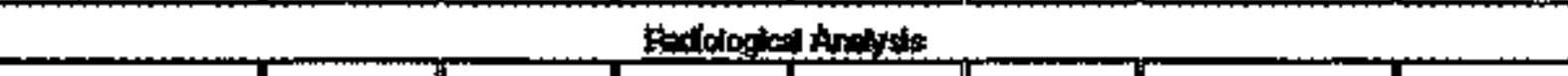 } \\
\hline 89.90 $5 \mathrm{r}$ & 34 & g1.6 & 46,3 & 36 & 9.3 & 40 & an \\
\hline $1 \% 7 \mathrm{cs}$ & 0.92 & 0.92 & 1.1 & 1.04 & 0.78 & 0.78 & 대 \\
\hline $99,90 \mathrm{Sr}$ & 129 & $\mathrm{~s} 0 \mathrm{~s}$ & 175 & 138 & 35 & 151 & ard \\
\hline Py & $1.25 \mathrm{C}_{02}$ & 2.80ED2 & 1.66E01 & t.26E01 & 4.94502 & & gin \\
\hline
\end{tabular}




\begin{tabular}{|c|c|c|c|c|}
\hline \multicolumn{5}{|c|}{ Waste Tank 102-A } \\
\hline \multicolumn{5}{|c|}{ Analyis of Tark Waste } \\
\hline \multicolumn{5}{|c|}{ Jily 17, 198d } \\
\hline CONRDNENT & $\begin{array}{l}\text { LAB VALUE } \\
\text { T-1243 } \\
\text { Istufagel }\end{array}$ & $\begin{array}{c}\text { LAB VALUE } \\
\text { T-1244 } \\
\text { (4taet) }\end{array}$ & $\begin{array}{c}\text { LAB VALUE } \\
\text { T-1245 } \\
\text { 7teen }\end{array}$ & LAB UNT \\
\hline $\operatorname{sog}$ & 1554 & $1157 ?$ & 11554 & \\
\hline $\mathrm{H}-2 \mathrm{2O}$ & 82.330 & 91.B2 & 82.10 & $\%$ \\
\hline \multicolumn{5}{|c|}{ Pas } \\
\hline $\mathbf{A}$ & 0.268 & 0.291 & 0.410 & $\mathbf{M}$ \\
\hline $\mathrm{OH}$ & 0.738 & 0.622 & 0.622 & $\mathbf{M}$ \\
\hline NOL & 0.594 & 0.603 & 0.589 & $\mathbf{M}$ \\
\hline Nos & 0.942 & 0.879 & $0.99 B$ & $M$ \\
\hline $\cos$ & 0.110 & 0.120 & 0.110 & $\mathbf{M}$ \\
\hline PO4 & 0.0346 & 0.0674 & 0.0962 & M \\
\hline $\mathrm{T} \propto \mathrm{c}$ & $\mathrm{MF}$ & 4,060 & 3.38 & g는 \\
\hline \multicolumn{5}{|c|}{ 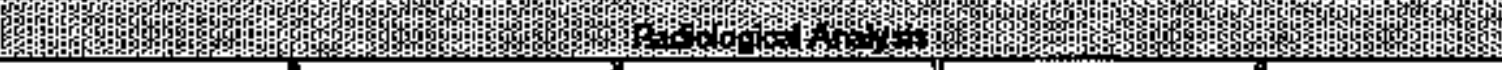 } \\
\hline $\mathrm{Pu}$ & 3.64E05 & 2.94E005 & 3.60 E0S & gالو \\
\hline Cs 137 & $2.016+05$ & 2.04E+ 05 & 2.00E 05 & ucth \\
\hline Spr 99.90 & $1.00 E+04$ & $1.20 E+04$ & $7.74 E+03$ & uCitL \\
\hline 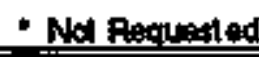 & & & & \\
\hline
\end{tabular}


WHC-SD-WM-ER-308, Rev, 0

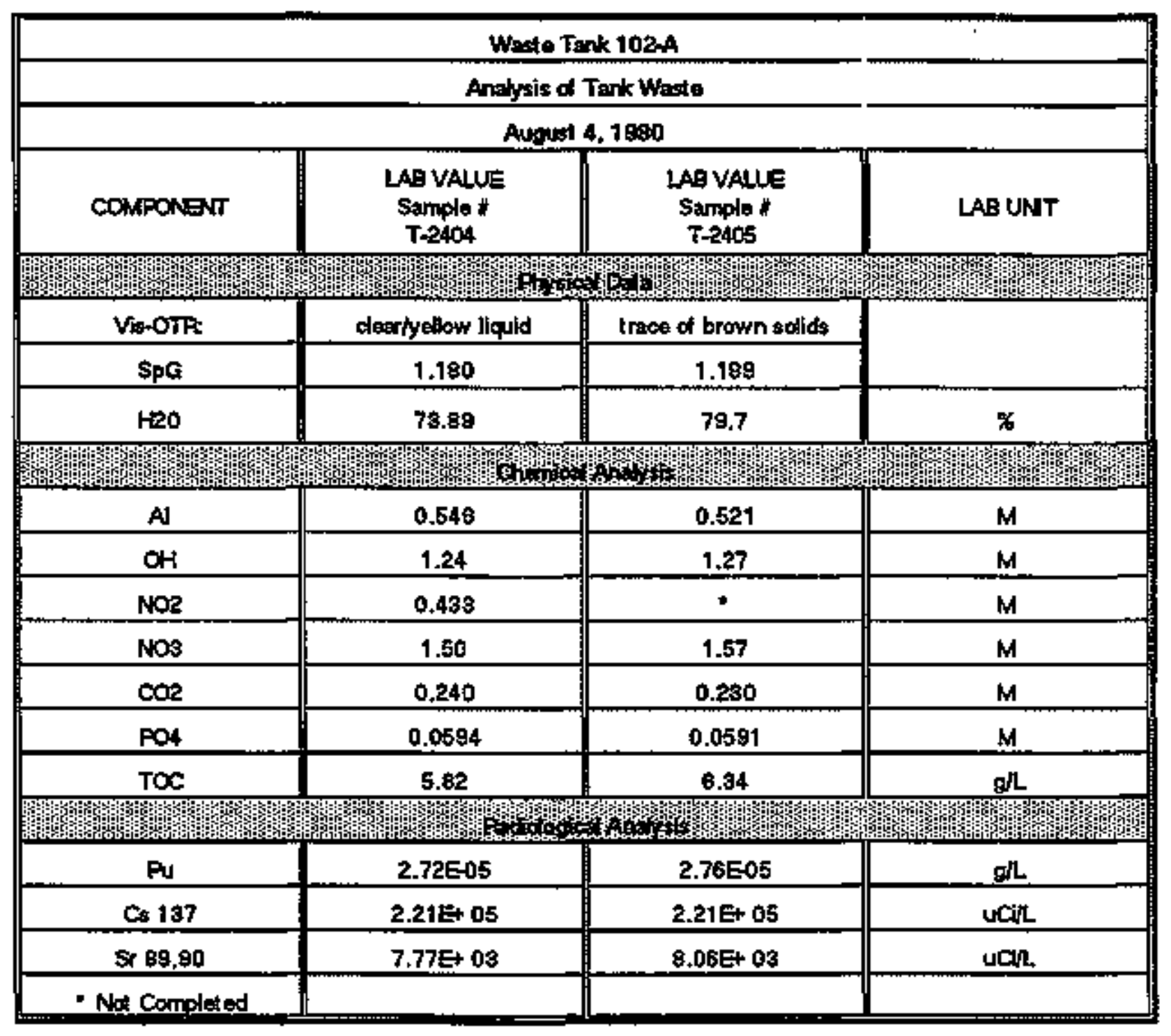


WHC-SD-WM-ER-308, Rev. 0

\begin{tabular}{|c|c|c|c|}
\hline \multicolumn{4}{|c|}{ Wate Tank $102 \mathrm{~A}$} \\
\hline \multicolumn{4}{|c|}{ Compoestion of Tink 102A Wets } \\
\hline \multicolumn{4}{|c|}{ Doep Sarngle } \\
\hline \multicolumn{4}{|c|}{ Deoumber 23,1990} \\
\hline & \multicolumn{2}{|c|}{ FLTRATE } & SOUDS \\
\hline COMPONENT & MOAFITY (MOLEATUTEF) & WEGH $\%$. & W曰GT ж" \\
\hline 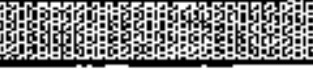 & \multicolumn{2}{|c|}{ 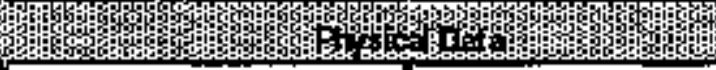 } & 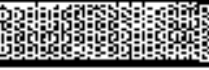 \\
\hline XHEO & \multicolumn{2}{|r|}{47.69} & \\
\hline SpG & \multicolumn{2}{|c|}{1.44} & \\
\hline 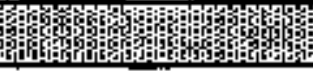 & \multicolumn{2}{|c|}{ 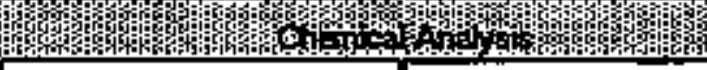 } & 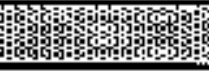 \\
\hline NeAlOZ": & \multicolumn{2}{|r|}{13.77} & \\
\hline $\mathrm{NaOH}$ & \multicolumn{2}{|r|}{9.19} & \\
\hline NeNOS & \multicolumn{2}{|r|}{15.43} & \\
\hline NaNOS & \multicolumn{2}{|r|}{12.16} & 84 \\
\hline $\mathrm{NazCOS}$ & \multicolumn{2}{|r|}{1.09} & \\
\hline NasPO4 & 0.04 & 0.48 & 13 \\
\hline$T O C$ & 1.79 & 0.37 & \\
\hline \multicolumn{4}{|c|}{ 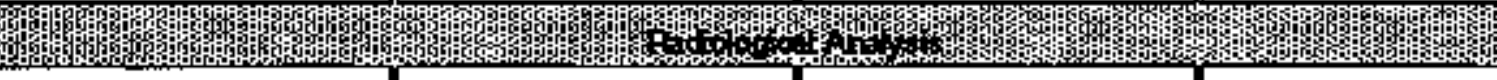 } \\
\hline 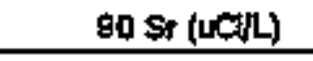 & \multicolumn{2}{|l|}{$4.196+03$} & \\
\hline $137 \mathrm{C}(\mathrm{( \omega O \textrm {g }}$ & \multicolumn{2}{|l|}{$6.305+05$} & \\
\hline 60 Co $(U C H L)$ & \multicolumn{2}{|l|}{ Not Defecled } & \\
\hline $306 \mathrm{Au}(\mathrm{UCAR})$ & \multicolumn{2}{|l|}{ Not Dotected } & \\
\hline Pu (gllL) & \multicolumn{2}{|l|}{ 3.3BE-05 } & \\
\hline \multicolumn{3}{|c|}{ Doos not include radionuclities } & \\
\hline - Calbedaked by Difle & & & \\
\hline
\end{tabular}




\begin{tabular}{|c|c|c|c|}
\hline \multicolumn{4}{|c|}{ Waske Tank tor-A } \\
\hline \multicolumn{4}{|c|}{ Composation of Tisk 102A Watle } \\
\hline \multicolumn{4}{|c|}{ Shellow Sanple } \\
\hline \multicolumn{4}{|c|}{ Dexember 23, 1980} \\
\hline & \multicolumn{2}{|c|}{ RLTRATE } & SOUDS \\
\hline COMPONENT & MOLARTY (MOLSILTEA) & Wectr x" & WeGrT \%" \\
\hline \multicolumn{4}{|c|}{ 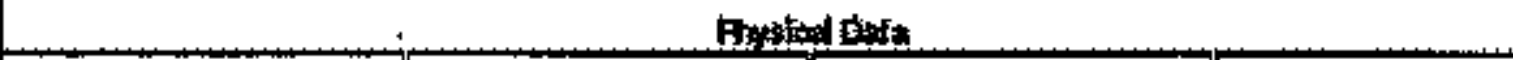 } \\
\hline$\% \mathrm{H} 2 \mathrm{O}$ & & 41.46 & \\
\hline Spt & \multicolumn{2}{|c|}{1.452} & \\
\hline \multicolumn{4}{|c|}{ Chimlon Anshyis: } \\
\hline NaAlo2 & 1.92 & 10.8 & $\therefore$ \\
\hline $\mathrm{NaOH}$ & $\$ .12$ & 8.6 & $11^{* * *}$ \\
\hline Navae & 8.13 & 94.9 & 26 \\
\hline Nancos & 1.93 & 11.2 & 59 \\
\hline $\mathrm{Na} 2 \mathrm{OOS}$ & 0,14 & 1 & \\
\hline $\mathrm{Na}_{3} \mathrm{PO}_{4}$ & 0.07 & D.B & 3 \\
\hline $\operatorname{TOC}(\alpha / L)$ & Not Available & Nol Available & Not Available \\
\hline \multicolumn{4}{|c|}{ 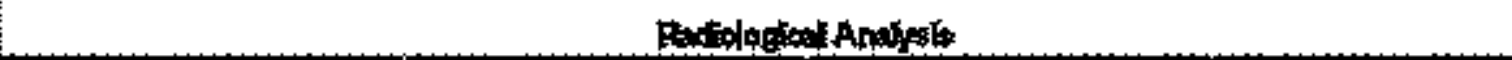 } \\
\hline $90 \mathrm{se}$ (uCils) & $3.196+03$ & & \\
\hline $137 \mathrm{Cs}(\mathrm{NOH})$ & 6.31 E 05 & & \\
\hline $69 \mathrm{Co}(\mathrm{LCN})$ & $1.535+02$ & & \\
\hline t06 Pu (uCill) & 4.37E ds & & \\
\hline$P_{N}(g / L)$ & 9.00E05 & & \\
\hline \multicolumn{4}{|c|}{ E Does not inchude radiomutolidus } \\
\hline \multicolumn{2}{|c|}{ * Calculated by Difieremes. } & & \\
\hline$* *$ Should not prect & & & \\
\hline
\end{tabular}


WHC-SD-WM-ER-308, Rev, 0

\begin{tabular}{|c|c|c|}
\hline \multicolumn{3}{|c|}{ Wasle Tank 102-A } \\
\hline \multicolumn{3}{|c|}{ Pu and Am Amalyses of Various Tank Farm Samples } \\
\hline \multicolumn{3}{|c|}{ May 28, 1981} \\
\hline COMPONENT & LAB VALUE & LAE UNIT \\
\hline \multicolumn{3}{|c|}{ 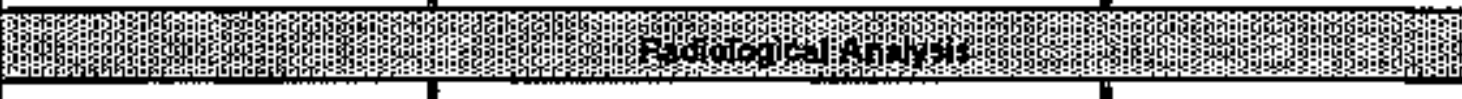 } \\
\hline Pu" & 1.32E-05 & gil of fjltrake \\
\hline Am" & $8.70 E-07$ & g/l of filtrale \\
\hline * Aosul & given in grams per liger of filtuate & \\
\hline
\end{tabular}


WHC-SD-WM-ER-300, REV. 0

\begin{tabular}{|c|c|c|c|}
\hline \multicolumn{4}{|c|}{ Waste Tank 102-A } \\
\hline \multicolumn{4}{|c|}{$\begin{array}{l}\text { L129 Analydical Dats } \\
\text { Aqueous Sampless }\end{array}$} \\
\hline \multicolumn{4}{|c|}{ July 14,1986} \\
\hline COMPONENT & $\begin{array}{l}\text { LABVALUE } \\
\text { La' No. \$1XD }\end{array}$ & $\begin{array}{l}\text { LAB VALUE } \\
\text { Lab No. } 9200\end{array}$ & LAB UNIT \\
\hline \multicolumn{4}{|c|}{ Pardiglogiforis Analysis } \\
\hline $1-129$ & $2.20 E-04$ & $2.10 \mathrm{E}-04$ & $\mathrm{uCilmI}$ \\
\hline
\end{tabular}




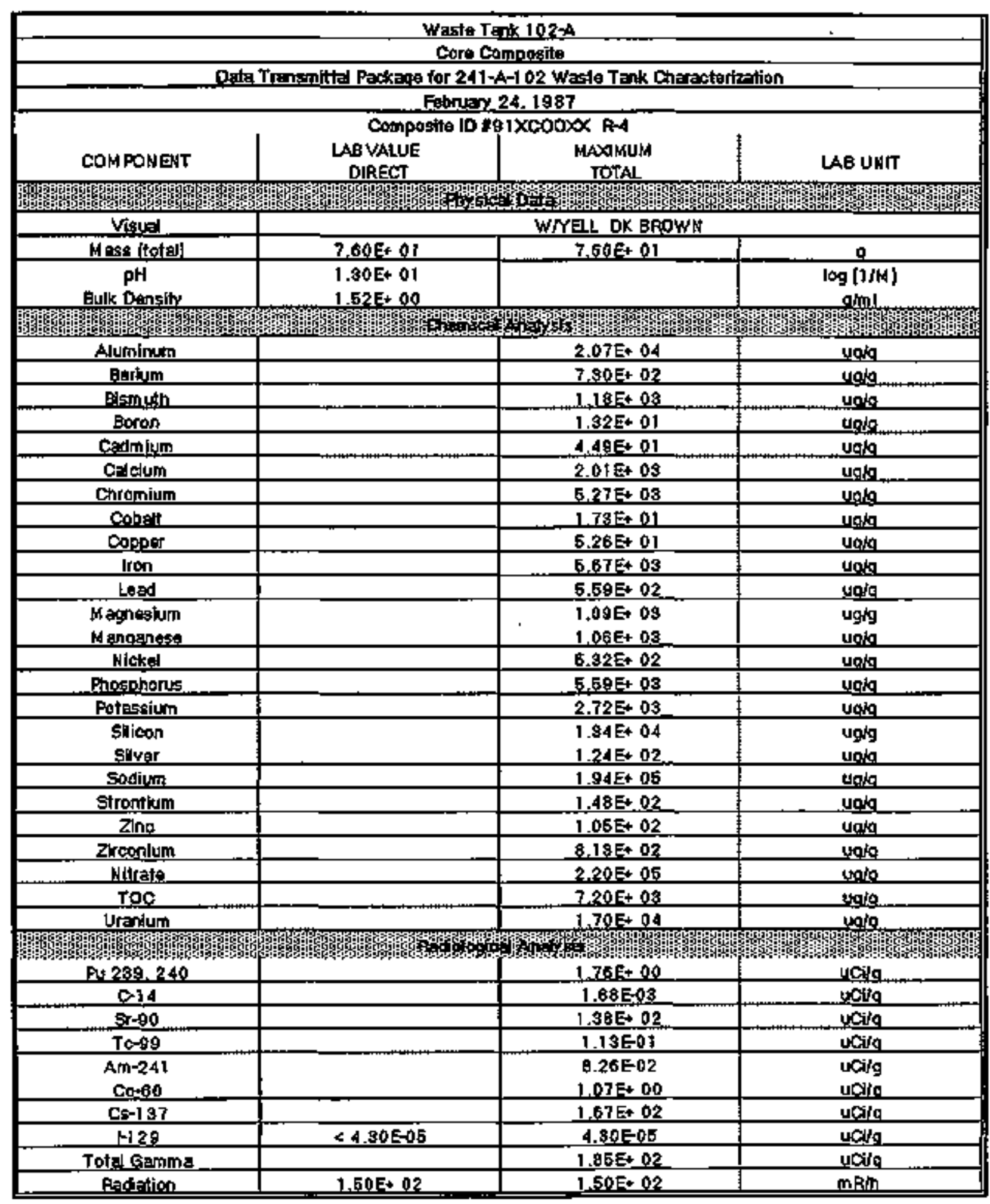




\begin{tabular}{|c|c|c|c|}
\hline \multicolumn{4}{|c|}{ Wasta Tank-102-A } \\
\hline \multirow{2}{*}{\multicolumn{4}{|c|}{ 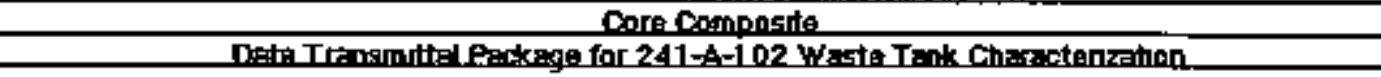 }} \\
\hline & & & \\
\hline & & & \\
\hline & Compos & $x \times 14$ & \\
\hline COM PONENT & $\begin{array}{l}\text { LABVALUE } \\
\text { DARECT }\end{array}$ & $\begin{array}{l}\text { MAXINUN } \\
\text { TOFAL }\end{array}$ & LAB UNIT \\
\hline \multicolumn{4}{|c|}{ 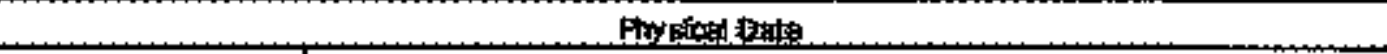 } \\
\hline YIsual & & DK BA & \\
\hline Mass (total) & $462+01$ & $\triangle 52 E+01$ & q \\
\hline $\mathrm{pH}$ & $130 \mathrm{E}+01$ & & loatidu) \\
\hline Elyk Density & $166 E+00$ & & gikn! \\
\hline \multicolumn{4}{|c|}{ 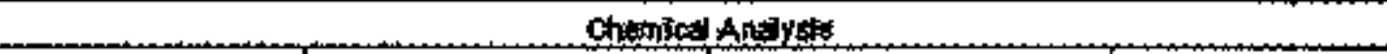 } \\
\hline Aluminum & & $258 E+04$ & uㅗ요요 \\
\hline Eanum & & $1035+03$ & Lain \\
\hline Basmuth & & $229 E+03$ & ugig. \\
\hline Bpmn & & $152 \mathrm{E}+01$ & uplo \\
\hline Cadmium & & $849 E+01$ & ugla \\
\hline Cgalcum & & 317E+03 & س \\
\hline chromium & & 6 32E4 03 & 096 \\
\hline Cobstit & & $3.11 E+01$ & yolg \\
\hline Cooper & & $111 E+02$ & uqla \\
\hline tron & & $228 E+04$ & ugk \\
\hline Lead & & $B \mathrm{BIE}+03$ & uqu \\
\hline $\begin{array}{l}\text { Magnesilm } \\
\text { Mengoners }\end{array}$ & & $\begin{array}{l}158 E+03 \\
324 E+03\end{array}$ & $\begin{array}{l}\text { ugkg } \\
\text { ugig. }\end{array}$ \\
\hline Nickel & & $620 E+02$ & uoli \\
\hline Fhosphorus & & $489 E+09$ & ugk \\
\hline Potessum & & $291 \mathrm{E}+03$ & uakg \\
\hline $\begin{array}{l}\text { Silicen } \\
\text { Silver }\end{array}$ & & $\begin{array}{l}197 \mathrm{E}+0 \mathrm{~d} \\
370 \mathrm{~B}+02\end{array}$ & $\begin{array}{l}\text { ugkg } \\
\text { ugig }\end{array}$ \\
\hline sodmum & & $180 \mathrm{E}+05$ & unia. \\
\hline Strontum & & $471 E+01$ & Loig \\
\hline . Zne & & $0,005+00$ & Lotgits \\
\hline Zirconuming & & $207 E+03$ & موزئي \\
\hline Nitrate & & $137 E+05$ & Laig \\
\hline TOC & & $794 \mathrm{H}_{+} 03$ & مـاموبي. \\
\hline Urenum & & $208 E+03$ & wola \\
\hline \multicolumn{4}{|c|}{ 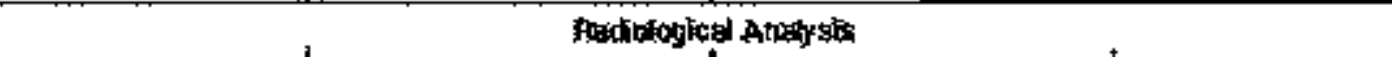 } \\
\hline Pts 239. 240 & & $235 E^{2}+00$ & LCikg. \\
\hline$C-14$ & & $823 E-04$ & uola \\
\hline$s r-90$ & & $1075+03$ & ualo \\
\hline Tc-g 9 & & 1 74E02 & ucila \\
\hline $\begin{array}{c}\text { Am-241 } \\
\text { Co-60 }\end{array}$ & & $\begin{array}{l}234 E+00 \\
600=01\end{array}$ & ucilg \\
\hline$C x-137$ & & $1125+02$ & uCilo \\
\hline $1-129$ & $<350505$ & $360 E-05$ & _uct:lo \\
\hline Totel Gemma & & $158 E+02$ & UCWg \\
\hline Fathetion & $100 \mathrm{E}+0 \mathrm{3}$ & $1005+03$ & $\mathbf{m R / h}$ \\
\hline
\end{tabular}




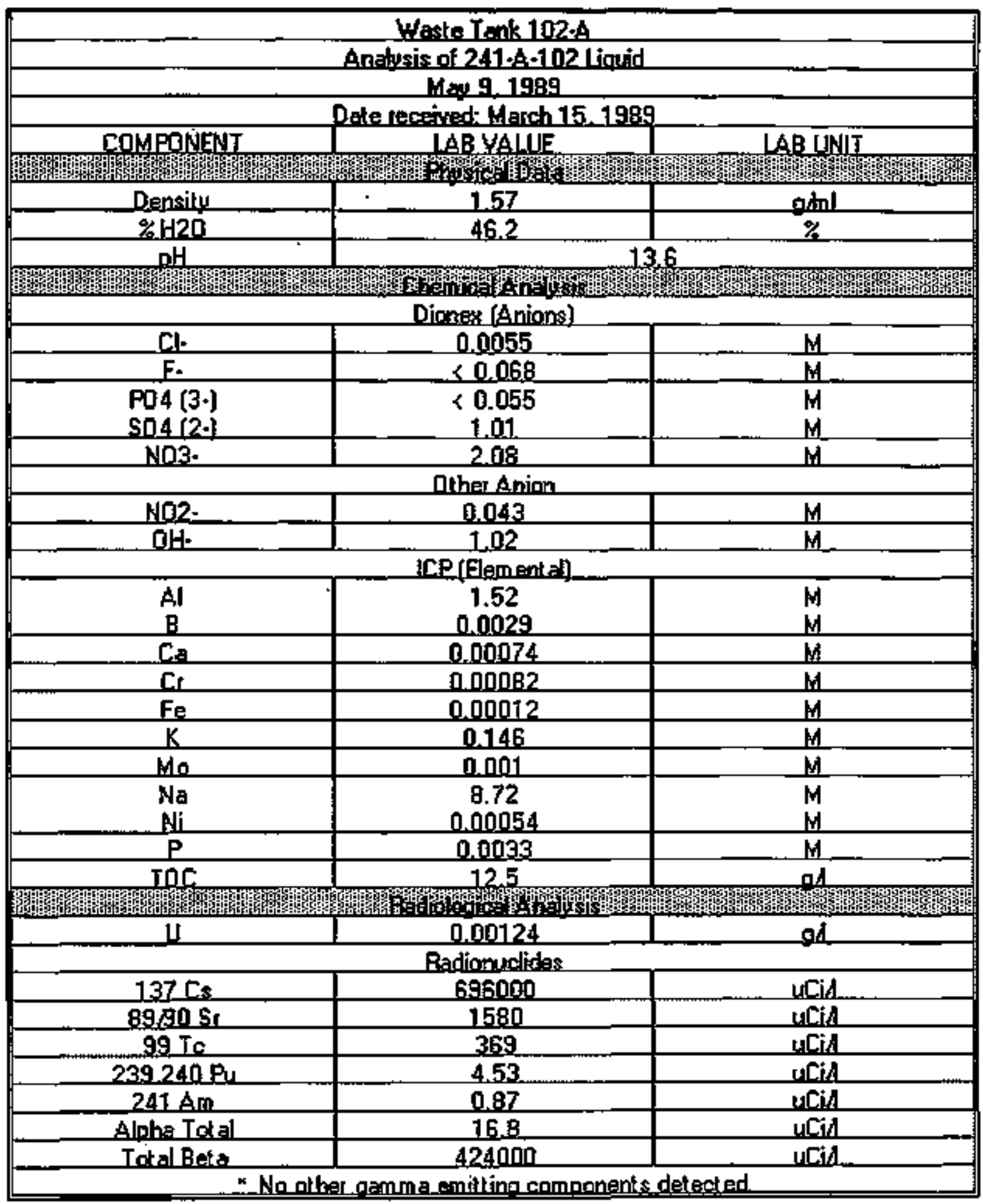


WHC-SD-WM-ER-308, Rev. 0

\begin{tabular}{|c|c|c|c|c|c|c|}
\hline \multicolumn{7}{|c|}{ Waste Tank 103-A } \\
\hline \multicolumn{7}{|c|}{ Tank $108-A$ SLUDEE CHARACTERZATION } \\
\hline \multicolumn{7}{|c|}{ Decombtr 18, 1972} \\
\hline COMPONGNT & LAB VALUE & LAB UNIT & LAB VALUE & LAB UNTT & LAB VALUE & LAB UNT \\
\hline & SUPESNATANT & & $\begin{array}{l}\text { HARD } \\
\text { SLIDGE }\end{array}$ & & FNES & \\
\hline \multicolumn{7}{|c|}{ 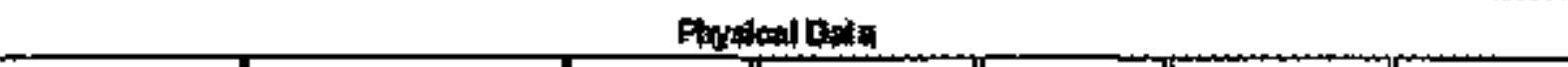 } \\
\hline Wet walgin & & & 1.9671 & g & $1,038 E+00$ & g \\
\hline Dry wolgnt & & & 1.7845 & 9 & 5,004501 & G \\
\hline Wea volume & & & 1 & $\mathbf{m}$ & 7.000501 & mi \\
\hline Dry volume & & & 0.8 & ml & 5.000E01 & $\mathbf{m}$ \\
\hline $\operatorname{spg}$ & 1.175 & & & & & \\
\hline \multicolumn{7}{|c|}{ Chemiond Antrats } \\
\hline$F_{\mathbf{q}}$ & $\leq 1,81609$ & $\underline{M}$ & 9.70E-02 & oldg & 7.200502 & glg \\
\hline Al & 2,60E-02 & $\underline{M}$ & $<6.755002$ & $g$ & $3.690 \mathrm{~B} 02$ & gig \\
\hline si & $<1.96 E_{03}$ & $M$ & $1,0 B E 02$ & وفئ & $9.490 \mathrm{E}-03$ & $\mathrm{~g} g$ \\
\hline Mn & 1.94E-0S & $M$ & & & & \\
\hline $\mathrm{Na}$ & 3.47 & & 0.288 & $g / g$ & 9.900002 & gig \\
\hline $\mathrm{OH}$ & 0.904 & & & & & \\
\hline \multicolumn{7}{|c|}{ Parieloctiol Anahrots } \\
\hline $89+90 \mathrm{sr}$ & $1.19 \mathrm{E}-02$ & cill & 1.64E-02 & Cile & 1,7600002 & Org \\
\hline $137 \mathrm{cs}$ & 0.49 & $\mathrm{CH}$ & $2.94-03$ & 이요 & 6.550504 & $\mathrm{C} / \mathrm{g}$ \\
\hline
\end{tabular}


WHC-SD-WM-ER-308, Rev. O

\begin{tabular}{|c|c|c|c|c|}
\hline \multicolumn{5}{|c|}{ Whil Tank 108-A } \\
\hline \multicolumn{5}{|c|}{ Taxk 103-A Shudgo Characteriention } \\
\hline \multicolumn{5}{|c|}{ Decemper 19, 1972} \\
\hline COMPONENT & LAB VALUE & LAB VALUE & LAS VALLE & LAB UNT \\
\hline & & 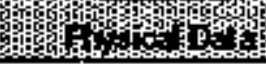 & & 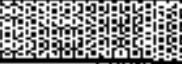 \\
\hline \multicolumn{5}{|c|}{ Focelatant } \\
\hline & CONIRAL & MACNALC & Xuow & \\
\hline Actd phase & $47.0 \%$ & $60.3 \%$ & $68.2 \%$ & $\%$ \\
\hline Water wash & $10.2 \%$ & $4.9 \%$ & $1.8 \%$ & $\%$ \\
\hline Splids & $42,0 \%$ & $34.8 \%$ & $29.9 \%$ & $\%$ \\
\hline Vol. of Sol. & 3.6 & 10.6 & 6.0 & $\mathbf{m}$ \\
\hline 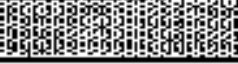 & 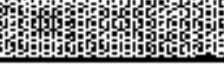 & 然 & 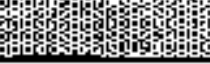 & 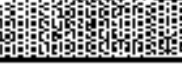 \\
\hline $\mathrm{H}+$ & 0.218 & 0.172 & 0.359 & $\underline{M}$ \\
\hline Fo & 2.77E-02 & $1.67 \mathrm{E} 02$ & 0.111 & $\underline{M}$ \\
\hline A & 0.172 & 0.132 & 0.235 & $\underline{M}$ \\
\hline $\mathbf{\$}$ & 0.109 & 6.585002 & 0.144 & $\underline{M}$ \\
\hline Mn & $0.83 \mathrm{E} 03$ & 2.67E03 & $1,130 E-02$ & $\underline{M}$ \\
\hline 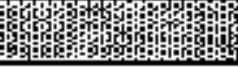 & 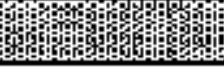 & $\mathrm{H}^{2}$ & 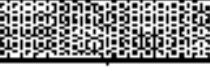 & \\
\hline $137 C_{s}$ & 1.12E 05 & 2.ઐ 8 O5 & $1,89 E 0 \%$ & 嵌 \\
\hline $89+90 \$ r$ & 1.76502 & 3.05E02 & 4.04E 02 & $\mathrm{Cs}$ \\
\hline
\end{tabular}




\begin{tabular}{|c|c|c|c|}
\hline \multicolumn{4}{|c|}{ WASTE TANK 103-A } \\
\hline \multicolumn{4}{|c|}{ SUMMARY OF SLUDGE ANALYSIS } \\
\hline \multicolumn{4}{|c|}{ February 12, 1976} \\
\hline \multicolumn{4}{|c|}{ Date Sampled: Nov. 1972} \\
\hline COM FONENT & $\begin{array}{l}\text { LAG VALUE } \\
\text { (Soft-TOD) }\end{array}$ & $\begin{array}{c}\text { LAB VALUE } \\
\text { IHard-Bottoml }\end{array}$ & LAB UNIT \\
\hline \multicolumn{4}{|c|}{ Radiological A alatysłs } \\
\hline Sr 90 & 25.9 & 32.3 & Cilliter \\
\hline CS 137 & 0.97 & 5.78 & Cilliter \\
\hline
\end{tabular}




\begin{tabular}{|c|c|c|c|c|c|}
\hline \multicolumn{5}{|c|}{ Wate Tank 10S-A } & - \\
\hline \multicolumn{6}{|c|}{ ANALYSS OF 109 A TANK FESIDUAL SUUDCE } \\
\hline \multicolumn{6}{|c|}{ tanutr 24, 1977 } \\
\hline \multicolumn{6}{|c|}{ Dade Peosived: Docember 10, 1976-lanuary 10, 1977} \\
\hline COMPONENT & $\begin{array}{l}\text { LAB VALUE } \\
\text { SAMPLE I } 89\end{array}$ & $\begin{array}{l}\text { LAB VALUE } \\
\text { SAMPLE } 190\end{array}$ & $\begin{array}{l}\text { LAB VALLE } \\
\text { SAMPLE I } 667\end{array}$ & $\begin{array}{l}\text { LAB VALLE } \\
\text { SAMPLE I TOP }\end{array}$ & LAB UNT \\
\hline sat on or & & & & & \\
\hline ost ol & 8,0 & 64.0 & 24.0 & 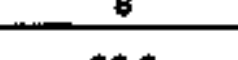 & Qsiner \\
\hline $89+90 \mathrm{Sr}$ & 34,0 & $\mathbf{9 2 . 0}$ & 90.0 & $\mathbf{3 0 . 0}$ & Clga \\
\hline $137 \mathrm{Cs}_{s}$ & 0.59 & 0.69 & 2.8 & 0.87 & ctine \\
\hline Pu & 6.35E-02 & $6.72 E-02$ & 0.121 & $\$ .62 E 02$ & ofliter \\
\hline
\end{tabular}




\begin{tabular}{|c|c|c|c|}
\hline \multicolumn{4}{|c|}{ Waste Tank 103-A } \\
\hline \multicolumn{4}{|c|}{ FAD ANALYSIS OF TANK 108-A } \\
\hline \multicolumn{4}{|c|}{ Augeat 2, 1979} \\
\hline CONPONENT & LABVALUE & WECAT $\%$ & LAB UNT \\
\hline \multicolumn{4}{|c|}{ 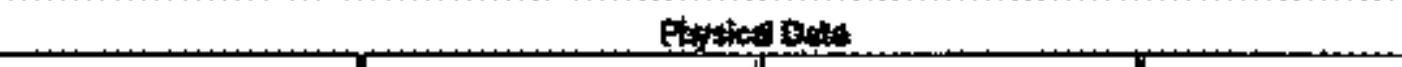 } \\
\hline $\operatorname{spg}$ & 1.398 & & \\
\hline$\%+20$ & 53.00 & & \\
\hline \multicolumn{4}{|c|}{ Chemited Ahalysit } \\
\hline $\mathrm{A}+\mathbf{B}$ & 0.889 & 5.21 & $\underline{M}$ \\
\hline$\alpha+$ & 1.48 & 4.28 & $M$ \\
\hline $\mathrm{NO2}$. & 1.68 & a.29 & $M$ \\
\hline Nos- & 3 & 18.2 & $\underline{M}$ \\
\hline $\cos 2-$ & 0.8 & 6.07 & $\mathbf{M}$ \\
\hline $\mathrm{PO} 43$ & $0.0 \% 5$ & 0.46 & $\underline{M}$ \\
\hline 5042 & $<0.080$ & & $\underline{M}$ \\
\hline Toc & 16.4 & 1.10 & of \\
\hline Troils' Wetah: Percent & & 98.6 & $\not{x}$ \\
\hline - Tolal Orgaric Carbon & & & \\
\hline
\end{tabular}


WHC-SD-WM-ER-308, Rev, 0

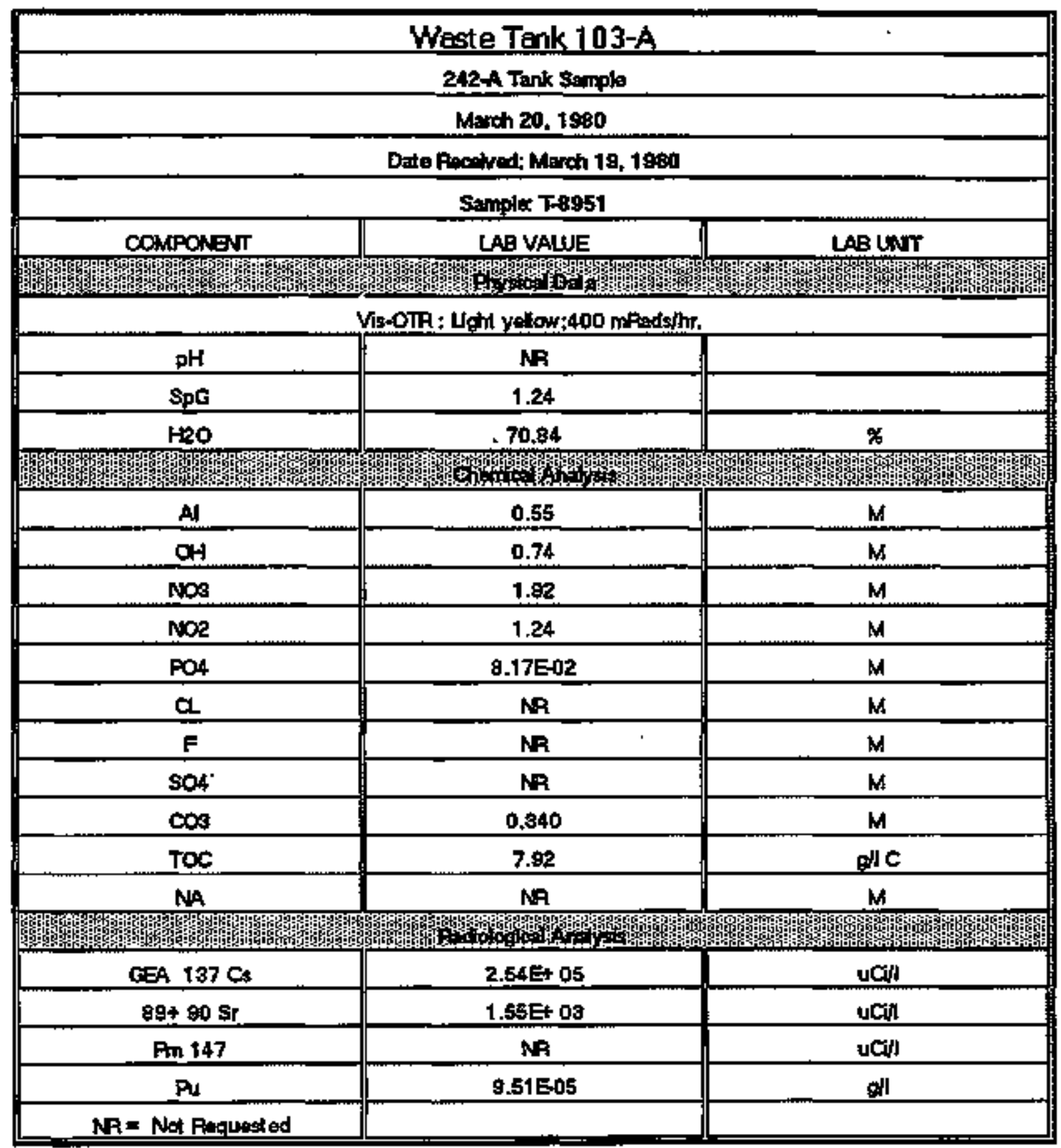




\begin{tabular}{|c|c|c|}
\hline \multicolumn{3}{|c|}{ Waste Tank 1 03-A } \\
\hline \multicolumn{3}{|c|}{ 242A Tank Sample } \\
\hline \multicolumn{3}{|c|}{ March 20. 1980} \\
\hline \multicolumn{3}{|c|}{ Date Recolvet Aprd 7, 1980} \\
\hline \multicolumn{3}{|c|}{ Samples: T-9536 } \\
\hline COMPONENT & LAB VALUE & LAB UNIT \\
\hline \multicolumn{3}{|c|}{ 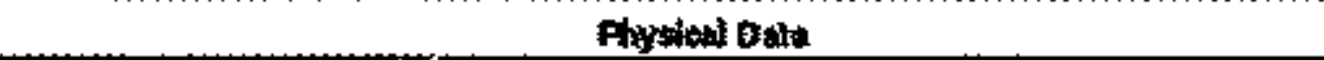 } \\
\hline \multicolumn{3}{|c|}{ Vis-OTR :Opaqut- yellow ; $1.2 \mathrm{mRad} s / \mathrm{hr}$} \\
\hline $\mathrm{pH}$ & NR & \\
\hline SpG & 1.417 & \\
\hline $\mathrm{HzO}$ & 51.4 & $\%$ \\
\hline \multicolumn{3}{|c|}{ Chenichal Analystis } \\
\hline Al & 1.14 & M \\
\hline $\mathrm{OH}$ & 1.67 & M \\
\hline NO3 & 3.17 & $\mathbf{M}$ \\
\hline NO2 & 4.89 & $\mathbf{M}$ \\
\hline PO4 & $7.07 \mathrm{E}-02$ & $\mathbf{M}$ \\
\hline CL & $\mathbf{N R}$ & M \\
\hline$F$ & $\mathbf{M P}$ & $\mathbf{M}$ \\
\hline $\mathbf{s 0 4}$ & $\mathbf{M R}$ & $\mathbf{M}$ \\
\hline $\cos$ & 0.521 & $\mathbf{M}$ \\
\hline TOC & NR & gil c \\
\hline NA & NR & $\mathbf{M}$ \\
\hline \multicolumn{3}{|c|}{ Patiologioal thatiostion } \\
\hline GEA $137 \mathrm{Cs}$ & $4.48 \mathrm{E}+05$ & uCijl \\
\hline $89+90 \mathrm{Sr}$ & $2.41 E+0 B$ & uCA \\
\hline $\operatorname{Pm} 147$ & $\mathbf{N R}$ & UCWI \\
\hline Pu & 9.35605 & gh \\
\hline NA = Not Recuesi & & \\
\hline
\end{tabular}




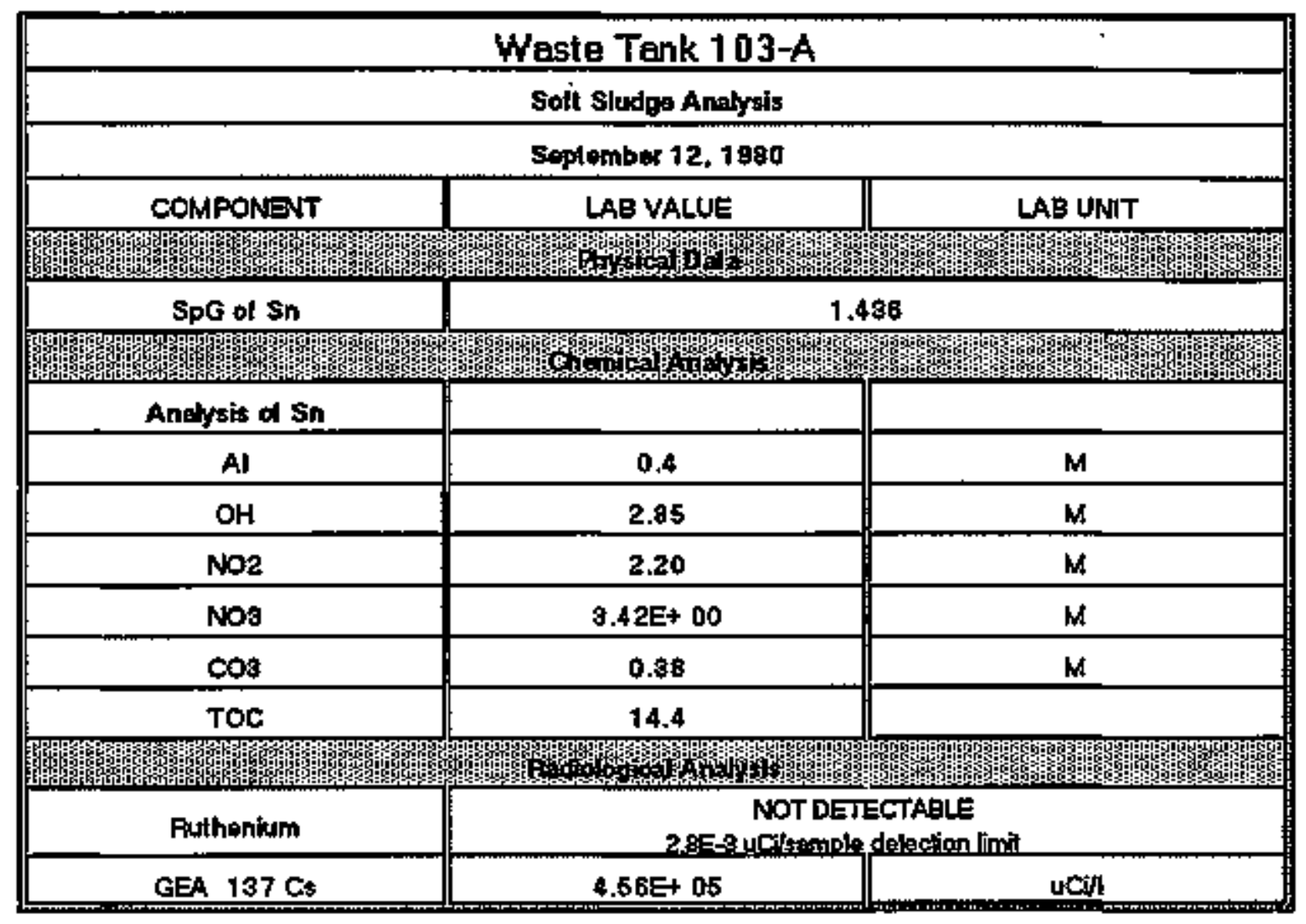




\begin{tabular}{|c|c|c|c|c|}
\hline \multicolumn{5}{|c|}{ Waste Tark 103-A } \\
\hline \multicolumn{5}{|c|}{ DOMPOSMON $G=$ TANK 103A WASTE } \\
\hline \multicolumn{5}{|c|}{ Seplember 22, 1980} \\
\hline & \multicolumn{3}{|c|}{ Supematard } & "Sctids \\
\hline COMPONENT & LAB VALUE & LAB UNT & WتGT $\%$ & \\
\hline \multicolumn{5}{|c|}{ 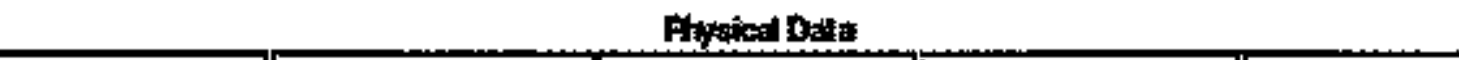 } \\
\hline$\% \mathrm{H} 20$ & & & 50.17 & \\
\hline \multirow{2}{*}{\multicolumn{5}{|c|}{ Chenicotenthits }} \\
\hline & & & & \\
\hline A & 0.400 & M & 2.20 & \\
\hline OH & 2.86 & $M$ & 7.94 & \\
\hline $\mathrm{NO} 2$ & 2.20 & $\mathbf{M}$ & 10.57 & \\
\hline Nos & 3.42 & $M$ & 20.24 & \\
\hline $\cos$ & 0.380 & $\mathbf{M}$ & 2.81 & \\
\hline PO4 & 0.083 & $\mathbf{M}$ & 0.72 & \\
\hline Toc & 14.4 & ant & 2.88 & \\
\hline TOTAL. & & & 97.71 & \\
\hline \multicolumn{5}{|c|}{ 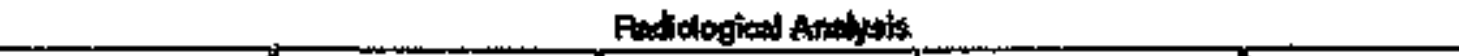 } \\
\hline 1376 & 4.56505 UCAL & & & \\
\hline 108 As & $\begin{array}{l}<28 E-08 \\
\text { uCtksmple }\end{array}$ & & & \\
\hline & 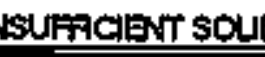 & & & \\
\hline
\end{tabular}




\begin{tabular}{|c|c|c|c|c|}
\hline \multicolumn{5}{|c|}{ Waste Tank 103-A } \\
\hline \multicolumn{5}{|c|}{ COMPOSIION CFTAKK 103A WASIE } \\
\hline \multicolumn{5}{|c|}{ October 2, 198d } \\
\hline & \multicolumn{3}{|c|}{ SUFETATANT } & SOUDS \\
\hline COMFONBNT & LAB VALUE & LAE UNT & WEctrT & WECHT \% \\
\hline 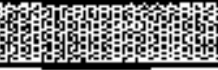 & 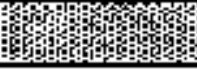 & 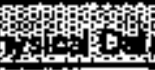 & 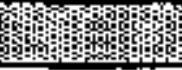 & 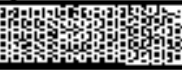 \\
\hline \% Waker. & & & 41.66 & \\
\hline SpG & \multicolumn{3}{|c|}{1,402} & \\
\hline \multicolumn{5}{|c|}{ 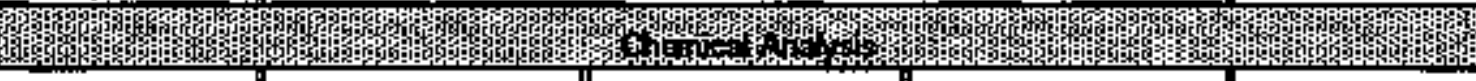 } \\
\hline $\mathbf{A}$ & 1.21 & $\mathbf{M}$ & 7.1 & 0 \\
\hline OH & 1.96 & $M$ & 5.8 & 0 \\
\hline $\mathrm{NO2}$ & 1.65 & $\mathbf{M}$ & B.1 & 20 \\
\hline Nos & 3.28 & $\mathbf{M}$ & 19.5 & 4 \\
\hline $\cos$ & 0.31 & $\mathbf{M}$ & 2.3 & 69 \\
\hline PO4 & $0.58^{\circ}$ & $M$ & 6.8 & 0 \\
\hline$T \alpha$ & 4.65 & gl & 1,1 & 0 \\
\hline
\end{tabular}




\begin{tabular}{|c|c|c|c|}
\hline \multicolumn{4}{|c|}{ Waste Tank 108-A } \\
\hline \multicolumn{4}{|c|}{ Coro Composiste } \\
\hline \multicolumn{4}{|c|}{ February 24, 1987 } \\
\hline \multicolumn{4}{|c|}{ 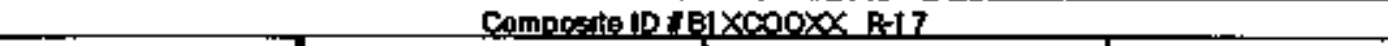 } \\
\hline CON PONENT & $\begin{array}{c}\text { LAB VALUE } \\
\text { Otrect }\end{array}$ & MAXIM UM TOTAL & LAB UNIT \\
\hline \multicolumn{4}{|c|}{ 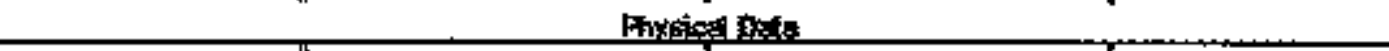 } \\
\hline $\mathrm{pH}$ & $=1$ B3E+01 & & $\log (1 / \mathrm{N})$ \\
\hline V|퇴비 & GREFH|SH EROY & & \\
\hline Hess totoly & $268 \mathrm{E}+0 \mathrm{~B}$ & 263.6*0. & هـ \\
\hline Fulk Densty & $134 E+00$ & $134 E+00$ & gimL \\
\hline \multicolumn{4}{|c|}{ 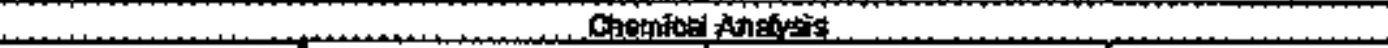 } \\
\hline Aluminum & & $161 F+04$ & ugig. \\
\hline 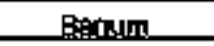 & & E 37E+02 & ـ ولوب \\
\hline 8fmulk & & $150 \mathrm{E}+02$ & unio \\
\hline Boron & & $326 E+01$ & 노영 \\
\hline Cadmum & & $764 E+01$ & ugig \\
\hline Csglcysm & & $160 \mathrm{E}+03$ & ب919.9. \\
\hline Chromium & & $134 E+03$ & uata \\
\hline Cobet & & $1.30 \mathrm{E}+0.0$ & ugda \\
\hline Coppor & & $185 E+01$ & uqla. \\
\hline Iron & & $328 E+02$ & ung \\
\hline $\begin{array}{c}\text { Loat } \\
\text { Magnesumm }\end{array}$ & & $\begin{array}{l}338 E+02 \\
737 E+02\end{array}$ & ugkg \\
\hline Mengentse & & $953 E+01$ & 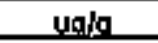 \\
\hline Nlokel & & $823 E+01$ & ugla \\
\hline $\begin{array}{l}\text { Phosphorus } \\
\text { Potassinmm }\end{array}$ & & $\begin{array}{l}105 E+08 \\
257 E+03 \\
\end{array}$ & $\begin{array}{l}\text { ugkg } \\
\text { ughg. }\end{array}$ \\
\hline Silicon & & $120 E+04$ & ugla. \\
\hline Siver & & $176 E+01$ & unto \\
\hline Sodium & & $202 E+05$ & Lolg \\
\hline Strontıum & & 소 24E+ 00 & tolg \\
\hline Zinc & & $81 E+01$ & tolg \\
\hline Arconium & & $146 \mathrm{E}+02$ & toing \\
\hline Uranrum & & 1_60E+03 & Heig \\
\hline Mrtuate & & $114 E+05$ & ugko \\
\hline TOC & & $B 04 E+03$ & uqg \\
\hline \multicolumn{4}{|c|}{ 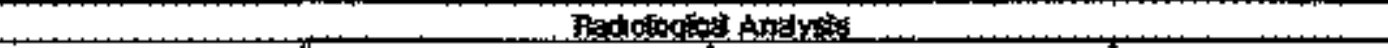 } \\
\hline Pur-209.40 & & $125 E 01$. & uCilg \\
\hline $0-14$ & & $269 E 03$ & yCug \\
\hline SS-90 & & $481 \mathrm{E} \cdot 01$ & uCwg \\
\hline Tc-9g & & $122 \mathrm{E01}$ & ucidg \\
\hline An-241 & & 9.67E02 & LCH \\
\hline Co-Bo & & $920=02$ & ucia \\
\hline $\mathrm{Cs}-137$ & & $202 E+02$ & . \\
\hline 1129 & $000 \mathrm{E}+00$ & $000 \mathrm{E}+00$ & volo \\
\hline Tota Gamma & & $2235+02$ & ualn \\
\hline Prodighon & $100 E+02$ & $1005+02$ & $m R h$ \\
\hline
\end{tabular}




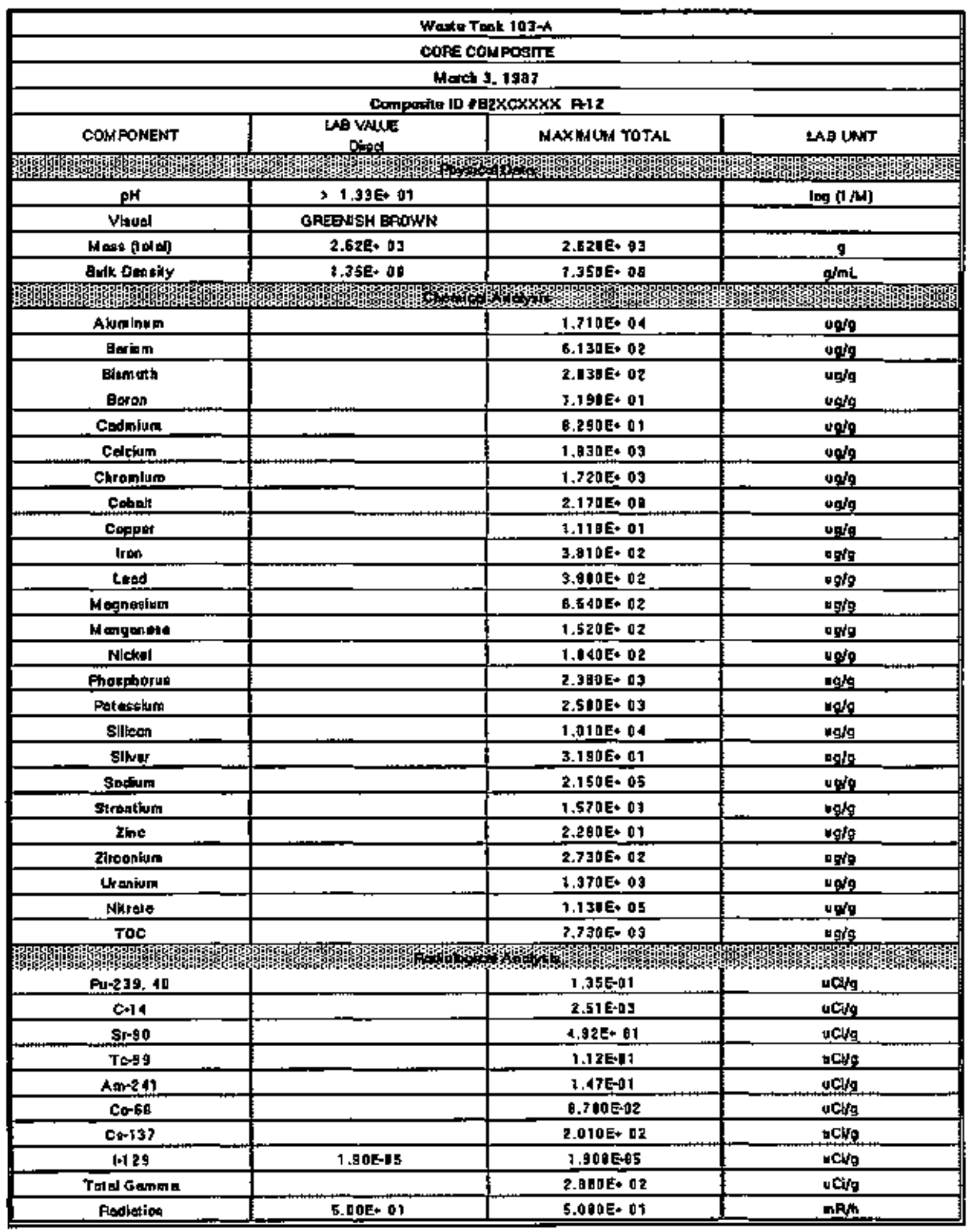




\begin{tabular}{|c|c|c|c|}
\hline \multirow{2}{*}{\multicolumn{4}{|c|}{ Waste Tank 103-A }} \\
\hline \multirow{2}{*}{\multicolumn{4}{|c|}{ Draned Sludge }} \\
\hline & & & \\
\hline \multicolumn{4}{|c|}{ August 18, 1807} \\
\hline \multicolumn{4}{|c|}{ Fackired March 9.1987} \\
\hline \multicolumn{4}{|c|}{ 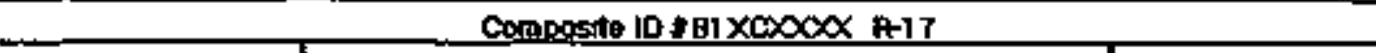 } \\
\hline COMPONENT & & & LAB UNIT \\
\hline \multicolumn{4}{|c|}{ 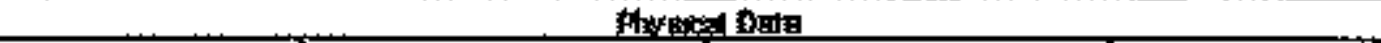 } \\
\hline & REPQRT & MAXIMUM & \\
\hline Euyk Densty & $134 E+00$ & $134 E+00$ & gim I \\
\hline \multicolumn{4}{|c|}{ 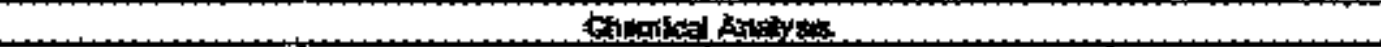 } \\
\hline Aluminum & $265 E+07$ & $255 E+07$ & g \\
\hline Barium & $046 \mathrm{E}+05$ & $85 \$ E+05$ & … \\
\hline Ensmuth & $101 E+00$ & $238 E+06$ & Q \\
\hline Boron & $518 E+04$ & $518 E+04$ & q \\
\hline cadmum & 000.00 & 12.1.E+ 05 & . \\
\hline Colatum & $254 E+06$ & $254 E+0 B$ & a \\
\hline Ghiomum. & $212 E+06$ & $212 E+06$. & 9 \\
\hline Cobatt & $207 E+08$ & $207 E+03$ & 9 \\
\hline Copper & $214 \mathrm{E}+04$ & $214 E+04$ & ㅇ. \\
\hline Iron & $511 E+05$ & $521 E+05$ & $q$ \\
\hline Loed & $123 E+06$ & $539 E+06$ & q \\
\hline Magnesintn & $1,17 E+06$ & $117 \mathrm{E}+08$ & g \\
\hline Manqanesa & $108 \mathrm{E}+05$ & $168 E+05$ & _ \\
\hline Mlckel & $181 E+05$ & $131 E+05$ & a \\
\hline Phosphons: & $312 E+0 B$ & $512 k+0 B$ & q \\
\hline Pot호헤느. & $408 \mathrm{E}+06$ & $408 \mathrm{E}+06$ & a \\
\hline Silnon & $190 \mathrm{E}+07$ & $190 E+07$ & 9 \\
\hline Silver & $2,48 \mathrm{E}+04$ & $2.79 E+04$ & 오 \\
\hline Sodium & $321 E+0 B$ & $321 E+08$ & g \\
\hline Stconitiun & $131 E+04$ & $131 E+04$ & g. \\
\hline ZnRC & $135 E+05$ & $1 \$ 6 E+05$ & s \\
\hline Zurcoruuin & $208 E+05$ & $230 E+05$ & q \\
\hline Uranaum & $2 \$ B E+06$ & $288 E+06$ & a \\
\hline Kingta & $1.81 \mathrm{E}+08$ & $181 E+08$ & a \\
\hline Toc & $128 E+0 ?$ & $12 B E+0 ?$ & q \\
\hline \multicolumn{4}{|c|}{ 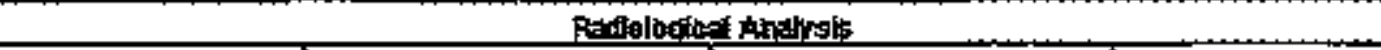 } \\
\hline Ar 239240 & $198 \mathrm{E}+02$ & $198 E+02$ & $\mathrm{C}_{\mathrm{l}}$ \\
\hline $0-14$ & $4185+00$ & $41 \mathrm{BE}+00$ & C. \\
\hline \$r-90 & $764 E+04$ & $764 E+04$ & $\mathrm{C}_{1}$ \\
\hline$T<-90$ & $194 E+02$ & $184 E 02$ & $C_{1}$ \\
\hline Afm-241 & $140 \mathrm{E}+02$ & $152 \mathrm{E}+02$ & 0 \\
\hline $\cos 6$ & $147 \mathrm{E}+02$ & $147 E+02$ & $a$ \\
\hline 0 & $320 E+05$ & $320 \mathrm{E}+05$ & a \\
\hline $1-128$ & $000 E+00$ & $000 E+00$ & D \\
\hline Totgl Gamma & $3535+05$ & $353 \mathrm{E}+05$ & ca. \\
\hline
\end{tabular}




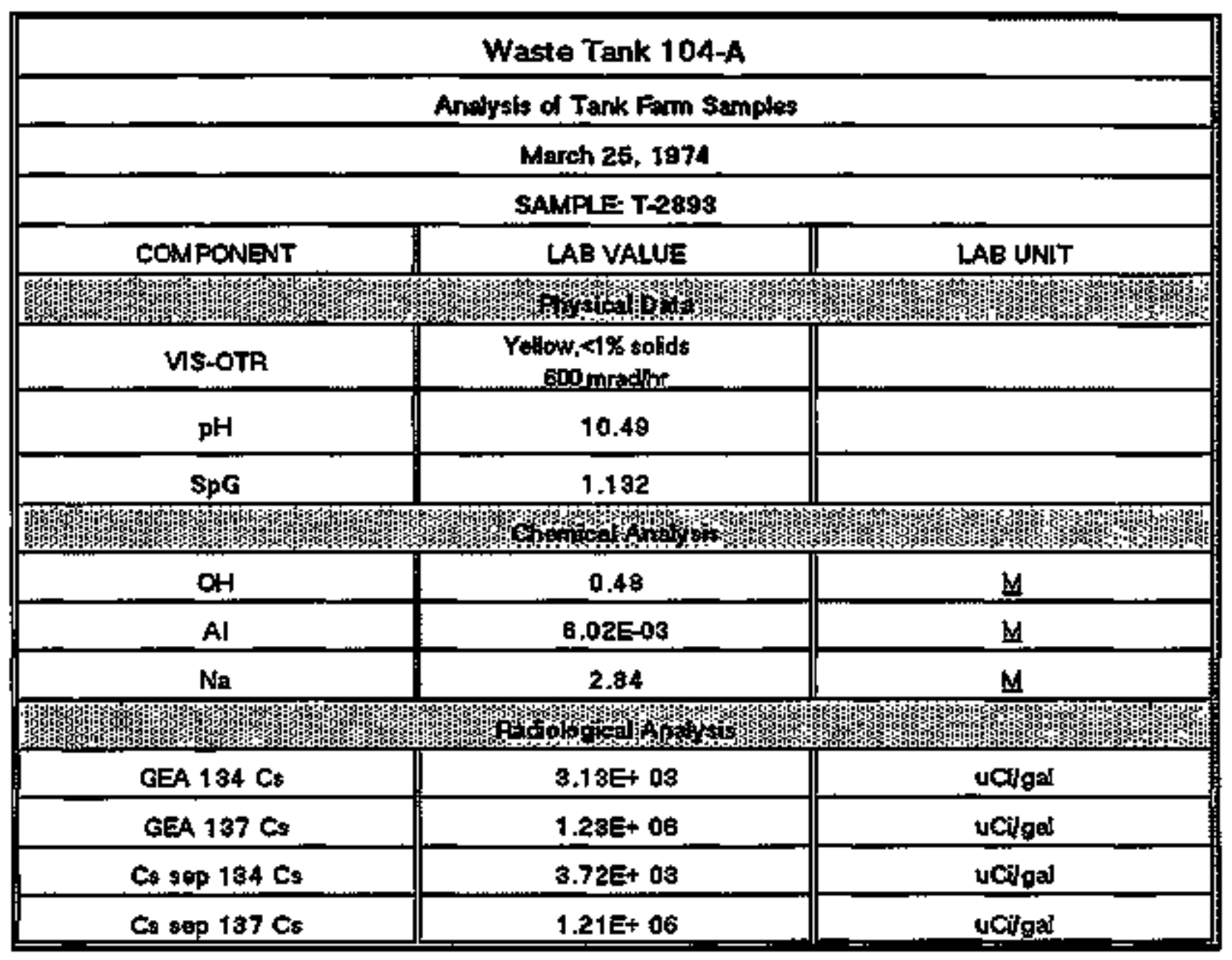




\begin{tabular}{|c|c|c|c|}
\hline \multicolumn{4}{|c|}{ Waste Tank 10 - -A } \\
\hline \multicolumn{4}{|c|}{ ANualysts OF 104A SLUDCE } \\
\hline \multicolumn{4}{|c|}{ Sepkerber 4, 1974} \\
\hline COMPONENI & LAB VALUE & LAB UNT & WEG $\%$ \\
\hline \multicolumn{4}{|c|}{ Atesoldtata } \\
\hline Densily & 1.64 & $g+c$ & \\
\hline Water & & & 42.3 \\
\hline \multicolumn{4}{|c|}{ 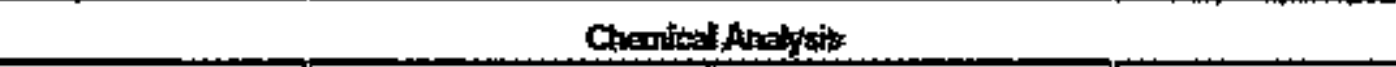 } \\
\hline Al & 2.82 & moles! & 4.6 \\
\hline s & 0.88 & motesfl & 1.4 \\
\hline $\mathrm{Fa}$ & 4.99 & medesl & 17.0 \\
\hline$M_{g}$ & 0.11 & molesil & 0.2 \\
\hline $\mathrm{Ca}$ & 0.41 & moiles & 1.0 \\
\hline Ea & 0,009 & moles!l & 0.1 \\
\hline Wh & 0.71 & moles & 2.4 \\
\hline sr & $<0.056$ & moles & \\
\hline \multicolumn{4}{|c|}{ Pedicloy ical Anatysis } \\
\hline $89+90 \mathrm{~s}$ & $4.395+07$ & الئى & \\
\hline $137 \mathrm{cs}$ & $1.15 E+06$ & بctin & \\
\hline $134 \mathrm{C:}$ & 3.33E+ 04 & vân & \\
\hline $60 \mathrm{Co}$ & $5.94 \mathrm{E}+04$ & $\mathrm{uCM}$ & \\
\hline $125 \mathrm{sb}$ & $5.95 \mathrm{~F} 05$ & ucin & \\
\hline 154 E & $1.30 \mathrm{E}+05$ & uaf & \\
\hline $239 \mathrm{Pu}$ & 0.11 & ئl & \\
\hline
\end{tabular}




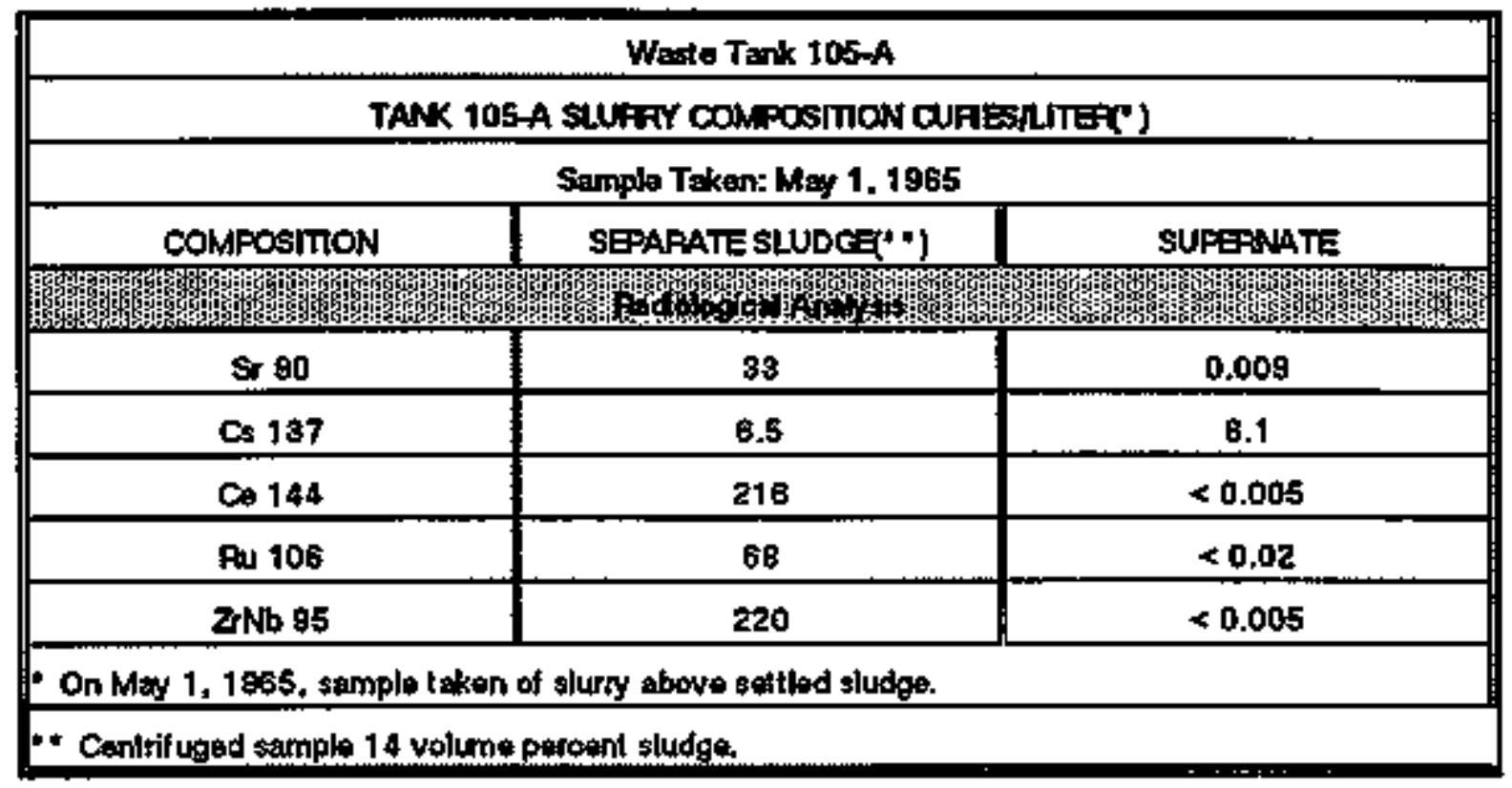




\begin{tabular}{|c|c|c|c|c|}
\hline \multicolumn{5}{|c|}{ Waste Tank 105-A } \\
\hline \multicolumn{5}{|c|}{ COMPOSFION AND DENSITY OF 105-A TANK SOLIDS } \\
\hline \multicolumn{5}{|c|}{ Jarktay 12,1872} \\
\hline \multirow[t]{2}{*}{ COMPONENT } & \multicolumn{3}{|c|}{ LAB VALUE } & LAB UNIT \\
\hline & WET SOUDS & \multicolumn{2}{|c|}{ DRY SOLIDS } & \\
\hline \multicolumn{5}{|c|}{ 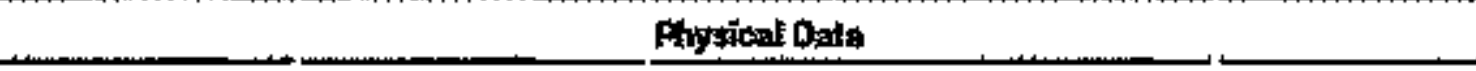 } \\
\hline \multicolumn{5}{|c|}{ Vis-OTR: hard, dry, brown lumps } \\
\hline TAP Denshy & 1.43 & \multicolumn{2}{|c|}{1.54} & $\mathrm{~g} / \mathrm{ml}$ \\
\hline Perticle Densily & 2.04 & \multicolumn{2}{|c|}{$1.22 "$} & $\mathrm{~g} m \mathrm{~m}$ \\
\hline \multicolumn{5}{|c|}{ Chombat Analy st: } \\
\hline & ANALYSTS $2 b$ & ANALYSIS 1a & ANALYS1S 1a & \\
\hline $\mathrm{Na}$ & 0.31 & 0.24 & 0.22 & do \\
\hline $\mathrm{F}$ & 0.081 & 0.058 & 0.13 & ofo \\
\hline Al & 0.089 & 0.032 & 0.046 & gla \\
\hline$M \mathbf{n}$ & 0.0026 & 0.0025 & 0.004 & alg \\
\hline si & 0.022 & 0.018 & 0.028 & glg \\
\hline \multicolumn{5}{|c|}{ 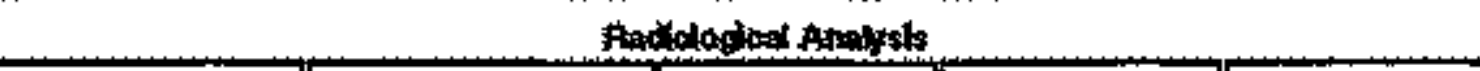 } \\
\hline Pu & $2.80 \mathrm{E}-05$ & $2.80 \mathrm{E}-05$ & $7.90 E-05$ & g/g \\
\hline 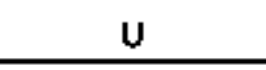 & $1.20 \mathrm{E}-0 \mathrm{~S}$ & - & - & $g / g$ \\
\hline $90 \mathrm{Sr}$ & 25.700 & 13.400 & 49,300 & uClg \\
\hline $144 \mathrm{C}$ & 2,060 & 610 & 2,900 & uCH/g \\
\hline $137 \mathrm{C}_{4}$ & 960 & 500 & 1660 & ucig \\
\hline $108 \mathrm{Ru}$ & - & 420 & 1400 & ucilo \\
\hline $125 \mathrm{sb}$ & 410 & 230 & 540 & uCl/g \\
\hline $154 \mathrm{Bu}$ & 160 & 85 & 290 & ucilo \\
\hline
\end{tabular}




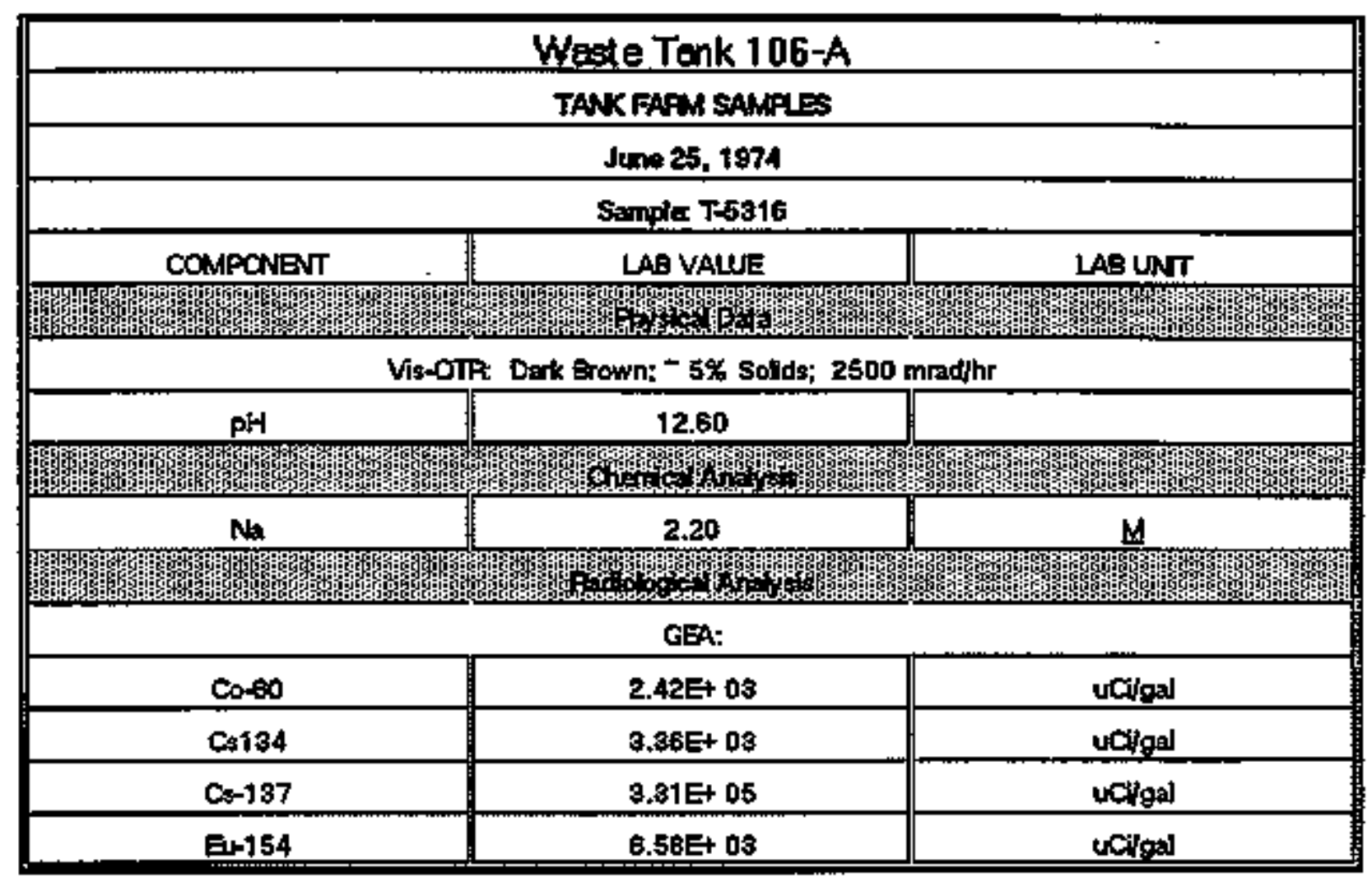




\begin{tabular}{|c|c|c|}
\hline \multicolumn{3}{|c|}{ Woste Tank 106-A } \\
\hline \multicolumn{3}{|c|}{ TANK FAFM SAMPLES } \\
\hline \multicolumn{3}{|c|}{ Dale receivect Otcher 27, 1979} \\
\hline \multicolumn{3}{|c|}{ Sarple T-4818 } \\
\hline COMPONENT & LAB VALUE & LAB LNT \\
\hline \multicolumn{3}{|c|}{ 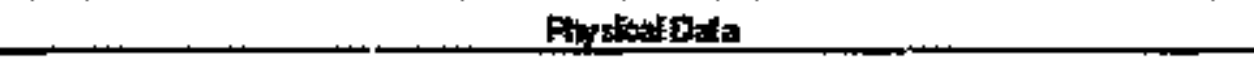 } \\
\hline \multicolumn{3}{|c|}{ Vis-OTR: Clear yellow; to solfés; $190 \mathrm{mrad}$} \\
\hline $\mathrm{pH}$ & NR & \\
\hline $\mathrm{SpG}$ & 1.194 & \\
\hline HeO & 75.48 & w \% \\
\hline \multicolumn{3}{|c|}{ Chería:Andx } \\
\hline Al & $7.24 E-01$ & M \\
\hline OH & 1.01 & $M$. \\
\hline Nos & $1.3 \$ 1$ & $M$ \\
\hline $\mathrm{NO2}$ & 0.739 & $M$ \\
\hline $\mathbf{P O 4}$ & 8.69E-02 & $\mathrm{M}$ \\
\hline \multicolumn{3}{|c|}{ 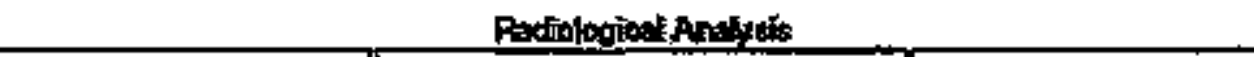 } \\
\hline Pm 147 & $\mathbf{M}$ & ucil \\
\hline Sr 89.90 & $2.70=04$ & uCA \\
\hline Pu & 2.87505 & gll \\
\hline CEA $C_{5-137}$ & $1.78 E+05$ & ucll \\
\hline
\end{tabular}




\begin{tabular}{|c|c|c|c|}
\hline \multicolumn{4}{|c|}{ WASTE TANK 106-A } \\
\hline \multicolumn{4}{|c|}{ Anahysis of Tank tob-A Fead Liqua } \\
\hline \multicolumn{4}{|c|}{ Mar 23, 1980} \\
\hline \multicolumn{4}{|c|}{ Sample: T-12* } \\
\hline CONPONENT & LAB VALUE & LAB UNT & Wh \\
\hline \multicolumn{4}{|c|}{ 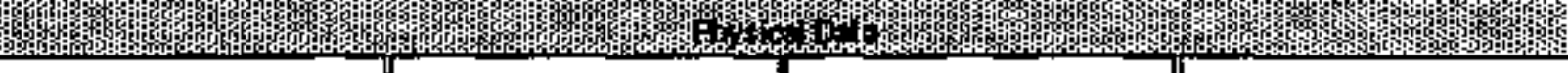 } \\
\hline \$pG & 1.39 & olm & \\
\hline $\mathrm{H} 20$ & & & $61.9 \%$ \\
\hline \multicolumn{4}{|c|}{ 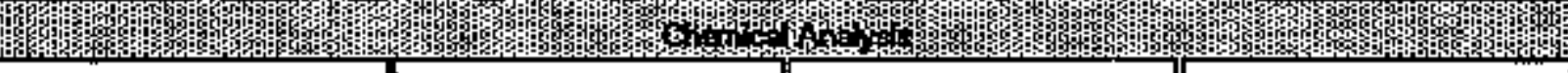 } \\
\hline NaAlo2 & 1.00 & Molarty (MR) & 5.49 \\
\hline $\mathrm{NaOH}$ & 2.35 & Molavily (ML) & 6.80 \\
\hline $\mathrm{NaNOO2}$ & 3.10 & Molarily (M) & 15.41 \\
\hline NaNos & 1.40 & Mokarily (MH) & 8.57 \\
\hline $\mathrm{Na2}$ & $0.90 " \cdot$ & Molarily (M/L) & 6.87 \\
\hline $\mathrm{Na3PO4}$ & 0.129 & Molarliy (M니) & 1.52 \\
\hline \multicolumn{2}{|l|}{ Total } & & 106.88 \\
\hline \multicolumn{4}{|c|}{ 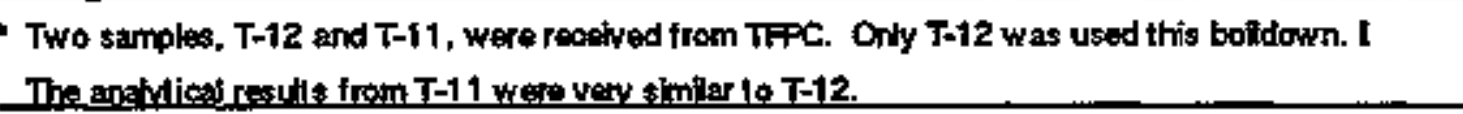 } \\
\hline
\end{tabular}




\begin{tabular}{|c|c|c|c|}
\hline \multicolumn{4}{|c|}{ Maste Tank 106-A. } \\
\hline \multirow{2}{*}{\multicolumn{4}{|c|}{ Core Composil: }} \\
\hline & & & \\
\hline \multicolumn{4}{|c|}{ 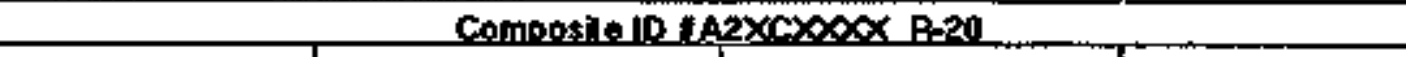 } \\
\hline COMPONENT & $\begin{array}{l}\text { LAB VALLE } \\
\text { DEECT }\end{array}$ & MAXIMUM TOTAL & LAB UNST \\
\hline \multicolumn{4}{|c|}{ 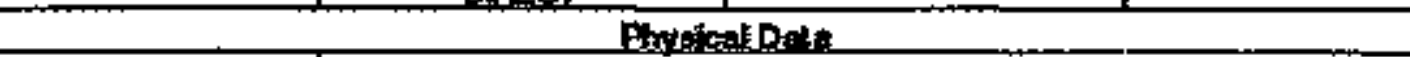 } \\
\hline V:나에 & \multicolumn{3}{|c|}{ DARK CHOCOLATEWITH A SANDY TIXX } \\
\hline Bulk Densily. & $1.54 E+00$ & $1.54 E+00$ & almL \\
\hline $\mathrm{pH}$ & $1.20 E+01$ & & $\log (1 / M)$ \\
\hline Aluminurn & & . & molo \\
\hline Barium. & & $2.355+03$ & ungla \\
\hline BismLth & & 2.75E+ 02 & yola. \\
\hline Boron & & 1.BGE+ 01 & ugth \\
\hline Cadmisum & & $4.595+01$ & ugla \\
\hline Calcium & & 5.7 gE +03 & ugin. \\
\hline Chromingm & & 5.5.E+ 03 & uels \\
\hline Cobalt & & $1.62 E+01$ & 4.당 \\
\hline Copper & & $1.02 E+02$ & 19. \\
\hline Iron & & $3,48 F+04$ & لofle \\
\hline Load & & $1.47 E+08$ & ugla \\
\hline $\begin{array}{l}\text { Megnesium } \\
\text { Manganese }\end{array}$ & & $\begin{array}{l}3.05 E+03 \\
2.00 E+03\end{array}$ & ugig \\
\hline Nickol & & $8.16=02$ & 1atio \\
\hline Phosphoris & & $1.78 E+04$ & ugle \\
\hline Polassium & & $2.15 E+03$ & unig \\
\hline Silicon & & $4.03 E+04$ & letels \\
\hline Siker & & $3.93 E+02$ & upla \\
\hline Sodilum & & $1.79 E+05$ & unido. \\
\hline Strontium & & $8.26 \mathrm{E}+01$ & vols \\
\hline Thropinitum & & $1605+09$ & unde \\
\hline TOC & & $7.15 E+03$ & chald \\
\hline Nitrate & & $7.505+04$ & ungle \\
\hline Uranium & & 1.67E+ 02 & ugfo \\
\hline Pu 239 . 240 & & $2.85 . E+00$ & UCStio \\
\hline $\mathrm{c}-14$ & & $5.93=04$ & ucilg \\
\hline Sr-90 & & $1,49 F+03$ & $\triangle C D$ \\
\hline To-99 & & $\{.09 E-0.1$ & ucilg \\
\hline Am-241 & & $1.01 \mathrm{E}+0 \mathrm{O}$ & بclis \\
\hline 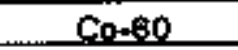 & & 9.36E-01 & ucilg. \\
\hline$C_{5-137}$ & & $9.19 E+01$ & uclio \\
\hline $\mathrm{l}-12 \mathrm{~g}$ & $1.40=-04$ & 8.20E-05 & ucla. \\
\hline Tolal Grimma & & $1,215+02$ & ucilig \\
\hline Aadịtion & $1.00 E+08$ & $100 E+03$ & $\mathrm{mPlh}$ \\
\hline
\end{tabular}




\begin{tabular}{|c|c|c|c|}
\hline \multicolumn{4}{|c|}{ Wate Tonk 106-A } \\
\hline \multicolumn{4}{|c|}{ Core Composile } \\
\hline \multirow{2}{*}{\multicolumn{4}{|c|}{$\begin{array}{c}\text { Janvary } 5,1988 \\
\text { COMPOSTIE ID } 1 A 1 \times C \times 0 \times \text { R-17 }\end{array}$}} \\
\hline & & & \\
\hline COMPONENT & $\begin{array}{l}\text { LAB VALLE } \\
\text { DIBECT }\end{array}$ & MAXAMUM TOTAL & LAB UNIT \\
\hline 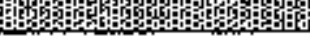 & 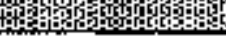 & 5 & \\
\hline Vișugl & \multicolumn{3}{|c|}{ WCAYIDK \& LTER } \\
\hline Mass Antail & $4.7 A F+02$ & $4.78 E+02$ & ? \\
\hline Bulk Donsity & 1.56⿸厂二 00 & $1.56 \mathrm{E}+00$ & $\mathrm{~g} / \mathrm{ml}$ \\
\hline $\mathrm{PH}^{\mathrm{H}}$ & 1.20 +01 & & $\log (1 / \mathrm{M})$ \\
\hline Aluminum & & $1.92 F+04$ & ugin \\
\hline Barium & & $1.95 E+08$ & Uefa. \\
\hline Eismulh & & $1.88 E+02$ & 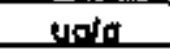 \\
\hline Poron & & $1.90 E+01$ & ugig \\
\hline Cadmium & & $3,11 E+01$ & 4019 \\
\hline Calcium & & $4.76 E+03$ & unto \\
\hline Chromlum & & $4.55 E+08$ & ugfta \\
\hline Cobalt & & 1.08E+01 & uglo \\
\hline Cogner & & B.54F+ 01 & noto \\
\hline Iron. & & $1.79=+04$ & uglg \\
\hline Lead & & $6.91 E+02$ & ugin \\
\hline $\begin{array}{l}\text { Magnestum } \\
\text { Manganeste }\end{array}$ & & $\begin{array}{l}2.62 E+08 \\
1.91 E+08\end{array}$ & $\begin{array}{l}\text { ugig } \\
\text { uolg }\end{array}$ \\
\hline Niokel & & 4. $285+02$ & -ugito \\
\hline Phasphonss & & 1.73E+ 04 & 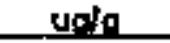 \\
\hline Potestium & & $2.35 E+00$ & Uetg \\
\hline Silipon & & 3. $655+04$ & yola \\
\hline Silver & & $1.71 E+0 p$ & wolg \\
\hline Sodium & & $6.48 E+04$ & unio \\
\hline $\begin{array}{l}\text { Strontium } \\
\text { Zno }\end{array}$ & & $\begin{array}{l}4.19 E+01 \\
3.70 E+00\end{array}$ & $\begin{array}{l}\text { ugig } \\
\text { unigl }\end{array}$ \\
\hline Treonium & & $8.49 E+02$ & engig \\
\hline TOPC & & $6.23 E+0.9$ & usig \\
\hline Nitmate & & B.1.2E+04 & ugio \\
\hline & & & 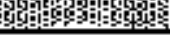 \\
\hline Uranium & & $1.44 E+09$ & ugilg \\
\hline Pu289.240 & & $1.295+00$ & 는ㄱㅇㅛ \\
\hline c-14 & & $1.02 E-03$ & uci/g \\
\hline St-90 & & $8.44 E+02$ & yciff \\
\hline Tose & $=$ & $1.15 \bar{E}=01$ & uCila \\
\hline Am $=241$ & & $0.97 E 01$ & y Give \\
\hline $\mathrm{Com}_{0} 60$ & & $4.72 E 01$ & LCsiln \\
\hline C5-137 & & $2.03 E+02$ & uCla \\
\hline $1-129$ & $1,40=04$ & $1.40=-04$ & uCilg \\
\hline Tolal Gamma & & $2.23 E+02$ & ucilg \\
\hline Radiation & $3.50 \mathrm{E}+02$ & $3.50 \mathrm{E}+02$ & minth \\
\hline
\end{tabular}


TCRC-3

Atmatic wild Honford Company

WHC-SD-WM-ER-308, Rev. D

Alol.

il

Date: June 25, 1974

To:

From:

R. L. Walser

Subject: TAAK FARM SAHPLES

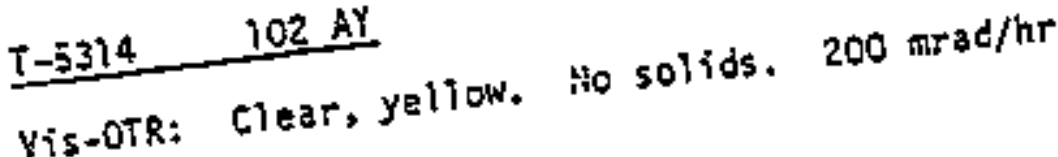

pH:

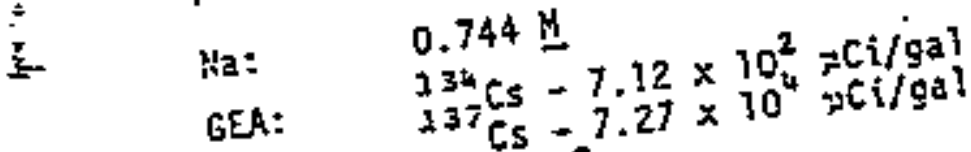

$T=5315-101 A$

pit: $\quad 11.15$

Ha: $\quad 3.60 \mathrm{H}$

GEA:

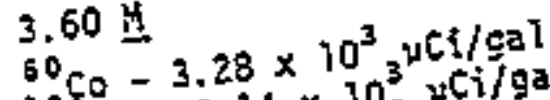

$125_{5 b}-8.14 \times 10_{3}^{3} \mathrm{pCi} / \mathrm{ga}$

$374 \mathrm{Cs}-7.27 \times 10_{5} \mathrm{yCi} / \mathrm{ga}$

$1377_{\mathrm{CS}}-7.04 \times 10_{3}^{5} \mathrm{vilgal}$

$35 \mathrm{Eu}-4.69 \times 10^{3} \mathrm{pCi} / \mathrm{gz}$

$3500 \mathrm{argd} / \mathrm{hr}$

T-5315 $106-\mathrm{A}$

Vis-OTR: Dark brown, $-5 \%$ solids. 2500 arad/hr

ph:

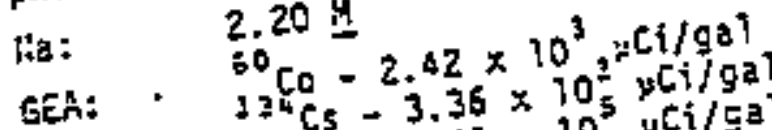

12.60

GEA: $\quad 124 \mathrm{Cs}-3.36 \times 10_{5}^{2} \mathrm{yCi} / \mathrm{gal}$

$13 \mathrm{CS}^{-3}-3.31 \times 10^{5} \mathrm{pCi} / \mathrm{Ga}$
$\left.15 \mathrm{Cs}-3.38 \times 10^{3} \mathrm{yC} / \mathrm{ga}\right]$

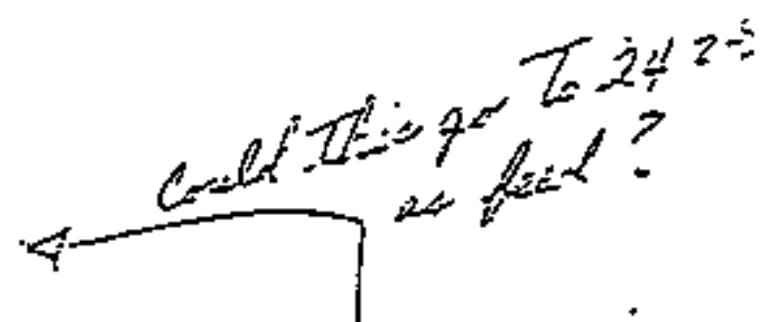

REW:jd

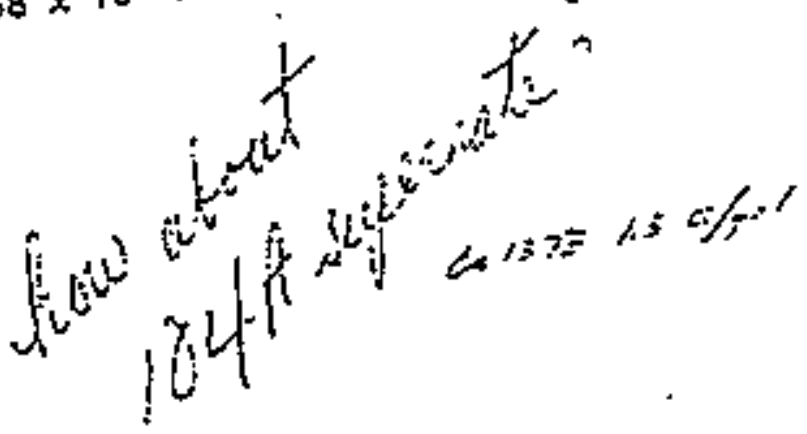


Date: December 17,1974

To: R. L. Walser

Fron: R. E. Wheeler forthueln

Subject: AMALYSIS OF TANK FARN SAMPLES

SAMPLE: T-8936 10]-A Rec. 10/14/74

T.8936 'lot-A

Vis-OTR: Reddish, brown.

$5 \mathrm{Rad} / \mathrm{he}$

pH: $\quad>9.8$

Spt: $\quad 1.2890$

OH: $\quad 0.525 \mathrm{M}$

A]: $2.52 \times 10^{-2}$ 프

Na: 2.40 픈

$\mathrm{NO}_{2}: 0.252 \mathrm{H}$

$\mathrm{NO}_{3}:-2.96 \mathrm{H}$

Pu: $\quad 1.65 \times 10^{-2} \mathrm{gm} / 921$

פTA: Large exotherm at $240^{\circ} \mathrm{C}$

50. : Cancelled by R. L. Walser

$\mathrm{PO}_{4}: \quad 1.72 \times 10^{-2} \underline{\mathrm{H}}$

F: $\quad 1.65 \times 10^{-3} \underline{M}$

$\mathrm{CO}_{3} ; \quad 0.402$ 프

GEA: $\quad{ }^{134} \mathrm{Cs}=2.88 \times 10_{5}^{3} \mathrm{HCi/ga1}$

Q9, $90^{2} ;: 9.99 \times 10^{5} \mathrm{y}[\mathrm{i} / \mathrm{gaj}$

-.. Hater: $69.31 \% \cdots . . .-$

Nhis: $\quad<7.15 \times 10^{-3} \underline{M}$ Cooling eurve: $40^{\circ} \mathrm{C}$ for $60^{\circ}$.min. Ho solids.
$35^{\circ} \mathrm{C}$ for 45 min. No solids. $30^{\circ} \mathrm{C}$ for $45 \mathrm{~min}$. No solids. $25^{\circ} \mathrm{C}$ for 45 min. Ho solids. $20^{\circ} \mathrm{C}$ for $45 \mathrm{~min}$. No solids. $15^{\circ} \mathrm{C}$ for 45 min. No sotids. $10^{\circ} \mathrm{C}$ for 45 min. No solids. $5^{\circ} \mathrm{C}$ for 45 min. Ho solids.

RDW:jd

te: ds suckingham

WR Christensen

RM Ginestet

RE Wheeler

JC Womack. 
Atlantic Richfield Hantord Cormpary

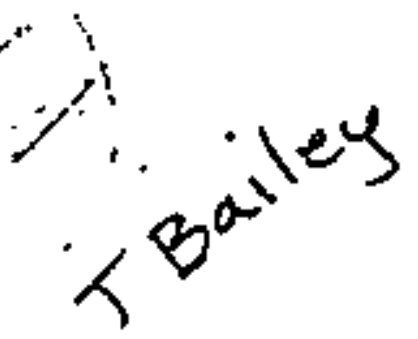

Date: Agrif 9,1976

To:

D. H. Miyasaki

$\nabla$

From:

J. E. Hotะon

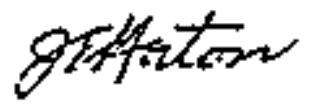

Subject: ANALYSIS OF 201-ג TANK RESIDUAD SLUDGS IOI-A TANK

A sample from Tank 101-A was rectived on April I, 1976. This is the fisst samplo of a series to be taken from Tank IOI-A. The sample had courge granular-1ike crystals "iike sand" intermixed with small partioles and hard chunks about the size of a quartis. The hard chunks would not soften in water. The course granular crystals were grayish in color, while the snall particles vere redaish brown.

The calculated heat generation rate for the 101-A sludge, based on $89+9055$ and $137 \mathrm{cs}$ content was $1.30 \times 10^{-2}$ watts/1iter siudge. The chemical andysis showed that thers was about three tines es much aluminum as silicon.

sludge analyses were made by fusing 2.0 miliiliters centrifuged as received solids with KOH, and dissolving the melt with concentrated HCl and alluting to a volume of $500 \mathrm{milliliters}$ with water. Analyses are ropozted in Table I. If you have any guestions, please call me.

JE到:jf

スヒさ。

ce: $w / a t+$,

H Babad

J5 guckingham

LQ Fong

DG Harlow

DC Lini

ORH Rasmigsen

RC Roal

He Shaw

RJ Thompson

RL Walser

Process Aild (7)

File

IB 


\section{TABLE}

ANALYSTS OF 101-A SUUDGE 푼 3901

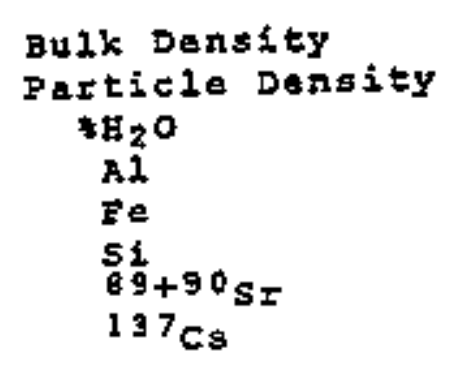

$1.365 / 40$

2.78 g/te

9.1

9.95 moles/1iter

0.5 moles/liter

3.95 noles/liter

$1.85 \times 10^{6}$ HCi/liter $=1.26 \times 10^{-2}$ watts/liter

$2.68 \times 10^{5}$ jci/liter $=1.28 \times 10^{-3}$ watts/liter

$$
\therefore \frac{1+2}{\operatorname{lin} 400}
$$




$$
\begin{aligned}
& \text { IL L r L - J } \\
& \text { Atlentic Fichfield Henford Compon } \\
& T C P C-8
\end{aligned}
$$

WHC-SD-WM-ER-308, ReV. 0

Date:

To:

From

sobject:

Ref arence:
April 30,1976

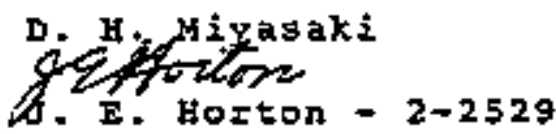

ANALYSES OF ADDITIOHAI RESIDUAL SIUDGE SAMPLES FROK TANX 1O1-A TANK IOI-A

Letter, April $\%, 1976, J$. E. Horton to D. H. Miyasaki, "Analysis of 10l-A Tanx Restiual siudge"

Chemscel Technology Iaboratory received a request For strontium and cesium analysis to doteriolse heat genezation in rosidual sludgo from Tank lol-n.

A number of samplos weze taken ftom taxk 101-A and idestified as follows. Sample No. 4355 riser 19 north, No. 4365 ol3 sluice pit, No. 4364 riger 19 southside (did not have enough solids to analyza), No. 4554 riser 19 , and No. 4555 riser 20.

The calculated heat genezation rate for samplo No. 4355 fiset 19 north, based on $89+9055$ and $137 \mathrm{Cs}$ content was $1.78 \times 10^{-1}$ wats/21ter.

The falculated heat generation rate for sampla No. 4365 ola 514 ç 马it, basad on $9+90 \mathrm{~s}$ and $137 \mathrm{Cs}$ content was $1.60 \times 10^{-1}$ watts/litez.

The calculated heat genezation rate for samole No. 4554 R29, based on $89+90^{\circ}$ and $137 \mathrm{Cs}$ contant was $4.11 \times 10^{-2}$ watts/liter.

The calculatod heat ganeration fate for sample No. 4555 R20, based on 94+50s and 137 cs content was $1.07 \times 10^{-1}$ watts/litex.

Antiyste were made by fusing a known volume of solids sxom ach sample with xoH, dissolving each melt with concentratad HCI, and diduting each sample to a known volute with water. Aralyses for all samples are reported in the tollowing tablo. 
D. H, Miyasaki

Fage 2

Apxil 30,1976

TABLE I

ANALYSIS OF RESIDUAL SLUDGES FROM TANK 101-A

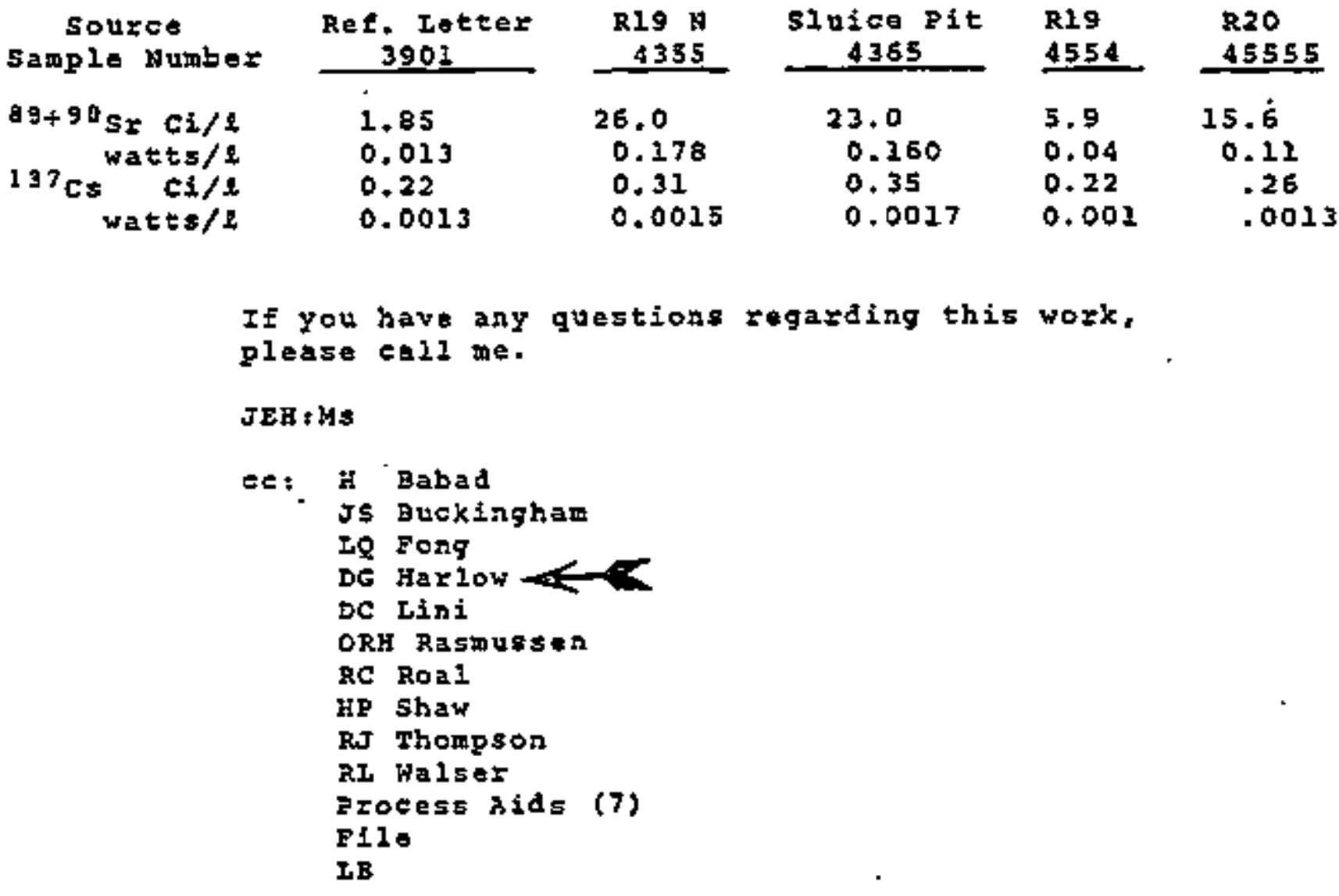

H-63 
TCRC-B Internal Lette:

$$
\begin{aligned}
& \therefore \text {. WHC-SD-WM-ER-308, Rev. } 0
\end{aligned}
$$

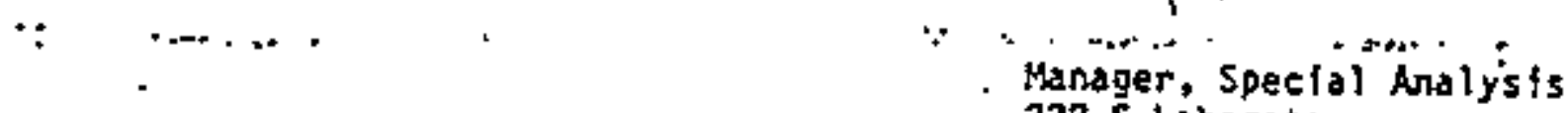

$$
\begin{aligned}
& \begin{aligned}
-. \quad & .222+5 \text { Laboratory } \\
. & 200 \text { Hest Area } \\
& 2-2449
\end{aligned} \\
& \therefore \text { Analyses of Tonk Fara Safoles } \\
& \text { Serial No. } T-3970 \text { Tank } \frac{1 C 1-A}{7 A n K / 0 /-A} 242-A B
\end{aligned}
$$

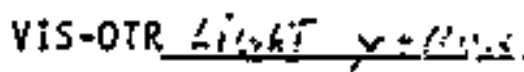

ist nirdithr

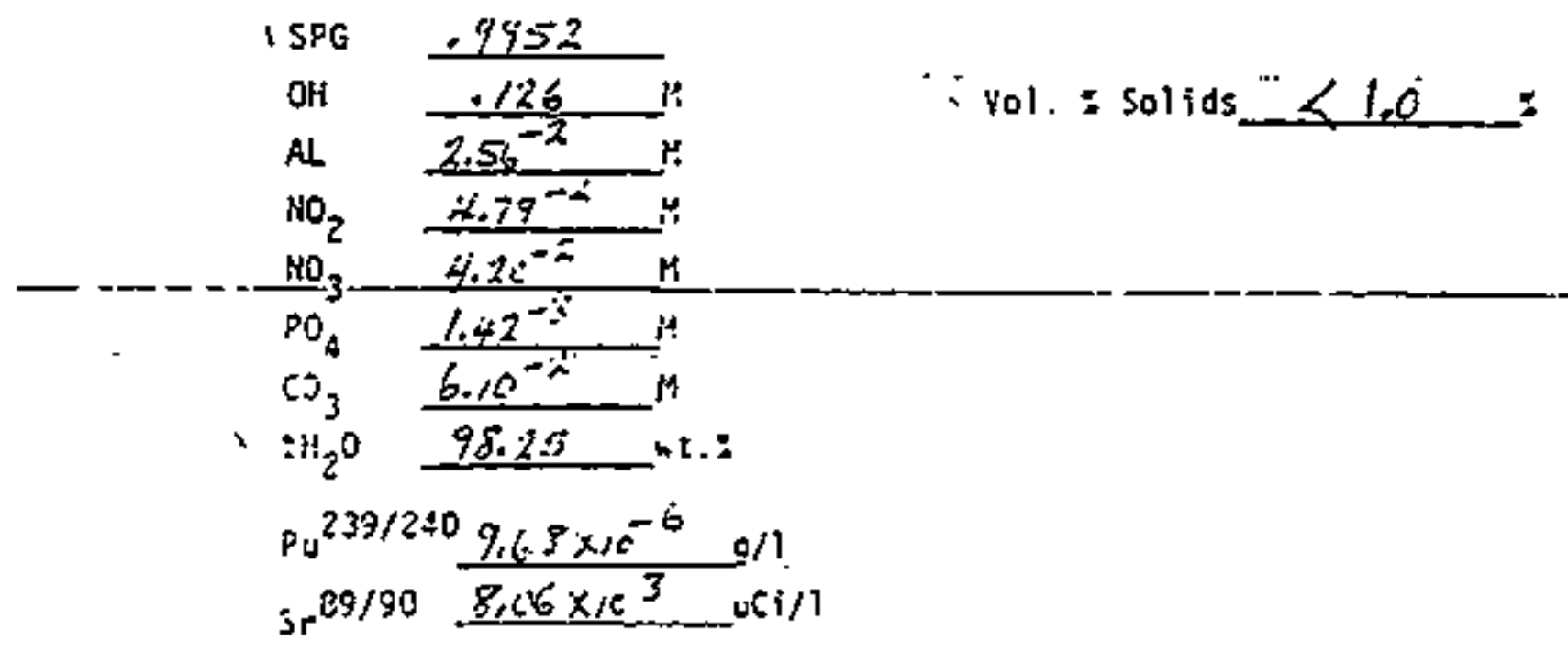

GEA

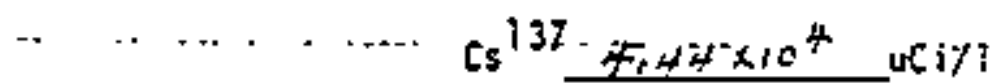

Cs 134 $u[i / 2$

$\mathrm{Co}^{60}$ ufi/.

$E^{154}$

$u C 1 / 1$

$\operatorname{CePr}^{144}$

biti?

$50^{125}$

uti/l

$u C i / 1$

H-64 


\section{1
$T C R C-16$ \\ Internal Letter}

Ost4. Novenber 2, 1979

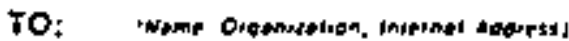

. H. J. Eding

- Waste Processing Technology

. 2750-E, 200 East
WHC-SD-WM-ER-308, Rev. 0

no. 65124-79-005

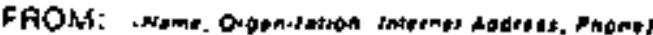

C. H. Delegard.

Chemical Sciences Group

222-5, 200 West

$2-1571$

suopect: Hot Boiłdown of Tank 101-A Waste Sample

Ref: Letter, October 16, 1979, K. J. Eding to C. K. Delegard, hot Boildowns of Wastes in Tanks 10I-A and 101-AY

In response to your referenced letter, a hot boildown of Tank 103-A waste was done. Boiling point versus pressure data were requested and are presented in Jable 1. The boildown was conducted at 40 Torr pressure. Nucleation occurred at 77 percent WVR!' At this point, the slurry was about 50 volume percent solids. Upon cooling to room temperature, the slurry became about 90 volume percent solids. Centrifugation compacted the soljids resuiting in a slurry of about 45 volume percent solids. No further WVR was done for this feed. The solid particles were fine, but settled readily. This behavior is characteristic of non-complexed feed.

The composition of the feed, the product liquor, and the product 501 ids are shown in table II. The soljos seem to be entirely $\mathrm{HaNO}_{2}$. This analys is is suspect oue to the relatively low concentration of $\mathrm{NaNO}_{2}$ in the original feed.

Further analyses of the solids are not possible; however, the solids are toore likely to be $\mathrm{NaNO}_{3}$, not $\mathrm{NanO}_{2}$.

Please call me if you have any questions regarding this work.<smiles>C=CC(C)CCCC</smiles><smiles>CC(C)(C)C</smiles>

C. H. Delegard, Aúvanced Chenist

Waste Chemistry Unit

- CHD:jkr

- Attachments 2

Information: d. S. Buckingham

G. T. Dukelow

F. M. Jungfleisch (w/o attach)

T. A. Lane

D. L. Merrick

R. D. Prosser

Process Aids (8)

File Code: KFSSE 
Table I

TEMPERATURE AND PRESSURE AT BOILING VERSUS WVR FOR TANK 1OI-A WASTE LIQUOR

Botling Point

\section{Percent WVR}

0

50

60

70

leation

end point)
Temperature $\left({ }^{\circ} \mathrm{C}\right)$

35.4

Pressure (Torr)

42.6

40

48.0

80

37.0

40

44.2

60

50.0

80

38.0

40

46.2

60

52.4

80

40.4

40

48.4

60

54.8

80

44.2

40

51.8

60

58.0

80 


$$
\begin{aligned}
& W H C-S D-W M-E R-308, \text { Rev. } 0 \\
& \text { HIO } 1.5
\end{aligned}
$$

Tabie II

ANALYSES OF FEED AND PROOUCT SLURRY

\begin{tabular}{|c|c|c|c|c|}
\hline Component & $\begin{array}{c}\text { Feed } \\
\text { Molarity } \\
\end{array}$ & Molaritty & $\begin{array}{l}\text { Liquor } \\
\text { Weight Percent }\end{array}$ & $\begin{array}{l}\text { Product Solid } \\
\text { Weight Percent }\end{array}$ \\
\hline $\mathrm{NaA1O}$ & 0.274 & 1.26 & 7.50 & $-0-$ \\
\hline $\mathrm{NaOH}$ & 0.518 & 2.31 & 6.70 & $-0-$ \\
\hline $\mathrm{NaNO}_{2}$ & 0.0772 & 1.56 & 7.82 & 104.5 \\
\hline $\mathrm{NaNO}_{3}$ & 0.421 & 2.27 & 14.00 & $-0-$ \\
\hline $\mathrm{Na}_{2} \mathrm{CO}_{3} \cdot \mathrm{H}_{2} \mathrm{O}$ & 0.0610 & 0.750 & 6.75 & $-0-$ \\
\hline $\mathrm{Na}_{3} \mathrm{PO}_{4} \cdot 12 \mathrm{H}_{2} \mathrm{O}$ & 0.02117 & 0.037 & 0.98 & $-0-$ \\
\hline$T O C *(g / 1)$ & -- & 20.0 & 1.45 & $-0-$ \\
\hline $5 \rho G(g / m 1)$ & 1.052 & j. 378 & -- & - \\
\hline $2^{0}(w \mathrm{wt})$ & 96.70 &.$^{--}$ & 50.25 & $-0-$ \\
\hline Total & & & 95.45 & 104.5 \\
\hline
\end{tabular}

Tank 101-A

Total Organfe Carbon 
TCKC-14

linternal Las

WHC-SD-WM-ER-308, ReV , 0

;

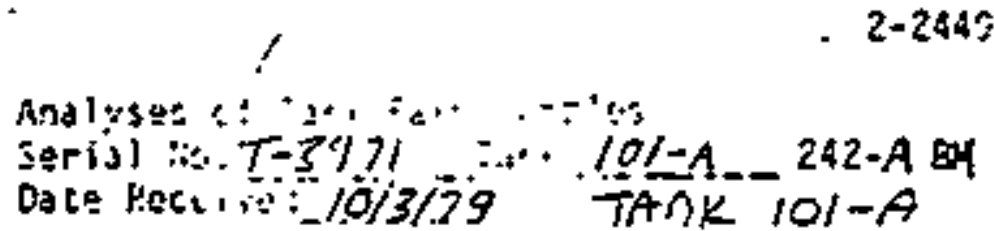

- llanager. Spectal Analists

$\left.A\right|^{0^{2 \alpha \nu^{-}}}$

A $\left.\right|^{0}$ 222-5 Laboracory
$222-5$ Laboracor
200 kase frod

- 2-2443

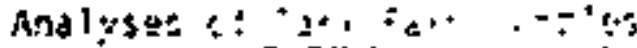

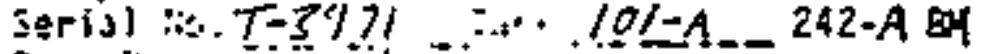

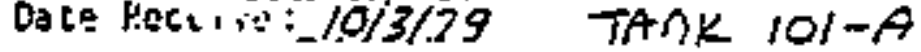

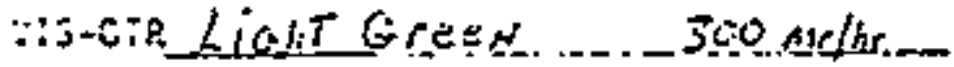

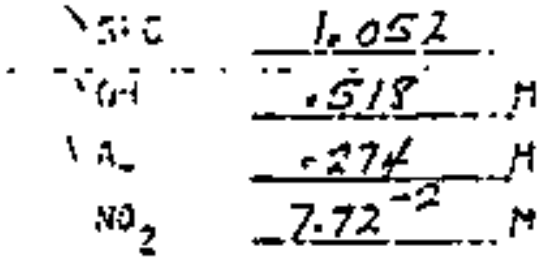

$\mathrm{NO}_{2}=-\frac{.421}{2.1 \hat{A}^{-3}}-\frac{M}{M}$

$0_{2} \quad-0.10 \div \bar{*}$

$. \pi_{2} \mathrm{O}-.96 \cdot \mathrm{I}_{-} . w \%$

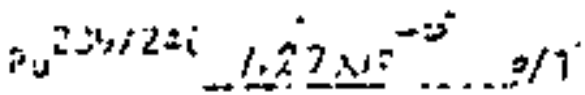

$\therefore 2.9 \mathrm{~J} \quad-159.10 \%$ \% $61 / 7$

GEA.

$\mathrm{cs}^{15} .2 .08 \times 1 c^{5} \mathrm{u} i / 1$

(i):

$: 0^{6}$

$\therefore+$

G.:.: :.:

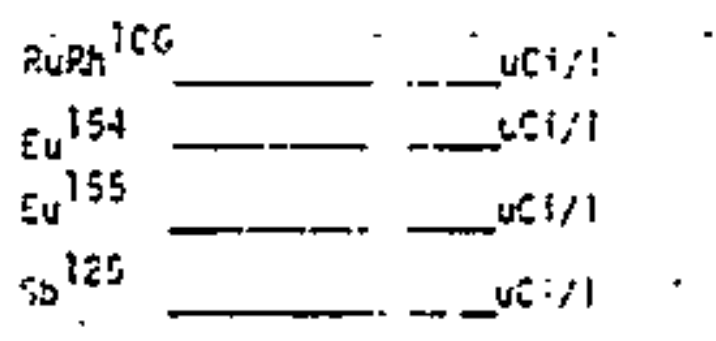




\section{TCRC-17 \\ iniernal Letter}

sstet * August 22, 1980

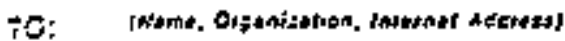

- M. C. Teats

- Plant Engineering

- 2750E/200E

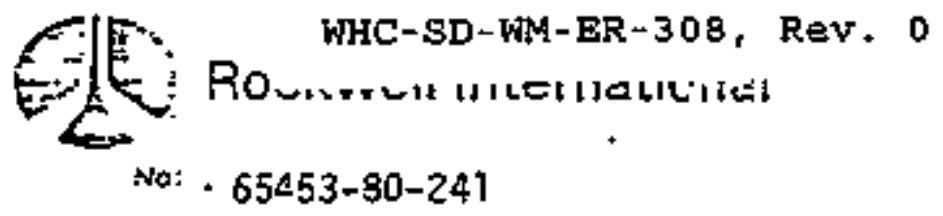

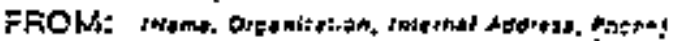

- M. T. Jansky

- Separations Process Development

- 2225/M0-037/200H

- 3-1571

5-opel: : 101A Hot Boildown

A hot bofldown has been completed on waste samples from Tank 101A. The ideste was a dilute complexed waste which has been cross-site transfered. Final nucleation occurred at 50 percent waste volutie reduction (WVR). The final product appeared to be composed of two solid phases most itkely offferentiated by crystal sizes. Kigh Performance Liquid Chromatography (KPLC) techniques did not find sufficient. EOTA or HEDSA concentrations to explain the "compiexed" nature of the waste. There may be sone other "complexant" present interfering with the desired nucleation behavior of the waste. Possible candidates are degradation products from HEDTA and/or ETTA destruction, citric acid, or some other organic carbon source. Labaratory details are. dişucssed below.

Tto samples from Tank ioiA, T-2691 and T-2692, were analyzed by Analyited Laboratories. The results are shown in Table $1 . A$. 50 fi composite was prepared for a hot boi ldown. The dark brown solution made determination of the nucleation point difficult. At 30 percent wV, a slight color chence was noticed, and at 40 percent WWR the color was even darker: At jo cercent WR, however, the sample suddeniy turned a "pea soup green," indicating final nucleation. WVR versus temperature data are shown in Table II. The sroduct was allowed to 5 tand for approximately one hour, when 95 volume Fercent solids were observed. Aliquots of mixed product were centrifuged. ihe centrifuged product contajned 48 volume percent supernatant, 27 percent solid phase A, and 25 percent solid phase B. The phases A and B were distinguished visually, apparently having two different crystal sizes. The, supernatant was separated from the solids, and the two phases were submitted to AL Tor analyses. Note that the solids were easily poured into sample vials. The product analyses are shown in Table III. A mass balance of the solids only eccounted tor $70 \%$ of the weight, based on AL andyses. The ralues shown in Table Ifl for soljd wejght percent are normalized to joos.

in aliquot of the 50:50 composite wes subritted for complexant analyses using HPLC. The results are shown in iable IV. Hote the large discrepancy ir $g / \mathrm{L}$ it the totai organic carbon (JOC) is attributed solely to HEDTA/ETTA. There acy be some other source of organic carbon, such as citrete or YEDTA/EOTA degradation prodtucts. 
H. C. Teats

WHC-SD-WM-ER-300, Rev, 0

Page 2

August 27, 1980

Please call if you have any questions regarding this work.

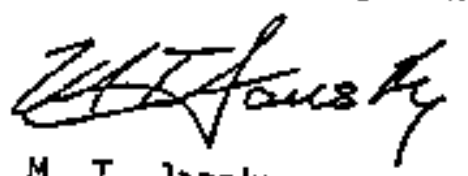

M. T. Jansky

Chemist

MTJ/pap

ce:

D. E. Bowers

l. C. Brown

J. S. Buckinghan_J $B$

H. J. Eding

L. A. Gale

T. A. Lane

H. E. Ogres

T. B. Venezizno

File code KF5 SE

Process Aids (8)

I 


\section{TABLE I}

ANALYTICAL LABORATORIES RESULTS OH TAYK YOIA WASTE SAMPLES

tomponent

A1.

OH:

$\mathrm{NO}_{3}$

$\mathrm{NO}_{2}$

$\mathrm{CO}_{3}$

$\mathrm{PO}_{4}$

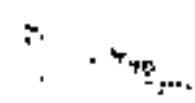

TOC $(g / L)$

Pu $(0 / L)$

${ }^{137} \mathrm{Cs}(\mu \mathrm{Si} / \mathrm{L})$

$89,90_{5 \mathrm{r}}(\mu \mathrm{Cj} / \mathrm{L})$

SpG

$\approx \mathrm{H}_{2} \mathrm{O}$

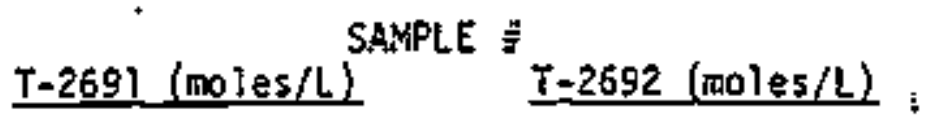

0.374

0.383

0.603

0.612

2.41

2.54

0.956

0.947

0.570

0.462

0.045

0.045

35.16

43.79

$1.01 \times 10^{-4}$

$1.24 \times 10^{-4}$

$2.73 \times 10^{5}$

$7.75 \times 10^{5}$

$4.85 \times 10^{4}$

$4.27 \times 10^{4}$

1.2814

1. 3077

63.92

65.86 
 \\ - Internal Letter}

Dxis: - September 22, 1980

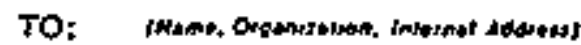

- M. C. Teats

- Plant Engineering

. 2750E/A]00/200 East

$$
\text { Un- 101A waste }
$$

WHC-SD-WM-ER-308, Rev. 0

No: . 65453-80-267

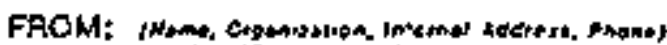

. K. T. Jansky

: Separations Process Developrient L'

- 222-5/N0-037/200 West

- 3-157?

Suoject: Solids in lolA Waste, FIRSt PASS PRODLCT

Solids present in 101A waste may effect processing parameters in the 242A evaporator. The Separation's Process Develogment linit deternined that the most prevalent solid prasent consists of carbonates.

Analyses of three samples taken fron three different areas of Tank IolA show that at the surface most of the solids are carbonates ( 68 percent). ilear the sludge layer 45 percent of the solids are carbonates and 45 percent are nitrates. The sludge itself is 43 percent carbonate, 33 percent nitrate, and 14 percent aluminate. The data are shown in Tables I, II, and III.

It should be pointed out that the carbonate levels reported here are most likely low. Complexants, as jetermined by Total Organie Carbon (TOC), should not precipitate. The TOC measurement includes carbonate, which is subtrected to give TOC. The high carbanate concentrations may have affected the analysis.

Please call if you have any questions.

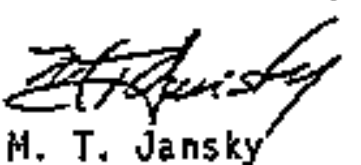

Advanced Chemist

MTJ/naj

Attachments

Distrfbution:

D. E. Bowers

L. C. Brown

J. S. Buckingham

H. S. Eding

L. A. Ga7e

D. L. Herting

T. A. Lane

H. C. Teats

T. B. Veneziano

o. G. Hijkins

Fjle Code: XF55E

Process Aids: (8)

Letterbook 
So-WM. P.

APPENDF.

TABLE I

WHC-SD-WM-ER-308, Rev. 0

Composition of Tank 10IA Waste Sample 4218

(Near Surface Sample)

Supernatant

Solids

Component

Molartty

Wt.\%

Wt. $\%$

A]

1.364

8.32

0

$\mathrm{OH}$

3.06

9.11

0

$\mathrm{NO}_{2}$

2.04

10.47

2.72

17.20

0

$\mathrm{NO}_{3}$

0.98

7.72

0

$\mathrm{CO}_{3}$

.0 .036

0.20

$16.24 \mathrm{~g} / \mathrm{h}$

3.59

68

$\mathrm{PO}_{4} \quad \therefore$.

TOC

${ }^{\alpha} \mathrm{H}_{2} \mathrm{O}$

50.56

SpG

1.344

TOTAL

107.77

100 


$$
\begin{array}{r}
\text { SUML } \\
\text { APPENDF }
\end{array}
$$

WHC-SD-WM-ER-308, ReV, 0

\section{- $\cdot$ tABLE II}

Composition of Tank J01A Waste Samp?e 54378

(Near sludge Sample)

\section{Component}

\section{A1}

애

$\mathrm{NO}_{2}$

$\mathrm{NO}_{3}$

$\mathrm{CO}_{3}$

$\mathrm{PO}_{4}$

TOC

${ }^{2} \mathrm{H}_{2} \mathrm{O}$

SpG
Supernatant

Molarity

1.758

3.15

2.565

2.298

0.156

0.330

$10.74 \mathrm{~g} / \mathrm{L}$
Wt. $\%$

12.60

1).0T

15.47

17.07

I. 45

4.73

2.63

41.56

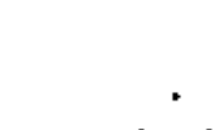

Soljds

Wt. $\%$.

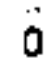

0

0

45

45

0

10

TOTAL

\subsection{4}



Dxis: - October 13, 1980

To:

- it. C. Teats
: P)ant Engineering
- 2750E/Al00/200 East

4 WHC-SD-WM-ER-308, ReV. 0

ob IOIA LOASt

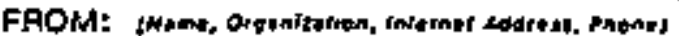

- H. T. Jansky

- Separations Process DeveJopmen:

- 222-5/\$10-037/200 best

3- 357]

subiet: . Composition of lola kaste, FrNAL PRoduct

Two samples of waste from Tank 101A were received by the Separations Process Develooment Unit (SPOU). The soljos were separated from the superatant in both samples and the phases were analyzed. The solids were approximately 50 percent sodiua nitrate, with sodium carbonate and sodiun phosphate present as well. The results are shown in Tables I and II. Laboratory details are discussed below.

Two sertiples of i01A waste received by SPDU contained solids. One sample. taken one foot befow the surface, contained more than twenty percent crystalline solids on the bottom of the bottle. The solids would not shake into a sluryy when the bottle was shaken, but could be broken up with a 5 patula. Aliquats of the mixture were poured into centrifuge cones. The soljds settled rapidly. The eliquots were centrifuged, resulting in three phases: 78 volume percent supernatant, a "paper-thin" Jayer of "dark green goo". and 22 volume percent light green solids. The supernatant was decanted. The supernaţant and solids were subaitted to Analytical Laboratories (AL). For analyses. The resuits are shown in Table $I$.

A second sample was taken-six feet below the surface. The sample conteined more thon twenty percent crystalline solids. The sample was mixed with a spatula, and aliquots were transferred to centriruge cones. The aliquots were centrifuged, resulting in four phases: 85 volume percent supernatant, a paper-thin layer of "green goo", and two more phases of soljds, each 7.5 ". percent by volune. The two botton phases of solids were distinguished by two different shades of green. The supernatant was decanted. The solids were inixed and subritted to Al for enaiyses, as well as the supernatant. The results are shown in Table II.

The dissoived solids were anolyzed for sodium, chromium, uranium, fron, and other metais. The sodium ion content stoichiometricaliy matches the sur of all the anion concentrations. This indicates that material baiance caiculations based on sodium salts are valid.

Pleasa call if you have any questions or comments.

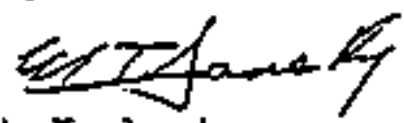

M. T. Jansky

Advanced Chemist

HTJ $/$ naj

Attachinents

Distribution:

D. E. Bowers

ง. S. Buckinghen

H. J. Eding

L. A, Gale
D. L. Herting

T. A. Lane.

T. B. Veneziano*

D. G. Wilkins
Fi]e Code: IHB612

Process Aids: (B) Letteroook 


\section{TABLE I}

Cempasition of Tank lota Waste

One Foat Below striface

Corogonent

$\mathrm{NaAlO}_{2}$

$\mathrm{NaOH}$

$\mathrm{NaNO}_{2}$

$\mathrm{NanT}_{3}$

$\mathrm{Na}_{2} \mathrm{CO}_{3}$

$\mathrm{Na}_{3} \mathrm{PO}_{4}$

IOC

$\mathrm{y}_{2} \mathrm{o}$

spG

\section{Molaiㄷㄴ}

2.54

4.20

3.79

2.12

0.24

0.08

$10.71 \mathrm{c} / \mathrm{L}$

Supernatane

Helghty

15.95

22.96

19.34

13.73

1.95

1.00

2.44

45.42
Solids*

Weighe $z$

.

0

0

$\cdot 0$

50.7

26.1

23.4

0

*Aralycical resuits Indfeated that complexants (measured by IOC) had prectpitated. This gave trelghe pereedis of: $\mathrm{NaNO}_{3} / 45.9, \mathrm{Ha}_{2} \mathrm{CO}_{3} / 21.3$; $\mathrm{Na}_{3} \mathrm{FO}_{4} / 18.6$, and TOC/14.3. Complezants should not preeipitate, houever; therefore the soltas veighc percent treze adjitsed to discount Toc. 
$S D-W M-P$

APPENDI WHC-SD-WM-ER-308, ReV, 0

TABLE II

Composition of Tank I01A Naste

S1x Feat Below Surface

Component

$\mathrm{NaAlO}_{2}$

NaOE

$\mathrm{NanO}_{2}$

$\mathrm{Nation}_{3}$

$\mathrm{Na}_{2} \mathrm{CO}_{3}$

$\mathrm{xa}_{3} \mathrm{PO}_{4}$

TOC

$\mathrm{H}_{2} \mathrm{O}$

Spg
Supernatane

oㅣ다녀

2.185

3.51

3.26

2.02

0.06

0.23

11.52
Wetrhex

14.03

13.46

17.61

13.47

0.77

1.87

2.68

45.49
Soltds

Weightz

0

0

0

46.8

17.7

35.4

0 


\section{Internal Letter}

Oalc: . November 13, 1980

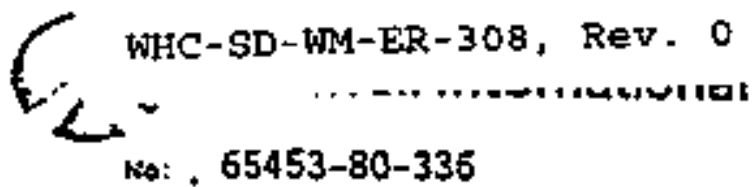

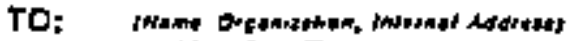

H. C. Teats

* Plant Engineering

. 2750E/AiDOO/200 East

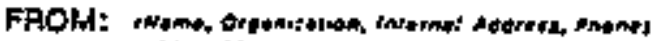

H. T. Jansky

Separations Process Development $\mathrm{Ha}$

. 222-5/10-037/200 Hest

$3-157 \mid$

Sucject: . I01A Waste Sample

A sample of Tank TOlA waste was received by 5P ov on 0etober 22, 1980. The sarpla was filtered at room temperature, and the solids and jiquids submitted to Analytical Laboratories for analyses. The results are shown in iable I.

The data show that the solids were primarity sodium nitrate and sodium carbonate. The high percentage of sodium hydroxide in the solids may be attributed to a iarge volune of interstitial fiquor trapped within the solids.

Please call if yot have any questions or cominents regarding this work.

i. T. jansky

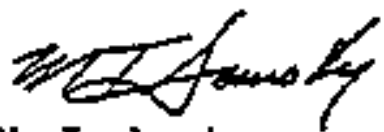

Advanced Chemist

$M T J / n a j$

Attachment

Distribution:

J. E. Bowers

J. 5. Buckinghan .

H. J. Eding

L. A. Gale

๑. 1. Herting

T. A. Lane

0. A. Reynolds

T. 8. Veneziano

D. G. Wfikins

Fj\}e Code: WB612

Process Aids:

let terbook 
WHC-SD-WM-ER-308, Rev, O

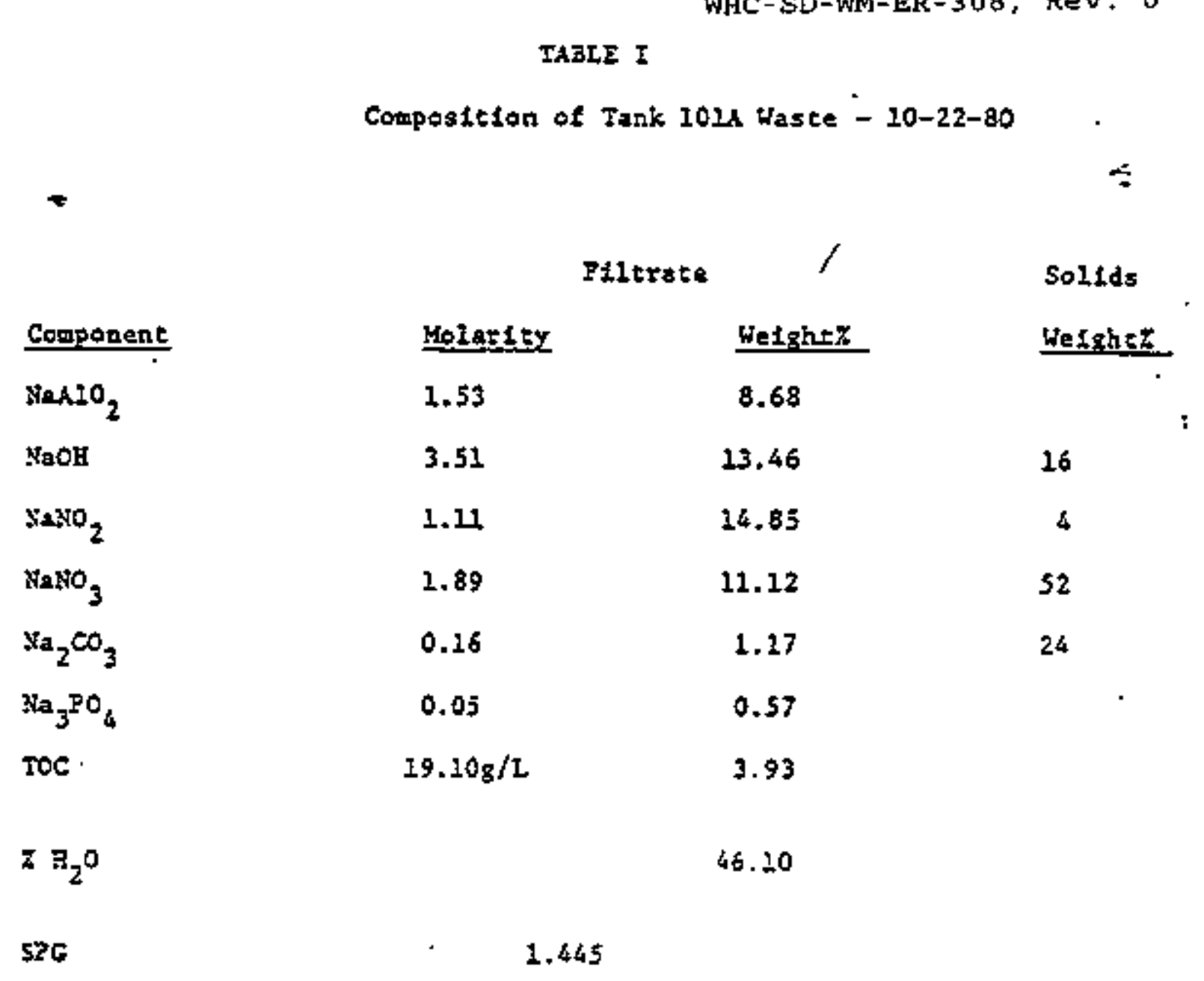


$T \angle R C-21$

Internal Letier

Dz:e: - November 17, 1980

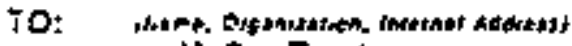

- H.C. Teats

- Plant Engineering

2750E/A100/200E
WHC-SD-WM-ER-308, Rev. 0

No: . $\quad 65453-80-337$

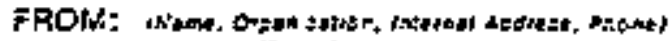

- H.T. Jansky

- Separations Process Development Ur:

- 2225/M0-037/200W

- 3-1571

stopect: , 101A Waste Sample Characteristics

Tank 301A is scheduled for deactivation to meet the 1-i-81 Rockwell miflestone. Prior to deactivation, some knowledge of the waste contajned" in the tank is necessary. In lieu of this, the Separations Process Development Unit (SPDU) received three difierent sampios from $101 \mathrm{~A}$ this week. All three samples contained a significant volume of solids when received. The solids have been characterized as sodium carbonete, as shown in Tables I, III, and $Y$. Viscosity messurements on all three samples show that even at elevated temperatures $\left(70^{\circ} \mathrm{C} / 158^{\circ} \mathrm{F}\right)$ the waste is in excess of 100 centipoise viscosity. This indicates that ettempting to transfer the waste via pumping may plug the transier lines. . Additionally, is the waste is cooled, solids precipitate out. Viscosity versus. temperature data are shoten in Tables II, IV, and VI. Laboratory detajls are discussed below.

Two samples from 101A were received by SPDI the evening of the tenth. Soth samples were at room temperature. They were placed into a $63^{\circ} \mathrm{C} /$ $145^{\circ} \mathrm{F}$ water bath for one-half hour. The following observations were noted:

Tho Foot Beiow Surtiace

The sample bottle was less then half full when received. At room temperature approximately 75 volume percent solids were observed. A portion of tine sample was vacuuti $f 17$ tered at $65^{\circ} \mathrm{C}$ and solids and filtrate subai tted to Analytical Laboratories for enaiyses. The flitered waste was 20-30 volume percent solids and 31 weight pertent solids. An aliquot of the slurry was submitted to Al as well. The deta are shoten in Table I. Viscosity versus temperature data on the as-received slurry sample are show in Table 11. The viscosity was measured with a Brookfieid cup-and-cone microviscometër.

\section{Four Foot Below Suriace}

At roon temperature this sample contained ninety-five yolume percent solids. As above, soljos and liquid were separated by vacuum filtration at $65^{\circ} \mathrm{C}$, with al iquots of esch (and tote] slurry) subnitted to AI. The oata are shown in Table III. The fijtered waste was 30 volume percent soilds and 47 veight percent solids. Viscosity versus tenperature deta are shown in Table IV.

A third sample, Tabeled four foot below surface, was received iate. Tuesday (November 17, 1980). This sample was in a heated pig, and vas immediately transferred to a $59^{\circ} \mathrm{C}$ water bath. The material contained loosety suspended solio's when received, but did not readily pour out of the sample bottle; it plopped. Large grainy particles were present in the waste. Using the same procedure as for the two earlier samples, solids, liquid, and slurry were submitted for analysis. The results 
Page 2

M.C. Teats

November 17, 1980

are shown in Table $Y$. The filtered waste was 33 volume percent solids and 54 weight percent solids. Viscosity versus temperature data for both the filtrate and totai slurry are shown in Table VI.

This laboratory study on Tank $101 \mathrm{~A}$ waste shows that the waste is quite viscous and would need to be difuted if a transfer were attenpted. The dato so indicates that if the waste were allowed to cool, solids wotld precipitate. Finaliy, the solids present in the waste are sodiura carbonate.

Please call if you have any questions or comments regarding this work.

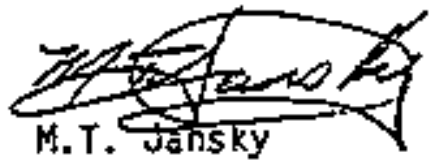

Advanced Chemist

$i \mathrm{~T} J / v 10$

Distribution: D.c. Bowers

J.S. Buckinghạm

H.J. Eding

L.A. Gale

D.L. Herting

D.R. Jorgensen

T.A. Lane

T.B. Veneziano

D.G. Wilkins

File tode: WBEl2

process Aids: (8)

letterbook 


\section{TABLE I}

WHC-SD-WM-ER-308, Rev. O

Composition of Tank 101A Wase

Two Foot Semple 11/10/80

\begin{tabular}{|c|c|c|c|c|c|}
\hline Conponent & $\begin{array}{c}\text { Toral } \\
\underline{\underline{M}}\end{array}$ & $\begin{array}{c}\text { slurry* } \\
\text { kt. }\end{array}$ & $\begin{array}{l}\bar{z} \text { ilt } \\
\underline{M}\end{array}$ & $\begin{array}{l}a t e^{*} \\
t \underline{t} . z\end{array}$ & $\begin{array}{c}\text { Solids } \\
\text { 드." }\end{array}$ \\
\hline $\mathrm{KaAlO}_{2}$ & 1.51 & 7.84 & 1.21 & 6.96 & 4 \\
\hline $\mathrm{AaOH}$ & $2.45^{\circ}$ & 6.20 & 2.31 & 6.46 & \\
\hline $\mathrm{NeNO}_{2}$ & 2.22 & 9.69 & 2.22 & 10.40 & \\
\hline $\mathrm{NaNO}_{3}$ & 2.77 & 14.90 & 2.57 & 15.30 & \\
\hline $\mathrm{Na}_{2} \mathrm{CO} / 5 / 3$ & 1.33 & 8.92 & 0.31 & 2.30 & 94 \\
\hline $\mathrm{Na}_{3} \mathrm{PO}_{4}$ & 0.08 & 0.83 & 0.09 & 1.02 & 2 \\
\hline $\mathrm{Ne}_{2} \mathrm{SO}_{4}$ & 0.13 & 1.17 & 0.01 & 0.13 & \\
\hline $\operatorname{Toc}(\mathrm{g} / \mathrm{E})$ & 3.98 & 0.75 & 6.86 & 1.43 & \\
\hline${ }^{137} \mathrm{Cs}(\nu \mathrm{CL} / \mathrm{I})$ & 1.172 & $10^{-5}$ & 4.55 & $30^{5}$ & \\
\hline${ }^{90} \mathrm{St}(\mathrm{yCz} / \mathrm{L})$ & $1.23 x$ & $10^{4}$ & 4.25 & $10^{3}$ & \\
\hline$F u(g / L)$ & $5.67 x$ & $10^{-5}$ & 1.08 & $20^{-5}$ & \\
\hline $\mathrm{FH}_{2} \mathrm{O}$ & & 42.37 & & 48.26 & \\
\hline$\$ p G$ & 1.5 & & 1. & & \\
\hline
\end{tabular}

HWeight percent does not include ${ }^{137} \mathrm{Cs},{ }^{90} \mathrm{Sr}$, or $\mathrm{Pu}$. 


\title{
TABLE I I
}

WHC-SD-WM-ER-308, Rev. O

\begin{abstract}
Viscosfty versus Iegperature of Tenk 101A Waste
\end{abstract}
Two Foot Sample $11 / 10 / 80$

\section{Temperature $\left({ }^{\circ} \mathrm{C}\right)$}

80*

70

60

50

40

30

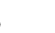

$\underline{\text { RPM }}$

12

6

3

1.5

0.6

6

3

1.5

0.6
0.6

0.3 viscosicty (cps)

\section{2}

117

197

332

**

185)

318)

)

541) more solids

precipitacing $* *)$

)

572)

)

927) more solids

) precipitating

623)

979)

1180) more solitis

1008) more solids I088)
I 


\section{TABLE III}

Compostcion of Fank 101A Waste

Faur Foor Sanple 11/10/90

Componenc

$\mathrm{NaAlO}_{2}$

saot

$\mathrm{NaNO}_{2}$

$\mathrm{NaNO}_{3}$

$\mathrm{Na}_{2} \mathrm{CO}_{3}$

$\mathrm{Na}_{3} \mathrm{PO}_{4}$

$\mathrm{Na}_{2} \mathrm{SO}_{4}$

$\operatorname{Toc}(\mathrm{g} / \mathrm{L})$

$137 \mathrm{es}(\mathrm{HCi} / \mathrm{L})$

$90_{\mathrm{Sr}}(\mu \mathrm{Ci} / \mathrm{L})$

Pu $(\mathrm{g} / \mathrm{L})$

$z \mathbf{H}_{2} \mathrm{O}$

Spg
$\operatorname{Total}_{M}$ Slurry*

$1.59 \quad 7.90$

$2.45 \quad 5.94$

$2.2 B$

9.53

$3.07 \quad 15.32$

1.50

9.64

0.09

0.39

0.15

1.29

9.31

2.71

5. $19 \times 10^{5}$

$1.97 \times 10^{2}$

$1.99 \times 10^{-t}$

31.34
Filtrate*

느‥

$1.67 \quad 9.32$

$2.51 \quad 6.84$

$2.40 \quad 11.26$

$3.24 \quad 15.71$

$0.34 \quad 2.47$

$0.08 \quad 0.94$
Solids

We. 7

90

6

$7.51 \quad 1.52$

$7.69 \times 10^{5}$

$4.50 \times 10^{3}$

$1.34 \times 10^{-5}$

45.47

1.47

1.65

${ }^{137} \mathrm{C}_{5},{ }^{90} \mathrm{st}$, or $\mathrm{Fu}$. 
TABIE $\because *$

Viscosicy vergus Tetpe: WHC-\$D-WM-ER-30B, Rev.

Four Foot Sample $11 / 10 / 80$

Temperacture $(* \mathrm{C})$

$77 *$

70

60

50

40

- 30

\section{표므}

3

1.5

12

6

3

3.5

0.6

3

1.5

0.6

0.3

0.6

0.3

1.5

0.6

0.3
$\mathrm{Viscos} \operatorname{cy}(\mathrm{cps})$

233

277

80

225

205

255

342

368

533

716

966

925

1040

618

899

1012

Very isiscots

*Sore solids dissolved, and concinually gret in as the temperature uns lowered. 
Composicion of Tank lolA Wasce'

$21 / 2$

Joer Foot Sample $11 / 11 / 80$

Component

Total SIurry*

II We.Z

Filtrace*

프 트.

Solids

hrc.z

$\mathrm{NaAlO}_{2}$

$1.95 \quad 8.64$

$2.08 \quad 11.80$

NaOH

$3.51 \quad 7.59$

$3.22 \quad 8.64$

$\mathrm{NaNO}_{2}$

$3.01 \quad 11.23$

$2.94 \quad 14.02$

$\mathrm{NaNO}_{3}$

$3.66 \quad 26.82$

$3.48 \quad 20.45$

$\mathrm{Na}_{2} \mathrm{CO}_{3}$

$2.31 \quad 13.24$

$0.13 \quad 0.93$

95

$\mathrm{Na}_{3} \mathrm{PO}_{4}$

$0.12 \quad 1.06$

$0.13 \quad 1.53$

$\mathrm{Na}_{2} \mathrm{SO}_{4}$

Analyses Roc Nvailable

$\operatorname{TOC}(\mathrm{g} / \mathrm{L})$

$15.61 \quad 2.52$

$9.94 \quad 2.04$

${ }^{137} \mathrm{Cs}$ ( $\mathrm{HCI}_{\mathrm{CI}}$ )

$5.95 \times 10^{5}$

6. $22 \times 10^{5}$

$9_{5 \%}(\mathrm{NCL} / \mathrm{L})$

$9.55 \times 10^{4}$

$4.19 \times 10^{3}$

$P u(g / t)$

$5.39 \times 10^{-4}$

$3.96 \times 10^{-5}$

$\mathrm{ZH}_{2} \mathrm{O}$

34.19

34.86

$\$ p G$

1.85

1.45

tWeight percent does noc include ${ }^{137} \mathrm{Cs},{ }^{90} \mathrm{~S}_{\mathrm{r}}$, or Pu. 
TABIE VI

Viscosity versus Temperacure WHC-SD-WM-ER-308, Rev. 0

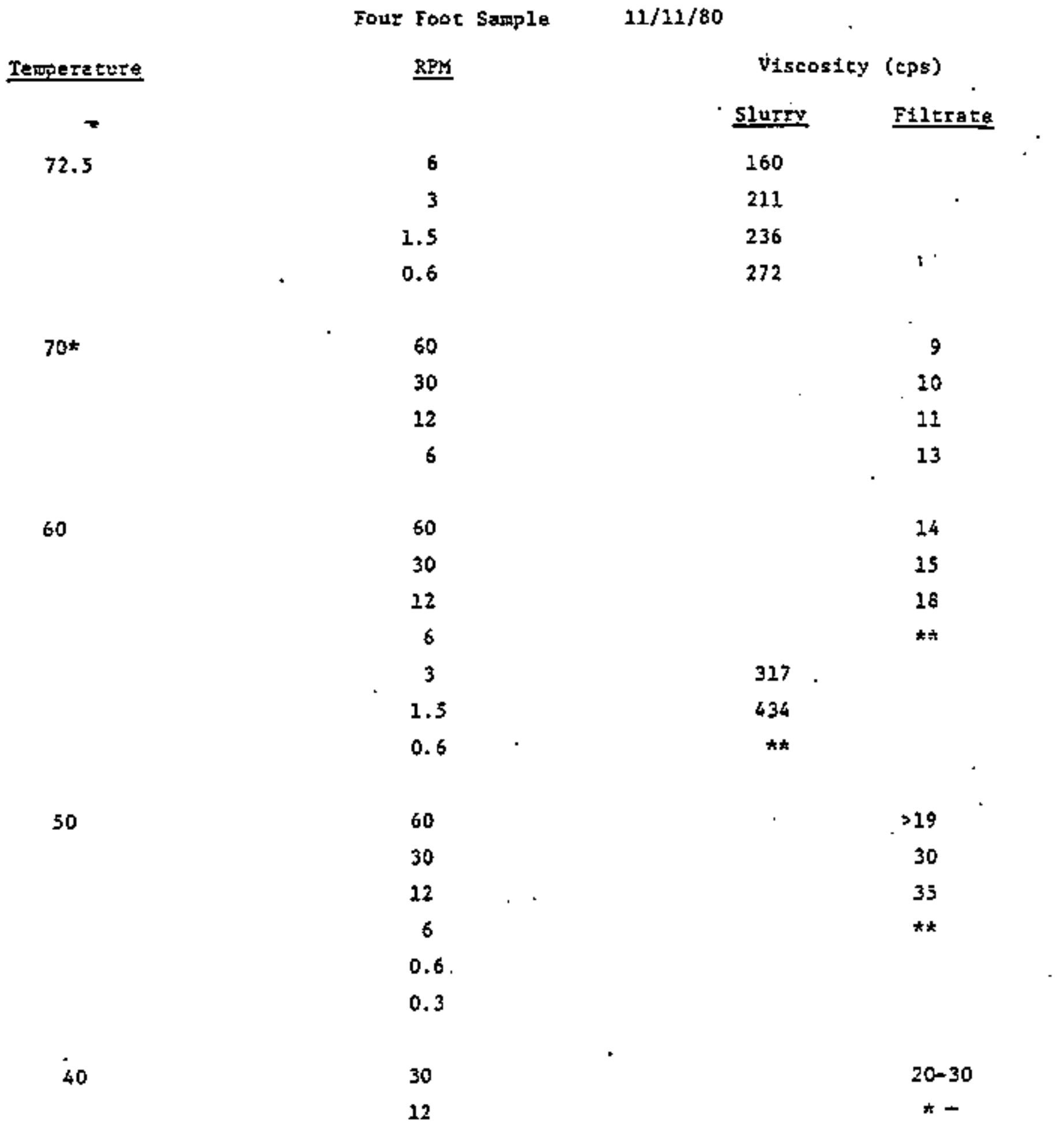

35

\$98 volume $z$ solids

* Some solids dissolved or melted and coneinually grew in as terperature was lowered

* Erratic reabings 
$T C R C-27$

\section{Internal Letter}

osk. January 3, 1984

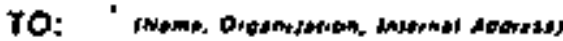

. T. D. Kirkpatrick

- Stabilization, Isolation, \&

- Surveillance

2750E/A103/200E
No. . 65453-84-003

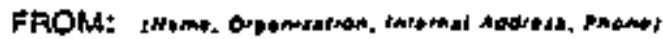

- M. T. Jansky

- Chenical Laboratory

. 2225/40-037/200W

. 3-1571

subjec:. Waste Samples from Tank $101 \mathrm{~A}$ (7879 \& 7898)

Ref: Letter $\$ 65453-80-302$, October 13, 1980, M. T. Jansky to H. C. Teats, "Composition of 101A Waste"

Two waste samiles from Tank 2018 were received by the Chemical Laboratory Unit (CLU) in October. Although the jnformation gathered from those samples has been verbally transmitted to engineering personnel, the purpose of this Tetter is to more formally document the data.

Two waste samples from $101 \mathrm{~A}$ were received by CLU. One, 7879 , was dated october 10, 1983. The other sample, 77898 , was dated October 11, 1983. Both samples were treated in the same fashion. Each was heated to $65^{\circ} \mathrm{C}$ for 30 mitnutes and observed. Aliquots of each were centrifuged, and physical data were gathered. Supernatant and dissolved solids were analyzed, as well as an aliquot of the total sturry. Viscosity versus temperature data were gathered on 7879 . Relevant data are shown in the attached Tables.

The data indicate that the two 101A samples are dramaticaliy different. Even though the solids of each contain a signjficant amount of aluminum (as sodium aluminate), $\$ 7879$ has more organic carbon and less nitrate in the solids. Also, \$7898 has a significant3y more concentrated supernatant liquor, based on both analyses and volume percent solids.

An interesting phenomenon was observed for both samples. Both samples contajned a significant amount of organic carbon. The weight percents given in the Tables are based on organic carbon from complexants (HEDTA/EDTA). It is interesting to note that sigilar results were observed in Tank 101A waste analyses in 1980 (Reference). This finding way bear further inyestigation.

Please call if you have questions, comrents, or require further work with 101 A samples.

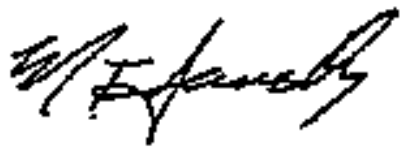

H. T. Jansky

Chenist

KT.J/pjm
cc: R. B. Bendixsen
J. S. Buckingham $35 \mathrm{~B}$
P. J. Certa
K. G. Carothers
H. I. Finemran
D. L. Herting
K. P. Me]son
D. A. Reynoids
L. H. Rodgers
M. C. Teats
Process Aids
D. W. Lindsey
File" Code HB 
TAQLE I

PHYSICAL DATA

Parameters

$\$ 7879$

Solids - Volume $\%$ Settled at $65^{\circ} \mathrm{C}$

- Volume * Centrifuged

- Weight $\%$ Centrifuged

Specific Gravity • Slurry

- Supernatant

- Centrifuged Solids*
425

6.4

6.3

1.3597

1. 3617

1.3307
$A-10 \%$

$\$ 7898$

n9

75.4

74.7

1.6400

1.6847

1.6253

*Includes entrained interstitial liquor, but excess supernatant removeo. 
TABLE II

COMPOSITION OF TANK IOIA WASTE

SAMPLE 7879

Concentration

\section{Component}

Al

$\mathrm{OH}$

$\mathrm{NO}_{2}$

$\mathrm{NO}_{3}$

$\mathrm{CO}_{3}$

TOC $(g / 2)$

$\mathrm{PO}_{4}$

$\mathrm{SO}_{4}$

$\mathrm{Cs}^{137}(\mu \mathrm{Cs} / \mathrm{L})$

$5 r^{90}(\mu \mathrm{Ci} / L)$

Pu $(g / L)$
Supernatant (M)

0.01

4.11

*

7.02

0.13

0.20

$\star \star$

$3.49^{4}$

$1.88 \times 10^{-5}$
0.90

1.43

Solids (wt. q)

33

1.57

2.59

NA

5.23

44

0.02

0.05

23

* Dissolved in Acio.

**Below Detection Limits.

NA - Not Availaoble. 
WHC-SD-WTH-ER-308, Rev. O

TABLE III

COMPOSITION OF TANK IOLA WASTE

SAMPLE 7898

Concentration

\begin{tabular}{|c|c|c|c|}
\hline Component & Slurry (16) & Supernatant (M) & Solids (wt $x$ ) \\
\hline 11 & 1.59 & 2.27 & 27 \\
\hline JW & * & 3.86 & \\
\hline $\mathrm{NO}_{2}$ & 0.01 & 2.53 & \\
\hline $\mathrm{NO}_{3}$ & 5.08 & 3.26 & 62 \\
\hline $\mathrm{CO}_{3}$ & * & NA & \\
\hline $\operatorname{roc}(g / L)$ & 9.78 & 11.0 & 11 \\
\hline $0_{4}$ & $\star \star$ & ** & \\
\hline $50_{4}$ & $\star \star$ & 0.19 & \\
\hline $\mathrm{s}^{137}(\mu \mathrm{C} / \mathrm{L})$ & ** & $\star \star$ & \\
\hline$x^{90}\left(\mu \mathrm{Ci}_{1} / \mathrm{L}\right)$ & $6.11 \times 10^{4}$ & $8.11 \times 10^{4}$ & \\
\hline$u\langle g / L\rangle$ & $6.52 \times 10^{-4}$ & NA & \\
\hline
\end{tabular}

* Dissolved in Acid.

**Below Detection Limits.

NA - Not Available. 
TABLE IV

VISCOSITY verSUS TEMPERATURE OF TANK 1OIA WASTE SAMPLE 7879

\begin{tabular}{ccc} 
Temperature $\left.{ }^{\circ} \mathrm{C}\right)$ & $\frac{\text { RPM }}{\text { Viscosity (cps)* }}$ \\
\hline 0 & 60 & 164 \\
& 30 & 178 \\
10 & 60 & 53 \\
20 & 30 & 60 \\
30 & 60 & 17 \\
40 & 30 & 26 \\
& 60 & 10
\end{tabular}

*Brookfield LVT spindle viscometer, $\frac{52}{\pi 2}$ spindle. 


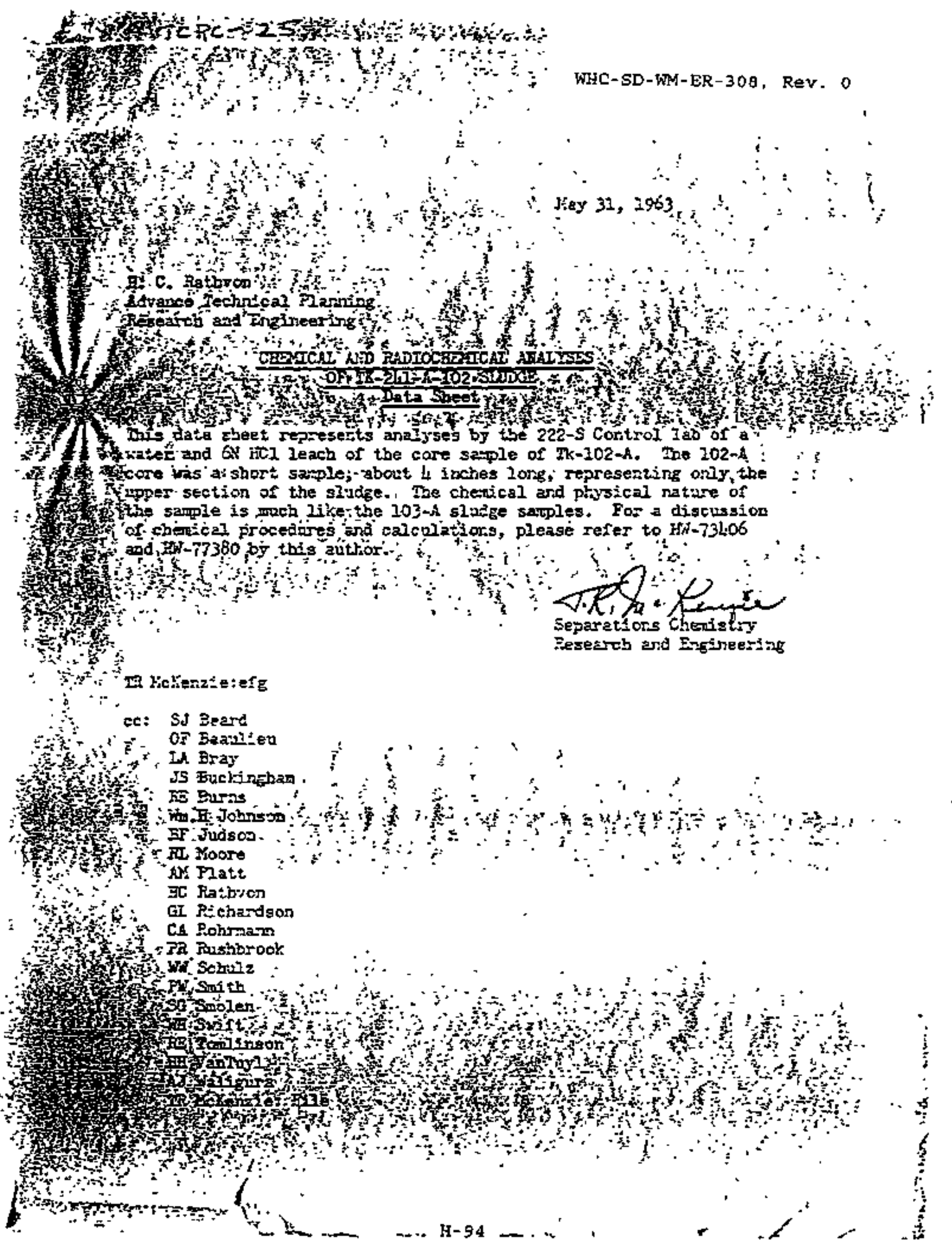


WHC-SD-WM-ER-30B, ReV , 0

프르돌

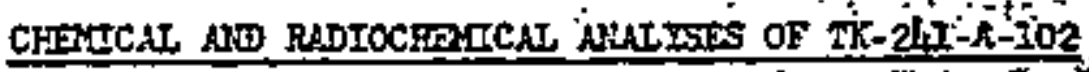

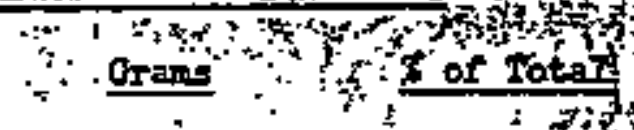

Sabole it.

Sample wt. d1gsolved by $\mathrm{H}_{2} \mathrm{O} \quad \therefore \quad 3.20$

Feafdue dissoived by $\mathrm{ECI} 20.95$

Restdue insolubie in $\mathrm{FCl}$

Residue lost by polatilization

.16

lestdue lost by rolatilization $\quad .45$

$\because \div 100$

Fit

保

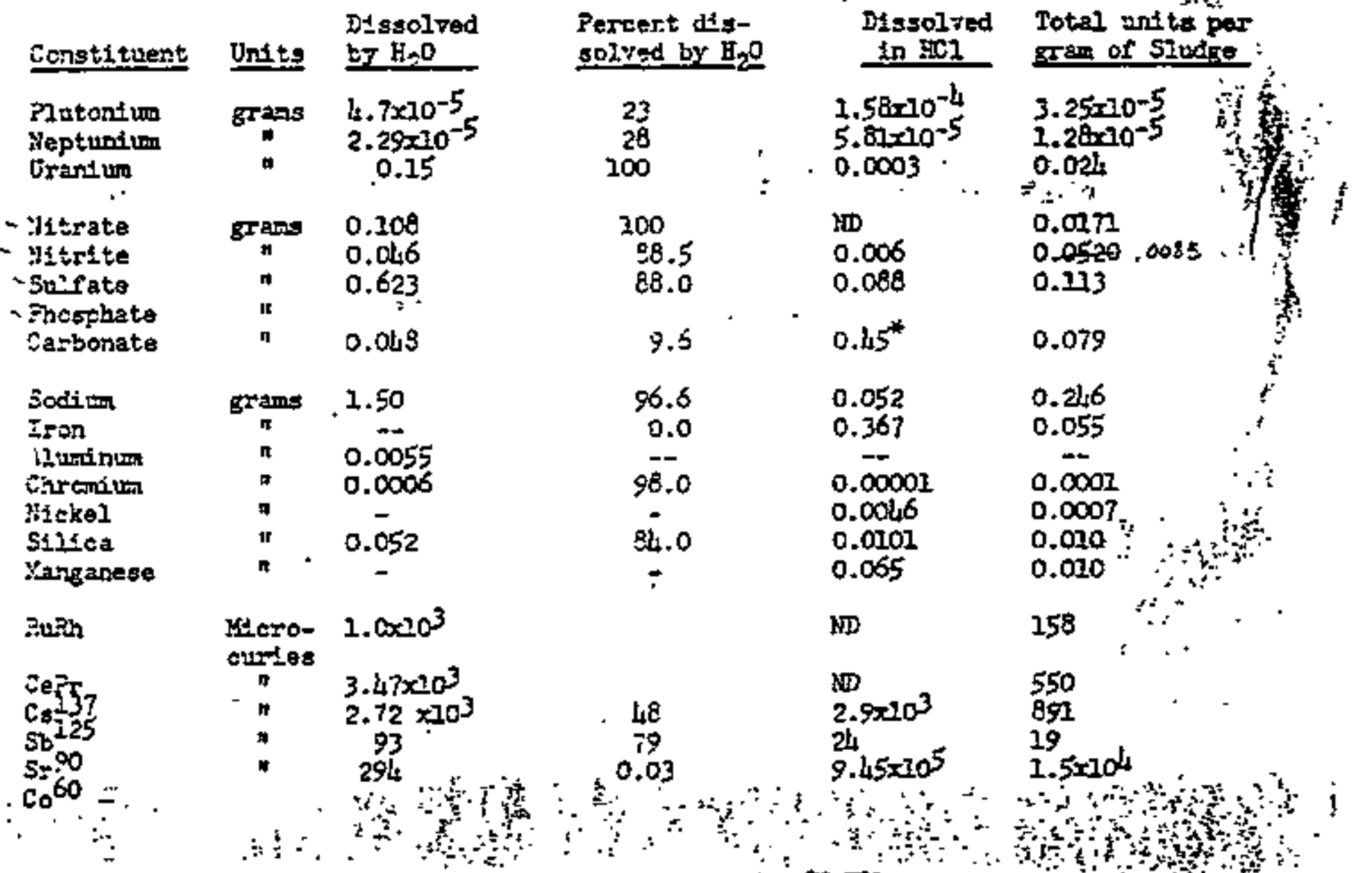

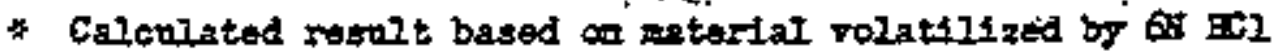

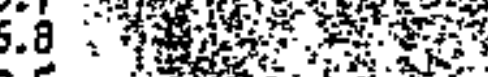

5

$+217 \div=$

$=4+i=?$ 
TCRC- 3

WHC-SD-WM-ER-308, Rev. 0

\author{
Date: January 16,1373 \\ $A 102 \cdot 6$ \\ 20: \\ S. C. Homack \\ zon: \\ D. A. Doda

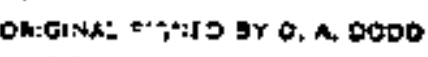

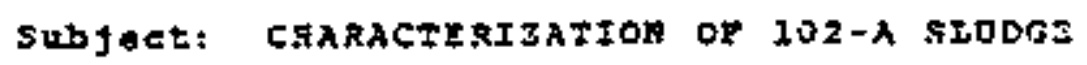
Pot consistency of the rasults, a nar allatot of

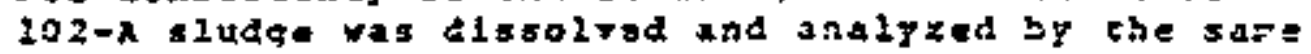

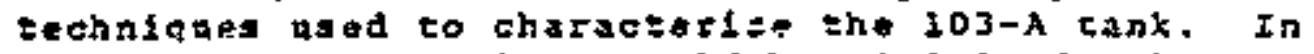 thts way any raxiatlons coult be mintgizen other ihan the possibilief of non-konogeneoug sindge. The rolume. to vefght ratio and analysas basod on ralghta rerk lot

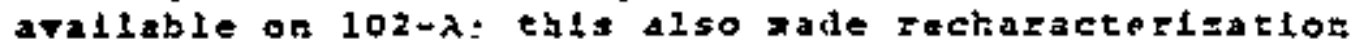 pecesang -
The andigeo hare nov beas completed and aze as follows calculated os a wet efitgleuged wetght basis.

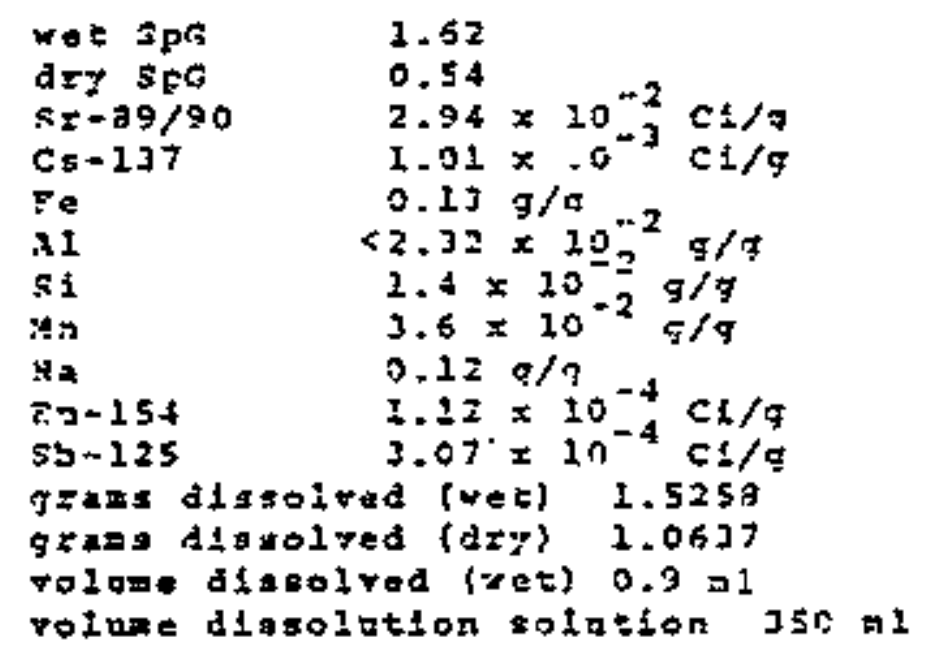

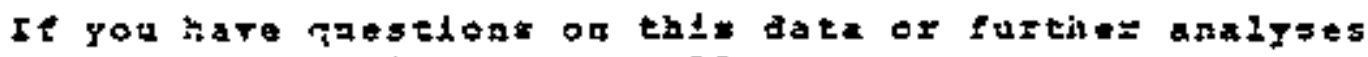 ot latereat reol frat to call.
DAD : $\mathrm{nq} \leqslant$
CE: GL gozathe
Js 30ckinghan-t. -
xt campbe11
Da Larkin
Procea $11 \mathrm{ds}$
$\forall$ ite




$$
\begin{aligned}
& T C R=-11 \\
& \text { A } 10^{2}, \text { WHC-SD-WIM-ER-308, Rev. } 0 \\
& \text { Date: } 12-8-79 \\
& ; \ldots \cdots, \ldots, \ldots \ldots \ldots+\ldots \\
& \text { Manager, Services } \\
& \text {. 222-5 Laboratory } \\
& 200 \text { s } 2965 \text { or } 2-2435
\end{aligned}
$$

Analytical Results

$$
102 \text { - } A
$$

- talk sample number JO R-A 242-A-BM

$$
\text { AL SERIAL HLHEER T-6/76 }
$$

DATE RECEIVED

$$
12.8 .79
$$

DILUTION FACTOR"

.175 Ref hr

ANALYSIS

$\mathrm{PO}_{4}$

$\mathrm{Cl}$

F

$\mathrm{SO}_{4}$

$\mathrm{CO}_{3}$

Targe

NA
RESULTS

$7.22^{-3}$ is

-NR_ $M$

$-N R$

NR $"$

.360 .14

$4.87-9 / 1 \mathrm{c}$

$N R \quad:$

Cool ing Curve

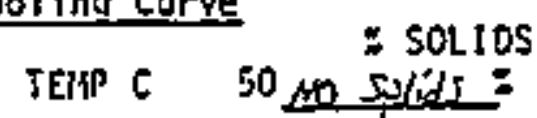

$\frac{N R}{\frac{5.62^{3}}{2.655 \times 10^{-6}} u(1 / 1}$

EA

Cs -137

$2.0<\times 10^{5}$ vii/

C5-134

uci/1

$u c t / 1$
WC $/ 1$

UCi/1.

url $/ 1$

UR = Hot Requested NO= Hot Detected. "The dilution factor is included in the H-97 calculations. 
$\therefore \quad T=2 .-4$

WHC-SD-WM-ER-308, ReV. O

Date: netober 20, 1975

To:

R. L. Walser

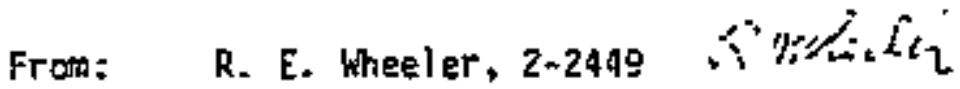

$\begin{array}{ll}\text { Subject: } & \text { ALALYSIS OF TANK FAPM SAHPI, } \\ \text { Sample: } \mathrm{i}-7252 & \text { Tank: } 102-A\end{array}$
A, O2. $y^{\prime}$

Vis-otk: Brown No solids $350 \mathrm{mrad} / \mathrm{hr}$

ph: $\quad 20.3$

SpG: $\quad 1.087$

กH: $<9.50 \times 10^{-3}$.1.

Al: $\quad 0.13 \times 10^{-3} \underline{M}$

$\mathrm{Na}: \quad 1.58 \mathrm{M}$

$\mathrm{HO}_{2}: \quad 0.112 \underline{\mu}$

$\mathrm{irO}_{3}: \quad 1.07 \mathrm{M}$

Pu: $\quad 1.85 \times 10^{-3} \mathrm{~g} / \mathrm{gal}$

D.T.A.: No exetherm belon $200^{\circ} \mathrm{C}$ Smol! exotherm of $235^{\circ} \mathrm{C}$

50. : Cancelies

$\mathrm{PO}_{4}: \quad \quad 0.03 \times 10^{-2} \underline{\mathrm{M}}$

Cl: $\quad 5.10 \times 10^{-4} \mathrm{H}$

F: $\quad 2.19 \times 10^{-4} \underline{M}$

$90,90 \mathrm{Sr}: 4.73 \times 10^{5} \mathrm{wij/gal}, 5$.

$\mathrm{CO}_{3}: \quad 0.775 \underline{\mathrm{H}}$

GEA: $\quad{ }_{30}^{60} \mathrm{CO}=0.18 \times 10^{2} \mathrm{p}[\mathrm{j} / \mathrm{ga}]$

$125 \mathrm{Sb}-8.75 \times 10_{5}^{3}$ wtj/gEi

${ }_{154}^{137} \mathrm{Cs}-2.18 \times 10^{5} \mathrm{ycj/0zl} \sim 2$

$154 \mathrm{Ev}-2.05 \times 10^{3} \mathrm{pCj/60)}$

${ }^{134} \mathrm{Cs}-1.71 \times 10^{3} \mathrm{yCi} / \mathrm{cel}$

H-98 
R. L. Walser

Page 2

Oetober 20, 1975

Hater: 88.91 :

Cooling Curve: $35^{\circ} \mathrm{C}$ for $30^{\circ} \mathrm{min}$. No solids. $30^{\circ} \mathrm{C}$ for 30 min. Ho soljds. $25^{\circ} \mathrm{C}$ for 30 min. Ho solids. $20^{\circ} \mathrm{C}$ for $30 \mathrm{~min}$. Ho solids. $15^{\circ} \mathrm{C}$ for $30 \mathrm{~min}$. No solids. $10^{\circ} \mathrm{C}$ for $30 \mathrm{~min}$. No solids. $5 \circ \mathrm{C}$ jor $30 \mathrm{~min}$. No solids.

REH:sYW

cc: J5 Buckingham

WR Christonsen

RM Ginestet

RE theeler

JC Noneck $<<-2$ 


$$
102-A
$$

Lota: Fitery 12,1975

$$
\text { ริ): } 3 . \text { E. : } 315
$$

From: S. 5. :

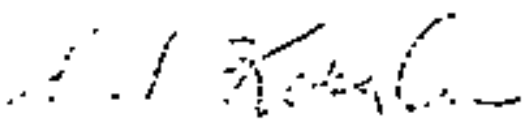

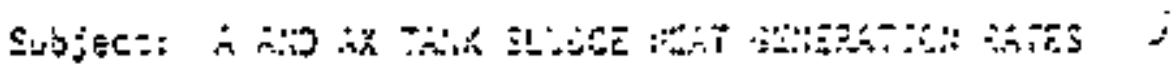

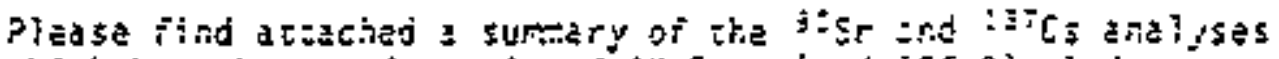

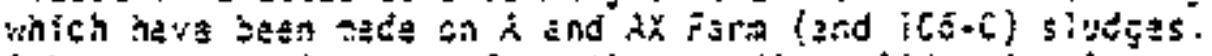

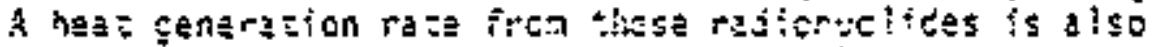

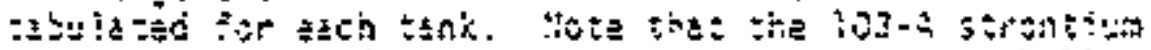

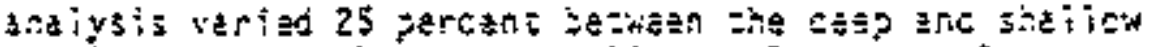

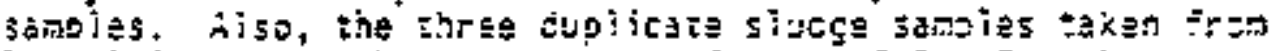

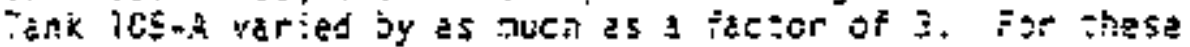

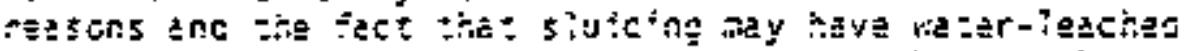

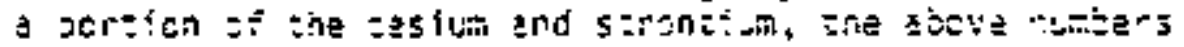

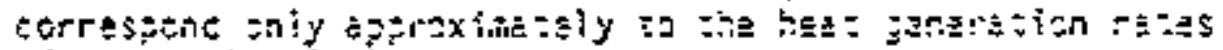
of the resicua s sugess.

jast: vink

$\therefore i$.

ce w/az:s: of Hariow.

\# sinew

?t Toe

aj Thomason

?三 Yen cer reck

:

$\because+$

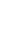

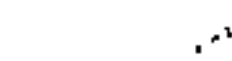


WHC-SD-WM-ER-308, Rev. 0

$\because$

\begin{tabular}{|c|c|c|c|c|}
\hline Iff: & $\begin{array}{l}\text { DATE } \\
\text { SisfirtLD }\end{array}$ & $\begin{array}{l}36 \mathrm{Sr} \text { CONTËHT } \\
\text { (ci/1itar) }\end{array}$ & $\begin{array}{l}137 \mathrm{CS} \text { CONTENT } \\
\text { [Cj/Jiter) }\end{array}$ & 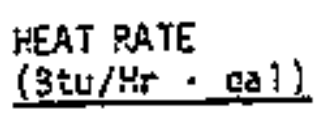 \\
\hline $101-\dot{A} x$ & Suly 1975 & 28.2 & 0.45 & 2.42 \\
\hline$i e 2-i x$ & A.g & 9.3 & 1.26 & 0.87 \\
\hline $103-A x$ & Sept 1974 & 22.4 & 3.11 & 2.08 \\
\hline $104-A x$ & $\therefore: 1974$ & 93.5 & 2.02 & 8.05 \\
\hline $102-A$ & Stgt 1972 & 49.3 & 1.71 & 4.33 \\
\hline (SOAT-7op) & Hov 1972 & 25.9 & 0.97 & 2.26 \\
\hline$(\forall \in c c-3 c \div-5,3)$ & fiow 1972 & 32.3 & 5.78 & 3.10 \\
\hline $104-A^{*}$ & suly 1574 & 43.9 & i. $1 \overline{3}$ & 3.79 \\
\hline $105-h^{2}=$ & $\tan 1972$ & $\div 4.3$ & 2.24 & 3.94 \\
\hline$! 05-C$ & $D C=1974$ & 18.8 & 0.36 & 1.55 \\
\hline Jaterial i & an $101-A]$, & $32-A$, and $10 E-A$ & siuficing & \\
\hline
\end{tabular}




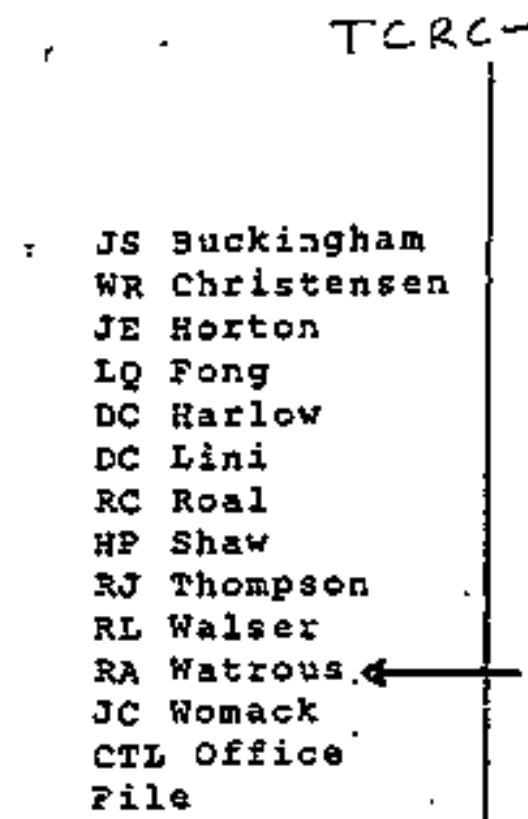

Iculate:

CrL Exempt

2a:

CTL-123
WHC-SD-WM-ER-308， Rev. 0

To: D. C. Ini From; J. E. Horton

\section{ENGINEERING ASSESTANGE}

Budget Number:

$\mathrm{x}+3 \mathrm{C} 7$
Contributors:

$$
\begin{aligned}
& \text { J. E. Horton } \\
& \text { E. L. Rithards }
\end{aligned}
$$

\section{ABSTRAC:}

Iaboratory analyses were zequestea for stzonting and cesium on all residual slutge samples from Tanks 101-AX end fankfioz-A.

The calculated heat. generation rate due to B9+90sr and $137 \mathrm{Cs}$ content from 101-AX restalul sludge samples was $\$ 5817,2.06 \times 10^{-1}$ watts/liter: \#5818, $2.48 \times 10^{-1}$ watts/1iter; $5819,2.53 \times 10^{-1}$ watts/1iter, and $\frac{\pi}{7} 6160,2.43 \times 10^{-1}$ wattg,liter.

Tho calculatod heat generation rate due to 日9090 and ${ }^{337} \mathrm{Cs}$ content from $102-\mathrm{A}$ residual sludge samples was $16038,2.35 \times 10^{-1}$ watts/3itas;

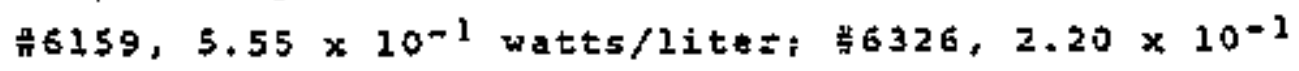
watts/1iter, f6327, $2.60 \times 10^{-1}$ watts/liter; 45693, 6.70 $610^{-2}$ watts/1iter; and $47279,2.73 \times 10^{-2}$ wat $\$ 8 / 11$ tar.

\section{PROGRESS DURING REPORT PERIOD}

We received a group of samplas tron Tank 101-AX on July 23, and August 16, 1976. Analyses were made by tusing known volume of solids from each samplath xor, dissolving each melt with concentrated fCl and diluting each sample to a known volune with water. Analyses are reported in Table I. 
We received a group of samples from rank $102-\mathrm{A}$ in August 1976. Analyses were by fusing a known volume of solids from each sample with XOH, Afsolving each meit with concentrated HCl, ane diluting oach sample to a known volume with water. Analyses are reported in Table II.

\section{BZANS FOR NEXT REROETING PERIOD}

To ovaluate any new samples taken.

\section{CONCLUSIONS}

We have completed and reported ail the work on samples received from Tanks $101-A X$ and $102-A$.

\section{BIBLIOGRAPHY}

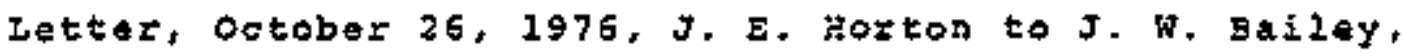

"Analysis of 101-AX Tank Rosidual squages."

Lettor, September 28. 1976, J. Z. Hozron to J. W. Bailey, "Analysis of 10z-A Regidual szudge." 


\section{- TAEIEE}

ANALYSES OF RESIDUAL 1O1-AX TANK SAMPLES

\section{5amole Number}

$$
9+90_{\mathrm{SI}} \mathrm{Ci} / \mathrm{L}
$$$$
\text { watts/2 }
$$

${ }^{37} \mathrm{CF} \mathrm{CI} / \mathrm{L}$ Watt $\$ / L$ $9 \div 90$ s $61 / 9 a l$

su $9 / 2$

\section{7}

28.0

$2.90 \times 10^{-1}$

3. 3

$2.57 \times 10^{-2}$ 206.0

$5.45 \times 10^{-2}$
5016

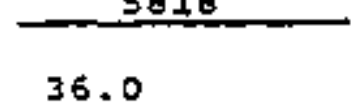

$2.41 \times 10^{-1}$

1.4

$6.77 \times 20^{-3}$

136.3

$6.75 \times 10^{-2}$
5819

36.0

$2.44 \times 10^{-1}$

1. 9

$9.02 \times 10^{-3}$

136.3

$4.73 \times 10^{-2}$
6150

35.1

$2.39 \times 10^{-}$

0.9

$4.44 \times 11=$

132.8

$2.91 \times 10$ 


$$
+10^{2}
$$

\section{TADLE II}

I

, ANALYSES OF RESIDURL d02-A TANK SAMPIES

\section{Sample Number}

$09+90 \mathrm{~S} \times \mathrm{Cl} / 2$

wäts /

$137 \mathrm{Cs} \mathrm{ci} / \mathrm{s}$

wats / t

$69+90 \mathrm{srct} \cos$

Pu $g / t$

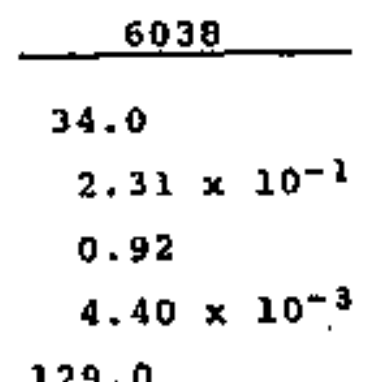

$1.25 \times 1.02$

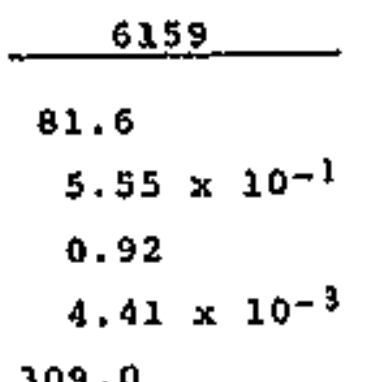

09.0

$2.80 \times 10^{-2}$

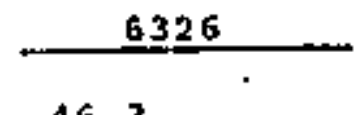

46.3

$3.15 \times 10^{-1}$

1.1

$5.26 \times 10^{-3}$

175.0

$1.66 \times 10^{-1}$

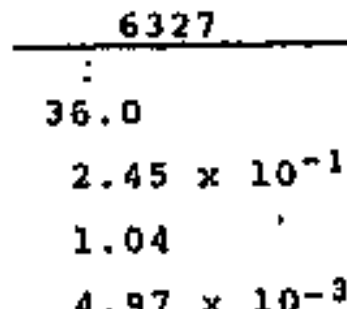

136.0

$1.26 \times 10^{-1}$

$\frac{5693}{9.3}$
$6.93 \times 10^{-2}$
0.73
$3.48 \times 10^{-3}$

35.0

$4.94 \times 10^{-2}$
7279

40.0

$2.70 \times 10^{\circ}$

0.73

$3.48 \times 10^{-}$ 151.0

号 


$$
T-25-i 3
$$

\section{internal Letter}

Da1e: . July 17, 1980

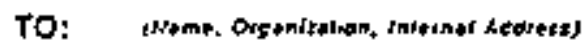

- D. E. Bowers \& H. C. Teats

- Plant Eng íneering

- 2750E/AJ0O

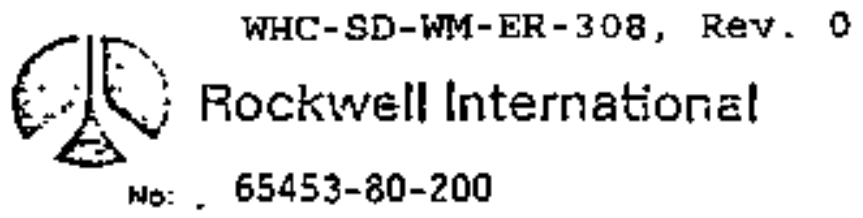

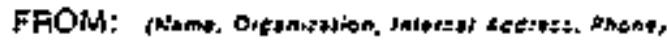

- H. T. Jansky \& D. L. Herting

- Separations Process Development Unit 222-5/M0-037/200 West

3-1571 and 3-3612

Subjet: . Hot boildown of 102A slaste Liquor

Three samples of 102A waste were received by Analytical Laboratories (AL), *

The waste was feed for the $242-A$ evaporator. The samples were and lyzed

and the data are shown in Iable I. Note that the analyses are very similar.

The samples were then transferred to the Separations Process Development

Unit (SPOU) for hot boildowns. The waste consistentiy nucleated instantaneously at $>70 \%$ waste volune reduction (WWR). This supports analytical deta indicating relative homogeneity of uncomplexed waste. Labotatory details are discussed below.

Tank 102A Sample ET-1243 contained $=5$ volume percent solids as recelved by SPDU. This agrees with AL visual observation but contrasts with cooling curve data. The sample was taken trom the suritce of the waste. Teble II shows LVR versus temperature data. Aatter nucleation at $70 \%$ WVR, the phases were separated by centrifugation end submitted to $\mathrm{AL}$, ior analyses. The product analyses are shown in teble III.

Samples fT-1244 (4 ieet bolow the suriace) and $T-1245$ ( 7 feet below the surTace) there combined in a 50:50 composite. Table IV contains UVR versts temperature data. Nucleztion occurred instantaneously at $73^{*}$ WVR, which is within experimental error of the earl ier $70 \%$ WVR. Ito product analyses tere reques ted.

Please call if you have eny questions regarding this work.

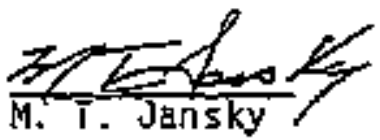

Advenced Chemist

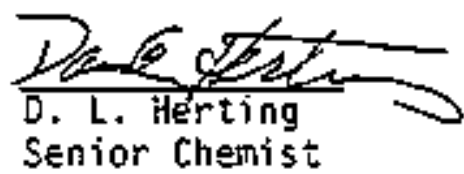

MTJ: DLK: naj

At tachinents

Distribution:

L. C. Brown

J. 5. 3uckingham (xifinis:

T. A. Lane

4. E. Ooren

$\bar{T}$. B. Veneziano

Process Ajds:

File Code: KF55E

Letterbook 


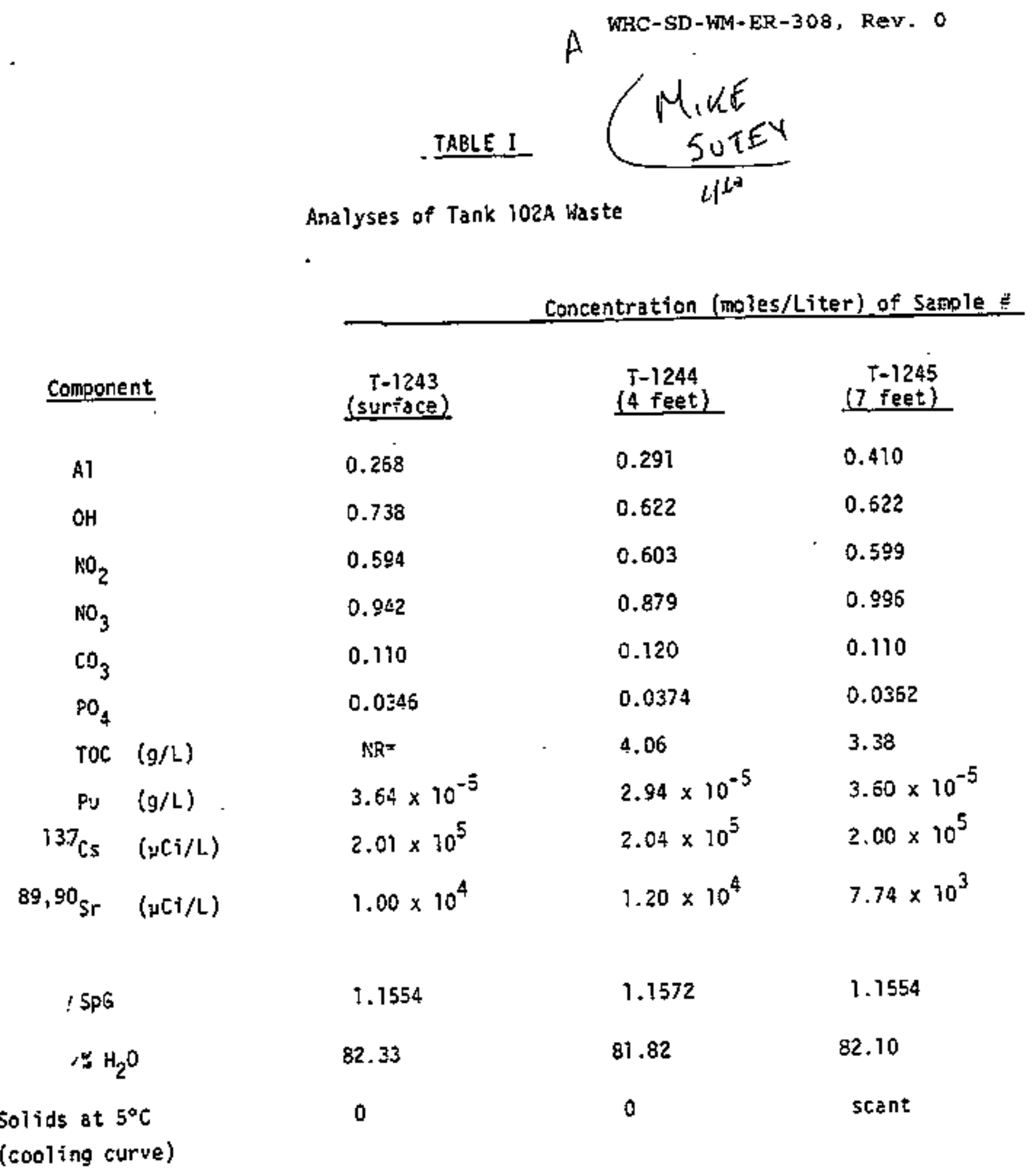

* Not Requested 
WHC-SD-WM-ER-308, Rev, 0

\section{TABLE II}

WVR versus Temperature for J02A Waste (ET-1243)

WYR

0

10

20

30

40

50

60

$69.6 *$
Temperature $\left({ }^{\circ} \mathrm{C} /{ }^{\circ} \mathrm{F}\right)$

$35.8 / 96.6$

$43.6 / 170.4$

$49.2 / 120.6$

$36.2 / 97.2$

$44.4 / 111.8$

$49.6 / 121.4$

$36.8 / 98.4$

$44.8 / 112.6$

$50.6 / 123.0$

$33.6 / 101.8$

$45.4 / 113.4$

$51.0 / 124.0$

$39.3 / 103.2$

$46.5 / 715.8$

$52.0 / 725.6$

$40.0 / 104.0$

$48.2 / 119.0$

$54.4 / 130.0$

$42.8 / 109.2$

$50.8 / 123.4$

$57.4 / 135.4$

$52.2 / 126.0$
Pressure (torr)

40

60

80

40

60

80

40

60

80

40

60

80

40

60

80

40

60

80

40

60

80

40 
TABLE III $\quad N^{0}$.

Product Analyses of Tank 102A hot Boildown (5ample gT-1243)

\begin{tabular}{|c|c|c|c|c|}
\hline Component & Molarit & $\begin{array}{c}\text { Supernatant } \\
y \text { (moles/liter) }\end{array}$ & Wt. $g$ & $\begin{array}{l}\text { Solids* } \\
\text { wt. "ֶ }\end{array}$ \\
\hline A1 & 1.73 & & 30.2 & 0 \\
\hline $\mathrm{OH}$ & 2.57 & & 7.4 & 5.7 \\
\hline $\mathrm{NO}_{2}$ & 2.19 & & 10.9 & 0 \\
\hline $\mathrm{NO}_{3}$ & 2.79 & & 17.1 & 69.4 \\
\hline $\mathrm{CO}_{3}$ & 0.31 & & 2.4 & 21.1 \\
\hline $\mathrm{PO}_{4}$ & 0.12 & & 1.4 & 3.8 \\
\hline TOC & 11.02 & & $2.35^{\pi *}$ & 0 \\
\hline $\mathrm{H}_{2} \mathrm{O}$ & & & 45.8 & 0 \\
\hline$S p G$ & & 1.39 & & \\
\hline Tota 1 & & . & 98.6 & 100.0 \\
\hline $\begin{array}{l}\text { *Ana jysis } \\
\text { wt: shown } \\
\text { in soiuti }\end{array}$ & $\begin{array}{l}\text { i TOC for solid phase } \\
\text { here are based on the } \\
\text { n. }\end{array}$ & $\begin{array}{l}\text { was apparently } \\
\text { assumption that }\end{array}$ & $\begin{array}{l}\text { in error. } \\
\text { ai) corsio }\end{array}$ & $\begin{array}{l}\text { The solids } \\
\text { lexants rema ined }\end{array}$ \\
\hline
\end{tabular}


WV versus Temperature for 102A Haste *

WR

0

Temperature $\left({ }^{\circ} \mathrm{C} /{ }^{\circ} \mathrm{F}\right)$

$36.8 / 98.4$

$43.4 / 110.2$

$49.4 / 120.8$

$36.4 / 97.2$

$44.0 / 111.2$

$49.6 / 121.4$

$36.6 / 98.0$

$44.2 / 111.6$

$49.8 / 121.8$

$37.2 / 98.8$

$44.6 / 112.4$

$50.6 / 123.0$

$38.2 / 100.8$

$45.4 / 113.6$

$51.4 / 124.6$

$39.8 / 103.6$

$46.8 / 116.4$

$52.8 / 127.2$

$41.6 / 106.8$

$49.2 / 120.6$

$55.2 / 131.4$

$47.6 / 117.6$

$55.2 / 131.4$

$61.6 / 142.8$
$50.2 / 122.4 \quad \mathrm{H}-110$

Pressure (torr)

40

60

80

40

50

80

40

60

80

40

50

80

40

60

80

40

60

80

40

60

30

40

60

80

0

0

0

0

0

40

80

0

0

0




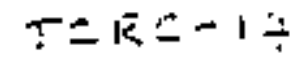

\section{- Internal Letter}

Date: . August 4, 1980

TO: Wame, Organ zalon, mivend Aoeress]

- H. C. Teats

- Plent Engineering

- 2750E/AIOO
WHC-SD-FM-ER-308, Rev. 0

nulavell filersadiorial

No. , 65453-80-213

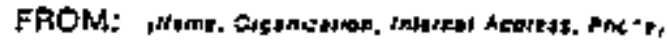

- M. T. Jansky

- Separations Protess Developrent Unit

. 222-S/M0-037/200 Hest

. 3-1571

Sudeer:. Hot Boi jown of Tank 102A Waste

A hot bofldown of Tank J02A waste was perforned by Separations Process Development Unit (SPBU). The waste was an uncomplexed $2 ₫ 2 A$ feed. Instantaneous nucleation occurred at $60 \%$ waste volume reduction (WVR). Laboratory details are discussed below.

Two J02A samples were received by SPDU iron Analytical Laboratories. The anaiytical data are shown in iable I. Sample $\bar{T}-2405$ had a trace of brown settled solids when received by SPDU, while sample IT-2404 was a clear, yellow liquid. Fifty al alquots of each were combined for a composite boildown. WVR yersus tenperăture data are shown in Tabie II. Nucledtion occurred instantêneously at 50\% WWR, indicazing a non-complexed behavior of the waste. The siurry was allowed to setitle resulting in $=95^{\circ}$ settied soiids etter 10 minutes, $90 \%$ settled solids after an hour, and $=80 \%$ settled solids after sitting overnight. The product solids visually appeared to be a mixture of large and small crystals. As we discussed over the phone, the product phases were not analyzed.

Please call tif you have any questions regarding this work.

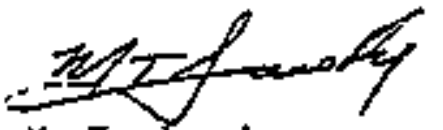

M. T. Jansky

Advanced Chemist

$\sim^{\top} \mathrm{T} J / n a j$

Attachments - (2)

Distribution:

D. E. Bowers

L. C. Brown

J. 5. Buckingham_ 153

H. J. Eding

4. A. Gale

T. A. Lene

W. E. Ogren

T. B. Veneziano

File Code: KF55

Process Aids: (8)

Letierbook 
WHC-SD-WM-ER-30日, Rev. 0 TABLE I H AIO $2.1 \%$

Analytical Data for Jank 102-A llaste

\section{Component}

( A)

, OH

- $\mathrm{NO}_{2}$

, $\mathrm{NO}_{3}$

, $\mathrm{CO}_{2}$

, $\mathrm{PO}_{4}$

, joC (g/L)

, Pu $(g / L)$

137 CS ( $\mathrm{WCT} / \mathrm{L})$

$.89,90 \mathrm{sr}(\mathrm{u} C 5 / \mathrm{L})$

, $\mathrm{ar}_{2} \mathrm{O}$

, SpG

Cooling Curve**
Molarity (moles/Liter)

Sample 市
$\underline{\mathrm{T}-2404}$

0.546

1.24

0.433

1.50

0.240

0.0594

5.62

$2.72 \times 10^{-5}$

$2.21 \times 10^{5}$

$7.77 \times 10^{3}$

73.89

1.180

No solids
$\underline{T-2405}$

0.521

1.27

*

1.57

0.230

0.0591

6.34

$2.76 \times 10^{-5}$

$2.27 \times 10^{5}$

$8.05 \times 10^{3}$

79.7

1.199

No Solids

* Not completeo

$* *$ From $50^{\circ} \mathrm{C}$ down to $0^{\circ} \mathrm{C}$ 
IABLE II

WVR versus Temperature for Tank 102A Haste

क्wWR

Temperature $\left({ }^{\circ} \mathrm{C} /{ }^{\circ} \mathrm{F}\right)$

Pressure(torr)

0

$37.2 / 99.0$

40

$45.0 / 113.0$

60

$50.8 / 723.4$

80

10

$38.0 / 100.6$

$4 \mathrm{C}$

$45.6 / 114.0$

60

$51.4 / 124.6$

80

20

$38.4 / 101.2$

40

$46.2 / 115.0$

60

$52.2 / 125.8$

80

30

$40.4 / 104.6$

40

$47.4 / 117.4$

60

$53.2 / 127.8$

80

40

$47.2 / 706.2 \quad 40$

$49.4 / 120.8$

60

$55.2 / 73) .4$

80

50

$44.2 / 131.6$

40

$52.2 / 126.0$

60

$58.2 / 736.8$

80

$60^{*}$

$49.6 / 127.4$

40

$57.6 / 135.4$

60

$63.6 / 746.4$

80

* Instantaneous nuclestion et $60 \%$ WWR 
CCCM TCRC-15 $102+A$

\section{internal Letter}

Cate: - December 23, 1980

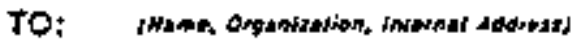

- M. C. Teats

- Plant Engineeríng

- 2750E/A100/200 East
WHC-SD-WM-ER-308, Rev. O

No: . 65453-80-395

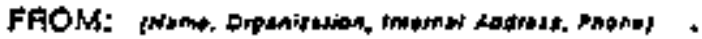

- H. T. Jansky

- Separations Process Development Unit

- 222-5/MO-037/200 Wes 6

3-3571

Sublect: Composition of Waste in Tank 102A

Waste samples from Tank 102A were received by the Separations Process Development linit (SPOU). The waste had been transferred to $102 \mathrm{~A}$ from iolA due to "tank growth". The sanple phases were separated and prepared for anatyses by SPDS. The enalyses are shown in Tabies I and II. It should be noted that the low concentration of aluminum in the samples required the use of inogerative equipment. Therefore the aluminuri values show were determined by difference. The andyses indicate that the 501ids present in the waste are primarily sodiun aitrate. Laboratory detatis are discussed below.

Iwo samples of Tank $102 \mathrm{~A}$ waste were received by SPOJ. Both samples appeared to have approxinately thirty volume percent large crystalline soliss. Tine samples were eastiy stirred to obtain representative aliquots, and the aliquots were $i$ iltered via vacuut. . Keedle-like solids werg obsarved for the deep sample as well as la roe, grainy crystals. The solids were dissolved and subnitted for enalyses, along with the filtrate. The ijnal sompositions are shown in Tables I and II. Note that the deep semple contained approximateiy fifteen wefght percent soltus, end the shallow sample contained approximately nine weight percent solids.

The low concentrations of aluminum in the semples required the use of the atomic absorption spectrometer. Since the unit was inoperative, the aluminum values shcwn in the Taoles are calculated by difierence. Table It shows that the solids are eleven weight percent soditum hydroxioe. Sodtum hydroxide should not precipitate, naking the percentages of the other components really higher.

The data show that the solids are prinarily sodiug nitrate. This conitrms visual observations.

Please carl if you have any questions or comments.

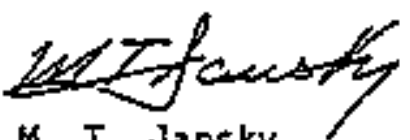

M. T. Jansky

Advanced Cherist

MTJ/naj

Ofstribution:

D. E. 8owers

J. S. Buckingham

File Code: WC667

1. A. Ga]e

D. L. Herting

Process Aids:

T. A. Lane

D. A. Reynolds

D. G. Wilkins

T. B. Veneziano 


\section{TABLE I}

WHC-SD-WM-ER-30B, Rev, 0

Composition of Tank 102A Was ce - Deep Sample

Filerace

Solids

Companenc

Nolazicy (Woles/Liter) Weighcz*

heighcz

$\mathrm{NaAlO}_{2}$

NaOB

$\mathrm{NaNO}_{2}$

NaNo $_{3}$

$\mathrm{He}_{2} \mathrm{CO}_{3}$

$\operatorname{ToC}(g / \mathrm{L})$

$\mathrm{Na}_{3} \mathrm{PO}_{4}$

$9 \mathrm{~S}_{\mathrm{SI}}(\mu \mathrm{CI} / \mathrm{\tau})$

${ }^{137} \mathrm{Cs}(\mu \mathrm{Ci} / \mathrm{L})$

$60 \mathrm{Co}(\mu \mathrm{Ci} / \mathrm{L})$

$106_{R \Psi}(\mu \mathrm{Cl} / \mathrm{L})$

Pu $(\mathrm{g} / \mathrm{L})$

$\approx \mathrm{H}_{2} \mathrm{O}$

SpG $2.42^{\circ}$

3.31

3.22

2.06

0.14

1.79

0.04

$4.19 \times 10^{3}$

$6.30 \times 10^{5}$

NoF Defected

Not Detected

$3.38 \times 10^{-5}$
13.77

9.19

15.43

12.16

1.03

0.37

0.46

13 


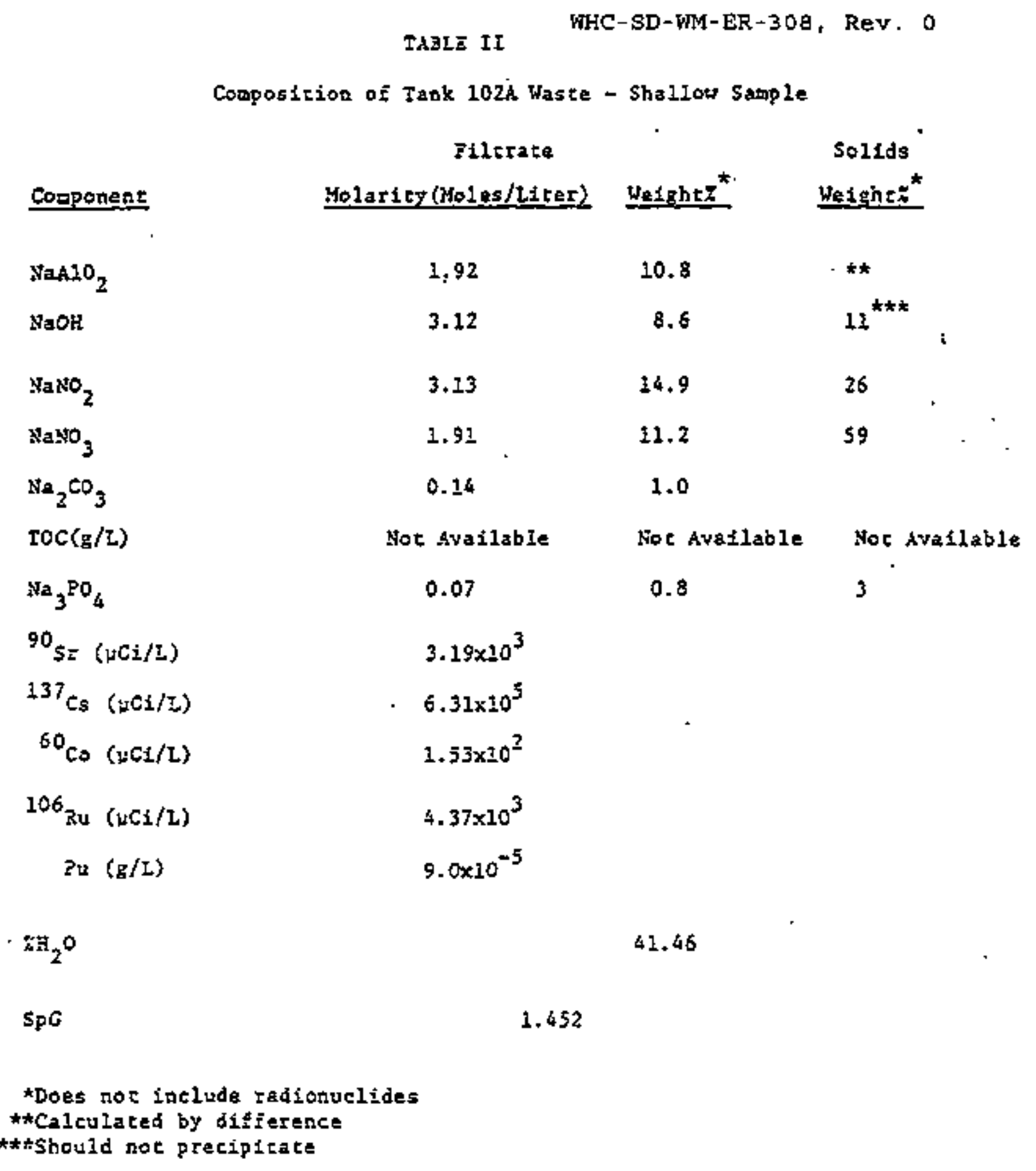


$\operatorname{TCR}-16$

$\therefore$ Infornal Letter

Ou:e; . Nay 26, \$981

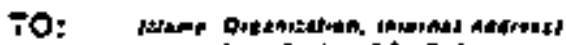

- J.S. Schotietd

- Sys tens Engineering

. 2750E/AJ19/200E
WHC-SD-WM-ER-308, Rev. 0

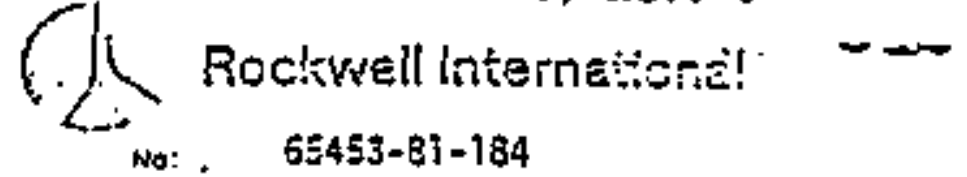

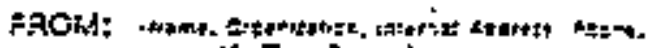

h. T. iansky

- Separations process Developuent:

- 222\$/M0-037/720

. $3-157 \mathrm{j}$

Sueject: Pu and Afr Analyses of Various Tank Fara Samples it pạ-

Reference:

Letter

:

in

j.S. Schofield, "Dejay in $P_{y}$ and An Anajys of Varieus $\cdots ;$ Tank Farah Sanples."

The reterenced Ietter explained thet a delay would ofcir before Py and Aft analytical resuits could be fonurded to you. As of this lritinge sayeral analyses heve been completed. The outstending anaiyses utill be reported at a latar date. The avalibble results are shown in Table I.

All samples were archived spot sampies. The sampies were sitiered on a

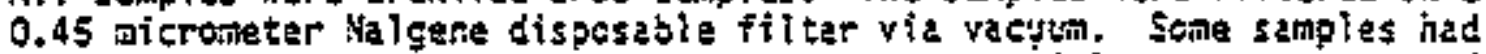
solidisjed, end required waming in a water bath to loosen then vo. Arttar filtration the tiltrates were irrediately sutmittad to AL for enalyses. Solids were dissolved and subvisted tor analys es es welt, when sutificient solfds were retzined on the filtar.

The data shown in Tabie I indicatas a strong trand towards low Fo levels in the fjitrates. As expected, filtration renoving even a trace of solids significantly decreased the Pu detected. A simblar trand treuld be expected for $\mathrm{Am}$, but sufficient evidence is not availebla to verify this.

As stated farlier, the rotaining rasults will be forwarcied to you when SPQU refsives then. Please call if you have any çuestions or coments.

M. T. Jans ky

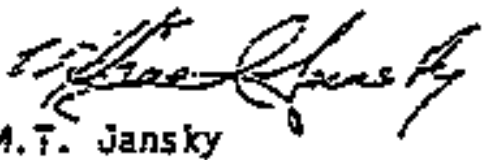

Senior Chentst

HTS/ 10

cc: J.S. 3uckinghan

C.H. Delegars

D.L. Herting

J.R. Wetch

File Code: W966̄

$p+w+\lambda i$ 


\section{TABLE I}

\section{$P u$ and An Analyses in Various Tank Fam Samoles}

Tank IO

$1097 x$

$1 / 07 / 81$

jO2A Sottoms

$12 / 02 / 80$

$10 \mathrm{t}$ AX 4' Below

$10 / 31 / 80$

10IA 4' Selow*t

$11 / 11 / 80$

DSS Frodust***

$11 / 05 / 80$

101A 4. 3elow

$11 / 10 / 80$

102A Botzons

$12 / 02 / 8$ ]

spiked with HEDTA

\author{
Pu* \\ $1.45 \times 10^{-5}$ \\ $\mathrm{Am}$ \\ $5.03 \times 10^{-6}$ \\ $1.32 \times 10^{-7}$ \\ $5.79 \times 10^{-7}$ \\ $1.58 \times 10^{-5}$ \\ $3.87 \times 10^{-8}$ \\ $1.02 \times 10^{-5}$ \\ $8.32 \times 10^{-7}$ \\ (Solids analyses noc svailable)

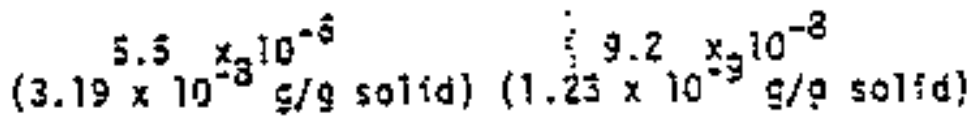 \\ Not siveilaole \\ Hot Avaliable
}

tResults giyen in grams per liter of filtrate * Assume SpG $* 3.45$

th-As sume sog $=7.6$ 


\section{Internal Letter}

Dare. July 14, 1986

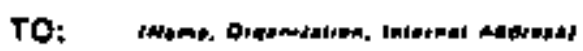

- R. L. Heiss

- MO-037, 200W

- 3-1972

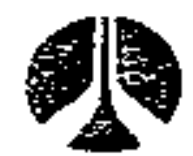

Rockwell International

No. 65452-86-100

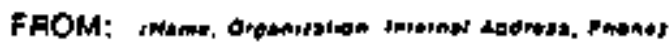

- R. G. Clark

- M0-037, 200W

- 3-2271

Subpet, I-129 Analytical Data

The Analytical Process Development Unit analyzed the following samples for I-129 concentrations. About half of the samples were solids and the rest were clear aqueous solutions. The solid sample sizes ranged from 0.9 to 3.0g. One ml of an aqueous sample was analyzed in each instarce. The counting instrument could detect about $2 \times 10^{-5}{ }_{\mu C j}$ of $1-129$.

Potassium fodioe carrier was added to each sample at the beginning of the anslytical procedure to provide a recovery factor for correcting the I-129 counting data to 200 percent recpvery. The iodide recoveries for the solit samples ranged from 55 to 75 percent and 70 to 95 percent for the aqueous samples.

The analytical data from the first eight solid samples in this series were reported in June.

Please tontact D. A. Dodd or mysel $i \frac{1}{t}$ there are any questions concerning the attached data.

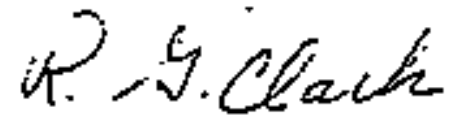

R. G. Clerk

Chemist

RGC/CSE

Attachinent

Ec: D. R. Bratze]

J. P. Sioughter 


$$
\text { A 10\%+', }
$$

R. L. Heíss

Page 2 - Attachment

July 14, 1986

$50 L I D S A M P L E S$

늘 No.

$B 1 \times C$

$B 2 \times C$

FIXC

F $2 \times C$

DIXC

$C \mathrm{IXC}$

G1XC

Lab Nㅏㅇㅡ.

91XD

$92 \times 0$

$31 \times 0$

$B 2 \times D$

FIXD

F2XD

$71 \times 0$

$72 \times 0$

$81 \times 0$

$82 \times 0$

CIXD

G1X0

AIXD

A2XD
Tank No. 니 I-129/G

$103 A$

$103 \mathrm{~A}$

$103 C$

$103 \mathrm{C}$

$104 C$

$105 \mathrm{C}$

$106 C$
$1.9 \times 10^{-5}$

$2.3 \times 10^{-5}$
$1.9 \times 10^{-5}$

$<3.3 \times 10^{-5}$
$1.2 \times 10^{-4}$

$8.1 \times 10^{-5}$

A OUEOUS SAMPLES

Tank No.

102A

$102 \mathrm{~A}$

2034

$103 \mathrm{~A}$

$103 C$

$103 \mathrm{C}$

$1048 X$

$1043 X$

$1058 \mathrm{x}$

$1053 X$

$105 C$

$106 \mathrm{C}$

106A

106A vici $1-129 / \mathrm{ml}$

$2.2 \times 10^{-4}$

$2.1 \times 10^{-4}$

$7.9 \times 10^{-5}$

$1.2 \times 10^{-4}$

$2.7 \times 10^{-4}$
$4.0 \times 10^{-5}$

$1.1 \times 10^{-4}$
$1.1 \times 10^{-4}$

$4.6 \times 10^{-5}$

$5.6 \times 10^{-5}$

$2.7 \times 10^{-4}$

$1.8 \times 10^{-4}$

$1.8 \times 10^{-4}$ 


\section{Aristing}

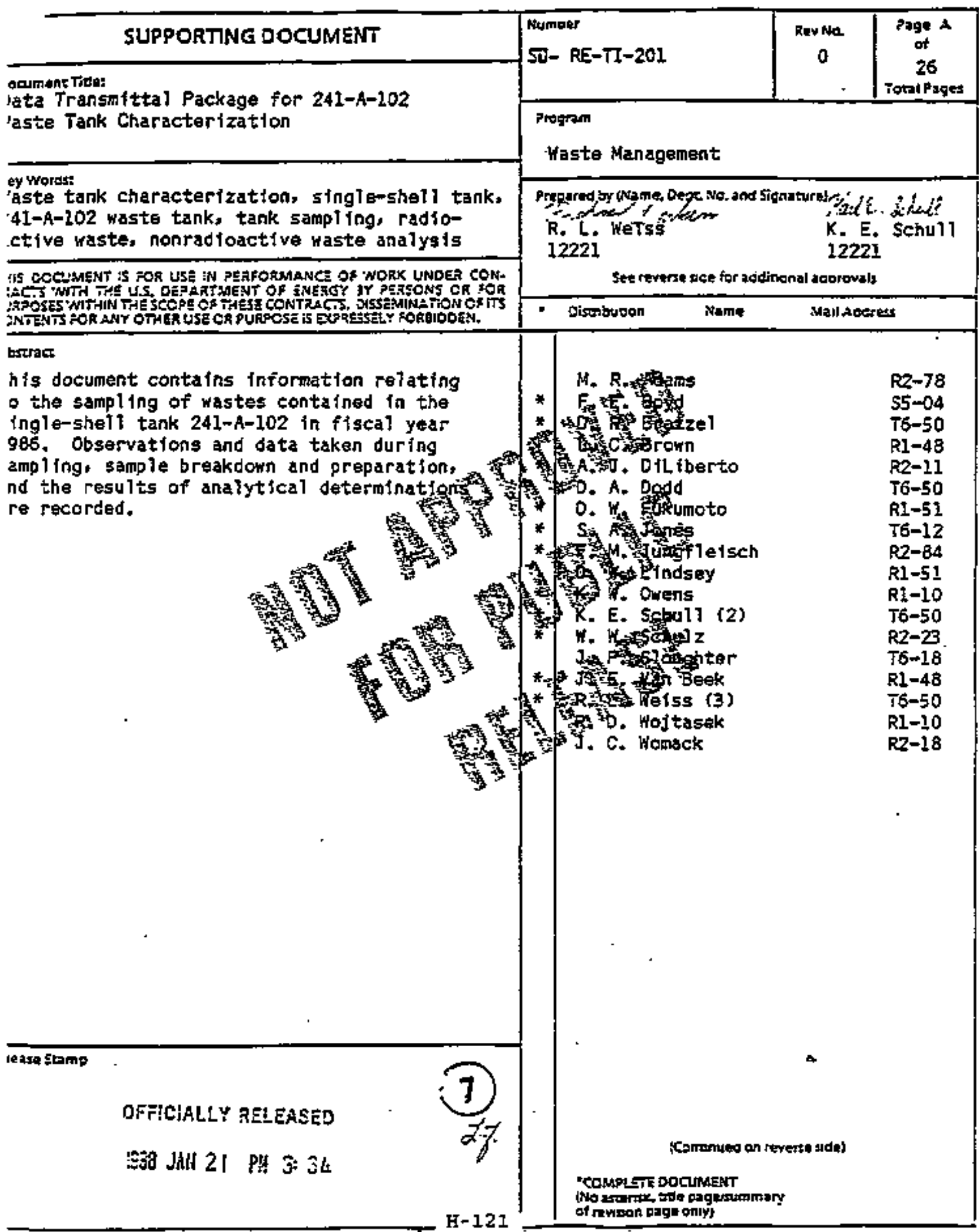


$201-6 E-T I-201$

Rev. 0

\section{ANALYTICAL SAMPLE PREPARATION DATA}

The actual weights and volumes generated during analytical sample preparation (so) uttion Teaching) for the cors composites from tank 241- $h-102$ are shown. Peptization of the solid nay occur in some of the water ieaches (third contact). This forms an unseparable sollo-liquid suspension broken by adding concentrated $\mathrm{HCl}$ to the leach to bring the acid concentration to $5 \mathrm{M}$. In these cases, effectively no third water leach occurred and the acidified solution becomes the first acid leach.

No analytical sample preparation was rsquired, except fsttering, for the drainable I fquor core composites.

\section{Compesite. ID $\$ 91 \times$ conox}

Initial weight of sample Yolume

Residual weight:

- Water leach - $1.155 g^{p}$

- Acid leach $=0.485 \mathrm{~g}$

- $\mathrm{HNO}_{3}-\mathrm{HF}-\mathrm{HCl}$ dissolution - 0 .

Final solution volume:

- Water 1each -- $35 \mathrm{~mL}$

- Acid leach - 25 m

- $\mathrm{HNO}_{3}-\mathrm{HF}-\mathrm{HCl}$ dissolution -- $25 \mathrm{~mL}$.

- Acid leach and $\mathrm{HNO}_{3}-\mathrm{HF}-\mathrm{HCl}$ fraction sombined for analysis.

D Sample peptized on third water wash -- concentrated HCl becoming first water wash. 
SD-RE-TI-201

Rev. 0

Composite ID $\$ 92 \times c 00 \times x$

Initfal reight of sample

$4.984 \mathrm{~g}$

Volume

$3.0 \mathrm{~mL}$

Residuai yeight:

- Hater leach $-2.250 \mathrm{~g}^{\mathrm{p}}$
- Acid leach - $1.517 \mathrm{~g}$
HNO $-\mathrm{HF}-\mathrm{HCl}$ dissolutíon $=0$.

Final solution valume:

- Water leach $=35 \mathrm{~mL}$

- Acia leach - 25 mL

- HNO $-H F-H C I$ dissolution -- 25 mL.

- 10.55 ml of acid Jeach combined with 25 mL $\mathrm{HNO}_{3}-\mathrm{HF}-\mathrm{HCl}$ dissolution and submitted for analysis.

P Sample peptized on third water wash -- concentrated HCI beconing firsi water wash.

$10.54 \mathrm{~g}$ carried on to dissolution step. 


\section{NAL TICAL RESULTS}

A spreadsheet program on a bedfcated microcomputer is used to convert raw analytical data to concentration values for the components in the water. The following are coples of the spreadsheet printouts for the core composite samples from tank 24i-A-102. For the solid phase core composites, concentration values from the individuel leaching solutions (water, HCl-HNO $-\mathrm{HF}$ pressure dissolution) are reported along with overall results. Two overall results are reported: REPORT TOTAL - sum of all values reported greater than the 1 imit of confirmation ("less then values" not sumined) and MAXIMUM TOTAL suar of all values reported with init of confirmation values taken as actual value (worst case).

Physical data deterainad are shown on the spreadsheet reports. 
r.

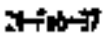

SO-RE-TI-201

Rev. 0

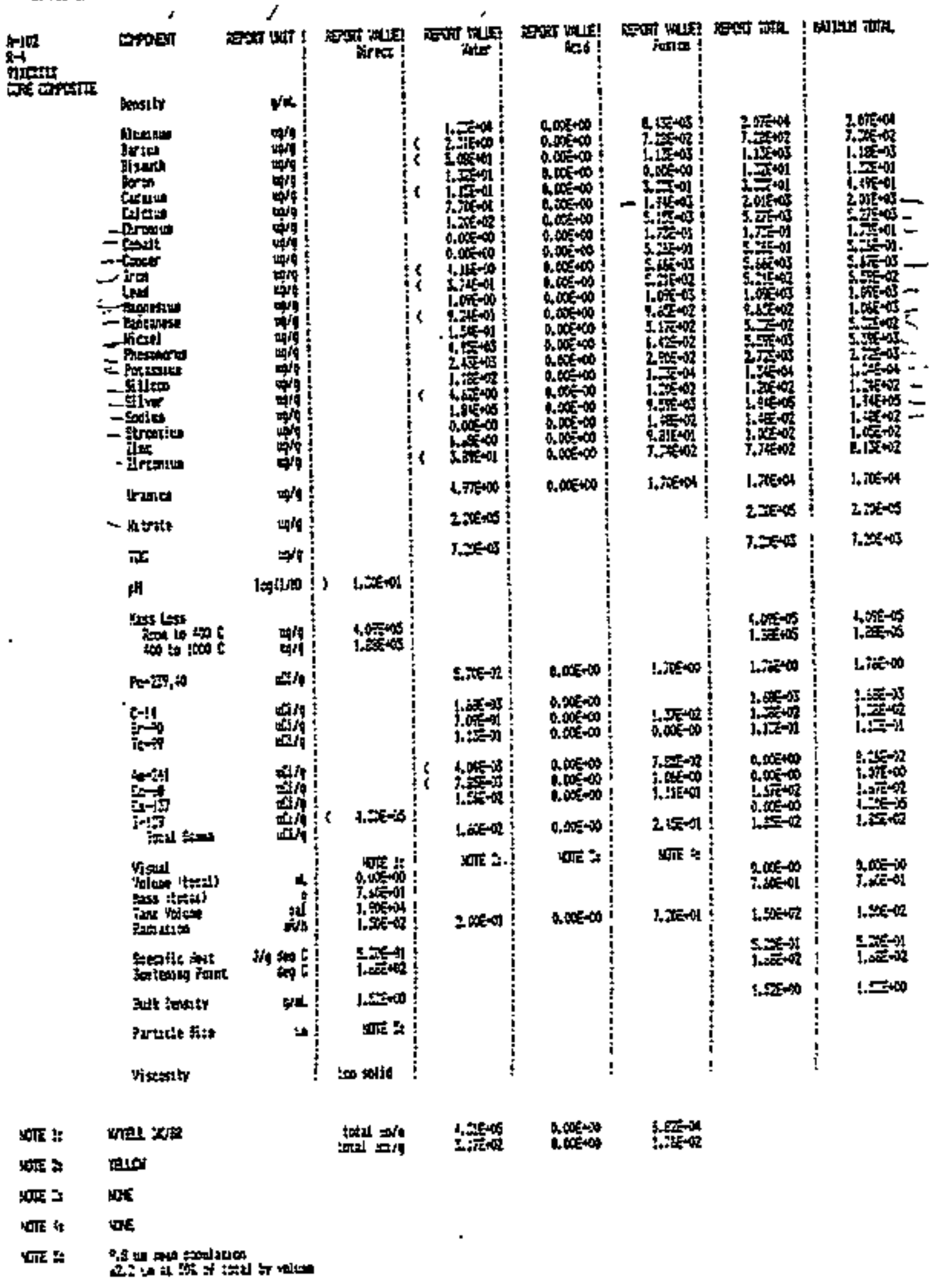


WHC-SD-WM-ER-308, Rev, O

SD-RE-TI-201

Rev. 0

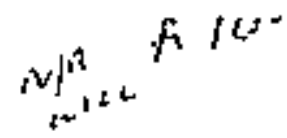

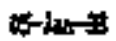

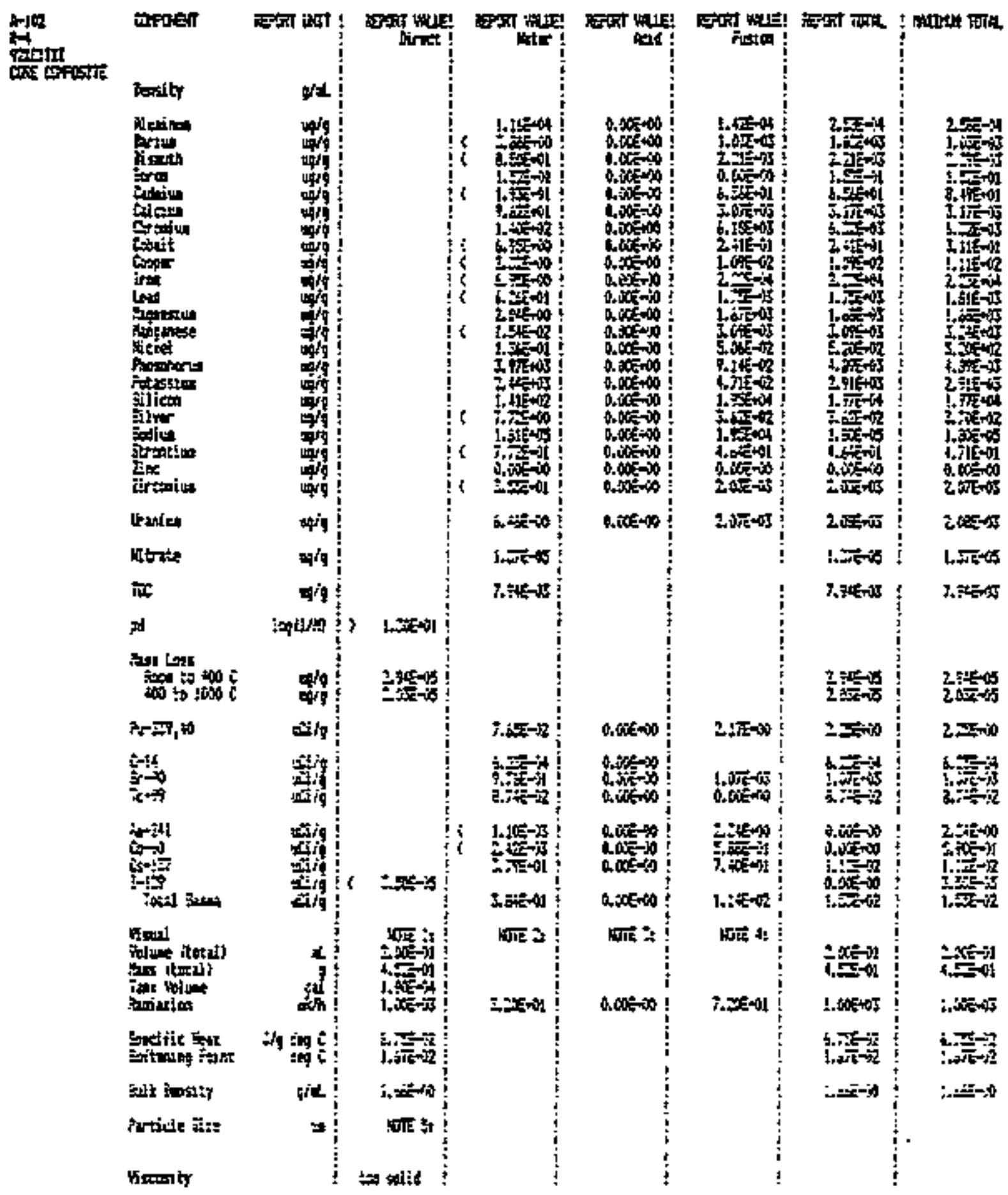

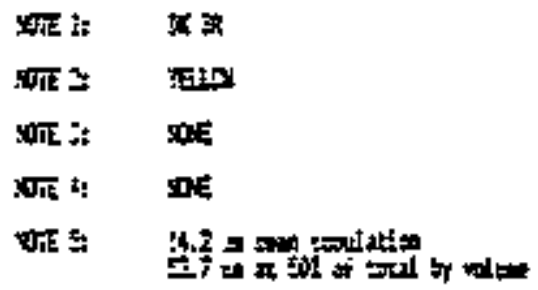

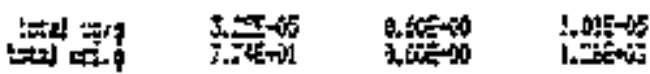




$$
\text { T长こ-23 }
$$

Westinghouse

WHC-SD-WM-ER-308, ReV. 0

Hanfort Company

From: Process Chemistry Laboratories

12712-PCL89-112 Rev. 1

Phome: 3-1972 HD-032 200W T6-50

Date: May 9, 1989

SUbJect: ANALYSIS OF LIQUID SAMPLE FRON TANK 241-A*102

To: V. C. Boyles
cc:
D. R. Bratzel
D. E. Baide
J. E. Bramson
$\mathrm{T} 5-40$
Rl-51
P7-50
K. G. Carothers
R2-51
A. J. Di†iberto
$\mathrm{R} 2-12$
6. L. Dunford
RI-51
R. E. Raymond
R2-40
D. E. Scully
$\mathrm{Rl}-51$
D. D. Higgins
RLW $F j]_{e} / L B$
Ṛl-5̣l..

On Narch 15, 1989, a sample of supernatant 1iquid frant tank 241-A-102 was received for analysis. The sample was identified as R4656, sampled at 13:00 on March 14. The sample was loaded into the 1E-1 hotcell and an aliquot of the liquid renoved and submitted for analysis. The results of the analysis are shown in Table 1 .

Please feel free to call me with any questions.

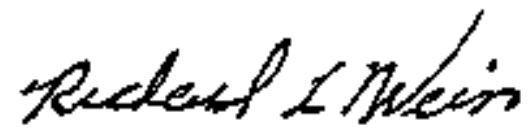

R. 1. Weiss

Senior Scientist

omj

Attachment 
WHC-SD-WM-ER-308，Rev. O

12712-PCL89-1]2 Rev. I

TabTe 1

Analysis of 241-A-102 Liquid

Component

Concentration

Unit

Dionex (Anions

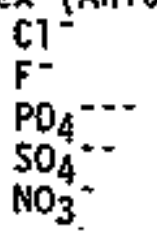

0.0055

$<.068$

$<.055$

1.01

2.08 .

$M$
$M$
$M$

Other Anions

$\mathrm{NO}_{2}{ }^{2}$

0.043

1.02

M

$M$

ICP (Elenenta ])
A7
1.52
0.0029
Ca
0.00074
$\mathrm{Cr}$
0.00082
$\mathrm{Fe}$
0.00012
K
0.146
0.0010
isa
8.72
0.00054
0.0033
0.00124

$M$
$M$
$M$
$M$
$M$
$M$
$M$
$M$
$M$
$M$
$M$

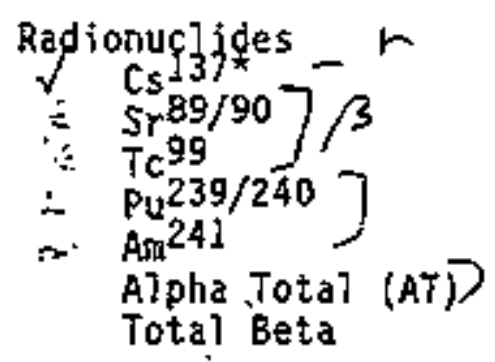

696000

נ580" $"$

369

4.53

0.87

16.8

424000

UC $1 /$ !

$\mathrm{uCi} / \mathrm{q}$

$\mathrm{UC}[\mathrm{i} / \mathrm{T}$

U[i/]

UCi/?

$\mathrm{UC} \mathbf{j} / \mathrm{P}$

$u C j / 1$

Other Analyses

Tota] Organic Carbon

Density

$\mathrm{pH}$

12.5

1.57

13.6

\%water

46.2

$g / 1$

$g / m l$

$\%$

* No other gamma emitting components detected 


$$
T \in R<-4
$$

Date: Decerober 18, 1972

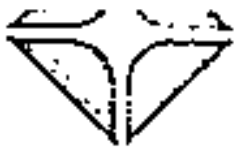

Subject: TANX 103-A SLUDGE CHARAGTERIZATION

Reference: Letter, J. C. Womack, G. L. Borshein to

H. H. Canpbe11, seme subjece, November 7, 1972

The laboratory was requested to ldentiay the operetional characteristics of the 103-A waste tank as it is sluiced, scidified and processed through solvent extraction.

The work perfored shows definite advantages of the flocculating ggents fa sddicion to setfing races. Forcy-seven (47) percent of the stronciun $89+90$ wss found in the acid phase of the prepared PAS when no flocculanc wes used. ragnifloc itproved this leach to 60 percent, but the experimencal Dow jlocced fraction contained 60 percenc in the acid pbase. The resaining stronciun tas distributed in the wacer wish of the solfas and in the solids.

then the flocculants axa actiod a chersical change occurs with spparencly anjarces the otrontilu leact rate. The experianend Dos flocculant also sectles the solids tigbely enough to allow gors of the interstitial liquor co be decented orf.

A core sanple of the 103-d sludge was received and put into the cubicle on incvemiber 13,1972 . tọon examingtion five incties in the borchin end ot the core barrel was very hard while the renatitag six inches ws ftre sofe chocolate brown material. The tine poserial appested co be ver, while the herd meterial apparently conteined littie isterstitial 1iouor. To test this observation and to deternine scrontium discribution, aliquors were tekan of the two phases, weighed

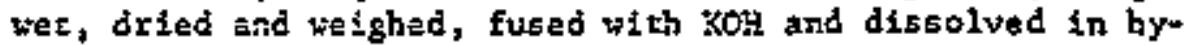
drochloric acid and water. An aifquot of the suparnatant which was received ac the sage time vas also characterized. The aaslyses of the fractions are: 
J. c. Wouneck

Page 2

December 18, 1972

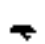

Supernatant

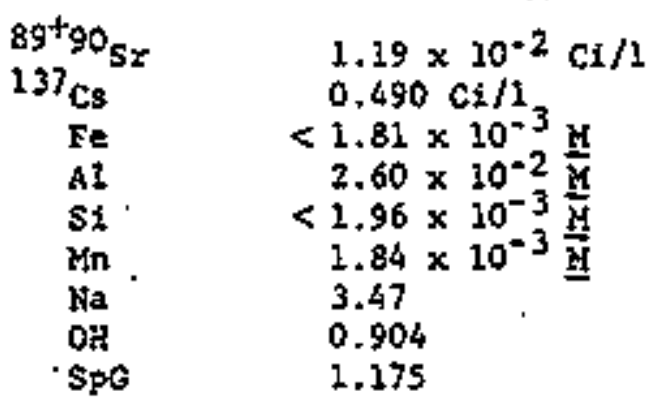

Wet weight

Dry weight

Wet volume

Dry volume ktard

Sludge

\begin{tabular}{|c|c|c|c|}
\hline $\begin{array}{r}1.64 x \\
2.96 \times \\
8.70 \times \\
66.75 \times \\
1.06 \times\end{array}$ & $\begin{array}{l}10^{-2} \mathrm{ci} / \mathrm{g} \\
10^{-3} \mathrm{Ci} / \mathrm{g} \\
10^{-2} \mathrm{~g} / \mathrm{g} \\
10^{-2} \mathrm{~g} / \mathrm{g} \\
10^{-2} \mathrm{~g} / \mathrm{g}\end{array}$ & $\begin{array}{l}1.75 \times 10^{-2} \\
6.55 \times 10^{-4} \\
7.23 \times 10^{-2} \\
3.69 \times 10^{-2} \\
9.49 \times 10^{-3}\end{array}$ & $\begin{array}{l}\mathrm{Ci} / \mathrm{g} \\
\mathrm{Ci} / \mathrm{g} \\
\mathrm{g} / \mathrm{g} \\
\mathrm{g} / \mathrm{g} \\
\mathrm{g} / \mathrm{g}\end{array}$ \\
\hline 0.288 & $g / g$ & $9.90 \times 10^{-2}$ & $g / g$ \\
\hline $\begin{array}{l}1.9671 \\
1.7845 \\
1.0 \mathrm{ml} \\
0.8 \mathrm{ml}\end{array}$ & $\begin{array}{r}1.97 \\
.\end{array}$ & $\begin{array}{l}1.0360 \mathrm{~g} \\
0.50048 \\
0.7 \mathrm{ml} \\
0.5 \mathrm{ml} .\end{array}$ & $4: z^{\prime}$ \\
\hline
\end{tabular}

The solits analyses are calculated on a wet, centrifuged weight basis. These andyses indfeate thac there is no significanc difference in the strontiun distribution between the hard core and fines on a wet basis.

To decemine che solubility of the hard cake, a plece about $3 / 8$ inch olaneter was submerged in about $10 \mathrm{ml}$ or water and ellowed to set undisturbed for 20 hours. The rock had softent on the otitside, however a hard core approxinately 3 inch diameter remained. A second piece was submerged in about $20 \mathrm{ml}$ of water and azitaned with low keat for a weekend. At the end of titis tise perfod, all of the hard sludge had erzoted to the consistency and appearance of the finst. Anocher aliourot of the berd 5 ludge was contacted wich dilute nitric acid. A very vigorous evolurion or g\$s was appazert, eausirg a fotming action which carried some of che sludge out of the vial used to contain tive nixture. The rescion is cypical if cha concentracion of the car* bonate fon were high, although an analysis has noc been made. The work indicates tiat even wich che hard sludge it should be posgible co sluice cise sludge our of the waste tank by continuous recirculation of che supernatent or taber to produce egitation. Withowt agitacion che sluicing progrea would be prohibatively slow.

The raftertince proeecure was followed through with tho settling races with and without tlocculeting agants. These dita are reported under separace cover.+ 
J. c. Wonack

Page 3

Docember 1B, 1972

The decanted superatant after contact with the sludge was analyzed. The results indicate there was no significast consentration chagge in the infloceed or floced aliquots except that the strontiun comcentration appeared to be less in the aliciuoc contacted with the experimental Dow tlocculanc. The hydroxide concencration was also lowered to 0.786 in this flocculation step conpared to 0.916 in the oegniflocted aliquot. Ont possible explanazion for this phenomenon is that all dispersed solids containing scrontiun have been settled, although no solids wete visible in any of the decanted fractions.

A water wash was gade of the solids to thich flocevlants were also abded. The decanted fraction after sereling contained 80 to 90 percent less strontín then the orig. . inal supernacant concained. This quantity is just a dilucion of the supernacant lefe frod the previous step. There is no stgificent difference beveen flocced and wizloceed al iquots.

The distribution oi strontitu azter tije gciditication of sludge varies by more than 20 percenc with the flocculanc. The percentages of stronciun $89 \div 90$ arte:

\begin{tabular}{|c|c|c|c|}
\hline & Cont $=01$ & 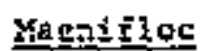 & $x-D$ ow \\
\hline $\begin{array}{l}\text { Acld phase } \\
\text { Ho wash } \\
\text { Soldas }\end{array}$ & $\begin{array}{l}47.0 \% \\
10.2 \% \\
42.8 \%\end{array}$ & $\begin{array}{r}60.3 \% \\
4.9 \% \\
34.5 \%\end{array}$ & $\begin{array}{r}68.27 \\
1.87 \\
29.9 \%\end{array}$ \\
\hline
\end{tabular}

Analyses of the constitients in tise tcid piese include:

$137 \mathrm{csci} / \mathrm{l}$. $39+90 \mathrm{st}$ Ci $/ \mathcal{A}$

$\mathrm{H} \div \mathrm{x}$

Fis

AI 1

$5 \leq x$

频

Vol: of sol. (ml) 3.6
$1.12 \times 10^{-5}$

$1.76 \times 10^{-2}$

0.220

$2.77 \times 10^{-2}$

0.172

0.109

$3.53 \times 10^{-3}$
$2.38 \times 10^{-5}$

$3.05 \times 10^{-2}$

0.172

$1.67 \times 10^{-2}$

0.132

$6.53 \times 10^{-2}$

$2.67 \times 10^{-3}$

10.6
$1.89 \times 10^{-3}$

$4.04 \times 10^{-2}$

0.359

0.111

0.235

0.144

$1.13 \times 10^{-2}$

6.0

Duplicate aliquots of anch of the above PAS acidified feed were renoved for solvenc extraction canpacability tests.

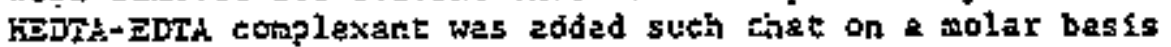
115 percene and 150 percenc of the sio or the iron, aluninutr and matganese was added. 
J. C. Womsek

Fage 4

Decèmber 18,1972

An equal volume of aqueous feed and organic laX was used. The scxontiun was sirippad tron the organic wich an equal volume of $2 \mathrm{M} \mathrm{HNO}_{3}$ for salysis. Matertal balnoes of $92.6 \%$ on the unfloceed aliquor, $91 \mathrm{z}$ on the megnifloc aliquoc and $97.2 \%$ were obtained througi the solvent extration study. The retnainder was probably not stripped iron the organic phas*.

The experimental Dow flocculant apparently had no deleterlous affects on the solvent extrection characteristics. The extraction coefficients, $\bar{E} / 2$ on a curie ratio are:

$\begin{array}{ccc}\text { Control } & \text { Megnitiloe } & \text { X-Dow } \\ 4.9 & 6.3 & 8.0\end{array}$

The differences noted represent very small concentration changes but the face thac tie flocced feeds are boch slightly higher than the control is signiricent.

Two additional batches of PAS were prepared for stronciun leach scudies by a caustic strike of che regidual solids followed by ze-acidificacion. The settled PAS slurry wes decanted to a $1.5 \mathrm{ml}$ jeel. The heel was cencrifuged contacted wich $0.3 \mathrm{ml}$ of 50 percent caustic for 10 minutes. While agteating $4.8 \mathrm{ml}$ of 12 II nieric acid was added. When che caustic was addeo the slưge becand vezy viscose appeared to have no froe 1 isfuted assoclaced wich it, the consistency of mid. The color changed izon light chocolate brown to dark brown. When the sid was added the thocolace brow color returred and the solices settled rspidiy fron tine acid phese.

Analyses of the re-xcidicied acteous phase end solids phese shows that the stronctum sssoclazed with the solids are reduead frem 40 percenc in tie PAS solids to 14 percent by the eaustic scritio. A 30 percent reduccion in the value of sludge remining aiter reaciditicacion was noted.

The taustic strike is Afificule to aceouraty mix with the Actipent evaileble in place. Itis tay afcount for che

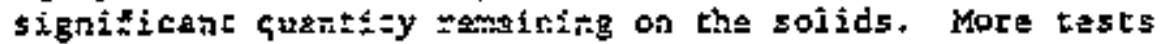
will be perfomed to detrroine optiats leaching condifions with inproved techaiques.

DAD: ch

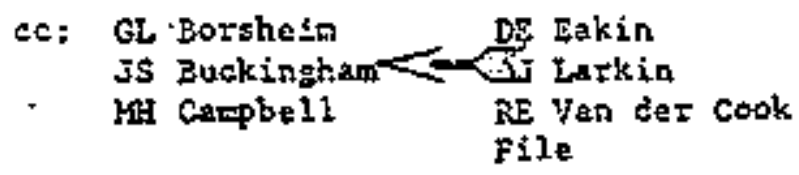


Date: February 12,1976

To: R. E. Felt

From: S. S. Koeģ $\grave{\epsilon}$ r, 942-2214

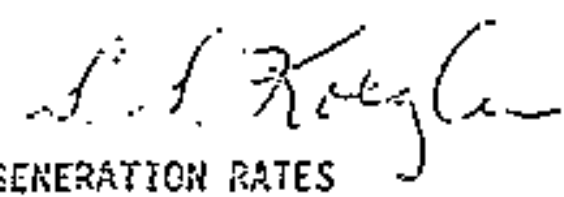

SUbject: A AND AX TANK SLUDGE HEAT EENERATTON R,TES

Please find attached a sunmary of the ${ }^{50} \mathrm{Sr}$ and $137 \mathrm{Cs}$ andlyses which heve been mede on $A$ and $A X$ Farm (and 106-C) siudges. A heat generation rate from these radionuclides is also tabulated for each tank. iote that the 103-A strontium analysis varied 25 percent between the deep and shallow samples. Aiso, the three duplicate sludge samples táken from Tenk 105-A varied by as much as a factor of 3. For these reasuns and the fact that sluicing anay have water-lesched a portion of the cesium and strontium, the above numbers correspond onjy approxinately to the heat generstion rates of the residual sludges.

SSK: vmk

Att.
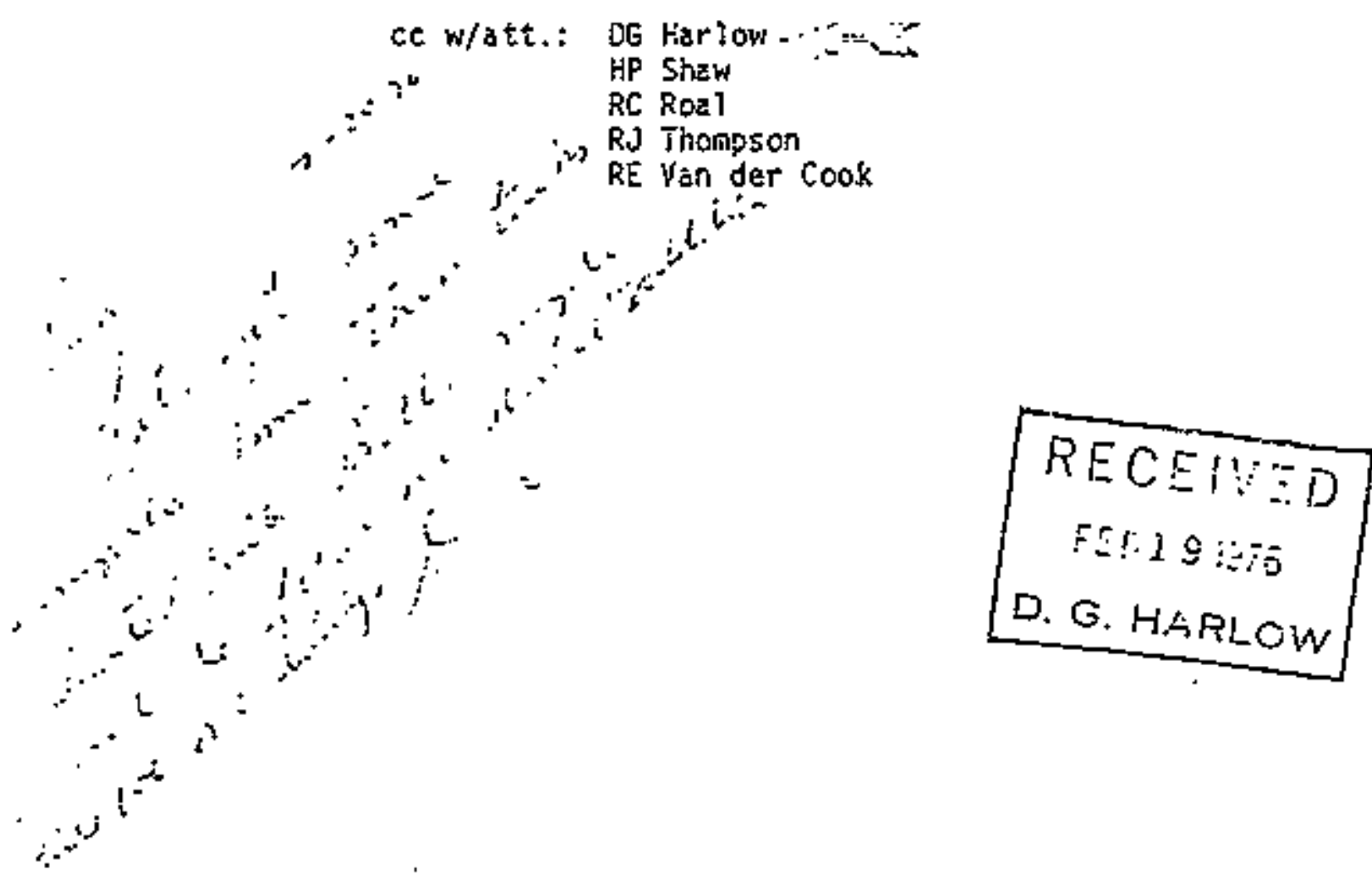
WHC-SD-WM-ER-308, Rev. 0

SU'i-

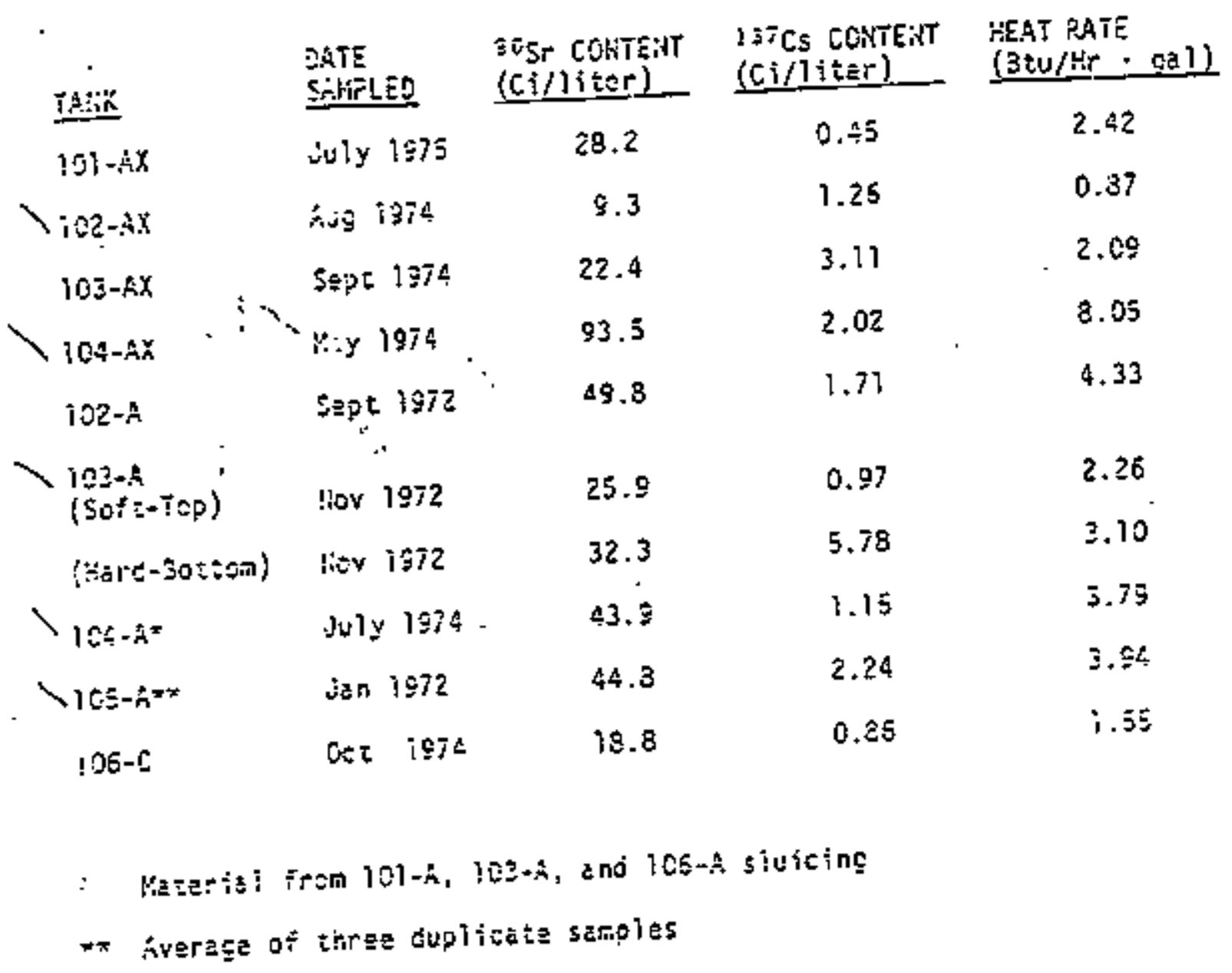


Atlantic Richfield Hanlord Company

$$
T<R(-1)
$$

Date: January 24,1977

To:

J. H. Bailey

From:

J. E. Borton - 2-2529

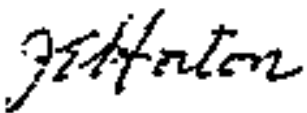

Subject: ANALYSIS OF 103-A TANK RESIDUAL SIDDGE

Chemical Technology Laboratory received a request for strontium and cesium analyses to aetermine heat genexation in residual sludge from Tank 103-A.

Four samples ware recefved from Tank 103-A betwen December 10, 1976 to Januaxy 10, 1977. They were identified as $\# 89$, $\$ 90$, \#667, and $\$ 732$.

The calculated heat genexation rate for sagle based on 69+90s and $137 \mathrm{Cs}$ content, was $6.07 \times 10^{-2}$ watts/litor.

The calculatod heat gineration rate for 5 ample $\$ 90$, based on B9+905x and 13768 content, was $1.68 \times 10^{-1}$ watts/liter.

Tho calculated hat gereration rate for sampl 5667 based on $9+90 \mathrm{se}$ and $137 \mathrm{Cs}$ content, was $1.74 \times 10^{-1}$ wats/liter.

The calculated heat generation rato for sample 73 i, based on $89+905$ and 137 Cs content, was $5.97 \times 10^{-2}$ watts/1iter.

Anglyges ware made by fusing a known volume of centrifuged sluage solids from each sample with KOH, dissolving each melt with concentrated HC2, and diluting each to a known volume with water. Analyses are zeported in Table I.

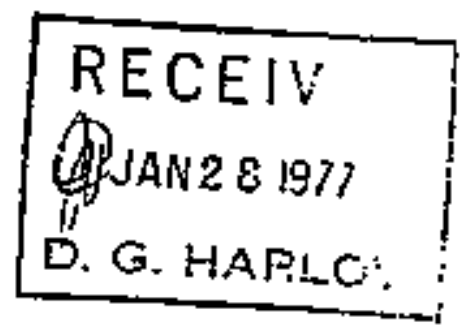


ง. พ. Bailey

Page 2

January 24,3977

TABLE I

ANALYSIS OF IO3-A TANK RESIDUAL SLUDGE

sample

SI $\mathrm{Ci} / 1$ iter watts/3iter oci/gal

Cs Ci/liter watts/3iter Pu g/liter

$\begin{array}{cc}99 & -90 \\ 5.79 \times 10^{-2} & 1.65 \times 10^{-1} \\ 34.0 & 92.0 \\ 0.59 & 0.69 \\ 2.93 \times 10^{-3} & 3.33 \times 10^{-3} \\ 6.35 \times 10^{-2} & 6.72 \times 10^{-2}\end{array}$

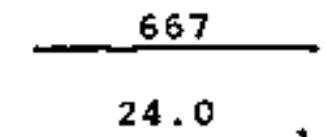

$1.61 \times 10^{-1}$ 90.0

2.8

$1.33 \times 10^{-2}$

0.221

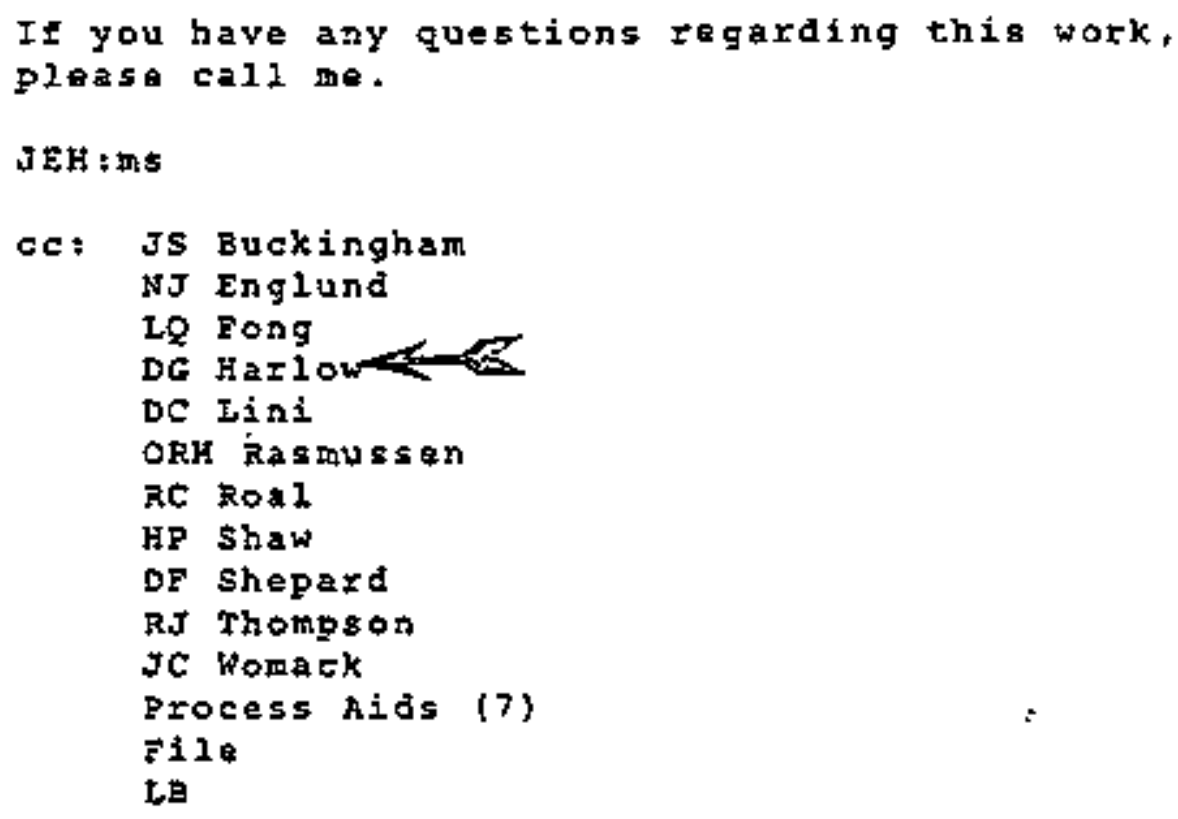




\section{Intcrnal Letter}

Dare. August 2, 1979

To:

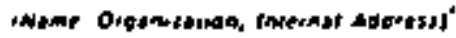

D. R. Jorgenson

- Waste Processing Technology

. 2750-E, 200 East

2-78Il, Ext. 229
RRC $R=17$<smiles>CC(C)C(C)C</smiles>

WHC-SD-WM-ER-308, Rev. 0 No. $65120-79-123 \mathrm{~J}$

FROM:

. T. A. Lane

- Chemical Sciences Group

. 222-5, M0-037, 200 Hest

. 2-1571

Supeel: . Tank 103-A "Hot" BojIdown

A "hot" boildown of Tank 103-A material was requested to verify the temperature-pressure curve and general flowsheet. The temperature versus waste volume reduction (WNR) data is shown in Table I. This material should not be processed as uncomplexed waste. The slurry behavior is similar to that of complexed waste. Experimental details are below.

A single sampte of 103-A material was received and sent to Analytical Laboratories (AL) for analysis. See Tabie II for results. A batch bolidown was run at 40 torr. The PREOICT program showed the nucleation point should have been $5.8 \mathrm{per}-$ cent WVR, however, nucleation was observed at 7.4 percent IWR. The boildown was stopped at 22 percent WVR because the materiai slurry had thickened. A solios/liquid separation was made by centrifuging. Aliquots of both were sent to AL for anajyses. The anayses are shown in Table III. The solids were 43 percent of the slurry.

A test to determine complexant strength remains to be done. The resuits of this test will be reported later.
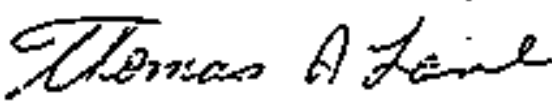

- T. A. Lane, Advanced Chemist Waste Chemistry injt

JAL: jkr

Attachment 2

Information: J. S. Buckinghara

C. H. Delegard

S. C. Estrada

F. M. Jungfleisch 7 ,

L. P. McRae

D. L. Merrick

G. A. 01 sen 


\section{Table I}

WHC-SD-WM-ER-308, Rev. 0

\section{TEMPERATURE VERSISS WASTE VOLUAAE REOUCTION}

AT CONSTANT PRESSURE (40 TORR)

\section{Percent WWR}

0

7.4

22.1

\section{Temperature ${ }^{\circ} \mathrm{C}$}

43.8

46.2 (nucleation)

50.2

\section{Table II}

A 103.17

FEED ANALYSIS OF TANK 103-A

\begin{tabular}{|c|c|c|c|}
\hline & $\frac{\text { Analysis }}{:}$ & Wejght_Percent & \\
\hline SpG & 7.398 & & \\
\hline Percent $\mathrm{H}_{2} \mathrm{O}$ & 53.00 & & $\vdots$ \\
\hline$A 1^{+3}$ & $0.888 \underline{\text { II }}$ & 5.21 & : \\
\hline $\mathrm{OH}^{-}$ & $1.48 \mathrm{M}$ & 4.23 & \\
\hline $\mathrm{NO}_{2}^{-}$ & $1.68 \mathrm{M}$ & 8.29 & \\
\hline $\mathrm{NO}_{3}-$ & $3.00 \quad \underline{M}$ & 18.20 & \\
\hline $\mathrm{CO}_{3}{ }^{2-}$ & 0.80 프 & 6.07 & \\
\hline $\mathrm{PO}_{4}{ }^{3-}$ & $0.039 \underline{\mathrm{N}}$ & 0.45 & \\
\hline $50_{4} 2=$ & $<0.030 \mathrm{U}$ & & \\
\hline \multirow[t]{2}{*}{ Tot* } & $15.4 \mathrm{~g} / 1$ & 1.10 & \\
\hline & & $96.60 \%$ & \\
\hline
\end{tabular}

* Total Organic Carbon 
WHC-SD-WM-ER-308, Rev, 0

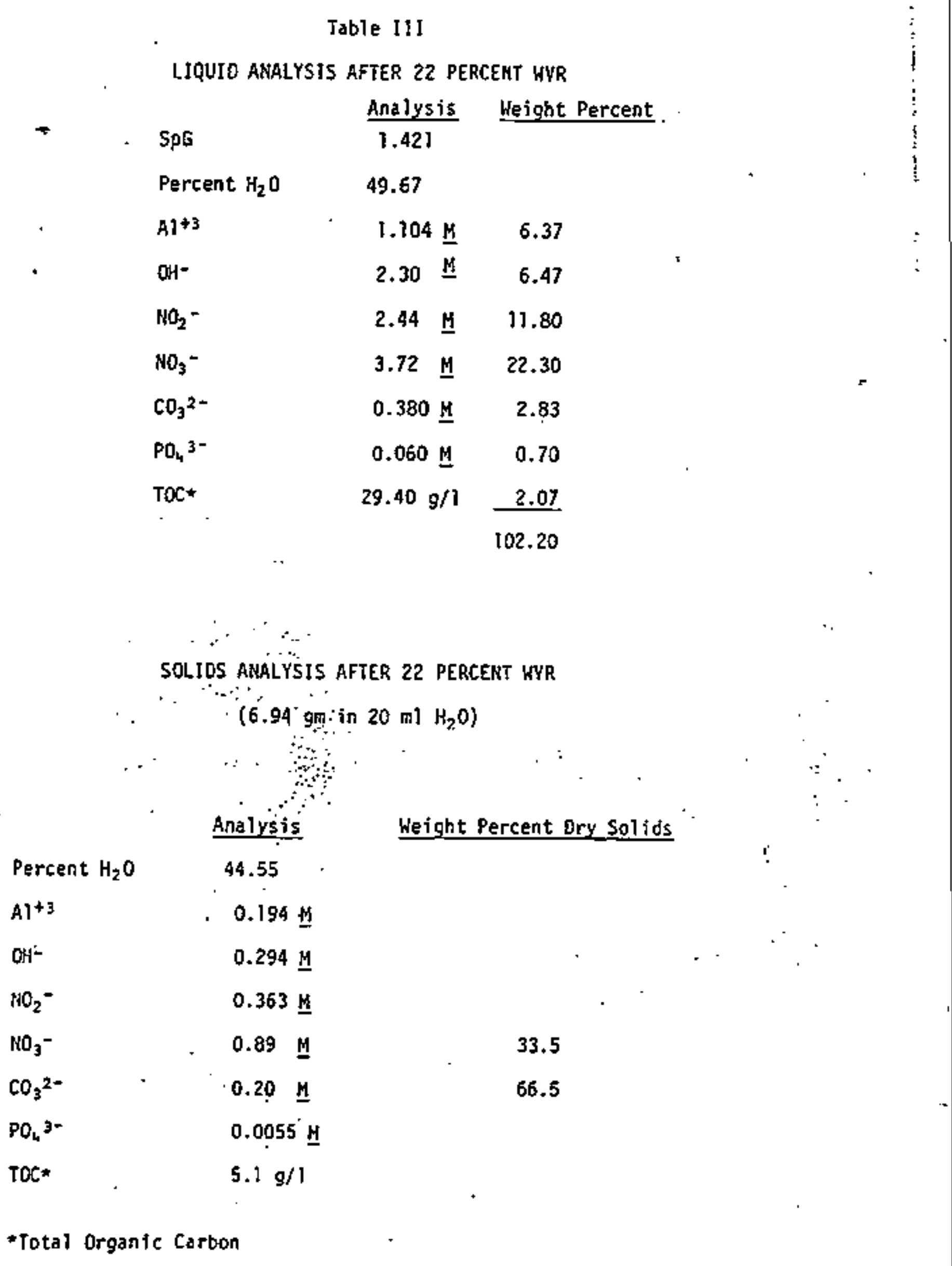




$$
\begin{aligned}
& \text {. TCRL-IS } \\
& \text { WHC-SD-WM-ER-308, Rev, O } \\
& \text { Manager. Services } \\
& \text { - 222-5 Laboratory } \\
& 2004 \\
& \text { 2-2965 or 2-2435 }
\end{aligned}
$$

TANK SAMPLE NUKEER 103-A 2H2-A-BMI

AL SERIAL NUTBER T 8951

DATE RECEIYED $3 / 19 / 80$

OLLUTION FACTOR" NONE

YIS-0TR $L T$. yellow $400 \mathrm{mRed/th}$

ANALYSIS

RESULTS

ANALYSIS

RESELTS

pH

$N R$

$\mathrm{PO}_{4}$

$8.17^{-2}$ i

Spe

1.240.

CL

$-N R^{\circ}$ i

$: \mathrm{H}_{2} \mathrm{O}$

70.84 wt:

$\mathbf{F}$

NR_M

DIA

NA

$5 \mathrm{O}_{4}$

$\therefore N S \quad \|$

A]

., $55^{4}$

$\mathrm{CO}_{3}$

ForgC

.340

$\mathrm{OH}$

$.74 . \mathrm{H}$

$7.929 / 1 \mathrm{c}$

$\mathrm{HO}_{3}$

$1.92-4$

KA

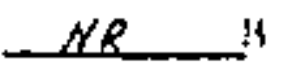

$\mathrm{HO}_{2}$

1.24

Coolina Curve

, Pm 147

TESPP $\mathrm{C}$

* SOLIDS

50 Alove :

$\checkmark$ Sr BS.90

$$
\frac{N E}{1.55^{3}} \text { U6\%/1 }
$$

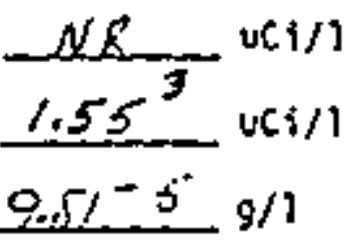

t $\mathrm{Pu}$

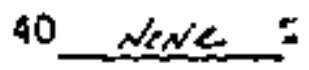

30 Texis.
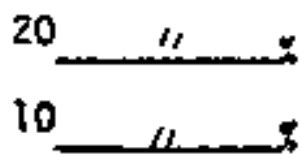

토소

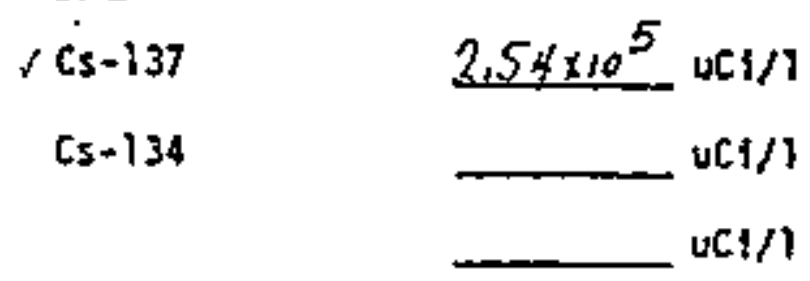

$u C H / 1$

vel/1 $4 \mathrm{CI} / 1$

HR - Hot Requested MD - Hot Detected

The dilution factor is included in the calculations. 


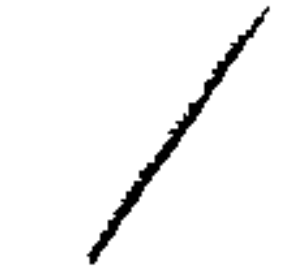

TCRC-17

$$
\because \ldots:: \ldots . \ldots
$$

$$
T C R C-17
$$

।
WHC-SD-WM-ER-308, ReV. 0 A $x^{2-}$

Kanager. Services 222-5 Laboratory 200 2-2965 or 2-2435

- Analytical Results

TAMK SAMPLE MULEER $103-A \cdot 242-A-B M$

AL SERIAL NURBER $T-9536$

DATE RECEIYED 4/7/80

VIS-0TR OQAQVE - yedow 1.2Rad/he

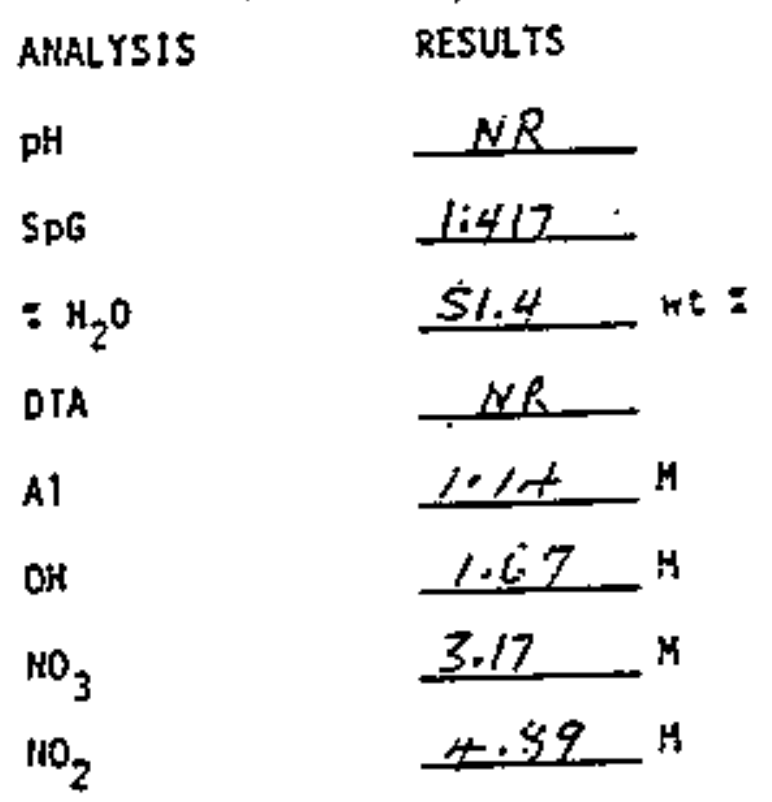

Pm 147

Sr 89,90

Pu

$\frac{N R}{\frac{N \cdot 41^{3}}{9.35^{-5}}} v \delta / / 1$

GEA

[5-13?

$4.48 \times 10^{5}$ uct/1

$[s-] 34$

บโI/ 1

uC $1 / 1$

NR - Hot Requested MD = Rot Detected
DILUTIOH FACTOR 2.368

ANALYSIS

RESULTS

$\mathrm{PO}_{4}$

$\therefore 7.07^{-2} \mathrm{M}$

CL

$f$

$\mathrm{SO}_{4}$

$\mathrm{CO}_{3}$

Torgc

KA

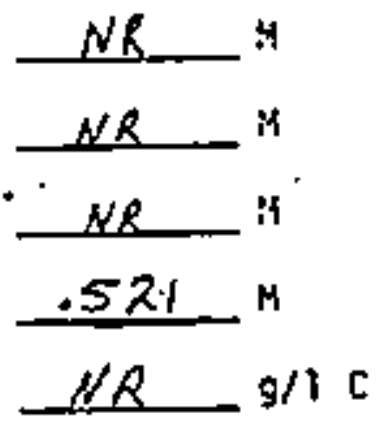

NR

:1.

Cooling Curve

TEEPP $C$

So 18 SOL10S

$40+18=$

$30+18=$

$20 \quad 18$

$10 \quad 18$

$518=$

otill

uC $1 / \mathrm{I}$

$u[1 /]$

The dilution factor is inciuded in the calculations. 


\section{Internal Letter}

$$
T L R L-21
$$

Dale: . September 22, 1980

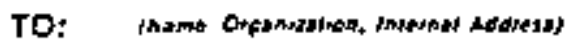

- M. C. Teats

- Plant Engineering

. 2750E/A100/200 East
WHC-SD-WM-ER-308, Rev. 0

$$
\text { No . } 65453-80-266
$$

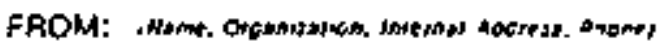

- M. T. Jansky

- Separations Process Development Unit

- 222-5/MO-037/200 liest

. 3-1571

Subjet: . Analyses of Tank 103A Waste

The orocessing of waste from Tank 103A requires a knowledge of the composition of the waste. Solids are believed to be present in the waste; however, two samples of $103 \mathrm{~A}$ waste received by Separations Process Development Unit (SPOU) contained only a trace of sojids. A sample taken one foot bejow the surface contained no salids, while a sample taken six feet below the surface contained only 3 volume percent centrifuged solids.

The liquor was analyzed and the results are shown in Table I. There were insufficient solids for a solids analysis. Both samples of Tank $103 \mathrm{~A}$ waste are in storage.

Please call if you have any questions or comments regarding this work.

M. T. Jünsky

Advanced Chelnis $\mathrm{c}$

MTJ/naj

Distribution:

D. E. Sowers

L. C. Brown

J. S. Buckingham

H. J. Eding

L. A. Gale

D. L. Herting

T. A. Lane

T. B. Veneziano

C. H. Walker

D. G. Wi kins

File Code: KF55E

Process Aiós: (B)

Letterbook 


\section{TABLE I}

\section{Composftion of Tank 103A Haste}

Component Supernatant Weight\%
Molarity

Solids

\begin{tabular}{|c|c|c|c|}
\hline $\mathrm{Al}$ & 0.400 & 2.28 & \multirow{12}{*}{ 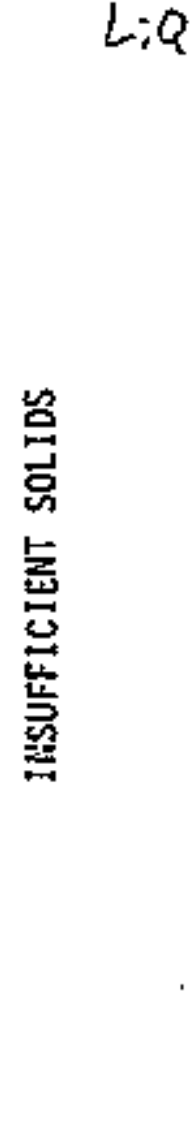 } \\
\hline $\mathrm{OH}$ & 2.85 & 7.94 & \\
\hline $\mathrm{NO}_{2}$ & 2.20 & 10.57 & \\
\hline $\mathrm{NO}_{3}$ & 3.42 & 20.24 & \\
\hline $\mathrm{CO}_{3}$ & 0.380 & 2.81 & \\
\hline $\mathrm{PO}_{4}$ & 0.053 & 0.72 & \\
\hline $\operatorname{TOC}(g / L)$ & 14.4 & 2.98 & \\
\hline${ }^{137} \mathrm{Cs}$ & \multirow{2}{*}{\multicolumn{2}{|c|}{$\begin{array}{l}4.56 \times 10^{5} \mu \mathrm{CH} / \mathrm{L} \\
<2.8 \times 10^{-3} \mu \mathrm{Ci} / \mathrm{sample}\end{array}$}} & \\
\hline $106_{R J 1}$ & & & \\
\hline $\mathrm{SH}_{2} \mathrm{O}$ & \multirow{2}{*}{\multicolumn{2}{|c|}{50.17}} & \\
\hline SpG & & & \\
\hline TOTAL & \multicolumn{2}{|r|}{$\overline{97.71}$} & \\
\hline
\end{tabular}




\section{Internal Letter}

Onle - October 2, 1980

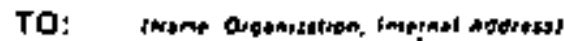

- H. C. Teats

- System Engineering

- $2750 E / A 100$
№. $65453-80-287$

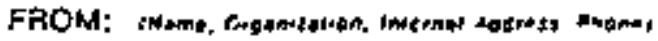

. M. T. Jansky

- Separations Process Qevelopment Unit

. 222-5/M0-037/200 West

. 3-1571

Subpat: Solids in Tank J03A Waste

Ref: Letter, $\frac{5}{5} 65453-80-266, M$. T. Jansky to M. C. Teats

"Analyses of Tank J03A Waste", dated September 22, 1980

Earlier waste samples from Tank 103A, discussed in the referenced letter, contained virtually no solids. Pumping behavior and general characteristics indicate that this is not true. Another sample was taken recently to verify the nature of the waste.

The recent sample, taken three feet below the surface of the waste, contained twenty percent well-settled solids which were easfly mixed into a slurry and poured into centrifuge cones. Centrifugation resulted in 18 volume percent centrijuged solids. The phases were separated and submitted to Analytical Laboratories for enalyses. The results are shown in Table 1.

The data show that the solids are primarily carbanates with signiricant amounts of nitrite. The high concentration of phosphate reported for the supernatant is deiniteily erroneous. The maximum solubijity of piosphate is 0.17 molar. A 0.5 molar solution would definitely have phosphates present in the solids, which was not the case.

Please call if you have aryquestions or comments regarding this work.

i. T. Jansky

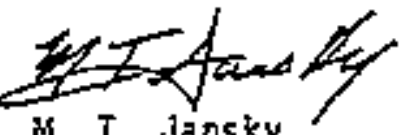

Advanced Chęnist

HTI $/$ กaj

\section{Attachment}

Distribution:

J. S. Buckinghan

D. E. Bowers

H. J. Eding

L. A. Gale

0 . L. Herting

T. A. Lane

T. B. Veneziano

o. G. Hijkins

Letterbook 


\section{TABLE I}

Composition of Tank 103A Waste

Supernatant

Component

Al

OH

$\mathrm{NO}_{2}$

$\mathrm{NO}_{3}$

$\mathrm{CO}_{3}$

$\mathrm{PO}_{4}$

TOC

$\mathrm{H}_{2} \mathrm{O}$

SpG

*Too high, most likely an error

$4.95 \mathrm{~g} / \mathrm{L}$
Wt Percent

solids Liq/solits.

Wt.

7.1

5.6

8.1

19.5

2.3

6.8

1.1

41.66
0

0

20

4

69

0

0 


\section{YI. ANALYTICAL SAMPLE PREPARATION DATA}

The actual weights and volumes generated during anatytical sample preparation (solution leaching) for the core composites from tank 241-A-103 are shown. Peptization of the solid may occur in some of the water leaches (third contact). This forms an unseparable solid-1iquid suspension broken by adding concentrated $\mathrm{HCl}$ to the leach to bring the acid concentration to $5 \mathrm{H}$. In these cases, effectively no third water leach occurred and the acidified solution becones the first actd jeach.

No anaiyticai sample preparation was required, except filtering, for the drainable 1 iquor core composites.

\section{Composite ID SBIXCOOXX}

Initial welght of sample

Volume

Residual weight:

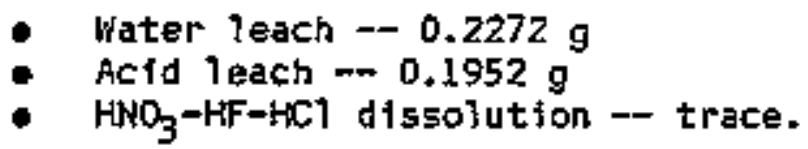

Finat solvtion volume:

- Water jeach $-40 \mathrm{~mL}$

- Ació leach - $25 \mathrm{~mL}$

- $\mathrm{HNO}_{3}-\mathrm{HF}-\mathrm{HCl}$ dfs5olution -- $20 \mathrm{~mL}$.

\section{Comosite ID A A2XCO0XX}

Initial weight of sample Volume

Residuat weight:

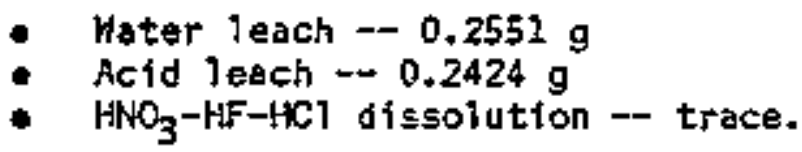

Final sotution volume:

- Wazer leach -- 40 ml

- Acid leach -- $25 \mathrm{~mL}$

- HNO $-\mathrm{HF}-\mathrm{HCl}$ dissolution $-20 \mathrm{~mL}$. 
Rev. 0

\section{ANALYTICAL RESULTS}

A spreadsheet program on a dedicated microcomputer is used to convert raw andytical data to concentration values for the components in the water. The following are copies of the spreadsheet printouts for the core composite samples from tank 241-A-103. For the solid phase core composites, concentra$t$ fon values from the indivioual leaching solutions (water, $\mathrm{HCT}-\mathrm{HNO}_{3}-\mathrm{HF}$ pressure dissolution) are reported along with overall results. Two overall results are reported: REPORT TOTAL - sum of all values reported greater than the 1 imit of confirmation ("less than values" not summed) and MAXIMUM TOTAL sum of all values reported with 1 imit of confination values taken as actudi value (worst case).

Phystcal data detarmined are shown on the spreadsheet reports. 


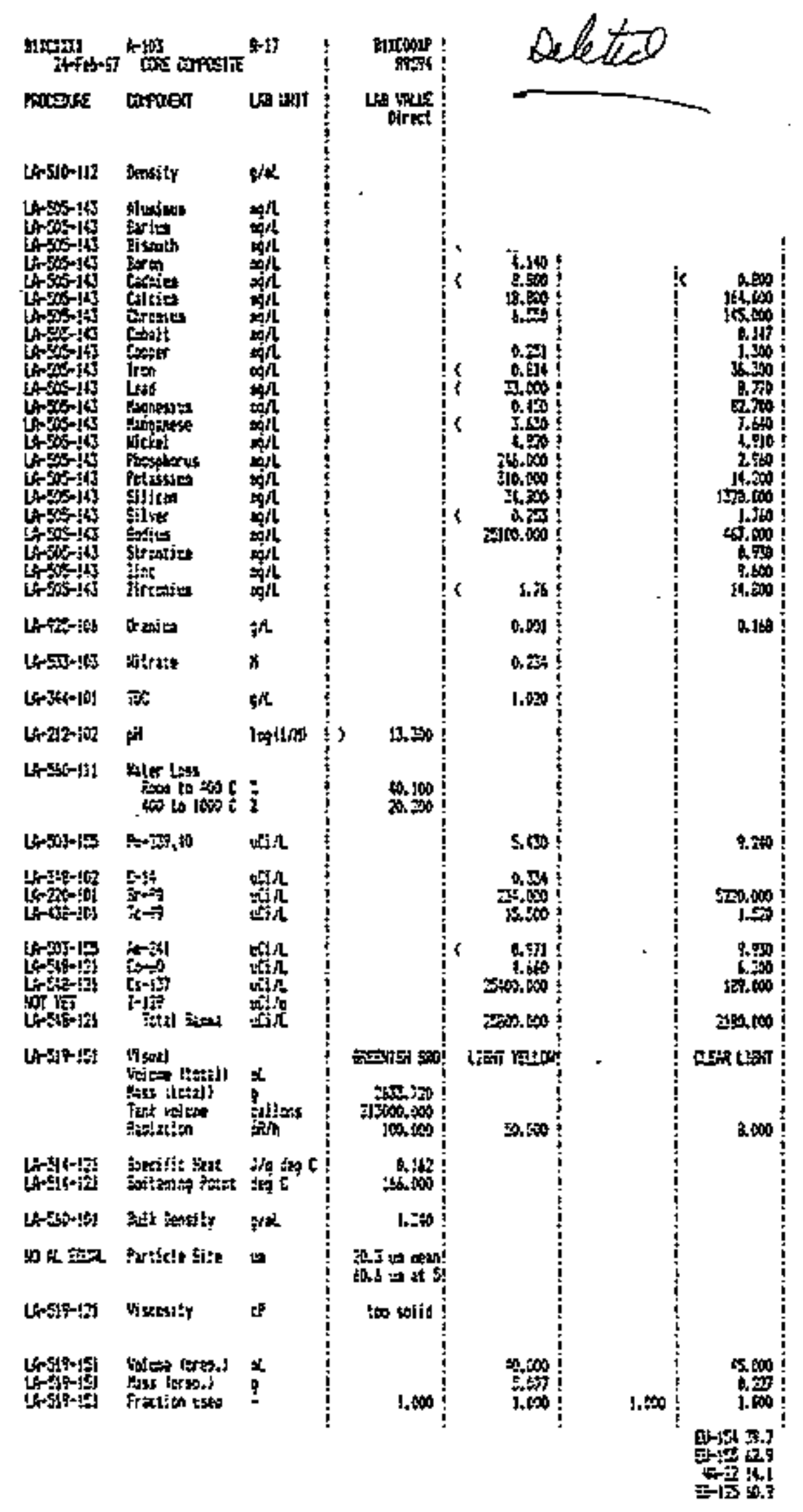

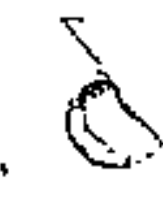


하낵ㄱ

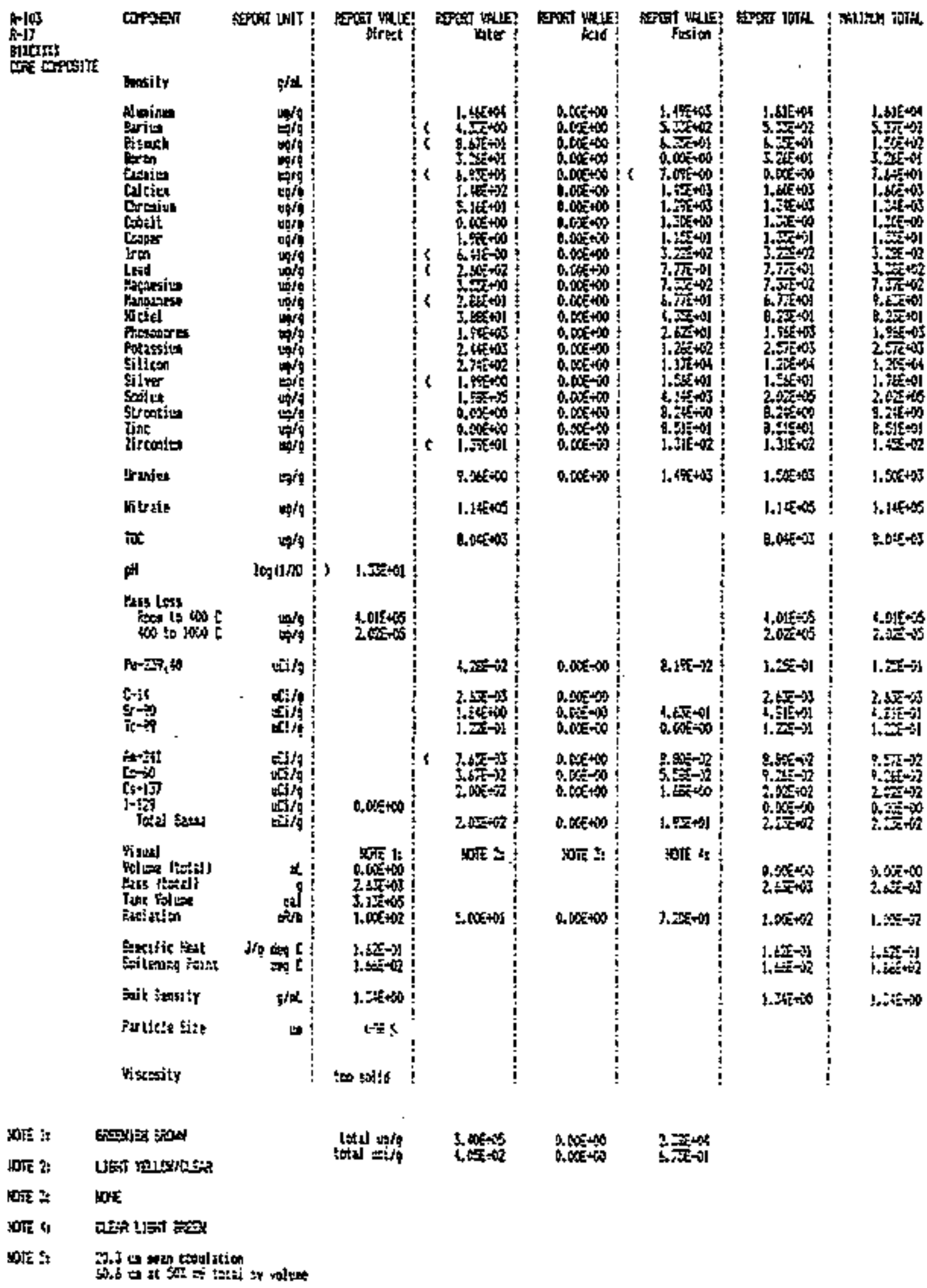



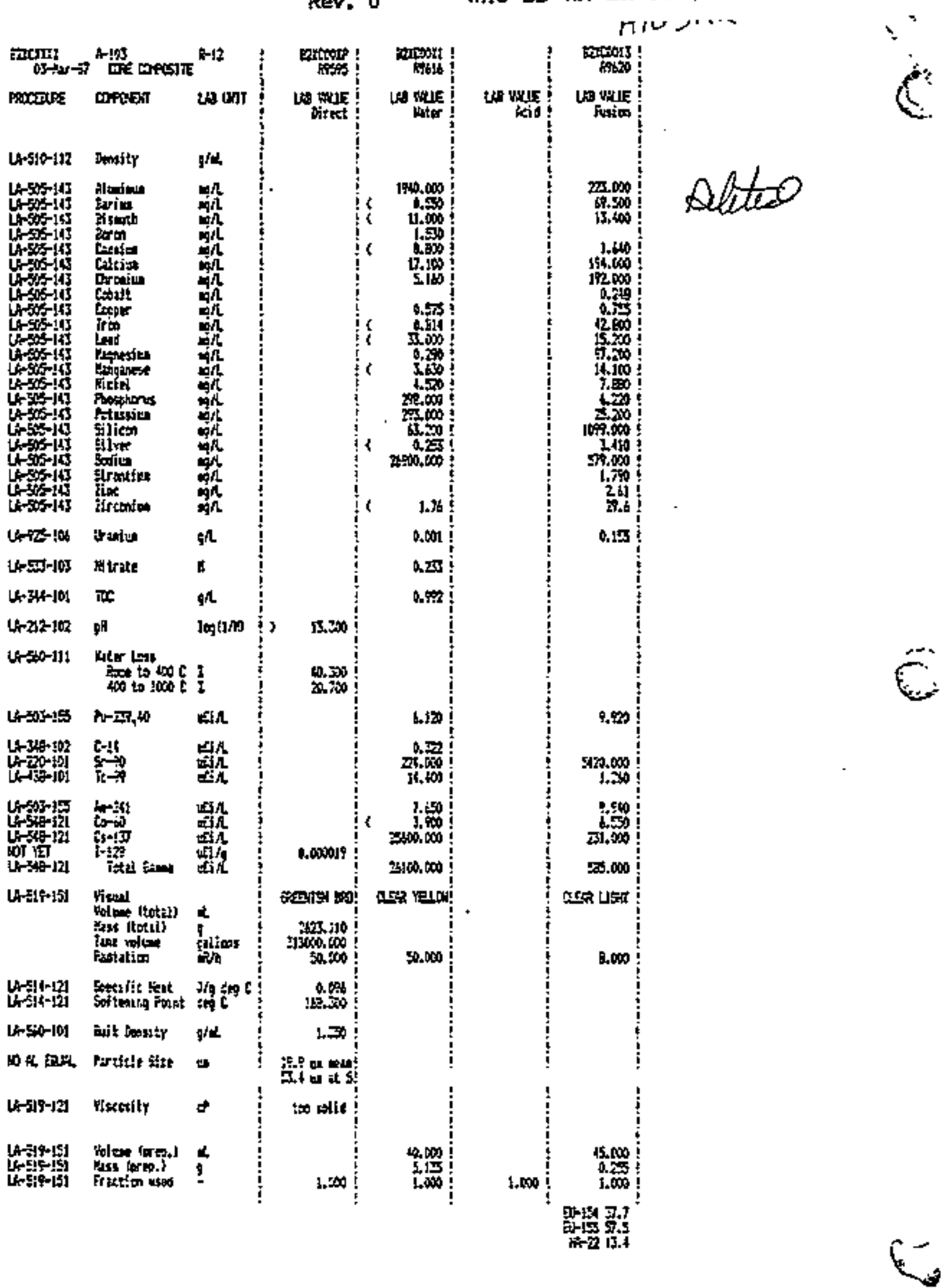
$05 \div 42-3$

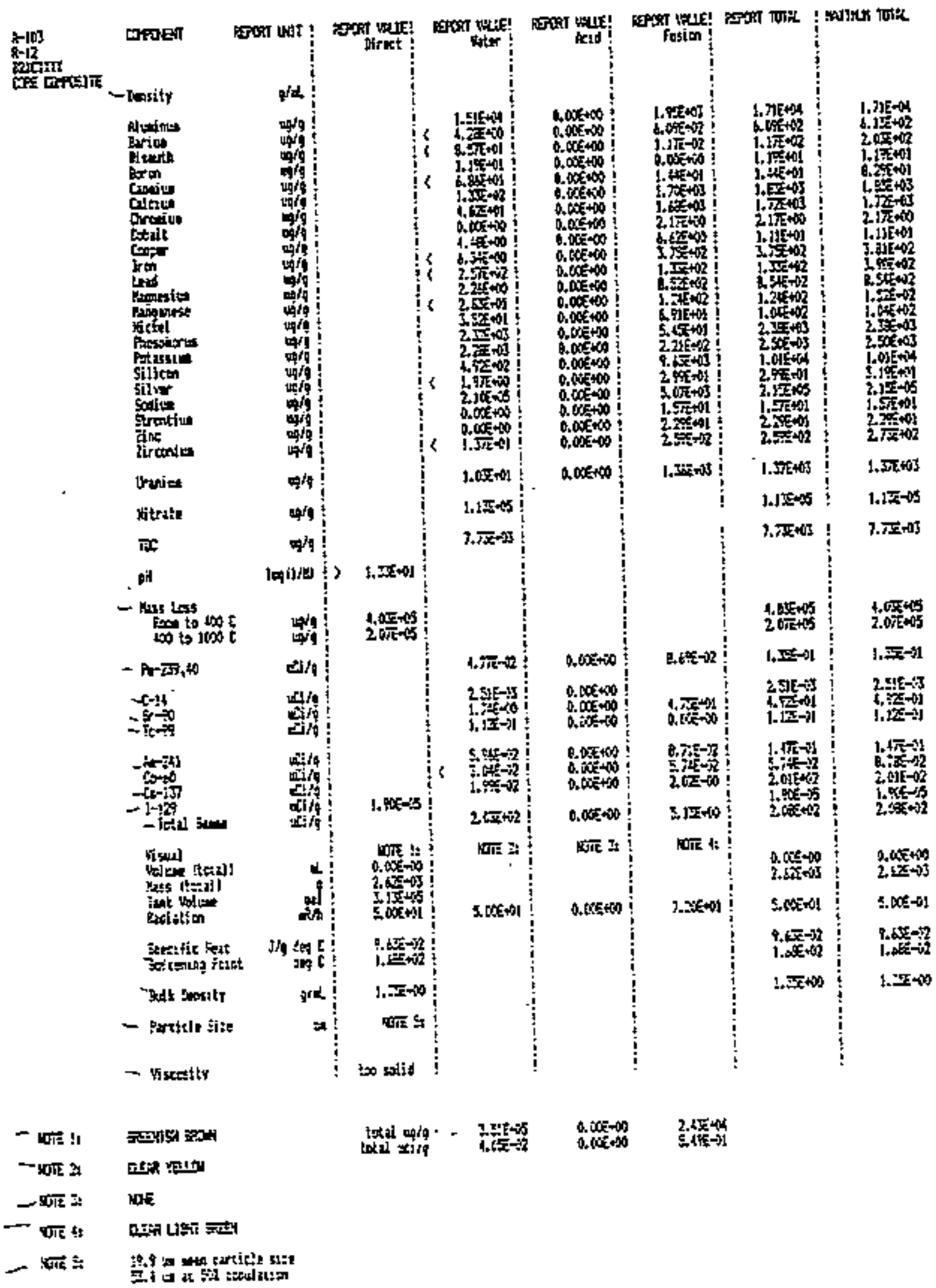


From: Process Chemistry Laborator jes

$65453-87-11 B$

Phone: 3-1972/MO-037/200 West Area

Date: August 18, 1987

Sulvect:

INTERIM REPORT ON WASTE TAKK CHARACTERIZATION ANALYSIS OF TANK $241-k-103$

To:

SL Lindberg . 2750E/200E 3-1847

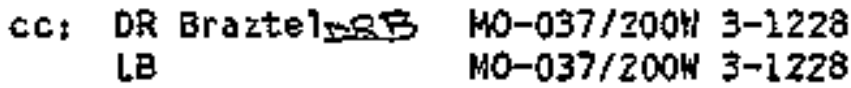

The included tables contain the results of the radionuclide and chemical analysis perforined on the core samples recovered from tank 241-A-103. These cores were recovered and analyzed as part of the FYa6 waste tank characterization effort. The magnitude of the errors (raste Inhowogenefty, sampling, preparetion, and anaysis) sssociated with these andyses have not been fully defined. These results should be considered to be ofly approximations of waste composition in the tanke.

Two cores were recovered, one from riser 12, the other from $r$ sser 17. Concentration values for the soljos fraction of each core are contained in the jast two colutins in Tables 1 and 3 . The column Report Tota? sums only those results havfing values greater than the linit of confirmation. The Haxinua Total column sus al) reported values, including results below the iinit of confirmation (less than) using the less than value for sufnation. Tables 2 and 4 contain the results of andiysis on the drainable liquid fraction associated with each core sample.

A complation of all information regarding the sampling and analyses of these core samples will be issued as a supporting document data package before the end of 1997.

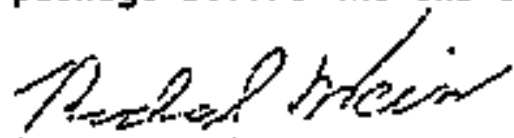

RL Heiss, Cheníst

Rt. W/cse

Attachients 


\begin{tabular}{|c|c|c|c|c|c|}
\hline & tarten & Luls & & & WHC-SD-WM-ER-30B， Rev. 0 \\
\hline Sifint & & & EPTit & 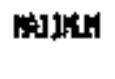 & \\
\hline
\end{tabular}

\begin{tabular}{|c|c|c|c|c|}
\hline & 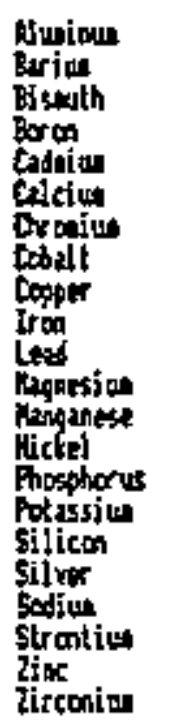 & 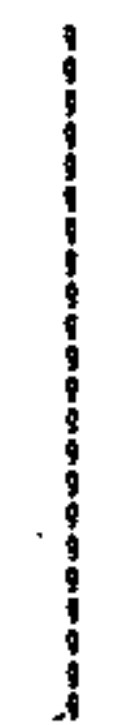 & 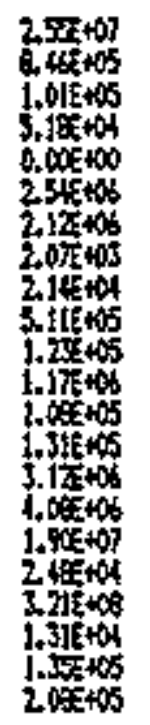 & 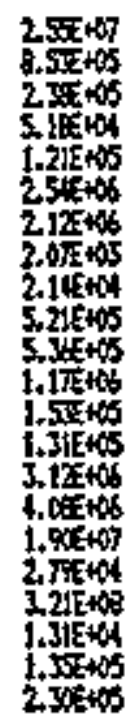 \\
\hline & Iratitu & 9 & 2,3 我玨 & 2.3. \\
\hline & sitoter & 9 & $1.81 E+C B$. & $1.81 t+18$ \\
\hline & $\pi$ & 1 & $5,27+07$ & 1.羍 407 \\
\hline & pH & - & & \\
\hline & 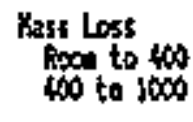 & 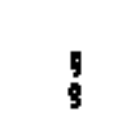 & $\frac{6.5 E+\infty}{3.21 E+\infty}$ & 6. \\
\hline & $A \mathrm{r}-2 \mathrm{~S}_{1}, 40$ & $\mathbf{H}$ & $1.5 x+02$ & $4.5+02$ \\
\hline & $\begin{array}{l}6-14 \\
8 x-90 \\
\text { fe-th }\end{array}$ & $\begin{array}{l}\mathbf{j} \\
\mathbf{t} \\
\mathbf{6}\end{array}$ & 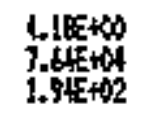 & 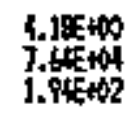 \\
\hline & 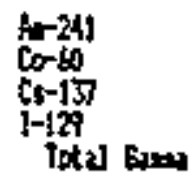 & 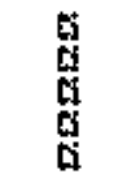 & 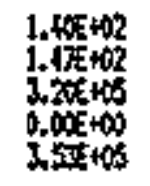 & 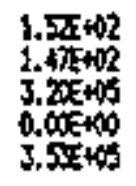 \\
\hline & Tunt volum & getions & $3.1 \bar{x}+05$ & 3. $15+05$ \\
\hline & Adt tonsity & $g / 1 t^{2}$ & $1.34+06$ & 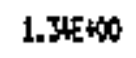 \\
\hline
\end{tabular}


TABLE 3

coptari

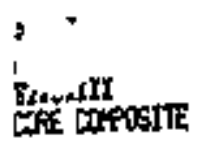

Dasijty

At녀ำ

Briver

trm

Cánjar

Ansin

Dreailti

Cotal:

Coppes

Iran

dagnesian

Painowest

bicke]

Phosphorus

Potussitua

siltem

Silver

Soditutitis

If $x$

Zirconiu

Itunitu

Kitorite

itc

git

Hass Loss

Rocat to $\sin \mathrm{c}$ 100 to $1000 \mathrm{C}$

P 2039,0

C-14

$x_{i \rightarrow 0}$

|-241

$\cos \theta$

[s- $\{3\}$

- 12

totel trans

W5y!!

Wolus (total)

Nass (totili)

Tank Volvese

Ridiation

Specifit Heat

softening ioint

Devit bersity

Purtic]e Ėe

Viscosity

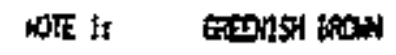

porte 2

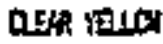

HoTt 3: WE

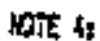

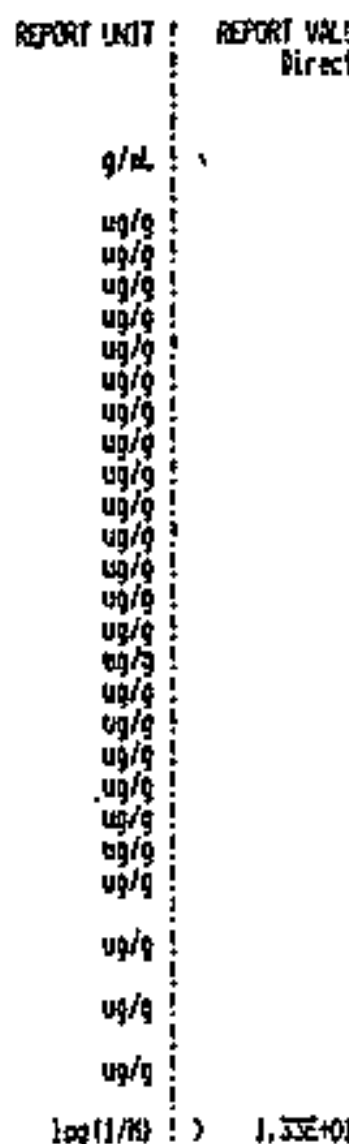

1.51E+ok

4. $x+\infty+\infty$

is $8.5 / 2+01$

i 1.5\%+01

(6) $6.85+01$

$1.5+5+2$

$4.02+01$

$0,00+\infty)$

4.

I 6. J J

i) $2.51+02$ i

is $2,245+60$

2. 8 x +01 :

$2.57+03$

2. $20 \mathrm{x}+03$

4. 4 还 02

(1) 1.97

$2.100+\infty 5$

$0.00<+\infty$ !

$0.00 t+\infty$

i $1, \sqrt[5 / 6]{101}$

$\$ .6 \overline{5} \div 01$

1.1풍

$7.75+103$

logtd/at?

$1, \overrightarrow{3 x}+01$

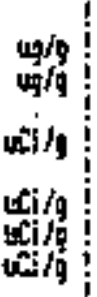

1.05:T05

$2.00+4$

uitis!

ucilo:

ufilo:

ufilg

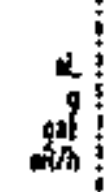

$J / 4$ ded

얻ㄴ?

gits

네

tos soljd

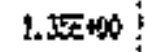

따토 5 :

$4, \pi \pm-\Omega$

$0.00+100$

2.5:5-1]

$1,74=00$

1. $1 \frac{1}{2}-7$

5. 995-62!

$3.04-32$;

$1,4+02$ !

$205+02$

गाE 2:

以ण度

0. $00=400$ !

了, $3+05$ !

5. 00E+ +9]

$9.65-02$

J.

$0.00=0 \mathrm{~s}$

$0.00=00$

$0.05 E+00$ !

$0.005+00 ?$

$0.00+00 !$

$0,02+01$ ?

0. $005+00$

HIE 3

$5,00=01$

$0.003+00$

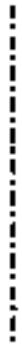

tokal ogis

total usis

$3.5 \mid \underline{E}+03$

1. $05 x+02$

$0.00+\infty$

$0.0 x+10$

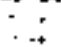

WHC-SD-WM-ER-308, Rev.

0

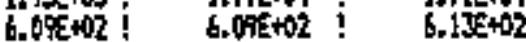

$1.17+02 ! \quad 1.57+02 ; \quad 2.05+402$

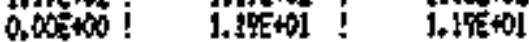

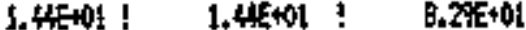

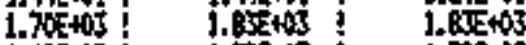

1. $6 x+03$ ! j.7t+03! 1.7t +03

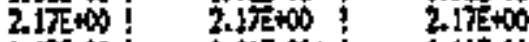

b. $6 x+00$ 1.j15401 1.115+01

$3 . \sqrt{5}+02$ ! 3.75+42! 3.15402

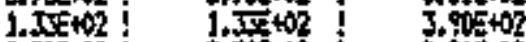

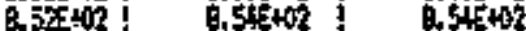

1. $\hat{2}+5+02 ! \quad 1.205+02 ! 1.525+02$

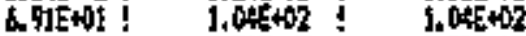

$5.45+01 !$

$2+\mathrm{xic}+03$

$2.30+03$

2.2]E+02

$2,52+05$

$2.50[+03$

9.65

$1.05 E+04$

$1.0 \mathrm{E}+\mathrm{H}$

5.07

2. $P$ TE+1

I. JoEt+is?

$1,5 \mathrm{Tt}+4 \mathrm{H}$

$2.25+65$

$215+15$

1.5Tस

5.5Et

2. 주넨

2,20001

$2.26[+0$

2. Stcto

$25+42$

2. $\sqrt{x} \times 02$

1.겆이

1.3505

1.JIE+03

1. J54 5

1. 1]E+2S

$7.7 x+63$

7. T50 403

8.65:-02

1.

$2.07+50$

1.

4. 7 空+ 01

2.51E-A

2.515-73

$0.00+\infty$

4. 就 401

4. 920

0.71E- 02 !

1. 2 20

1.125-11

5. $74=-62$

1. $17-4$

1.7][-3]

2.0 0 E+

S.

5. 1 EEt +0

1. 915

.

1. 90 if

$2.0 x+02$

DDE 4

$2.05+102$

$0.00 \div 0$

$0.005+00$

$2.62 x+63$

$7.20 E+01$

5. $(00 \pm+0)$

5 .cotol

9.175-62

9. 150.02

1. 6002

$1.8 x+02$

$1 . \overline{F E}+10$

1.

$2.15+4$
$5.45+01$ 
T A B L E 4

DW: ${ }^{\prime}:$ WHC-SD-WM-ER-308, Rev. 0

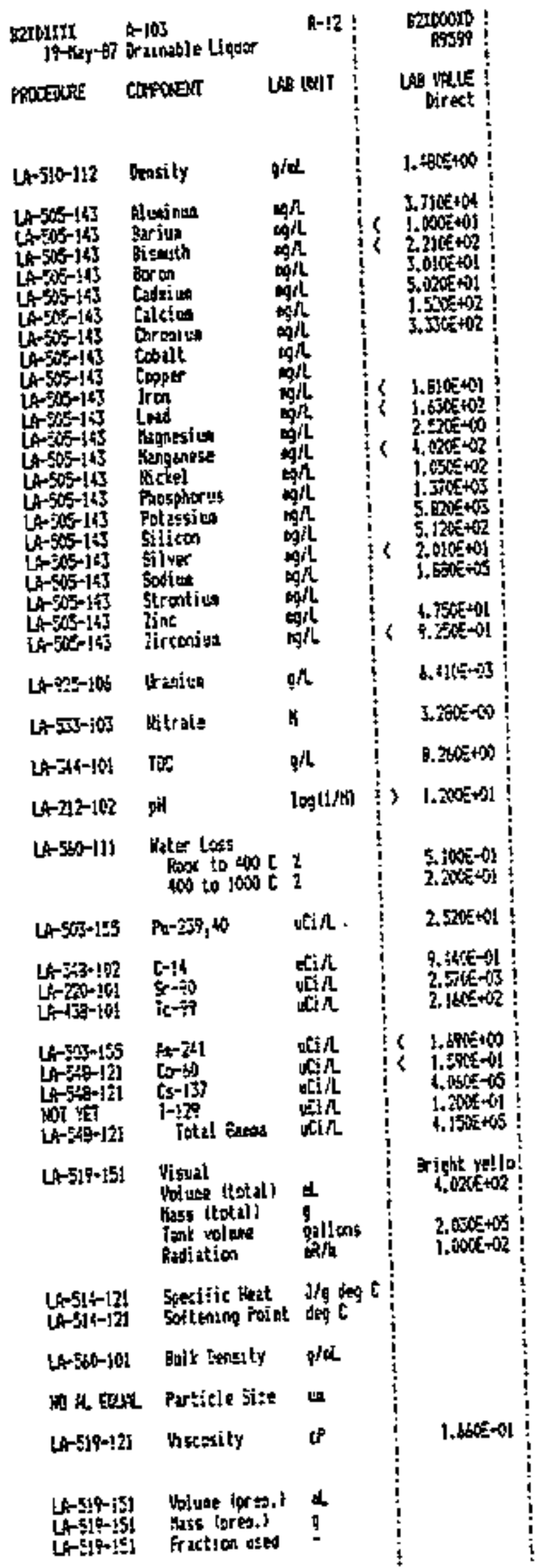


Atlantice Ifield Hantord Conpary : .

$$
\text { TCRC-2 }
$$

$$
\text { WHC-SD-WM-ER-308, Rev. } 0
$$

A104.1 - VV

Dete: March 25,1974

To:

R. L. Walser

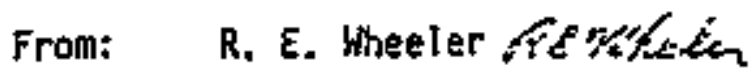

Subject: ANALYSIS OF TANK FARH SAMPLES

SAMPLE: T-2893 TARK 104-A

VIS-OTR: Yeilow, $<1 \%$ solids $500 \mathrm{mrad} / \mathrm{hr}$.

pH: $\quad 10.49$

SpG: $\quad 1.132$

OH: $\quad 0.480 \mathrm{M}$

A1: $\quad 6.02 \times 10^{-3} \underline{\mathrm{M}}$

$\mathrm{Na}: \quad 2.84 \underline{\mathrm{H}}$

GEA: $\quad{ }^{134}{ }^{\mathrm{C} 5}-3.13 \times 10^{3} \mathrm{WCi} / \mathrm{gal}$

Cs sep: $\quad{ }_{134}^{134} \mathrm{Cs}-3.72 \times 10^{3} \mathrm{HCi} / \mathrm{gel}$

REW: $5 d$

cc: LG Johnson (2)

14-4000-010 119-\$b)

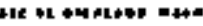

H-157 
At antic Fichfleld Hanford Compary

Date: $\quad$ September 4, 1974

To: J. C. Womack

From: $\quad$ T. S. Buckingham $/$. E. Horton

SUbject: CHARACTERIZATION OF SLUDGE FROM TANK 104-A

Tank 104-A was used to store Purex Sludge Supernatant (PSS) generated during the sluicing of tanks 106-A, $107-A$, and 103-A. Much of the sludge in the tank consists of material that did not settle in tank 002-AR when 106-A, 101-A and 103-A were siviced.

A sample of sludge from 104-A showed that the material was quite fluid having a consistency similar to heavy cream. The $89+905 r$ content of the sludge was $43.9 \mathrm{Ci} / 1$ iter. Particle size distribution showed 4 weight percent of the sludge was less than $10 \mathrm{um}$ and 90 percent was less than $50 \mathrm{um}$. Purex Acid Sludge (PAS) made from a 30 percent slurry of sludge and supernatant liquio contained 70 percent of the sfrosr. The PAS gelled in five to seven days due to the high silica content. Purex Acid Sludge made from 20 percent siturry had not gelled after 13 days. Details of the laboratory work are discussed below.

The "as received" 104-A siudge was analyzed by fusing 1.7 milliliters with $\mathrm{XOH}$, ojs50]ving the melt with $\mathrm{HC} 1$, and difuting to 320 milliliters. Density and moisture contents were measured on a separate portion of the sludge. Results are shown in Tabie 1 , attached.

Particle size distribution of the 104-A siudge as determined on a HIAC particie counter is shown in Tabie II, attached. Particle size distribution for siudges from 102-A, 10j-A, 102-AX and 106-C are also shown for comparison.

Two batches of PAS were made up from 30 volume percent siudge slurries. One slurry was made using supernatant liquid from 103-AX and the other slurry was made using supernatant liquid from $104-\mathrm{A}$ In both cases, $10 \mathrm{millj-}$ liters of the 30 percent slurry were added to 1.5 milliliters of $12 \mathrm{M} \mathrm{HNO}_{3}$. Severe foaming accompanied by the evolution of $N 0_{x}$ occurred during acidification. The $\mathrm{pH}$ of the resulting PAS was around 3 . Adoitional acid was needed to adjust the ph to less then 0.3 . Batch 1 required two additional mitilititers of acid (total $3.5 \mathrm{mi}] 111 \mathrm{iters}$ ). The amount of additional 
J. C. Womack

Page 2

- Septenber 4, 1974

acid needed for Batch i was not recorded, but it was about the same. It should be noted that after standing a few days, the pH of the PAS increases slightly, and a few additional drops of acid are required to again bring the pht to less than 0.3 .

Purex Acid sludge Batch i gelled in five days before all analyses could be completed. Batch 2 gelled in seven days. The PAS anatyses are shown in Table III, attached. A] so shown in Table III are volumes of sjudge and supernatant liquid used to make the PAS, the volume of PAS, and the volume of centrifuged undissolved solids.

A third batch of PAS was made to see if diluting the PAS 10, 15, 20, 25, and 50 percent would prevent gelling. The PAS was aiso to be analyzed for ammonium ion, manganese, ${ }^{90} \mathrm{Sr}$ and differential thermal analyses. The a +90 Sr analys is was lower than Batch 1 and 2 by a factor of 3 Indicatitg that we did not have a good make-up. The work will be repeated and the results reported later:

Acidification does not dissolve all of the sludge. Previous work bas shown a considerable amount of strontium associated with the insoivbie sludge. Treating the acid insoluble sludge with an equal volume of 50 percent HaOH then acidifying with $12 \mathrm{M} \mathrm{HNO}_{3}$ removes additional strontivm. Tables $I V$ and $V$ show the anount of strontiutal dissolved by acid for Batch 1 and 2 , respectively. Table IY, attached, shows the amount of strontium remaved by caustic acid treatment. Unfortunately, solids remaining after the caustic atid treatment were lost; however, the data indicate that the caustic acid treatment removed alt of the remaining strontium,

The tables also show the centrifuged volume of solids at each step in the treatment and the volume percent solids based on the original volume of sludge.

A caustic acid treatment was not made on the solids renaining from the acidification of Batch 2; however, the amount of strontium remaining on the solids was analyzed. Data for the treatment of Batch 2 are shown in Table $V$.

Gellation of PAS was thecked by making PAS from 10, 20, and 30 volume percent siurries. Results are shown in Tabie VI, attached. 
J. C. Homack

Page 3

Septenber 4,1974

As mentioned earljer, edijtional getlatjon work is underway in which PAS made from 30 percent 5 iurry is being diluted.

That work will be reported on later.

Piease call us if you have any questions regarding this work.

JSB : JEH : dU

Att. (3)

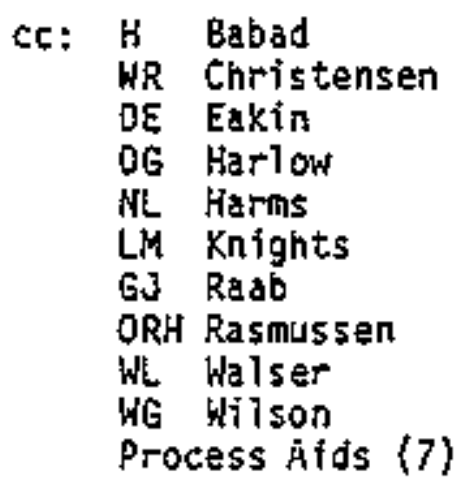




\section{TABLE I}

WHC-SD-WM-ER-308, Rev, 0

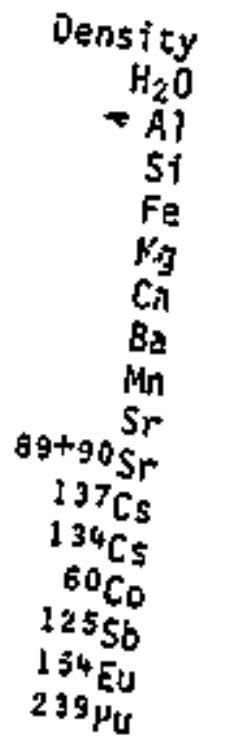

$1.54 \mathrm{~g} / \mathrm{cc}$

2.82 moles $/ 1$

0.83 moles $/ 1$

4.99 notes/1

0.11 moles $/ 1$

$0.41 \mathrm{met} i \mathrm{is} / \mathrm{l}$

0.009 moles $/ 1$

0.71 males $/$ ?

$<0.055$ moles $/ 1$

$4.39 \times 10^{7}+\mathrm{Ci} / 1$
$\left.1.15 \times 10^{6} \mathrm{\mu C} / 1\right]$

$3.33 \times 10^{4} \mathrm{bCl} / 7$

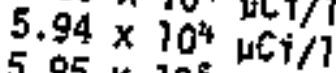

$5.95 \times 10^{5} \mathrm{HCH} / \mathrm{T}$

$1.30 \times 10^{5} \mathrm{wCi} / 1$

$\sin 2 x$ $4.6 \%$
$1.4 \%$$\quad A 104.2$

$17.0 \%$

0.28

$1.0 \%$

$0.1 \%$

2.4\%

\section{IABLE II}

PARTICLE SIZE DISTRIBUTION IN SLUDGES

Particle
Size, 㧘
5 5

10

8.2

i02-A 103-A Weight Percent

20

16.5

30

25.9

35.7

40

45.5

1.7

9.5

104-A 102-Ax

$\underline{106-C}$

33.3

4.3

$46.7 \quad 34.7$

34.0

14.1

17.6

11.1

55.4

60

70

65.4

1.4

0.7

5.2

80

90

75.3

85.3

100

95.3

$: 100$

0.0

$0.1 \cdot 1.0$

$0.1 \quad 0.9$

0.0

17.0

32.5

7.0

12.3

4.7

3.1

$2.0 \quad 1.0$

35.0

45.7

14.3

3.1

1.1

0.4

75.3

17.7

$22.8 / 4 / 04.3$

21.4 等

12.4

5.0

0.3

3.8

0.5

0.1

0.2

2.4

0.1

1.8

3.5 
WHC-SD-WM-ER-308, Rev, O

8.4 Sludge and Supernate Anajyses

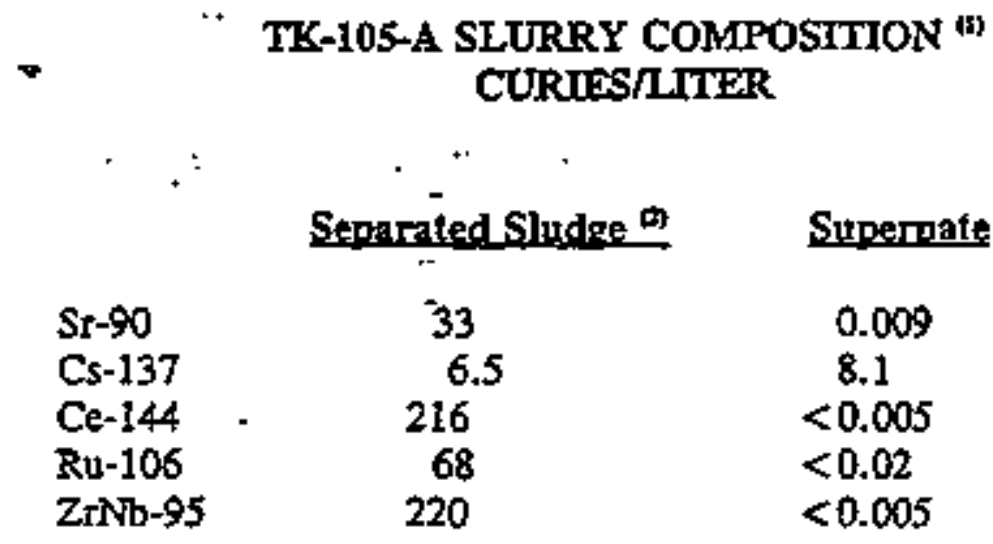

(1) On May 1, 1965, sample taken of slurry above settled studge.

(n) Centrifuged sample 14 volume percent sludge.

The above data are from Reference 3. 


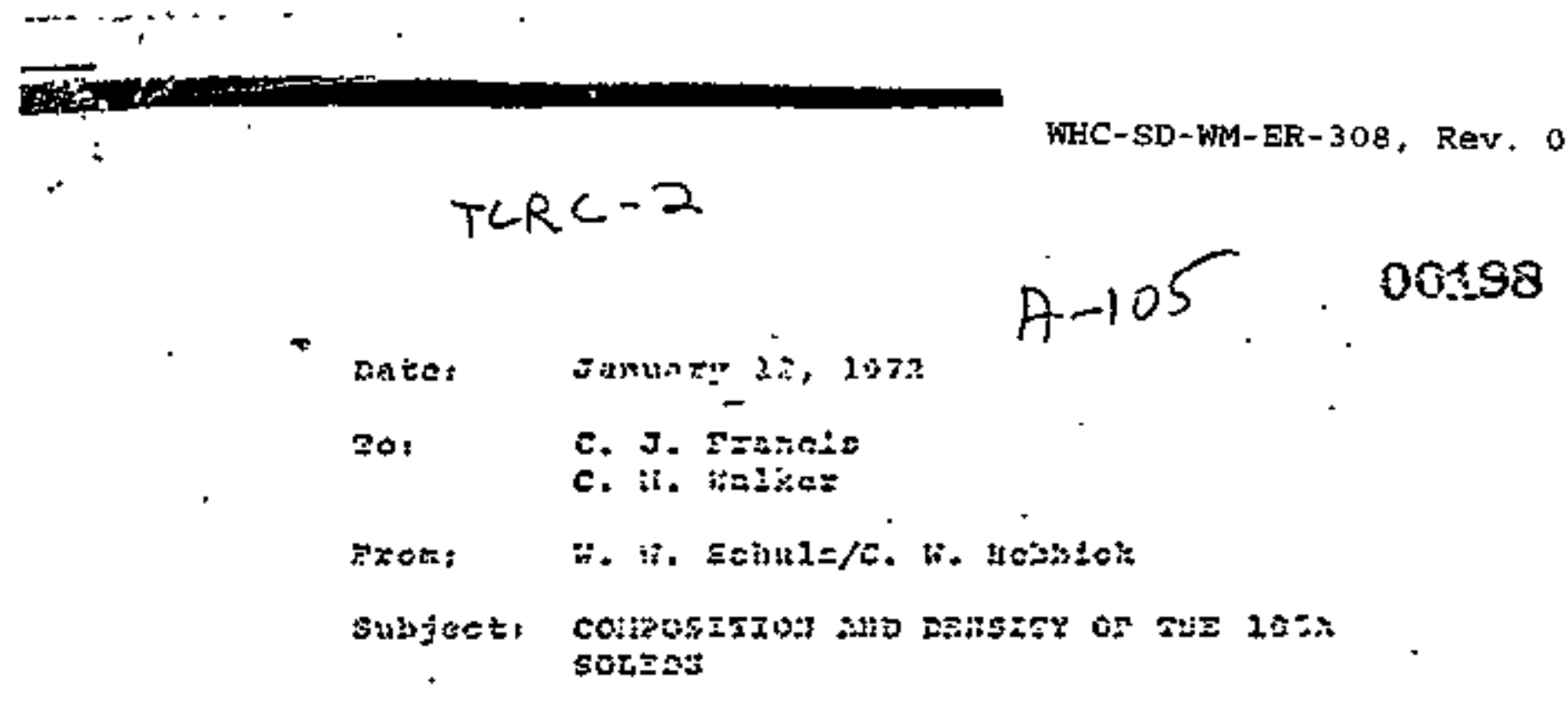

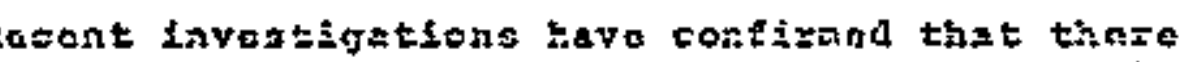
is a bulg in the bottor os tho 205A tatx. Fians

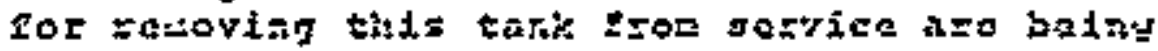

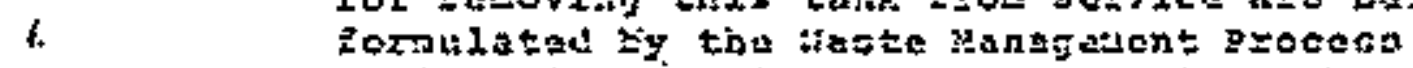

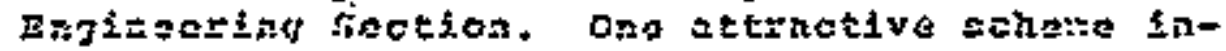
volves rafteval of tho reatteal liguid Fron tis

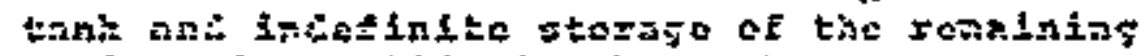
radionctive solids it tho tark.

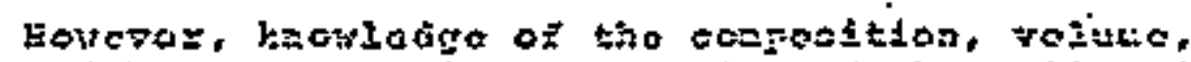
and heat jerezation pioyertita of the folls in

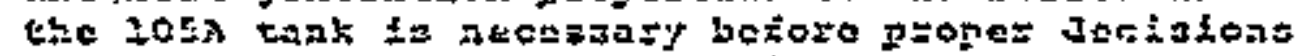

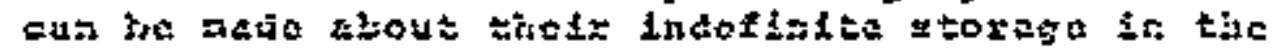

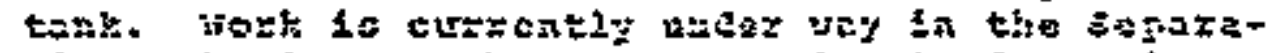

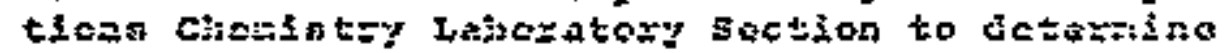

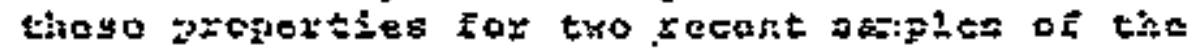

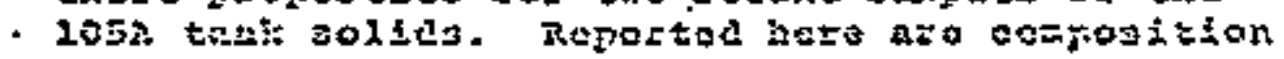

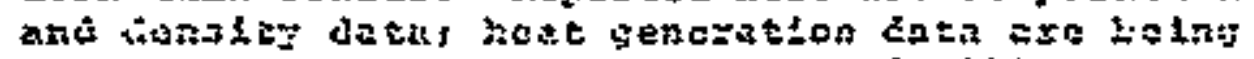
fatioged by G. 0 . Jarsey of SCl sat will be sepoztod sagtzatedy.

\section{RESLLTS}

Corgaritios a

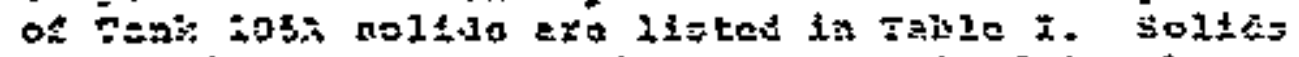

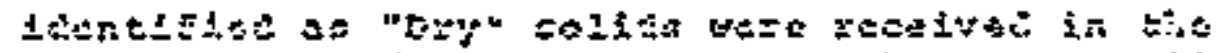
Eor: u=

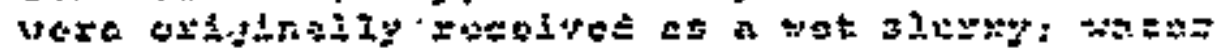

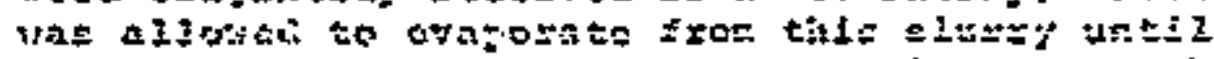

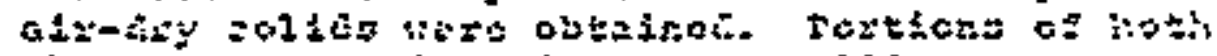
the "DF" a

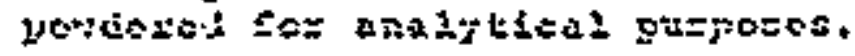

जิva

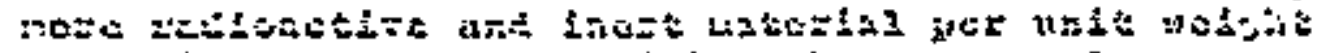

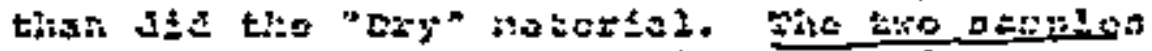

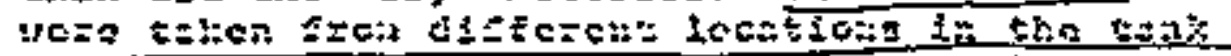

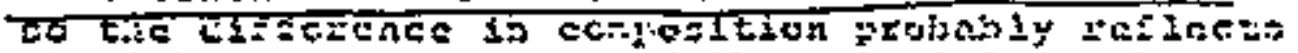

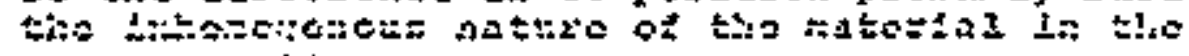

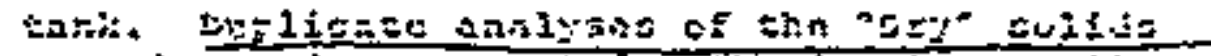

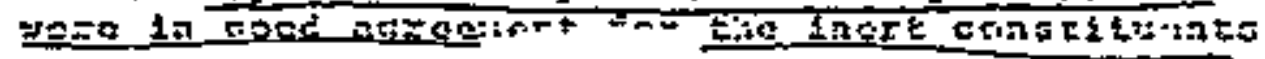


WHC-SD-WM-ER-309, Rev. 0

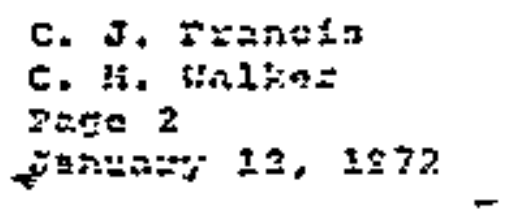

A105.1

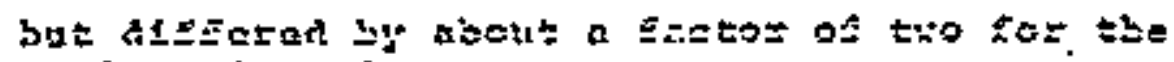

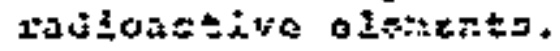

\section{TALIE_ I}

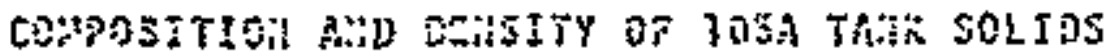

P:apex=y

rap ansity, $y /=1$

Patticlo denaity, $5 / n I$

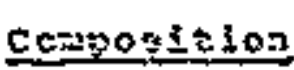

$\mathrm{HO} 3, \mathrm{~s} / \mathrm{q}$

$\mathrm{re}, \mathrm{g} / \mathrm{g}$

$\lambda I, \quad S / J$

line $3 / 9$

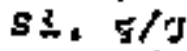

Fis, $g / t$

$0,5 / 5$

IVIS, $\mu \mathrm{C} i / \mathrm{g}$

$2+4 \mathrm{Co}, 1 \mathrm{Ci} / \mathrm{T}$

$437 \mathrm{Cs}, \mathrm{pCi} / \mathrm{s}$

$105 \mathrm{so}, \mathrm{ped} / \mathrm{T}$

$22353, \mu \mathrm{Ci} / \mathrm{s}$

$254 \mathrm{su}, \mu \in i / 9$

13,500

610

500

420

230

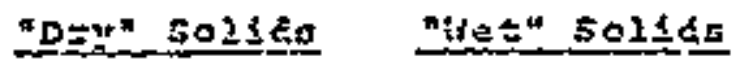

3.54

1. 21 (2)

$\sqrt{25}$

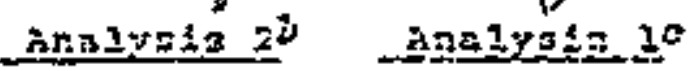

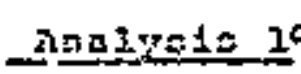

0.31

0.001

0.21

0.058

$0.032^{\circ}$

0.032

0.0025

0.013

$2.3=10^{-5}$

0.0026

$2.3 \times 10^{\circ}$

$3.3 \% 10^{-5}$

25,700

2,000

960

410

200
3.43

2.04

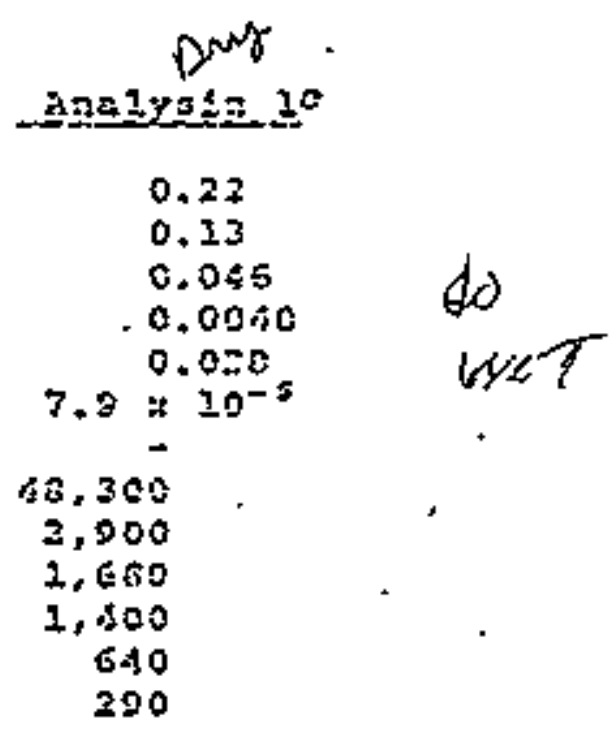

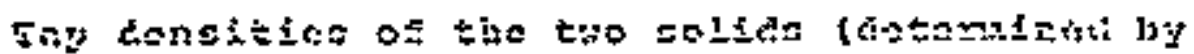

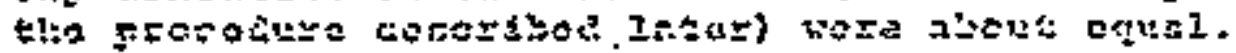

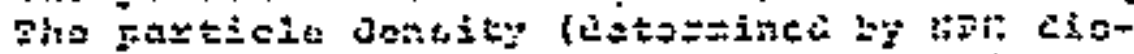

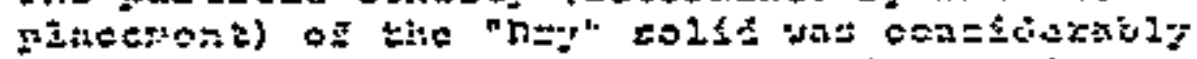

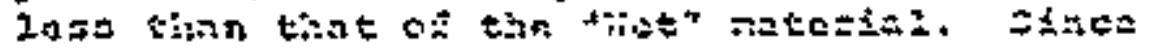

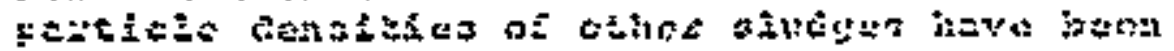

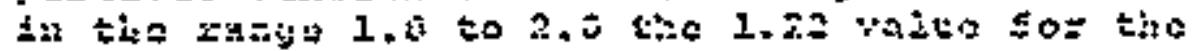

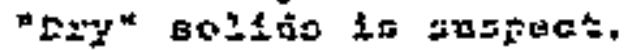



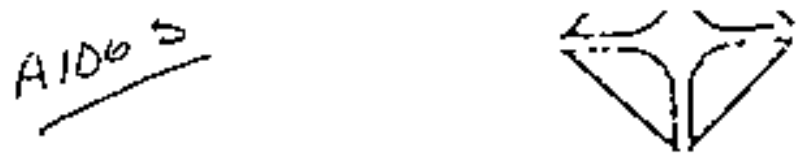

Dote: June $25, j 974$

To:

From:

R. L. Walser

Subject: TAHK FARH SAMPLES

$T-531 d$

102 AY

vis-0TR: Ciear, yellow. Ho solios. $200 \mathrm{mrad} / \mathrm{hr}$

$\mathrm{pH}$ :

11.2

Mra:

$$
0.744 \text { 然 }
$$

GEA: $\quad \begin{aligned} & 234 \\ & 237 \mathrm{Cs}-7.12 \times 10^{2}+\mathrm{H}[\mathrm{Cs} / \mathrm{ga}]\end{aligned}$

\section{$T-53 / 5-101 A$}

I-5315

$$
\text { 10)-A }
$$

Vis-0TR: Dark Brown. 5\% soljids.

$\mathrm{pH}$ :

11.15

Ha:

GEA:

$$
3.60 \mathrm{H}
$$

$3250-3.28=10^{3} \mathrm{NCj} / \mathrm{gz}$

$3 \pi 550-8.10 \times 30_{2}^{3} 06 i / 921$

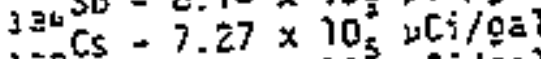

$157 \mathrm{Cs}-7.0 \mathrm{C} \times 10^{5} \mathrm{yCi} / \mathrm{ge}$

$154 \mathrm{Eu}=4.69 \times 10^{3} \mathrm{w}[j / \mathrm{ca}]$

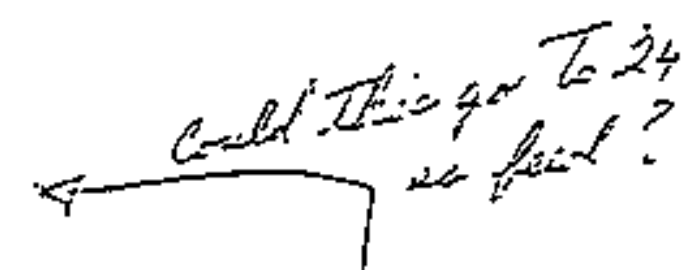

T-5316 7B6-A

Vis-0TR: Derk brown, -5: solios. 2500 mico/hr

pH:

12.60

He: $\quad 2,20 \underline{M}$

GEA: $\quad$ J $500-2.42 \times 10^{3}, y[$ [ $/ 02]$

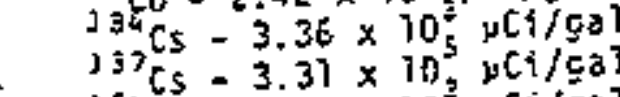

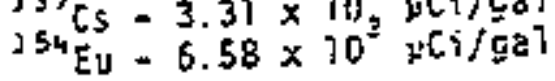

RES: $5 d$

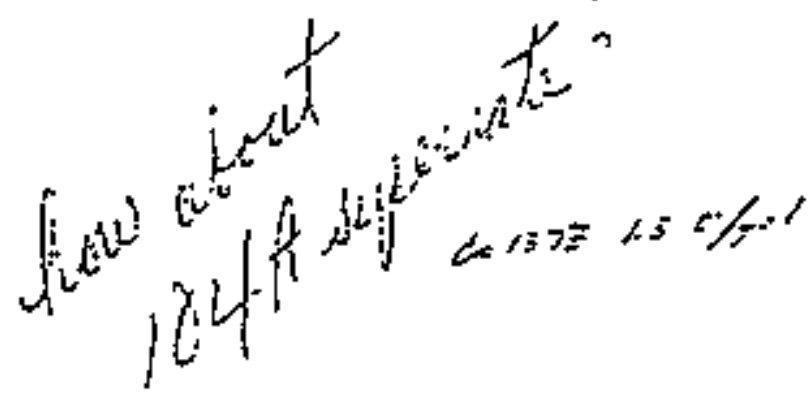


TCRC -6

$$
10-27-79 \ldots
$$

A106.6

Manager, Services 222-5 Laboratory

200 it

2-2965 or 2-2435

Analytical Results

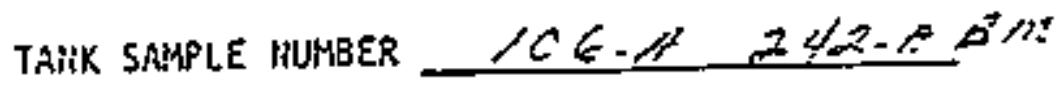

AL SERIAL HMMER TE $4 / 6 / 8$

DATE RECEIYED $10 / 27 / 29$

DILUTION FACTOR

Nase

V/S-OTL

AHALYSIS

$\mathrm{pH}$

SPG

$\because \mathrm{H}_{2} \mathrm{O}$

TTA

A1

$\mathrm{OH}$

$\mathrm{NO}_{3}$

$\mathrm{HO}_{2}$

Pm 147

Sr 89,90

$\mathrm{Pu}$

GEA

Cs-i37

$\because \quad[s-134$
RESULTS

NiE

104

$75,4 S^{\prime}$ w:

$-N R$

$7.20-1$.

1.01

$1,33 \mid$.

$.739{ }^{M}$

${\frac{N C_{0}^{4}}{2.57-5}}_{2 / 1}^{u c i / 1}$

$1.76^{5-5} u[i / 1$

$\mathrm{uCi} / \mathrm{T}$

$\mathrm{uC} \mathfrak{j} / \mathrm{I}$
ANALYSIS

$\mathrm{PO}_{4}$

$\mathrm{CL}$

' $F$

$\mathrm{SO}_{4}$

$\mathrm{CO}_{3}$

Torgh

NA
- RESULTS

$8.69^{-2} M$

NR

$-N E{ }^{H}$

NE "

$1.7^{-1} M$

NR $9 / 1 \mathrm{C}$

$N R-4$

Cooling Curve

$\checkmark \alpha 7501$ is

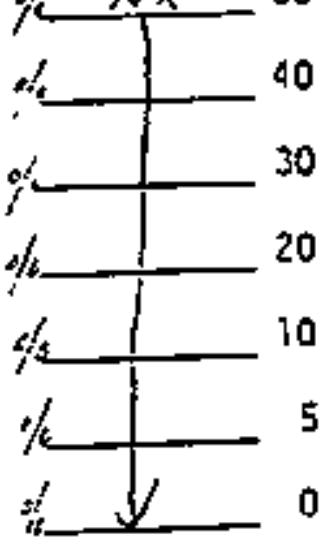

$4 \mathrm{Cj} / \mathrm{I}$

u[i/1

UC? $/$ )

$H R=$ Hot Requested $H D=$ Not Detectec H-167 dilution factor is inciuded in the -ulations. 


\section{Internal Letter TCRC-T}

Ca:ę: - May 23, 1980

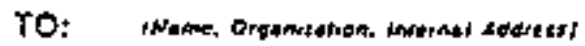

- H. J. Eding

- Systens Engineering

- 2750E/A-227, 200 East
WHC-SD-WM-ER-309, Rev, 0

No: . 65453-080-156

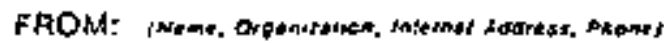

- M. T. Jansky and D. L. Herting

- Separations Process Development Unit

- 222-5, MO-037, 200 llest

. 3-1571 and 3-1541

Subjer: Hot Botidown of Tank 106A Waste

Ref: Letter, Aprit 25, 1980, R. 8. Bendixsen to H. J. Eding "Concentration ō Customer Waste into Double Shell Slurry"

As requested in the reiterenced letter, a hot boijdown of Tank 106A waste was completed. The waste was customer waste concentrated during the first quarter of FY80. The purpose ot this boildown was to provide injormation concerning the suitability of Tank $706 \mathrm{~A}$ waste for processing in a double shell slurry run. We found that nucleation occurred at $12 \%$ waste volume reduction (WVR). Wucleation was quite rapid (a non complexed behavior), al though no settling was observed (a complexed behavior). Laboratory details are described below.

The as-received sample was $=90 \%$ solids and viscous. It should be pointed out that the AL cooling curve showed <3\% solids at $5^{\circ} \mathrm{C}$. Agitation and gentle heating were susficient to allow transier of the sample. The viscosity of the sample at room temperature is shown in Table I, along with viscosities at the initial botiling point and at nucleation. Teble It shows WVR versus temperature data. After nucleation, the phases were separated by centrifugation and stibntited to AL for analyses.

The Feed liquor analyses are shown in Table III. fable iv shows boildown product analyses. Note the good agreenent between the analyzed product slurry, the carculated product slurry, and the analyzed product supernatant liquor. The data shows:

(a) Analytical results are consistent and probably refiable (except where noted in Table IV).

(b) The similarity between the analyzed product slurry and enalyzed product supernatant indicate that $\mathrm{HaA}_{3} \mathrm{O}_{2}$, $\mathrm{NaOH}, \mathrm{HaNO}_{2}$, and $\mathrm{NaNO}_{3}$ did not precipitate.

(c) The feed was virtually saturated in $\mathrm{Ha}_{2} \mathrm{CO}_{3}$ and Na $\mathrm{PO}_{4}$, and at least $25 \%$ below saturation in all other components.

(d) The product slurry resemiled a suspension of Nat $\mathrm{PO}_{4}-12 \mathrm{H}_{2} \mathrm{O}$ needles. In addition, the thisotropic nature oi the product slurry (Table I) is characteristic of $\mathrm{Na}_{3} \mathrm{PO}_{4}-12 \mathrm{H}_{2} \mathrm{O}$ suspensions.

Thus: (e) The solid product phase is most likely $\mathrm{Na}_{3} \mathrm{PO}_{4}$ 


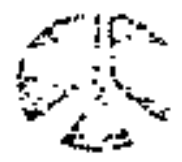

H. J. Eding

Page 2

May 23, 1980

and $\mathrm{Na}_{3} \mathrm{CO}$ (Analyses of these two components in the product phases ore unavajlable due to equigment malfunction\}.

Please call if there are any questions or comments regarding this work.
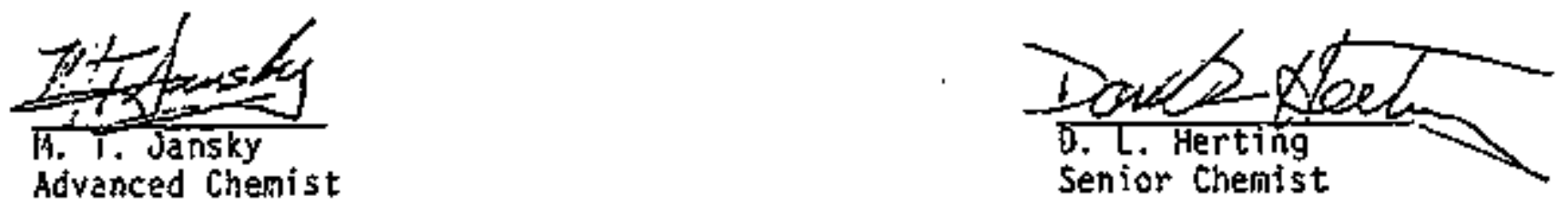

MTJ : DLL H: naj

Attachments

Information:

R. B. Bendixsen

L. C. Brown

D. E. Bowers

d. 5. Buckingham $\overline{15 B}$

T. A. Lane

H. E. Ogren

0. A. Reynolos

H. E. Teats

T. B. Veneziano

Process Aides:

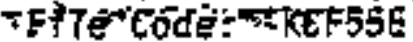

Letterbook 
TABLE I

Viscostty Data for Tank 106A Bot Boildown

2ORPM

Room Temperature

46

49
SORPM

187

26

263
10ORPY

155

31

197

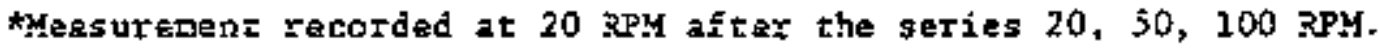

This indicaces thixortopic benavior. 
WHC-SD-WM-ER-308, ReV, 0

\section{TABLE II}

WVR vs. Temperature for Tank 106A hot Boildown

F WVR Temperature $\left({ }^{\circ} \mathrm{C} /{ }^{\circ} \mathrm{F}\right)$

Fressure (Torr)

0

$$
44.2 / 111.8
$$

40

1

$45.8 / 114.4$

40

1

$59.2 / 138.8$

$80 *$

10

$48.2 / 118.6$

40

12

$48.6 / 119.6$

40

*Due to rapid nucleation and iigh radistion exposure, only one peasuramenc ar 80 Torr was made. 
WHC-SD-WM-ER-30B, Rev, 0

TABLE III

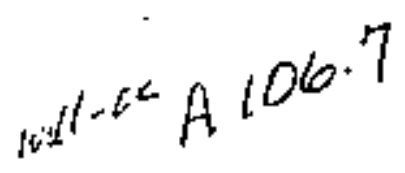

Analyses of Tank 106A Feed Liquor (T-12)*

Component

ITaAlo 2

$\mathrm{NaOH}$

$\mathrm{NasiO}_{2}$

$\mathrm{NaNO}_{3}$

$\mathrm{Sa}_{2} \mathrm{CO}_{3}$

$\mathrm{Na}_{3} \mathrm{PO}_{4}$

$\operatorname{TOC}(g / \mathrm{L})$

$\mathrm{H}_{2} \mathrm{O}$

Tocal

SpG $(g / \pi \mathrm{H})$
Molaricy (M/L)

Wct

1.00

5.49

2.36

6.80

3.10

15.41

1.40

8.57

$0.90 * *$

6.87

0.129

1.52

$.61 .8 * *$

106.88

*Two samples, $I-12$ and $T-11$, were recefved firon TRTC. Only T-12 vss used in this boildown. The analytical results frow $\mathrm{T}-11$ vere very similar to $\mathrm{T}-12$.

*t Values sre most likely high. 
Product Analyses of Tank 106A Hot Boxldown

\section{Product Slurry}

Component

$\mathrm{NaAlO}_{2}$

SaOH

$\mathrm{NaNO}_{2}$

$\mathrm{NaNO}_{3}$

$\mathrm{Na}_{2} \mathrm{CO}_{3}$

$\mathrm{Na}_{3} \mathrm{PO}_{4}$

$\operatorname{soc}(g / L)$

$\mathrm{H}_{2} \mathrm{O}$

soral

Sp.G. $(g / m I)$
Calculated
Molarity

1.14

2.68

3.52

1,59

1.0

0.15

$-$
Analyzed

Molarizty

1.25

2.12

3.52

1.68

$-$

$-$

14.4

45.34

$92.56^{a, 3}$

9.92

2.21

$a, b$
Analyzed Product Phases

Anazyzed Supernatane Supernatant Soljo

Molaxity

Wt. $\bar{z}$

Wt $z$

7.91

2.18

6.23

3.59

27.69

1.81

10.99

2.27

$-$

2.3

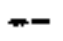

2.34

15.4

3.27

48.18

98.88

1.40

a) Assuming saturation concentrations for $\mathrm{Na}_{2} \mathrm{CO}_{3}(0.3 \mathrm{M})$ and $\mathrm{Na}_{3} \mathrm{PO}_{4}(0.2 \mathrm{M})$

3) If $\mathrm{Na}_{2} \mathrm{CO}_{3}$ and $\mathrm{Ka}_{3} \mathrm{PO}_{4}$ waro the pringry soifds in the slurty, these values wovld be significancly higher, and the total would be closer to $100 \%$.

c) Calculated values.

d) As pointed out in the text, the solids are probebly $100 \% \mathrm{Na}_{3} \mathrm{PO}_{4}$ and $\mathrm{Na}_{2} \mathrm{CO}_{3}$. 


SUPPORTING DOCUMENT
samentita: -
ata Trangmittat Package for 241-A-10s -
aste Tank Characterization

by Worcs

aste tank characterization, single-shell tank, 41-A-106 waste tank, tank sampling. radioctive weste, nonradioactive waste enelysis

HS DOCAMENT IS FOA USE IN PEFRORMANCE OF WORX UNDER CDN. JALS WTH THE US, DEFARTMENT OF ENETESY DY PSRSONS OR FOR

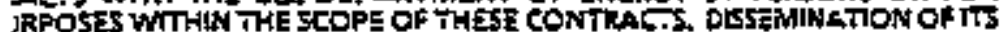

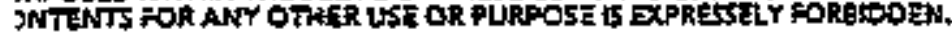

\section{Exter}

his document contains information relating o the sampling of rastes contalned in the ingle-shel1 tank 241-k-106 in fiscal year 986. Observatfons and data taken during ampling, sample breakoown and preparation, nd the results of analytical determinations re recorded. rease 5tmp

OFFCIALIY FELEASED

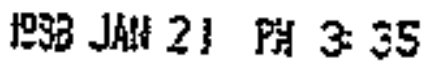

\begin{tabular}{|l|c|c|}
\hline Number & Rev No. & Pagk A \\
So-RE-TI-200. . . . & 0 & 31 \\
& & 31 \\
\hline
\end{tabular}

Program

Haste Management

Propared by tome, Deoc No. and Signaturet)

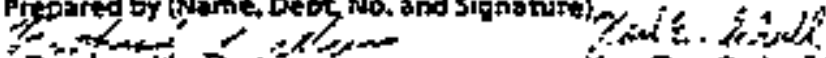
R. L. HeTss K. E. Schuj 12223 22272

... . Ses riverse side for adoitional apgrovals ... -

- Distgigugar Mame Moldadres

M. R.rutams

R2-78

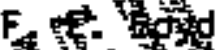

S5-04

T6-50

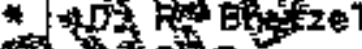

RI-48

R2-11

T5 -50

R1-51

TS-32.

R2-84

R2-51

$\mathrm{Rl}-10$

T6-50

$\mathrm{R} 2-23$

T6-18.

Rl-48

T6-50

Rl-10

RZ-18 


\section{SD-RE-TI- $\bar{I}-\bar{O} 0$ \\ Rev. 0}

WHC-SD-WM-ER-308, REV. 0

\section{ANALYTICAL SAYPLE PFEPARATICN DATA}

The actual wefghts and volumes gerierated during analytical sample preparation (solution leaching) for the core composites from tank 241-A-10s are shown. Peptization of the solid may occur in some of the water leaches (third contact). This forms an unseparable solid-1 iquid suspension broken by adding concentrated $\mathrm{HCl}$ to the leach to bring the actd concentration to $5 \mathrm{M}$. In these cases, effectively no third water leach oceurred and the acidiffed solution becones the first actd leacis.

No analytical sample preparation was required, except fittering, for the drafnable liquor core composjtes.

\section{Compos the ID $4 A 1 \times \operatorname{cog} x$}

Inttial weight of sample $4.677 \mathrm{~g}$ Volume

Rosidual weight:

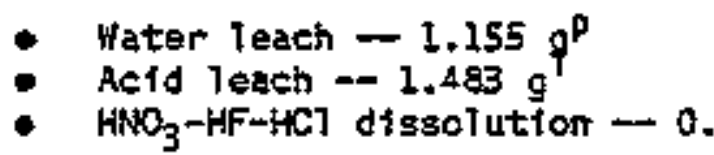

Final soiution volumes. ... then...

- Nater leach - $35 \mathrm{mi}$

- Acto Teach - $25 \mathrm{~mL}$

- $\mathrm{HNO}_{3}$-HF-Fit dissolution - $25 \mathrm{mt}$.

- $8.09 \mathrm{~mL}$ of acfd leach added to $25 \mathrm{~mL}$ of HNO $\mathrm{O}_{3}$-HF-HCT dissolution : and submitted for andysis...

P Sample peptized on thitd water wash -- concentrated HCl becoming first water wash.

$10.48 \mathrm{~g}$ carriad on to dissolution step. 


\section{Composite In AA2X0OOXX}

Initial reight of sample Volume

Resioual weight:

- Water Jeach - $1.565 \mathrm{~g}^{\mathrm{P}}$

- Acid Teach - 2.0BD $\mathrm{g}^{\mathrm{m}}$

- $\mathrm{HNO}_{3}-\mathrm{HF}-\mathrm{HCl}$ dissolution - 0 .

Finai solution volume:

- Hater 7each - 35 mL

- Acid Teach - $25 \mathrm{~mL}$

- $\mathrm{HNO}_{3}-\mathrm{HF}-\mathrm{HCl}$ dissolution - $25 \mathrm{mi}$.

- 6.25 al of acid leach added to $25 \mathrm{~mL}$ of $\mathrm{HHO}_{3}-\mathrm{HF}-\mathrm{HCT}$ dissolution and subatted for anolysis.

P Sample peptized on third water wash - concentrated HCi becoming first water wesh.

$m 0.52 \mathrm{~g}$ carried on to djssolution step. 


\section{$\operatorname{Sn}-\mathrm{RE}-\mathrm{TI}-200$ \\ WHC-SD-WM-ER-308, Rev. 0 \\ Rev. 0 \\ VII. NWYTICAL RESULTS}

A spreadsheet program on a dedicated microcomputer is used to convert rav anelytical data to concentration values for the for the core composite

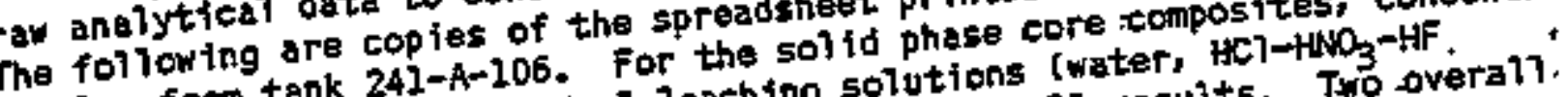

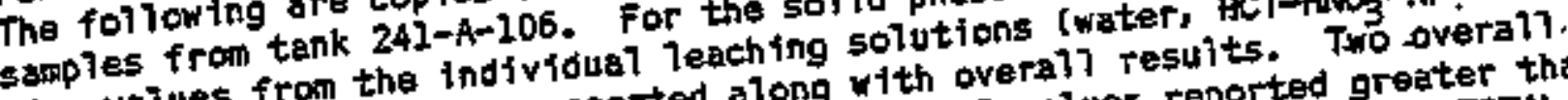
tion values from the indith are reported along with overallues reported greater than pressure dissolvtions REPORT TOTAL - stm of all not stmmed) and MAXINHM TOTAL results are reportiontion (")ess than values" not sion values taken as actual

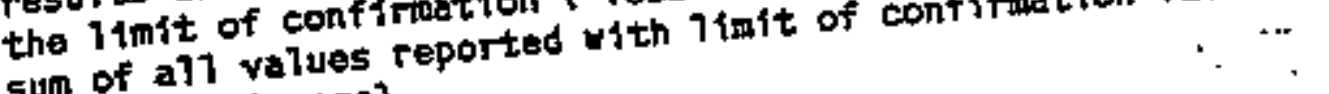
value (worst case).

Physical oata determined are shown on the spreadsheet reports. 
SO-RE-TI-200

Rer. 0
WHC-SD-WM-ER-308, Rev, 0

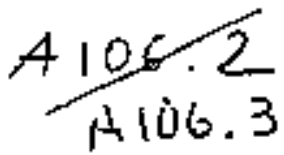

$10-1 \times 20$

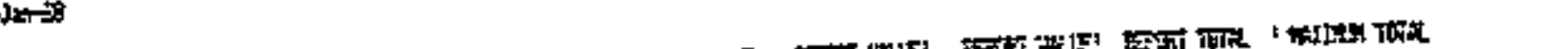

\section{$\sin _{i=10}^{\infty}$ \\ 21:}

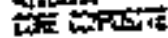

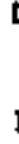

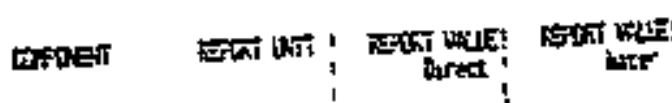

Denter

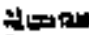

irian

红政

inn

int?

ixiti

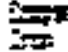

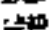

Patsit

Fis

hate?

phempors

filletter

ti)

Sorla

trority

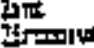

tryand

ㅊt하료

两

W"

Lers Los:

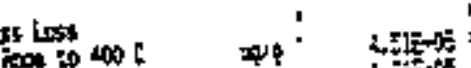
$i_{200}=0$ iocit

$=5.20$

ind

ix

孛

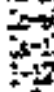

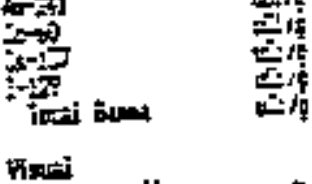

Kh⿻上丨

miss itsed?

itax the

paration

secic the

E-It Besty

parafle tain

hxosety

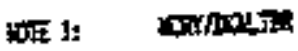

खण 2 田

जाए 3 Mas

WTI : NOE

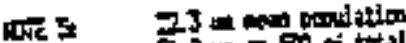

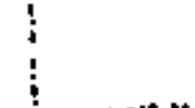

等

แั

(5)

i

4

imina:

$=-5-7)$

क

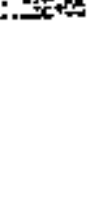

1.40 45

WOE!:

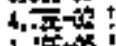

然+3 is

i) 1 ,

is $1 .=-1=01$

itcil

ts 4,040

is

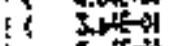

is

i' 1 1.

, 质

1,40

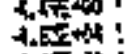

$++x_{0=0}$

is $200+0$,

at

Fosim:

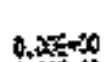

$0.2 x=0$

$0,00=-10$

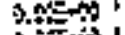

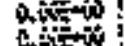

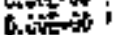

P.

b. 0 atos

a.t.

. .40

$0,0+\infty$

$0,0 \times+0$

$0,0=0+\infty$

$\log _{0,0}, 0$

$8.10 \rightarrow 0$

$0,00=0$

atis:

$0.000 \times 0,1$

a. $x=-\infty$

o. 0.00

$0 . x \in=-\infty$

$0.0 \mathrm{E}-70$ :

$1=5-\infty$ ?

토단

$\therefore=25$ !

$5035-3$

10

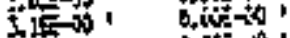

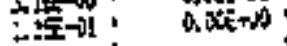

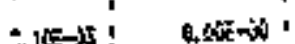

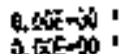

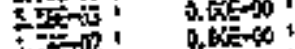

I.t?

1. $-7=-12$ :

a.ters i

प्रा:

ज्ञाह 21

Uा:

$=\infty$

$\cos =0$

7.xis.011

a.

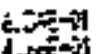

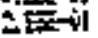

$\sqrt{4}\left[\mathrm{x}+\mathrm{w}^{3}\right.$

चाE की

and

1.505

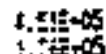

1.

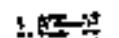

然

1.

?.1.

$0.0=0$

1.

4,조기

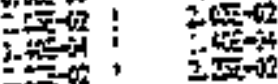

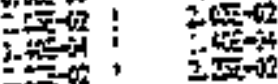

4.NEST

: $2=0$

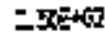

1.

(1)

:

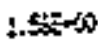




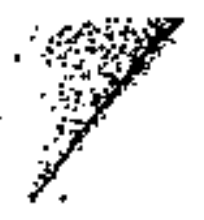

$\because:$

WHC-SD-WM-ER-308, Rev, 0

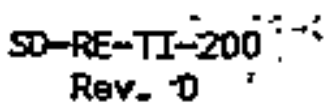

A. $10^{6.4}$
田新茯

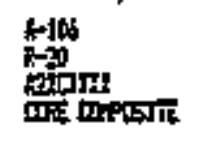

Drist

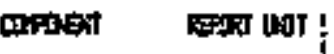

Desity

Aluminen

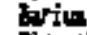

켜눈.

Pritivis

Evith:

Divi:

Entit

Cono:

ime.

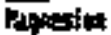

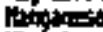

ifolit

prophor

pissivin

牙的

collot.

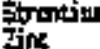

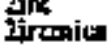

Iraia

|xtraip

तtr.

pi

Hor Ins

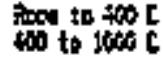

ति要,

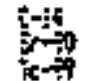

int

ton

it來

19.

Viand

Vhime datud

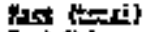

ipal the

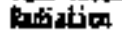

boctilie that

sortening 70 tot

aut Ditsity

Fa하랕 퇴하

visengity

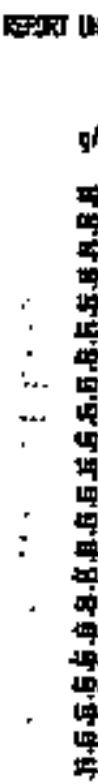

4

4 is

口4 :

lę̣l1/m

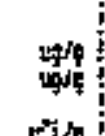

تئ1

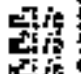

ثأ

装年

포곤

i

㓍

if and $^{2}$

천ㄷㄴ

trat

v

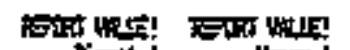
Dinst

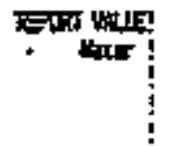

$7 x+105$

? 2 .

<.

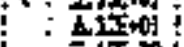

(4.0

; $\cdots 4$, the

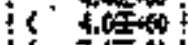

if $36 \sigma^{-0}$ !

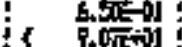

i i.t.

$5, \frac{1}{5}+0$

Inster

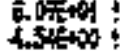

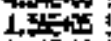

$0,00=$

$2.00=0$

is -20

$-1+[5+01 !$

T.t즘에

7.15003

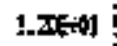

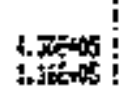

4.

ㄱ.르됴

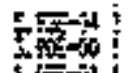

f.

<

2:

호는ㅎㅇ

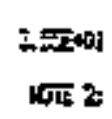

प्या:

tis

i.

1.

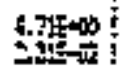

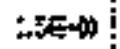

LDF 5 ?

tonstion

$\pm \cos _{0+01}$

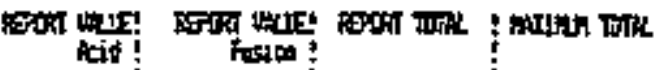

$0.00=0$

0.00

0. $100-10$

0.004

$0.010+4$

$0.0 \mathrm{x}=0$

o. ut

A.

$0.0 \mathrm{~s}+00$ !

4.

b.

o.

o.

$0 . x=1$

0.00 ?

0.

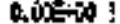

$0,0=+\infty$ !

Q.

6.

a, $00+00$ !

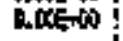

D.potwo

$\sin x$

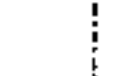

i

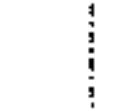

$0, M E=0$

i.

vitis

$0,3 x-\infty$

6.

$0,0 \mathrm{cos}$

a. $005+10$

U⿴囗十丁:

0,0000

Q.$$
5=40
$$

1. 位来

$1-\cos 20$

대다어

라무두에 :

NUE di

$7 . x+m$

I.

siteon

$2+20$

- . 1.50

i.5 $5+2+\infty)$

WIE $\mathrm{d}$

tot the

.

WWE 2

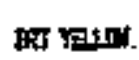

WHE Tis aE

IOTE A: WE

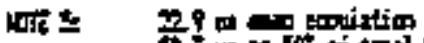

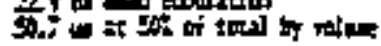




\section{A Tank Farm}

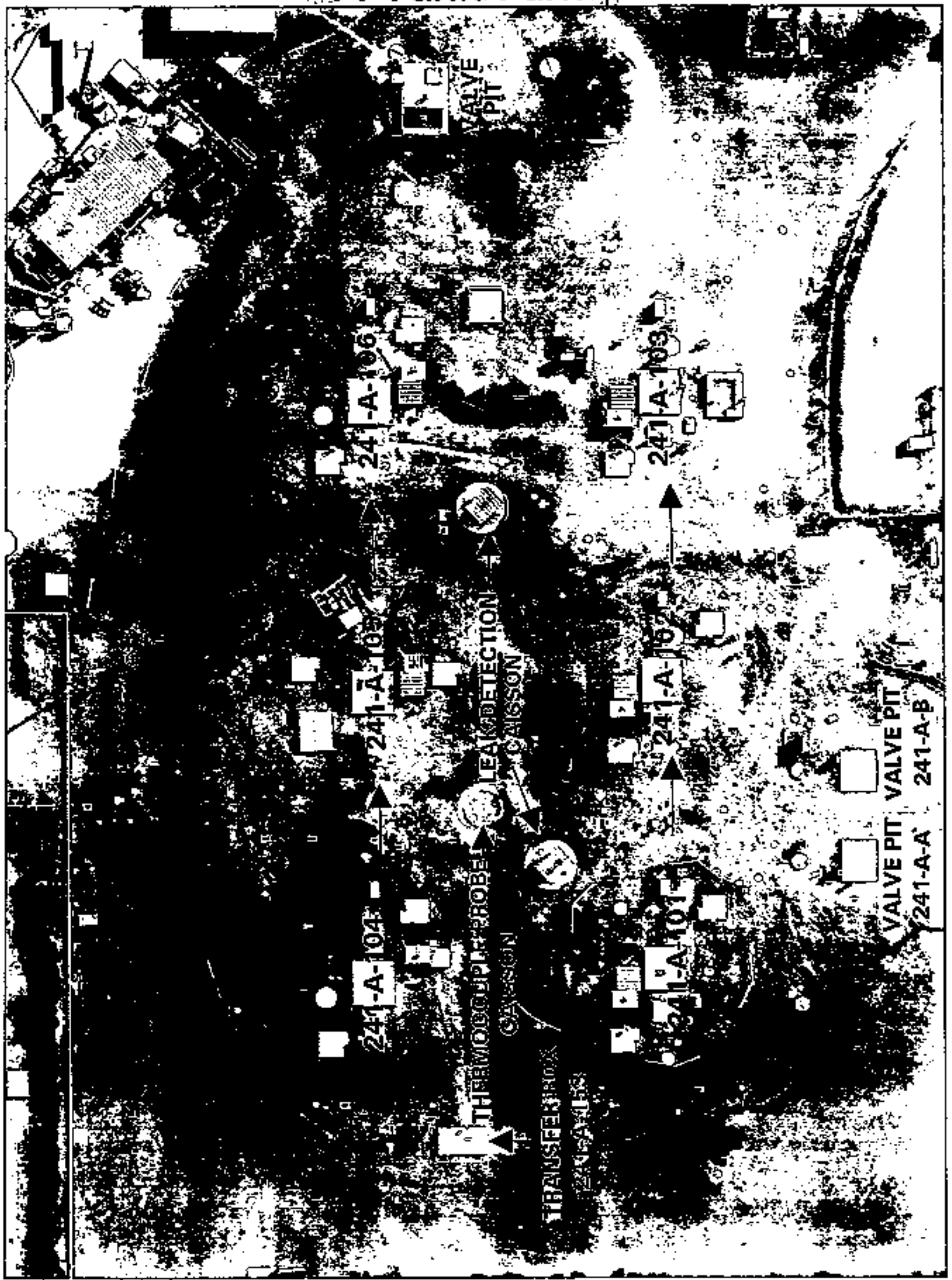




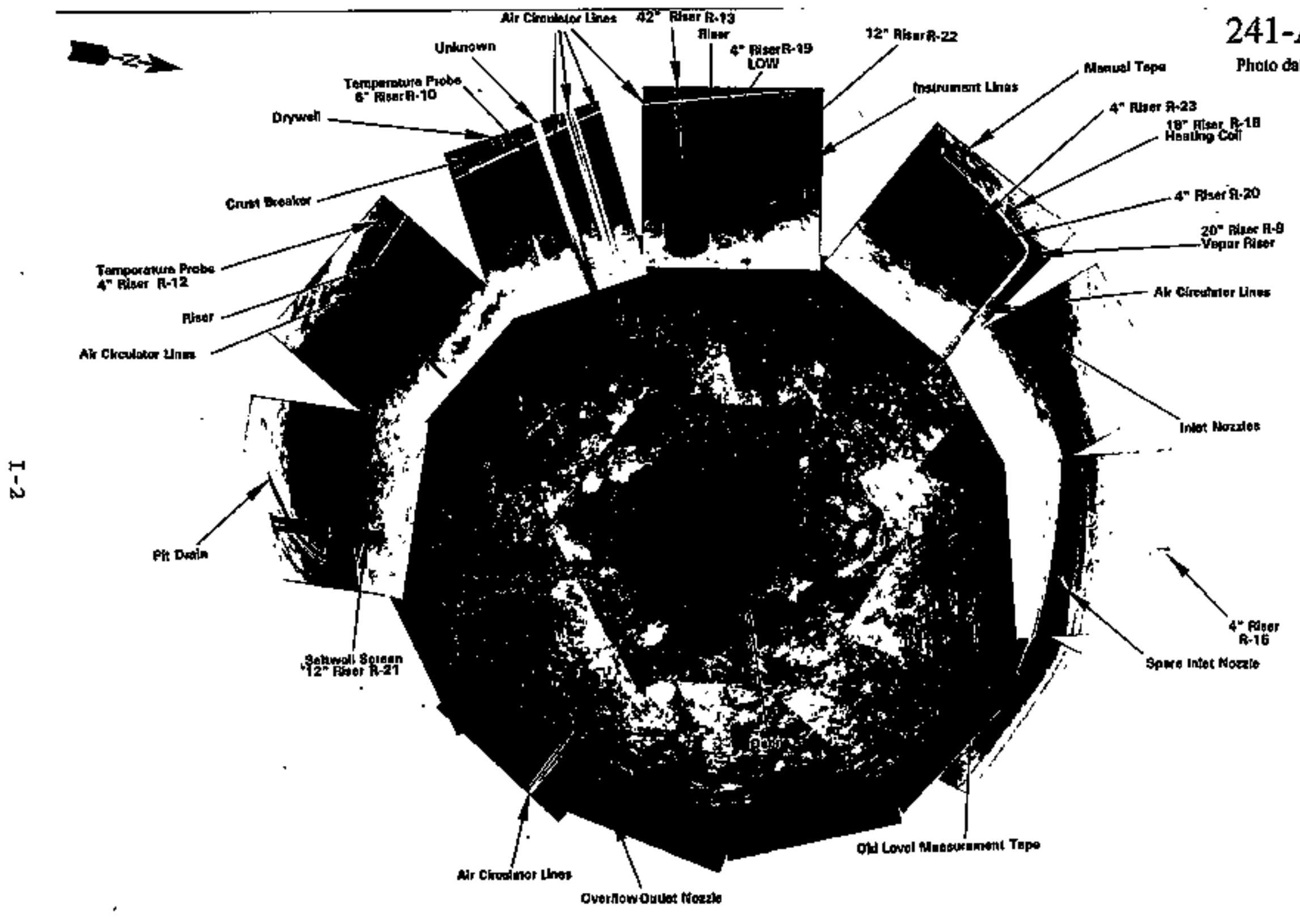




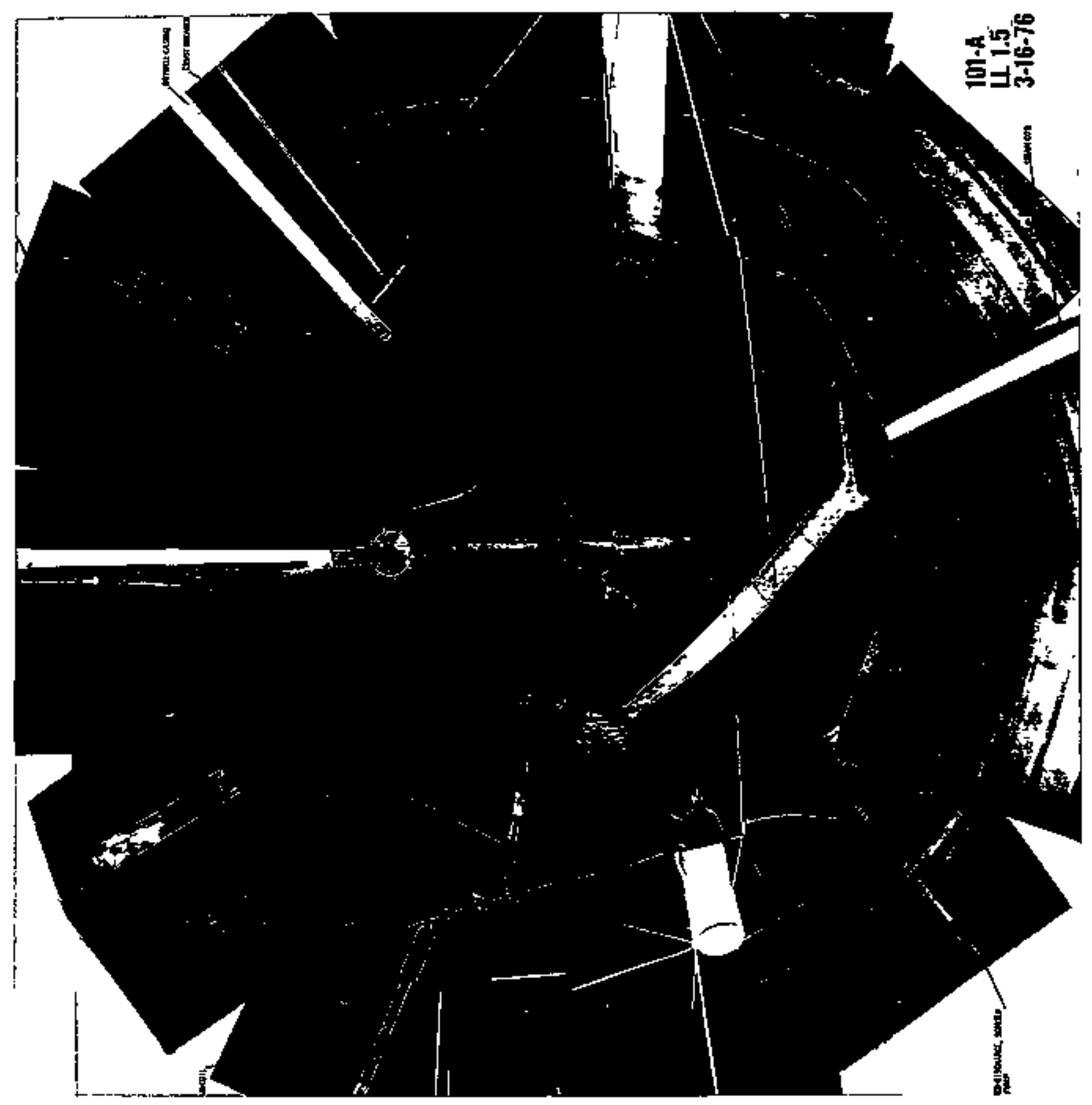




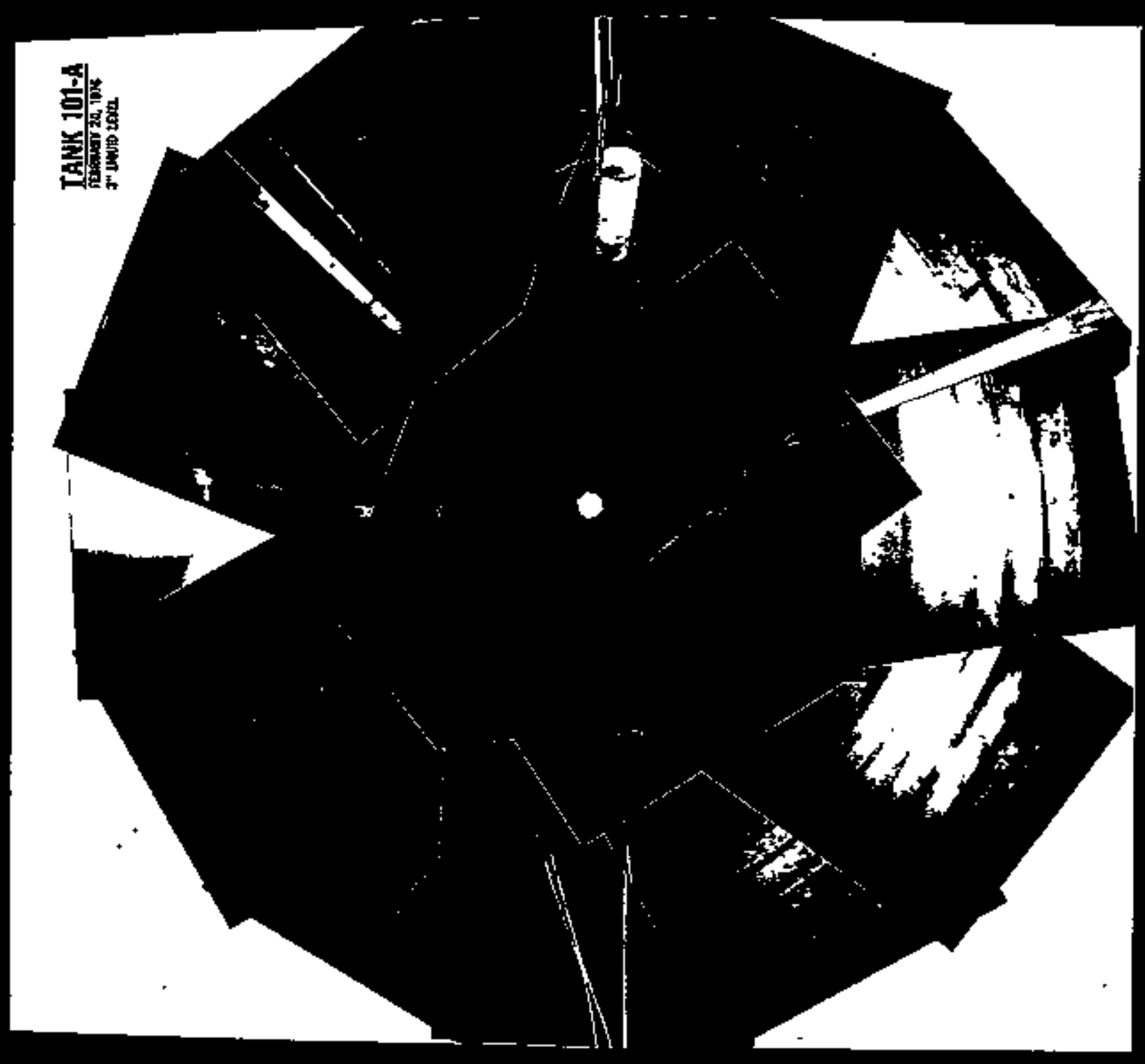




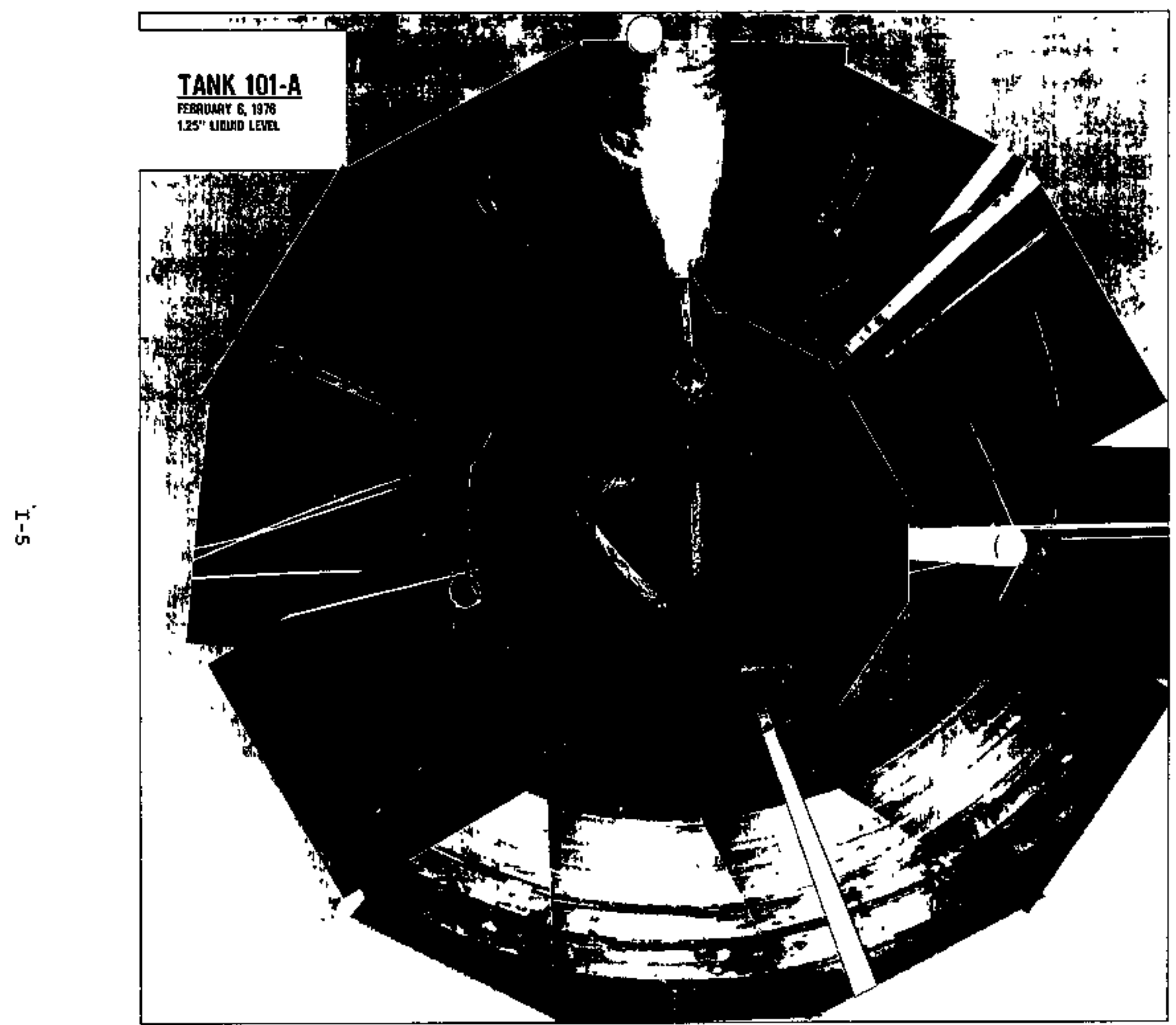




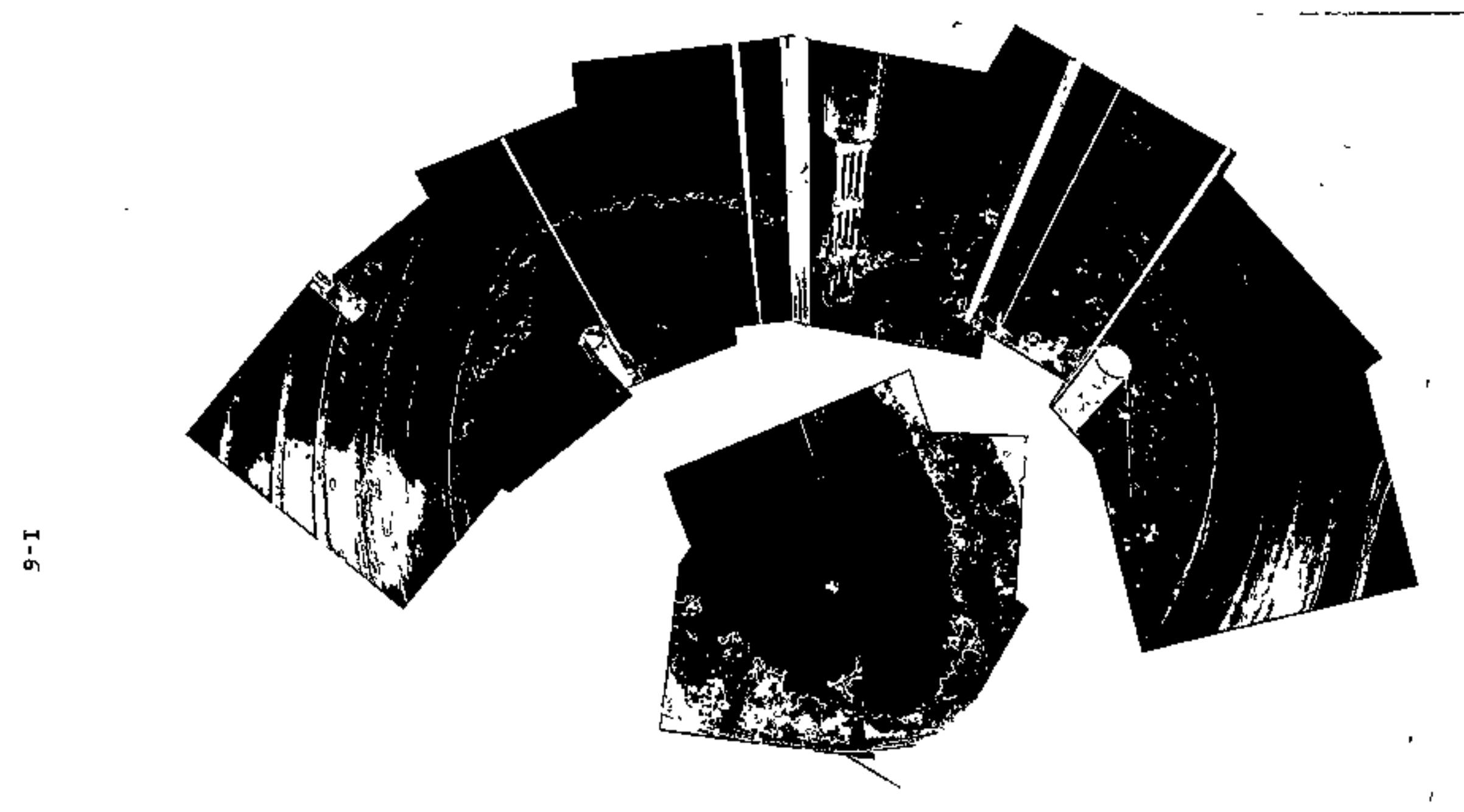

TANK 101-A

LIQUID LEVEL 17"

10-25-68 


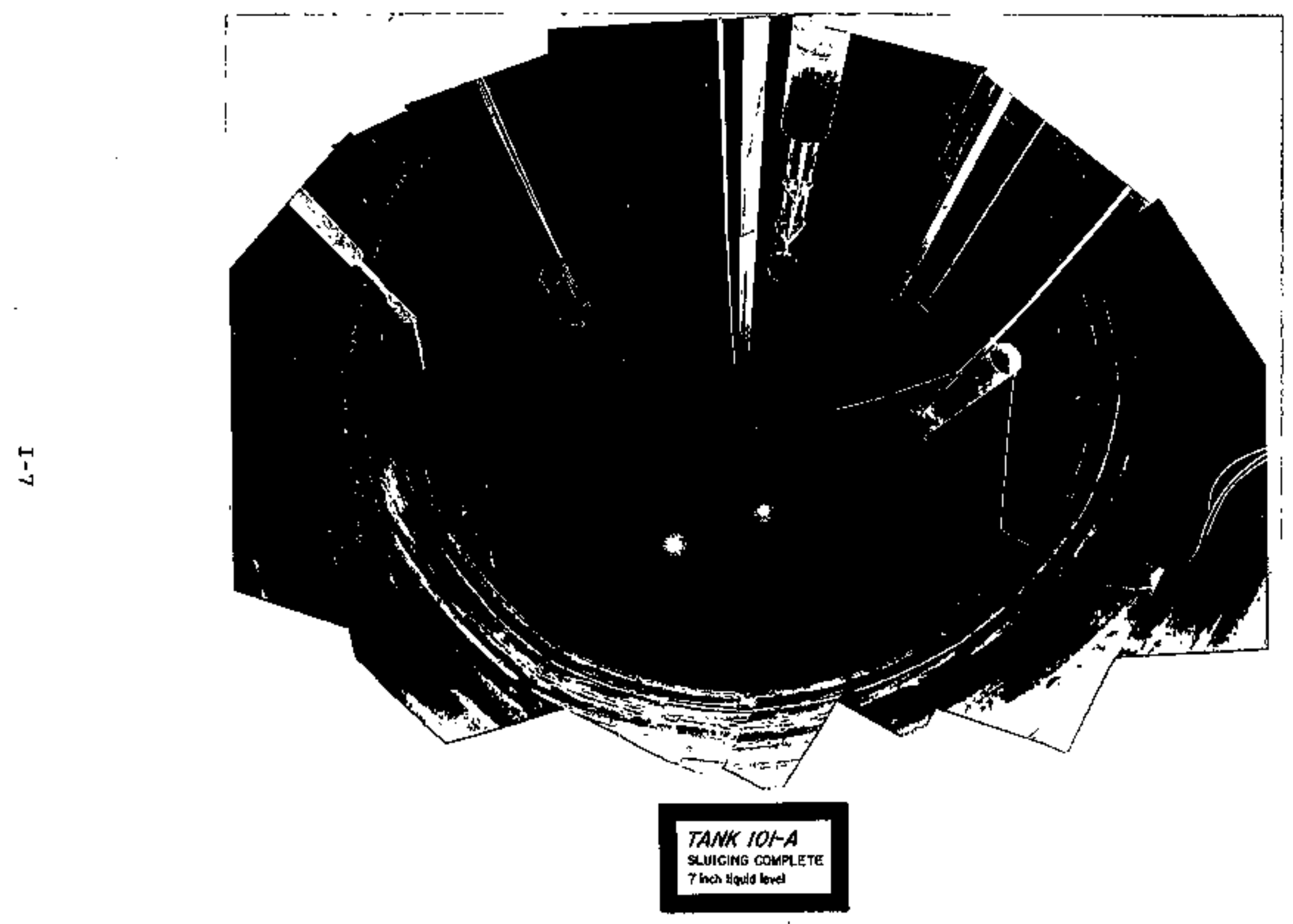

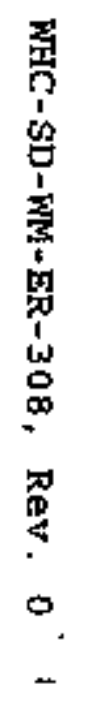




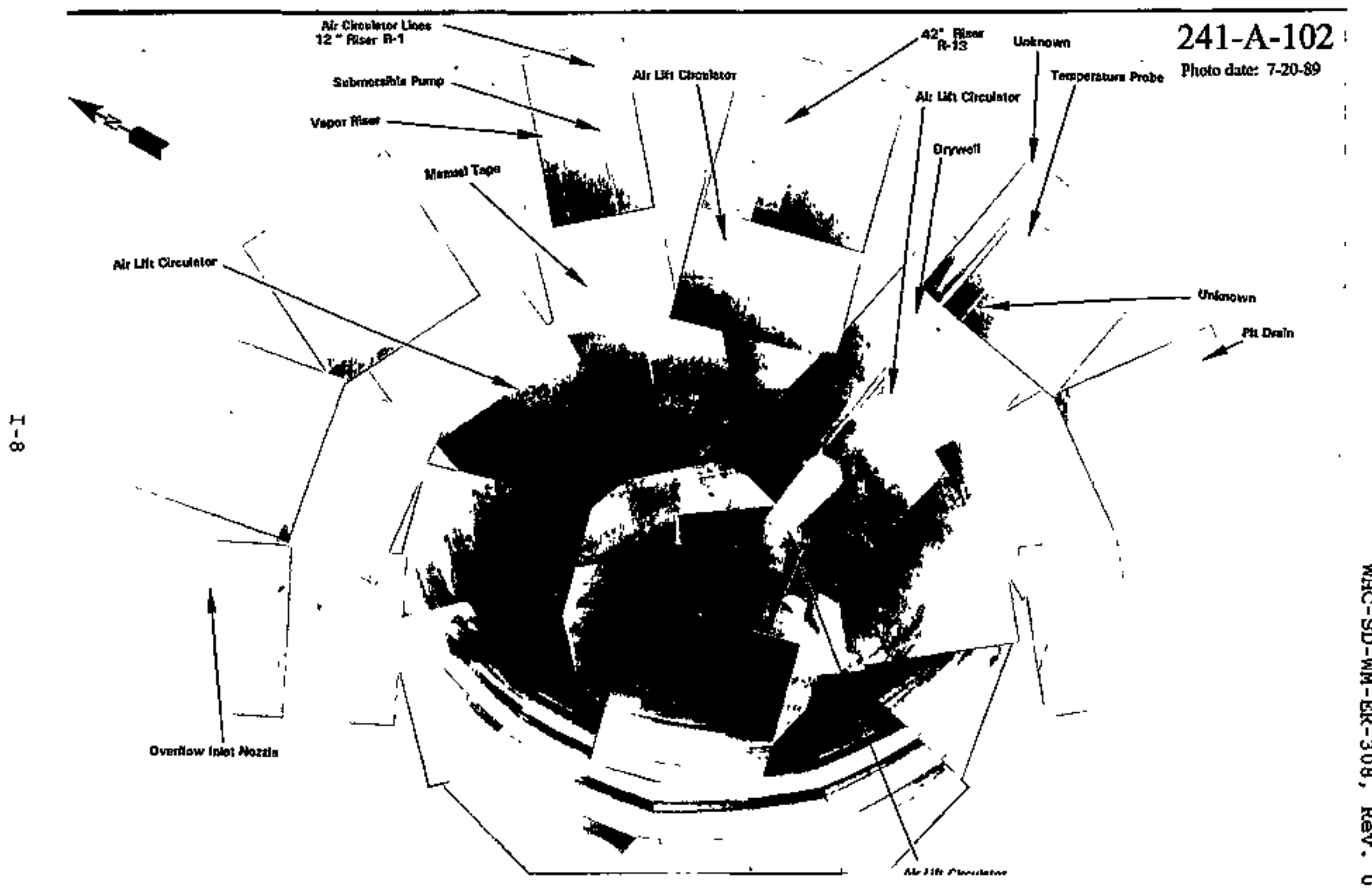

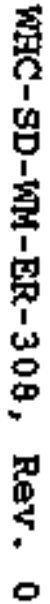




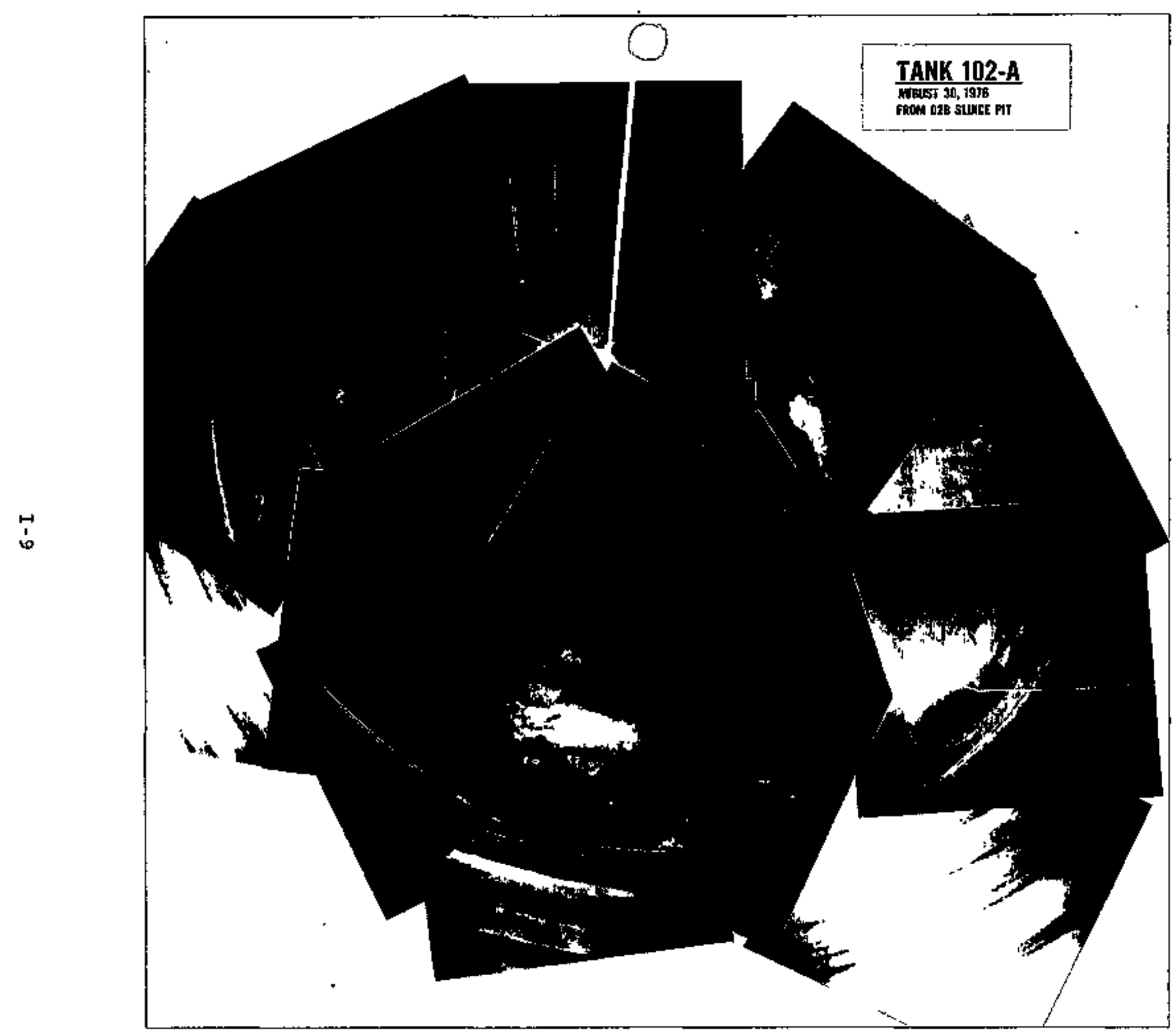


WHC-SD-WM-ER-308, Rev. O

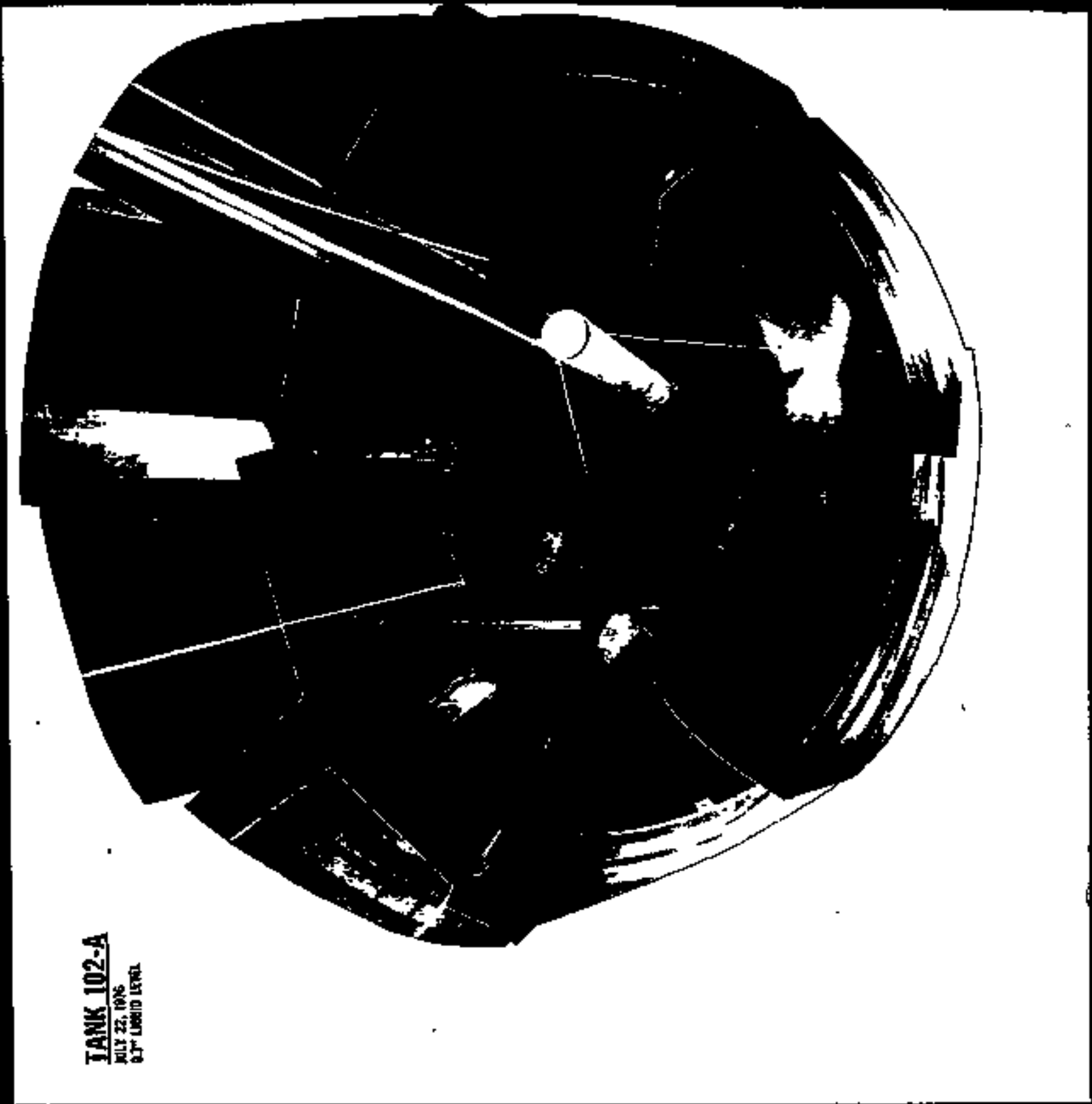

$I-10$ 

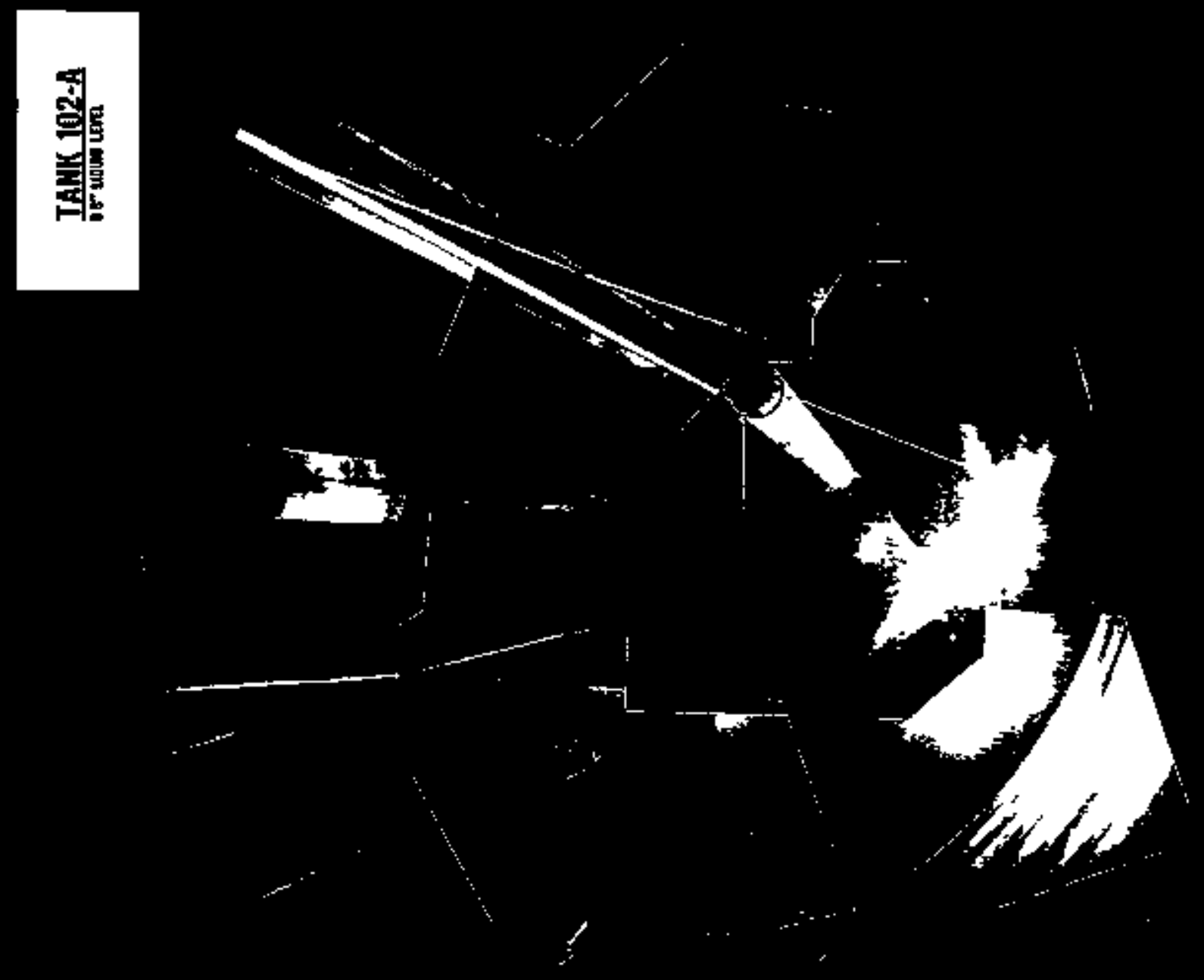

$$
\dot{I}-11
$$




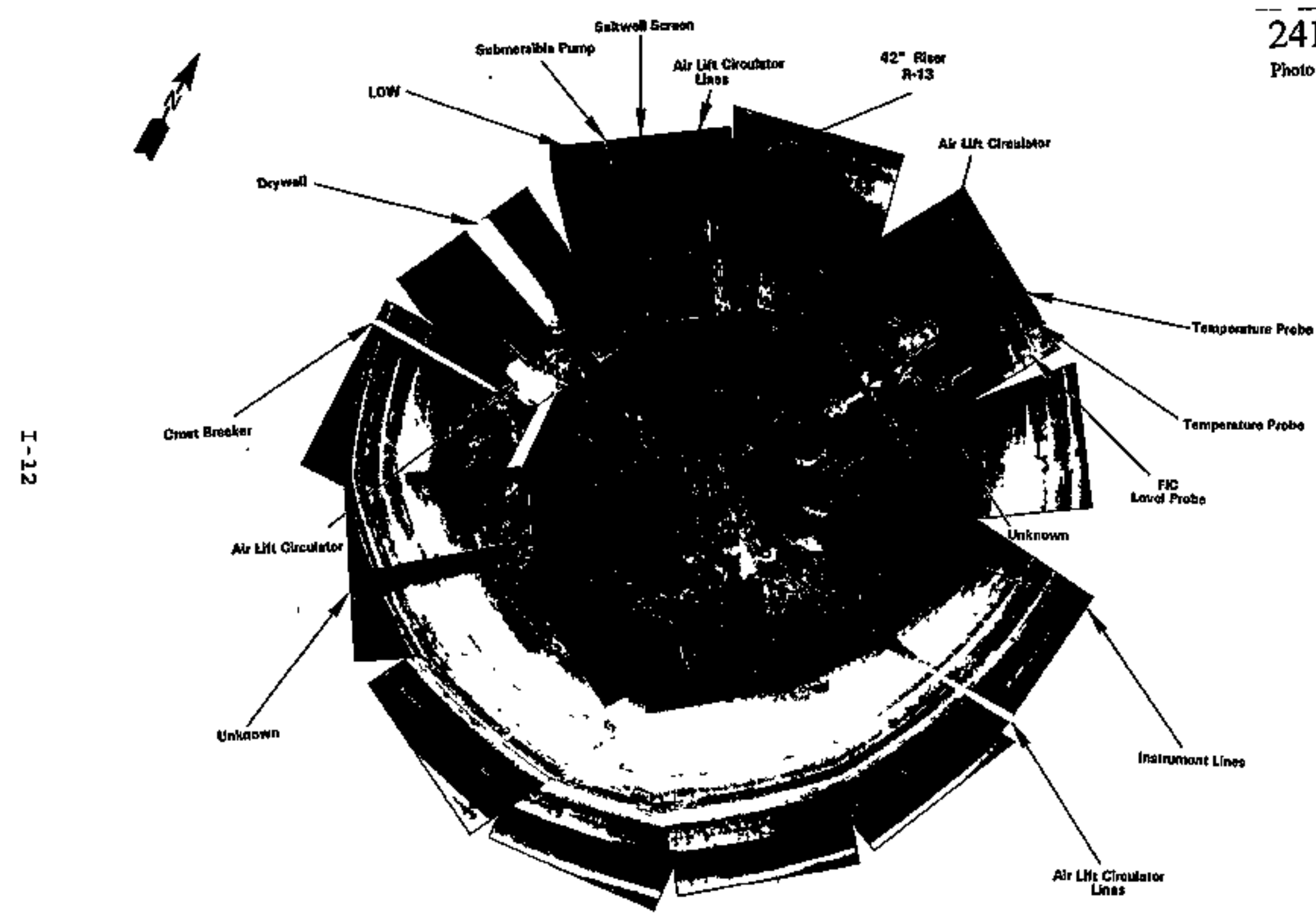

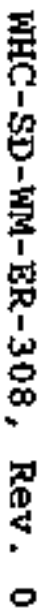


WHC-SD-WM-ER-308, Rev. 0

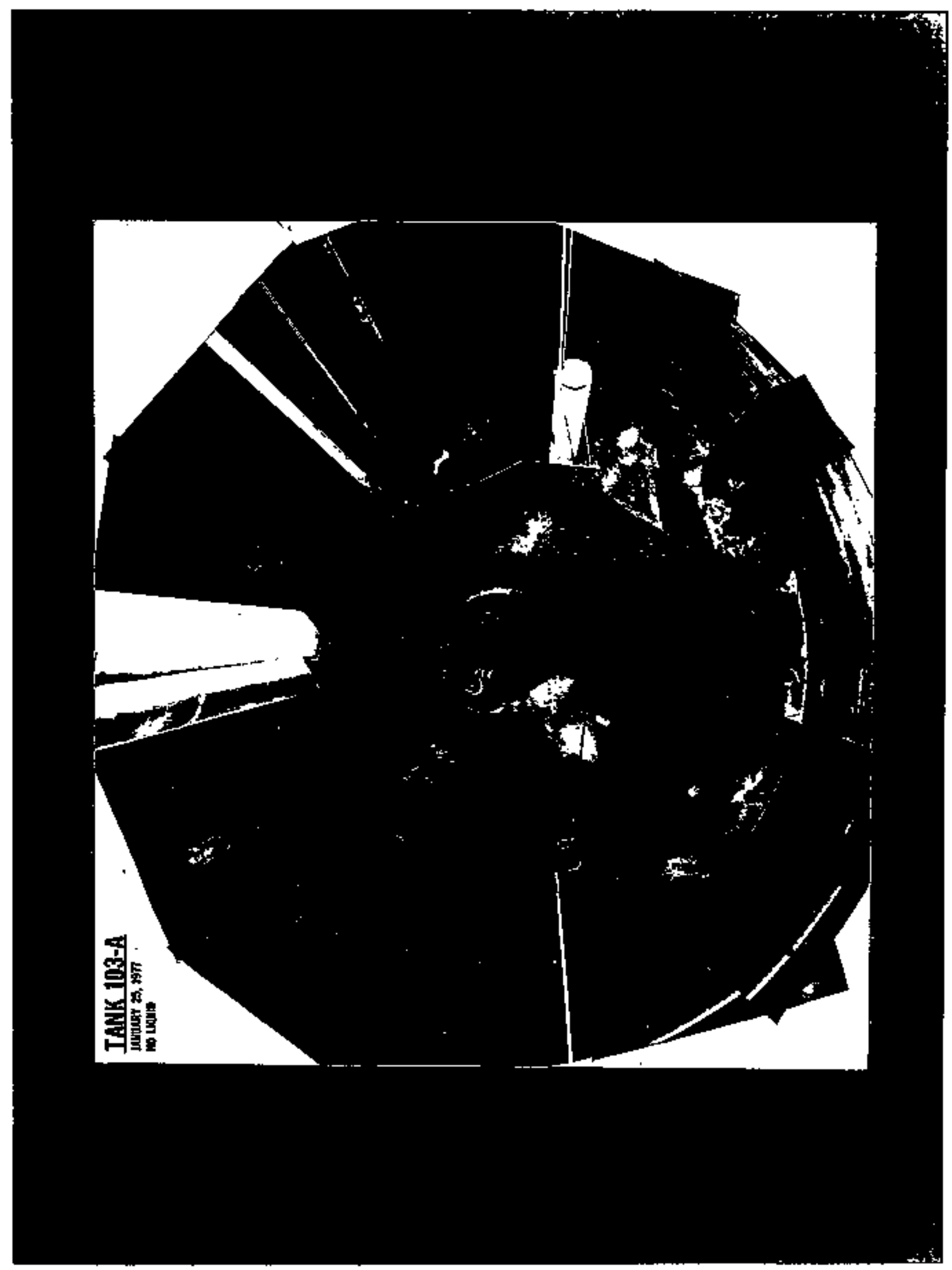




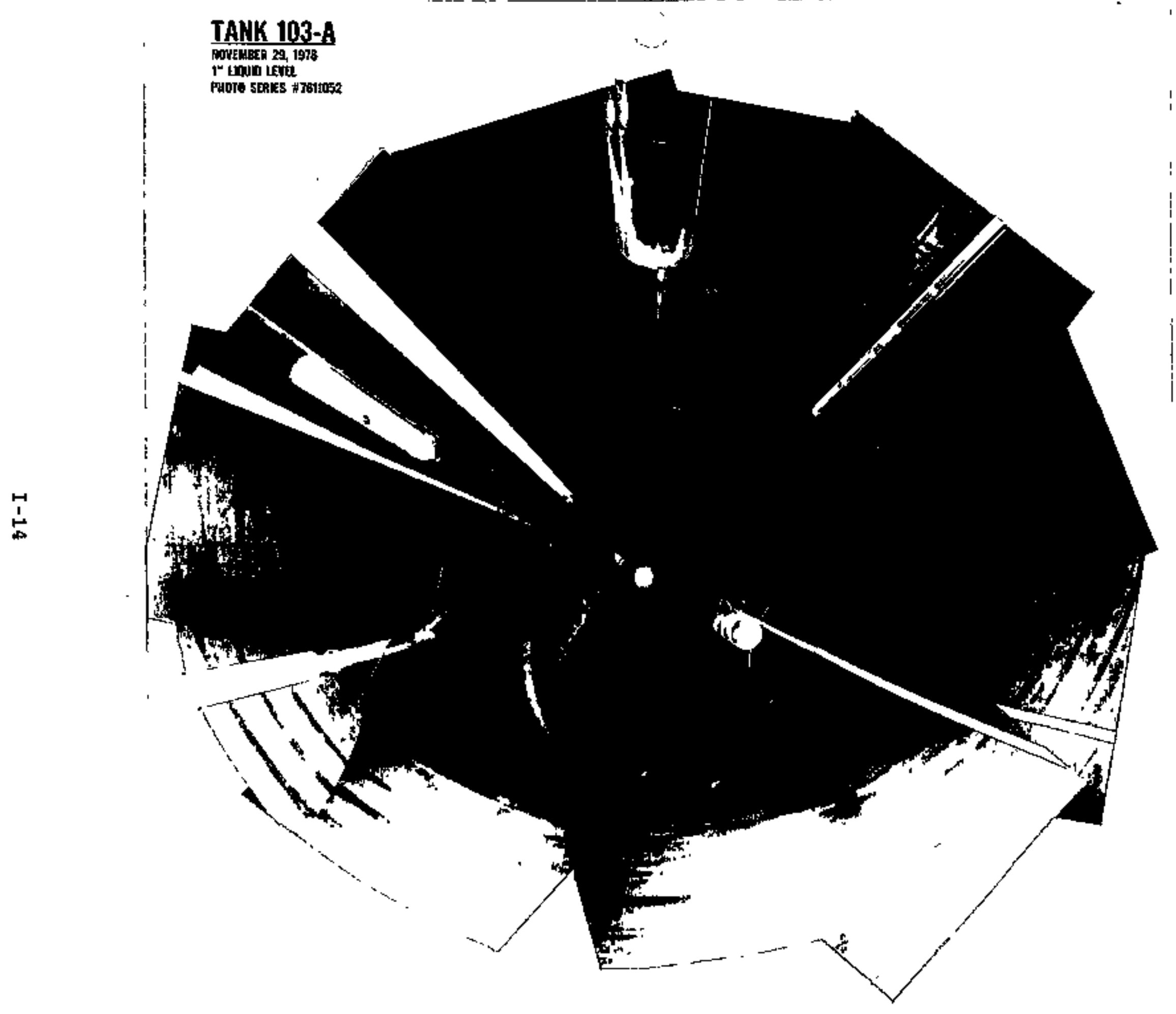


WHC-SD-WM-ER-308, ReV , 0

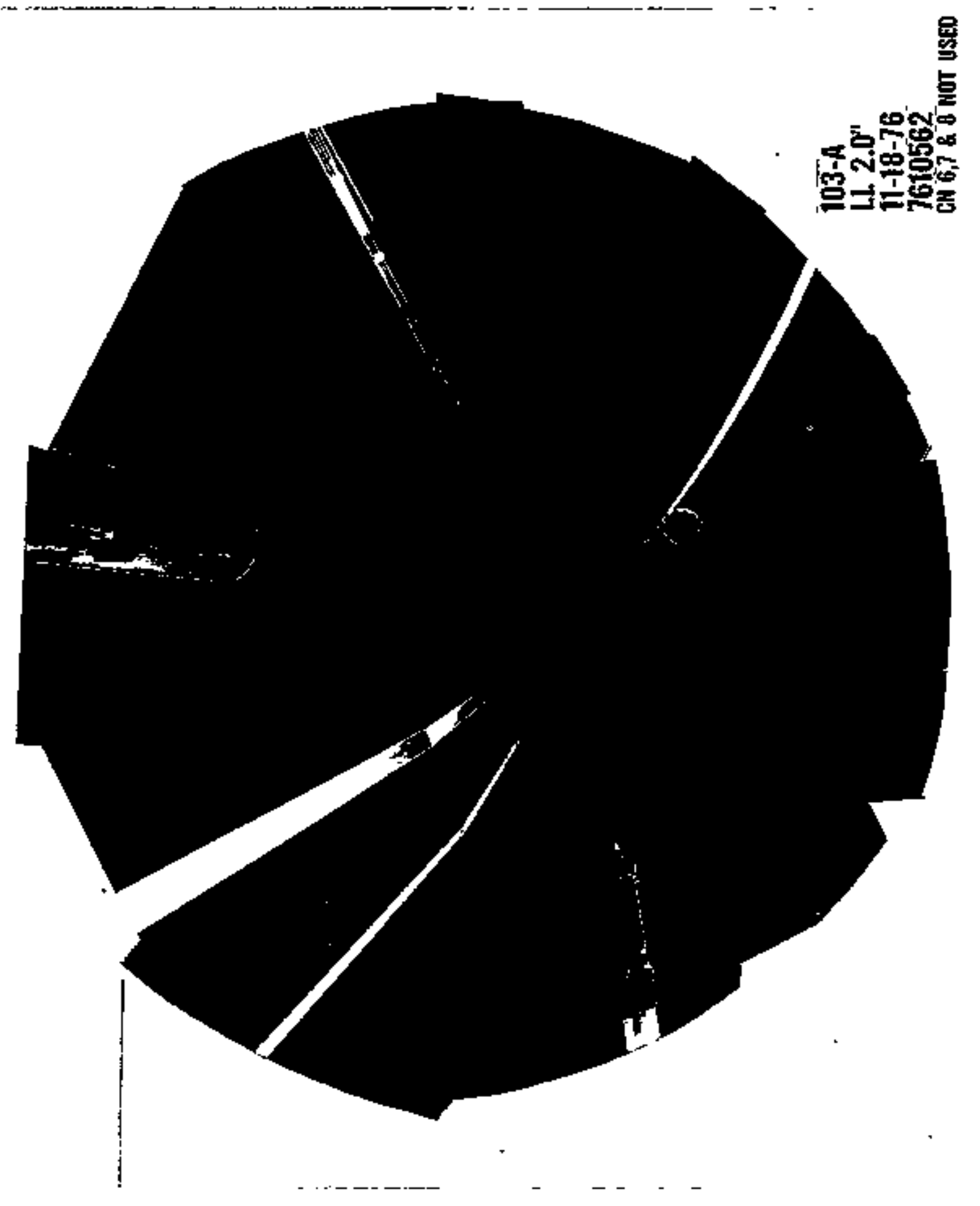

$I-15$ 
WHC-SD-WM-ER-308，Rev. 0

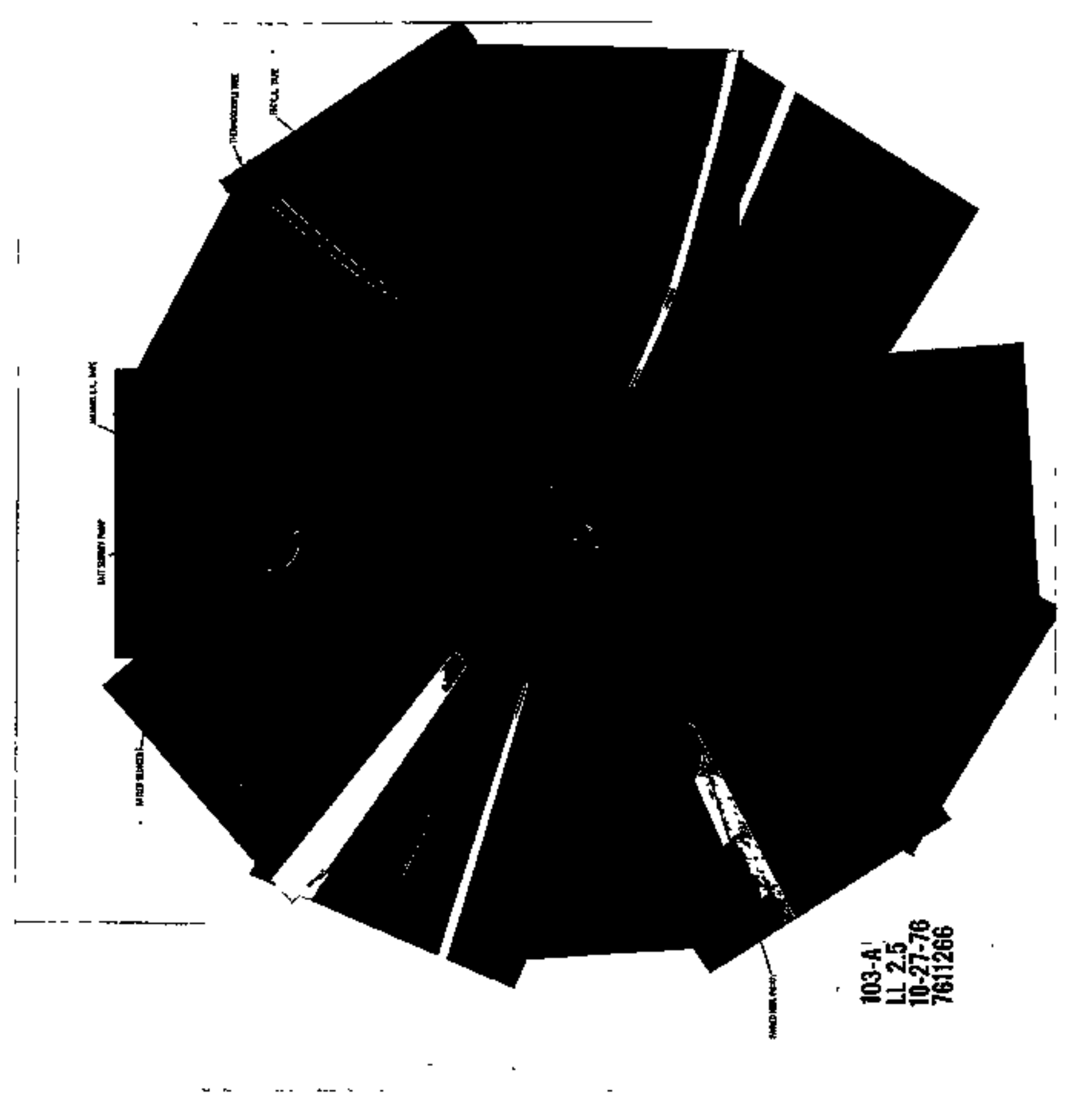

$I-16$ 


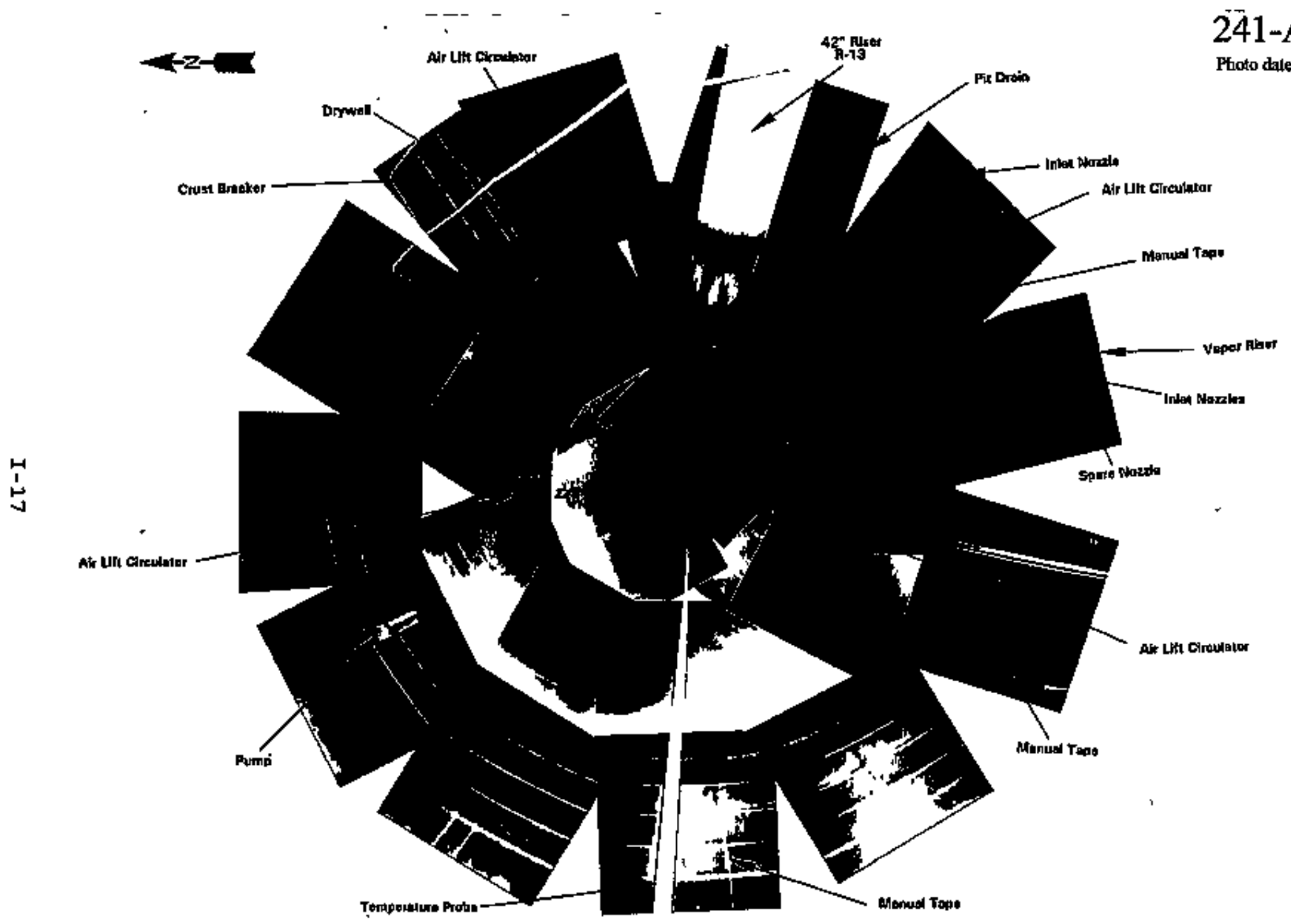




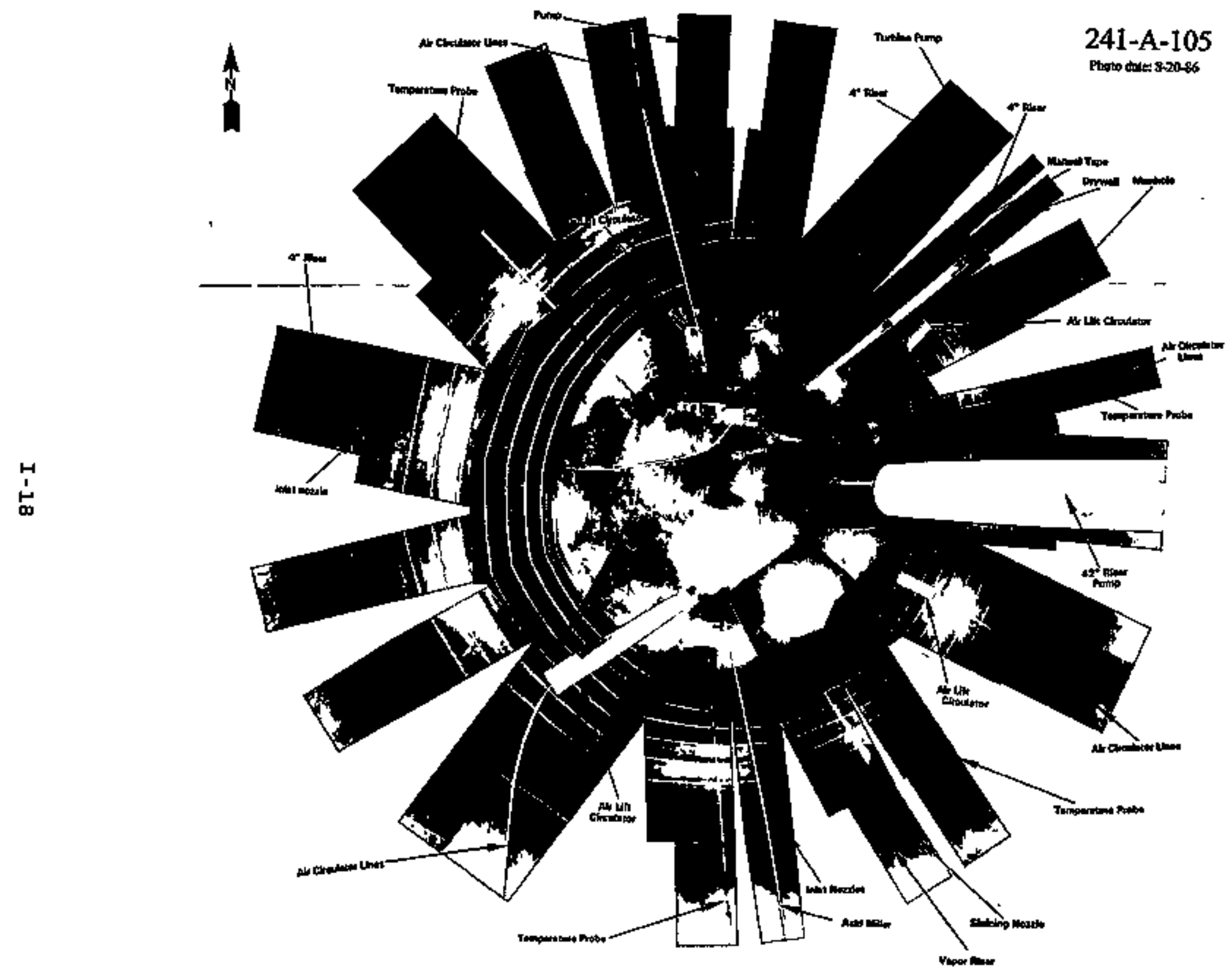

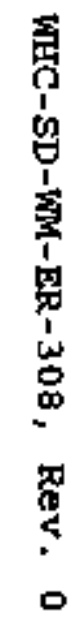




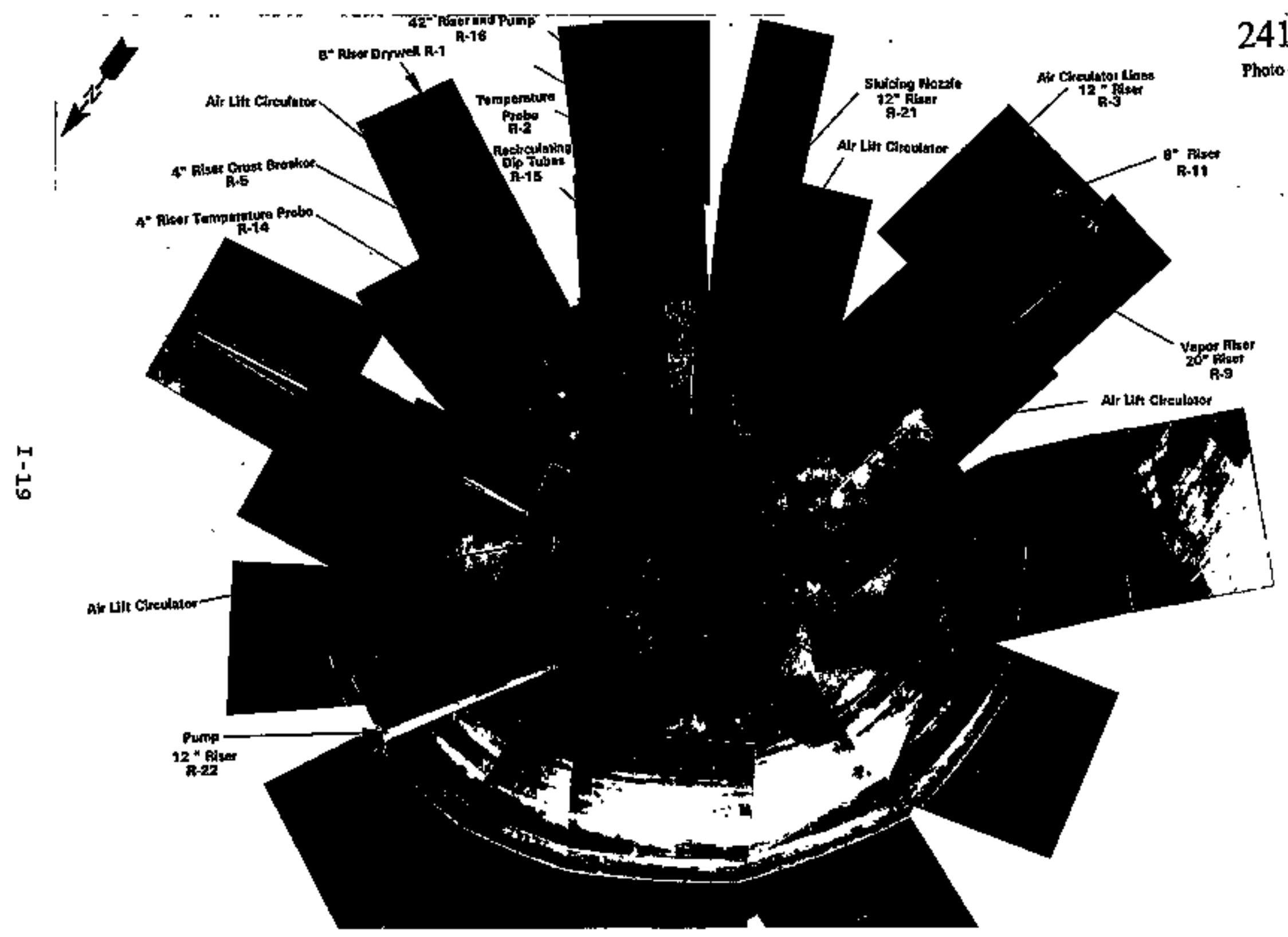


WHC-SD-WM-ER-308, ReV. 0

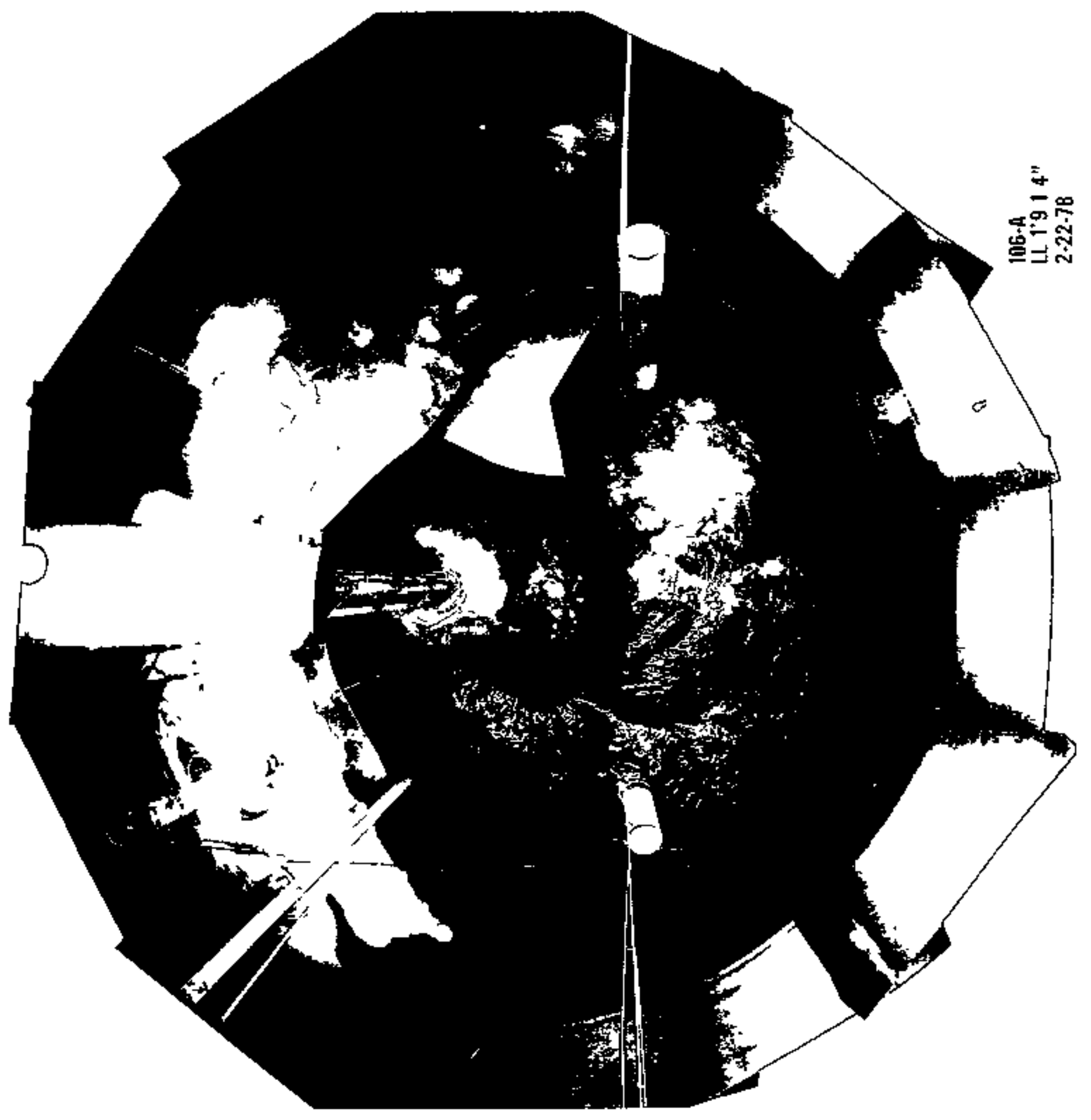




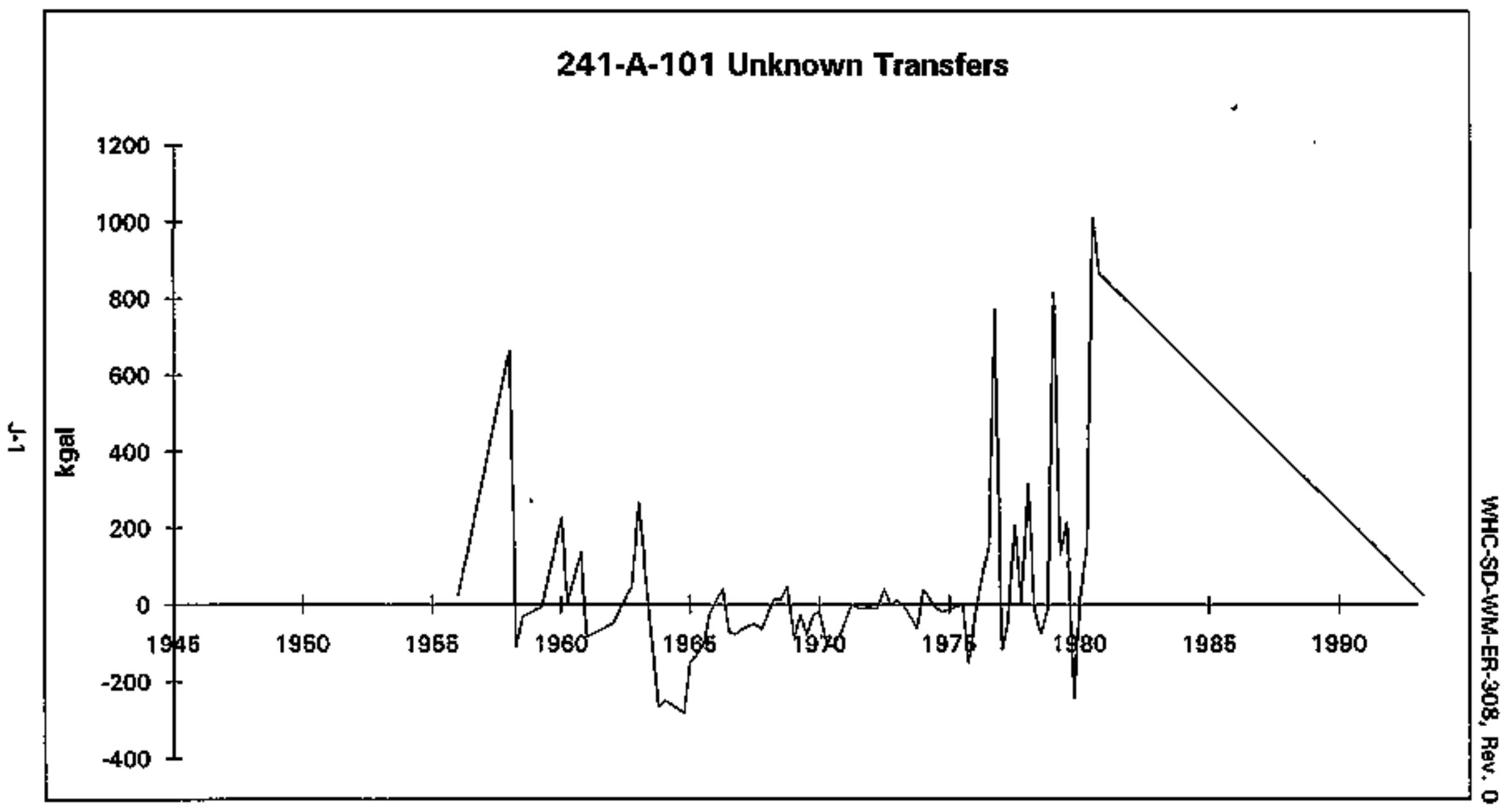

Derived from LANL Waste Status and Transaction Record Summary V2.0b (Agnew 1994) 


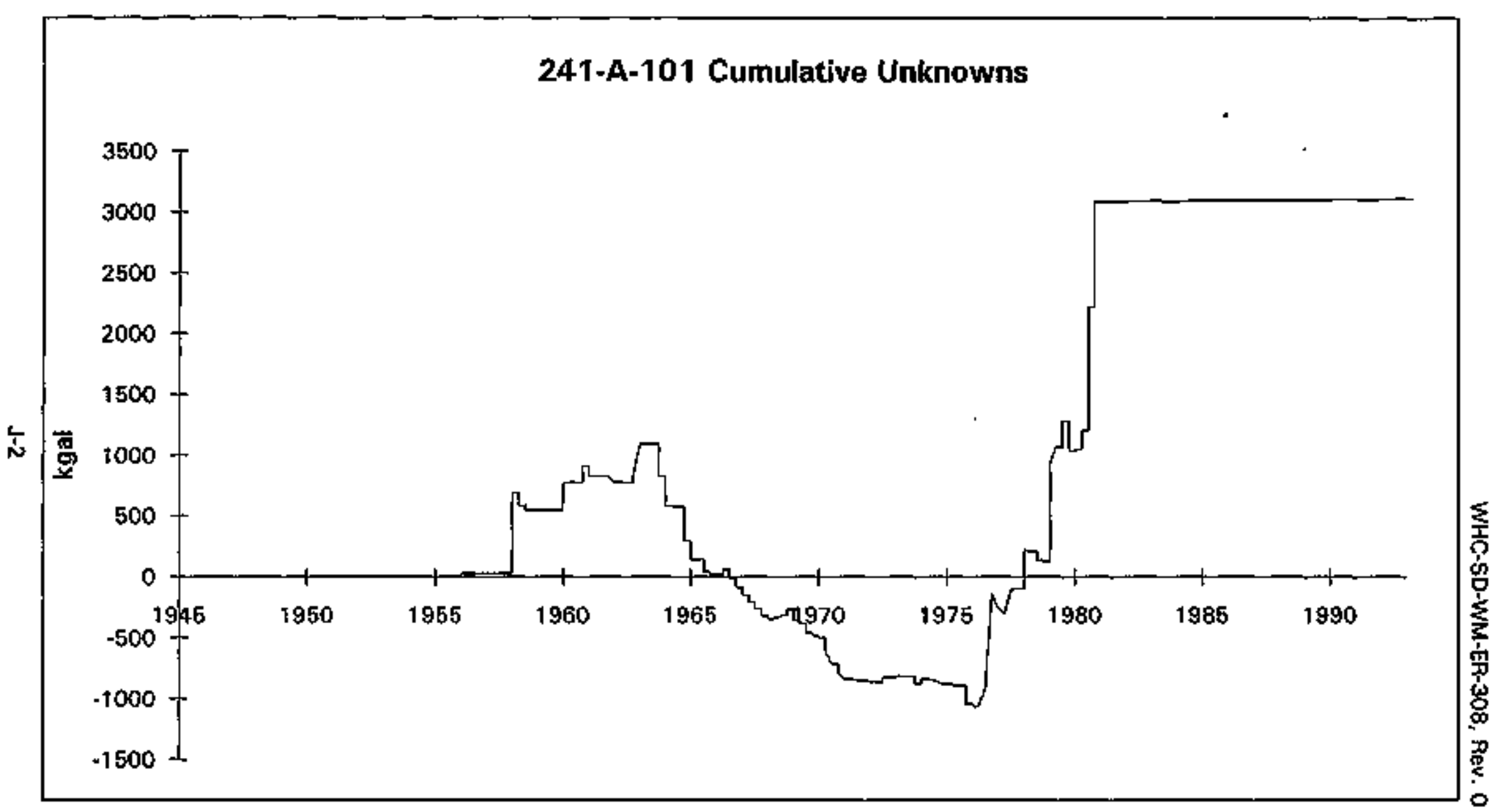

Derived from LANL Waste Status and Transaction Record Summary V2.0b (Agnew 1994) 


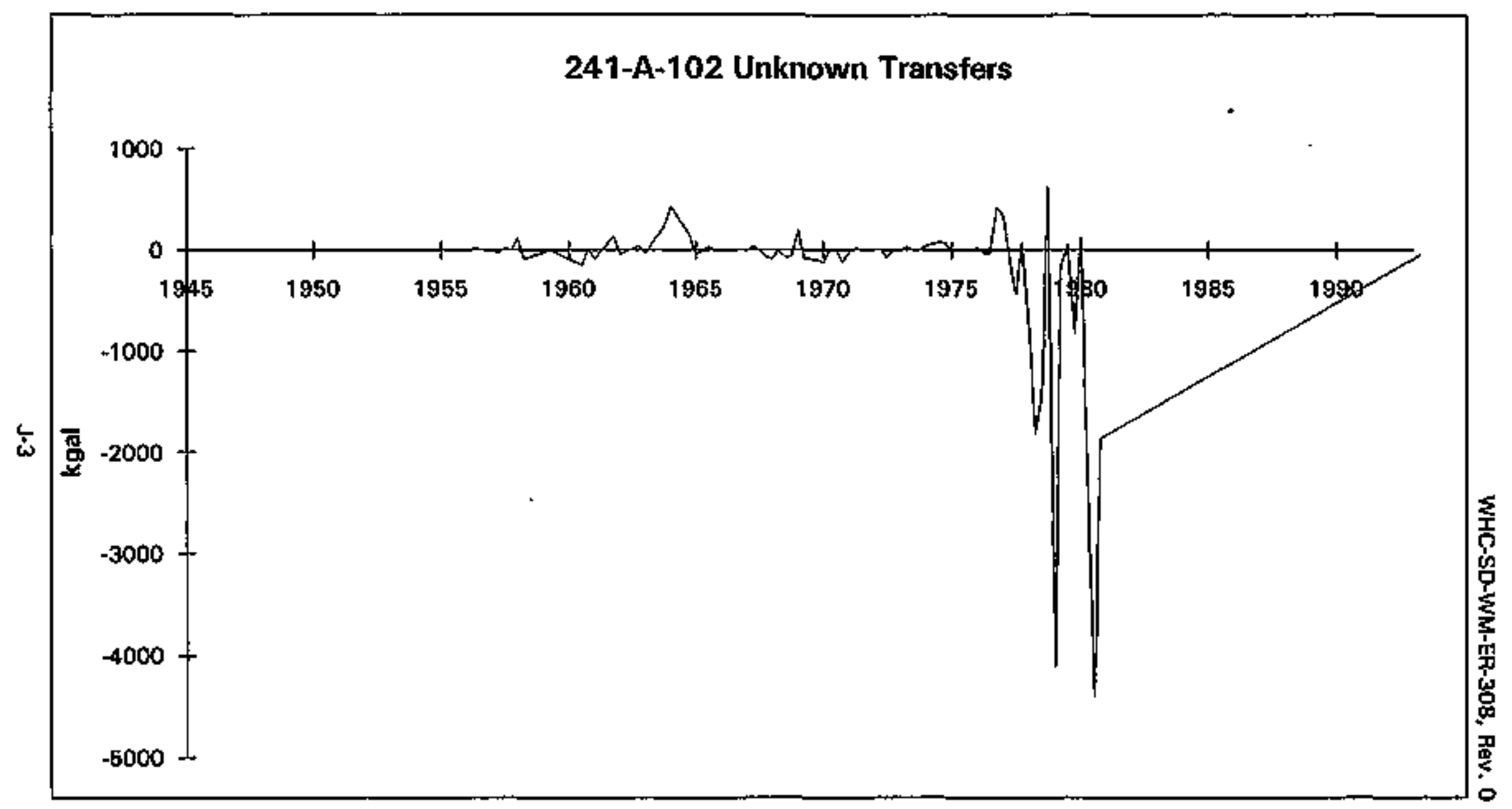

Derived from LANL Waste Status and Transaction Record Sumrnary V2.Ob (Agnew 1994) 


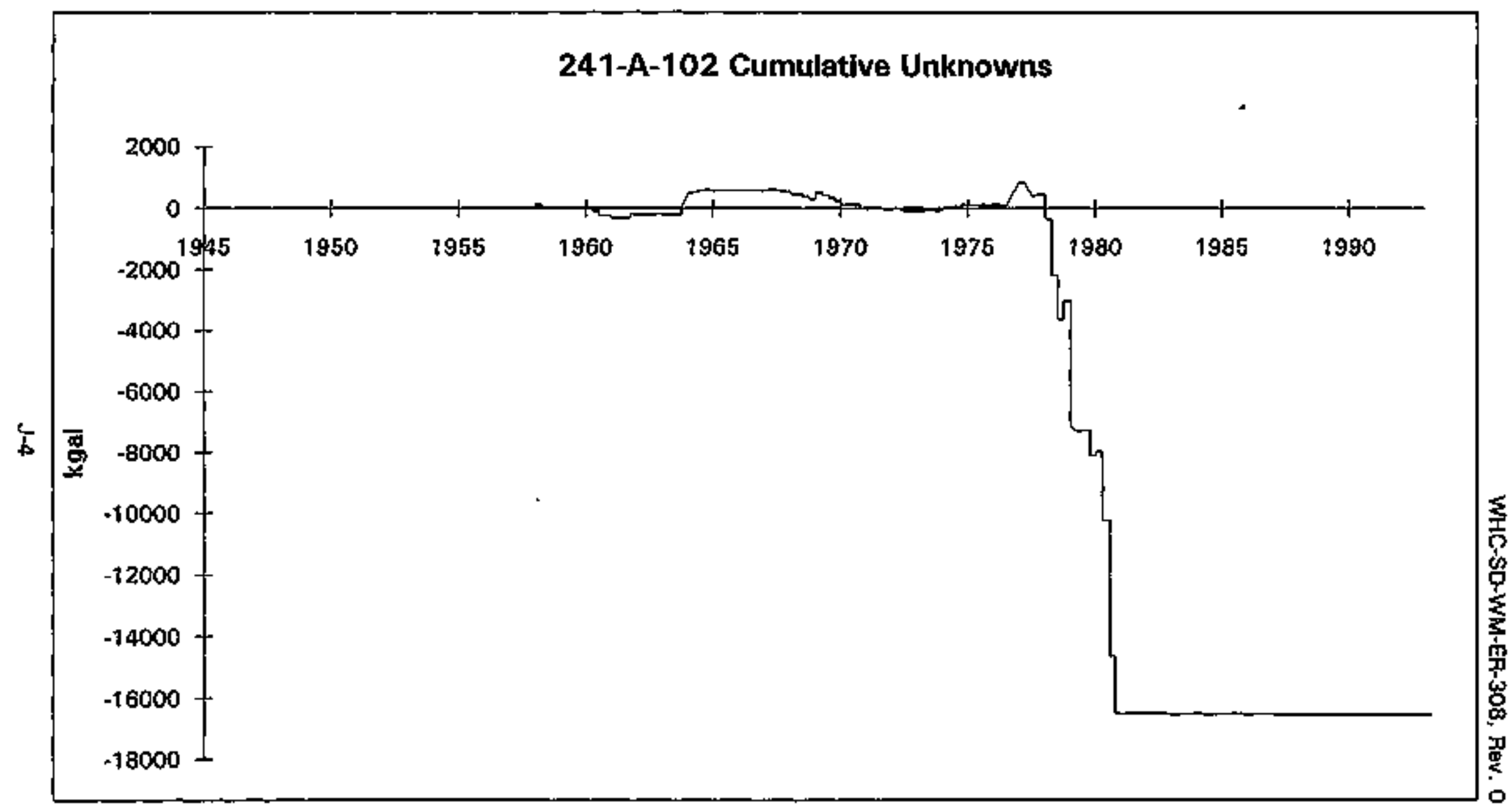

Derived from LANL Waste Status and Transaction Record Summary V2.0b (Agnew 1994) 


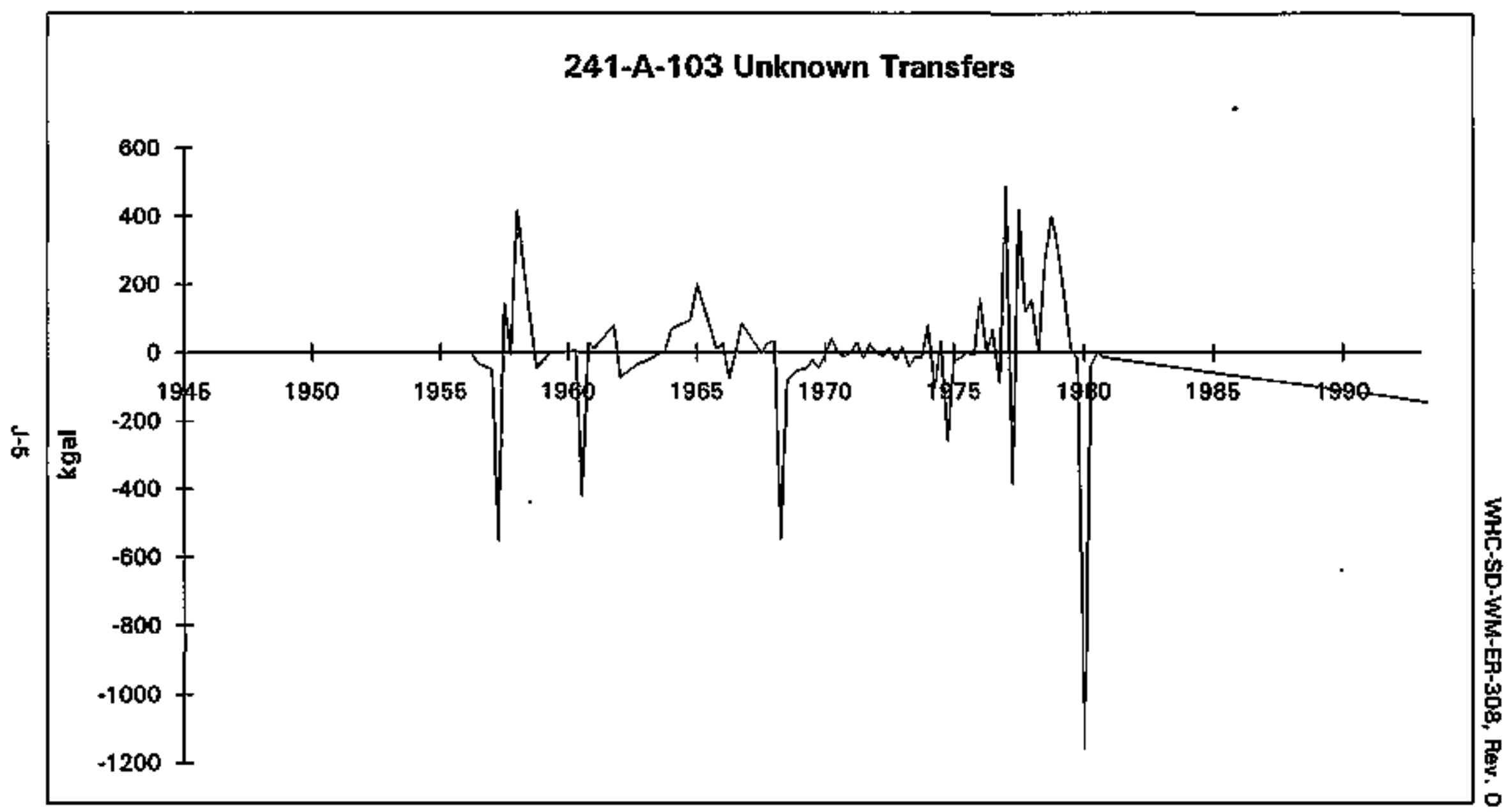




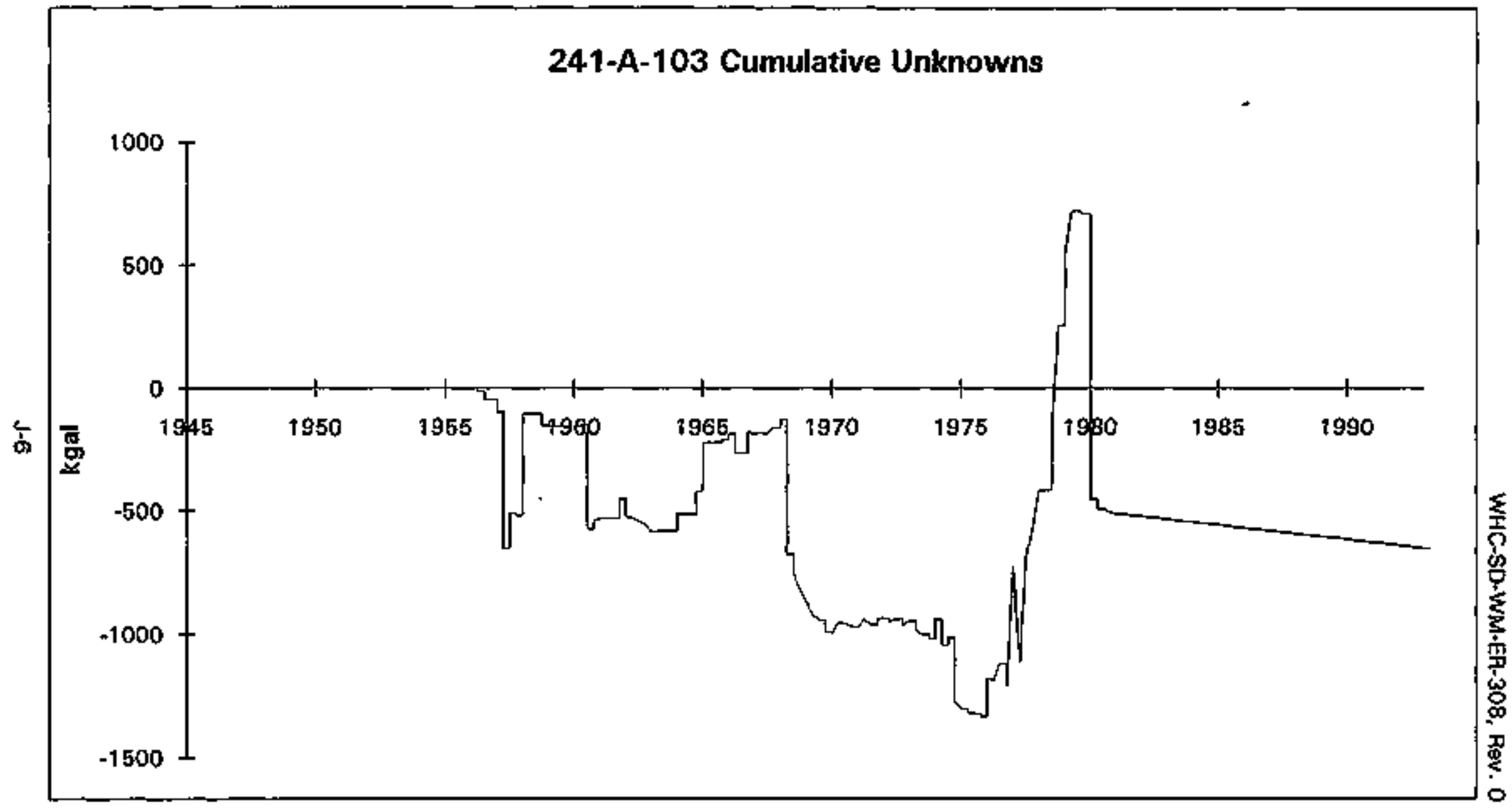

Derived from LANL Waste Status and Transaction Fecord Summary V2.0b (Agnew 1994) 


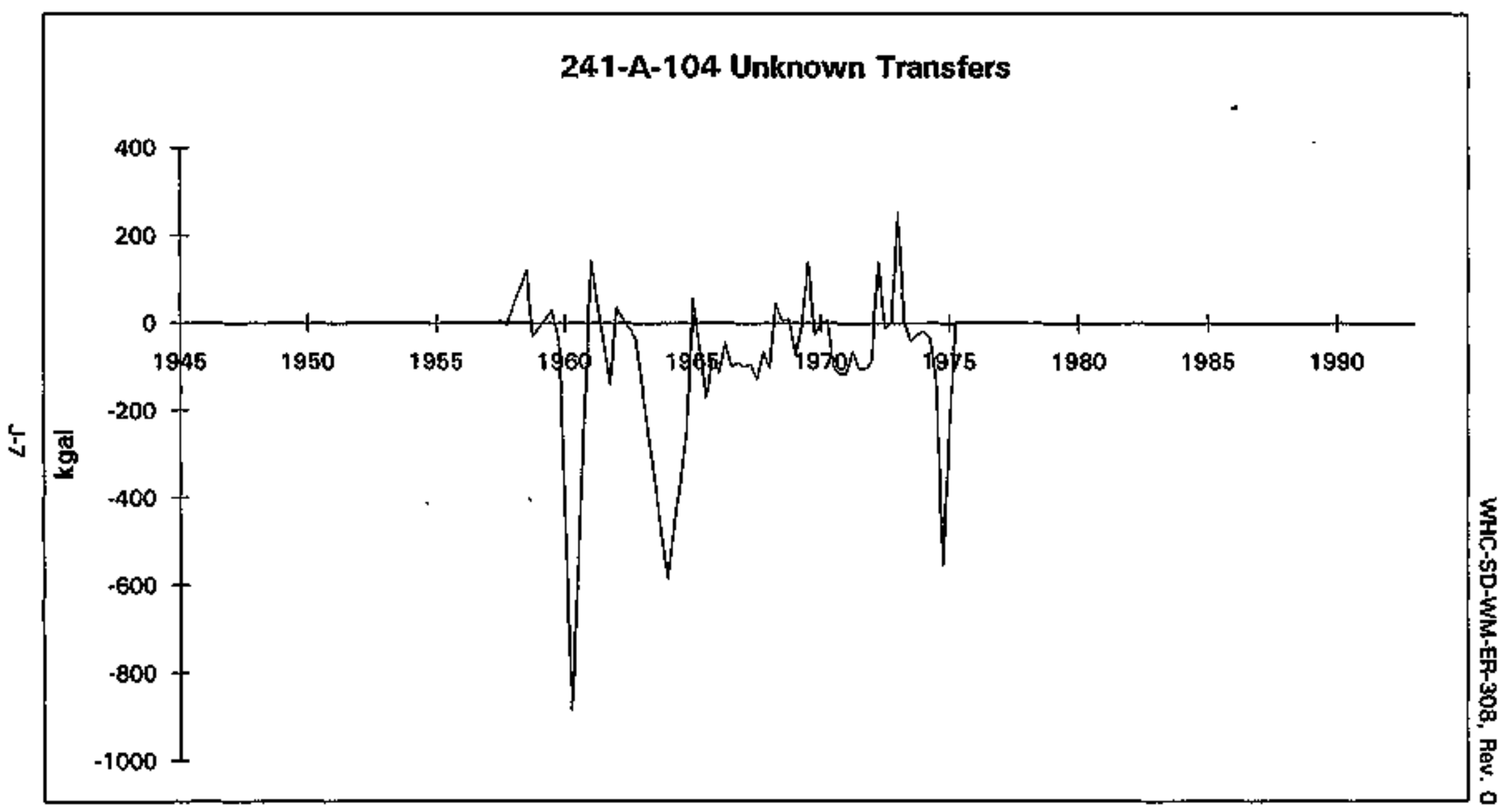

Derived from LANL Waste Status and Transaction Record Summary V2.0b AAgnew 1994| 


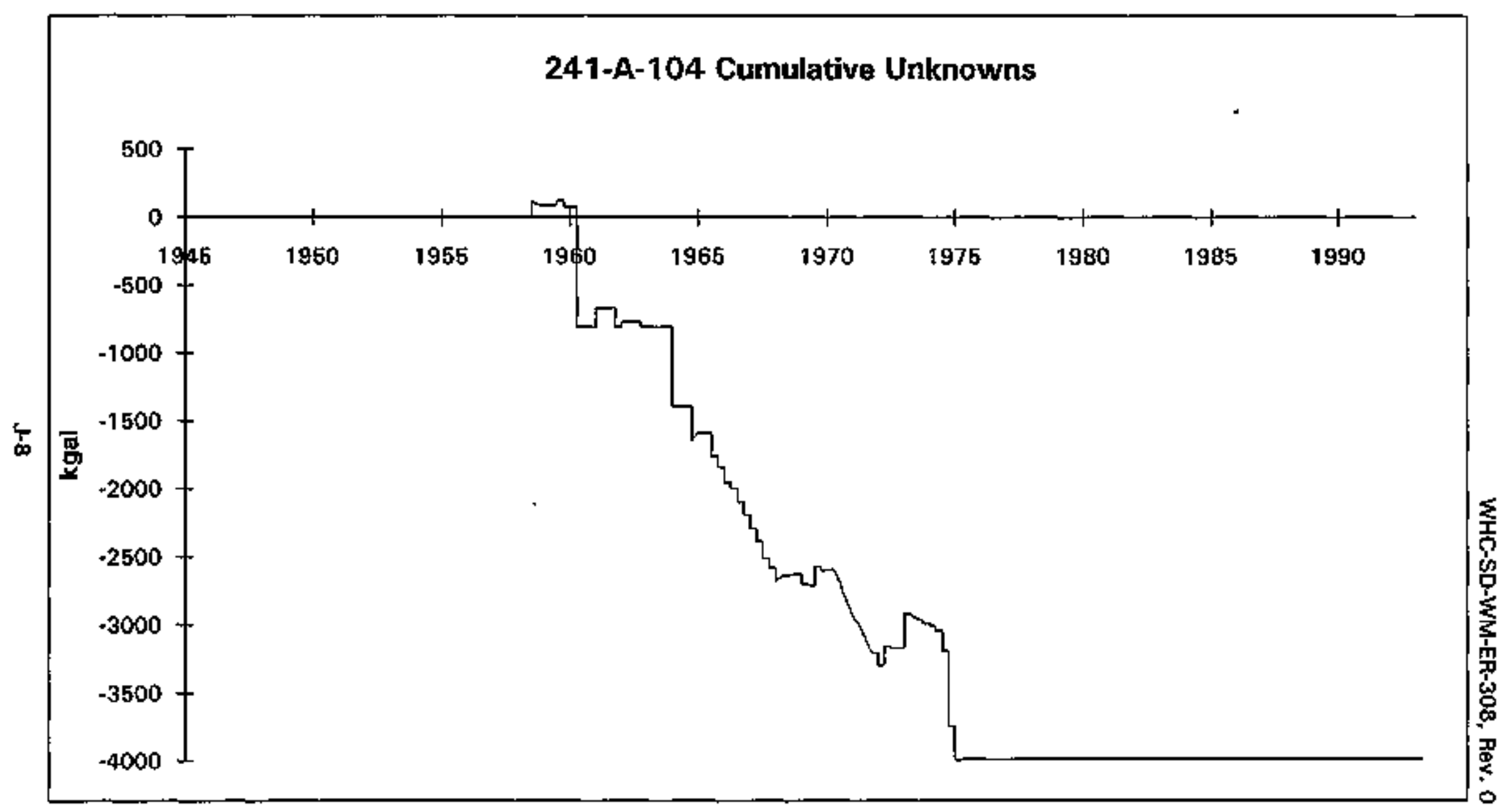

Derived from LANL Waste Status and Transaction Record Summary V2.Ob (Agnew 1994) 


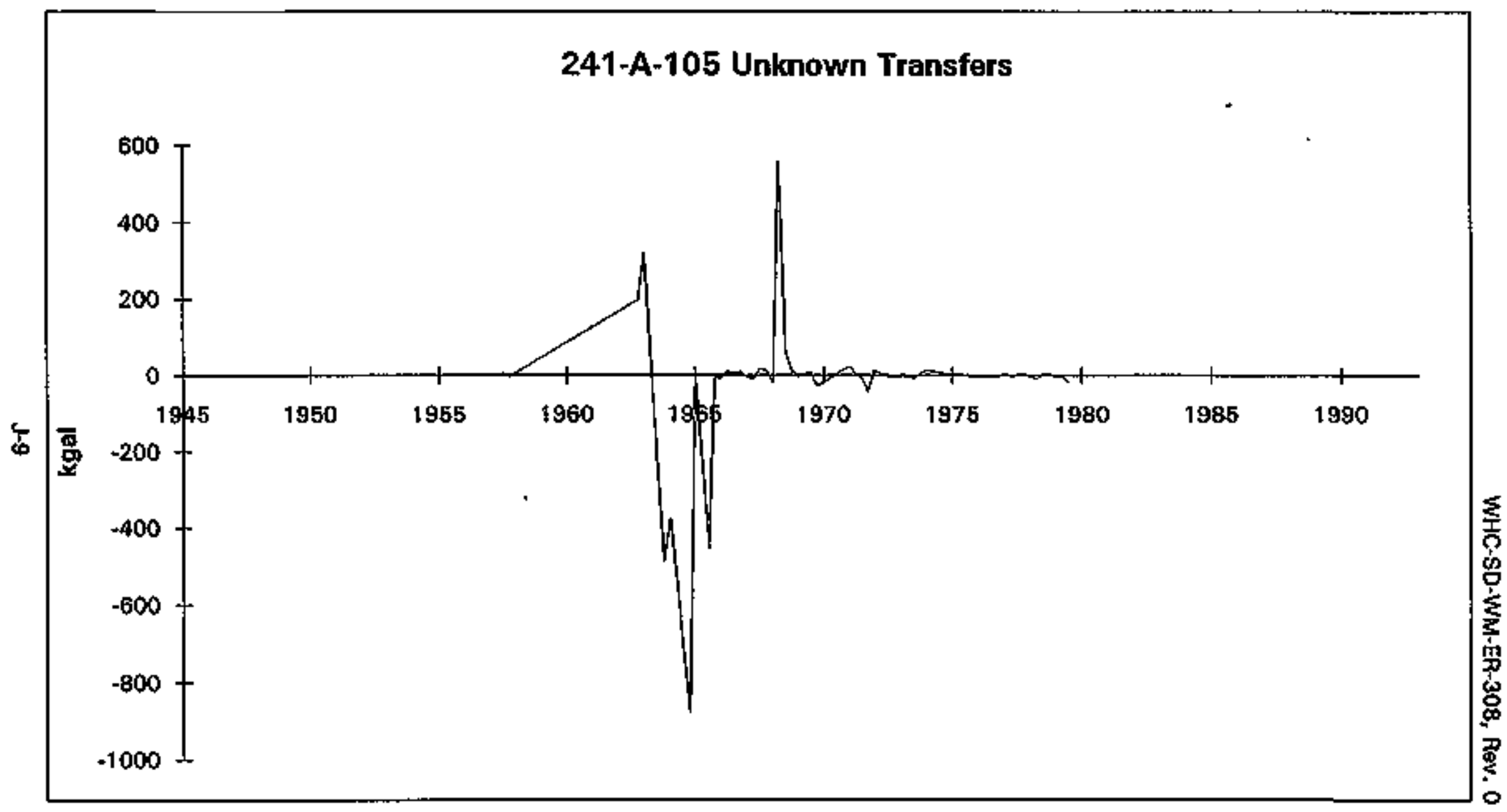

Derived from LANL Waste Status and Transaction Record Summary V2.0b (Agnew 1994) 


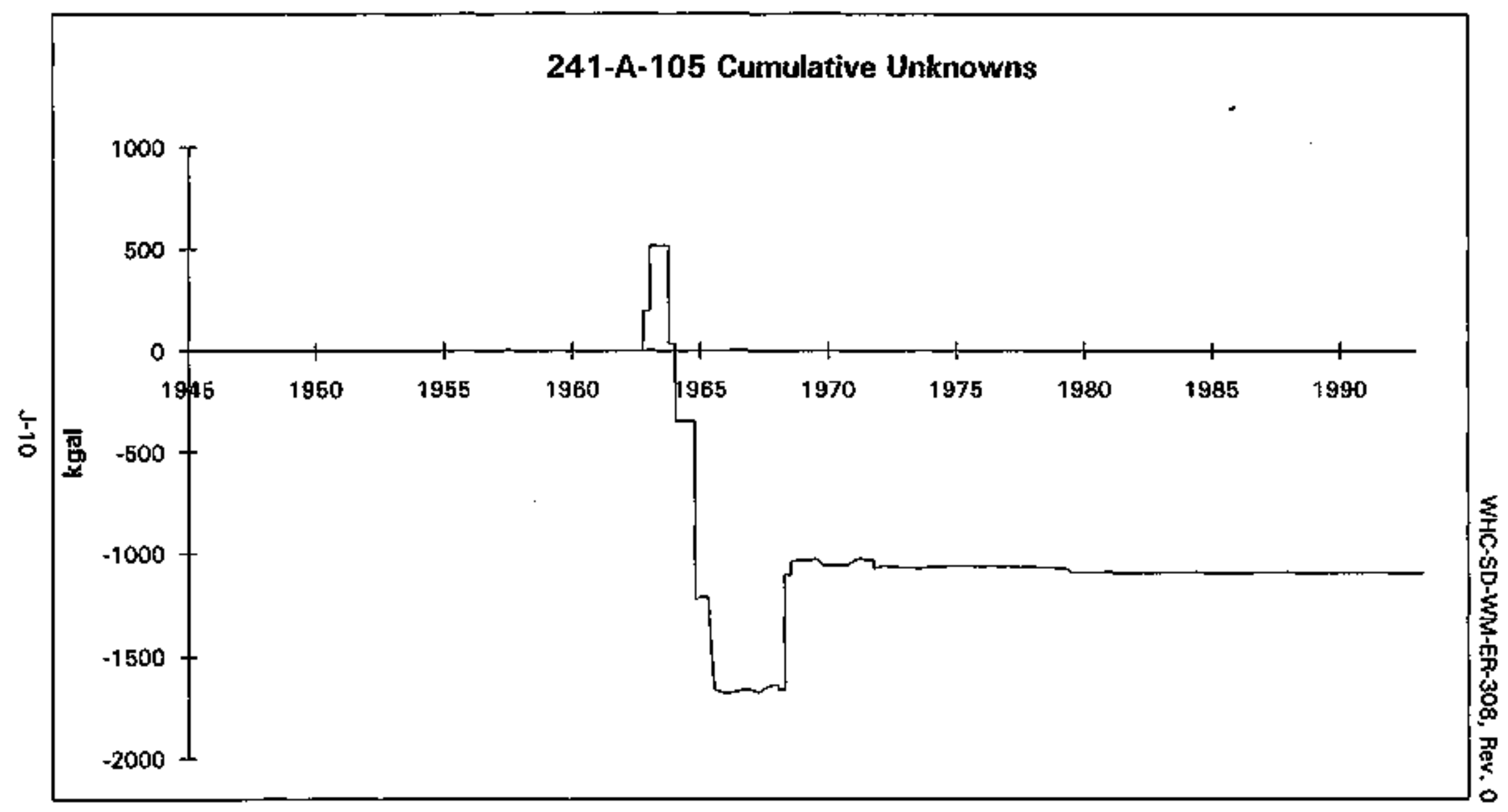

Derived from LANL Waste Status and Transaction Record Summary V2.0b (Agnew 1994) 


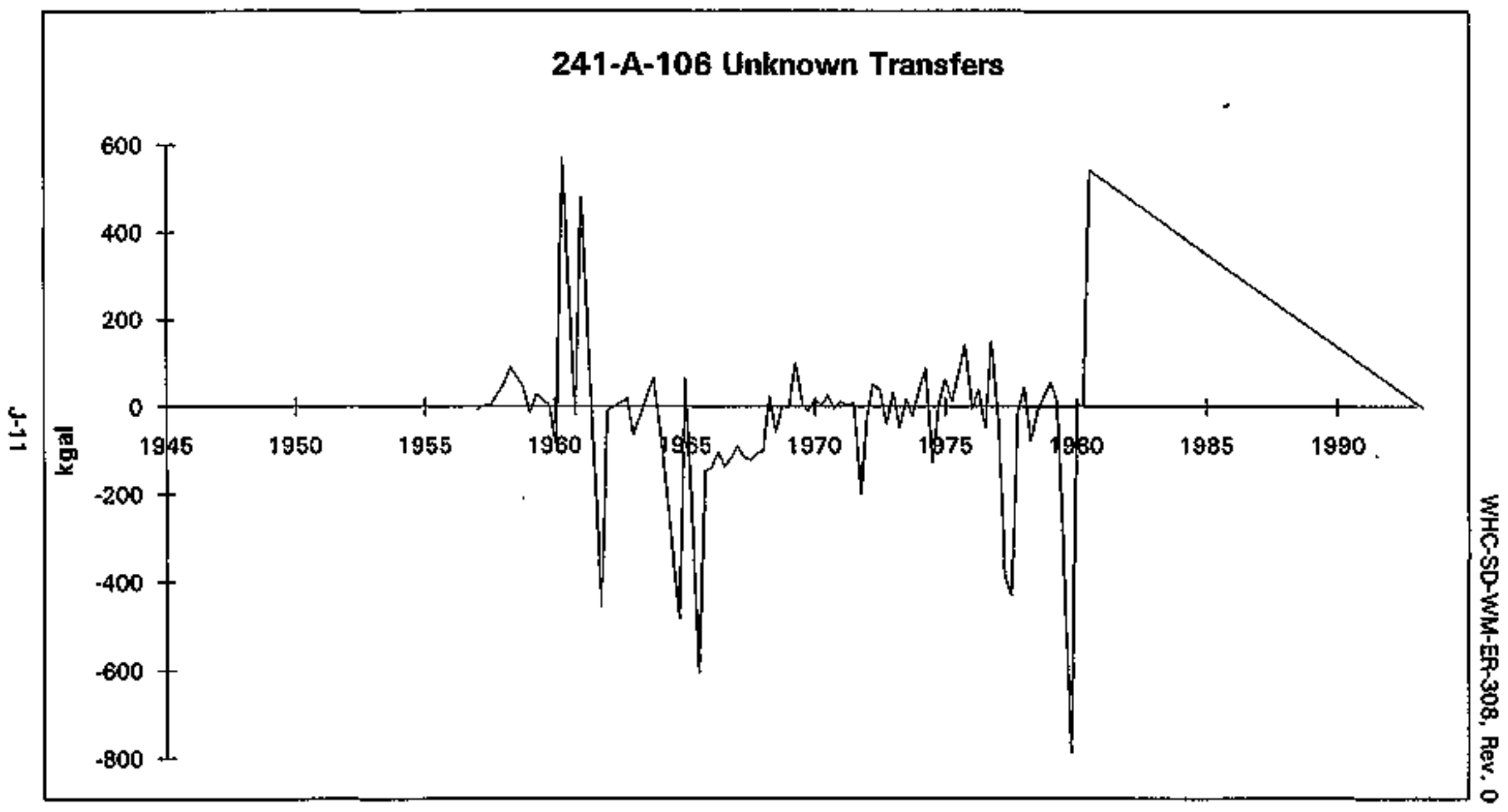

Derived from LANL Waste Status and Transaction Record Summary V2.0b (Agnew 1994) 


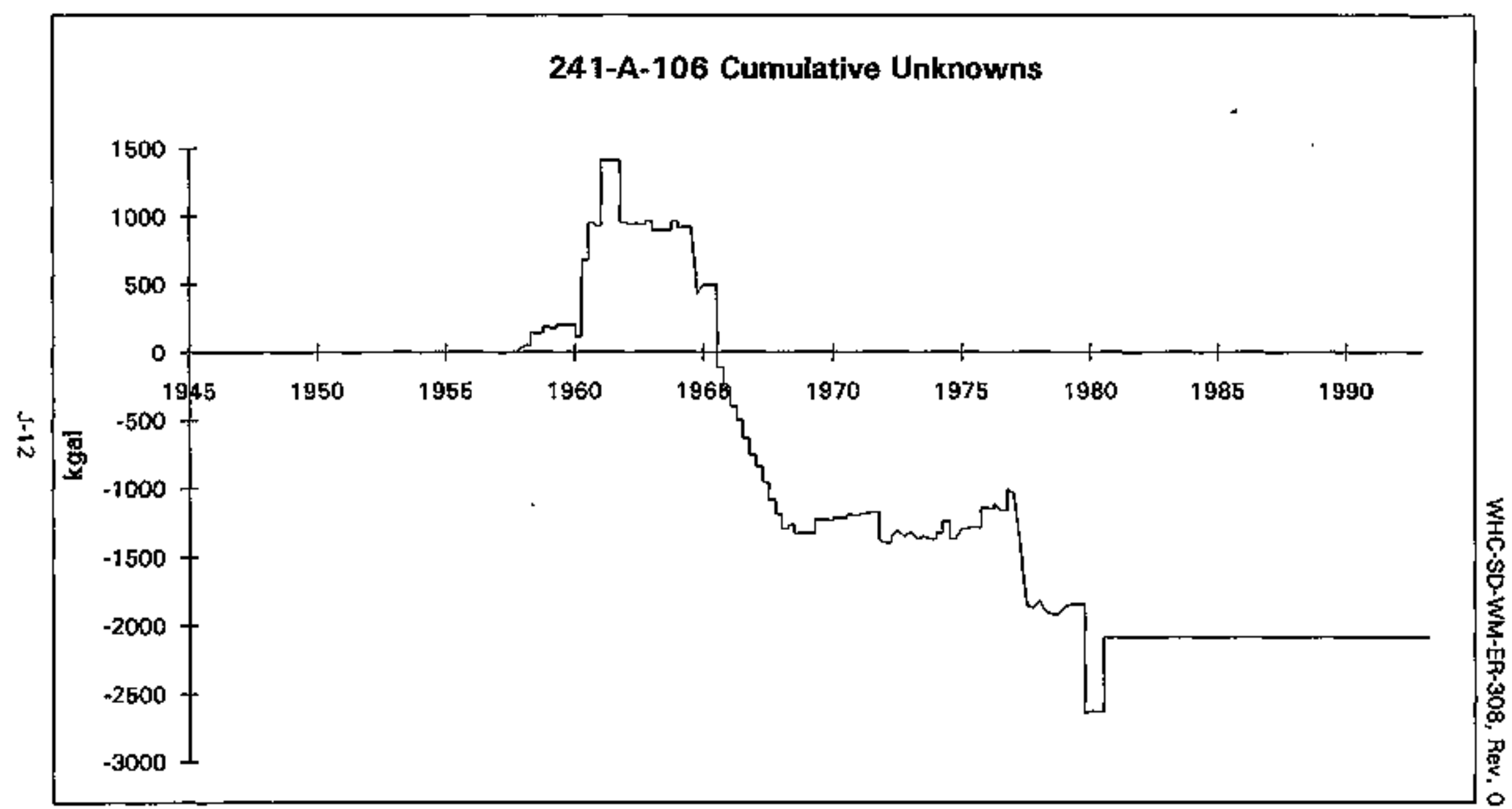




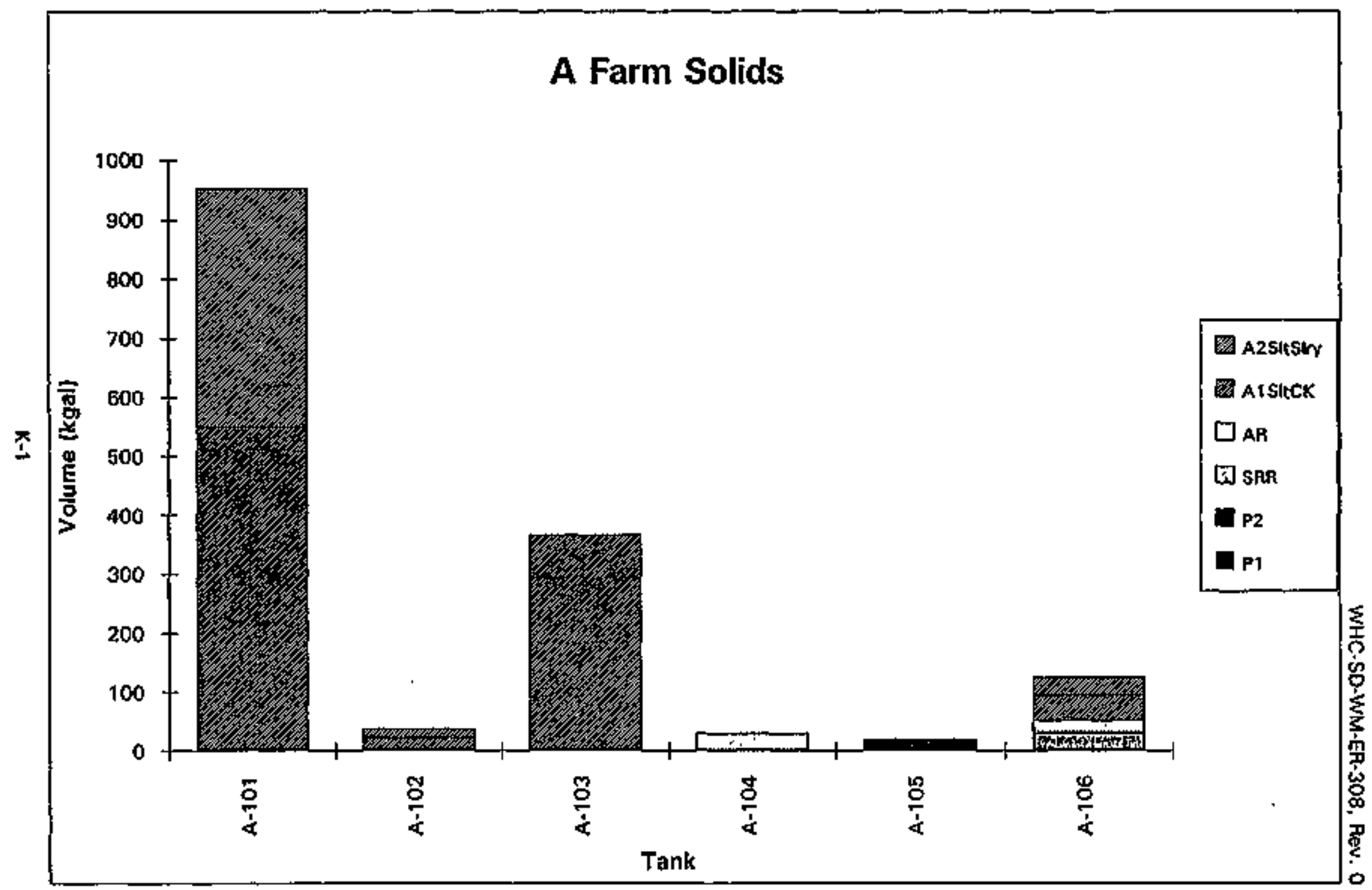

Derived from LANL Waste Stotus and Transaction Record Summary V2.0b \{Agnew 1994\} 
WHC-SD-WM-ER-308, Rev, O

\begin{tabular}{|c|c|c|c|c|c|c|c|}
\hline Tank & Year & Qtr & $\begin{array}{l}\text { Meas. } \\
\text { solidss }\end{array}$ & $\begin{array}{l}\text { Solids } \\
\text { Change }\end{array}$ & $\begin{array}{c}\text { Predicted } \\
\text { Layer }\end{array}$ & $\begin{array}{l}\text { Layer } \\
\text { Type }\end{array}$ & $\begin{array}{l}\text { Waste } \\
\text { Volume } \\
\text { (K Gal) }\end{array}$ \\
\hline$A-101$ & $19 \overline{6}\}$ & 1 & & 105 & & $P$ & 4754 \\
\hline & $19 \overline{65}$ & 1 & 72 & -33 & & & \\
\hline & 1966 & 3 & & 3 & & P2 & 70 \\
\hline & 1967 & 3 & 79 & & & & \\
\hline & 1967 & 4 & 80 & & & & \\
\hline & 1968 & 1 & 83 & 8 & & & \\
\hline & 1968 & 2 & & & & & \\
\hline & 1968 & 2 & 50 & & & & \\
\hline & 1969 & 1 & & & & & -9 \\
\hline & 1969 & 1 & & & & & .33 \\
\hline & 1969 & 1 & 8 & -75 & & & \\
\hline & 1969 & 3 & 3 & & & & \\
\hline & 1969 & 4 & 11 & & & & \\
\hline & 1970 & 4 & & 1 & & $P$ & 36 \\
\hline & $19 \overline{72}$ & 4 & 16 & & & & \\
\hline & 1973 & 1 & & 53 & & SRR & 1066 \\
\hline & 1973 & 4 & & 0 & & $P L$ & 5 \\
\hline & 1974 & 2 & 33 & & & & \\
\hline & $19 \overline{75}$ & 2 & 11 & & & - & \\
\hline & 1975 & $\overline{3}$ & & 0 & & SAR & 6 \\
\hline & 1975 & 4 & & & & & -11 \\
\hline & 1975 & 4 & 8 & & & & \\
\hline & 1976 & 1 & 1 & & & & \\
\hline & 1976 & 2 & 3 & -59 & 3 & $\mathrm{P} 1$ & \\
\hline & $19 \overline{77}$ & 3 & 85 & & & & \\
\hline & 1978 & 1 & 377 & & & & \\
\hline & 1978 & 4 & 490 & & & & \\
\hline & 1979 & 1 & 415 & & & & \\
\hline & 1979 & $\overline{4}$ & 333 & & & & \\
\hline & 1980 & 2 & 316 & & & & \\
\hline & 1980 & 4 & 550 & 547 & 547 & Asltck & \\
\hline & 1993 & 2 & 953 & 403 & 403 & Asltsslry & \\
\hline & & & & & & & \\
\hline & & & & & & & \\
\hline & & & & & & & \\
\hline & & & & & & & \\
\hline & & & & & & & \\
\hline & & & & & & & \\
\hline & & & & & & & \\
\hline
\end{tabular}


WHC-SD-WM-ER-308, ReV. O

\begin{tabular}{|c|c|c|c|c|c|c|c|}
\hline Tank & Year & Qtr & $\begin{array}{l}\text { Meas. } \\
\text { sollds }\end{array}$ & $\begin{array}{l}\text { Solfds } \\
\text { Change }\end{array}$ & $\begin{array}{c}\text { Predicted } \\
\text { Layer }\end{array}$ & $\begin{array}{l}\text { Layer } \\
\text { Type }\end{array}$ & $\begin{array}{l}\text { Waste } \\
\text { Volume } \\
\text { (K Gal) }\end{array}$ \\
\hline A-102 & 1961 & 3 & & 164 & & $\bar{P}$ & 7436 \\
\hline & 1965 & 1 & 77 & & & & \\
\hline & 1967 & 3 & 102 & -62 & & & \\
\hline & 1967 & 4 & 99 & & & & \\
\hline & 1968 & 2 & & & & $P, P 2$ & 33 \\
\hline & 1968 & 2 & 124 & 22 & & & \\
\hline & 1968 & 3 & 127 & & & & \\
\hline & 1969 & 1 & & & & $\mathrm{P}$ & 33 \\
\hline & 1969 & 1 & 146 & & & & \\
\hline & 1969 & 2 & 154 & 30 & & & \\
\hline & 1969 & 3 & $1 \overline{49}$ & & & & \\
\hline & 1970 & 1 & 138 & & & & \\
\hline & 1970 & 2 & 146 & & & & \\
\hline & 1970 & 4 & 154 & & & & \\
\hline & 1972 & 4 & & & & $\mathrm{P}$ & -85 \\
\hline & 1972 & 4 & 69 & & & & \\
\hline & 1973 & 1 & & & & $\mathbf{P}$ & -40 \\
\hline & $197 \overline{3}$ & 1 & 39 & & & & \\
\hline & 1973 & 2 & & & & $\mathbf{P}$ & -24 \\
\hline & 1973 & 2 & 15 & & & & \\
\hline & 1974 & 1 & & & & $P$ & -15 \\
\hline & 1974 & 1 & 0 & -154 & & & \\
\hline & 1974 & 4 & & 8 & & SRR & 150 \\
\hline & 1974 & 4 & 171 & 9 & & & \\
\hline & 1975 & 1 & & 3 & & AR & 78 \\
\hline & 1975 & 2 & & 12 & & SRR & 243 \\
\hline & 1975 & 2 & & 1 & & AR & 17 \\
\hline & 1976 & 1 & & & & & -16 \\
\hline & 1976 & 1 & 1 & & & & \\
\hline & 1976 & 2 & 2 & & & & \\
\hline & 1976 & 3 & 6 & & & & \\
\hline & 1976 & 4 & 3 & -30 & 3 & SRR & \\
\hline & 1977 & 4 & 6 & & & & \\
\hline & 1978 & j & 8 & & & & \\
\hline & 1978 & 2 & 17 & & & & \\
\hline & 1978 & 3 & 11. & & & & \\
\hline ' & 1978 & 4 & 17 & & & & \\
\hline & 1980 & 4 & 22 & 19 & 19 & Asttck & \\
\hline & 1993 & 2 & 37 & 15 & 15 & Asltsiry & \\
\hline
\end{tabular}

Derived from LANL Waste Status and Transaction Record Summary V2.Ob (Agnew 1994] 
WEAC-SD-WM-ER-30日, REv. O

\begin{tabular}{|c|c|c|c|c|c|c|c|}
\hline Tank & Year & Qtr & $\begin{array}{l}\text { Meas. } \\
\text { solids }\end{array}$ & $\begin{array}{c}\text { Solids } \\
\text { Change }\end{array}$ & $\begin{array}{c}\text { Predicted } \\
\text { Layer }\end{array}$ & $\begin{array}{c}\text { Layer } \\
\text { Type } \\
\end{array}$ & $\begin{array}{l}\text { Waste } \\
\text { Volume } \\
\text { (K Gal) }\end{array}$ \\
\hline \multirow{3}{*}{ A-103 } & 1960 & 3 & & 85 & & $\mathrm{P}$ & 3855 \\
\hline & 1964 & 1 & & & & $\bar{P}$ & -50 \\
\hline & 1964 & 4 & & & & $P$ & -100 \\
\hline & 1966 & 1 & 0 & -85 & & & \\
\hline & 1966 & 4 & & & & & -100 \\
\hline & 1967 & 4 & 22 & & & & \\
\hline & 1968 & 1 & 61 & & & & \\
\hline & 1968 & 2 & & & & & 98 \\
\hline & 1968 & 2 & 52 & & & & \\
\hline & 7968 & 3 & & & & & 49 \\
\hline & 1968 & 3 & 102 & 102 & & & \\
\hline & 1969 & 2 & 91 & & & & \\
\hline & 1969 & 3 & 121 & & & & \\
\hline & $1969^{\circ}$ & 4 & 102 & & & & \\
\hline & 1973 & 2 & & & & & 24 \\
\hline & 1974 & 2 & & & & & -80 \\
\hline & 1974 & 2 & 22 & & & & \\
\hline & 1974 & 3 & & & & & -22 \\
\hline & 1974 & 3 & 0 & -102 & & & \\
\hline & 1974 & 4 & 14 & 11 & & AR & 266 \\
\hline & 1975 & 2 & 6 & & & & \\
\hline & 1975 & 2 & & 1 & & AR & 29 \\
\hline & 1975 & 3 & 14 & & & & \\
\hline & 1975 & 4 & & 7 & & SRR & 141 \\
\hline & 1975 & 4 & & 5 & & AR & 118 \\
\hline & 1976 & 1 & 6 & -18 & & & \\
\hline & 1976 & 2 & 16 & & & & \\
\hline & 1976 & 3 & 17 & & & & \\
\hline & 1976 & 4 & & & & & -8 \\
\hline & 1976 & 4 & 3 & -3 & 3 & AR & \\
\hline & 1977 & 3 & 105 & & & & \\
\hline & 1978 & 1 & 206 & & & & \\
\hline & 1978 & 2 & 275 & & & & \\
\hline & 1978 & 4 & 303 & & & & \\
\hline & 1980 & 3 & 499 & & & & \\
\hline & 1980 & 4 & 516 & 513 & & & \\
\hline & 1993 & 2 & 366 & -150 & 363 & Asltck & \\
\hline & & & & & & & \\
\hline & & & & & & & \\
\hline
\end{tabular}

Derived from LANL Waste Status and Transaction Record Summary V2.Ob (Agnew 1994) 
WHC-SD-WM-ER-308, Rev. 0

\begin{tabular}{|c|c|c|c|c|c|c|c|}
\hline Tank & Year & Qtrr & $\begin{array}{l}\text { Meas. } \\
\text { solids }\end{array}$ & $\begin{array}{l}\text { Solids } \\
\text { Change }\end{array}$ & $\begin{array}{c}\text { Predicted } \\
\text { Layer }\end{array}$ & $\begin{array}{l}\text { Layer } \\
\text { Type }\end{array}$ & $\begin{array}{l}\text { Waste } \\
\text { Volume } \\
\text { (K Gal) }\end{array}$ \\
\hline \multirow[t]{2}{*}{ A-104 } & 1958 & 1 & 0 & & & & \\
\hline & 1961 & 1 & 3 & 78 & & P & 3564 \\
\hline & 1961 & 4 & & 70 & & $P$ & 3184 \\
\hline & 1965 & 1 & 146 & & & & \\
\hline & 1967 & 3 & 165 & & & & \\
\hline & 1967 & 4 & 168 & 20 & & & \\
\hline & 1968 & 1 & 182 & & & & \\
\hline & 1968 & 2 & 171 & & & & \\
\hline & 1968 & 3 & 165 & & & & \\
\hline & 1968 & 4 & 171 & & & & \\
\hline & 1969 & 2 & & & & & .97 \\
\hline & 1969 & 2 & 74 & -94 & & & \\
\hline & 1969 & 3 & & & & & .63 \\
\hline & $\longdiv { 1 9 6 9 }$ & 3 & & & & & -35 \\
\hline & 1969 & 3 & 11 & & & & \\
\hline & 1969 & 4 & 3 & & & & \\
\hline & 1970 & 1 & 1 & -73 & 1 & $P_{1}$ & \\
\hline & 1972 & 3 & 8 & 3 & & AR & 84 \\
\hline & 1973 & 1 & & 35 & & AR & 883 \\
\hline & 1973 & 1 & 80 & & & & \\
\hline & 1974 & 2 & & 51 & & AR & 1273 \\
\hline & 1974 & 3 & & & & & -23 \\
\hline & 1974 & 3. & 57 & -33 & & & \\
\hline & 1974 & 4 & & & & & -32 \\
\hline & 1974 & 4 & 39 & & & & \\
\hline & 1975 & 2 & 25 & 32 & & AR & 809 \\
\hline & 1977 & 4 & 28 & -61 & 27 & AR & \\
\hline & 1993 & 2 & 28 & & & & \\
\hline & & & & & & & \\
\hline & & & & & & & \\
\hline & & & & & & & \\
\hline & & & & & & & \\
\hline & & & & & & & \\
\hline & & & & & & & \\
\hline & & & & & & & \\
\hline & & & & & & & \\
\hline & & & & & & & \\
\hline & & & & & & & \\
\hline & & & & & & & \\
\hline
\end{tabular}

Derived from LANL Waste Status and Trantaction Record Summary V2.Ob (Agnew 1994) 
WHC-SD-WM-ER-308, Rev. 0

\begin{tabular}{|c|c|c|c|c|c|c|c|}
\hline Tank & Year & $\mathrm{Qtr}$ & $\begin{array}{l}\text { Meas. } \\
\text { solids }\end{array}$ & $\begin{array}{c}\text { Solids } \\
\text { Change }\end{array}$ & $\begin{array}{l}\text { Predicted } \\
\text { Layer }\end{array}$ & $\begin{array}{l}\text { Layer } \\
\text { Type }\end{array}$ & $\begin{array}{l}\text { Waste } \\
\text { Volume } \\
\text { (K Gal) }\end{array}$ \\
\hline \multirow[t]{2}{*}{$\mathrm{A}-105$} & 1957 & 4 & 17 & & & & \\
\hline & 1961 & 1 & 50 & & & & \\
\hline & 1964 & 4 & & $\overline{114}$ & & IWW & 2932 \\
\hline & 1966 & 1 & 72 & & & & \\
\hline & 1967 & 3 & $1 \overline{10}$ & -4 & & & \\
\hline & 1968 & 1 & 180 & & & & \\
\hline & 1968 & 2 & & & & & -98 \\
\hline & 1968 & 2 & 82 & & & & \\
\hline & 1968 & 3 & & & & & -60 \\
\hline & 1968 & 3 & & & & & -49 \\
\hline & 1968 & 3 & 33 & -77 & & & \\
\hline & 1969 & 2 & 52 & & & & \\
\hline & 1969 & 3 & 63 & & & & \\
\hline & 1969 & 4 & 33 & & & & \\
\hline & 1979 & $\overline{2}$ & 19 & & & & \\
\hline & 1993 & 2 & 19 & -14 & 19 & P2 & \\
\hline & & & & & & & \\
\hline & & & & & & & \\
\hline & & & & & & & \\
\hline & & & & & & & \\
\hline & & & & & & & \\
\hline & & & & & & & \\
\hline & & & & & & & \\
\hline & & & & & & & \\
\hline & & & & & & & \\
\hline & & & & & & & \\
\hline & & & & & & & \\
\hline & & & & & & & \\
\hline & & & & & & & \\
\hline & & & & & & & \\
\hline & & & & & & & \\
\hline & & & & & & & \\
\hline & & & & & & & \\
\hline & & & & & & & \\
\hline & & & & & & & \\
\hline & & & & & & & \\
\hline & & & & & & & \\
\hline & & & & & & & \\
\hline & & & & & & & \\
\hline
\end{tabular}

Derlved from LANL Waste Status and Transaction Record Summary V2.0b (Agnew 1934) 
WHC-SD-WM-ER-308, REV, 0

\begin{tabular}{|c|c|c|c|c|c|c|c|}
\hline Tank & Year & Qtr & $\begin{array}{l}\text { Meas. } \\
\text { solids }\end{array}$ & $\begin{array}{c}\text { Sollds } \\
\text { Change }\end{array}$ & $\begin{array}{l}\text { Predicted } \\
\text { Layer }\end{array}$ & $\begin{array}{l}\text { Layer } \\
\text { Type }\end{array}$ & $\begin{array}{l}\text { Waste } \\
\text { Volume } \\
\text { (K Gal) }\end{array}$ \\
\hline$A-106$ & 1962 & 4 & & 78 & & $\mathrm{P} 1$ & 3550 \\
\hline & 1963 & 1 & & 6 & & P2 & 152 \\
\hline & 1965 & 1 & 118 & 34 & & & \\
\hline & 1967 & 3 & 140 & & & & \\
\hline & 1967 & 4 & 176 & & & & \\
\hline & 1968 & 1 & 173 & & & & \\
\hline & 1968 & 2 & 171 & & & & \\
\hline & 1968 & 3 & 168 & & & & 60 \\
\hline & 1968 & 4 & 176 & & & & \\
\hline & 1969 & $\overline{1}$ & 175 & & & & 2 \\
\hline & 1969 & 2 & 227 & & & & 19 \\
\hline & 1969 & $\overline{3}$ & 254 & & & & $\overline{48}$ \\
\hline & 1969 & 4 & 234 & 116 & & & \\
\hline & 1970 & $\overline{1}$ & 195 & & & & -28 \\
\hline & 1970 & $\overline{2}$ & 185 & & & & -10 \\
\hline & 1970 & 3 & 163 & & & & -22 \\
\hline & 1970 & 4 & 124 & & & & .39 \\
\hline & 1971 & 1 & 98 & & & & -26 \\
\hline & 1972 & 1 & 96 & & & & \\
\hline & 1972 & $\overline{2}$ & 19 & & & & -77 \\
\hline & 1972 & 3 & 22 & & & & \\
\hline & 1972 & 4 & 11 & & & & \\
\hline & 1973 & 4 & 6 & & & & \\
\hline$\cdot$ & 1974 & 1 & 0 & -234 & & & \\
\hline & 1974 & 1 & & 26 & & SRR & 525 \\
\hline & 1974 & 2 & & 0 & & $\mathrm{AR}$ & 7 \\
\hline & 1974 & 3 & 12 & \begin{tabular}{|c|}
-14 \\
\end{tabular} & $\mid 12$ & SRR & \\
\hline & 1974 & 3 & & 23 & & AR & 23 \\
\hline & 1974 & 3 & & 20 & & SRR & 407 \\
\hline & 1974 & 4 & & 32 & & AR & 32 \\
\hline & 1974 & 4 & 11 & & & & \\
\hline & 1975 & 3 & & 3 & & SRR & 53 \\
\hline & 1975 & 4 & & 11 & & SRR & $\overline{11}$ \\
\hline & 1976 & 1 & & 16 & & & 16 \\
\hline & $\overline{1976}$ & $\overline{1}$ & 91 & -26 & & & \\
\hline & 1976 & $\overline{4}$ & & 8 & & & 8 \\
\hline & 1976 & 4 & 102 & 3 & & & \\
\hline & 1977 & 3 & 80 & -22 & & & \\
\hline & 1977 & 4 & 52 & -28 & 17 & SRR & \\
\hline
\end{tabular}

Derived from LANL Waste Status and Trensaction Record Summary V2.Ob (Agnew 1994) 
WHC-SO-WM-ER-308, ReV. O

\begin{tabular}{|c|r|r|r|r|r|r|r|}
\hline Tank & Year & atr & $\begin{array}{c}\text { Meas. } \\
\text { solids }\end{array}$ & $\begin{array}{c}\text { Solids } \\
\text { Change }\end{array}$ & $\begin{array}{c}\text { Predicted } \\
\text { Layer }\end{array}$ & $\begin{array}{c}\text { Waste } \\
\text { Layer } \\
\text { Type }\end{array}$ & $\begin{array}{c}\text { Volume } \\
\text { (K Gal) }\end{array}$ \\
\hline & 1978 & 2 & 50 & -2 & 21 & AR & \\
\hline & 1980 & 2 & 96 & & & & \\
\hline & 1980 & 3 & 94 & 44 & 44 & A1sltck & \\
\hline & 1993 & 2 & 125 & 31 & $31 \mid$ A2sltsiry & \\
\hline
\end{tabular}

